\title{
Automated Energy Distribution and Reliability System Status Report
}

Subcontract Report NREL/SR-581-42265

October 2007

D.L. Buche

Northern Indiana Public Service Co.

Merrillville, Indiana

S. Perry

SSP Innovations, LLC

Parker, Colorado

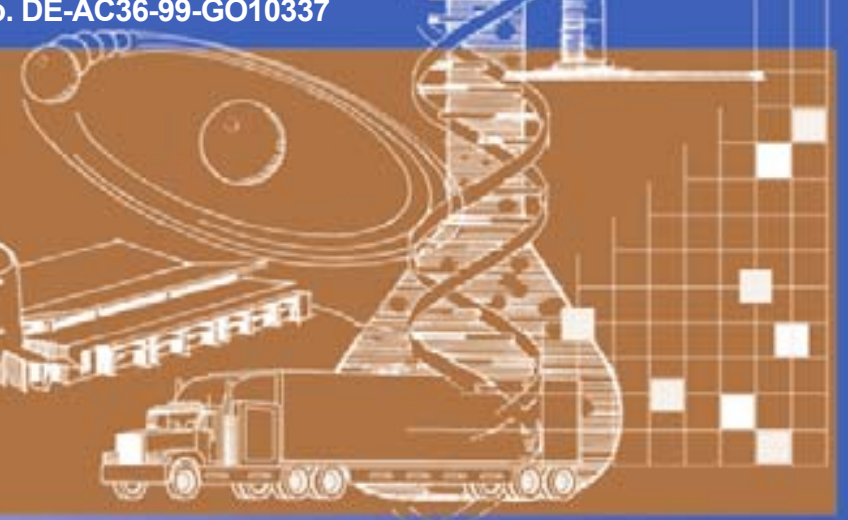




\section{Automated Energy Distribution and Reliability System Status Report}

D.L. Buche

Northern Indiana Public Service Co.

Merrillville, Indiana

S. Perry

SSP Innovations, LLC

Parker, Colorado

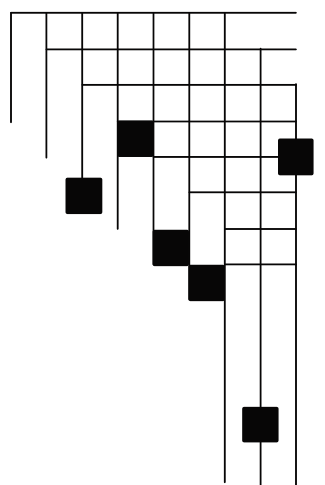

NREL Technical Monitor: Thomas Basso

Prepared under Subcontract No. RAX-4-33652-01 
This publication was reproduced from the best available copy Submitted by the subcontractor and received no editorial review at NREL

\section{NOTICE}

This report was prepared as an account of work sponsored by an agency of the United States government. Neither the United States government nor any agency thereof, nor any of their employees, makes any warranty, express or implied, or assumes any legal liability or responsibility for the accuracy, completeness, or usefulness of any information, apparatus, product, or process disclosed, or represents that its use would not infringe privately owned rights. Reference herein to any specific commercial product, process, or service by trade name, trademark, manufacturer, or otherwise does not necessarily constitute or imply its endorsement, recommendation, or favoring by the United States government or any agency thereof. The views and opinions of authors expressed herein do not necessarily state or reflect those of the United States government or any agency thereof.

Available electronically at http://www.osti.gov/bridge

Available for a processing fee to U.S. Department of Energy and its contractors, in paper, from:

U.S. Department of Energy

Office of Scientific and Technical Information

P.O. Box 62

Oak Ridge, TN 37831-0062

phone: 865.576 .8401

fax: 865.576 .5728

email: mailto:reports@adonis.osti.gov

Available for sale to the public, in paper, from:

U.S. Department of Commerce

National Technical Information Service

5285 Port Royal Road

Springfield, VA 22161

phone: 800.553 .6847

fax: 703.605.6900

email: orders@ntis.fedworld.gov

online ordering: http://www.ntis.gov/ordering.htm 


\section{Preface}

In 1998, the Department of Energy (DOE) established the Distributed Power Program to address systems integration issues and market barriers that may prohibit the widespread deployment of distributed power technologies. Initial efforts under the program involved creating national technical interconnection standards, establishing research and development programs to address system integration technologies documenting regulatory and institutional market barriers, and working with industry and state and federal policymakers to remove barriers. At that time, the National Renewable Energy Laboratory (NREL) led these research activities for the DOE. Under this subcontract, Northern Indiana Public Service Company (NIPSCO), a NiSource, Inc. Company, has developed a modernized Automated Energy Distribution and Reliability System (AEDR) based on geographical information system (GIS) technology. This integrated geographical database serves to enhance energy supply reliability and security by improving the integrity and accessibility of location data, while fostering public safety through sharing of utility location information with authorized government entities and other organizations. This modernization of the gas \& electric infrastructure helps to assure safe, reliable, and affordable service to homes and businesses.

This report details the efforts undertaken to implement the AEDR at NIPSCO and provides the interim status of the subcontract work. A future final status report will complete the documentation for this subcontract. While specific to NIPSCO's requirements, there is sufficient information to provide individuals in the initial stages of their own GIS project with a conceptual strategy, answers to some common questions and enable the re-use of some of the materials provided.

NIPSCO wishes to express sincerest gratitude for the funding assistance provided by the Department of Energy and the technical support received by the National Renewable Energy Laboratory. This project would not have been possible without their support. 
Table of Contents

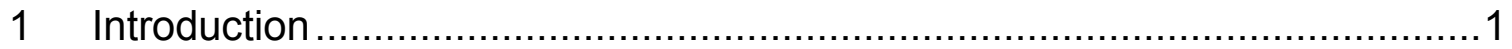

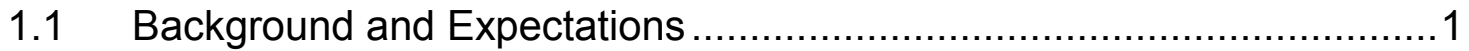

1.2 AEDR Project Structure and Status Summary .................................

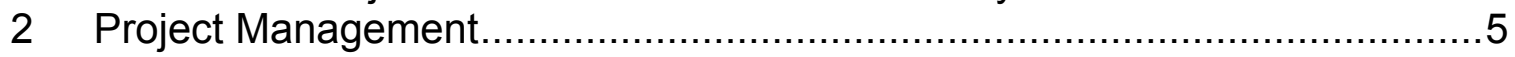

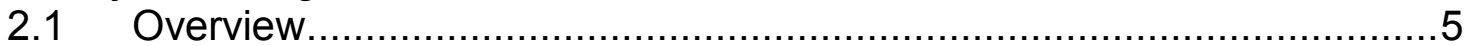

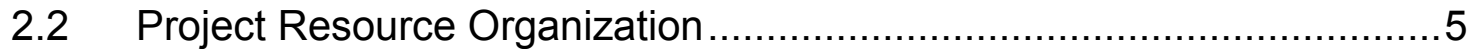

2.2.1 Project Resource Responsibilities ..........................................

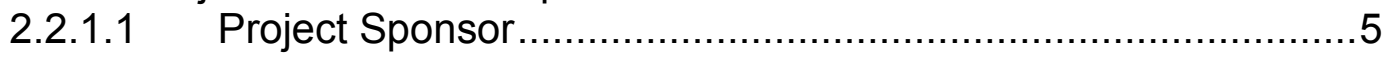

2.2.1.2 Project Manager ...........................................................

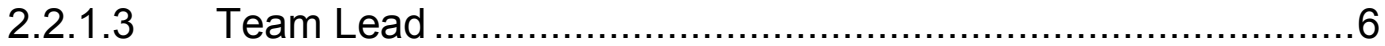

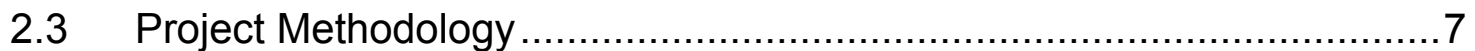

2.3.1 Project Activities and Schedule Development .............................

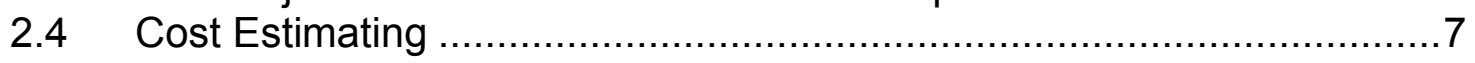

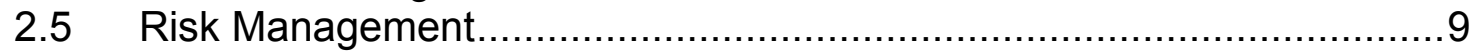

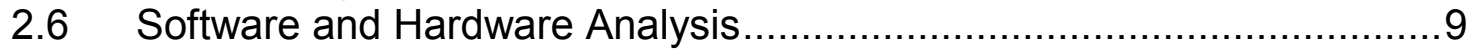

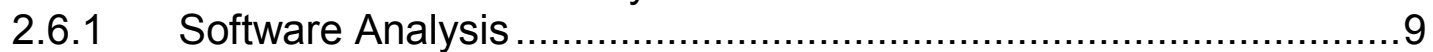

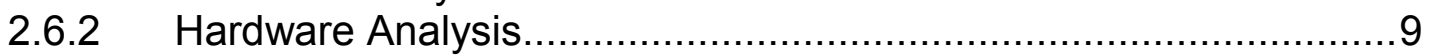

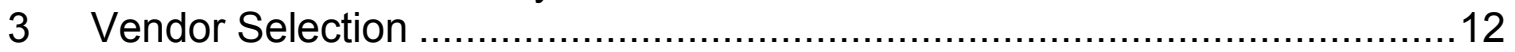

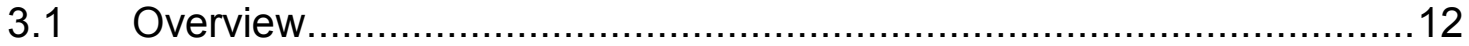

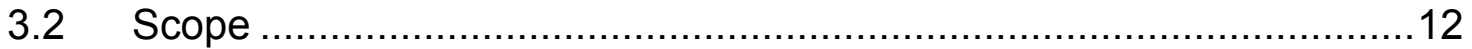

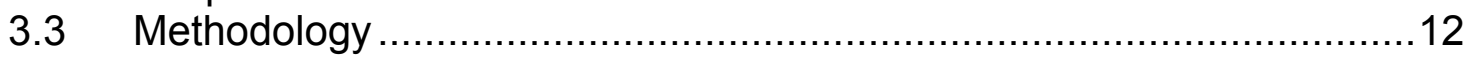

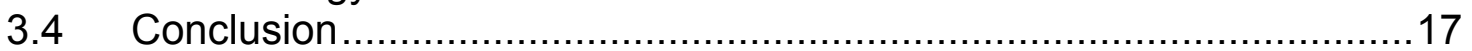

4 Immediate Conceptual Architecture (Phase I) ......................................... 19

4.1 Detailed Functional Analysis.................................................... 19

4.1.1 Objectives of Functional Analysis ...........................................19

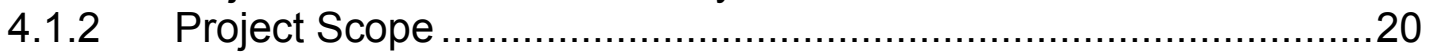

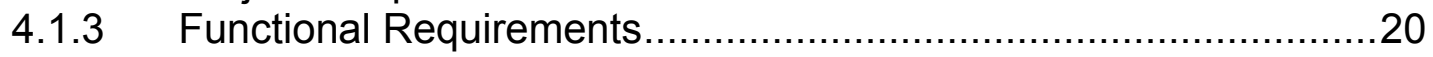

4.1.3.1 Functional Requirements Summary ................................21

4.1.4 System / Software Requirements .....................................22

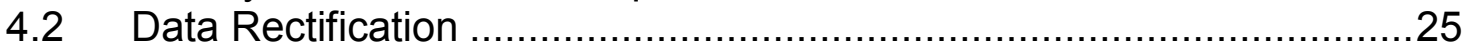

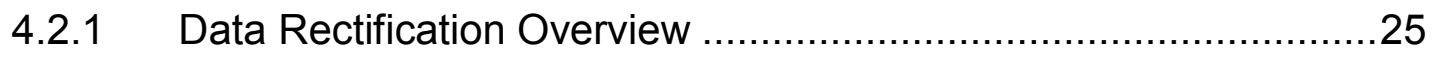

4.2.2 Imagery and Coordinate System ..........................................25

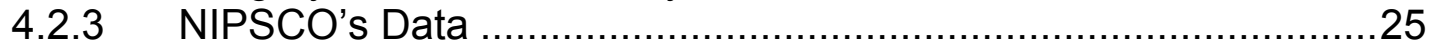

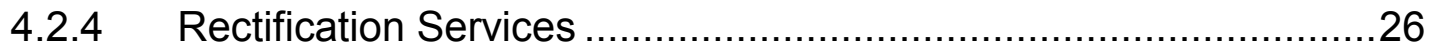

4.2.4.1 Landbase Data Requirements.......................................26

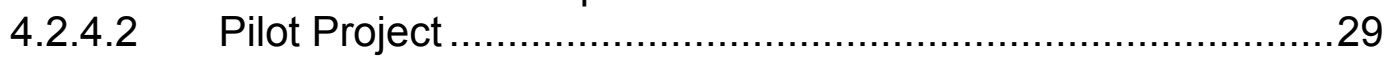

4.2.4.3 Data Rectification Process .................................................. 30

4.2.5 Data Rectification Conclusion..................................................

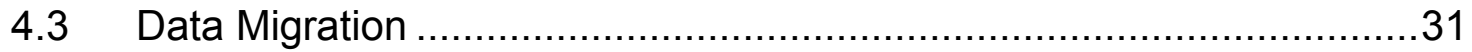

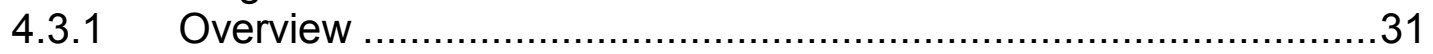

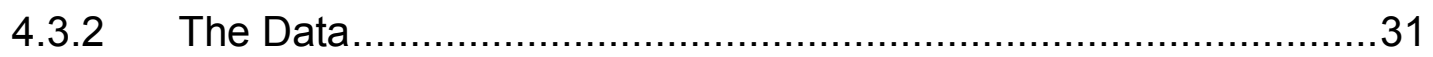

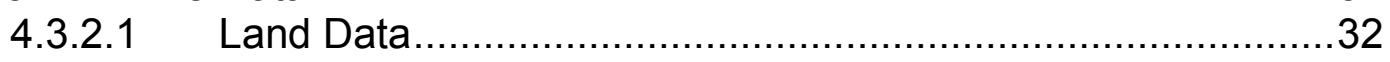

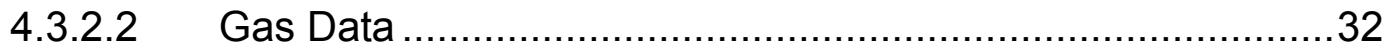

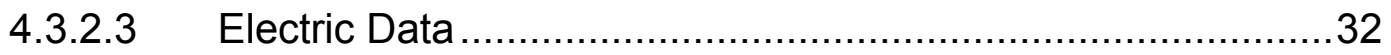




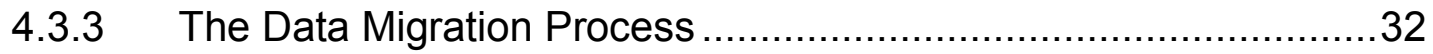

4.3.3.1 The Iterative Approach to Data Migration ...............................33

4.3.3.2 Acceptance Criteria ........................................................ 34

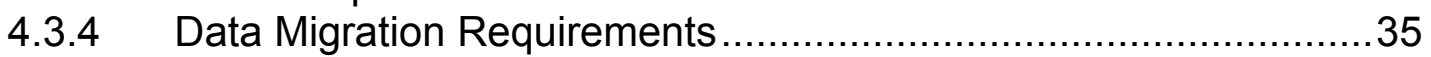

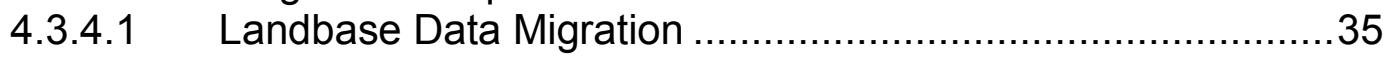

4.3.4.2 Gas Facilities Data Migration .......................................... 35

4.3.4.3 Electric Facilities Data Migration .......................................36

4.3.4.4 AutoCAD-EDFS Match Keys and Mismatch Reporting ...........36

4.3.5 Data Migration Plan ............................................................... 37

4.3.5.1 QA (Quality Assurance) Process .......................................... 37

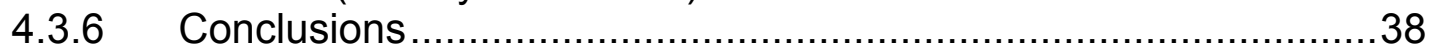

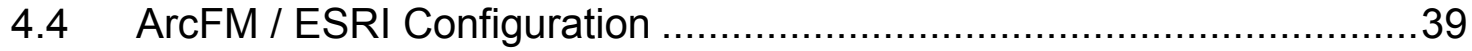

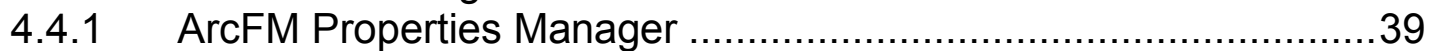

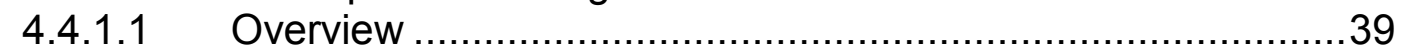

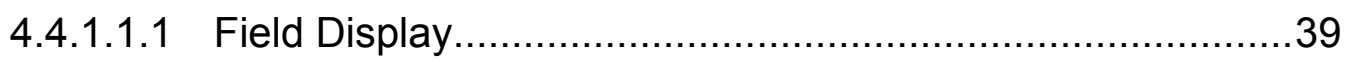

4.4.1.1.2 Object Info..................................................... 41

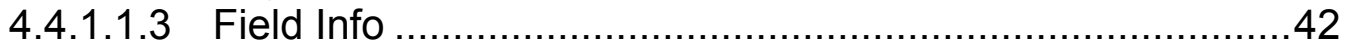

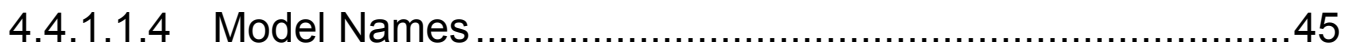

4.4.1.1.5 Field Model Names .....................................................46

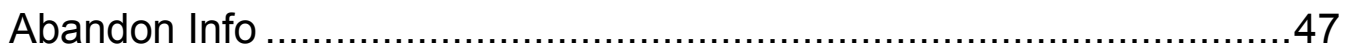

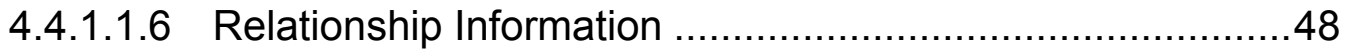

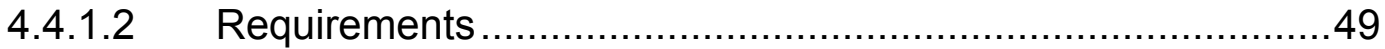

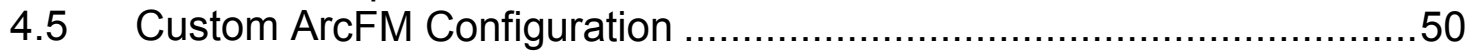

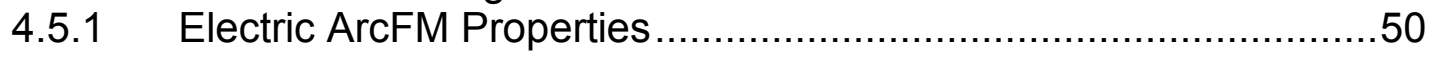

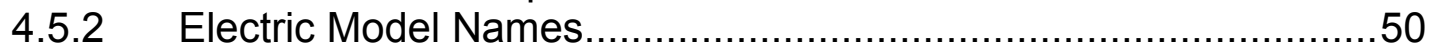

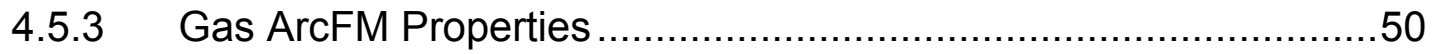

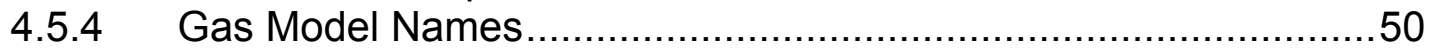

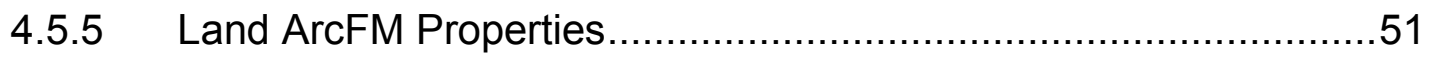

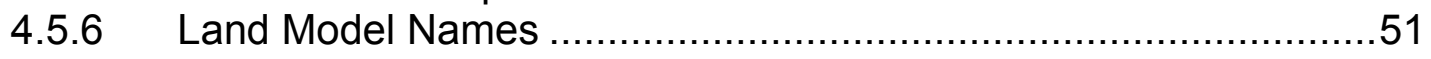

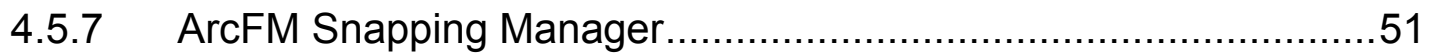

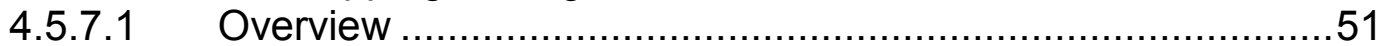

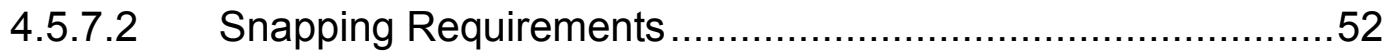

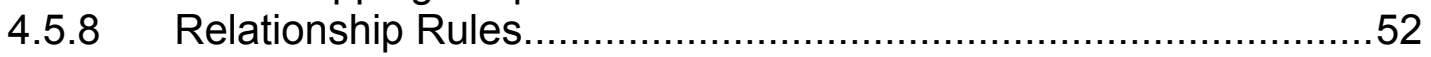

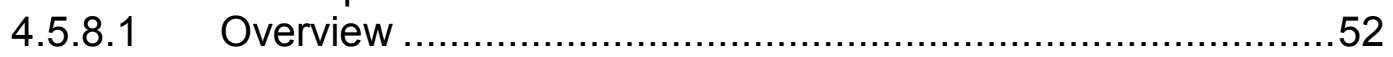

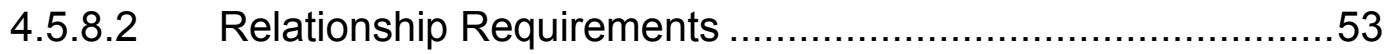

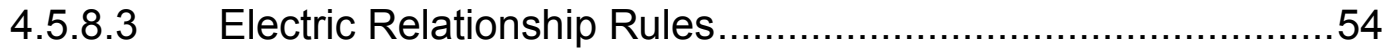

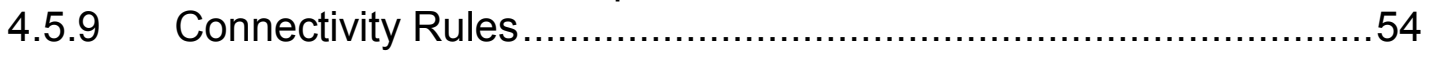

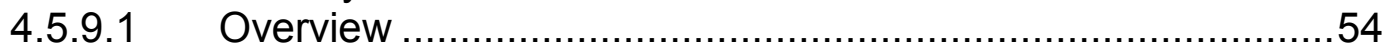

4.5.9.2 Connectivity Requirements ..........................................55

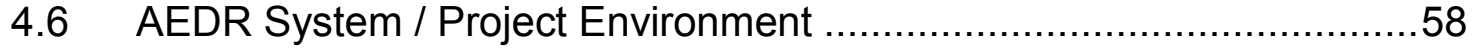

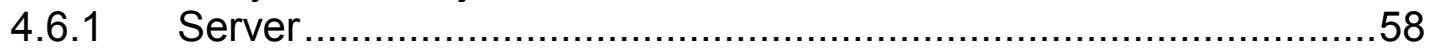

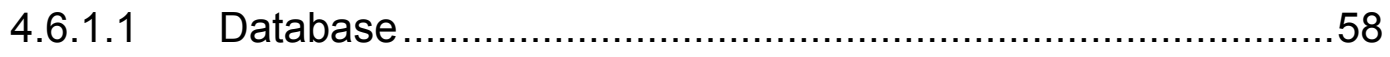

4.6.1.1.1 Target Database Environment .......................................58

4.6.1.1.2 Database Permissions .................................................59

4.6.1.1.2.1 Database Privilege Strategy ..................................59

4.6.1.1.2.2 User Accounts and Roles .......................................60 
4.6.1.1.2.3 Standard User Permissions ......................................60

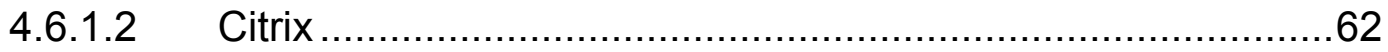

4.6.1.2.1 Citrix Application Components ........................................63

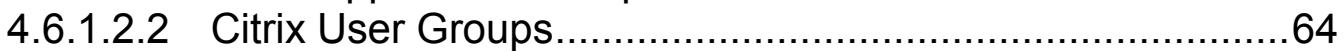

4.6.1.3 Management of the Server Environment............................65

4.6.1.3.1 ArcSDE Environment Descriptions...................................65

4.6.1.3.1.1 Delta ArcSDE Environment .....................................65

4.6.1.3.1.2 Migration ArcSDE Environment ................................66

4.6.1.3.1.3 Development ArcSDE Environment............................66

4.6.1.3.1.4 Test ArcSDE Environment (Test Server) ....................67

4.6.1.3.1.5 Test ArcSDE Environment (Production Server)............67

4.6.1.3.1.6 Production ArcSDE Environment...............................67

4.6.1.3.2 Citrix Server Environments............................................68

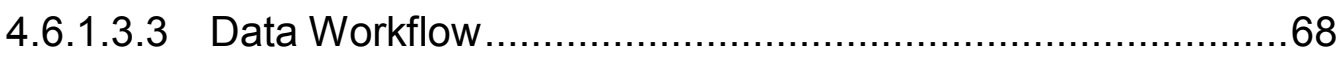

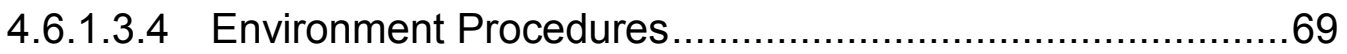

4.6.1.3.4.1 Environment Status Log .......................................69

4.6.1.3.4.2 Creating a Clean Model - Delta Environment...............69

4.6.1.3.4.3 Configuration Steps - Migration Environment .............69

4.6.1.3.4.4 Configuration Steps - Development Environment ........69

4.6.1.3.4.5 Configuration Steps - Test (aedrhamgis01)

Environment ..................................................... 70

4.6.1.3.4.6 Configuration Steps - Test (aedrdbsqlp01) .................70

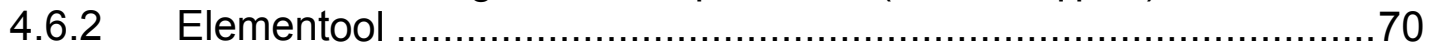

4.6.2.1 Discovering / Researching an Issue ...................................71

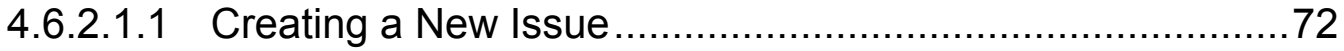

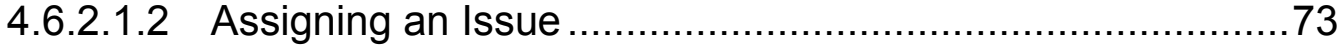

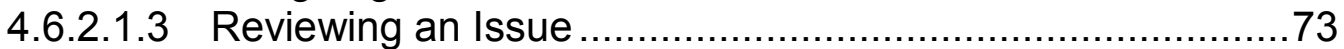

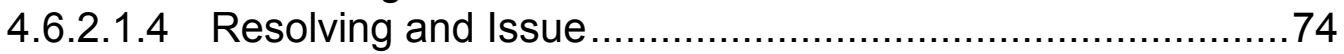

4.6.2.1.5 Re-Assigning an Issue ............................................. 74

4.6.2.1.6 Review and Rework ............................................... 74

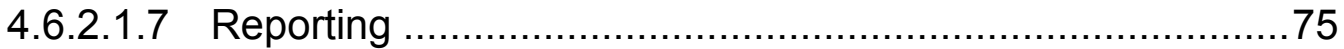

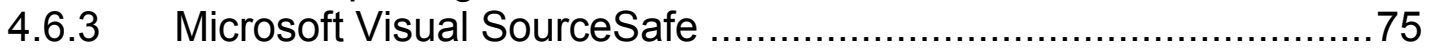

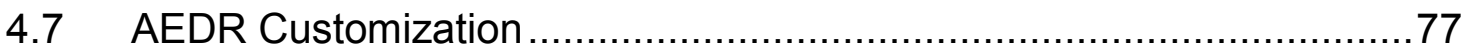

4.7.1 EDFS (Electric Distribution Facility Services) Integration Including EDFS Data Modeling ............................................77

4.7.1.1 EDFS Integration Objectives ........................................

4.7.1.2 EDFS Background.................................................... 78

4.7.1.3 EDFS GIS Data Model Changes ..........................................78

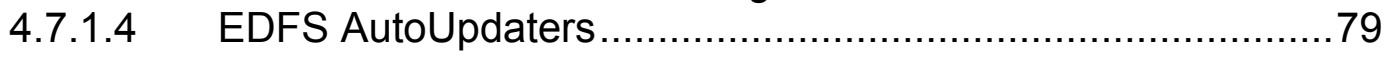

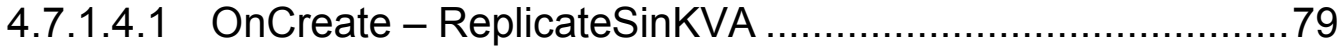

4.7.1.4.1.1 Assign to Class .................................................. 79

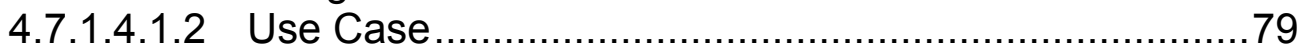

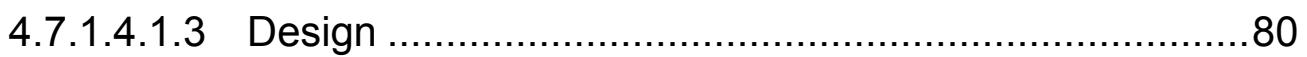

4.7.1.4.2 OnUpdate - UpdateSinKva ........................................ 80

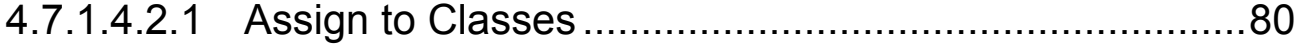

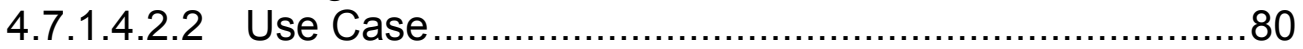




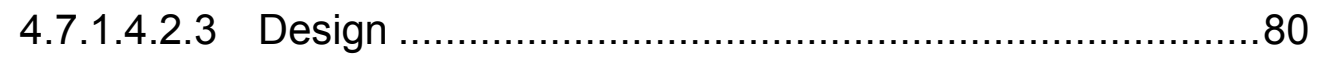

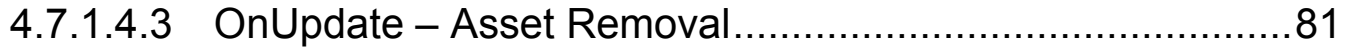

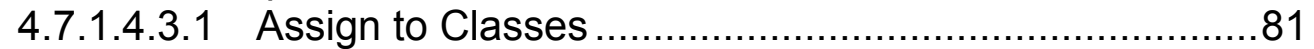

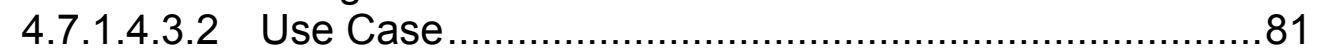

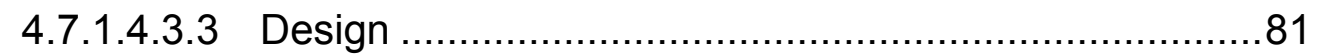

4.7.1.4.4 OnUpdate - Capacitor Removal .................................... 83

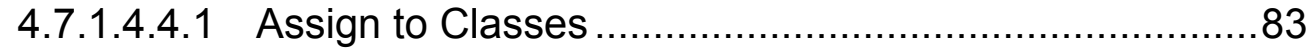

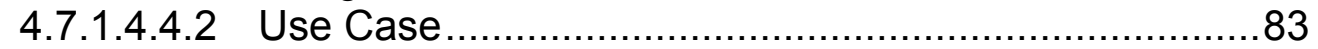

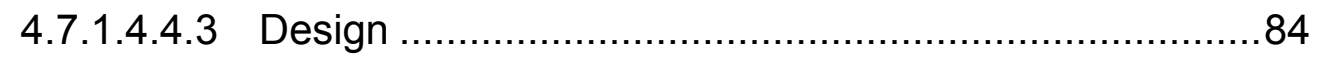

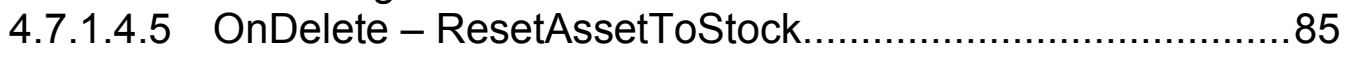

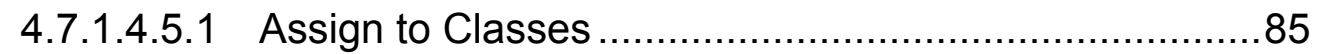

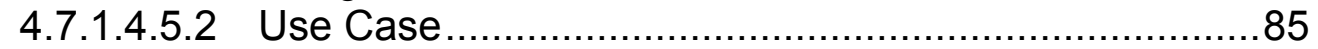

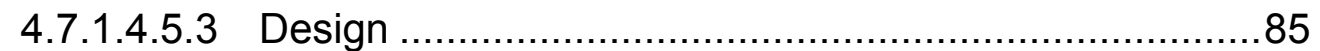

4.7.1.4.6 OnDelete - ResetAssetTolnstalled ................................86

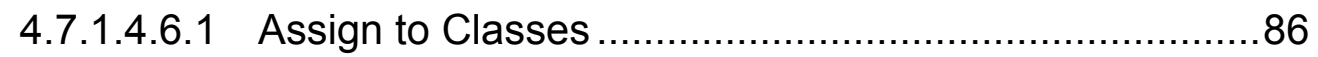

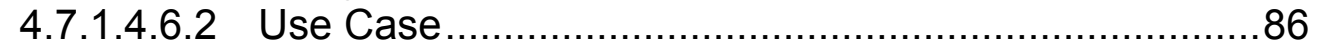

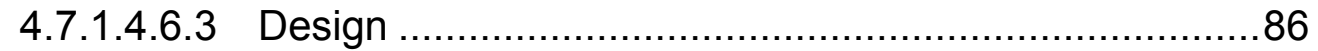

4.7.1.4.7 OnCreate, OnUpdate - ValidateWorkOrderNumber ...........86

4.7.1.4.7.1 Assign to Classes .................................................... 87

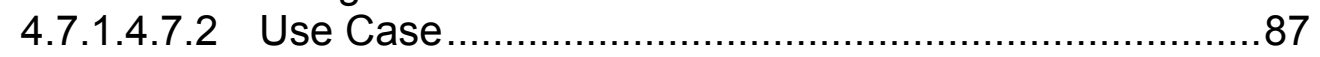

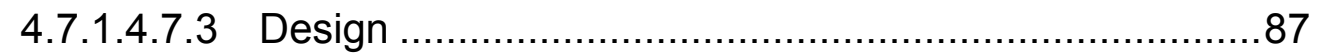

4.7.1.4.8 OnCreate, OnUpdate - ValidateAssembly Number .............88

4.7.1.4.8.1 Assign to Classes ..................................................... 88

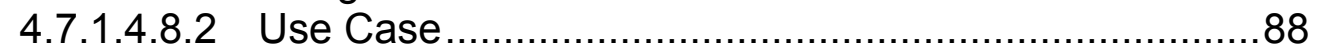

4.7.1.4.8.3 Design ........................................................... 88

4.7.1.4.9 OnCreate, OnUpdate - Validate Referenced Assembly .....89

4.7.1.4.9.1 Assign to Classes .....................................................89

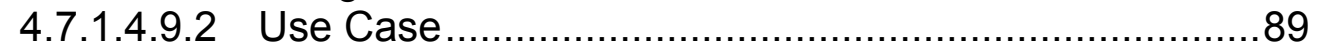

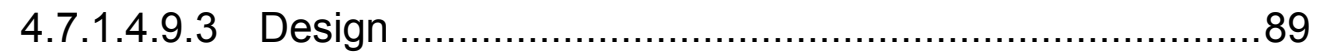

4.7.1.4.10 OnCreate, OnUpdate - ValidateConductorProperties.......90

4.7.1.4.10.1 Assign to Classes .................................................. 90

4.7.1.4.10.2 Use Case..............................................................

4.7.1.4.10.3 Design .......................................................... 91

4.7.1.4.11 OnRetire, OnDelete - PreventDeletionRelatedAssets ......92

4.7.1.4.11.1 Assign to Classes ...............................................92

4.7.1.4.11.2 Use Case ...........................................................92

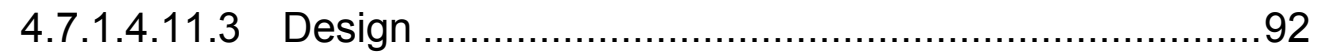

4.7.1.4.12 OnRetire - SupportStructureRetirement ........................93

4.7.1.4.12.1 Assign to Classes .................................................93

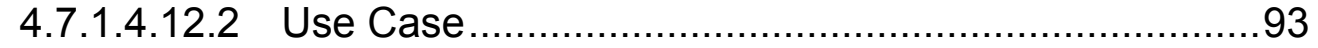

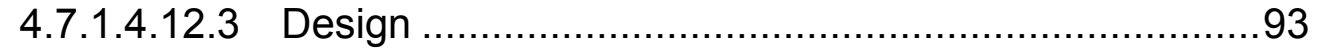

4.7.1.4.13 OnRetire - ReplicateRetirementData..............................99

4.7.1.4.13.1 Assign to Classes and Required Data ........................ 94

4.7.1.4.13.2 Use Case............................................................94

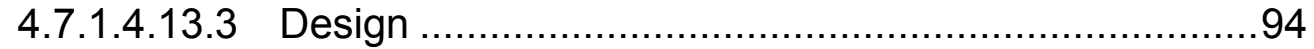

4.7.1.4.14 OnRetire - ReplicateSpatialData ...................................95 
4.7.1.4.14.1 Assign to Classes and Required Data ......................95

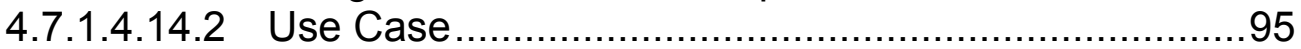

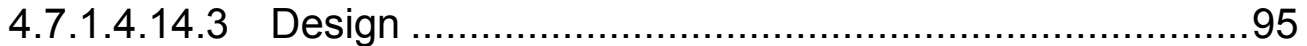

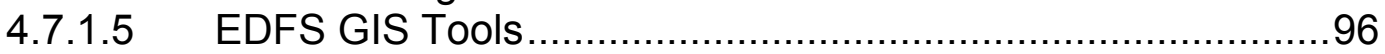

4.7.1.5.1 SupportStructure and PadMount Replace Tool..................96

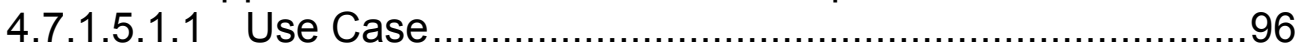

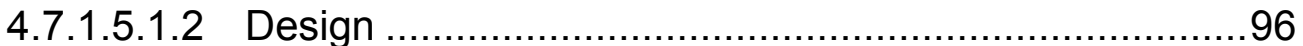

4.7.1.5.2 LegacyConductorInfo - Retirement / Migrate \& Delete .......97

4.7.1.5.2.1 Use Case .............................................................97

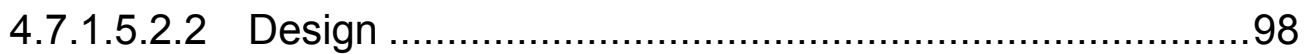

4.7.1.6 EDFS Batch Management and Reporting Applications ........100

4.7.1.6.1 MAPPS / GIS Transaction Processing ...........................100

4.7.1.6.1.1 Use Case ............................................................101

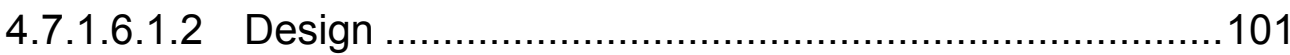

4.7.1.6.2 Batch Report Generator ............................................106

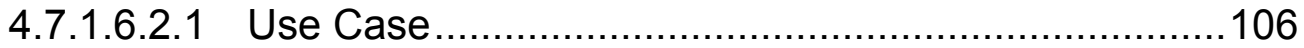

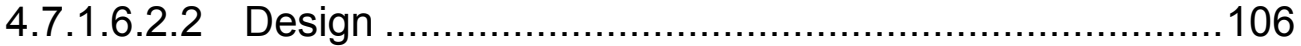

4.7.1.6.3 Interface Management ............................................... 107

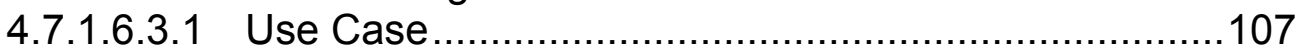

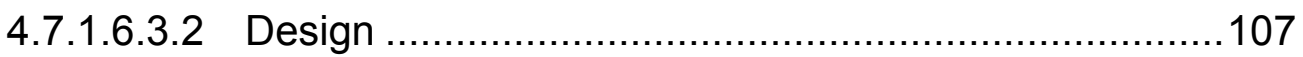

4.7.1.6.4 Structure to Conductor Join.........................................107

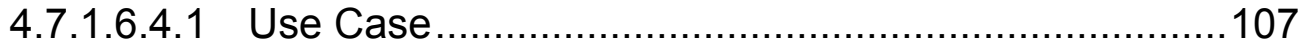

4.7.1.6.4.2 Design ........................................................... 108

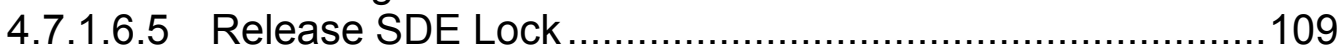

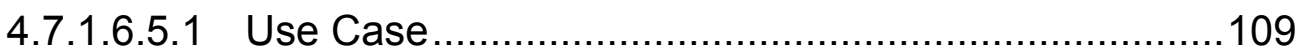

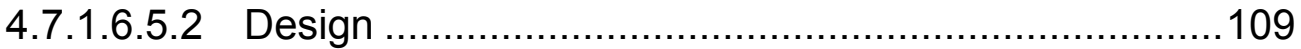

4.7.1.6.6 Domain Synchronization ............................................109

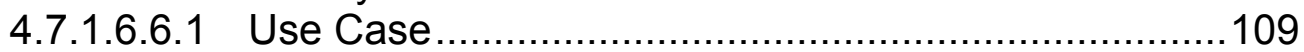

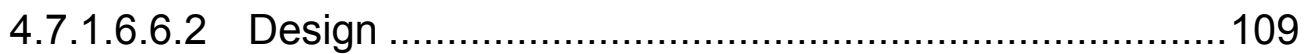

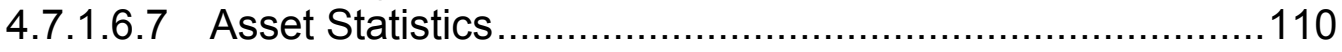

4.7.1.6.7.1 Use Case .............................................................110

4.7.1.6.7.2 Design ................................................................ 110

4.7.1.6.8 Session Reporter .....................................................110

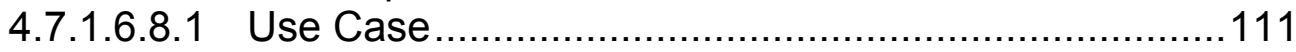

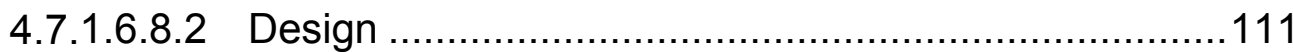

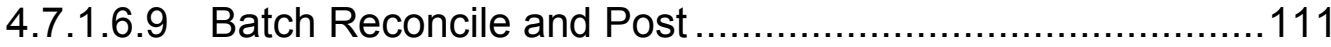

4.7.1.6.9.1 Use Case .............................................................111

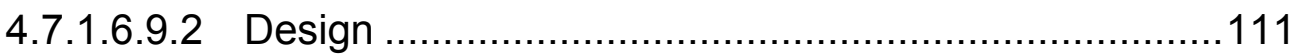

4.7.1.6.10 Batch Reconcile and Compress ...................................111

4.7.1.6.10.1 Use Case ..........................................................111

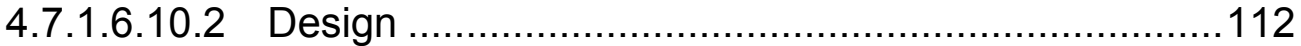

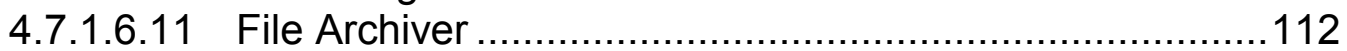

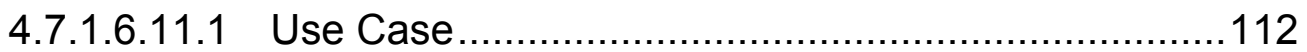

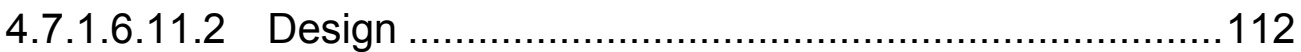

4.7.1.6.12 Condemned Asset Cleanup ......................................112

4.7.1.6.12.1 Use Case ........................................................113 
4.7.1.6.12.2 Design ...............................................................113

4.7.1.6.13 On Demand Reports Cleanup .....................................113

4.7.1.6.13.1 Use Case .......................................................113

4.7.1.6.13.2 Design ............................................................. 113

4.7.1.6.14 Street Centerline Intersection Creator ...........................113

4.7.1.6.14.1 Use Case........................................................114

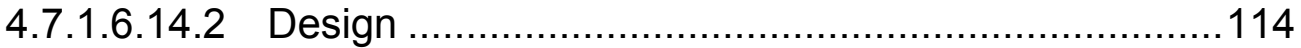

4.7.1.7 SAGE Architecture ......................................................114

4.7.1.7.1 SAGE Multi-Versioned View Update Manager ..................115

4.7.1.8 SAGE Authentication and Authorization............................116

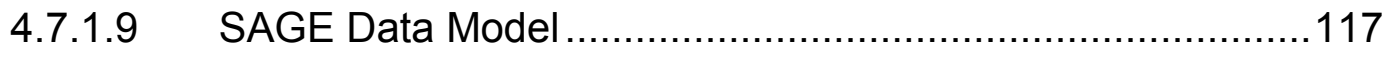

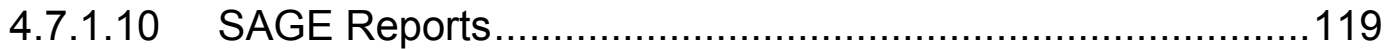

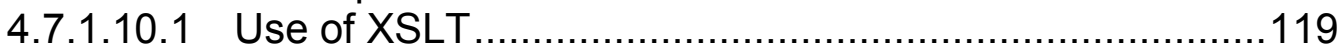

4.7.1.10.2 Report Generation....................................................119

4.7.1.10.2.1 Request for Authority to Condemn Transformers and

Regulators .......................................................119

4.7.1.10.2.2 Line-Transformer / Voltage-Regulator Company Use

Report..............................................................121

4.7.1.10.2.3 FERC For-1 Report of Line Transformers.................121

4.7.1.10.2.4 Report Showing Transformers and Poles.................123

4.7.1.10.2.5 Annual Count of Transformers................................124

4.7.1.10.2.6 Annual Count of Transformers by County.................125

4.7.1.10.2.7 Transformer Failure Report ...................................126

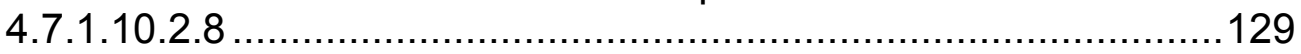

4.7.1.10.2.9 Transformer \& Regulator Stores Item Number

Description Report ............................................130

4.7.1.10.2.10 Transformers In Stock Inventory Report .................131

4.7.1.10.2.11 Annual Count of Capacitors ..................................132

4.7.1.10.2.12 Capacitor Failure Report.....................................133

4.7.1.10.2.13 Capacitor Stores Item Number Description Report.136

4.7.1.10.2.14 Capacitors In Stock Inventory Report ....................137

4.7.1.10.2.15 Securities and Exchange Commission Form 10K (EDB5032)...............................................138

4.7.1.11 SAGE Screen Design ...................................................139

4.7.1.11.1 Transformer / Regulator Screens ...............................139

4.7.1.11.1.1 Add New Transformer / Regulator ..........................139

4.7.1.11.1.2 Query by company Number / Serial Number ............139

4.7.1.11.1.3 Query by Location ............................................141

4.7.1.11.1.4 Edit Existing Transformer Manufacturer and Warranty

Data .................................................................142

4.7.1.11.1.5 Transformer Installation .....................................143

4.7.1.11.1.6 Transformer Removal .......................................144

4.7.1.11.1.7 Transformer Deletion .............................................145

4.7.1.11.1.8 Property Transfer..............................................146

4.7.1.11.1.9 Transformer Status ..............................................147

4.7.1.11.1.10 Replace a Transformer .....................................148 


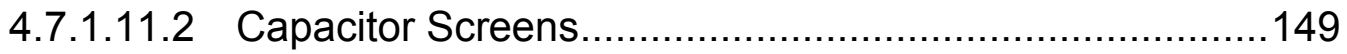

4.7.1.11.2.1 Add New Capacitor..............................................149

4.7.1.11.2.2 Query By Company Number / Serial Number ...........150

4.7.1.11.2.3 Query by Location ..............................................151

4.7.1.11.2.4 Edit Existing Capacitor Manufacturer and Warranty Data ............................................................ 152

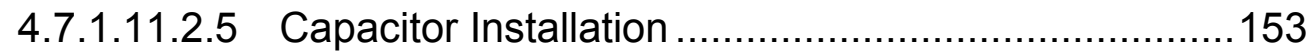

4.7.1.11.2.6 Capacitor Removal ............................................154

4.7.1.11.2.7 Capacitor Deletion .............................................155

4.7.1.11.2.8 Capacitor Property Transfer ….............................156

4.7.1.11.3 Structure Span Screens ............................................156

4.7.1.11.3.1 Structure Span Lengths Add / Edit / Delete ..............157

4.7.1.11.3.2 Structure Span Lengths Query ..............................158

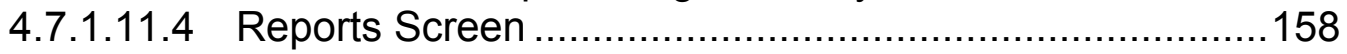

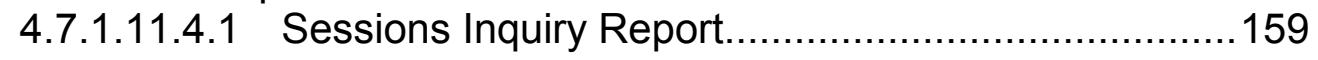

4.7.1.11.4.2 Capital Assets for Sale Report...............................161

4.7.1.11.4.3 Pole / Pad Mounting Inquiry Report..........................164

4.7.1.11.4.4 Padmount Inspection Form Report.........................166

4.7.1.11.4.5 Batch Reports Screen...........................................168

4.7.1.11.5 Coordinator Screens ................................................169

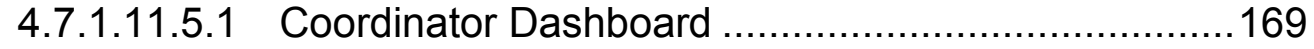

4.7.1.11.5.2 SAGE Security Report .....................................170

4.7.1.11.5.3 Add New SAGE User.........................................171

4.7.1.11.5.4 Manage SAGE Roles .........................................172

4.7.1.11.5.5 Unique Distribution Reference Administration ..........173

4.7.1.11.5.6 Reset Condemnations .......................................174

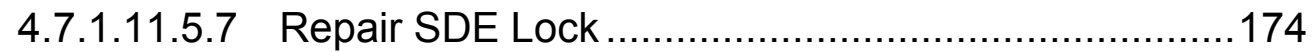

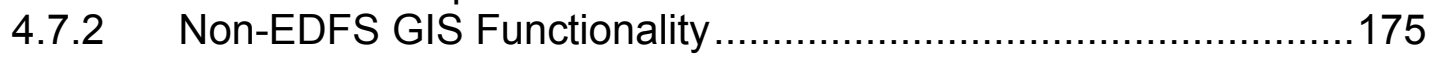

4.7.2.1 Core GIS and Outfield Replacement...............................175

4.7.2.1.1 Copy Value to Related Object...................................... 175

4.7.2.1.1.1 Assign to Classes .............................................175

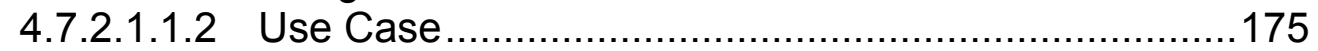

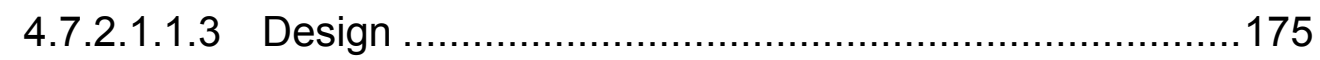

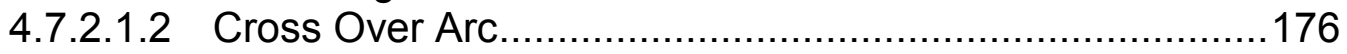

4.7.2.1.2.1 Assign to Classes ...............................................176

4.7.2.1.2.2 Use Case ............................................................176

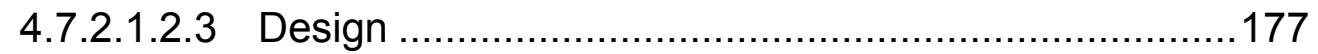

4.7.2.1.3 Session Manager Do Not Post Subtask ..........................177

4.7.2.1.3.1 Assign to Classes ................................................177

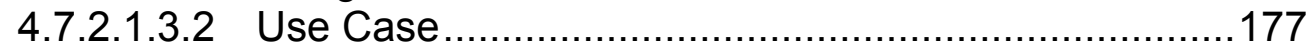

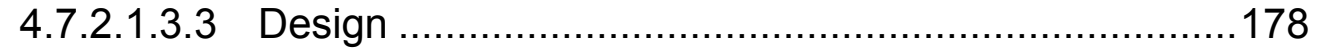

4.7.2.1.4 Field Concatenation AU ............................................. 178

4.7.2.1.4.1 Assign to Classes ................................................178

4.7.2.1.4.2 Use Case ........................................................178

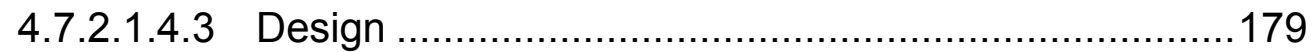

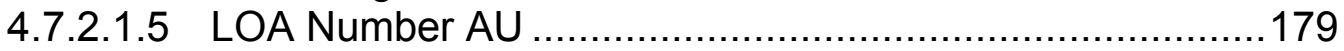




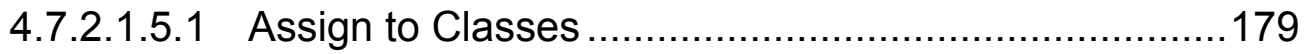

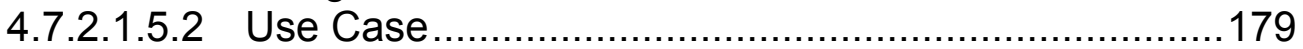

4.7.2.1.5.3 Design ..........................................................179

4.7.2.1.6 Network Edge Split at Tap Point ..................................179

4.7.2.1.6.1 Assign to Classes ................................................180

4.7.2.1.6.2 Use Case .......................................................... 180

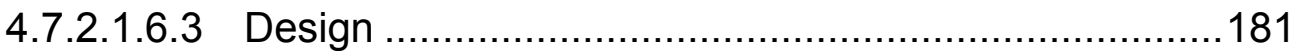

4.7.2.1.7 Normal Position Symbology AU ...................................182

4.7.2.1.7.1 Assign to Classes ...............................................182

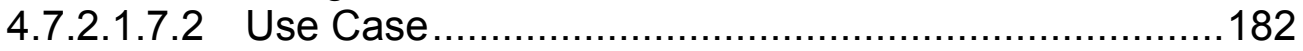

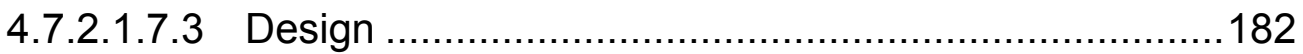

4.7.2.1.8 Prevent Delete if Object has Related Objects ...................183

4.7.2.1.8.1 Assign to Classes ...................................................183

4.7.2.1.8.2 Use Case ..............................................................183

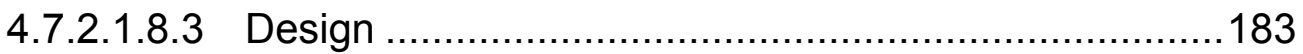

4.7.2.1.9 Reference Features Remove Button ............................. 183

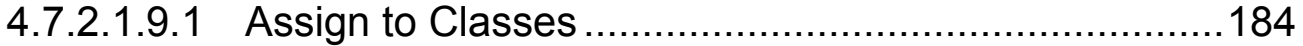

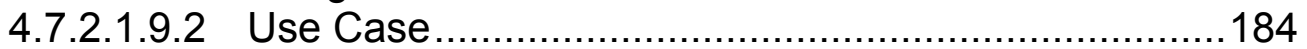

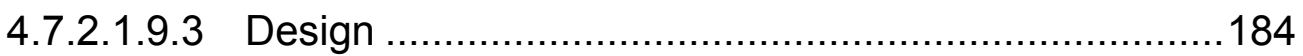

4.7.2.1.10 Feature Offset Edit Task .......................................... 184

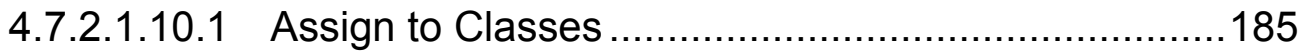

4.7.2.1.10.2 Use Case......................................................185

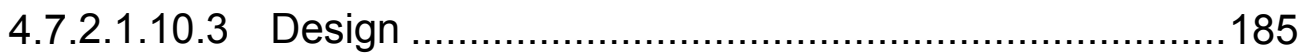

4.7.2.1.11 Unique Distribution Reference AU .............................186

4.7.2.1.11.1 Assign to Classes .............................................187

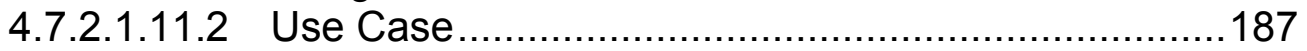

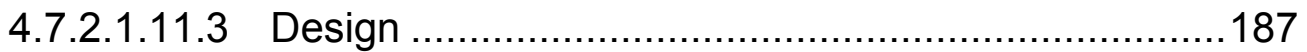

4.7.2.1.12 FeederAll Open Point Deletion AU .................................188

4.7.2.1.12.1 Assign to Classes .............................................. 188

4.7.2.1.12.2 Use Case........................................................188

4.7.2.1.12.3 Design ..................................................... 188

4.7.2.1.13 Transformer Lead Edit Task.......................................188

4.7.2.1.13.1 Assign to Classes ..............................................189

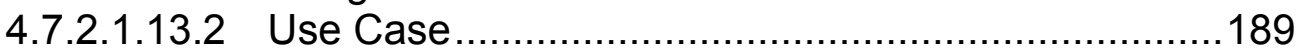

4.7.2.1.13.3 Design ........................................................190

4.7.2.1.14 Delete Attached Transformer Lead ..............................191

4.7.2.1.14.1 Assign to Classes ...............................................191

4.7.2.1.14.2 Use Case ............................................................191

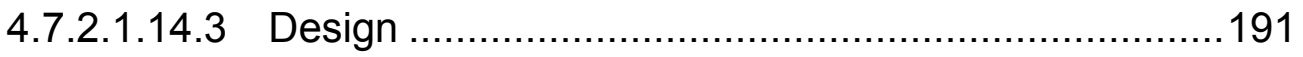

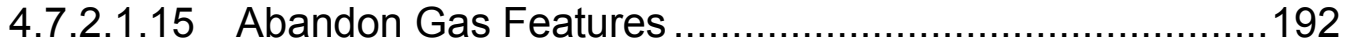

4.7.2.1.15.1 Assign to Classes .................................................192

4.7.2.1.15.2 Use Case........................................................192

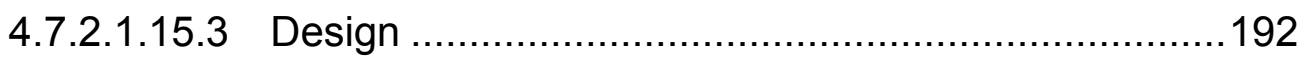

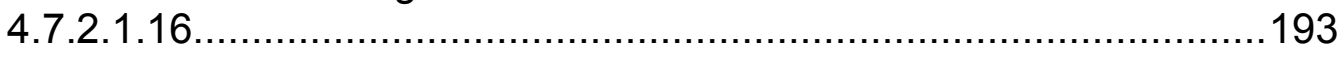

4.7.2.1.16.1 Assign to Classes ................................................193

4.7.2.1.16.2 Use Case.......................................................193 


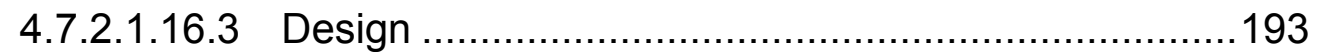

4.7.2.1.17 Place and Relate Gas Main Edit Task..........................194

4.7.2.1.17.1 Assign to Classes ................................................. 194

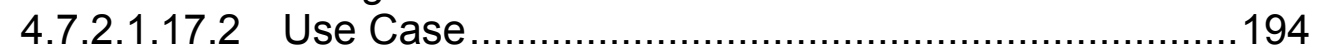

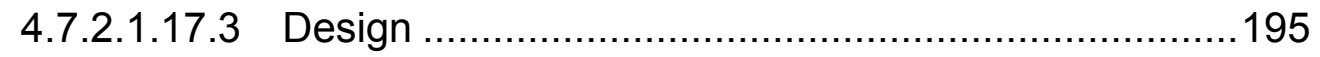

4.7.2.1.18 Retrieve ObjectID of Closest Gas Main.........................195

4.7.2.1.18.1 Assign to Classes ...............................................195

4.7.2.1.18.2 Use Case.........................................................196

4.7.2.1.18.3 Design .........................................................196

4.7.2.1.19 CP Section - Corrosion Control Number .......................196

4.7.2.1.19.1 Assign to Classes ................................................196

4.7.2.1.19.2 Use Case........................................................196

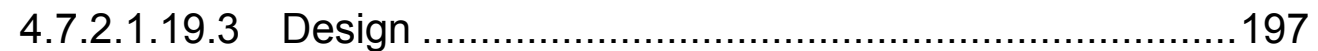

4.7.2.1.20 CP Section - Maintenance Tool ................................197

4.7.2.1.20.1 Assign to Classes ..............................................198

4.7.2.1.20.2 Use Case..................................................198

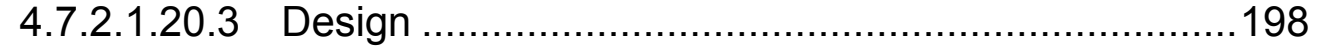

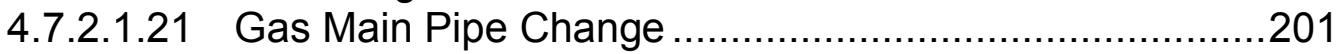

4.7.2.1.21.1 Assign to Classes ...............................................202

4.7.2.1.21.2 Use Case.........................................................202

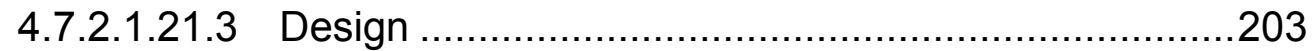

4.7.2.1.22 Emergency Valve Indicator ...................................204

4.7.2.1.22.1 Assign to Classes ..............................................204

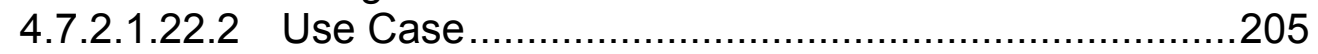

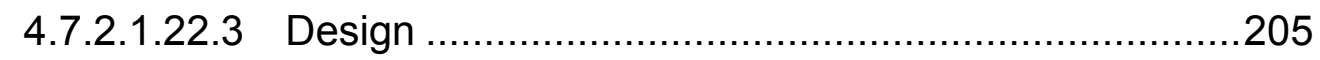

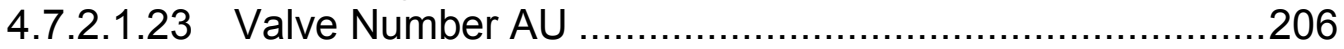

4.7.2.1.23.1 Assign to Classes ..............................................206

4.7.2.1.23.2 Use Case........................................................206

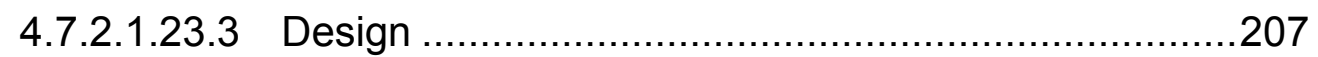

4.7.2.1.24 Custom Identify Tool ............................................207

4.7.2.1.25 Custom Structure Relate .......................................208

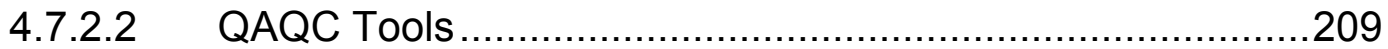

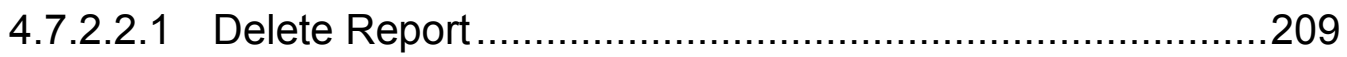

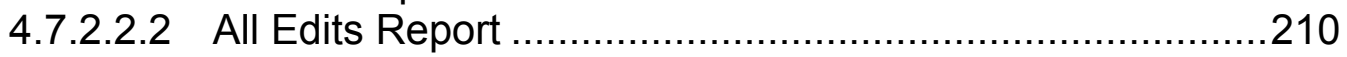

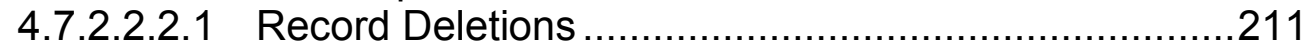

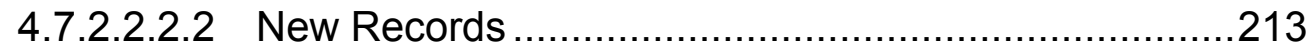

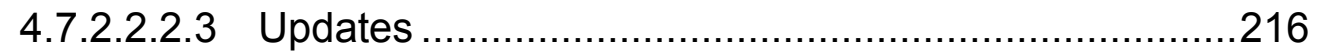

4.7.2.2.2.4 All Edits Options ...................................................219

4.7.2.2.2.5 All Edits Tools ......................................................219

4.7.2.2.2.5.1 Visualize Report...........................................220

4.7.2.2.2.5.2 Zoom to Selected ........................................221

4.7.2.2.2.5.3 Flash Selected ............................................221

4.7.2.2.2.5.4 Visualize Selected and View Attributes ................221

4.7.2.2.2.5.5 Visualize Clear ..............................................222

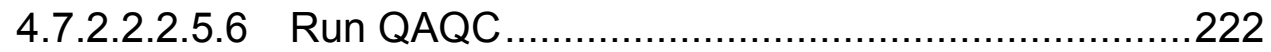

4.7.2.2.2.5.7 Copy to Clipboard .........................................223 


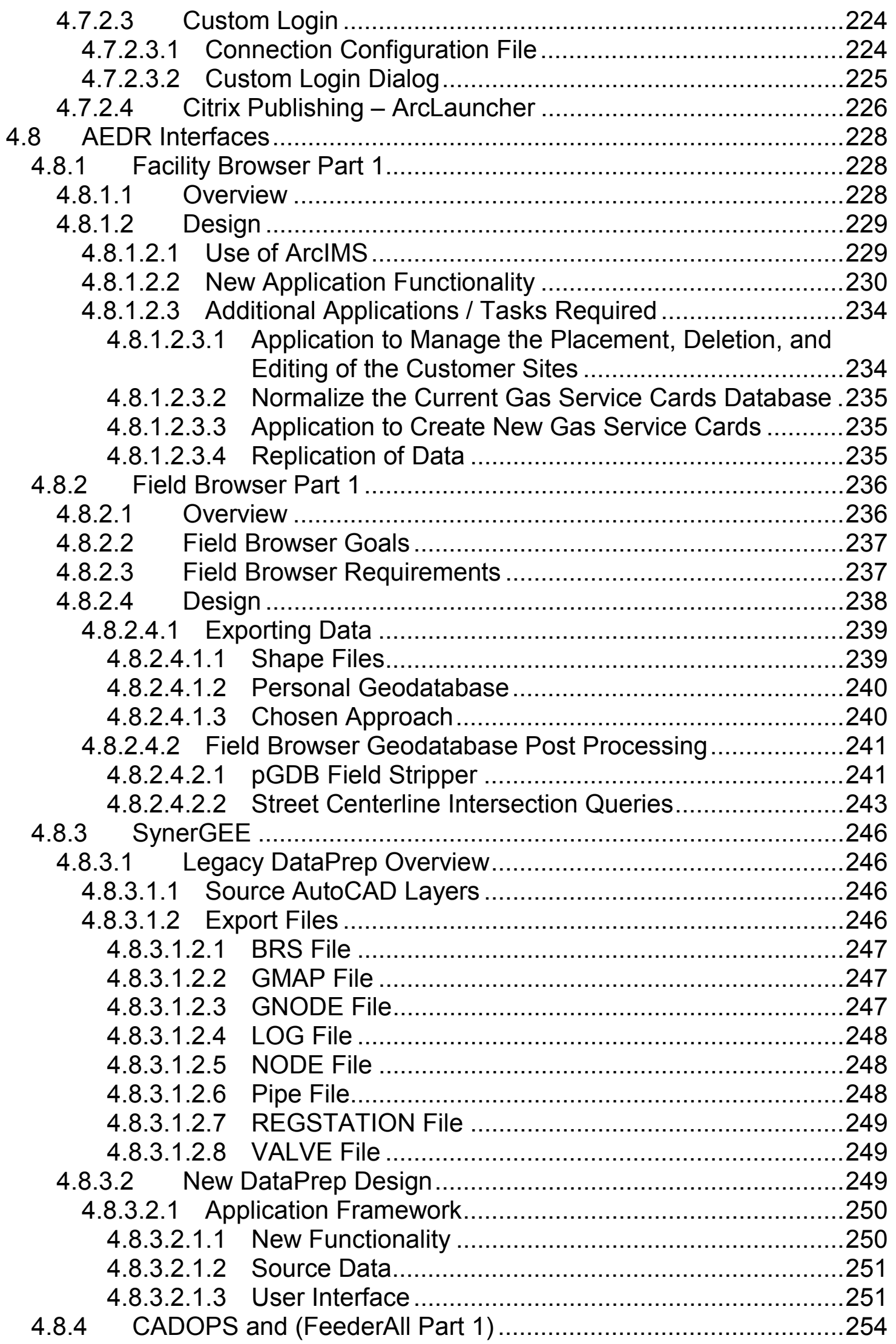




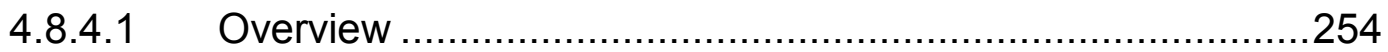

4.8.4.1.1 ArcFM Network Adapter Architecture ...........................255

4.8.4.1.2 Extensible Stylesheet Language Transformation (XSLT)..256

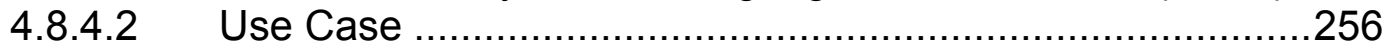

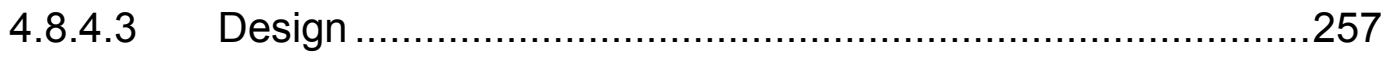

4.8.4.3.1 Feature and Object Class Mappings .............................257

4.8.4.4 Fields Requiring Additional Processing ............................258

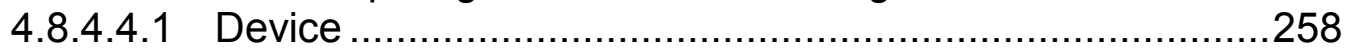

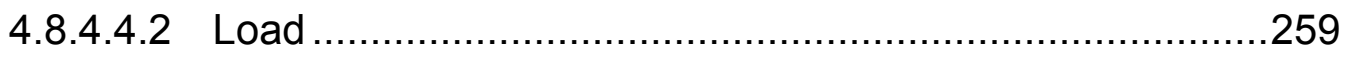

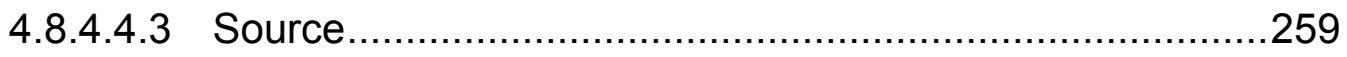

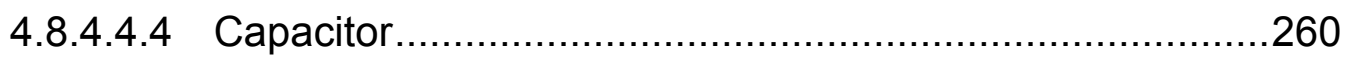

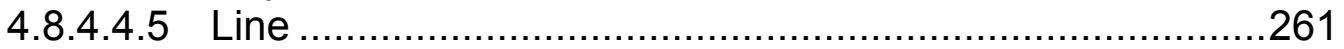

4.8.4.5 ABB Category, Type, Voltage, and Symbolization Fields.....261

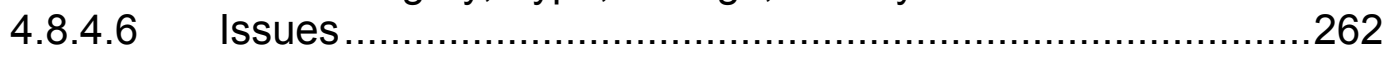

4.8.4.6.1 Modeling of VoltageRegulator .....................................262

4.8.4.6.2 New Business Process for Modeling CustomerGenerator 262

4.8.4.6.3 Utilization of ABB Path Points .......................................262

4.8.4.6.4 GIS Modeling of SubstationBusbar ..............................262

4.8.4.6.5 Maintaining List of Node IDs at LOA Boundaries .............263

4.8.4.6.6 Export of Transmission ............................................263

4.8.4.6.7 Tracking of a Single Source for Multiple Feeders.............263

4.8.4.6.8 Modeling of a Single Phase Transformer on a 3-Phase

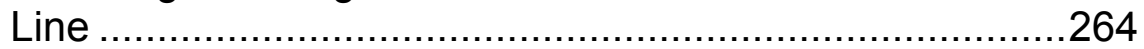

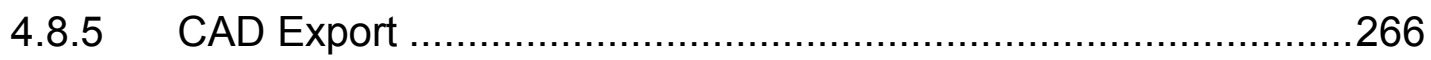

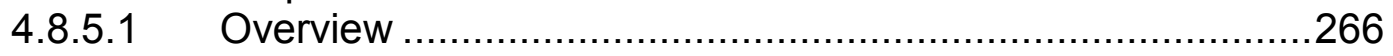

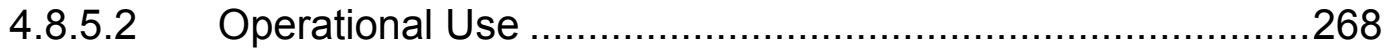

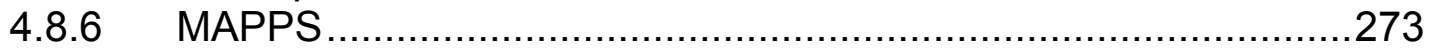

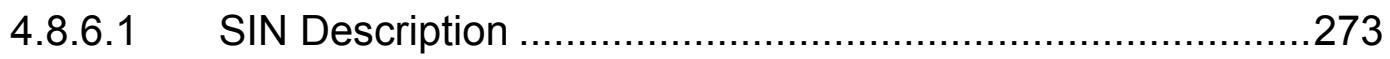

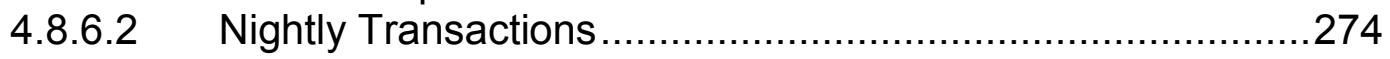

4.8.6.3 Near Real Time Asset Push .........................................275

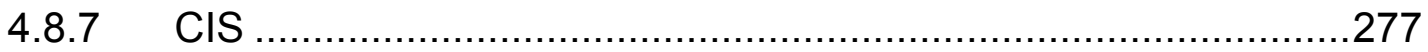

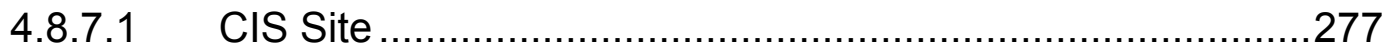

4.8.7.2 CIS Installed Service .................................................279

4.8.7.3 Transformer Install and Removal ...................................280

4.8.7.4 SupportStructure and Padmount Installation .......................280

4.8.7.5 Installed Service Coordinate Update ...............................281

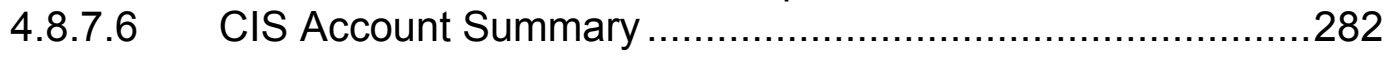

4.8.7.7 CIS Service Address Summary .....................................282

4.8.7.8 CIS Life Support Summary ..........................................283

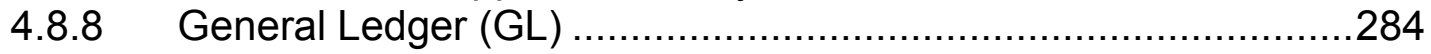

4.8.9 Engineering Accounts Information File (EAIF).......................28

4.8.10 Material and Labor Online Guide (MLOG) ...............................285

4.9 System Performance Evaluation / Stress Testing ..............................287

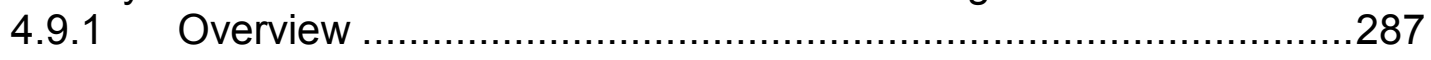

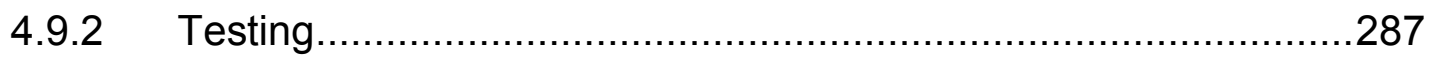

4.9.2.1 Hardware Size Testing and Tuning ….............................287 
4.9.2.2 First Performance Testing and Tuning ..............................287

4.9.2.3 Second Performance Testing and Tuning ..........................288

4.9.2.4 Final Performance Testing and Tuning .............................289

4.9.3 Base Performance Configuration .........................................289

4.9.4 ArcSDE Geodatabase Performance Maintenance ....................290

4.9.4.1 SQL Server Log Maintenance ..........................................290

4.9.4.2 ArcSDE Compress Schedule .......................................291

4.9.4.3 ArcSDE Compress Success ...........................................291

4.9.4.4 Analyze Command Schedule ..........................................291

4.9.4.5 Rebuild Field Index...................................................291

4.9.4.6 Evaluate Spatial Index Grids (SIG).................................291

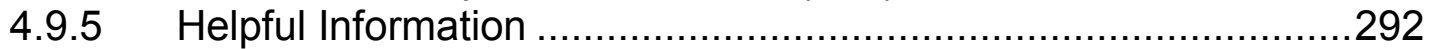

4.10 AEDR Online Help Documentation .............................................293

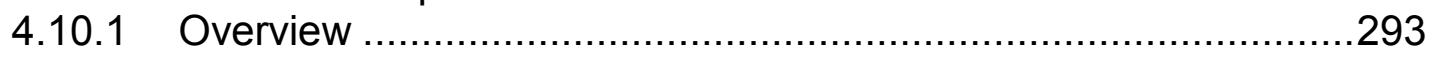

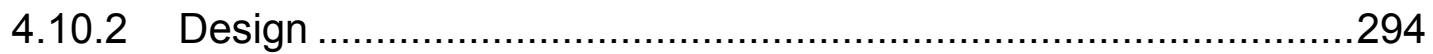

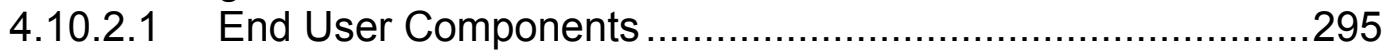

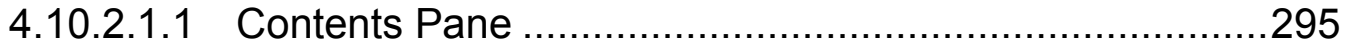

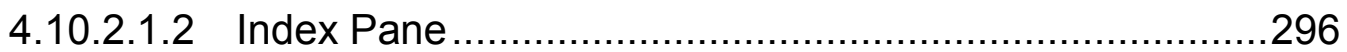

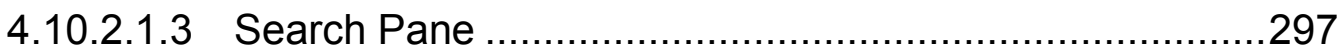

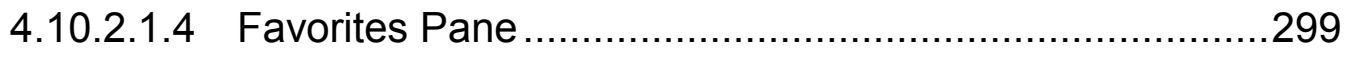

4.10.2.2 Administration Components ............................................301

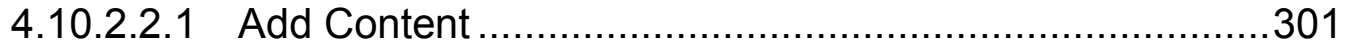

4.10.2.2.2 Image Management ..................................................302

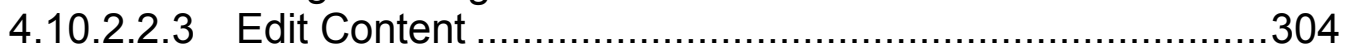

4.10.2.2.4 Contents Management..............................................305

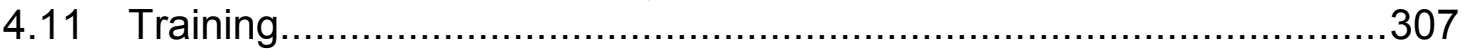

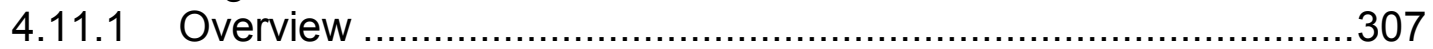

4.11.1.1 Training Needs Assessment..........................................307

4.11.1.2 GIS Project Team Training ............................................307

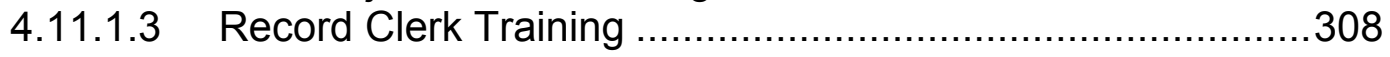

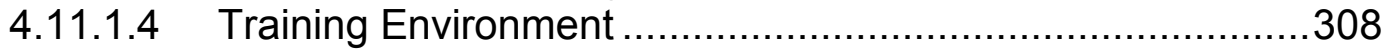

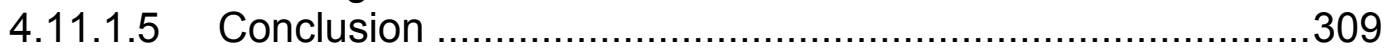




\section{Introduction}

This report describes the NIPSCO project efforts and status of their Automated Energy Distribution and Reliability (AEDR) system. A future final status report will complete the documentation.

\subsection{Background and Expectations}

Modernizing the electric-gas utility infrastructure by implementing a Geographic Information System (GIS) technology results in a number of benefits for both the utility as well as its customers. Traditionally residing partly in an AutoCAD-based system and partly in a relational database system, NIPSCO facility information (gas, electric and land) was served by distinct and not readily interoperable technologies. Thus it was time consuming and sometimes cumbersome to readily maintain the integrity of the data and disseminate facility information to those requiring it. These technical inadequacies could be significantly improved by the implementation of a Geographic Information System (GIS) technology.

The purpose of this project was to implement a database-driven GIS solution that would manage all of NIPSCO's gas, electric and landbase objects (Figure 1-1).

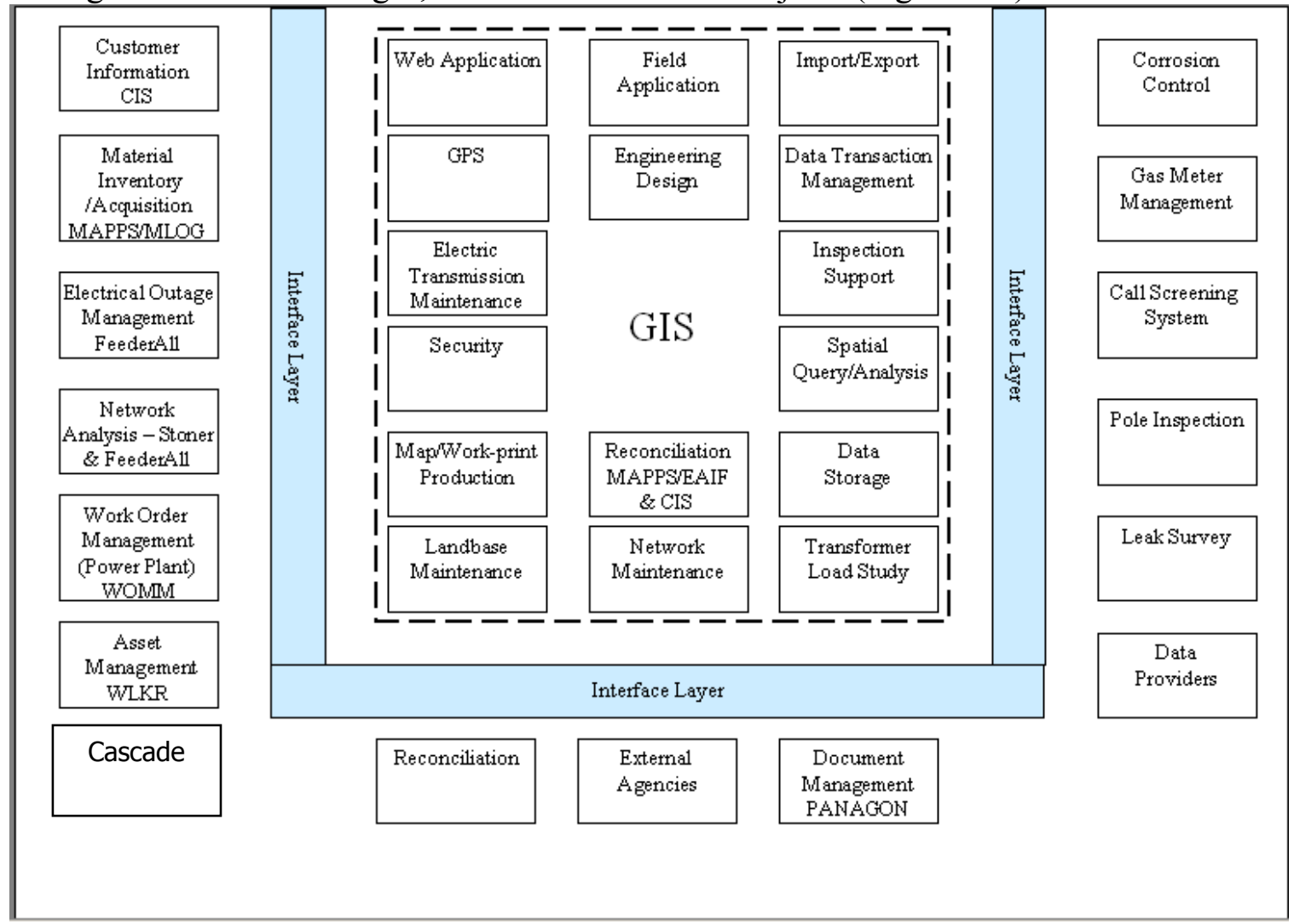

Figure 1-1. NIPSCO GIS approach to AEDR 
The goals of this project included:

- Elimination of redundant data and systems

- Implementation of a business rule-driven application to manage GIS data

- Improvement of data quality and integrity

- Gas and electric load study integration

- Establishment of improved and integrated information to satisfy external entities' needs and identify opportunities such as

1. Identification of critical habitats of threatened or endangered species

2. Exploration of alternative energies, such as wind energy, combined heat and power (CHP) units and distributed resources/generation (DR/DG)

3. Impact future installation of facilities located near the Indiana Dunes National Lakeshore to enable environmentally sound decisions.

- Gather and provide information to, and support participation in IEEE standards development work groups.

\subsection{AEDR Project Structure and Status Summary}

Each section within this report details the effort undertaken to implement the AEDR at NIPSCO.

The Environmental Systems Research Institute (ESRI)'s GIS technology platform was selected to replace NIPSCO's legacy systems, and the Automated Energy Distribution and Reliability (AEDR) system was born. The AEDR problem and solution domain are presented in the following graphic (Figure 1-2).

\begin{tabular}{|l|l|}
\hline The problem of & $\begin{array}{l}\text { A limited automated mapping application } \\
\text { that does not provide all of the data all of } \\
\text { the time }\end{array}$ \\
\hline Affects & $\begin{array}{l}\text { Records, Engineering, Corrosion Control, } \\
\text { Gas Meter Management, Gas Systems } \\
\text { Engineering, Locate Screening, Field } \\
\text { Crews, Analysis Engineering and Maps \& } \\
\text { Records }\end{array}$ \\
\hline The impact of which is & $\begin{array}{l}\text { Business decisions made on questionable } \\
\text { or insufficient data and the length of time it } \\
\text { takes to perform key operational activities. }\end{array}$ \\
\hline A successful solution would & $\begin{array}{l}\text { Promote safety, enable sound business } \\
\text { decisions, increase efficiencies, increase } \\
\text { the visibility of data, increase the user base } \\
\text { and compliance with governmental } \\
\text { requirements. }\end{array}$ \\
\hline
\end{tabular}

Figure 1-2. The NIPSCO AEDR problem-solution domain 
Spatial analysis capabilities that were limited in the legacy environment became available and redundant data and systems were eliminated. Further, there was a focused effort on data accuracy improvement and insertion of business rules to maintain the improved data resulting in significant improvements in data integrity and the ability to access the data.

The capacity to conduct spatial analysis of NIPSCO facilities and service territory directly provides organized information on which to base sound business decisions and supports enhanced physical safety for both NIPSCO employees and the general public.

Gas load information has been provided in a manner that truly supports gas load studies. By re-routing the method in which the ABB application, FeederAll receives data, additional and critical information will be provided to enhance electric load studies. When the project is completed, all users of the AEDR such as Corrosion Control, Gas Meter Management, Field Crews, Rate Department, Engineering, Maps \& Records, Electric System and Services Operations, Gas Operations Integration, External Contractors, Facility Locators, Government entities, etc. will be provided with all of the information they require from the AEDR system. And, the wind energy study undertaken in partnership with the National Renewable Energy Laboratory will provide NIPSCO management with the feasibility of producing clean energy in proximity to NIPSCO's grid.

The project is governed by the subcontract Statement of Work (SOW) consisting of seven tasks. The project was structured for completion in three phases:

- The Preliminary Phase includes "Task 1 - Selection of GIS Software Platform" and "Task 2 - GIS Project Definition and Demonstration of Integration"

- Phase I includes "Task 3 - Immediate Conceptual Architecture Implementation"

- Phase II includes "Task 4 - Future Conceptual Architecture Implementation" "Task 5 - AEDR Studies"

"Task 6 - Post Implementation Review of AEDR" will be conducted at the close of Phase II.

"Task 7 - Standards Development and Implementation" was conducted throughout Phase I by active participation in the standards development process that resulted in publication of the IEEE Std 1547.3 ${ }^{\mathrm{TM}}$ (2007) Guide for Monitoring, Information Exchange and Control of Distributed Resources Interconnected with Electric Power Systems. That standards process work included attending meetings, gathering information, developing, reviewing and providing feedback on the draft IEEE 1547.3 standard that is one in the series related to the IEEE Std $1547^{\mathrm{TM}}$ (2003) Standard for Interconnecting Distributed Resources with Electric Power Systems.

The Preliminary Phase Tasks 1 and 2 have been completed and the focus for this report is Phase I and Task 3 Immediate Conceptual Architecture Implementation. The Preliminary Phase material has been included in Section Three and in Appendix A-1. Future reports will include the remaining Tasks governed by the SOW. 
Phase I was successfully implemented into production use on June 12, 2006. In summary, Phase I involved implementing the "Immediate Conceptual Architecture" according to NIPSCO requirements and criteria. The Phase I implementation objectives were:

- Implement a database-driven GIS solution that will manage all of NIPSCO's gas, electric and land-base objects

- Replace the core mapping maintenance product (Outfield), merge the Electric Distribution Facility Services (EDFS) data into the new GIS and maintain or improve the existing interfaces to the Customer Information System (CIS), Engineering Accounts Information File (EAIF), Material Accounts Payable and Purchasing System (MAPPS), Advantica Gas SynerGEE and ABB CADOPS

- Correct positional inaccuracies through a data rectification process

- Develop a stable system environment in which to develop and test the system

- Migrate the data to the new GIS platform

- Configure the software.

- Customize the software as needed to improve user interaction

- Ensure adequate system performance

- Generate user-friendly help documentation

- Train the users, and

- Implement the Immediate Conceptual Architecture into the production environment rendering it useful to all. 


\section{Project Management}

\subsection{Overview}

As with any successful large-scale implementation, the AEDR project required highly organized project management skills, strength in project sponsorship and adherence to system development industry standards. Some of the items that enabled the success of this project are: Project scope and critical path components were monitored, project budget and timeline were maintained, quality assurance was of prime focus especially on the externally contracted data migration and rectification processes, detailed test cases were thoroughly exercised for every application, and risks were managed throughout the project. Problems were tracked and resolved as timely as possible and resources were assigned team lead roles to ensure ownership.

\subsection{Project Resource Organization}

The AEDR development project team was composed of a combination of external vendors and internal NIPSCO employees.

Project sponsors, a project manager, GIS developers, GIS analysts, GIS migration vendors, IT database support, IT server support and subject matter experts in Maps \& Records, Engineering and individuals responsible for interfacing systems all participated in the development of various components of the AEDR system.

\subsubsection{Project Resource Responsibilities}

\subsubsection{Project Sponsor}

As a member of the steering committee, the project sponsors set the direction for the project, approved the scope and objectives and communicated the priorities. The project sponsors actively monitored project progress and provided cross-department/enterprise conflict resolution and served as a reporting mechanism to executive management.

\subsubsection{Project Manager}

At a high level, the project manager was responsible for development of the project definition and scope as defined in the Vision document, project budget estimation, creation and maintenance of the project plan to ensure that all project tasks were completed on time and within budget and that milestones and deliverables were met. Project management responsibilities also included project guidance, managing project resources, licensing agreements, vendor contracts, project budget maintenance, identification and management of project risks, change control, and communicating project status to the project sponsor, NREL and the project team. The project manager maintains similar responsibilities throughout all phases of the project. 


\subsubsection{Team Lead}

Under the direction of the project manager, the team lead coordinated the tasks needed to complete the work for a particular area of the project. The team lead reported status and issues to the project manager. Team leads were assigned to the following areas:

- ArcFM Configuration: Annotation, AU assignments, Connectivity, Display Fields, Favorites, Map Templates, Model Names \& Symbology

- ArcFM Customization: Customization candidate list, component specifications, coding, code reviews and code standards.

- Data Migration and Rectification: Migration, Rectification, Data vendor management, Data Matrix, Sample data, Migrated Data, Rectification and Migration QA Procedures, Rectification Rules, Scoring Spreadsheets

- Data Modeling: Build NIPSCO's data model in accordance with the data model procedures and guidelines.

- Development, Testing, and Production Environments: Environment setup and administration, software installs, software patches, server setup, SDE admin, FTP server

- Documentation \& Training

$>$ System Documentation: Administrator guide documentation

> User Documentation: Typical editing scenarios, user guides, Quick Start Guide, on-line help

$>$ Training: Create training materials, training plan, conduct training

- Elementool Support: Configuration, daily backups, security administration

- Interface Development: Requirements, design, implementation, testing and documentation for the following interfaces/components:

$>$ CADOPS/Feederall

$>$ CIS/EAIF (Inc. EDFS)

$>$ Facility Browser

$>$ Field Browser

$>$ GIS to DXF

$>$ MLOG

$>$ General Ledger

$>$ SynerGEE

- Source Safe Administration: Create new Source Safe directories, security administration

- System Architecture: Architecture discussions, system context diagram

- Testing / QA / QC: Test plan, write test cases, perform test cases, document results, report testing metrics

At AEDR implementation, a Help Desk was established to assist users with the transition to the new system, provide future Help Desk support and ensure quality control. 


\subsection{Project Methodology}

The Rational Unified Process (RUP) was recommended by a vendor project team member who reportedly used it successfully on other projects. The RUP methodology was implemented, however it was soon determined to be an unwieldy methodology and abandoned in favor of the traditional waterfall methodology. This explains the references to RUP and the iterative process throughout the first half of the project.

\subsubsection{Project Activities and Schedule Development}

Project activities and schedules were governed by the Project Plan. Project tasks and their associated target dates were defined using Microsoft Project. Project team meetings were held weekly (at a minimum) during which the project team members contributed to the creation and maintenance of the project tasks and schedules. Three separate project plans were developed to manage the various Phases; Preliminary Phase: Inception and Elaboration Phase Project Plan (part of RUP and also known as Pre-Planning), Construction Phase I and Deployment Phase 1. The detailed Project Plans are shown in Appendices A-1, A-2 and A-3, respectively.

\subsection{Cost Estimating}

Project costs were estimated during the Pre-Planning stage of the project. The various components and interfaces had been predefined during the analysis phase. Figure 2-1 was developed to assist with the estimating process. Vendor rates were averaged and contingency was added based on the confidence to estimate the total cost for each component. 


\section{AEDR GIS Project \\ Estimating Worksheet}

$<$ Name of Interface $>$

$<$ Date $>$

\section{Overview of Approach}

Please provide an overall description of the technical approach that will be used. This should include a textual description, pros/cons of the approach, plus one or more diagrams that describe the proposed solution.

Overall Description: <insert text>

Pros for this approach: <insert text $>$

Cons for this approach: $<$ insert text $>$

Diagram for this approach: $<$ insert diagram(s) and supporting text $>$

List of Assumptions: $<$ insert text $>$

\section{Risks for this Approach}

Please describe the technical risks that exist for the proposed solution. For each risk, describe the probability of this risk occurring (percentage) and the impact that would be expected if the risk actually occurs.

\begin{tabular}{|l|l|l|}
\hline Risk & \% Probability & Impact \\
\hline & & \\
\hline & & \\
\hline
\end{tabular}

\section{Construction Tasks / Estimates / Confidence}

Please describe the Construction Phase tasks, estimates, and confidence (\%) for each of the estimates.

\begin{tabular}{|l|l|l|}
\hline Construction Task (w/ Expertise Needed) & $\begin{array}{l}\text { Duration } \\
\text { (person hrs) }\end{array}$ & $\%$ Confidence \\
\hline & & \\
\hline TOTAL & $<$ Total estimate $>$ & $\begin{array}{l}<\text { Overall } \\
\text { confidence }>\end{array}$ \\
\hline
\end{tabular}

Figure 2-1. The overview, risks and percentage of confidence were factored in to the estimated costs for each component and interface 


\subsection{Risk Management}

Project risks were identified, documented and managed throughout the system development lifecycle to help ensure a successful implementation. Weekly meetings were held and tasks assigned to address and resolve/mitigate each project risk. A simple spreadsheet was developed with a worksheet for Technical Project Risks and General Project Risks. All risks had a detailed description, impact, mitigation plan, ranking, severity code, indicators, probability factor and owner. Each owner was responsible for managing assigned risks until that risk was completely mitigated.

\subsection{Software and Hardware Analysis}

\subsubsection{Software Analysis}

Following the software vendor selection results, we requested trial licenses for a proof of concept activity before entering into a perpetual licensing arrangement. We were subsequently granted a trial license to conduct the activities detailed in the Inception and Elaboration Phase Project Plan located in Appendix A-1. The results of the Elaboration Phase provided sufficient proof of concept to continue the project.

\subsubsection{Hardware Analysis}

Legacy system features were listed, counted and sized (in bytes), the projected user environment (Table 2-1) was documented and together with ESRI application architecture requirements, this information was used as a baseline for an initial hardware requirements study.

Table 2-1. Projected User Environment

\begin{tabular}{|l|r|}
\hline Total number of CITRIX desktop clients & 131 \\
\hline Total number of ArcMap desktop users & 29 \\
\hline Total number of Web users & 0 \\
\hline Total number of users & 160 \\
\hline $\begin{array}{l}\text { Estimated number of concurrent CITRIX } \\
\text { desktop clients }\end{array}$ & 50 \\
\hline $\begin{array}{l}\text { Estimated number of concurrent ArcMap } \\
\text { desktop users }\end{array}$ & 18 \\
\hline Estimated number of concurrent Web users & 0 \\
\hline Estimated number of total concurrent users & 68 \\
\hline
\end{tabular}


The following configurations were recommended:

Table 2-2. ArcSDE Database Server Configuration

\begin{tabular}{|l|l|}
\hline No. of Servers & 1 \\
\hline No. of CPUs & 2 \\
\hline CPU type & Intel Xeon 3.06 GHz 512 MB L3 Cache \\
\hline Memory & 4 GB \\
\hline SPECfp_rate2000 & 16.7 \\
\hline
\end{tabular}

Performance testing was conducted at NIPSCO on Nov 19, 2003 with the current dual $3.06 \mathrm{GHz}$ server. The testing showed that the ArcSDE/SQL Server 2000 server can provide optimal performance to 80 concurrent users. This exceeds the estimated concurrent user number of 68 .

Table 2-3. Storage requirements

\begin{tabular}{|l|l|}
\hline Type & Hardware based RAID \\
\hline Data Storage & $100 \mathrm{~GB}$ \\
\hline Application Storage & $60 \mathrm{MB}$ for ArcSDE \\
\hline
\end{tabular}

Table 2-4. Application architecture

\begin{tabular}{|l|l|}
\hline Supported Operating Systems & $\begin{array}{l}\text { Windows 2003 - Standard, Enterprise and Data } \\
\text { Center Editions } \\
\text { Windows 2000 - Server, Advanced Server and Data } \\
\text { Center Editions } \\
\text { Windows NT 4.0 Server with Service Pack 6a. }\end{array}$ \\
\hline $\begin{array}{l}\text { Supported Versions of SQL Server } \\
2000\end{array}$ & $\begin{array}{l}\text { SQL Server 2000 Standard Edition SP2 or SP3 } \\
\text { SQL Server 2000 Enterprise Edition SP2 or SP3 }\end{array}$ \\
\hline Supported Version of ArcSDE & ArcSDE 8.3 for SQL Server by ESRI, Inc. \\
\hline
\end{tabular}

Table 2-5. Network requirements

\begin{tabular}{|l|l|}
\hline Protocol & TCP/IP \\
\hline Bandwidth required per user & $18-45 \mathrm{KBS}$ \\
\hline Application Storage & $60 \mathrm{MB}$ for ArCSDE \\
& $200 \mathrm{MB}$ for SQL Server 200 \\
\hline
\end{tabular}

Based on ESRI, Inc. sizing charts, a load of 50 concurrent users requires a cumulative SPECfp_rate2000 value of 56 or higher. 
Table 2-6. CITRIX server farm configuration

\begin{tabular}{|l|l|}
\hline Operating System & Windows 2000 Terminal Server \\
\hline Number of CPUs & 2 \\
\hline CPU type & Intel Xeon 2.8 GHz, 1 MB L3 Cache \\
\hline Memory & 4 GB \\
\hline SPECfp_rate2000 & 13.2 \\
\hline
\end{tabular}




\section{Vendor Selection}

\subsection{Overview}

This section describes the methodology used by the GIS project team to arrive at the selection of GIS software.

A formal Request for Proposal (RFP) process was initiated on March 3, 2003 to begin searching for GIS software that would initially solve the redundant data issues, improve the mechanism for data integrity and integrate the SynerGEE gas model. The long-term goal is to build on these initial requirements using a software platform that is flexible enough to easily satisfy new user requirements and powerful enough to support a growing user community over time. The target to issue the RFP was March 30, 2003.

Previously, in the fall of 2001 this same process was started. User requirements were defined and two vendors demonstrated their products. At that time, the vendors were selected from a broad range of GIS providers based on the desire to preserve our Autodesk software, application, and data investment. Two months into the project, it was put on hold.

The methodology for this RFP process involved leveraging the 2001 user requirements, the RFP process and the engagement of consulting services to preserve the previous investment. The user requirements were re-validated and re-prioritized. The RFP process was updated as needed and included the use of a newer technology tool to support a more methodical selection process.

\subsection{Scope}

A GIS project involves vendor software selection, data migration, conversion and cleanup, integration with interfacing applications and GIS software installation \& configuration.

This RFP process phase was limited in scope to the selection of vendor software only. However, the above GIS project criteria were taken into consideration, to the degree possible to further validate the investment in this large-scale project.

\subsection{Methodology}

The validation and reprioritization of the 2001 requirements produced the Immediate and Future Conceptual Architectures. The project team interviewed key users from Engineering, Records, Construction \& Maintenance, Information Technology, Gas Systems Planning, Distribution Services, Distribution Reliability, Transmission, Operations and Corrosion Control. The user requirements gathered during these sessions were integrated into the Immediate and Future Conceptual Architectures and became the scope of requirements for the comprehensively constructed RFP. 
Four candidate vendors were selected from a combination of GITA conference demonstrations, a technology report \& summary of potential candidates provided by a consulting vendor and a desire to preserve our Autodesk investment if at all possible. The vendors were selected based on but not limited to:

- the potential to meet NIPSCO's functional requirements

- having both a gas \& electric data model

- open architecture

- experience in the gas/electric utility industry

- network modeling capabilities

- scalability

The RFP was delivered to four candidate vendors: one of which was Environmental Systems Research Institute (ESRI) on April 9, 2003. The vendors were given 26 calendar days in which to provide responses to the RFP. All four vendors responded.

Evaluation criteria was established (see Figure 3-1) and weighted (see Figure 3-2) by the project team using Expert Choice, decision support software to aid in a collective and justifiable software decision. The foundation of Expert Choice is the Analytic Hierarchy Process (AHP), "highly regarded and proven process designed to reflect the way people actually think".

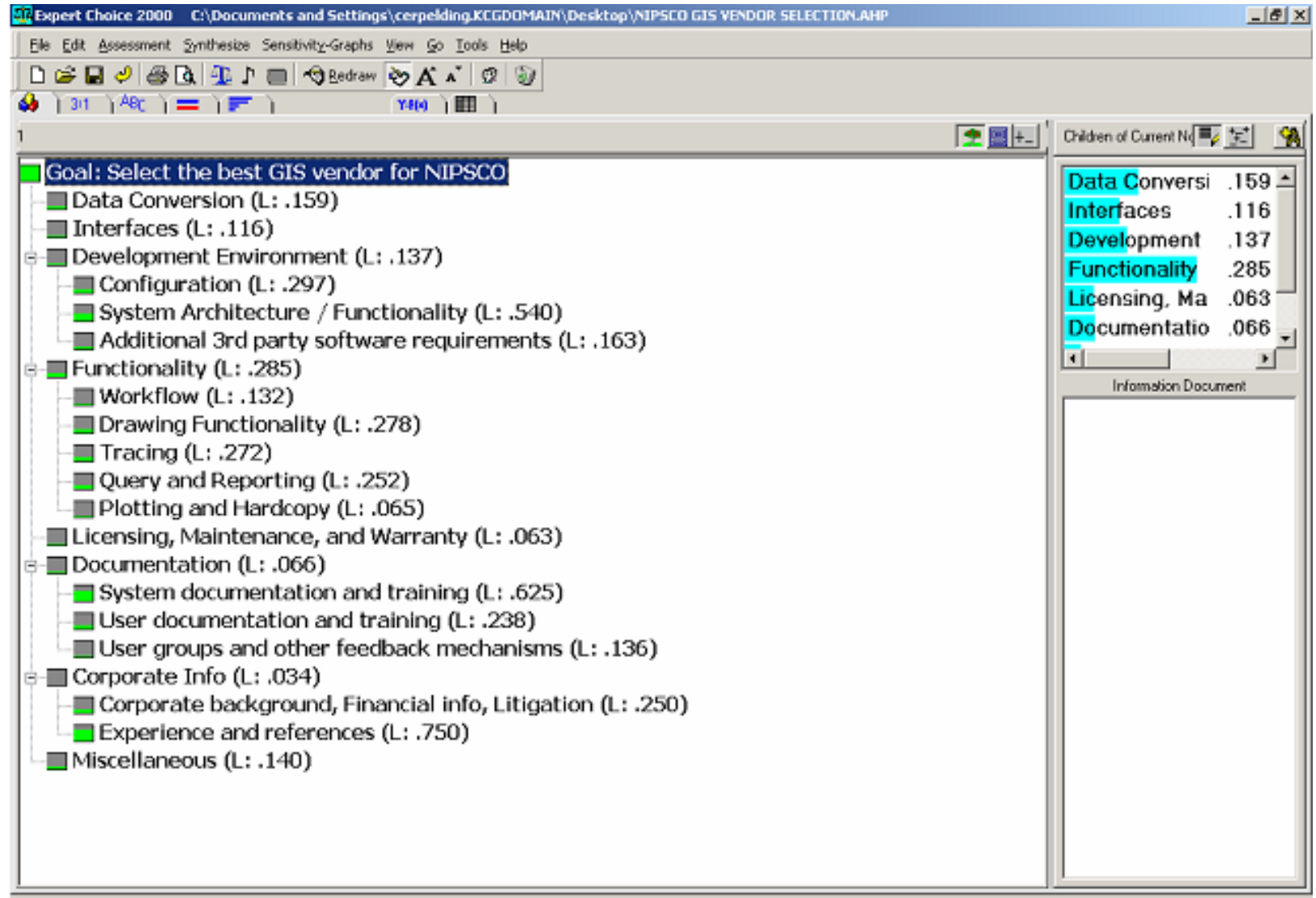

Figure 3-1. 


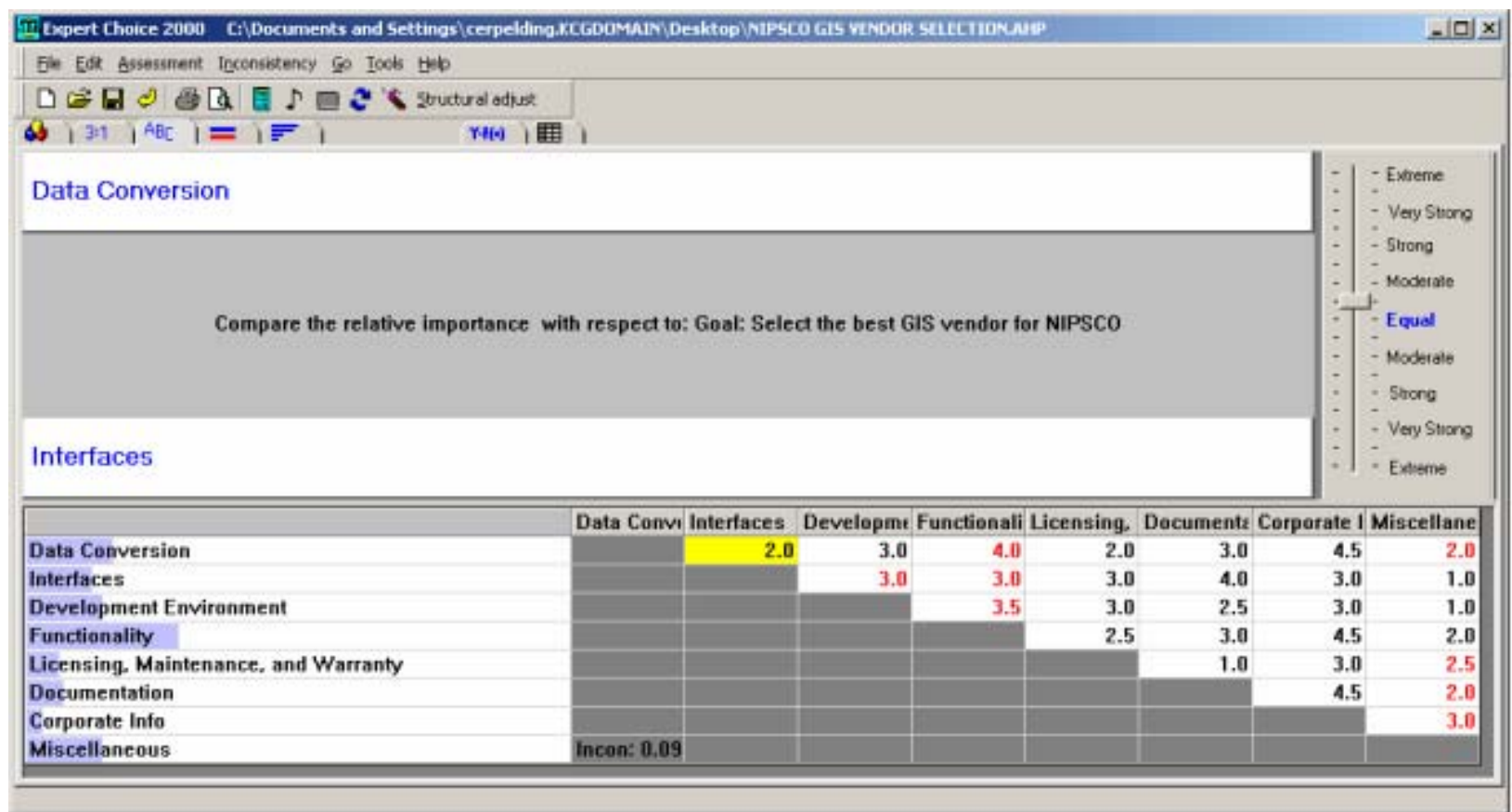

Figure 3-2.

Figure 3-3 shows the results of the pairwise comparisons that determined the most important criteria.

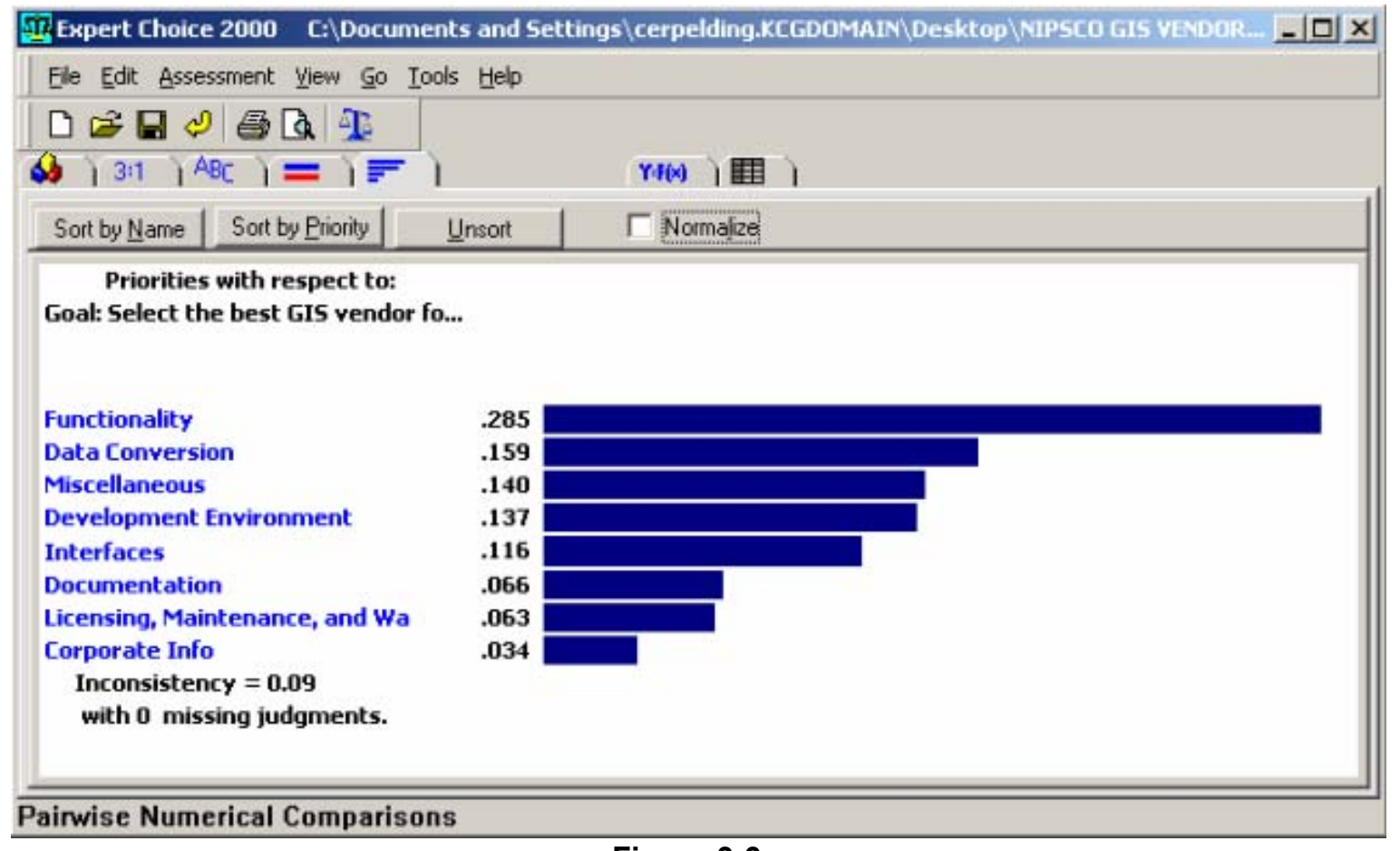

Figure 3-3. 
Next, vendor software was compared using the same scoring scale as the evaluation criteria (see Figure 3-4). Prior to each scoring session, roundtable discussion helped to bring out the positive and negative points of each vendor's software, which aided in the scoring process.

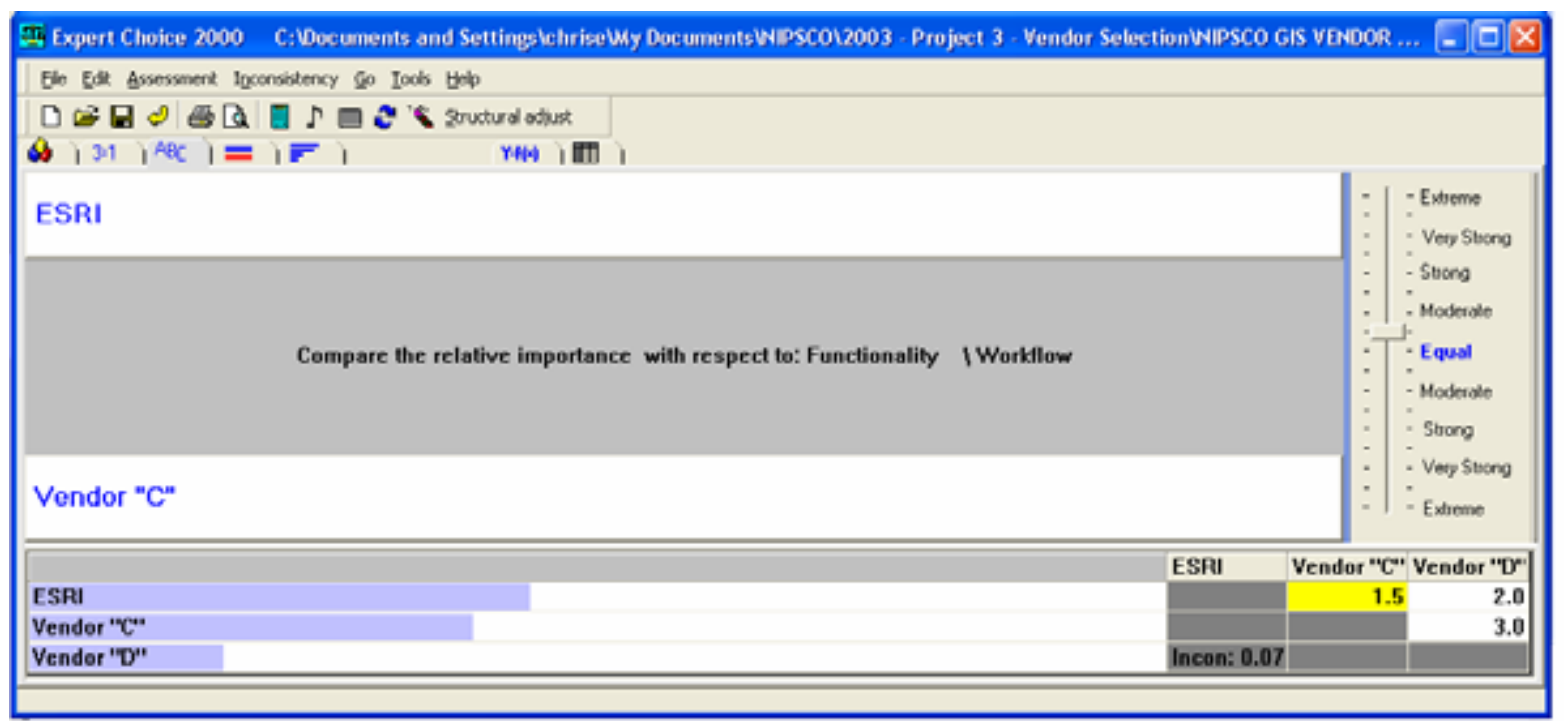

Figure 3-4.

The project team spent several days reviewing the responses and scoring the vendors and software. Scoring of criteria that was demonstration-specific was postponed until the vendors conducted the software demonstrations.

The project team reconvened after each vendor demonstration and, factoring in the feedback from the users who also attended the demonstrations, completed the scoring of the vendor software. In addition, a shortlist was established. Following the demonstration, it was made clear to the project team that Vendor " $\mathrm{A}$ " should be removed from the shortlist based on their response to the RFP and, finally the demonstration.

The graph in Figure 3-5 shows the performance of the remaining vendors based on the criteria. 


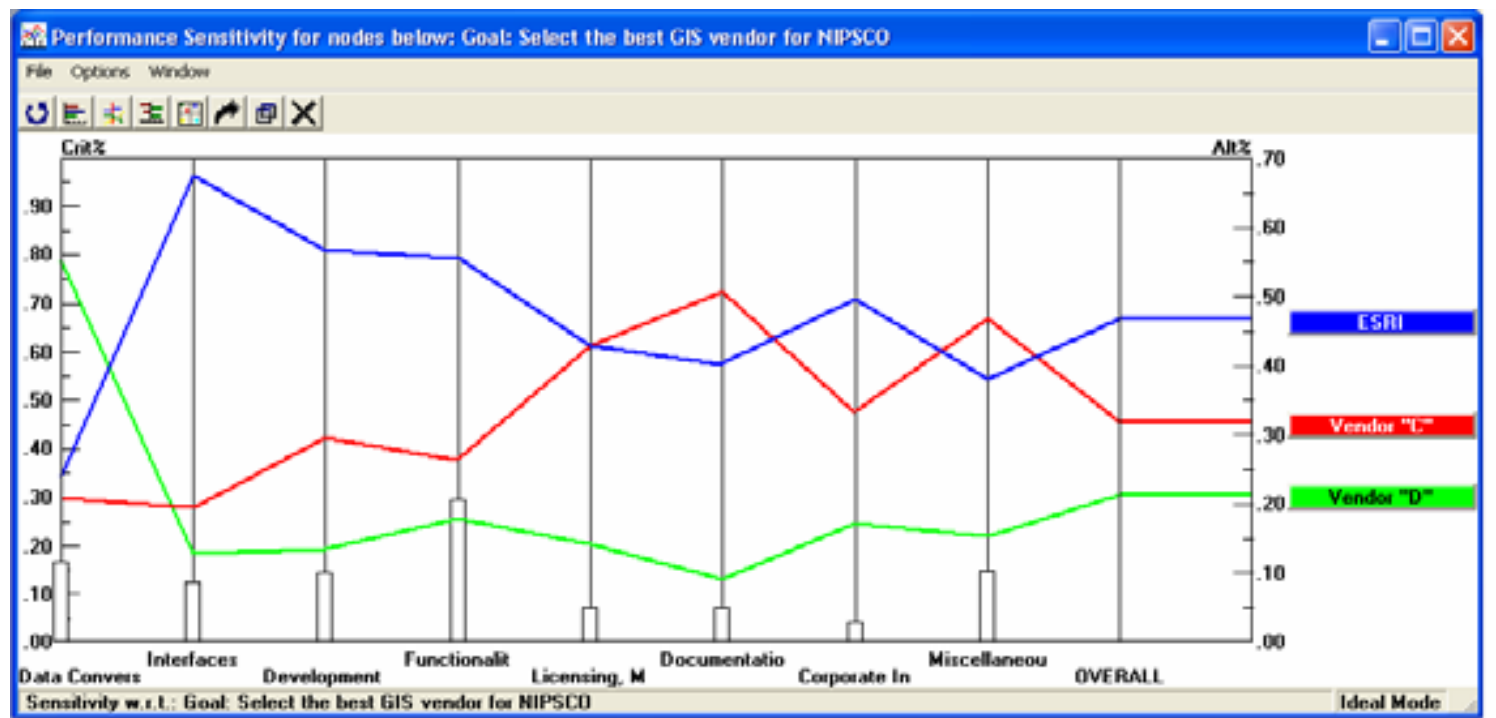

Figure 3-5.

Figure 3-6 represents the overall scoring percentage for each vendor. The weighted criteria are shown to the left.

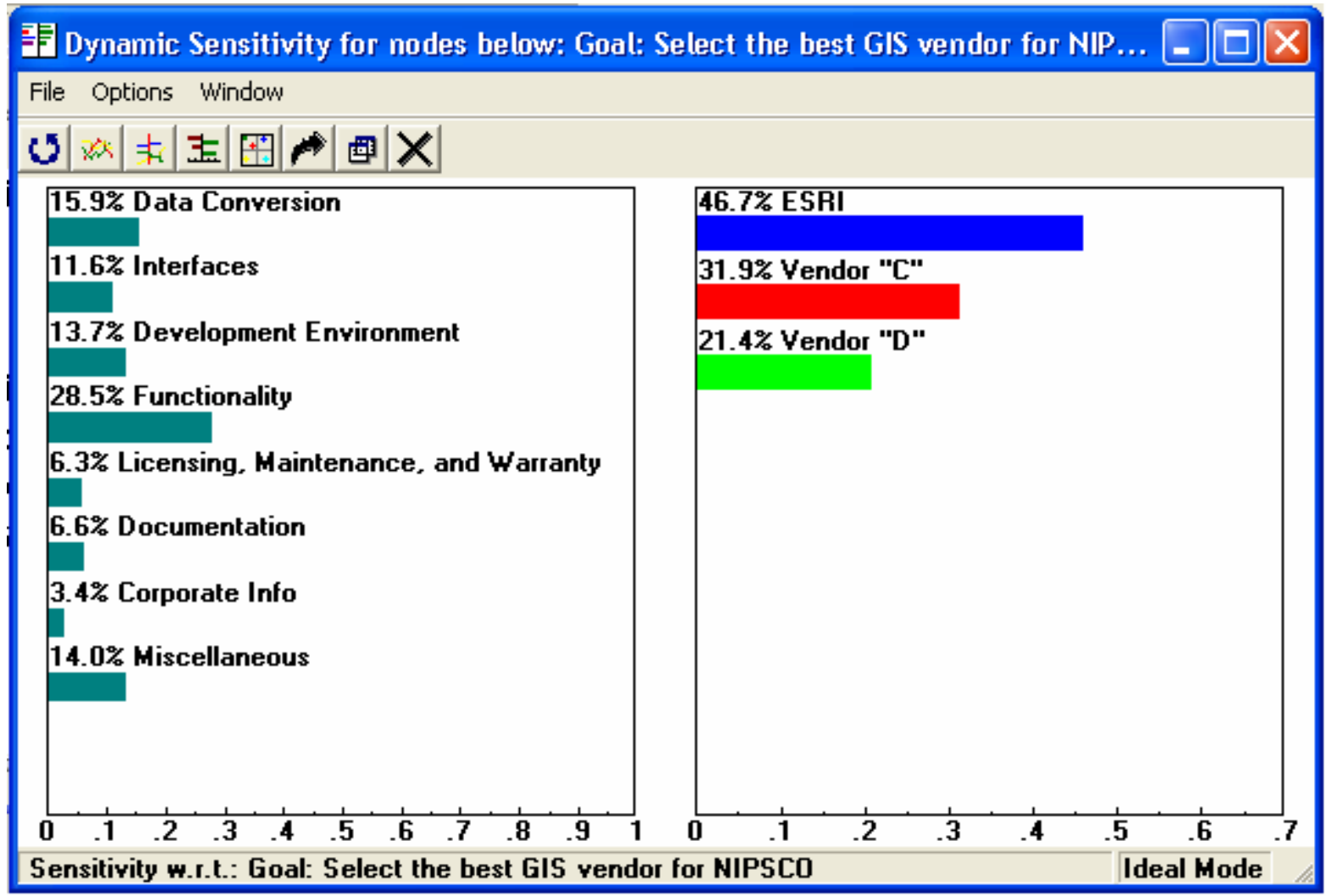

Figure 3-6. 
Finally, a value-per-dollar analysis was performed which, over a 5 year period, shows how each vendor fared in providing the most value for the dollar (see Figure 3-7a \& 3$7 b)$.

\begin{tabular}{|c|c|c|c|c|}
\hline & ESRI & Vendor " $\mathrm{C}$ " & Vender "D" & Notes \\
\hline \multirow[t]{2}{*}{ Expert Choice Score } & 0.467 & 0.319 & 0214 & Higher vilue is better \\
\hline & ESRI & Vendor ${ }^{\circ} \mathrm{C}^{\circ}$ & Vendor " $\mathrm{O}^{\circ}$ & \\
\hline \multirow[t]{2}{*}{ Normalized Cost Ratio } & 0.926 & 1.000 & 0.860 & Lower cost is better \\
\hline & ESRI & Vendor ${ }^{\circ} \mathrm{C}$ " & Vendor " $\mathrm{O}$ " & \\
\hline \multirow[t]{2}{*}{ Value per dollar ratio } & 0.504 & 0.319 & 0249 & Higher vilue per dollar is better \\
\hline & ESRI & Vendor " $\mathrm{C}$ " & Vendor " $\mathrm{O}$ " & \\
\hline \multirow[t]{2}{*}{ Dollars per value unit } & $\$ 1.90$ & $\$ 9.13$ & $\$ 4.02$ & Lower cost per value unit is better \\
\hline & ESRI & Vendor ${ }^{\circ} \mathrm{C}$ " & Vendor " $\mathrm{O}$ " & \\
\hline \multirow[t]{2}{*}{ How much more than the best value? } & $0.00 \%$ & $58.15 \%$ & $102.71 \%$ & Zero is the best value. The rest are $x \times \%$ more expensive per value unit. \\
\hline & ESRI & Vendor "C" & Vendor " $\mathrm{D}$ " & \\
\hline RANK & 1 & 2 & 3 & Which GIS Vendor is best for NIPSCO? \\
\hline
\end{tabular}

Figure 3-7a.

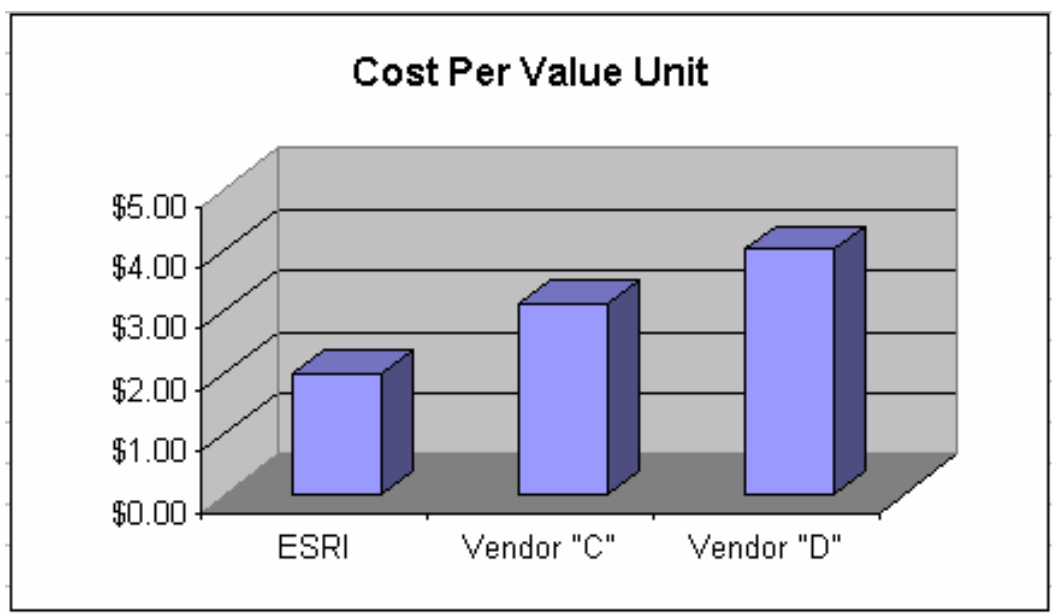

Figure 3-7b.

\subsection{Conclusion}

The GIS project team spent many hours:

- On user requirements, refining original user requirements, interviewing users for their updated requirements and designing an RFP that best describes NIPSCO's requirements

- Designing a demonstration script that allows the project team to accurately and fairly compare each vendor's product offering

- Reviewing and discussing responses to the RFP

- Attending and discussing vendor \& software performance at the demonstrations

- Scoring each vendor against the evaluation criteria via a pairwise comparison

ESRI not only ranked the highest in pairwise comparisons, but also provides the highest value per dollar over a 5 year period. See (Figure 3-7c). 


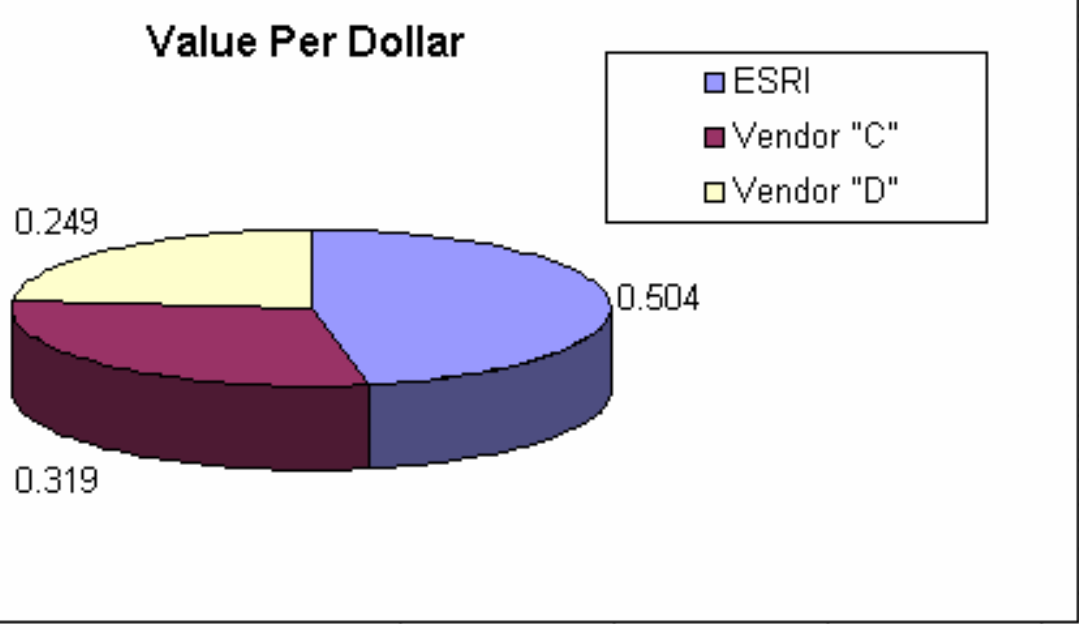

Figure 3-7c.

The project team has chosen ESRI as the preferred software vendor for all of the reasons described above.

The next steps were to negotiate a contract with ESRI; however should contract negotiations fail to adequately decrease the associated costs over time, Vendor " $\mathrm{C}$ " and Vendor "D" may have been revisited. 


\section{Immediate Conceptual Architecture (Phase I)}

\subsection{Detailed Functional Analysis}

This section describes the objectives of the detailed functional analysis that was performed during the various stages of the project along with the process that was used to perform the analysis. Completion of detailed functional analysis is key to the overall success of any project including user acceptance and change management.

Functional analysis began with a broad look at the project goals and then refinement of each area within the project with additional detail, functional requirements, software requirements, and finally a functional software design. As the process surrounding each area of the project became more detailed, it was tied back to the higher level components to ensure consistency, scope, and coverage.

\subsubsection{Objectives of Functional Analysis}

The objectives of performing functional analysis centered on defining what the new system will be and how it is expected to function to meet the requirements of the organization. The objectives of the AEDR functional analysis are defined as follows:

- Define High Level Goals and Scope - This high level vision serves as the core mission of the project and defines the true benefit of the project to upper management and the greater organization. The rest of the project initiatives ultimately contribute to meeting these goals and fit within this initial scope. These high level goals were developed based on input and agreement from the project sponsors and the key stakeholders/users.

- Functional Requirements - The high level goals and scope were broken down into functional areas. Each of these areas was defined by conducting workshops with the stakeholders and/or users that interact with the functional area. Many studies confirm that inadequate stakeholder/user involvement is a leading cause of failure in software projects. Getting the users involved at this early stage and keeping them involved throughout the development of software requirements was crucial.

- System Requirements - Depending on the functional area, this stage was often an exploratory activity. The core project team determined the required software functionality based on the documented functional requirements. Some requirements were met by out-of-the-box software while others required custom design and development. The proposed solutions were reviewed extensively with the stakeholders/users and refined as necessary. The end result was a wellrounded set of system requirements which defined the new system.

- Detailed Software Design - Once the software requirements were determined, refined, documented, and approved by both the users and the project team, the detailed software design process began. To the extent possible, the detailed design was created by the team member(s) with the most extensive knowledge of both the functional area and of the software technologies to be used. Each component 
of the design was tied either directly or indirectly to a software requirement. The design documents the way the software will be used to meet the requirement and the custom work that was completed. The level of detail in the design was sufficient to communicate what the end result will look like and how it will operate.

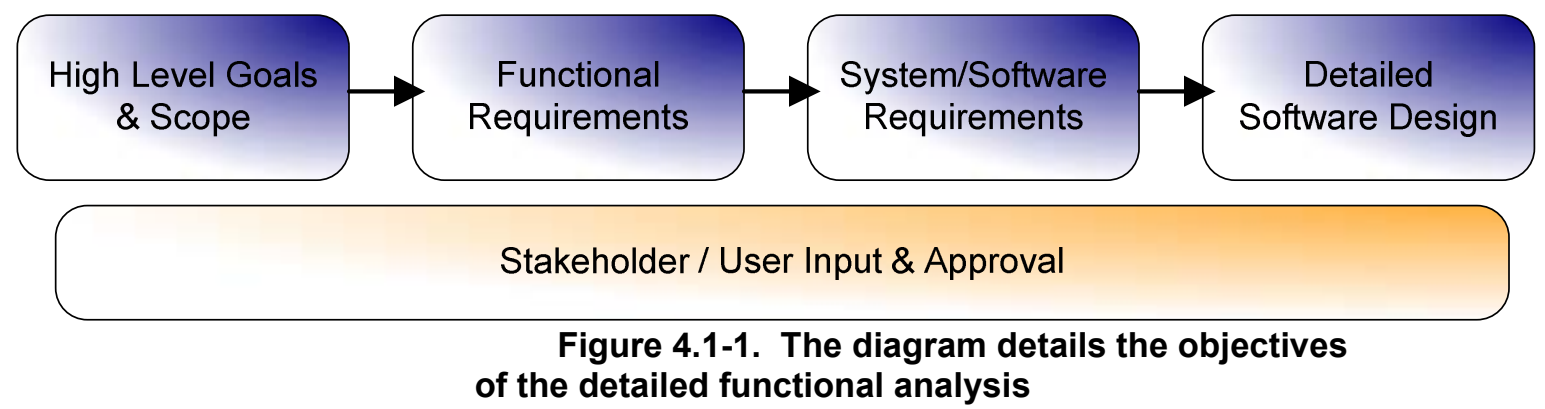

Effective requirements definition enabled the team to determine the mix of product capabilities that best delivered end user value. Adequately exploring and crafting requirements into a set of product features and attributes and refining these during early project work, helped to ensure that the end user needs were met throughout the project lifecycle.

\subsubsection{Project Scope}

The scope of Phase I was defined as:

- Implement a database-driven GIS solution that will manage all of NIPSCO's gas, electric and land-base objects

- Replace the core mapping maintenance product (Outfield), merge the Electric Distribution Facility Services (EDFS) data into the new GIS and maintain or improve the existing interfaces to the Customer Information System (CIS), Engineering Accounts Information File (EAIF), Material Accounts Payable and Purchasing System (MAPPS), Advantica Gas SynerGEE and ABB CADOPS

- Correct positional inaccuracies through a data rectification process

- Develop a stable system environment in which to develop and test the system

- Migrate the data to the new GIS platform

- Configure the software

- Customize the software as needed to improve user interaction

- Ensure adequate system performance

- Generate user-friendly help documentation

- Train the users, and

- Implement the Immediate Conceptual Architecture into the production environment rendering it useful to all.

\subsubsection{Functional Requirements}

The next phase of the functional analysis was to expand on the project goals and scope by identifying the functional areas that would be developed, conducting user and stakeholder interviews, and documenting functional requirements. The functional areas are listed with 
their requirements in Appendix D. Note: The functional requirements were reviewed and fine-tuned as described in Section 4.1.4 System / Software Requirements.

\subsubsection{Functional Requirements Summary}

Extensive interviews were conducted with the identified stakeholders and/or users for all of the above areas. From the interviews, use cases were developed to define the desired workflow and interaction in each of these areas. This then drove the creation of functional requirements which were organized and documented into spreadsheets. An individual worksheet was used for each functional area and the requirements were documented in the standard "The application shall..." format. The requirements were purely functional and contained no implementation details. These requirements were entered into a product called System Architect which generated Figure 4.1-2, the functional system diagram.

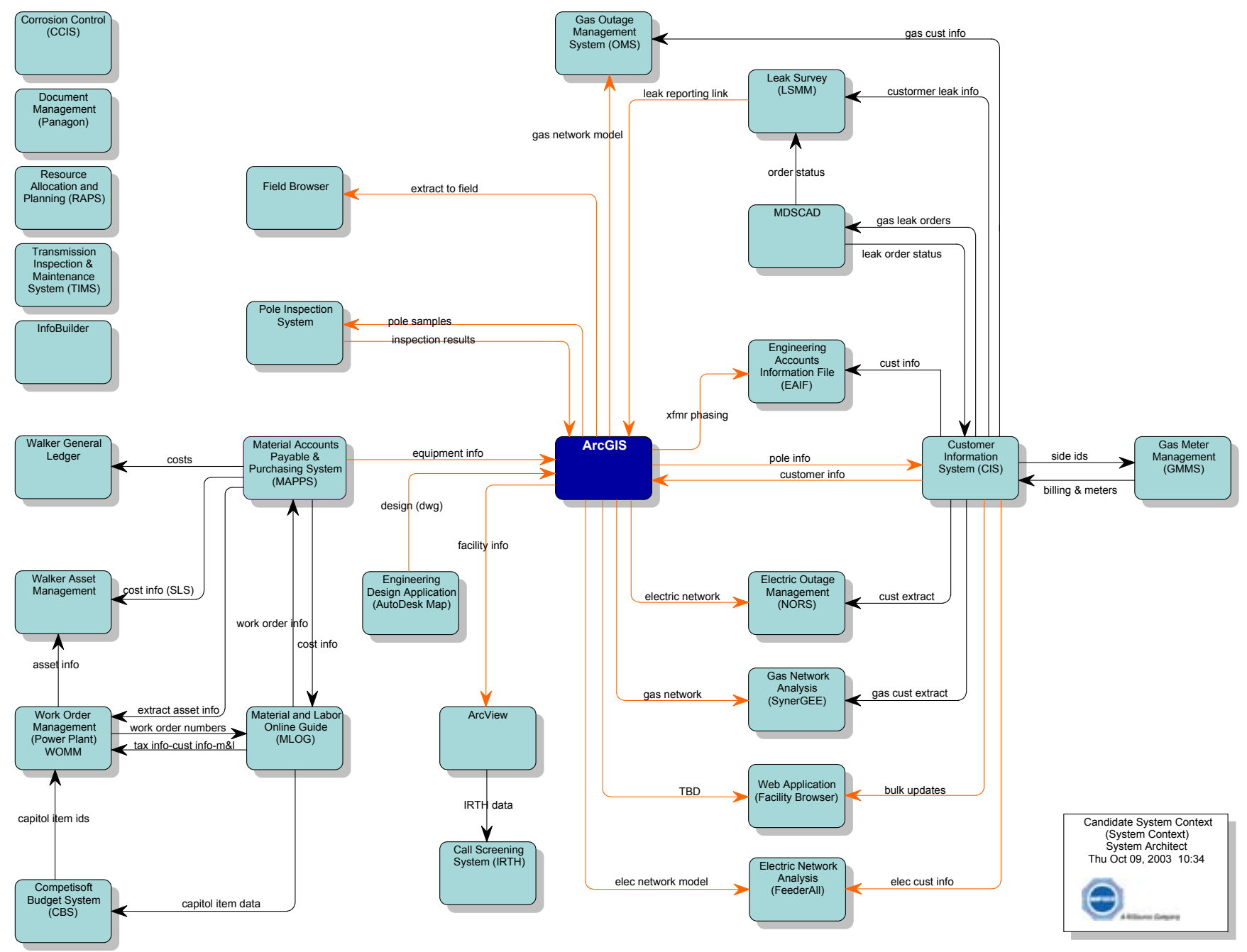

Figure 4.1-2. Functional requirements diagram 
There are several systems listed on this diagram that are not included within the functional areas of this project. These are included to completely define the systems surrounding this project.

\subsubsection{System / Software Requirements}

The process of defining the AEDR system requirements encompassed using the functional requirements to determine how a combination of out-of-the-box and custom software would meet those requirements. Several governing technology guidelines that had been established previously came into play at this point in the process:

- GIS Platform - ESRI (ArcGIS, ArcSDE, ArcIMS, etc)

- GIS Platform Extension for Utilities - Miner \& Miner ArcFM

- DBMS - Microsoft SQL Server

- Primary Development Language: Microsoft C\#.Net

- Gateway to Legacy Mainframe Systems: IBM DB2 Connect

- Application Deployment Environment: Citrix on Windows 2003 Server

- Web Server - Microsoft IIS

The various functional areas were broken up and a core project team member was assigned as the lead for each area. In some cases the system requirements were handled directly by the core project team while others were handled by third party vendors. In all cases, software-specific concepts were developed with input from the stakeholders / users and in the context of the above guidelines. Once agreed upon, these concepts provided the basis for the system requirements for each functional area which detailed how the system would operate, the intended user interaction, any custom development that was required, and which technological approaches would be used. Estimate documents were created for each task detailing a high level approach, the effort involved, pros, cons, and any risk areas. In some cases multiple estimate documents were created to present alternative approaches to satisfying the functional requirements.

The NIPSCO project team subsequently reviewed each of the estimate documents and made decisions as to how to proceed. The software construction tasks, including the creation of the detailed software design, were added to the project plan and assigned to the appropriate team members. The Table 4.1-1 defines the chosen system/software approach for each functional area:

Table 4.1-1. This table describes the final approach for the development of the AEDR based on the functional requirements

\begin{tabular}{|l|l|}
\hline Functional Area & Approach (determined from System Requirements) \\
\hline AutoCAD (Outfield) & $\begin{array}{l}\text { ArcGIS and ArcFM will largely fulfill the mapping requirements from } \\
\text { Outfield. Specific gaps have been identified and will be met by custom } \\
\text { development of ArcFM Autoupdaters (AU's) and tools to be made } \\
\text { available directly within the GIS. }\end{array}$ \\
\hline
\end{tabular}




\begin{tabular}{|c|c|}
\hline EDFS Replacement & $\begin{array}{l}\text { ArcGIS and ArcFM will partially fulfill the asset management requirements } \\
\text { of EDFS. The remaining requirements will be met by custom development } \\
\text { in three different areas: } \\
\text { - ArcFM Autoupdaters (AU's) and tools to be made available directly } \\
\text { within the GIS. } \\
\text { - A Standalone Geodatabase Editor (SAGE) website to allow for the } \\
\text { editing, management, and reporting of asset data outside of the } \\
\text { GIS. } \\
\text { - A suite of nightly batch applications to handle GIS maintenance, } \\
\text { processing, and interfaces with other systems. }\end{array}$ \\
\hline CADOPS (NORS) & $\begin{array}{l}\text { A customized version of the ArcFM Network Adapter product will be used } \\
\text { to export the electric network data from the GIS and to translate the data } \\
\text { into the format expected by the CADOPS load processes. }\end{array}$ \\
\hline FeederAll & $\begin{array}{l}\text { A customized version of the ArcFM Network Adapter product will be used } \\
\text { to export the electric network data from the GIS and to translate the data } \\
\text { into the format expected by the FeederAll load processes. }\end{array}$ \\
\hline EAIF & $\begin{array}{l}\text { The existing EAIF application will remain unchanged. The new GIS must } \\
\text { feed transformer installation data into the EAIF data stores during a nightly } \\
\text { interface between GIS SQL Server and EAIF DB2. }\end{array}$ \\
\hline SynerGEE & $\begin{array}{l}\text { A custom gas network export application will be written to trace out a gas } \\
\text { system based on specified parameters and to export the results in the } \\
\text { format expected by SynerGEE Middlelink which is responsible for loading } \\
\text { the data into SynerGEE. This custom export application replaces } \\
\text { SynerGEE's DataPrep. }\end{array}$ \\
\hline Facility Browser & $\begin{array}{l}\text { A custom ESRI ArcIMS (Internet Mapping Server) web site will be } \\
\text { developed to serve the GIS data via the NIPSCO intranet. The target } \\
\text { functionality will be defined by the current Map Info Facility Browser and } \\
\text { custom tools will be built as needed. }\end{array}$ \\
\hline Field Browser & $\begin{array}{l}\text { ESRI ArcReader will be used to display the NIPSCO data in a } \\
\text { disconnected format. ArcReader is free software and can display map } \\
\text { documents published directly from the back office ESRI GIS. }\end{array}$ \\
\hline MAPPS Interface & $\begin{array}{l}\text { The new GIS will interface to MAPPS by passing data back and forth } \\
\text { between GIS SQL Server and MAPPS DB2. The interface will include the } \\
\text { following components: } \\
\text { - Nightly synchronization and processing of MAPPS asset } \\
\text { transactions by the new GIS. } \\
\text { - Nightly synchronization of MAPPS Stores Item Number } \\
\text { Descriptions into the GIS. } \\
\text { - Near real-time synchronization of asset/unit data from the GIS into } \\
\text { MAPPS. }\end{array}$ \\
\hline IRTH & $\begin{array}{l}\text { ESRI ArcView will be used to provide full read-only access to the GIS } \\
\text { data. This will allow the Facility Locate Screeners to utilize the data for } \\
\text { their analysis requirements. }\end{array}$ \\
\hline
\end{tabular}




\begin{tabular}{|l|l|}
\hline MLOG Interface & $\begin{array}{l}\text { The new GIS will interface to MLOG on a nightly basis by synchronizing } \\
\text { Assembly Compatible Unit information from MLOG DB2 into GIS SQL } \\
\text { Server. ArcFM Autoupdaters (AU's) will be written to perform real time } \\
\text { validation of assembly numbers within the GIS. }\end{array}$ \\
\hline CIS Interface & $\begin{array}{r}\text { The new GIS will interface to the CIS on a nightly basis by passing data } \\
\text { back and forth between GIS SQL Server and CIS DB2. The interface will } \\
\text { include the following components: } \\
\text { Nightly synchronization of new and updated CIS Site data from } \\
\text { CIS into GIS. } \\
\text { Nightly synchronization of new and updated CIS Installed Service } \\
\text { data from CIS into GIS. }\end{array}$ \\
$\begin{array}{l}\text { Nightly synchronization of new transformer installations and } \\
\text { removals from the GIS into CIS. }\end{array}$ \\
$\begin{array}{l}\text { Nightly synchronization of new and updated pole and pad data } \\
\text { from the GIS into CIS. } \\
\text { Nightly synchronization of updated Installed Service X/Y } \\
\text { coordinate data from the GIS into CIS. }\end{array}$ \\
\hline DXF Export for Design & $\begin{array}{l}\text { The new GIS must be able to export AutoCAD DXF files with enough } \\
\text { detail for the engineering department to complete its work design process. }\end{array}$ \\
\hline Pipeline Integrity & $\begin{array}{l}\text { The new GIS should handle the external mandated requirements of } \\
\text { managing the integrity of NIPSCO's gas pipelines. }\end{array}$ \\
\hline
\end{tabular}

The chosen approach for each of the above components lead directly into the development of a detailed design. The detailed design for each of these components is defined later in this document within the appropriate area under the AEDR Customization and/or Interfaces sections. 


\subsection{Data Rectification}

\subsubsection{Data Rectification Overview}

NIPSCO's objective in pursuing the data rectification project was to improve the positional accuracy of its AutoCAD data prior to implementing a new GIS.

The landbase data within NIPSCO's legacy AutoCAD files was converted from sources of moderate positional accuracy and variable currency. The facility data within NIPSCO's legacy AutoCAD files was converted over the originally converted landbase data. Since the initial conversion, NIPSCO updated and maintained the land and facility data through the use of a variety of sources. The resultant AutoCAD data was therefore of varying positional accuracy. Some landbase and facility feature positions were essentially correct, many land and facility feature positions were modestly incorrect when compared to more accurate sources, and some are more dramatically misplaced.

\subsubsection{Imagery and Coordinate System}

NIPSCO examined a number of options with regard to obtaining a control target for the positional enhancement. In part because Digital Orthophoto Quarter Quadrangle (DOQQ) data provided for consistent comprehensive coverage of the NIPSCO service territory, DOQQ imagery was determined to be the most appropriate positional control source.

As part of the data rectification effort, the legacy source coordinate system and datum (Indiana State Plane West NAD27) was re-projected to the Indiana State Plane West NAD83 coordinate system.

\subsubsection{NIPSCO's Data}

NIPSCO's AutoCAD datastore was (and still is) composed of AutoCAD Map DWG files. Each file covers an area approximately 3 Public Land Survey Section (PLSS) x 3 PLSS. Each DWG file is uniquely identified with a 5-character name. Within the name, the first 2 characters identify the grid, the third and fourth characters identify the quadrant within the grid, and the last character identifies the file as a land, gas, or electric file. For example, the file named BFSEL.dwg is the land file covering the southeast quadrant of grid area $\mathrm{BF}$.

NIPSCO's land data is generally composed of road centerlines, approximated road Right of Ways (ROWs), street name annotation, bridges, easements, railroads, hydrography, political boundaries, political boundary annotation, company boundaries, company boundary annotation, a limited number of parcel/lot lines, and an assortment of miscellaneous lines, symbols and annotation for features such as power plants, shopping centers, schools, etc.

NIPSCO's land data is stored within $\sim 1361$ DWG files. 
NIPSCO's gas data is generally composed of mains, valves, regulator stations, pipe change symbols, dead end symbols, and associated annotation.

NIPSCO's gas data is stored within 862 DWG files.

NIPSCO's electric data contains structures, transmission facilities, substations, overhead $(\mathrm{OH})$ and underground (UG) primary facilities, UG secondary/streetlight/service conductors, $\mathrm{OH}$ primary facilities, and a subset of $\mathrm{OH}$ secondary/streetlight/service facilities.

NIPSCO's electric data is stored within 703 DWG files. In addition to the AutoCAD data, NIPSCO's electric facilities attribute data was stored within EDFS.

\subsubsection{Rectification Services}

A Request for Proposal (RFP) was developed to externally contract the effort of adjusting NIPSCO's data to achieve better positional accuracy. This RFP was a combined request for both data rectification and data migration services. Section 4.3 contains the migration process and services.

The size of this effort was significant in that NIPSCO did not have the manpower to achieve this task within the scope of this project and complete the implementation within the required timeframe. The rectification process was required to be completed prior to the data migration process. At different times during the rectification process, there were upwards of one hundred contracted personnel moving the data into better positional accuracy.

\subsubsection{Landbase Data Requirements}

NIPSCO's requirements for rectification of landbase data were as follows:

1. The delivered orthophoto imagery must be re-projected to the target projection, coordinate system, and datum.

2. All landbase features in the source data must be present in the deliverable rectified data.

3. Street intersections in the deliverable rectified landbase data shall be positioned such that the mathematical center of the vector-depicted intersection area is within $25^{\prime}$ of the visual center of the street intersection as depicted within the reprojected DOQQ imagery.

4. Road Right of Way (ROWs) in the deliverable rectified landbase data shall be positioned such that at no point along the road, does more than $50 \%$ of the roadway, as depicted in the re-projected DOQQ imagery, appear outside of the vector ROW depiction. This result must be achieved without materially altering the depicted width of the vector road ROW.

5. Except as appropriate and based on industry-standard application of cartographic displacement rules, hydrography features in the delivered rectified landbase data 
shall be positioned such that all feature vertices are located within $25^{\prime}$ of the visible hydrography feature in the re-projected DOQQ imagery. This result must be achieved without materially reducing the number or vertices in the feature and without materially altering the location of vertices along the feature.

6. Except as appropriate and based on industry-standard application of cartographic displacement rules, railroad features in the delivered rectified landbase data shall be positioned such that all vertices are located within $25^{\prime}$ of the visible railroad feature in the re-projected DOQQ imagery. This result must be achieved without inappropriately reducing the number or vertices in the feature and without inappropriately altering the location of vertices along the feature.

7. Rectified bridge locations shall be appropriately related to rectified locations for any associated road ROW, railroad (RR), and hydrographic features.

8. Political and company boundaries within the deliverable rectified landbase data shall be positioned such that any coincidence with planimetric or other boundary features in the source data is preserved in the deliverable data. In areas where political or company boundaries are not coincident with other features, rectification of the boundary locations shall be rationally consistent with the rectification of proximate planimetric features.

9. Rectified easement locations shall be appropriately related to rectified locations for any associated land and facility features.

10. Rectified miscellaneous landbase feature locations shall be appropriately related to rectified locations for any related or proximate landbase and facility features.

11. Within the deliverable rectified landbase data, annotation associated with any landbase feature shall be positioned such that its positional relationship to its associated feature is essentially the same as that in the source data. In cases where landbase annotation is not associated to a specific feature, its rectified position shall be rationally consistent with the rectification of proximate landbase features. Annotation that did not overstrike other features or annotation in the source data shall not overstrike features or annotation in the deliverable data.

12. The relative locations of discrepancy flags to their associated feature(s) shall be preserved in the rectified deliverable data.

13. All landbase feature and network connectivity in the source data, whether characterized by positional coincidence, network topology, or both, must be preserved in the deliverable data. This requirement includes preservation of graphic connectivity (snapping) at source Outfield/AutoCAD files edges.

14. All feature attribute values in the source data must be preserved in the deliverable data.

NIPSCO's requirements for rectification of facility data were as follows:

1. All facility features in the source data must be present in the deliverable rectified data. 
2. Facility features located within any street intersection in the source data must be located within the same rectified street intersection in the deliverable data. Additionally, the logical configuration and relative positional relationships of all facilities within any street intersection must be preserved in the deliverable rectified data.

3. The positional relationships of facility features to land features in the source data must be preserved in the deliverable rectified data. Facility features located within a road ROW in the source data shall be located within the same road ROW in the deliverable rectified data. Facility features located outside the road ROW in the source data shall be located outside the ROW in the deliverable rectified data. Additionally, the logical location of facilities within or just outside of a road ROW shall be preserved within the deliverable rectified data. For example, pole symbols shown adjacent to the southern edge of a ROW in the source data shall be adjacent to the southern edge of the road ROW in the deliverable data, and gas mains shown running near the eastern edge of a road ROW in the source data shall run near the eastern edge of the road ROW in the deliverable rectified data. Further, facility features shown as proximate to a significant landbase feature in the source data must be similarly located in the deliverable rectified data. For example, a pole shown in the source data as being located at a significant curve in a road must be similarly depicted in the deliverable rectified data.

4. Facility features shown in the source data as located along a road but between two significant landbase features must be located in the deliverable rectified data based on the $(+/-10 \%)$ proportionate relationship of the source and target distances between the two closest significant landbase features.

5. The rectification of facility features which are not located within or along road ROWs shall be rationally consistent with the rectification of proximate planimetric and facility features.

6. Within the deliverable rectified facility data, annotation associated with any facility feature shall be positioned such that its positional relationship to its associated feature is essentially the same as that in the source data. In cases where facility annotation is not associated to a specific feature, its rectified position shall be rationally consistent with the rectification of proximate facility features. Annotation that did not overstrike other features or annotation in the source data shall not overstrike features or annotation in the deliverable data.

7. All facility feature and network connectivity in the source data, whether characterized by positional coincidence, network topology, or both, must be preserved in the deliverable data. This requirement includes preservation of graphic connectivity (snapping) at source AutoCAD files edges.

8. All feature attribute values in the source data must be preserved in the deliverable data.

9. In addition to complying will all facility data rectification requirements listed above, whenever any facility feature is clearly located incorrectly within an AutoCAD Map source DWG file (i.e. when a pole is located within a water body, 
highway, or building), the Contractor will be required to use their best judgment in modifying the facility location to correct the problem, and to place a discrepancy flag.

\subsubsection{Pilot Project}

Prior to the initiation of full production data rectification work, the contractor was required to undertake and successfully complete a pilot data rectification effort. The objectives of the pilot project were as follows:

- To test the project's documented requirements and specifications and serve as a contained environment within which the requirements and specifications can be refined

- To prove the understandings and capabilities of the contractor

- To allow the contractor to refine its methodology and procedures prior to the initiation of full production work

- To allow both NIPSCO and the contractor to test the source and deliverable data processing procedures and to make any appropriate refinements

- To allow both NIPSCO and the contractor to test the project's administrative procedures and to make any appropriate refinements.

For the purposes of full production data rectification work, NIPSCO divided the source and deliverable data into a series of data batches. Each batch was composed of a contiguous area of land, gas, and electric data. NIPSCO utilized its twelve Local Operating Areas (LOAs) in the definition of data rectification batches.

Delivered rectified data was subjected to a series of automated and interactive quality acceptance tests. Acceptance or rejection of the delivered data was determined based upon the results of the acceptance tests. The testing regime combined 100\% examination relative to certain acceptance criteria and examination of a random sample of the delivered data relative to certain other acceptance criteria.

NIPSCO utilized the following criteria in determining the acceptability of each data rectification delivery:

\section{Criteria tested through $100 \%$ Verification}

- Delivery Format Compliance - 100\% Accuracy Required

- System Compatibility/Data Loadability - 100\% Accuracy Required

- Delivery Completeness - 100\% Accuracy Required

- Peripheral Deliverable Report Presence

- Data Delivery Extents

\section{Criteria tested through Random Sample Verification}

- Landbase Feature and Annotation Position - 98\% Accuracy Required 
- Gas Feature and Annotation Position - 98\% Accuracy Required

- Electric Feature and Annotation Position - 98\% Accuracy Required

\subsubsection{Data Rectification Process}

Project preparation began in June 2004. A three day workshop was held with the vendor for the purposes of vendor education. Throughout the next six months, rectification rules were refined, automated QA processes were developed and tested, QA procedures were established, the NIPSCO QA team was trained, ancillary issues were identified and processes established to manage the rectification process. Modifications to the preparation planning continued for the next six months.

The pilot project began three months into the project preparation phase. Refinements continued throughout the next two months until the pilot was completed.

Following the pilot project, the remainder of the rectification process commenced. Data was "frozen" in batches in the legacy production environment, rectified, QA'd and returned to the production datastore after passing the QA. This process continued until the entire NIPSCO AutoCAD DWG files were rectified and placed back into production. The details of the process are shown in Appendix A-2 under the Rectification Section.

\subsubsection{Data Rectification Conclusion}

The data rectification process was difficult, cumbersome and required a full-time resource committed to ensuring the contractor delivered quality data, delivered the data as defined within schedule and addressed the quality concerns.

Overall, NIPSCO's data was re-projected to the correct coordinate system, and the positional accuracy has been much improved. 


\subsection{Data Migration}

\subsubsection{Overview}

Data migration entails moving NIPSCO's source land, gas and electric data from the legacy format into NIPSCO's ArcGIS/ArcFM data model. More specifically, the migration of graphic and attribute data from the AutoCAD files, partially replicated Microsoft Access database and the EDFS database to NIPSCO's ArcGIS/ArcFM data model to support loading the data to NIPSCO's future ArcGIS/ArcFM system and the use of the data within that system. Additionally, the migrated data must comply with the ArcGIS/ArcFM system's symbology and topological and facility feature connectivity requirements.

NIPSCO worked with its software and service supplier partners to design, develop, and configure the ArcGIS/ArcFM system and the associated data model. Following completion of the data model development and ArcGIS/ArcFM configuration work, NIPSCO's existing source data was migrated to the ArcGIS/ArcFM geodatabase format.

The data migration and data rectification processes required significantly more effort than could be accomplished by the GIS project team developing AEDR, and therefore was packaged together as an external contracted data migration/data rectification component.

The fundamental deliverables associated with the data migration effort was land, electric, and gas data in ArcGIS/ArcFM geodatabase format. Since the data migration and data rectification tasks are closely linked, there is some overlap in some sections of this report. Section 4.2 describes the rectification effort.

\subsubsection{The Data}

NIPSCO's source data was housed in the following datastores:

- AutoCAD DWG files

- Microsoft Access database - subset of the AutoCAD files (containing some of the same information but in an MS Access format)

- Electric Distribution Facilities System (EDFS) data stored in mainframe DB2 (relational database) format

The challenge was to determine which of the two data stores (AutoCAD/EDFS) contained the most accurate information, eliminate the duplicate data, report on mismatched information for future investigation and cleanup, and migrate the accurate data to the target.

The EDFS served as a consolidated and centralized repository for pole card data, transformer life record card data, underground electric facilities data, street light location and dusk-to-dawn lighting record cards. EDFS' ability to interface with the customer information, material and supply, material and labor estimating, wood pole treatment and 
general ledger account validation systems eliminated numerous entries of duplicate information.

Operations as well as several user departments had access to this system for preparing monthly and/or annual property unit reports, transformer history and test data, pole treatment and reinforcement data and environmental documentation. User departments benefiting from EDFS include: Purchasing, Material Services, Environmental, Transformer Shop, Rate Base, General Accounting, Resource Planning and Engineering. EDFS data was used in conjunction with AutoCAD data in the migration of electric facilities data to the ArcGIS/ArcFM system.

\subsubsection{Land Data}

NIPSCO's land data is generally composed of road centerlines, approximated road ROWs, street name annotation, bridges, easements, railroads, hydrography, political boundaries, political boundary annotation, company boundaries, company boundary annotation, a limited number of parcel/lot lines, and an assortment of miscellaneous lines, symbols and annotation for features such as power plants, shopping centers, schools, etc.

The land data was stored within $~ 1361$ DWG files within the AutoCAD system.

\subsubsection{Gas Data}

NIPSCO's gas data is generally composed of mains, valves, regulator stations, pipe change symbols, dead end symbols, and associated annotation.

The gas data was stored within $\sim 862$ DWG files within the AutoCAD system.

\subsubsection{Electric Data}

NIPSCO's electric data contains structures, transmission facilities, substations, $\mathrm{OH}$ and UG primary facilities, UG secondary/streetlight/service conductors, OH primary facilities, and a subset of $\mathrm{OH}$ secondary/streetlight/service facilities.

The electric data was stored within $\sim 703$ DWG files within the AutoCAD system. In addition to the AutoCAD data, NIPSCO's electric facilities attribute data is stored within EDFS.

\subsubsection{The Data Migration Process}

Data migration specifications were developed using a migration matrix (spreadsheet) of source to target directions for the data. Finely detailed specifications were developed for derived data processes, that is those processes that were not simply a one for one move, but involved merging and or conversion of the data into a different format.

Miscellaneous migration specifications such as device edge splitting requirements, geometry alterations and secondary conductor migration were developed in addition to the migration matrix and included with each delivery of data to the migration vendor. 


\subsubsection{The Iterative Approach to Data Migration}

The migration process followed the Rational Unified Process (RUP) iterative process methodology.

NIPSCO worked with the data migration vendor to refine and demonstrate the suitability of the data migration specifications and processes through the following iterative approach to data migration:

- The pilot was the first of several iterative demonstrations of the readiness of the data migration specifications and processes. The pilot data migration entailed the migration of a subset of significant land, gas and electric features and objects within a contained geographic area

- The second data migration iteration entailed the migration of all land, gas and electric feature and objects contained within a geographic area. The objectives of the second migration were to:

1. demonstrate that any refinements to the specifications identified through iteration one have been incorporated in the migration

2. provide migrated data of all feature and object types

3. demonstrate the functionality of the full production data migration process and technology

4. allow for the identification of necessary refinements to the migration specifications and technologies

- The third data migration iteration entailed the migration of all land, gas, and electric feature and object types within NIPSCO's complete service territory. The vendor was required to use the planned full migration process and technology solution in accomplishing iteration three data migration. The objectives of the third migration iteration:

1. demonstrate that any refinements to the specifications identified through iteration two have been incorporated in the migration processes and technologies

2. demonstrate the functionality and performance of the full production data migration process and technology

3. allow for the identification of further requirements for refinement of the migration specifications and technologies

- The fourth data migration iteration entailed the migration of all land, gas and electric feature and object types within NIPSCO's complete service territory. The vendor was required to use the planned full migration process and technology solution in accomplishing iteration four data migration. The objectives of the fourth migration iteration were to:

1. demonstrate that any refinements to the specifications identified through iteration three have been incorporated in the migration processes and technologies

2. demonstrate the functionality and performance of the refined full production data migration process and technology

3. allow for the identification of any final requirements for refinement of the migration specifications and technologies 
- The final data migration iteration involved the migration of all NIPSCO land, gas and electric data from the AutoCAD/EDFS format to the ArcGIS/ArcFM geodatabase format. The effort was not to commence until the following conditions were met:

1. development of the ArcGIS/ArcFM system and its interfaces have been appropriately completed

2. the suitability of the data migration specifications and processes have been assured

3. full production data rectification work has been completed.

\subsubsection{Acceptance Criteria}

Delivered migrated data was subjected to a series of automated and interactive quality acceptance tests. Acceptance or rejection of the delivered data was determined based upon the results of the acceptance tests. The testing regime was to combine $100 \%$ examination relative to certain delivery characteristics and examination of a random sample of the delivered data relative to certain other delivery characteristics.

Criteria used in the examination and acceptance testing of each of the iterative data migration deliveries was defined to be consistent with the stated and agreed objectives for the relevant iteration, with the recognition that the overall objective of the iterative approach was to confirm and demonstrate appropriate incremental improvement.

Iterative migration deliveries demonstrating a clear failure to meet the objectives of the relevant iteration were rejected.

Requirements for improvement of the migration process or technology that were identified through review of any preceding iterative delivery that were not demonstrated within a subsequent iterative delivery were rejected.

NIPSCO utilized the following criteria in determining the acceptability of the final full data migration delivery:

Criteria tested through $100 \%$ Verification

- Delivery Format Compliance - 100\% Accuracy Required

- System Compatibility/Data Loadability - 100\% Accuracy Required

- Delivery Completeness - 100\% Accuracy Required

- Peripheral Deliverable Report Presence

- Data Delivery Extents

- Automated Integrity Verification - 100\% Accuracy Required

- Feature and Object Quantities

- Attribute Validity

- Feature and Object Relationship Validity

- Topological and Facility Network Connectivity Validity 
Criteria tested and scored separately for land, gas, and electric data, through Random $\underline{\text { Sample Verification }}$

- Feature and Object Presence - $100 \%$ Accuracy Required

- Attribute Accuracy - 99.5\% (of Features and Objects) Accuracy Required

- Feature and Object Relationship Accuracy - 99.5\% (of Features and Object) Accuracy Required

- Connectivity Accuracy - 99.5\% (of Features) Accuracy Required

\subsubsection{Data Migration Requirements}

NIPSCO's AutoCAD data served as the sole data source for the migration of landbase data to NIPSCO's ArcGIS/ArcFM system. The mapping of features, objects, and attributes between the AutoCAD landbase files and NIPSCO's ArcGIS/ArcFM data model was described within the Land Data Migration matrix. The matrix included source and target definitions as well as rules to be employed in the migration of the data. The vendor was advised that even though AutoCAD data is the only source to be used in the migration of landbase data, the vendor was required to deliver landbase data that fully complies with the structural, topological, and relational requirements of NIPSCO's ArcGIS/ArcFM system and data model. Given this requirement, the vendor was required to segment, aggregate, associate, and/or manipulate the landbase data in order to create appropriately segmented, joined, related, connected, and populated GIS features and objects.

\subsubsection{Landbase Data Migration}

NIPSCO's AutoCAD data served as the sole data source for the migration of landbase data to NIPSCO's ArcGIS/ArcFM system. The mapping of features, objects, and attributes between the AutoCAD landbase files and NIPSCO's ArcGIS/ArcFM data model was described within the Land Data Migration matrix. The matrix included source and target definitions as well as rules to be employed in the migration of the data. The vendor was advised that even though AutoCAD data is the only source to be used in the migration of landbase data, the vendor was required to deliver landbase data that fully complies with the structural, topological, and relational requirements of NIPSCO's ArcGIS/ArcFM system and data model. Given this requirement, the vendor was required to segment, aggregate, associate, and/or manipulate the landbase data in order to create appropriately segmented, joined, related, connected, and populated GIS features and objects.

\subsubsection{Gas Facilities Data Migration}

NIPSCO's AutoCAD data served as the sole data source for the migration of gas facilities data to NIPSCO's ArcGIS/ArcFM system. The mapping of features, objects, and attributes between the AutoCAD system gas files and NIPSCO's ArcGIS/ArcFM data model was described within the Gas Data Migration matrix. The matrix included source and target definitions as well as rules to be employed in the migration of the data. The vendor was advised that even though Outfield system data is the only source to be used in the migration of gas facility data, the vendor was required to deliver gas data that fully complies with the structural, topological, and relational requirements of NIPSCO's 
ArcGIS/ArcFM system and data model. Given this requirement, the vendor was required to segment, aggregate, associate, and/or manipulate the gas facility data in order to create appropriately segmented, joined, related, connected, and populated GIS features and objects.

\subsubsection{Electric Facilities Data Migration}

NIPSCO's AutoCAD data and EDFS data served as the fundamental data sources for the migration of electric facilities data to NIPSCO's ArcGIS/ArcFM system.

The following principles describe the use of AutoCAD and EDFS data in the electric facilities data migration process:

- For active electric facilities, feature presence and location was migrated based exclusively on AutoCAD graphics.

- EDFS records for retired facilities served as the source for the migration of these retired facilities to the object class within the ArcGIS/ArcFM database.

- For active electric facilities, match-keys were used to associate AutoCAD and EDFS records. AutoCAD/EDFS mismatches were reported.

- Since retired facilities are recorded only in EDFS, no mismatch reporting related to retired facilities were required.

- Rules within the Electric Data Migration matrix were used to populate feature and object attributes, and to govern creation and population of child records and relationship attributes from AutoCAD and matched EDFS data sources.

- GIS Connectivity will be set based on AutoCAD connectivity and ArcGIS/ArcFM rules.

The mapping of features, objects, and attributes between the AutoCAD system electric files and NIPSCO's ArcGIS/ArcFM data model was described within the Electric Data Migration matrix. The matrix included source and target definitions as well as rules to be employed in the migration of the data.

The vendor was advised that in addition to using AutoCAD and EDFS data in the migration of electric facility data, the vendor was also be required to deliver electric data that fully complies with the structural, topological, and relational requirements of NIPSCO's ArcGIS/ArcFM system and data model. Given this requirement, the Contractor may be required to segment, aggregate, associate, and/or manipulate the electric facility data in order to create appropriately segmented, joined, related, connected, and populated GIS features and objects.

\subsubsection{AutoCAD-EDFS Match Keys and Mismatch Reporting}

The migration of electric facility data required the association and integration of data currently stored in NIPSCO's AutoCAD and EDFS systems. Both Match Keys and special relationships were used to associate individual electric facility features in 
AutoCAD with each other, to associate individual records stored in EDFS with each other, and to associate features stored in AutoCAD with records stored in EDFS.

In addition to delivering migrated ArcGIS/ArcFM data, the vendor was required to deliver reports detailing feature-level mismatches between the AutoCAD and EDFS source data. These reports describe AutoCAD features that could not be matched to EDFS records and EDFS records (for active electric facilities) for which no corresponding AutoCAD feature was identified.

\subsubsection{Data Migration Plan}

A highly detailed data migration plan was developed during migration delivery four and followed for each migration delivery thereafter. The project team was required to review and update the migration plan prior to it's execution for each migration iteration. The Plan provided direction and helped to ensure that no steps were missed during the complex preparation of the data and environment for each migration delivery.

\subsubsection{QA (Quality Assurance) Process}

The Quality Assurance Plan Checklist in Appendix B was also developed during the fourth migration delivery that guided the QA process to ensure that no steps were missed and also served as a timing mechanism for each subsequent QA process as the timeframes were recorded on the QA Plan spreadsheet. QA team members were required to record their start and stop times and note any comments on the sheet. This Plan proved highly valuable in determining subsequent QA timeframes.

The following set of tracklists (in spreadsheet form) were developed to guide and document the QA process:

- $\quad$ Automated QA

- Connectivity QA

- Interactive QA

- Object Existence QA

- Ready for Review QA

\section{Automated QA}

A suite of automated test routines were developed to help quickly identify data anomalies such as null or un-populated fields, invalid data relationships, invalid or null domain fields, etc. All failed tests were loaded into the Automated QA tracklist, reviewed and commented on. Some failures result from unpopulated source data, others result from data not being populated correctly elsewhere such as in the relationships which may have an impact on multiple data fields. Others were valid failures and required attention.

\section{Connectivity QA}

For electric, all circuits were verified via "Trace All Feeders" and for gas, "Find Connected" and "Find Stopping Trace" were run to determine connectivity errors. 


\section{Interactive QA}

Interactive QA employs a visual inspection of a sample of the data. Data was migrated in batches by the vendor so the Interactive QA entailed a visual inspection of a sample of data from each batch. All features in the geodatabase were visually inspected and documented. A minimum of nineteen feature occurrences, one for each batch, were visually inspected.

\section{Object Existence QA}

This high level QA commenced upon loading the data into NIPSCO's ArcGIS/ArcFM database and was typically the first QA process to be exercised. If the Object Existence QA contained any failures, the delivery was rejected on the basis that it did not meet the $100 \%$ presence criteria. The remainder of the QA effort for that delivery was subsequently cancelled until the vendor re-delivered a correctly migrated set of data.

\section{Ready for Review QA}

Data migration corrections from past deliveries were immediately inspected following the passing of the Object Existence QA. All corrections requested from the vendor were documented and tested prior to moving on to the rest of the QA process.

\subsubsection{Conclusions}

The third and fourth data migrations revealed that the migration process was not progressing as intended. During the third and fourth iteration, the QA routines had been significantly improved and it was realized that the vendor's migration process would require substantial refinement.

Data migration was very difficult from both the NIPSCO and vendor perspectives. Prior to the last migration delivery, it was determined that, the segmenting, aggregating, associating, and/or manipulation of the data in order to create appropriately segmented, joined, related, connected, and populated GIS features and objects along with some fairly simple migration proved too challenging for the migration vendor. In order to complete the migration and implement the AEDR, NIPSCO internal resources wrote in excess of forty migration routines that migrated NIPSCO's data where the migration vendor could not. 


\subsection{ArCFM / ESRI Configuration}

ArcFM and ESRI Configuration consisted of configuring a combination of the out-of-thebox and custom ArcFM Properties, ArcFM Snapping, ESRI Relationship Rules, and ESRI Connectivity Rules. While this exercise may seem trivial on the surface, the time invested in these tasks has an enormous impact on the end user experience as well as the quality and integrity of the data managed by the systems. The participants in the base configuration included core team members, end user representatives, and consulting and product vendors who had specialized knowledge in the conceptual and practical implementation of the configuration.

\subsubsection{ArcFM Properties Manager}

\subsubsection{Overview}

ArcFM is highly configurable which allows the out of the box tools to be utilized on a variety of different data models. The configuration is achieved with an extension to the ESRI ArcCatalog application called ArcFM Properties Manager. The configurable components of the ArcFM Properties Manager are discussed briefly below.

\subsection{Field Display}

The Field Display tab is used to change the order of the attribute fields as they are seen on all of the ArcFM tools including the Attribute Viewer/Editor, Attribute Locator, etc. It is also used to assign a Display Name Object or to change the ArcFM Display Field. Both of these options control how the feature/object is represented in the ArcFM tools when only summary identification is shown. 


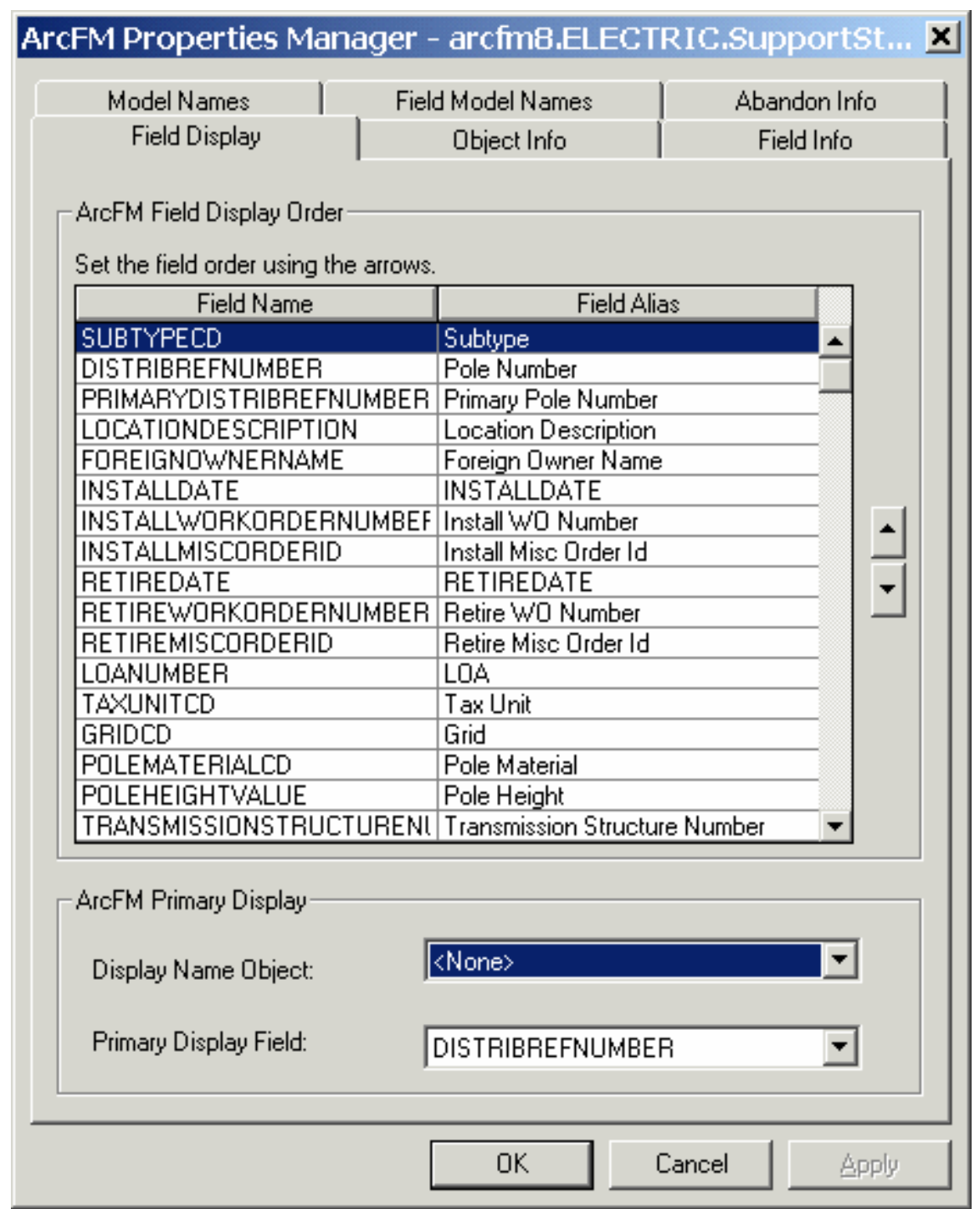

Figure 4.4-1. On Support Structure, the DistribRefNumber (pole number) is shown 


\subsection{Object Info}

The Object Info tab shown in Figure 4.4-2 configures AutoUpdaters, Edit Tasks and Validation Rules for both the feature and object classes. These components can be configured at either the feature class or at a subtype level. Configuring at the subtype level allows different code components to be used for different types of the class. For example, an overhead transformer has certain components while an underground transformer has others.

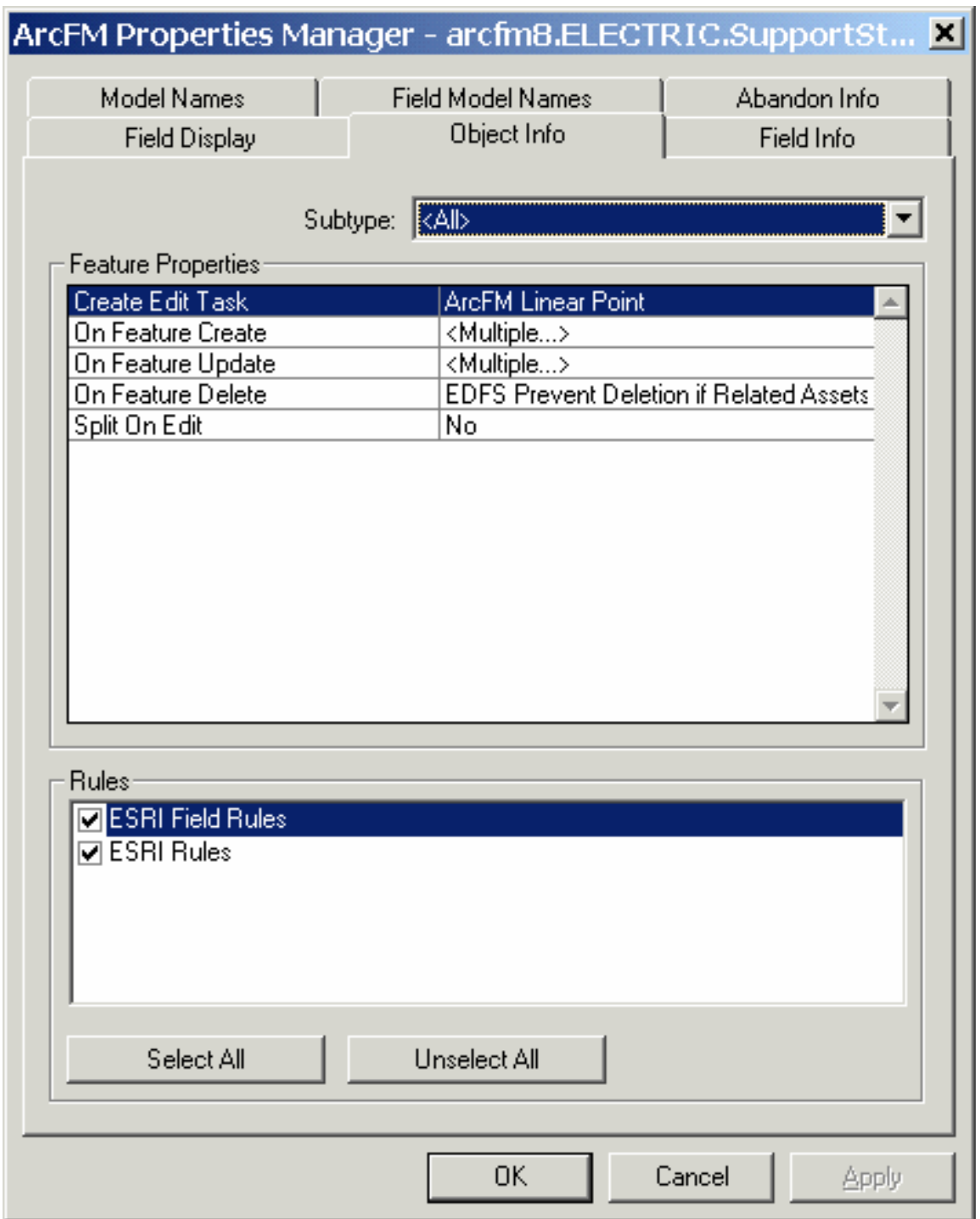

Figure 4.4-2. 
When " $<$ Multiple... $>$ " is shown for any of the event fields, it implies that there is more than one AutoUpdater configured for that event.

\begin{tabular}{|l|l|}
\multicolumn{1}{l}{$\begin{array}{c}\text { Table 4.4-1. Descriptions of the feature } \\
\text { properties available on the object info tab }\end{array}$} \\
\begin{tabular}{|l|l|}
\hline Property & Function \\
\hline Create Edit Task & Supports user interaction when placing a feature. \\
\hline On Feature Create & Assign AutoUpdaters to perform action when feature is created. \\
\hline On Feature Update & Assign AutoUpdaters to perform action when feature is edited. \\
\hline On Feature Delete & Assign AutoUpdaters to perform action when feature is deleted. \\
\hline Before Feature Split & $\begin{array}{l}\text { Only available on linear features. Assign AutoUpdaters to perform action } \\
\text { before the feature is split. }\end{array}$ \\
\hline On Feature Split & $\begin{array}{l}\text { Only available on linear features. Assign AutoUpdaters to perform action } \\
\text { when the feature is split. }\end{array}$ \\
\hline After Feature Split & $\begin{array}{l}\text { Only available on linear features. Assign AutoUpdaters to perform action } \\
\text { after the feature is split. }\end{array}$ \\
\hline Split on Edit & $\begin{array}{l}\text { Only available on point features. When Yes is specified, the line is split } \\
\text { first and then the point feature is placed (for improved performance). }\end{array}$ \\
\hline
\end{tabular}
\end{tabular}

\subsection{Field Info}

The Field Info tab adjusts the settings for a specific attribute field on a feature or object class. 


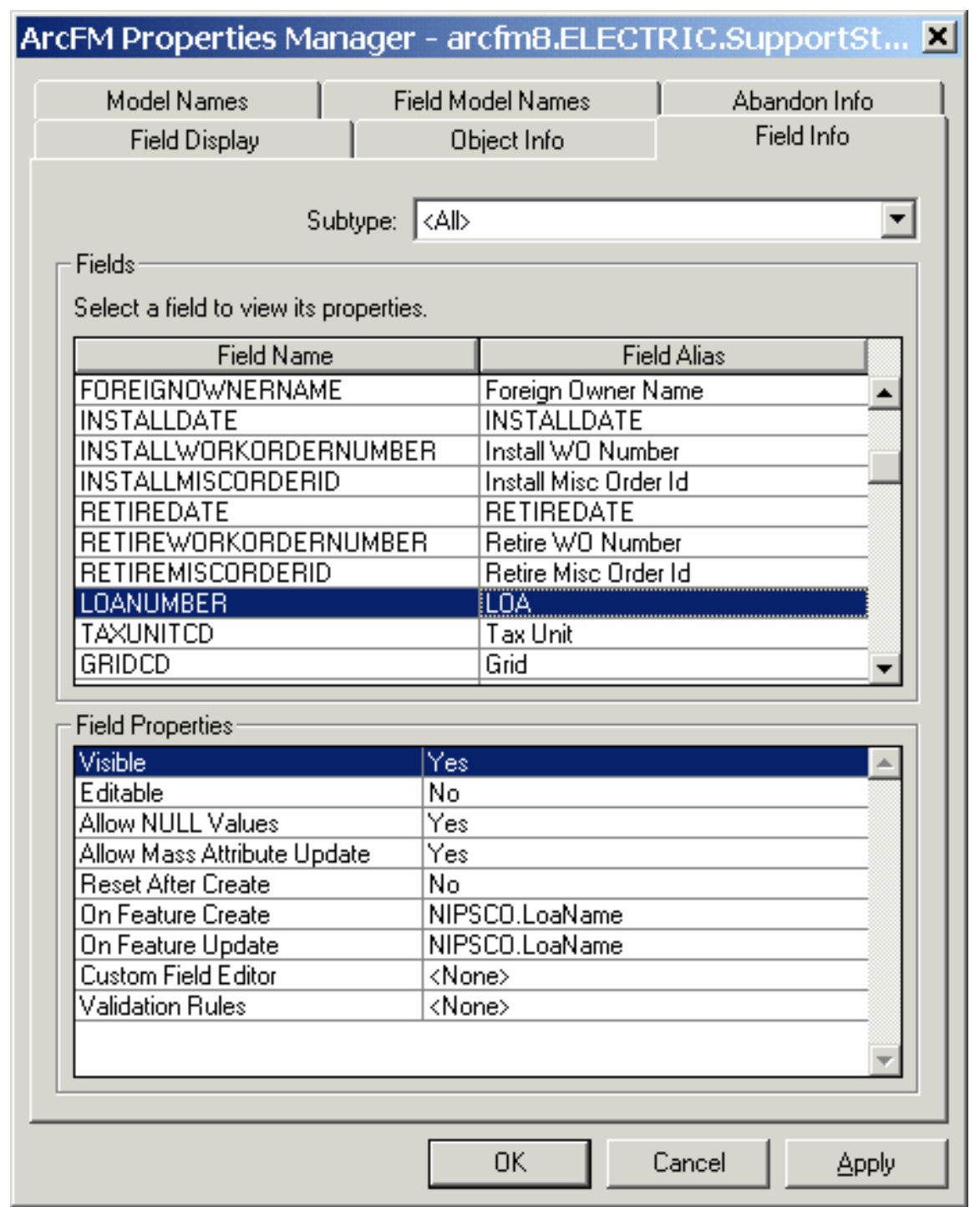

Figure 4.4-3. The settings can be applied to the entire feature class or a specific subtype of the feature class

Table 4.4-2. Definition of field properties that can be altered on this tab

\begin{tabular}{|l|l|}
\hline Property & Function \\
\hline Visible & $\begin{array}{l}\text { Controls the visibility of the field in all ArcFM controls. Allows system fields } \\
\text { to be hidden from the user. }\end{array}$ \\
\hline Editable & Controls whether a field is editable in all ArcFM controls. \\
\hline Allow NULL Values & $\begin{array}{l}\text { Controls whether or not a field will be required on all ArcFM controls. } \\
\text { Allows a field to be defined as nullable at the database level but still be } \\
\text { required by the application. }\end{array}$ \\
\hline $\begin{array}{l}\text { Allow Mass } \\
\text { Attribute Update }\end{array}$ & $\begin{array}{l}\text { Enables Mass Attribute Update for the field. If set to Yes, multiple features } \\
\text { can be selected and this attribute updated on all features in one edit. }\end{array}$ \\
\hline
\end{tabular}




\begin{tabular}{|l|l|}
\hline Reset After Create & $\begin{array}{l}\text { Determines whether the value in the field is reset after the feature is } \\
\text { created. After a feature is placed via the ArcFM Targets Tab this field will } \\
\text { be nulled out forcing the user to input a new value. }\end{array}$ \\
\hline $\begin{array}{l}\text { On Feature Create, } \\
\text { On Feature Update }\end{array}$ & $\begin{array}{l}\text { Allows the configuration of field autoupdaters. ArcFM field autoupdaters } \\
\text { automatically generate an attribute value at the onset of a create or an } \\
\text { update event on the corresponding feature or object. }\end{array}$ \\
\hline $\begin{array}{l}\text { Custom Field } \\
\text { Editors }\end{array}$ & $\begin{array}{l}\text { Allows the configuration of a custom editor that will be used whenever the } \\
\text { user edits this attribute field. An example would be a date/time picker } \\
\text { control. }\end{array}$ \\
\hline Validation Rules & $\begin{array}{l}\text { Field Validation Rules can be configured to perform custom validation on } \\
\text { the value of the attribute via the ArcFM QAQC application. }\end{array}$ \\
\hline
\end{tabular}




\subsection{Model Names}

The Model Names tab allows the user to assign model names to feature and object classes. Class Model Names allow ArcFM to run on top of a data model without knowing the actual names of the classes.

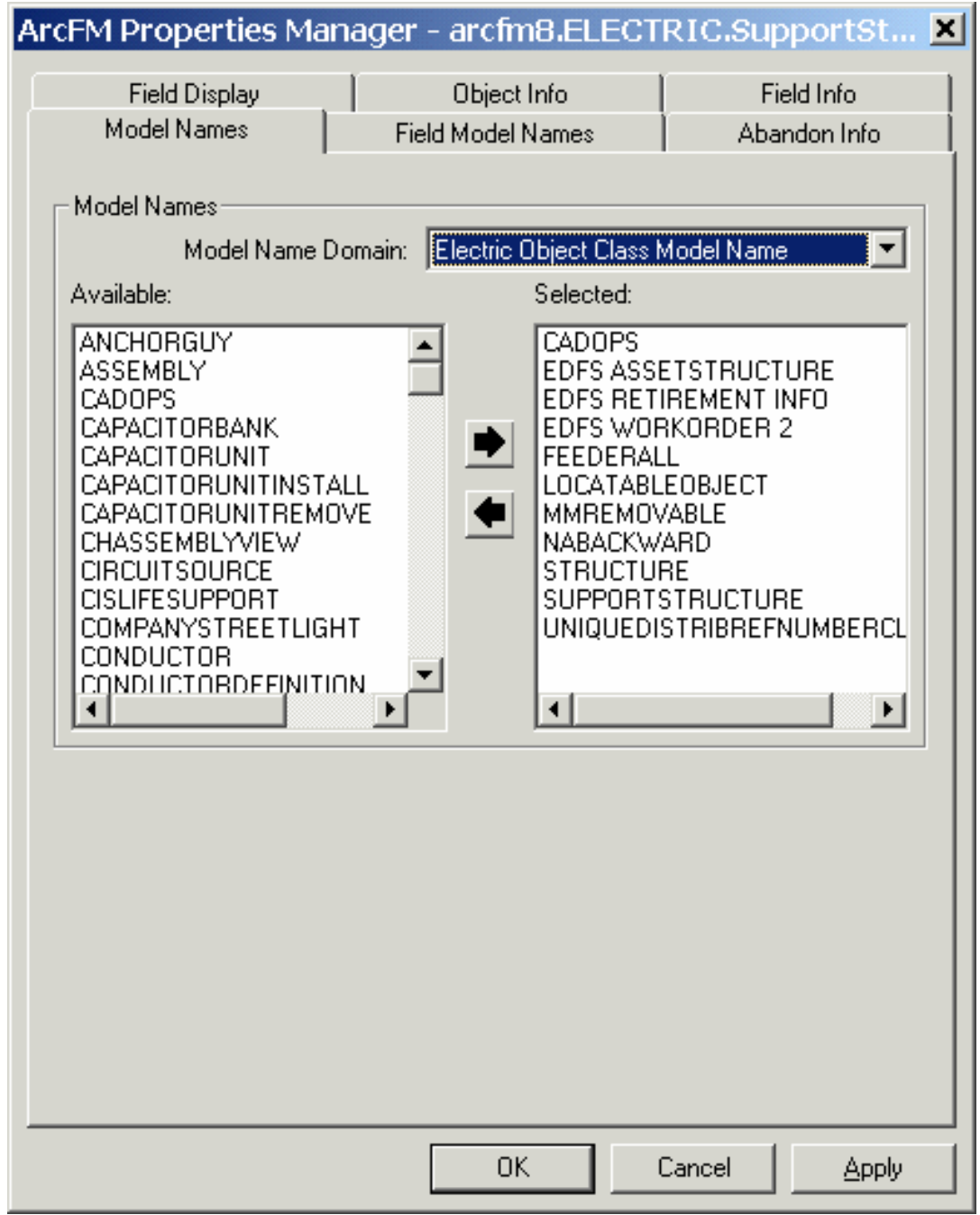
Figure 4.4-4. The Model Name Domain is used to choose the type of model name to apply which then filters the available list of model names 


\subsection{Field Model Names}

The Field Model Names tab allows the user to assign model names for the attribute fields of feature and object classes. Field Model Names allow ArcFM to run on top of a data model without knowing the actual names of the underlying fields.

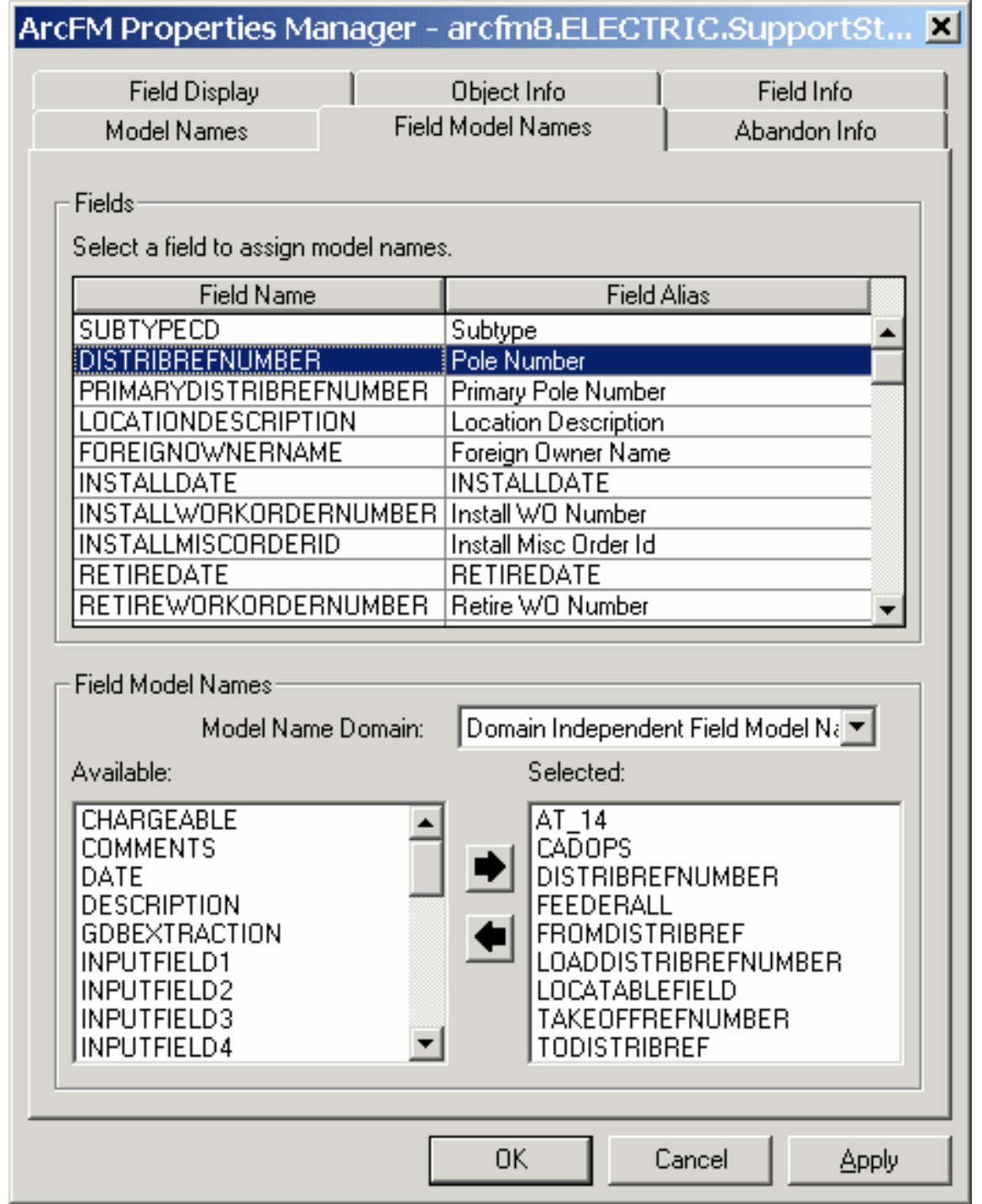

Figure 4.4-5. The Model Name Domain is used to choose the type of model name to apply which then filters the available list of model names 


\section{Abandon Info}

The Abandon Info tab shown in Figure 4.4-6 is used to determine how a feature acts when it is abandoned (or abandoned and removed) via the ArcFM abandon tools.

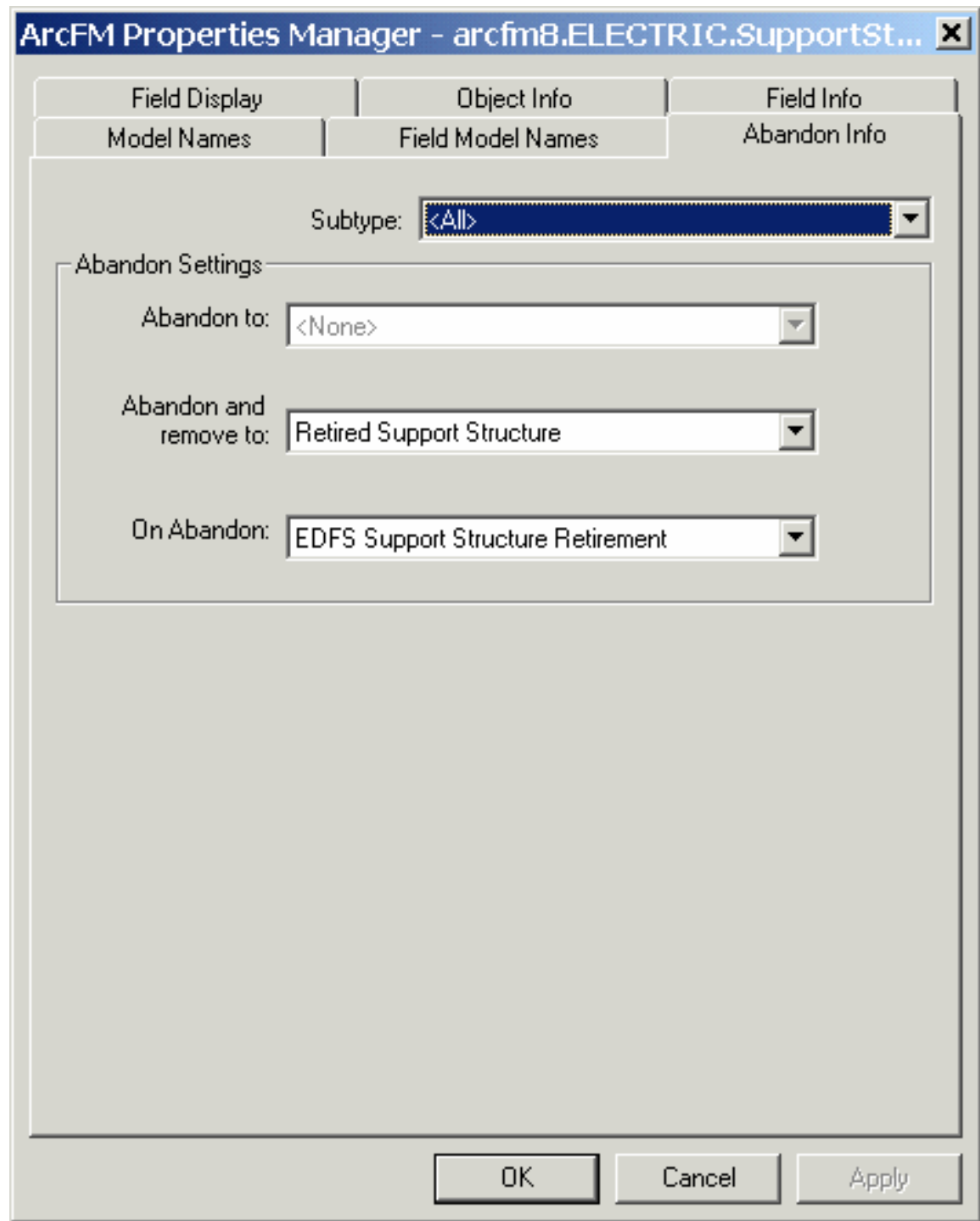

Figure 4.4-6. The abandonment can be defined at the class or at the subtype level

The options are defined in Table 4.4-3. 
Table 4.4-3.

\begin{tabular}{|l|l|}
\hline Property & Function \\
\hline Abandon to: & $\begin{array}{l}\text { Configures a Feature class where the feature will be } \\
\text { moved to upon retirement. The abandoned feature } \\
\text { class includes the shape of the feature. }\end{array}$ \\
\hline Abandon and remove to: & $\begin{array}{l}\text { Configures an Object class where the feature will be } \\
\text { moved to upon retirement. The shape field is not } \\
\text { maintained in this case. }\end{array}$ \\
\hline On Abandon: & $\begin{array}{l}\text { Allows for the configuration of an AutoUpdater that } \\
\text { will fire when the abandonment/retirement takes } \\
\text { place. }\end{array}$ \\
\hline
\end{tabular}

\subsection{Relationship Information}

ESRI Relationship classes have a different ArcFM Properties Manager interface. The Relationship interface is used to set AutoUpdaters for the create or delete event of the relationship class. In addition, the Validate Related Objects checkbox is checked to include the destination relationship class whenever the ArcFM QAQC application is run on an instance of the original relationship class.

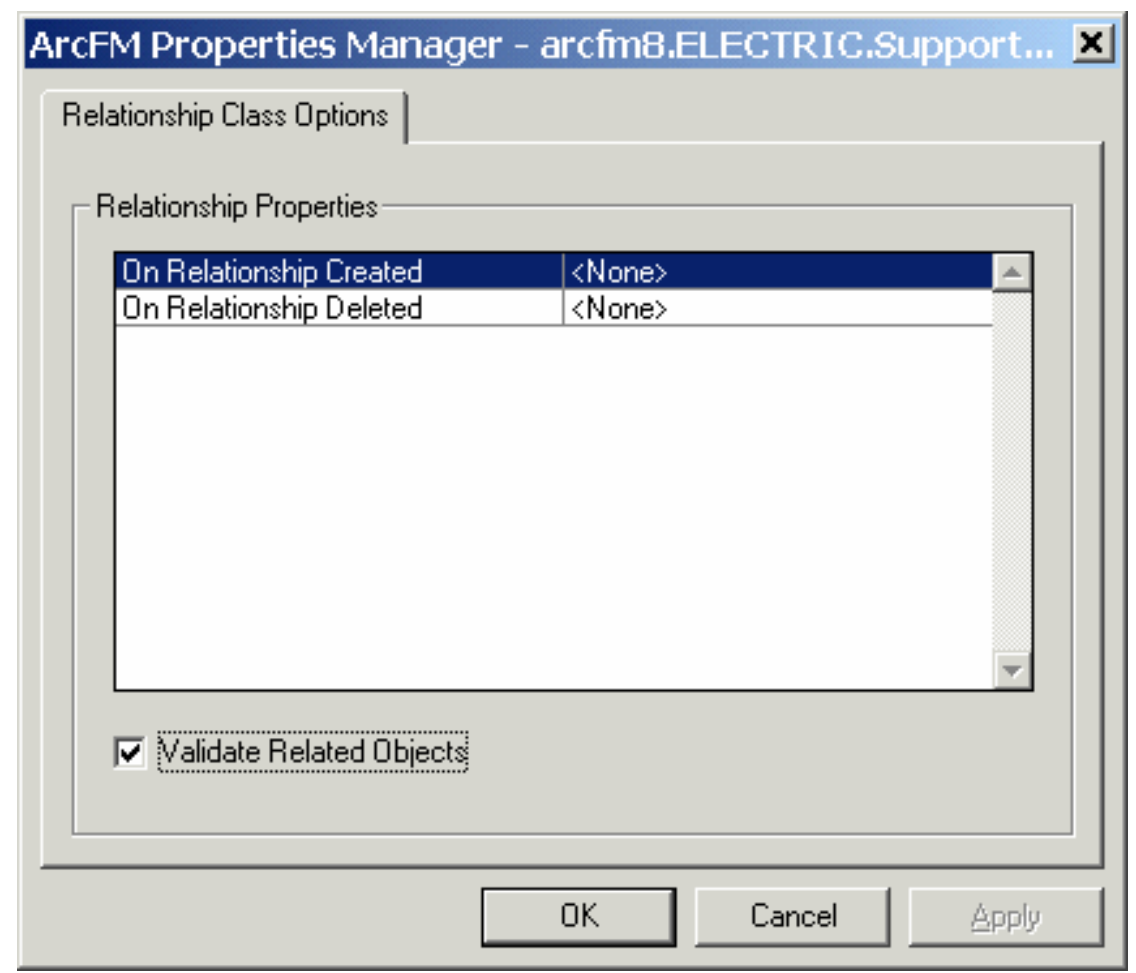

Figure 4.4-7. 


\subsubsection{Requirements}

The baseline ArcFM Properties requirements were captured using a two step approach. First, the configuration that constituted end user preferences were captured during a series of interview sessions with key end users. This configuration included items such as primary display field, order of fields, and basic AutoUpdater assignment. The second set of baseline configuration included configuring the out-of-the-box ArcFM tools and AutoUpdaters to run on the NIPSCO data model. This was accomplished by using the published ArcFM configuration help files.

In both instances, the configuration was entered directly into the ArcFM Properties Manager during the sessions as this is the easiest way to capture and retain this information. Once captured, this information was exported and saved in standard XML files. These XML files were maintained in Visual SourceSafe. When needed, XSLT is applied to the XML files to format them into an easy to read format. 


\subsection{Custom ArcFM Configuration}

Once the base configuration is complete, the configuration for all custom software components must also be applied to the geodatabase via the ArcFM Properties. Each of the custom installations includes some form of an Operations Guide which details the custom configuration for both the Model Names and the component assignment. These are applied in the same manner as the base configuration using the ArcFM Properties tools described above.

\subsubsection{Electric ArcFM Properties}

Tables E-1 and E-2 in Appendix E detail the core ArcFM Properties of all Electric features and tables. For brevity, only the default configuration has been displayed (no subtype-specific configuration is included). The table includes both the base configuration as well as the custom configuration. Any ArcFM component assignments reference out-of-the-box tools while both NIPSCO and EDFS component assignments reference custom tools. More information is provided on the custom tools in the later sections of this document.

\subsubsection{Electric Model Names}

The model names allow the software components to access the database without any knowledge of the underlying table or column naming conventions. Many model names indicate their use. For example, the "CADOPS" model name indicates that the class/field is used by CADOPS. Any "AT_*" model names are used for the ArcFM Abandon / Retirement mapping. Other model names simply indicate the class or field name to the software. Model name assignments for both the base and custom configuration can be found in Table E-3 in Appendix E.

\subsubsection{Gas ArcFM Properties}

Table E-4 in Appendix E details the core ArcFM Properties of all Gas features and tables. For brevity, only the default configuration has been displayed (no subtype-specific configuration is included). The table includes both the base configuration as well as the custom configuration. Any ArcFM component assignments reference out-of-the-box tools while both NIPSCO and EDFS component assignments reference custom tools. More information is provided on the custom tools in the later sections of this document.

\subsubsection{Gas Model Names}

The model names allow the software components to access the database without any knowledge of the underlying table or column naming conventions. Many model names indicate their use. Any "AT_*" model names are used for the ArcFM Abandon / Retirement mapping. Other model names simply indicate the class or field name to the software. 


\subsubsection{Land ArcFM Properties}

Table E-6 in Appendix E details the core ArcFM Properties of all Land features and tables. For brevity, only the default configuration has been displayed (no subtype-specific configuration is included). The table includes both the base configuration as well as the custom configuration. Any ArcFM component assignments reference out-of-the-box tools while both NIPSCO and EDFS component assignments reference custom tools. More information is provided on the custom tools in the later sections of this document.

\subsubsection{Land Model Names}

The model names allow the software components to access the database without any knowledge of the underlying table or column naming conventions. Many model names indicate their use. Other model names simply indicate the class or field name to the software. Table E-7 in Appendix E contains the model name assignments for Land

\subsubsection{ArcFM Snapping Manager}

\subsubsection{Overview}

The ArcFM base configuration also includes the configuration of the default snapping rules. As shown in Figure 4.5-1, ESRI typically requires the user to select the snapping rules manually when a feature is being created. ArcFM extends this functionality by allowing each feature class to have a configured list of snapping preferences.

Subsequently, when a user places a feature, the snapping preferences are automatically loaded and applied.

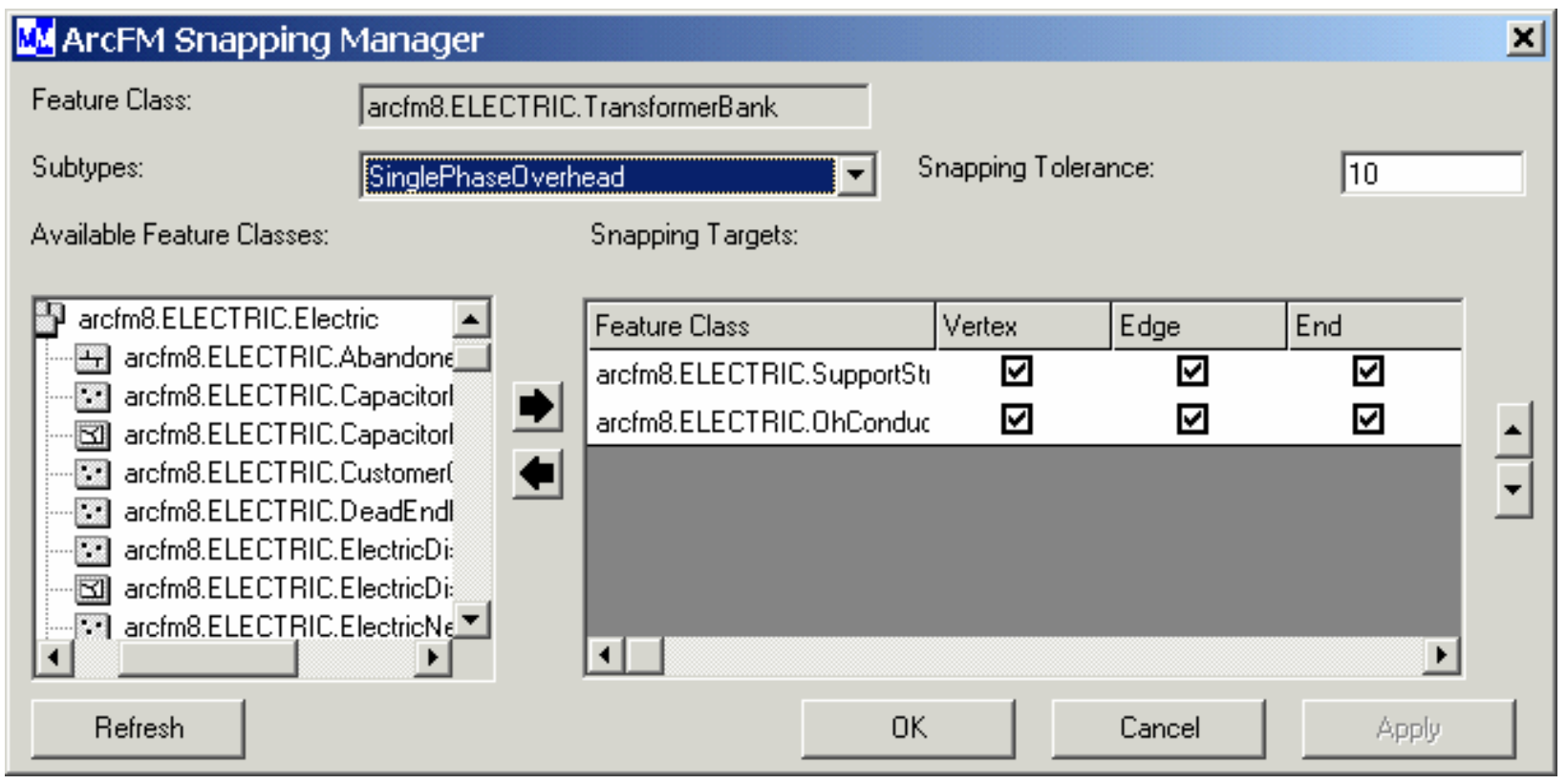

Figure 4.5-1. The snapping manager interface 
Snapping is configured at the subtype level. This means that each subtype can snap to a different set of features with different options and tolerance. The snapping tolerance defines how close the user's mouse must be to the target feature before it will snap to the feature. The snapping targets can be any feature class in the database. The order they are listed in the display box is the order in which they will be snapped to if two snapping targets are found at the exact same distance from the user's mouse.

The snapping configuration must only be performed once but will greatly streamline a user's interaction with the system when they are placing/creating new features.

\subsubsection{Snapping Requirements}

The snapping requirements were initially compiled by the core project team. They were then later reviewed with key end users to confirm any issues or gaps. The initial snapping was input directly into the ArcFM Snapping Manager and was maintained there throughout the project. The ArcFM XML Export utility was used to export the snapping settings into an XML file which was managed in SourceSafe. And XSLT template can be applied to the XML to display the snapping settings in an easy to read format. The snapping properties are detailed in Appendix E: Electric Snapping Configuration - Table E-8; Gas Snapping Configuration - Table E-9; and Land Snapping Configuration - Table E-10.

\subsubsection{Relationship Rules}

\subsubsection{Overview}

Relationship rules are defined for each relationship class in the database. Though these rules are optional, when implemented they are used with the ArcFM QAQC application to strengthen the integrity of the data. 


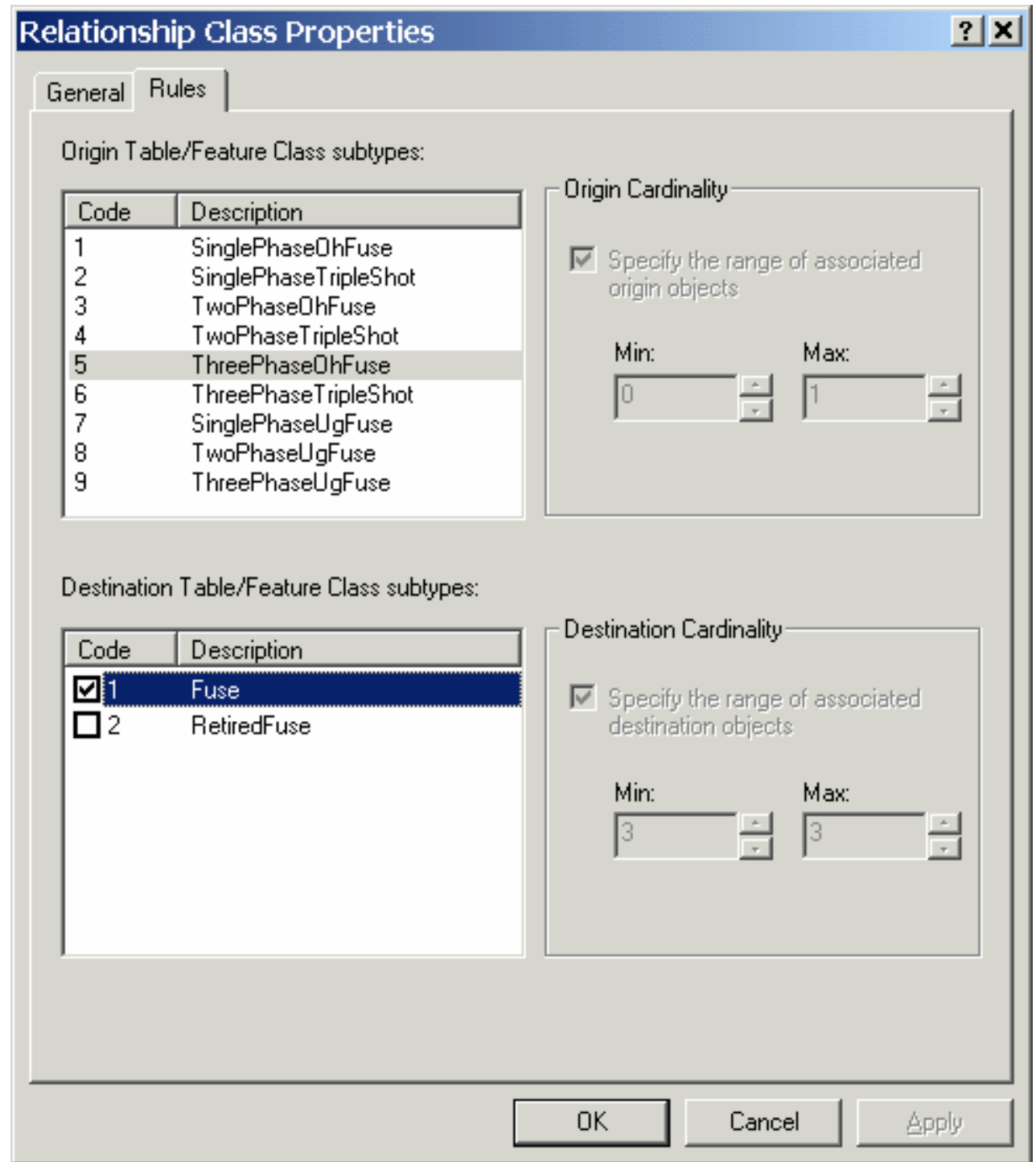

Figure 4.5-2. The relationship rules are defined by ESRI in the properties dialog on the rules tab for all relationship classes

The rules are defined at the subtype level and determine which subtypes on the origin class can be related to which subtypes of the destination class. The cardinality of the relationship can then be explicitly defined. In the above screenshot from the FuseCutouBank_FuseUnit relationship, a FuseCustoutBank.ThreePhaseOhFuse is related to a FuseUnit.Fuse (cannot be related to the RetiredFuse subtype). Further, a single ThreePhaseOhFuse is related to a mandatory three Fuse units and a single FuseUnit can only be related to a single ThreePhaseOhFuse. These rules will then raise QAQC errors if a ThreePhaseOhFuse is only related to a single Fuse unit.

\subsubsection{Relationship Requirements}

The relationship rules were established by the core team members at the beginning of the project and were refined as the project progressed. They were documented in a spreadsheet that was managed in SourceSafe. 


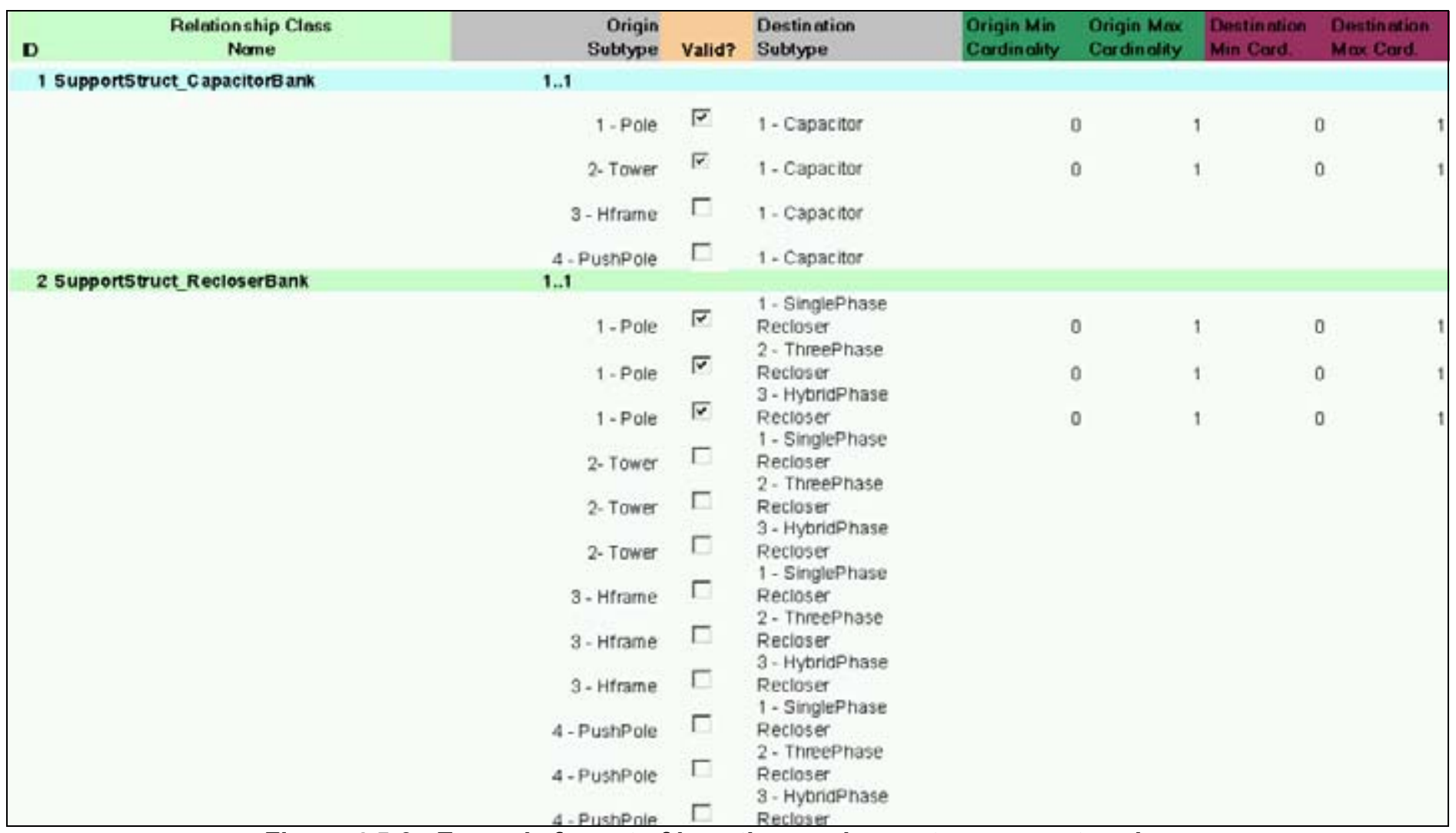

Figure 4.5-3. Example format of how the requirements were captured

These rules were then maintained in the geodatabase on a regular basis. In production the rules are easily exported and viewed using the ESRI Geodatabase Designer tool. The current rules are detailed in the following sections.

\subsubsection{Electric Relationship Rules}

Table E-11 in Appendix E shows only the electric relationships where relationship rules have been defined in the production AEDR geodatabase.

\subsubsection{Connectivity Rules}

\subsubsection{Overview}

Connectivity rules are defined for all networked features in the geodatabase. These rules are also optional but when implemented they are used with the ArcFM QAQC application to strengthen the integrity of the data. 


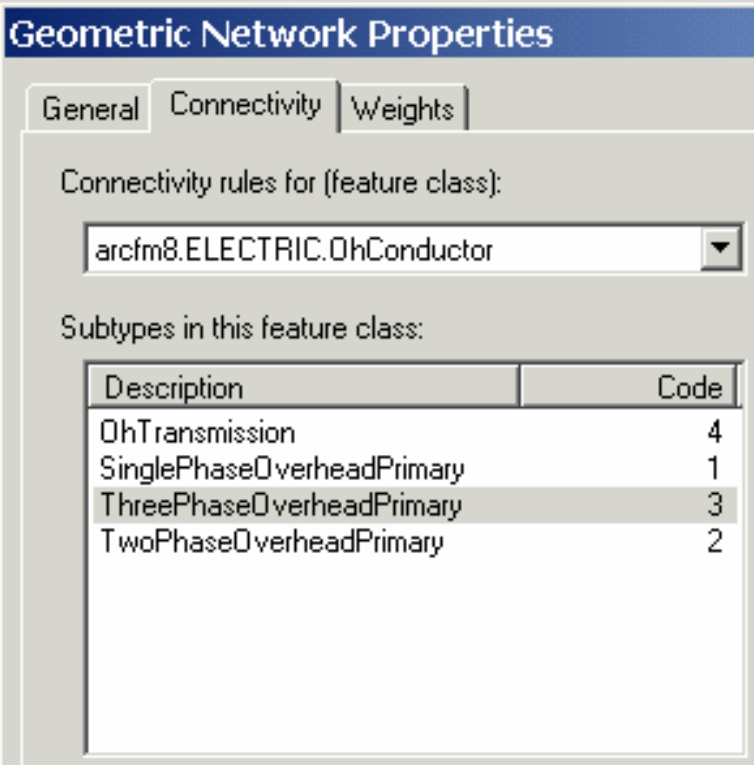

Subtypes in the Network:
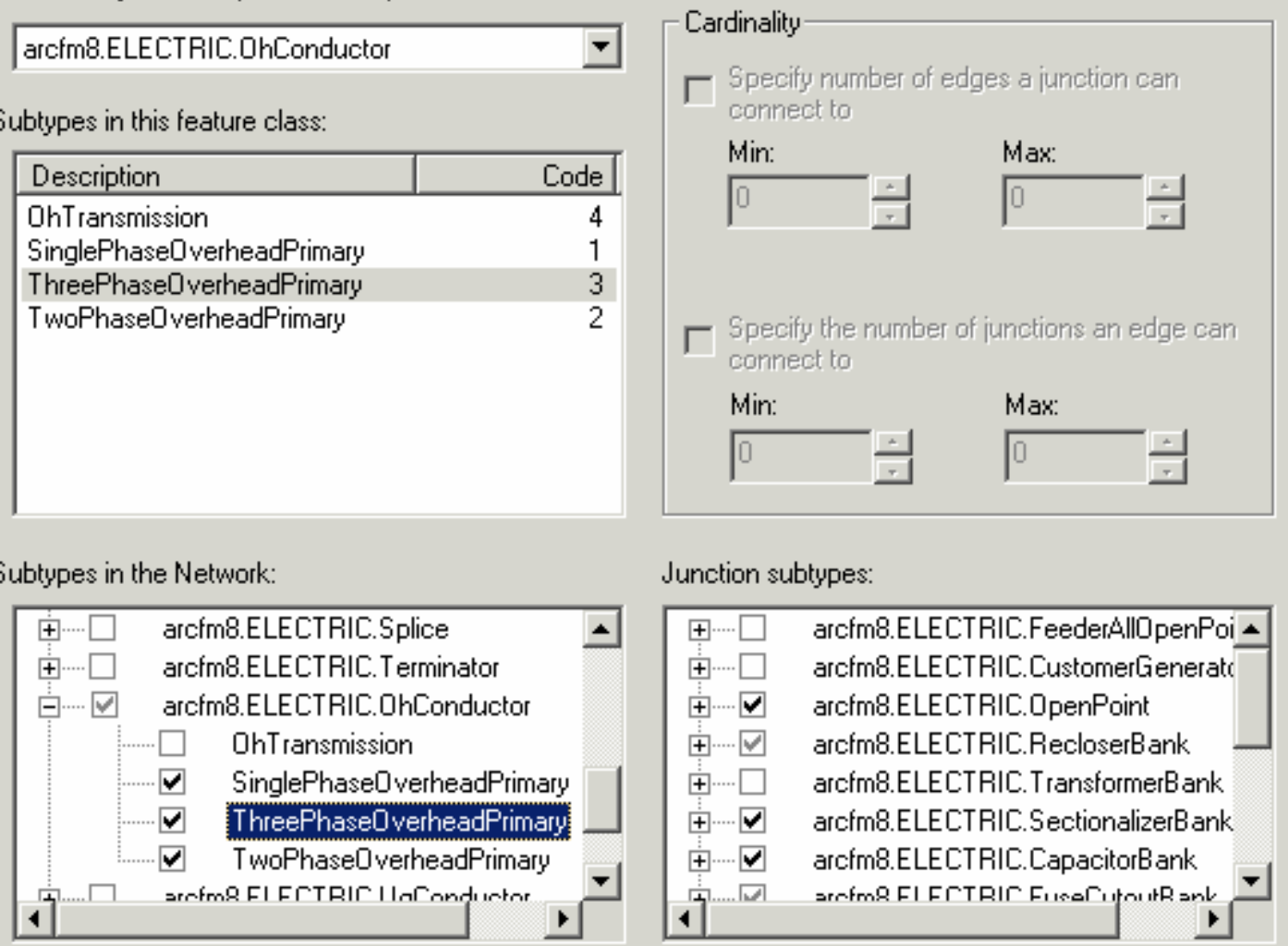

Junction subtypes:

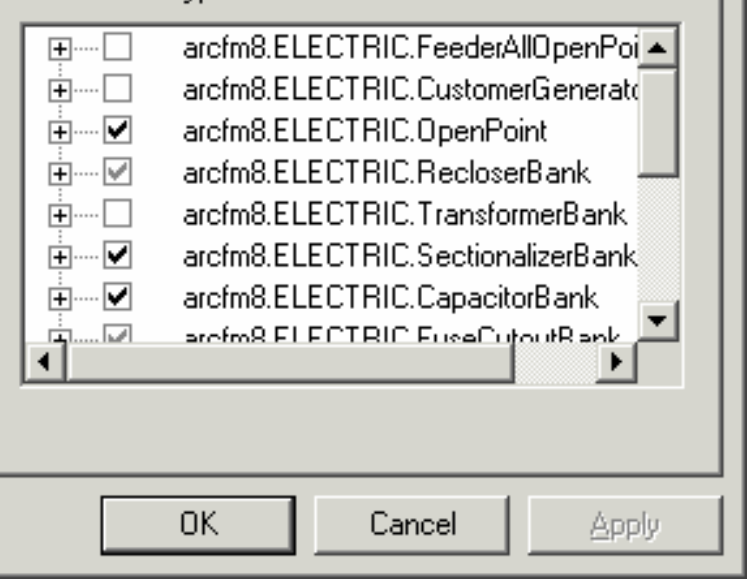

\section{Figure 4.5-4. The connectivity rules are defined by ESRI in the geometric network properties dialog on the connectivity tab}

The rules are defined at the subtype-to-subtype level and include both edge-edge rules as well was edge-junction rules. When defining edge-edge rules (as in the example above), the configuration specifies which junctions are used to connect two edges. When defining edge-junction rules, the minimum and maximum cardinality is configured. This allows the user to specify how many edge connections can be connected to a single junction and vice versa. This extensive configuration is labor intensive but provides great value to the integrity of the data.

\subsubsection{Connectivity Requirements}

The connectivity rules requirements were gathered at the beginning of the project and were subsequently refined during the rest of the project. They were documented in a spreadsheet that was maintained in SourceSafe. 


\begin{tabular}{|c|c|c|c|c|c|c|c|c|c|c|c|c|}
\hline \multicolumn{3}{|c|}{ Edge-Junction Rules } & \multicolumn{4}{|c|}{\begin{tabular}{|c|c|} 
Eages to & Junctrons to \\
Connect to & Connect to
\end{tabular}} & & & & & & \\
\hline \multirow{2}{*}{\begin{tabular}{|l} 
Junction Type \\
Junction Subtype \\
\end{tabular}} & \multirow{2}{*}{$\begin{array}{l}\text { JEdge Type } \\
\text { |Edge Subtype }\end{array}$} & \multirow[b]{2}{*}{ OK? } & \multicolumn{2}{|c|}{$\begin{array}{l}\text { Connect to } \\
J-E\end{array}$} & \multirow{2}{*}{$\begin{array}{l}E-J \\
\text { Min } \\
\end{array}$} & \multirow{2}{*}{$\begin{array}{l}\text { Ev } \\
\text { Max }\end{array}$} & \multicolumn{4}{|c|}{ Snapping (by Feature Class) } & & \\
\hline & & & $\begin{array}{r}J-2 \\
\text { Min } \\
\end{array}$ & $\begin{array}{c}J-E \\
\text { Max } \\
\end{array}$ & & & \multicolumn{2}{|c|}{\begin{tabular}{l|l} 
Vertex & Edge \\
\end{tabular}} & \multirow[t]{2}{*}{ End } & Tolerance & & \\
\hline \multirow{16}{*}{$\begin{array}{c}\text { Fuse Cutout Bank } \\
\text { OH Fuse }\end{array}$} & BusBar & & & & & & & & & & Split?] & Notes \\
\hline & $\begin{array}{l}\text { Switchgear Bus } \\
\text { Substation Bus }\end{array}$ & $\begin{array}{l}\text { No } \\
\text { No }\end{array}$ & & & & & & & & & & \\
\hline & Overhead Primary & & & & & & $\bar{\sigma}$ & $\checkmark$ & $\sqrt{2}$ & 5 & $\sqrt{2}$ & \\
\hline & Single Phase $\mathrm{OH}$ Pri & $\checkmark$ & 0 & 2 & 0 & 2 & & & & & & \\
\hline & Two Phase OH Pri & $\checkmark$ & 0 & 2 & 0 & 2 & & & & & & \\
\hline & Three Phase OH Pri & $\checkmark$ & 0 & 2 & 0 & 2 & & & & & & \\
\hline & Overhead Transmission & & & & & & & & & & & \\
\hline & Overhead Tranmission & No & & & & & & & & & & \\
\hline & Tie Wire & & & & & & $\checkmark$ & $\checkmark$ & $\checkmark$ & 5 & $\checkmark$ & \\
\hline & Tie Wire & $\checkmark$ & 0 & 2 & 0 & 1 & & & & & & See Data Migration Procedures \\
\hline & Underground Primary & & & & & & & & & & & \\
\hline & Single Phase $\mathrm{OH}$ Pri & No & & & & & & & & & & \\
\hline & Two Phase OH Pri & No & & & & & & & & & & \\
\hline & Three Phase OH Pri & No & & & & & & & & & & \\
\hline & Underground Trans. & & & & & & & & & & & \\
\hline & UG Transmission & No & & & & & & & & & & \\
\hline
\end{tabular}

Figure 4.5-5. Spreadsheet format for edge-junction rules

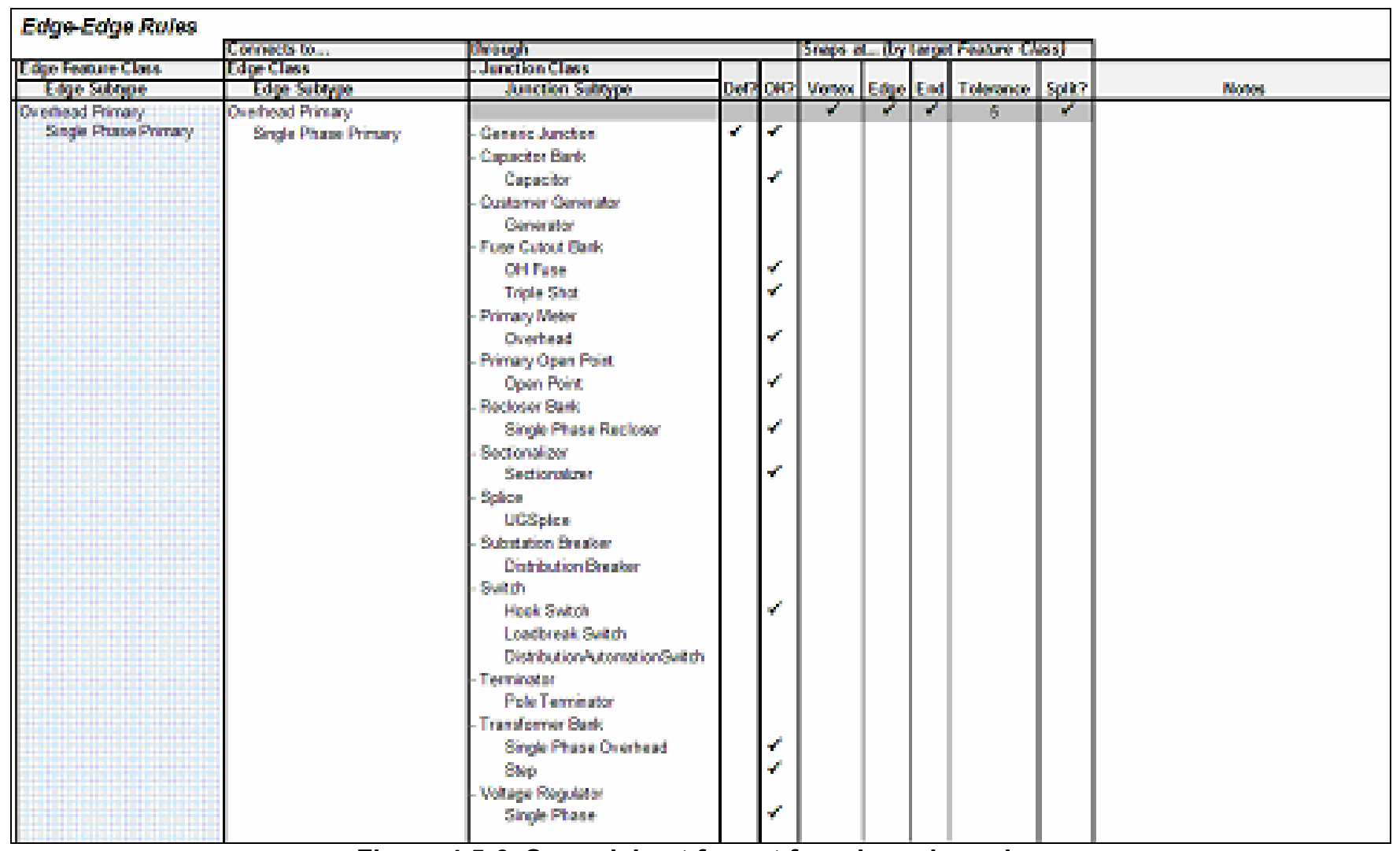

Figure 4.5-6. Spreadsheet format for edge-edge rules 
These rules were then maintained in the geodatabase on a regular basis. In production the rules can be easily exported and viewed using the ESRI Geodatabase Designer tool. The current rules are detailed in Appendix E, Tables E-13, E-14, E-15 and E-16. 


\subsection{AEDR System / Project Environment}

\subsubsection{Server}

This section details the specifications that are in use for the various server components within the AEDR system. The two major server components include the database server and the Citrix server farm. The database server hosts the actual data stores for the project as well as the ArcSDE server components that provide spatial access to the data stores. The Citrix servers exist as application service providers that allow the end users to harness the power of a server while using the thick client GIS applications. This section also includes a review of the various server environments that were employed during the construction phase of the AEDR project.

\subsubsection{Database}

Microsoft SQL Server was chosen as the underlying RDBMS. ESRI ArcSDE sits on top of the database and manages all GIS interaction with the database. The following subsections detail the installation, configuration, and use of the database components of the AEDR project.

\subsection{Target Database Environment}

The following specifications define the target database server environment for the AEDR project:

- Software Config:

i. Windows Server 2003

ii. SQL Server 2000 Enterprise Edition Sp3a

iii. ArcSDE 8.3 for SQL Server Sp1 (Includes ArcSDE for SQL Server Performance Patch)

- Databases

i. SDE: Manages all ArcSDE database components and the M\&M system components

1. Initial Size: $100 \mathrm{MB}$

2. recovery model: full

3. Datafile: e:|program files $\backslash$ Microsoft SQL Server\MSSQL ldatalsdedata.mdf

ii. ARCFM8: Contains all Electric, Gas, Land, and CIS data tables

1. Initial Size: $400 \mathrm{MB}$

2. recovery model: full

3. Datafile: e: program files $\backslash$ Microsoft $S Q L$ Server $M S S Q L \backslash$ data $\backslash$ arcfm8data.mdf

iii. PX: Contains all Session Manager data and administration tables

1. Initial Size: $100 \mathrm{MB}$

2. recovery model: full

3. Datafile: e: $\mid$ program files $\backslash$ Microsoft $S Q L$ Server $M S S Q L \backslash d a t a \mid p x d a t a . m d f$ 
iv. RASTER: Contains spatial imagery tables

1. Initial Size: $1 \mathrm{~GB}$

2. recovery model: simple

3. Datafile: e: program files $\backslash$ Microsoft SQL Server $\backslash M S S Q L \backslash d a t a \mid r a s t e r d a t a . m d f$

v. NIPSCOSage: Contains all system and authorization tables for the SAGE application

1. Initial Size: $100 \mathrm{MB}$

2. recovery model: full

3. Datafile: e: program files $\backslash$ Microsoft SQL ServerlMSSQLldatalsagedata.mdf

- ArcSDE Configuration

i. Installation

1. Location: E: $\mid$ arcgis $\mid a r c s d e l s q l e x e$

ii. Server Configuration

1. Port: 9999

2. Service name: esri_sde

iii. Service Packs and Patches

1. ArcSDE 8.3 SQL Server Service Pack 1

2. ArcSDE 8.3 SQL Server Performance Patch

\subsection{Database Permissions}

The following section outlines the strategy that was taken in defining the database privileges for the AEDR project:

\subsection{Database Privilege Strategy}

All users requiring access to the GIS system must have permissions set on each database object.

- Users to the system have to be identified by their NiSource domain user ID. All components of the AEDR system use windows authentication so that the end user is never required to type a password. Windows authentication allows the applications to use the credentials of the user that logged onto the computer where the application is being used.

- Roles for the system have been identified and are identified in the next section.

- For all Roles created within the database, permissions can be set on Roles within ArcCatalog. This is the preferred approach when dealing with a versioned database because ESRI handles assigning the permissions on all 'add', 'delete', and other system tables. In addition, for each versioned feature class there often exist several related tables that require permissions. By assigning permissions via ArcCatalog, these other related tables automatically receive the proper permission settings. This not only saves time, but ensures consistent permissions are set on underlying objects. 
- Once the Users and Roles have been identified and created, the Users must be assigned the appropriate Role(s). The standard assignment of roles is detailed in a below section.

\subsection{User Accounts and Roles}

- SQL Server User Accounts

O SDE: Key system user that owns the SDE database and has permissions across the GIS tables

- SAGE: Owns all NIPSCOSAGE tables and stored procedures

- Electric: Owns all electric tables

- Gas: Owns all gas tables

- Landbase: Owns all land tables

- CIS owns all CIS tables.

- SQL Server Roles

- SDE Database

- ArcFM_user - Grants appropriate access to Miner \& Miner tables for ArcFM users

- ArcFM_admin - Grants read/write access to all Miner \& Miner tables in the SDE database.

- ArcFM8Database

- Electric_viewer - Grants read-only access to electric data

- Gas_viewer - Grants read-only access to gas data

- Landbase_viewer - Grants read-only access to Landbase data

- CIS_viewer - Grants read-only access to CIS data

- Electric_editor - Grants read/write access to Electric data

- Gas_editor - Grants read/write access to Gas data

- Landbase_editor - Grants read/write access to Landbase data

- Cis_editor - Grants read/write access to CIS data

- PX Database

- SM_User - Grants read/write access to all session management tables

- Raster Database

- viewer - Grants read-only access to raster data

- NIPSCOSage

- N/A - only the SAGE user has access to the NIPSCOSage objects.

\subsection{Standard User Permissions}

- Standard Record Clerk: Responsible for the input and maintenance of all electric and gas GIS data per work orders and work design.

- ArcFM8 Database

- Electric_Editor

- Gas_Editor

- Landbase_Viewer

- CIS_Viewer

- SDE Database 
- ArcFM_User

- Db_ddladmin - system role granted temporarily to allow the ArcSDE process to create individual log and transaction tables for each user when they first log into the system. This permission is revoked after the user has logged in at least once.

- PX Database

- SM_User

- Land Record Clerk: Responsible for the input and maintenance of all land GIS data as well as rectifying electric and gas data to match the landbase.

- ArcFM8 Database

- Electric Editor

- Gas_Editor

- Landbase Editor

- CIS_Viewer

- SDE Database

- ArcFM_User

- Db_ddladmin - system role granted temporarily to allow the ArcSDE process to create individual log and transaction tables for each user when they first log into the system. This permission is revoked after the user has logged in at least once.

- PX Database

- SM_User

- ArcView Users: A varied group of users who need view only access into the GIS data. This includes call screening, design engineers, outage management, etc.

- ArcFM8 Database

- Electric_Viewer

- Gas_Viewer

- Landbase_Viewer

- CIS_Viewer

- SDE Database

- ArcFM User

- Db_ddladmin - system role granted temporarily to allow the ArcSDE process to create individual log and transaction tables for each user when they first log into the system. This permission is revoked after the user has logged in at least once.

- CIS Editors: A small group of individuals responsible for the updating of CIS Installed Service locations. They do not manage their work via Session Manager and therefore have no permissions in the PX database.

- ArcFM8 Database:

- Electric_Viewer

- Gas_Viewer

- Land̄base_Viewer

- CIS_Editor

- SDE Database 
- ArcFM_User

- Db_ddladmin - system role granted temporarily to allow the ArcSDE process to create individual log and transaction tables for each user when they first log into the system. This permission is revoked after the user has logged in at least once.

- GIS Administrators: Members within the GIS department who are responsible for general maintenance within the system as well as data analysis for varied external parties.

- ArcFM8 Database:

- Electric_Editor

- Gas_Editor

- Landbase_Editor

- CIS_Editor

- SDE Database

- ArcFM Admin

- Db_ddladmin - system role granted temporarily to allow the ArcSDE process to create individual log and transaction tables for each user when they first log into the system. This permission is revoked after the user has logged in at least once.

- PX Database

- SM_User

\subsubsection{Citrix}

Windows Terminal Server (WTS) is a multi-user server operating system that provides the ability to host multiple simultaneous thin-client sessions on remote client devices. All client processing is performed locally at the Terminal Server and only display, keystroke, and mouse commands are transmitted over the network to the client device. Citrix MetaFrame is a Windows Terminal Server add-on that significantly enhances the functionality, manageability, and ease of deployment of Terminal Server solutions. MetaFrame relies on the Independent Computing Architecture (ICA) protocol. One of the key aspects of ICA is that it allows non-Windows clients to access Terminal Server. Therefore, ICA allows for the efficient use of existing hardware in a heterogeneous environment.

Windows Terminal Server and Citrix implementations have a number of advantages:

- Applications and data access are moved to the computer room, reducing network bandwidth requirements

- Relieves the need to regularly upgrade client workstations to keep up with increasing software system demand

- Administration is centralized resulting in a reduction in administration costs

- Ability to rapidly deploy applications, including Web based deployment options

- Since the applications run on a powerful central server, end-client performance increases are often observed

- Provides a secure computing environment since all data can be protected in the server room while only displays are sent to the client device. 


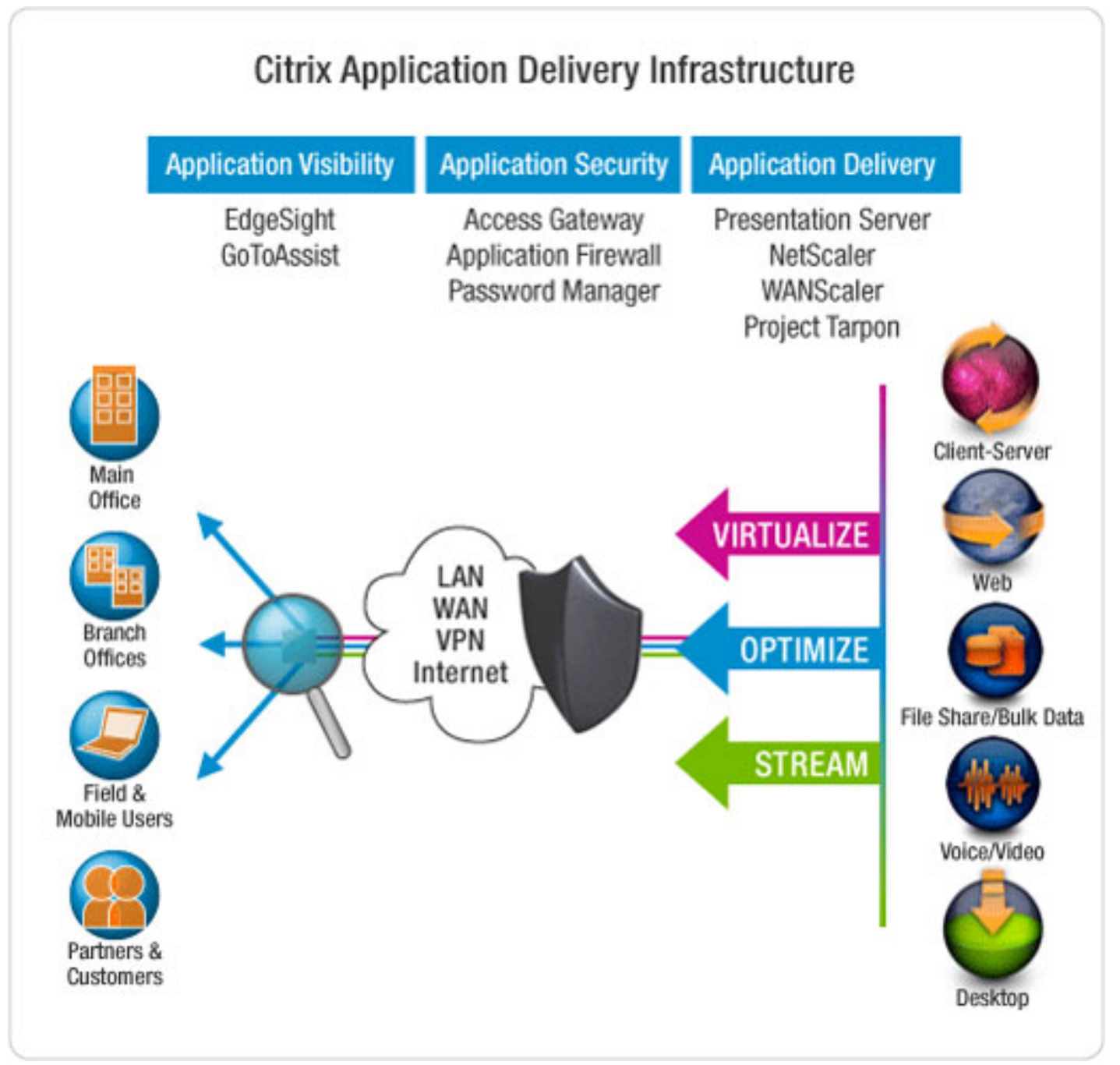

Figure 4.6-1. Diagram illustrates the Citrix infrastructure

\subsection{Citrix Application Components}

The AEDR project utilizes a production Citrix farm including 4 Windows 2003 servers as well as a single development / test Citrix server. These Citrix machines are loaded with all of the end user software installs as detailed below:

- Microsoft Internet Explorer 6.0 Service Pack 1

- Used for accessing any of the AEDR help systems as well as SAGE and Facility Browser.

- ESRI ArcGIS 8.3 Service Pack 3

○ This is the core ESRI GIS software.

- ESRI ArcObjects Developer Kit

- This installation includes Microsoft .Net wrappers for all ESRI programming objects and is required to support ArcFM on top of ArcGIS. 
- Microsoft .Net Framework

○ The .Net framework version 1.1 was used in the development of all custom code for the AEDR project and it must be installed prior to installing any custom components.

- Miner \& Miner ArcFM 9.01 Service Pack 2

- This is the core ArcFM GIS software.

- NIPSCO ArcLauncher:

- This application was defined specifically for the Citrix environment and it allows the same published Citrix applications to be used by groups with differing permissions.

- NIPSCO Custom Components

- This installation contains custom functionality to allow ArcGIS to match the effectiveness of AutoCAD / Outfield.

- NIPSCO EDFS Tools

- This installation provides all GIS replacement components for the legacy EDFS mainframe system.

- NIPSCO Font File

$\circ$ This font is used to display all of the GIS symbols.

- NIPSCO AutoUpdaters

- This installation contains upgrades to core Miner \& Miner AutoUpdaters that are required for their use at NIPSCO.

- NIPSCO Custom Login

- This installation allows ArcGIS, ArcFM, and Session Manager to all be used with Microsoft Windows Authentication thereby eliminating the need for the user to ever log into the system as long as they have logged onto the Citrix server with an authorized domain account.

- NIPSCO Tools

- This installation contains additional GIS tools that will be used in the day to day management and QAQC of the work being performed.

With these application components in place, the Citrix machines allow the NIPSCO users all the benefits of a high powered end user terminal.

\subsection{Citrix User Groups}

Three groups of Citrix users were defined with varying access. The groups were defined as Windows Active Directory Groups and were then used to set the access level for each of the published Citrix applications. This ensures that only authorized users can access each application with each defined permission level. The same applications are made available to more than one group but the permission level is changed depending on the authorization of the user. The permissions can be altered for both ESRI and Miner \& Miner applications by changing the license available to the user.

The ESRI license can be updated by setting an environment variable as follows:

- ArcView: ESRI_SOFTWARE_CLASS = Viewer

- ArcEditor: ESRI_SOFTWARE_CLASS = Editor

- ArcInfo: ESRI_SOFTWARE_CLASS = Professional 
The Miner \& Miner license can be updated by setting specific registry keys as follows:

- ArcFM Viewer: HKEY_CURRENT_USERISoftwarelMiner and Miner|Desktop Manager $\backslash$ Products $\backslash$ ArcFM Viewer $=1$

- ArcFM: HKEY_CURRENT_USERISoftwarelMiner and MinerlDesktop Manager $\backslash$ Products $\backslash$ ArcFM $=1$

Both of these license changes are automated via the NIPSCO ArcLauncher application. It is a command line application that accepts parameters for the application to be run (i.e. ArcMap, ArcCatalog, etc) and the license level to be used. It then sets both the ESRI license environment variable and the Miner \& Miner registry keys appropriately before starting the application.

Within this context, the following three Citrix user groups have been established:

i. Viewer

a. ArcMap with ArcView and ArcFM Viewer

ii. Clerk / Editor

a. ArcMap with ArcEditor and ArcFM

iii. Administrator / Manager

a. ArcMap with ArcEditor/ArcInfo and ArcFM

b. ArcCatalog with ArcFM

All AEDR Citrix users are assigned into one of these groups and the respective permissions along with the user's database roles effectively limit their access into the application and data.

\subsubsection{Management of the Server Environment}

This section defines the use and management of the server environment during AEDR data migration, application development, and testing. Six SQL Server instances split out over two database servers were utilized to create well-defined separation between the project initiatives. This allowed the project team to manage database and application updates and testing in a methodical manner while allowing multiple major initiatives to be pursued in parallel.

\subsection{ArcSDE Environment Descriptions}

\subsection{Delta ArcSDE Environment}

This environment is used for the initial creation of the physical ArcFM8 and SDE databases from the master physical Visio data model. This environment will never contain any actual data (i.e. only contains the latest database schema). The ArcFM8 and SDE databases will be deleted and recreated via the XMI export tools available in Visio and ArcCatalog. 
Table 4.6-1. Delta instance

\begin{tabular}{|l|l|}
\hline SQL Server/SDE Server Name & aedrmapgis01 \\
\hline SQL Server Instance Name & aedrmapgis01 \\
\hline ArcSDE Instance Name & 999A \\
\hline ArcSDE Instance Port & 9991 \\
\hline Physical drive location & e:larcgislarcsdelsqlexelbinlgiomgr.exe \\
\hline
\end{tabular}

\subsection{Migration ArcSDE Environment}

This environment is a repository for the data returned from the data migration vendor. This instance will be populated from a SQL back-up file (*.bak) received from the data migration vendor. This instance will not have any changes made to it other than relinking users. It will also serve as the environment that automated migration validation routines are run against. Each subsequent migration delivery will over write the schema and data from the previous restore.

Table 4.6-2. Migration instance

\begin{tabular}{|l|l|}
\hline SQL Server/SDE Server Name & aedrmapgis01 \\
\hline SQL Server Instance Name & aedrmapgis01/mig \\
\hline ArcSDE Instance Name & $999 \mathrm{~B}$ mig \\
\hline ArcSDE Instance Port & 9992 \\
\hline Physical drive location & e:larcgislarcsdelsqlexe_miglbinlgiomgr.exe \\
\hline
\end{tabular}

\subsection{Development ArcSDE Environment}

This environment is for the development and initial installation of all custom configuration, schema changes, and code development. The application code changes and unit testing takes place on a "thick" client machine and may reside on several machines as necessary. SDE/SQL configuration and data model changes are allowed in this environment to support the code development. Any changes that need to be made permanent are rolled back into the appropriate Visio or XML configuration files so that they are not lost when this environment is loaded with an updated version of the data. This environment is typically loaded with data from the Migration environment after the data has gone through the QAQC process. Once loaded, the latest configuration files (ArcFM Model Names, Properties, etc) are applied before development is resumed. The users of this environment are limited to the ongoing development and configuration tasks.

Table 4.6-3. Development instance

\begin{tabular}{|l|l|}
\hline SQL Server/SDE Server Name & aedrmapgis01 \\
\hline SQL Server Instance Name & aedrmapgis01ldev \\
\hline ArcSDE Instance Name & $999 \mathrm{C}$ dev \\
\hline ArcSDE Instance Port & 9993 \\
\hline Physical drive location & e:larcgislarcsdelsqlexe_devlbinlgiomgr.exe \\
\hline
\end{tabular}




\subsection{Test ArcSDE Environment (Test Server)}

This environment is used for the next level of testing and incorporates all the changes in custom code as well as the data model changes that have been approved to be promoted from the Development environment. In addition, the custom code and OOTB applications are tested in the "thin" Citrix environment against this environment. The Citrix test environment closely models the production hardware and software environment.

Table 4.6-4. Test instance on the test server

\begin{tabular}{|l|l|}
\hline SQL Server/SDE Server Name & aedrmapgis01 \\
\hline SQL Server Instance Name & aedrmapgis01/test \\
\hline ArcSDE Instance Name & 999D_test \\
\hline ArcSDE Instance Port & 9994 \\
\hline Physical drive location & e:larcgislarcsdelsqlexe_testlbinlgiomgr.exe \\
\hline
\end{tabular}

\subsection{Test ArcSDE Environment (Production Server)}

This environment was used for additional testing and typically matched the Test environment on aedrmapgis01. This test environment existed on the production server machine and was disabled before the system was taken into production to ensure that all system resources are made available to the production SDE environment.

Table 4.6-5. Temporary test instance on the production server

\begin{tabular}{|l|l|}
\hline SQL Server/SDE Server Name & aedrmdbsqlp01 \\
\hline SQL Server Instance Name & aedrmdbsqlp01ltest \\
\hline ArcSDE Instance Name & 999E_test \\
\hline ArcSDE Instance Port & 9995 \\
\hline Physical drive location & e:larcgislarcsdelsqlexe_testlbinlgiomgr.exe \\
\hline
\end{tabular}

\subsection{Production ArcSDE Environment}

This is the full production environment. When the system was rolled into production, the SQL/SDE database server and the Citrix server became solely dedicated for production use. Before production, this environment was used for miscellaneous other tasks including an additional load environment for migration data. Only fully tested and approved cuts from the Test environment are promoted into the Production environment.

Table 4.6-6. Production instance on the production server

\begin{tabular}{|l|l|}
\hline SQL Server/SDE Server Name & aedrmdbsqlp01 \\
\hline SQL Server Instance Name & aedrmdbsqlp01 \\
\hline ArcSDE Instance Name & esri_sde \\
\hline ArcSDE Instance Port & 9996 \\
\hline Physical drive location & e:larcgislarcsdelsqlexelbinlgiomgr.exe \\
\hline
\end{tabular}




\subsection{Citrix Server Environments}

The following Citrix machines will be used for the application test environment:

1. aedrmapcrx01

The following Citrix machines will be used for the application production environment:
2. aedrmapcrx02
3. aedrmapcrx03
4. aedrmapcrx04
5. aedrmapcrx05

\subsection{Data Workflow}

This section details the ideal ArcSDE workflow beginning with the Visio data model and ending with the rollout of the data into the production environment. Figure 4.6-2 illustrates this process including the workflow steps that occur within each environment and the steps to move the data between the environments. The processes are noted at a high level. More detail is provided in later sections.

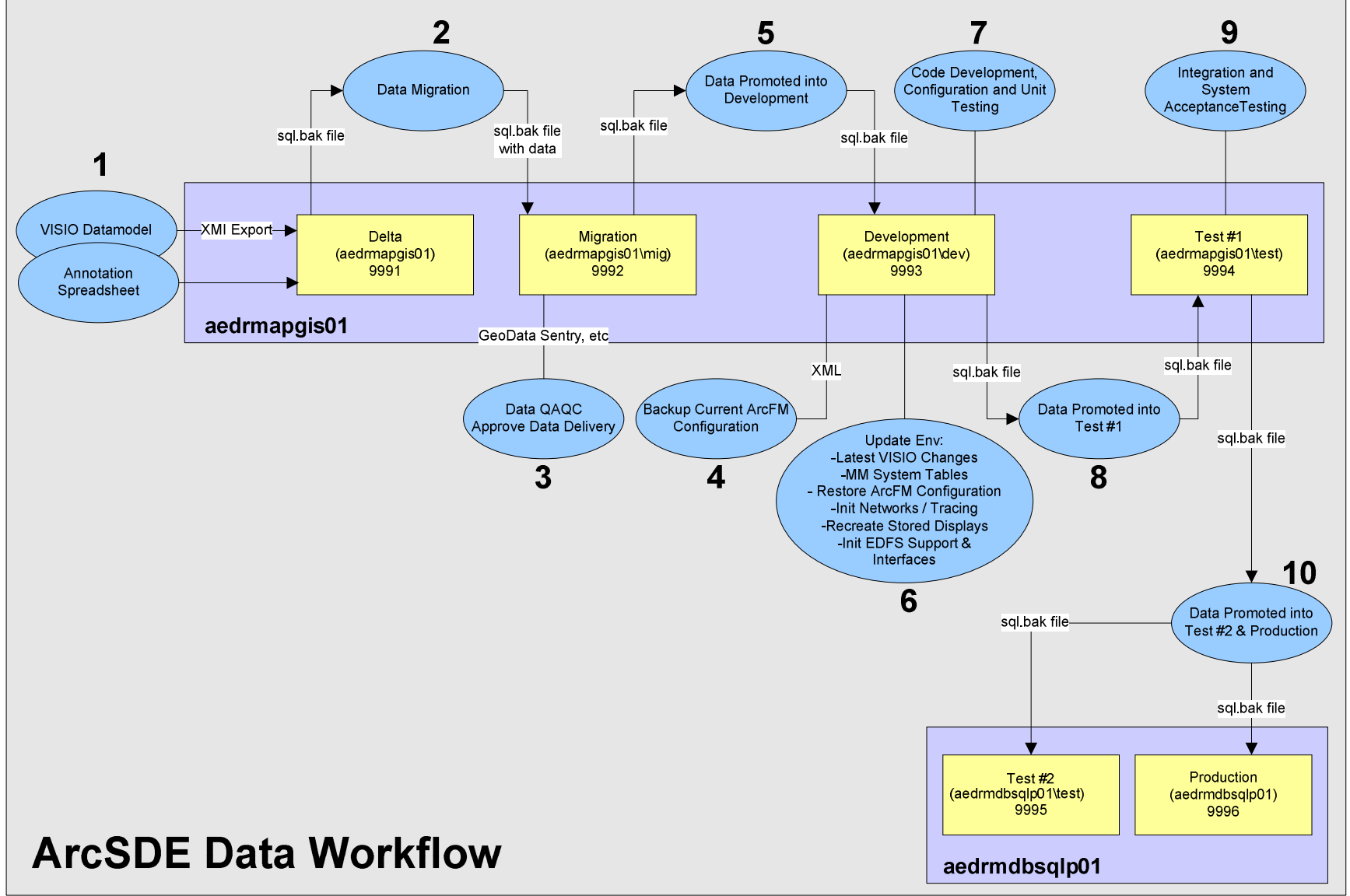

Figure 4.6-2. 


\subsection{Environment Procedures}

This section details various procedures for creating/loading/configuring data within the various environments.

\subsection{Environment Status Log}

Whenever any update is made to an environment (including restoring data, configuration, development, testing, etc) the update is added to the "NIPSCO Database Instance Status Log.xls" spreadsheet (stored within SourceSafe under "\$ $\$$ Administrative Documents $\backslash A r c S D E l$ "). This document contains a worksheet for each database environment and is used as a team-wide tool for tracking and identifying the status of any given environment at any point in time. This document is also referenced before any significant change is made to an environment to ensure that the change will not adversely affect another process that might be running against that environment.

\subsection{Creating a Clean Model - Delta Environment}

1. Stop SDE on the Delta instance. Have the responsible DBA drop and recreate the SDE and ArcFM8 databases and their users.

2. Run a reinstall of the SDE tables and data tables following the naming convention by using ArcSDE for Microsoft SQL server Post Installation located in programs on the SDE server. Choose the custom installation and click "next". Un-check the create ArcSDE Service and click "next"

3. Import the Visio XMI exports (electric, gas, and electric) into the SQL instance. Logon as the user Electric to import the electric.xml, Gas to import the Gas.xml and Landbase to import the Landbase.xml

4. Import the Annotation spreadsheet into the SQL instance. Logon as the user Electric to import electric annotation, Gas for Gas, and Land for Land.

5. Set SQL Server roles as described in SQL Server User Roles.

\subsection{Configuration Steps - Migration Environment}

1. Download the latest ArcFM8 and SDE SQL backup files from the data migration vendor website and put them in a folder named as the delivery date on server aedrmapgis01 physical drive E: $\backslash \mathrm{SQL}$ Backup deliveries from the data migration vendor.

2. Fill out a DBA request form for the responsible DBA to do a restore to the migration instance aedrmapgis01 $\backslash \mathrm{mig}$ specifying the file locations and names.

3. The DBA will need to run E: $\backslash$ ArcGis $\backslash$ ArcSDE $\backslash S S U$ serRelink.sql in Query analyzer to re-link the user accounts.

4. Set SQL Server roles as documented in user role matrix.xls.

5. If there is a dbtune view in the restored ArcFM8 table, delete it.

\subsection{Configuration Steps - Development Environment}

1. Do a backup of the ArcFm8 and SDE databases in the Migration instance.

2. Export the current Development environment ArcFM configuration into XML files and save. 
3. Restore the bak created in step one into the Development environment.

4. Re-link the users with the SSUserRelink.sql.

5. Import the latest Visio XML exports into the SQL instance. Logon as the user Electric to import the Electric.xml, Gas to import the Gas.xml and Landbase to import the Landbase.xml. This will ensure that any schema changes that have occurred since the start of the current data migration iteration are included in the development environment.

6. Create MM system tables.

7. Convert to ArcFM objects.

8. Set SQL Server roles as documented in user role matrix.xls.

9. Initialize users on the system.

10. Revoke ddladmin access after for each user after step 8 is complete.

11. Import the ArcFM XML configuration files back into the Development environment.

12. Initialize Feeder Manager and Gas Tracing which includes initializing the circuit source table, initializing trace weights, re-building the electric and gas geometric networks, etc.

13. Recreate standard Stored Displays from the mxd files.

14. Initialize EDFS Data Schema Support via EDFS Operations Guide (views, procs, SAGE db, Inteface Tables, etc).

\subsection{Configuration Steps - Test (aedrhamgis01) Environment}

1. Do a backup of the ArcFm8 and SDE databases in the Development instance.

2. Restore the bak created in step one into the Test environment.

3. Re-link the users with the SSUserRelink.sql.

4. Set SQL Server roles as documented in user role matrix.xls.

5. Initialize users on the system.

6. Revoke ddladmin access for each user.

\subsection{Configuration Steps - Test (aedrdbsqlp01)}

1. Do a backup of the ArcFm8 and SDE databases in the Test instance.

2. Restore the bak created in step one into the Production environment.

3. Re-link the users with the SSUserRelink.sql.

4. Set SQL Server roles as documented in user role matrix.xls.

5. Initialize users on the system.

6. Revoke ddladmin access for each user.

\subsubsection{Elementool}

The AEDR project team determined that a centralized issue tracking system was needed to track design questions, software bugs, enhancements, and other software or data issues. After considering several options, the web-based product 'Elementool' was selected to fill this requirement. Elementool provides its service for a monthly subscription fee and offers several advantages over other comparable tools in a similar price range: 
- No software or hardware is required: Elementool hosts its bug tracking application on its website. By using Elementool, NIPSCO did not need to install a database server, web server or client application to manage issues.

- Web based: Elementool is fully web based which was an important feature to support the offsite contractors that were/are involved on the AEDR project.

- Unlimited Users: There is no limit to the number of users who can access the NIPSCO Elementool account. This allowed the project team to include all aspects of the project in the system.

- Fully customizable: Elementool is a fully customizable system, which enabled the NIPSCO GIS project team to fully customize the forms and reports to meet specific needs.

- Downloadable database for self-backup: A Microsoft Access database is downloaded daily as a backup of the system/account.

- Email Notification: Automatic email messages are sent to different account users, informing them about Issues that are assigned to them.

- Advanced Reports: Enables searching the Issue list for different words and phrases, creates focused reports with only the Issues of interest (using AND/OR/NOT), set the fields that should be included in the Report Query and set the fields that should be displayed on the Issue Report.

- File Attachments: Enables file attachment such as screenshots, images and text documents to issues. The attached files provide other users with a better description of the specific issue.

- History Trail: Tracks and displays changes made by users in each issue throughout its life cycle.

The following procedures detail the life cycle of an Elementool issue from the time the issue is discovered to the time the issue has been resolved. These procedures ensure that all issues are entered into Elementool with the appropriate detail and each issue is addressed by the appropriate resources.

\subsubsection{Discovering / Researching an Issue}

During the initial phase of the life cycle the analyst / tester first becomes aware of an issue or problem with the GIS System. The analyst / tester should spend some time gathering adequate details concerning the problem and perform a search in Elementool to determine if an existing issue record already covers the problem.

If the analyst / tester finds an existing issue, any new details are added to the existing Elementool record. Or the analyst / tester can create a new record that lists related records as Links. If no issue has been logged the analyst / tester should create a new record and add all information collected so far. 


\subsection{Creating a New Issue}

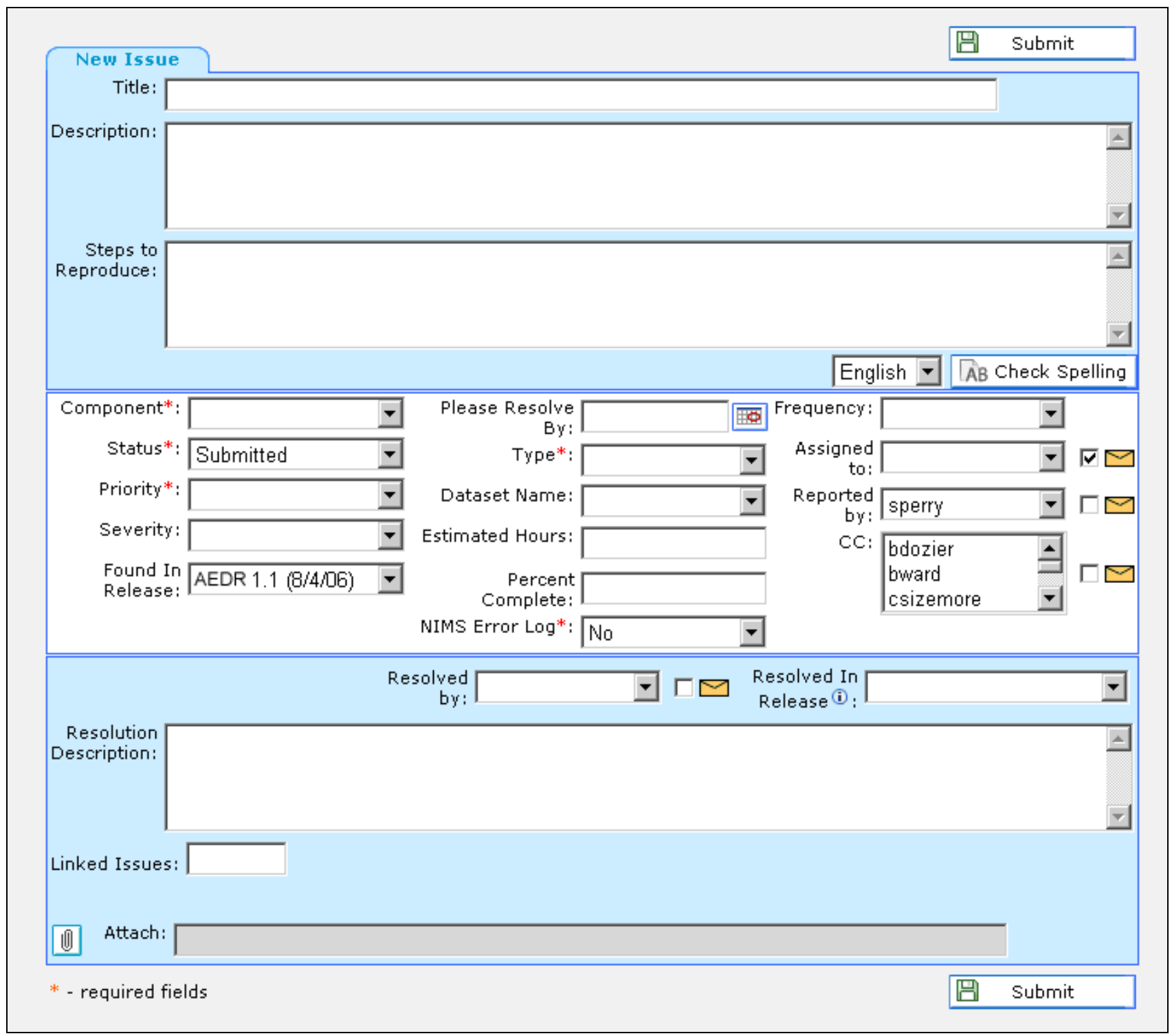

Figure 4.6-3. Image shows the form used to create a new issue

The following procedures were developed by the NIPSCO GIS project team for submitting a new issue:

1. Fill out the Title field with a high level summary of the problem. Try to limit the text to 80 characters for readability. If the issue relates to a specific table/attribute, make sure to include the table and attribute name to allow for easier search-ability.

2. Add details to the Description field that outlines the history and circumstance surrounding the occurrence of the issue. This field can hold a lot of text so the more details the better. 
3. In the Steps to Reproduce section add step by step instructions that describe how to make the problem occur again. This will also help the tester ensure that the problem has been resolved.

4. Select the option under the Component drop down list that best describes the area in which the issue was discovered.

5. Leave the Status field set to Submitted.

6. Choose an appropriate Priority, for example select Immediate if this issue is a showstopper that prevents the project from moving forward. On the other hand, select Low if the issue has little impact on functionality or progress of the project. If you select Immediate, notify the team leader assigned to that area right away about the issue.

7. Do not select anything for Severity. The Severity will be determined during the daily team review.

8. Select the project phase in which the issue was discovered. This will help as time goes by to know what state the system was in when the issue was discovered.

9. For the Type field select Question if you think the issue may not be a problem but simply requires clarification or may lead to a problem in the future. Select Bug if the issue adversely affects the performance of system and if the issue is related to a coding / configuration defect. Select Data Model Change if the issue requests that a Data Model Change be made. Do not select Enhancement when submitting an issue. The analyst who reviews the issue record will set the Type to Enhancement if the issue requests functionality that is beyond the scope of the project. Select AutoUpdater if the issue is related to an AutoUpdater. Select Data as the type if the issue is related to source data issue (i.e., data cleanup will be required).

10. Select the appropriate data set from the Dataset Name field.

11. Leave the Assigned to field blank. This issue will be assign be a daily team review process.

12. Uncheck any of the mail boxes on the right hand side of the screen.

13. Click the Submit button.

\subsection{Assigning an Issue}

Each morning, the Elementool team leader, and any other appropriate resources will discuss new issues that have been logged into Elementool but have not been assigned. An analyst will be selected to work on each unassigned issue. The Elementool team leader will make the changes to the Elementool Assigned to field. The new Elementool issues will be added to the project plan with the assigned analyst and an initial hours estimate.

\subsection{Reviewing an Issue}

1. An analyst will be notified that they have had an issue assigned to them

2. The analyst will visit the issue record in Elementool that same day.

3. While in Elementool set the Status field to In Progress.

4. Ensure that all fields are set, including Found in Release, Severity, and Frequency.

5. Read over all the information in the issue record to ensure the issue has been properly assigned and that adequate details exist in the record. 
6. If the issue has not been assigned correctly set the Status field to Need to ReAssign. Provide a short description of why a re-assignment is required in the Solution Description field.

7. Perform an Elementool search to ensure that no duplicate records have been entered. If a duplicate record is found, set the Status field of the new record to Duplicate and enter the ID number of the original issue record in the Links field.

8. Review the Vision Document to make sure that the changes requested or required by the issue are with in the scope of the project. If the request is out of scope, set the status field to Out of Scope and the type field to Enhancement.

9. Click the Update button.

\subsection{Resolving and Issue}

1. Add all details involving the solution of the issue in the Resolution Description field.

2. Set the status field to Resolved.

\subsection{Re-Assigning an Issue}

An analyst may find that a problem can only be partially resolved within their particular area of expertise. In this case the analyst cannot resolve the issue because additional work is required by someone else working on the project. The analyst should set the Status to In Progress and set the Assigned to field to the appropriate name. Notify the analyst that the new issue has been assigned. Be careful about delegating work to project resources, some work may require approval from the project manager. If in doubt, verify that the issue should be re-assigned first.

A change to the data model must be carried through each step of the data model change path. The issue cannot be marked as resolved until all steps in the path are complete. The data model change path is outlined below.

1. Alter the physical model and validate via Semantics Checker.

2. Alter the logical model to match the physical model.

3. Import the physical model changes into the sample data set.

4. Alter the data migration matrix spreadsheets to match the changes to the physical model.

5. Update ArcFM settings, connectivity rules, and symbology settings where necessary.

6. Update any Autoupdater or customer code impacted by the changes.

An analyst should complete all steps of the path that fall within their realm of responsibility. A note indicating that the step has been completed should be added as a Resolution Description on the issue record. Once these are complete, set the Status to Need to Re-Assign. The issue will be re-assigned the following morning.

\subsection{Review and Rework}

The "In Review", "Ready for Review", and "Needs Rework" status settings are used for issues that are worked on by multiple resources. The example below demonstrates how these status settings might work. 
1. A team member identifies a bug in the GIS Tool.

2. The team member searches Elementool for existing issues related to the problem.

3. When no matching issues are found, a new Elementool issue is entered.

4. The Elementool team lead assigns an initial resource to the issue and sets the issue Status field to "Assigned".

5. The analyst assigned to work on the task sets the "Status" field to "In Progress", researches the problem, and identifies a solution.

6. The analyst implements the solution, sets the issue Status field to "Ready for Review", and sets the "Assigned to" field to the name of a reviewer.

7. The reviewer opens the issue, sets the "Status" field to "In Review", and checks that the solution resolves the problem stated in the issue.

8. If the solution is good, the "Status" field is marked as Resolved. However, if the solution does not resolve the problem, the "Status" field is set to "Needs Rework" and notes are added about how the solution falls short of resolving the problem.

9. The original analyst sets the "Status" field back to "In Progress" and continues work on the issue. The process repeats until the issue has been resolved.

\subsection{Reporting}

A report of all issues having a Status of Submitted or Need to Re-Assign will be generated each morning. This report will be reviewed and all issues will be appropriately assigned.

\subsubsection{Microsoft Visual SourceSafe}

Version control systems are a means of tracking various versions of a set of files. It was determined early on that the AEDR project required a system to track both software development materials and project documentation. Microsoft Visual SourceSafe was chosen because it is the standard among Microsoft based development groups. SourceSafe deals with the issue of interaction between developers and other project members.

SourceSafe operates in the following manner:

- First, a database must be set up on a centralized server.

- Next files will be added to the centralized database. This is the $A d d$ operation.

- Once files exist in the database, they are available for modification. This is accomplished via the Checkout operation. Using the Checkout operation stores a copy of the file on a users' local drive and prevents other users from attempting to checkout the file for modification.

- The Undo Checkout command reverts to the last state of the file.

- The Checkin operation copies the updated file back in to the database, removes the lock and the file is once again made available for Checkout.

- The Get operation allows retrieval of older versions of a file or groups of files.

- Rename and Delete operations allow file management. 
- Version control systems can generate various useful reports about files. These are the Difference, History, Properties and Status operations. 


\subsection{AEDR Customization}

\subsubsection{EDFS (Electric Distribution Facility Services) Integration - Including EDFS Data Modeling}

This section describes the plan that was used to integrate the NIPSCO EDFS functionality into the new ArcGIS/ArcFM environment. The implementation was accomplished with a blend of data model and database updates, customization to ArcFM, and a custom Stand Alone Geodatabase Editor (SAGE), which exists independently of the GIS applications. The goals of this implementation include both the replacement of the EDFS functionality as well as the enhancement of both the technology and the business processes to provide a more efficient and effective solution for all concerned users of the system. There was a significant amount of documentation from the time when the EDFS system was originally implemented in 1986. Much of the specific application data management documentation still applied to the AEDR and was used whenever possible. This document provides EDFS documentation reference IDs whenever possible to allow for the easy location of the original documents.

This section details the following components that were used to implement the EDFS functionality within the new AEDR/ArcFM system:

- GIS Data model changes

- EDFS Data Migration

- ArcFM Auto Updaters

- Batch Data Management and Reporting Applications

- External System Interfaces

- SAGE Architecture

- SAGE Authentication and Authorization

- SAGE Data Model

- SAGE Screen Design

- SAGE Reports

\subsubsection{EDFS Integration Objectives}

Create functionality in the new ArcGIS/ArcFM system to satisfy all business needs related to the current EDFS system:

- Lifecycle management of Units of Property including Transformers, Regulators, and Capacitors

- Lifecycle management of all other standard overhead and underground assets including Poles, Pads, Vaults, Assemblies, Protective Devices, Conductor, and Lighting

- Current and legacy reporting on assets for both internal and external entities

- Interfaces to other NIPSCO systems including MAPPS (Materials, Purchasing and Accounts Payable), MLOG (Compatible Units), General Ledger, CIS (Customer Information System), and Tax Department. 
To improve the efficiency and effectiveness of the manner in which the data is accessed:

- Integrating asset data storage into the GIS

- Integrating asset data management into ArcFM (installation, removal, retirement, etc)

- Providing non-GIS data access to users who do not rely on the spatial aspect of the data

- Removal of unnecessary batch reporting while maintaining business value

- Improving workflow of targeted users by making data updates more efficient and straightforward by leveraging more modern technologies.

\subsubsection{EDFS Background}

The NIPSCO Electric Distribution Facilities System (EDFS) was a stand-alone asset management application that managed the utility's electric assets including transformers, regulators, capacitors, primary, secondary, and service conductor, poles, pads, streetlights, traffic lights, and miscellaneous data surrounding these assets.

EDFS was responsible for managing the life cycle of these assets from the time of their acquisition through retirement or scrapping. This life cycle can include multiple installations, retirement, condemnation, sale, and/or lease. In addition to managing these assets, EDFS was responsible for generating many reports based on the current and/or historical states of the assets. These reports are used by management for the assessment of the current assets as well as tax reporting purposes.

EDFS was built using the mainframe COBOL programming language and runs on top of a mainframe Customer Information Control System (CICS). It utilized standard COBOL text-based screens to interact with the user and utilizes SQL to read and write to the underlying DB2 database. There was no integration between EDFS and NIPSCO's mapping tool, a custom CAD-based application called Outfield. EDFS data records were manually matched to Outfield geographical records based on unique IDs including Company Number.

\subsubsection{EDFS GIS Data Model Changes}

The old EDFS system ran on top of a mainframe DB2 database that was completely separate from the Outfield graphics system. In the AEDR, this data structure was integrated directly into the GIS database. To accomplish this, the GIS data model was significantly updated to handle the EDFS data. These changes are primarily centered on the unit and conductor info object classes within the model but also included a few feature classes including support structure, pads, and switchgears. In addition, many edits to the relationships were required.

An extensive review of the proposed NIPSCO data model was conducted to identify the changes that were required to implement the EDFS functionality within the AEDR. The EDFS data model changes have been systematically detailed below. Appendix F provides the step-by-step edits that were made to the current proposed data model to allow it to handle the migration of the data including both abstract and concrete classes. 
While these recommended changes handled $95 \%$ of the needed changes for the EDFS functionality, there were minor additional changes that arose during development. These minor changes were documented during the development phase within Elementool and were presented to NIPSCO at the completion of the development phase. These minor changes did not affect data migration. Appendix F details out the changes by asset area.

The rest of the EDFS section refers to the functionality built on top of a data model that included all of the recommended changes.

\subsubsection{EDFS AutoUpdaters}

Much of the EDFS functionality was handled by out-of-the-box (OOTB) ArcFM tools within the AEDR including the ArcFM Attribute Editor. This tool allows the users to update the various EDFS records within the AEDR but does not handle any custom validation and/or field population. To accomplish these automated tasks, several simple ArcFM Auto Updaters (AUs) were created. These AUs are detailed in the following sections. When appropriate, the related EDFS screen number has been included in the description to provide additional background on the functionality that was created.

\subsection{OnCreate-ReplicateSinKVA}

ArcFM contains OOTB functionality to update the Transformer Bank KVA based on a summation of all child unit KVA values. For this to work correctly, model names must be set correctly on both the TransformerBank feature class and the related unit object class. In the NIPSCO model, the TransformerUnitInstall object class represents the unit object class that has relationships to the TransformerBank. NIPSCO uses a Stores Item Number (SIN) lookup table, and TransformerRegulatorStoresItem to manage standard KVA values for transformer units. The TransformerRegulatorStoresItem table is related to the TransformerUnit table which it turn is related to the TransformerUnitInstall table. For the OOTB ArcFM AU to work correctly, the SIN standard KVA values were required to be replicated directly on the TransformerUnitInstall record. This AU handles this replication and was configured to execute before the "ArcFM Update Transformer Bank Rated KVA" AU.

\subsection{Assign to Class}

TransformerUnitInstall

\subsection{Use Case}

Upon receiving a transformer install ticket back from the field, the user locates an existing TransformerUnit record by searching on the company number and then creates a new related TransformerUnitInstall record with the field installation data. When the new record has been created and saved the KvaValue field is automatically populated. The user can traverse the relationships from TransformerUnitInstall to TransformerUnit to TransformerRegulatorStoresItem to verify that the KvaValue on the TransformerUnitInstall record matches the value in the related TransformerRegulatorStoresItem table. 


\subsection{Design}

The following actions are performed upon the creation of a TransformerUnitInstall record:

1. Use the appropriate relationship classes to trace the relationship from the TransformerUnitInstall table to the TransformerUnit table to the TransformerRegulatorStoresItem table.

2. Read the value from the TransformerRegulatorStoresItem.KvaValue field.

3. Insert this value into the TransformerUnitInstall.KvaValue field.

\subsection{OnUpdate - UpdateSinKva}

Per the section above, if the SIN value on a TransformerUnit is updated to a new value, the related KVA values may need to be updated on any related TransformerUnitInstall records. Because each unique transformer SIN entry in the TransformerRegulatorStoresItem table has an assigned KVA value, these values must be replicated whenever the SIN is updated.

\subsection{Assign to Classes}

TransformerUnit

\subsection{Use Case}

The user locates an existing TransformerUnit record that has existing TransformerUnitInstall records. The user manually validates the current value of the TransformerUnitInstall.KvaValue field. The user then updates the TransformerUnit.RelStoresItemNumber field either manually or by using "ArcFM Locate and Relate" functionality. Once this update has been saved, the user should look at the related TransformerUnitInstall records once again and should validate that the KvaValue field has been updated and matches the value in the related TransformerRegulatorStoresItem.KvaValue field.

\subsection{Design}

The following actions are performed upon the update of a TransformerUnit record:

1. Determine if the RelStoresItemNumber field has been updated.

2. If an update has been made, use the appropriate relationship class to trace from the TransformerUnit table to the TransformerRegulatorStoresItem table.

3. Read the value from the TransformerRegulatorStoresItem.KvaValue field.

4. Use the appropriate relationship class to trace from the TransformerUnit table to all related TransformerUnitInstall records.

5. Update the TransformerUnitInstall.KvaValue field of each related record with the value from TransformerRegulatorStoresItem.KvaValue. 


\subsection{OnUpdate - Asset Removal}

This functionality was derived from the screen ED43 specifications. When an installation unit is disassociated from a bank, it constitutes a removal of the asset. There are several actions that need to happen when this occurs. A removal record should be created that captures the removal date, the material ticket number, and the removal reason code. A new stock record should be created and the status on the unit table (TransformerUnit, Regulator Unit) should be set to "in stock". The related FeederId, structure Distribution Reference Number, Local Operating Area (LOA) Number, Tax Unit Code, and Grid Code is recorded on the UnitInstall record so the information is available going forward in a historical context. And finally, a removal transaction record needs to be recorded in the GisMappsTransactionQueue table so that it can be matched to a MAPPS transaction during the nightly batch processing. A custom AU was created to automate this process as much as possible.

\subsection{Assign to Classes}

- TransformerUnitInstall

- RegulatorUnitInstall

\subsection{Use Case}

The user locates a TransformerBank or VoltageRegulator feature that has a unit that needs to be removed or retired. The user selects the related TransformerUnitInstall or RegulatorUnitInstall record, right-clicks, and selects the "Unrelate" menu option. The user is then prompted with a form that asks for the Removal Date, the Material Ticket Number, and a removal Reason Code. The user enters the requested information and clicks OK. The install record is then disassociated from the feature, a new removal record is created, a new stock record is created, the status on the unit record is set to "S - In Stock", the circuit number, distribution reference number, and LOA Number on the install record are updated correctly, and a new transactional record is created to be matched to MAPPS. The user also has a cancel button on the form that prevents the disassociation operation from being completed.

\subsection{Design}

The following actions are performed upon the update of a TransformerUnitInstall and RegulatorUnitInstall record:

1. Determine if the TransformerUnitInstall.RelTransformerBankObjectId or RegulatorUnitInstall.RelVoltageRegulatorObjectId has been updated from a valid long value to null, which would indicate a disassociation from the feature: 


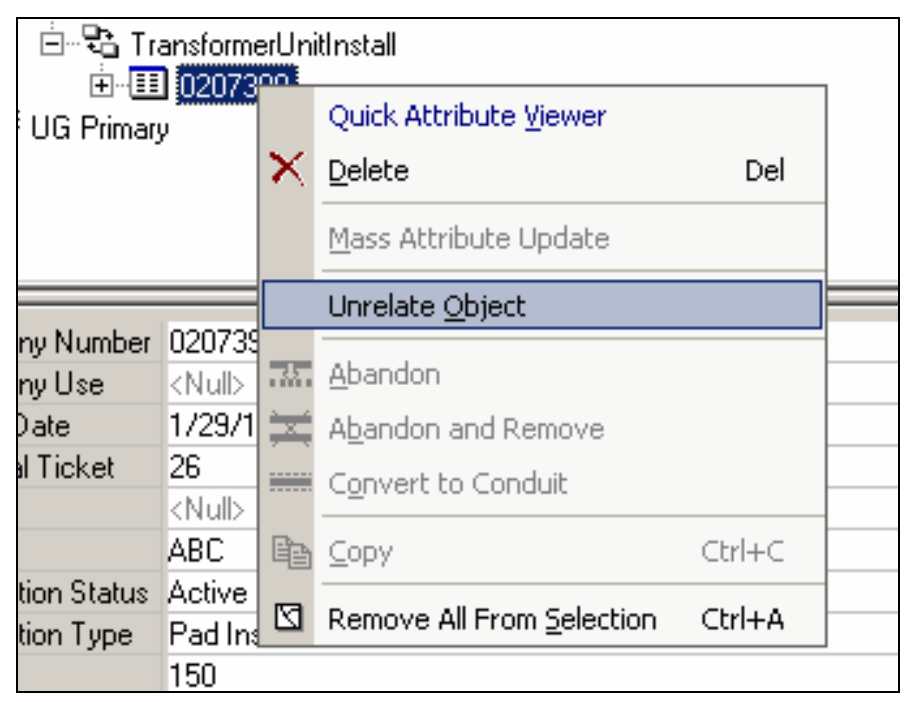

Figure 4.7-1. Unrelate transformer/regulator

2. Present the user with a custom form that prompts them for a Removal Date (using a date picker control), the material ticket number, and the reason code (drop down with valid domain for Transformer and Regulator).

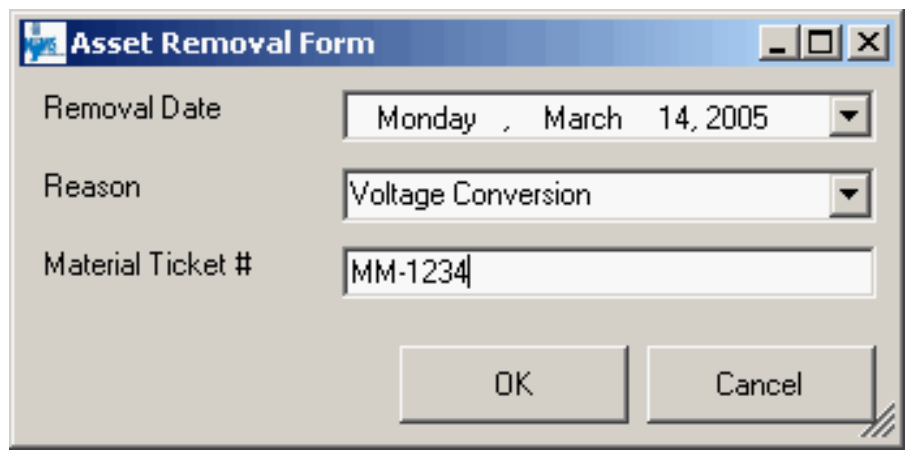

Figure 4.7-2. Remove the transformer/regulator

3. The user enters the information and clicks $\mathrm{OK}$ to continue with the operation or clicks Cancel to roll back the operation.

4. A new TransformerUnitRemove or RegulatorUnitRemove record is created and populated with the above gathered information. The RemovalTimestamp field is also populated with the system date and the CompanyNumber is populated from the CompanyNumber field of the install record.

5. A new TransformerUnitStock or RegulatorUnitStock is created. The StockTimestamp should match the RemovalTimestamp and the StockDate should match the RemovalDate. The CompanyNumber should be set from the installation record and the RelStoreRoomNumber should be set to the installation record's LoaNumber value.

6. The related TransformerUnit or RegulatorUnit record's StatusCd field should be set to "S" (in-stock) and the StatusTimestamp should be set equal to the RemovalTimestamp. 
7. Use the appropriate relationship classes to trace from the UnitInstall record to the TransformerBank or the VoltageRegulator feature. Retrieve the FeederId and insert this value into the UnitInstall.CircuitNumber field.

8. Use the appropriate relationship classes to trace from the UnitInstall record to the TransformerBank or VoltageRegulator feature to the SupportStructure or Pad feature. Retrieve the DistribRefNumber, LoaNumber, TaxUnitCd, and GridCd and insert these values into the UnitInstall.DistribRefNumber, UnitInstall.LoaNumber, UnitInstall.TaxUnitCd, and UnitInstall.GridCd respectively.

9. Insert a new record into GisMappsTransactionQueue with the following structure: insert into GisMappsTransactionQueue (CompanyNumber, TransactionType, LoaNumber, StoresItemNumber, TransasctionDate) values ([UnitInstall.CompanyNumber], "RMV", [UnitInstall.LoaNumber], [Unit.StoresItemNumber], [user-entered Removal Date]).

\subsection{OnUpdate - Capacitor Removal}

This functionality was derived from the screen ED32 specifications. When an installation unit is disassociated from a capacitor bank it constitutes a removal of the asset. There are several actions that happen when this occurs. A removal record is created that captures the removal date, the Work Order Number or the Miscellaneous Order Id, and the removal reason code. Because capacitors can no longer be installed more than once, a new stock record will not be created. Finally, the related FeederId and Support Structure Distribution Reference Number, LOA Number, Tax Unit Code, and Grid Code are recorded on the CapacitorUnitInstall record so the information is available going forward in a historical context. A custom AU was created to automate this process as much as possible.

\subsection{Assign to Classes}

CapacitorUnitInstall

\subsection{Use Case}

The user locates a CapacitorBank feature that has a unit that needs to be retired. The user selects the related CapacitorUnitInstall record, right-clicks, and selects the "Unrelate" menu option. The user is then prompted with a form that asks for the Removal Date, the Work Order Number OR the Miscellaneous Order ID, and a removal Reason Code. The user enters the requested information and clicks OK. The install record is then disassociated from the feature, a new removal record is created, the status on the unit record is set to "C - Out of Service", if the removal reason code was " $\mathrm{B}$ -

Sold/Exchanged REMC" or "E - Lost/Stolen", the unit record disposition code should be set to "C - Conveyed to REMC" or "L - Lost or Stolen" respectively, and the circuit number, distribution reference number, LOA Number, Tax Unit Code, and Grid Code on the install record are updated correctly. The user should also have a cancel button on the form that prevents the disassociation operation from being completed. 


\subsection{Design}

The following actions are performed upon the update of a CapacitorUnitInstall record:

1. Determine if the CapacitorUnitInstall.RelCapacitorBankObjectId has been updated from a valid long value to null, which would indicate a disassociation from the feature:

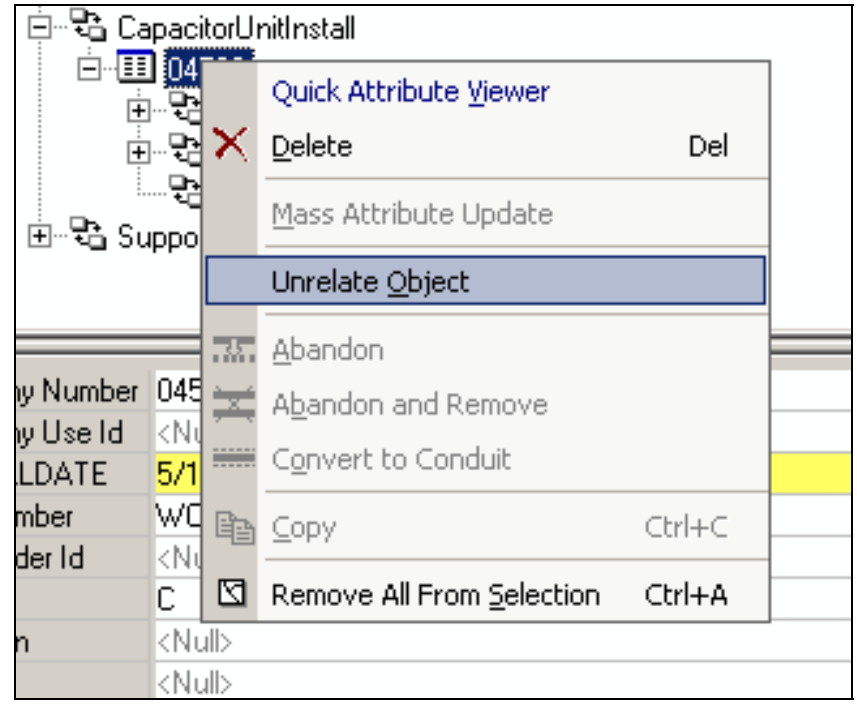

Figure 4.7-3. Unrelate the Capacitor

2. Present the user with a custom form that prompts them for a Removal Date (using a date picker control), the Work Order Number OR the Miscellaneous Order Id (an either/or choice, must begin with "WO" or "MO" respectively), and the reason code (drop down with valid domain for Capacitors):

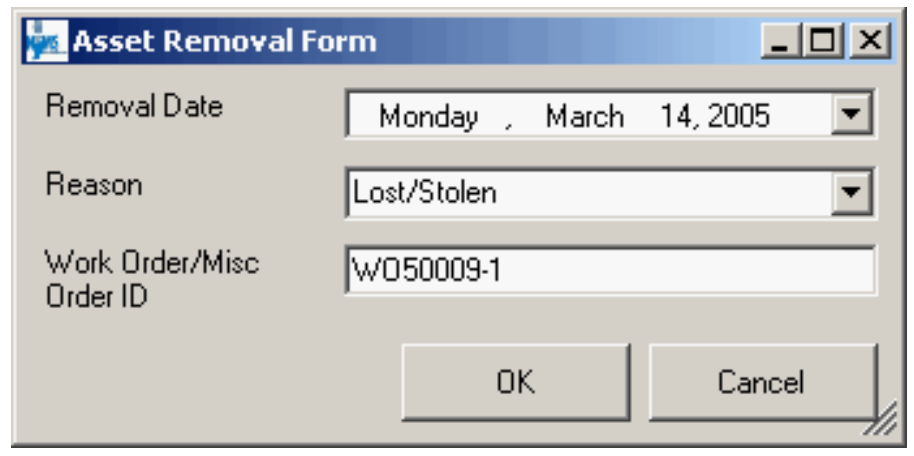

Figure 4.7-4. Remove the capacitor

3. The user enters the information and clicks OK to continue with the operation or clicks Cancel to roll back the operation.

4. A new CapacitorUnitRemove record is created and populated with the above gathered information. The RemovalTimestamp field is also populated with the system date and the CompanyNumber is populated from the CompanyNumber field of the install record. 
5. The related CapacitorUnit record's StatusCd field should be set to "C" (Out of Service) and the StatusTimestamp should be set equal to the RemovalTimestamp.

6. If the removal reason code was "B - Sold/Echanged REMC" or "E - Lost/Stolen", the unit record disposition code should be set to "C - Conveyed to REMC" or "L Lost or Stolen" respectively.

7. Use the appropriate relationship classes to trace from the CapacitorUnitInstall record to the CapacitorBank feature. Retrieve the FeederId and insert this value into the UnitInstall.CircuitNumber field.

8. Use the appropriate relationship classes to trace from the CapacitorUnitInstall record to the CapacitorBank feature to the SupportStructure feature. Retrieve the DistribRefNumber, LoaNumber, TaxUnitCd, and GridCd and insert these values into the CapacitorUnitInstall.DistribRefNumber, CapacitorUnitInstall.LoaNumber, CapacitorUnitInstall.TaxUnitCd, and CapacitorUnitInstall.GridCd respectively.

\subsection{OnDelete - ResetAssetToStock}

When a UnitInstall record is deleted, the status and timestamp of the corresponding Unit record must be updated so that it shows as being back in stock.

\subsection{Assign to Classes}

- TransformerUnitInstall

- RegulatorUnitInstall

- CapacitorUnitInstall

\subsection{Use Case}

The user locates a UnitInstall record that needs to be deleted, right-clicks it, and selects delete. When the record is deleted from the database, the related Unit record's status is updated from "I - Installed" to "S - In Stock" and the status timestamp is updated to be equal to the stock timestamp on the most recent stock record.

\subsection{Design}

The following actions are performed upon the delete of any of the above-mentioned records:

1. Update the related Unit record (TransformerUnit, RegulatorUnit, CapacitorUnit), setting the StatusCd = "S - Stock".

2. Query the UnitStock table (TransformerUnitStock, RegulatorUnitStock, CapacitorUnitStock) for the timestamp of the most recent stock record (i.e. select $\max ($ StockTimeStamp) from UnitStock where CompanyNumber=[Unit.CompanyNumber]).

3. Set the StatusTimestamp of the Unit record equal to the value found in step 2. 


\subsection{OnDelete-ResetAssetTolnstalled}

When a UnitRemove record is deleted, the status and timestamp of the corresponding Unit record must be updated so that it shows as being back installed. The corresponding UnitStock record (which was added during the removal operation) must also be deleted.

\subsection{Assign to Classes}

- TransformerUnitRemove

- RegluatorUnitRemove

- CapacitorUnitRemove

\subsection{Use Case}

The user locates a UnitRemove record that needs to be deleted, right-clicks it, and selects delete. When the record is deleted from the database, the related Unit record's status is updated to "I - Installed" and the status timestamp is updated to be equal to the install timestamp on the most recent UnitInstall record. The most recent UnitStock record is also deleted.

\subsection{Design}

The following actions are performed upon the delete of any of the above-mentioned records:

1. Update the related Unit record (TransformerUnit, RegulatorUnit, CapacitorUnit), setting the StatusCd = "I - Installed".

2. Delete the most recent UnitStock (TransformerUnitStock, RegulatorUnitStock) record, which has a StockTimestamp equal to the RemovalTimestamp of the UnitRemove record that is being deleted. NOTE: This step only applies to Transformers and Regulators.

3. Query the UnitInstall table (TransformerUnitInstall, RegulatorUnitInstall, CapacitorUnitInstall) for the timestamp of the most recent stock record (i.e. select $\max ($ InstallTimeStamp) from UnitInstall where CompanyNumber=[Unit.CompanyNumber $]$ ).

4. Set the StatusTimestamp of the Unit record equal to the value found in step 3.

\subsection{OnCreate, OnUpdate - ValidateWorkOrderNumber}

This functionality was derived from the screen ED20 specifications. This AU first validates the format of the entered Work Order Number of Miscellaneous Order Id. The AEDR has a real time interface to the General Ledger system that provides this AU with the ability to verify that a user-entered work order number exists within the General Ledger system (see details in the interface section of this document). There is no validation for Miscellaneous Order Ids. Because both the Work Order and Miscellaneous Order Id fields are mandatory fields, they must both contain values even if only one has a valid Id. If the user enters a valid entry in the Work Order field, they should enter a value of "WO" in the Miscellaneous Order Id field. If the user enters a valid entry Miscellaneous Order Id field, they should enter "MO" in the Work Order field. This AU does not attempt to validate values of "MO" against the General Ledger system. 


\subsection{Assign to Classes}

- CapacitorUnitInstall

- CapacitorUnitRemove

- OhConductorInfo

- UgConductorInfo

- SecondaryConductorInfo

- LegacyOhConductorInfo

- LegacyUgConductorInfo

- LegacySecondaryConductorInfo

- ServiceConductorInfo

- CompanyStreetlight

- StreetLightSwitch

- SectionalizerUnit

- SwitchUnit

- RecloserUnit

- FuseUnit

- SupportStructure

- PadMount

- SwitchGear

- Pedestal

- RetiredSupportStructure

- RetiredPadMount

- RetiredSwitchGear

\subsection{Use Case}

The user enters a value into the Work Order field or the Miscellaneous Order Id on any of the above-listed classes. When the record is saved, the system verifies that the format of the entry is correct and then validates work order numbers against the General Ledger system. If the entered number is a valid number it will be accepted and the record will be saved. If it is not a valid number, the user should be warned and prompted to fix it. The user can then reenter a valid number.

\subsection{Design}

The following actions are performed upon the creation or update of any of the above mentioned records:

1. If a Work Order Number or Miscellaneous Order Id was entered by the user, verify the format is correct.

2. If the number is not in the correct format and is not equal to "MO" or "WO" respectively, warn the user with the message "The [Work Order Number / Miscellaneous Order ID] that was entered was not in the correct format. Please reenter the number and save again." 
The wo Number / Misc Order ID combination that was entered [Wo10004-52 / MO] was not in the correct format. Please re-enter and save again.

Note:

-If a wo Number is entered, Misc Order Id must equal 'Wo'.

-If a Misc Order Id is entered, wo Number must equal 'MO'.

See the NIMS help system for more details on valid wo/mo formats.

$$
\text { OK }
$$

Figure 4.7-5. Work order validation error message

3. Next, if the user entered a Work Order Number that is not equal to "MO", query against the General Ledger database view to see if the number exists in the view (i.e. select count $\left(^{*}\right)$ from GeneralLedgerView where WONumber=[user value]).

4. If the number exists in the General Ledger view, do nothing further and allow the record to be saved.

5. If the number does not exist in the General Ledger view, warn the user with the message "You have entered a Work Order Number that does not exist in the General Ledger system. Please correct and attempt to save the record again."

Work Order [W012224-52] does not exist in the General Ledger system. Please correct and attempt to save the record again.

$$
\text { OK }
$$

Figure 4.7-6. Work order validation error message

6. The user can then reenter the Work Order number and then resave the record.

\subsection{OnCreate, OnUpdate - ValidateAssemblyNumber}

This functionality was derived from the screen ED21 specifications. The AEDR has a real time interface to the MLOG system that provides this AU with the ability to verify that a user-entered assembly number exists within the MLOG system.

\subsection{Assign to Classes}

Assembly

\subsection{Use Case}

The user creates or updates an Assembly record with a new value in the AssemblyId field. When the record is saved, the AEDR system validates the AssemblyId against the MLOG system. If the entered id is valid, it will be accepted and the record will be saved. If it is not a valid number, the user should be warned and prompted to fix it. The user can then reenter a valid number.

\subsection{Design}

The following actions are performed upon the creation or update of an Assembly record: 
1. If the AssemblyId field was populated or edited, query against the MLOG database view to see if the number exists in the view (i.e. select count(*) from MLOGAssemblyView where AssemblyId=[user value]).

2. If the number exists in the MLOG database view, do nothing further and allow the record to be saved.

3. If the number does not exist in the MLOG database view, warn the user with the message "You have entered an Assembly Id that does not exist in the MLOG system. Please correct and attempt to save the record again."

The Assembly Id that was entered (TCN2A045) does not exist in MLOG. Please correct and attempt to save the record again.

$$
\text { OK }
$$

Figure 4.7-7. Assembly ID validation error message

4. The user can then reenter the AssemblyId number and then resave the record.

\subsection{OnCreate, OnUpdate - Validate Referenced Assembly}

When a company streetlight is created, it tracks an assembly for its mounting. This streetlight assembly is not added to the related pole in addition to the assemblies already tracked in the Assembly object class, but instead must reference one of the related assemblies from the Assembly object class that has already been associated to the pole. So, when an AssemblyId and AssemblyIdCount are added to a CompanyStreetlight record, they must already exist as valid Assembly records in the Assembly table that are related to the same pole as the CompanyStreetlight.

\subsection{Assign to Classes}

CompanyStreetlight

\subsection{Use Case}

The user creates or updates a CompanyStreetlight record with new values in the AssemblyId and/or the AssemblyIdCount fields. When the record is saved, the AEDR system validates that the AssemblyId entered matches an existing Assembly record that is related to the pole that the CompanyStreetlight is mounted on. The system also verifies that the number entered in the AssemblyIdCount field is not greater than the available value in the Assembly.AssemblyCount field. If the values entered are valid, they will be accepted and the record will be saved. If either value does not match, then the user will be warned and prompted to fix the problem. The user can then reenter valid data.

\subsection{Design}

The following actions are performed upon the create or update of a CompanyStreetlight record:

1. If the AssemblyId field or the AssemblyIdCount field was populated or edited, query against the Assembly table to verify that the values are valid (i.e. select AssemblyId, AssemblyCount from Assembly where RelSupportStructureObjectId 
$=[$ CompanyStreetlight. RelSupportStructureObjectId $]$ and AssemblyId $=[$ userentered AssemblyId]).

2. If the AssemblyId is matched and the available Assembly.AssemblyCount is greater than or equal to the CompanyStreetlight.AssemblyIdCount, then do nothing further and allow the record to be saved.

3. If the AssemblyId field is not matched, warn the user with the message "The Assembly Id that was entered does not match an existing Assembly on this pole. Please correct and attempt to save the record again."

\section{Streetlight Assembly}

The Assembly Id that was entered (TCN111N4) does not match an existing assembly on this pole.

Please correct and save the record again.

$$
\text { OK }
$$

Figure 4.7-8. Assembly ID validation error message

4. If the AssemblyIdCount field is greater than the available AssemblyCount value, warn the user with the message "The chosen Assembly Count (QTY) is greater than the available Assemblies with Assembly Id related to this pole. Please enter a lower number and attempt to save the record again."

The entered Assembly Count for Assembly ID (TCN1A040) is greater than the available Assemblies with Assembly Id (TCN1A040) related to this pole. Please enter a lower number and save the record again.

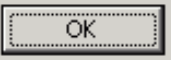

\section{Figure 4.7-9. Assembly ID validation error message}

5. In either case, the user can reenter the AssemblyId or the AssemblyIdCount and attempt to resave the record.

\subsection{OnCreate, OnUpdate - ValidateConductorProperties}

When the conductor properties for wire size, material, and type are entered for a conductor, the properties are restricted by domains. This AU further validates the user's entry by validating that the combination of these values is valid. The validation is made against a lookup table that is managed by the system coordinators.

\subsection{Assign to Classes}

- OhConductorInfo

- UgConductorInfo

- SecondaryConductorInfo

- ServiceConductorInfo

LegacyOhConductorlnfo

- LegacyUgConductorInfo

- LegacySecondaryConductorInfo 


\subsection{Use Case}

The user enters the wire size, material, and type on any of the above-mentioned object classes and saves the record. The system validates the combination of these fields along with the values for $\mathrm{OH} / \mathrm{UG}$, and the category (primary, secondary, or service) against the ConductorDefinition table. If the combination is valid, the record is saved successfully. If the combination is not valid, the user is warned with a message and prompted to reenter the values. The user can then update the values and save again.

\subsection{Design}

The following actions are performed upon the create or update of any of the above mentioned records:

1. The needed variables are gathered including wire size, material, and type, overhead vs. underground, and the category (primary, secondary, service - based on the object class).

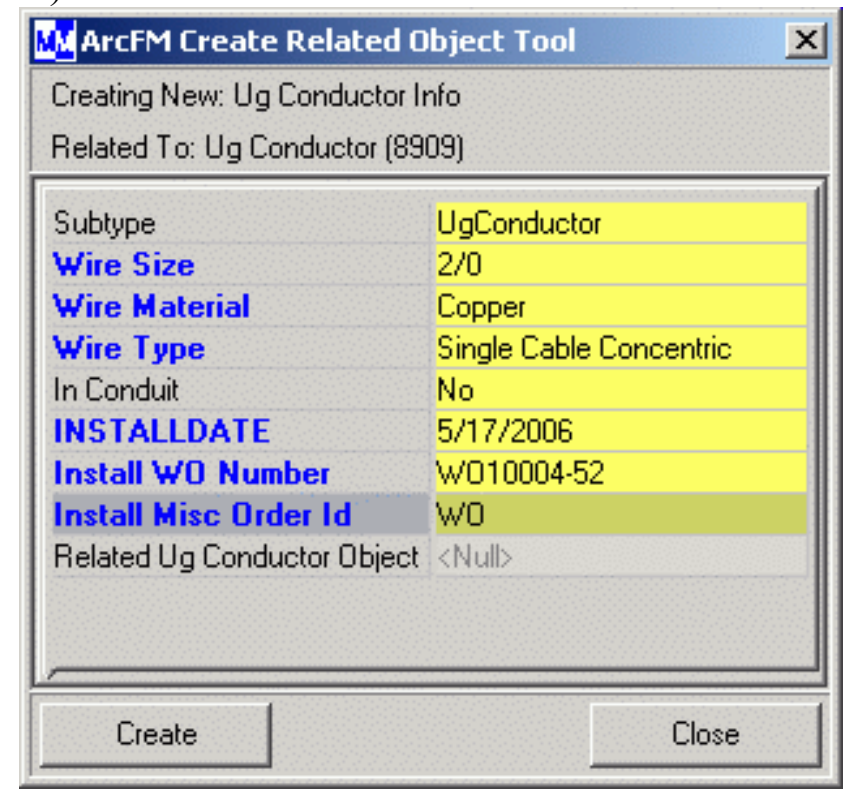

Figure 4.7-10. Attribute entry

2. Query table ConductorDefinition with these variables to see if the combination is valid (i.e. select count $(*)$ from ConductorDefinition where LocationCd $=[\mathrm{O} / \mathrm{U}]$ and CategoryCd $=[1,2, \mathrm{~S}]$ and WireType $\mathrm{Cd}=[$ user value $]$ and WireSize $=[$ user value] and WireMaterialCd=[user value $]$ ).

3. If the combination is valid, allow the edits to be saved to the database.

4. If the combination is not valid, warn the user with the message "The entered combination of wire size, material, and type is not valid. Please correct and attempt to resave." 


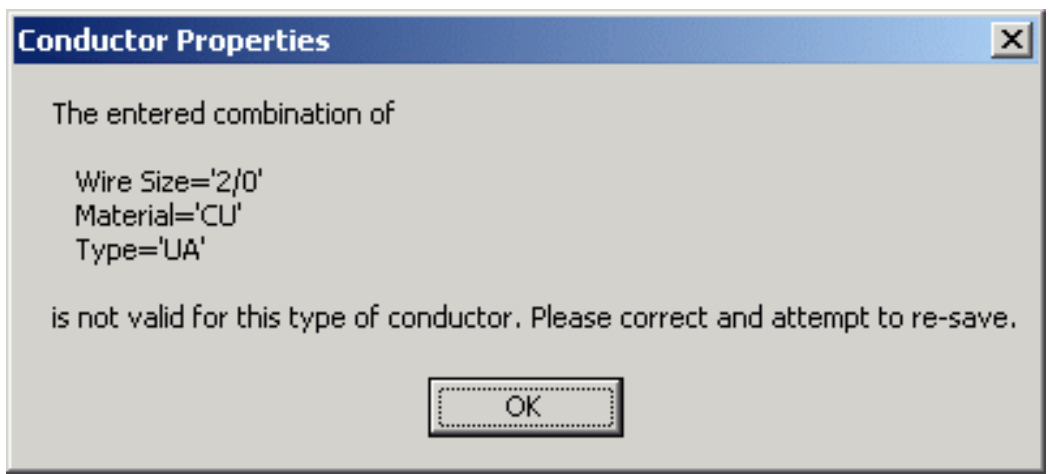

Figure 4.7-11. Conductor attribute combination error message

5. The user can then reenter the wire size, material, and type and then resave the record

\subsection{OnRetire, OnDelete - PreventDeletionRelatedAssets}

If a structure has any related Transformers, Regulators, or Capacitors OR if the pole has life support attached (from the CIS view), it cannot be retired or deleted. The devices must first be removed before the structure can be retired or deleted.

\subsection{Assign to Classes}

- SupportStructure

- PadMount

- Pedestal

\subsection{Use Case}

The user selects a structure that has related Transformers, Regulators, or Capacitors OR that has life support attached to it. The user attempts to retire or delete the structure but a message is displayed to the user explaining why the structure cannot be retired or deleted and subsequently prevents the action from occurring. If the problem was that the structure had Transformers, Regulators, or Capacitors attached to it, these devices could be removed from service and subsequently the pole could be successfully retired or deleted.

\subsection{Design}

The following actions are performed upon the retire or delete of any of the above mentioned records:

1. Check the appropriate relationship classes to see if any Transformers, Regulators, or Capacitors are related to the structure.

2. Check the CIS view to determine if the selected Distribution Reference Number is marked as a life support structure.

3. If neither of the above cases proves to be true then allow the retirement or deletion to continue.

4. If either of the above cases proves to be true, display a message to the user indicating the problem: "The structure cannot be retired/deleted because it has related Transformers/Regulators/Capacitors that must first be removed." Or "The structure cannot be retired/deleted because it has life support equipment attached to it." 
The structure cannot be retiredjideleted because it has related EDFS assets that must be retired or removed first.

Structure Relationships Preventing Retire/Delete:

-Support Structure: [Capacitor, CompanyStreetlight, CustomerStreetlight, Fuse, Recloser, Sectionalizer, ServiceConductor, StreetlightSwitch, Switch, Transformer, Regulator]

-Pad / Vault: [CustomerStreetlight, ServiceConductor, SwitchGear, Transformer]

-Pedestal: [ServiceConductor]

\section{OK}

Figure 4.7-12. SupportStructure relationship error message

5. Prevent the edit from completing.

\subsection{OnRetire - SupportStructureRetirement}

When a pole is retired, several additional actions need to be taken. First, if the pole has any related JointUseAttachment records, the company name of those attachments needs to be captured in the appropriate RetiredSupportStructure fields (TelephoneCompanyCd, FirstCableCompanyCd, SecondCableCompanyCd, UtilityCompanyCd, FiberCompanyCd, OtherCompanyCd). Second, if the pole has any related, Assemblies, they need to be retired as well into RetiredPoleAssembly subtype of the Assembly object class.

\subsection{Assign to Classes}

SupportStructure

\subsection{Use Case}

The user selects a pole, right-clicks on it, and selects the retire option. If the pole has any related joint use attachments, their company names are captured in the appropriate fields on the RetiredSupportStructure object class. If the pole has any related Assemblies, they will be retired in the same operation.

\subsection{Design}

The following actions are performed upon the retire of a SupportStructure record:

1. Check to see if there are any related Assembly records.

2. If there are, initiate the retirement of the related Assemblies into the RetiredPoleAssembly subtype.

3. Check to see if there are any related JointUseAttachments.

4. If there are, assign the values from their AttachmentCompanyName field to the correct fields on the RetiredSupportStructure record that is created as follows:

a. First Attachment of subtype Telephone $\rightarrow$ TelephoneCompanyCd

b. First Attachment of subtype Cable $\rightarrow$ FirstCableCompanyCd

c. Second Attachment of subtype Cable $\rightarrow$ SecondCableCompanyCd

d. First Attachment of subtype Utility $\rightarrow$ UtilityCompanyCd

e. First Attachment of subtype Fiber $\rightarrow$ FiberCompanyCd 
f. First Attachment of subtype Other $\rightarrow$ OtherCompanyCd

5. Delete any JointUseAttachments that were related to the SupportStructure because they do not exist on their own in a retired state.

\subsection{OnRetire - ReplicateRetirementData}

Many EDFS object classes have access to location and installation information by tracing through relationships to feature classes. For example, a SwitchUnit object can determine the circuit it is installed on by traversing to the related Switch feature and reading the FeederId field. The location and installation data is only available, however, while the EDFS object classes are "installed" and have an active relationship to a feature. When the EDFS object is retired, it is disassociated from the feature and loses the ability to reference the location and installation information. This AU captures the location and installation data that needs to be retained at the time of retirement by traversing relationships for various EDFS object classes. Some of this data acquisition has been documented within other AUs.

\subsection{Assign to Classes and Required Data}

Table 4.7-1. Relationships to feature classes

\begin{tabular}{|c|c|c|c|}
\hline Destination Class & Fields & Related Source Class & Fields \\
\hline SectionalizerUnit & $\begin{array}{l}\text { CircuitNumber } \\
\text { DistribRefNumber }\end{array}$ & $\begin{array}{l}\text { SectionalizerBank } \\
\text { SupportStructure/PadMount }\end{array}$ & $\begin{array}{l}\text { Feederld } \\
\text { DistribRefNum }\end{array}$ \\
\hline SwitchUnit & $\begin{array}{l}\text { CircuitNumber } \\
\text { DistribRefNumber }\end{array}$ & $\begin{array}{l}\text { Switch } \\
\text { SupportStructure/PadMount }\end{array}$ & $\begin{array}{l}\text { Feederld } \\
\text { DistribRefNum }\end{array}$ \\
\hline RecloserUnit & $\begin{array}{l}\text { CircuitNumber } \\
\text { DistribRefNumber }\end{array}$ & $\begin{array}{l}\text { RecloserBank } \\
\text { SupportStructure/PadMount }\end{array}$ & $\begin{array}{l}\text { Feederld } \\
\text { DistribRefNum }\end{array}$ \\
\hline FuseUnit & $\begin{array}{l}\text { CircuitNumber } \\
\text { DistribRefNumber }\end{array}$ & $\begin{array}{l}\text { FuseCutoutBank } \\
\text { SupportStructure/PadMount }\end{array}$ & $\begin{array}{l}\text { Feederld } \\
\text { DistribRefNum }\end{array}$ \\
\hline OhConductorlnfo & CircuitNumber & OhConductor & Feederld \\
\hline UgConductorInfo & $\begin{array}{l}\text { CircuitNumber } \\
\text { InConduitCd }\end{array}$ & $\begin{array}{l}\text { UgConductor } \\
\text { UgConductor }\end{array}$ & $\begin{array}{l}\text { Feederld } \\
\text { InConduitInd }\end{array}$ \\
\hline ServiceConductorInfo & $\begin{array}{l}\text { LoadDistribRef } \\
\text { Number } \\
\text { TakeOffRefNumber }\end{array}$ & $\begin{array}{l}\text { SupportStructure/PadMount } \\
\text { SupportStructure/PadMount/ } \\
\text { Pedestal }\end{array}$ & $\begin{array}{l}\text { DistribRefNumber } \\
\text { DistribRefNumber }\end{array}$ \\
\hline CompanyStreetlight & DistribRefNumber & SupportStructure & DistribRefNumber \\
\hline StreetlightSwitch & DistribRefNumber & SupportStructure & DistribRefNumber \\
\hline Assembly & DistribRefNumber & SupportStructure & DistribRefNumber \\
\hline
\end{tabular}

\subsection{Use Case}

The user locates any of the objects in 4.7-1, right clicks, and chooses the Abandon and Remove option. These objects will all be retired to subtypes within the same object class. When this occurs, the various fields mentioned above are automatically populated by traversing the relationships and retrieving the values from the related features.

\subsection{Design}

The following actions are performed upon the retire event of any of the above mentioned records: 
1. Use the appropriate relationship classes to traverse from the object class to the related feature class.

2. Read the values as described in the above table.

3. Insert the values into the object class as described in the above table.

\subsection{OnRetire-ReplicateSpatialData}

Many EDFS object classes have access to location and installation information by performing spatial searches based on their related feature classes. For example, a ConductorInfo object can determine the poles closest to its endpoints by traversing to the related Conductor feature and then doing a spatial buffer search against the support structure feature class based on the Conductor's end points. This location and installation data is only available, however, while the EDFS object classes are "installed" and have an active relationship to a feature. When the EDFS object is retired, it is disassociated from the feature and loses the ability to reference the location and installation information via a spatial buffer search. This AU captures the location and installation data that needs to be retained at the time of retirement by performing a spatial buffer search.

\subsection{Assign to Classes and Required Data}

\begin{tabular}{|l|l|l|l|}
\hline \multicolumn{4}{|c|}{ Table 4.7-2. Relationships } \\
\hline Destination Class & Fields & Related Source Class & Fields \\
\hline OhConductorlnfo & $\begin{array}{l}\text { FromDistribRefNumber } \\
\text { ToDistribRefNumber }\end{array}$ & $\begin{array}{l}\text { SupportStructure } \\
\text { SupportStructure }\end{array}$ & $\begin{array}{l}\text { DistribRefNum } \\
\text { DistribRefNum }\end{array}$ \\
\hline UgConductorlnfo & $\begin{array}{l}\text { FromDistribRefNumber } \\
\text { ToDistribRefNumber }\end{array}$ & $\begin{array}{l}\text { SupportStructure, PadMount, } \\
\text { Pedestal } \\
\text { SupportStructure, PadMount, } \\
\text { Pedestal }\end{array}$ & $\begin{array}{l}\text { DistribRefNum } \\
\text { DistribRefNum }\end{array}$ \\
\hline SecondaryConductorlnfo & $\begin{array}{l}\text { FromDistribRefNumber } \\
\text { ToDistribRefNumber }\end{array}$ & $\begin{array}{l}\text { SupportStructure, PadMount, } \\
\text { Pedestal } \\
\text { SupportStructure }\end{array}$ & $\begin{array}{l}\text { DistribRefNum } \\
\text { DistribRefNum }\end{array}$ \\
\hline
\end{tabular}

\subsection{Use Case}

The user locates any of the above objects, right clicks, and chooses the Abandon and Remove option. These objects will all be retired to subtypes within the same object class. When this occurs, the various fields mentioned above are automatically populated by traversing the relationships to their corresponding features, performing a spatial buffer search to the source class, and retrieving the values from the source features.

\subsection{Design}

The following actions are performed upon the retire event of any of the above mentioned records:

1. Use the appropriate relationship classes to traverse from the object class to the related feature class.

2. Perform a spatial buffer search against the source class.

3. Read the values as described in the above table.

4. Insert the values into the object class as described in the above table. 


\subsubsection{EDFS GIS Tools}

This section defines additional ArcFM tools that have been developed to assist with the user's edits within the GIS.

\subsection{SupportStructure and PadMount Replace Tool}

This tool allows the user to replace either a SupportStructure or PadMount feature in the AEDR without having to complete individual retire, create, and associate (transformers, regulators, etc) tasks. This functionality is derived from the screen ED21 and ED22 specs.

\subsection{Use Case}

The user selects a pole, pad, or vault that they want to replace and clicks the replace tool button (which can be added to the ArcFM toolbar). The tool prompts the user for the replacement date, retires the selected structure, creates a new structure with identical attributes, and re-relates any attached devices, attachments, or assemblies to the new structure.

\subsection{Design}

The following steps are executed when the user selects a SupportStructure or PadMount and clicks the Replace tool:

1. The process first validates that only a single SupportStructure of PadMount is selected.

2. The process then prompts the user with an input form that requests the replacement date (via a calendar date picker control).

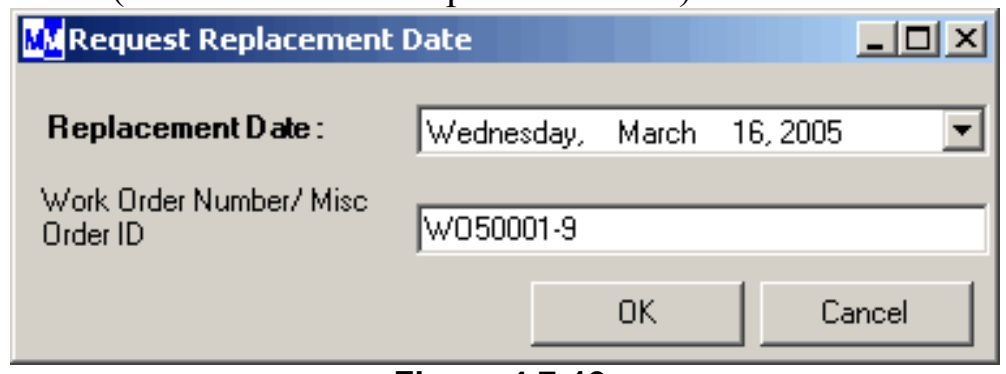

Figure 4.7-13.

3. The process un-relates any attached devices, service wire, or lighting records from the structure while retaining their object ids.

4. If the structure is a SupportStructure, the attribution of any related Assemblies or Joint Use Attachments are copied into memory.

5. The geometry of the selected structure is saved in memory.

6. The process sets the RetireDate on the structure to the date collected in step 2 above.

7. The ArcFM Retire function is called for the selected structure, which will effectively move it into the appropriate retired object class and fire any retirement AUs including "SupportStructureRetirement" (detailed in the AU section), which handles the retiring of the Assemblies and Joint Use Attachments for SupportStructures. 
8. The process next creates a new structure using the geometry collected in step 5 above.

9. The attributes are set identical to the structure that was previously retired, the retire date is set to null, and the install date is set to the value collected in step 2 above.

10. If the structure is a SupportStructure, new Assembly and Joint Use Attachment records will be created and related to the structure based on the data collected in step 4.

11. Any devices, service wire, or lighting records that were previously related to the retired structure will be re-related to the new structure.

\subsection{LegacyConductorInfo - Retirement/Migrate \& Delete}

A set of tools were created to assist the users with managing the LegacyOhConductorInfo and LegacyUgConductorInfo records that were migrated from EDFS. These records must be handled during the retirement or spatial editing of a conductor feature that was originally migrated from Outfield. Because these legacy records are not related directly to conductor features that were migrated from Outfield, they would not be automatically updated when an edit is made to the OhConductor or UgConductor feature class. For this reason, these tools allow the user to easily locate the related legacy records based off of a spatial search against poles, pads, and pedestals via distribution reference number.

\subsection{Use Case}

Retirement of Legacy Conductor Info Records

1. The user selects a single OhConductor or UgConductor feature. A command button residing on the Conductor Info Utilities toolbar will enable, allowing the user to launch the retire legacy conductor info objects functionality.

2. The component performs a spatial search on support structure, pad mount, and pedestal features that are within a configured buffer distance of the selected conductor feature. The component obtains distribution reference numbers from the located features and uses these distribution reference numbers to query the legacy $\mathrm{OH}$ and $\mathrm{UG}$ conductor info tables.

3. The returned records are presented to the user in a grid format. The grid is sorted by the 'from' and 'to' distribution reference numbers. Records with the same 'from' and 'to' distribution reference numbers are colored alike to assist the user in visually identifying the records. Clicking on each record in the grid will flash the corresponding 'from' and 'to' structures in the GIS.

4. The grid includes a column of checkboxes that allow the user to indicate which legacy conductor info records should be retired.

5. From the graphical display of the attribute information, the user chooses which records will be retired by clicking a checkbox on the individual rows.

6. The user inputs values for retirement work order number or miscellaneous order id and the retirement date.

7. The user clicks an execute button.

8. The selected objects to be retired are updated to signify retirement via the subtype. 
9. The user will then delete or abandon the OhConductor of UgConductor feature as needed based on business rules.

Migration of Legacy Conductor Info Records into ArcFM Conductor Info Records to Allow Spatial Editing of the related OhConductor/UgConductor features:

1. The user selects a single OhConductor or UgConductor feature. A command button residing on the Conductor Info Utilities toolbar enables, allowing the user to launch the migrate legacy conductor info objects functionality.

2. The component performs a spatial search on support structure, pad mount, and pedestal features that are within a configured buffer distance of the selected conductor feature. The component obtains distribution reference numbers from the located features and uses these distribution reference numbers to query the legacy $\mathrm{OH}$ and $\mathrm{UG}$ conductor info tables.

3. The returned records are presented to the user in a grid format. The grid is sorted by the 'from' and 'to' distribution reference numbers. Records with the same 'from' and 'to' distribution reference numbers are colored alike to assist the user in visually identifying the records. Clicking on each record in the grid flashes the corresponding 'from' and 'to' structures in the GIS.

4. If the attribution differs between adjacent groups of legacy conductor info records, a button is enabled to allow the user to split the conductor feature at all attribution changes. This tool can then be re-launched on the individual smaller conductor features.

5. The grid includes two columns of checkboxes that allow the user to indicate which legacy conductor info records should be migrated and which should be deleted after the migration.

6. Once the user has an acceptable set of legacy conductor info records in the grid, they select the records to be migrated into ArcFM conductor info records. Any migrated records should be deleted to avoid duplicate data. In addition, other legacy conductor info records can be deleted as well if they are not required to be migrated (in the case of duplicate data in the legacy tables).

7. The selected legacy conductor info records are migrated into the corresponding ArcFM conductor info table and the new ArcFM conductor info records will be related to the selected conductor feature.

8. Any legacy conductor info records that are marked for deletion will be permanently deleted from the legacy conductor info table(s).

9. The user can then edit the conductor feature using standard ArcFM tools and the new ArcFM conductor info records will be managed appropriately.

\subsection{Design}

This functionality consists of two user-forms designed to aid the user in retiring conductor units, and migrating conductor units preparing them for manipulation within the ArcFM toolset.

1. For retiring conductor information records, the tool presents the user with the appropriate related data, and offering checkboxes for each record returned. This 
allows the user to determine which records shall be retired. The user also populates the retirement work order or miscellaneous order id and the retire date.

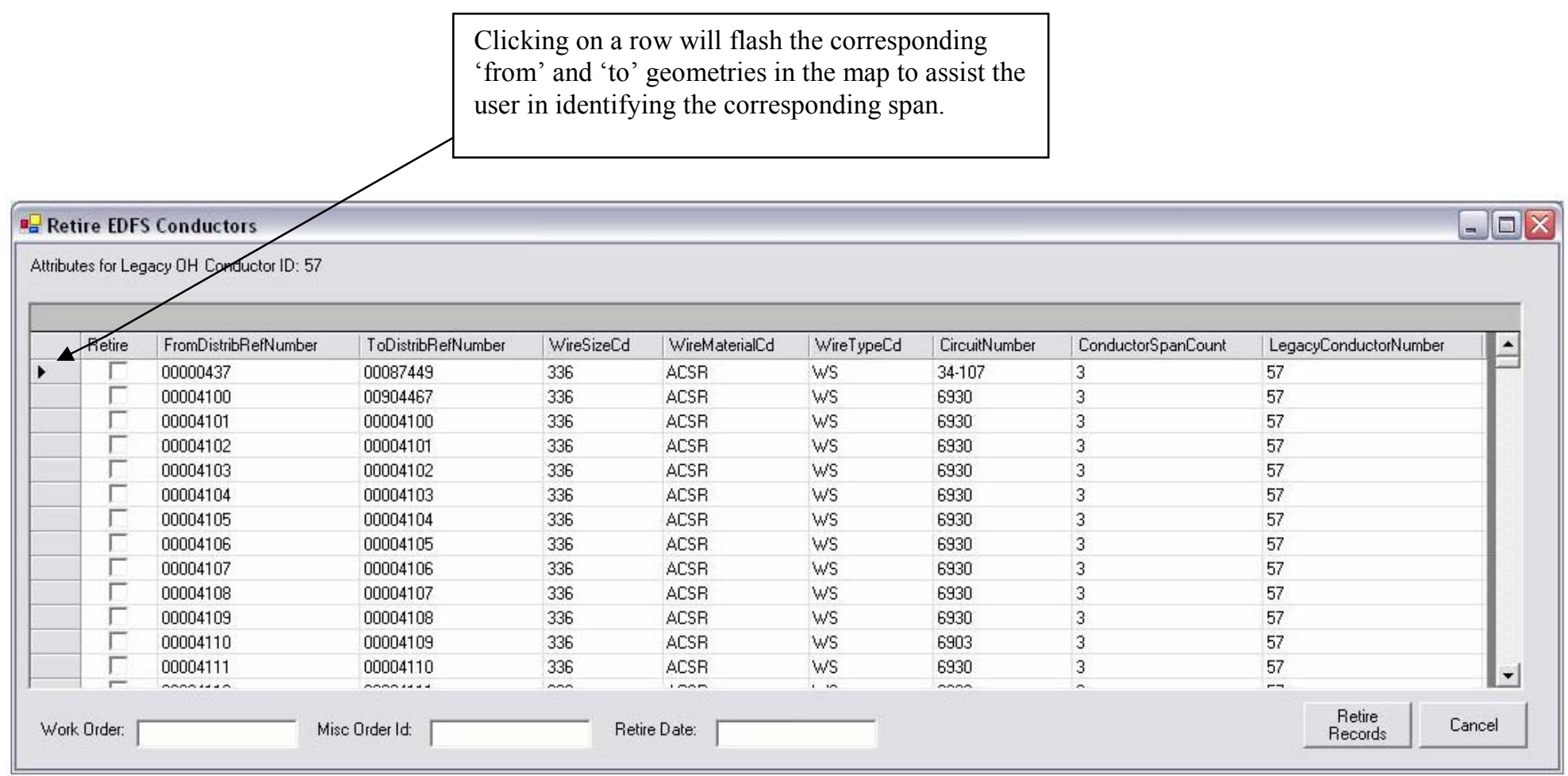

Figure 4.7-14. Retire EDFS conductor tool 
2. The second tool assists the user in manually migrating and deleting legacy conductor info records. When the tool is launched, the user is presented with a list of records returned from attributes gathered via the spatial search of Support Structure, Pad Mount, and Pedestal records. The user then is able to migrate and delete selected records. This interface additionally allows the user to split conductors by like info records, creating spans with like attribution.

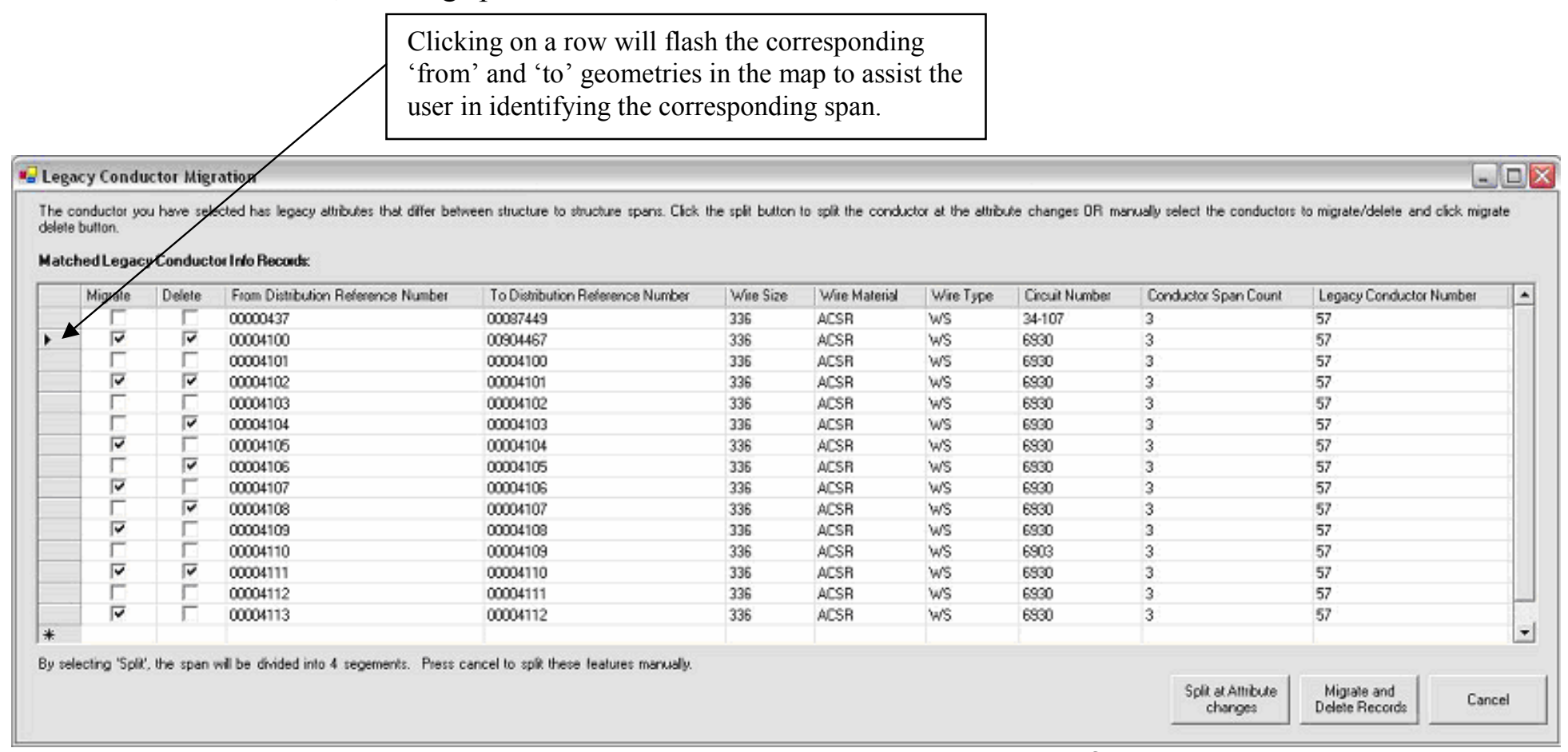

Figure 4.7-15. Migrate and delete legacy conductor info records

After Split is clicked, the selected span is split, creating several smaller spans all having similar attribution. The tool can then be re-run on each individual sub span.

\subsubsection{EDFS Batch Management and Reporting Applications}

Several batch applications were written to manage the EDFS data. These applications were written as .Net applications that are scheduled to run via the Windows scheduler. The following sections describe the applications and how they interact with the database.

\subsection{MAPPS / GIS Transaction Processing}

This functionality was derived from the screen EDB5050 and EDB5051 specifications. This application is responsible for processing the MAPPS transactions that are published to the AEDR system on a nightly basis. In the old EDFS system, these transactions were passed to the EDFS application in a flat file. In the AEDR system, the MAPPS system makes these transactions available via a database table view. This application reads these transactions, attempts to match them to AEDR transactions, and either handles them or moves them to a holding table where the processing will be attempted again the next 
night. The results of this application are the basis for the information contained in the Coordinator Dashboard.

\subsection{Use Case}

This application has been configured to run nightly. It reads in the new daily transactions from the MAPPS system, reads the new daily transactions from the AEDR system, combines them with any carried transactions from the previous night, and attempts to match and/or process the transactions. Various tables are updated with the results of the processed transactions.

\subsection{Design}

The following steps are executed when this batch application is run:

1. The process first reads all carried transactions from the previous night from the table MAPPSCarriedTransaction within the SAGE database (Figure 4.7-16). These transactions are stored in memory.

\begin{tabular}{|ll|}
\hline \multicolumn{2}{|c|}{ MAPPSCarriedTransaction } \\
\hline PK Transactionld & NUMERIC \\
CompanyNumber & VARCHAR(7) \\
TransactionType & VARCHAR(3) \\
Department & VARCHAR(3) \\
SystemCode & VARCHAR(1) \\
StoresttemNumber & VARCHAR(7) \\
TransactionDate & DATETIME \\
CondemnationNumber & VARCHAR(3) \\
TransferReceivingDepartment & VARCHAR(3) \\
TransferNumber & VARCHAR(7) \\
FailedGISArgument & VARCHAR(7) \\
ToBeDeleted & BIT \\
FK ReiRejectionCode & NUMER/C \\
\hline
\end{tabular}

Figure 4.7-16. MAPPS transactions and data types

2. If the ToBeDeleted column or any of these rows is equal to 1 (yes) then this indicates that a coordinator has chosen to manually delete the record and it should be discarded at this time.

3. The process next reads all new MAPPS transactions from the InterfaceMAPPSTransactions table. Only transactions of type 47 (transfer), 69 (condemn), 60 (issue), and 40 (return) will be retained. All other transactions are discarded. These transactions are added to the list of carried transactions in memory. 


\begin{tabular}{|ll|}
\hline \multicolumn{2}{|c|}{ InterfacehAPPSTransaction } \\
\hline PK Transactionld & NUMERIC \\
CompanyNumber & VARCHAR(7) \\
TransactionType & VARCHAR(3) \\
Department & VARCHAR(3) \\
SystemCode & VARCHAR(1) \\
StoresternNumber & VARCHAR(7) \\
TransactionDate & DATETIME \\
TransferReceivingDepartment & VARCHAR(3) \\
TransferNumber & VARCHAR(7) \\
CondernnationNumber & VARCHAR(3) \\
\hline
\end{tabular}

Figure 4.7-17. MAPPS interface transactions database table

4. The process next reads all new AEDR system transactions from the GISMappsTransactionQueue table within the GIS. These transactions are added to the list of carried transactions in memory.

5. The process sorts the list by the company number field, which is carried on all transactions from all systems.

6. The process attempts to process the transactions as follows:

a. 47-Transfer: Follow details in step 1 of the logic section of EDB5051.

b. 69-Condemn: Follow details in step 4 of the logic section of EDB5051.

c. 60-Issue, INS/CI - Install: Attempt to match the MAPPS '60' transaction to a GIS 'INS' or 'CI' transaction. If a match is made, both records can be considered complete. See details in step 5 of the logic section of EDB5051.

d. 40-Return, RMV - Removal: Attempt to match the MAPPS '40' transaction to a GIS 'REM' transaction. If a match is made, both records can be considered complete. See details in step 5 of the logic section of EDB5051.

7. Purge tables: MAPPSCarriedTransaction, InterfaceMAPPSTransactions, and GISMappsTransactionQueue.

8. For all transactions that were matched and/or completed, insert a corresponding record into MAPPSProcessedTransHistory recording all applicable fields. 


\begin{tabular}{|ll|}
\hline \multicolumn{2}{|c|}{ MAPPSProcessedTransHistory } \\
\hline PK ProcessedTransactionld & NUMERIC \\
ProcessedDate & DATETIME \\
CompanyNumber & VARCHAR(7) \\
TransactionType & VARCHAR(3) \\
Department & VARCHAR(3) \\
SystemCode & VARCHAR(1) \\
StoresttemNumber & VARCHAR(7) \\
TransactionDate & DATETIME \\
CondemnationNumber & VARCHAR(3) \\
TransferReceivingDepartment & VARCHAR(3) \\
TransterNumber & VARCHAR(7) \\
\hline
\end{tabular}

Figure 4.7-18. Completed MAPPS transactions are inserted into the history file

9. In Figure 4.7-19, all transactions that were not matched and/or completed, a corresponding record is inserted into MAPPSCarriedTransaction including the appropriate rejected reason code so that an attempt will be made the next night to process the record.

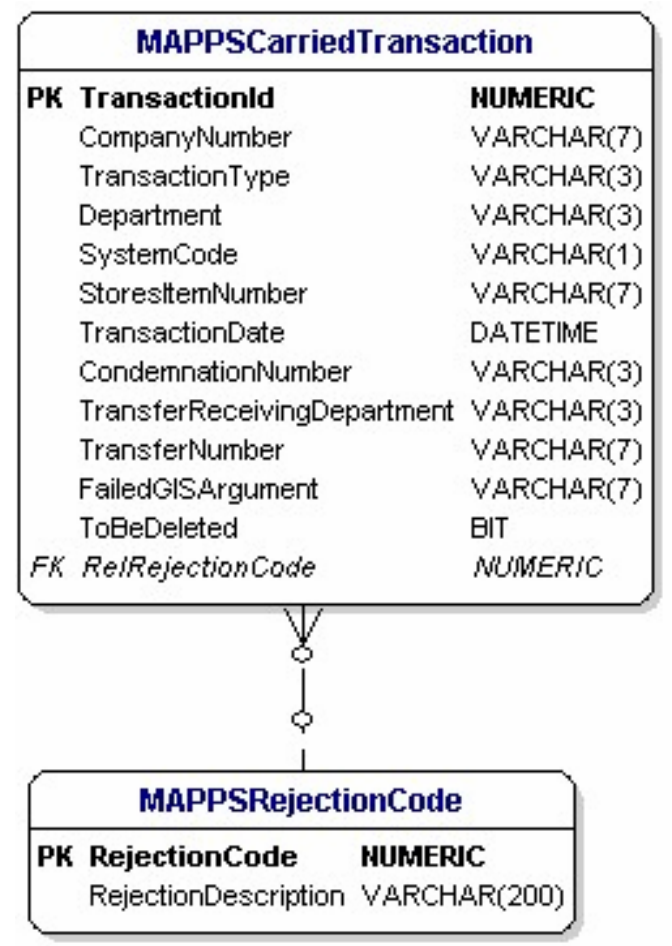

Figure 4.7-19. Incomplete MAPPS transactions are posted to the carry file

10. Insert a new record into MAPPSNightlyStatistics 


\begin{tabular}{|ll|}
\hline \multicolumn{2}{|c|}{ MAPPSNightlyStatistics } \\
\hline PK TransactionDate & DATETIME \\
CarryOverCount & INTEGER \\
ManualDeletions & INTEGER \\
NewMAPPSTransters & INTEGER \\
NewMAPPSCondernnations & INTEGER \\
NewMAPPSIssues & INTEGER \\
NewMAPPSReturns & INTEGER \\
TotalMAPPSTransactions & INTEGER \\
NewGISTRInstalls & INTEGER \\
NewGISTRRemovals & INTEGER \\
NewGISCapacitorlnstalls & INTEGER \\
TotalGISTransactions & INTEGER \\
ProcessedTransters & INTEGER \\
OutstandingTransfers & INTEGER \\
ProcessedCondernnations & INTEGER \\
OutstandingCondernnations & INTEGER \\
Matchedlssuesinstalls & INTEGER \\
MatchedReturnsRemovals & INTEGER \\
UnMatchedlssues & INTEGER \\
UnMatchedReturns & INTEGER \\
UnMatchedTRInstalls & INTEGER \\
UnMatchedTRRemovals & INTEGER \\
UnMatchedCapacitorlnstalls & INTEGER \\
TotaIProcessedSuccess & INTEGER \\
TotalOutstanding & INTEGER \\
TotalProcessed & INTEGER \\
\hline
\end{tabular}

Figure 4.7-20. And the nightly statistics are updated

Record the statistics from the processing that was performed including the following:

a. TransactionDate: The system date when the process was run.

b. CarryOverCount: The count of the records that was extracted from MAPPSCarriedTransaction in step 1.

c. ManualDeletions: The count of records from MAPPSCarriedTransaction that were discarded due to the ToBeDeleted column being equal to 1 (step 2 above).

d. NewMappsTransfers: The count of transfer records that was extracted from the MAPPS transaction view in step 3.

e. NewMappsCondemnations: The count of condemnation records that was extracted from the MAPPS transaction view in step 3.

f. NewMappsIssues: The count of issue records that was extracted from the MAPPS transaction view in step 3.

g. NewMappsReturns: The count of return records that was extracted from the MAPPS transaction view in step 3.

h. TotalMappsTransactions: The sum of the counts from d-g above.

i. NewGISTRInstalls: The count of 'INS' records that was extracted from GISMappsTransactionQueue in step 4 above. 
j. NewGISTRRemovals: The count of 'RMV' records that was extracted from GISMappsTransactionQueue in step 4 above.

k. NewGISCapacitorInstalls: The count of 'CI' records that was extracted from GISMappsTransactionQueue in step 4 above.

1. TotalGISTransactions: The sum of the counts from i-k above.

m. ProcessedTransfers: The count of the transfer transactions that was completed and subsequently entered into MAPPSProcessedTransHistory in step 8.

n. OutstandingTransfers: The count of the transfer transactions that was not completed and was subsequently entered into MAPPSCarriedTransaction in step 9.

o. ProcessedCondemnations: The count of the condemn transactions that was completed and subsequently entered into MAPPSProcessedTransHistory in step 8.

p. OutstandingCondemnations: The count of the condemn transactions that was not completed and was subsequently entered into MAPPSCarriedTransaction in step 9.

q. MatchedIssuesInstalls: The count of the matched MAPPS issues and GIS installs (each match counts for two entries, one from MAPPS and one from GIS).

r. MatchedReturnsRemovals: The count of the matched MAPPS returns and GIS removals (each match counts for two entries, one from MAPPS and one from GIS).

s. UnmatchedIssues: The count of the issue transactions that was not matched and was subsequently entered into MAPPSCarriedTransaction in step 9.

t. UnmatchedReturns: The count of the return transactions that was not matched and was subsequently entered into MAPPSCarriedTransaction in step 9.

u. UnmatchedTRInstalls: The count of the GIS transformer and regulator install transactions that was not matched and was subsequently entered into MAPPSCarriedTransaction in step 9.

v. UnmatchedTRRemovals: The count of the GIS transformer and regulator removal transactions that was not matched and was subsequently entered into MAPPSCarriedTransaction in step 9.

w. UnmatchedCapacitorInstalls: The count of the GIS capacitor install transactions that was not matched and was subsequently entered into MAPPSCarriedTransaction in step 9.

x. TotalProcessedSuccess: The sum of the counts from $\mathrm{m}, \mathrm{o}, \mathrm{q}$, and $\mathrm{r}$ above.

y. TotalOutstanding: The sum of the counts from $\mathrm{n}, \mathrm{p}, \mathrm{s}, \mathrm{t}, \mathrm{u}, \mathrm{v}$, and $\mathrm{w}$ above.

z. TotalProcessed: $(\mathrm{x}+\mathrm{y})$ and/or $(\mathrm{b}-\mathrm{c}+\mathrm{h}+\mathrm{l})$ from above. These two calculations should both be run to ensure that they match.

11. Finally, purge all records from MAPPSProcessedTransHistory where column TransactionDate $<$ SystemDate -6 months. 


\subsection{Batch Report Generator}

The majority of the NIPSCO reports are generated in a batch fashion. This application allows the users to configure the reports to be run and spooled to a windows printer on a daily, weekly, monthly, or annual basis. The reports are created using XML and XSLT (see the "SAGE Reports" section for more detail). The batch creation of these reports is accomplished by having the application manage an html viewer where each report can be loaded and then spooled to a printer. Each report can be spooled to multiple printers as needed and certain reports are configurable so that only certain sections of the report will be spooled to certain printers. An example of this would be for report "EDB5045 Transformers In Stock Inventory Report". This report details the transformers that are currently in stock in the various LOAs. The desired functionality is to have the report sorted by LOA. Then only the stock records for each individual LOA are spooled to that respective LOA's printer. Sub-reports may be run for each LOA that only contain the needed data. These sub-reports are then easily spooled to the correct printer at each LOA. It should also be noted that all printers that are used with this application are required to be registered as valid printers on the server where this application is run.

\subsection{Use Case}

The user configures the reports to be generated along with their frequency and their printer(s) via an XML application configuration file. The application is then run on a nightly basis. Based on the configuration, the application determines which reports need to be generated and printed and does so using an html viewer. The data source for the reports is the SAGE multi-versioned view of the "NIPSCO_SAGE" version. The reports are available on the printers in the correct locations by the opening of business the following day.

\subsection{Design}

The following steps are executed when this batch application is run:

1. The application accesses the configuration file to determine which reports need to be generated and printed as follows:

a. Daily: The reports are generated every night.

b. Weekly: The reports are generated on each Sunday night.

c. Monthly: The reports are generated on the last night of each month.

d. Annually: The reports are generated on the last night of each year.

2. Each report that is scheduled for generation is loaded into an html viewer. Certain reports may require processing to be executed against the database as part of the report generation. This processing will occur at this time.

3. Once loaded, each report is spooled to the configured Windows printer(s).

4. An entry is made in the BatchReportLog table in the SAGE application database recording the date, report id, printer, success indicator (yes/no, 1/0), and the error if any occurred. 


\subsection{Interface Management}

The AEDR system must maintain interfaces to many other NIPSCO systems. This section defines the application that manages and executes each of these interfaces on a nightly basis.

\subsection{Use Case}

The interface management is run on a nightly basis. It checks a configuration file for the specific interfaces that will be executed. Each interface is run, the results logged, and any errors sent to a list of configured parties.

\subsection{Design}

The following steps are executed when this batch application is run:

1. The application reads a list of interfaces to be executed from a configuration file. The configuration file contains assembly and class information so that each interface can be created and executed via reflection. This ensures that the interface management is completely configurable and extensible.

2. The application opens database connections to all required systems so that the connections can be shared between all of the interfaces. The database connections are maintained in a collection that can be easily passed to each interface.

3. Each configured interface is run. The specific interface defines the exact steps to be taken to complete the processing.

4. The application logs the start and stop times of each interface and catches and logs any errors if they occur.

5. If any errors occur within an interface, the error and any additional detail is emailed to a configured list of administrators. This ensures that the problem is fixed as soon as possible. The timing of many of these interfaces is critical.

\subsection{Structure to Conductor Join}

The Pole/Mounting Inquiry report requires the system to generate data on primary and secondary conductors based on the distribution reference number of a pole, pad, vault, or pedestal. Because there is only a spatial relationship between these structures and the conductors in the GIS, this data cannot be retrieved in the purely tabular manner that the SAGE and reporting tools require (there is a tabular join between the structures and the service wire, hence it is not included in this batch application). For this reason, this batch application was created to establish a tabular join between distribution reference numbers and conductors on a weekly basis. This relationship is not an explicit database relationship but instead captures the needed relational data in a table based in the SAGE database. This data is only used by the SAGE reports. It should be noted that this application was written with ArcObjects as it requires the ability to make spatial GIS queries.

\subsection{Use Case}

This report runs on a weekly basis over the weekend as it is relatively time consuming due to the number of spatial joins that are required. It loops through the structure features 
containing distribution reference numbers including poles, pads, vaults, and pedestals. It conducts a spatial buffer search for each structure feature against the primary and secondary feature classes. It then creates join records in the StructureConductorJoin table capturing a tabular relationship between these features and the conductor. The reports then utilize this table when it is necessary to identify conductor records attached to the structure features.

\subsection{Design}

The following steps are executed when this batch application is run:

1. The application purges the existing data from the StructureConductorJoin table.

2. The application connects to the Default version within the GIS database via ArcObjects.

3. The application loops through each feature within the SupportStructure, PadMount, and Pedestal feature class that has a valid distribution reference number.

4. For each feature, the application conducts a spatial buffer search against the OhConductor, UgConductor, and Secondary feature classes.

5. When a matching conductor feature is returned, the application traverses the appropriate relationship class to its related conductor info records.

6. A new record is created in StructureConductorJoin table (Figure 4.7-21) capturing the joined information between the distribution reference number and the conductor info record as follows:

a. DistribRefNum: Set to the Distribution Reference Number of the structure.

b. CategoryCd: This corresponds to 1 for Primary and 2 for Secondary.

c. LocationCd: This corresponds to $\mathrm{O}$ for Overhead and $\mathrm{U}$ for Underground.

d. ConductorObjectId: This corresponds to the object id of the conductor info record.

\begin{tabular}{|ll|}
\hline \multicolumn{2}{|c|}{ StructureConductorJoin } \\
\hline DistribRetNum & VARCHAR(8) \\
CategoryCd & INTEGER \\
LocationCd & VARCHAR(1) \\
ConductorObjectld & INTEGER \\
\hline
\end{tabular}

Figure 4.7-21.

7. When the reporting applications need to access this data, they use the above information to determine how to locate the conductor info record as follows:

e. For CategoryCd=1, Location $\mathrm{Cd}=\mathrm{O}$, query OhConductorInfo with the ConductorObjectId.

f. For CategoryCd=1, Location $\mathrm{Cd}=\mathrm{O}$, query UgConductorInfo with the ConductorObjectId.

g. For CategoryCd=2, query SecondaryConductorInfo with the ConductorObjectId. 


\subsection{Release SDE Lock}

A large portion of the EDFS custom functionality utilizes ESRI multi-version views. A common problem when using multi-version views heavily from many different processes is that the view becomes locked if there is ever an error within any of the processes. In basic terms, each application opens the view for editing, performs its edits, and then closes the view. If the application does not close the view, then it is effectively locked and is not available to any other processes/applications. This application was created to ensure that the view is unlocked correctly during batch processing even if any errors occur. This ensures that an error in any one process will not cause the rest of the processes to fail as well.

\subsection{Use Case}

This batch application runs multiple times during the nightly batch run. It is scheduled in between all other significant applications that use multi-version views. It is responsible for unlocking the view using any and all database user accounts that ever access the multi-version view.

\subsection{Design}

The following steps are executed when this batch application is run:

1. The application attempts to open the multi-version view.

2. If any errors are raised due to the view being locked, the application will then unlock the view with all configured user accounts.

\subsection{Domain Synchronization}

The SAGE application is an ASP.Net application that uses standard .Net controls to gather user input. In following standard .Net development concepts, these controls should be data-bound directly to a lookup table in the database. Unfortunately, ESRI stores its domain values in a binary format that cannot be directly bound to standard COM or .Net controls. So, this application has been written to synchronize configured ESRI domains into standard flat database tables on a nightly basis.

\subsection{Use Case}

Standard database lookup tables (ID and Description columns) have been established for all domains that are used by the SAGE application. This application synchronizes the values from the ESRI binary domain into these tables on a nightly basis to ensure that the lookup values match in both the GIS and SAGE.

\subsection{Design}

The following steps are executed when this batch application is run:

1. The application connects to the geodatabase using ArcObjects.

2. The application reads a list of the domains and their corresponding tables to be synched from a configuration file.

3. The application opens each domain within the geodatabase.

4. The application truncates each corresponding database table. 
5. The application writes each value found in the domain into the corresponding database table shown in Figure 4.7-22.
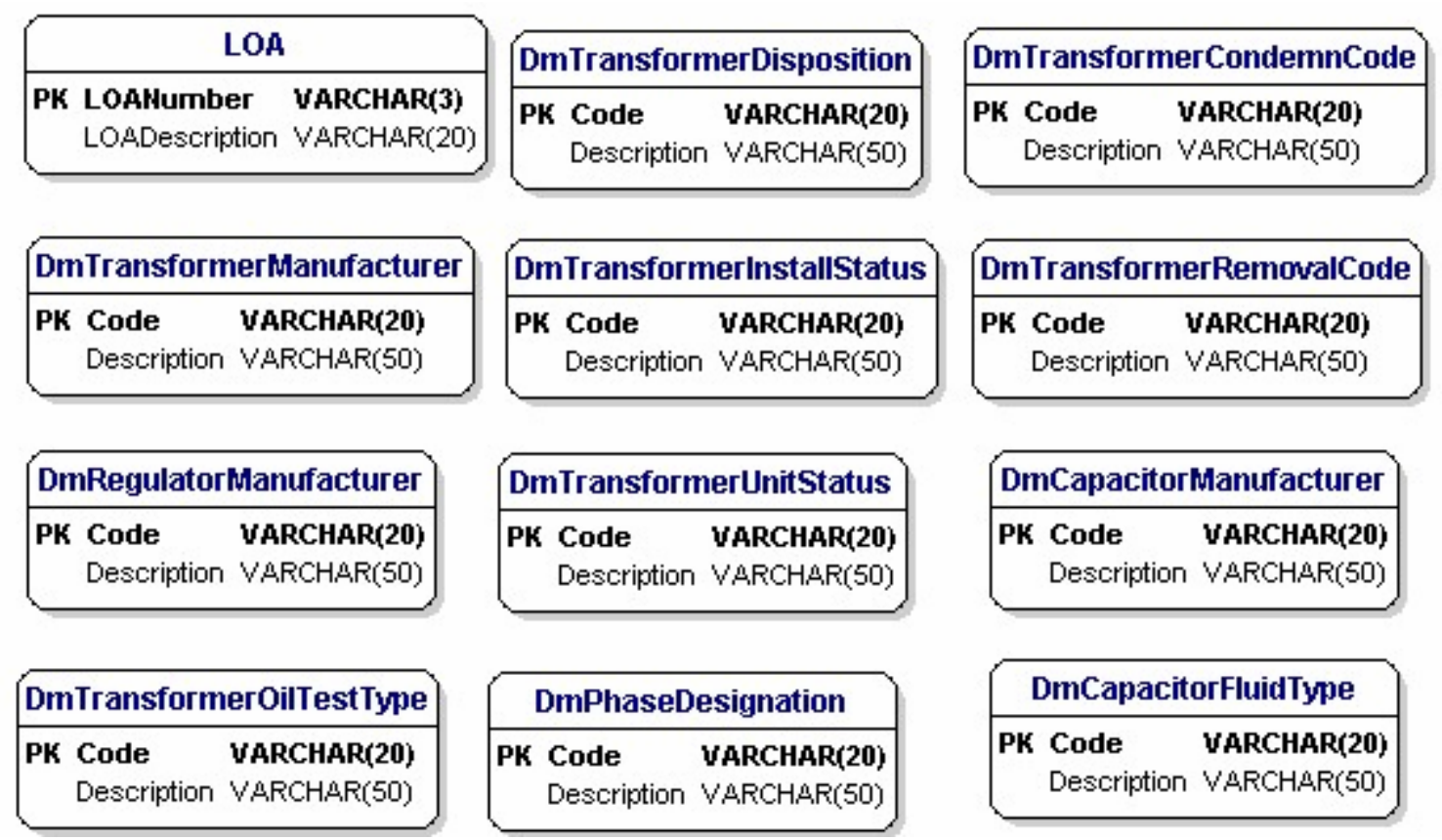

Figure 4.7-22. Database tables updated by Domain Synchronization

\subsection{Asset Statistics}

SAGE administrator users can access the SAGE Coordinator Dashboard. This web page shows key statistics for the company's capital assets (transformers, regulators, and capacitors). These statistics include the quantity, capacity, and install cost for each of the capital assets sub grouped by their status (in stock, installed, condemned).

\subsection{Use Case}

Because these statistics are pulled via multi-versioned views and they touch on a wide range of tables, they take considerable time to execute. This batch application queries the data on a nightly basis and stores the results in a single summary table that can then be used by the SAGE application to display the data very quickly. The data is current as of the night before which is acceptable to the users.

\subsection{Design}

The following steps are executed when this batch application is run:

1. The application executes the SQL queries to gather the required asset statistics.

2. The application removes the previous night's statistics from the summary table.

3. The application writes the statistics to the summary table.

\subsection{Session Reporter}

The management of Maps \& Records requires a nightly view capture of the work that is currently outstanding within the department. Work within the AEDR system is managed 
within sessions. Therefore, a nightly report of all sessions and their details are generated to meet this requirement.

\subsection{Use Case}

This application queries the database for all active sessions, organizes the results into a report, and e-mails the report out to key users.

\subsection{Design}

The following steps are executed when this batch application is run:

1. The application executes SQL against the session manager database to gather key information on all active sessions.

2. The application organizes the sessions by those that have been outstanding more than 30 days, between 7 and 30 days, and those opened within the last 7 days.

3. The application creates a report containing the session name, the create user, the current user, and the status.

4. The application e-mails the report out to a list of key users configured in a configuration file.

\subsection{Batch Reconcile and Post}

Certain versions within the AEDR system are used only by system processes. These versions never need to be QAQC'd and are posted up to SDE.Default on a regular basis. A batch application reconciles and posts these system versions on a nightly basis.

\subsection{Use Case}

The application reconciles down all edits in SDE.Default and then posts up any system edits from a version.

\subsection{Design}

The following steps are executed when this batch application is run:

1. The application reads a list of configured versions to be reconciled and posted from a configuration file.

2. The application reconciles each version against SDE.Default.

3. If any conflicts are present, the application writes the details to a log file.

4. The application posts each version into SDE.Default.

5. The application writes the details of the process to a log file.

\subsection{Batch Reconcile and Compress}

System performance is typically a struggle in any enterprise level ESRI GIS.

Performance is directly tied to the size of the ESRI state table which tracks outstanding edits across all versions. An application is required to reconcile all posted edits from SDE.Default down into ALL of the outstanding versions. This allows an SDE compress to be effective in minimizing the size of the ESRI state table.

\subsection{Use Case}

This application loops over all outstanding versions and attempts a reconcile against SDE.Default. If a successful reconcile cannot be achieved, the details are written to a log 
file. Once all versions have been reconciled, a compress is performed to remove any unneeded records from the ESRI state table.

\subsection{Design}

The following steps are executed when this batch application is run:

1. The application retrieves a list of all versions from the SDE geodatabase.

2. The application attempts to reconcile each of the versions against SDE.Default.

3. If any errors OR conflicts are encountered for any version, the details are written to a $\log$ file.

4. The application checks a configuration file to see if a compress should be attempted. This is configurable because a compress performed via ArcObjects is much less stable than a compress performed via the SDE command line. The compress option was disabled after a command line job was scheduled on the server to compress the SDE database on a nightly basis.

5. All results are written to a log file.

\subsection{File Archiver}

The Request for Authority to Condemn / Reverse-Condemn batch report is run nightly and made available the following day under the batch reporting SAGE page. The clerks can then locate the report for their district and print out the report for signatures.

Occasionally, a clerk has issues accessing SAGE or printing out the appropriate report. When this occurs, the report is overwritten the following night and the condemnation report is effectively lost. The File Archiver batch application was developed to archive the nightly condemnation reports for up to one week to account for any trouble accessing or printing the reports. The archived reports are also made available on the SAGE batch reports page.

\subsection{Use Case}

The File Archiver runs after the batch reports have been generated. It copies the current set of Request for Authority to Condemn / Reverse-Condemn reports to a configured archive folder. The archive folder is then published to a configured location on the webserver. Finally, the process deletes any archived reports that are over a week old.

\subsection{Design}

The following steps are executed when this batch application is run:

1. The application copies all of the current Request for Authority to Condemn / Reverse-Condemn reports from the configured reports directory into an archive subfolder named with the current date.

2. The application copies the entire archive folder to a configured UNC path on the webserver to make it available to SAGE users.

3. The application deletes any archive folders with a date greater than one week previous to the current date.

\subsection{Condemned Asset Cleanup}

It is possible for an installed Transformer or Regulator to be condemned. After the condemnation report is signed and the approved condemnation is sent back to the AEDR 
system by MAPPS, the condemnation status on the asset is updated by the MAPPS Synch batch process. The Condemned Asset Cleanup application is then responsible for dissolving any active relationships between the condemned asset record and any GIS feature (TransformerBank or VoltageRegulator respectively).

\subsection{Use Case}

The Condemned Asset Cleanup simply updates the system to null out any foreign keys between the asset tables and any active GIS feature classes.

\subsection{Design}

The following steps are executed when this batch application is run:

1. The application queries the database for any assets that are in the approved condemned state that are showing in the installed state and are related to a GIS feature class (TransformerUnitInstall $\rightarrow$ TransformerBank and RegulatorUnitInstall $\rightarrow$ VoltageRegulator).

2. The application then nulls out the foreign key on any install records that are returned by the above search (TransformerUnitInstall.RelTransformerBankObjectId or RegulatorUnitInstall.RelVoltageRegulatorObjectId respectively).

3. The application writes the results of the cleanup to a log file.

\subsection{On Demand Reports Cleanup}

The SAGE website allows users to interactively create three different on-demand reports (Session Inquiry, Pole/Pad Inquiry, and Padmount Inspections). These reports are generated as html files and are stored in a folder on the web server where SAGE is hosted. These reports are consumed by the user either by printing or saving the report after it has been generated. Over time, the number of these reports on the SAGE web server grows and the amount of disk space used becomes undesirable. The On Demand Reports Cleanup batch application was written to delete all on demand reports off of the web server after a specified number of days. This avoids any disk storage issues and maintains a much cleaner on-demand reports folder.

\subsection{Use Case}

The On Demand Reports Cleanup batch application deletes all on-demand reports from the configured web server directory that are older than a configured number of days.

\subsection{Design}

The following steps are executed when this batch application is run:

1. The application connects to the SAGE web server on-demand folder and query for all files that are older than a configured number of days.

2. The application then deletes any found files.

\subsection{Street Centerline Intersection Creator}

The record clerks manage street centerlines in the AEDR system by drawing the linear features and assigning the various road name attributes. Though a user could search on the street centerline features, they could not search on the spatial intersection of two 
street centerline intersections which is a much more valuable search. The Street

Centerline Intersection Creator batch application was created to process all existing street centerline features to locate their intersections and then create a street centerline intersection feature at the intersection attributed with both road names to allow for easy querying.

\subsection{Use Case}

The Street Centerline Intersection Creator application queries all street centerline features, locates their intersections, creates new point intersection features, and attributes the new features with both road names.

\subsection{Design}

The following steps are executed when this batch application is run:

1. The application connects to the SDE database via ArcObjects.

2. The application truncates the StreetCenterlineIntersection table to purge all existing records.

3. The application then queries the StreetCenterline table for all centerline features.

4. The application parses through all of the StreetCenterline features performing spatial searches for coincident end points.

5. When a coincident end point is found, a new StreetCenterlineIntersection point feature is created at that location.

6. The new feature is attributed with the road names from both source StreetCenterline features (feature1 and feature2).

7. A second StreetCenterlineIntersection feature is then created at the same location with the road name attributes reversed (feature 2 and feature 1 ) to allow the user to search for the intersection in either order.

8. The StreetCenterlineIntersections are committed to the SDE database.

\subsubsection{SAGE Architecture}

The Stand Alone Geodatabase Editor (SAGE) tool allows for the updating of the versioned GIS data without running ArcFM/ArcGIS. This is accomplished via ActiveX Directory Object (ADO) database access and use of ESRI's multi-versioned views. The ESRI versioning structure for the SAGE tool includes a single "NIPSCO_SAGE" version that exists under the SDE.DEFAULT version. This version is automatically reconciled and posted to DEFAULT on a nightly basis. All SAGE edits are non-spatial, asset-based edits and are made available to the enterprise in a relatively real-time fashion.

The SAGE application was written as a C\#.Net ASP.Net Web Application. All of the SAGE functionality is run as a stand-alone application and is handled in a linked-form manner (as identified by the current COBOL implementation of EDFS). This functionality easily fits into the scope of a web application. With today's advancements in C\#.Net Web Applications, we have the ability to provide a secure, feature-rich user interface in much the same manner as a fat-client client-server application. This is accomplished while retaining the major benefits of centralized access and maintenance. Figure 4.7-23 illustrates the architecture of the ASP.Net SAGE solution: 


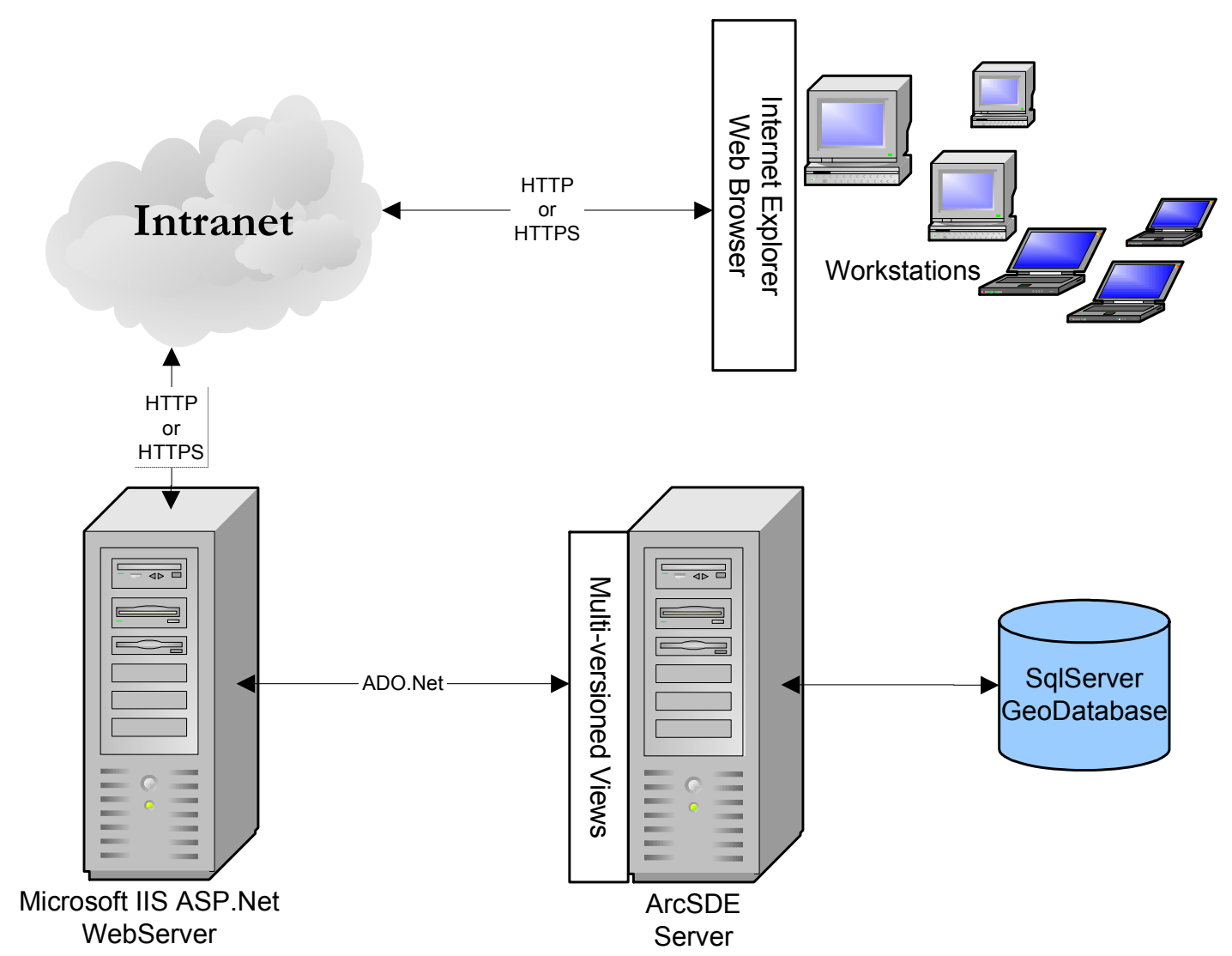

Figure 4.7-23. SAGE architecture

\subsection{SAGE Multi-Versioned View Update Manager}

The SAGE website uses a single database connection string which connects it to a single ESRI multi-versioned view. When multiple users attempt to access the database at the same time, there can be locking problems at the version level (state IDs).

To avoid this issue, SAGE writes all insert, update, and delete SQL statements to a MVV Queue table and notifies the SAGE Multi-Versioned View (MVV) Update Manager windows service by writing a SAGE Generate Update (.sgu) file on the web server. The MVV Manager Web Service is installed on the web server and monitors a configured directory for .sgu files. When notified, the service executes the queued SQL and deletes the SGU file. With this in place, there is only one process that updates the MVV which ensures there are never any locking issues.

The processing includes the following steps:

1. The SAGE application checks all SQL executed against the database (MVV) for insert, update, or delete statements.

2. If found, SAGE inserts the SQL statements into a queue table, ArcFM8.Electric.MVVUpdateQueue, instead of actually executing them against the database. Each SQL statement is tagged with a unique connection id that is specific to the calling SAGE session.

3. SAGE then writes a SGU file to a configured directory on the web server. 
4. The MVV Update Manager web service monitors the configured directory on the web server waiting for the creation of SGU files.

5. When a SGU file is created, the web service polls the MVVUpdateQueue table for all queued SQL statements. It then attains an edit lock on the MVV and executes each statement.

6. If a statement processes without error, the queued record is deleted from the MVVUpdateQueue table.

7. Once all queued statements have been executed, the windows service checks the configured for any additional SGU files.

8. If no additional SGU files are found, the edit lock is released on the MVV.

9. After initially creating the SGU file, the SAGE application monitors the MVVUpdateQueue table. Once all SQL statements tagged with the current connection id have been deleted from the table, the SAGE application assumes success and returns control to the user.

The above process executes in sub-second time and also handles the case where multiple users execute SAGE transactions at the same time. All SAGE transactions are then processed in the same edit lock.

\subsubsection{SAGE Authentication and Authorization}

The AEDR ArcFM/ArcGIS system use Windows Authentication to allow users to authenticate. This allows the users to utilize their NT username and password to connect to the system. The ASP.Net SAGE application uses the same authentication model by using .Net's built in Windows Authentication modules. This allows the users to connect to the web application without accessing a logon screen. The user's authentication credentials are read from the client machine via the connecting http request and the authentication transaction appears seamless to the user.

The user's username is extracted from the security credentials and is used to query the SAGE security tables to access the user's authorization role. The authorization role is used to grant or deny the user access to each area of functionality within the SAGE application.

Figure 4.7-24 details the SAGE security tables. 


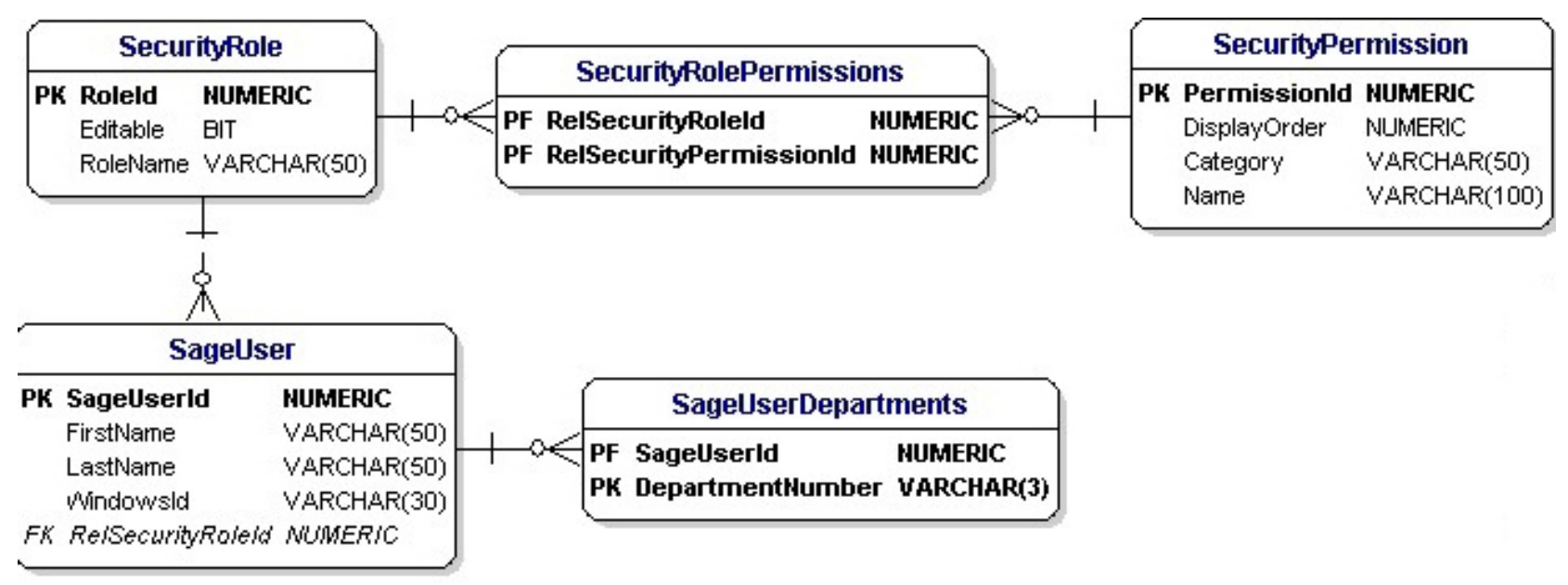

Figure 4.7-24. SAGE security tables

\subsubsection{SAGE Data Model}

The SAGE application requires a small database to manage the non-GIS data aspects of the application. These areas include the authorization model of the SAGE application, various tables relating to the nightly processing of the MAPPS transactions, and a join table that relates the distribution reference number of poles, pads, and pedestals to conductors (overhead and underground primary and secondary) for reporting purposes. This database is managed as a separate database within the same SQL Server instance as the GIS. This simplifies the management of this data as all of the tables are only accessed by SAGE applications and do not need to include the complexity of defining user permissions or GIS versioning. The data model for the SAGE application is detailed here: 


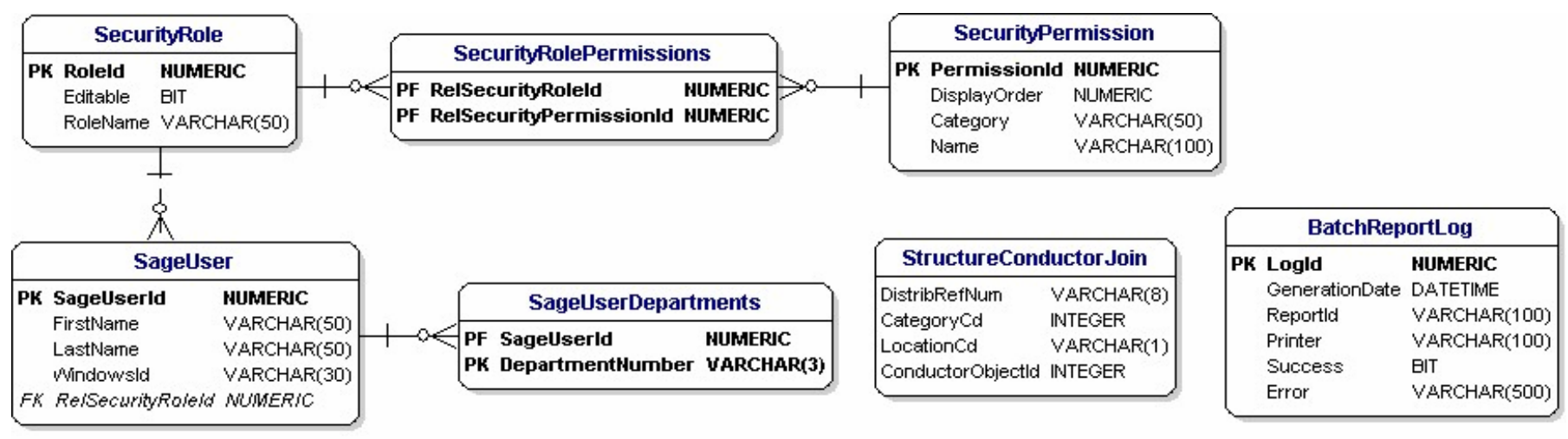

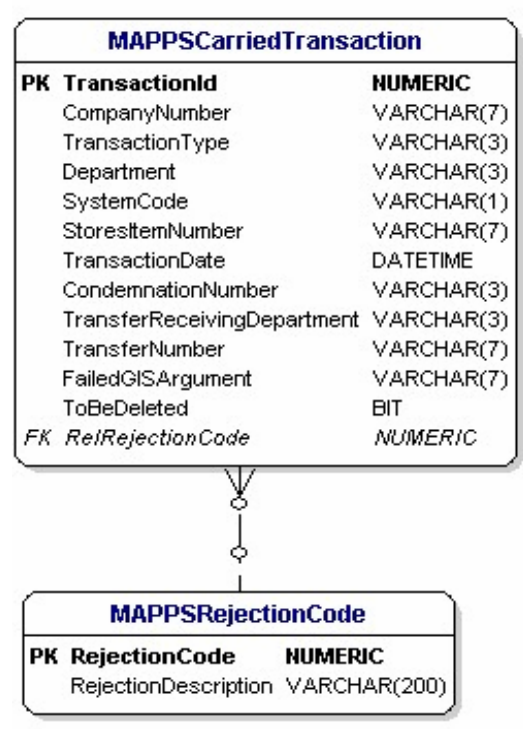

\begin{tabular}{|ll|}
\hline \multicolumn{2}{|c|}{ InterfacehAPPSTransaction } \\
\hline PK Transactionld & HUMERIC \\
CompanyNumber & VARCHAR(7) \\
TransactionType & VARCHAR(3) \\
Department & VARCHAR(3) \\
SystemCode & VARCHAR(1) \\
StoresitemNumber & VARCHAR(7) \\
TransactionDate & DATETIME \\
TransferReceivingDepartment & VARCHAR(3) \\
TransferNumber & VARCHAR(7) \\
CondemnationNumber & VARCHAR(3) \\
\hline & \\
\hline \multicolumn{2}{|c|}{ MAPPSProcessedTransHistory } \\
\hline PK ProcessedTransactionld & HUMERIC \\
ProcessedDate & DATETIME \\
CompanyNumber & VARCHAR(7) \\
TransactionType & VARCHAR(3) \\
Department & VARCHAR(3) \\
SystemCode & VARCHAR(1) \\
StoresttemNumber & VARCHAR(7) \\
TransactionDate & DATETIME \\
CondemnationNumber & VARCHAR(3) \\
TransferReceivingDepartment & VARCHAR(3) \\
TransferNumber & VARCHAR(7) \\
\hline
\end{tabular}

\begin{tabular}{|ll|}
\hline \multicolumn{2}{|c|}{ MAPPSNightlyStatistics } \\
\hline PK TransactionDate & DATETIME \\
CarryOverCount & INTEGER \\
ManualDeletions & INTEGER \\
NewMAPPSTransfers & INTEGER \\
NewMAPPSCondemnations & INTEGER \\
NewMAPPSIssues & INTEGER \\
NewMAPPSReturns & INTEGER \\
TotalMAPPSTransactions & INTEGER \\
NewGISTRInstalls & INTEGER \\
NewGISTRRemovals & INTEGER \\
NewGISCapacitorlnstalls & INTEGER \\
TotalGISTransactions & INTEGER \\
ProcessedTransters & INTEGER \\
OutstandingTransfers & INTEGER \\
ProcessedCondernnations & INTEGER \\
OutstandingCondemnations & INTEGER \\
Matchedlssuesinstalls & INTEGER \\
MatchedReturnsRemovals & INTEGER \\
UnMatchedlssues & INTEGER \\
UnMatchedReturns & INTEGER \\
UnMatchedTRInstalls & INTEGER \\
UnMatchedTRRemovals & INTEGER \\
UnMatchedCapacitorlnstalls & INTEGER \\
TotalProcessedSuccess & INTEGER \\
TotalOutstanding & INTEGER \\
TotaIProcessed & INTEGER \\
\hline
\end{tabular}
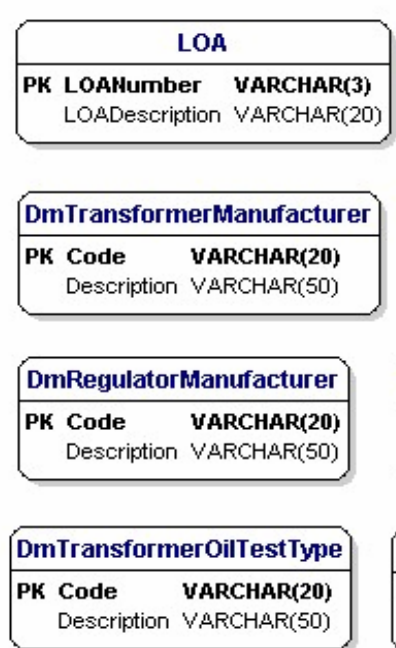
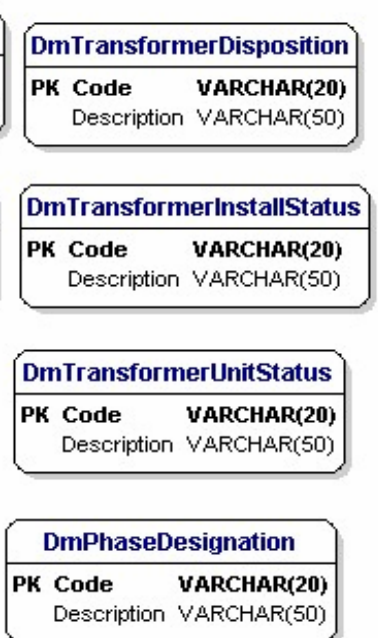

Figure 4.7-25. SAGE data model
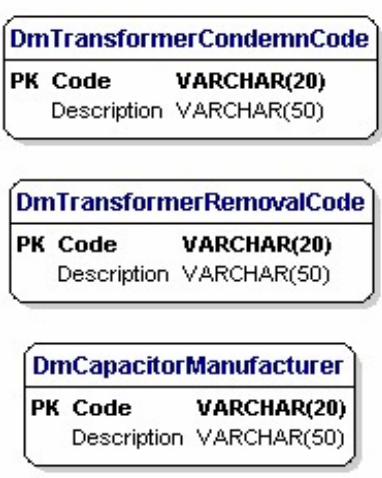

\begin{tabular}{|cc|}
\hline \multicolumn{2}{|c|}{ DmCapacitorFluidType } \\
\hline PK Code & VARCHAR(20) \\
Description VARCHAR(50)
\end{tabular}

RCHAR(20) 


\subsubsection{SAGE Reports}

The SAGE Reports are modeled after the EDFS reports in regard to the data they display and the layout. The data for the reports were gathered by using SQL queries against the SAGE multi-versioned view. The queries and logic for the reports were derived from the original EDFS design specifications. The data was converted into an XML format using .Net tools and then transformed into the desired format by using XSLT stylesheets.

\subsection{Use of XSLT}

An XSLT stylesheet can be used to transform an XML document into another form. In this implementation, the XML document was transformed into an HTML document, which can be easily displayed and/or printed from a web browser. XSLT stylesheets work as a series of templates, which produce the desired formatting effect each time a given element is encountered. XSLT can also control the order in which elements and attributes are displayed. XSLT is itself an implementation of XML

The stylesheets, or "transformation templates", are written primarily with declarative constructs though procedural techniques can be employed as needed. The stylesheet asserts the desired behavior of the XSLT processor based on conditions found in the source XML. It supplies examples of how each component of the result is formulated and indicates the conditions of the source that trigger which component is added to the result.

\subsection{Report Generation}

The reports can be generated in one of two ways. First, any of the batch reports can be configured to execute and print via the batch .Net process that was described in the "Batch Data Management and Reporting Applications" section of this document. In addition the reports can be generated in a real-time fashion via the SAGE tool. This approach allows the user to generate the report in a web browser. The report can then be spooled to any windows printer that is configured on the user's machine. Further, the report can be saved locally to the user's machine and can be attached to an email. For demonstration purposes, the following batch reports contain fictitious information. The batch reports that are available in the AEDR system include:

\subsection{Request for Authority to Condemn Transformers and Regulators}

Transformer and regulator condemnations are entered into SAGE (see the SAGE screen design section of this document for more detail). The following night, this batch report is generated. It contains the details for all condemnations or reverse-condemnations for each department. These reports are then printed the following day and submitted to management for approval signatures. 


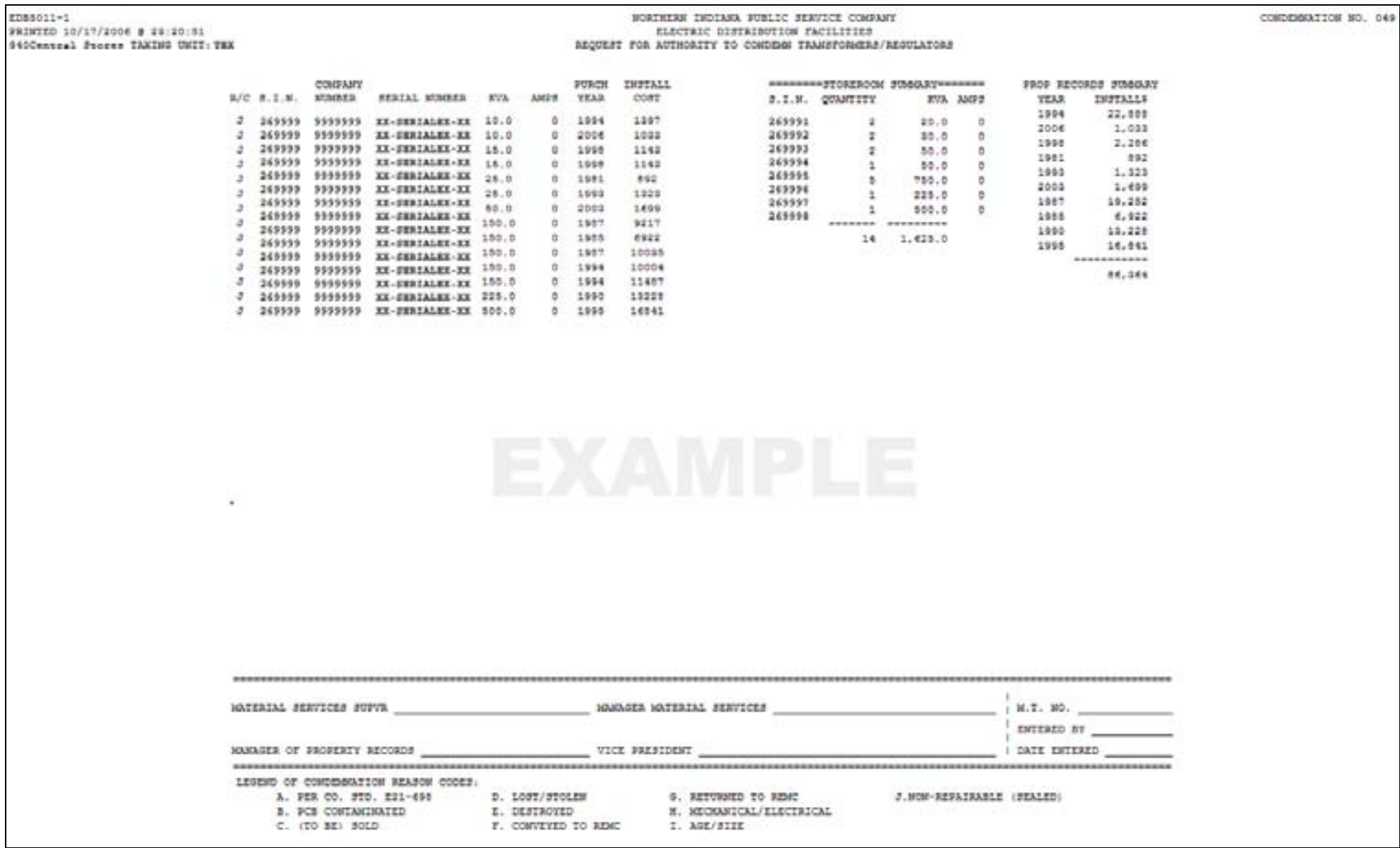

Figure 4.7-26. Detailed condemnation/reverse condemnation report 


\subsection{Line-Transformer / Voltage-Regulator Company Use Report}

This report is run monthly and details all company use installations of transformers and regulators during the previous month.

EDB 5020-1

PRIMTED 09/30/2005 23:00:14

OgOAMGOLA

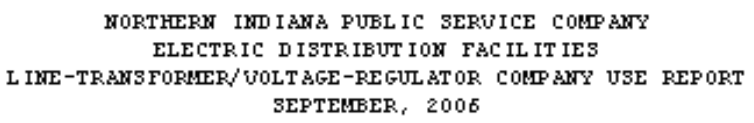

999

\begin{tabular}{|c|c|c|c|c|c|c|c|}
\hline $\begin{array}{l}\text { MATER I AL } \\
\text { T I CKET T }\end{array}$ & $\begin{array}{l}\text { COMP ANT } \\
\text { MUMEER }\end{array}$ & $\begin{array}{l}\text { SER I AL } \\
\text { MUMEER }\end{array}$ & KUA & MAP S & $\begin{array}{l}\mathrm{I} / \\
\mathrm{R}\end{array}$ & $\begin{array}{l}\text { PRCH } \\
\text { YE AR }\end{array}$ & $\begin{array}{l}\text { INST ALL } \\
\text { COST }\end{array}$ \\
\hline 999 & 1234567 & XX-SERIAL-EX & 250.0 & 328 & I & 1997 & 12888 \\
\hline
\end{tabular}

TAX IMG UN IT TOT BLS:

TR.AMS FORMERS IMST ALLED

REGUL ATORS IMST ALLED

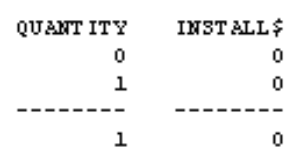

TRAMS FORMERS REMOUED

REGUL ATORS REMOUED

T OT AL

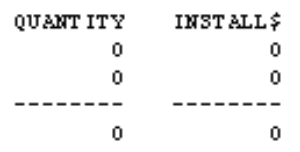

Figure 4.7-27. Line-Transformer / Voltage-Regulator Company Use report

\subsection{FERC For-1 Report of Line Transformers}

This report shown in Figure 4.7-28 is run annually and provides a summary of all transformer and regulator additions and reductions for the previous year. 


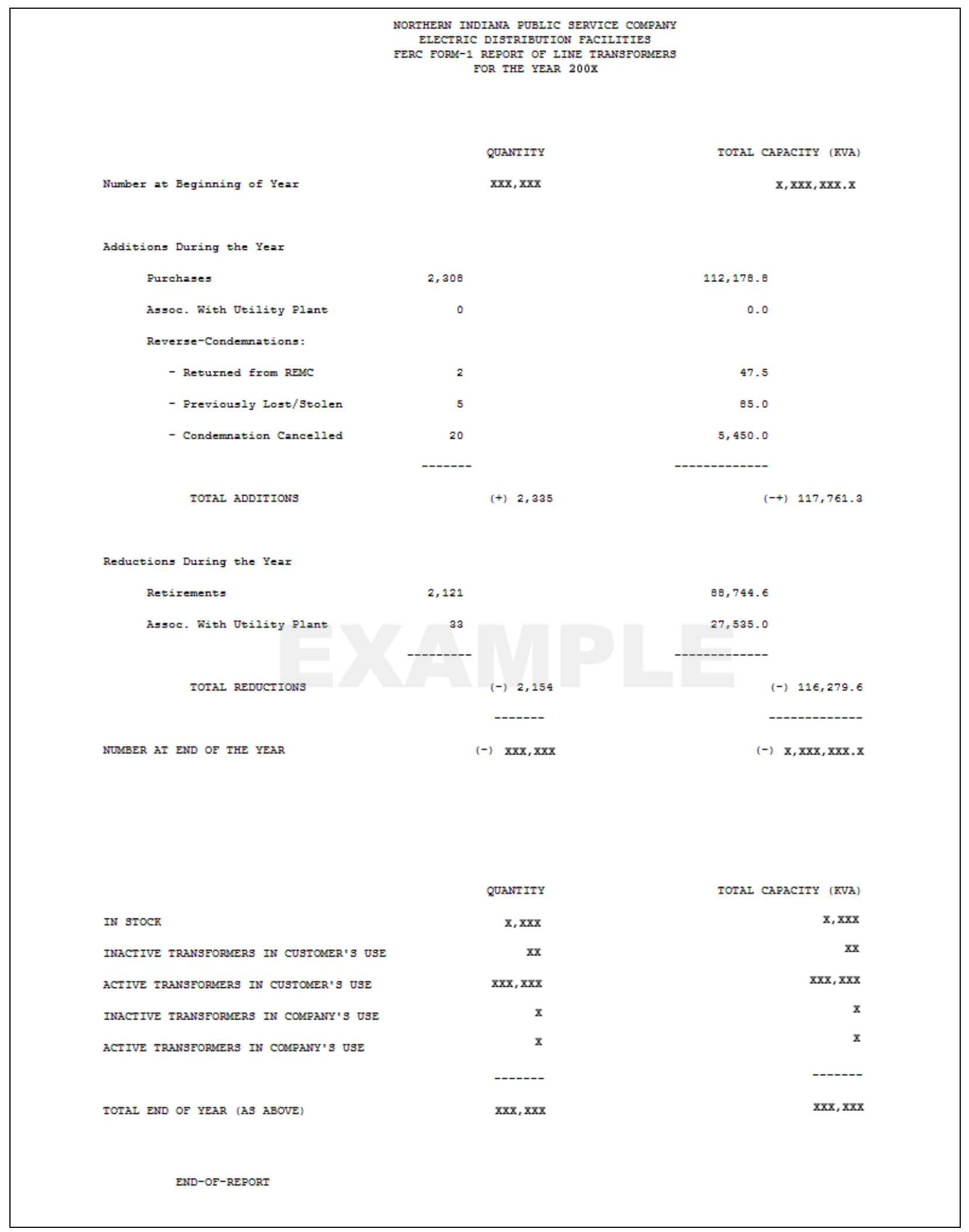

Figure 4.7-28. FERC Form-1 report of line transformers 


\subsection{Report Showing Transformers and Poles}

This report shown in Figure 4.7-29 is run annually and shows details for the installation of transformers, streetlight switches, and poles for the previous year. It is used by the engineering department.

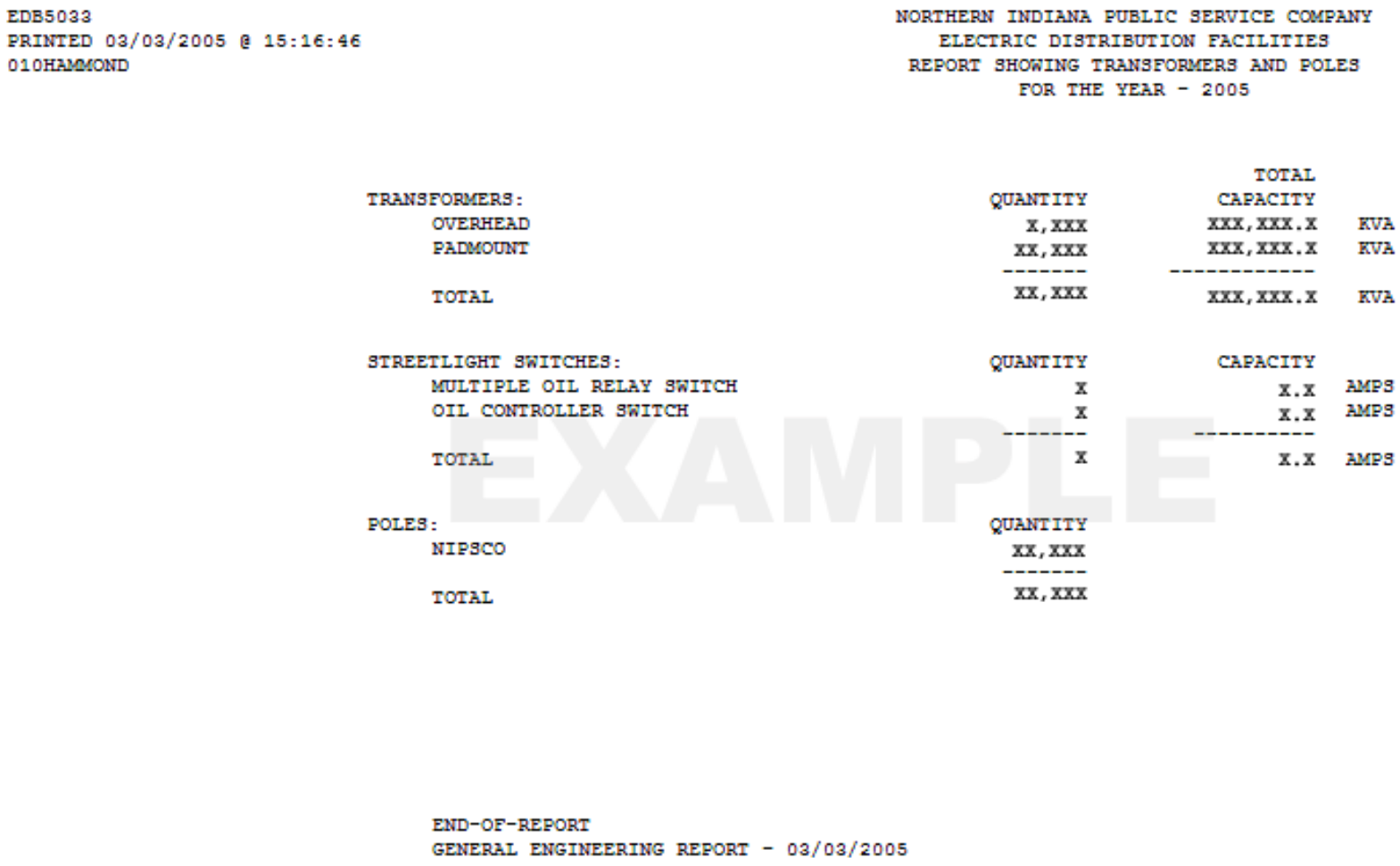

Figure 4.7-29. Annual report of transformers and poles 


\subsection{Annual Count of Transformers}

This report is run nightly and reports on up to date statistics of installed transformers in both company use and customer use. Figure 4.7-30 is grouped by department and sorted by taxing unit.

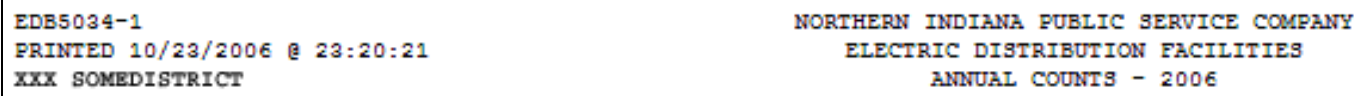

TAXING UNIT

RVA

INSTALIS

QUANTITY

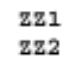

$\mathrm{zz2}$

$\mathrm{zz} 3$
$\mathrm{zz} 4$

$\mathrm{zz} 4$
$\mathrm{zz} 5$

$\mathrm{zz} 5$
$\mathrm{zz} 6$

$\mathrm{zz} 7$

$$
\mathrm{ZZ} 8
$$

229

$\mathrm{zz} 1$

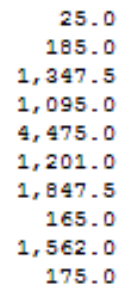

1, 315

11,610

47,656

42,923

206,777

38,803

60,860

9,489

61,352

DISTRICT TOTALS:

$12,078.0$

487,455

EDB5034-1

PRINTED 10/23/2006 23:20:21

NORTHERN INDIANA PUBLIC SERVICE COMPANY

ELECTRIC DISTRIBUTION EACILITIES

XXX SOMEDISTRICT

TRANGFORMERS IN CUSTOMER USE:

TAXING UNIT

$\mathrm{zz1}$
$\mathrm{zz} 2$
$\mathrm{zz} 3$
$\mathrm{zz4}$
$\mathrm{zz5}$
$\mathrm{zz6}$
$\mathrm{zz7}$
$\mathrm{zz8}$
$\mathrm{zz9}$
$\mathrm{zz1}$
$\mathrm{zz2}$
$\mathrm{zz3}$
$\mathrm{zz4}$
$\mathrm{zz5}$
$\mathrm{zz6}$
$\mathrm{zz} 7$
$\mathrm{zz} 8$
$\mathrm{zz} 9$

DISTRICT TOTALS:

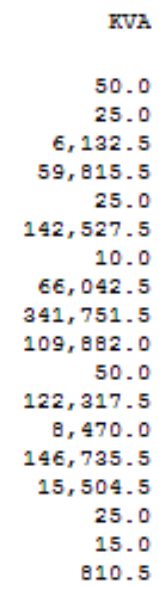

$1,020,189.5$

$$
\begin{array}{r}
\text { INSTALLS } \\
775 \\
1,579 \\
155,708 \\
2,475,000 \\
1,793 \\
3,504,362 \\
1,095 \\
2,136,178 \\
8,538,282 \\
3,214,849 \\
1,702 \\
3,727,146 \\
291,500 \\
5,973,367 \\
368,101 \\
2,200 \\
271
\end{array}
$$$$
19,348
$$

$30,413,256$

QUANTITY

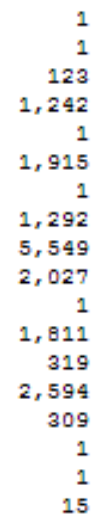

17,203

Figure 4.7-30. Annual count of transformers 


\subsection{Annual Count of Transformers by County}

Figure 4.7-31 report is run nightly and returns up to date statistics of installed transformers in both company use and customer use. It is grouped by county and sorted by taxing unit.

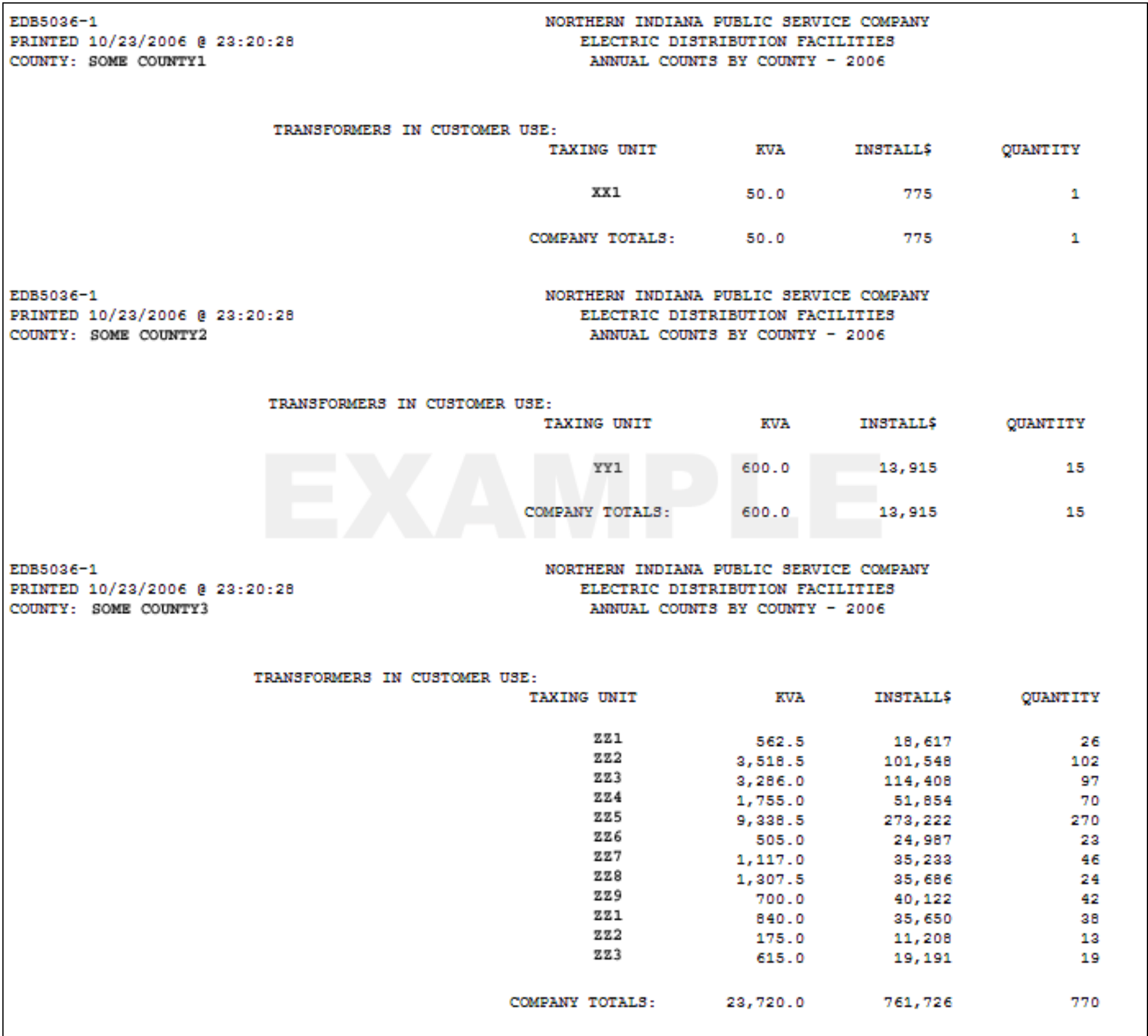

Figure 4.7-31. Annual count of transformers by county 


\subsection{Transformer Failure Report}

This report is run annually and provides a summary of transformer and regulator failures for the previous year. It presents the data in several different formats.

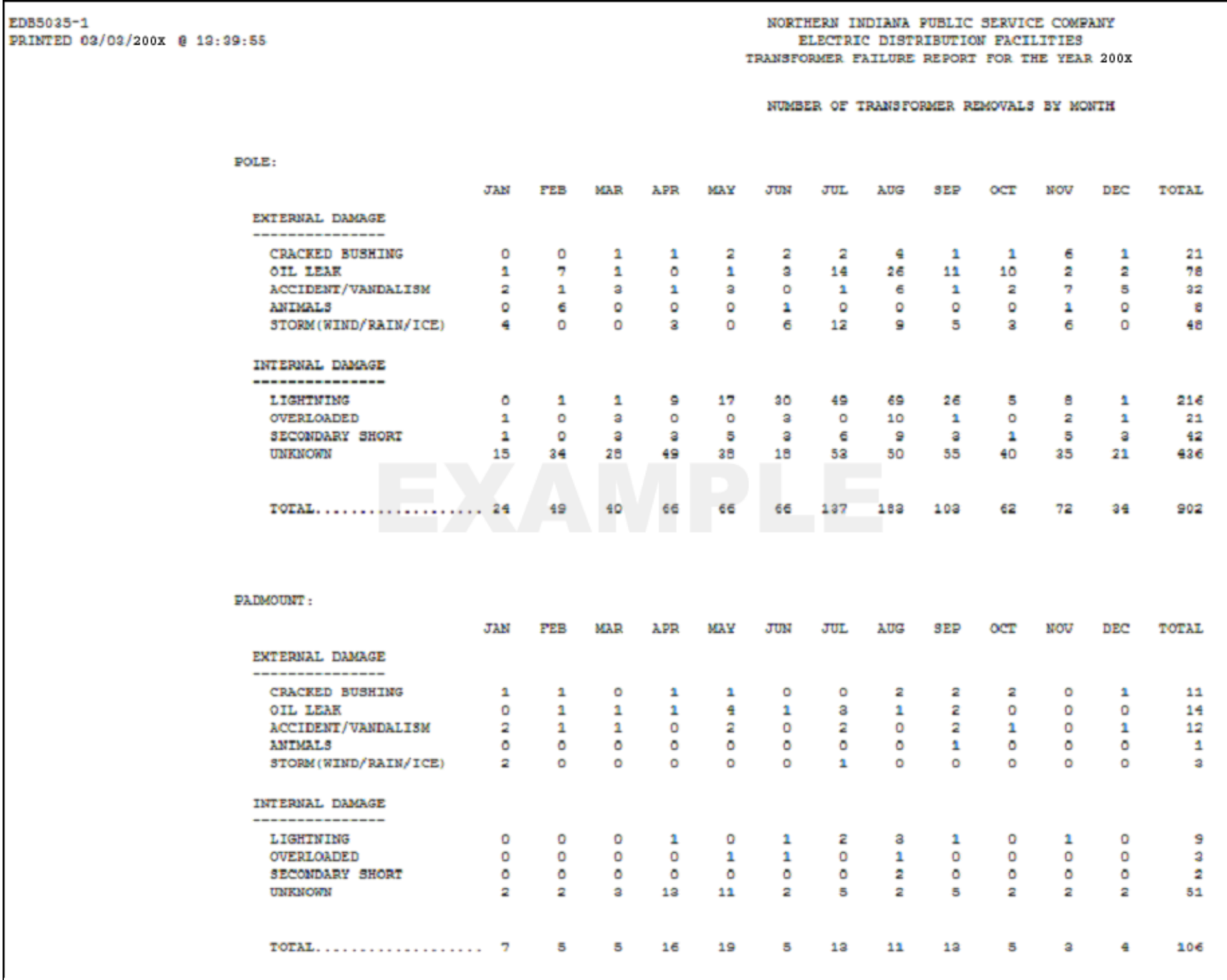

Figure 4.7-32. Transformer failure report. The first page is formatted with failures by month 


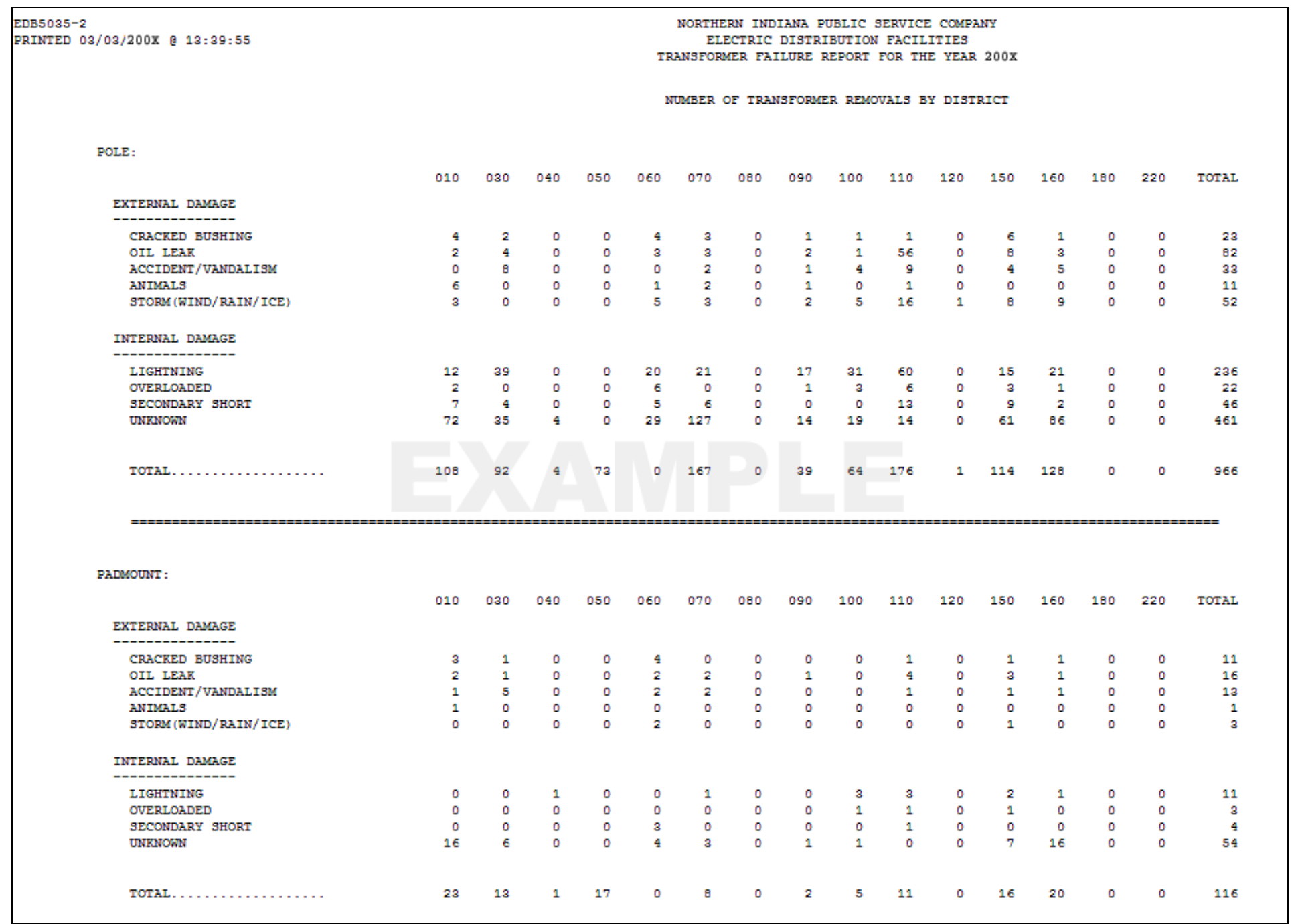

Figure 4.7-33. Transformer failure report. The second page is formatted with failures by department/district 


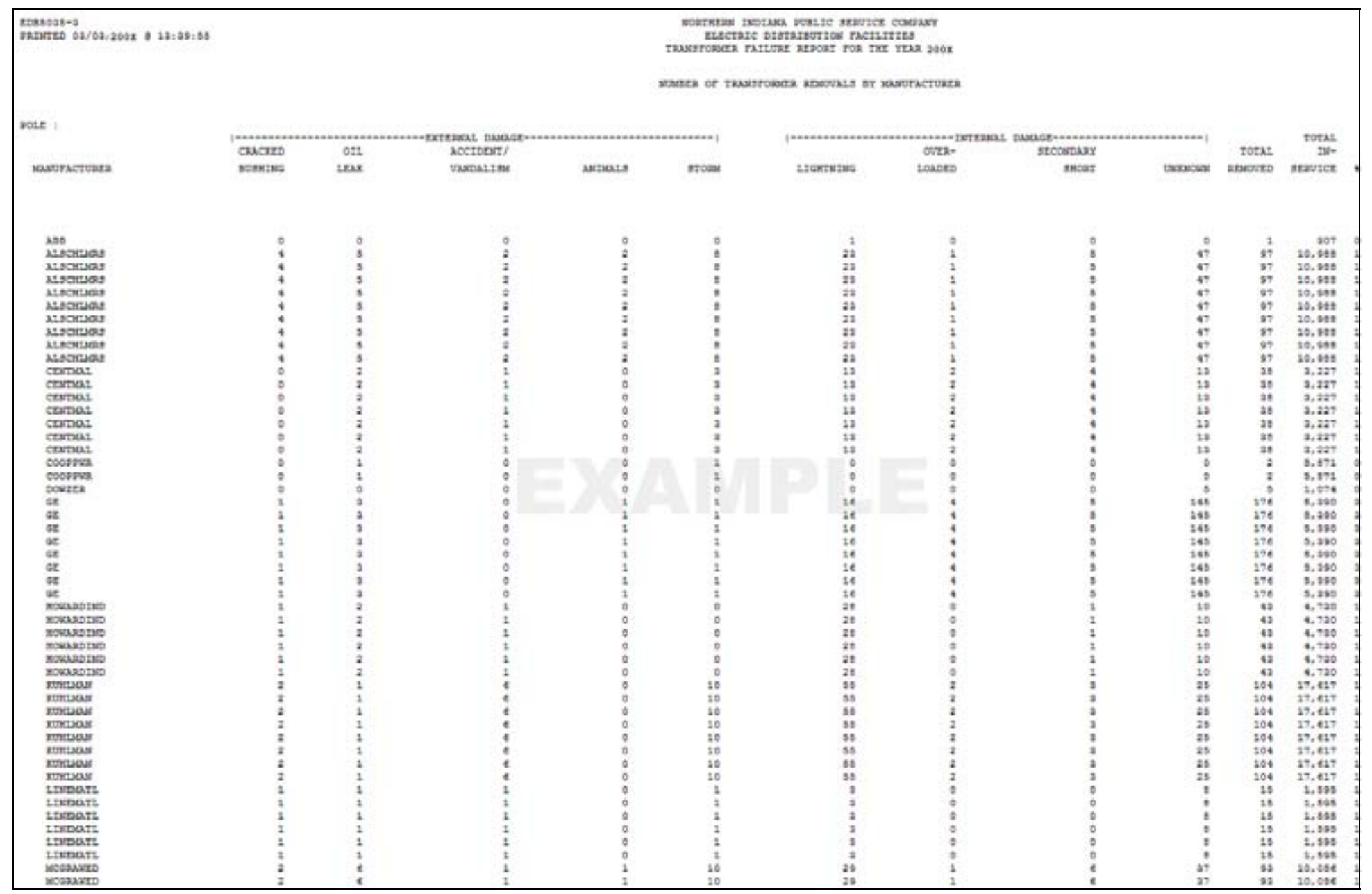

Figure 4.7-34. The third page is formatted with failures by manufacturer 


\subsection{8}

Temporary and Inactive Transformer and Regulator Installations - Aged Three Months This report is run nightly and reports on transformer and regulator installations that were marked as temporary and/or inactive that have been in the field for over three months. This helps NIPSCO in ensuring that these types of installations are not abandoned or lost in the field.

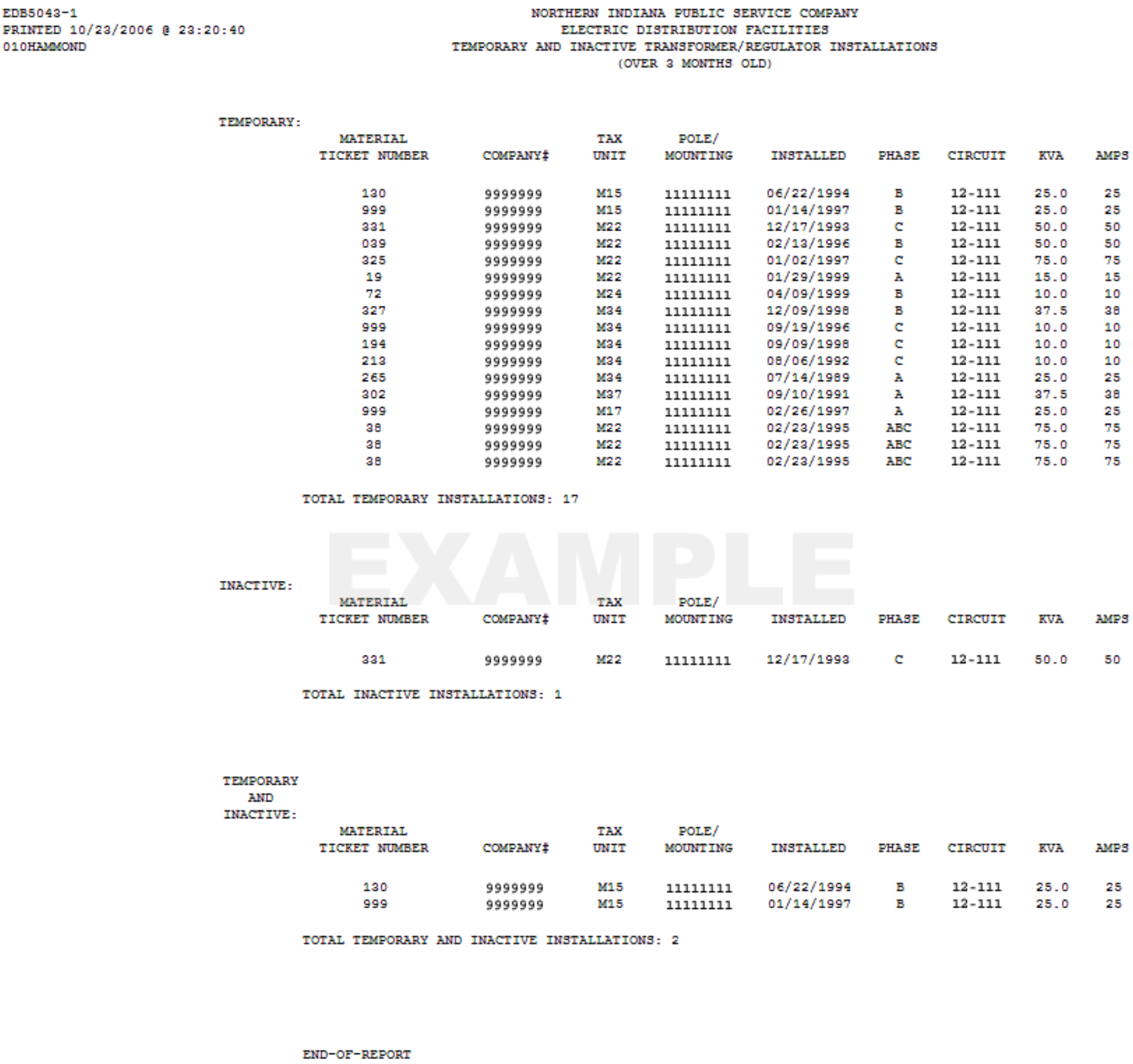

TOTAL TEMPORARY INSTALLATIONS: 17

9999999

M22

1111111

$12 / 17 / 1993$

TOTAL INACTIVE INGTALIATIONS: 1

END-OF-REPORT

Figure 4.7-35. Temporary and inactive

transformer and regulator installations aged 3 months 


\subsection{Transformer \& Regulator Stores Item Number Description Report}

This report is run nightly and shows the key details for all transformer and regulator stores item numbers. This is a useful report for anyone referencing these numbers.

\begin{tabular}{|c|c|c|c|c|c|c|c|}
\hline \multirow[t]{2}{*}{$\begin{array}{l}\text { EDB5044-1 } \\
\text { PRINTED } 10 / 23 / 2006 \text { \& } 2006\end{array}$} & \multicolumn{7}{|c|}{$\begin{array}{c}\text { ELECTRIC DISTRIBUTION FACILITIES } \\
\text { RANSFORMER/REGULATOR STORES ITEM NUMBER DESCRIPTION REPORT }\end{array}$} \\
\hline & S.I.N. & TYPE & KVA & AMPS & PHASE & PRIMARY VOLTAGE & SECONDARY VOLTAGE \\
\hline & 2666661 & $\mathrm{~T}$ & 4.0 & 0 & 1 & 2400 & $120 / 240$ \\
\hline & 2666662 & $\mathrm{~T}$ & 1.5 & 0 & 1 & 2400 & $120 / 240$ \\
\hline & 2666663 & $\mathrm{~T}$ & 2.0 & 0 & 1 & 2400 & $120 / 240$ \\
\hline & 2666664 & $\mathrm{~T}$ & 3.0 & 0 & 1 & 2400 & $120 / 240$ \\
\hline & 2666665 & $\mathrm{~T}$ & 5.0 & 0 & 1 & 2400 & $120 / 240$ \\
\hline & 2666666 & $\mathrm{~T}$ & 7.5 & 0 & 1 & 2400 & $120 / 240$ \\
\hline & 2666667 & $\mathrm{~T}$ & 10.0 & 0 & 1 & 2400 & $120 / 240$ \\
\hline & 2666668 & $\mathrm{~T}$ & 15.0 & 0 & 1 & 2400 & $120 / 240$ \\
\hline & 2666669 & $T$ & 25.0 & 0 & 1 & 2400 & $120 / 240$ \\
\hline & 2666610 & $\mathrm{~T}$ & 37.5 & 0 & 1 & 2400 & $120 / 240$ \\
\hline & 2666611 & $\mathrm{~T}$ & 50.0 & 0 & 1 & 2400 & $120 / 240$ \\
\hline & 2666612 & $\mathrm{~T}$ & 75.0 & 0 & 1 & 2400 & $120 / 240$ \\
\hline & 2666613 & $T$ & 100.0 & 0 & 1 & 2400 & $120 / 240$ \\
\hline & 2666614 & $\mathrm{~T}$ & 150.0 & 0 & 1 & 2400 & $120 / 240$ \\
\hline & 2666615 & $T$ & 200.0 & 0 & 1 & 2400 & $120 / 240$ \\
\hline & 2666616 & $T$ & 167.0 & 0 & 1 & 2400 & $120 / 240$ \\
\hline & 2666617 & $\mathrm{~T}$ & 250.0 & 0 & 1 & 2400 & $120 / 240$ \\
\hline & 2666618 & $\mathrm{~T}$ & 333.0 & 0 & 1 & 2400 & $120 / 240$ \\
\hline & 2666619 & $T$ & 5.0 & 0 & 1 & 2400 & $240 / 480$ \\
\hline & 2666620 & $\mathrm{~T}$ & 7.5 & 0 & 1 & 2400 & $240 / 480$ \\
\hline & 2666621 & $\mathrm{~T}$ & 10.0 & 0 & 1 & 2400 & $240 / 480$ \\
\hline & 2666622 & $T$ & 15.0 & 0 & 1 & 2400 & $240 / 480$ \\
\hline & 2666623 & $T$ & 25.0 & 0 & 1 & 2400 & $240 / 480$ \\
\hline & 2666624 & $\mathrm{~T}$ & 37.5 & 0 & 1 & 2400 & $240 / 480$ \\
\hline & 2666625 & $\mathrm{~T}$ & 50.0 & 0 & 1 & 2400 & $240 / 480$ \\
\hline & 2666626 & $T$ & 75.0 & 0 & 1 & 2400 & $240 / 480$ \\
\hline & 2666627 & $T$ & 100.0 & 0 & 1 & 2400 & $240 / 480$ \\
\hline & 2666628 & $\mathrm{~T}$ & 150.0 & 0 & 1 & 2400 & $240 / 480$ \\
\hline & 2666629 & $T$ & 167.0 & 0 & 1 & 2400 & $240 / 480$ \\
\hline & 2666630 & $T$ & 250.0 & 0 & 1 & 2400 & $240 / 480$ \\
\hline & 2666631 & $\mathrm{~T}$ & 333.0 & 0 & 1 & 2400 & $240 / 480$ \\
\hline & 2666632 & $T$ & 500.0 & 0 & 1 & 2400 & $240 / 480$ \\
\hline & 2666633 & $T$ & 50.0 & 0 & 1 & 2400 & $277 / 480$ \\
\hline & 2666634 & $\mathrm{~T}$ & 75.0 & 0 & 1 & 2400 & $277 / 480$ \\
\hline & 2666635 & $T$ & 100.0 & 0 & 1 & 2400 & $277 / 480$ \\
\hline & 2666636 & $T$ & 167.0 & 0 & 1 & 2400 & $277 / 480$ \\
\hline & 2666637 & $\mathrm{~T}$ & 250.0 & 0 & 1 & 2400 & $277 / 480$ \\
\hline & 2666638 & $T$ & 333.0 & 0 & 1 & 2400 & $277 / 480$ \\
\hline & 2666639 & $T$ & 500.0 & 0 & 1 & 2400 & $277 / 480$ \\
\hline & 2666640 & $\mathrm{~T}$ & 25.0 & 0 & 1 & 2400 & $120 / 240$ \\
\hline & 2666641 & $T$ & 50.0 & 0 & 1 & 2400 & $120 / 240$ \\
\hline & 2666642 & $T$ & 75.0 & 0 & 1 & 2400 & $120 / 240$ \\
\hline & 2666643 & $\mathrm{~T}$ & 100.0 & 0 & 1 & 2400 & $120 / 240$ \\
\hline & 2666644 & $\mathrm{~T}$ & 200.0 & 0 & 3 & 4000 & 2300 \\
\hline & 2666645 & $\mathrm{~T}$ & 167.0 & 0 & 1 & 2400 & $120 / 240$ \\
\hline & 2666646 & $\mathrm{~T}$ & 75.0 & 0 & 3 & $4160 / 2400$ & $120 / 208$ \\
\hline & 2666647 & $\mathrm{~T}$ & 150.0 & 0 & 3 & $4160 / 2400$ & $120 / 208$ \\
\hline & 2666648 & $\mathrm{~T}$ & 225.0 & 0 & 3 & $4160 / 2400$ & $120 / 208$ \\
\hline & 2666649 & $T$ & 300.0 & 0 & 3 & $4160 / 2400$ & $120 / 208$ \\
\hline & 2666650 & $T$ & 500.0 & 0 & 3 & $4160 / 2400$ & $120 / 208$ \\
\hline & 2666651 & $T$ & 750.0 & 0 & 3 & $4160 / 2400$ & $120 / 208$ \\
\hline & 2666652 & $\mathrm{~T}$ & 1000.0 & 0 & 3 & $4160 / 2400$ & $120 / 208$ \\
\hline & 2666653 & $\mathrm{~T}$ & 225.0 & 0 & 3 & $4160 / 2400$ & $277 / 480$ \\
\hline & 2666654 & $\mathrm{~T}$ & 500.0 & 0 & 3 & $4160 / 2400$ & $277 / 480$ \\
\hline & 2666655 & $T$ & 750.0 & 0 & 3 & $4160 / 2400$ & $277 / 480$ \\
\hline & 2666656 & $\mathrm{~T}$ & 1000.0 & 0 & 3 & $4160 / 2400$ & $277 / 480$ \\
\hline & 2666657 & $\mathrm{~T}$ & 1500.0 & 0 & 3 & $4160 / 2400$ & $277 / 480$ \\
\hline & 2666658 & $\mathrm{~T}$ & 264.0 & 0 & 3 & $4160 / 2400$ & $\mathrm{xxxxxxx}$ \\
\hline & 2666659 & $\mathrm{~T}$ & 75.0 & 0 & 3 & $12.5 / 7.2 \times 4 / 2.4$ & $120 / 208$ \\
\hline & 2666660 & $\mathrm{~T}$ & 150.0 & 0 & 3 & $12.5 / 7.2 \times 4 / 2.4$ & $120 / 208$ \\
\hline & 2666661 & $\mathrm{I}$ & 225.0 & 0 & 3 & $12.5 / 7.2 \times 4 / 2.4$ & $120 / 208$ \\
\hline
\end{tabular}

Figure 4.7-36. Transformer and regulator stores item numbers report showing voltage 


\subsection{Transformers In Stock Inventory Report}

This report is run nightly and reports on all transformers and regulators that are in stock on that particular date.

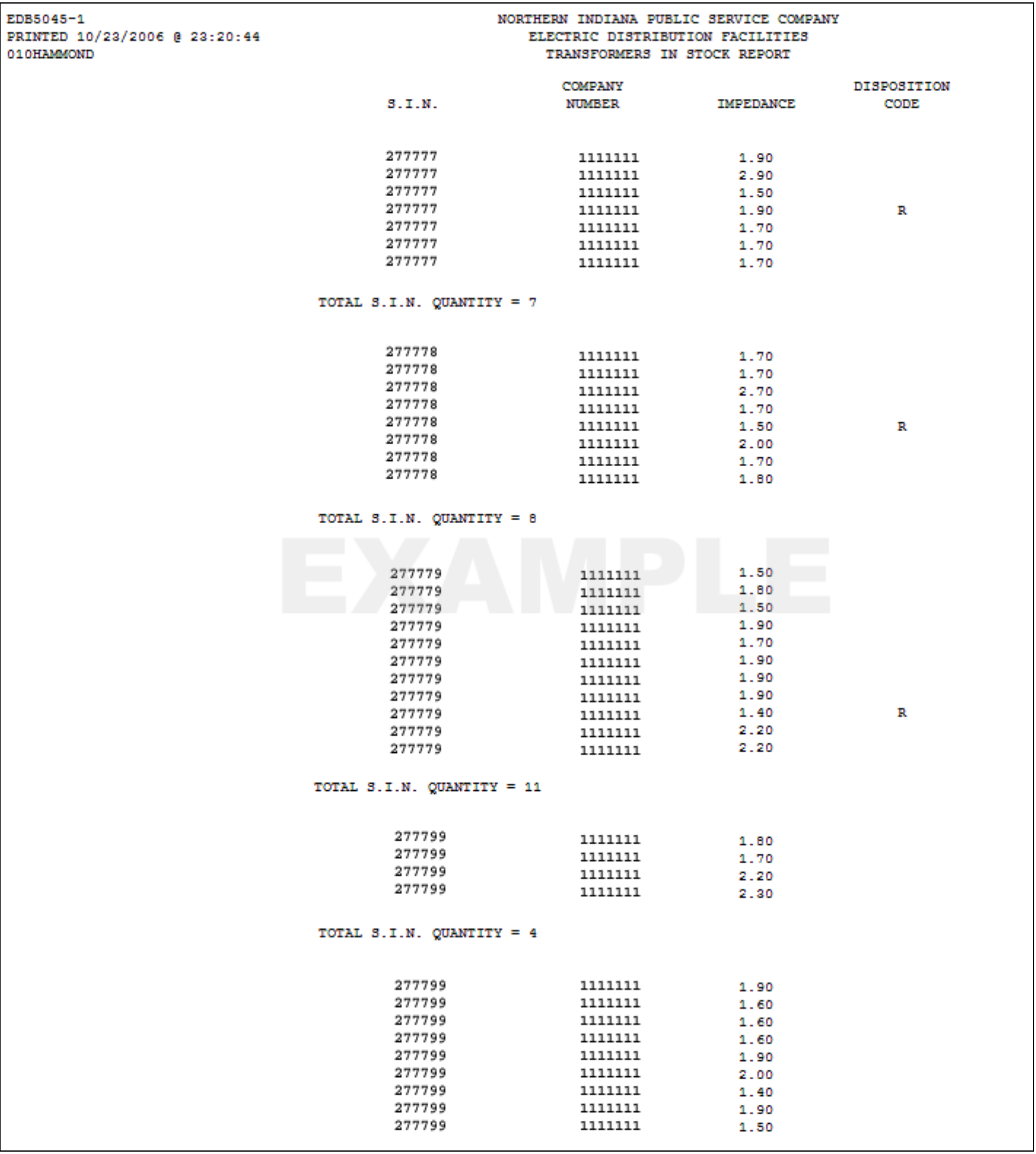

Figure 4.7-37. The Transformers in stock report is a widely used report by storeroom workers, accounting departments, and management 


\subsection{Annual Count of Capacitors}

This report is run nightly and reports on up to date statistics of installed capacitors in both company use and customer use.

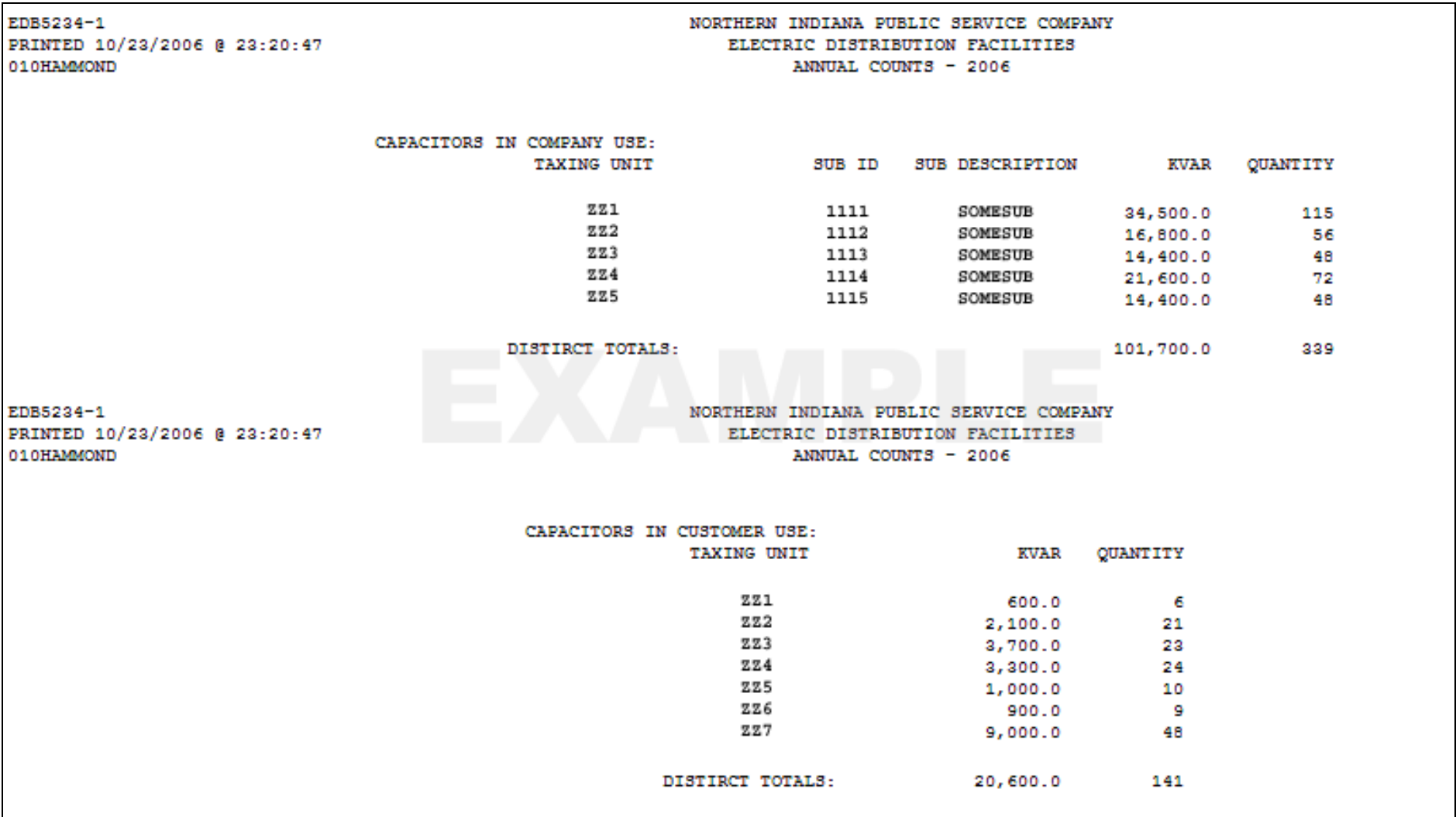

Figure 4.7-38. The annual count of capacitors report is grouped by department and sorted by taxing unit 


\subsection{Capacitor Failure Report}

This report is run annually and provides a summary of capacitor failures for the previous year. It presents the data in several different formats.

\begin{tabular}{|c|c|c|c|c|c|c|c|c|c|c|c|c|c|c|}
\hline \multirow{3}{*}{\multicolumn{2}{|c|}{$\begin{array}{l}\text { EDB5235-1 } \\
\text { PRINTED } 03 / 03 / 200 \mathrm{X} \text { \& } 14: 26: 22\end{array}$}} & & & & \multicolumn{10}{|c|}{$\begin{array}{l}\text { NORTHERN INDIANA PUBLIC SERVICE COMPANY } \\
\text { ELECTRIC DISTRIBUTION FACILITIES } \\
\text { CAPACITOR FAILURE REPORT FOR THE YEAR } 200 \mathrm{X} \\
\text { NCRBER OE CAPACITOR REMOVALS BY MONTH }\end{array}$} \\
\hline & & & & & & & & & & & & & & \\
\hline & & JaN & FEB & Mar & APR & MaY & Jon & JUL & AUG & SEP & oct & nov & $\mathrm{DEC}$ & TOTAL \\
\hline \multicolumn{15}{|c|}{ EXTERNAL DAMAGE } \\
\hline & CRACKED BUSHING & 0 & 0 & 0 & 0 & 0 & 0 & 0 & 0 & 0 & 0 & 0 & 0 & 0 \\
\hline & OIL IEAR & 0 & 0 & 0 & 0 & 0 & 0 & 0 & 0 & 0 & 0 & 0 & 0 & 0 \\
\hline & ACCIDENT/VANDALISM & 0 & 0 & 0 & 0 & 0 & 0 & & 0 & 0 & 0 & 0 & 0 & 0 \\
\hline & ANTMALS & 0 & 0 & 0 & 0 & 0 & 0 & 0 & 0 & 0 & 0 & 0 & 0 & 0 \\
\hline & STORM (WIND/RAIN/ICE) & 0 & 0 & 0 & 0 & 0 & 0 & 0 & 0 & 0 & 0 & 0 & 0 & 0 \\
\hline \multicolumn{15}{|c|}{ INTERNAL DAMAGE } \\
\hline & IIGHTNING & 0 & 0 & 0 & 0 & 0 & 0 & 0 & 0 & 0 & 0 & 0 & 0 & 0 \\
\hline & UNRaNOWN & 0 & 0 & 0 & 0 & 0 & 0 & 0 & 0 & 0 & 0 & 0 & 0 & 0 \\
\hline & $\operatorname{ToraI} \ldots \ldots \ldots \ldots \ldots$ & 0 & 0 & 0 & 0 & 0 & 0 & 0 & 0 & 0 & 0 & 0 & 0 & 0 \\
\hline \multicolumn{15}{|c|}{ SUBSTATION: } \\
\hline & & JaN & FEB & Mar & APR & $\operatorname{MaY}$ & Jons & JUL & AUG & SEP & oct & nov & DEC & TOTAL \\
\hline \multicolumn{15}{|c|}{ EXTERMAL DAMAGE } \\
\hline & CRACKED BUSHING & 0 & 0 & 0 & 0 & 0 & 0 & 0 & 0 & 0 & 0 & 0 & 0 & 0 \\
\hline & OII LEAR & 0 & 0 & 0 & 0 & 0 & 0 & 0 & 0 & 0 & 0 & 0 & 0 & 0 \\
\hline & ACCIDENT/VANDALISM & 0 & 0 & 0 & 0 & 0 & 0 & 0 & 0 & 0 & 0 & 0 & 0 & 0 \\
\hline & ANTMALS & 0 & 0 & 0 & 0 & 0 & 0 & 0 & 0 & 0 & 0 & 0 & 0 & 0 \\
\hline & STORM (WIND/RATN/ICE) & 0 & 0 & 0 & 0 & 0 & 0 & 0 & 0 & 0 & 0 & 0 & 0 & 0 \\
\hline \multicolumn{15}{|c|}{ INTERNAL DAMAGE } \\
\hline & LIGHTNING & 0 & 0 & 0 & 0 & 0 & 0 & 0 & 0 & 0 & 0 & 0 & 0 & 0 \\
\hline & UNRaNONN & 0 & 0 & 0 & 0 & 0 & 0 & 0 & 0 & 0 & 0 & 0 & 0 & 0 \\
\hline & TOTaL.......... & 0 & 0 & 0 & 0 & 0 & 0 & 0 & 0 & 0 & 0 & 0 & 0 & 0 \\
\hline
\end{tabular}

Figure 4.7-39. Capacitor failure report. The first page is formatted with failures by month 


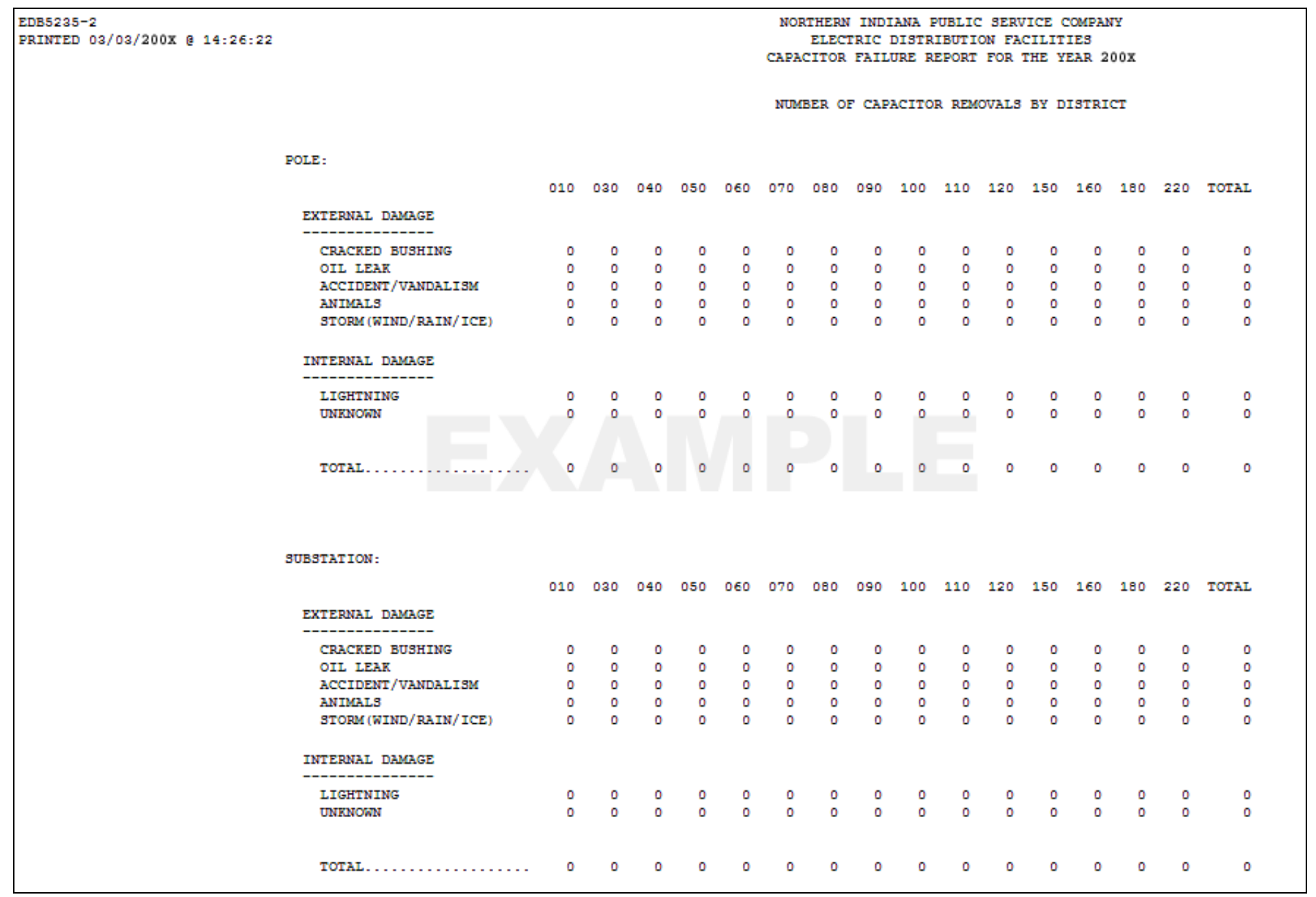

Figure 4.7-40. The second page of the capacitor failure report is formatted with failures by department/district 


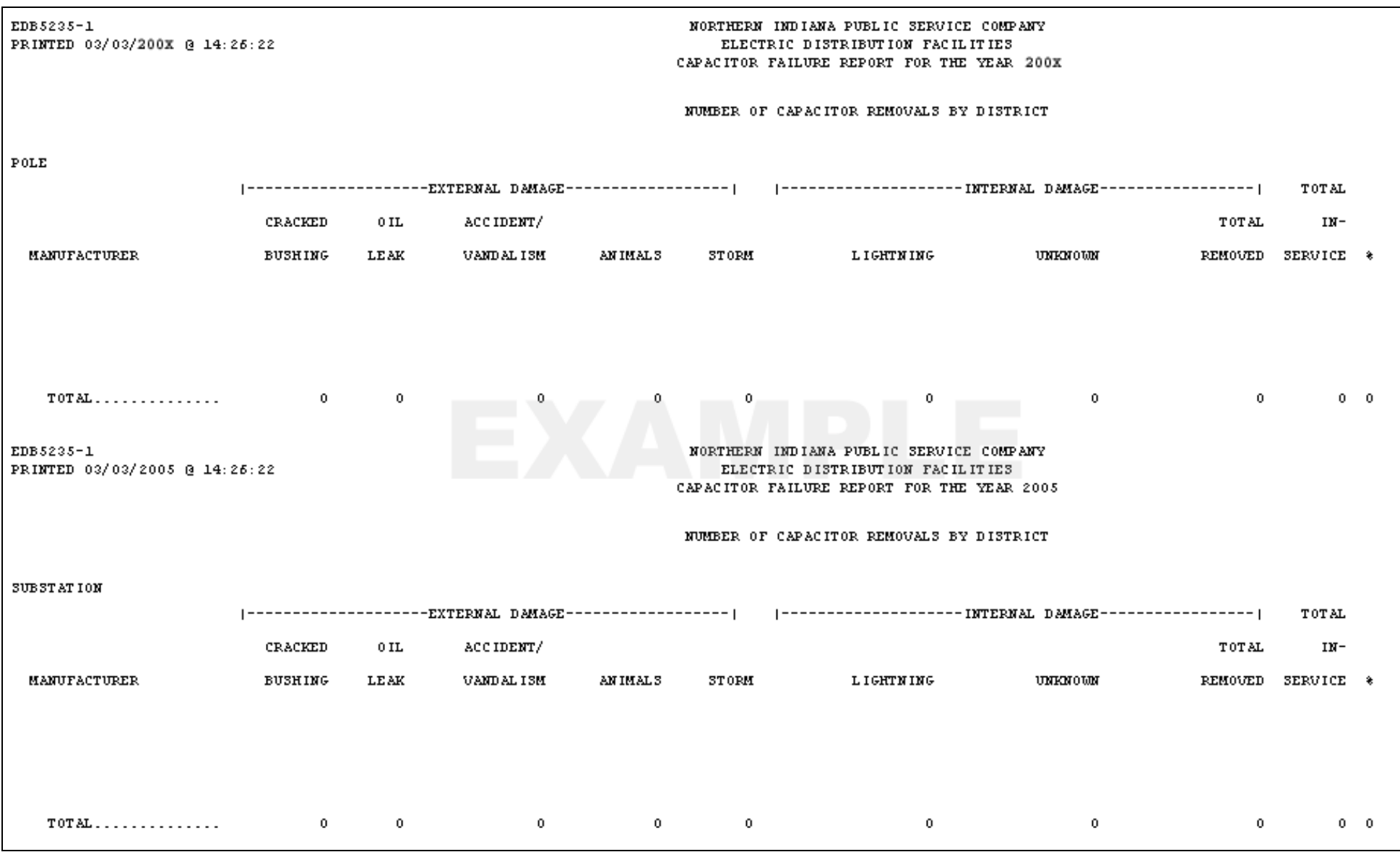

Figure 4.7-41. The third page of the capacitor failure report is formatted with failures by manufacturer 


\subsection{Capacitor Stores Item Number Description Report}

This report is run nightly and shows the key details for all capacitor stores item numbers.

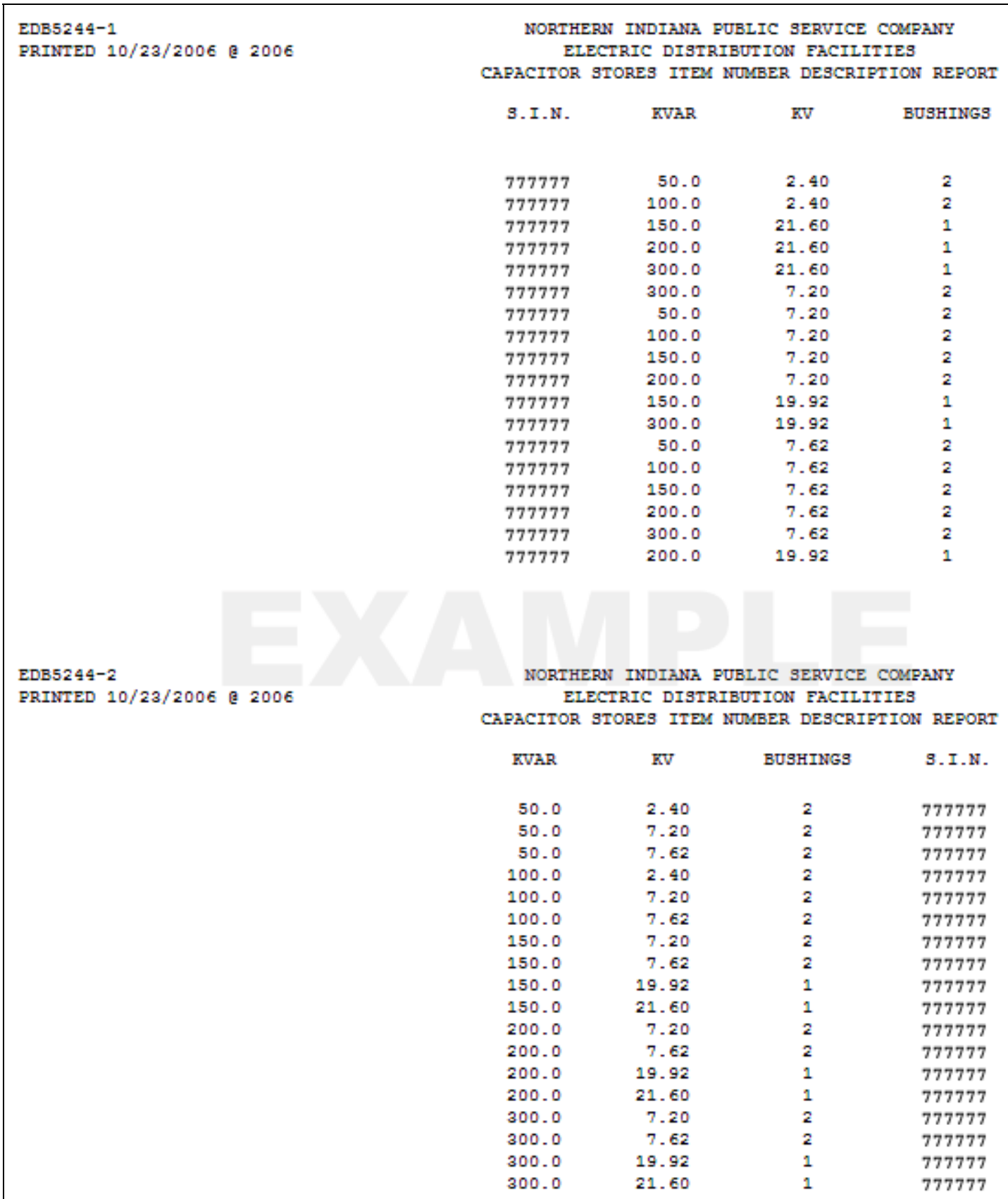

Figure 4.7-42. The capacitor stores item description report is a useful report for anyone referencing these numbers 


\subsection{Capacitors In Stock Inventory Report}

This report is run nightly and reports on all capacitors that are in stock on that particular date.

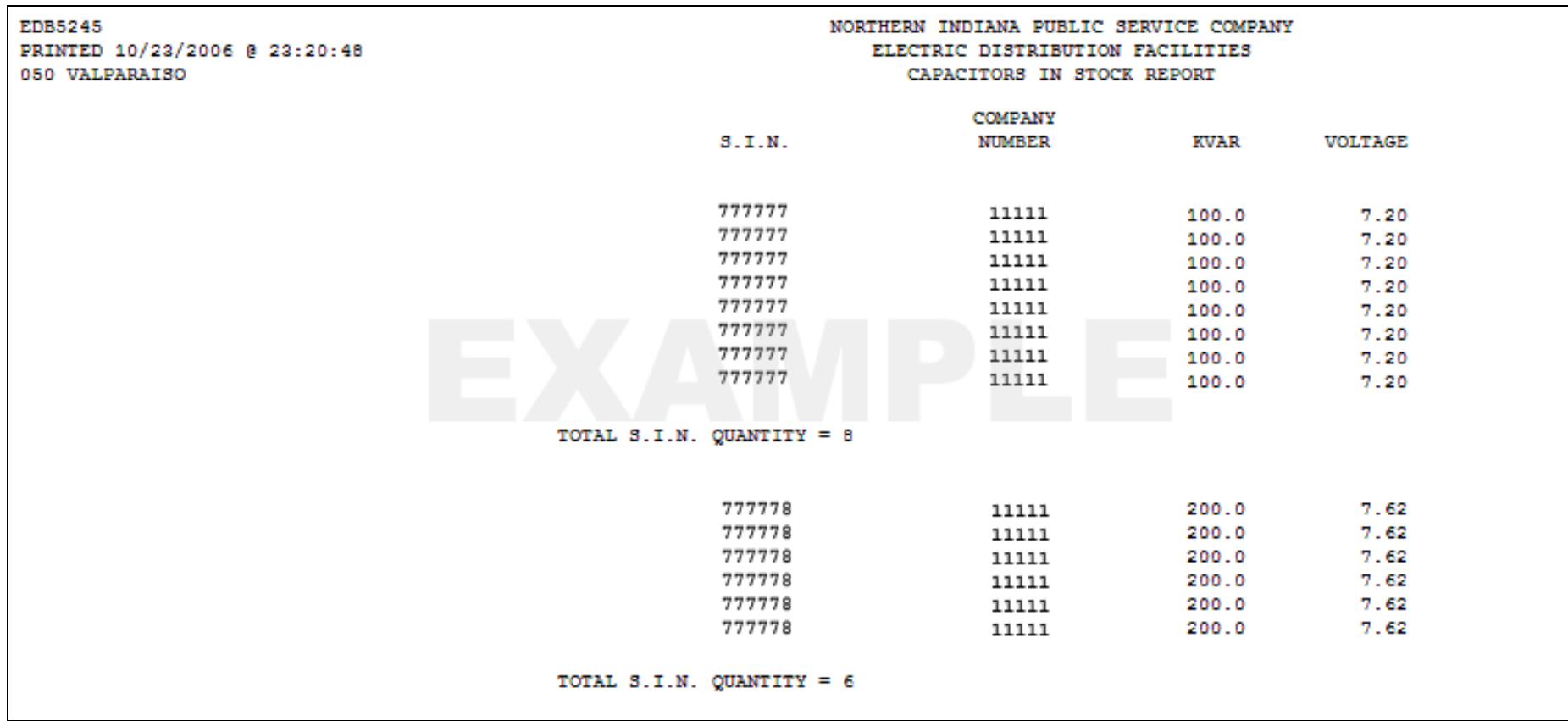

Figure 4.7-43. Capacitors in stock inventory report is a widely used report by storeroom workers, accounting departments, and management 


\subsection{Securities and Exchange Commission Form 10K (EDB5032)}

This report is run annually and is submitted to the federal government.

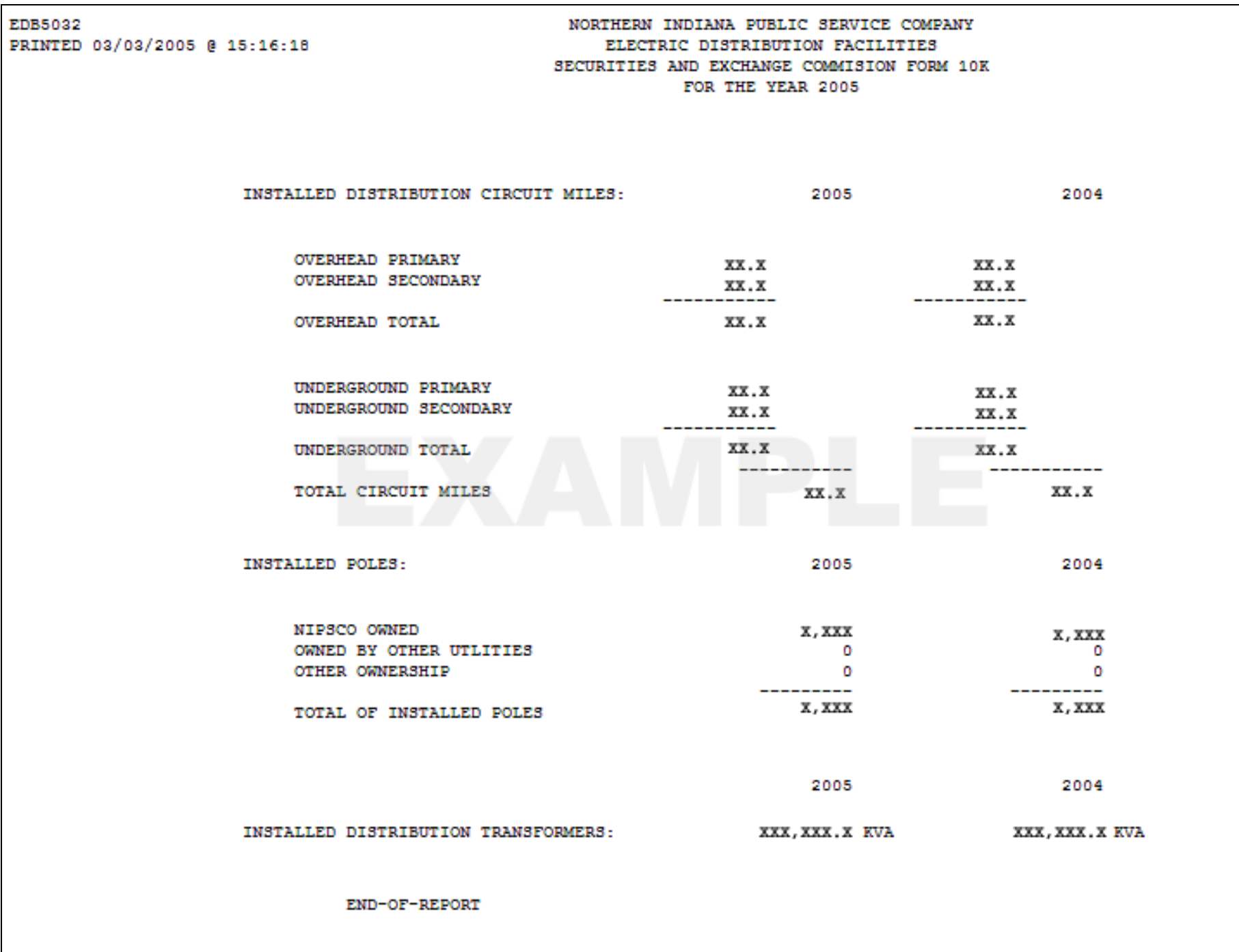

Figure 4.7-44. The Securities and Exchange Commission Form 10k captures high level statistics on the quantity of installed conductor, poles, and transformers over the previous year 


\subsubsection{SAGE Screen Design}

The SAGE screen design is organized into functional areas and is driven by the drop down menus at the top of the screen.

\subsection{Transformer/Regulator Screens}

Most screens for Transformers and Regulators have the exact same design and layout. Therefore, only the transformer screens are shown here. Any notable differences are explained in their respective sections.

\subsection{Add New Transformer / Regulator}

Figure 4.7-45 allows the central storerooms to add newly purchased assets into the system including manufacturer and warranty data.

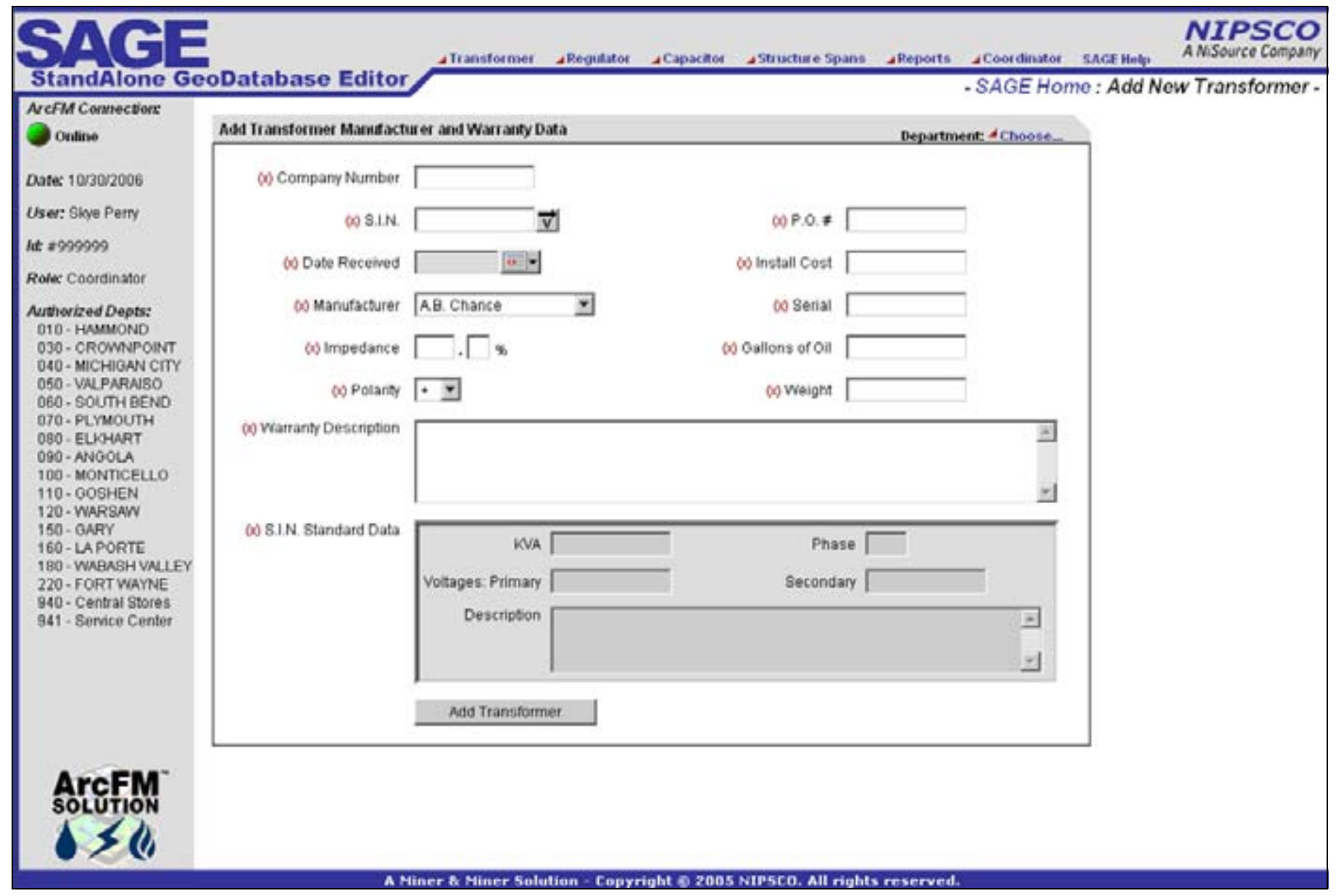

Figure 4.7-45. Add Transformer Manufacturer and Warranty Data Screen

\subsection{Query by company Number / Serial Number}

Figure 4.7-46 allows users to query for assets based on the company number and/or serial number. Depending on the user's permissions they can then perform various operations 
on the asset including edit, install, removal, delete, property transfer, status, or replace. These operations are described in more detail in the following sections.

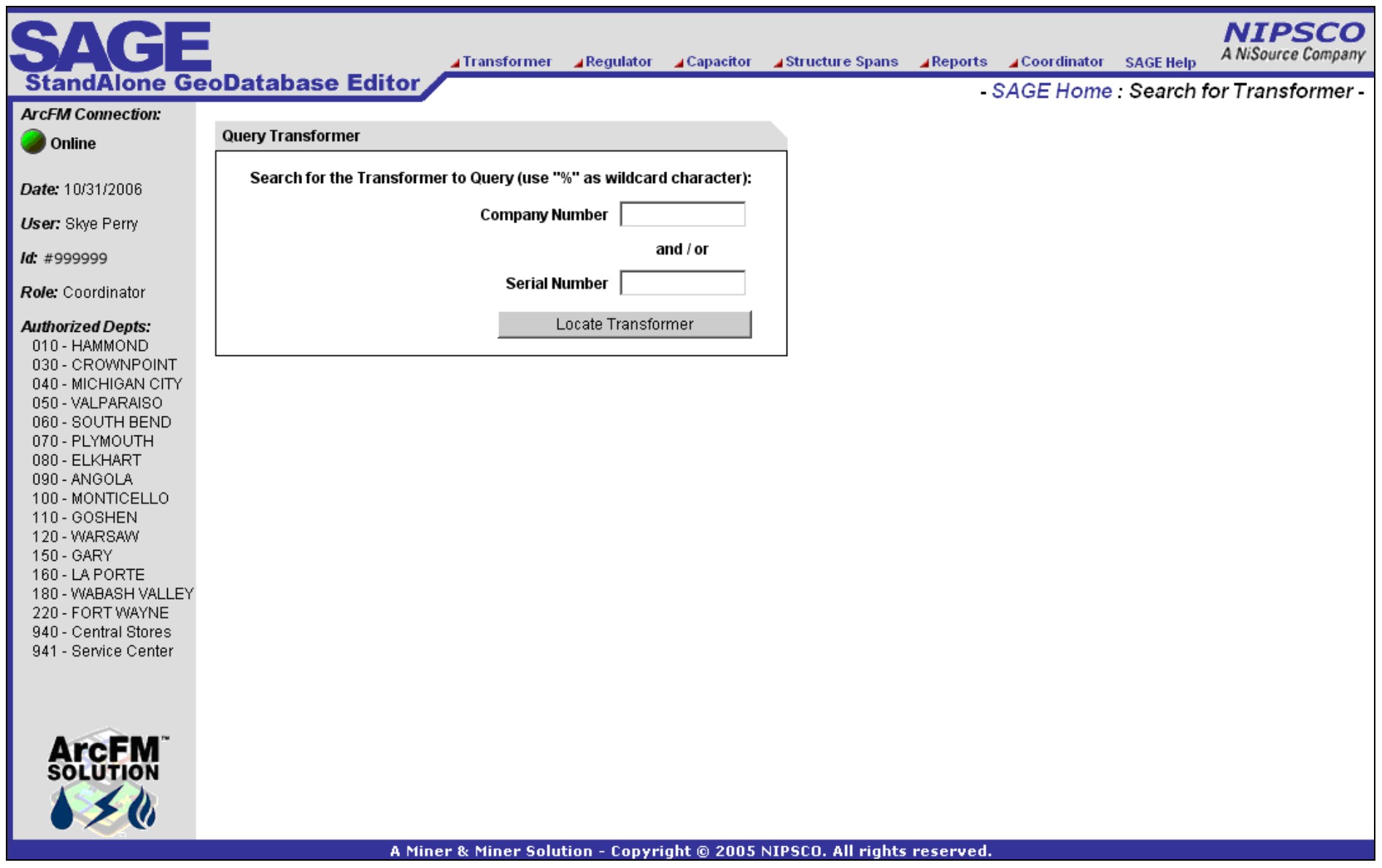

Figure 4.7-46. Query Transformer by company number / serial number screen 


\subsection{Query by Location}

Figure 4.7-47 allows users to query for assets based on the pole/pad number or company use location id. Depending on the user's permissions they can then perform various operations on the asset including edit, install, removal, delete, property transfer, status, or replace. These operations are described in more detail in the following sections.

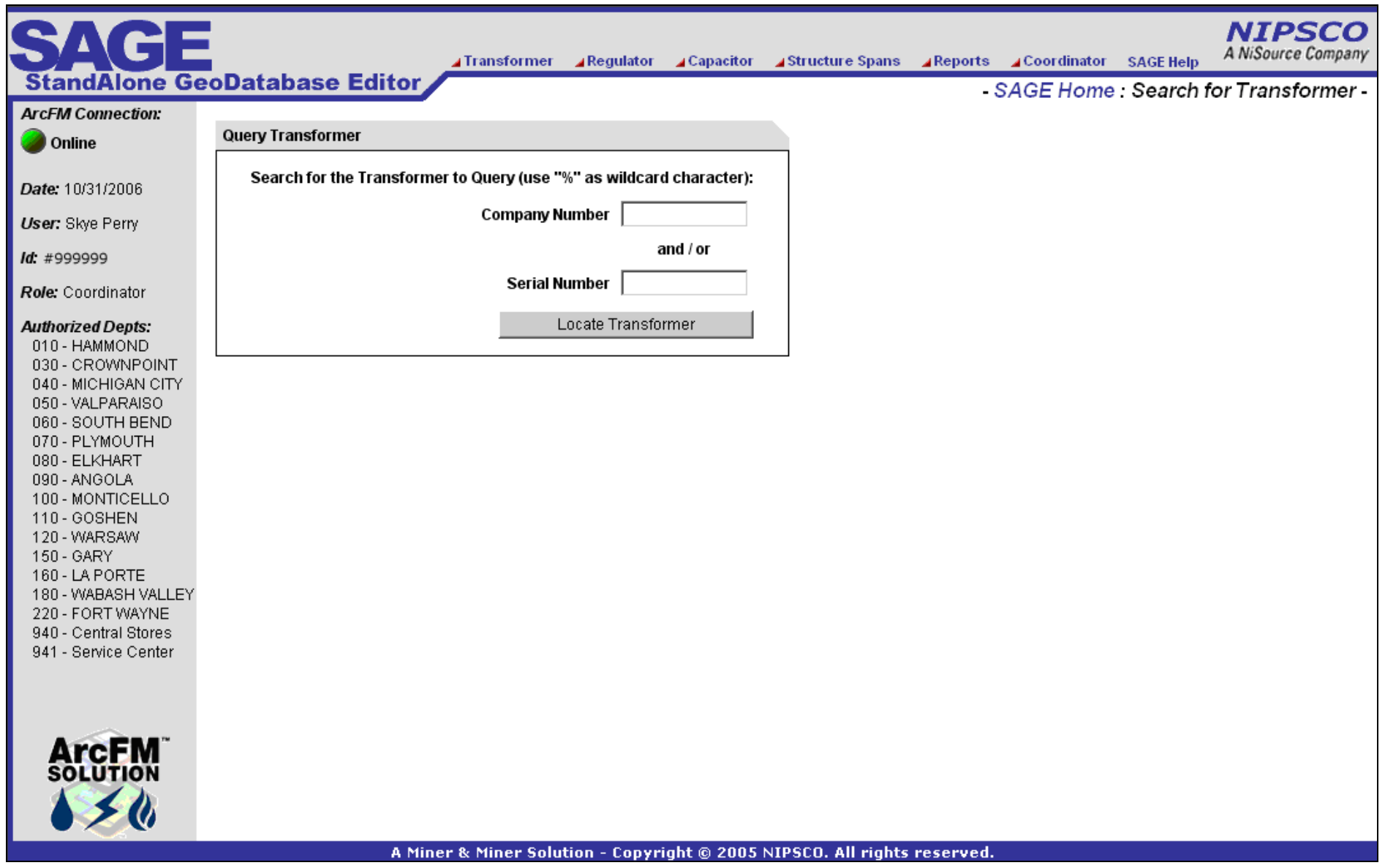

Figure 4.7-47. Query for a transformer by location 


\subsection{Edit Existing Transformer Manufacturer and Warranty Data}

Figure 4.7-48 allows the central storeroom clerks to update core manufacturer and warranty data as well as to enter function and oil test data.

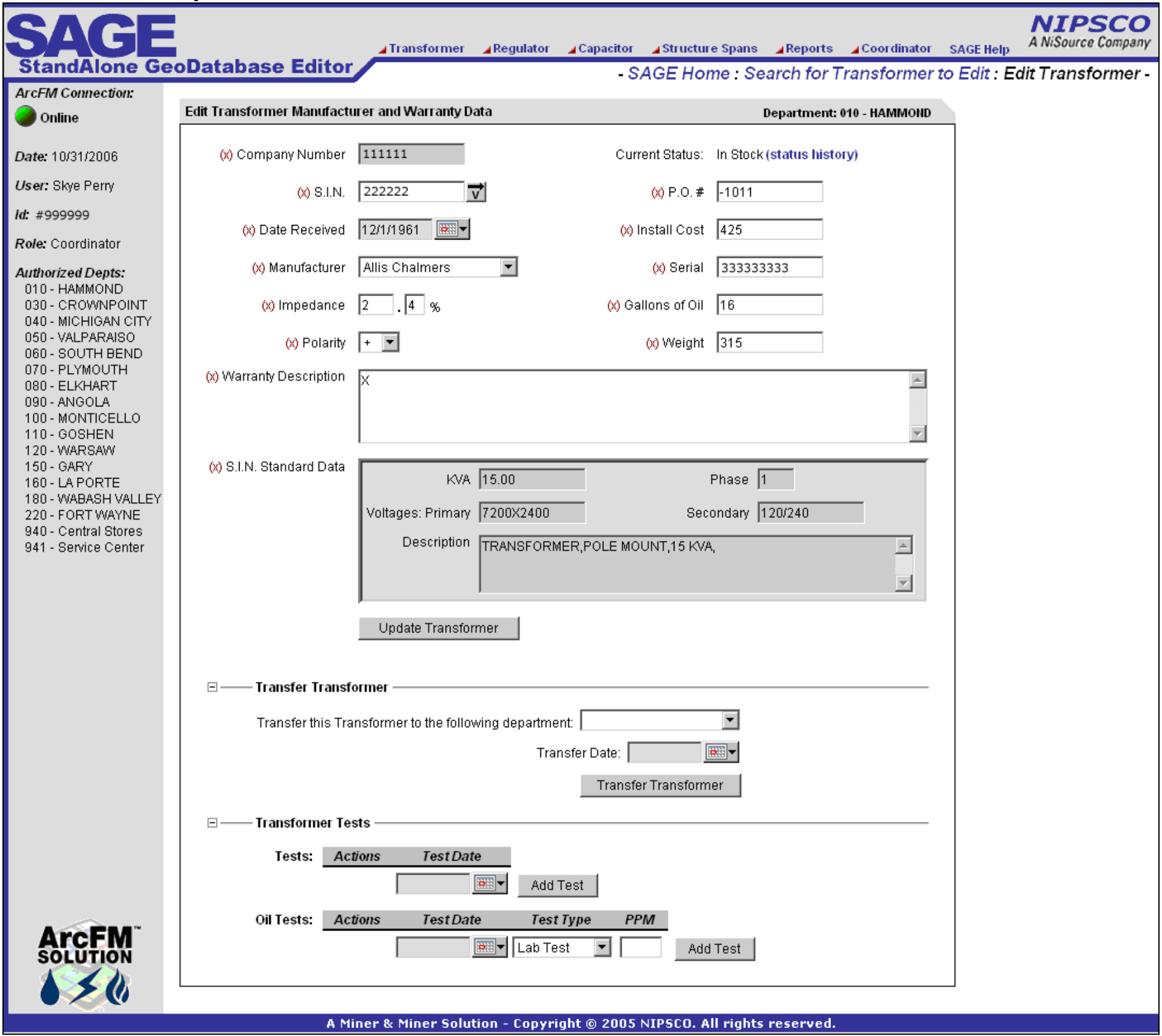

Figure 4.7-48. The edit existing transformer manufacturer and warranty data also allows users with the administrator role to transfer assets 


\subsection{Transformer Installation}

Figure 4.7-49 allows a record clerk to enter the installation information from a ticket. This is performed before work prints are returned from the field and captures all key tabular data about the installation.

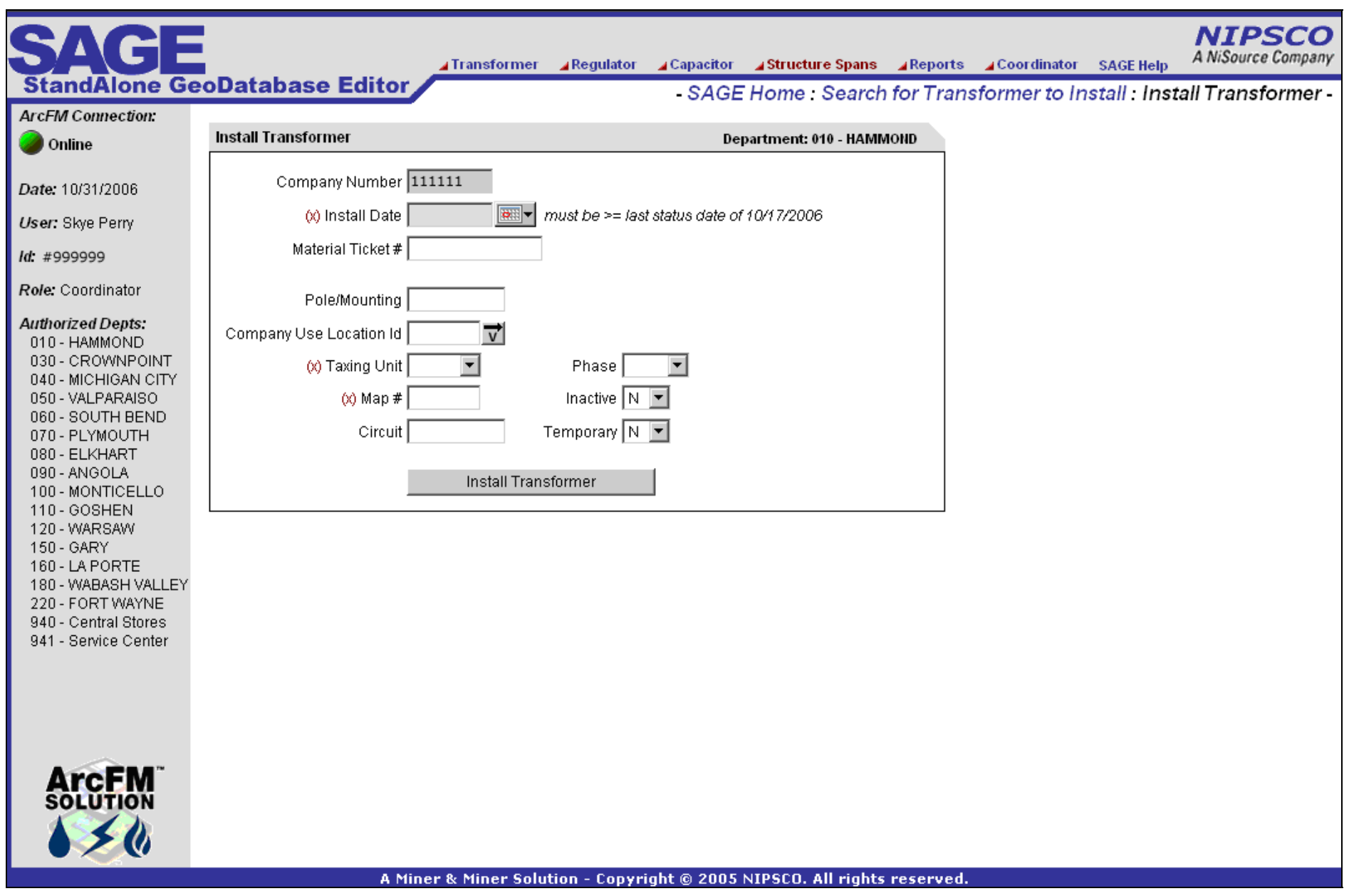

Figure 4.7-49. Install transformer screen 


\subsection{Transformer Removal}

Figure 4.7-50 allows a record clerk to remove assets that are currently installed but are not related to a GIS feature. This occurs regularly in company use / substation installations.

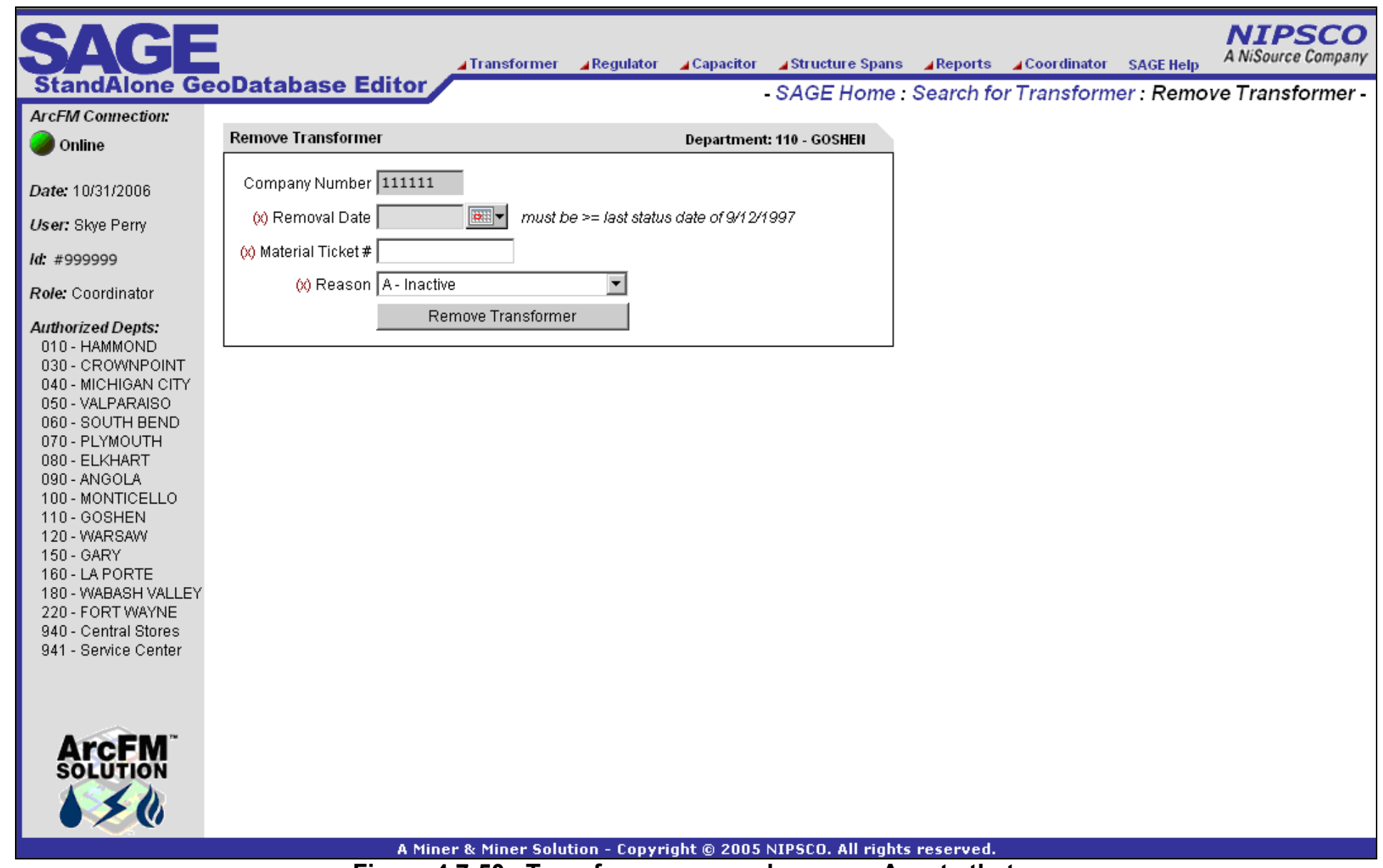




\subsection{Transformer Deletion}

Figure 4.7-51 allows the central storeroom clerks to remove all asset data from AEDR if an asset was entered in error.

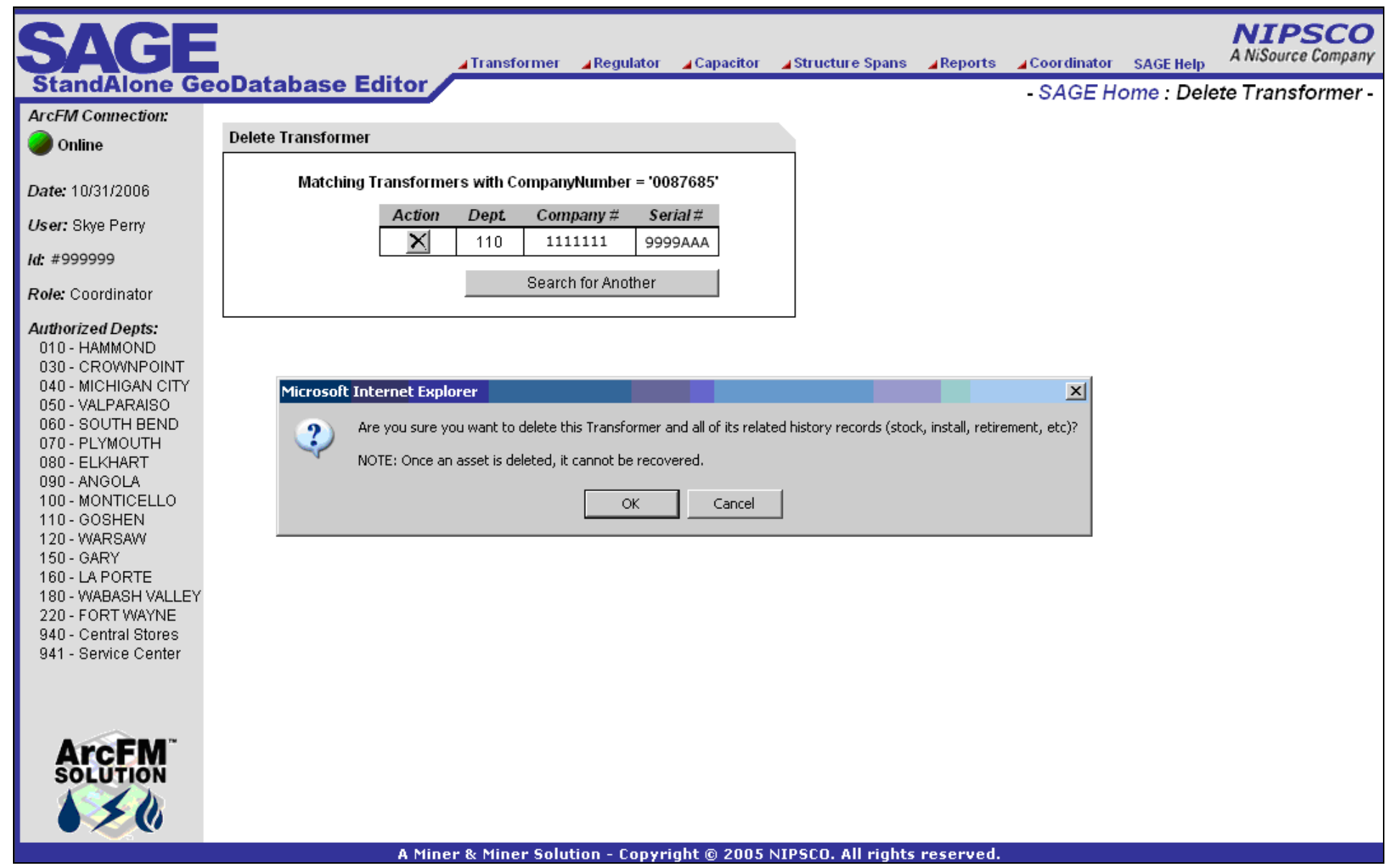

Figure 4.7-51. Delete transformer screen 


\subsection{Property Transfer}

Figure 4.7-52 allows an admin clerk to transfer installed assets from one department (LOA), company use location, tax district, and/or map number to another.

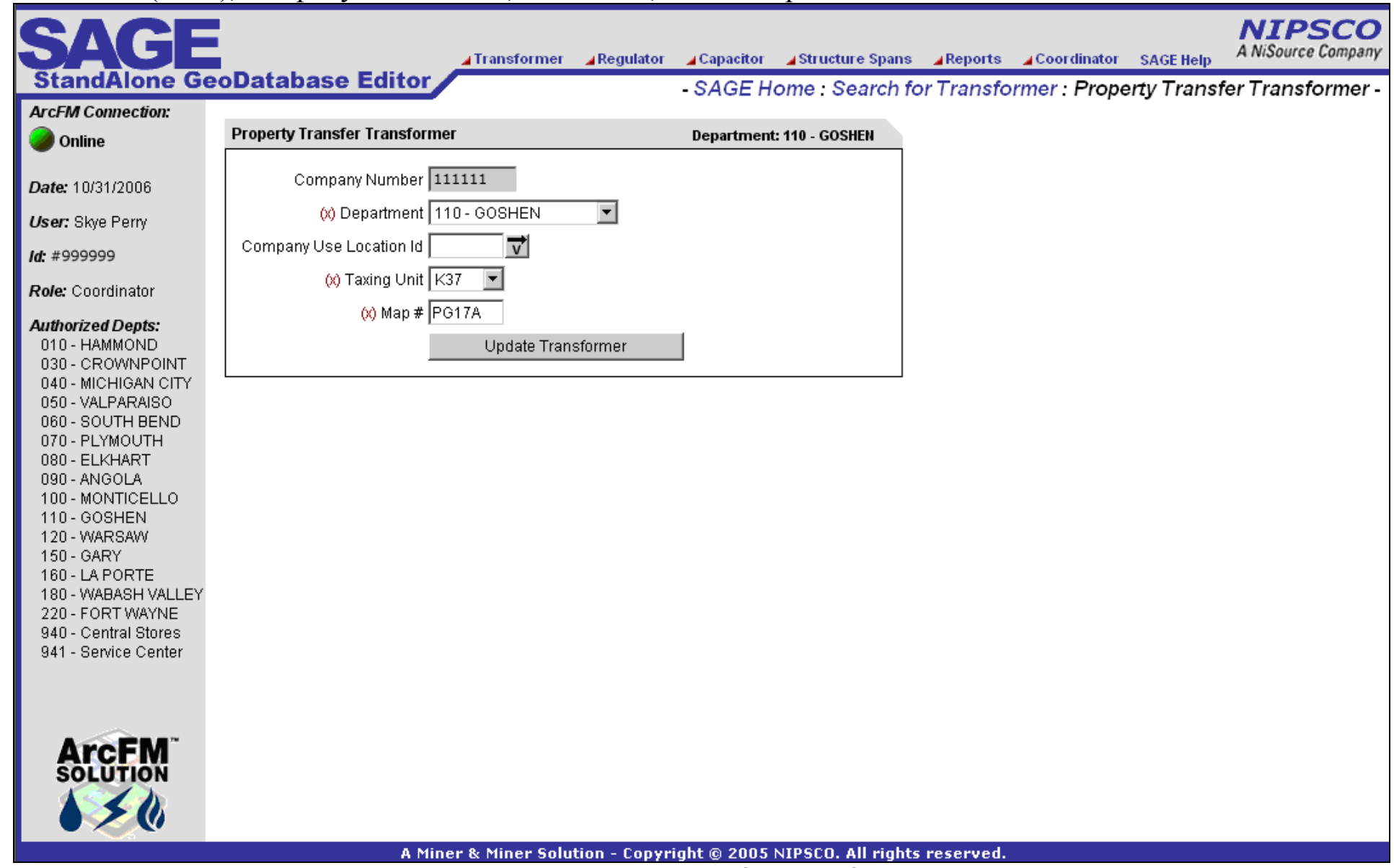

Figure 4.7-52. The property transfer (transformer) is used

when district lines are updated, re-organized, etc. 


\subsection{Transformer Status}

Figure 4.7-53 allows a record clerk to view the entire life cycle of an asset.

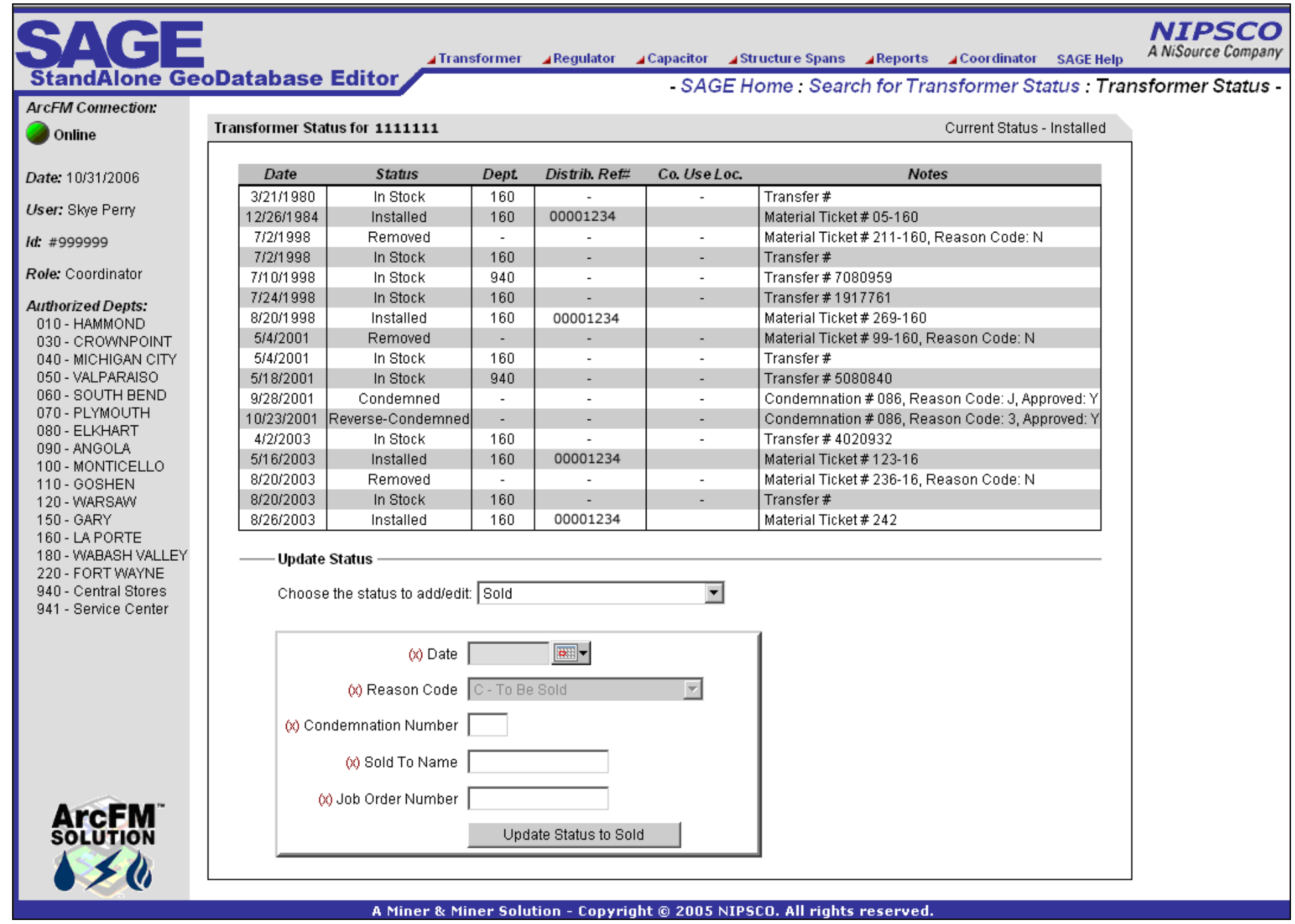

Figure 4.7-53. The transformer status screen also enables processing of condemnations, reverse-condemnations, sales, and leases 


\subsection{Replace a Transformer}

Figure 4.7-54 allows a record clerk to replace a transformer install with a new transformer. The first transformer is removed back into stock and the new transformer is installed and updated with all properties and relationships of the original transformer.

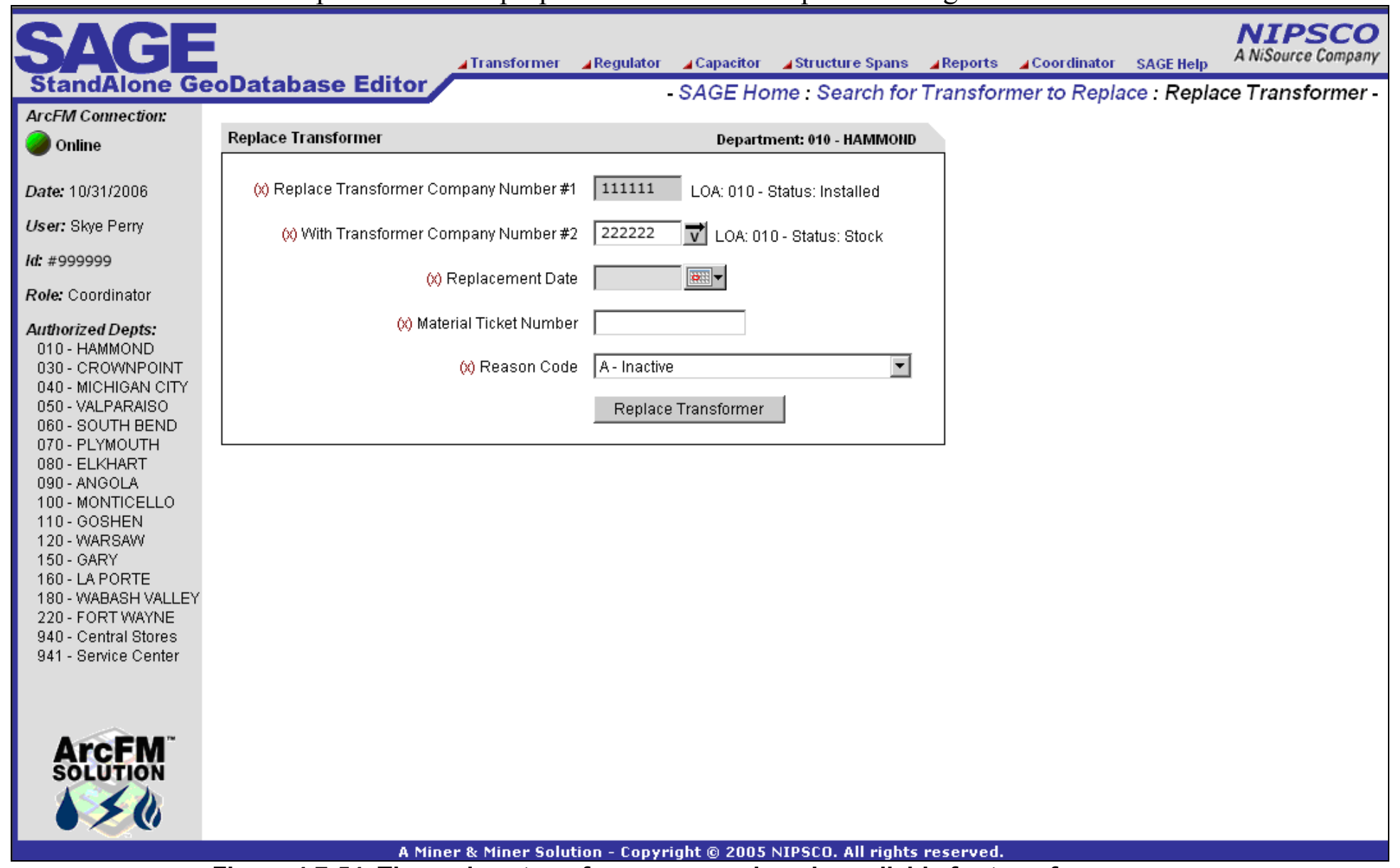

Figure 4.7-54. The replace transformer screen is only available for transformers 


\subsection{Capacitor Screens}

Capacitors are treated somewhat differently than transformers and regulators because they can only be installed once whereas transformers and regulators can be installed and removed many times throughout their life cycle. Therefore, the capacitor functionality is more limited than the transformer and regulators.

\subsection{Add New Capacitor}

Figure 4.7-55 allows the central storerooms to add newly purchased assets into the system including manufacturer and warranty data.

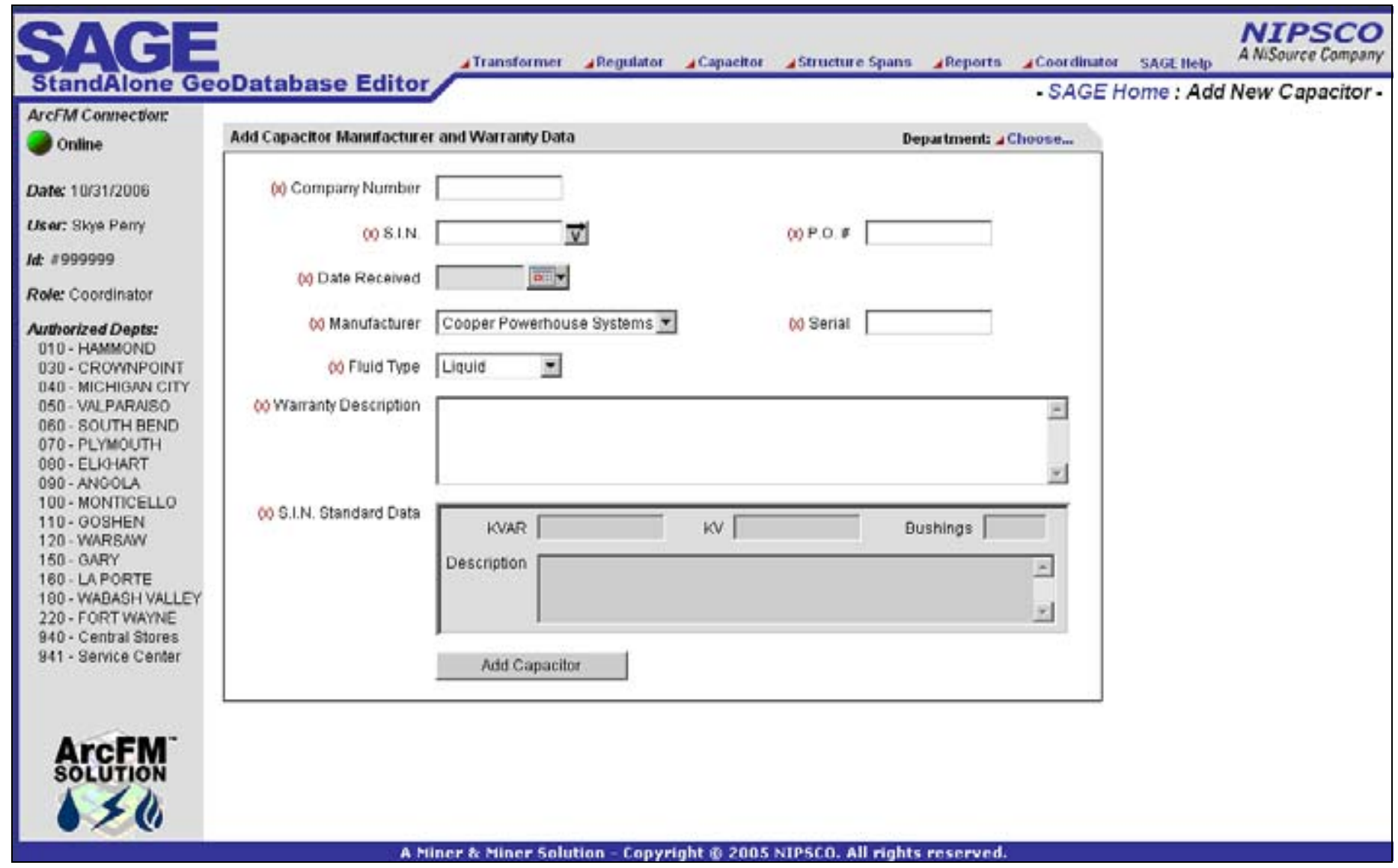

Figure 4.7-55. Add new capacitor screen 


\subsection{Query By Company Number / Serial Number}

Figure 4.7-56 allows users to query for assets based on the company number and/or serial number. Depending on the user's permissions, they can perform various operations on the asset including edit, install, removal, delete, or property transfer. These operations are described in more detail in the following sections.

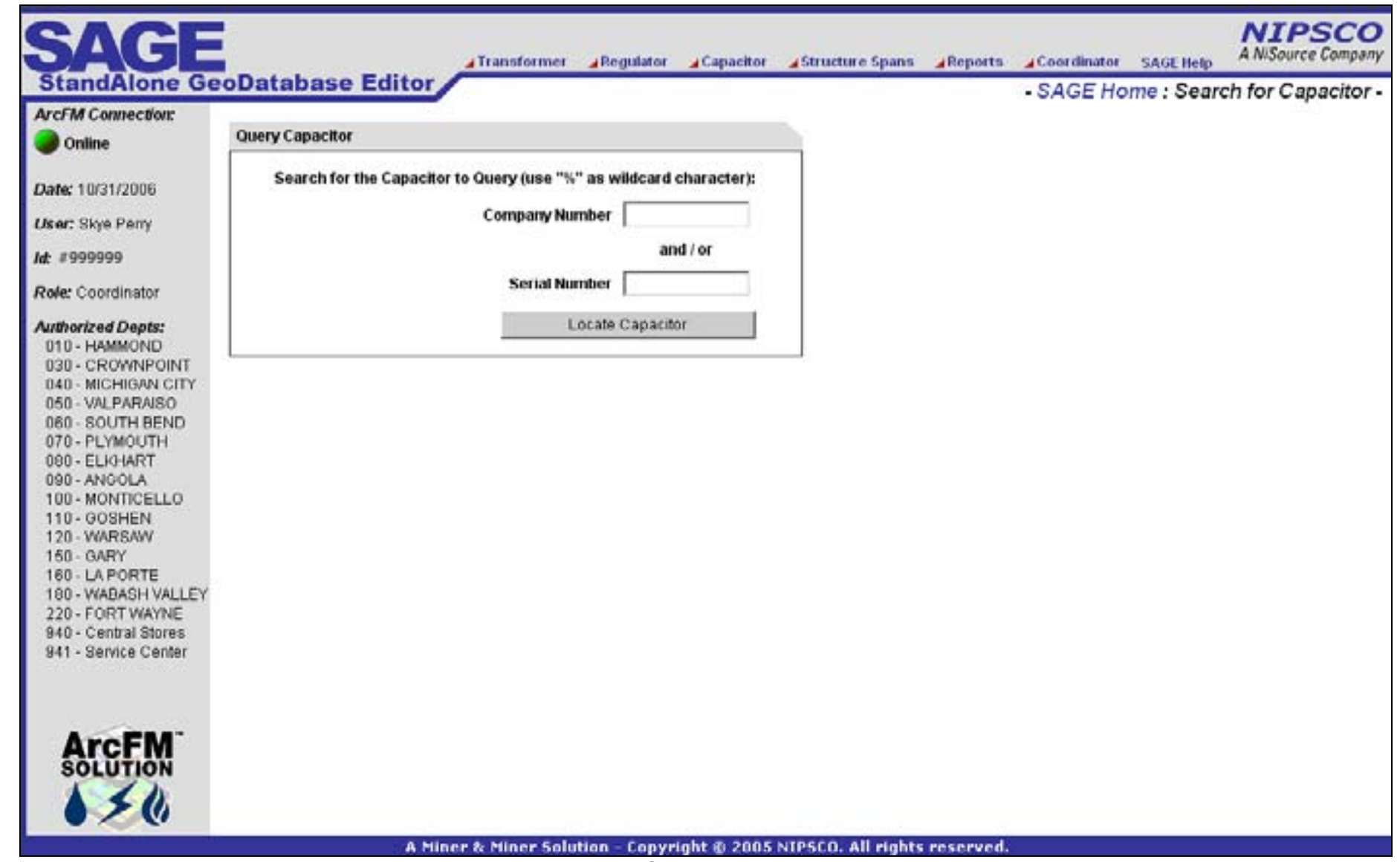

Figure 4.7-56. Query the capacitor screen 


\subsection{Query by Location}

Figure 4.7-57 allows users to query for assets based on the pole/pad number or company use location id. Depending on the user's permissions, they can perform various operations on the asset including edit, install, removal, delete, or property transfer. These operations are described in more detail in the following sections.

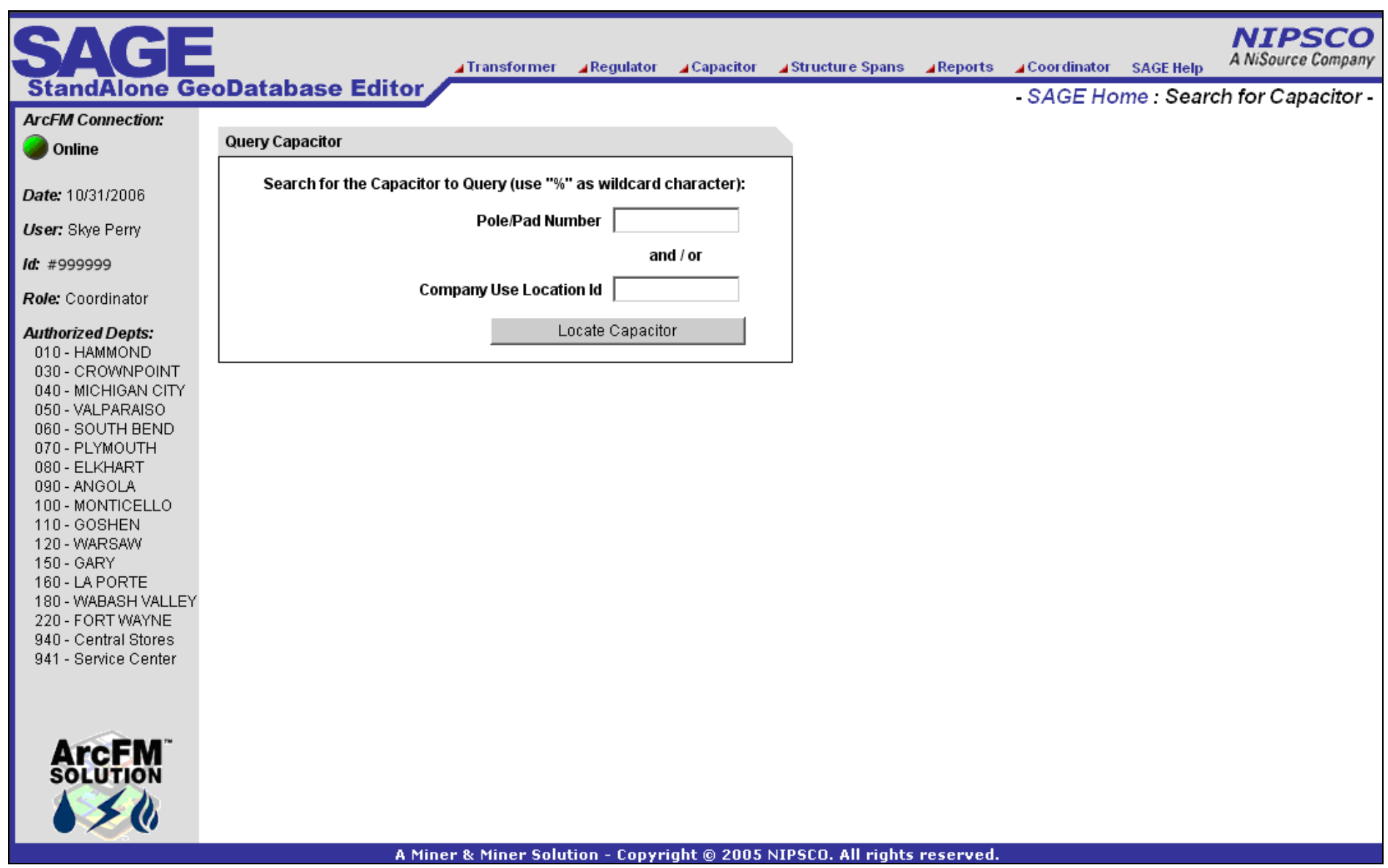

Figure 4.7-57. Query the capacitor by location screen 


\subsection{Edit Existing Capacitor Manufacturer and Warranty Data}

Figure 4.7-58 allows the central storeroom clerks to update core manufacturer and warranty data.

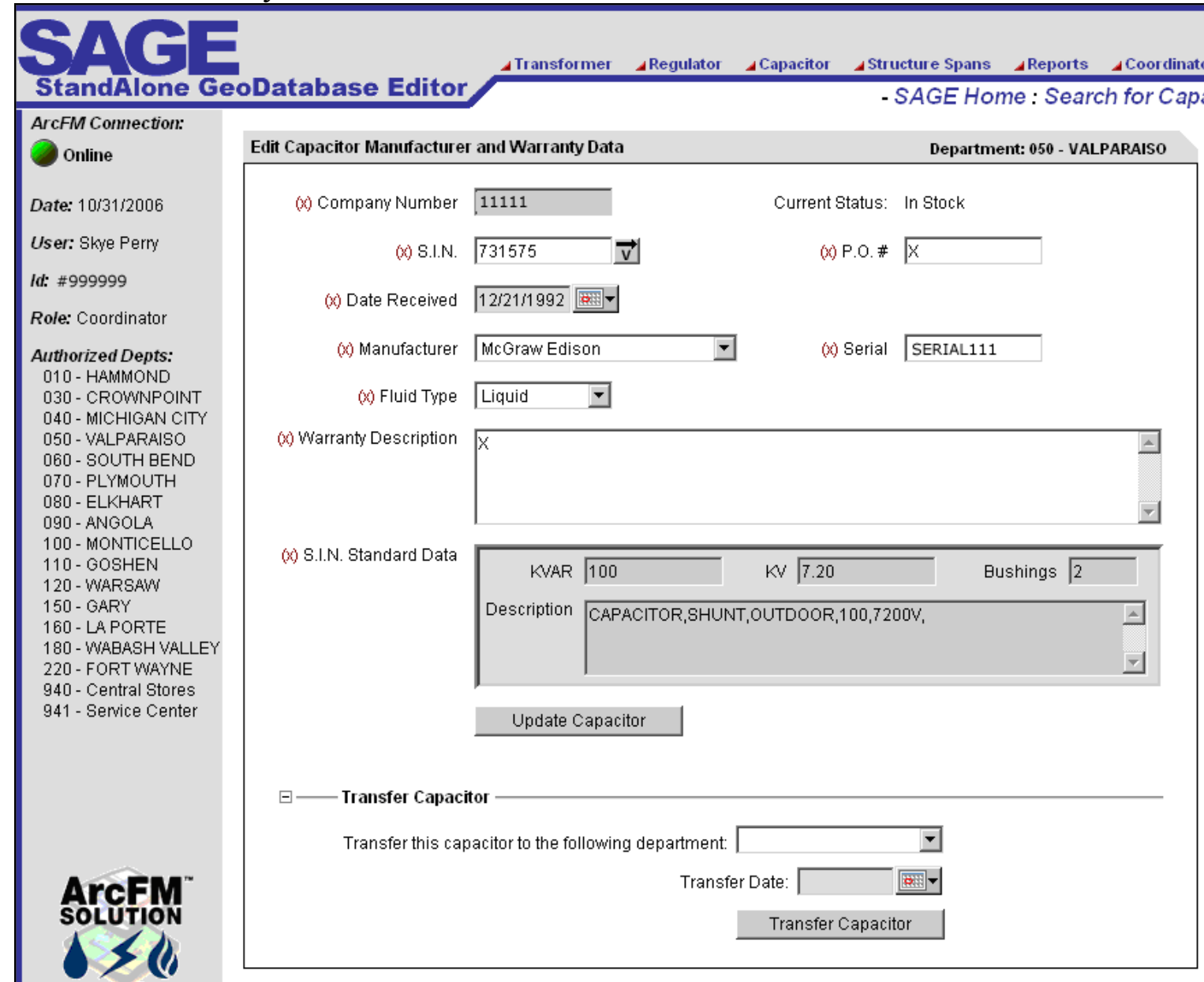

NIPSCO

A NiSource Company

StandAlone GeoDatabase Edito

ArcFM Connection:

A Miner \& Miner Solution - Copyright (c) 2005 NIPSC0. All rights reserved.

Figure 4.7-58. The edit capacitor manufacturer and warranty screen also allows users with the administrator role to transfer assets 


\subsection{Capacitor Installation}

Figure 4.7-59 allows a record clerk to enter the installation information from a ticket. This is performed before work prints are returned from the field and captures all key tabular data about the installation.

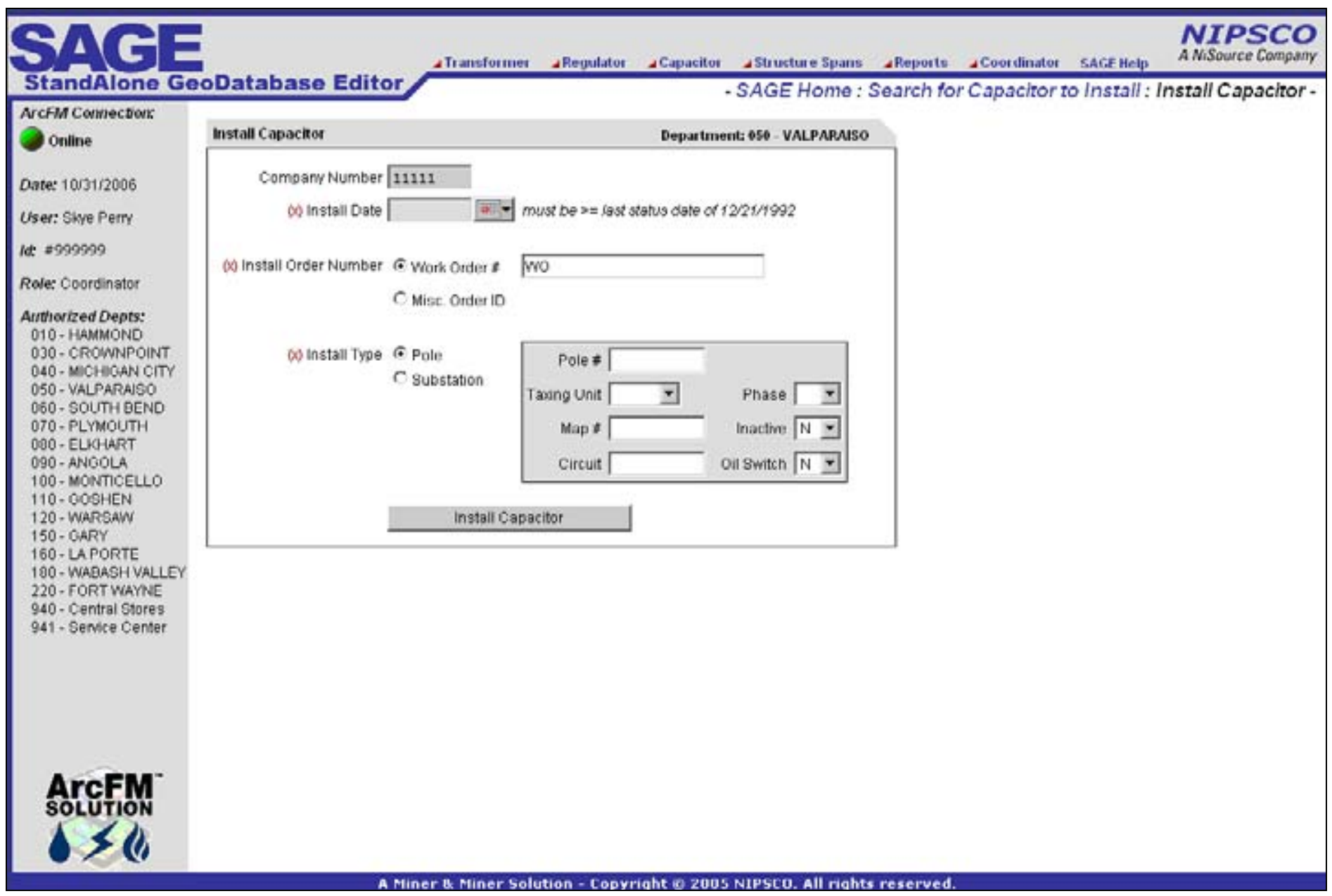

Figure 4.7-59. Install capacitor screen 


\subsection{Capacitor Removal}

Figure 4.7-60 allows a record clerk to remove assets that are currently installed but are not related to a GIS feature. This occurs regularly in company use / substation installations.

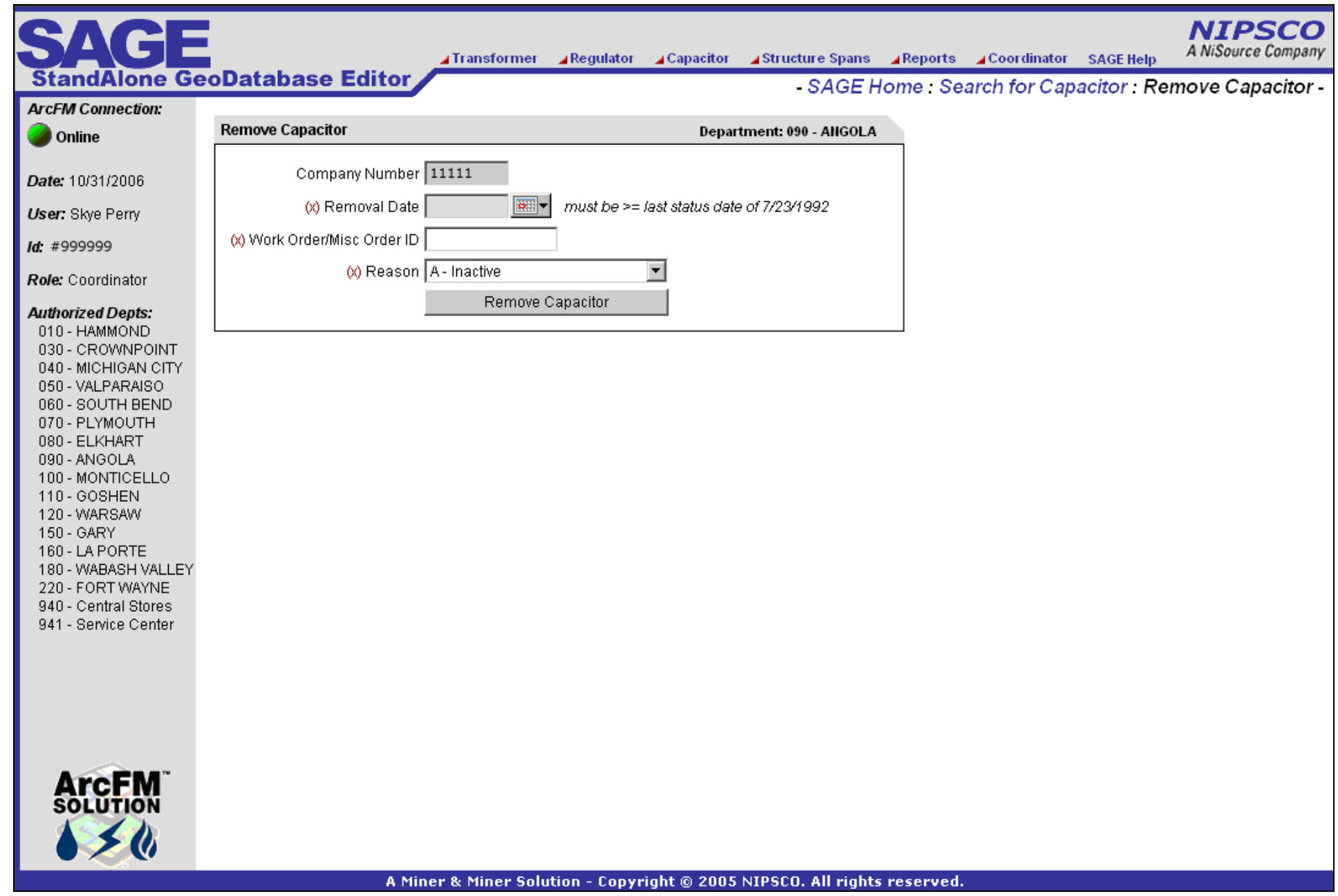

Figure 4.7-60. Remove capacitor screen. Assets that ARE related to a GIS feature are removed within the GIS 


\subsection{Capacitor Deletion}

Figure 4.7-61 allows the central storeroom clerks to remove all asset data from AEDR if an asset was entered in error.

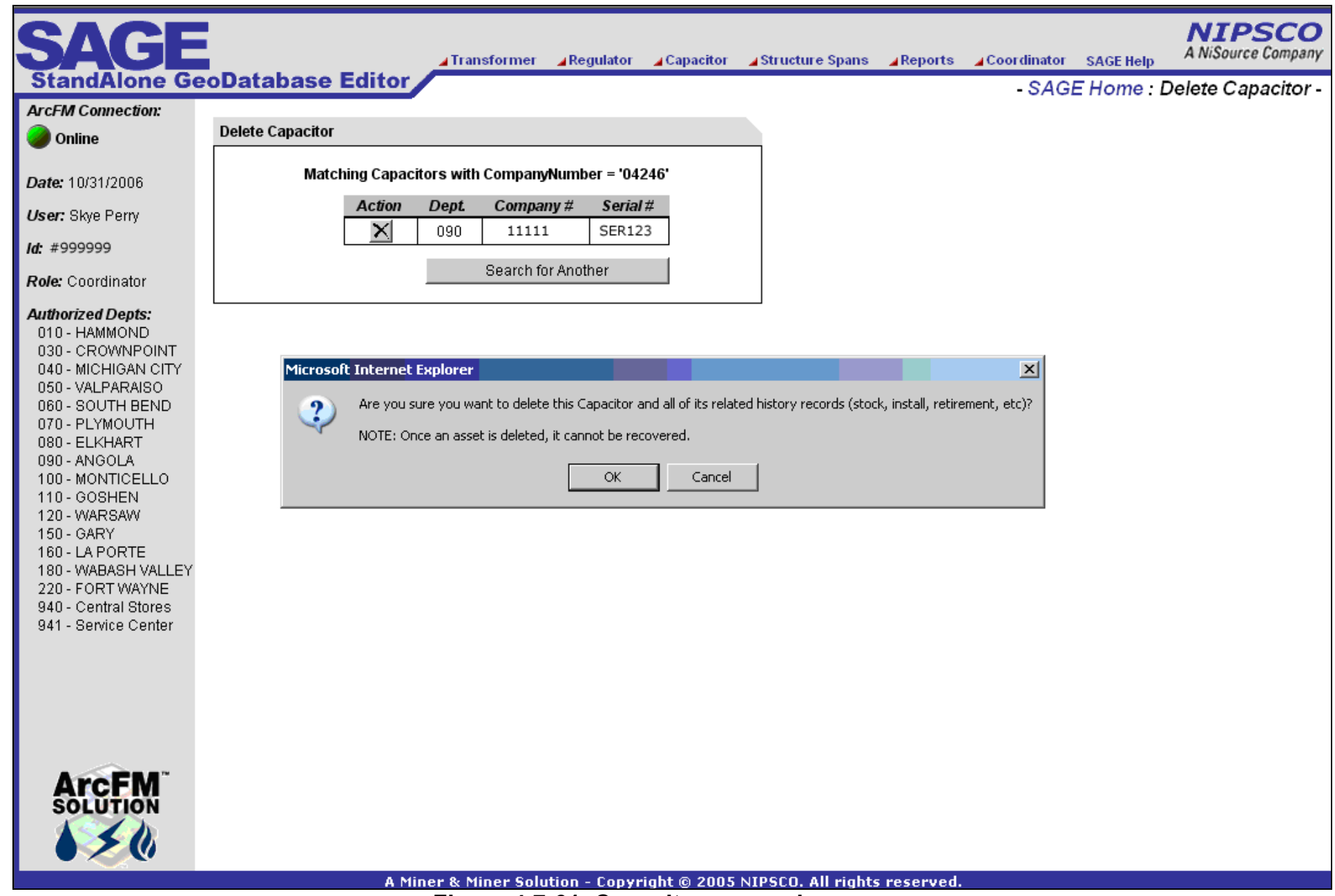

Figure 4.7-61. Capacitor removal screen 


\subsection{Capacitor Property Transfer}

Figure 4.7-62 allows an admin clerk to transfer installed assets from one department (LOA), company use location, tax district, and/or map number to another.

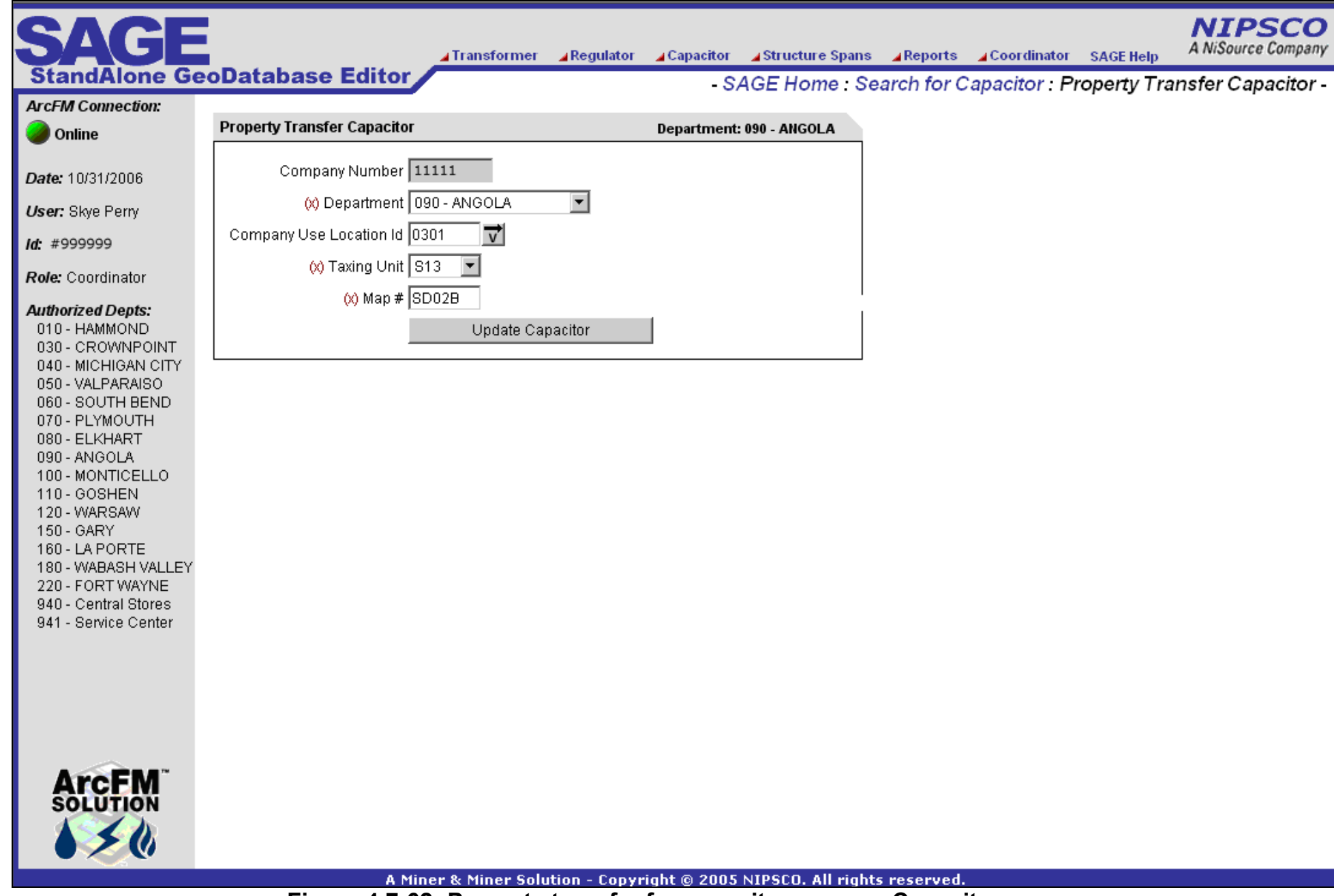

Figure 4.7-62. Property transfer for capacitor screen. Capacitors are transferred when district lines are updated, re-organized, etc.

\subsection{Structure Span Screens}

The structure span screens manage the field distances between various structures. The distances can then be used to determine how much conductor is in the field in a particular location. This data is tracked in a flat tabular manner as opposed to the GIS for two reasons. First, most conductor in the GIS is drawn as super spans (spans multiple poles) and does not track the length of conductor between the interior poles. And second, the GIS map length is not accurate enough for the analysis that this data is used for. Actual field lengths based on returned work orders is required. 


\subsection{Structure Span Lengths Add / Edit / Delete}

Figure 4.7-63 allows a record clerk to manage the captured WO lengths between any two structures (poles/pads/pedestals/terminals) in the field.

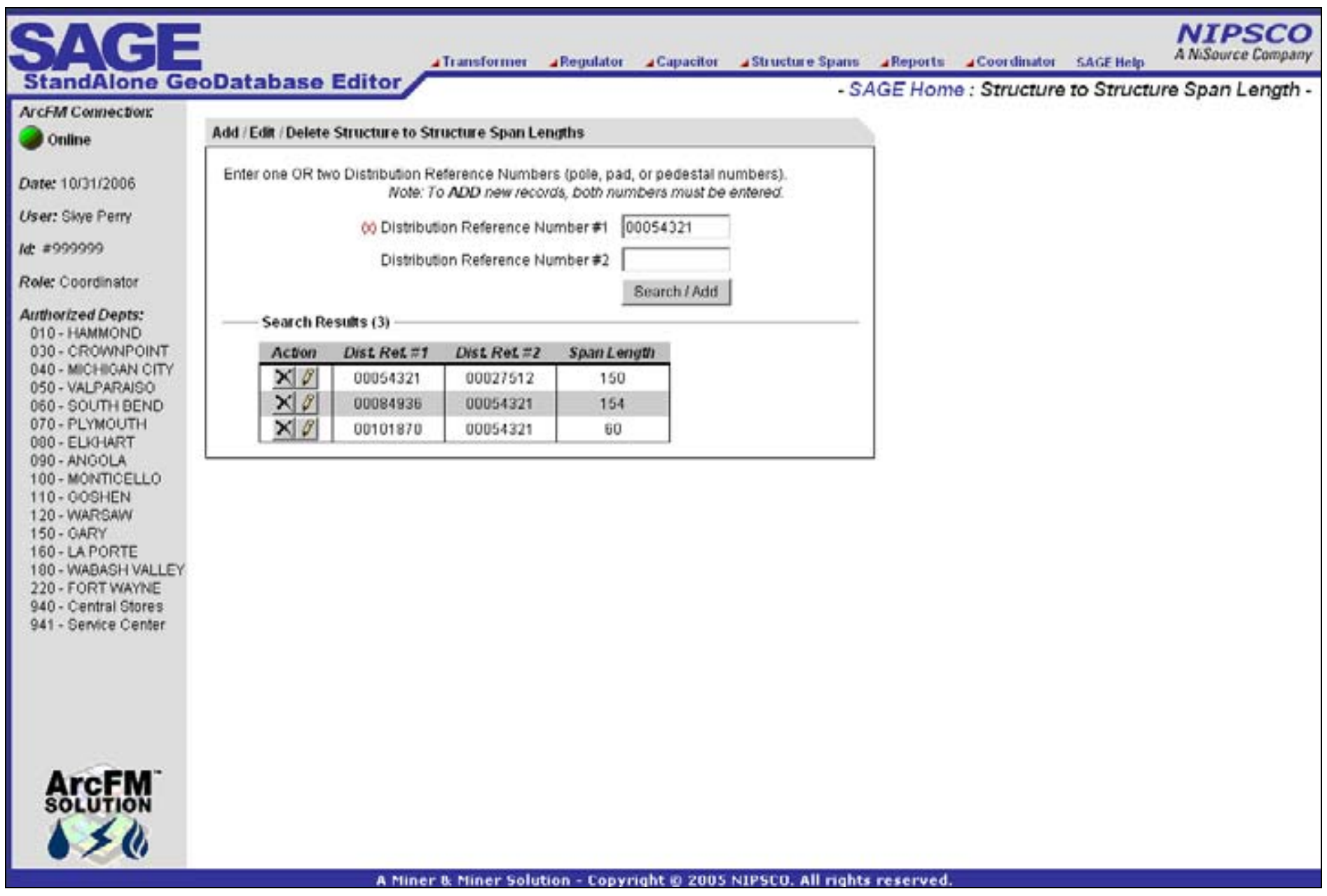

Figure 4.7-63. The add / edit / delete structure to structure span lengths screen allows AEDR to track actual distances between structures outside of the conductor features which are often drawn as super spans 


\subsection{Structure Span Lengths Query}

Figure 4.7-64 allows all users to query the structure span lengths by querying on any structure ID.

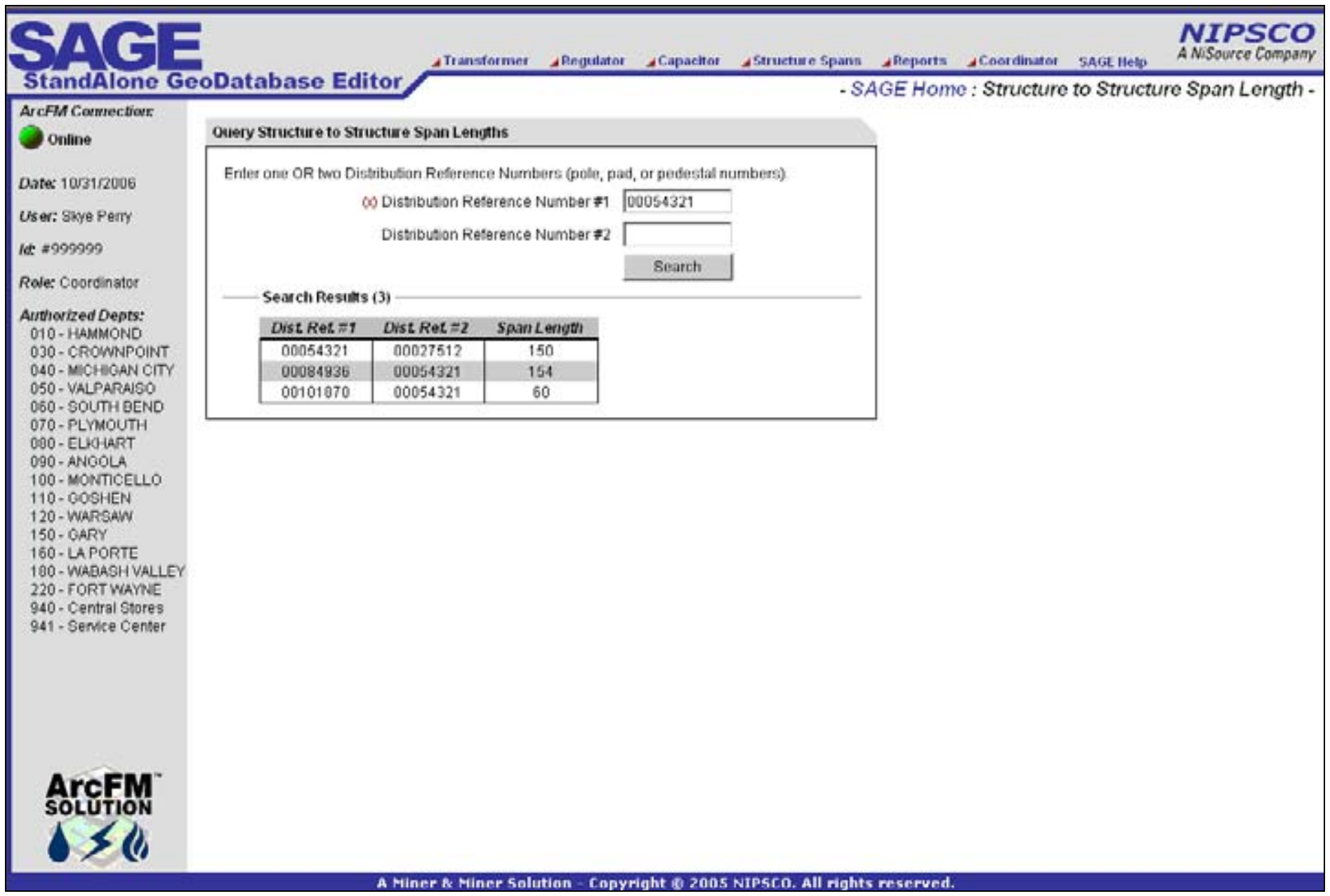

Figure 4.7-64. Structure to structure span length query screen

\subsection{Reports Screen}

The SAGE reports section is the main portal for all of NIPSCO to access asset management reports. It consists of both on demand reports that are generated based on user input as well as links to the batch reports that are generated by the EDFS batch suite on a nightly, monthly, or annual basis. All of the batch reports have been detailed in the batch suite section of this document. This section details the SAGE on demand reports as well as the access to the batch reports. 


\subsection{Sessions Inquiry Report}

Figure 4.7-65 allows an admin user to dynamically create a session manager query to display a report on a group of sessions by user/dates, status, etc.

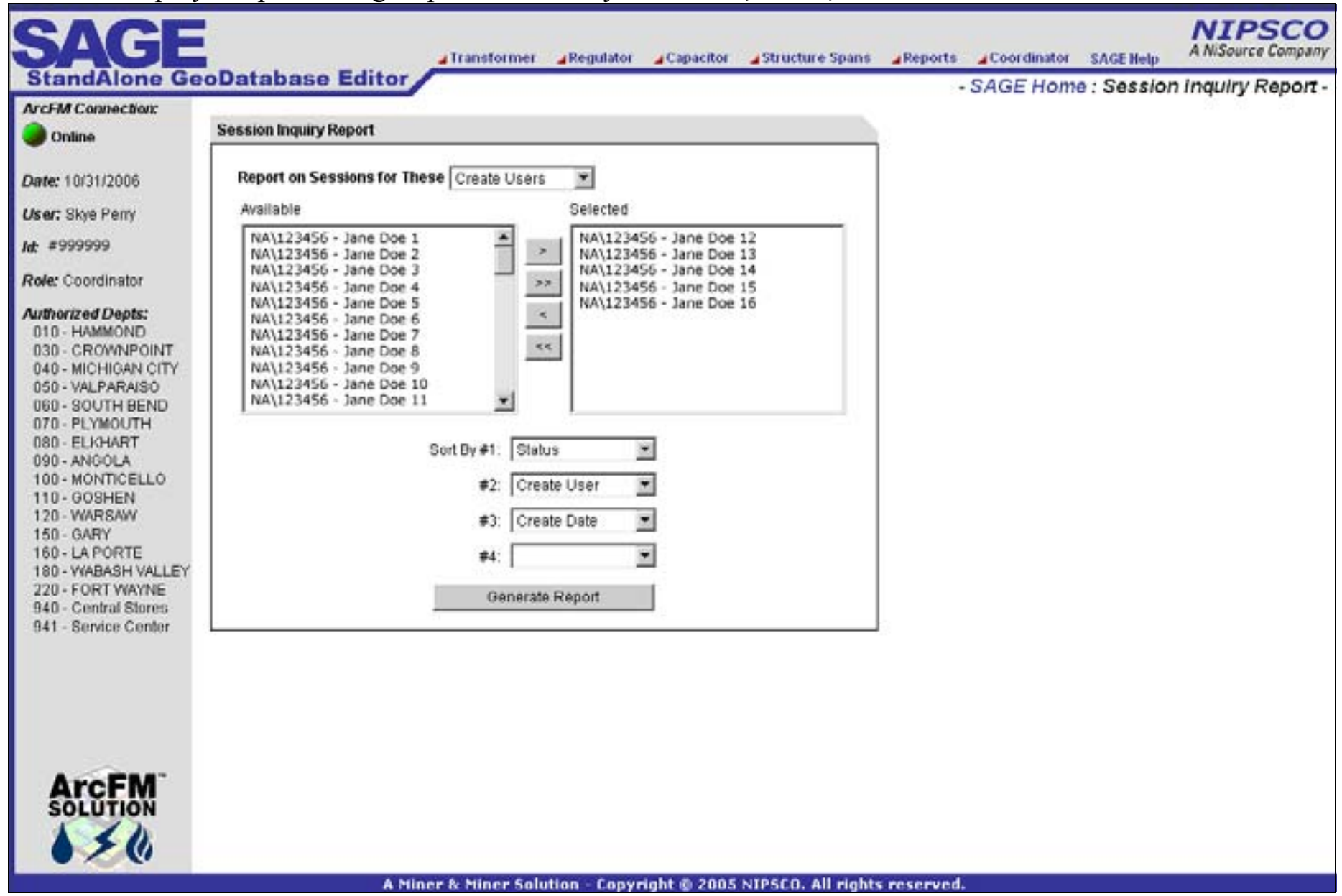

Figure 4.7-65. The session inquiry report is considered an on demand report because it is generated based on user input values 
Clicking the Generate Report button dynamically queries the session manager database and creates a report similar to Figure 4.7-66.

\begin{tabular}{|c|c|c|c|c|c|}
\hline \multicolumn{4}{|c|}{ Session Inquiry Report - Count [14] } & \multicolumn{2}{|c|}{ Generated 10/31/2006 10:11:37 $\mathrm{AM}$} \\
\hline Create Date & ID & Name & Created By & Current 0wner & Status \\
\hline $10 / 30 / 2006$ & 4150 & WOG 3382-050 Shenandoah springs & Jane Doe 1 & Supervisor 1 & Pending Approval \\
\hline $10 / 30 / 2006$ & 4162 & Wo 43477-5 Center Minor Sub & Jane Doe 2 & Supervisor 1 & Pending Approval \\
\hline $10 / 30 / 2006$ & 4167 & ธ043490-5 288 S CR $368 \mathrm{E}$ & Jane Doe 3 & Supervisor 1 & Pending Approval \\
\hline $10 / 31 / 2006$ & 4177 & to 43583-5 01de Totme square & Jane Doe 4 & Supervisor 1 & Pending Approval \\
\hline $10 / 31 / 2006$ & 4183 & to 53499-5 NICTD - East Tamarack & Jane Doe 5 & Supervisor 1 & Pending Approval \\
\hline $10 / 19 / 2006$ & 3924 & W049037-59\& \$043507-16 & Jane Doe 6 & Supervisor 1 & Pending Approval \\
\hline $10 / 30 / 2006$ & 4166 & WO\# 43428-6 ARS 10/30/06 & Jane Doe 7 & Jane Doe 7 & In Progress \\
\hline $10 / 16 / 2006$ & 3843 & to 53769-3 Summit Crossings & Jane Doe 8 & Jane Doe 8 & In Progress \\
\hline $10 / 16 / 2006$ & 3830 & พ0 53985-15 E-Law Building - HOLD 10/16/2006 & Jane Doe 9 & Jane Doe 9 & In Progress \\
\hline $10 / 31 / 2006$ & 4185 & W053558-16 Lifestyle Homes & Jane Doe 10 & Jane Doe 10 & In Progress \\
\hline $10 / 30 / 2006$ & 4176 & Carlson Estates & Jane Doe 11 & Jane Doe 11 & In Progress \\
\hline $10 / 25 / 2006$ & 4060 & w053520-11/ kp / 10-25-06 & Jane Doe 12 & Jane Doe 12 & In Progress \\
\hline $10 / 26 / 2006$ & 4104 & ธ050619-5/ kp / 10-26-06 & Jane Doe 13 & Jane Doe 13 & In Progress \\
\hline $10 / 31 / 2006$ & 4186 & W043551-5 & Jane Doe 14 & Jane Doe 14 & In Progress \\
\hline
\end{tabular}

Figure 4.7-66. Session inquiry report 


\subsection{Capital Assets for Sale Report}

Figure 4.7-67 allows a business group within NIPSCO to generate a specific formatted report on company numbers of assets that will be potentially sold to other utilities/organizations. The report accepts up to 500 company numbers at once and the resulting report can be dumped into Excel very easily.

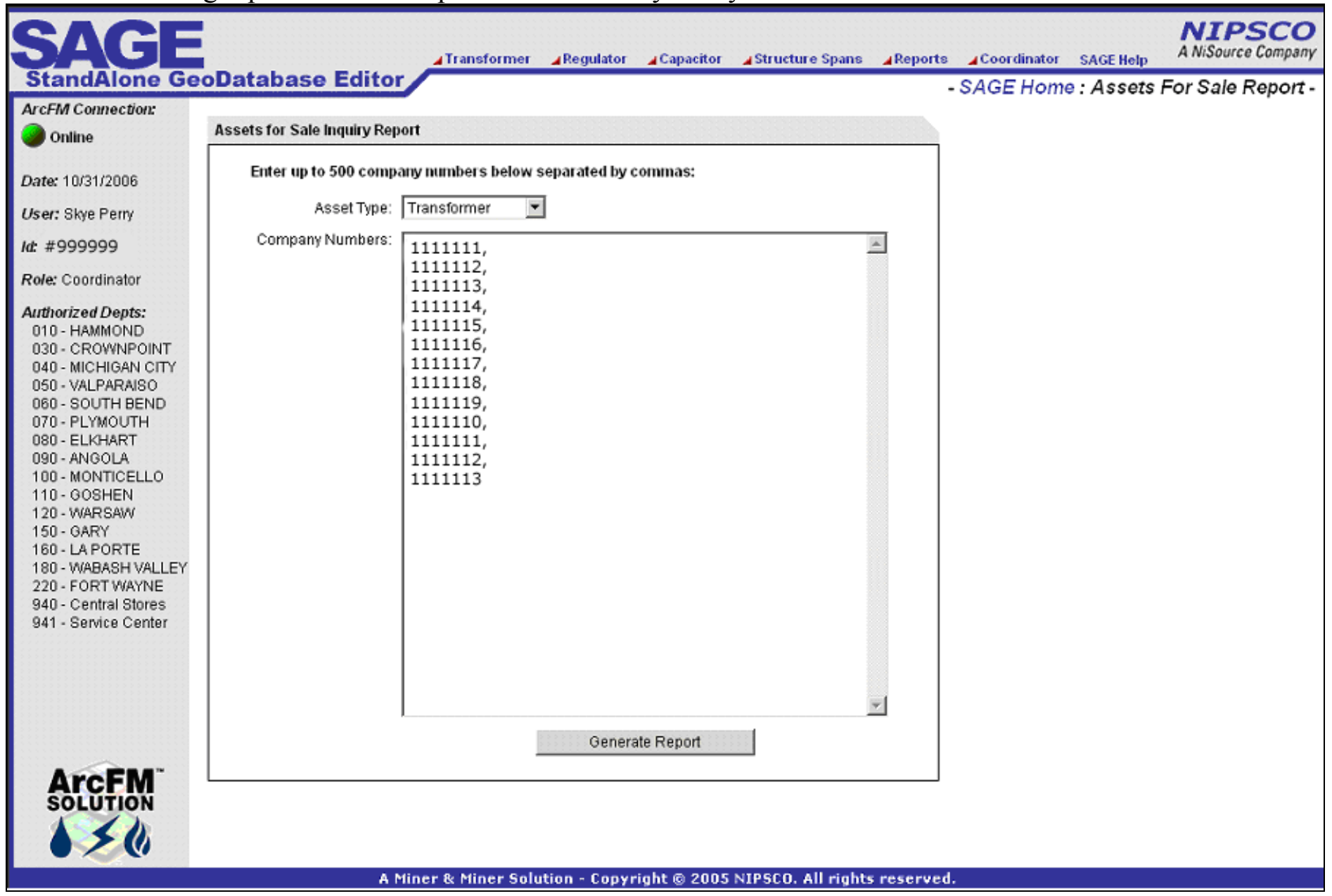

Figure 4.7-67. The assets for sale inquiry report is considered an on demand report because it is generated based on user input values 
Clicking the Generate Report button will dynamically query the asset tables and create a report similar to Figure 4.7-68.

\begin{tabular}{|c|c|c|c|c|c|c|c|c|c|c|c|c|}
\hline \multicolumn{5}{|c|}{$\begin{array}{l}\text { PRNTED } 10 / 31 / 2006 \\
010: 17: 21\end{array}$} & \multicolumn{4}{|c|}{ 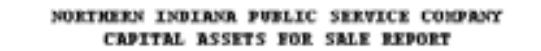 } & \multirow[b]{3}{*}{ SERTAL. } & \multirow[b]{3}{*}{ BCVD DATE } & \multirow[b]{3}{*}{ WCE PPIK } & \multirow[b]{3}{*}{ PCE TEST } \\
\hline \multicolumn{9}{|c|}{ SOKTED EY COICANY wneER (eount-12) } & & & & \\
\hline соровихи & ITEM & TrPE & kVa & PRTIGarY & SECOXDANY & phase & no & gDVUractVRER & & & & \\
\hline 1111111 & 090009 & DOLE MONT & 15.06 & $3200 \times 2400$ & $120 / 240$ & 1 & 2.40 & alise Chalnexs & 999999 & $03 / 01 / 1961$ & & \\
\hline 1111111 & 999999 & poLs sowr & 15. 00 & $7200 \times 2400$ & $120 / 240$ & 1 & 2.40 & an1is Catinexs & 999999 & $12 / 01 / 1961$ & & \\
\hline 1111111 & 999999 & poLs movrt & 15. 00 & $7200 \times 2400$ & $120 / 240$ & 1 & 2.10 & RTE Corporation & 999999 & $04 / 01 / 1962$ & & \\
\hline 1111111 & 999999 & poLx sowrt & 15.00 & $7200 \times 2400$ & $120 / 240$ & 1 & 1. 70 & kTE Corporation & 999999 & $09 / 12 / 1962$ & & \\
\hline 1111111 & 999999 & DOLF MONTT & 15.00 & $7200 \times 2400$ & $120 / 240$ & 1 & 1.70 & ETE Corporation & $\$ 00000$ & $09 / 01 / 1963$ & & \\
\hline 1111112 & 999999 & POLE MอทัT & 15.00 & $7200 \times 2400$ & $120 / 240$ & 1 & 2.70 & Al1is Chalnexs & 9989ง9 & $01 / 01 / 1961$ & 49 & $02 / 03 / 1992$ \\
\hline 11111112 & 999999 & POLE MOVT & 15.00 & $7200 \times 2400$ & $120 / 240$ & 1 & 1. 70 & RTE Corporation & ร9จ9จ9 & $01 / 01 / 1963$ & & \\
\hline 1111111 & 990909 & DOLE MONT & 15.06 & $2200 \times 2400$ & $120 / 240$ & 1 & 2.00 & kTE Corporatien & 999999 & $12 / 09 / 1959$ & & \\
\hline 1111111 & 999999 & pOLE sourt & 15. 00 & $7200 \times 2400$ & $120 / 240$ & 1 & 1.50 & Metrau ratison & 999999 & $01 / 01 / 1974$ & 49 & $01 / 23 / 1997$ \\
\hline 1111111 & 990999 & poLs sowT & 15. 00 & $7200 \times 2400$ & $120 / 240$ & 1 & 2.00 & Cenexal nectric & 999999 & $02 / 27 / 1975$ & 49 & $03 / 19 / 1997$ \\
\hline 1111111 & 999999 & POLX Mowst & 15.00 & $7200 \times 2400$ & $120 / 240$ & 1 & 1. 70 & kTX Corporation & 999999 & $11 / 09 / 1972$ & 1 & $12 / 27 / 2000$ \\
\hline 1111111 & 999999 & DOLF MONT & 15.00 & $7200 \times 2400$ & $120 / 240$ & 1 & 1.80 & ETE Corporation & $\$ 0 \$ 0000$ & 63/67/1977 & 1 & $06 / 26 / 2001$ \\
\hline \multicolumn{13}{|c|}{ SOKTED EY STORES ITEC NDare. (covent-12) } \\
\hline rrzich & cosenawn & TrmE & kVa & Prmary & SECONDANY & noasE & no & MENUYACTVRER & SERTALII & ECV DATE & NCE mere & PCE TEST \\
\hline \multicolumn{13}{|c|}{ 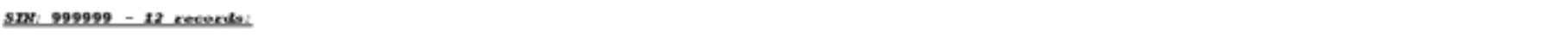 } \\
\hline 999999 & 1211112 & POLE MOVTT & 15.00 & $7200 \times 2400$ & $120 / 240$ & 1 & 2.40 & auss Chalners & 999999 & $07 / 01 / 1961$ & & \\
\hline 090909 & 1111111 & DOLE MONST & 15.06 & $2200 \times 2400$ & $120 / 240$ & 1 & 2.40 & ailise Chalnexs & 999999 & $12 / 01 / 1961$ & & \\
\hline 929993 & 1111111 & POLX monT & 15. 06 & $7200 \times 2700$ & $120 / 240$ & 1 & 2.10 & NTE Corporation & 999999 & $04 / 01 / 1962$ & & \\
\hline 999999 & 1111111 & DOLE mowr & 15. 00 & $7200 \times 2400$ & $120 / 240$ & 1 & 1. 20 & ETI Corporation & 999999 & $09 / 12 / 1962$ & & \\
\hline 999999 & 1111111 & POLX nowrt & 15.00 & $7200 \times 2400$ & $120 / 240$ & 1 & 1. 70 & kTE Corporation & 999999 & $09 / 01 / 1963$ & & \\
\hline 999999 & 1111111 & DOLE MOET & 15.00 & $7200 \times 2400$ & $120 / 240$ & 1 & 2.70 & Divis Chalmexs & 999999 & $01 / 01 / 1961$ & 49 & $02 / 03 / 1992$ \\
\hline 999999 & 1111111 & POLE ตอที & 15.00 & $7200 \times 2400$ & $120 / 240$ & 1 & 1. 70 & ETE Cerporatien & 99929s & $01 / 01 / 1963$ & & \\
\hline 999999 & 1111111 & POLE MOVKT & 15.00 & $7200 \times 2400$ & $120 / 240$ & 1 & 2.00 & ETE Corporatien & 999999 & $12 / 09 / 1959$ & & \\
\hline 990909 & 1111111 & DOLE MoบnT & 15. 06 & $2200 \times 2400$ & $120 / 240$ & 1 & 1.50 & Heckat Edisen & 999999 & 01/01/1974 & 49 & $48 / 23 / 1997$ \\
\hline 929599 & 1111111 & DOLX $=0 \mathrm{MT}$ & 15.06 & $7200 \times 2700$ & $120 / 240$ & 1 & 2.00 & General nectric & 999999 & $02 / 27 / 1975$ & 49 & $03 / 19 / 1997$ \\
\hline 999999 & 1111111 & DOLE mows & 15. 00 & $7200 \times 2400$ & $120 / 240$ & 1 & 1.20 & RTE Corporation & 999999 & $11 / 09 / 1972$ & 1 & $12 / 22 / 2000$ \\
\hline 999999 & 1111111 & pOLX sowr & 15. 00 & $7200 \times 2400$ & $120 / 240$ & 1 & 1. & KTE Corporation & 999999 & $03 / 07 / 1977$ & 1 & $06 / 26 / 2001$ \\
\hline
\end{tabular}

Figure 4.7-68. Capital assets for sale report 
This data can then be copy and pasted directly into Excel which is the preferred format of the NIPSCO business unit that uses this report.

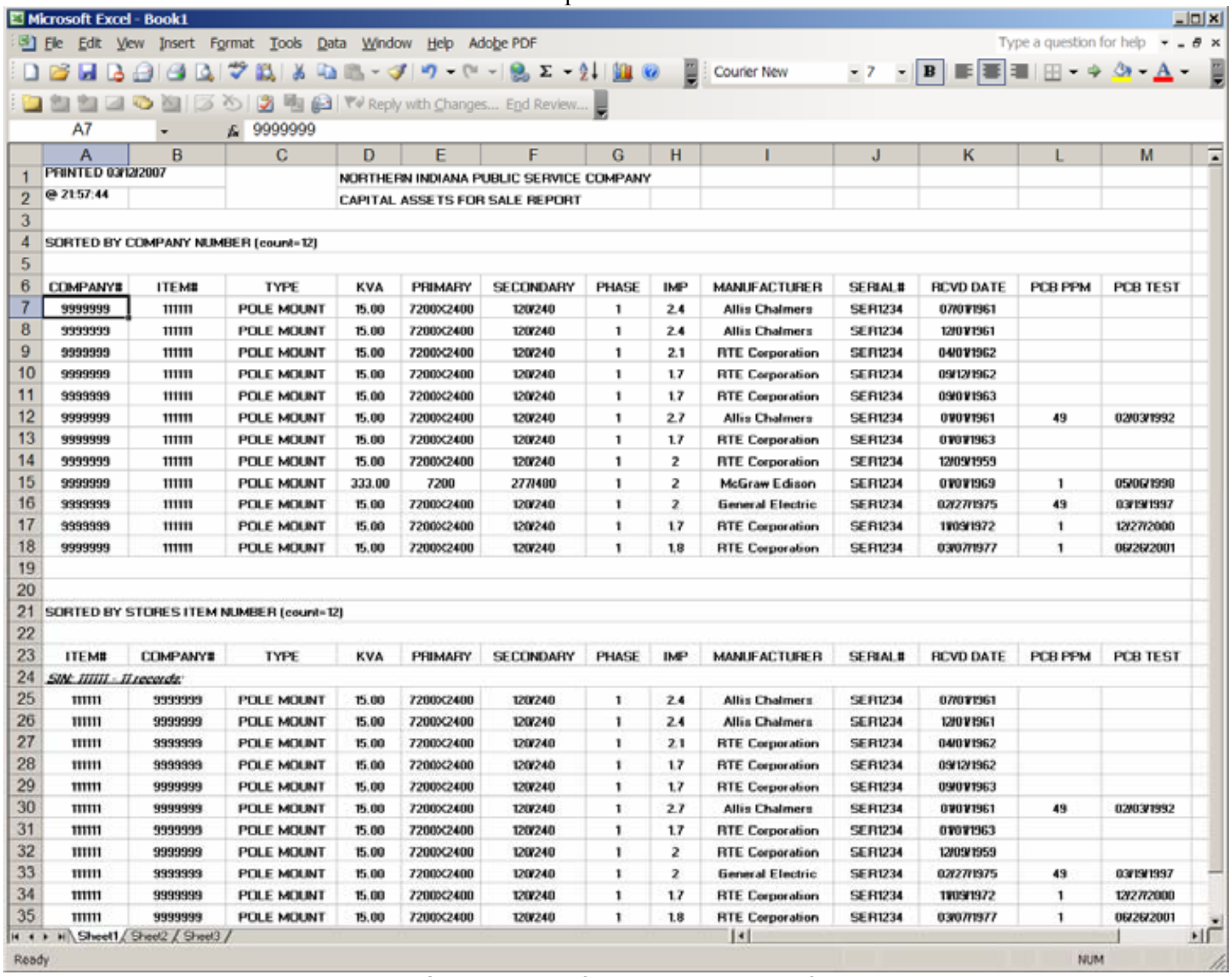

Figure 4.7-69. Capital assets for sale spreadsheet formatting is maintained from the html report into Excel 


\subsection{Pole / Pad Mounting Inquiry Report}

Figure 4.7-70 allows all users to run a pole/pad card report which presents a comprehensive view of all the details of a pole/pad/pedestal including all related conductor, devices, services, CIS records, etc. This report is used heavily throughout NIPSCO.

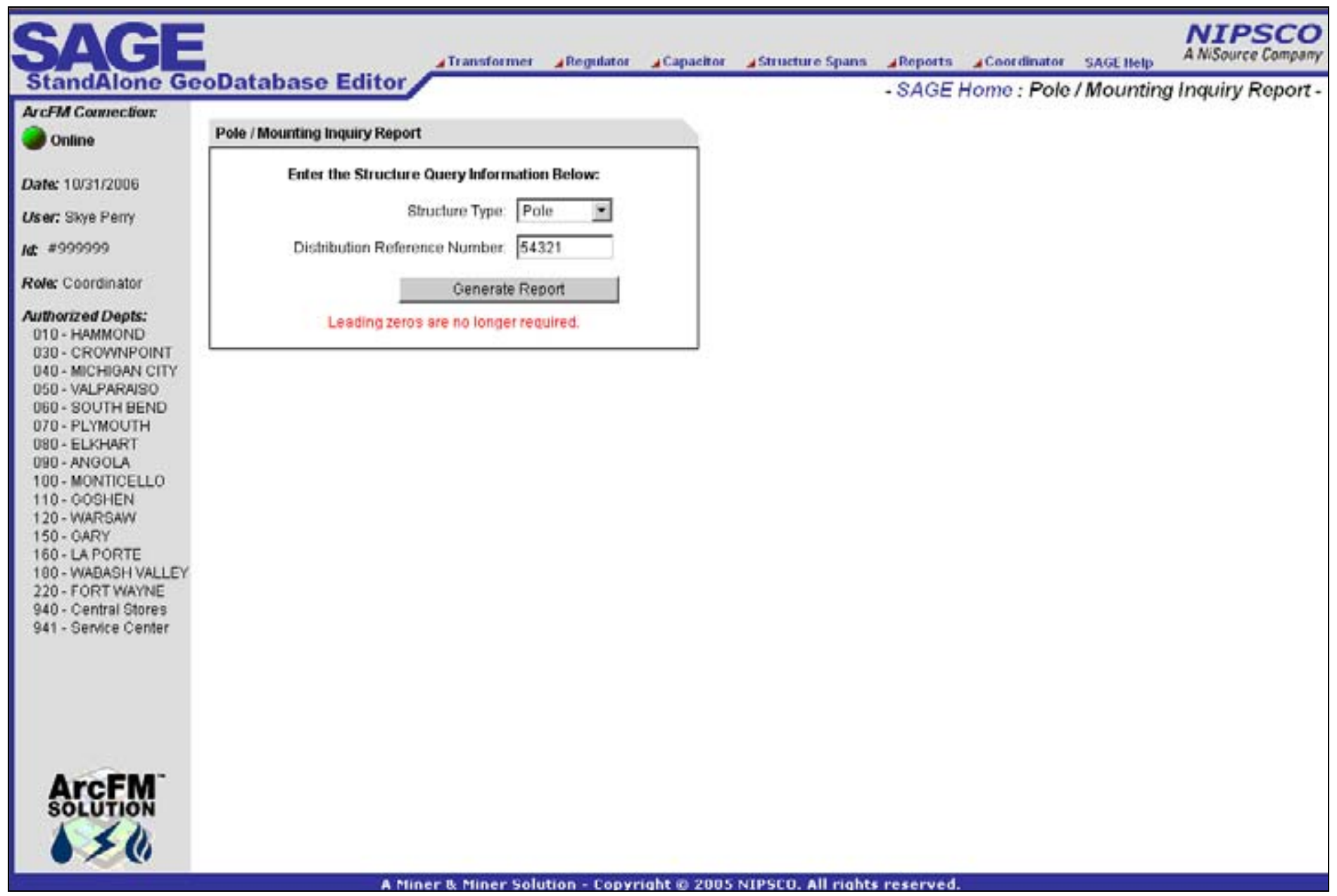

Figure 4.7-70. The pole / pad mounting inquiry report is considered an on demand report because it is generated based on user input values 
Clicking the Generate Report button will dynamically query the asset tables and will return a report similar to Figure 4.7-71.

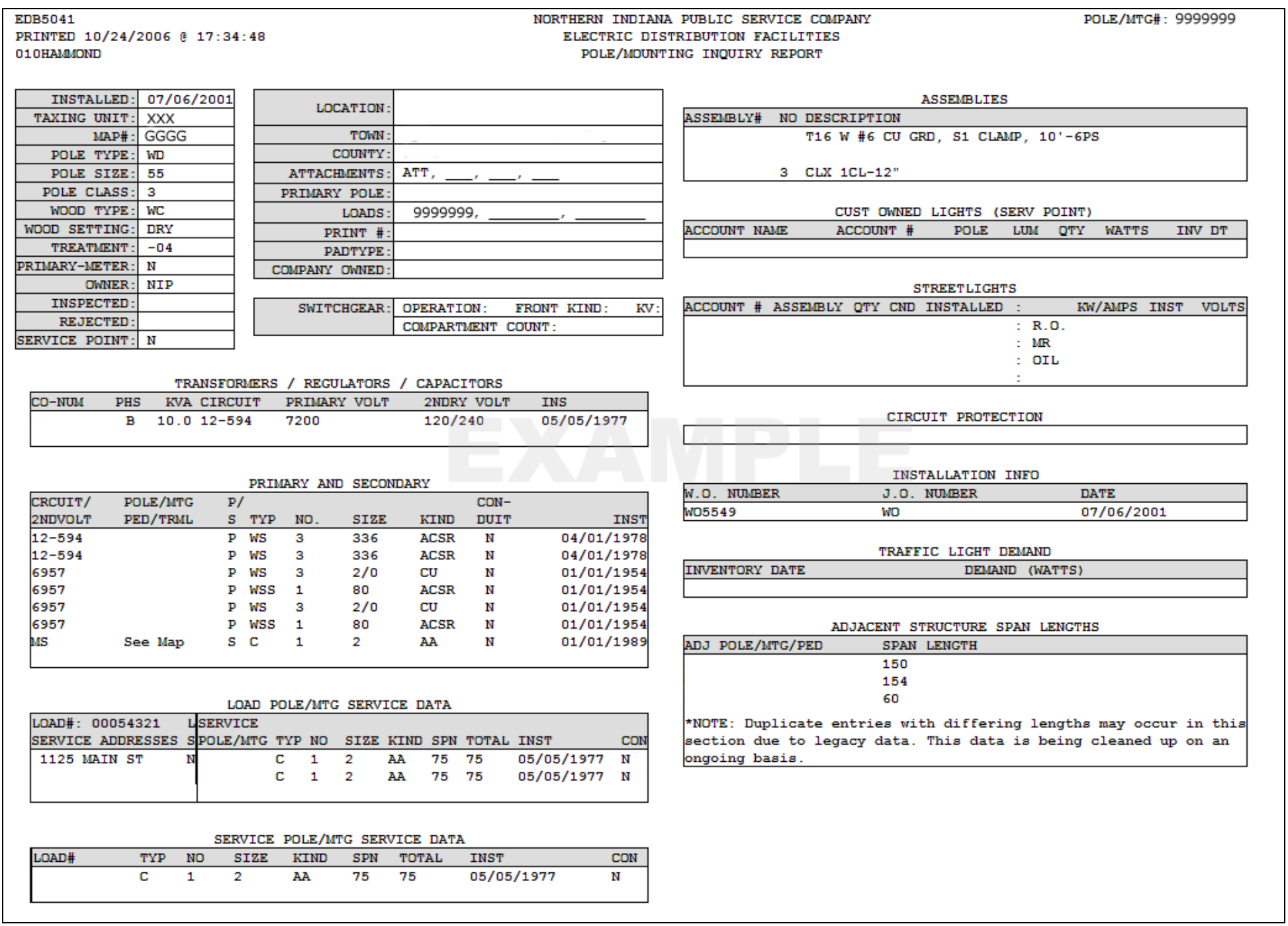

Figure 4.7-71. Pole / mounting inquiry report 


\subsection{Padmount Inspection Form Report}

Figure 4.7-72 allows all users to query the system for all pad-mounted transformers or switchgears based on grid information and device size. The report returns a formatted ready to print template for field inspections of all of these devices.

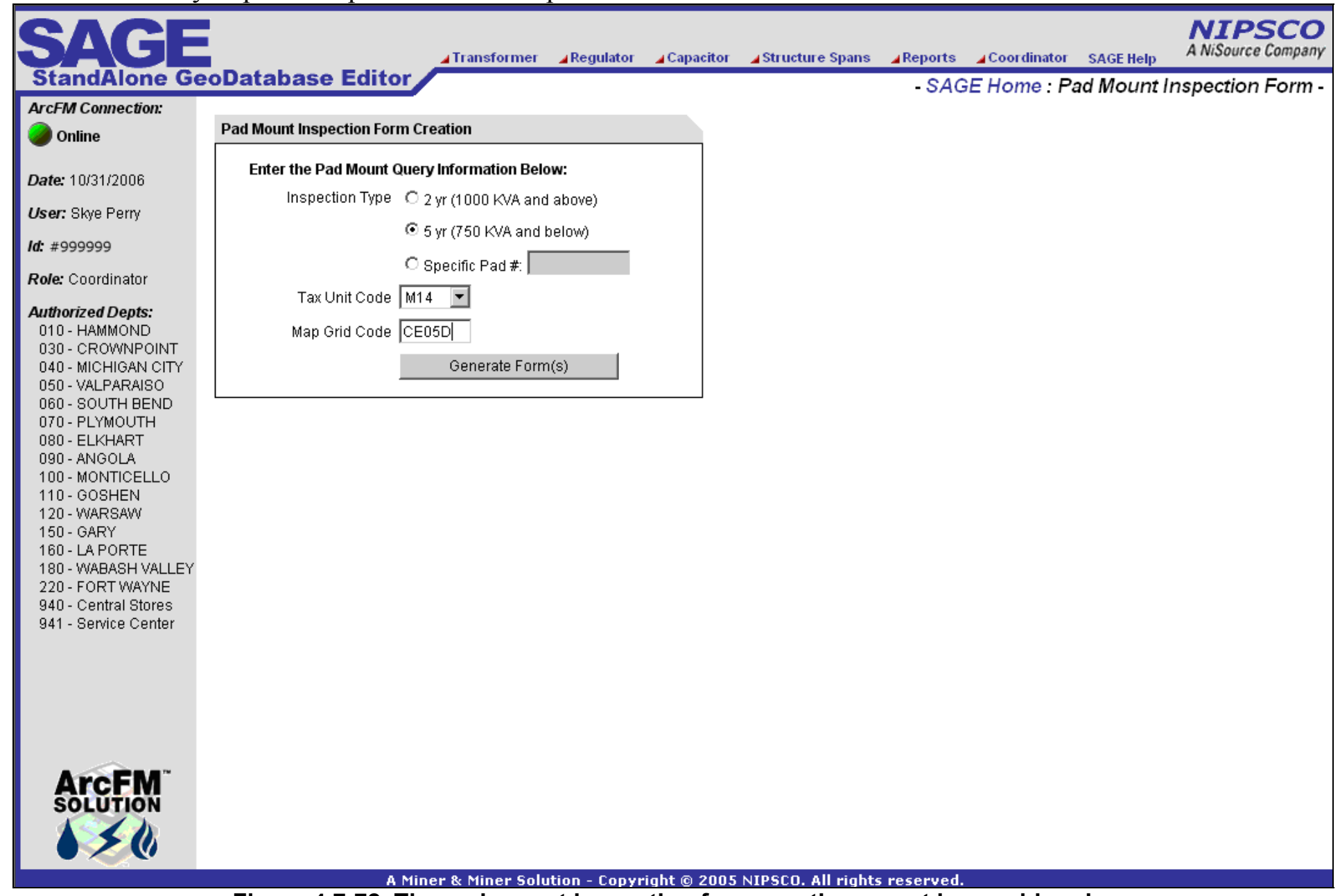

Figure 4.7-72. The pad mount inspection form creation report is considered an on demand report because it is generated based on user input values 
Clicking the Generate Form(s) will dynamically query the asset tables and will return a matching inspection form similar to Figure 4.7-73.

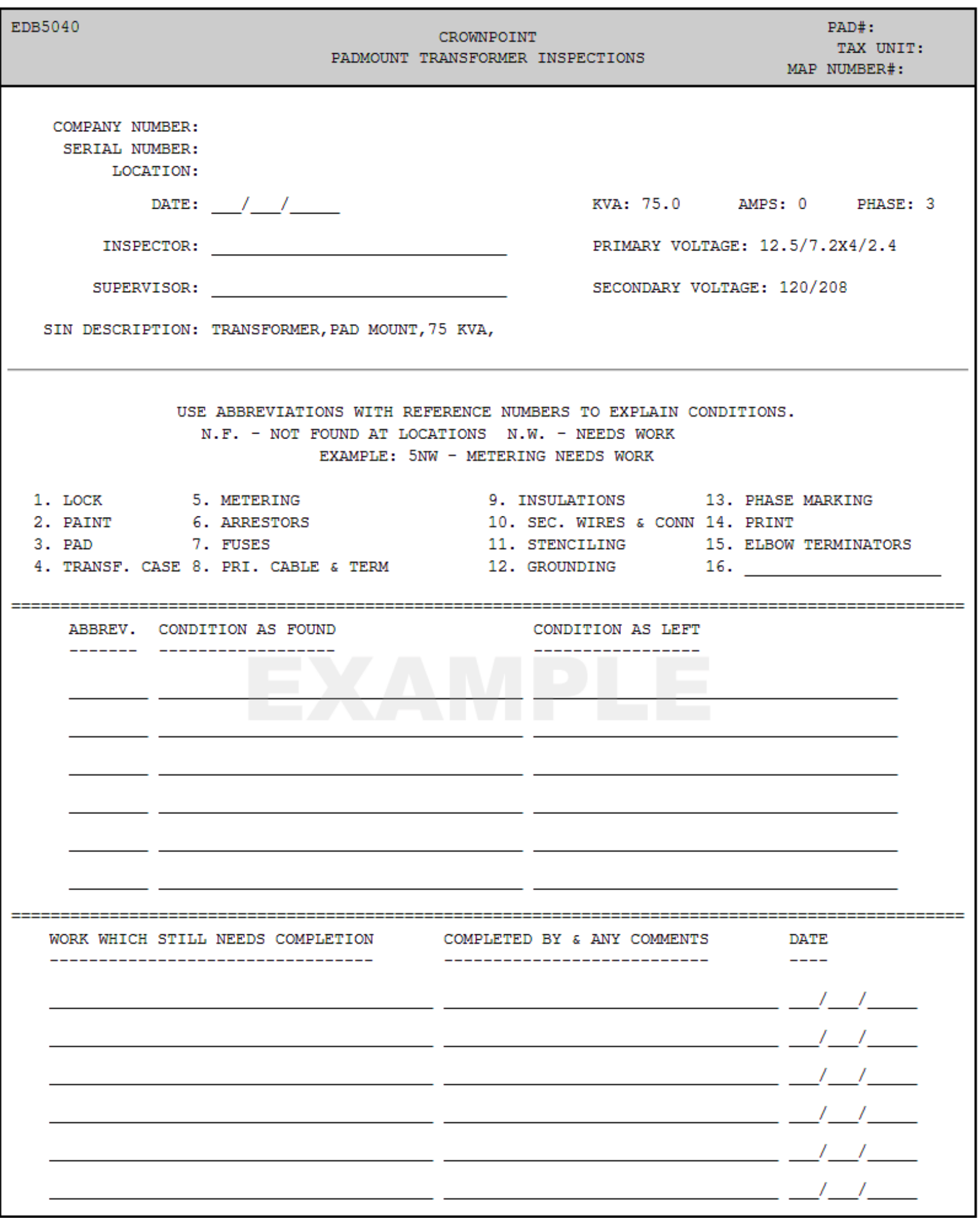

Figure 4.7-73. Padmount transformer inspections form 


\subsection{Batch Reports Screen}

Figure 4.7-74 allows all users to view the batch reports: Condemnations Needing Approval (including archives), XFR/VR Company Use Report, FERC XFR/VR Report, Annual XFR/VR \& Pole Report, Annual Count of XFR/VR/CAPs, Annual Count of XFR/VRs by County, XFR/VR/CAP Failure Report, Temporary \& Inactive XFR/VR installation report, SIN Report, In Stock XFR/VR/CAP by LOA Report, SEC Form 10K Report.

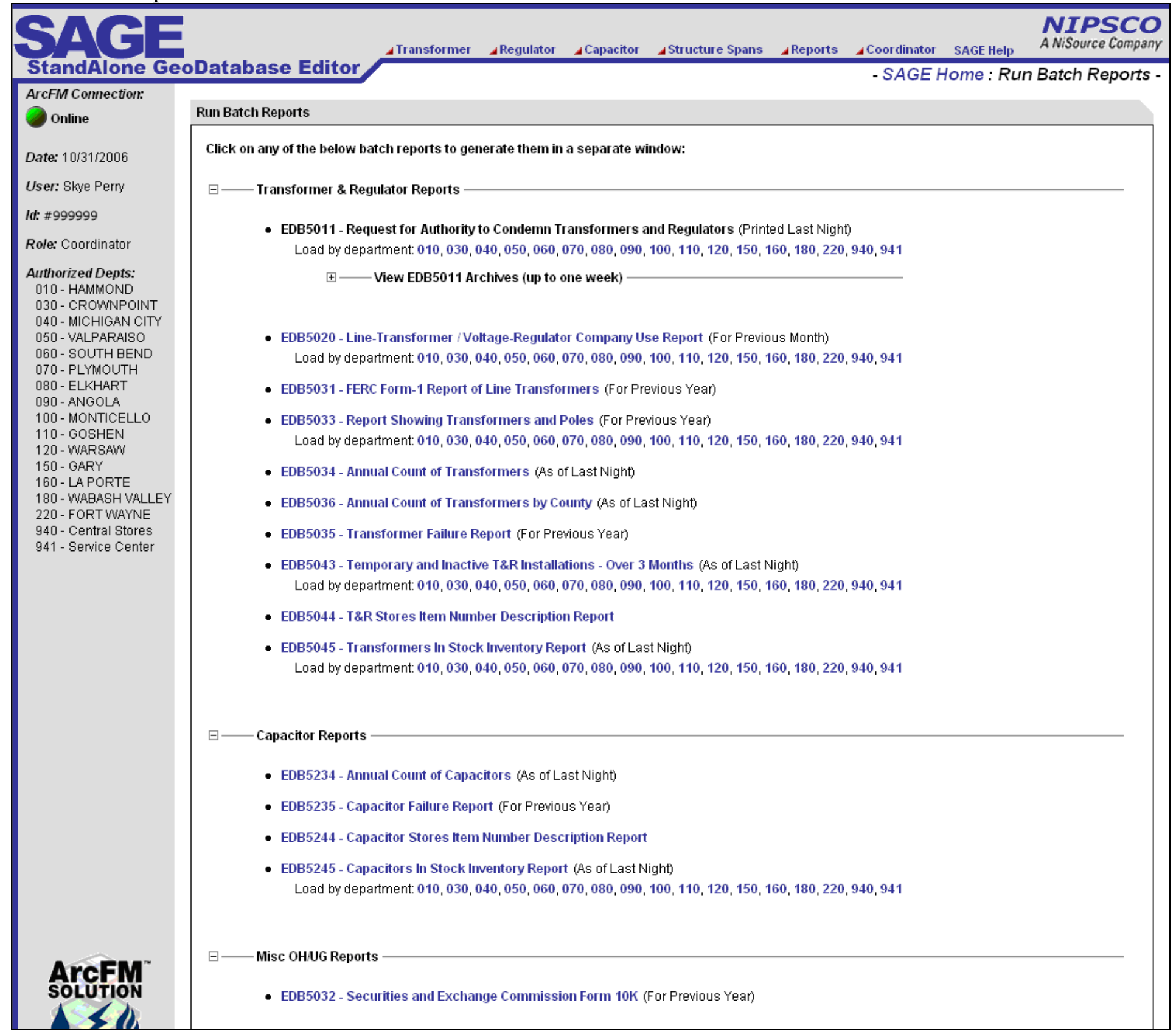

Figure 4.7-74. Batch reports screen 


\subsection{Coordinator Screens}

\subsection{Coordinator Dashboard}

- Viewing of KPI capital asset statistics

- View details of MAPPS/AEDR Synch (transfers, Condemns, issues, Returns)

- Allows management of outstanding MAPPS/AEDR Synch transactions

- Allows querying of processed MAPPS/AEDR Synch transactions

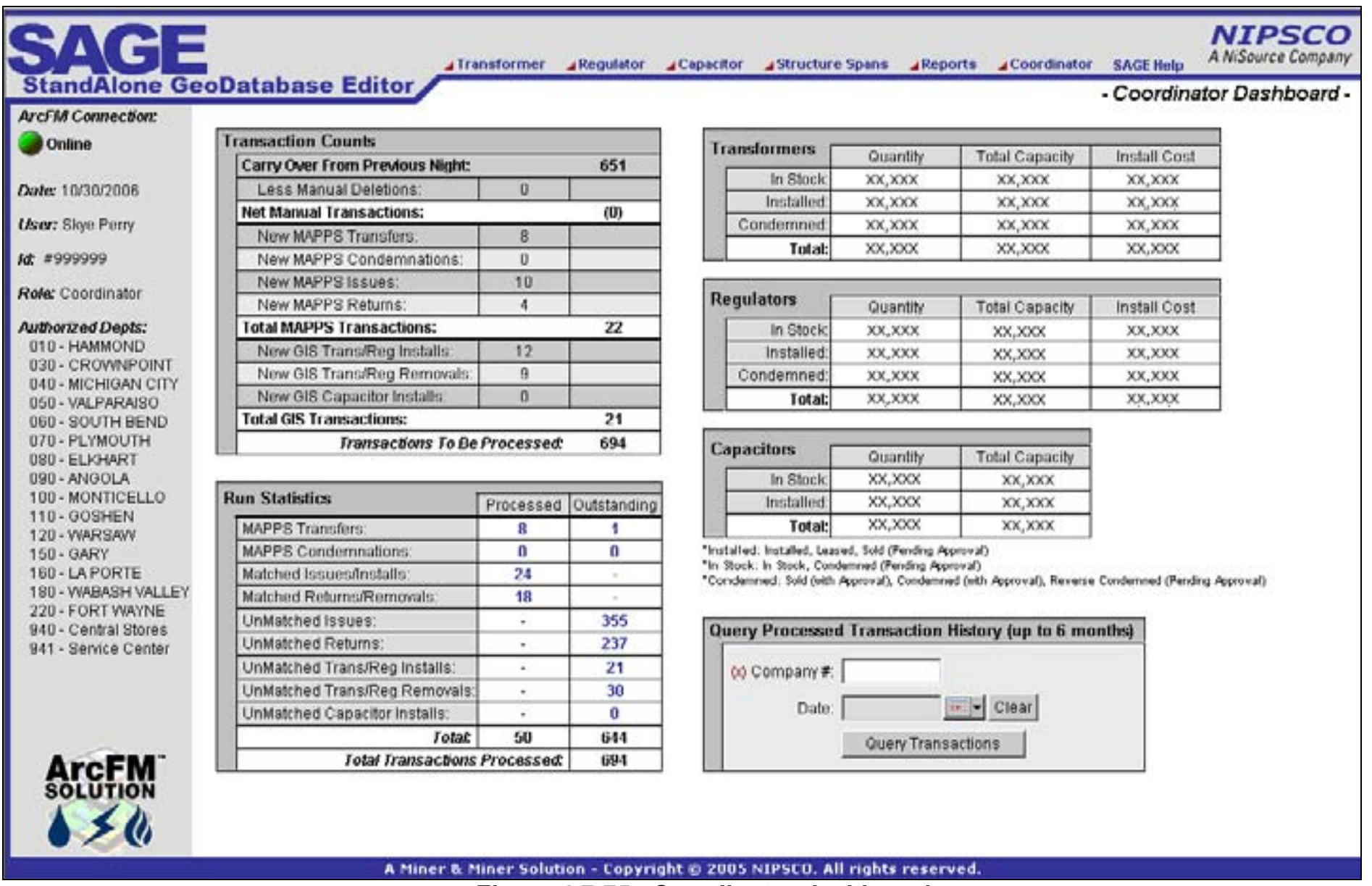

Figure 4.7-75. Coordinator dashboard 


\subsection{SAGE Security Report}

Figure 4.7-76 provides a comprehensive view of all SAGE users, their assigned roles, etc.

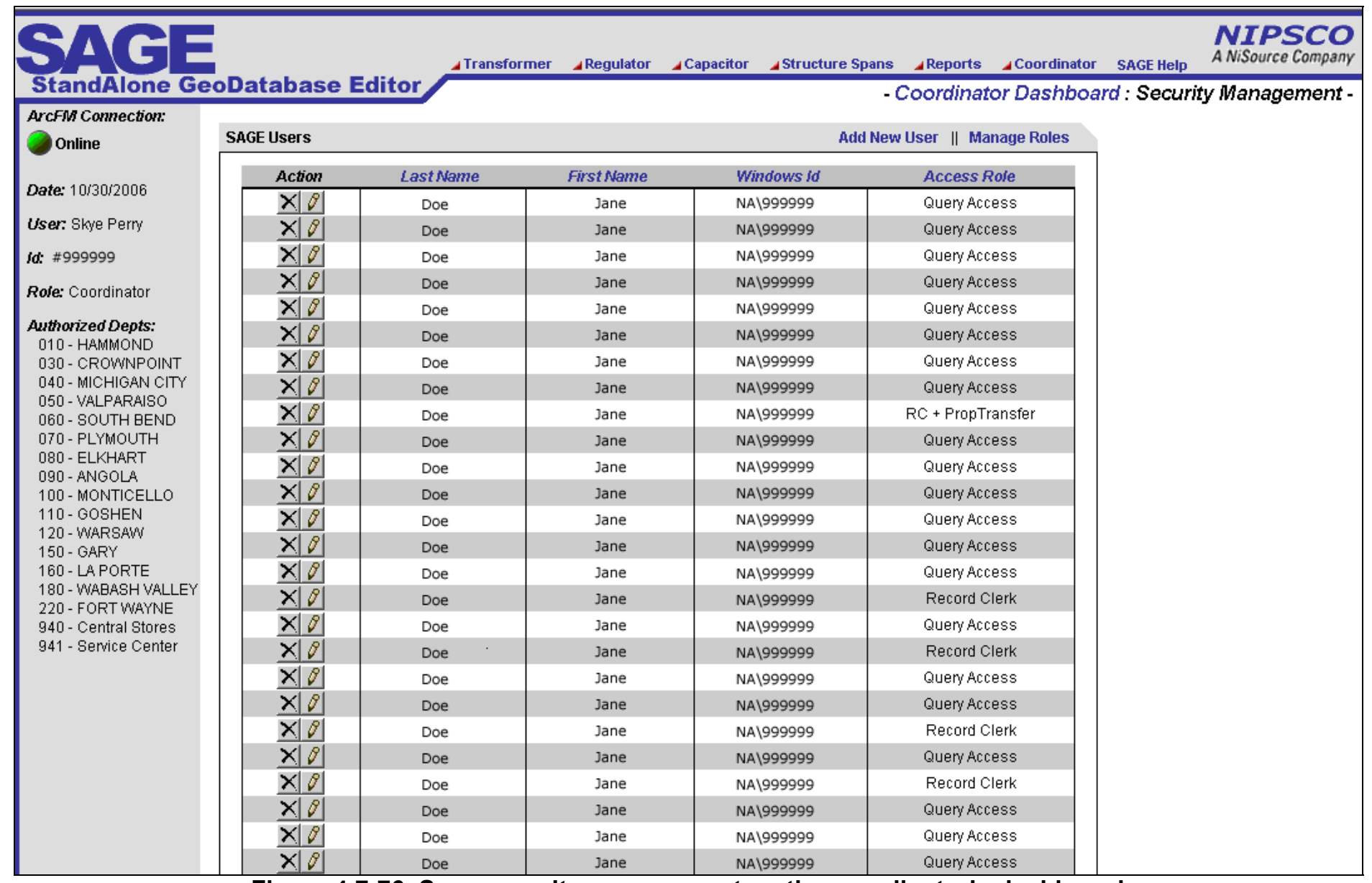

Figure 4.7-76. Sage security management on the coordinator's dashboard 


\subsection{Add New SAGE User}

Figure 4.7-77 shows that an administrator user is provided functionality to add a new SAGE user account, to specify the permissions role, and specify which LOAs the user is authorized to access.

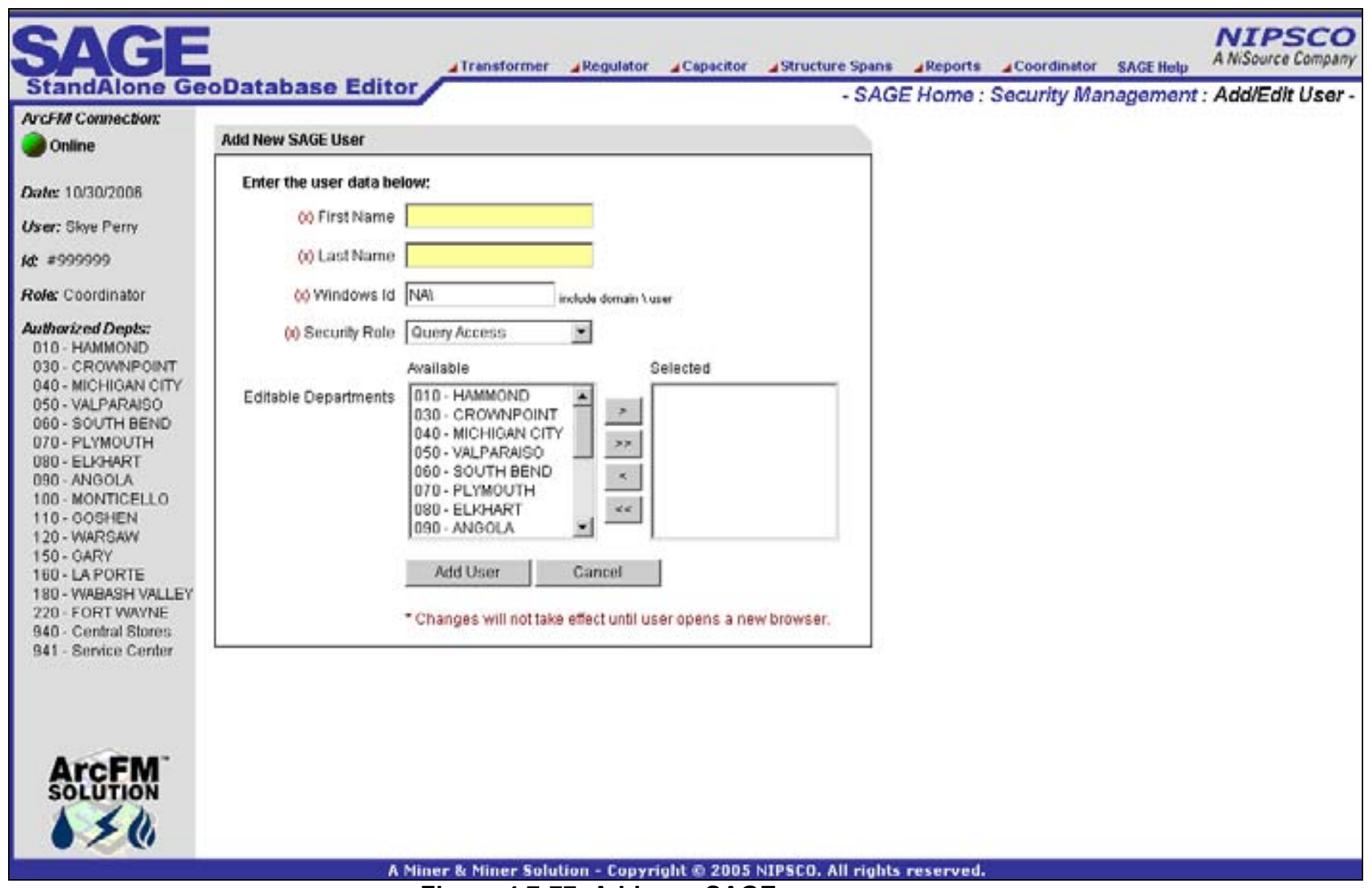

Figure 4.7-77. Add new SAGE user screen 


\subsection{Manage SAGE Roles}

Administrator user will add, update, and delete SAGE system roles as shown in Figure 4.7-78. A role contains a defined list of SAGE permissions. Several system roles exist as well including Coordinator, Record Clerk, Stores Clerk, Query User, etc.

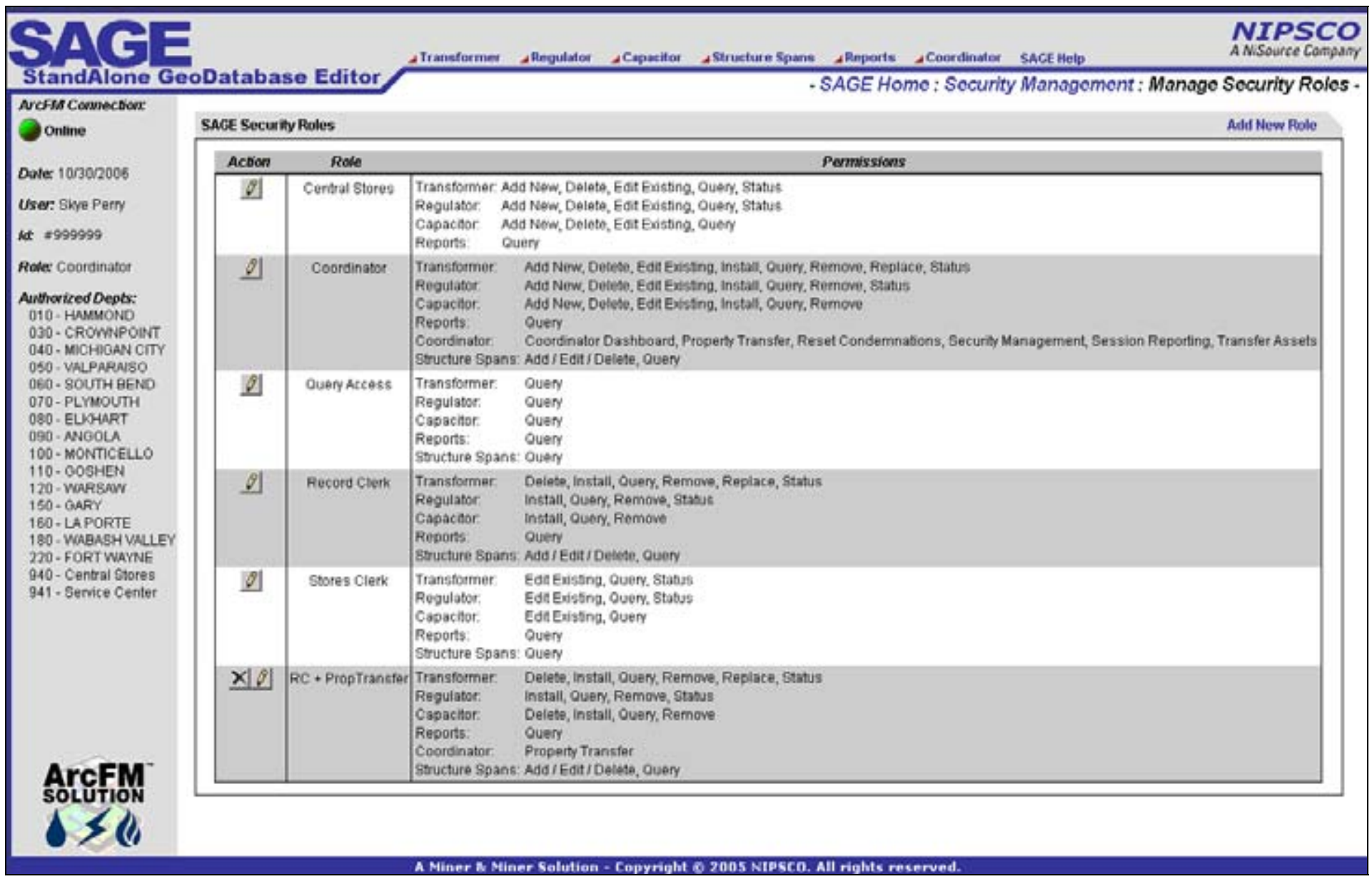

Figure 4.7-78. SAGE security roles screen 


\subsection{Unique Distribution Reference Administration}

The AEDR GIS code contains functionality to prevent a user from entering a pole, pad, or primary pedestal with a duplicate distribution reference number. This SAGE admin application allows an administrator user to add, update, or delete entries from the unique table which is used by the GIS to determine if a number has been used.

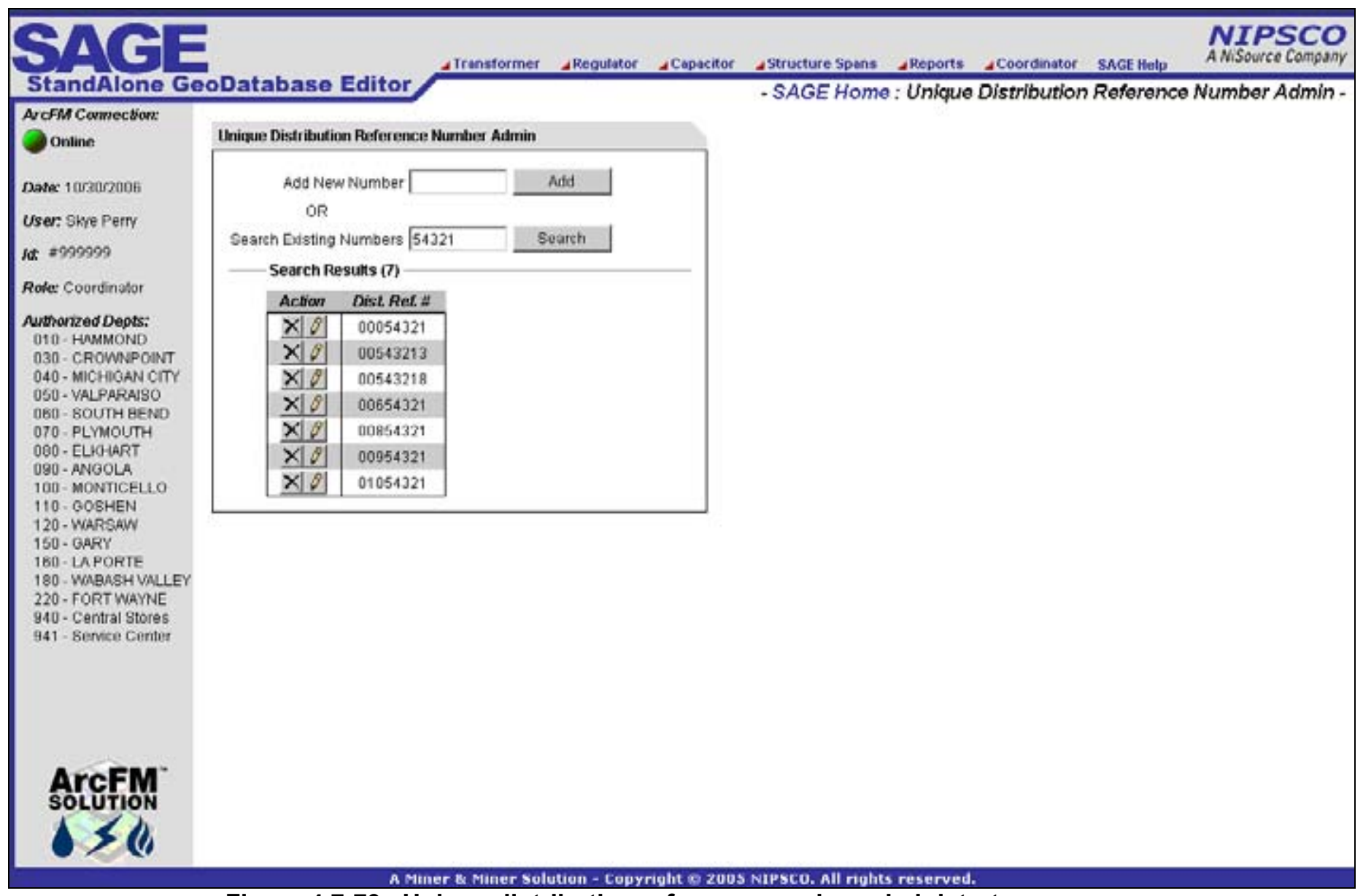

Figure 4.7-79. Unique distribution reference number administrator screen 


\subsection{Reset Condemnations}

If there is ever a problem with a printer or a user is not able to print a condemnation report for approval signatures, the condemnations can be reset so they are added to the report again on the next nights run.

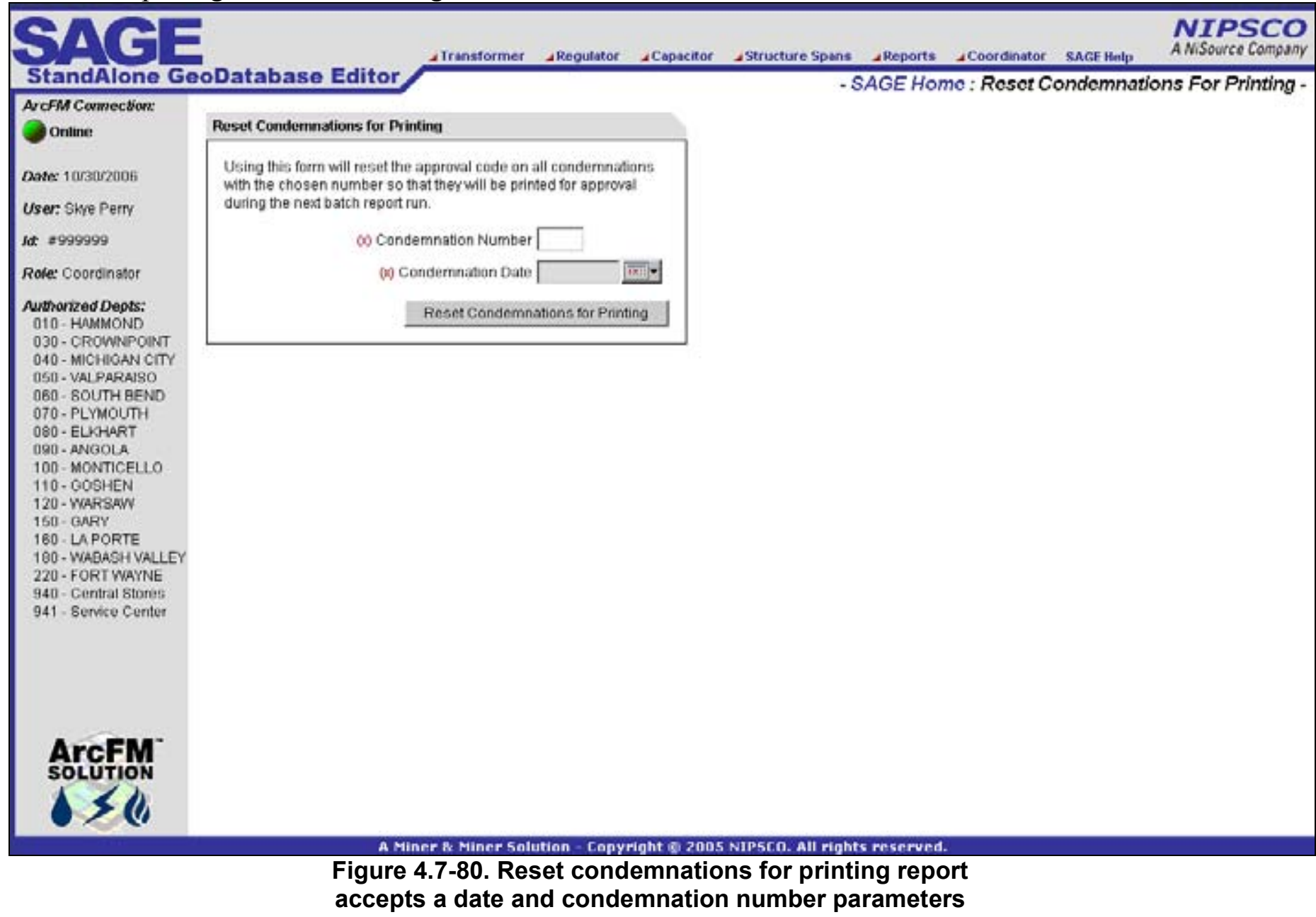

\subsection{Repair SDE Lock}

ESRI multi versioned views are used for the SAGE application. In the past, there have been issues where a MVV version becomes locked by the system. This functionality runs the admin code to unlock a locked version. There is no screen for this page. 


\subsubsection{Non-EDFS GIS Functionality}

Various software requirements were gathered to enhance the ArcGIS / ArcFM functionality to make it better meet the needs of the NIPSCO end users. Much of this was driven from the review of required functionality within the previous AutoCAD Outfield system. Other areas were implemented to streamline the user's interaction with the system, ensure data integrity, or to auto-populate data required by a new system interface. This section outlines all AutoUpdaters, Edit Tasks, Subtasks, and tools that were created outside the scope of the EDFS replacement.

\subsubsection{Core GIS and Outfield Replacement}

\subsection{Copy Value to Related Object}

The goal was to create an AU that copies a source value from one feature over to the target field of a related feature. This occurs on either the create or update event of the source feature. The source feature is determined by the class the component is assigned to. The source field, target class and target field are all determined using model names.

This AU originated from the specific case of a Gas Valve and a related Emergency Valve Marker. When an EmergencyValveMarker feature originally is created, via the 'CreateRelateRotateEmergencyValveMarkerAU' assigned to the GasValve feature class, the valve number from the GasValve feature is copied to the newly created EmergencyValveMarker feature. Therefore, this document only addresses the situation where a GasValve's valve number is updated. This AU is generic and will only work if the source and destination field data types match. As well, the target class must be related to the object to which is AU is assigned. In essence, we are copying a value from the parent to all children with assigned model names and appropriate data type.

\subsection{Assign to Classes}

- GasValve

- CapacitorBank

- TransformerBank

- VoltageRegulator

\subsection{Use Case}

This is an example use case. The same functionality is able to be configured anywhere a value must be duplicated to a related object. A user updates the 'valve number' attribute on a GasValve feature. Once this occurs, any related EmergencyValveMarker features have their 'label text' attribute updated with the name value.

\subsection{Design}

The following actions are performed upon the update of any of the above-mentioned records:

1. Select a GasValve feature which has a related EmergencyGasValve feature. 
If none can be found, change an existing GasValve 'emergencyvalveind' field to 'yes'. This will create a related EmergencyValveMarker for you (assuming configuration has been completed for this functionality).

2. Update the 'valvenumber' attribute on the GasValve feature

3. Check to see that the 'labeltext' value on all related EmergencyValveMarker feature is the same as the 'valvenumber' value on the GasValve.

\subsection{Cross Over Arc}

The goal was to create an Edit Task that automatically alters the shape of Line (OHConductor, UGConductor, GasMain) features at the point at which they cross over other Line features.

The requirements can be summarized as follows:

- An AU that alters the shape of a Line as it passes over another Line

- The alteration of the shape must occur automatically, without the intervention of the user.

- The alteration must occur when a Line is placed over another Line.

- Users can use ArcMap editing tools to adjust the humps in those cases where adjacent humps do not line up or similar cartographic esthetics are not met by the automatic placement.

This component works for the majority of situations. In situations where a line crosses several lines at once, or where an arc cannot be created due to limited space, this component may not be able to create a crossover arc as desired. If a situation arises where arcs are not created as desired, end user can manually adjust shapes to give the desired appearance.

\subsection{Assign to Classes}

- OhConductor

- UgConductor

- GasMain

\subsection{Use Case}

A user places a Line feature that crosses over another Line (using standard ArcMap/ArcFM tools). At the point in which the two lines cross, the newly added Line has its shape altered to resemble an arc. 


\subsection{Design}

Figure 4.7-81 shows an example of a cross over arc indicating the conductors are not connected:

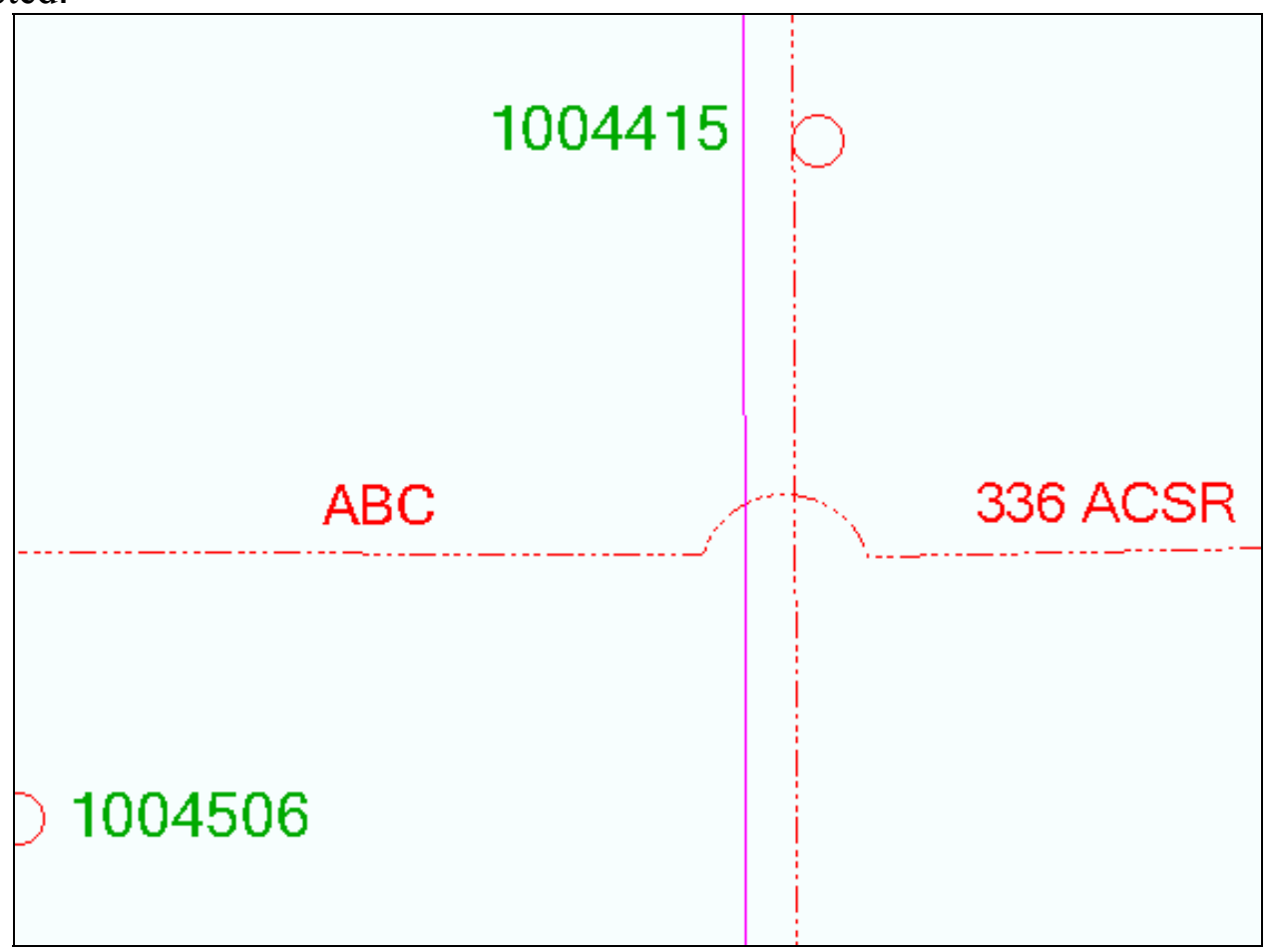

Figure 4.7-81. Conductor is not connected at the crossover arc

\subsection{Session Manager Do Not Post Subtask}

This component prevents features with a model name of DoNotPost from being posted to the parent version. Although this is a generic component, it was initially being developed for the ReferenceLine feature class and ReferencePoint feature class.

The requirements are summarized as follows:

- Create a component that prevents configured features from being posted.

- Enable the component to be configured for use on any feature and/or object class.

- Invoke the component before a Session is posted.

\subsection{Assign to Classes}

This is a custom subtask that was configured as part of the process framework. It is applied to the following classes:

- ReferenceLine

- ReferencePoint

\subsection{Use Case}

A user approves/posts a version using the MM Session Manager tool. Before the post, all non- postable features are removed. The Session is then posted to its parent without nonpostable features. 


\subsection{Design}

1. Create a Session.

2. Start Editing.

3. Add one or more non postable features, such as a ReferenceLine or ReferencePoint feature.

4. Save your edits.

5. Submit your Session.

6. Have the Session Approved.

7. Reconcile the Session.

8. Post the Session.

9. Open the Sessions parent version and search for the non postable feature created above. None should be present.

\subsection{Field Concatenation AU}

A generic AU was created that is used to take a variable number of fields, concatenate their values and enter the result into an output field. If a field has an associated domain, the domain value will be used versus its description.

The fields designated as input fields have been identified by model names such as InputField1, InputField2, InputField3, etc. The fields are concatenated in the order in which they are numbered. The output field is identified by the model name OutputField.

Whoever uses this AU must be aware of the different combination of values generated and configure accordingly. Of course, improper assignment of model names, changes in domains, domain values, etc, are handled manually via configuration versus updating of code.

This change was needed to allow for the concatenation of values within a feature class on any number of fields. This AU is also reusable across several feature classes due to it dependence on configuration versus code.

\subsection{Assign to Classes}

Gas Valve

\subsection{Use Case}

Since this AU is used on a variety of feature classes, the outcome of using this AU is dependant on upon configuration. Each feature class that uses this AU has a configuration document containing the following:

- The input fields that make up the concatenated output value

- The output field

- All possible combinations of input field values and their resulting value

Based on an individual feature class configuration document, a user creates a feature and then is able to observe if the resulting value is correct. 


\subsection{Design}

1. Create a feature that has this generic $\mathrm{AU}$ assigned.

2. The output value should correspond to the documentation for the feature class in question.

\subsection{LOA Number AU}

This AU populates an LoaName field with the name of the LOA that it resides within. If a feature resides within more than one LOA, the field will be populated with only one LOA name. This change was needed to fully alleviate the user from manually entering this information. Ultimately this information is required for tax reasons.

The requirements can be summarized as follows. Take the shape of the feature being added, spatially determine which LOA polygon features it resides within, capture the LoaName and persist this information in the field that the AU is associated with. Fields that are designed to hold LoaName information have a length of 3 . If a feature resides within more than one LOA feature the code will not concatenate the LoaNames together. Currently this AU will only return the first LOAName on the first LOA feature it finds. If requirements change in the future, code will need to be updated.

\subsection{Assign to Classes}

All electric and gas feature classes that carry the LOANumber field

\subsection{Use Case}

GIS End User creates and/or updates a feature as they are performing their daily tasks. All fields that have the LoaNameAU assigned to them become populated with the LoaName of the LOA Feature it resides within. No user intervention is required.

\subsection{Design}

1. Assigned the AU to a field as noted above.

2. Add or update a feature in question.

3. Observe the LoaName that was populated within the field the AU is assigned to.

4. Add the LOA feature class to your map.

5. Select the identify tool.

6. Click the feature that you added/updated.

7. A form will pop up. Select the LOA feature and note its LoaName.

8. This number should be the same as the one populated within the features attribute.

9. An update that triggers Feeder Manager should be run to verify that this AU is not triggered by only an attribute update.

\subsection{Network Edge Split at Tap Point}

AU that allows a network edge feature (including OhConductor, UgConductor, TieBus and GasMain) to split another network edge feature(s) at the to/from tap point(s). This splitting occurs only where an end point does not currently exist, which is along a Network edge. A user may tap a network edge at both the start and end points of a newly 
created Conductor or Gas Main (this would look like an $\mathrm{H}$ ). At both tap points, the tapped Network edge is split. Note: Secondary is modeled as a feature class, but does not participate in the network. This feature will not be affected by this AU.

Once it's been determined that a Network edge is to be split, code must ensure data integrity. When a split occurs, the shape of the Network edge being split is altered to form one side of the split line. A new Network edge is created from the other side of the split line. After a Network edge is split we must ensure relationships are properly maintained. Custom code handles the maintenance of existing relationships and whether or not they are updated. This change is needed to ensure the network is maintained in a consistent manner and to support the network models of external interfaced applications including CADOPS and FeederAll.

The requirements are summarized as follows:

- To automate this functionality using an AU

- To have a network edge split at the point where another network edge taps off of it. This could occur only at the To/From points of a newly created network edge feature. When this is done, the network edge being tapped will have its shape changed so it represents one side of the split network edge. The other side of the split network edge is represented by a newly created network edge feature

- To retain the original attributes of the network edge being split and have them applied to each of two segments after the split occurs

- To ensure objects related to the tapped network edge are correctly re-related as deemed appropriate. Situations where the Network edge feature in the relationship is the destination feature class will be treated differently than if the Network edge is the origin feature class. In the case of the Network edge being the destination feature class, the related objects will retain their relationship with the original Network edge and also be related to the new Network edge. In the case of the Network edge being the origin feature class, the related objects will queried to determine which Network edge they are closest to, the original or the new one. If it's the original, nothing is changed. If it's the new one, the relationship between the original Network edge is removed and a relationship to the new Network edge is created.

\subsection{Assign to Classes}

- OhConductor

- UgConductor

- TieBus

- GasMain

\subsection{Use Case}

- A user places a new Network edge. The From point resides on an existing end point and the To point ends at a generic junction. Since the From and To points do not tap off of an existing Network edge's edge, no split is made.

- A user places a new Network edge. The From point is placed on the edge of an existing Network edge and the To point ends at a generic junction. Since the 
From point is placed on the edge of an existing Network edge, it is effectively tapping off of that Network edge. In this case, the Network edge being tapped must be split. Once the split is complete, all relationships that once existed must be evaluated and re-established if necessary.

- A user places a new Network edge. Both the From and To points are placed on the edge of an existing Network edge. Therefore, they are effectively tapping off of each Network edge. In this case, both Network edge's being tapped must be split. Once the split is complete, all relationships that once existed must be evaluated and re-established if necessary.

\subsection{Design}

1. Place a new Network edge with it's From point on the edge of an existing Network edge and it's To point not connected to any other network feature

2. The Network edge being tapped should be split and all relationships maintained.

3. Place a new Network edge with it From and To points on the edge of an existing Network edge

4. Both Network edge being tapped should be split and all relationships maintained.

5. Place a new Network edge with it From point at the end of an existing Network edge and it's To point not connected to any other network.

6. This scenario should not cause any Network edge to be split and the edit should stand as is.

Figure 4.7-82 shows an example of an un-split conductor. The conductor is selected to show that it is not split at the pole:

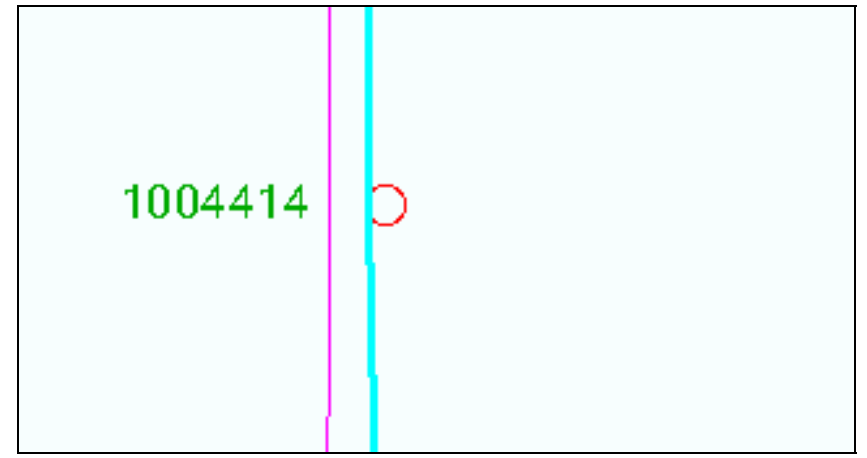

Figure 4.7-82. Conductor is not split at the pole

Figure 4.7-83 shows the same area after a new conductor has been placed tapping off of the existing conductor at the pole. Note the original conductor has been automatically split: 


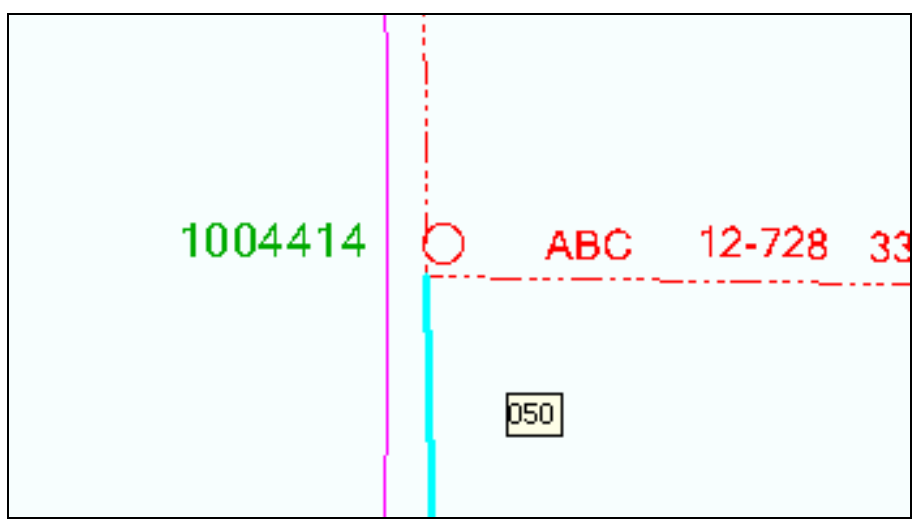

Figure 4.7-83. New conductor is tapped off existing conductor at the pole

\subsection{Normal Position Symbology AU}

An AU is required to populate the SymbologyConfigurationCode attribute of a feature class from which symbology will be driven. The code that is populated into this field is dependant upon the NormalStatus_A, NormalStatus_B and NormalStatus_C fields. When a feature is created or updated, the AU fires and, based upon the combination of these attributes, determines the appropriate code to populate the field (1-Closed, 0-Open).

Code will look at the three NormalStatus fields. If all are set to 'Open', then the SymbologyConfigurationCode field would be set to 'Open'. If any of the of these fields are set to 'Closed' then the SymbologyConfigurationCode would be set to 'Closed'. Open is represented by a 0 , while Closed is represented by a 1 .

\subsection{Assign to Classes}

- FuseCutoutBank

- Switch

- SectionalizerBank

- RecloserBank

- OpenPoint

- CustomerGenerator

\subsection{Use Case}

- A user adds or updates a FuseCutoutBank feature and sets NormalPositionA, NormalPositionB or NormalPositionC fields to 'Open'. The AU fires and the SymbologyConfigurationCd field is updated to 'Open' (0).

- A user enters adds or updates a FuseCutoutBank feature and sets one or more of the NormalPositionA, NormalPositionB or NormalPositionC fields to 'Closed. The AU fires and the SymbologyConfigurationCd field is updated to 'Closed' (1).

\subsection{Design}

1. Add a new FuseCutoutBank feature with the fields NormalPositionA, NormalPositionB and NormalPositionC set to 'Opened'.

2. The SymbologyConfigurationCd field should be set to 'Opened'. 
3. Add a new FuseCutoutBank feature with one or more of the fields NormalPositionA, NormalPositionB and NormalPositionC set to 'Closed.

1. The SymbologyConfigurationCd field should be set to 'Closed'.

\subsection{Prevent Delete if Object has Related Objects}

This AU prevents a feature from being deleted if it has objects related to it.

\subsection{Assign to Classes}

- CapacitorBank

- VoltageRegulator

- TransformerBank

- OhConductor

- UgConductor

- FuseCutoutBank

- Switch

- Secondary

- RecloserBank

- SectionalizerBank

\subsection{Use Case}

A user attempts to delete a feature. If it has objects related to it, the user is warned and the deletion not allowed.

\subsection{Design}

1. Assign the AU to an object.

2. Delete a feature.

3. If the feature has related objects, a warning should appear and the edit not allowed.

4. If the feature does not have related objects, it should be deleted.

Figure 4.7-84 is an example of the message box that will be displayed.

The Fuse Cutout Bank, w/ OID of 21125, has related object(s). Please delete all related objects and try again.

$$
\text { OK }
$$

Figure 4.7-84. Warning message indicating related objects exist

\subsection{Reference Features Remove Button}

A user selects an area on the map and clicks a button to remove all ReferencePoint and ReferenceLine features found within the current selection. ReferencePoint and ReferenceLine features only exist to aid in the creation of other features and therefore will not be posted to the parent version. Because of this, removing them from the active view has the effect of cleaning up the map, but has no impact on data itself. 
The requirements can be summarized as follows:

1. To create a command button that can be placed within the ArcMap interface.

2. When this command button is selected, it goes out to the current selection set, finds all the ReferencePoint and ReferenceLine features and deletes them.

\subsection{Assign to Classes}

This is a command button that is available in the user interface. It operates on the following classes:

- ReferencePoint

- ReferenceLine

\subsection{Use Case}

- A user clicks the ReferenceFeaturesRemovalButton. Since no features are selected, nothing happens (i.e., no error messages come up).

- A user selects several features including some Reference Features(either line or point) and clicks the ReferenceFeaturesRemovalButton. All ReferenceLine and ReferencePoint features that are selected will be deleted.

- A user selects several features, none of which are Reference Features(either line or point) and clicks the ReferenceFeaturesRemovalButton. Nothing happens( i.e. no error messages come up) since no Reference Features are in the selection set.

- A user clicks the button and the ReferenceLine and ReferencePoint layers are not in the document. Nothing happens (i.e. no error messages come up).

\subsection{Design}

1. The interface consists of an ArcMap button with an icon of a lightening bolt. If this button does not currently exist on a toolbar, it must be manually placed there. All other GUIbuttons/tools are standard ArcMap or ArcFM tools.

2. No forms or message boxes are envisioned.

3. This button will always be enabled. The reason for this is it eliminates the need for code to continuously run behind the scenes to determine if it should/should not be enable. Also, if a button is disabled, the user may have no idea as to why it's in this disabled state. If a button is enabled and a user selects it when the state of the application is not set properly, the user will be informed of the problem and therefore be able to rectify the situation.

\subsection{Feature Offset Edit Task}

An edit was created to allow for a feature to be offset from a SupportStructure versus being created directly on top of it. A user snaps to a conductor then clicks on or close to the point they wish the feature to be placed. Code determines if a SupportStrucure feature is nearby. If so, the $\mathrm{AU}$ will place the feature 15 feet down the conductor from the SupportStructure. If a feature needs to be related to the nearest support structure, the ArcFM Structure Relate AU is applied to the feature class in question.

The requirements are summarized as follows:

- When a user snaps to a conductor and clicks to place a Feature, the Feature will be placed 15 feet off of the nearest SupportStructure 
- A SupportStructure must exist within the vicinity ( $250 \mathrm{ft})$ of the point of placement.

- A Feature splits the conductor at the location it's placed. This functionality was tested with the feature configured to split the conductor as this is a requirement for NIPSCO. Split functionality is not provided by this AU, but by proper configuration of the database using ArcCatalog.

This edit task will also ensure offset features do not reside on top of each other. For example, if a capacitor is placed and offset from a pole, it will reside 15 feet down from the pole. If a switch is to be placed on the same side of the pole, it will be placed 30 feet down from the pole. In do this, it will ensure the integrity of the network.

\subsection{Assign to Classes}

- FuseCutoutBank (OH subtypes only)

- RecloserBank

- Switch (OH subtypes only)

- VoltageRegulator

- CapacitorBank

\subsection{Use Case}

- A user places a Feature by first selecting the feature from the Features tab. A user then places the cursor over the map and snaps to a conductor at a point that's near a SupportStructure. When the user clicks to place the feature, it's offset from the nearest SupportStructure by 15 feet and auto rotated.

- A user places a Feature before snapping to a conductor. In this case, the feature is not placed.

- A user places a Feature but at a distance not close to any SupportStructure. In this case, the feature is not placed.

\subsection{Design}

1. Start editing, select the Feature from the features tab, using the Targets tab set the features attributes, sketch the feature by snapping to a conductor at a position close to a SupportStructure.

2. The feature is added and offset a set distance from the pole.

3. Start editing, select the Feature from the features tab, using the Targets tab set the features attributes, sketch the feature without first snapping to a conductor.

4. A warning is given and the edit not allowed.

5. Start editing, select the Feature from the features tab, using the Targets tab set the features attributes, sketch the feature by snapping to a conductor at a position not close to a SupportStructure.

6. A warning is given and the edit not allowed.

7. In all cases, the placement of a Feature splits the conductor. 
In Figure 4.7-85, the user would have clicked the conductor very close to the pole. The offset edit task then places the switch at a fixed distance up from the pole:

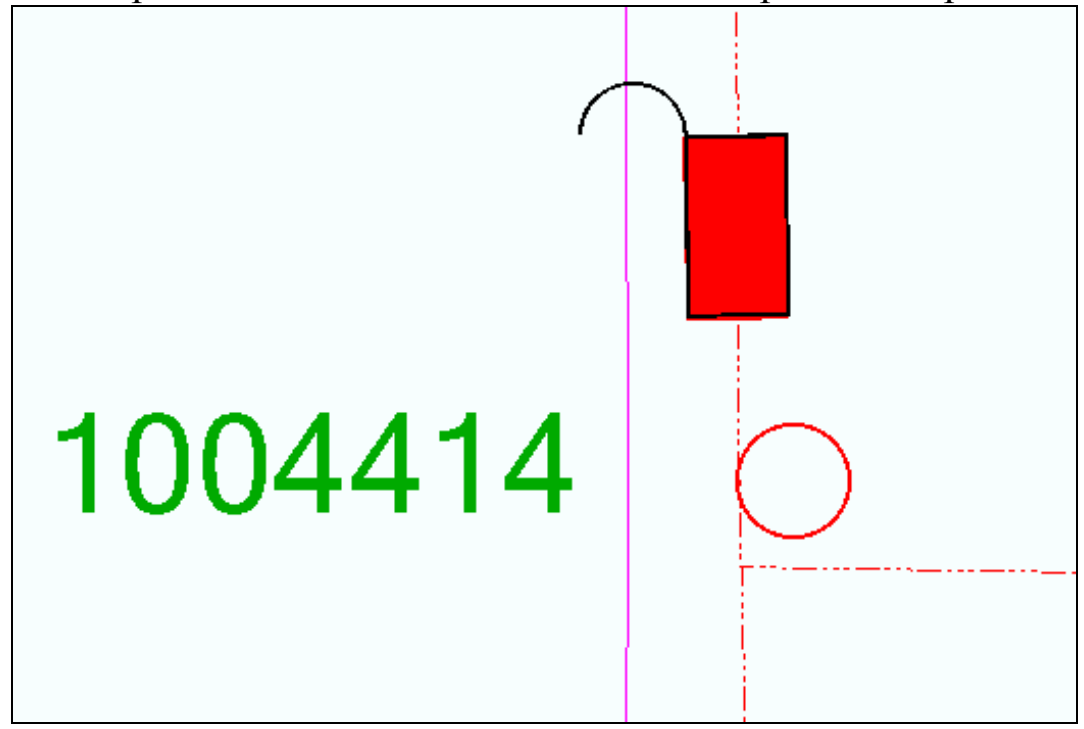

Figure 4.7-85. Feature offset

\subsection{Unique Distribution Reference $A U$}

The AU developed is associated with the following feature classes; SupportStructure, Pedestal and Padmount.

When a user creates or updates any of the above listed features, the AU will determine if the DistribRefNumber is unique. If a DistribRefNumber is found to be a duplicate, the user will be warned and the edit disallowed.

Uniqueness of DistribRefNumbers is enforced on a company wide basis, therefore this component checks all versions on all appropriate feature classes to ensure uniqueness. Instead of performing this work each time a new DistribRefNumber is entered, the following approach is taken. An un-versioned table is created and populated with all DistribRefNumbers currently in the database. This table is checked to determine if newly entered DistribRefNumbers are duplicates. This table is updated with newly entered, unique DistribRefNumbers. A tool has been provided that allows for the manual editing of records in this table, if needed.

The requirements can be summarized as follows:

1. To provide a mechanism that generates and populates a table, 'UniqueDistribRefNumbers', with all DistribRefNumbers currently in the GDB. This is achieved via an admin command button within ArcCatalog. This tool gathers all existing DistribRefNumbers from the SupportStructure feature class, PadMount feature class, Pedestal feature class and RetiredSupportStructure object class.

2. To provide an AU, that pads all distribrefnumbers out to 8 digits. 
3. To provide an AU, that when assigned to an object's oncreate or onupdate events, will check the UniqueDistribRefNumbers table for duplicates.

4. To prevent edits when duplicate DistribRefNumbers are entered

5. Before validation is done, this AU checks the feature to which it's assigned for a field with a model name of 'Owner'. If that field exists, the code checks the value in this field. If that valid is "C" (for customer) then the validation will not be performed. If no field with the model name of 'Owner' exists, or the field exists but the value is not set to ' $\mathrm{C}$ ', validation to ensure a unique distribrefnumber will be performed. This requirement was intended to address the PadMount feature where the Owner model name would be assigned to the PadMound.OwnerInd field. This configuration could be applied to other features as well.

6. To update the unique UniqueDistribRefNumbers table when new DistribRefNumbers are entered

Since the UniqueDistribRefNumbers table is non-versioned, changes to it will not be rolled back in the cases where an edit to an object is undone/rolled back. Therefore, this AU must be assigned to fire last within the list of class level AU's assigned to any one object.

\subsection{Assign to Classes}

- SupportStructure

- PadMount

- Pedestal (only Primary Pedestal subtype)

\subsection{Use Case}

- A user creates a new feature (SupportStructure, Pedestal, Padmount) and enters a unique DistribRefNumber. The feature is successfully stored.

- A user creates a new feature (SupportStructure, Pedestal, Padmount) and enters a non unique DistribRefNumber. The user is presented with an error message explaining the error and the edit is disallowed. At this point it's up to the user to manually reenter the correct DistribRefNumber.

- A user enters a non unique DistribRefNumber for a Padmount feature. If the Padmount OwnerInd field is set to Customer, validation does not occur and the edit is allowed. If the OwnerInd field is not set to Customer, validation occurs and the edit is not allowed.

- A user executes the process that generates the UniqueDistribRefNumbers table. The table is generated and populated with all DistibRefNumbers currently in the GDB.

\subsection{Design}

This tool consists of three components, the AU assigned to DistribRefNumber attribute, a tool to generate the UniqueDistribRefNumbers table and a SAGE tool to allow for the alteration of the UniqueDistribRefNumbers table.

1. The AU does not provide an interface beyond a message to the user that a duplicate DistribRefNumber has been entered. 
2. The code to generate the UniqueDistribRefNumbers table consists of a button within ArcCatalog or a stand alone executable. When successfully executed, a standard message box indicating success is shown.

3. The tool that allows for the editing of a UniqueDistribRefNumber (see the SAGE section of this document).

Figure 4.7-86 is an example of the message box that will be displayed when a duplicate pole number is input.

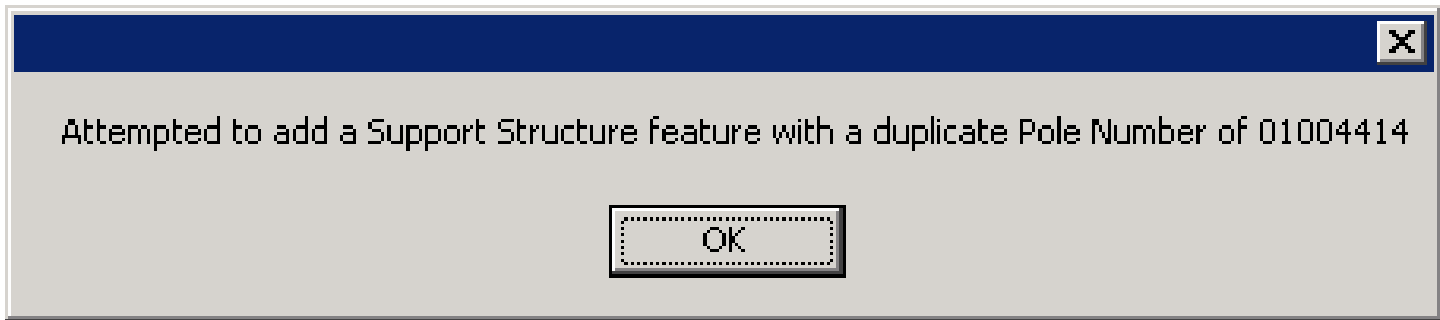

Figure 4.7-86. Attempted use of a duplicate pole

\subsection{FeederAll Open Point Deletion AU}

A composite relationship does not exist between UgConductor and FeederAllOpenPoint. Because of this, deleting an UgConductor feature will leave any connected FeederAllOpenPoint features in place, or in effect orphaned. To prevent orphaned FeederAllOpenPoints from existing, this AU deletes them as part the deletion of an UgConductor deletion.

\subsection{Assign to Classes}

UGConductor

\subsection{Use Case}

A user deletes an UgConductor feature that has FeederAllOpenPoints connected to it. When the conductor is deleted, the FeederAllOpenPoints are also deleted.

\subsection{Design}

1. Add a FeederAllOpenPoint feature to an UgConductor.

2. The UgConductor is split by the FeederAllOpenPoint.

3. The FeederAllOpenPoint is created successfully.

4. Delete an UgConductor which has a FeederAllOpenPoint residing on it.

5. If, after the conductor is deleted, the FeederAllOpenPoint will also be deleted if no other UgConductor is connected to it.

6. If another UgConductor is connected to it then the FeederAllOpenPoint will not be deleted.

\subsection{Transformer Lead Edit Task}

TransformerBank and OhConductor are connected via a TieBus (subtype

TransformerLead). This provides connectivity within the Electric Network since

OhConductor is being offset varying distances from poles (and therefore

TransformerBanks). When a TransformerBank is placed, the edit task requires a user 
click a second time on an OhConductor. Upon creation of the TransformerBank, the TieBus (TransformerLead) feature is also created, spanning from the TransformerBank to the selected OhConductor. Within the Stored Displays, the TransformerLeads themselves will not be visible.

The plan at NIPSCO was to have the Manual Angle Setter edit task applied to TransformerBanks. This edit task already requires two clicks from the user. The first to determine the location of the geometry and the second to determine the angle of rotation for the TransformerBank. Since this custom edit task will override this functionality and use the second click to establish the connection to an OhConductor, we made it three clicks. 1 -geometry, 2 - connection to a OhConductor and 3 - rotation angle.

It should also be noted that per the "Conductor Split at Tap Edit Task", the transformer lead splits the OhConductor at the tap point therefore maintaining the electrical integrity of the network in regard to the $\mathrm{ABB}$ programs.

Note that TransformerLead features are not required for UgConductor/Transformer connectivity since the UgConductor is not being offset from its surface structure. The requirements can be summarized as follows:

- Automatically create a TieBus (TransformerLead) feature when a new Overhead TransformerBank feature is placed.

- Require a 3 click edit sketch. The first click identifies where to place the TransformerBank, the second click indicates which OhConductor it's connected to, and the third click is used to determine the rotation angle of the TransformerBank.

- Connect the TieBus from the TransformerBank feature to the selected OhConductor feature.

- Create the TieBus feature's From/To points based upon the first and second click

- Set the TransformerBank's SymbolRotationValue based upon the angle between the first and third click.

- Automatically populate the TieBus attributes (OnCreate only). Attributes are populated as follows:

- OpenClosedStatusCd is set to Closed $=1$

- PrimaryOperatingVoltage is set to its default

- PhaseDesignationCd matches that of the OHConductor to which it is connected.

- SubtypeCd is set to 3 (TransformerLead)

All other attributes are populated automatically by other AUs, primarily Feeder Manager.

\subsection{Assign to Classes}

TransformerBank (OH Subtypes only)

\subsection{Use Case}

- A user places an Overhead TransformerBank. The first click is on a pole the TransformerBank is to be placed. The second click is on an OHConductor that the user intends the bank to be connect to. The third click determines the rotation 
angle of the bank. After the third click, the TieBus feature is created, it's attributes populated and is then placed between the TransformerBank and the OHConductor. The bank is rotated according to the angle between the first and third click.

- A user places a TransformerBank. On the first click the user does not snap to a pole. The edit is cancelled.

- A user places a TransformerBank. On the second click, the user does not select an OHConductor. The edit is cancelled.

- A user places a TransformerBank. On the second click, the user selects an OHConductor at a point that is not near the first point clicked. The edit is cancelled.

\subsection{Design}

1. Place an $\mathrm{OH}$ TransformerBank by snapping/clicking on a pole, then snapping/clicking on an OHConductor and finally, clicking to set the TransformerBank's rotation angle.

2. The TransformerBank is created and rotated correctly.

3. A TieBus (subtype TransformerLead) feature is created and placed between the TransformerBank and the selected OHConductor.

4. The TieBus attributes are populated correctly.

5. Connectivity exists between the TransformerBank, TieBus and OHConductor.

6. Place an $\mathrm{OH}$ TransformerBank by first clicking somewhere other than on a pole.

7. The edit is cancelled since a pole was not snapped to.

8. Place an $\mathrm{OH}$ TransformerBank by first snapping/clicking on a pole, and second by clicking anywhere but on an OHConductor.

9. The edit is cancelled since an OHConductor was not snapped to.

10. Place an $\mathrm{OH}$ TransformerBank. Click the second time at a distance further than 250 feet from the first click.

11. The edit is cancelled since the distance between the two points is too far apart.

12. Place an UG TransformerBank by connecting it to UG conductor lines.

13. The TransformerBank is created correctly (no TransformerLead). 
Figure 4.7-87 depicts an overhead transformer bank installation. The Transformer Lead is represented by the red line that connects the $\mathrm{OH}$ Primary conductor to the insertion point of the transformer which is at the center of the pole.

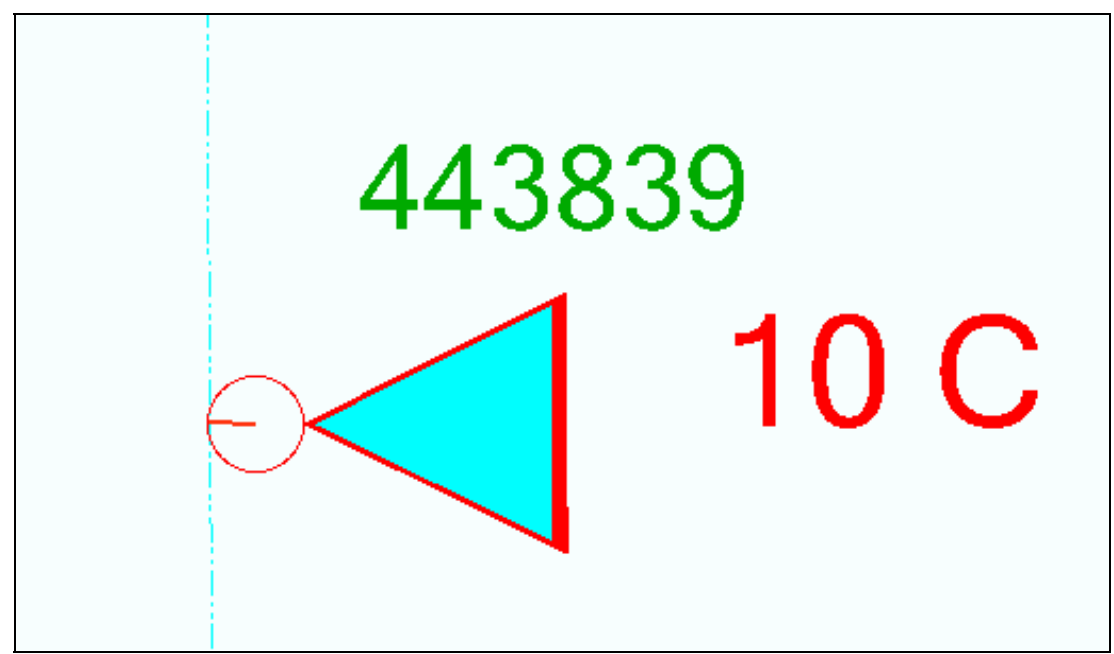

Figure 4.7-87. Overhead transformer bank

\subsection{Delete Attached Transformer Lead}

Transformer Lead features are not visible on the standard NIPSCO stored displays. They are maintained in the data for connectivity purposes but are not shown because the desired symbology is that the transformer is snapped to the pole offset from the conductor. Because the transformer lead is not visible, users are not able to select and delete it when they are deleting a transformer. Therefore this AutoUpdater will automatically delete any attached transformer lead features whenever a transformer bank feature is deleted in the GIS.

\subsection{Assign to Classes}

TransformerBank

\subsection{Use Case}

A user deletes an overhead TransformerBank feature that is connected to the overhead conductor via a TransformerLead feature (subtype of TieBus). When the transformer is deleted, the TransformerLead should also be deleted.

\subsection{Design}

1. Add a new Overhead TransformerBank by using the TransformerBank Edit Task $-1^{\text {st }}$ click on the pole, $2^{\text {nd }}$ click on the conductor at the tap point, $3^{\text {rd }}$ click to indicate the rotation of the TransformerBank.

2. The TransformerBank is created on the pole and attached to the existing overhead conductor via a TieBus. TransformerLead feature.

3. Select only the TransformerBank feature.

4. Delete the TransformerBank feature. 
5. The TransformerLead feature connecting the TransformerBank to the overhead conductor is also deleted from the map.

\subsection{Abandon Gas Features}

The Miner and Miner Abandon Tools are used in the process to abandon gas features. The RetireWONumber, RetireDate, and RetireType of a retired gas feature being collected by this component do not exist before abandonment. Therefore they must be collected at the time of retirement.

The requirements can be summarized as follows. When a gas feature is abandoned, it is moved from its originating feature class to its corresponding Retired feature class. For example, a Drip feature will be moved to the RetiredDrip feature class upon abandonment.

During the abandon process, a form is presented to the user. This form allows the user to fill in the applicable RetireWONumber, RetireDate, and RetireType fields. Once done, the user clicks ' $\mathrm{OK}$ ' and the Miner and Miner abandon process will take over, delete the original feature and create the new feature using the values as input by the user.

\subsection{Assign to Classes}

- Casing

- Drip

- DeadEndGas

- PressureControlFitting

- GasMain

- GasValve

- PipeChange

- RegulatorStation

- TakeStation

\subsection{Use Case}

A user abandons one of the above listed gas features. During this process, the shape of the originating feature plus any attributes configured to be transferred is copied over to its corresponding Retired feature. Along with this, the user is prompted for a RetireWorkOrderNumber, RetireDate, and RetireType information. This information, once collected, will be used to populate the corresponding attributes on the retired feature.

If the user does not indicate the RetireType, Abandoned will be populated as the default.

\subsection{Design}

1. Abandon a gas feature (i.e. Drip, DeadEnd, PressureControlFitting, GasValve, RegulatorStation) using the MM Abandon tools.

2. A form prompts the user for RetireWorkOrderNumber, RetireDate, and RetireType.

3. The feature is abandoned to its corresponding Retired feature class. 
4. The newly created Retired feature's attributes are populated with the data from the form.

Figure 4.7-88 shows the form that is used to collect the work order and abandonment type.

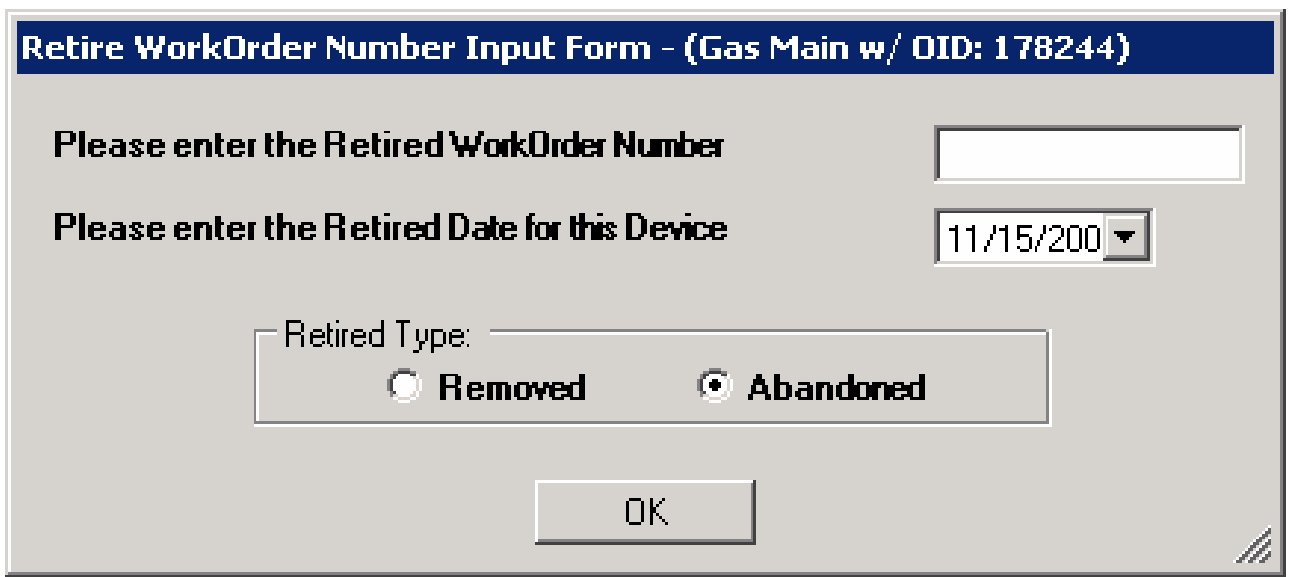

Figure 4.7-88. Form to abandon a feature

\subsection{6}

An AU determines the GasMain that the Casing feature has been snapped to and populates the RelMainObjectId field with the GasMain's OID.

When a user creates/updates a Casing feature, the user will snap it to a GasMain. The AU will populate the RelMainObjectId field of the Casing with the ObjectID of the GasMain.

\subsection{Assign to Classes}

- Casing

- Others as needed

\subsection{Use Case}

A user creates a new Casing feature and places it on a GasMain. The RelMainObjectId field of the Casing feature is populated with the OID of the Main it was snapped to. If the Casing overlies more than one GasMain, the related OID will be based on the last point the user clicked on.

\subsection{Design}

1. Create a Casing feature and place it on a GasMain.

2. The RelMainObjectId field of the Casing feature is populated with the OID of the GasMain on which it resides.

3. Update the Casing feature by moving it to a different GasMain feature.

4. The RelMainObjectId field of the Casing feature is populated with the OID of the GasMain on which it resides.

5. Place a casing feature over more than one GasMain feature. 
6. The RelMainObjectId field of the Casing feature is populated with the OID corresponding to the GasMain that the last sketch point was set on.

\subsection{Place and Relate Gas Main Edit Task}

The edit task is available to be applied to any feature class. This edit task requires two clicks during each sketch. The first click determines where the feature is to be placed. The second click determines which GasMain is to be considered the related GasMain. The OID of the designated GasMain is persisted within the 'RelatedGasMainOID' field. An AutoUpdater will fire twice. First, after the Edit Task executes and second after the ArcFM Segment Split AU executes. The Feature AU will check to see if the OID of the Related GasMain is valid (since the ArcFM Segment Split AU may have deleted it). If it is valid then nothing else is done. If it's not valid, the AU will determine the valid related GasMain based on the second point of the edit sketch. If for some reason the AU is unable to determine this, the value entered into each RelatedGasMainOID field will be zero.

The user is locked into a two click sketching process during the creation of the configured feature. The first click determines where the feature is placed. The second click is used to determine the OID of the related GasMain. The second click uses the point selected and creates an invisible buffer in an effort to find a single feature containing the model name of GasMain. If a feature with the model name GasMain is found, the OID from the GasMain is saved into the 'RelatedGasMainOID' (or similar) field. If this GasMain is then split (deleted and two new GasMains added), the newly created GasMain will be determined and it's ObjectId populated into the RelatedGasMainOID field. If a GasMain cannot be determined, a zero will be placed in the fields and later flagged for manual correction via a QA rule. If a related ObjectId is determined but the GasMain it belongs to is later split, then the ObjectId will not longer be valid. The validation rule will also flag this error for correction.

\subsection{Assign to Classes}

- RegulatorStation

- PipelineMarker

- GasMainLocation

\subsection{Use Case}

- User places a Feature on the edge of a GasMain. The user first clicks on the location where the feature will be placed. The second click is on or near the GasMain that the user deems to be the related GasMain. The Feature is created and the appropriate RelatedGasMainOID populated with the appropriate OID.

- User places a Regulator Station feature at the end of a GasMain. The user first clicks on the location where the feature will be placed. The second click is on or near the GasMain that the user deems to be the related GasMain. The Feature is created and the appropriate RelatedGasMainOID populated with the appropriate OID.

- User places a Regulator Station feature at the end of a GasMain. The related GasMain has yet to be created so the user simply clicks in an area where no other 
GasMains reside. The new feature is created, but neither RelatedGasMainOID field will be populated since it did not exist at the time of creation. The user will either fix this manually or be prompted to do so when QA is run.

- User places a Regulator Station feature and then selects the related GasMain. The data is valid until a later time when a user splits the related GasMain as determined above. Since the GasMain was split, it was effectively deleted and two new GasMains created. Because of this, the original ObjectId as determined during placement of the Feature is no longer valid. When a user runs QA on the Feature in question, it will be flagged as an error since the Objectld as originally assigned is no longer valid.

\subsection{Design}

1. Ensure both the Edit Task and AU are installed and configured according to this document.

2. Start editing and place a Feature within the MM Attribute Editor's Targets Tab.

3. Place a Feature by first clicking on the location where the Feature will reside. The Edit Task is expecting two clicks so click again on a GasMain feature which you intend to be designated as the related GasMain. Once done, the Edit Task will complete and the new feature will be added.

4. Check to ensure RelatedGasMainOID value of the newly created Feature corresponds to the OID of the GasMain designated as the related GasMain and is contained within the correct attribute.

5. Try creating a new Feature at the end of a GasMain and select the GasMain itself as the related GasMain. Repeat step \#4.

6. Try creating a new Feature anywhere between the endpoints of a GasMain and then click again to designate either side as the related GasMain. Repeat step \#4.

7. Try creating a new Feature at the end of a GasMain then select a point that is not near any other GasMain. In this situation, the end user is not designating a related GasMain since it may not yet exist.

8. Check to ensure that the new Feature's RelatedGasMainOID fields contain a value of zero.

9. Note: behind the scenes, the AU is working to ensure that the correct OID is being assigned to the RelatedGasMainOID field depending on whether or not the GasMain in question is being split by the MM Segment Split AU.

\subsection{Retrieve ObjectID of Closest Gas Main}

This AU, when assigned to a field will populate the field with the OID of the GasMain that resides closest to it.

\subsection{Assign to Classes}

- GasMainLocation

- PipeExposure

- PipelineMarker

- Drip

- LeakReports 


\subsection{Use Case}

A user creates/updated a feature with a field where this AU is assigned. The field is populated with the OID of the closest GasMain.

\subsection{Design}

1. Create any of the above features.

2. Once created, the feature should automatically be related to the Gas Main that is closest to it on the map.

\subsection{CP Section-Corrosion Control Number}

When a CPSection feature is created/updated, the DG_GridNumber field will be populated with the 12 digit DGGridNumber of the DGGrid feature it resides within. To build this information, the AU will perform a spatial query to determine which DGGrid the CPSection (point feature) resides within. From this, the DGGridNumber is retrieved and placed into the DG_GridNumber field.

The CorrosionSectionNumber will be populated manually by the end user.

The CorrosionControlNumber field will then be populated based upon the concatenation of the DG_GridNumber along with a dash (-) and the 3 digit CorrosionSectionNumber. Note that if a user enters a CorrosionSectionNumber that is less than three digits, this AU will need to pad out the CorrosionSectionNumber to 3 digits before its concatenated.

The requirements can be summarized as follows:

1. To automate the maintenance of the DG_GridNumber field.

2. To automate the maintenance of the CorrosionControlNumber field.

3. To determine the DGGrid that the CPSection lies within.

4. To determine the DGGridNumber of the DGGrid that the CPSection resides within.

5. To populate the DG_GridNumber field with the number retrieved from the DGGrid feature.

6. To pad out the CorrosionSectionNumber to 3 digits

7. To concatenate the DG_GridNumber, a dash and the CorrosionSectionNumber field value.

8. To take this concatenated value and populate the CorrosionControlNumber field. To execute this functionality when a CPSections is either created or updated.

\subsection{Assign to Classes}

CPSection

\subsection{Use Case}

- A user creates a CPSection feature. During this process, they manually populate the three-digit CorrosionSectionNumber field. When the AU fires, the DG_GridNumber field is populated. Along with this, the CorrosionControlNumber field is populated based upon the concatenation of the 
DG_GridNumber field, a dash and the CorrosionSectionNumber number(padded out to 3 digits).

- A user updates a CPSection feature. During this update, they may change the three-digit CorrosionSectionNumber field. When the AU fires, the DG_GridNumber is updated (if the feature has moved), then the CorrosionControlNumber field is updated with a concatenation of the DG_GridNumber, a dash and the CorrosionSectionNumber number.

\subsection{Design}

1. A user adds a new CPSection feature.

2. During this addition, the CorrosionSectionNumber is manually populated with a number up to three digits in length.

3. The DG_GridNumber is auto populated with the DGGrid number of the DGGrid feature it resides within.

4. The CorrosionControlNumber field is then auto populated with a concatenation of the DG_GridNumber, a dash and the CorrosionSectionNumber. This concatenated value should contain no spaces.

5. A user updates a CPSection feature.

6. During this update, the CorrosionSectionNumber is changed to a number up to three digits in length.

7. The DG_GridNumber is auto populated with the DGGrid number of the DGGrid feature it resides within.

8. The CorrosionControlNumber field is then updated with a concatenation of the DG_GridNumber, a dash and the CorrosionSectionNumber. This concatenated value should contain no spaces.

\subsection{CP Section-Maintenance Tool}

The CP Section tool relates a CPSection to one or more lines of GasMain. A user selects a CPSection, clicks a button and then selects a GasMain. The trace begins at the point where the user clicked the Main. This trace continues until it comes to a point where electricity would no longer be able to flow, such as an insulated fitting or plastic pipe. Once the trace is complete, the pipes found within the trace are highlighted with a red graphic. The user is then prompted to accept or reject the results. If the user accepts the results, the CPSection is related to the pipe(s) found by the trace. If the user rejects the results, the tool exits.

The trace used in this customization leverages the ArcFM CP Trace task and GasTraceWeights.

The requirements can be summarized as follows:

1. Create a tool that automatically relate a CPSection feature to the appropriate GasMain feature(s)

2. Utilize the off-the-shelf ArcFM CP Trace task and GasTraceWeights

3. Implement this functionality via an ESRI Tool(allowing the user to interact with the map). 
Allow a user the choice of accepting or rejecting the results of this tool. The trace determines which GasMain features make up a specific CP section. If this appears incorrect to the user, they can reject the results as needed.

\subsection{Assign to Classes}

This is implemented as a Command. It functions by building relationships between the following two classes:

- GasMain

- CPSection

\subsection{Use Case}

- The end user selects one CPSection feature. The user then clicks the custom tool with a tool tip of 'CPSection Maintenance Tool'. The tool then waits for the user to click within the selection tolerance of a GasMain feature. When done, this initiates the ArcFM CP Trace task. This trace task is dependant upon the GasTraceWeight field which is updated automatically by the ArcFM Solution. Effectively, the trace task identifies all sections of GasMain that are part of one $\mathrm{CP}$ Section. The results are displayed as a red graphic over top of the GasMain feature(s) found within the trace. A user has the ability to determine if a relationship should be created between the CPSection and the results of the CP Trace. A form is presented asking if the user would like to accept or reject the results. If the user accepts the results, the GasMain features found by the trace will be related to the CPSection feature. This is accomplished by populating the GasMain's RelCpSectionObjectId attribute with the OID of the CPSection feature in question. If the user rejects the results no relationships are created.

- The end user updates a CPSection feature by performing the same steps as found in \#1.

- The end user places an insulated fitting between a section of GasMain that used to be one continuous CPSection. The end user then selects the original CPSection feature and follows the steps as outlined in \#1. A new CPSection feature is placed and associated with the appropriate GasMain by following the steps as outlined in $\# 1$.

\subsection{Design}

Standard ESRI tool command button and message boxes are used. The button is always enabled. Only when the user selects the button does code fire to determine if there's a problem. If so, the user is warned and given an appropriate warning. For example, if more than one CPSection is selected, then the tool will give a warning then exit. If no CPSection has been selected, the tool will give the user is given a warning then exits.

Select a CPSection, click the 'Update CPSection' tool, click on a GasMain to start the trace. Observe the red graphics highlighting the results of the trace, respond to 'Accept' or 'Reject' the results. Check to ensure that the GasMain found in the trace now has its RelCpSectionObjectId attribute populated with the OID of the CPSection feature in question. 
Figure 4.7-89 shows a selected CP Section feature and the cursor when the CP Section Maintenance tool is invoked:

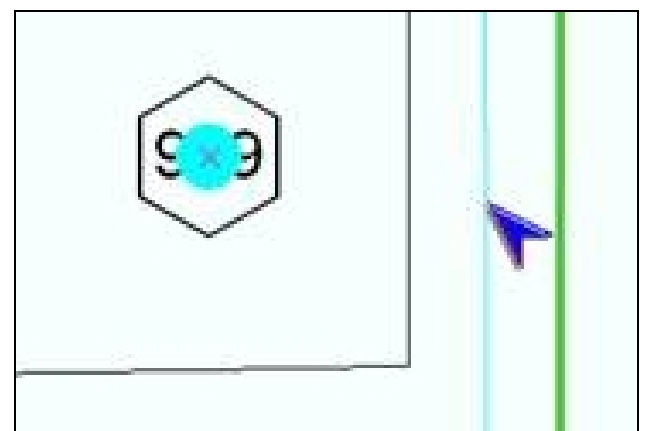

Figure 4.7-89. Standard selection set 
The user then clicks on the Gas Main and the CP trace runs. Figure 4.7-90 shows a completed trace along with the confirmation dialog:

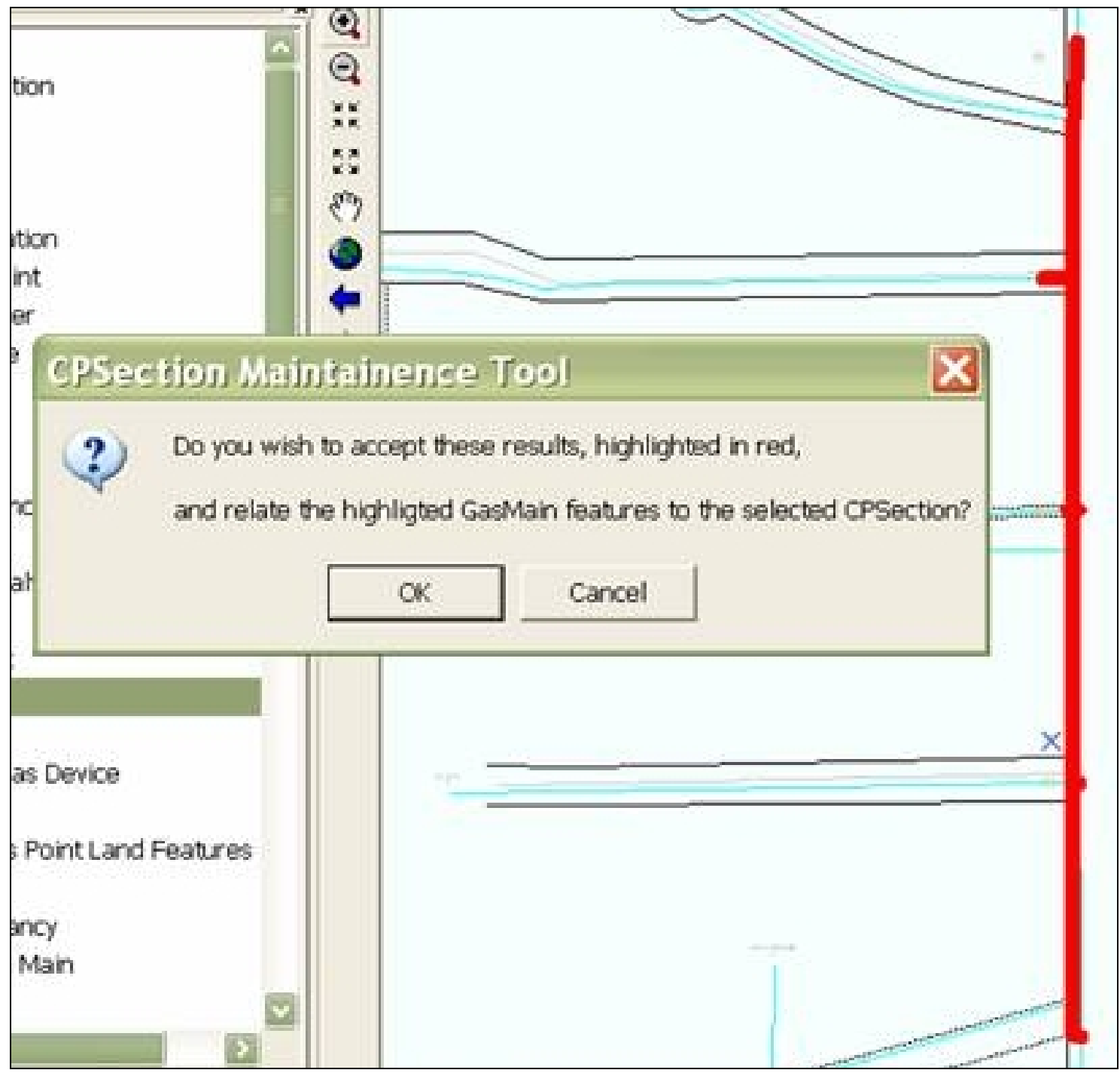

Figure 4.7-90. Completed trace 
After the user clicks OK, the system will create the relationships and then will display a confirmation similar to Figure 4.7-91.

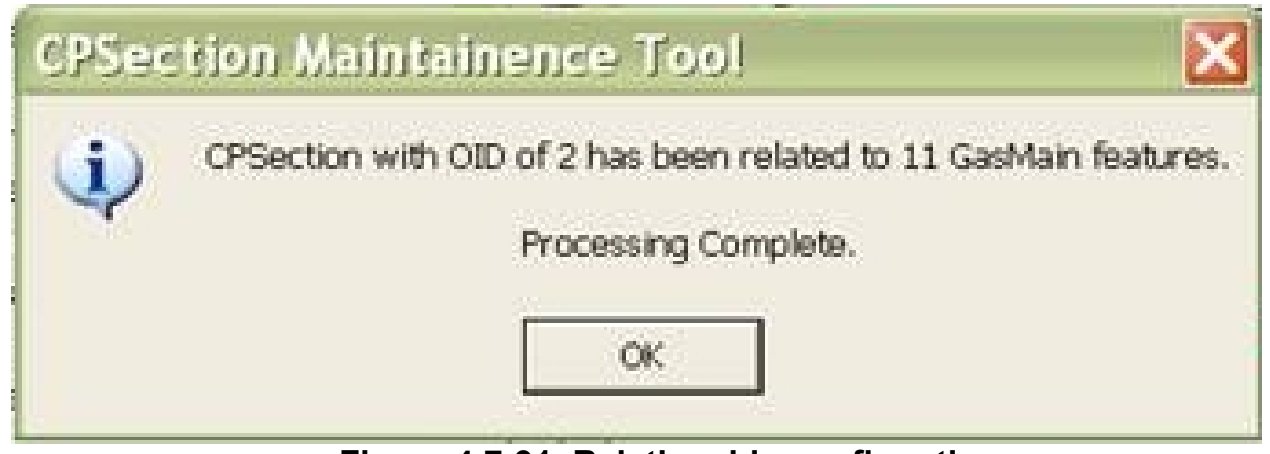

Figure 4.7-91. Relationship confirmation

\subsection{Gas Main Pipe Change}

Functionality was created to automatically detect when the size or material of a newly added or updated GasMain is different from that of the GasMain(s) to which it is connected. If this is found to be the case, the added/updated GasMain creates a new PipeChange feature and places it 6 feet from the intersection of the differing pipe. The PipeChange feature itself rotates and relates itself to the GasMain it resides on.

Functionality was created to automatically detect when the size or material of an updated GasMain is the same as that of connected GasMain. If this is found to be the case and a PipeChange feature currently exists, it will be deleted.

The functionality mentioned above is separated between two autoupdaters as follows. One AutoUpdater (PipeChangeAddorDeleteAU) resides on the GasMain's OnCreate and OnUpdate events. The AU determines if a PipeChange is required and then creates and places it. This AU also determines if a PipeChange is no longer required and deletes it. A second AutoUpdater (PipeChangeRotateRelateAU) resides on the PipeChange's OnCreate and OnUpdate events. This AU rotates the PipeChange so that it's perpendicular to the GasMain it resides on. It also relates itself to the GasMain it resides on.

This AU ensures that PipeChange features are properly created, related, and placed as necessary. Also, when a GasMain is deleted, all related PipeChange features are deleted as well since the relationship between the two is composite.

When the size or material of connected GasMains do not match, a PipeChange feature is required to provide a visual indication. The PipeChange feature is created and placed 6 feet from the point where the pipes intersect (on the added/updated GasMain). This can occur when a new GasMain is being created and taps an existing Main or when the attributes of an existing Main are updated. Also, if a GasMain is updated and no longer different in size or material from the GasMain it's connected to, then any existing PipeChange feature will be deleted. 
The PipeChange is a non-network feature placed 6' down the length of an added/updated pipe.

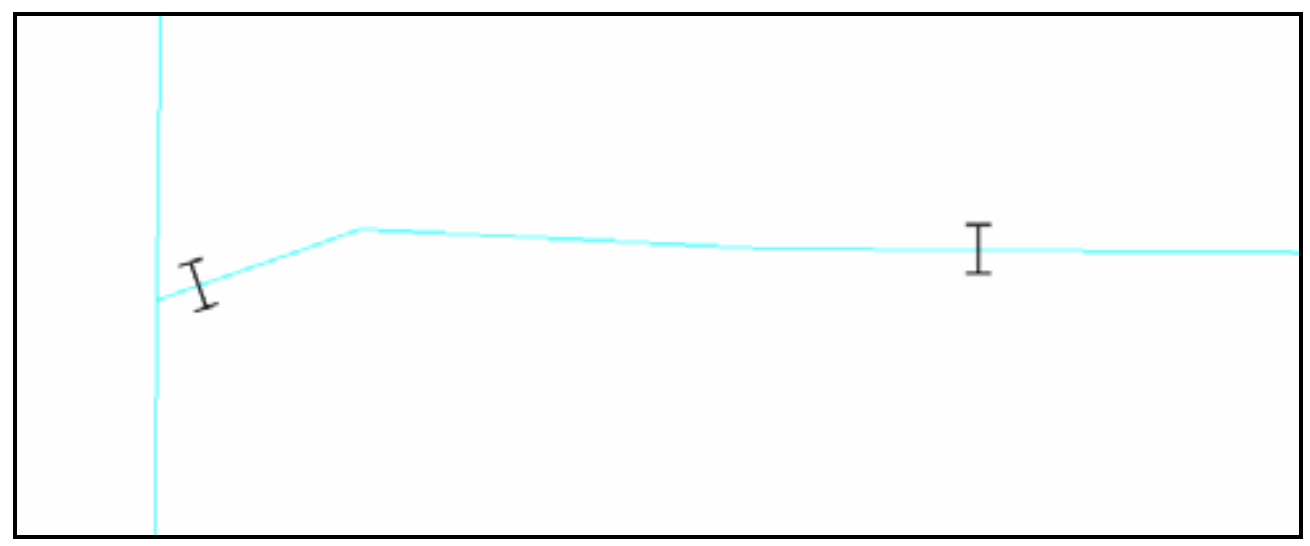

Figure 4.7-92. Pipe change

The pipe change on the left in Figure 4.7-92 shows a pipe that taps into another-the pipe running vertically has a different material than the pipe that taps into it so the PipeChange feature is placed on the horizontal pipe in this case.

This customization also rotates the Pipe Change feature to orient it perpendicular with the underlying GasMain. Since the Pipe Change feature is a non-network feature, the out-ofthe-box ArcFM Auto Angle Setter AU cannot be used because it is designed to work on network features. There is a developer's sample AU (and source code) that ships with ArcFM that does rotate a non-network feature, and that AU does work fine with the PipeChange and GasMain.

\subsection{Assign to Classes}

- GasMain - PipeChangeAU

- PipeChange - RotateRelateAU

\subsection{Use Case}

1. A user adds a GasMain feature, which ultimately is connected to another GasMain. If the GasMain that's added is different in size or material from any GasMain at either (from or to) junction, a PipeChange feature is created and added 6' down from the junction where the change exists. The PipeChange is rotated so that it's perpendicular to the GasMain and it's also related to the GasMain.

2. A user updates an existing GasMain feature by moving it or changing its attribute values. After this occurs, if the GasMain that's updated is different in size or material from GasMain at any junction, a PipeChange feature is created and added 6' down from the junction where the change exists. The PipeChange is rotated so that it's perpendicular to the GasMain and it's also related to the GasMain.

3. A user updates an existing GasMain feature by moving it or changing its attribute values. After this occurs, if the GasMain that's updated is no longer different in 
size or material from GasMain at any junction and a PipeChange feature exists 6' down from the junction where the change used to exist, the PipeChange feature is deleted.

\subsection{Design}

Adding a New GasMain Feature of a different Size:

- Add a new GasMain and ensure its size is different than that of the GasMain it will be connected to. Connect it to the end of an existing GasMain.

- A PipeChange feature is created.

- The PipeChange feature is related to the GasMain it resides on.

- The PipeChange feature is placed on the newly added GasMain.

- The PipeChange feature offset 6 feet from the end of the GasMain.

- The PipeChange feature is rotated so that its symbology is perpendicular to the GasMain it resides on.

Adding a New GasMain Feature of a different Material:

- Add a new GasMain and ensure its material is different than that of the GasMain to which it will be connected. Connect it to the end of an existing GasMain.

- A PipeChange feature is created.

- The PipeChange feature is related to the GasMain it resides on.

- The PipeChange feature is placed on the newly added GasMain.

- The PipeChange feature is offset 6 feet from the end of the GasMain.

- The PipeChange feature is rotated so that its symbology is perpendicular to the GasMain it resides on.

Updating a GasMain Feature to a different Size:

- Update an existing GasMain and ensure its material is different than that of the GasMain it's connected to.

- A PipeChange feature is created if it did not already exist.

- The PipeChange feature is related to the GasMain it resides on.

- The PipeChange feature is placed on the newly added GasMain.

- The PipeChange feature is offset 6 feet from the end of the GasMain.

- The PipeChange feature is rotated so that its symbology is perpendicular to the GasMain it resides on.

Updating a GasMain Feature to a different Material:

- Update an existing GasMain and ensure its material is different than that of the GasMain it's connected to.

- A PipeChange feature is created if it did not already exist.

- The PipeChange feature is related to the GasMain it resides on.

- The PipeChange feature is placed on the newly added GasMain.

- The PipeChange feature is offset 6 feet from the end of the GasMain.

- The PipeChange feature is rotated so that its symbology is perpendicular to the GasMain it resides on. 
Updating a GasMain Feature to the same Size and Material:

- Update an existing GasMain and ensure its size and material are the same as that of the GasMain to which it is connected. Also ensure that the GasMain being updated currently has a PipeChange feature on it.

- The PipeChange feature is deleted.

Deleting a GasMain:

When a Gas Main with PipeChange features is deleted, all PipeChange features are also deleted.

\subsection{Emergency Valve Indicator}

The AutoUpdater will automatically create, relate and place an EmergencyValveMarker feature on the map when a GasValve feature is created with or has its EmergencyValveInd field update to 'Yes'. The AU will automatically delete an EmergencyValveMarker feature from the map when a GasValve feature has its EmergencyValveInd field updated to 'No'. This AU will also place the EmergencyValveMarker symbol and its associated annotation in a location that is considered a best fit.

Benefits of this AU:

1. This AU saves the end user from having to remember to:

- create and place the EmergencyValveMarker feature each time one is required

- relate the GasValve in question to the newly created emergencyValveMarker feature

- place the related EmergencyValveMarker annotation

- delete the EmergencyValveMarker feature when needed.

2. Since EmergencyValveMarker features are automatically added and removed when necessary, this AU effectively maintains the proper map display without having to rely on the end user memory and manual input.

3. Since EmergencyValveMarker features are automatically added and removed when necessary, this $\mathrm{AU}$ effectively maintains data integrity without having to rely on the end user memory and manual input.

It should be noted that the GasValves which have their EmergencyValveInd field set to 'Yes' are one of the most critical infrastructure items within the gas model. Therefore, maintaining properly related and displayed EmergencyValveMarker features along with their corresponding annotation should be considered equally as important.

\subsection{Assign to Classes}

GasValve 


\subsection{Use Case}

- GIS end user places/changes a GasValve feature so that its EmergencyValveInd is set to 'Yes'. When this occurs, a EmergencyValveMarker feature and its associated annotation is placed/rotated in an appropriate position near the GasValve in question.

- GIS end user changes a GasValve feature so that its EmergencyValveInd is set to 'No'. This will cause any related EmergencyValveMarker feature to be deleted.

\subsection{Design}

When a Gas Valve feature's EmergencyValveInd is set to 'Yes', an EmergencyValveMarker feature is automatically created, related to the Gas Valve in question and place near the GasValve in question. Along with placing the EmergencyValveMarker feature, its related annotation is also placed within the EmergencyValveMarker's symbol and rotated. When a GasValve's EmergencyValveInd is set to 'No, its associated EmergencyValveMarker feature is automatically deleted. The primary purpose is to alleviate the end user from having to remember to create/delete these features and to eliminate the manual steps that go along with this process. This AU may not place annotation in the most optimal location and therefore may require user interaction to do so.

When a GasValve feature's EmergencyValveInd attribute is changed, an attribute AU will fire. First, the AU determines if the GasValve is being set as an emergency GasValve. If it is being set to an emergency GasValve, the AU checks if the GasValve has an EmergencyValveMarker feature related to it. If it does not(most likely), the AU will create an EmergencyValveMarker feature and populate its values. The shape of this feature will be similar to the GasValve with the exception that it's offset from the Gas Valve to avoid overlapping. The SymbolRotationValue will be set and adjusted appropriately so that it resides at one of the following angles(45, 135, 225 or 315 degrees). Its LabelText will be populated with the ValveNumber of the GasValve. Once this is done, the EmergencyValveMarker will have it's corresponding annotation created and placed as per standard ArcMap functionality. The GasValve will be related to the EmergencyValveMarker which in turn will be related to its annotation. The annotation will also be rotated in a similar fashion to the EmergencyValveMarker and reside within this feature.

Because functionality executes within an $\mathrm{AU}$, there is no user interaction while the features are being created. After the placement is complete, a user is free to adjust the placement using standard ArcMap functionality.

If a GasValve is no longer designated an emergency valve, the AU will find all related EmergencyValveMarker features and delete them. This will automatically remove any annotation related to the EmergencyValveMarker as well. 
Note: ArcMap's automatic screen refresh does not always execute as it should. Therefore the AU does a screen refresh for the user after the above noted changes have been made. This makes for a much cleaner and less confusing map display while editing.

\subsection{Valve Number AU}

An AutoUpdater is required to maintain the LabelText field value on the EmergencyValveMarker feature class. The EmergencyValveMarker feature has featurelinked annotation that is driven from the LabelText field. Figure 4.7-93 shows an emergency valve marker.

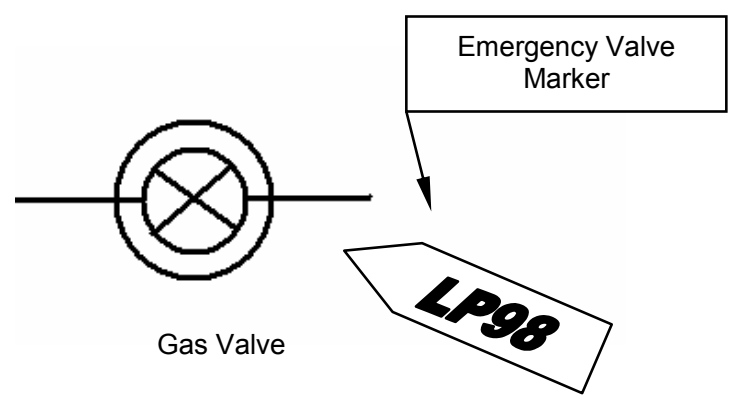

Figure 4.7-93. Emergency valve marker

This LabelText field reflects the ValveNumber of the Gas Valve feature that it's related to. When the ValveNumber field of the GasValve feature is updated, this update is propagated to the LabelText field of any related EmergencyValveMarkers.

This AU will compliment the CreateRelateRotateEmergencyValveMarkerAU. The CreateRelateRotateEmergencyValveMarkerAU fires when a GasValve's EmergencyValveInd field is set to 'Yes'. This AU is set on the OnUpdate event and the OnCreate event of the GasValve feature class. When this occurs, an EmergencyValveMarker feature is created and related to the GasValve and the Label text field of the related EmergencyValveMarker is populated with the GasValve's valve number. The ValveNumberAU only addresses the situation where a GasValve's valve number is updated. This update is propagated to the EmergencyValveMarker's label text field as discussed above.

\subsection{Assign to Classes}

GasValve

\subsection{Use Case}

A user updates the 'valvenumber' attribute on a GasValve feature causing any related EmergencyValveMarker features to update the 'labeltext' attribute with the name value. 


\subsection{Design}

When the 'valvenumber' field of a GasValve feature is updated, this change is propagated to the 'labeltext' field of any related EmergencyValveMarker features.

1. Select a GasValve feature which has a related EmergencyGasValve feature.

If none can be found, change an existing GasValve 'emergencyvalveind' field to 'yes'. This will create a related EmergencyValveMarker.

2. Update the 'valvenumber' attribute on the GasValve feature.

3. Verify the 'labeltext' value on all related EmergencyValveMarker feature is the same as the 'valvenumber' value on the GasValve.

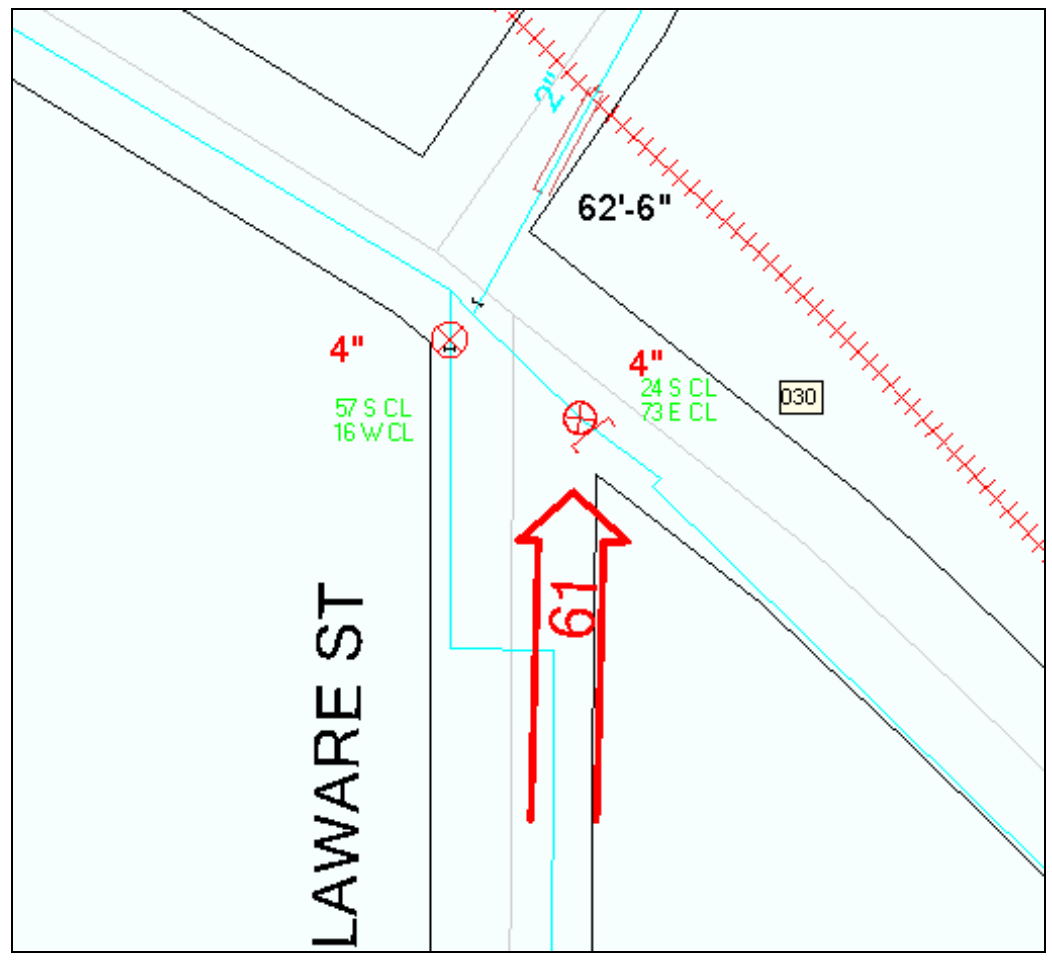

Figure 4.7-94. Emergency valve marker installation in the AEDR system

\subsection{Custom Identify Tool}

The ESRI product contains a standard feature Identify Tool. This tool is used to click on any location on the map and display all features at that location along with their attributes. This tool works very well but it displays all attributes of the features including the system fields that were meant to be hidden via the ArcFM configuration. For this reason, a custom Identify Tool was developed for the AEDR system. It works in an identical manner to the ESRI tool but it honors the ArcFM configuration and does not display any hidden fields. Figure 4.7-95 shows the custom Identify Tool. 


\begin{tabular}{|c|c|c|}
\hline AEDR Identify Tool & & \multirow[t]{2}{*}{ 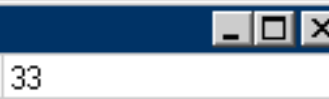 } \\
\hline \multirow{20}{*}{ 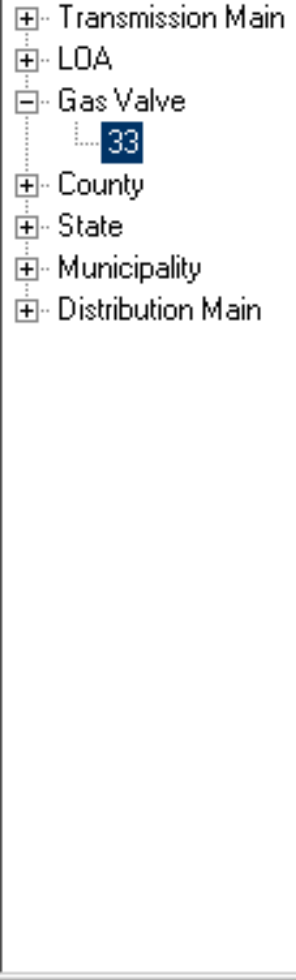 } & OBJECTID & \\
\hline & INSTALLDATE & \\
\hline & Install Work Order & UNK \\
\hline & LOA Name & SOUTH BEND \\
\hline & Tax Unit & \\
\hline & Grid Code & \\
\hline & Pressure System Status Cod & No \\
\hline & Emergency Isolation System & No \\
\hline & Gas System Status Code & No \\
\hline & Subtype Code & DistributionValve \\
\hline & Valve Number & 52 \\
\hline & Valve Size & 3 \\
\hline & Location Description 1 & $120 \mathrm{E} \mathrm{CL}$ \\
\hline & Location Description 2 & $49 \mathrm{~S} \mathrm{CL}$ \\
\hline & Insulated Indicator & No \\
\hline & Open/Closed & Open \\
\hline & Emergency Valve & Yes \\
\hline & Installation Type & Buried \\
\hline & Install Misc Order Id & wo \\
\hline & \multicolumn{2}{|l|}{1} \\
\hline
\end{tabular}

Figure 4.7-95. Custom identify tool

The attributes for a feature are viewed by selecting the object id of the feature in the left hand pane.

\subsection{Custom Structure Relate}

The ArcFM product contains a Structure Relate AutoUpdater with the following description:

Searches within a tolerance for features with a model name of 'Structure' (default tolerance is 25 map units). If found, then a new relationship is created between the placed feature and the structure feature. The search tolerance is 25 map units. This value is hard-coded and may be modified by customizing the shared product code.

When this was tested at NIPSCO, it was found that the product code actually used a 25 map unit square (measured on the diagonal) to do the spatial search instead of a 25 map unit radius of a circle as expected. This resulted in dead spots within the 25 map unit radius of a circle which caused some structures to not be related correctly. The product code was attained from Miner \& Miner and two custom versions of this AutoUpdater were implemented with different (larger) map units. The first was used for all standard structure relates (devices/etc to pole/pad). The second was implemented to relate a substation breaker to the substation feature. 


\subsubsection{QAQC Tools}

NIPSCO employs two QAQC users to validate, approve, and post the work of over sixty editors. This amounts to a very large amount of work. Two tools were developed to assist the QAQC users in this task.

\subsection{Delete Report}

The delete was created as a very simple way to pull a list of all features that have been deleted in the current version/session. Without this step in place, there was no way for the QAQC users to know if anything had been deleted from the current session. This tool was written before the "All Edits Report" (see next section) which includes more detailed information on the deletions. The Delete Report was a quick and dirty way to extract deletions from the system. It operates by querying the ESRI versioning "Delete" tables for any edits committed in the current version/session. The report is available by clicking an ArcMap button while editing any version/session. An example of the report is shown in Figure 4.7-96. 


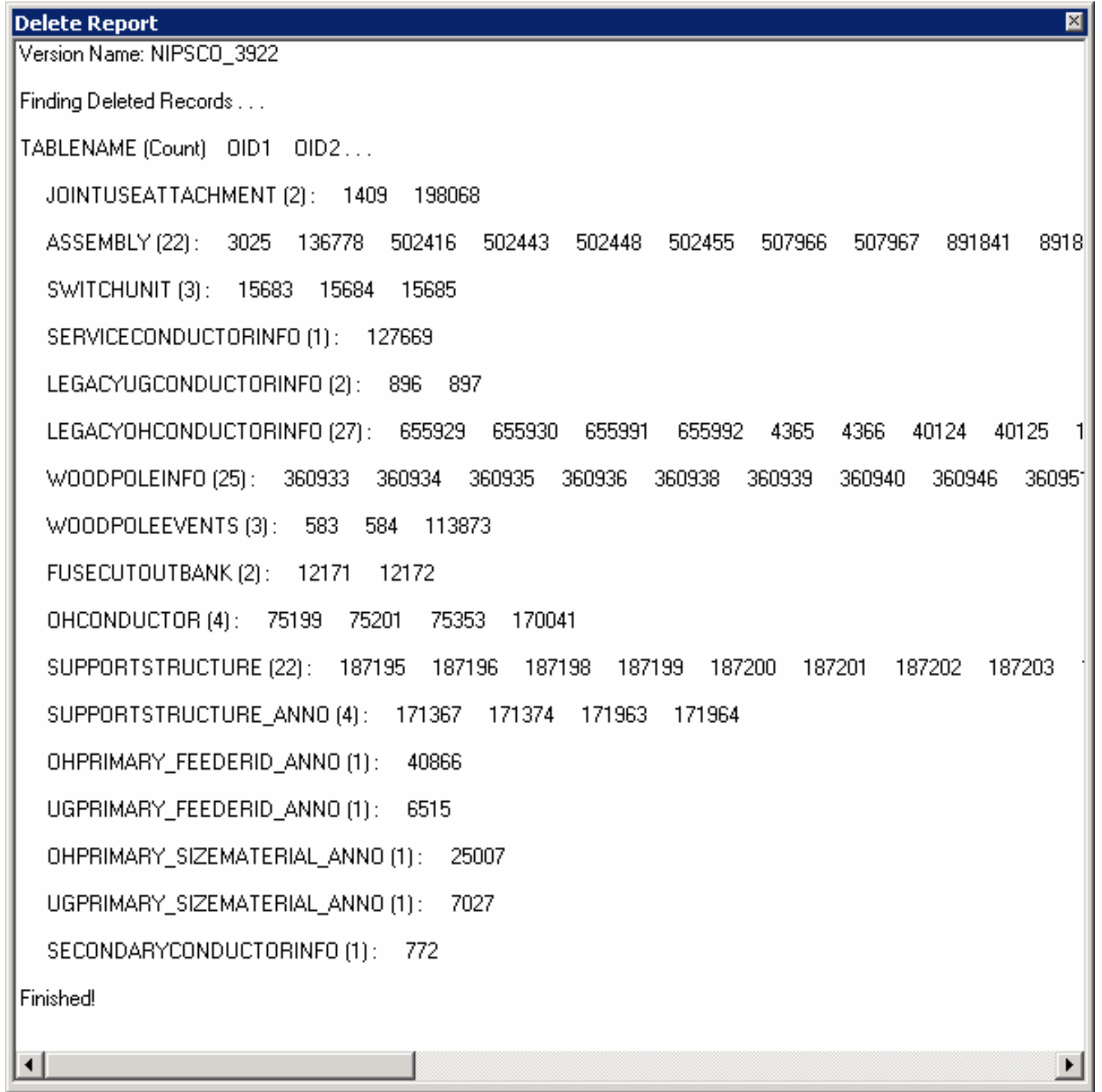

Figure 4.7-96.

The report simply displays the table name, the count of deleted records, and the object id of the deleted records. This works as a simple approach but does not provide an easy way to find more information on the deleted records or to export the report. These more advanced requirements were rolled into the creation of the All Edits Report.

\subsection{All Edits Report}

The All Edits Report was developed as a single point of access for the QA/QC of any session/version. It includes both basic and advanced tools to view all new records, updated records, and deleted records. The report utilizes an ESRI reconcile operation to obtain a list of all edited tables in the current version/session. Then each table is queried with a difference cursor to locate the details of each individual edit. The report is 
launched by clicking an ArcMap button. The basic user interface is shown below and the tools are detailed in the following sections.

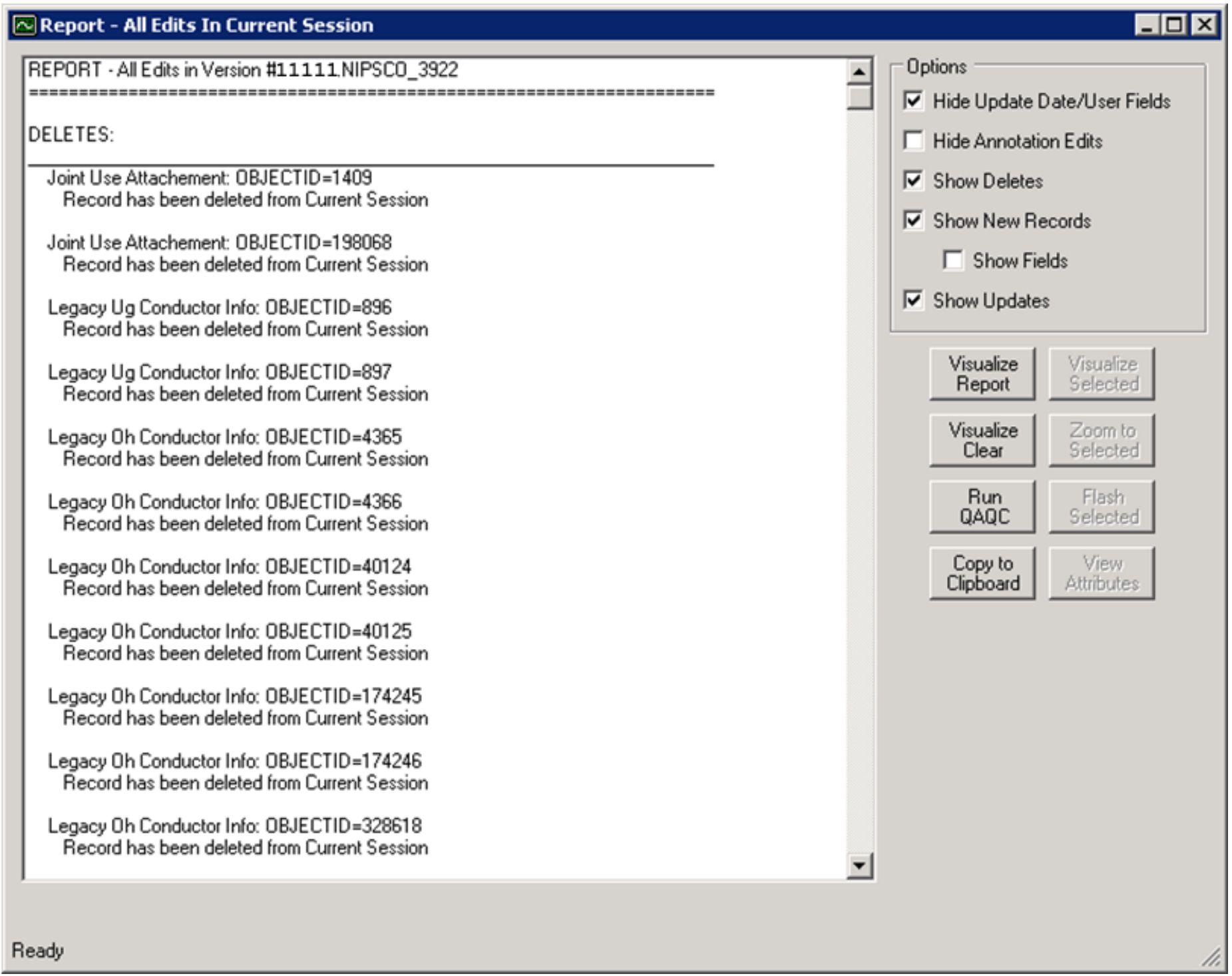

Figure 4.7-97. All edits report

The basic report is text based and is categorized by the edit type (delete, new record, or update). At the root, the display includes the table name, the object id, and the details of the edit.

\subsection{Record Deletions}

The initial display of a deletion includes only high level information including the table name and the object id. An example of a deleted record would look like Figure 4.7-98.

DELETES :

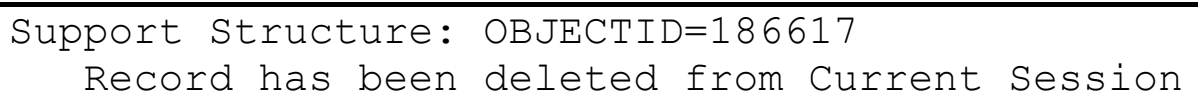

Figure 4.7-98. Record deletion 
The user can then select the entry in the report and click the "View Attributes" button. This will launch the edit report attribute details form shown in Figure 4.7-99 which displays the attributes of the feature from the parent version and shows that the record has been deleted in the current version.

\begin{tabular}{|c|c|c|}
\hline \multicolumn{3}{|c|}{ 圆Edits Report - Attribute Details } \\
\hline \multicolumn{3}{|c|}{$\begin{array}{l}\text { Support Structure: OBJECTID }=186617 \\
\text { Record has been deleted from Current Session }\end{array}$} \\
\hline Attribute & Current Session & Parent Session \\
\hline OB.JECTID & DELETED & 186617 \\
\hline CreationDate & DELETED & 5/11/2006 11:49:59 AM \\
\hline Created $\mathrm{By}$ & DELETED & Migration \\
\hline UPDATEDATE & DELETED & 4/11/2000 12:00:00 AM \\
\hline Updated By & DELETED & FDSI \\
\hline Legacy Ees Number & DELETED & 31570231 \\
\hline LOA & DELETED & HAMMOND \\
\hline Grid & DELETED & BD16A \\
\hline INSTALLDATE & DELETED & 11/27/1979 12:00:00 AM \\
\hline Install W0 Number & DELETED & MO \\
\hline Install Misc Order Id & DELETED & M08588-1 \\
\hline RETIREDATE & DELETED & \\
\hline Retire WO Number & DELETED & \\
\hline Retire Misc Order Id & DELETED & \\
\hline Tax Unit & DELETED & M34 \\
\hline LEGACYWRINSTALLTIME & DELETED & 2/11/1993 1:35:24 PM \\
\hline LEGACYWRREMOVALTIM & DELETED & \\
\hline Symbol Rotation & DELETED & 0 \\
\hline Graphics Scale Factor & DELETED & 1 \\
\hline Subtype & DELETED & Pole \\
\hline Location Description & DELETED & E.S. US 41 5TH P.S. US 30 \\
\hline Pole Height & DELETED & 40 \\
\hline Pole Material & DELETED & Wood \\
\hline Primary Pole Number & DELETED & \\
\hline Foreign Dwner Name & DELETED & NIPSCO \\
\hline Service PointType & DELETED & No Service Point \\
\hline Transmission Structure Num & DELETED & \\
\hline INSTALLTIMESTAMP & DELETED & 2/11/1993 1:37:13 PM \\
\hline REMOVALTIMESTAMP & DELETED & \\
\hline Pole Number & DELETED & 00094401 \\
\hline
\end{tabular}

Figure 4.7-99. Record deletion report

The user can use this data to discover all of the key asset information about the deleted asset. This is crucial in discovering if the asset was deleted in error. With the report line still selected, the user can then click the "Visualize Selected" button if the deletion was a feature (i.e. not an object). The map will be automatically zoomed to the location where the feature previously existed and the previous point or linear geometry will be drawn in red as shown in Figure 4.7-100. 


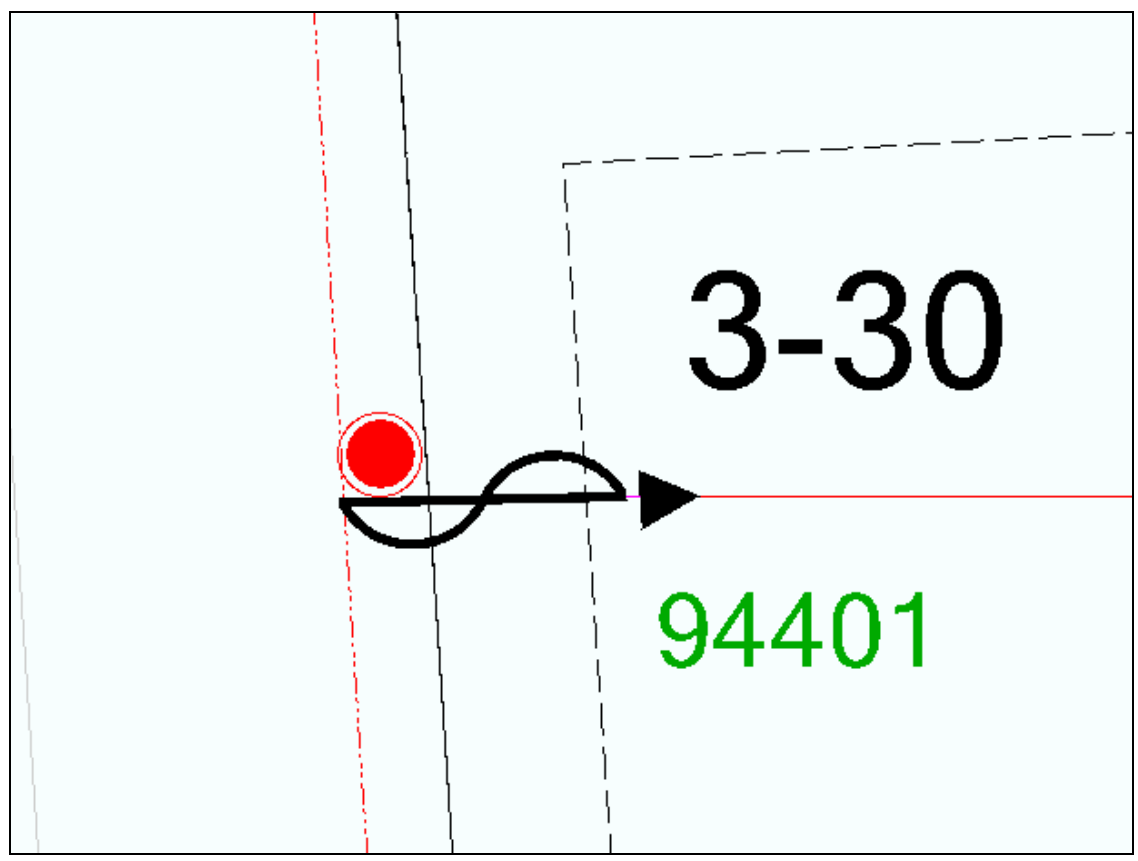

Figure 4.7-100. Visual indicator to the user as to where the asset was located before it was deleted

\subsection{New Records}

The initial display of a new record includes only the high level information including the table name and the object id. Figure 4.7-101 shows an example of a new record.

\section{NEW RECORDS:}

Oh Conductor: OBJECTID $=203272$

NEW record was created in Current Session

Figure 4.7-101. Record added

With New Records, the user can check the "Show Fields" check box to add all textual fields to the report as shown in Figure 4.7-102.

\section{Show New Records}

ॠ Show Fields

Figure 4.7-102. Show

fields checkbox 
Figure 4.7-103 shows the same record in the report expanded.

NEW RECORDS:

Oh Conductor: OBJECTID $=203272$

NEW record was created in Current Session

OBJECTID = 203272

Enabled $=$ True

CreationDate $=10 / 20 / 2006$ 12:00:00 AM

Created By $=\# 999999$

UPDATEDATE = 3/17/2006 12:00:00 AM

Updated By $=$ \#999999

Legacy Node $1=41481970$

Legacy Node $2=31570556$

Legacy Ees Number $=41481971$

LOA Number $=$ HAMMOND

Grid $=$ BD17D

Measured Length $=66.88$

Electric Trace Weight $=1879048192$

Feeder Manager Non-Traceable $=$

Feeder Information $=7$

Circuit Number $=12-175$

Circuit Number $2=$

Primary Operating Voltage $=12.5 \mathrm{KV}$

Phase $=A B C$

Owner $=$ NIPSCO

Legacy Circuit Number $=12-175$

Subtype $=$ ThreePhaseOverheadPrimary

Wire Size $=2$

Wire Material $=$ Aluminum Alloy

SHAPE.len $=66.8875459633555$

Figure 4.7-103. New record expanded when

the "show fields" checkbox is enabled

This added detail provides the QAQC user with much more information on the edit. In this case we can see that this new record was likely the result of a split operation on an existing conductor. This is evident by noting that several migration fields are populated (an end user would not have access). 
Alternatively, the user could also click the "View Attributes" button to launch the All Edits Attribute Details form.

\begin{tabular}{|c|c|c|c|}
\hline \multicolumn{3}{|c|}{ Edits Report - Attribute Details } & $\square \times$ \\
\hline \multicolumn{4}{|c|}{$\begin{array}{l}\text { Oh Conductor: OBJECTID }=203272 \\
\text { NEW record was created in Current Session }\end{array}$} \\
\hline Attribute & Current Session & Parent Session & \\
\hline OBJECTID & 203272 & $N / A$ & \\
\hline Enabled & True & $N / A$ & \\
\hline CreationDate & 10/20/2006 12:00:00 AM & $N / A$ & \\
\hline Created By & & $N / A$ & \\
\hline UPDATEDATE & 3/17/2006 12:00:00 AM & $N / A$ & \\
\hline Updated By & & $N / A$ & \\
\hline Legacy Node 1 & 41481970 & $N / A$ & \\
\hline Legacy Node 2 & 31570556 & $N / A$ & \\
\hline Legacy Ees Number & 41481971 & $N / A$ & \\
\hline LOA Number & HAMMOND & $N / A$ & \\
\hline Grid & BD17D & $N / A$ & \\
\hline Measured Length & 66.88 & $N / A$ & \\
\hline Electric Trace Weight & 1879048192 & $N / A$ & \\
\hline Feeder Manager Non-Trace & & $N / A$ & \\
\hline Feeder Information & 7 & $N / A$ & \\
\hline Circuit Number & $12-175$ & $N / A$ & \\
\hline Circuit Number 2 & & $N / A$ & \\
\hline Primary Operating Voltage & $12.5 \mathrm{KV}$ & $N / A$ & \\
\hline Phase & $\mathrm{ABC}$ & $N / A$ & \\
\hline Owner & NIPSCO & $N / A$ & \\
\hline Legacy Circuit Number & $12 \cdot 175$ & $N / A$ & \\
\hline Subtype & ThreePhase0 verheadPrimar & $N / A$ & \\
\hline Wire Size & 2 & $N / A$ & \\
\hline Wire Material & Aluminum Alloy & $N / A$ & \\
\hline SHAPE.len & 66.8875459633555 & $N / A$ & \\
\hline
\end{tabular}

Figure 4.7-104. All edits attribute details form depicts the same data for the new record and shows that the record does not exist in the parent session 
With the report line still selected, the user can then click the "Visualize Selected" button if the new record was a feature (i.e. not an object). The map will be automatically zoomed to the location where the feature has been created and the point or linear geometry will be drawn in blue as shown in Figure 4.7-105.

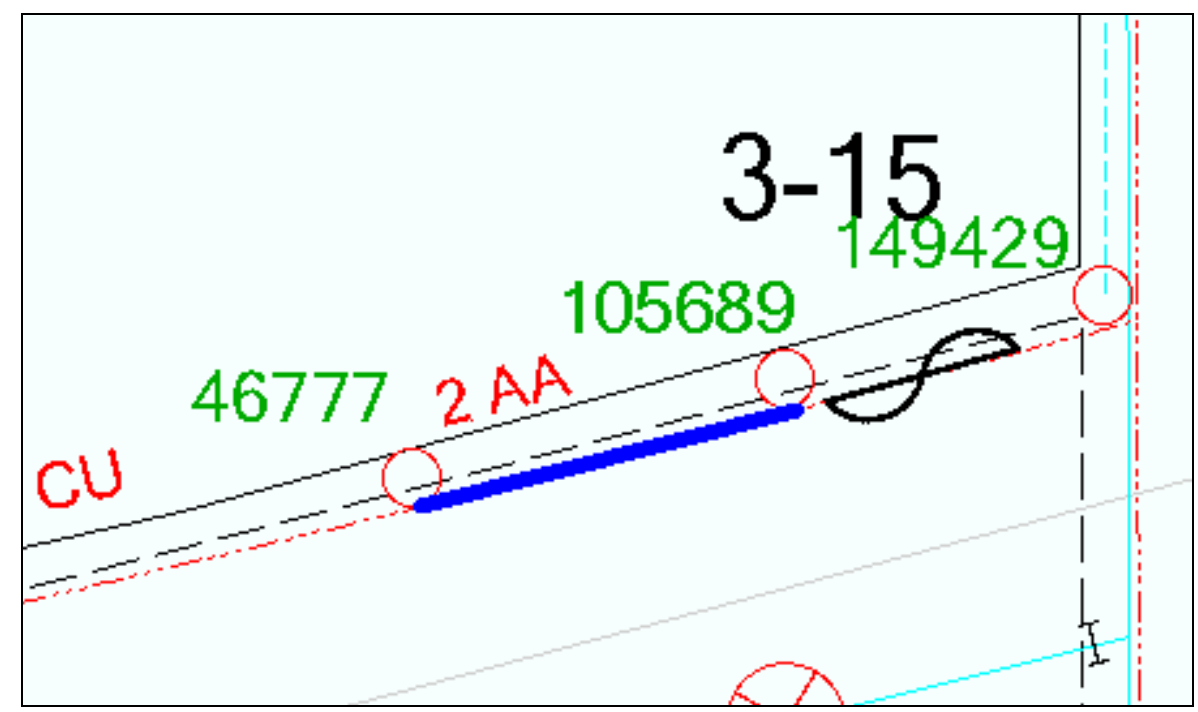

Figure 4.7-105. Visualized selected provides a visual indicator to the user showing where the asset was originally located

\subsection{Updates}

The initial display of an updated record includes the high level information including the table name and the object id. It also includes the attribute level details of the update. An example of a updated record is shown in Figure 4.7-106.

UPDATES:

Legacy Oh Conductor Info: OBJECTID=655992

Record has been updated in Current Session

* Field UPDATEDATE changed:

- value in Parent Session =

- value in Current Session = 10/20/2006 12:00:00 AM

* Field Updated By changed:

- value in Parent Session =

- value in Current Session = \#999999

* Field RETIREDATE changed:

- value in Parent Session $=$

- value in Current Session = 1/15/2005 7:36:45 AM

* Field Retire WO Number changed:

- value in Parent Session =

- value in Current Session = WO53529-1

* Field Retire Misc Order Id changed:

- value in Parent Session =

- value in Current Session $=$ WO

${ }^{*}$ Field Subtype changed:

- value in Parent Session $=$ OhConductor 
This detail tells the QAQC user exactly what was updated on the record. In this case, we can easily tell that this record was retired by noting the change in Subtype as well as the populated retire date and retire work order.

If the QAQC user needs additional information on the record, they can select the entry in the report and click the "View Attributes" button. This will launch the edit report attribute details form which shows the attributes of the feature from the current session AND the parent session side by side as shown in Figure 4.7-107.

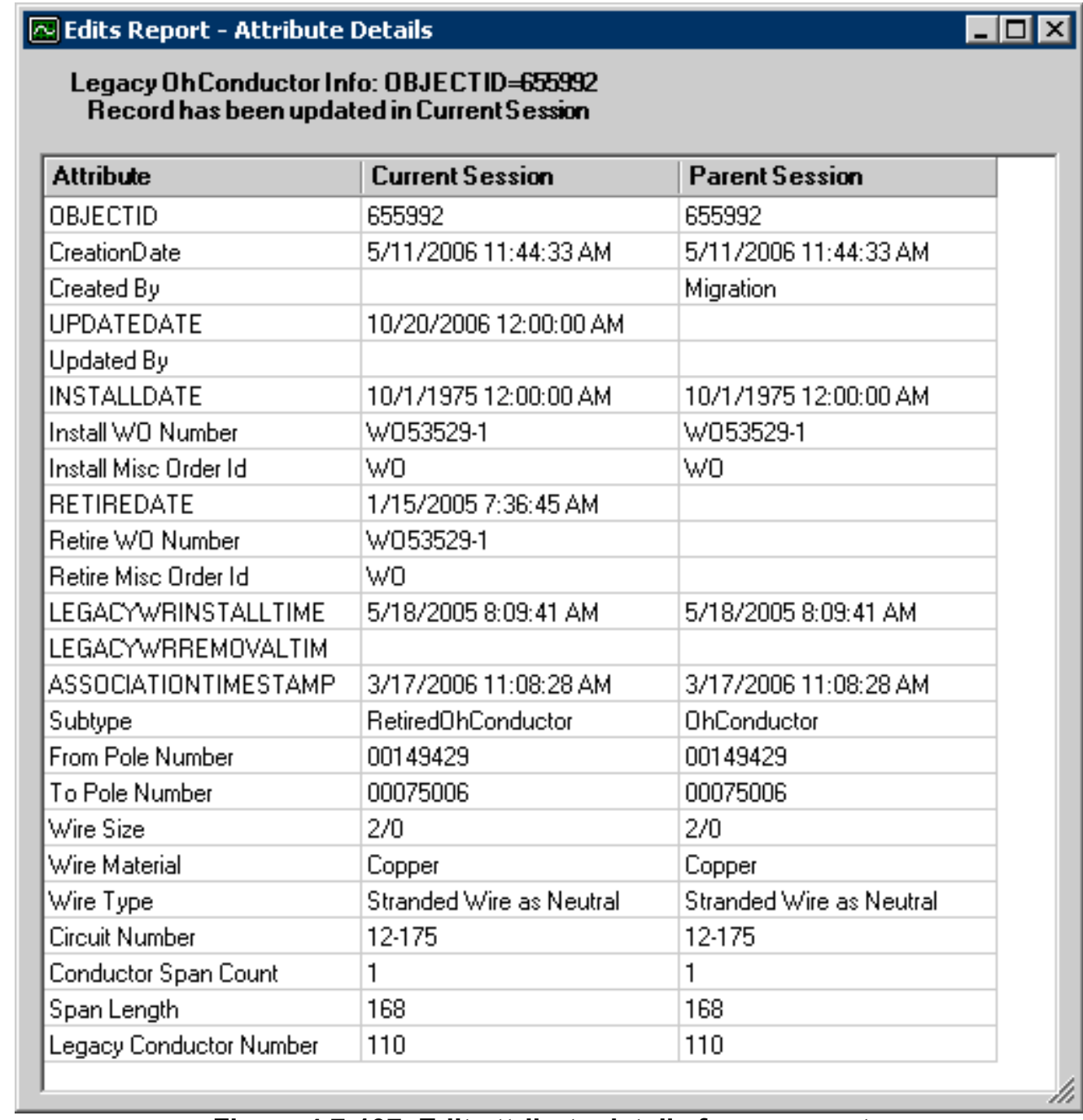

Figure 4.7-107. Edit attribute details form presents an easy to read format for the QAQC user to review all information about the edit

If the updated record is a feature, the user can click the "Visualize Selected" button to view the location of the updated feature. The map will zoom to the location and the updated feature will be displayed in green as shown in Figure 4.7-108. 


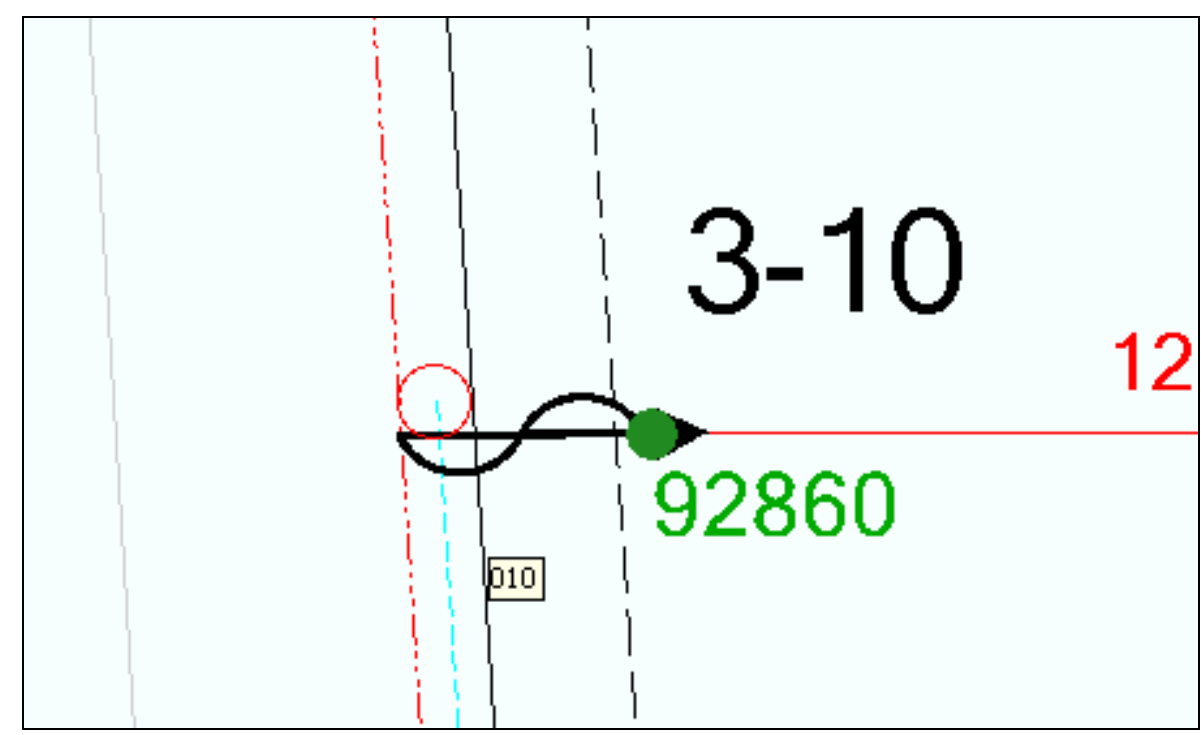

Figure 4.7-108. Updated fuse cutout bank

If a feature has been spatially edited, the textual update entry will include an entry that specifies "Geometry has changed". Then when the user clicks the "Visualize Selected" button, the original geometry from the parent version is shown in orange and the new geometry from the current version is shown in green.

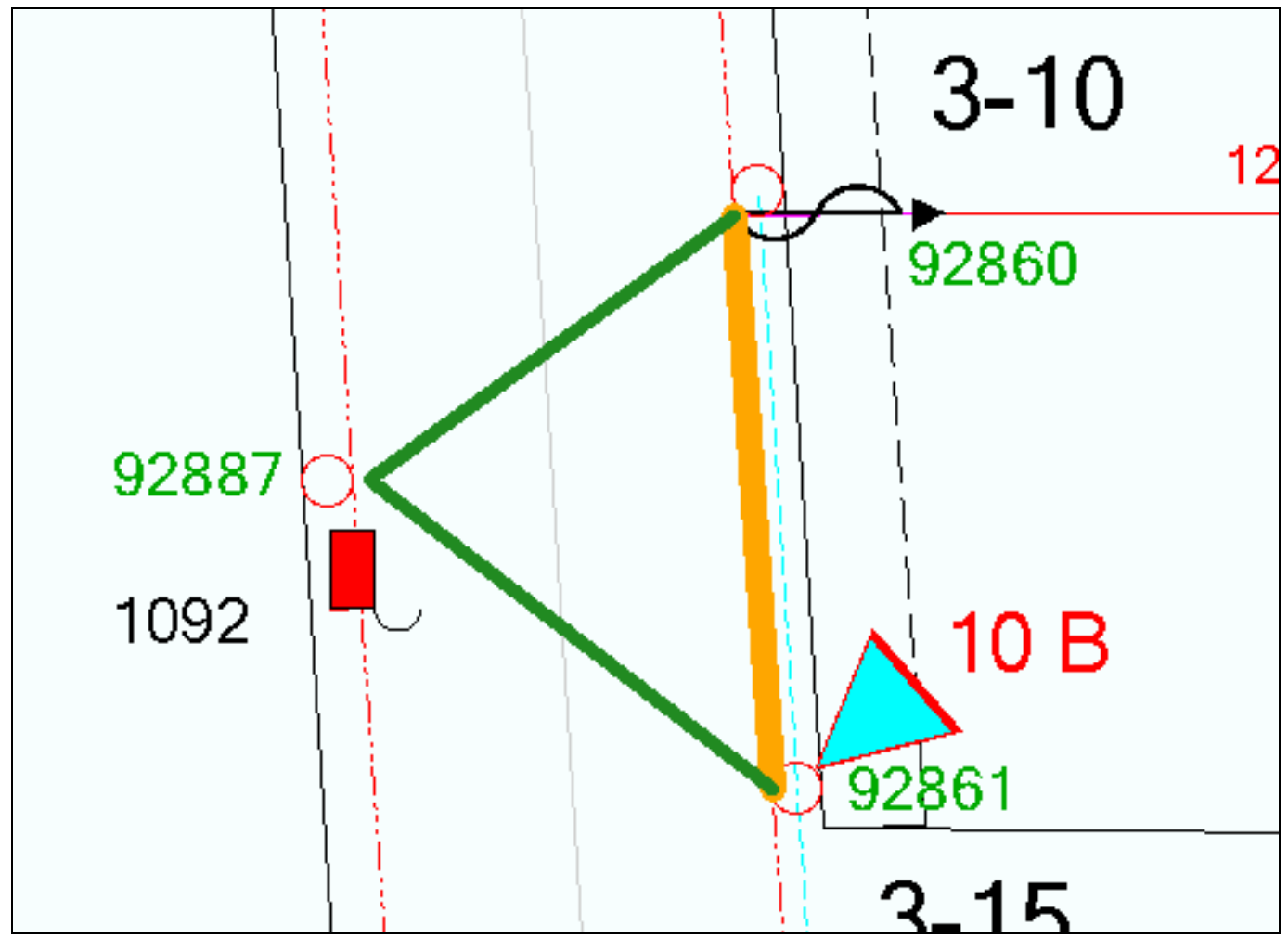

Figure 4.7-109. Conductor has been re-drawn to cross the street (shown in green)

This makes it very easy for the QAQC user to determine exactly what edits have taken place. 


\subsection{All Edits Options}

Several options are available with the All Edits report to give the QAQC users more control over their interaction with the edits:

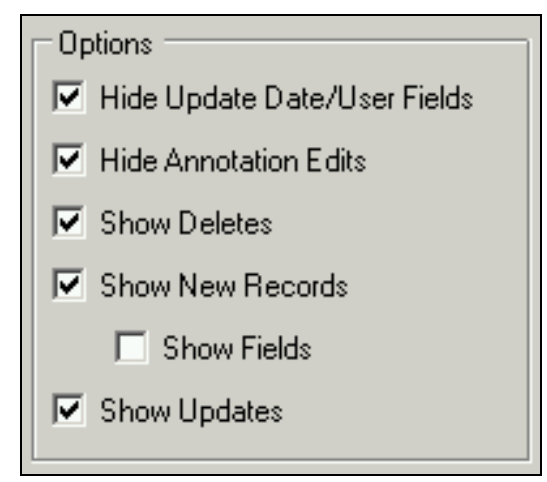

Figure 110. All edits options

The "Hide Update Date/User Fields" check box will remove any of the following fields from the 'new records' or 'updates' section of the report:

- CreationUserId

- CreationDate

- UpdateDate

- UpdateUserId

This can help to de-clutter a report with a significant amount of data. While important, this data is often not the information that the QAQC users want to view because these fields are set by field AutoUpdaters as a result of other edits made on the features.

The "Hide Annotation Edits" check box removes any annotation edits from the report. This can also help to de-clutter a report with a significant amount of data. AEDR annotation is primarily feature-linked which means that it is auto-generated by the system and is not explicitly edited by the user. Therefore it is often not necessary to QA the annotation as long as the related feature is QA'ed.

The "Show Delete", "Show New Records", and "Show Updates" check boxes toggle on and off their respective sections of the report. This is yet another way to de-clutter a report with a significant amount of data. Each type of edit can be reviewed individually making the report easier to manage.

\subsection{All Edits Tools}

Several tools are included with the All Edits report to make the QA experience more valuable. Some have already been discussed in previous sections. The others are discussed here. 


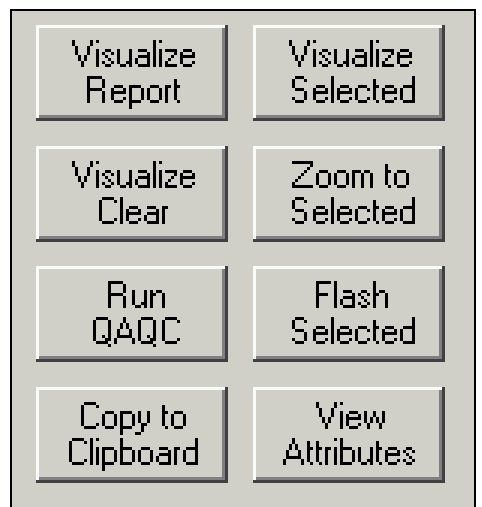

Figure 4.7-111. Other useful tools included with the all edits report

\subsection{1 Visualize Report}

This button visualizes all feature edits from the report on the map. The deletions are shown in red, the new records in blue, and the updates in green/orange. This tool is extremely useful in determining the spatial extent of the edits performed in the session. Figure 4.7-112 is an example of this tool.

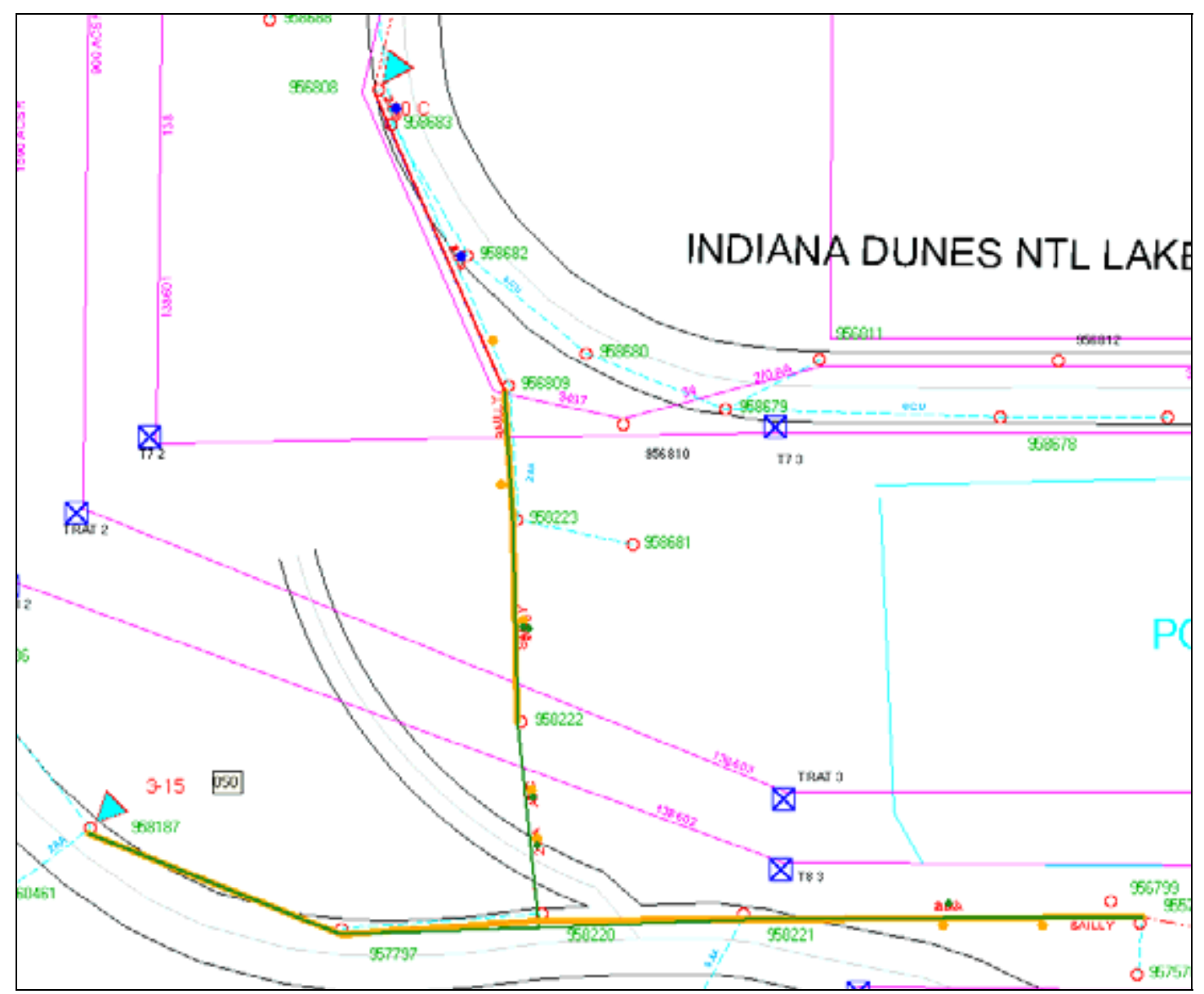

Figure 4.7-112. Visualize report 
If this tool zooms the map out to a very large scale, it is indicative that the editor has performed edits spanning many different areas within the service territory. This can occasionally be a red flag to the QAQC user because most edits should be occurring within a fixed area within a single operating area.

If the QAQC user is having a hard time seeing the edits, the feature layers on the map can be turned off so that only the edit graphics remain. This makes the location of the edited features very evident regardless of the scale of the map. Figure 4.7-113 shows the same report with the layers turned off.

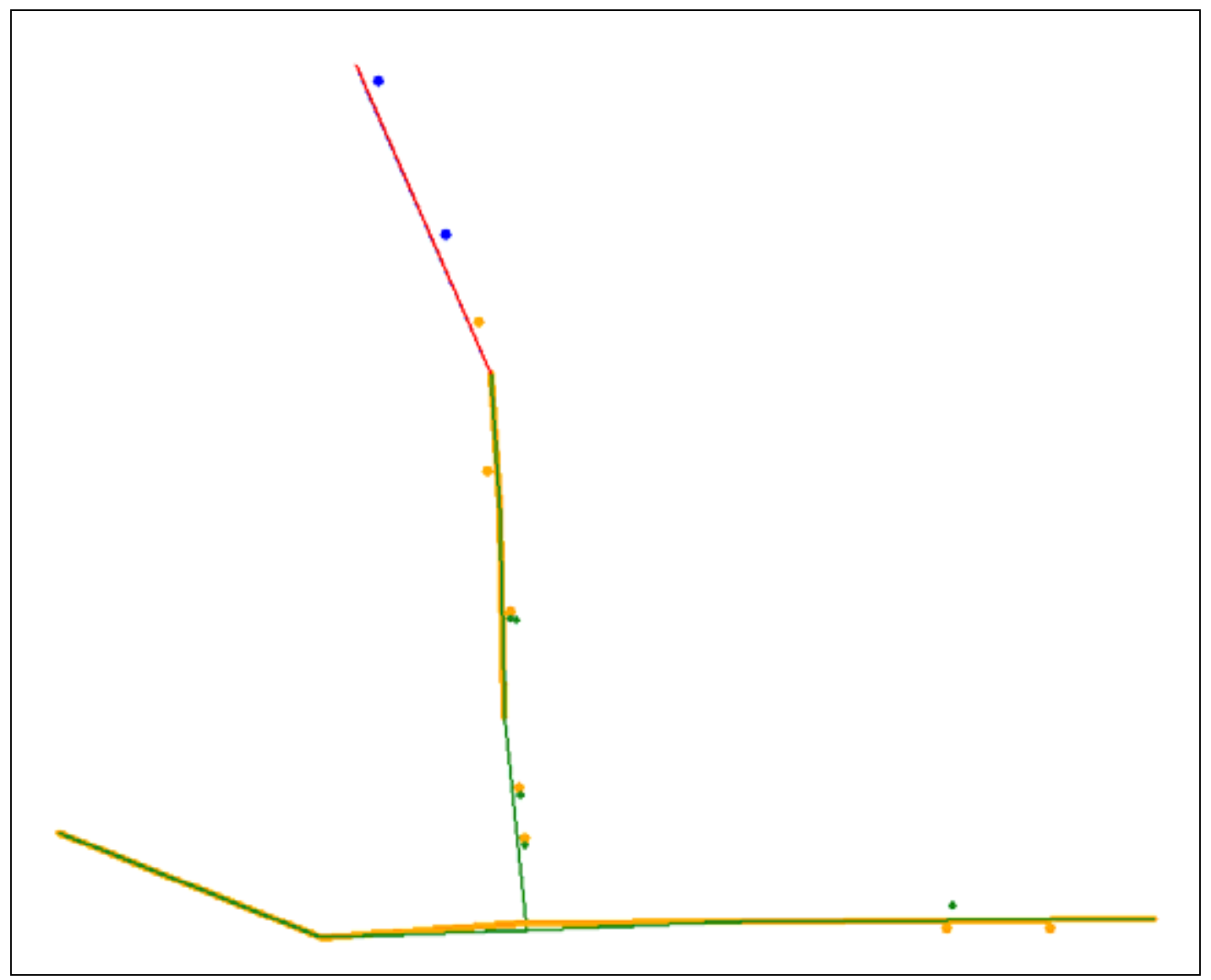

Figure 4.7-113. Layers toggled off for clarity

\subsection{2 Zoom to Selected}

This button will zoom the user to the extent of the feature corresponding to the selected entry on the All Edits report. It can be very helpful after using the Visualize Report button to locate individual features.

\subsection{3 Flash Selected}

This button will flash the feature corresponding to the selected entry on the All Edits report. It can be very helpful after using the Visualize Report button to locate individual features within the larger picture of all edited features.

\subsection{4 Visualize Selected and View Attributes}


These tools were discussed in earlier sections with regard to their use when performing QAQC on the individual deletions, new records, or updates.

\subsection{5 Visualize Clear}

This button simply clears any current All Edits visualizations on the map generated from either the Visualize Report or Visualize Selected tools. All visualizations will also be removed from the map when the All Edits report is closed.

\subsection{6 Run QAQC}

This button automates several tasks for the QAQC user that would otherwise have to be performed manually:

1. The report selects all edited features on the map.

2. The report then selects features related to any edited objects on the map (the AEDR system uses the ArcFM "Validate Related Objects" option whenever available which will allow the ArcFM QAQC to run on object classes related to selected features even if the features themselves were not edited).

3. The report will add new feature layers for feature classes that are required for QA but that were not previously on the map. For example, if the current display only included the electric layers but the user had edited a Gas Main, the report would add a new layer for Gas Main and would then select any edited features.

4. The report will then open the ArcFM Attribute Editor if it is not already open.

5. The report will invoke the ArcFM QAQC routine for all selected features. The QAQC will run and will return the results in the standard fashion on the QA/QC tab of the Attribute Editor as shown in figure 4.7-114. 


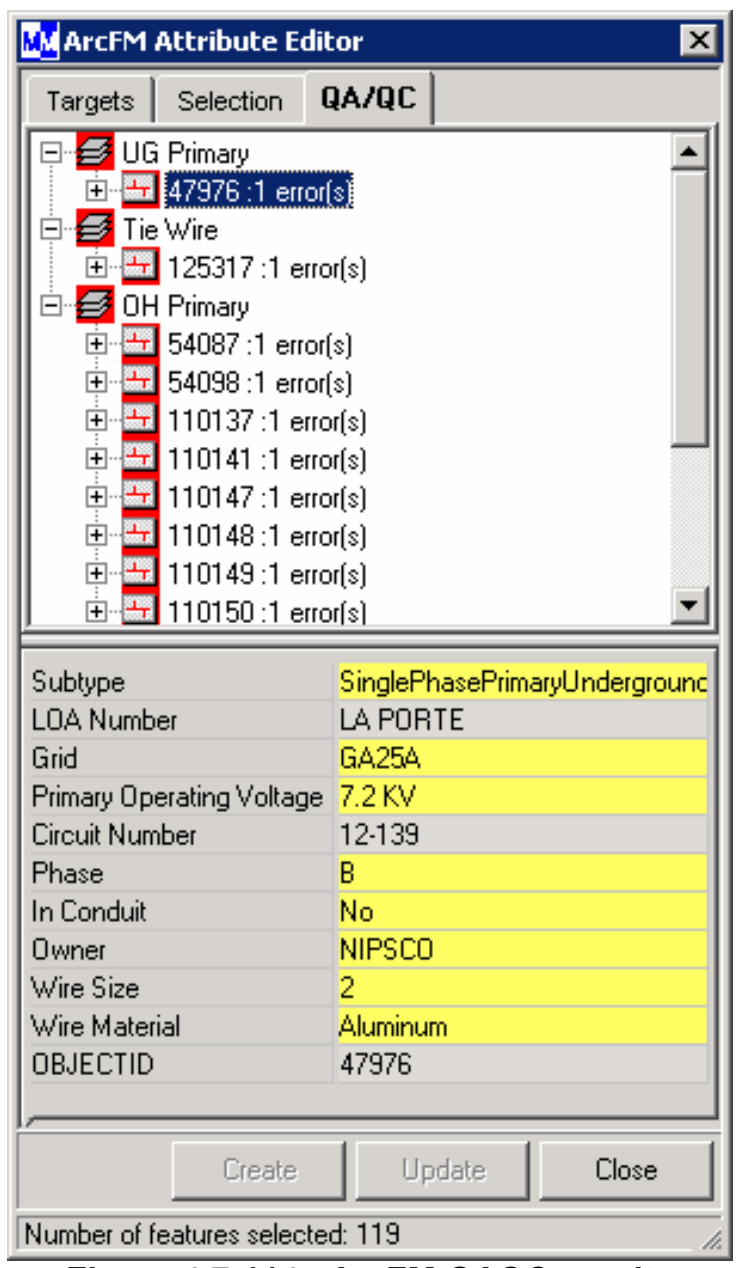

Figure 4.7-114. ArcFM QAQC results

6. The user can then proceed with using the standard QAQC tools.

This tool ensures that the QA/QC routine is run for all available features and objects and saves the QAQC user time by locating and selecting all edited features.

\subsection{7 Copy to Clipboard}

NIPSCO developed an advanced requirement to be able to back up a file based representation of the work performed in any session. This tool copies the current representation of the All Edits report to the system clipboard where it can then be pasted into any text editor. The QAQC users typically first set the report options as shown in Figure 4.7-115. 


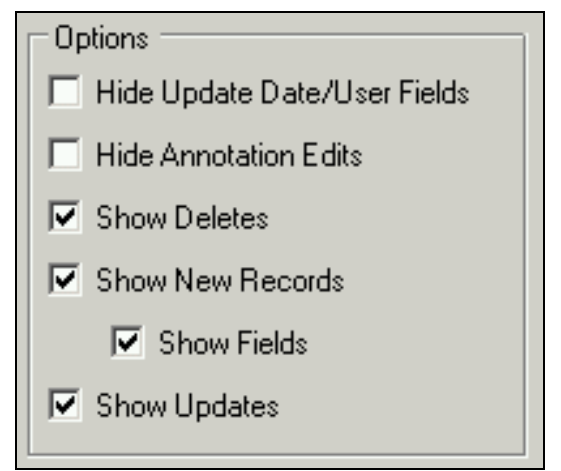

Figure 4.7-115. Report options

Then they use the Copy to Clipboard tool. This ensures that the maximum amount of data is included on the report. This process has become crucial as a combination of ESRI and M\&M bugs have caused the loss of session data during the posting process. With the exported report in hand, it is much easier for an editor to reconstruct the edits that occurred in a session.

\subsubsection{Custom Login}

This section describes the NIPSCO ArcFM Login Utilities. This custom login component enables NIPSCO users to connect to an ArcFM geodatabase and Process Framework (Session Manager) database using Windows authenticated accounts on SQL Server databases. The custom login component must be installed on ArcFM client machines as necessary. While the default ArcFM login prompts users for connection information each time the ArcMap application is started, the NIPSCO custom login will attempt to read connection information from a configuration file, bypassing the default ArcFM Login dialog. If the connection configuration file is missing or contains incorrect parameters, then users will be prompted to supply the connection information.

\subsection{Connection Configuration File}

When ArcMap is started, the NIPSCO custom login component attempts to locate the configuration file containing connection information. The component will read the connection information from the file and attempt to connect to the specified geodatabase. The Process Framework (Session Manager) connection information is cached and used when the "Open Session Manager" button is clicked from within ArcMap. The configuration file must be located in the same folder where the custom login assembly (NIPSCOLogin.dll) is installed. The name of the configuration file is NIPSCOConnections.Xml. The configuration file is an XML document that has the values for the geodatabase server, geodatabase database, geodatabase service, Process Framework server, and Process Framework database that will be used to make appropriate connections.

The following is an example of a NIPSCOConnections.xml file with connection parameters specifying the geodatabase server (SQL), the SQL Server database location of the geodatabase (arcfm8), the SDE service (5151), the Process Framework server (SQL), and the SQL Server database location of the Process Framework data (px): 
$<$ ?xml version="1.0" encoding="UTF-8"?>

$<$ !-- Sample NIPSCO Custom Login Connection information configuration file.

Must be installed in the same folder as the NIPSCOLogin.dll -->

$<$ NIPSCOLogin>

$<$ SDEServer>aedrdbsqlp01</SDEServer $>$

$<$ SDEDatabase $>$ arcfm8 $</$ SDEDatabase $>$

$<$ SDEService>9999</SDEService>

$<$ PxServer>aedrdbsqlp01</PxServer $>$

$<$ PxDatabase $>$ px $<$ /PxDatabase $>$

$<$ /NIPSCOLogin >

Figure 4.7-116. Example NIPSCO custom login connection

The NIPSCO custom login assumes that the databases being connected to are running on the SQL Server platform and are able to use Windows authentication to validate login names. The current Windows user name will always be used when making database connections.

\subsection{Custom Login Dialog}

If the NIPSCOConnections.xml file is missing or has incorrect parameter names for the geodatabase login, then the user will be prompted with a dialog asking them to provide the information necessary to connect to the ArcFM geodatabase and the Process Framework (Session Manager) database.

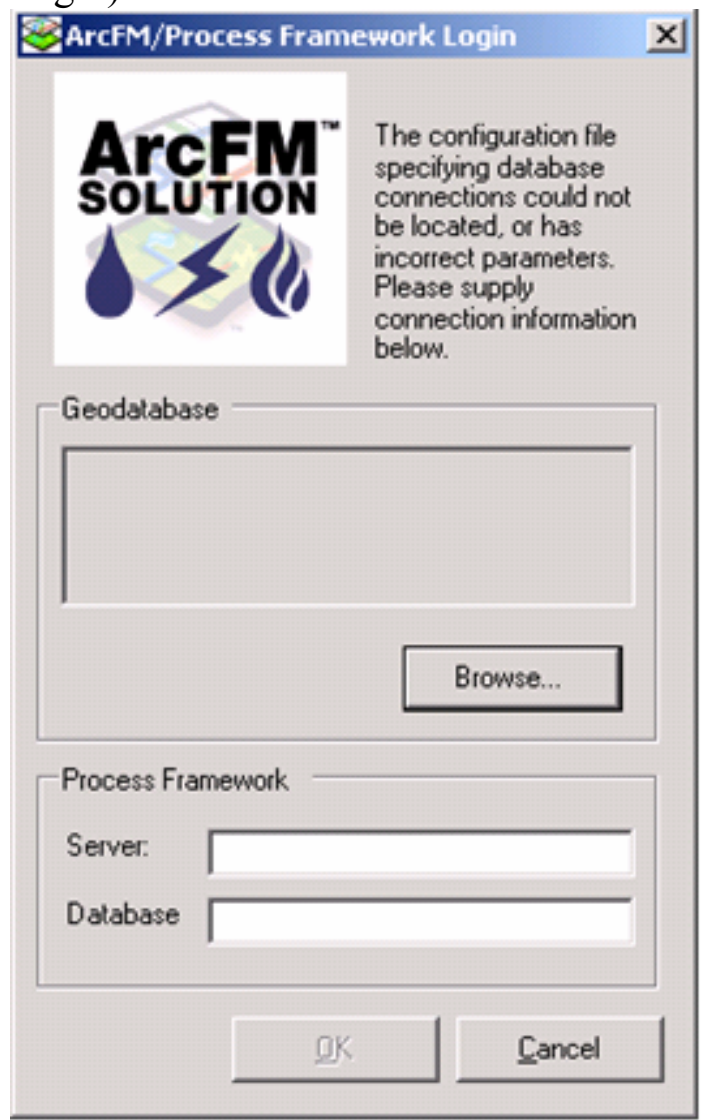

Figure 4.7-117. Login dialog box 
Clicking the "Browse" button on the "Geodatabase" frame will open up an ESRI Catalog browser, enabling the user to choose an established workspace connection. Once a valid connection has been selected, its properties will appear in the box above the Browse button. The "Process Framework" frame allows users to type the Process Framework server and Process Framework SQL Server database used for the Session Manager connection. The "OK" button will become enabled when a valid geodatabase connection has been selected. Note that it is not necessary to indicate the Process Framework server or database in order to enable the OK button and proceed with the geodatabase login, but users will not be able to access the Session Manager from ArcMap. Clicking the "Cancel" button will bypass the ArcFM geodatabase login and Process Framework login. ArcMap will start as normal, but there will be no access to Session Manager or ArcFM functionality such as Stored Displays, Page Templates, and Stored Documents. In this case, the default ArcFM Login may still be used to connect to a geodatabase to access ArcFM functionality by clicking the "Login To ArcFM" button on the ArcFM toolbar in ArcMap.

\subsubsection{Citrix Publishing - ArcLauncher}

Both ESRI ArcMap and Miner \& Miner ArcFM can be used with different licensing schemes. This means that the same installed applications provide different functionality based on the license that is used by the user. The following are the available licenses in use at NIPSCO for both products:

\section{ESRI ArcMap:}

- ArcView: Provides view only access to the data via the ArcMap product.

- ArcEditor: Provides edit access to the data via the ArcMap product.

- ArcInfo: Provides full edit and analysis to the data via the ArcMap product.

M\&M ArcFM:

- ArcFM Viewer: Provides use of ArcFM tools within ArcMap within a read only context.

- ArcFM: Provides full editing with ArcFM tools within ArcMap.

NIPSCO has purchased licenses of all of the above types but different licenses are intended for different groups of users. The ArcView/ArcFM Viewer is intended for the engineers, call screening, and outage management groups. They are provided with read only access for analysis purposes. The ArcEditor/ArcFM is intended for the Maps \& Records clerks. They are the primary editors of the GIS data. The ArcInfo license is only used by the GIS group for advanced data analysis purposes.

Allowing different users to use different licensing combinations in a Citrix environment is a somewhat complicated task because there is only one single installation of the software. Typically the license preferences are set by the desktop administrator programs which store the setting in the registry. This setting then applies to ALL connections that are made to the server. It was necessary to find a way to dynamically set the license to be used on user basis. 
The ESRI license can be dynamically updated by setting an environment variable as follows:

- ArcView: ESRI_SOFTWARE_CLASS = Viewer

- ArcEditor: ESRI_LSOFTWARE_CLASS = Editor

- ArcInfo: ESRI_SOFTWARE_CLASS = Professional

The Miner \& Miner license can be updated by setting specific registry keys as follows:

- ArcFM Viewer: HKEY_CURRENT_USER/Software\Miner and Miner|Desktop Manager Products $\backslash A r c F M$ Viewer $=1$

- ArcFM: HKEY_CURRENT_USERISoftwarelMiner and MinerlDesktop Manager\Products $\backslash$ ArcFM $=1$

Both of these license changes are automated via the NIPSCO ArcLauncher application. It is a command line application that accepts parameters for the application to be run (i.e. ArcMap, ArcCatalog, etc) and the license level to be used. It then sets both the ESRI license environment variable and the Miner \& Miner registry keys appropriately before starting the application.

All AEDR Citrix applications have been published using ArcLauncher to handle the licensing issues. The three core applications are published as follows:

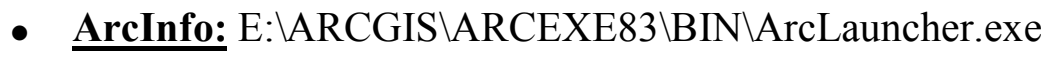

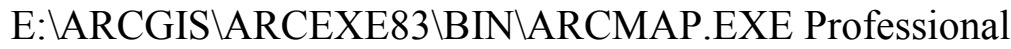

- ArcEditor: E: $\backslash A R C G I S \backslash A R C E X E 83 \backslash B I N \backslash A r c L a u n c h e r . e x e$

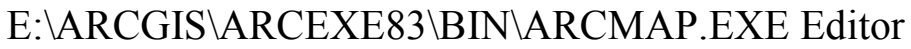

- ArcViewer: E: $\backslash A R C G I S \backslash A R C E X E 83 \backslash B I N \backslash A r c L a u n c h e r$.exe

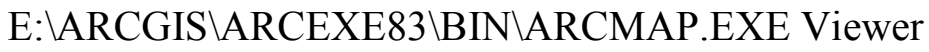

This allows a single user to access the same application via different Citrix publishing and all licensing is set correctly. 


\subsection{AEDR Interfaces}

This section defines the interfaces between the AEDR system and all of the surrounding applications and systems. Some of the interfaces are minimal while others are very in depth. Details and illustrations are provided for each interface in the following sub sections.

\subsubsection{Facility Browser Part 1}

\subsubsection{Overview}

The Facility Browser Part 1 encompasses the design only of the Facility Browser and is discussed in this report. The actual Facility Browser implementation will be part of Phase II.

The legacy version of Facility Browser used MapGuide as its Mapping Engine. Additional tools and components of Facility Browser are SQL Server as the database for point based-features, Spatial Data Files (SDF) as the linear spatial data, and Cold Fusion as the primary application development environment. There are also additional tables in the SQL Server database for information about customers and Gas Service Cards. Additional development tools being utilized are the MapGuide API, HTML, and JavaScript.

MapGuide is a web-based mapping solution from Autodesk. The SQL Server database is storing data in a flat-file type format where the spatial information is coming from XY coordinates. The SDFs require conversion from the AutoCAD drawings, and the Cold Fusion technology being used to drive the application has become outdated.

The legacy version of Facility Browser was initially implemented as a query and reporting tool on the web. It also allowed for the viewing of NIPSCO data as a seamless database versus using the tiled AutoCAD environment. Over the years, the seamless database remained in place, but Facility Browser became more than a query and reporting tool. Data entry and editing was implemented as some of the added functionality. Spatial data editing was also implemented for the addition, deletion, and movement of customer points. Additional functionality also allowed for the creation of Gas Service Cards, as well as the maintenance of some Gas Service Card data.

In the legacy version of Facility Browser the processing is executing on the client, so any of the geoprocessing for analysis of the map data is executing on the user's machine and not the server.

The new version of Facility Browser was designed using ArcIMS as the mapping engine and $\mathrm{ArcSDE}$ as the spatial data component. The data connections for querying and reporting on the spatial data, as well as making the various calls to ArcIMS was designed to use ArcXML. By using ArcXML, no interpretation of calls being made using a connector are necessary. The development tool to be used for the new version is ASP.NET in the C\#.NET environment. Both HTML and JavaScript will continue to be 
used for the client application. All custom functionality developed for Facility Browser (e.g. Gas Service Card viewer) will be built using C\#.NET.

The new version of Facility Browser will take a step back to its origins and become a query and reporting only tool. Any of the data editing, data maintenance, data creation, etc. tools and functionality that existed in the legacy version of Facility Browser will reside elsewhere.

Also, the new application will be using an ArcIMS Image Map Service. By using the Image Map Service, all of the geoprocessing and querying will take place on the ArcIMS Application/Spatial Server with the results being returned to the end user as a web page.

Figure 4.8-1 shows the main user interface for the new Facility Browser:

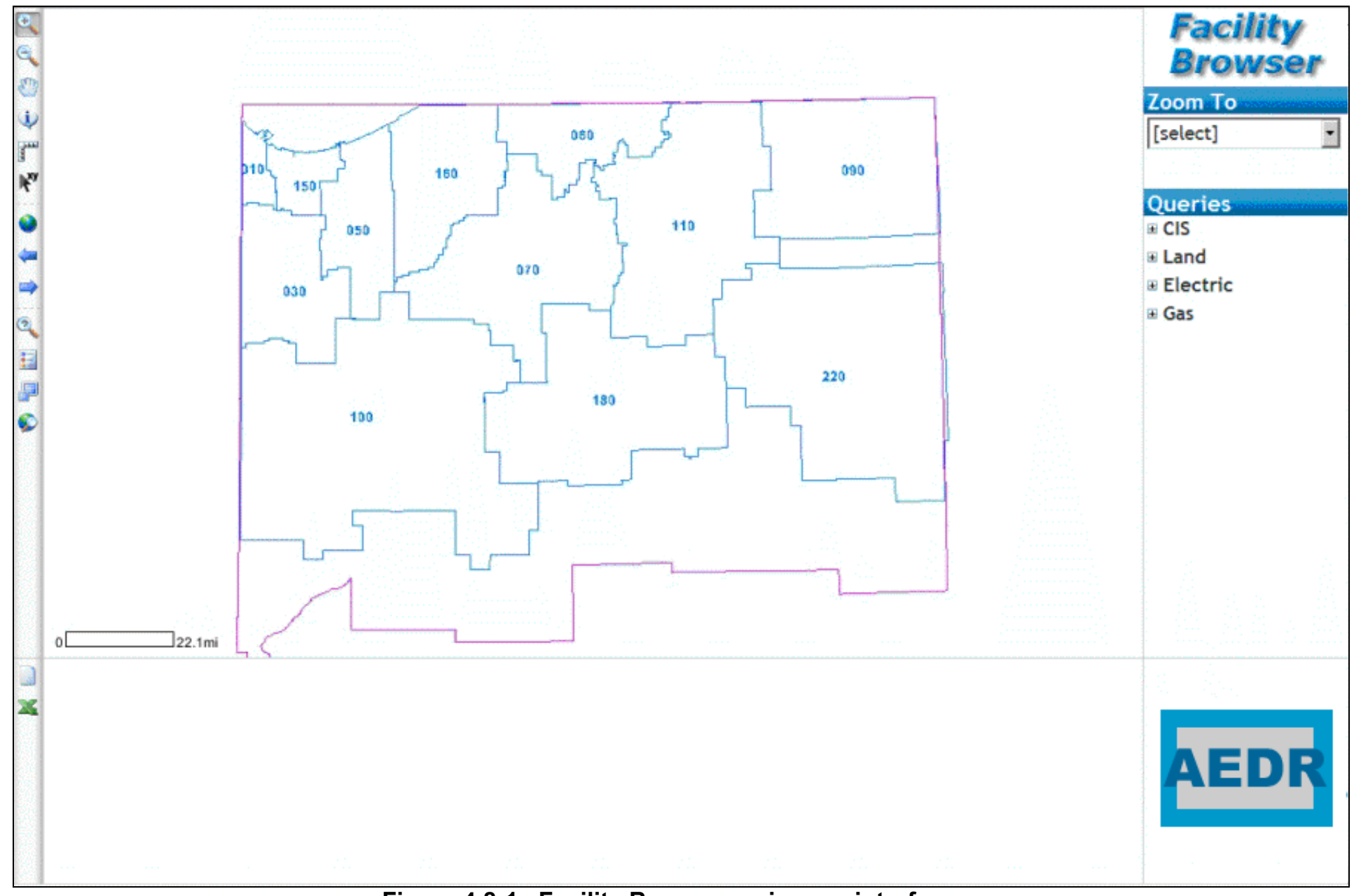

Figure 4.8-1. Facility Browser main user interface

\subsubsection{Design}

\subsection{Use of ArcIMS}

ArcIMS will be the solution for delivering dynamic maps and AEDR data and services via the Web. It provides a highly scalable framework for GIS Web publishing that meets 
the needs of corporate Intranets and demands of worldwide Internet access. ArcIMS services can be used by a wide range of clients including custom Web applications, the ArcGIS Desktop, and mobile and wireless devices.

With ArcIMS users can:

- Deliver dynamic maps and data via the web

- Create task-focused applications that use geographic content

- Develop custom applications using industry standard web development environments such as ASP.Net, and

Implement GIS portals.

\subsection{New Application Functionality}

The new Facility Browser will support all of the current map tools that were available to the users for interacting with the map. These include Zoom In, Zoom Out, Zoom Extent, Zoom Previous, Zoom Forward, Pan, Saved Views, Get XY Location, Select/Identify, Turn On/Off Map Layers, View Map with No Frames, View Legend.

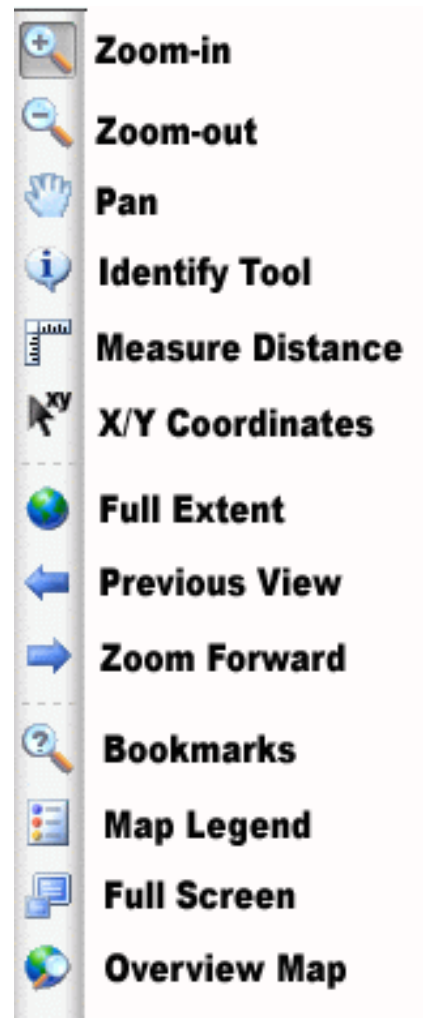

Figure 4.8-2. Map tools 
The new application will also have a locator map as shown in Figure 4.8-3.

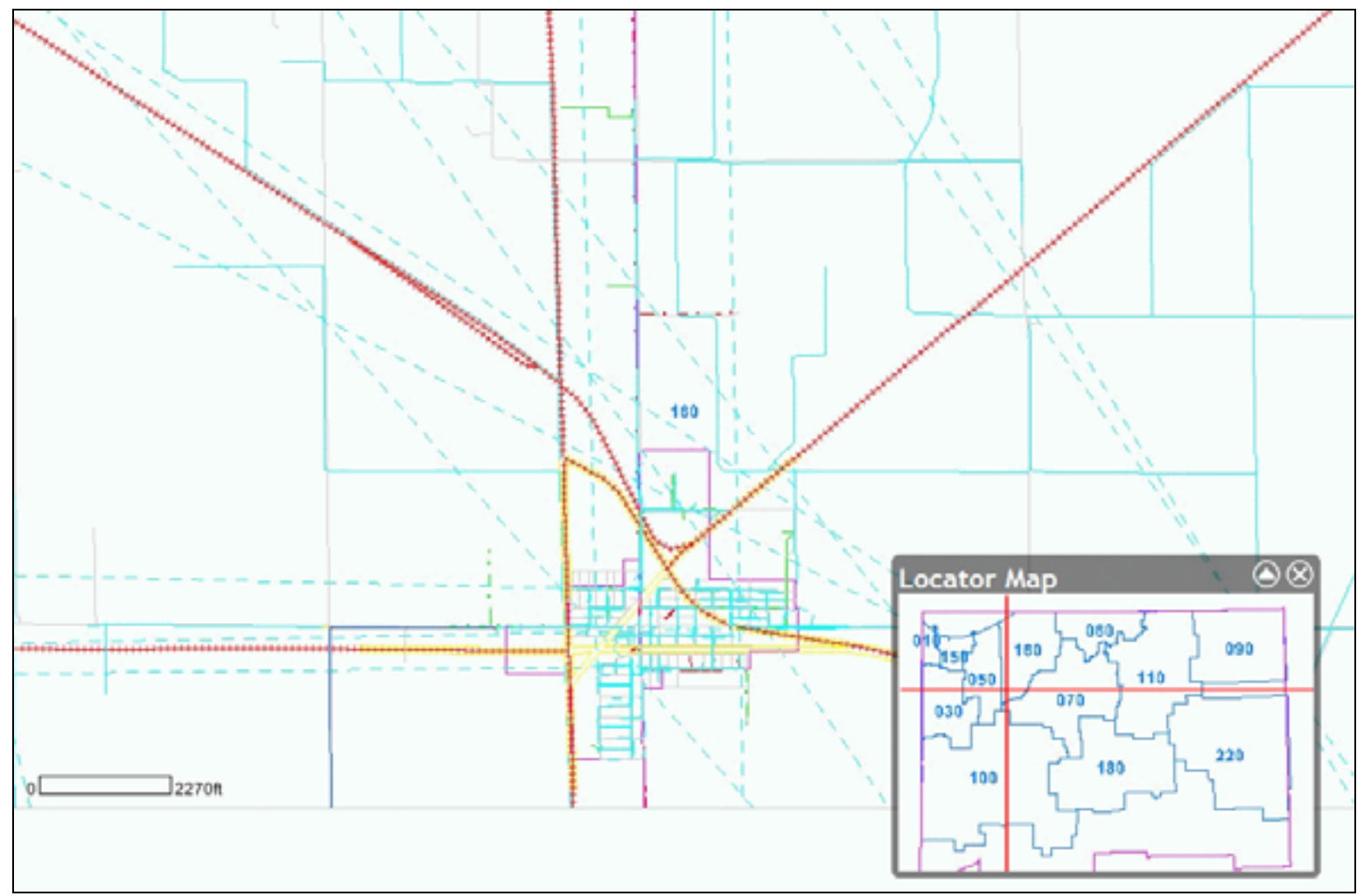

Figure 4.8-3. Locator map (in lower right corner) showing the current map location in relation to the entire NIPSCO Service Territory

Users will be able to perform queries on all of the Land, Gas, and Electric Facilities in the Geodatabase. These queries will function the same manner that the existing queries function. Once a set of features has been selected based on the criteria input by the user, a report is generated and the map is zoomed to the extents of the selection set. A user can then zoom to the individual features listed in the report, get more details about a feature, and send the reports to a delimited file for use in Excel. 
Figure 4.8-4 shows an example of executing a feature query:
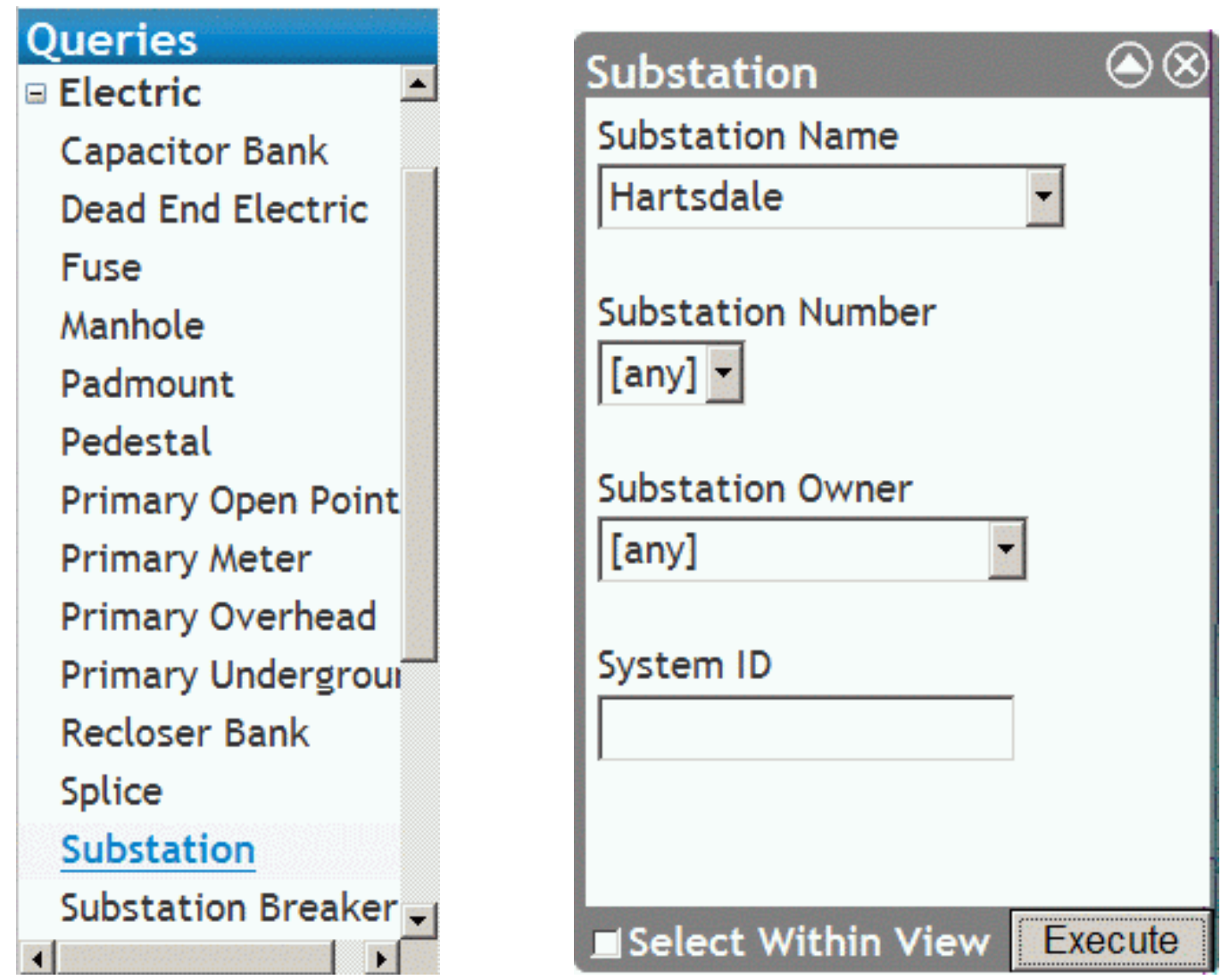

Figure 4.8-4. Query execution

Any matching records are returned in the results grid shown in Figure 4.8-5.

\begin{tabular}{|lllll|}
\hline & Substation Name & Substation Number & Substation Owner & System ID \\
\hline (i) $P$ Hartsdale & 33 & Nipsco Substation & 57 \\
\hline (i) $P$ Hartsdale & 33 & Nipsco Substation & 58 \\
\hline
\end{tabular}

Figure 4.8-5. Query results 
Users will also be able to perform queries on the Gas \& Electric Service Cards to retrieve a list of cards based on an LOA, City, and Street or via the site location. These cards will then be viewed with the new image browser developed. The new image browser will replace the legacy Pegasus ImagXpress tool as the technology has become outdated.

Figure 4.8-6 shows an example of querying a Gas Service Card:

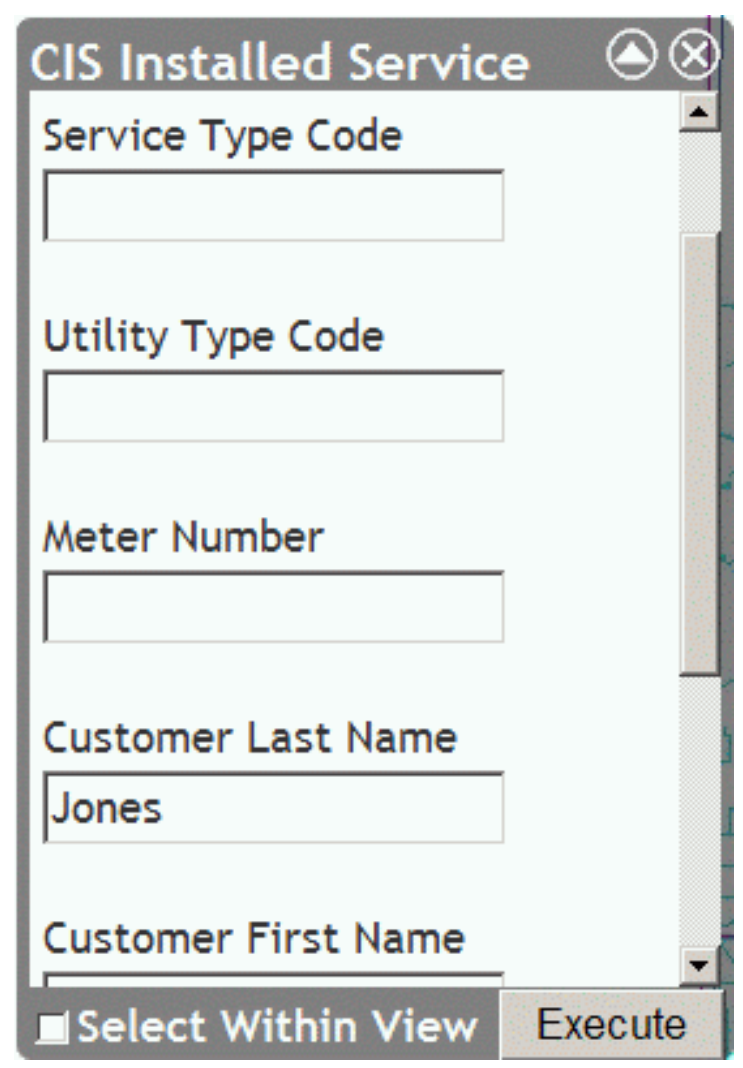

Figure 4.8-6. Query of service cards

Any matching records are returned in the results grid shown in Figure 4.8-7.

\begin{tabular}{|c|c|c|c|c|c|c|}
\hline & Site Identification Num... & Service Type C... & Utility Type C... & Meter Num... & Customer Last Name & Customer First 1 \\
\hline Q i r P & $\underline{28510008}$ & SVC & EL & & JONES & RENALDO \\
\hline$Q$ i) $P$ & 31874003 & SVC & GA & 9475374 & JONES & $\mathrm{JOHN}$ \\
\hline$Q$ i) $P$ & 947090002 & SVC & EL & 0472738 & JONES & ELLEN \\
\hline$Q$ i) $P$ & 983630004 & SVC & EL & 0557719 & JONES & BRUCE \\
\hline$Q$ i) $P$ & 926040003 & SVC & GA & 9407169 & JONES & GARY \\
\hline $\begin{array}{l}Q \text { i) } P \\
11\end{array}$ & 923850007 & SVC & GA & 0920473 & JONES & $\mathrm{JOHN}$ \\
\hline
\end{tabular}

Figure 4.8-7. Results of query 
Clicking the links above will launch the new Service Card Viewer shown in Figure 4.8-8.

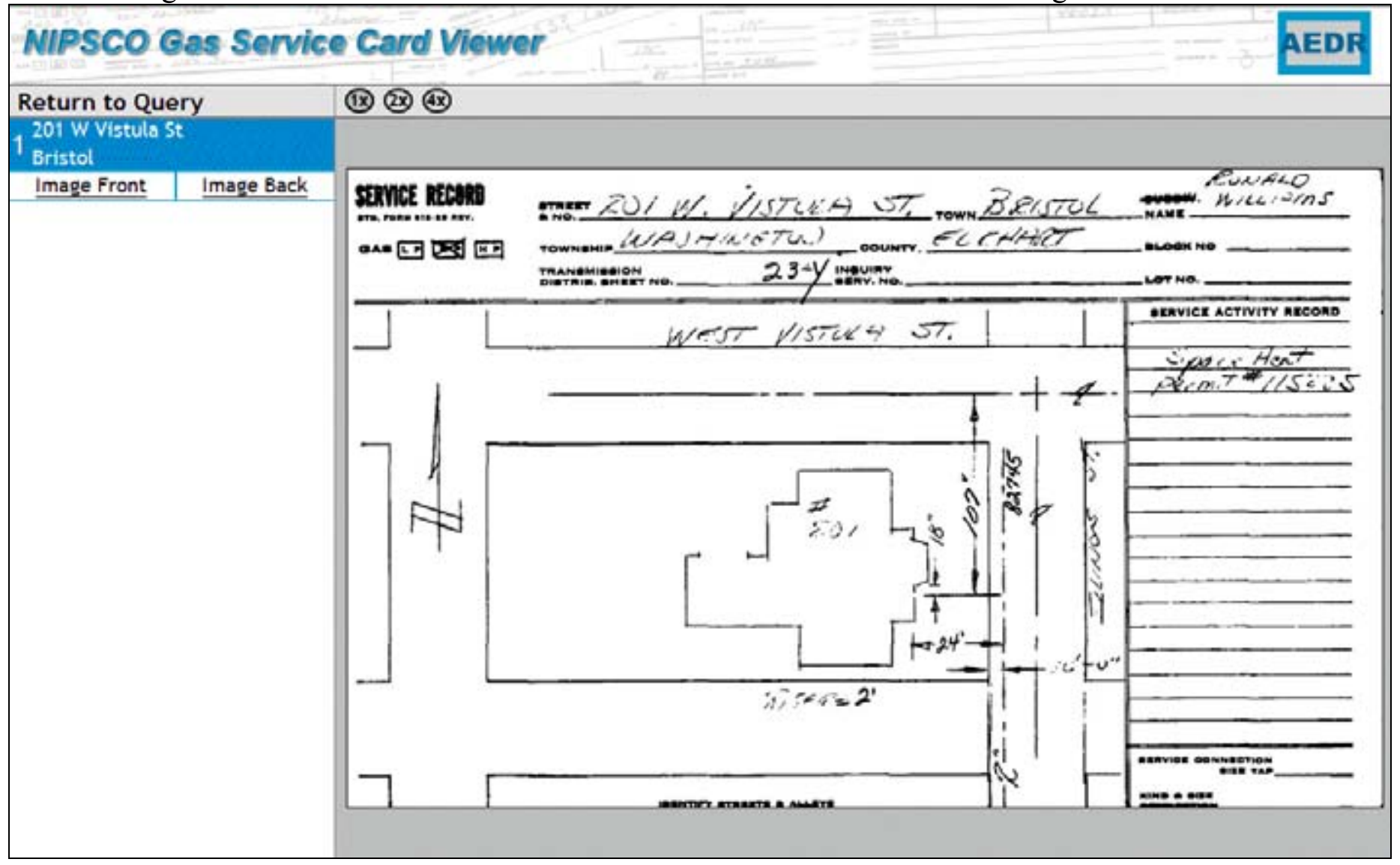

Figure 4.8-8. Service card

\subsection{Additional Applications / Tasks Required}

Because the new version of Facility Browser will revert to a query and reporting system only, some tools and functionality that previously existed were moved elsewhere.

\subsection{Application to Manage the Placement, Deletion, and Editing of the Customer Sites}

In the legacy Facility Browser, new customers are managed by storing the site locations as XY coordinates in a flat file. The new data model has a feature class for customers which enables the use of out-of-the-box ArcMap/ArcFM tools to manage the customer sites.

A new interface that imports the data from CIS into the Geodatabase must be developed. Currently, there is a process in place that moves the data from the CIS text file to SQL Server. This application will require some modifications but the process will remain the same. The difference is that the data will be imported into a Geodatabase Feature Class. When the import takes place, the new sites will be assigned the XY Coordinate of the Minor Grid Code within the site. By assigning this XY, the user creating the new data will be navigated to the general area of the customer location. Additionally, customers 
will need to be flagged as to whether or not they are a gas customer. In doing so, the process in which Gas Service Cards are created can come into play.

Placement functionality of the customers will be accomplished using out-of-the-box ArcFM tools. Some new custom forms will be created to allow the users to query and find the various customers that require placement, require a new service card, or need to be moved to a new location because of a poor geocoding score from the original geocoding process. This customization will be done using C\#.NET and ArcObjects.

\subsection{Normalize the Current Gas Service Cards Database}

The legacy Gas Service Cards database does not contain unique Image IDs throughout the company. Image IDs are only unique for the LOA, City, and Street where they are located. In order for a more straightforward maintenance, as well as a more efficient query/reporting process, assignment of unique Image IDs are required. This is known as "normalizing" the data. Normalizing the data can be accomplished via an automated fashion using a custom $\mathrm{C \#}$ application. If the data were to be normalized, the queries to access the Service Cards would have to be modified. Queries for the other features and facilities will be more or less translated from MapGuide-ColdFusion to ArcIMS-C\#.NET. Additionally, some discussion will be necessary to ensure the validity and appropriateness of the new naming conventions.

\subsection{Application to Create New Gas Service Cards}

The legacy Gas Service Cards were created using Facility Browser and the Pegasus ImageXpress tool. This solution is rather inelegant and cumbersome, but the business process and workflow is relatively sound. A new tool needs to be developed to manage the creation of the Gas Service Cards.

The new tool for creating and managing Gas Service Cards and their associated data will be integrated into the ArcMap/ArcFM framework using an ArcMap Layout. By creating and customizing an ArcMap layout for the end users, it will have the general look and feel of a paper Gas Service Card but will be editable. By moving the application from the web to the desktop, the users will have a more stable environment for editing and creating data. Plus, they will already have access to the software and data necessary to create the maps for the Service Cards. Additionally, they will have direct access to the necessary tables that are populated with Service Card data.

Customization will be required to build the application. This customization will be accomplished using ArcObjects and C\#.NET. The new tool will allow for the creation, insertion, and update of the map for the service card, as well as any data that needs to be updated in the various tables.

\subsection{Replication of Data}

The new Facility Browser will access ArcSDE to pull both spatial and attribute data. Because Facility Browser is a web application, it will be accessed by many users across the NIPSCO organization. This will generate a sizable load on the ArcSDE database server and when combined with the load from ArcEditor and ArcView users it will likely 
cause performance problems. Therefore it has been determined that a second production ArcSDE server will be purchased and configured to support the Facility Browser data access. This will separate the two major load components of the AEDR system and should provide significant improvements in performance for all system components.

All editing and maintenance of the data will take place on the production ArcSDE database server. The data will be replicated to the Facility Browser ArcSDE database on a nightly basis and will be executed via an SQL Server administration script.

The script will be responsible for the following actions:

- Take a backup (*.bak) of the production ArcSDE database

- Restore the .bak file to the Facility Browser ArcSDE database

- Apply Facility Browser-specific views to allow object table data to be joined directly to feature classes (ArcIMS does not allow the direct viewing of object tables)

- Apply the Facility Browser-specific indexes to improve the ArcIMS performance.

\subsubsection{Field Browser Part 1}

\subsubsection{Overview}

Field Browser Part 1 encompasses the design only of the Field Browser as discussed in this report. The actual Field Browser implementation will be part of Phase II.

The Field Browser is used both in the office and by field personnel. It is a self-contained installation of the AEDR data and a GIS viewer that does not require a software license of any sort. The legacy Field Browser application is a custom CAD-based solution which contained basic map viewing functionality. The Field Browser data is updated on the field units monthly by running an application called InfoBuilder. This application processes and groups the NIPSCO CAD map tiles into a *.dwf format. It also extracts all of the entity data from the CAD tiles into a Microsoft Access database. The legacy Field Browser solution has performed well for several years and the main goal of the new Field Browser implementation is to match the existing functionality on the ESRI platform.

ESRI ArcReader was chosen as the platform for the new Field Browser because of its easy deployment, lack of license, and native support for ESRI data formats. ArcReader is an ideal way to deliver interactive mapping capabilities that access a wide variety of dynamic geographic information. Using ArcReader, the field users can view the same data that exists in the back office ArcGIS applications after they have been converted using the ArcGIS Publisher extension. This extension adds simple map publication capabilities to ArcMap that allow any map document (*.mxd) to be converted to a published map file (*.pmf). These pmf files can then be copied locally to any computer along with an extracted set of data. ArcReader can then load the data with the configured layers and symbology without any connection to a network. ArcReader provides a comprehensive set of tools to view and query the data as well as a fairly customizable user interface that will allow different users to have individualized views of the data. 
Figure 4.8-9 shows the standard ArcReader "Find" window which will be used to query the AEDR assets.

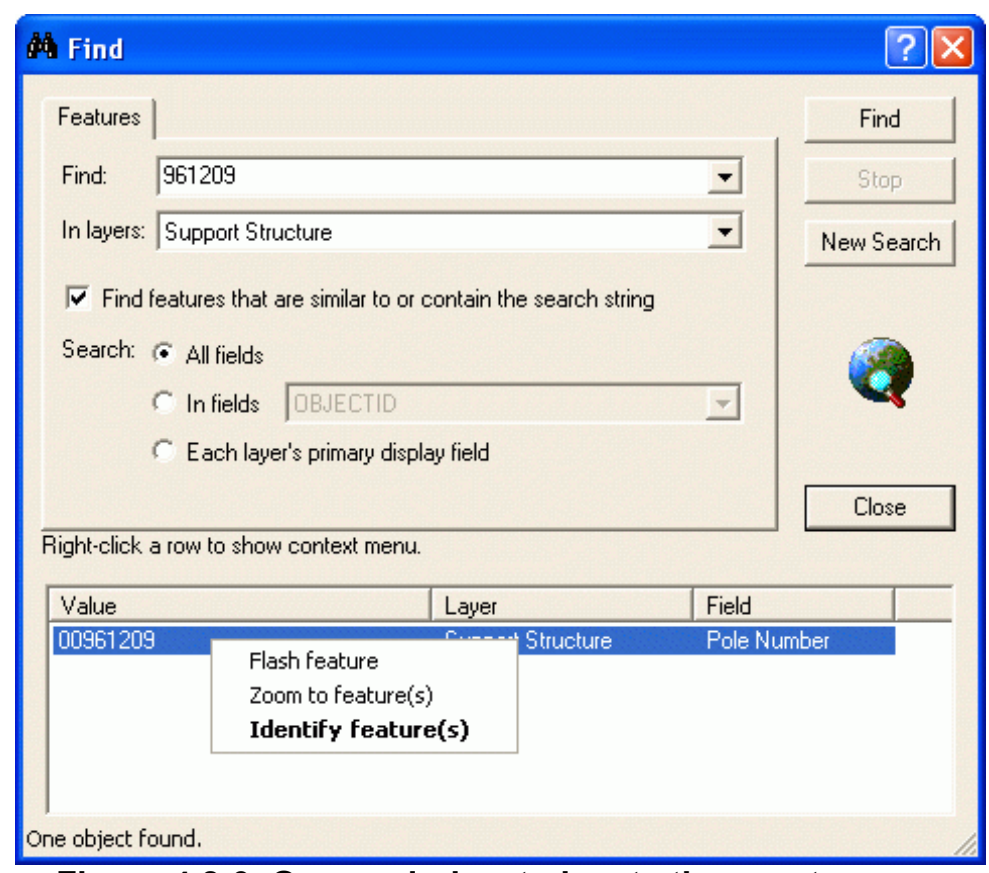

Figure 4.8-9. Query window to locate the assets

\subsubsection{Field Browser Goals}

The goal of the new Field Browser is to provide the end-user with at the least the same functionality that exists in the legacy Field Browser;

- a tool that allows NIPSCO employees to view electric and gas facilities over the top of an accurate land base

- the ability to query based on certain facility information including pole number, regulator station number, substation name, pad number, valve number, street intersection, etc, and

- the users must be able to view an entire LOA, seamlessly, without the need to jump from map to map.

\subsubsection{Field Browser Requirements}

The requirements for the new Field Browser center on replacing the current functionality:

- The end user is able to view all the facilities within the NIPSCO service area. The system provides the gas street, line, service personnel, corrosion control and locating contractors with electronic field access.

- The end user will need to be able to view a legend of the facilities with a description name.

- The end user needs to be able to use zoom functionality including pan, zoom window, zoom inlout, zoom full extents, zoom previous, and zoom forward.

- The end user is able to pull up the extents of any of our Local Operating Areas (LOA's). 
- The end user has full control on what facilities are viewed.

- The system provides full query access to the facility data by using any of the following fields: Street Intersection, Pole, Switch, Substation, Transformer Number, Pad Number, Regulator Station, Emergency Valve, Valve, and Corrosion Control Section.

- The end user can view facilities seamlessly.

- The Field Browser will load and open with minimal delay.

\subsubsection{Design}

The most notable design feature for the new Field Browser is the MS Explorer style view of the application. This feature is important because it allows the GIS team to manage the layers into data frames or groups. By creating groups like "electric", "gas" and "land", a user on the gas side of the company can easily toggle off the electric and or land facilities. It also allows the user to turn individual layers onloff, an option currently missing in the legacy Field Browser.

The background color of the new Field Browser will be white instead of the current black background. The new application will continue to run on the existing Field Browser laptop computers. Performance will be evaluated to insure the current hardware will support the new Field Browser. The GIS department will continue to provide Field Browser users with monthly updates via CD media. The updates will be created by exporting personal geodatabases.

Figure 4.8-10 shows the default interface of the new Field Browser. 


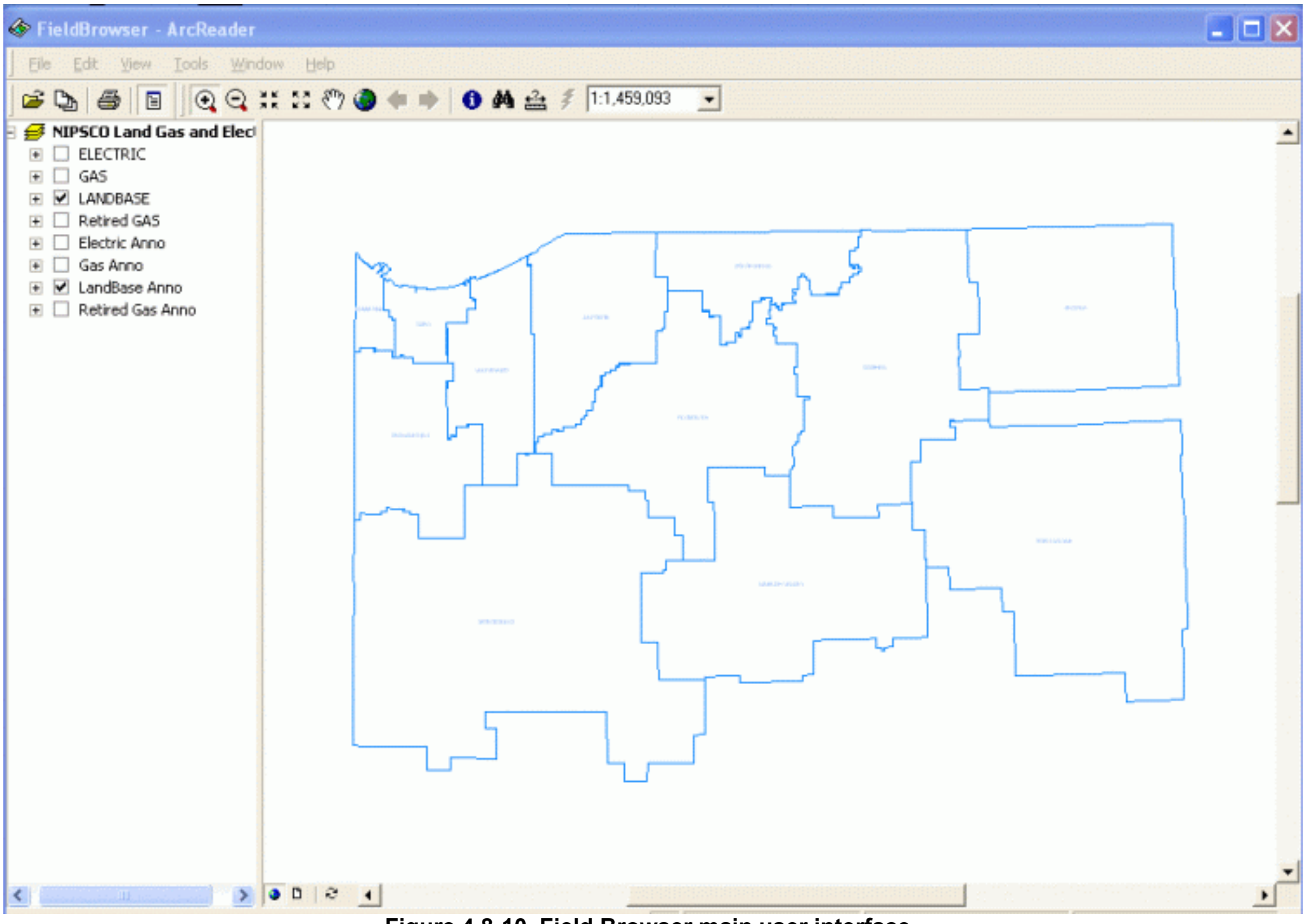

Figure 4.8-10. Field Browser main user interface

\subsection{Exporting Data}

There are a couple of ways to export data using ArcGIS tools. The method NIPSCO uses to export the data will depend on performance and efficiencies going forward to provide monthly updates to our field personnel. The two methods are exporting personal geodatabases and exporting shape files.

An ArcMap (.mxd) file is created by saving a copy of the production SDE database. This allows configuration and symbology maintenance of all the features within the database. Once the NewFB.mxd has been created, either a shape file or a personal geodatabase may be exported.

\subsection{Shape Files}

1. To export the shape file for each feature using the ArcCatalog exporting functionality, the location of the exported shape files will need to be created using relative paths. NIPSCO will use the following locations: 
$\mathrm{C}: \backslash$ FieldBrowser $\backslash$ Land $\rightarrow$ Land Feature Shape files

C: $\mid$ FieldBrowser $\mid$ Gas $\rightarrow$ Gas Feature Shape files

$\mathrm{C}: \mid$ FieldBrowser|Electric $\rightarrow$ Electric Feature Shape files

2. The next step is to create the link between the ArcMap .mxd and the exported shape files by "setting the data source" for each of the features. This is necessary because the .mxd was saved when the links to the data were pointing to the SQL database.

3. Next, some minor configurations changes will need to occur. The changes will include configuring labels, scale ranges for both the feature and label, setting the rotation of symbols, and making certain that the query is defined correctly.

4. Finally, the user can publish the Map using ArcMap's Publisher Extension and export to $\mathrm{c}:$ NewFieldBrowserlNewFB.pmf.

\subsection{Personal Geodatabase}

1. To export a personal geodatabase, the user opens ArcCatalog and creates four personal geodatabases in the following locations:

$\mathrm{C}: \backslash$ FieldBrowser $\backslash$ PersonalGDB $\backslash$ Electric.mdb

$\mathrm{C}: \backslash$ FieldBrowser $\backslash$ PersonalGDB $\backslash$ Gas.mdb

$\mathrm{C}: \backslash$ FieldBrowser $\backslash$ PersonalGDB $\backslash$ Landbase.mdb

$\mathrm{C}: \mid$ FieldBrowser $\backslash$ PersonalGDB $\backslash$ Annotation.mdb.

The purpose for the individual personal geodatabases are to ensure that no individual geodatabase exceeds the maximum size of $2 \mathrm{~GB}$. The NIPSCO dataset is too large to fit into a single personal geodatabase.

2. The user connects to the production database by right-clicking on a dataset or individual classes within a dataset, the user then selects copy, then pastes it into the appropriate new personal geodatabase created in step 1. Repeat for each dataset.

3. Finally, the user can publish the Map using ArcMap's Publisher Extension and export to c: \FieldBrowser\NewFB.pmf.

\subsection{Chosen Approach}

The personal geodatabase approach was chosen for the rollout of the Field Browser because a personal geodatabase retains much more metadata surrounding the raw data than a shapefile. For example, a geodatabase retains the alias information which allows the field names to be shown with common names as opposed to system names ("Pole Number" vs. "DistribRefNumber"). A shapefile loses this information and the published .mxd file must then be manually updated to replicate these settings. Another limitation with shapefiles was that the field names were truncated to eight characters. This caused heavy rework of the published .mxd file because all of the feature layer definitions referenced the longer back office field names. 
The main issue with using a personal geodatabase is the size of the database. It is ideal to keep the size of the Field Browser footprint as small as possible, especially because the monthly data updates need to be distributed via CD-ROM. When the datasets are copied from the ArcSDE geodatabase into the personal geodatabase, all table objects that are related to the feature classes are also copied across by default. ArcReader, however, does not allow a user to view related records so the existence of this data serves no purpose. For this reason, these tables will be removed from the personal geodatabases before they are distributed. Additional steps were also taken to minimize the size of the geodatabases and they are detailed in the next section.

\subsection{Field Browser Geodatabase Post Processing}

After the personal geodatabases have been created, several additional processes are used to reduce the size of the databases and to enhance the usability of the data. These processes are detailed in this section.

\subsection{1 pGDB Field Stripper}

As noted in an earlier section, all tables objects related to feature classes are deleted from the personal geodatabases. To keep the Field Browser footprint as small as possible, unnecessary back office columns of data will be removed from the feature class tables.

A new light-weight standalone windows application is planned to be created called "pGDB Stripper". This application will allow the Field Browser administrator to create strip profiles that define which fields should be removed from all tables and also which fields should be removed from specific tables. An administrator will be able to create multiple strip profiles if needed to accommodate the four different personal geodatabases. The user interface is expected to be easy to use and the strip profiles are planned to be text based and can be checked into source control.

pGDB Stripper will also handle executing a strip profile against a personal geodatabase. Figure 4.8-11 shows the pGDB Stripper main window. 


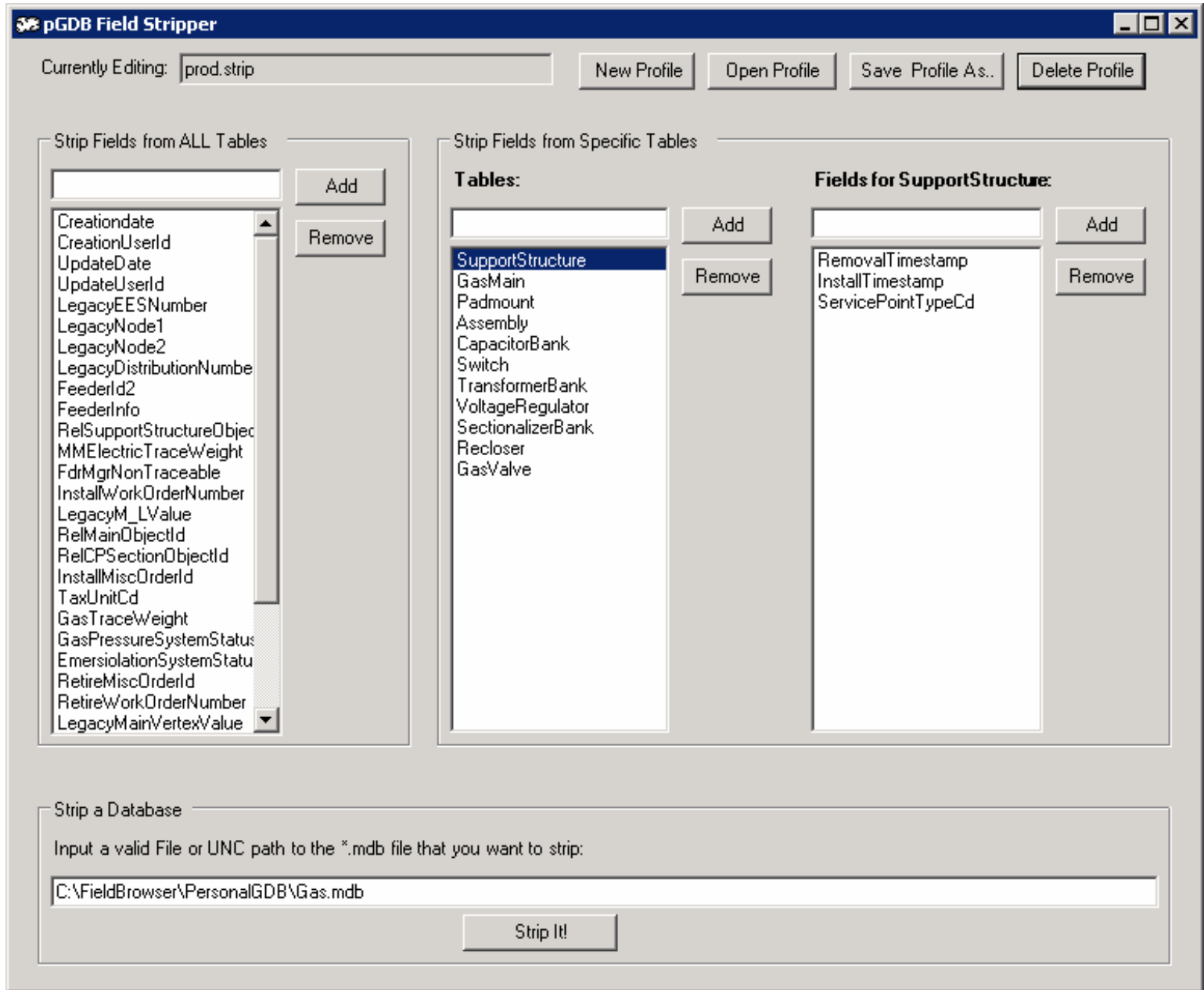

\section{Figure 4.8-11. The pGDB stripper parses \\ through each table in the database and deletes any matching fields}

Currently, the following fields have been approved for removal from all personal geodatabase tables:

- Creationdate

- CreationUserId

- UpdateDate

- UpdateUserId

- LegacyEESNumber

- LegacyNode1

- LegacyNode2

- LegacyDistributionNumber

- FeederId2

- FeederInfo

- RelSupportStructureObjectId

- MMElectricTraceWeight

- FdrMgrNonTraceable

- InstallWorkOrderNumber 
- LegacyM_LValue

- RelMainObjectId

- RelCPSectionObjectId

- InstallMiscOrderId

- TaxUnitCd

- GasTraceWeight

- GasPressureSystemStatusCd

- EmerisolationSystemStatusCd

- RetireMiscOrderId

- RetireWorkOrderNumber

- LegacyMainVertexValue

- RelGasMainObjectId

- LayerName

- Handle

- XdAppName

- ParentHandle

- GraphicScaleFactorValue

- ObjectId

Individual tables are still being reviewed for additional fields that are not necessary. Removing these fields from all tables will dramatically reduce the size of the personal geodatabases and using pGDB Stripper to manage the process will provide a consistent and reusable pattern to handle this task on a monthly basis.

\subsection{Street Centerline Intersection Queries}

A landbase feature class "StreetCenterlineIntersections" exists in the back office and defines spatial points at the intersection of all street centerline features. Each point tracks the attribution as shown in Figure 4.8-12.

\begin{tabular}{|l|l|}
\hline \multicolumn{1}{|c|}{ Field Name } & \multicolumn{1}{c|}{ Data Type } \\
\hline NAME1 & Text \\
\hline NAME2 & Text \\
\hline ZIPCODES & Text \\
\hline XCOORD & Double \\
\hline YCOORD & Double \\
\hline ROADNAME & Text \\
\hline SHA.PE & Geometry \\
\hline
\end{tabular}

Figure 4.8-12 Attribution of street centerlines

Most of these attributes are used for system purposes but the Name1 and Name2 fields will allow a user to query the AEDR for street intersections such as "Main" and "Broadway" or any other combination. A spatial location is returned and the user can then zoom directly to the intersection of the specified streets. This works very well for all of the back office applications including ArcGIS, ArcView, and Facility Browser.

However, ArcReader only allows a user to query on a single field so inputting both a Name1 and a Name2 is not possible. Street intersection searches are crucial to field users and a solution was needed. 
Figure 4.8-13 shows new light-weight windows application, "AddIntersectionToStreetIntersections" that will be created. It will contain a single prompt that allows the Field Browser to input a UNC path to the personal geodatabase where the extracted StreetCenterlineIntersections feature class resides:

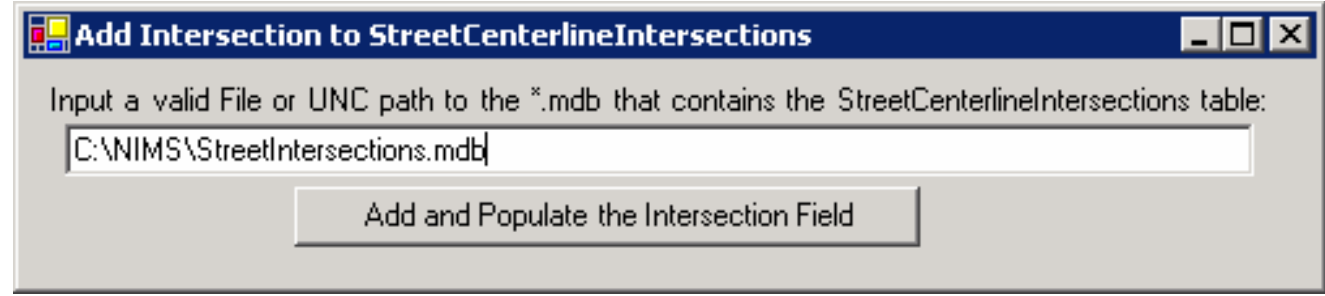

Figure 4.8-13. Street Centerline Intersection query

When the user clicks the "Add and Populate the Intersection Field" button, the process will access StreetCenterlineIntersections table to check for the existence of an "Intersection" column. If it is not found the application will automatically add this column to the table. The process will then proceed to concatenate the values for Namel and Name2 with an " $\&$ " in between and subsequently load this concatenated value into the Intersection column for all StreetCenterlineIntersections records. When this data is distributed to the field users, they can query on the single Intersection field with a parameter such as "Regency $\& 18^{\text {th }}$ " as shown in Figure 4.8-14.

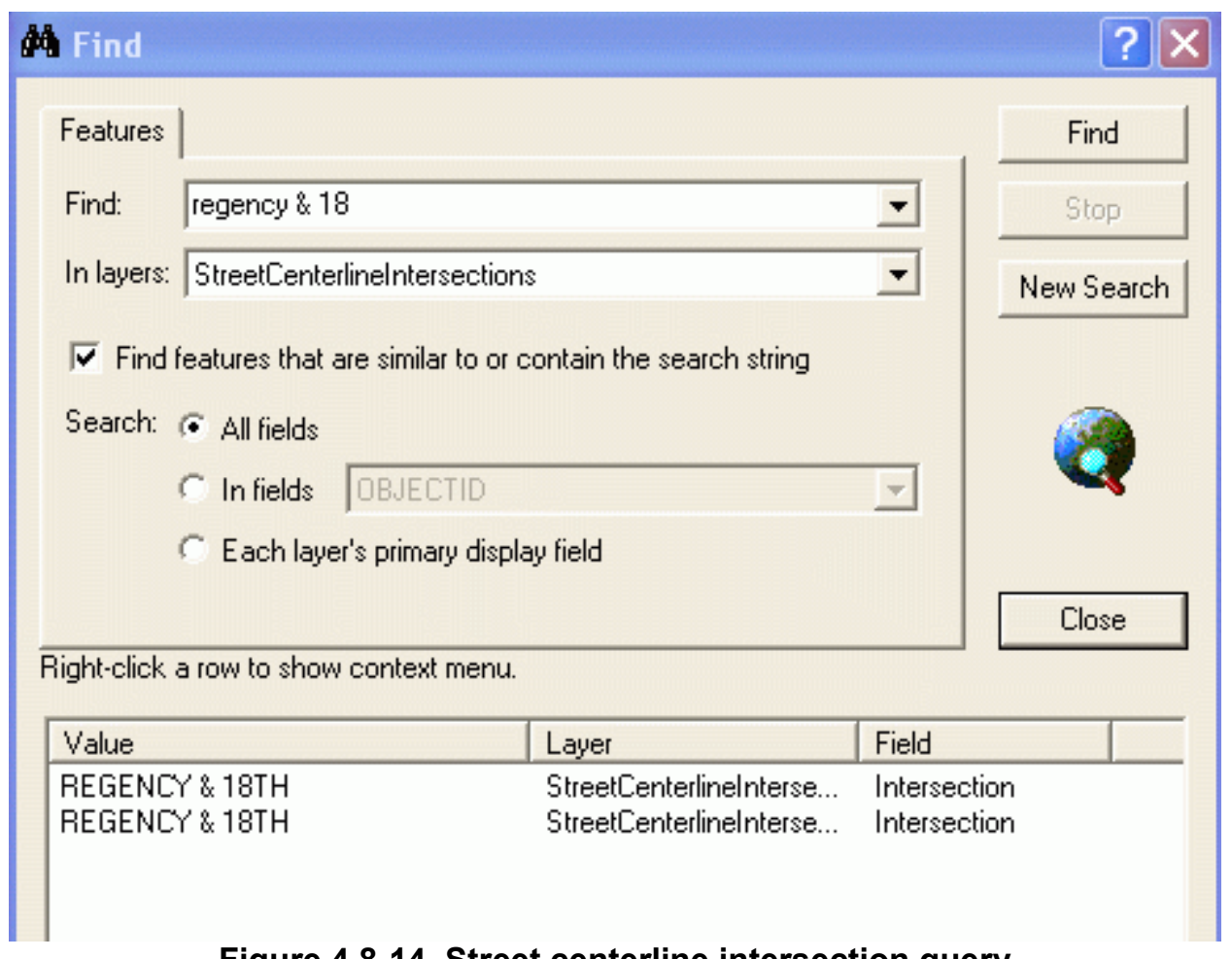

Figure 4.8-14. Street centerline intersection query 
This solution solves the problem and allows the field users to utilize the StreetCenterlineIntersections table in a very similar manner to back office users. 


\subsubsection{SynerGEE}

\subsubsection{Legacy DataPrep Overview}

This section provides an overview of the legacy DataPrep process. Legacy DataPrep was a component of an interface between the SynerGEE application and the legacy Outfield mapping system. Legacy DataPrep exported gas system data from NIPSCO's database of AutoCAD drawings as comma delimited text files. A second application called MiddleLink read these text files to create a SynerGEE database. Finally, the SynerGEE application used the database for gas system flow \& pressure calculation.

Legacy DataPrep exported gas mains, closed valves and regulator stations from each AutoCAD drawing specified by the user. Legacy DataPrep was designed to export open valves as well, however NIPSCO's legacy DataPrep only exported closed valves. Legacy DataPrep processed the facilities from each AutoCAD file contained within a target folder.

\subsection{Source AutoCAD Layers}

\begin{tabular}{|l|l|}
\hline Gas Mains & GLNLMAIXXXXXX \\
& GLNMMAIXXXXXX \\
& GLNHMAIXXXXXX \\
& GLNTMAIXXXXXX \\
& GLNTMAINIPXXX \\
\hline \multirow{2}{*}{ Valves } & GPTXVALEMGCLO \\
& GPTXVALREGCLO \\
\hline \multirow{2}{*}{ Regulator Stations } & GPTHREGSERXXX \\
& GPTMREGSERXXX \\
& GPTLREGSERXXX \\
& GPTHREGSTAXXX \\
& GPTMREGSTAXXX \\
& GPTLREGSTAXXX \\
\hline
\end{tabular}

Figure 4.8-15. Source AutoCAD Layers

\subsection{Export Files}

Legacy DataPrep generated eight text files for each AutoCAD drawing that was processed. Each of the eight file formats contain a header line, which contains comma delimited fields names, followed by many lines of comma delimited data values. When all the drawings were processed, Legacy DataPrep appended the files from each drawing into a single set of eight text files. The following file formats are generated by the application. 


\subsection{BRS File}

The BRS (browser) file contained data that was to be reviewed by the legacy DataPrep user after the process had been run. It contained references to regulator stations that do not have any connected pipes. The following header describes the fields exported to the browser file.

Drawing Name, Data Source ID, x1, y1, x2, y2, Error Code, Message

Drawing Name - No longer a need for this value

Data Source ID - The station EES number (Originally from "EES" EED Field) $\mathrm{x} 1-\mathrm{X}$ value for first point in regulator station $\mathrm{y} 1-\mathrm{Y}$ value for first point in regulator station $\mathrm{x} 2-\mathrm{X}$ value for second point in regulator station $\mathrm{y} 2-\mathrm{Y}$ value for second point in regulator station Error Code - Always "1"

Message - Always "Could not gather any pipes at insertion point of Regulator"

\subsection{GMAP File}

The GMAP file was written before any data is analyzed. It is static and contained the following lines:

[Version]

FmtFileVersion $=4.00$

\subsection{GNODE File}

The GNODE file contained all of the vertices for each gas main. The number of fields can vary due to the number of vertices. The following header describes the fields exported to the GNODE file.

¡Id,FromX,FromY,GNodeX,GNodeY,....ToX,ToY,

ID - EES number for the gas main (Originally from "EES" EED Field)

From $X-X$ value from the gas main's "From" point

From $Y-Y$ value from the gas main's "From" point

GNodeX - X value from one of a gas main's interior vertices (may repeat)

GNodeY $-\mathrm{Y}$ value from one of a gas main's interior vertices (may repeat)

ToX - X value from the gas main's "To" point

ToY - Y value from the gas main's "To" point 


\subsection{LOG File}

The log file contained messages generated by the application during the export process.

\subsection{NODE File}

The NODE file listed the nodes at the end of each selected gas main. The following header describes the fields exported to the NODE file.

;Id,X,Y,Name,Elevation,IsSupplyNode,SourcePressure,SecondaryPressureUnits,F low,SpecificGravity,HeatContent,Temperature,

ID - PCON NDE1 or NDE2 (Originally from PCON NDE1 or NDE2 EED Field)

$\mathrm{X}-\mathrm{X}$ coordinate for the node

$\mathrm{Y}-\mathrm{Y}$ coordinate for the node

Name - Empty

Elevation - Empty

IsSupplyNode - Always "False"

SourcePressure - Empty

SecondaryPressureUnits - Always "False"

Flow - Empty

SpecificGravity - Empty

HeatContent - Empty

Temperature - Empty

\subsection{Pipe File}

The PIPE file listed all of the gas mains selected by the user. The following header describes the fields exported to the PIPE file.

;Id,FNode,TNode,Name,Length,Material,NominalDiameter,InternalDiameter,Rou ghness,Pressure,Efficiency,Gravity,Temperature,FMMStatus,

ID - EES Number of the gas main (Originally from the EED "EES" field)

FNode - EES Number for the first node (Originally from PCON NDE1)

TNode - EES Number for the second Node (Originally from PCON NDE2)

Name - EES Number of the gas main (Originally from the EED "EES" field)

Length - Total length of the gas main (Calculated length)

Material - Material of the gas main (Originally from the EED "MAT" field)

NominalDiameter - Size of the gas main (Originally from the EED "SIZ" field)

InternalDiameter - Empty

Roughness - Empty

Pressure - Empty

Efficiency - Empty

Gravity - Empty

Temperature - Empty

FMMStatus - Always "U" 


\subsection{REGSTATION File}

The REGSTATION file listed all of the regulator stations selected by the user. The following header describes the fields exported to the REGSTATION file.

;Id,FNodeId, TNodeId,StationName,Name, Temperature,SetPressure,SetNodeId,Is Linear,Orientation,FMMStatus,

ID - EES Number of the reg station (Originally from the "EES" EED field)

FNodeID - EES Number for the first node (Originally from PCON NDE1)

TNodeID - EES Number for the second node (Originally from PCON NDE2)

StationName - Copied from the reg station's "NUM" EED field

Name - Concatenation of reg station's "NUM" and "SEQ" EED fields

Temperature - Empty

SetPressure - Empty

SetNodeID - EES Number for the second node (Originally from PCON NDE2)

IsLinear - Always "True"

Orientation - Empty

FMMStatus - Always "U”

\subsection{VALVE File}

The VALVE file contained data about closed valves only. The following header describes the fields exported to the VALVE file.

;Id,FNode,TNode,Name,RegulatorConstant,MaxRegulatorConstant,SetPressure, MinDP,ValveType,IsLinear,Orientation,FMMStatus,

ID - EES Number of the closed valve (Originally from the "EES" EED field)

FNode - EES Number for the first valve node (Originally from PCON NDE1)

TNode - Empty

Name - Empty

RegulatorConstant - Empty

MaxRegulatorConstant - Empty

SetPressure - Empty

MinDP - Empty

ValveType - Empty

IsLinear - Always "False"

Orientation - Empty

FMMStatus - Always "U”

\subsubsection{New DataPrep Design}

As previously mentioned in the overview, the NIPSCO GIS project team replaced Outfield; an AutoCAD based mapping system, with an ESRI based GIS. Because the facility data is now stored and maintained in a different technology platform, the legacy DataPrep component to the SynerGEE interface was re-written. The new custom DataPrep application creates the same text files in the same format as the legacy DataPrep application. The DataPrep export procedure was required to yield data values 
identical to the original Outfield data in order to support the target applications. However, instead of exporting the data from tiled AutoCAD drawings, the new application exports the data from a seamless ESRI Geodatabase.

Due to the change in technology, the following alterations to the application export process have occurred.

- Since the new DataPrep application only exports data from a single seamless data source, as opposed to multiple drawings, all text file headers occur only once in each file.

- The legacy Outfield managed field, EES, is no longer maintained in the new AEDR, as a result, the new DataPrep uses the ESRI managed ObjectId field instead as a unique identifier.

- The legacy Outfield managed fields NDE1, NDE2 are no longer maintained in the new AEDR either. The new DataPrep application replaced these values by generating node IDs at run-time. Like the NDE1 and NDE2 values, this new number uniquely identifies every exported node in the gas system.

- Because the original log file for legacy DataPrep was parsed per drawing and did not appear to have any data automation values, the new log file does not follow the same format as the original, but it does contain much of the same information.

- Regulator stations in the new system are one-point devices. In the legacy Outfield system regulators were two point devices with one of the points being identified as the high-pressure side of the regulator. The new DataPrep application exports the regulator stations from the AEDR so that the high-pressure side is identifiable by the SynerGEE application. The higher pressure side of the Regulator Station is identified in the REGSTATION file by the FnodeId field. The new application populates the FnodeId field by locating the geometrically coincident end Node of the GasMain feature identified in the RegulatorStation.RelUpstreamPipeOID field.

\subsection{Application Framework}

The DataPrep application is built as an ArcMap extension, which adds functionality to the out-of-the-box tools. The DataPrep extension adds a toolbar to the ArcMap graphic user interface, with a single command button. The button launches a wizard that guides the user through the export process. A number of configuration settings necessary for the DataPrep Application are configurable via an XML file.

\subsection{New Functionality}

The legacy DataPrep application does not export open gas valves. The source code that handles open valves in the legacy DataPrep application has been commented out. This 
exports open gas valves to an AIM file. The new DataPrep application exports the open gas valves to the AIM file.

\subsection{Source Data}

DataPrep extracts information from an ESRI Geodatabase and saves that information as a set of comma delimited text files. Below is a list of connection properties to the Geodatabase and the feature classes used for the export.

Server Name: aedrdbsqlp01

ArcSDE Instance Name: esri_sde

ArcSDE Instance port: 9999

Database: $\operatorname{arcfm} 8$

Version: Master Child

Feature Classes: arcfm8.gas.GasMain, arcfm8.gas.Gas Valves, and arcfm8.gas.RegulatorStations

Assumption: A single child version will be created to SDE.Default. This document refers to the child version as "Master Child". Master Child will be the only child version to SDE.Default. All other versions will be created as children to the Master Child version.

\subsection{User Interface}

The user launches the DataPrep application by double clicking on the DataPrep ArcMap document icon located on the user's Desktop shown in Figure 4.8-16. The DataPrep ArcMap Document contains all configuration settings necessary for the DataPrep Application and is contained in the ArcMap Document. Three layers comprise the map, "Gas Main", "Gas Valves", and "Regulator Stations".

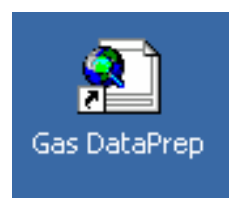

Figure 4.8-16.

The user clicks on the DataPrep button to launch the wizard dialog box.

Open Gas DataPrep

Figure 4.8-17. 
The first screen, Figure 4.8-18 of the DataPrep wizard asks the user to specify an export project name and directory. Once a valid path has been specified, the Next button will become enabled and the user clicks Next.

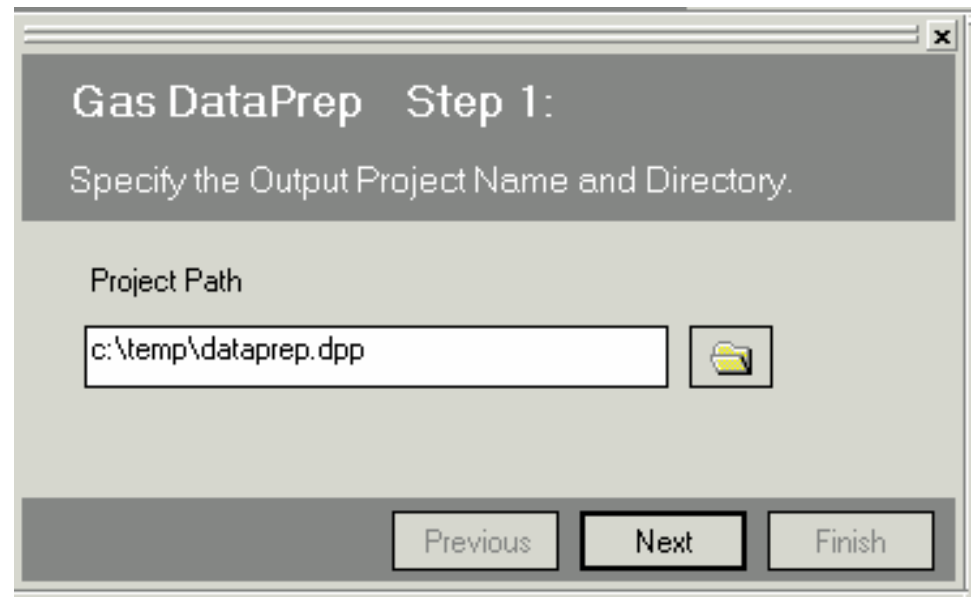

Figure 4.8-18. DataPrep output location

On the second wizard screen shown in Figure 4.8-19, the user can choose whether to export all features or features from a selection and clicks Next.

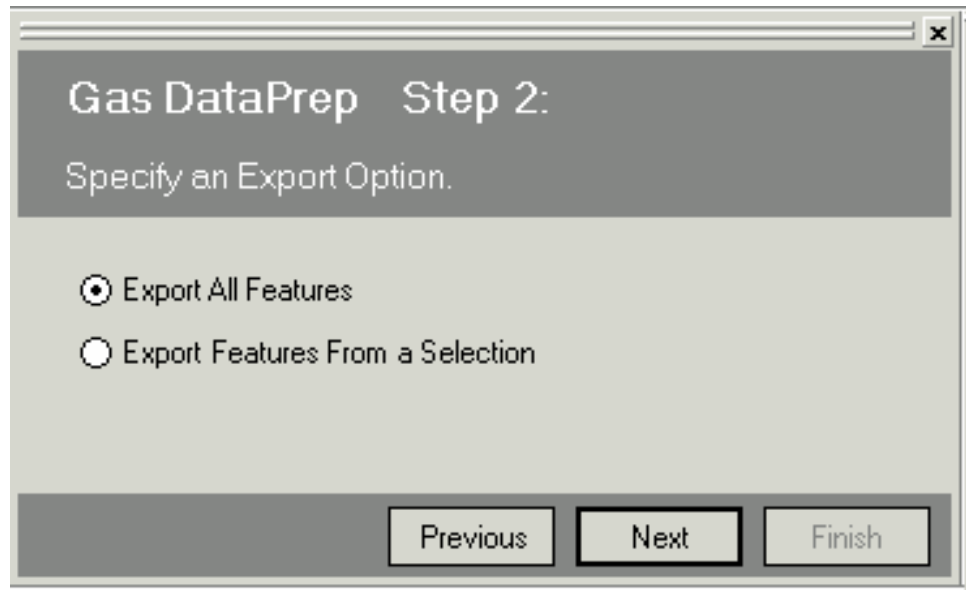

Figure 4.8-19. DataPrep export option 
If the user chooses to export all features, the Figure 4.8-20 is presented and the user clicks Finish.

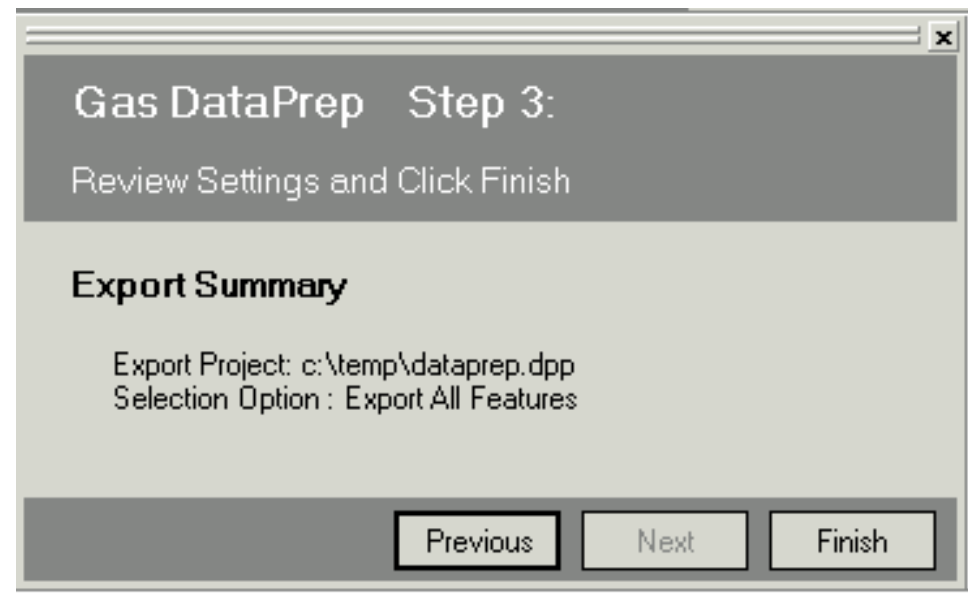

Figure 4.8-20. DataPrep export summary

If the user chooses to export features from a selection, Figure 4.8-21 the Feature Selection wizard screen is presented. The Feature Selection screen is a tabbed control offering several selection options including select by trace, select by attribute, and select by shape.

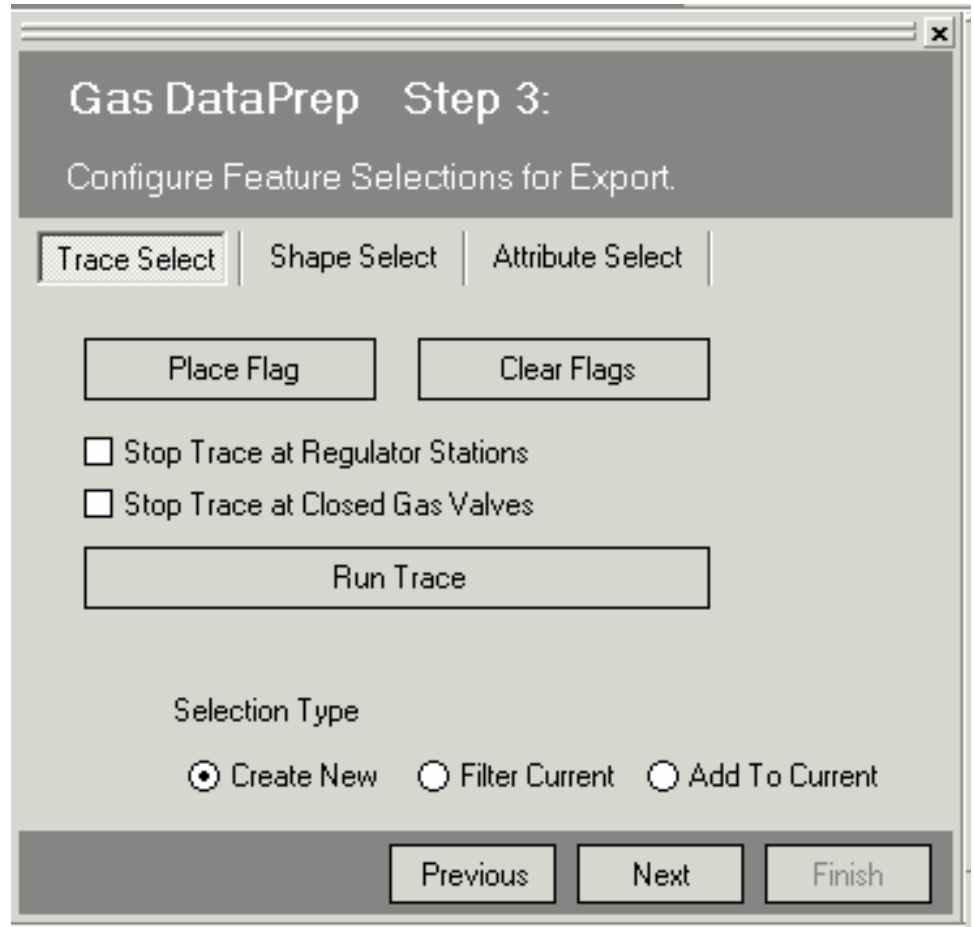

Figure 4.8-21. Feature selection configuration box 


\subsubsection{CADOPS and (FeederAll Part 1)}

\subsubsection{Overview}

For outage management in the electric distribution system, NIPSCO uses ABB CADOPS running on top of an Oracle database. This system is also referred to as the NIPSCO Outage Restoration System or NORS. Previously, NIPSCO used a tool called ABB OUT to export facility data from legacy Outfield into a comma-delimited file. ABB OUT was also responsible for assigning type numbers to all features to provide a link to characteristic tables (DEVICETYPE, LINETYPE) maintained in CADOPS. Operations then used Oracle SQL Loader to load the data from the comma-delimited file into the CADOPS Oracle tables where custom SQL scripts were then run to update additional information.

For engineering analysis in the electric distribution system, NIPSCO uses ABB FeederAll. In the legacy system, a copy of the CADOPS comma-delimited file is used to supply the data for FeederAll. Oracle SQL Loader is used to load the data from the comma-delimited file into the FeederAll Oracle tables where custom SQL scripts are then run to update additional information. Next, an ABB process was used to transfer the Oracle data into a Microsoft Access database, which supports the FeederAll application. Additional hand entry of data within the Access database was necessary to support the analysis aspects of FeederAll.

To support the new interface, facility features and the network hierarchy are exported into the required comma-delimited file formats for line, device, load, node, path, capacitor, source, and mpoint. This is accomplished using ArcFM Network Adapter to select either a section or a feeder of data for export. The export process uses the XML produced by the Network Adapter API and transforms it to create the aforementioned comma-delimited formats for CADOPS and FeederAll. Only facility network geometry and attributes are exported - no annotation is exported for facility information.

CADOPS was completed and installed into production in Phase I of the AEDR project. FeederALL implementation was postponed until Phase II. However, the two projects share the same application with slightly differing configurations and exports, therefore they are described as one application and the interfaces are differentiated within this section. 
The Figure 4.8-22 diagram illustrates the new interface:

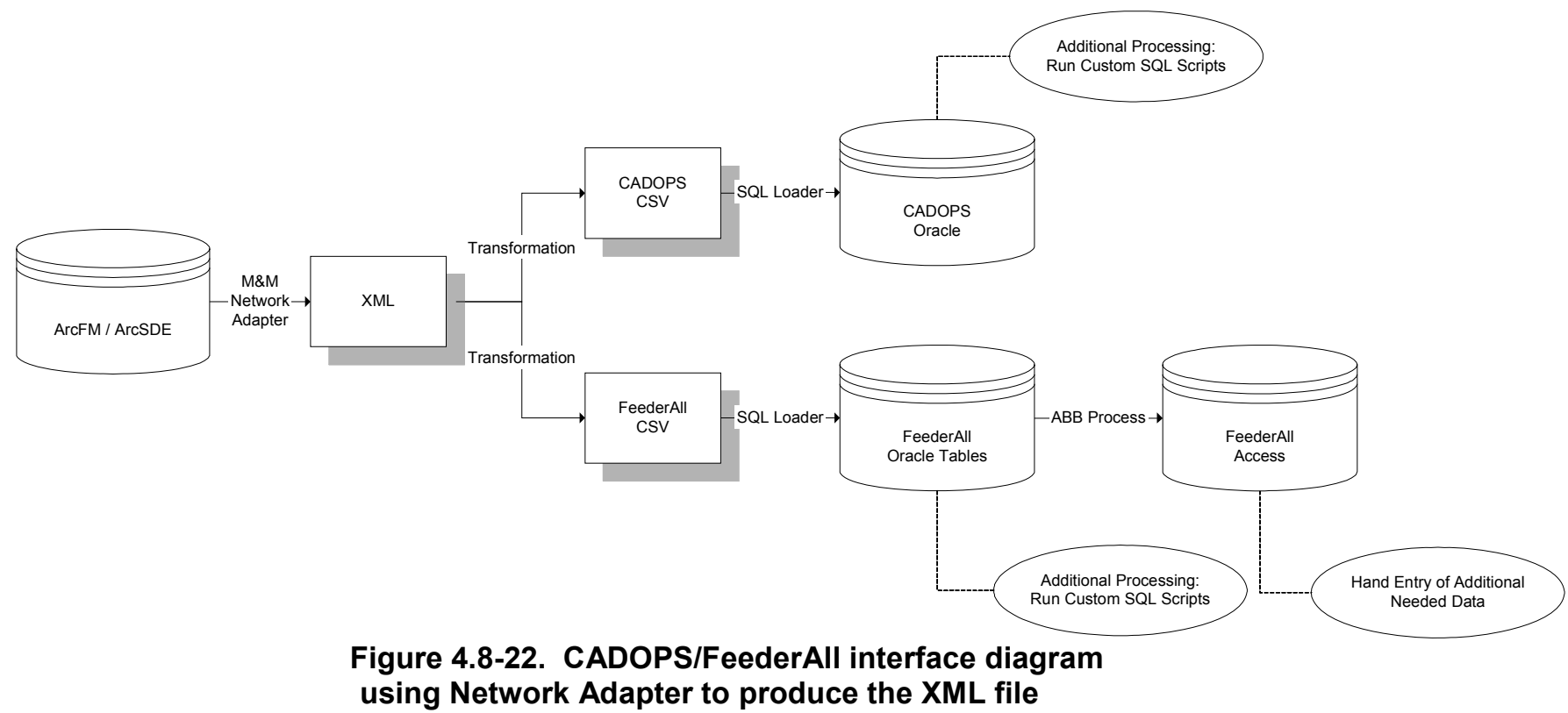

Once the XML is transformed into the CADOPS and FeederAll comma-separated-value (CSV) files, the new process will match the process that existed previously.

\subsection{ArcFM Network Adapter Architecture}

Network Adapter is a part of the ArcFM Solution and is based on ArcGIS 8 technology. The solution architecture is such that Network Adapter can be used with ArcFM, Designer, or Responder. The Network Adapter toolbar is available within ArcFM and allows a user's specific network model to be made available to external analysis engines.

The Network Adapter data flow framework consists of the following primary components:

- The Analysis API handles data flow between the Geodatabase in ArcFM/Designer and the data-specific XML document. The Analysis API is common to all analysis implementations and is a commercial off-the-shelf (COTS), COMcompliant component. It extracts features selected by the user-this means the user can create a selection set by Feeder or Feeders or using any spatial or logical query tool provided with ArcMap or ArcFM. The specific data items within the selected set that the API extracts are determined by the configuration of Model Names. The API has been optimized to produce the XML in a format that contains network connectivity and all the attributes configured through Model Names for extraction.

- As shown in Figure 4.8-23, the Implementation Specific Transformation which transforms data from the data-specific XML document produced by the Analysis API into the format required by a specific implementation. This transformation is accomplished through the use of XSLT. 


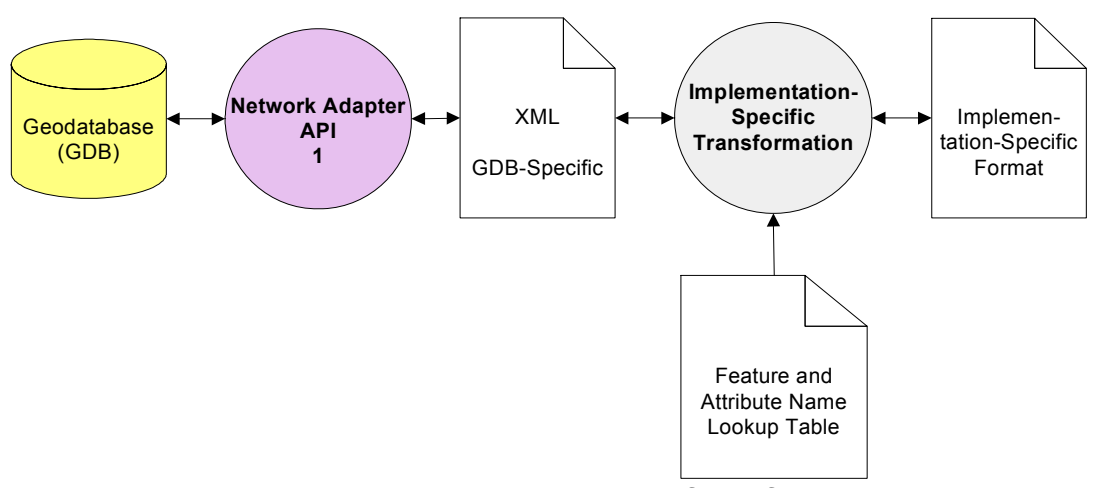

Figure 4.8-23. Network Adapter data flow framework

\subsection{Extensible Stylesheet Language Transformation (XSLT)}

An XSLT stylesheet is used to transform an XML document into another form. This could be another XML document, or a document in a different format altogether, such as PDF, HTML, or TXT. XSLT stylesheets work as a series of templates, which produce the desired formatting effect each time a given element is encountered. XSLT can also control the order in which elements and attributes are displayed. This means that tables of contents or indexes can be generated automatically on the basis of the content of a document. XSLT itself is an implementation of XML.

XSLT is sometimes defined as "Transformation by Example" whereas many other techniques would be defined as "Transformation by Program Logic". When using XSLT, a stylesheet (the example) is created that defines the desired end result. The XSLT processor is then responsible for transforming the source XML into that desired result.

The stylesheets, or "transformation specifications", are written primarily with declarative constructs though procedural techniques can be employed as needed. The stylesheet asserts the desired behavior of the XSLT processor based on conditions found in the source XML. It supplies examples of how each component of the result is formulated and indicates the conditions of the source that trigger which component is added to the result.

The use of XSLT within this interface will consist of the organizing and formatting of the Network Adapter XML into the appropriate CSV files to be imported into CADOPS and FeederAll.

\subsubsection{Use Case}

Within an ArcFM session, the user selects the desired features using any of the ArcFM / ArcMap tools (including select by attributes, select by Feeder, trace tools, rubber band selection, etc). The user opens the ArcFM Network Adapter toolbar and clicks the export button. The system exports the selected features into the Network Adapter XML file based on the configured Network Adapter class and field model names. The system then transforms the XML via XSLT into the CADOPS and FeederAll comma-delimited file formats that are used by the current system. The user loads the comma-delimited files into their respective Oracle databases using Oracle SQL Loader. The CADOPS and 
FeederAll interface process continues using the post-processing techniques and algorithms as they exist today.

\subsubsection{Design}

The detailed design of this interface outlines the feature classes and attributes that will be exported via Network Adapter and then the specifics of how they will be transformed via XSLT into the necessary comma-delimited files. The Oracle data models for CADOPS and FeederAll are identical which serves to simplify the process of transforming the data as well as allows for only a single set of Oracle SQL Loader control files to be maintained. However, the data modeling requirements for FeederAll differ from CADOPS significantly which has resulted in the need to have two separate exports for the two systems.

\subsection{Feature and Object Class Mappings}

The requirements for this interface state that eight key tables must be loaded for CADOPS and FeederAll to function correctly. These tables include Line, Device, Load, Node, Path, Capacitor, Source, and MPoint. The GIS feature classes that participate in the electric network (and their related object classes) have each been individually mapped into these seven ABB tables shown in Table 4.8-1.

Table 4.8-1. GIS feature and object class mapping

\begin{tabular}{|l|l|l|}
\hline GIS Feature Class & ABB Table & System \\
\hline Switch / SupportStructure & Device & FeederAll \& CADOPS \\
\hline $\begin{array}{l}\text { FuseCutoutBank / FuseUnit / SupportStructure } \\
\text { RecloserBank / RecloserUnit / } \\
\text { SupportStructure }\end{array}$ & Device & FeederAll \& CADOPS \\
\hline $\begin{array}{l}\text { SectionalizerBank / SectionalizerUnit / } \\
\text { SupportStructure }\end{array}$ & FeederAll \& CADOPS \\
\hline PrimaryOpenPoint & Device & \\
\hline $\begin{array}{l}\text { VoltageRegulator / VoltageRegulatorUnit / } \\
\text { VoltageRegulatorBank }\end{array}$ & Device & FeederAll \& CADOPS \\
\hline CustomerGenerator & Device & FeederAll \& CADOPS \\
\hline CustomerGenerator & Device & FeederAll \\
\hline $\begin{array}{l}\text { TransformerBank / TransformerUnit / } \\
\text { SupportStructure }\end{array}$ & Source & FeederAll \& CADOPS \\
\hline SubstationBreaker & Load & FeederAll \& CADOPS \\
\hline & Device & FeederAll \\
\hline SubstationBreaker / CircuitSource / Substation & Source & $\begin{array}{l}\text { FeederAll \& CADOPS } \\
\text { (with differing stype } \\
\text { values) }\end{array}$ \\
\hline SubstationBreaker & Mpoint & FeederAll \\
\hline PowerTransformer / SubstationBreaker(s) & Source & FeederAll \\
\hline CapacitorBank / CapacitorUnit & Capacitor & FeederAll \& CADOPS \\
\hline & Node \& & FeederAll \& CADOPS \\
\hline Network Junction & Path & \\
\hline OhPrimary / ConductorInfo & Line & FeederAll \& CADOPS \\
\hline
\end{tabular}




\begin{tabular}{|l|l|l|}
\hline UgPrimary / Conductorlnfo & Line & FeederAll \& CADOPS \\
\hline Busbar & Line & FeederAll \& CADOPS \\
\hline Tiewire & Line & FeederAll \& CADOPS \\
\hline PrimaryMeter & Load & FeederAll \& CADOPS \\
\hline OhTransmission / ConductorInfo & Line & FeederAll \\
\hline UgTransmission / Conductorlnfo & Line & FeederAll \\
\hline FeederAllOpenPoint (FAOP) & Device & FeederAll \\
\hline
\end{tabular}

It was determined that although terminators and splices also participate in the electric network within the GIS, they would not be mapped into the ABB interface, as their existence does not affect either CADOPS or FeederAll. Also, the transmission features will only be exported for FeederAll as CADOPS deals exclusively with the distribution network.

Many attributes of the feature/object classes are mapped directly across into ABB fields. Some fields require additional processing such as concatenation or other summarization. These fields are further detailed in the next section.

ArcFM was configured to export the needed tables and attributes via Network Adapter. This is accomplished by creating new class and field model names to identify the tables and attributes to be exported. For this interface, two class model names of "CADOPS" and "FEEDERALL" and two field model names of "CADOPS" and "FEEDERALL" were created and configured on the tables listed above. This configuration can be easily modified along with the XSLT in the future to configure additional fields to be exported.

\subsubsection{Fields Requiring Additional Processing}

\subsection{Device}

- DID: The purpose of this field is defined as containing a unique identifier. Because multiple feature classes are being mapped into the DEVICE table, a unique combination of feature class / feature IDs are necessary. Though this field's data type is defined as a VARCHAR, the data is later migrated into a NUMBER field which does not allow for any delimiting text such as a ".”. To satisfy these requirements, the FeatureClassId is concatenated with the ObjectId to create a single unique number. This ID allows the feature to be easily located in the GIS as long as the user is aware of the value of the FeatureClassId (this is a constant number determined when the geo-database schema is created).

- DNAME: The purpose of this field is to provide a name or description of the device. The desired format for this field is "CircuitId - SubstationId LoaNumber". To achieve this format, three GIS fields are concatenated. The circuit ID (or feeder ID) is read from the FeederId field, which is maintained by Feeder Manager on all electric network features. The SubstationId is acquired by traversing the relationships from any network feature to the CircuitSource. Finally, the LoaNumber exists on all features that are being exported.

- NORM_STATE: This field accepts the normal status of the device in the format of a 3-digit integer ( $\mathrm{ABC}-0$ indicates open, 1 indicates closed, ex. 101 - A \& C closed, 
B open). In the GIS, the normal state is tracked individually for phases A, B, and C. To populate this field in the ABB table, the values for the individual states are concatenated together to create the needed 3-digit integer.

- PHASE: This field accepts the phase on the device in the format of a 3-digit integer (ABC -1 present, 0 not present). In the GIS, phase is tracked as a domain of values for all available phase combinations. To populate this field in the $\mathrm{ABB}$ table, the phase within the GIS is mapped into the 3-digit format (i.e. $\mathrm{ABC}=111, \mathrm{AC}=101$, $\mathrm{B}=010$, etc). This phase designation does not imply the order of phases as they may appear on a pole or structure, such as $\mathrm{ABC}, \mathrm{CAB}, \mathrm{BCA}$, or other phase orientation combinations.

- DEVICESIZE: This field tracks the amperage rating of the device. In most cases this exact field is tracked on the device features in the GIS. In a few cases like Switch, this field is not tracked on the banked feature class object but is calculated by adding the amperage rating values of any related unit objects.

\subsection{Load}

- LOID: This purpose of this field is defined as containing a unique identifier. In the case that the load is related to an overhead device, this field is populated with the related SupportStructure's PrimaryReferencePoleNumber. In the case that the load is related to an underground device, this field is populated with LongPadNumber that exists directly on the device.

- LONAME: The purpose of this field is to provide a name or description of the load. The desired format for this field is "CircuitId - SubstationId LoaNumber". To achieve this format, three GIS fields are concatenated. The circuit ID (or feeder ID) is read from the FeederId field, which is maintained by Feeder Manager on all electric network features. The SubstationId is acquired by traversing the relationships from any network feature to the CircuitSouce. Finally, the LoaNumber exists on all features that are being exported.

- PHASE: This field accepts the phase on the device in the format of a 3-digit integer (ABC -1 present, 0 not present). In the GIS, phase is tracked as a domain of values for all available phase combinations. To populate this field in the $\mathrm{ABB}$ table, the phase within the GIS is mapped into the 3-digit format (i.e. $\mathrm{ABC}=111, \mathrm{AC}=101$, $\mathrm{B}=010$, etc).

- ABB_INT_ID: The purpose of this field is defined as containing a second unique identifier. Because multiple feature classes are being mapped into the load table, a unique combination of feature class / feature IDs are necessary. This field's data type is defined as a NUMBER(10) which does not allow for any delimiting text such as a ".”. To satisfy these requirements, the FeatureClassId is concatenated with the ObjectId to create a single unique number. This ID also allows the feature to be easily located in the GIS as long as the user is aware of the value of the FeatureClassId (this is a constant number determined when the geo-database schema is created).

\subsection{Source}

- ABB_INT_ID: The purpose of this field is defined as containing a unique identifier. Because multiple feature classes are being mapped into the source table, a unique 
combination of feature class / feature IDs are necessary. This field's data type is defined as a NUMBER(10) which does not allow for any delimiting text such as a ".." To satisfy these requirements, the FeatureClassId is concatenated with the ObjectId to create a single unique number. This ID also allows the feature to be easily located in the GIS as long as the user is aware of the value of the FeatureClassId (this is a constant number determined when the geo-database schema is created).

- SOID: The purpose of this field is to provide a name or description of the source. The desired format for this field is "CircuitId - SubstationId LoaNumber". To achieve this format, three GIS fields are concatenated. The circuit ID (or feeder ID) is read from the FeederId field, which is maintained by Feeder Manager on all electric network features. The SubstationId is acquired by traversing the relationships from any network feature to the CircuitSource. Finally, the LoaNumber exists on all features that are being exported. In the case of a customer generator, CustomerName should be substituted for SubstationId.

- SONAME: The desired format for this field is "SubstationId - SubstationNumber". The SubstationId is acquired by traversing the relationships from any network feature to the CircuitSource. The SubstationNumber is acquired by traversing the relationships from any network feature to the CircuitSource to the SubstationBreaker to the Substation. In the case of a customer generator, CustomerName and CustomerSubstationId are substituted for SubstationId and SubstationNumber respectively.

\subsection{Capacitor}

- CAID: The purpose of this field is defined as containing a unique identifier. To accomplish this, a unique combination of feature class / feature IDs are necessary. Though this field's data type is defined as a VARCHAR, the data is later migrated into a NUMBER field which does not allow for any delimiting text such as a ".". To satisfy these requirements, the FeatureClassId is concatenated with the ObjectId to create a single unique number. This ID also allows the feature to be easily located in the GIS as long as the user is aware of the value of the FeatureClassId (this is a constant number determined when the geo-database schema is created).

- CANAME: The purpose of this field is to provide a name or description of the capacitor. The desired format for this field is "CircuitId - SubstationId LoaNumber". To achieve this format, three GIS fields are concatenated. The circuit ID (or feeder ID) is read from the FeederId field, which is maintained by Feeder Manager on all electric network features. The SubstationId is acquired by traversing the relationships from any network feature to the CircuitSouce. Finally, the LoaNumber exists on all features that are being exported.

- PH3PRES: This field accepts the phase on the capacitor in the format of a 3-digit integer ( $\mathrm{ABC}-1$ present, 0 not present). In the GIS, phase is tracked as a domain of values for all available phase combinations. To populate this field in the ABB table, the phase within the GIS will need to be mapped into the 3-digit format (i.e. $\mathrm{ABC}=111, \mathrm{AC}=101, \mathrm{~B}=010$, etc). 


\subsection{Line}

- ID: The purpose of this field is defined as containing a unique identifier. Because multiple feature classes are being mapped into the LINE table, a unique combination of feature class / feature IDs are necessary. Though this field's data type is defined as a VARCHAR, the data is later migrated into a NUMBER field which does not allow for any delimiting text such as a ".". To satisfy these requirements, the FeatureClassId is concatenated with the ObjectId to create a single unique number. This ID also allows the feature to be easily located in the GIS as long as the user is aware of the value of the FeatureClassId (this is a constant number determined when the geodatabase schema is created).

- NAME: The purpose of this field is to provide a name or description of the line. The desired format for this field is "CircuitId - SubstationId LoaNumber". To achieve this format, three GIS fields are concatenated. The circuit ID (or feeder ID) is read from the FeederId field, which is maintained by Feeder Manager on all electric network features. The SubstationId is acquired by traversing the relationships from any network feature to the CircuitSource. Finally, the LoaNumber exists on all features that are being exported.

- PHASE_PERM: This field accepts the phases that are built on the line in the format of a 4-digit integer (representing $\mathrm{ABCN}$ ). A value of 0 indicates the phase is not present. The values of 1-4 are used to indicate the position of the phased conductor if it exists. For NIPSCO's purposes, the conductors are always in the order ABCN $(\mathrm{ABCN}=1234, \mathrm{AN}=1004, \mathrm{AB}=1200, \mathrm{ABN}=1204$, etc). In the GIS, phase is tracked as a domain of values for all available phase combinations. To populate this field in the ABB table, the phase within the GIS is mapped into the first three digits of the above format. To determine if a neutral conductor is present, the related ConductorInfo objects are searched for an object with ConductorTypeCd=WN, which indicates a neutral conductor.

\subsubsection{ABB Category, Type, Voltage, and Symbolization Fields}

Many of the ABB tables utilize lookup tables to determine the category, type, voltage level, and/or symbol to use within CADOPS and FeederAll. These values are set within the interface based on the properties of the features being exported. ABB maintains individual lookup tables for device category, device type, regulator type, line type, symbol, and voltage level. These tables have been consolidated into three text files in the current interface (Outfield $\rightarrow \mathrm{ABB}$ ). Within the new interface, these lookup tables will be mapped into a single XSL lookup files as follows:

Table 4.8-2. ABB lookup tables defining fields

\begin{tabular}{|l|l|}
\hline ABB Lookup Table & GIS Lookup Table \\
\hline DEV_CAT & ABBLookups.xsI \\
\hline DEVTYPE & ABBLookups.xsI \\
\hline REGTYPE & ABBLookups.xsI \\
\hline LINETYPE & ABBLookups.xsI \\
\hline SYMBOL & ABBLookups.xsI \\
\hline VOLTAGELV & ABBLookups.xsI \\
\hline
\end{tabular}




\subsubsection{Issues}

Many issues were raised during the workshop for this interface. These issues have been reviewed by the NIPSCO GIS and/or CADOPS and FeederAll teams and have been closed with a decision. These issues are documented below:

\subsection{Modeling of VoltageRegulator}

The GIS models two and three-phase VoltageRegulators on two and three poles respectively because of their physical size. These multi-phase VoltageRegulators are related to each other via a VoltageRegulatorBank object. For the ABB interface, any multi-phase VoltageRegulator is transferred as a single entry in the DEVICE table. The location of the entry in the DEVICE table is derived from any one of the phased VoltageRegulator features. The phasing on the single device entry was adjusted to include all available phases in the bank.

\subsection{New Business Process for Modeling CustomerGenerator}

Customer generators are new features that are included in the AEDR going forward. To support the effective use of customer generators in the CADOPS and FeederAll applications, a new business process was created for the modeling of customer generators within the AEDR. When placing a CustomerGenerator feature, it is offset from the main primary conductor. This is accomplished by creating a primary conductor stub between the main primary conductor line and the CustomerGenerator. The CustomerGenerator is always modeled as an open device to further facilitate the export to the ABB applications. (NormalPosition for A, B, and C set to 0 ).

\subsection{Utilization of ABB Path Points}

The legacy interface between Outfield and CADOPS and FeederAll did not utilize path points but instead modeled all line objects as node to node. To provide a more accurate representation of the GIS spatial information, the new interface utilizes path points (via the ABB PATH table) to define multi-segmented lines. To accomplish this, the electric network simple junctions are used as path points and lines are only broken by nodes where their originating features are broken in the GIS (at devices or attribution changes).

\subsection{GIS Modeling of SubstationBusbar}

The GIS model includes a feature class for Busbar with an available subtype of SubstationBusBar. This subtype has its FdrMgrNonTraceable property defaulted to true (1). This prevents the SubstationBusbar from being traced and included in any ArcFM feeders. The intended use of the SubstationBusbar is to model connections to a common point between multiple SubstationBreakers within a Substation. This data will be used by FeederAll to assist in re-modeling a common source for multiple feeders and should be included in any exports for FeederAll. It should be noted that because the SubstationBusbar is not included as part of an ArcFM feeder, it will not be selected when running a 'select by feeder' operation or by an ArcFM trace. Therefore, to include it in an export for FeederAll, the SubstationBusbar will need to be manually added to the selection before launching the Network Adapter export. SubstationBusbar was excluded from any CADOPS export. 


\subsection{Maintaining List of Node IDs at LOA Boundaries}

Part of the CADOPS import process is to run a script that creates sources on all nodes that exist at a LOA boundary. To accomplish this, the CADOPS administrator maintains a list of the IDs of those nodes at LOA boundaries. The concern was raised that the IDs of these nodes might not be constant if the electric network was dropped and recreated. While this is true, now that the AEDR data has been put into production and versioned, it is highly unlikely that the network will be dropped and recreated (therefore maintaining constant IDs).

\subsection{Export of Transmission}

The FeederAll application includes analysis for transmission features whereas the CADOPS application deals exclusively with distribution features. Since the Network Adapter export operates based on a selected set of features, the easiest way to handle exporting different sets of features to the different systems is to complete the export based off of different selected sets of features. To include transmission features in an export, the user should manually add the features to the selected set. It is acceptable to also include surrounding features in the selected set as they will not be exported unless they have been configured for the ABB export via the chosen model names. This approach also allows more specific exports to be run (i.e. transmission only) as needed.

\subsection{Tracking of a Single Source for Multiple Feeders}

The FeederAll application requires a single source for multiple feeders within a substation. A new feature class called PowerTransformer was added to the data model that acts as this single source and is tied to multiple SubstationBreakers by SubstationBusbar. The PowerTransformer was modeled as a simple point feature and will not be part of the electric network as it is not recognized as part of a feeder by Feeder Manager. An explicit relationship is created between the PowerTransformer and any downstream SubstationBreakers. This relationship will allow the export to use attribution from the SubstationBreakers instead of duplicating the data within the PowerTransformer feature class. In this manner, the PowerTransformer exists only as a geographic location for the FeederAll export. Figure 4.8-24 illustrates an example of a PowerTransformer connected to three Feeders: 


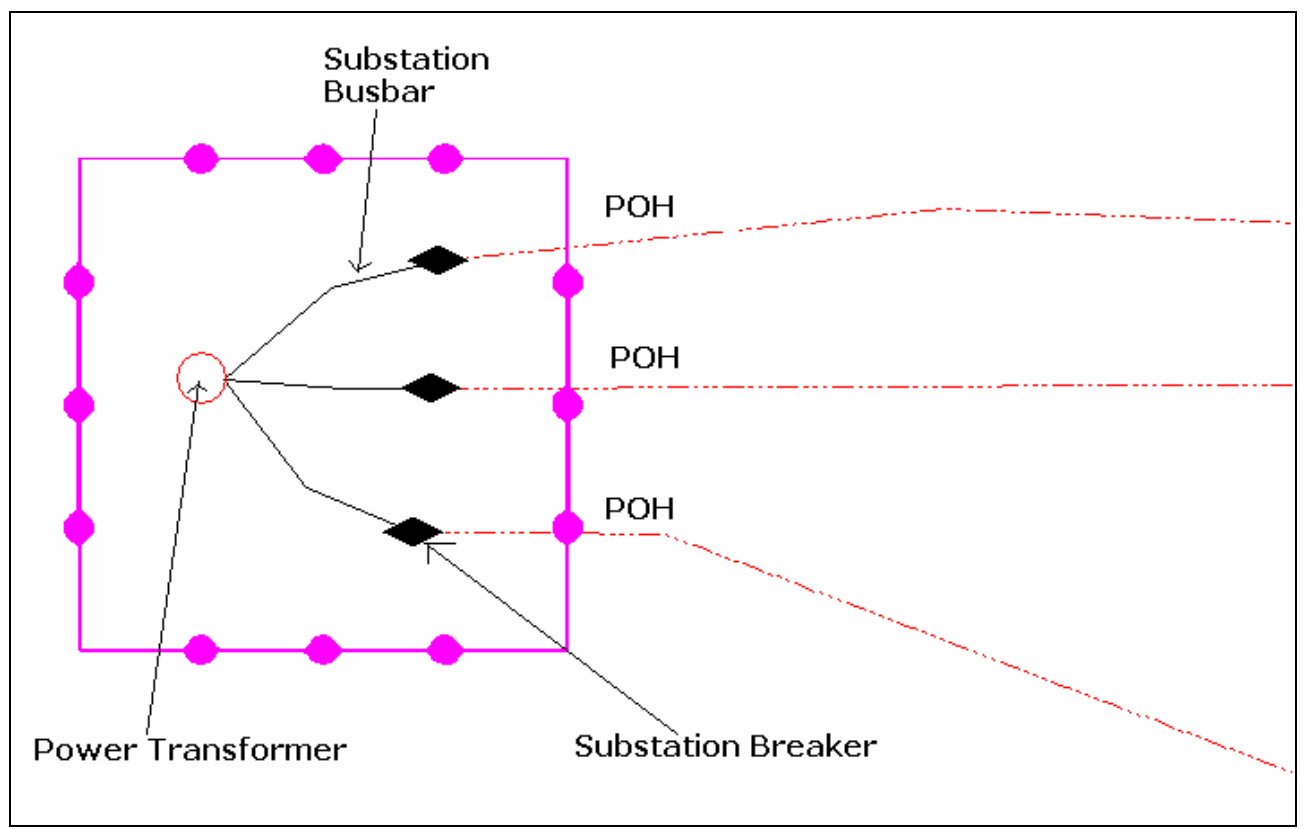

Figure 4.8-24. Feeder configuration within a substation

When these features are exported into FeederAll, an entry in the SOURCE table (with stype $=1$, designates as FeederAll source) is created at the node where the PowerTransformer exists. The SubstationBusbar will be exported as lines. And at the SubstationBreakers, three records are entered for each breaker as follows: 1) Entry is created in the Source table (with stype $=0$, designates as FeederAll feeder designation) 2) Entry is created in the MPOINT table (creates a FeederAll meter point) 3) The SubstationBreaker is entered as a device on the line. The handling of these features is significantly different from CADOPS and is necessary to run the distribution load analysis for a substation within FeederAll.

The required data model changes have been approved by the NIPSCO GIS project team. If this data model change had not been approved, the FeederAll administrator would have to continue with the legacy process, which included manually connecting related SubstationBreakers together via ABB lines and creating a single source connected at the intersection of the lines. It has been noted that because NIPSCO manages over 300 substations in the GIS, that this is a very time intensive process that would prevent the FeederAll users from gaining the desired benefits from the new interface.

\subsection{Modeling of a Single Phase Transformer on a 3-Phase Line}

The NIPSCO data has many cases where a 3-phase underground line feeds an area where a single-phase transformer is offset from the 3-phase line. In these cases, a single-phase conductor is split off from the 3-phase line, runs out to the transformer, and returns to join the 3-phase line. The segment of the 3-phase line where the single-phase line splits off is reduced to a 2-phase line. The graphic in Figure 4.8-25 illustrates this case: 


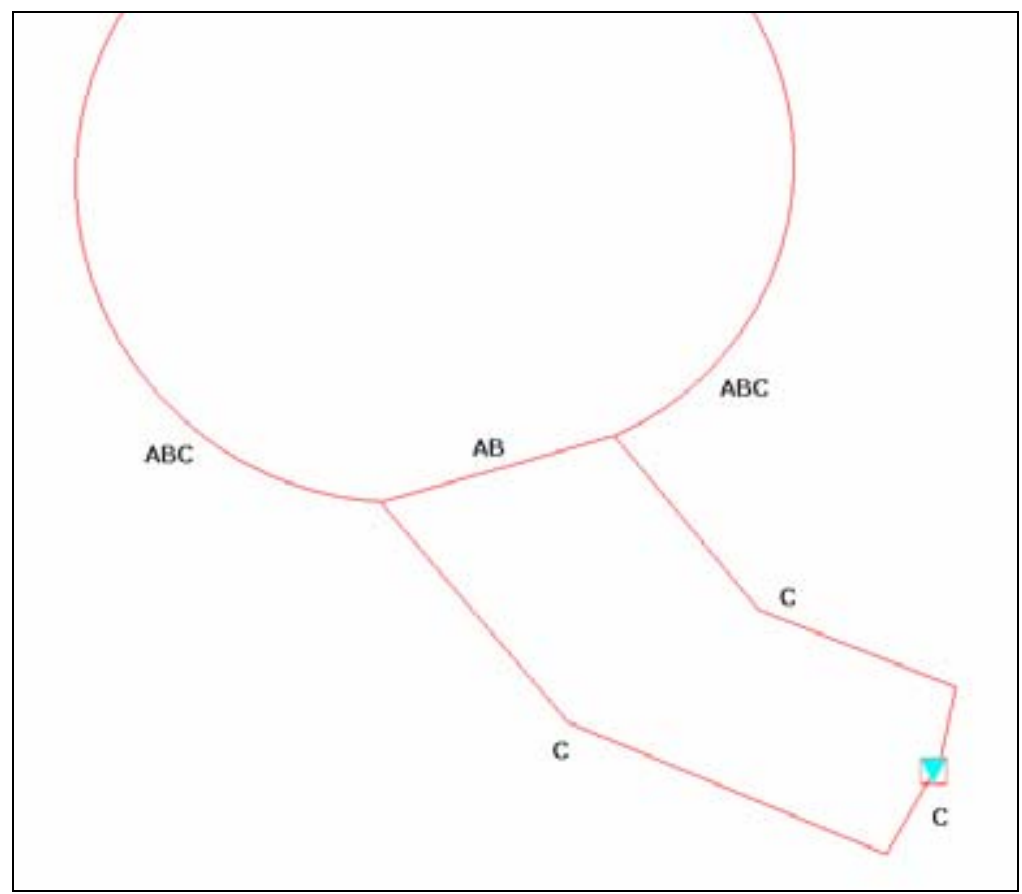

Figure 4.8-25. Single-phase transformer offset on a 3-phase line

This case accurately models the facilities as they exist in the field and is a valid scenario within the AEDR and within CADOPS. This case is not, however, supported by FeederAll. The FeederAll analysis sees this case as a loop condition and the administrator has to subsequently enter an open point on the single-phase line and update the two-phase line to 3-phase to correct the problem. To prevent this required manual update of the data by the FeederAll administrator, a new approach to this case was proposed. A new feature class called FeederAllOpenPoint should be created as part of the electric network within the GIS. This feature class should have no impact on FeederManager and will exist purely for the purposes of exportation to FeederAll. A FeederAllOpenPoint should be placed on the leg of the $C$ phase that is downstream from the pad-mounted transformer. During the export, this FeederAllOpenPoint will be exported as an open device into FeederAll.

Next, a new attribute was added to the underground conductor feature class called FeederAllPhase. This attribute references the 'Phase Designation' domain. It allows the conductor to carry a different phase for the FeederAll export than it does for any GISbased analysis. In the above example, the conductor with a phase of $\mathrm{AB}$ would have a FeederAllPhase of ABC. The export uses the FeederAllPhase if it is populated or PhaseDesignation if it is not. This change in conjunction with the FeederAllOpenPoint will allow the export to work correctly for the FeederAll analysis. The updated case is illustrated in Figure 4.8-26. 


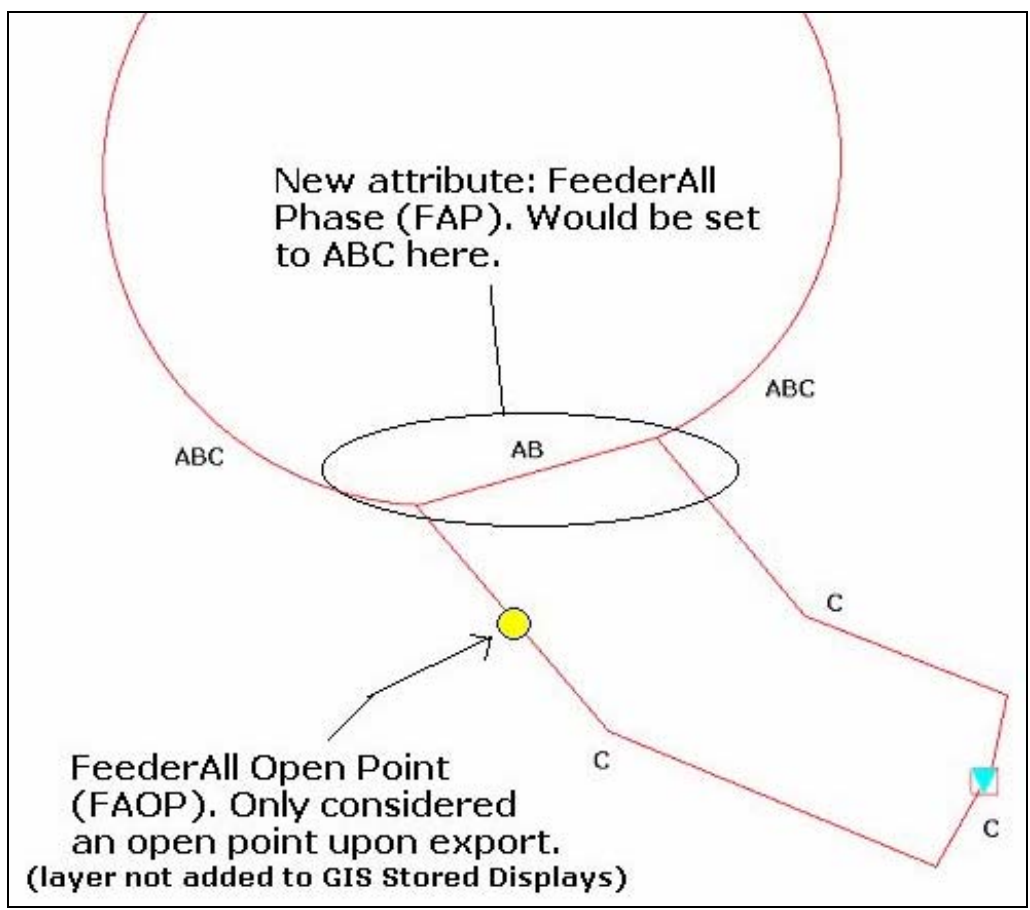

Figure 4.8-26. FeederAll Open Point

The required data model changes for FeederAllOpenPoint and FeederAllPhase have been approved by the NIPSCO GIS team. If this data model change had not been approved, the FeederAll administrator would have to continue with the process used today, which includes manually adding the open point and changing the phasing in all cases where this occurs. It has been noted that because there are hundreds, if not thousands, of locations where this case occurs, that this is a very time intensive process that would prevent the FeederAll users from gaining the desired benefits from the new interface.

\subsubsection{CAD Export}

\subsubsection{Overview}

NIPSCO required a CAD Conversion tool that would export GIS data from the NIPSCO Geodatabase to AutoCAD 2000 DWG and DXF files. A set of preexisting, non-productized tools developed by Miner \& Miner running on top of Safe Software's productized Feature Manipulation Engine (FME) were configured to generate the AutoCAD files.

The CAD Converter offered many of the characteristics that met NIPSCO functional requirements including:

- It presently works on ArcGIS 8.3.

- It exports graphics and labels/annotation.

- It exports to an AutoCAD DWG and DXF formats (presently to an AutoCAD 2000 format).

- It allows the user to specify a geographic area, and select layers to be exported. 
CAD Converter offered a user-interface that guides the technician through the process of mapping the GIS data to a CAD template, including options to save the data mapping configuration as a template, and then exports the GIS data to an AutoCAD 2000 DWG or DXF format.

CAD Converter translates geographic features from ArcGIS / ArcFM formats to DWG, allowing data to be viewed in AutoDesk applications, such as AutoCAD and AutoCAD Map.

CAD Converter offers a simple and comprehensive user-interface, employing several methods to export features to a chosen export file from existing CAD drawing templates. The tool allows exporting features based on 4 selection types: currently selected and displayed features, the screen extent, polygon feature from feature layer in map, and features that lie within a polygon graphic. CAD Converter requires the GIS features to be mapped to their associated CAD layer, and allows the mappings to be saved for later retrieval. CAD Converter supports AutoCAD blocks.

The tool was evaluated against the following NIPSCO set of requirements:

1. The tool must produce a DWG file from the ArcSDE Geodatabase.

2. The tool must export CAD Layer Name information from a "CADLayerName" field.

3. The tool must export CAD Block Name information from a "CADBlockName" field.

4. The tool must export Block Rotation values from a "CADBlockRotation" field.

5. The tool must export Block scale from a "CADBlockScale" field.

6. The tool must be configurable.

7. The tool must be able to schedule the export of the DXF files from the AEDR (i.e., must support batch processing).

8. The tool must specify color as "ByLayer" and "By Block".

9. The tool must support Line Weight.

10. The tool must be able to store and process multiple configurations.

11. The tool must support the export of True Arc Information a.k.a. "bulge factor" (i.e., "hops" over a gas main). AutoCAD and ArcSDE manage arcs and polylines differently.

The CAD Converter tool meets $95 \%$ of the above requirements. It eliminated training on FME which would have required great deal of resource time in training and configuration.

Additional tool customization was required to meet all of the requirements. Miner \& Miner provides the CAD Export tool as a developer sample tool only. It is not supported by Miner \& Miner and will not be upgraded or altered by Miner \& Miner without associated charges. 
During implementation, we were able to meet the requirements 2, 3 and 4 with the data mapping database (see Figure 4.8-27) which uses existing geodatabase fields to drive differing symbology, line types, layers and line weights. This required minimal configuration training and time compared to the learning curve of other products. Requirements 5 and 11 did not represent a significant problem in the day to day production of AutoCAD files. Requirement 7 was resolved by exporting larger sections of data resulting in a significant reduction in operator time required to complete the export of company wide data.

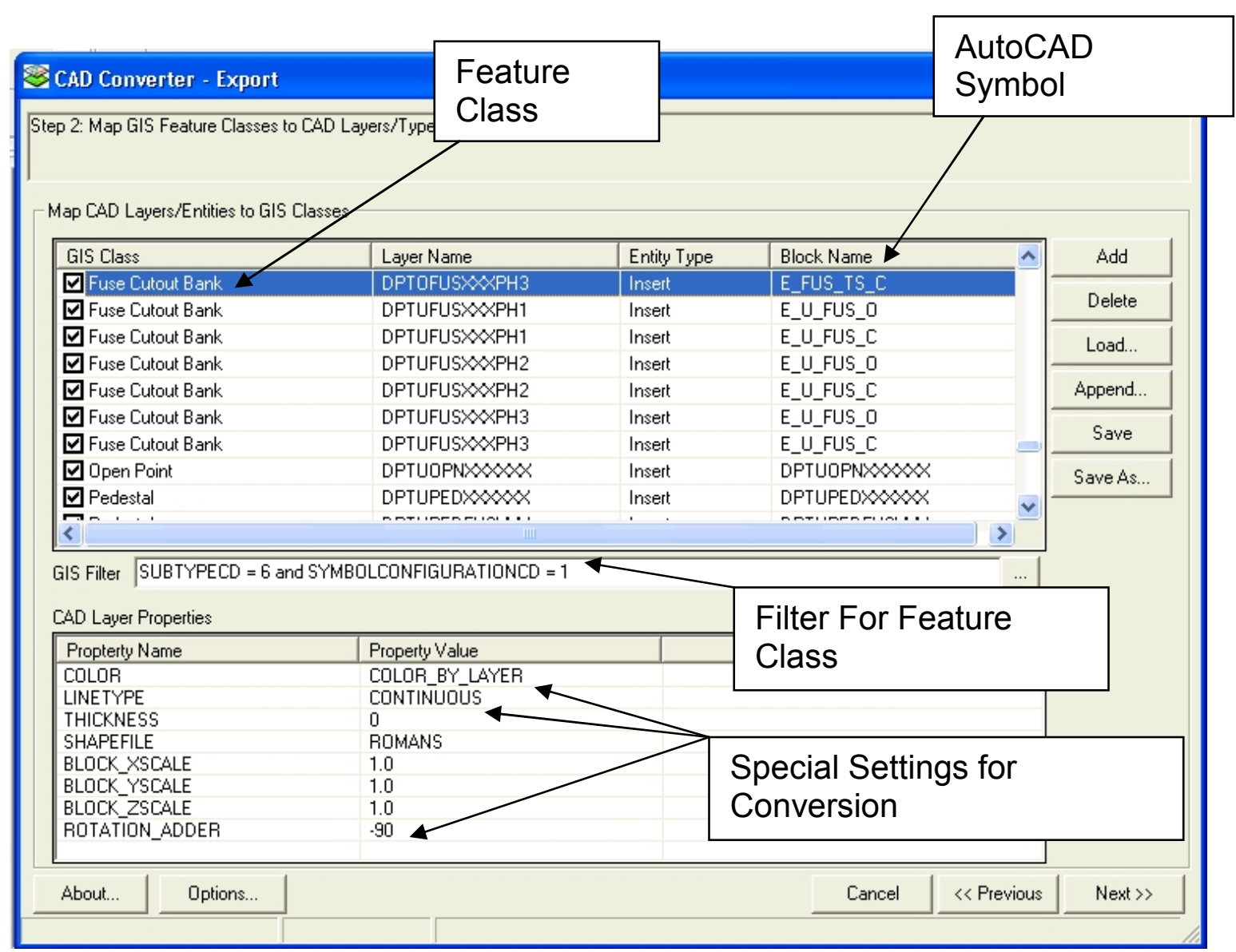

Figure 4.8-27. CAD Converter export mapping screen

\subsubsection{Operational Use}

To invoke the CAD Converter wizard, click the CAD Converter command on the CAD Converter toolbar as shown in Figure 4.8-28.

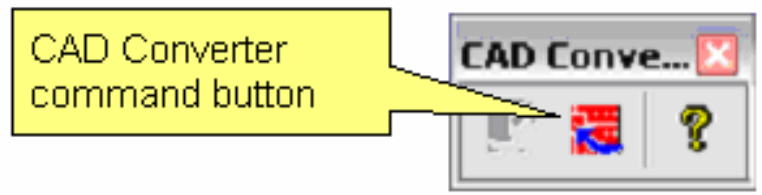

Figure 4.8-28. 


\section{Step 1:}

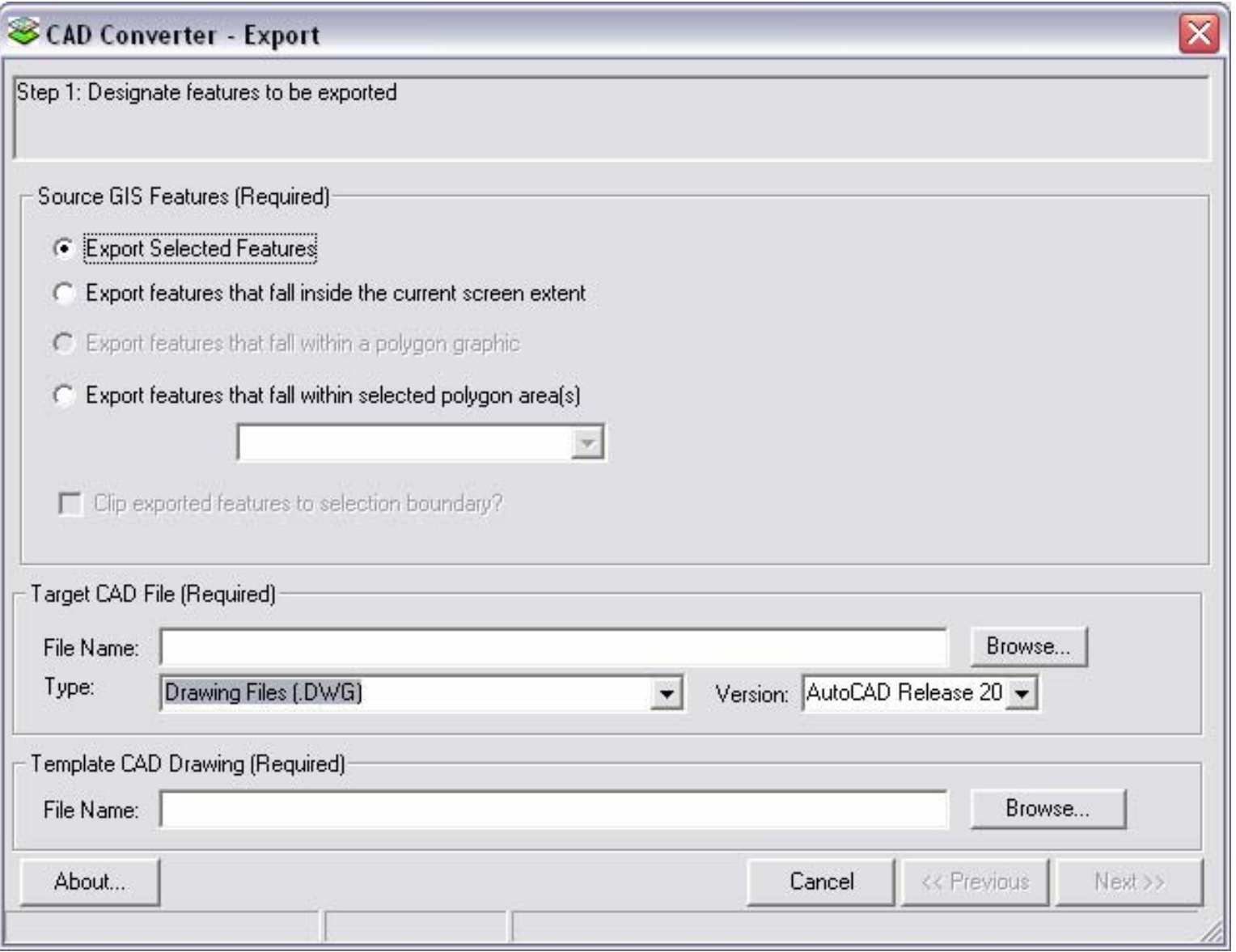

Figure 4.8-29. Feature selection wizard

\section{Selecting Output Features}

Figure 4.8-29 is the first of three wizard screens that allows the user to choose the feature selection type, designate an output file, and reference a mapping AutoCAD drawing.

CAD Converter exports features based on 4 selection types. Four radio buttons on the initial export wizard screen display these options:

\section{Export by Selected Features}

Exporting data by selected features requires features to be selected before the wizard is launched. It also depends on all features selected for an export to be visible in the map frame. This is due to ESRI functionality limiting selected features to those features currently in the view extent.

\section{Export by Screen Extent}

Exporting data from the current screen extent will export all mapped features that lie in the visible extent regardless of any view scale set. Checking the "Clip Features at Boundary" checkbox when selecting this option will ensure only features within the boundary are selected. 


\section{Export by Polygon Feature Extent}

Exporting by polygon features allows the user to export based on the extent of a polygon feature. This feature must belong to a feature class loaded in ArcMap.

\section{Export by Polygon Graphic}

Exporting by polygon graphic allows the user to export based on the extent of a polygon drawn to the graphics container.

\section{Clip Exported Features to Selection Boundary}

The user can check this box (shown in Figure 4.8-30) to clip features at the boundary, or leave it unchecked to export the full feature geometry.

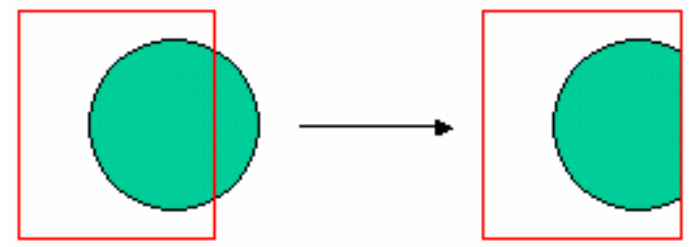

Export full feature geometry
Clip feature at boundary

Figure 4.8-30. Clip features checkbox

\section{$\underline{\text { Target CAD File }}$}

This option launches a Windows 'Save As' dialog. The user navigates to the destination location and either selects an existing file to overwrite, or types in the desired file name. To change between DXF and DWG files, the user selects the desired option on the combo dropdown labeled 'Type'. Click Save.

\section{Template CAD Drawing}

The export requires creating a GIS to CAD layer mapping, and uses an existing AutoCAD drawing for this purpose. This drawing must include all layers required in the output. Using the CAD Converter wizard, the user can then create the mappings between the GIS layer data and the CAD layers.

Clicking the Browse button in the Template CAD Drawing section of the wizard opens a Windows 'File Open' dialog. The user navigates to the .DWG file acting as the template and clicks Open.

This option saves the selected DWG template once it is set for future use. 


\section{Step 2:}

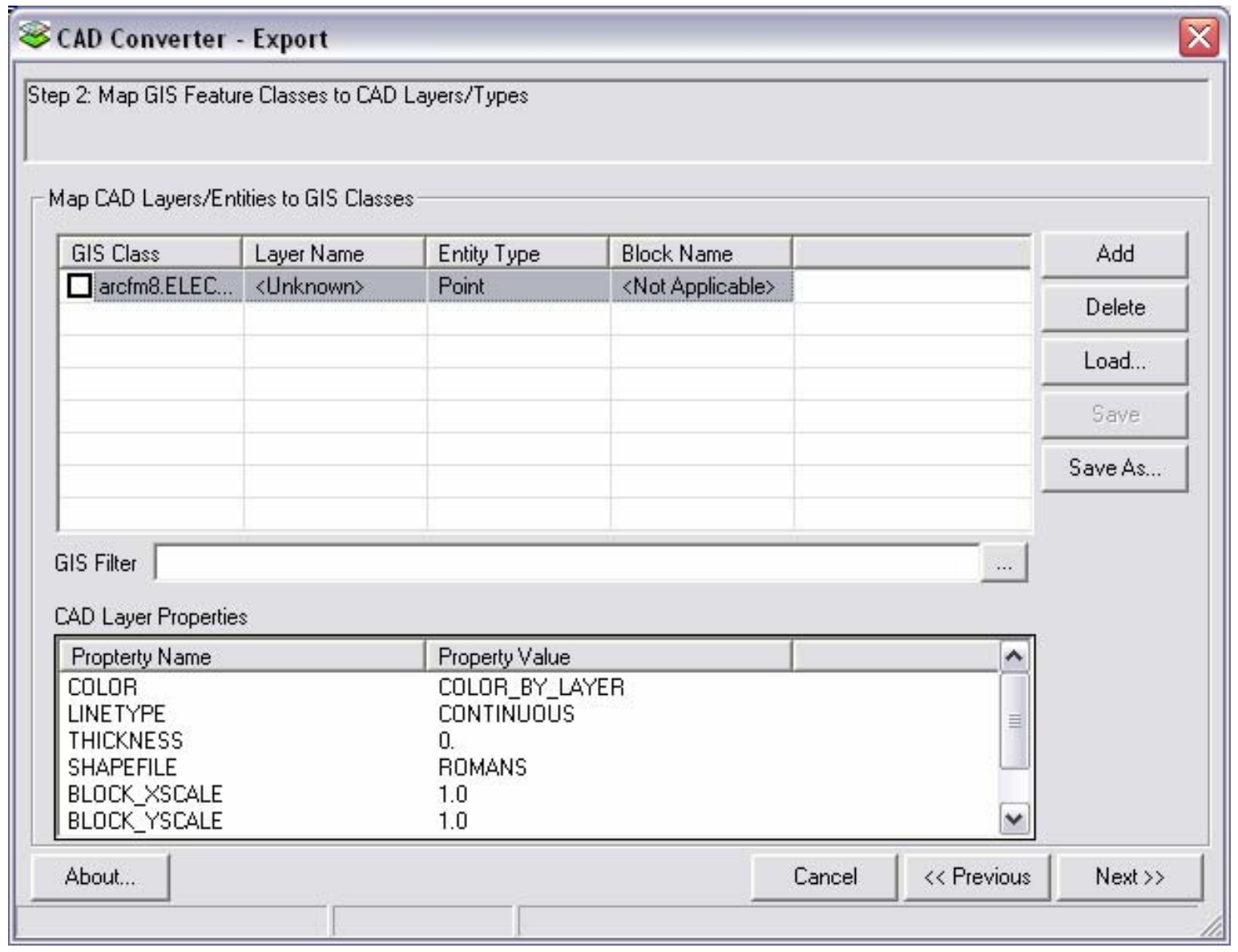

Figure 4.8-31. Mapping wizard

\section{Map Feature Layers to CAD Layers}

This step shown in Figure 4.8-31 allows the user to add feature layers present in the map document and map the feature layers to a CAD layer. For point feature types, AutoCAD block graphics are specified.

To map layers, the user clicks the Add button to the right of the grid. This will add a blank GIS to CAD mapping row. The feature layer column will have a checkbox in the cell, checking the box will export the layer.

Once a new layer is added, clicking in each cell will invoke a drop down menu. The first column represents the feature layer name, the second the CAD layer, then the feature type, and the block name. Table 4.8-3 describes how the fields will be populated. 
Table 4.8-3.

\begin{tabular}{|c|c|c|}
\hline GIS Class & \multicolumn{2}{|c|}{ The name of the feature class to export. } \\
\hline $\begin{array}{l}\text { CAD Layer } \\
\text { Name }\end{array}$ & \multicolumn{2}{|c|}{$\begin{array}{l}\text { The name of the layer (level) onto which features will be placed. } \\
\text { Multiple feature class/subtypes can be placed on the same CAD Layer. For } \\
\text { example, all conductors, overhead and underground primary and secondary, } \\
\text { can be placed on a single CAD Layer named 'WIRE'. }\end{array}$} \\
\hline \multirow{6}{*}{$\begin{array}{l}\text { CAD Entity } \\
\text { Type }\end{array}$} & \multicolumn{2}{|c|}{$\begin{array}{l}\text { The type of CAD entity to be created. The entity type is based on the geometry } \\
\text { type of the Arclnfo feature. Here are the types of entities that were created } \\
\text { from Arclnfo features: }\end{array}$} \\
\hline & Arclnfo Feature & Possible CAD Entity Types \\
\hline & Line & Polyline \\
\hline & Point & $\begin{array}{l}\text { Point } \\
\text { Block (Cell) }\end{array}$ \\
\hline & Annotation & Text \\
\hline & Polygon & $\begin{array}{l}\text { Polyline } \\
\text { Polygon }\end{array}$ \\
\hline Block Name & \multicolumn{2}{|c|}{ The name of the AutoCAD block to draw the exported feature. } \\
\hline Color & \multicolumn{2}{|c|}{$\begin{array}{l}\text { Colors of CAD entities were defined as a property of the entity itself, however, } \\
\text { colors can also be obtained from the color assigned to the level. For example, } \\
\text { all conductors on a single CAD Layer were named "WIRE" but still required } \\
\text { different colors for primary and secondary, so a specific color was assigned to } \\
\text { each feature class/subtype. However, if all entities in the WIRE layer were } \\
\text { required to have the same color, then the color could be set to "BYLAYER". }\end{array}$} \\
\hline Line Style & \multicolumn{2}{|c|}{$\begin{array}{l}\text { A line style can be designated for CAD Polyline and Polygon features such as } \\
\text { CONTINUOUS, DASHED, DASHDOT, etc. }\end{array}$} \\
\hline Line Type & \multicolumn{2}{|c|}{$\begin{array}{l}\text { A line weight from } 0 \text { to } 15 \text { can be designated for CAD Polyline and Polygon } \\
\text { features. }\end{array}$} \\
\hline Shapefile & \multicolumn{2}{|c|}{ Allows selecting the exported text font. } \\
\hline Block Scale & \multicolumn{2}{|c|}{ Controls the scale of the exported block. } \\
\hline Rotation & \multicolumn{2}{|c|}{$\begin{array}{l}\text { Allows setting a rotation for exported features. ESRI rotation is known to be off } \\
-90 \text { degrees from AutoCAD. Employing this constant will result in a consistent } \\
\text { feature look between the two systems. }\end{array}$} \\
\hline
\end{tabular}




\section{(Optional) Select existing Feature Layer / CAD Layer mapping template}

Once a mapping template has been created, it can be saved and reused at a later date.

This functionality expedites the export process by removing the layer-by-layer mapping of GIS to CAD data every time the tool is run.

A mapping template is created by running CAD Converter, mapping GIS to CAD layers, selecting Save $A s$, and naming the mapping template. Once a mapping template has been created, it becomes ready for use by selecting Load from the wizard button, navigating to the template, and opening it.

\section{Step 3:}

\section{Execute}

By clicking Finish, the designated features will be exported to the target output file(s). Progress is indicated on a bar that is shown at the bottom of the form while the export is in process.

\subsubsection{MAPPS}

Several interfaces exist between the new system and the Material Accounts Payable and Purchasing System (MAPPS). These interfaces are defined below.

\subsubsection{SIN Description}

The SAGE tool accesses data from the MAPPS Stores Item Number (SIN) Description table CORP_ITEM to display the SIN description to the user when the SIN value is added/updated on the transformer, regulator, or capacitor add/edit pages. This data exists in the MAPPS DB2 database. This data is replicated from DB2 into the AEDR database on a nightly basis so that it can be utilized quickly by the SAGE application. The query pulls both the SIN and the description field. This data is stored in the AEDR table ArcFM8.electric.InterfaceMAPPSSinDescription shown in Figure 4.8-32.

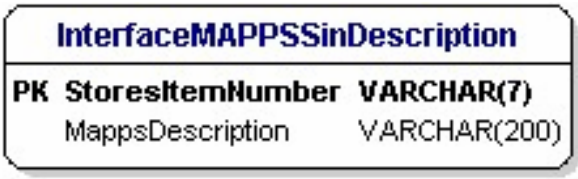

Figure 4.8-32. Stores Item Number data type

The process is illustrated in Figure 4-8.33. 


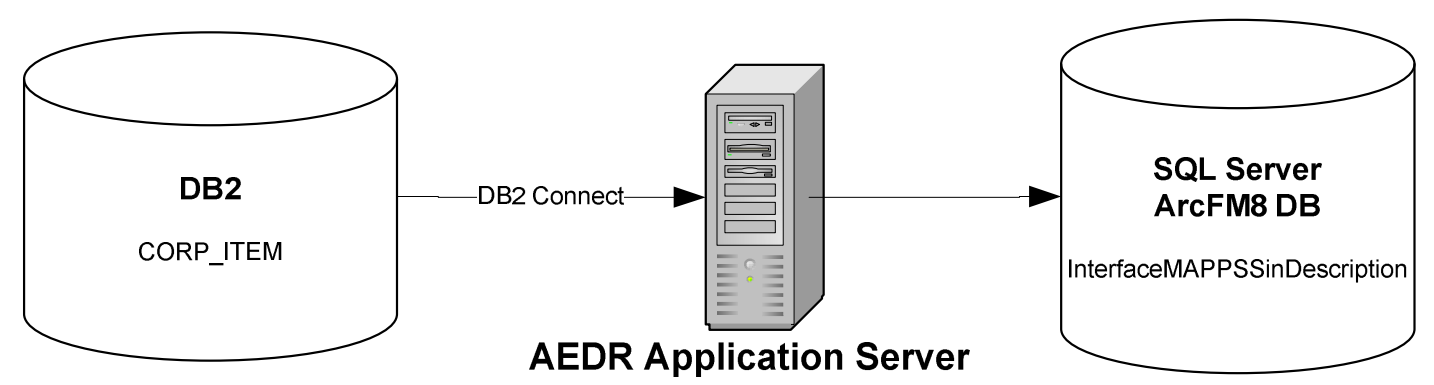

Figure 4.8-33. MAPPS/AEDR Interface

\subsubsection{Nightly Transactions}

The MAPPS system manages daily transactions relating to the issuing out, stores return, transfer, and condemnation of transformers, regulators, and capacitors. These transactions must be matched against the EDFS/AEDR transactions that occur in a given day. Previously these transaction records were written nightly to a flat file that was then parsed by the EDFS application. In the new ArcFM/SAGE AEDR system, the MAPPS system writes these transaction records to a DB2 table, IP_MAPPS_GIS that are made available to the AEDR system via DB2 Connect. This operation occurs on a nightly basis and the GIS system truncates the DB2 transaction table after the records have been parsed. This architecture change allows the AEDR system to access the transactions in a more efficient and real-time manner. The transaction data is copied over into the GIS table NIPSCOSage.Sage.InterfaceMAPPSTransaction shown in Figure 4.8-34.

\begin{tabular}{|ll|}
\hline \multicolumn{2}{|c|}{ InterfacehAPPSTransaction } \\
\hline PK Transactionld & HUMERIC \\
CompanyNumber & VARCHAR(7) \\
TransactionType & VARCHAR(3) \\
Department & VARCHAR(3) \\
SystemCode & VARCHAR(1) \\
StorestternNumber & VARCHAR(7) \\
TransactionDate & DATETIME \\
TransferReceivingDepartment & VARCHAR(3) \\
TransterNumber & VARCHAR(7) \\
CondernnationNumber & VARCHAR(3) \\
\hline
\end{tabular}

Figure 4.8-34. MAPPS interface transactions and data types

The process is illustrated in Figure 4.8-35.

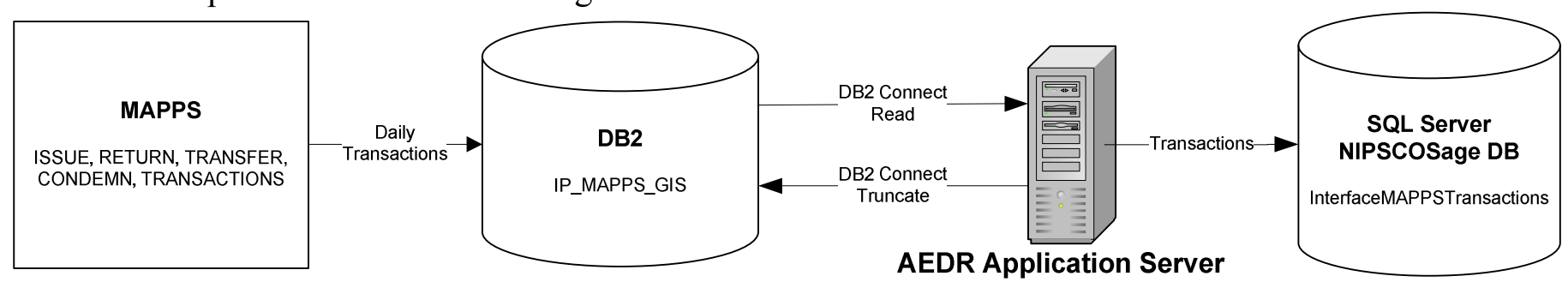

Figure 4.8-35. Batch transaction process 


\subsubsection{Near Real Time Asset Push}

Capital assets (transformers, regulators, and capacitors) are entered into the SAGE system. Whenever a new asset record is created, updated, or deleted it needs to be transmitted to MAPPS in near real time so that it can be issued out into the field. This is a critical task in a storm scenario. Because DB2 Connect is not always a reliable method of communication between .Net / SQL Server and the DB2 database, it was decided to not make this a synchronous operation when the asset is created in SAGE. Instead the transaction is written to a queue hosted in a SQL Server table MAPPSAssetPushQueue which contains two columns:

- ID - A system-generated unique ID

- AssetPushSQL - The SQL that needs to be executed against the DB2 database to update it with the latest asset data.

A windows service, MAPPSAssetPush, was then created on an application server. This service is responsible for polling the MAPPSAssetPushQueue table on a scheduled interval (every 30 minutes) to check for the existence of any records. If records are found, the service will then attempt to execute the SQL against the DB2 database. If the operation succeeds, the record will be removed from the MAPPSAssetPushQueue table. If it fails, the operation will be retried again at a specified time interval. The service also is configured to send out emails if failures occur so that the failure may be addressed as quickly as possible. The diagram in Figure 4.8-36 illustrates the MAPPS near real time asset push process: 


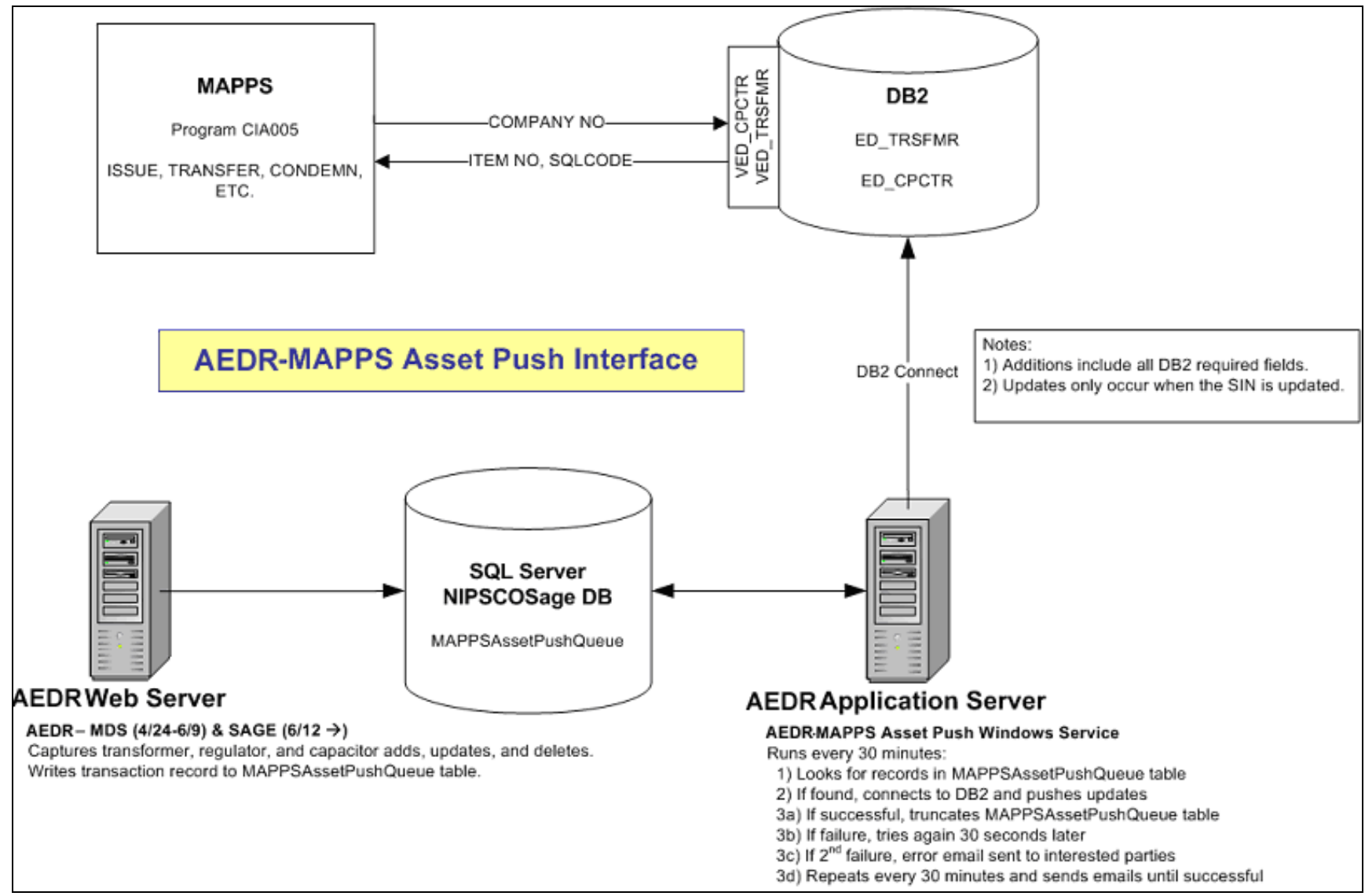

Figure 4.8-36. Asset Push process to transmit a new asset record to MAPPS 


\subsubsection{CIS}

There are several interfaces between the AEDR and the Customer Information System (CIS). Information is passed back and forth between the two systems on a nightly basis. These interfaces are defined below.

\subsubsection{CIS Site}

The CIS tracks attribute information for every site (location) where services are installed. This information includes address and location information. This information is used within the AEDR for validation, reporting, and analysis. The CIS contains transaction tables that accumulate changes to this site information during the day. Then, every night the transactions are moved from the CIS DB2 transaction table into the GIS InterfaceCISSite table shown in Figure 4.8-37 which contains current data for all CIS Site records. This information is then utilized by AEDR users and various reports. 


\begin{tabular}{|c|c|c|}
\hline \multicolumn{3}{|c|}{ InterfaceCISSite } \\
\hline PK & SIT_ID & INTEGER \\
\hline PK & GISST_CRET_TMSTMP & DATETIME \\
\hline & SITUSE_CD & VARCHAR(2) \\
\hline & DPT_CD & VARCHAR(3) \\
\hline & TXNCUT_CD & VARCHAR(3) \\
\hline & RVTMN_CD & VARCHAR(3) \\
\hline & GISST_STAT_CD & VARCHAR(2) \\
\hline & NIPSCO_MA,J_MAP_CD & VARCHAR(2) \\
\hline & N_INTER_MAP_CD & VARCHAR(2) \\
\hline & NIPSCO_MNR_MAP_CD & VARCHAR(1) \\
\hline & AREA_CD & VARCHAR(3) \\
\hline & PHN_NUM & VARCHAR(7) \\
\hline & PHN_EFTV_DT & DATETIME \\
\hline & A.DDR_DVLPMT_NM & VARCHAR(30) \\
\hline & ADDR_STT_NUM & VARCHAR(7) \\
\hline & A.DDR_BLDG_NUM & VARCHAR(8) \\
\hline & ADDR_RRL_RTE_NUM & VARCHAR(8) \\
\hline & ADDR_APT_NUM & VARCHAR(8) \\
\hline & ADDR_EX_NUM_TXT & VARCHAR(8) \\
\hline & ADDR_STT_NM & VARCHAR(25) \\
\hline & ADDR_LOT_NUM & VARCHAR(10) \\
\hline & ADDR_ZIP_PLUS_NUMM & VARCHAR(6) \\
\hline & ADDR_OVFL_AD & VARCHAR(23) \\
\hline & ADDR_STE_NUM & VARCHAR(8) \\
\hline & STTPFX_CD & VARCHAR(2) \\
\hline & STTSFX_CD & VARCHAR(4) \\
\hline & CNTY_CD & VARCHAR(2) \\
\hline & ST_CD & VARCHAR(2) \\
\hline & MNCPLT_NMM & VARCHAR(25) \\
\hline & ZIP_CD_NUMM & VARCHAR(5) \\
\hline & ADDR_FRCTN_TXT & VARCHAR(3) \\
\hline & ADDR_POST_BAR_CD & VARCHAR(12) \\
\hline & A.DDR_CARR_RTE_NUM & VARCHAR(6) \\
\hline & GISST_LS_INDCR & VARCHAR(1) \\
\hline & PHN_EXTN_NUM & VARCHAR(5) \\
\hline & AREA2_CD & VARCHAR(3) \\
\hline & PHN2_NUM & VARCHAR(7) \\
\hline & PHN2_EXTN_NUM & VARCHAR(5) \\
\hline & ORGDTL_NM & VARCHAR(50) \\
\hline & ADRTXT_ADTL_TXT & VARCHAR(240) \\
\hline & GISST_CRET_ID & VARCHAR(10) \\
\hline & GISST_RVSD_ID & VARCHAR(10) \\
\hline & GISST_RVSD_TMSTMP & DATETIME \\
\hline
\end{tabular}

Figure 4.8-37. CIS interface transactions and data types

This process is illustrated in Figure 4.8-38.

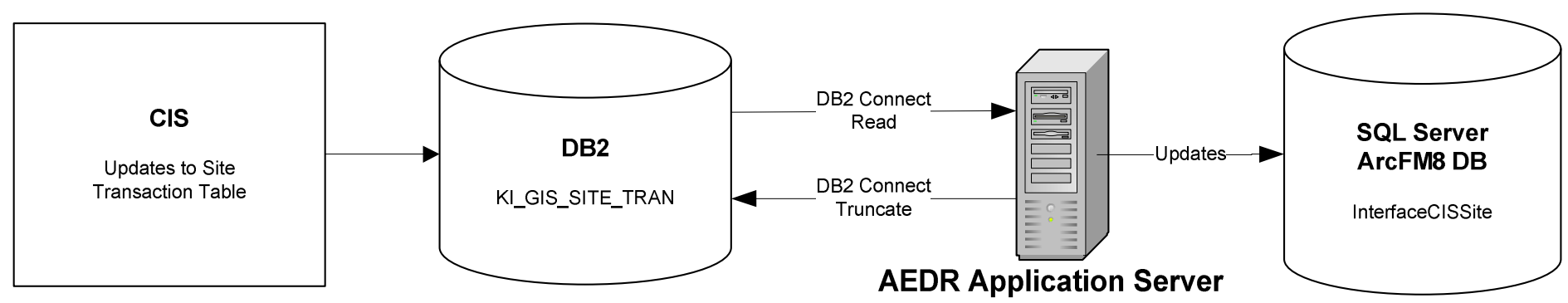

Figure 4.8-38. CIS interface batch process 


\subsubsection{CIS Installed Service}

The CIS tracks attribute information for every installed service located at each site (see previous section). This information includes customer and account information. This information is used within the AEDR for validation, reporting, and analysis. The CIS contains transaction tables that accumulate changes to this installed service information during the day. Then, every night the transactions are moved from the CIS DB2 transaction table into the GIS InterfaceCISInstalledService table as shown in Figure 4.839 which contains current data for all CIS Service records. This information is then utilized by AEDR users and various reports.

\begin{tabular}{|c|c|c|}
\hline \multicolumn{3}{|c|}{ InterfaceCISInstalledService } \\
\hline PK & SIT_ID & INTEGER \\
\hline PK & IS_SEQ_HUM & SMALLINT \\
\hline PK & GISIS_SEQ_HUM & INTEGER \\
\hline PK & GISIS_CRET_TMSTMP & DATETIME \\
\hline & SRVTP_CD & VARCHAR(3) \\
\hline & SRVTP_MTRD_INDCR & VARCHAR(1) \\
\hline & UTLL_TYP_CD & VARCHAR(2) \\
\hline & ISSTAT_CD & VARCHAR(2) \\
\hline & NUMEQ_COMP_NUM & VARCHAR(10) \\
\hline & MSL_CD & VARCHAR(2) \\
\hline & IRSERV_CD & VARCHAR(2) \\
\hline & GISIS_STAT_CD & VARCHAR(2) \\
\hline & CA_ID & INTEGER \\
\hline & SAA_ID & INTEGER \\
\hline & SAA_SEQ_NUM & SMALLINT \\
\hline & SRVPLN_ID & INTEGER \\
\hline & SPO_ID & INTEGER \\
\hline & $\mathrm{RC}_{-} \overline{\mathrm{CD}}$ & VARCHAR(2) \\
\hline & CUIN_LST_NM & VARCHAR(25) \\
\hline & CUIN_FST_NM & VARCHAR(25) \\
\hline & CUIN_MID_INIT_TXT & VARCHAR(1) \\
\hline & CUOR_CUST_NM & VARCHAR(50) \\
\hline & DSTR_RFRNC̄_ID & VARCHAR(8) \\
\hline & IS_LEG_NUMM & INTEGER \\
\hline & ISD_SEQ_NUM & INTEGER \\
\hline & $S I C \_C D$ & VARCHAR(4) \\
\hline & NAICS_CD & VARCHAR(6) \\
\hline & ACYC_NUM & VARCHAR(2) \\
\hline & CYC_FRQY_CD & VARCHAR(2) \\
\hline & GISIS_CRET_ID & VARCHAR(10) \\
\hline & GISIS_RVSD_ID & VARCHAR(10) \\
\hline & GISIS_RVSD_TMSTMP & DATETIME \\
\hline & PET_CD & VARCHAR(2) \\
\hline
\end{tabular}

Figure 4.8-39. CIS installed service transactions and data types

This process is illustrated in Figure 4.8-40. 


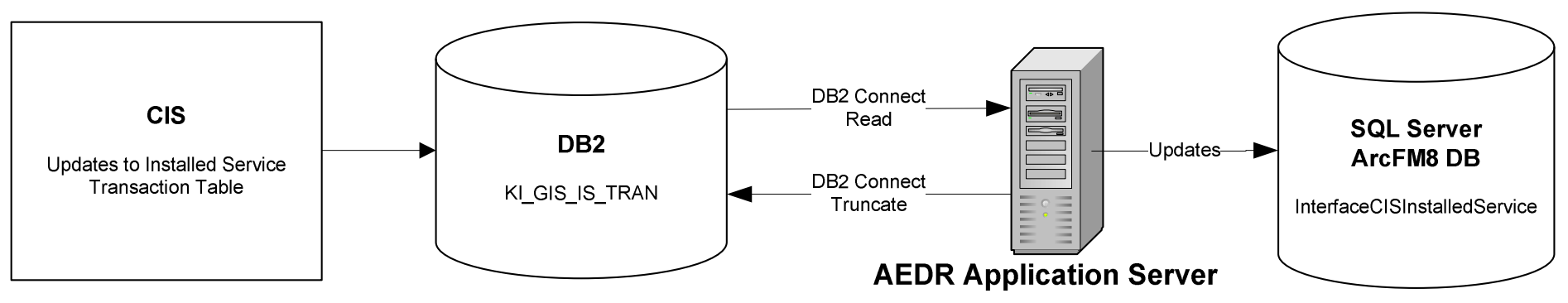

Figure 4.8-40. CIS installed service batch process

\subsubsection{Transformer Install and Removal}

Transformers are tracked as load points in the CIS and customers are tied to the load points. Because transformers are created, installed, and removed within the AEDR, this information needs to be passed to the CIS on a regular basis. Whenever these types of transactions occur in the AEDR, the records are tagged with creation and update timestamps. These timestamps are then used to query the system for all transformer installs and removals that have occurred in the past day. A nightly batch process pulls this information and then updates a CIS DB2 transaction table with the changes. These changes are then processed by the CIS to ensure the systems are always in sync. This process is illustrated in Figure 4.8-41.

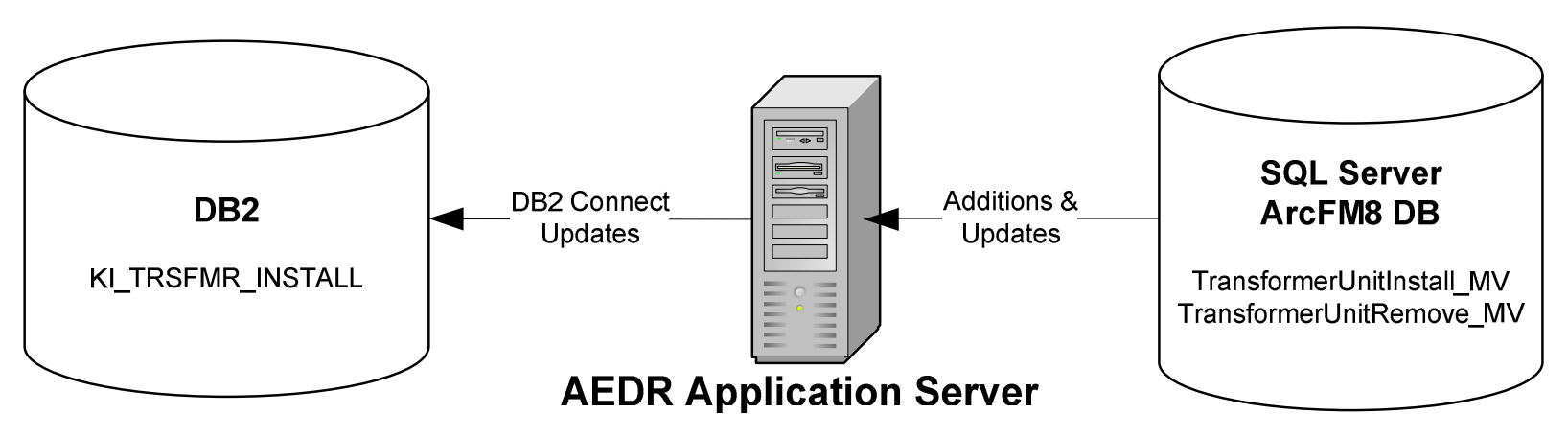

Figure 4.8-41. CIS transformer install and removal batch process

\subsubsection{SupportStructure and Padmount Installation}

Transformers are tracked as load points in the CIS. A transformer that feeds customers is always installed on a pole (SupportStructure) or a Padmount (pad or vault). Both of these structures are given a unique location ID within the NIPSCO territory. This information along with other key data is also used within the CIS to identify the location of a load point / transformer. Whenever a SupportStructure or Padmount is installed in the AEDR, the records are tagged with creation and update timestamps. These timestamps are then used to query the system for all installations that have occurred in the past day. A nightly batch process pulls this information and then updates a CIS DB2 transaction table with the changes. These changes are then processed by the CIS to ensure the systems are always in sync. This process is illustrated in Figure 4.8-42. 


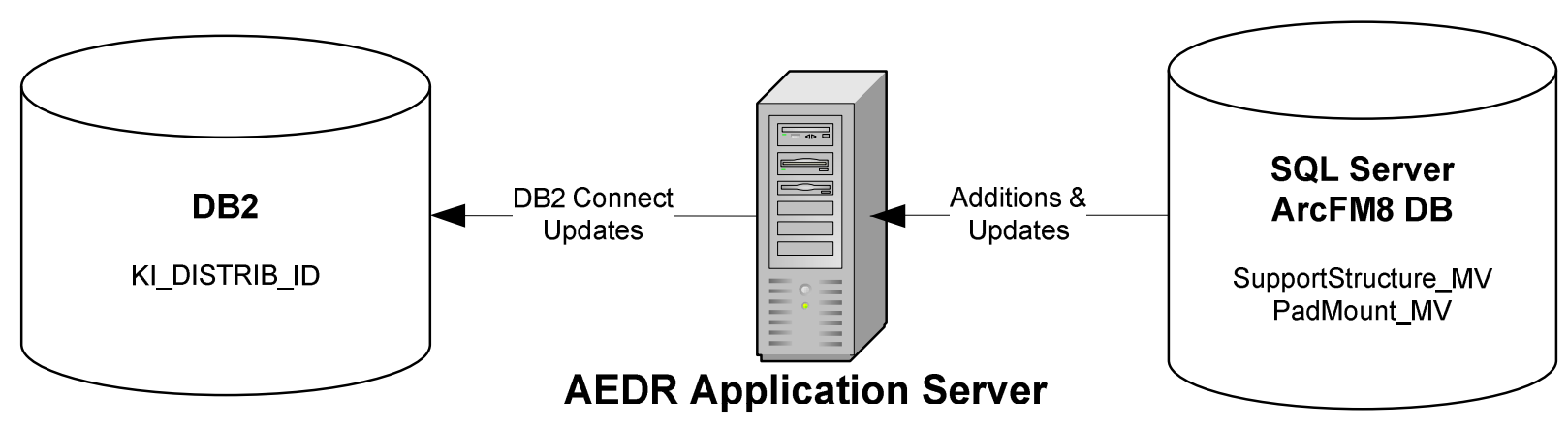

Figure 4.8-42. CIS support structure and padmount installation nightly batch process

\subsubsection{Installed Service Coordinate Update}

Each CIS installed service record contains an X, Y coordinate that is managed within the GIS. The records in the InterfaceCISInstalledService table are related to the CIS installed service features that exist in the GIS. A select group of editors have rights to update the location of these installed service points. Whenever an installed service point feature is moved or updated, the record is tagged with an update timestamp. This timestamp is used to pull the records that have been updated in the past day. A nightly ArcObjects batch process pulls the updated records, extracts $\mathrm{X}$ and $\mathrm{Y}$ coordinates from their shape field, and writes this information out to a staging table, InterfaceCoordinateTransfer as shown in Figure 4.8-43.

\begin{tabular}{|ll|}
\hline \multicolumn{2}{|c|}{ InterfaceCISCoordinateTransfer } \\
\hline PK SIT_ID & INTEGER \\
PK IS_SEQ_HUM & SHALLINT \\
PK GISIFT_CRET_TMSTMP & DATETIME \\
GISIFT_STAT_CD & VARCHAR(1) \\
DSTR_RFRNC_ID & VARCHAR(8) \\
GISIFT_X_COORD_VAL & DECIMAL(15,6) \\
GISIFT_Y_COORD_VAL & DECIMAL(15,6) \\
IS_LEG_NUM & INTEGER \\
ISD_SEQ_NUM & INTEGER \\
GISIFT_CRET_ID & VARCHAR(10) \\
GISIFT_RVSD_ID & VARCHAR(10) \\
GISIFT_RVSD_TMSTMP & DATETIME \\
\hline
\end{tabular}

Figure 4-8.43. CIS installed service transfer transactions and data types

A second nightly batch process pulls this information out of the InterfaceCoordinateTransfer table and then updates a CIS DB2 transaction table with the changes. The updated location information is then processed by the CIS to ensure the systems are always in sync.

This process is illustrated in Figure 4.8-44. 


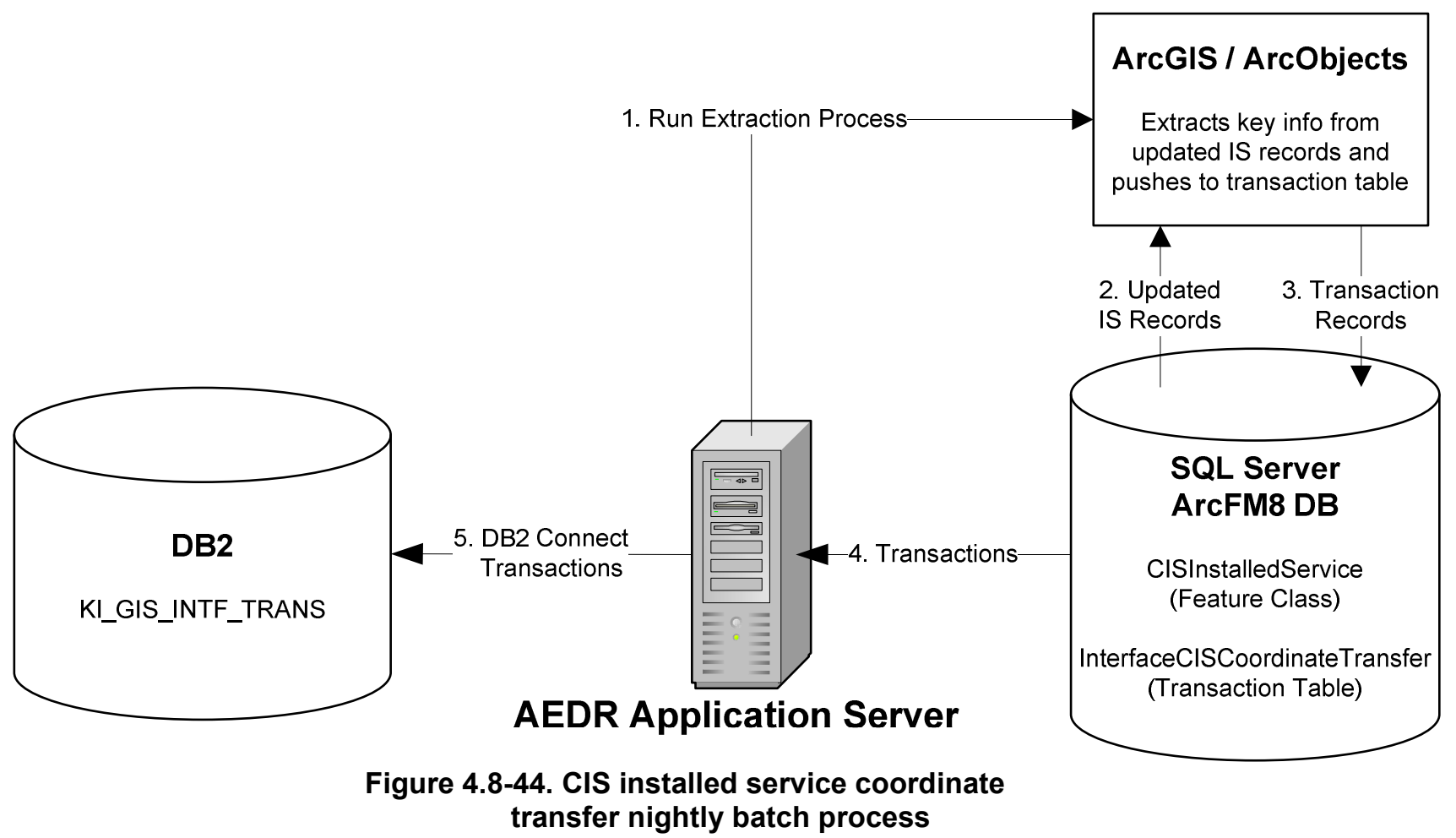

\subsubsection{CIS Account Summary}

The raw CIS Site and Installed Service tables are large, bulky, and not easy for an end user to traverse and/or analyze. Therefore a nightly batch process was written to extract the key CIS Account data into a much smaller table, InterfaceCISAccount, shown in Figure 4.8-45 which is very easy to access.

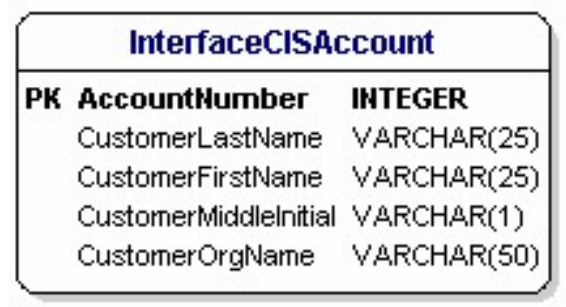

Figure 4.8-45. CIS account data transactions and data types

The AccountNumber field is the primary key. This key joins these account records to several GIS asset tables that track a CIS Account Number field including CompanyStreetlight and CustomerStreetlight. This link allows a user to create a client side join to easily display the CIS Account records related to the assets within the GIS.

\subsubsection{CIS Service Address Summary}

The raw CIS Site and Installed Service tables are large, bulky, and not easy for an end user to traverse and/or analyze. Therefore a nightly batch process was written to extract the key CIS Service Address data into a much smaller table, InterfaceCISServiceAddress, shown in Figure 4.8-46 which is very easy to access. 


\begin{tabular}{|ll|}
\hline \multicolumn{1}{|c|}{ InterfaceCISServiceAddress } \\
\hline LoadDistribRefNumber & VARCHAR(8) \\
StreetNumber & VARCHAR(7) \\
BuildingNumber & VARCHAR(8) \\
RuralRouteNumber & VARCHAR(8) \\
ApartmentNumber & VARCHAR(8) \\
BoxNumber & VARCHAR(8) \\
StreetName & VARCHAR(25) \\
LotNumber & VARCHAR(10) \\
ZipPlusNumber & VARCHAR(6) \\
Addressoverflow & VARCHAR(23) \\
SuiteNumber & VARCHAR(8) \\
StreetPrefixCode & VARCHAR(2) \\
StreetSuffixCode & VARCHAR(2) \\
CountyCode & VARCHAR(2) \\
StateCode & VARCHAR(2) \\
ZipCode & VARCHAR(5) \\
\hline
\end{tabular}

Figure 4.8-46. CIS service address transactions and data types

The LoadDistribRefNumber is the primary key. This key joins these address records to the pole or pad features (based on DistribRefNumber) that are related to the transformer that feeds the services at these addresses. This link allows a user to create a client side join to easily display the CIS service address records related to a pole or pad within the GIS.

\subsubsection{CIS Life Support Summary}

The raw CIS Site and Installed Service tables are large, bulky, and not easy for an end user to traverse and/or analyze. Therefore a nightly batch process was written to extract the CIS Life Support indicator per distribution reference number into a much smaller table, InterfaceCISLifeSupport, shown in Figure 4.8-47 which is very easy to access by users, validation, and reporting processes.

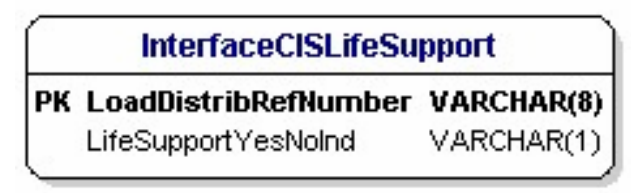

Figure 4.8-47. CIS life support interface transactions and data types

The LoadDistribRefNumber is the primary key. This key joins the life support Y/N indicator values to the pole or pad features (based on DistribRefNumber) that are related to the transformer that feeds the service with the life support customer. This link allows a user to create a client side join to easily display the CIS service address records related to a pole or pad with the GIS. This relationship is also used to prevent certain asset operations from occurring within the GIS to ensure that power is never disconnected to a life support customer. 


\subsubsection{General Ledger (GL)}

The GL system manages work order numbers within NIPSCO. Work Order numbers are entered when most AEDR assets are installed/created. When this occurs an EDFS validation runs and checks the user-entered number against GL values. To accomplish this, all valid GL work order numbers are duplicated from the GL DB2 table into the GIS table, InterfaceGLWorkOrderNumber shown in Figure 4.8-48.

\section{\begin{tabular}{|c|}
\hline InterfaceGLWorkOrderNumber \\
\hline PK WorkOrderWumber VARCHAR(15) \\
\hline
\end{tabular} \\ Figure 4.8-48. General Ledger work order validation data type}

The EDFS validation can then easily validate the user-entered numbers against this table directly within the AEDR database. This process is illustrated in Figure 4.8-49.

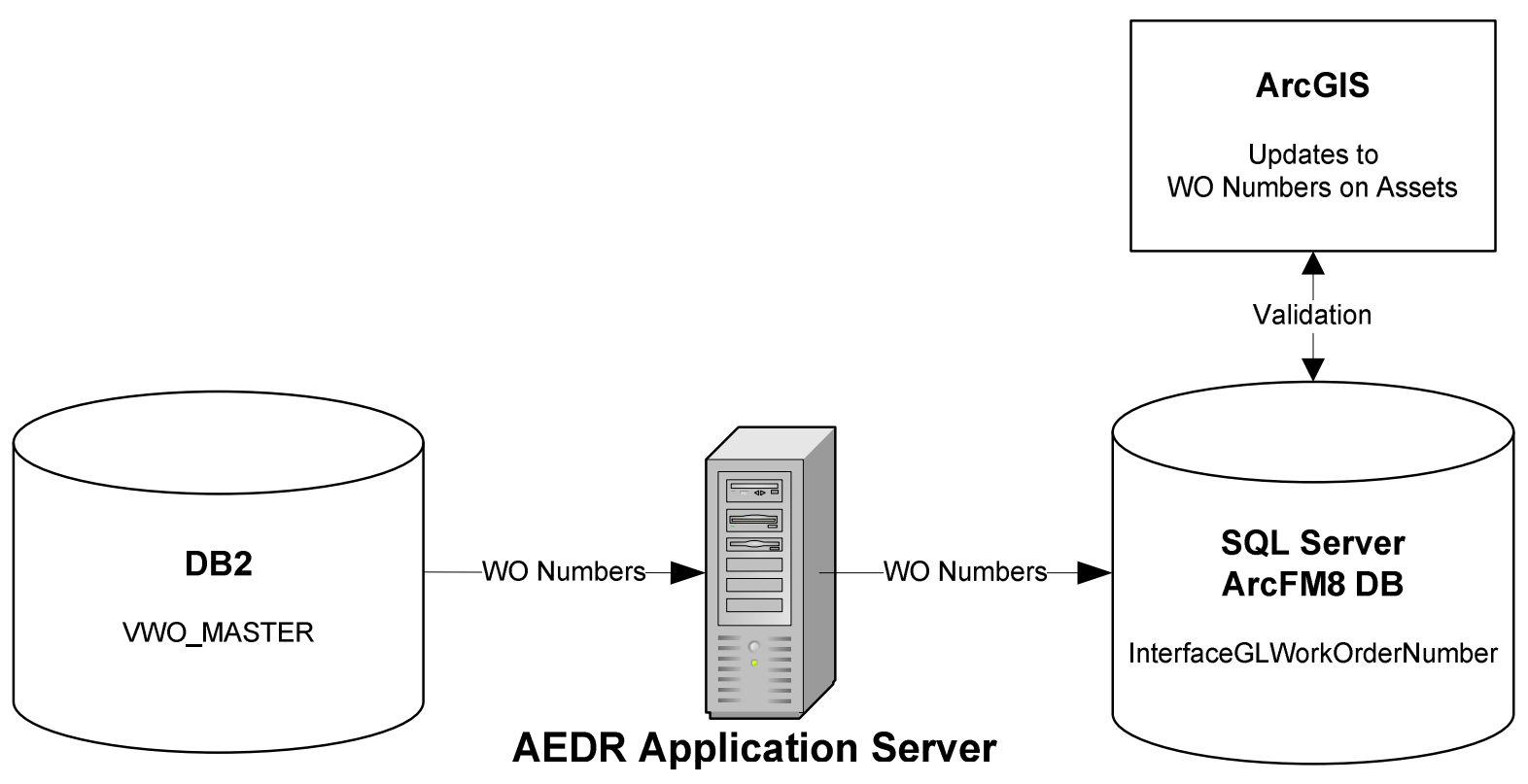

Figure 4.8-49. Work order validation process

\subsubsection{Engineering Accounts Information File (EAIF)}

The EAIF application allows engineers to perform load analysis on a specific transformer based on the properties of the transformer combined with the customer consumption data tracked in the CIS. Previously the EAIF application directly accessed the EDFS tables to get the transformer unit and installation data. In the new AEDR system, this information is managed within the GIS. When transformers are created, installed, and removed within the GIS, this information needs to be passed to the EAIF on a regular basis. This is now accomplished by passing this data via the CIS interface. EAIF was updated to read the transformer data directly from the CIS DB2 tables in place of the EDFS tables. 
Whenever a transformer is edited in the GIS, the records are tagged with creation and update timestamps. These timestamps can then be used to query the system for all transformer installs and removals that have occurred in the past day. A nightly batch process pulls this information and then updates a CIS DB2 transaction table with the changes. These changes are then processed by the CIS to ensure the systems are always in synchronization. EAIF then accesses the CIS DB2 tables directly for both the transformer data and the load consumption data.

This process is illustrated in Figure 4.8-50.

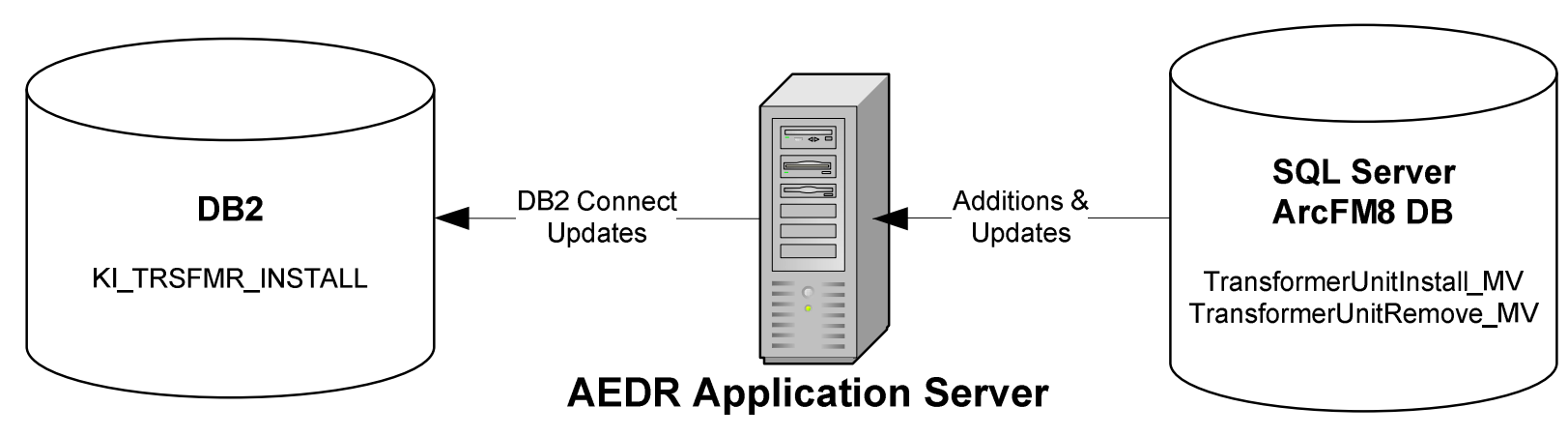

Figure 4.8-50. Transformer install \& removal batch process interface for EAIF synchronization

\subsubsection{Material and Labor Online Guide (MLOG)}

The Material and Labor Online Guide (MLOG) system manages compatible unit / assembly numbers within NIPSCO. Assembly numbers are entered when GIS assets including Assemblies and Company Streetlights are installed/created. When this occurs an EDFS validation runs and checks the user-entered number against MLOG values. To accomplish this, all valid MLOG assembly numbers are duplicated from the MLOG DB2 table into the GIS table, InterfaceCHAssemblyNumber as shown in Figure 4.8-51.

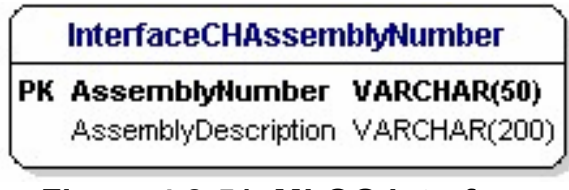

Figure 4.8-51. MLOG interface transaction and data type

The EDFS validation can then easily validate the user-entered assembly numbers against this table directly within the GIS database. The assembly description is also utilized in certain reports. This process can be illustrated in Figure 4.8-52. 


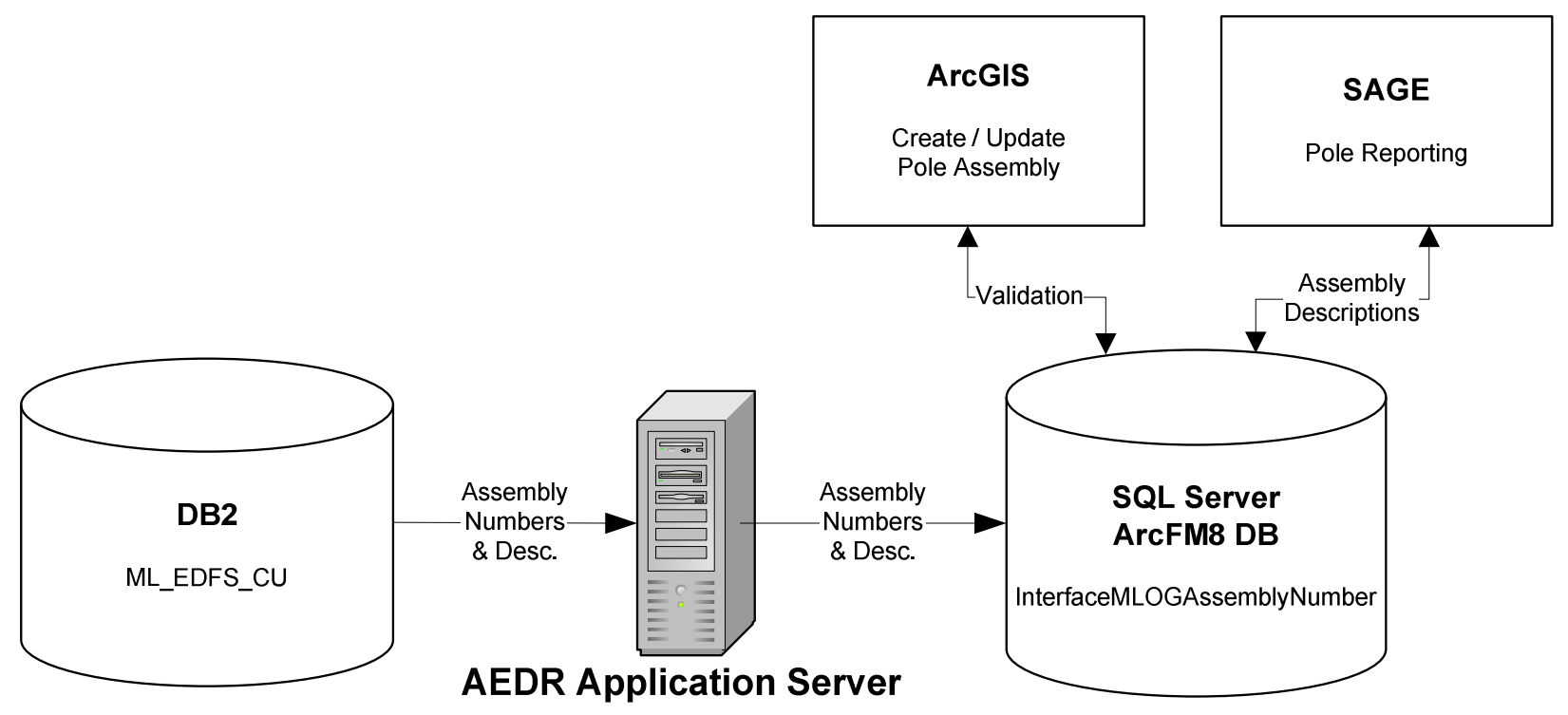

Figure 4.8-52. Assembly number validation process 


\subsection{System Performance Evaluation / Stress Testing}

\subsubsection{Overview}

In order to maximize the usability of AEDR, NIPSCO tuned the system for maximum performance. This document outlines the method employed for configuring and maintaining a faster performing system. Performance was defined as the application's impact on productivity. Therefore, the goal was to ensure a system performance level that complimented the end user workflow.

NIPSCO used the following strategies to achieve adequate system performance:

- Implement basic performance configurations prior to rollout.

- Run tests to evaluate the performance of the system and to identify potential performance enhancements prior to rollout.

- Based on performance testing, fine-tune the system prior to rollout.

- Conduct regular performance maintenance on the production system.

In tandem with system performance testing, performance testing of the ArcSDE/SQL Server database server was conducted. The performance testing report is located in Appendix C.

\subsubsection{Testing}

\subsubsection{Hardware Size Testing and Tuning}

Prior to the Construction phase of the project, NIPSCO conducted testing to validate that the hardware selected to run AEDR supported adequate performance. Since AEDR had not been built yet, a simple proxy was constructed using basic out-of-the-box ArcGIS/ArcFM and sample data imported from NIPSCO's existing Automated Mapping System. A script was used to apply a processing load to the system by automatically zooming in and out and panning around within NIPSCO map data. The script recorded the redraw times of a single user. While redraw times were being recorded for the first user, additional users performing redraw actions were incrementally added to simulate additional load on the system. In general these tests demonstrated that NIPSCO's network, client hardware, ArcSDE server hardware, and Citrix server hardware were more than adequate to handle the maximum concurrent user load.

\subsubsection{First Performance Testing and Tuning}

By December 2004, NIPSCO had completed some Construction Phase tasks and had received some preliminary deliveries from the data migration vendor; it was possible to evaluate the preliminary Development Environment for performance. Some prerequisite tuning steps were identified that would be completed before the more advanced testing and tuning could occur. 


\subsubsection{Second Performance Testing and Tuning}

With the exception of a few bug fixes and last minute tweaks, AEDR was fully designed and developed, including a Base Performance Configuration (see Section 4.9.3 Base Performance Configuration) by the spring of 2006. Additionally, the migration vendor that was moving NIPSCO's legacy data into the ArcSDE Geodatabase had provided a sample data set that was as close to production quality as possible. It was then possible to performance test AEDR under a production scenario.

NIPSCO created six testing scripts that included a cross section of day to day activities that AEDR would need to support in production. The test scripts emulated activities such as adding, deleting, or editing records, panning and zooming, zoom to feature by query, check out a new session (version), reconcile and post a session, system interface import, system interface export, run reports, etc.

Seven testers ran a test script simultaneously recording the duration for each script step in the form of start times and stop times. Then the step durations were summarized for each tester for the test script to create the Total Script Duration. The Total Script Durations for each tester were averaged and a standard deviation value calculated. Then a Quality Index was derived as an expression of the percent of standard deviation to the average. To increase confidence in the Average Script Duration, any total script duration that did not include all steps in the script was eliminated. Also, any total script durations that produced a statistical outlier was eliminated. And finally any Average Script Duration with a Quality Index greater than 33\% was eliminated. Figure 4.9-1 describes the results.

\begin{tabular}{|c|c|c|c|c|c|c|c|c|c|}
\hline Step & $\begin{array}{c}\text { Tester } \\
1\end{array}$ & $\begin{array}{c}\text { Tester } \\
2\end{array}$ & $\begin{array}{c}\text { Tester } \\
3\end{array}$ & $\begin{array}{c}\text { Tester } \\
4\end{array}$ & $\begin{array}{c}\text { Tester } \\
6\end{array}$ & $\begin{array}{c}\text { Tester } \\
7\end{array}$ & Average & $\begin{array}{l}\text { Standard } \\
\text { Deviation }\end{array}$ & Quality Index \\
\hline $\begin{array}{l}\text { Performance } \\
\text { Test Script \#1 }\end{array}$ & 77 & & & & & 83 & 80.00 & 4.24 & 5.303300859 \\
\hline $\begin{array}{l}\text { Performance } \\
\text { Test Script \#2 }\end{array}$ & 90 & 111 & & 148 & & 70 & 90.33 & 20.50 & 22.69597685 \\
\hline $\begin{array}{l}\text { Performance } \\
\text { Test Script \#3 }\end{array}$ & 58 & & & & 144 & 60 & 59.00 & 1.41 & 2.39697214 \\
\hline $\begin{array}{l}\text { Performance } \\
\text { Test Script \#4 }\end{array}$ & 28 & & 70 & & & 27 & 27.50 & 0.71 & 2.571297386 \\
\hline $\begin{array}{l}\text { Performance } \\
\text { Test Script \#5 }\end{array}$ & 24 & & 50 & & 25 & 24 & 24.33 & 0.58 & 2.372672339 \\
\hline $\begin{array}{l}\text { Performance } \\
\text { Test Script \#6 }\end{array}$ & 20 & & 33 & & 26 & 20 & 22.00 & 3.46 & 15.74591643 \\
\hline & & & ge 0 & $\begin{array}{l}\text { vera } \\
\text { uratic }\end{array}$ & otal & & 50.53 & & \\
\hline
\end{tabular}

Figure 4.9-1.

An average of 50.53 minutes per script was considered to show that the system could support adequate user productivity in production for seven concurrent users. No performance enhancements were identified. 


\subsubsection{Final Performance Testing and Tuning}

The last performance testing and tuning iteration took place a few weeks after the Second Performance Testing and Tuning session. The Final Performance Testing and Tuning session was similar to the Second Performance Testing and Tuning session except in two ways. First, more testers participated in the testing session; 32 testers instead of 7.

Secondly, the 6 testing scripts used were divided into 18 separate scripts. Each of the 18 scripts contained approximately the same amount of work which was about equal to an average Work Order that would be entered into the system on a day to day basis. The advantage to structuring the tests scripts in this manner is that the final average of the script durations was more meaningful.

After filtering out the data points where a reliable duration could not be obtained, 8 data points remained. The Average of Average Total Script Durations was 43.10 minutes. Since each script was determined to contain about the same amount of work as an average work order then by proxy the prediction was that an average Work Order would take 43.10 minutes to complete. NIPSCO determined that this meant the system could support adequate user productivity. See the Figure 4.9-2 for the results.

\begin{tabular}{|c|c|c|}
\hline Step & Average & Quality Index \\
\hline Performance Test Script \#1 & & \\
\hline Performance Test Script \#2 & & \\
\hline Performance Test Script \#3 & & \\
\hline Performance Test Script \#4 & & \\
\hline Performance Test Script \#5 & & \\
\hline Performance Test Script \#6 & & \\
\hline Performance Test Script \#7 & 109.00 & 30.57327011 \\
\hline $\begin{array}{l}\text { Performance Test Script \#8 } \\
\text { Performance Test Script \#9 }\end{array}$ & & \\
\hline Performance Test Script \#10 & 33.50 & 30.84973803 \\
\hline Performance Test Script \#11 & 32.00 & 28.25680012 \\
\hline Performance Test Script \#12 & 31.00 & 26.70797804 \\
\hline Performance Test Script \#13 & 29.50 & 22.49715154 \\
\hline Performance Test Script \#14 & 55.50 & 30.11435281 \\
\hline Performance Test Script \#15 & 32.33 & 23.76168994 \\
\hline Performance Test Script \#16 & & \\
\hline Performance Test Script \#17 & 22.00 & 26.42722314 \\
\hline Performance Test Script \#18 & & \\
\hline Average of Average Total Script Durations & 43.10 & \\
\hline
\end{tabular}

Figure 4.9-2.

\subsubsection{Base Performance Configuration}

This section is intended to provide a checklist of base configurations that must be in place before a comprehensive performance tuning or testing workshop could take place

- Identify the Performance Testing Platform

- ArcSDE Instance

o Citrix Environment 
○ Thick Clients

o Thin Clients

- Create a Test Dataset

- ArcSDE Geodatabase with the latest migration dataset

- Update ArcSDE with the latest schema

- Load CIS data into the Geodatabase

- Create and populate all non-Geodatabase interface support tables

- Load all aerial photography files on the test server

- Install Custom Application Components on the Test Server

- Custom AutoUpdaters

- EDFS Replacement Tool

- Session Manager

- AEDR Help System

- Conductor Management Tool

- SynerGEE Export

- CADOPS/FeederAll Export

- CIS and other interfaces

- Distribution Reference Number Validation Tool

- ArcFM / ESRI Configuration

○ Build the Geometric Networks and import connectivity rules

- Build Miner \& Miner System Tables

- Convert the database to Miner \& Miner Objects

$\circ$ Initialize Network Trace Weights

- Populate the Circuit Source table

- Initialize the Electric Network using Feeder Manager Trace a Feeder, at least in performance testing area

- Configure Snapping Rules

- Configure and assign all AutoUpdaters

- Configure Stored Displays

- Basic Performance Configuration

○ Apply Field Indexes

- Run and apply the Spatial Index Grid scripts

$\circ$ Build table statistics using the Analyze command

\subsubsection{ArcSDE Geodatabase Performance Maintenance}

Once the system is in production, regular performance maintenance steps will need to occur. Many of these steps are included in the basic configuration exercise. However, as the data set changes over time, certain configurations should be re-applied.

\subsubsection{SQL Server Log Maintenance}

Log files record each transaction completed by the database. If the database would need to be restored, these log files would be used to restore the database state as it was altered AFTER the last backup was performed. Therefore, transaction logs recorded BEFORE the last backup procedure are no longer needed since those transactions were included in the backup. 
In order to keep the log file size low, regular database backups should be performed. The $\log$ files should be truncated to mark unneeded log file records and shrunk to reduce the size of the log file.

\subsubsection{ArcSDE Compress Schedule}

ArcSDE versioning works by keeping track of the changes performed on the version.

Behind the scenes a version is a set of tables that track these changes known as the "Add" and "Delete" tables. Over time the size of the Add and Delete tables can grow very large causing degraded performance. The "Compress" command removes any database states that are no longer needed and moves states common to all versions to the base business table. Decreasing the size of the Add and Delete tables improves performance.

The Compress command should be run often. However, the ArcSDE Geodatabase must be taken out of production in order to run the Compress since the Compress command requires an exclusive lock on all tables. No users can be logged in.

\subsubsection{ArcSDE Compress Success}

Compress success, meaning the ability of the Compress command to reduce the size of the Add and Delete tables can impact performance. A Compress process that removes very few records from the Add and Delete tables will not improve performance much.

The effectiveness of the Compress command can be maximized by encouraging the user community to post their version changes to the parent version when possible and by automating a batch and reconcile routine. Posting and reconciling improves compression success by moving states from one version to another. Once a state becomes common to all versions, the Compress command can write the state to the business table and remove the state.

\subsubsection{Analyze Command Schedule}

The "Analyze" command causes the underlying RDBMS to rebuild table statistics. The Analyze command should be issued after major database updates such as a Compress.

\subsubsection{Rebuild Field Index}

Field index improves the performance of queries. Indexes should be updated as data changes.

\subsubsection{Evaluate Spatial Index Grids (SIG)}

SIG improves the performance of spatial queries, for example, to find which polygon features a point falls within, a candidate group of polygons is created by returning only the polygons that fall within the SIG that intersect the point. Subsequently each geometry in the candidate list is examined to determine if the point falls within the polygon. This is faster because the query does not have to examine every record in the feature class. SIG are most effective when sized properly to the geometries stored in a particular layer. The goal is to have as few geometries returned in a sub-candidate list as possible. 
The Spatial Index Grids will be built with the following criteria.

- $\quad$ SIG for Point Feature Class layers will equal 3.

- $\quad$ SIG for Line and Polygon Feature Class layers will equal 3 times the average of the average extent of geometries stored in the Feature Class. The average extent is the average of the height and width of the rectangle bounding each feature.

- Approximately $80 \%$ or more of a layer's features should fall within a single grid cell.

- The number of Grid Index record entries divided by the number of features in layer. This number will always be 1 or greater because every feature must belong to at least one grid. However, some features may belong to several grids and therefore would be represented in the grid table several times. 4 entries per feature should be the maximum ratio.

- Average features per grid should be low, with a maximum of 300 .

- Maximum features per grid should be low, with a maximum of 4000.

\subsubsection{Helpful Information}

- Analyze Data and Rebuild Indexes http://support.esri.com/index.cfm?fa=knowledgebase.techarticles.articleShow\&d= $\underline{24518}$

- $\quad$ Setting the Selection Threshold http://support.esri.com/index.cfm?fa=knowledgebase.techarticles.articleShow\&d= $\underline{22668}$

- HowTo: Tune the Multi-Level Grid Spatial Index http://support.esri.com/index.cfm?fa=knowledgebase.techarticles.articleShow\&d= $\underline{23407}$

- "ArcSDE Configuration and Tuning Guide for Microsoft SQL Server" Located on the ArcSDE installation CD's

- Performance Considerations for Run-Time Technologies in the .NET Framework http://msdn.microsoft.com/library/default.asp?url=/library/enus/dndotnet/html/dotnetperftechs.asp

- Improving .NET Application Performance and Scalability http://msdn.microsoft.com/library/en-us/dnpag/html/scalenet.asp

- How to improve ArcSDE Performance by analyzing data and rebuilding indexes http://support.esri.com/index.cfm?fa $=$ knowledgebase.techarticles.articleShow\&d= $\underline{24518}$ 


\subsection{AEDR Online Help Documentation}

\subsubsection{Overview}

The AEDR system is very large and encompasses many separate but related components. It was determined that a comprehensive and centralized online help documentation system was needed to provide a single source for all AEDR users to access for information and operational instructions. Initially a compiled html help document (*.chm) format was considered but this idea was eventually dismissed because of the inherent problems related to regularly distributing updated copies of the chm file to a large number of users.

The ideal system should handle real time updates and content distribution but would have the same format and functionality of a local chm file. It was determined that a custom solution would best meet this need. This solution is web based and is hosted at an easily accessible intranet web address. The solution contains sections for the following five main AEDR components:

- ArcEditor

- ArcView

- SAGE

- Facility Browser

- Field Browser

The end user functionality contains the following key components from a chm format:

- Contents: A tree view presentation of the help contents are used to allow a user to traverse a logical path of AEDR functionality to access the specific topic desired.

- Index: An alphabetic index of all topics are included with a type ahead text box to allow a user to locate various topics by typing common keywords or phrases that define the information requested.

- Search: A full text search engine is included that will search all help files. Any matching files are shown in a list format and the searched words are highlighted on the topic page when it is viewed.

- Favorites: The users are able to save a list of their favorite topics for easy repeated access. These favorites are stored centrally by the web application so that a user can access their favorites from any computer on the NIPSCO intranet. Each user's favorites are managed individually via their windows active directory authentication data.

Additionally, it was decided to include web-based authoring and management tools in the solution so that updates to the help content could be deployed by users with no knowledge of html, JavaScript, the web server, etc. This allows a standard administrator (admin) user to easily create and deploy help content in a seamless environment. The following tools have been provided:

- Add Content: An admin user is able to design html content files directly within the application using a WYSIWYG (what you see is what you get) html editor. 
The application handles the saving of the html content, the indexing of the topic title, and the indexing of the content text in the full text search engine.

- Edit Content: An admin user is able to edit any existing html content files directly within the application using a WYSIWYG (what you see is what you get) html editor. The application handles the saving of the html content, the re-indexing of the topic title, and the re-indexing of the content text in the full text search engine.

- Image Management: The application allows an admin user to upload and view images associated to the help topics. In an html based system, the images are not embedded directly in the html documents but are instead referenced from the html content files. The images reside in a common folder and are easily made accessible when editing the html content files.

- Contents Management: An admin user is able to edit the tree view contents layout including the order of topics, categories of topics, and the labels of the topics.

\subsubsection{Design}

The solution was designed using ASP.Net and JavaScript technologies. The end product is a user-friendly help system. Figure 4.10-1 shows the main user interface to the help system:

\begin{tabular}{|c|c|}
\hline 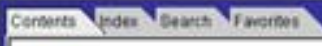 & AEDRONLINE HELP SYSTEM \\
\hline 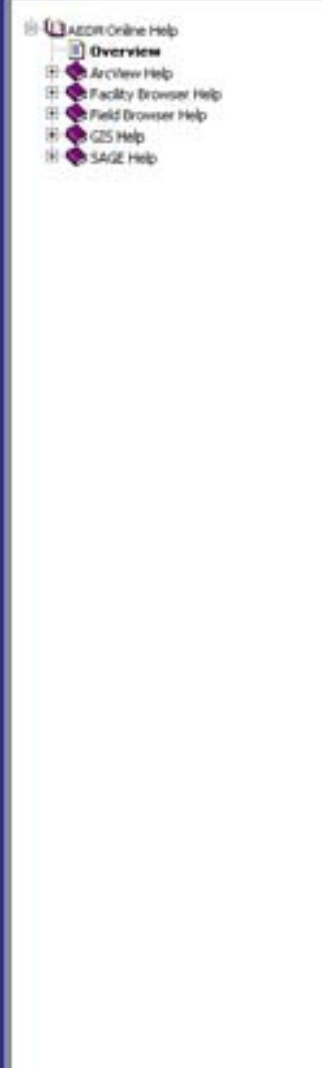 & 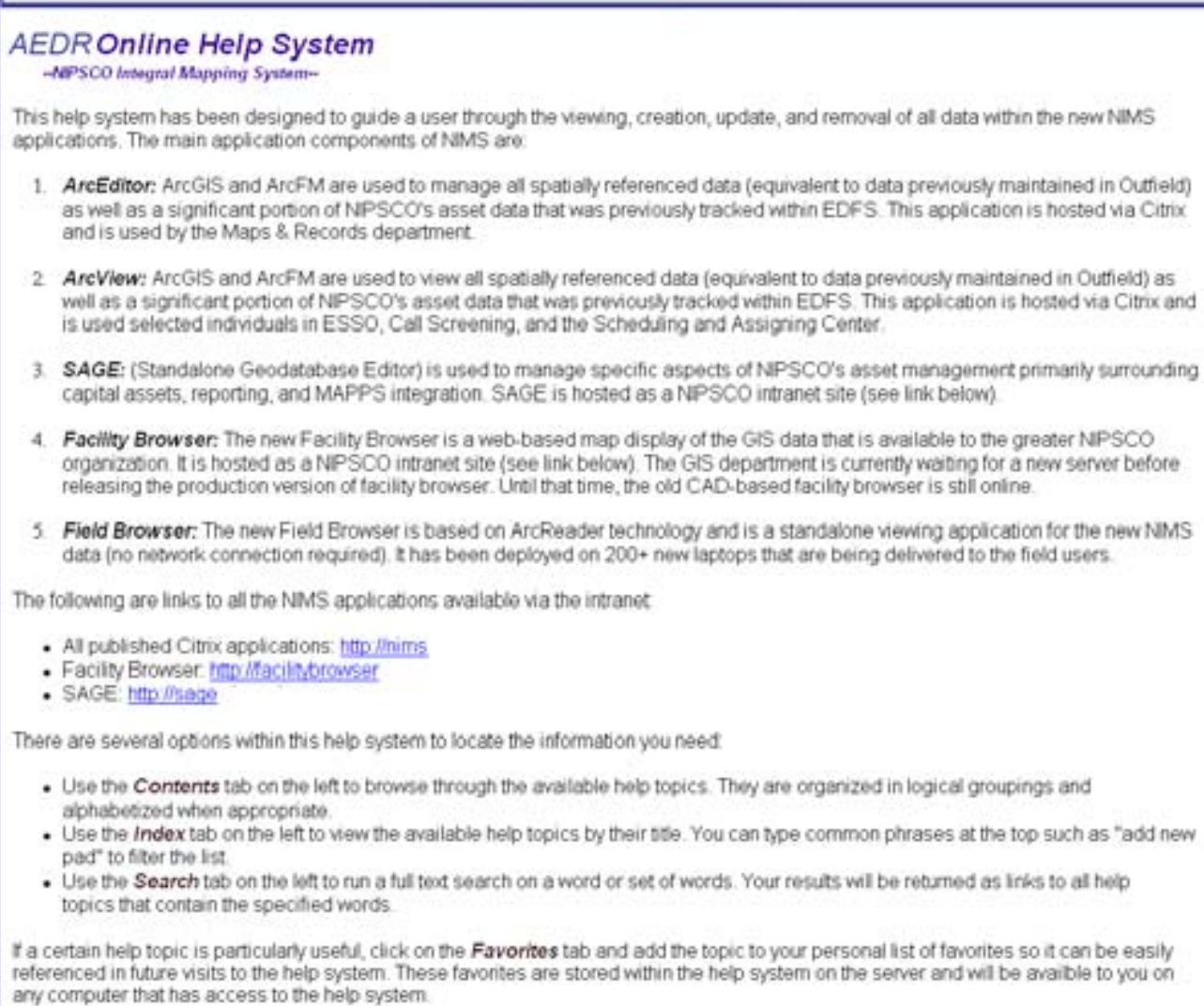 \\
\hline
\end{tabular}

Figure 4.10-1 


\subsubsection{End User Components}

\subsection{Contents Pane}

The goal of the contents pane is to utilize a tree view presentation of the help contents to allow a user to traverse a logical path of AEDR functionality to access the specific topic they are looking for. The content pane uses categories and sub categories to facilitate a drill-down approach to content files. In the following example, an edit user might be looking for instructions on adding a new fuse cutout bank in the GIS. They would drill down through GIS Help $\rightarrow$ Electric $\rightarrow$ Add New / Install $\rightarrow$ Add Fuse Cutout Bank:

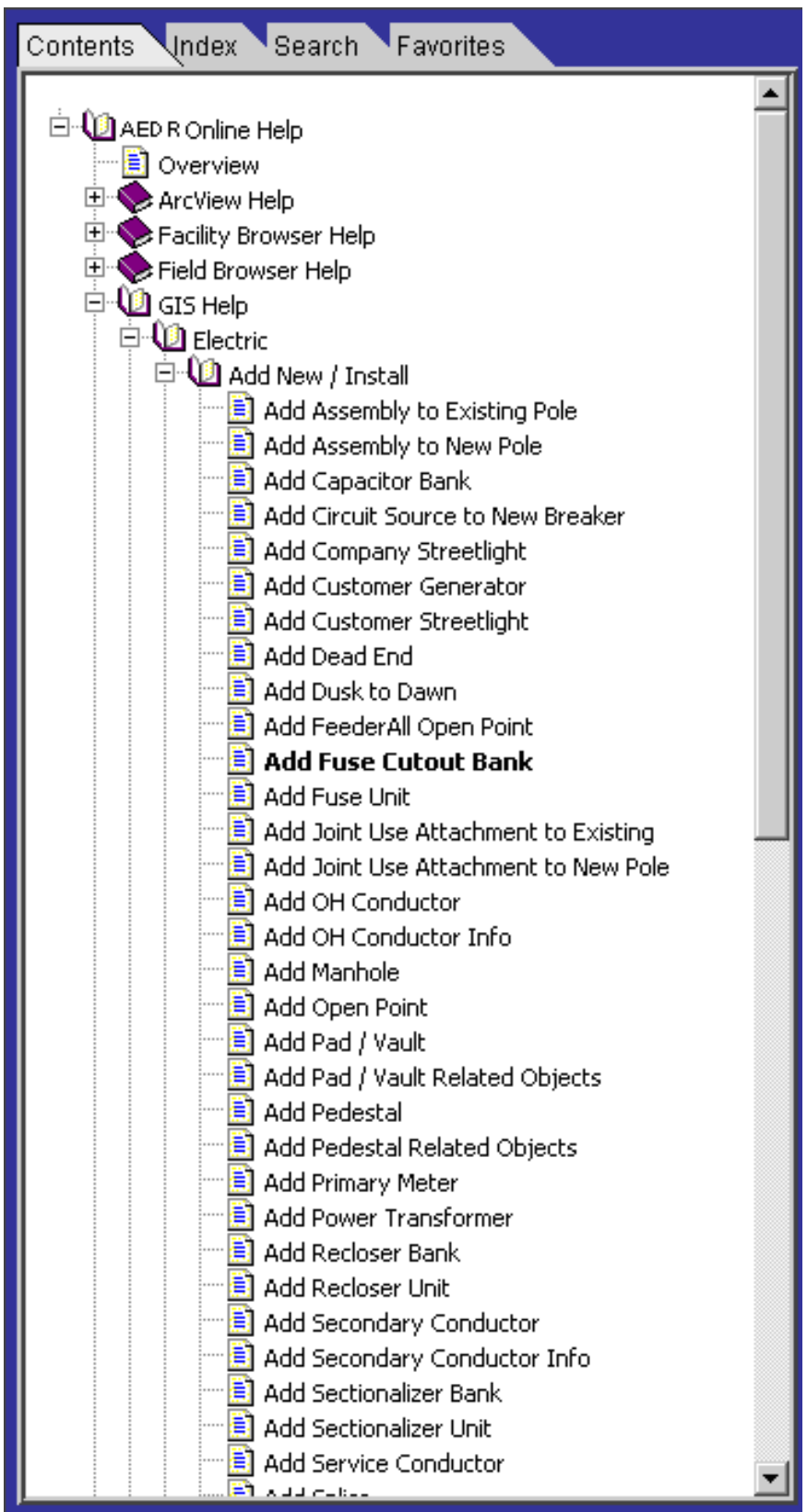

Figure 4.10.2 
A standard chm-style look and feel is used for the contents pane. This capitalizes on user's previous use of standard help files. Clicking on any of the topics loads the content in the reading pane of the help system.

\subsection{Index Pane}

The goal of the index pane is to present an alphabetic index of all topics with a type ahead text box to allow a user to locate various topics by typing common keywords or phrases that define the information they are looking for. As the user types the keywords or phrases, the application will dynamically search the list for matching topics. As matches are found, the application will automatically highlight the first match in the index list. In the following example, a user is searching for information on retiring a regulator unit. As the user types "Retire R" the application automatically matches the topic and highlights the matched entry: 


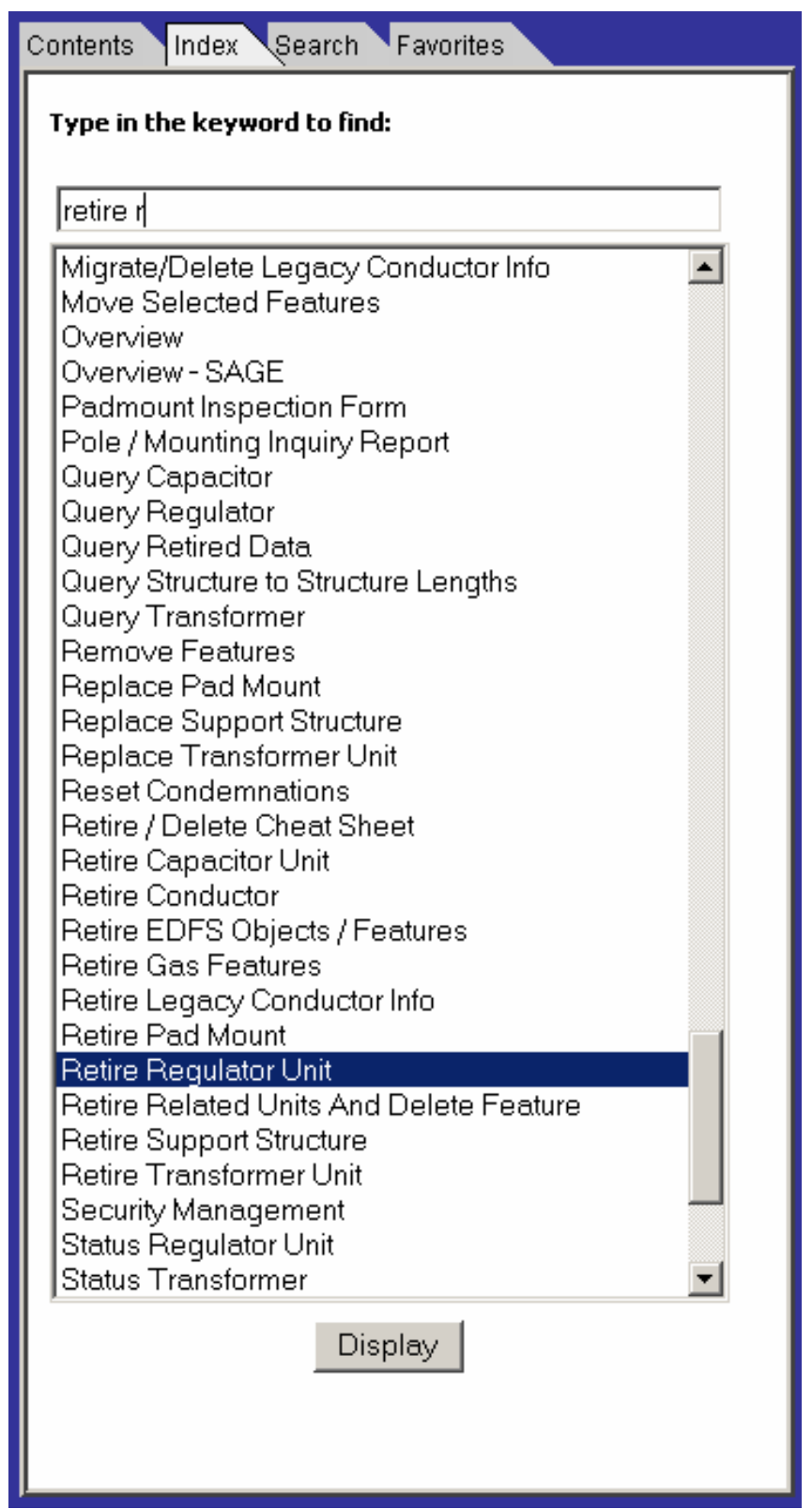

Figure 4.10-3.

The topic content can be displayed in the reading pane by either double clicking the index entry or clicking the "Display" button.

\subsection{Search Pane}

The goal of the search pane is to allow a user to perform a full text search against all help files. Any files containing ALL search words will be shown in a list format. In the following example, the user has searched on "install transformer" and several topics have been returned because they contain both the word "install" and "transformer": 


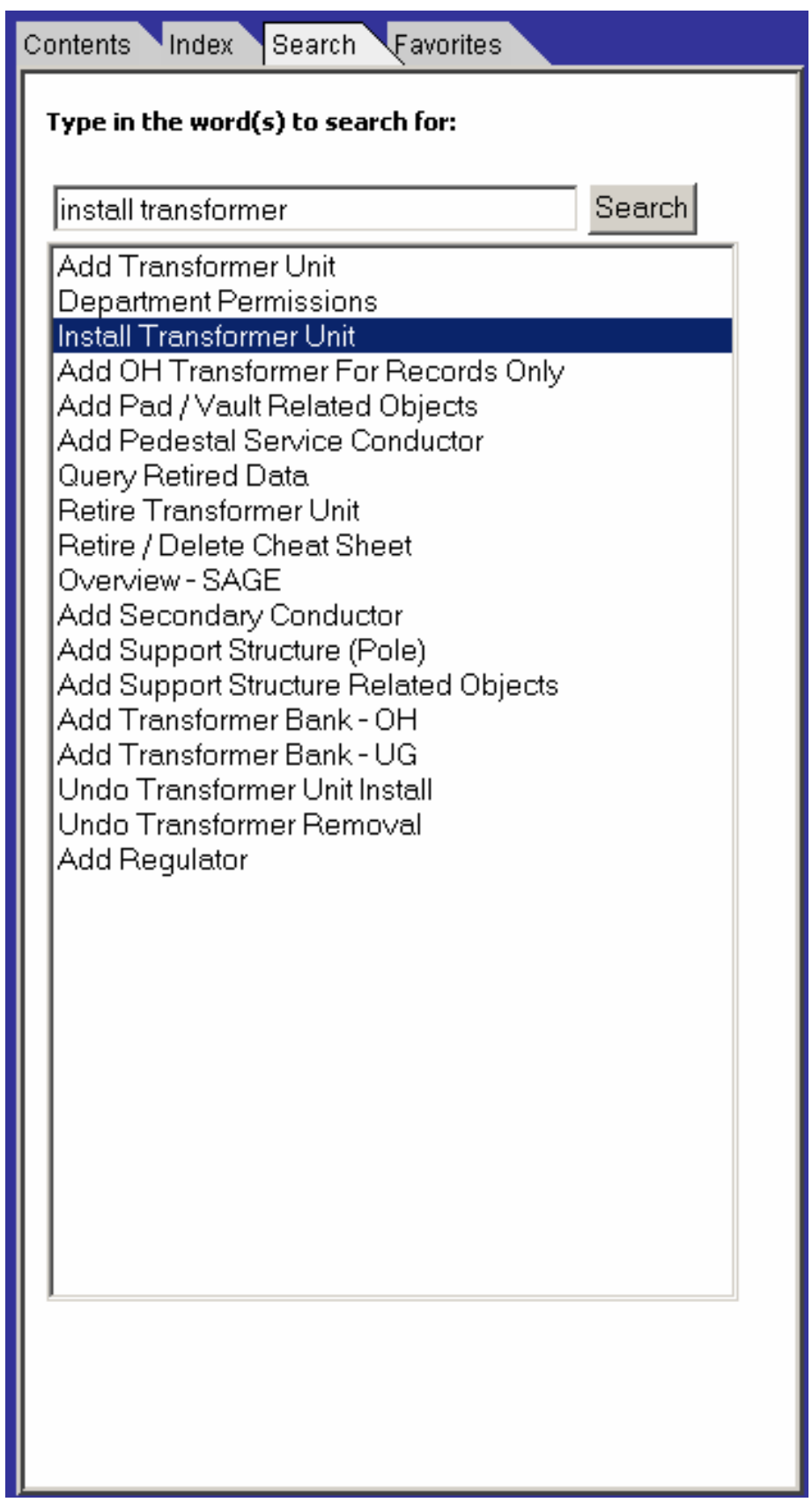

Figure 4.10-4.

Double clicking on any of the search results will load the topic content in the reading pane. When accessing topics from the search pane, the searched words will be highlighted on the topic page when it is viewed. In Figure 4.10-4 the user has double clicked on the "Install Transformer Unit" search result. The topic is displayed with the search words highlighted in yellow in Figure 4.10-5. 


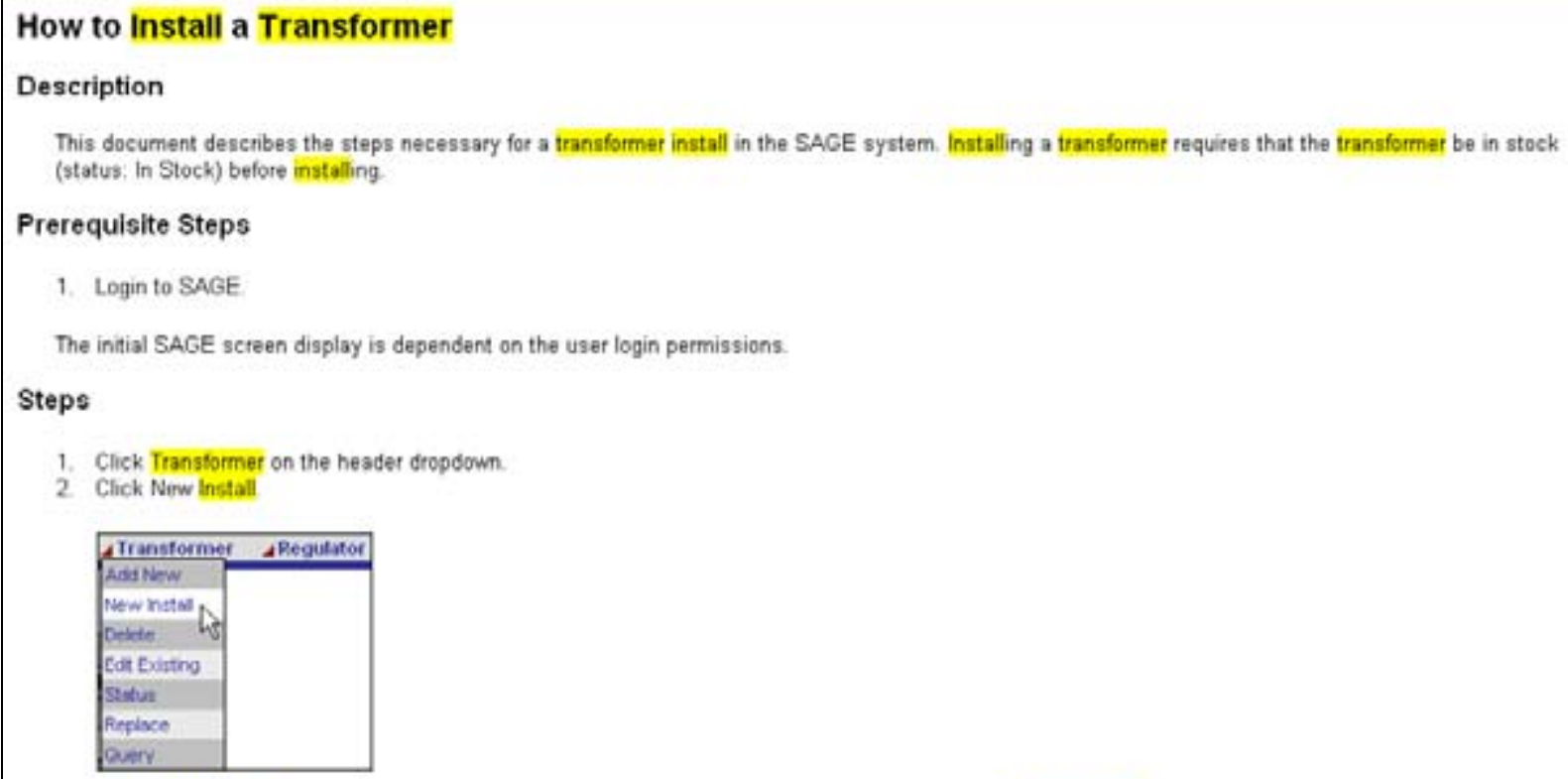

The Install Transformer display will appear and contains two specific search fields to find the existing transformer. The fields include the Company Number and/or the Senal Number

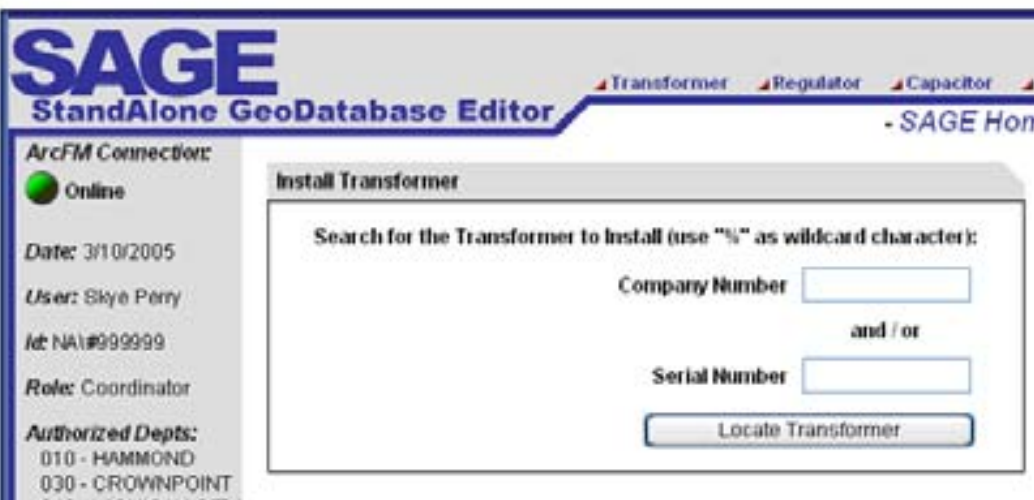

Figure 4.10-5

\subsection{Favorites Pane}

The goal of the favorites pane is for the users to be able to save a list of their favorite topics for easy repeated access. The user can click on the favorites pane with any content topic loaded. A text box will be automatically populated with the title of the current topic. The user can update the title and save the link as a favorite. In the Figure 4.10-6, the user is adding the "Install Transformer Unit" page to their favorites and is customizing the title that will be saved for the topic with a personal note: 


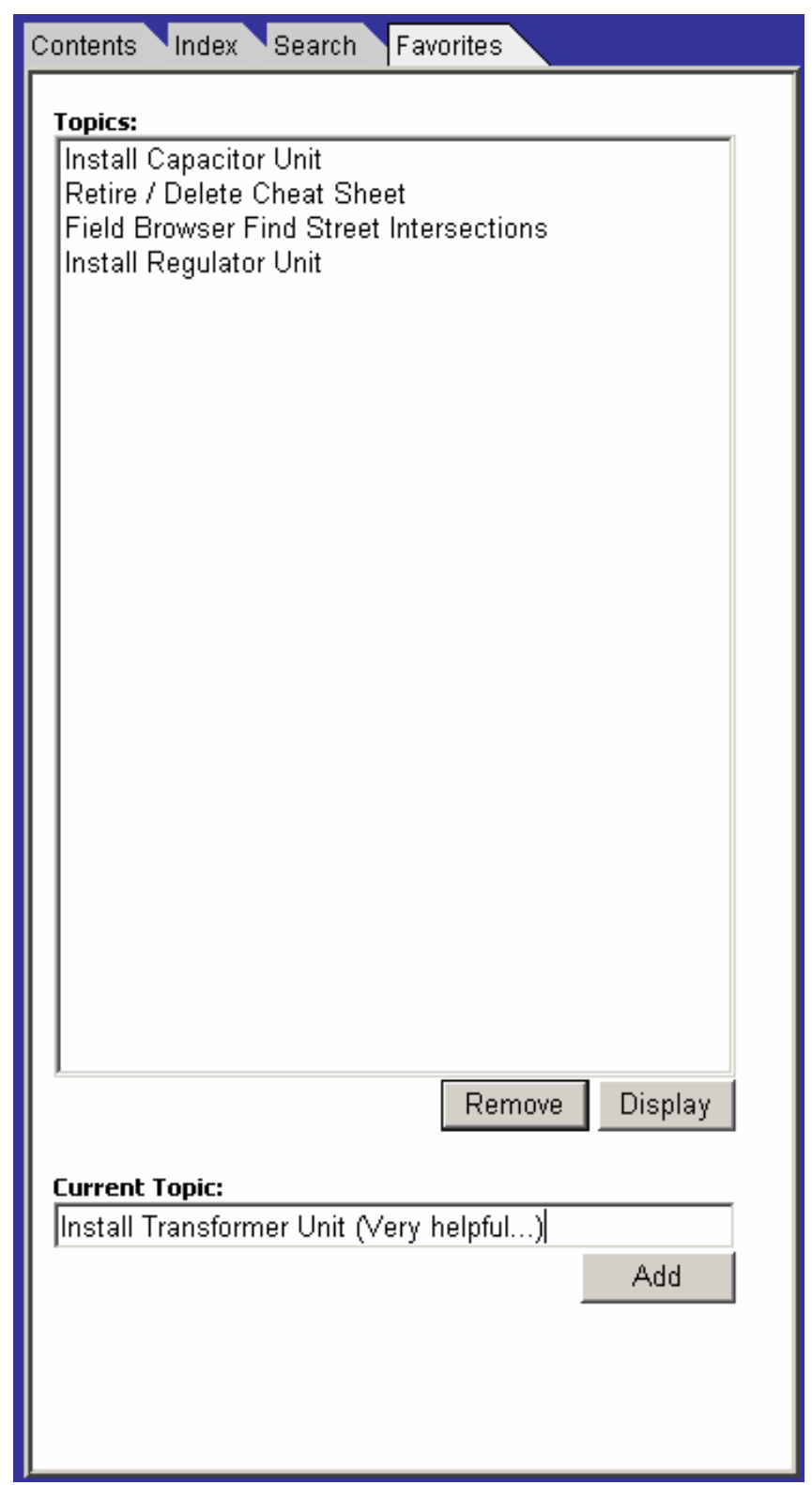

Figure 4.10-6.

The storage of these favorites occurs in an xml file stored on the web server. It is indexed by the user's windows active directory user id which allows for a user to access their unique favorites list from any computer on the NIPSCO intranet. An example of the xml storage is shown in Figure 4.10-7.

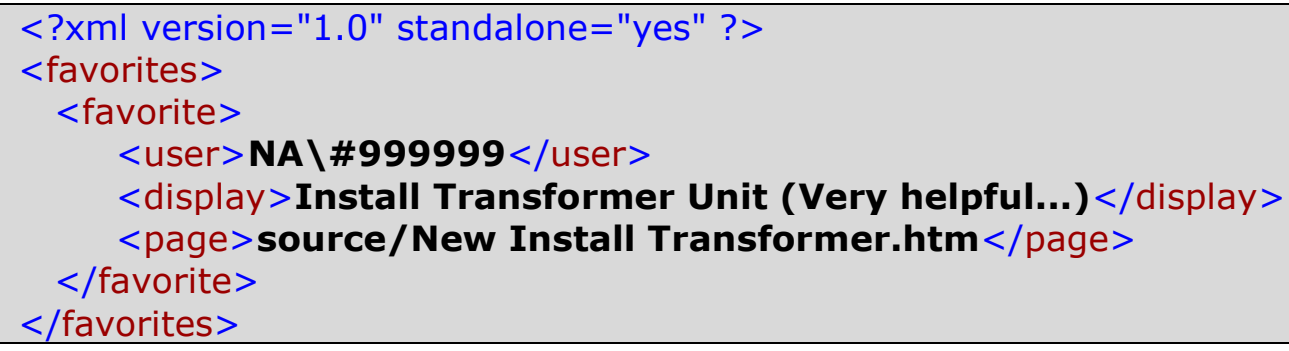

Figure 4.10-7 


\subsubsection{Administration Components}

An admin user accesses the same intranet website but is presented with additional options for managing the help system. In the upper right of the screen of Figure 4.10-8 three new links are available: Add New, Edit, and Manage Contents:

\begin{tabular}{|c|c|}
\hline 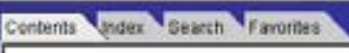 & AEDRONLINE HELP SYSTEM \\
\hline 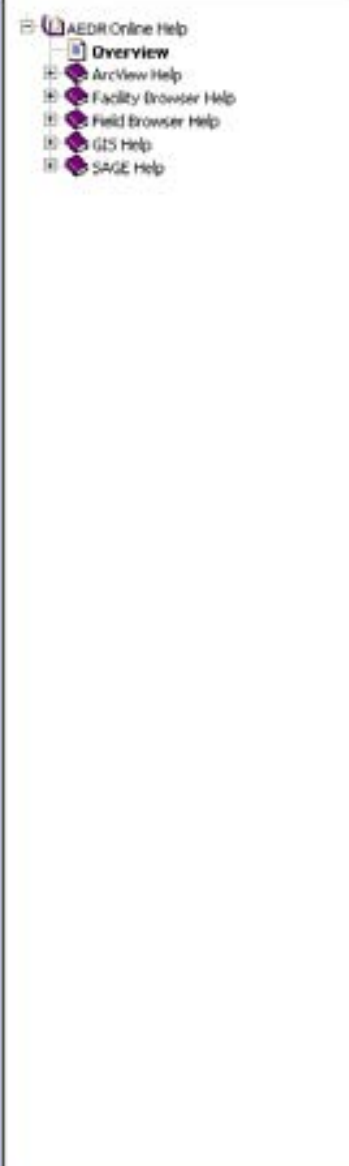 & 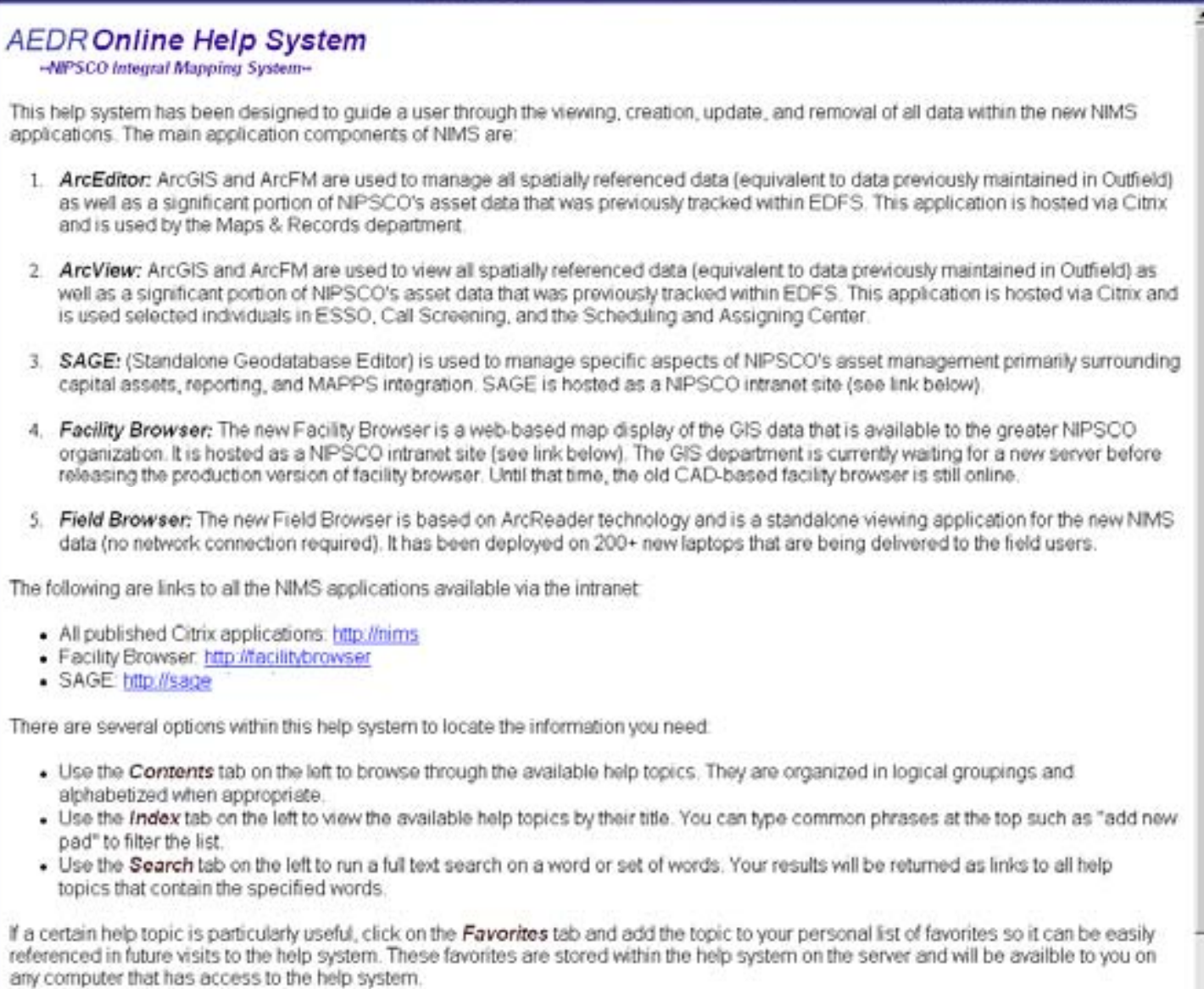 \\
\hline
\end{tabular}

\section{Figure 4.10-8.}

Links available via the intranet allow an admin user full capability to manage the content within the help system without knowing anything about the underlying technology.

\subsection{Add Content}

An admin user can click the "Add New" link to load the "Add a New Help Page" management page. This page allows the user to specify the name of the html file, the page title (used for indexing), and the content of the page. The html editing is all handled via a free WYSIWYG (what you see is what you get) editor supplied by www.InteractiveTools.com. This tool allows the creation of an html file using standard formatting tools equivalent to a subset of Microsoft Word tools. The end result is an intuitive user interface that allows for the accelerated development of help content shown in Figure 4.10-9. 


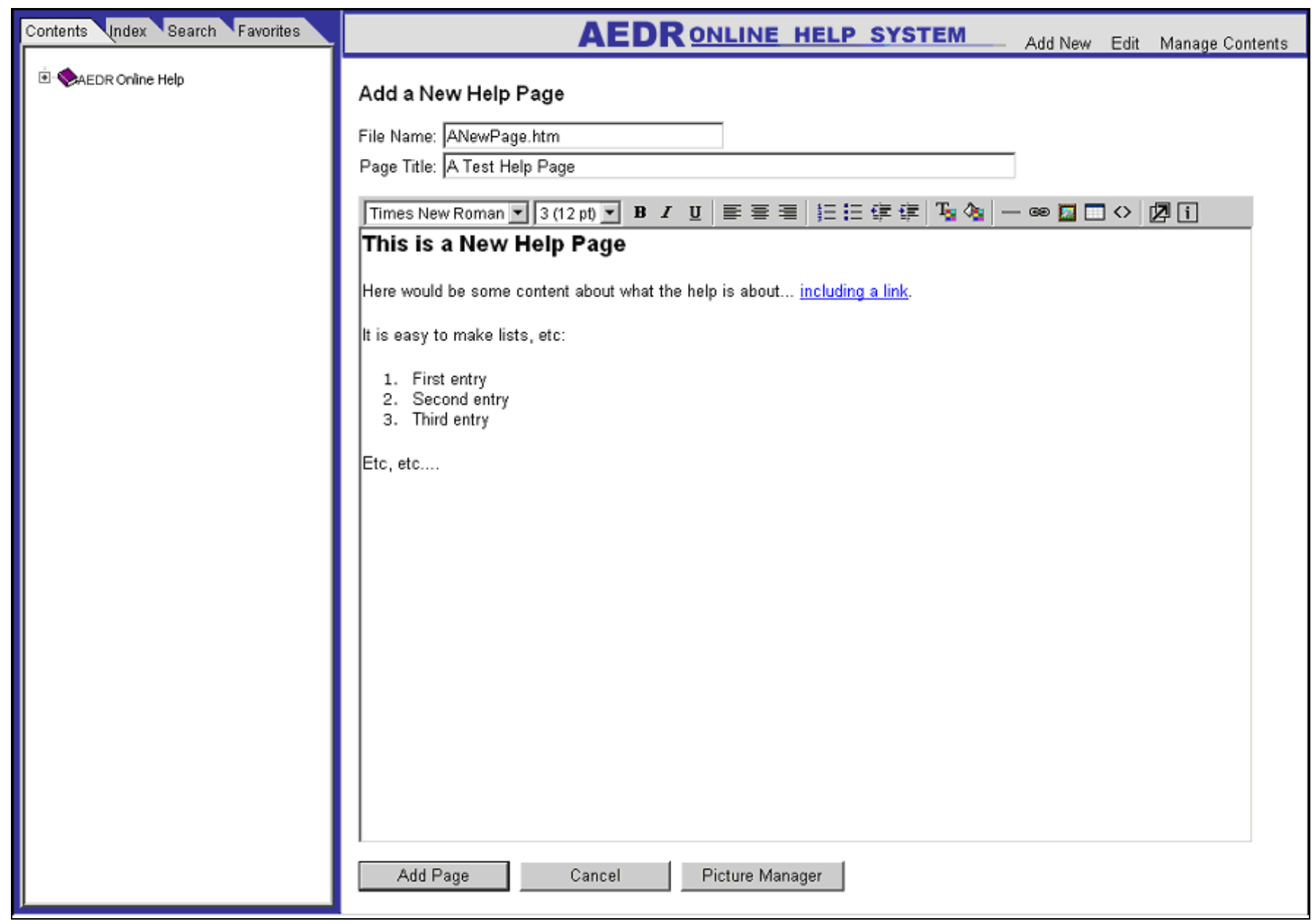

Figure 4.10-9.

When the user clicks the Add Page button, the html is automatically saved to the web server, the topic title is added to the help index file, and the content of the help file is added to the full text search engine.

\subsection{Image Management}

In an html based system, the images are not embedded directly in the html documents but are instead referenced from the html content files. To assist the user in managing these images, the application allows a user to upload and view images associated to the help topics. Clicking the "Picture Manager" button loads the Help Image Manager shown in Figure 4.10-10. 


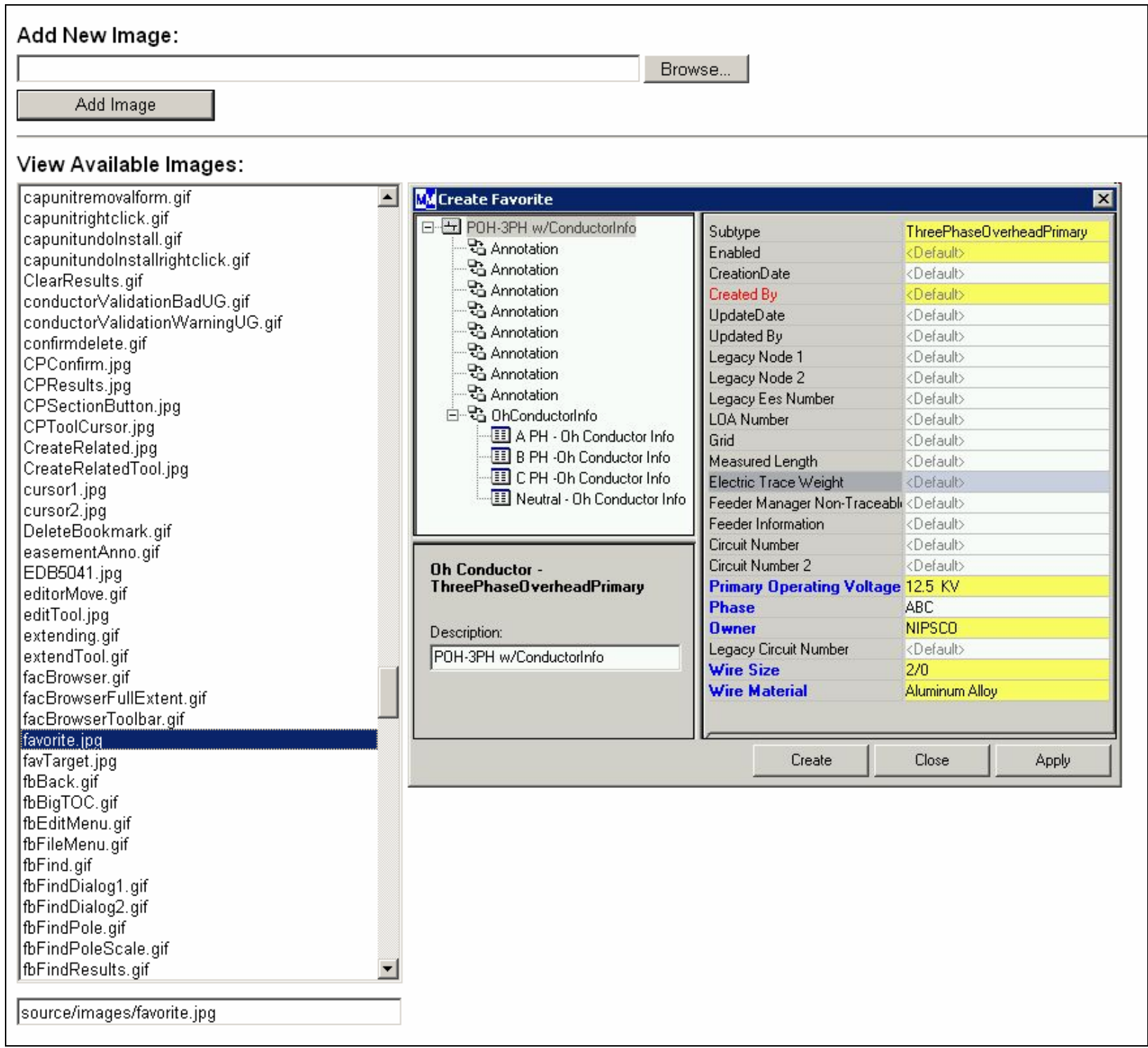

Figure 4.10-10.

The image manager provides several functions:

- A list is provided of all images that are currently part of the help system.

- The user can click on any image in the list and it will be displayed on the right hand side via dhtml.

- When an available image is selected, its full relative web server path is displayed at the bottom of this page. This is the path that is used to add a picture to a help content page.

- An "Add New Image" file upload box is provided to allow a user to browse to a picture on their local machine and subsequently upload it into the image manager.

The image manager makes it easy to view and locate images to be used in the help content pages. Within the add or edit content controls, the user just clicks the Insert 
Image button $\mathrm{D}$ and then pastes the path of the picture into the Figure 4.10-11 dialog box.

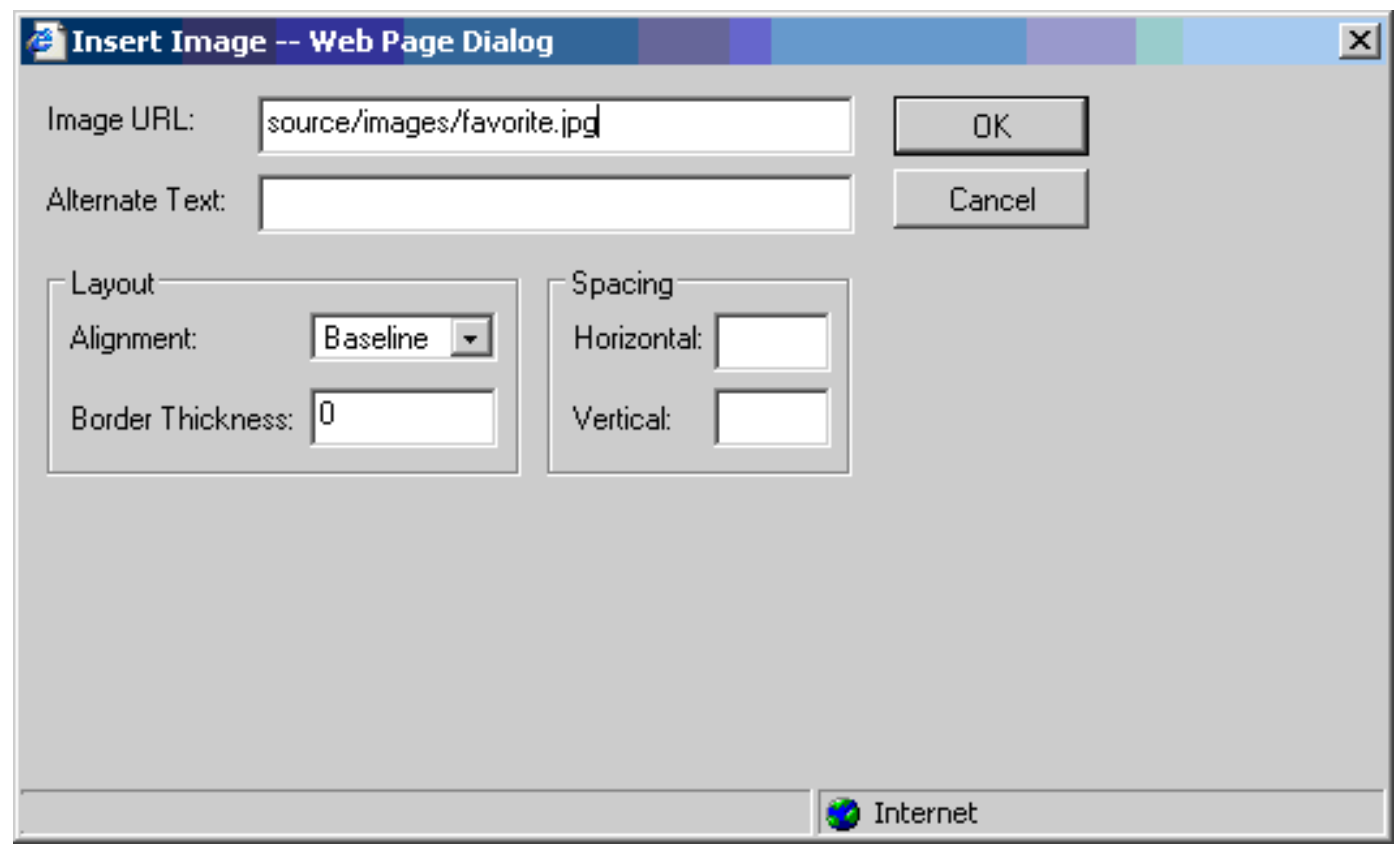

Figure 4.10-11.

The image is then added to the content page.

\subsection{Edit Content}

The Edit button loads a very similar page to the Add button but it can be used on any existing help content page. The user just loads the content page and then clicks the Edit button. The WYSIWYG editor is loaded with the current html file for editing including all formatting and images as shown in Figure 4.10-12. 


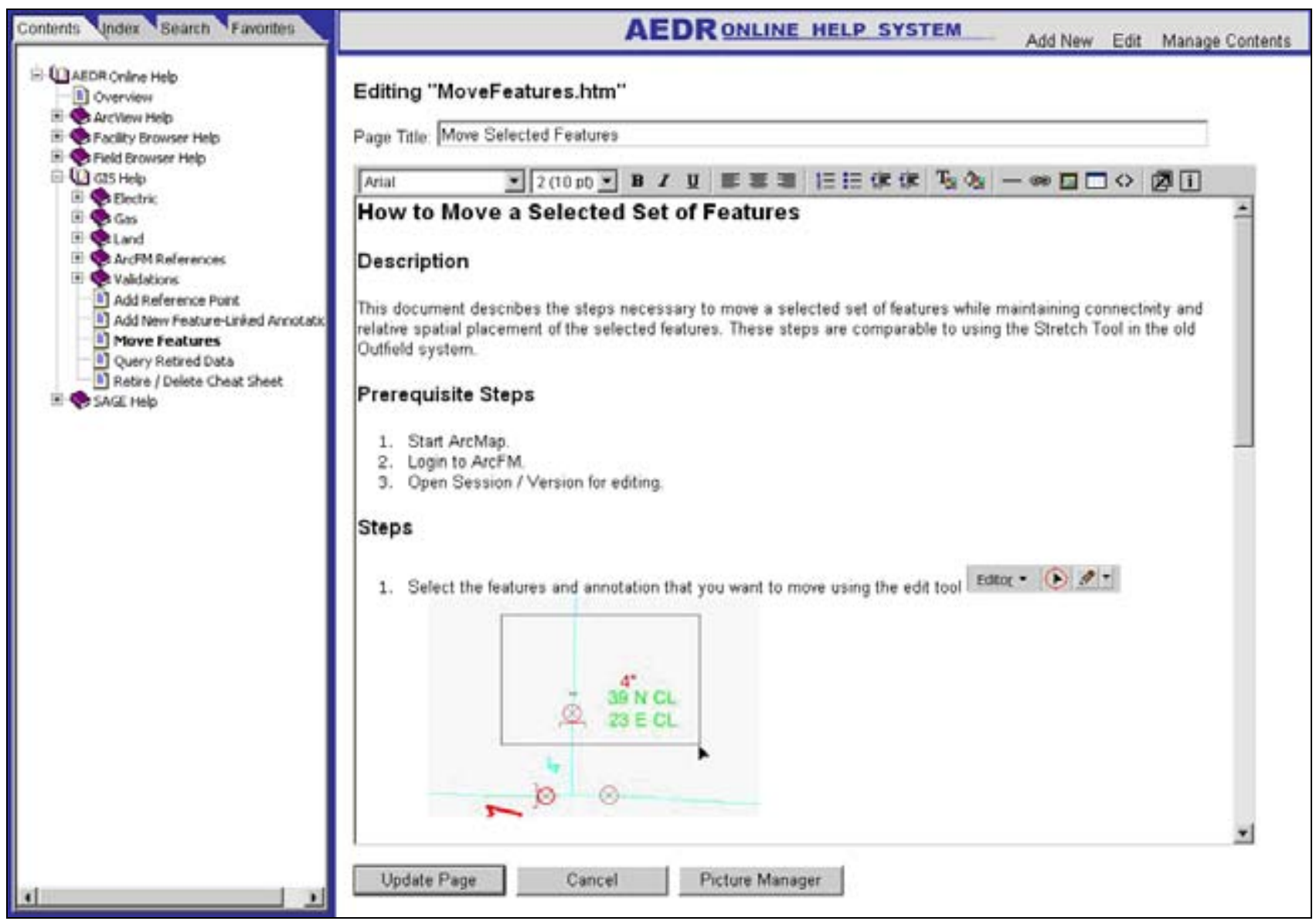

Figure 4.10-12.

The user can then apply any needed updates and save the page back to the server. Like the add page, the topic title is re-added to / updated in the help index file, and the content of the help file is re-indexed in the full text search engine.

\subsection{Contents Management}

A comprehensive contents manager control has also been provided to allow an admin user full control over the contents tree view that is displayed on the contents pane. This tool shows the design of the contents tree view in formatted list box. A "+" indicates a heading and a "_" indicates a content page. The indention of the entries shows the categorical structure of the contents in Figure 4.10-13. 


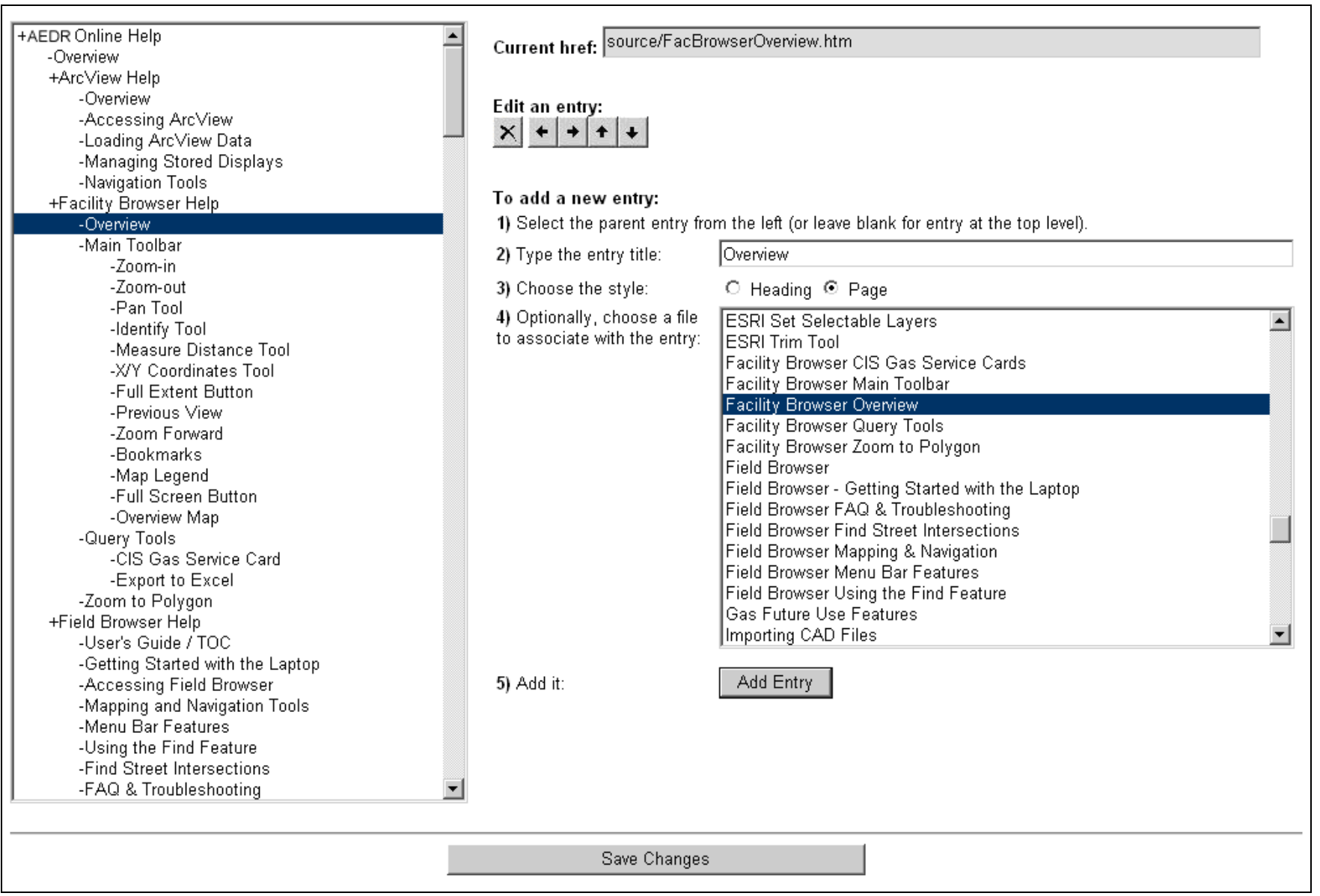

Figure 4.10-13.

Clicking on any entry in the contents list box will show the current path to the help content file in the "Current href" text box. Contents entry can be deleted, moved left or right (indention), and moved up and down in the list box using the edit controls:

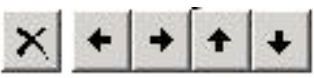

New contents entries can be easily added by following the steps provided on the screen. The entry title can be any value independent of the topic title. Clicking the Add Entry button adds a new entry with the specified parameters directly under the currently selected entry in the list box. It can then be moved using the edit controls as needed.

Once the new layout of the contents is acceptable, the user clicks the "Save Changes" button. The layout of the contents tree view is saved to the web server and is made immediately available to all users of the help system. All of these administration controls contribute to a highly effective and dynamic distribution of help information to a large audience of users. 


\subsection{Training}

\subsubsection{Overview}

The training audience is composed of the GIS project team and the end-user community; the record clerks. The GIS project team was trained from both a technical and functional perspective, while training for the end-users was purely functional. NIPSCO adopted the "Train the Trainer" concept of training GIS project team members to assist in training the record clerks. The training effort of planning, preparing and organizing began eight months in advance of site training.

\subsubsection{Training Needs Assessment}

This task was the cornerstone of the entire training plan. Miner \& Miner conducted an on-site visit to meet with a combination of NIPSCO GIS project team members and record clerk subject matter experts to accomplish the following:

- Discussed background, skill level, and computing literacy of the target training audience- record clerks

- Identified all job responsibilities of record clerks as they relate to tasks and functions in AEDR

- Reviewed implementation specific, custom functionality within AEDR

- Reviewed roles \& responsibilities, business processes, and work flows with regards to AEDR

- Discussed and establish skeleton outlines for training content, structure, and delivery mechanisms

- Established AEDR "best practices" for all record clerk oriented tasks for incorporation into all custom documentation development

- Reviewed and select a series of real world work orders, where applicable, to serve as the basis for hands-on exercise scenarios

- Open Q\&A, demonstration of ArcMap/ArcFM functionality

- Reviewed data options in support of course development and training delivery

- Discussed issues regarding training location preparations including: facility setups, hardware/software configurations, user roles and permissions, and on-site IT support.

\subsubsection{GIS Project Team Training}

During the pre-planning stages of the project (September 2003), the GIS project team received the standard (two weeks) ArcGIS and ArcFM training from ESRI and Miner \& Miner, respectively. Approximately nine weeks prior to production implementation, the GIS project team attended the ArcFM user training for the purposes of critiquing the class, serving as a dress rehearsal for Miner \& Miner trainers and preparing for the "Train the Trainer" program. Several GIS project team members were selected to augment the training program by assisting users in practice sessions following the formal Miner \& Miner ArcFM training program. 


\subsubsection{Record Clerk Training}

A half-day training session on the basics of ArcGIS/ArcFM as it relates to Window technology preceded the customized training on current day-to-day processing of work using real-life work orders and business materials. For simplicity, users were taught only one method instead of a multitude of methods to accomplish the same goal.

Training the user community presented unique challenges related to timing and logistics. Record clerks were spread across the northern third of Indiana, in fourteen locations often with only two or three record clerks located at a particular site. The logistics were further complicated in that business requirements prevented training all record clerks at once. Some employees were required to remain back in the office to process critical work while co-workers attended training.

Record clerks were trained seven weeks prior to production implementation after which they were provided approximately four to six weeks of mandatory half-day practice. Miner \& Miner training resources trained more than fifty record clerks in three different locations over a period of two weeks. Following the first week of record clerk training, NIPSCO trainers were dispersed to the newly trained record clerk locations to assist the record clerks in practice sessions while the second week of record clerk training commenced. Subsequent to completion of the second week of record clerk training, NIPSCO resources assisting Week 1 students were thinned out to the newly trained Week 2 students to continue assistance with the record clerk practice sessions.

\subsubsection{Training Environment}

Logistically, three training sites were set up across northwest Indiana located several hours drive apart. This presented challenges for setting up each training environment with the appropriate hardware as additional desktops were scarce.

The AEDR software was installed on the future production CITRIX farm serving simultaneous training classes. Using CITRIX as opposed to stand-alone implementations presented an ideal situation for the following reasons:

- Users would be trained on the exact environment they would be working in production. Logging on to CITRIX would be included as part of the training.

- The training PCs were of substandard horse power and would require an investment in hardware upgrades to allow the applications to run.

Thirty-two client machines were located in logistically challenged areas and would need to be fully configured with OOTB ESRI/ArcFM, service packs \& patches, custom font, EDFS and other custom installers. We saved approximately 600 man hours by using CITRIX where the applications were installed on a server farm. 


\subsubsection{Conclusion}

Training presented some significant challenges due to the complexity of the software, the number of users requiring training and the remote training locations. We had highly acceptable results using the CITRIX environment while containing the resource costs to support the training environment.

Developing the training program to include the minimum amount of information necessary for the users to perform their job function was critical for the success of the training. The ArcGIS/ArcFM platform is sophisticated software in that the same type of function can be accomplished many different ways. We attribute user acceptance of a new, complex system for the following reasons:

- Real-world business cases were used to train users

- Teaching only one method to perform a given function allowed the user to understand and perfect the function before using different tools to accomplish the same task

- Mandatory practice sessions every day for several weeks with required work submitted for review

- Onsite assistance during practice sessions

- A robust on-line help with detailed steps to perform a given function. 
Appendix A-1

Inception and Elaboration Project Plan 
NIPSCO GIS Inception and Elaboration Project Plan

\begin{tabular}{|c|c|c|c|c|}
\hline ID & (i) & Task Name & Start & Finish \\
\hline 1 & $\checkmark$ & NIPSCO Inception and Elaboration (Planning Stage) & Mon $7 / 21 / 03$ & Fri 12/19/03 \\
\hline 2 & $\checkmark$ & General Management Tasks & Fri $7 / 25 / 03$ & Fri 12/19/03 \\
\hline 3 & $\checkmark$ & Change Management (5\% of project) & Fri $7 / 25 / 03$ & Fri 12/19/03 \\
\hline 4 & $\checkmark$ & Project Management (15\% of project) & Fri $7 / 25 / 03$ & Fri 12/19/03 \\
\hline 5 & $\checkmark$ & Configuration Management (10\% of project) & Fri $7 / 25 / 03$ & Fri 12/19/03 \\
\hline 6 & $\checkmark$ & System Administration ( $10 \%$ of project) & Fri $7 / 25 / 03$ & Fri 12/19/03 \\
\hline 7 & $\checkmark$ & Inception Phase & Mon $7 / 21 / 03$ & Thu 10/16/03 \\
\hline 8 & $\checkmark$ & Develop the Business Case & Fri 8/1/03 & Tue $9 / 30 / 03$ \\
\hline 9 & $\checkmark$ & Define project objectives & Fri 8/1/03 & Fri 8/1/03 \\
\hline 10 & $\checkmark$ & Develop a financial forecast & Mon 8/4/03 & Tue 9/30/03 \\
\hline 11 & $\checkmark$ & Describe the project constraints & Wed $8 / 6 / 03$ & Wed $8 / 6 / 03$ \\
\hline 12 & $\checkmark$ & Establish the Initial Staffing / Commitments & Fri 8/1/03 & Tue 8/26/03 \\
\hline 13 & $\checkmark$ & Establish initial vendor staffing plan & Fri 8/1/03 & Fri $8 / 1 / 03$ \\
\hline 14 & $\checkmark$ & Get participation commitments from SMEs & Mon $8 / 4 / 03$ & Mon 8/4/03 \\
\hline 15 & $\checkmark$ & Schedule the core NIPSCO team commitments & Mon $8 / 4 / 03$ & Mon 8/4/03 \\
\hline 16 & $\checkmark$ & Create initial project org chart & Mon $8 / 25 / 03$ & Mon $8 / 25 / 03$ \\
\hline 17 & $\checkmark$ & Create project contact list & Tue $8 / 26 / 03$ & Tue $8 / 26 / 03$ \\
\hline 18 & $\checkmark$ & Plan team training & Thu $8 / 7 / 03$ & Fri 8/8/03 \\
\hline 19 & $\checkmark$ & Setup Elaboration Development Environment & Mon $7 / 21 / 03$ & Thu 10/16/03 \\
\hline 20 & $\checkmark$ & Investigate hardware requirements & Mon $7 / 21 / 03$ & Mon $7 / 21 / 03$ \\
\hline 21 & $\checkmark$ & Obtain hardware & Fri 8/22/03 & Mon $8 / 25 / 03$ \\
\hline 22 & $\checkmark$ & Install hardware & Mon $9 / 8 / 03$ & Wed $9 / 10 / 03$ \\
\hline 23 & $\checkmark$ & Investigate software requirements & Mon $7 / 21 / 03$ & Mon $7 / 21 / 03$ \\
\hline 24 & $\checkmark$ & Obtain software & Fri 8/1/03 & Tue $8 / 5 / 03$ \\
\hline 25 & $\checkmark$ & Install SourceSafe software & Wed $10 / 8 / 03$ & Wed $10 / 8 / 03$ \\
\hline 26 & $\checkmark$ & SDE Server Software & Wed 9/17/03 & Thu $10 / 9 / 03$ \\
\hline 27 & $\checkmark$ & Install SQLServer Enterprise Edition 8.00.760 SP3 & Wed $9 / 17 / 03$ & Wed $9 / 17 / 03$ \\
\hline 28 & $\checkmark$ & Install geodatabase for ArcFM & Fri $9 / 19 / 03$ & Fri $9 / 19 / 03$ \\
\hline 29 & $\checkmark$ & Install ArcGIS 8.3 SP1 Map \& Catalog & Thu $9 / 18 / 03$ & Thu 9/18/03 \\
\hline 30 & $\checkmark$ & Install ArcView 8.3 SP1 Map \& Catalog & Thu $9 / 18 / 03$ & Thu 9/18/03 \\
\hline 31 & $\checkmark$ & Install ArcReader 8.3 SP1 & Thu $9 / 18 / 03$ & Thu 9/18/03 \\
\hline 32 & $\checkmark$ & Move FlexLM to SDE server & Thu $10 / 9 / 03$ & Thu 10/9/03 \\
\hline 33 & $\checkmark$ & Citrix Server Software & Thu 9/18/03 & Fri $9 / 19 / 03$ \\
\hline 34 & $\checkmark$ & Install ArcGIS 8.3 SP1 Map \& Catalog & Thu $9 / 18 / 03$ & Thu $9 / 18 / 03$ \\
\hline 35 & $\checkmark$ & Install ArcView 8.3 SP1 Map \& Catalog & Thu $9 / 18 / 03$ & Thu 9/18/03 \\
\hline 36 & $\checkmark$ & Install ArcReader 8.3 SP1 & Thu $9 / 18 / 03$ & Thu 9/18/03 \\
\hline 37 & $\checkmark$ & Install ArcFM 8.3.1 & Fri $9 / 19 / 03$ & Fri $9 / 19 / 03$ \\
\hline 38 & $\checkmark$ & Test the installation \& uninstallation of ArcFM Designer 8.3.1 & Fri 9/19/03 & Fri $9 / 19 / 03$ \\
\hline 39 & $\checkmark$ & Desktop Installations & Thu $9 / 18 / 03$ & Fri 9/26/03 \\
\hline 40 & $\checkmark$ & Install ArcGIS 8.3 SP1 Map \& Catalog & Thu $9 / 18 / 03$ & Thu $9 / 18 / 03$ \\
\hline 41 & $\checkmark$ & Install ArcFM 8.3.1 & Fri 9/19/03 & Fri 9/19/03 \\
\hline
\end{tabular}


NIPSCO GIS Inception and Elaboration Project Plan

\begin{tabular}{|c|c|c|c|c|}
\hline ID & (i) & Task Name & Start & Finish \\
\hline 42 & $\checkmark$ & Install ArcFM 8.3.1 Designer on 3 of the elaboration desktops & Fri 9/19/03 & Fri 9/19/03 \\
\hline 43 & $\checkmark$ & Install ArcFM 8.3.1 on project team desktops & Mon $9 / 22 / 03$ & Fri 9/26/03 \\
\hline 44 & $\checkmark$ & Install starter database that ESRI created for the RFP demo & Tue $10 / 14 / 03$ & Tue $10 / 14 / 03$ \\
\hline 45 & $\checkmark$ & Set up the development, test environments & Tue $10 / 14 / 03$ & Thu $10 / 16 / 03$ \\
\hline 46 & $\checkmark$ & Create Development Environment Report & Mon 9/29/03 & Fri $10 / 3 / 03$ \\
\hline 47 & $\checkmark$ & Setup Document Collaboration & Mon $9 / 29 / 03$ & Mon 10/6/03 \\
\hline 48 & $\checkmark$ & Review Lotus QuickPlace as potential collaboration tool & Mon 9/29/03 & Mon 9/29/03 \\
\hline 49 & $\checkmark$ & Make decision on usefulness & Mon $10 / 6 / 03$ & Mon 10/6/03 \\
\hline 50 & $\checkmark$ & Inception Phase Kickoff & Wed $8 / 13 / 03$ & Wed 9/24/03 \\
\hline 51 & $\checkmark$ & Prep work for the conference call & Wed 8/13/03 & Wed 8/13/03 \\
\hline 52 & $\checkmark$ & Pre-inception conference call & Wed $8 / 13 / 03$ & Wed 8/13/03 \\
\hline 53 & $\checkmark$ & Prep work for the inception phase kickoff meeting & Wed $8 / 13 / 03$ & Thu 8/14/03 \\
\hline 54 & $\checkmark$ & Participate in the inception phase kickoff meeting & Thu 8/14/03 & Wed 8/20/03 \\
\hline 55 & $\checkmark$ & Document action items & Wed $8 / 20 / 03$ & Wed 8/20/03 \\
\hline 56 & $\checkmark$ & Schedule additional Inception Phase meetings & Wed $8 / 20 / 03$ & Wed 8/20/03 \\
\hline 57 & $\checkmark$ & Revise project plan based on results of the kickoff meeting & Tue $8 / 26 / 03$ & Wed 9/24/03 \\
\hline 58 & $\checkmark$ & Prepare the risk spreadsheet & Wed $8 / 6 / 03$ & Wed 9/24/03 \\
\hline 59 & $\checkmark$ & Create initial draft of the risk spreadsheet & Wed $8 / 6 / 03$ & Thu $8 / 7 / 03$ \\
\hline 60 & $\checkmark$ & Refine / revise / publish the risk spreadsheet (on-going) & Fri 8/8/03 & Wed 9/24/03 \\
\hline 61 & $\checkmark$ & Prepare QA plan & Tue $9 / 23 / 03$ & Wed 10/15/03 \\
\hline 62 & $\checkmark$ & Fill out the RUP template & Tue $9 / 23 / 03$ & Fri 9/26/03 \\
\hline 63 & $\checkmark$ & Review the document & Fri 9/26/03 & Mon 10/6/03 \\
\hline 64 & $\checkmark$ & Finalize the document & Mon 10/6/03 & Wed $10 / 15 / 03$ \\
\hline 65 & $\checkmark$ & Develop Sub-contractor Management Plan & Mon $9 / 22 / 03$ & Fri $9 / 26 / 03$ \\
\hline 66 & $\checkmark$ & Define organization & Mon $9 / 22 / 03$ & Mon 9/22/03 \\
\hline 67 & $\checkmark$ & Define methods for sub-contract management & Tue $9 / 23 / 03$ & Thu 9/25/03 \\
\hline 68 & $\checkmark$ & Define acceptance process & Fri 9/26/03 & Fri 9/26/03 \\
\hline 69 & $\checkmark$ & Capture System Details & Mon $9 / 22 / 03$ & Thu 10/2/03 \\
\hline 70 & $\checkmark$ & Create Project Encyclopedia & Mon $9 / 22 / 03$ & Fri 9/26/03 \\
\hline 71 & $\checkmark$ & Load existing requirements & Mon $9 / 22 / 03$ & Tue 9/23/03 \\
\hline 72 & $\checkmark$ & Load actor details & Tue $9 / 23 / 03$ & Wed 9/24/03 \\
\hline 73 & $\checkmark$ & Load system details & Wed $9 / 24 / 03$ & Wed 9/24/03 \\
\hline 74 & $\checkmark$ & Load interface details & Thu $9 / 25 / 03$ & Thu 9/25/03 \\
\hline 75 & $\checkmark$ & Load data store details & Fri 9/26/03 & Fri 9/26/03 \\
\hline 76 & $\checkmark$ & Load project org chart & Fri 9/26/03 & Fri 9/26/03 \\
\hline 77 & $\checkmark$ & Review information that has been gathered & Wed $10 / 1 / 03$ & Wed 10/1/03 \\
\hline 78 & $\checkmark$ & Make adjustments if needed & Wed $10 / 1 / 03$ & Thu 10/2/03 \\
\hline 79 & $\checkmark$ & Define Elaboration Phase Project Organization and Staffing & Tue $9 / 9 / 03$ & Mon $10 / 6 / 03$ \\
\hline 80 & $\checkmark$ & Evaluate staffing requirements based on scope & Tue 9/9/03 & Mon $10 / 6 / 03$ \\
\hline 81 & $\checkmark$ & Assign teams for each component & Thu $9 / 11 / 03$ & Wed 9/24/03 \\
\hline 82 & $\checkmark$ & Revise project org chart & Wed 9/17/03 & Wed 9/17/03 \\
\hline
\end{tabular}




\begin{tabular}{|c|c|c|c|c|c|}
\hline \multicolumn{6}{|c|}{ NIPSCO GIS Inception and Elaboration Project Plan } \\
\hline ID & ( & Task Name & Start & Finish & \\
\hline 83 & $\checkmark$ & Send out the org chart / review / revise (on-going) & Mon 9/22/03 & Mon 9/22/03 & \\
\hline 84 & $\checkmark$ & Secure commitments on project resources for the Elaboration Phase & Fri 10/3/03 & Fri 10/3/03 & \\
\hline 85 & $\checkmark$ & Miscellaneous Prep Tasks & Wed 9/17/03 & Tue 9/30/03 & \\
\hline 86 & $\checkmark$ & Gather all data sources & Wed 9/17/03 & Fri 9/19/03 & \\
\hline 87 & $\checkmark$ & Create agenda for demonstration of existing system & Tue 9/30/03 & Tue 9/30/03 & \\
\hline 88 & $\checkmark$ & Conduct Team Training & Mon 9/22/03 & Fri 10/3/03 & \\
\hline 89 & $\checkmark$ & ArcGIS I and II training & Mon 9/22/03 & Fri 9/26/03 & \\
\hline 90 & $\checkmark$ & ArcFM training & Mon 9/29/03 & Fri 10/3/03 & \\
\hline 91 & $\checkmark$ & Review Inception Phase Results & Fri 9/26/03 & Fri 9/26/03 & \\
\hline 92 & $\checkmark$ & Review estimated Elaboration schedule and cost & Fri 9/26/03 & Fri 9/26/03 & \\
\hline 93 & 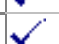 & Go / No-Go Decision & Fri 9/26/03 & Fri 9/26/03 & \\
\hline 94 & $\checkmark$ & Elaboration Phase & Tue $10 / 7 / 03$ & Fri 12/19/03 & \\
\hline 95 & $\checkmark$ & Data Modeling (4 weeks) & Tue $10 / 7 / 03$ & Mon 11/10/03 & \\
\hline 96 & $\checkmark$ & Land Data Modeling & Tue $10 / 7 / 03$ & Fri 10/10/03 & \\
\hline 97 & $\checkmark$ & Data modeling overview & Tue $10 / 7 / 03$ & Tue $10 / 7 / 03$ & \\
\hline 98 & $\checkmark$ & Land Data modeling workshop & Tue $10 / 7 / 03$ & Fri 10/10/03 & \\
\hline 99 & $\checkmark$ & Associations & Fri 10/10/03 & Fri 10/10/03 & \\
\hline 100 & $\checkmark$ & Full Team Kickoff Tasks & Mon 10/13/03 & Thu 10/16/03 & \\
\hline 101 & $\checkmark$ & Demos of the existing system & Mon 10/13/03 & Mon 10/13/03 & \\
\hline 102 & $\checkmark$ & EDFS demo & Mon 10/13/03 & Mon 10/13/03 & \\
\hline 103 & $\checkmark$ & Outfield demo & Mon 10/13/03 & Mon 10/13/03 & \\
\hline 104 & $\checkmark$ & Infobuilder, MapGuide demo & Mon 10/13/03 & Mon 10/13/03 & \\
\hline 105 & $\checkmark$ & Training / Overviews & Mon 10/13/03 & Tue 10/14/03 & \\
\hline 106 & $\checkmark$ & Hold Multi-user Versioning \& Designer workshop & Mon 10/13/03 & Mon 10/13/03 & \\
\hline 107 & $\checkmark$ & ESRI Data Modeling/Migration discussion & Tue $10 / 14 / 03$ & Tue $10 / 14 / 03$ & \\
\hline 108 & $\checkmark$ & SourceSafe training & Tue $10 / 14 / 03$ & Tue $10 / 14 / 03$ & \\
\hline 109 & $\checkmark$ & Collaboration tool training & Tue $10 / 14 / 03$ & Tue $10 / 14 / 03$ & \\
\hline 110 & $\checkmark$ & RUP overview & Tue $10 / 14 / 03$ & Tue $10 / 14 / 03$ & \\
\hline 111 & $\checkmark$ & General Data Model Interface Workshop & Wed 10/15/03 & Thu 10/16/03 & \\
\hline 112 & $\checkmark$ & Facility Browser Interface & Wed 10/15/03 & Wed 10/15/03 & \\
\hline 113 & $\checkmark$ & Field Browser Interface & Wed 10/15/03 & Wed 10/15/03 & \\
\hline 114 & $\checkmark$ & Autodesk Map Interface & Wed 10/15/03 & Wed 10/15/03 & \\
\hline 115 & $\checkmark$ & InfoBuilder Interface & Wed 10/15/03 & Wed 10/15/03 & \\
\hline 116 & $\sqrt{2}$ & $\mathrm{CIS}$ & Thu $10 / 16 / 03$ & Thu $10 / 16 / 03$ & \\
\hline 117 & $\checkmark$ & GPS & Thu $10 / 16 / 03$ & Thu 10/16/03 & \\
\hline 118 & $\checkmark$ & Electric Data Modeling & Thu 10/16/03 & Tue 10/28/03 & \\
\hline 119 & $\checkmark$ & Electric Data Modeling Workshop & Thu 10/16/03 & Thu 10/23/03 & \\
\hline 120 & $\checkmark$ & Electric data model general workshops & Thu 10/16/03 & Wed 10/22/03 & \\
\hline 121 & $\checkmark$ & EDFS data modeling issues & Thu $10 / 23 / 03$ & Thu 10/23/03 & \\
\hline 122 & $\checkmark$ & GM\&T data model issues & Fri 10/24/03 & Fri 10/24/03 & \\
\hline 123 & $\checkmark$ & Electric Data Model Interface Workshops & Mon 10/27/03 & Tue $10 / 28 / 03$ & \\
\hline & & & & & Fri 8/17/07 \\
\hline
\end{tabular}




\begin{tabular}{|c|c|c|c|c|c|}
\hline \multicolumn{6}{|c|}{ NIPSCO GIS Inception and Elaboration Project Plan } \\
\hline ID & (a) & Task Name & Start & Finish & \\
\hline 124 & $\checkmark$ & CADOPS Interface & Mon 10/27/03 & Mon 10/27/03 & \\
\hline 125 & $\checkmark$ & Feederall Interface & Mon 10/27/03 & Mon 10/27/03 & \\
\hline 126 & $\checkmark$ & IRTH Interface & Tue $10 / 28 / 03$ & Tue $10 / 28 / 03$ & \\
\hline 127 & $\checkmark$ & EAIF Interface & Tue $10 / 28 / 03$ & Tue $10 / 28 / 03$ & \\
\hline 128 & $\checkmark$ & MAPPS & Tue $10 / 28 / 03$ & Tue $10 / 28 / 03$ & \\
\hline 129 & $\checkmark$ & Walker General Ledger & Tue $10 / 28 / 03$ & Tue $10 / 28 / 03$ & \\
\hline 130 & $\checkmark$ & MLOG & Tue $10 / 28 / 03$ & Tue $10 / 28 / 03$ & \\
\hline 131 & $\checkmark$ & Gas Data Modeling & Wed 10/29/03 & Tue $11 / 4 / 03$ & \\
\hline 132 & $\checkmark$ & Gas data model - general workshop & Wed 10/29/03 & Mon 11/3/03 & \\
\hline 133 & $\checkmark$ & Gas Data Model Interface Workshops & Tue 11/4/03 & Tue $11 / 4 / 03$ & \\
\hline 134 & $\checkmark$ & Stoner & Tue $11 / 4 / 03$ & Tue $11 / 4 / 03$ & \\
\hline 135 & $\checkmark$ & Cathodic Protection/Corrosion Control & Tue $11 / 4 / 03$ & Tue $11 / 4 / 03$ & \\
\hline 136 & $\checkmark$ & Gas OMS & Tue $11 / 4 / 03$ & Tue $11 / 4 / 03$ & \\
\hline 137 & $\checkmark$ & Finish the Remaining Data Model Tasks & Wed 11/5/03 & Mon 11/10/03 & \\
\hline 138 & $\checkmark$ & Finish Remaining Landbase Data Modeling & Wed 11/5/03 & Thu 11/6/03 & \\
\hline 139 & $\checkmark$ & Land data model - Logical \& Physical model completion & Wed $11 / 5 / 03$ & Wed 11/5/03 & \\
\hline 140 & $\checkmark$ & Land data model - Construction phase estimates & Thu 11/6/03 & Thu $11 / 6 / 03$ & \\
\hline 141 & $\checkmark$ & Finish Remaining Electric Data Modeling & Wed 11/5/03 & Mon 11/10/03 & \\
\hline 142 & $\checkmark$ & Electric data model - Logical \& Physical model completion & Wed $11 / 5 / 03$ & Mon 11/10/03 & \\
\hline 143 & $\checkmark$ & Electric data model - Construction phase estimates & Mon 11/10/03 & Mon 11/10/03 & \\
\hline 144 & $\checkmark$ & Finish Remaining Gas Data Modeling & Wed 11/5/03 & Mon 11/10/03 & \\
\hline 145 & $\checkmark$ & Gas data model - Logical \& Physical model completion & Wed $11 / 5 / 03$ & Mon 11/10/03 & \\
\hline 146 & $\checkmark$ & Gas data model - Construction phase estimates & Mon 11/10/03 & Mon 11/10/03 & \\
\hline 147 & $\checkmark$ & Production Planning Hardware / Environment Architecture & Tue 11/4/03 & Fri $11 / 7 / 03$ & \\
\hline 148 & $\checkmark$ & Hardware configuration evaluation & Tue $11 / 4 / 03$ & Wed 11/5/03 & \\
\hline 149 & $\checkmark$ & Create system architecture blueprint & Wed $11 / 5 / 03$ & Thu 11/6/03 & \\
\hline 150 & $\checkmark$ & Estimate construction phase tasks & Thu 11/6/03 & Fri 11/7/03 & \\
\hline 151 & $\checkmark$ & Data Cleanup Tasks (3 weeks) & Mon 11/10/03 & Fri 12/5/03 & \\
\hline 152 & $\checkmark$ & Setup Tasks & Mon 11/10/03 & Tue 11/11/03 & \\
\hline 153 & $\checkmark$ & Establish test data / area & Mon 11/10/03 & Mon 11/10/03 & \\
\hline 154 & $\checkmark$ & Data cleanup approach / plan & Tue 11/11/03 & Tue 11/11/03 & \\
\hline 155 & $\checkmark$ & Corrosion Control Numbering & Tue 11/11/03 & Wed 11/12/03 & \\
\hline 156 & $\checkmark$ & Pre-migration cleanup & Tue 11/11/03 & Tue 11/11/03 & \\
\hline 157 & $\checkmark$ & Meet with user & Wed 11/12/03 & Wed 11/12/03 & \\
\hline 158 & $\checkmark$ & Valid Values (Parallel Task 1) & Wed 11/12/03 & Fri 11/14/03 & \\
\hline 159 & $\checkmark$ & List all known Outfield and EDFS attribute issues & Wed 11/12/03 & Thu 11/13/03 & \\
\hline 160 & $\checkmark$ & SQL against Outfield and EDFS to complete remaining issues list & Thu 11/13/03 & Thu 11/13/03 & \\
\hline 161 & $\checkmark$ & Determine transmission valid values & Fri 11/14/03 & Fri $11 / 14 / 03$ & \\
\hline 162 & $\checkmark$ & Positional Accuracy (Parallel Task 2) & Tue 11/11/03 & Thu 11/13/03 & \\
\hline 163 & $\checkmark$ & List all known positional accuracy issues & Tue $11 / 11 / 03$ & Tue $11 / 11 / 03$ & \\
\hline 164 & $\checkmark$ & SQL against Outfield and EDFS to determine remaining issues list & Wed 11/12/03 & Wed 11/12/03 & \\
\hline & & Pag & & & Fri 8/17/07 \\
\hline
\end{tabular}


NIPSCO GIS Inception and Elaboration Project Plan

\begin{tabular}{|c|c|c|c|c|}
\hline ID & (i) & Task Name & Start & Finish \\
\hline 165 & $\checkmark$ & Present results to entire team & Thu $11 / 13 / 03$ & Thu $11 / 13 / 03$ \\
\hline 166 & $\checkmark$ & Connectivity (Parallel Task 3) & Tue 11/11/03 & Wed 11/12/03 \\
\hline 167 & $\checkmark$ & List all known Outfield connectivity issues & Tue 11/11/03 & Tue $11 / 11 / 03$ \\
\hline 168 & $\checkmark$ & SQL against Outfield to complete remaining issues list & Wed $11 / 12 / 03$ & Wed $11 / 12 / 03$ \\
\hline 169 & $\checkmark$ & GPS & Thu 11/13/03 & Thu 11/13/03 \\
\hline 170 & $\checkmark$ & Identify all known GPS points & Thu $11 / 13 / 03$ & Thu 11/13/03 \\
\hline 171 & $\checkmark$ & Decision process & Thu 11/13/03 & Thu $11 / 13 / 03$ \\
\hline 172 & 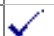 & Key fields & Fri 11/14/03 & Mon 12/1/03 \\
\hline 173 & 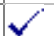 & Estimate remaining pole cleanup & Fri $11 / 14 / 03$ & Fri 11/14/03 \\
\hline 174 & $\checkmark$ & Estimate transformer cleanup & Fri $11 / 14 / 03$ & Fri 11/14/03 \\
\hline 175 & $\checkmark$ & Estimate padmount cleanup & Mon 11/17/03 & Mon 11/17/03 \\
\hline 176 & $\checkmark$ & Estimate remaining corrosion control section connectivity cleanup & Mon 11/17/03 & Mon 11/17/03 \\
\hline 177 & 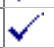 & Discuss how to deal with secondary network data (on linen). & Tue 11/18/03 & Tue $11 / 18 / 03$ \\
\hline 178 & $\checkmark$ & Planning / design of secondary prototype. Review data, etc & Tue $11 / 18 / 03$ & Tue $11 / 18 / 03$ \\
\hline 179 & $\checkmark$ & Present results to entire team & Wed $11 / 19 / 03$ & Wed 11/19/03 \\
\hline 180 & 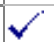 & Prototype conversion of some secondary data & Wed $11 / 19 / 03$ & Mon 12/1/03 \\
\hline 181 & $\checkmark$ & Run Standard ESRI/M\&M Validation Tools (Dependency on Database) & Tue 12/2/03 & Thu 12/4/03 \\
\hline 182 & $\checkmark$ & Run the scripts/tools & Tue 12/2/03 & Tue $12 / 2 / 03$ \\
\hline 183 & $\checkmark$ & Review gas results (parallel) & Tue 12/2/03 & Tue $12 / 2 / 03$ \\
\hline 184 & $\checkmark$ & Review electric results (parallel) & Wed $12 / 3 / 03$ & Wed $12 / 3 / 03$ \\
\hline 185 & $\checkmark$ & Review land results (parallel) & Thu $12 / 4 / 03$ & Thu $12 / 4 / 03$ \\
\hline 186 & & Estimate construction phase data cleanup tasks & Fri 12/5/03 & Fri 12/5/03 \\
\hline 187 & $\checkmark$ & Develop the Data Rectification RFP & Fri $12 / 5 / 03$ & Fri $12 / 5 / 03$ \\
\hline 188 & $\checkmark$ & Data Migration / Conversion Tasks ( 3 weeks) & Fri $10 / 17 / 03$ & Fri 11/21/03 \\
\hline 189 & $V$ & Prepare overview of data migration approach & Fri 10/17/03 & Fri $10 / 17 / 03$ \\
\hline 190 & $\checkmark$ & Complete overview of data migration approach & Mon 11/10/03 & Mon 11/10/03 \\
\hline 191 & 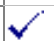 & Prepare rules for RFP & Tue 11/11/03 & Tue $11 / 11 / 03$ \\
\hline 192 & $\checkmark$ & Development work (actual migration tests) & Mon 11/10/03 & Fri 11/21/03 \\
\hline 193 & $\checkmark$ & Present results to entire team & Thu $11 / 13 / 03$ & Thu $11 / 13 / 03$ \\
\hline 194 & $\checkmark$ & Document the results (Migration Plan) & Fri $11 / 21 / 03$ & Fri $11 / 21 / 03$ \\
\hline 195 & $\checkmark$ & Develop the RFP for data migration & Fri 11/21/03 & Fri 11/21/03 \\
\hline 196 & $\checkmark$ & Performance Tasks & Mon 11/10/03 & Thu 11/20/03 \\
\hline 197 & $\checkmark$ & Develop performance testing plan & Mon 11/10/03 & Tue $11 / 11 / 03$ \\
\hline 198 & $\checkmark$ & Plan for production-like testing environment & Mon 11/17/03 & Mon 11/17/03 \\
\hline 199 & $\checkmark$ & Establish environment and run tests & Mon 11/17/03 & Thu $11 / 20 / 03$ \\
\hline 200 & $\checkmark$ & Estimate construction phase performance tasks & Thu $11 / 20 / 03$ & Thu $11 / 20 / 03$ \\
\hline 201 & $\checkmark$ & ArcGIS I ArcFM Configuration (2 weeks) & Fri 11/21/03 & Fri 11/21/03 \\
\hline 202 & $\checkmark$ & Complete overview of configuration approach & Fri 11/21/03 & Fri 11/21/03 \\
\hline 203 & $\checkmark$ & Review overview of configuration approach & Fri 11/21/03 & Fri 11/21/03 \\
\hline 204 & $\checkmark$ & Discuss the "Go / No-Go" Decision & Fri 12/19/03 & Fri 12/19/03 \\
\hline
\end{tabular}


Appendix A-2

AEDR Construction Phase I Project Plan 


\begin{tabular}{|c|c|c|c|c|c|}
\hline \multicolumn{6}{|c|}{ AEDR Construction Phase I Project Plan } \\
\hline ID & \multicolumn{2}{|r|}{ Task Name } & Start & Finish & \\
\hline 1 & \multicolumn{2}{|r|}{ NIPSCO AEDR - Phase 1} & Mon 3/1/04 & Wed 5/2/07 & \\
\hline 2 & \multicolumn{2}{|r|}{ General Project Tasks } & Mon 3/1/04 & Mon 6/12/06 & \\
\hline 3 & \multicolumn{2}{|r|}{ Project Management Tasks } & Mon 3/1/04 & Tue 11/1/05 & \\
\hline 4 & \multirow{2}{*}{\multicolumn{2}{|c|}{$\begin{array}{l}\text { Project Management Tasks } \\
\text { Miscellaneous Project Tasks }\end{array}$}} & Mon 3/1/04 & Tue $11 / 1 / 05$ & \\
\hline 5 & & & Mon 3/1/04 & Mon 6/12/06 & \\
\hline 6 & \multicolumn{2}{|r|}{ General team meetings / general communication tasks } & Mon 3/1/04 & Mon 6/12/06 & \\
\hline 7 & \multicolumn{2}{|r|}{ Elementool administration } & Mon 3/1/04 & Mon 6/12/06 & \\
\hline 8 & \multirow{2}{*}{\multicolumn{2}{|c|}{$\begin{array}{l}\text { Review / Mitigate Risks } \\
\text { Risk List Review Coordination }\end{array}$}} & Wed 6/9/04 & Thu $1 / 27 / 05$ & \\
\hline 9 & & & Wed 6/9/04 & Wed $6 / 30 / 04$ & \\
\hline 10 & \multicolumn{2}{|r|}{$\begin{array}{l}\text { Risk List Review Coordination } \\
\text { Establish draft schedule for reviewing / mitigating risks }\end{array}$} & Wed 6/9/04 & Wed 6/9/04 & \\
\hline 11 & \multicolumn{2}{|r|}{ Review and finalize the risk meeting / mitigation schedule } & Tue 6/15/04 & Tue 6/15/04 & \\
\hline 12 & \multicolumn{2}{|r|}{ Establish initial procedures for risk list reviews } & Wed 6/30/04 & Wed 6/30/04 & \\
\hline 13 & \multicolumn{2}{|r|}{ Risk Reviews - Repeated Until Risks are Mitigated } & Tue 6/15/04 & Thu $1 / 27 / 05$ & \\
\hline 14 & \multicolumn{2}{|r|}{ Technology review committee data migration/rectification risks } & Tue $6 / 15 / 04$ & Tue 6/15/04 & \\
\hline 15 & \multirow{2}{*}{\multicolumn{2}{|c|}{$\begin{array}{l}\text { Technology review committee data migration/rectification risks } \\
\text { Scope creep risks }\end{array}$}} & Fri 8/20/04 & Fri 8/20/04 & \\
\hline 16 & & & Thu $6 / 24 / 04$ & Thu 6/24/04 & \\
\hline 17 & \multicolumn{2}{|r|}{ Internal team risks } & Thu $6 / 24 / 04$ & Thu 6/24/04 & \\
\hline 18 & \multicolumn{2}{|r|}{ Current production support risks } & Thu 6/24/04 & Thu 6/24/04 & \\
\hline 19 & \multicolumn{2}{|r|}{ Resource dependency risks } & Fri 6/25/04 & Fri 6/25/04 & \\
\hline 20 & \multicolumn{2}{|r|}{ Application usability risks } & Wed 6/30/04 & Wed 6/30/04 & \\
\hline 21 & \multicolumn{2}{|r|}{ Quality risks } & Wed 6/30/04 & Wed 6/30/04 & \\
\hline 22 & \multicolumn{2}{|r|}{ Data maintenance process risks } & Thu $7 / 15 / 04$ & Thu $7 / 15 / 04$ & \\
\hline 23 & \multicolumn{2}{|r|}{ Test data risks } & Fri $7 / 23 / 04$ & Fri $7 / 23 / 04$ & \\
\hline 24 & $\checkmark$ & Field Browser risks & Wed 8/18/04 & Wed 8/18/04 & \\
\hline 25 & $\checkmark$ & Data migration / rectification risks & Wed 6/16/04 & Wed 6/16/04 & \\
\hline 26 & $\checkmark$ & Data migration / rectification risks & Fri 7/23/04 & Fri $7 / 23 / 04$ & \\
\hline 27 & $\checkmark$ & Annotation risks & Fri 7/16/04 & Fri 7/16/04 & \\
\hline 28 & $\checkmark$ & $\mathrm{CH}$ integration risks & Tue $7 / 27 / 04$ & Tue $7 / 27 / 04$ & \\
\hline 29 & $\checkmark$ & Real time interface risks & Fri $7 / 30 / 04$ & Fri $7 / 30 / 04$ & \\
\hline 30 & $\checkmark$ & Data cleanup risks & Wed 9/1/04 & Wed 9/1/04 & \\
\hline 31 & $\checkmark$ & Data migration / rectification risks & Wed 9/15/04 & Wed 9/15/04 & \\
\hline 32 & $\checkmark$ & Whitespace management risks & Wed 9/15/04 & Wed 9/15/04 & \\
\hline 33 & $\checkmark$ & Data Related Risks & Fri 10/22/04 & Fri $10 / 22 / 04$ & \\
\hline 34 & $\checkmark$ & Annotation risks & Wed 10/27/04 & Wed 10/27/04 & \\
\hline 35 & $\checkmark$ & Pan/zoom performance risks & Wed 10/27/04 & Wed 10/27/04 & \\
\hline 36 & $\checkmark$ & Data related risks & Fri 11/19/04 & Fri 11/19/04 & \\
\hline 37 & $\checkmark$ & General project risks & Mon 11/22/04 & Mon 11/22/04 & \\
\hline 38 & $\checkmark$ & Real time interface risks & Thu $12 / 9 / 04$ & Thu 12/9/04 & \\
\hline 39 & $\checkmark$ & Application startup performance risks & Thu $12 / 9 / 04$ & Thu $12 / 9 / 04$ & \\
\hline 40 & $\checkmark$ & Mapping product risks. & Fri 12/10/04 & Fri 12/10/04 & \\
\hline 41 & $\checkmark$ & $\mathrm{CH}$ integration risks & Fri 12/10/04 & Fri $12 / 10 / 04$ & \\
\hline 42 & $\checkmark$ & Data related risks & Wed $1 / 12 / 05$ & Wed 1/12/05 & \\
\hline 43 & $\checkmark$ & Real time interface risks & Thu $1 / 13 / 05$ & Thu $1 / 13 / 05$ & \\
\hline 44 & $\checkmark$ & Application startup performance risks & Thu $1 / 13 / 05$ & Thu $1 / 13 / 05$ & \\
\hline 45 & $\checkmark$ & Scope creep risks & Fri $1 / 14 / 05$ & Fri $1 / 14 / 05$ & \\
\hline 46 & $\checkmark$ & $\mathrm{CH}$ integration risks & Thu 1/27/05 & Thu $1 / 27 / 05$ & \\
\hline $\begin{array}{ll}\text { NIPS } \\
\text { Confic }\end{array}$ & $\begin{array}{l}\text { Co GIS } \\
\text { lential }\end{array}$ & Page 1 & & $\begin{array}{l}\text { Last Update } \\
\text { Printe }\end{array}$ & $\begin{array}{l}\text { ed on Tue } 7 / 3 / 07 \\
\text { ed on Fri 8/17/07 }\end{array}$ \\
\hline
\end{tabular}




\begin{tabular}{|c|c|c|c|c|c|}
\hline \multicolumn{6}{|c|}{ AEDR Construction Phase I Project Plan } \\
\hline ID & ( & Task Name & Start & Finish & \\
\hline 47 & $\checkmark$ & GIS Data Rectification & Mon 6/7/04 & Fri 1/6/06 & \\
\hline 48 & $\checkmark$ & GIS Data Rectification - Startup Tasks & Mon 6/7/04 & Wed 10/27/04 & \\
\hline 49 & $\checkmark$ & Migration and Rectification Preparation Tasks & Mon 6/7/04 & Thu 7/1/04 & \\
\hline 50 & $\checkmark$ & Review the data migration vendor proposal and update text as needed (for the contract) & Tue 6/8/04 & Thu $6 / 10 / 04$ & \\
\hline 51 & $\checkmark$ & Meeting to review rectification batches / pilot area & Thu 6/10/04 & Thu $6 / 10 / 04$ & \\
\hline 52 & $\checkmark$ & Put together map regarding the batches & Fri 6/11/04 & Fri 6/11/04 & \\
\hline 53 & $\checkmark(1)$ & Miscellaneous planning for the migration/rectification kickoff meeting & Mon 6/7/04 & Fri 6/11/04 & \\
\hline 54 & $\checkmark$ & Answer additional vendor questions & Wed 6/16/04 & Wed 6/16/04 & \\
\hline 55 & $\checkmark$ & Validate the feature counts for the data migration vendor contract & Thu $6 / 17 / 04$ & Thu 6/17/04 & \\
\hline 56 & $\checkmark$ & Meeting to review modeling of transformers / substations & Wed 6/30/04 & Wed 6/30/04 & \\
\hline 57 & $\checkmark$ & Review whitespace management of dual substations & Wed 6/30/04 & Wed $6 / 30 / 04$ & \\
\hline 58 & $\checkmark$ & Make sure that dual substation migration is covered in data migration vendor's SOW & Thu $7 / 1 / 04$ & Thu $7 / 1 / 04$ & \\
\hline 59 & $\checkmark$ & Data Migration and Rectification Contract & Wed 6/23/04 & Mon 8/9/04 & \\
\hline 60 & $\checkmark$ & Add level of effort and cost for optional tasks in contract & Fri 7/2/04 & Tue $7 / 20 / 04$ & \\
\hline 61 & $\checkmark$ & Finalize the data migration vendor contract & Wed 6/23/04 & Mon 8/9/04 & \\
\hline 62 & $\checkmark$ & Data Migration and Rectification Kickoff and Workshops & Mon 6/14/04 & Fri 6/18/04 & \\
\hline 63 & $\checkmark$ & Day 1 Workshops (afternoon only) & Mon $6 / 14 / 04$ & Mon 6/14/04 & \\
\hline 64 & $\checkmark$ & Vendors travel to NIPSCO & Mon 6/14/04 & Mon 6/14/04 & \\
\hline 65 & $\checkmark$ & Data migration and rectification kickoff meeting & Mon 6/14/04 & Mon 6/14/04 & \\
\hline 66 & $\checkmark$ & Review project / review scope of work & Mon 6/14/04 & Mon 6/14/04 & \\
\hline 67 & $\checkmark$ & Define contract expectations & Mon 6/14/04 & Mon 6/14/04 & \\
\hline 68 & $\checkmark$ & Define project administration and communication & Mon 6/14/04 & Mon 6/14/04 & \\
\hline 69 & $\checkmark$ & Day 2 Workshops & Tue 6/15/04 & Tue 6/15/04 & \\
\hline 70 & $\checkmark$ & Review pilot area & Tue $6 / 15 / 04$ & Tue $6 / 15 / 04$ & \\
\hline 71 & $\checkmark$ & Review rectification batches & Tue $6 / 15 / 04$ & Tue 6/15/04 & \\
\hline 72 & $\checkmark$ & Data rectification and migration - miscellaneous issues & Tue 6/15/04 & Tue 6/15/04 & \\
\hline 73 & $\checkmark$ & Outfield / Field Browser / Facility Browser demos & Tue $6 / 15 / 04$ & Tue $6 / 15 / 04$ & \\
\hline 74 & $\checkmark$ & Determine drafting standards / schema & Tue 6/15/04 & Tue 6/15/04 & \\
\hline 75 & $\checkmark$ & Documentation - AutoCAD schema & Tue $6 / 15 / 04$ & Tue $6 / 15 / 04$ & \\
\hline 76 & $\checkmark$ & Day 3 Workshops & Wed 6/16/04 & Wed 6/16/04 & \\
\hline 77 & $\checkmark$ & Data model and ArcFM status / issues & Wed 6/16/04 & Wed 6/16/04 & \\
\hline 78 & $\checkmark$ & EDFS migration strategies & Wed 6/16/04 & Wed 6/16/04 & \\
\hline 79 & $\checkmark$ & Review Source Materials & Wed 6/16/04 & Wed 6/16/04 & \\
\hline 80 & $\checkmark$ & Street light database & Wed 6/16/04 & Wed 6/16/04 & \\
\hline 81 & $\checkmark$ & Customer point data & Wed 6/16/04 & Wed 6/16/04 & \\
\hline 82 & $\checkmark$ & Demonstrate EDFS & Wed 6/16/04 & Wed 6/16/04 & \\
\hline 83 & $\checkmark$ & Outfield migration strategies & Wed 6/16/04 & Wed 6/16/04 & \\
\hline 84 & $\checkmark$ & Data matrix & Wed 6/16/04 & Wed 6/16/04 & \\
\hline 85 & $\checkmark$ & Fuzzy / buffered edges & Wed 6/16/04 & Wed 6/16/04 & \\
\hline 86 & $\checkmark$ & Rectification rules & Wed 6/16/04 & Wed 6/16/04 & \\
\hline 87 & $\checkmark$ & Day 4 Workshops & Thu 6/17/04 & Thu 6/17/04 & \\
\hline 88 & $\checkmark$ & Feature-level migration mapping rules & Thu $6 / 17 / 04$ & Thu $6 / 17 / 04$ & \\
\hline 89 & $\checkmark$ & Demo Elementool & Thu $6 / 17 / 04$ & Thu 6/17/04 & \\
\hline 90 & $\checkmark$ & Make decision on Elementool versus CRS usage for migration / rectification & Thu $6 / 17 / 04$ & Thu 6/17/04 & \\
\hline 91 & $\checkmark$ & Day 5 Workshops & Fri 6/18/04 & Fri 6/18/04 & \\
\hline 92 & $\checkmark$ & Miscellaneous issues / action items & Fri 6/18/04 & Fri 6/18/04 & \\
\hline $\begin{array}{l}\text { NIPS } \\
\text { Confi }\end{array}$ & $\begin{array}{l}\text { Co GIS } \\
\text { lential }\end{array}$ & Page 2 & & $\begin{array}{r}\text { Last Updat } \\
\text { Print }\end{array}$ & $\begin{array}{l}\text { ed on Tue } 7 / 3 / 07 \\
\text { ed on Fri 8/17/07 }\end{array}$ \\
\hline
\end{tabular}




\begin{tabular}{|c|c|c|c|c|c|}
\hline \multicolumn{6}{|c|}{ AEDR Construction Phase I Project Plan } \\
\hline ID & ( & Task Name & Start & Finish & \\
\hline 93 & $\checkmark$ & Get metrics on secondary migration issues & Fri 6/18/04 & Fri 6/18/04 & \\
\hline 94 & $\checkmark$ & Determine how to resolve EDFS secondary migration (graphic generation) & Fri 6/18/04 & Fri 6/18/04 & \\
\hline 95 & $\checkmark$ & Transfer any available sample migration code / migration reports to data migration vendor & Fri 6/18/04 & Fri 6/18/04 & \\
\hline 96 & $\checkmark$ & Resolve the Rectification Tickets from Elementool & Fri $7 / 30 / 04$ & Wed 8/18/04 & \\
\hline 97 & $\checkmark$ & Review and resolve Elementool tickets & Fri 7/30/04 & Wed 8/18/04 & \\
\hline 98 & $\checkmark$ & Establish Data Migration Vendors Project Web Site for NIPSCO & Mon 6/21/04 & Fri 6/25/04 & \\
\hline 99 & $\checkmark$ & Establish NIPSCO web site & Mon 6/21/04 & Fri 6/25/04 & \\
\hline 100 & $\checkmark$ & Establish NIPSCO logins for the web site & Fri 6/25/04 & Fri 6/25/04 & \\
\hline 101 & $\checkmark$ & Define the Delivery Batches for Rectified Data & Mon 6/21/04 & Fri 6/25/04 & \\
\hline 102 & 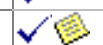 & Establish data rectification batch definition guidelines & Mon 6/21/04 & Mon 6/21/04 & \\
\hline 103 & $\checkmark$ & Create full schedule / map of data rectification batches & Mon 6/21/04 & Wed 6/23/04 & \\
\hline 104 & $\checkmark$ & Send the schedule / map to NIPSCO & Thu 6/24/04 & Thu $6 / 24 / 04$ & \\
\hline 105 & $\checkmark$ & Review the delivery batch schedule / map & Fri 6/25/04 & Fri 6/25/04 & \\
\hline 106 & $\checkmark$ & Define Migration Vendor Delivery QA and Acceptance Procedures & Mon 6/21/04 & Mon 7/12/04 & \\
\hline 107 & $\checkmark$ & Create migration vendor-specific data QA procedures and send to NIPSCO & Mon 6/21/04 & Fri $7 / 2 / 04$ & \\
\hline 108 & $\checkmark$ & Review migration vendor's QA procedures & Fri 7/9/04 & Fri $7 / 9 / 04$ & \\
\hline 109 & $\checkmark$ & Finalize migration vendor-specific data QA procedures & Mon 7/12/04 & Mon 7/12/04 & \\
\hline 110 & $\checkmark$ & Define NIPSCO Data Receipt and Acceptance Procedures & Wed 6/23/04 & Mon 7/12/04 & \\
\hline 111 & $\checkmark$ & Investigate possible data migration vendor's use of QA / QC routines from Outfield and ABB-OUT & Fri 6/25/04 & Fri 6/25/04 & \\
\hline 112 & $\checkmark$ & Gather all info / instructions from QA/QC routines from Outfield and ABB-OUT & Tue 6/29/04 & Fri 7/2/04 & \\
\hline 113 & $\checkmark$ & Create NIPSCO-specific rectification data receipt and acceptance procedures & Wed 6/23/04 & Tue $7 / 6 / 04$ & \\
\hline 114 & $\checkmark$ & Review the NIPSCO-specific rectification data receipt and acceptance procedures & Fri 7/9/04 & Fri $7 / 9 / 04$ & \\
\hline 115 & $\checkmark$ & Revise the NIPSCO-specific rectification data receipt and acceptance procedures & Mon 7/12/04 & Mon 7/12/04 & \\
\hline 116 & $\checkmark$ & Define the Rectification Rules & Thu $7 / 22 / 04$ & Wed 8/4/04 & \\
\hline 117 & $\checkmark$ & Present (or e-mail) rectification rules with examples & Thu $7 / 22 / 04$ & Thu $7 / 22 / 04$ & \\
\hline 118 & $\checkmark$ & Review the rectification rules and send back comments & Wed $7 / 28 / 04$ & Wed $7 / 28 / 04$ & \\
\hline 119 & $\checkmark$ & Update the rectification rules based on review comments & Fri 7/30/04 & Fri 7/30/04 & \\
\hline 120 & $\checkmark$ & Send the revised rectification rules to data migration vendor for review and revision & Fri 7/30/04 & Fri $7 / 30 / 04$ & \\
\hline 121 & $\checkmark$ & Review and revise the rectification rules & Mon 8/2/04 & Tue $8 / 3 / 04$ & \\
\hline 122 & $\checkmark$ & Update, finalize, and approve the rectification rules & Wed $8 / 4 / 04$ & Wed 8/4/04 & \\
\hline 123 & $\checkmark$ & Define Customer Point Rectification Process & Fri 6/18/04 & Tue 6/29/04 & \\
\hline 124 & $\checkmark b$ & Decide on options for freezing customer point data & Fri 6/18/04 & Fri 6/18/04 & \\
\hline 125 & $\checkmark$ & Determine resource / schedule options for rectifying customer point data & Wed 6/23/04 & Wed 6/23/04 & \\
\hline 126 & $\checkmark$ & Prepare draft proposal for customer point data & Thu $6 / 24 / 04$ & Thu 6/24/04 & \\
\hline 127 & $\checkmark$ & Review customer point proposal and determine next steps & Tue 6/29/04 & Tue 6/29/04 & \\
\hline 128 & $\checkmark$ & Miscellaneous Data Rectification Administrative Tasks & Mon 6/21/04 & Fri 8/6/04 & \\
\hline 129 & $\checkmark$ & Get decision on off-site data issues & Fri 8/6/04 & Fri 8/6/04 & \\
\hline 130 & $\checkmark$ & Start rectification pilot project & Mon 6/21/04 & Mon 6/21/04 & \\
\hline 131 & $\checkmark$ & Decide on rectification production schedule change (move up 3 weeks) & Fri 8/6/04 & Fri 8/6/04 & \\
\hline 132 & $\checkmark$ & Define the QC Automation Process & Fri 8/13/04 & Fri 10/1/04 & \\
\hline 133 & $\checkmark$ & Verify automated batch routines for QA/QC & Mon 8/16/04 & Mon 8/16/04 & \\
\hline 134 & $\checkmark$ & Identify QC automation process for feature count & Fri 8/13/04 & Thu 8/19/04 & \\
\hline 135 & $\checkmark$ & Identify QC automation process for DOQQ positional rectification & Fri 8/13/04 & Thu 8/19/04 & \\
\hline 136 & $\checkmark$ & Review automation process options and determine approach & Tue $8 / 24 / 04$ & Tue 8/24/04 & \\
\hline 137 & $\checkmark$ & Determine resources needed for automation process options & Fri 8/27/04 & Fri 8/27/04 & \\
\hline 138 & $\checkmark$ & Document the procedures to deliver \& accept tiles & Fri 10/1/04 & Fri 10/1/04 & \\
\hline $\begin{array}{l}\text { NIPS } \\
\text { Confic }\end{array}$ & $\begin{array}{l}\text { Co GIS } \\
\text { lential }\end{array}$ & Page 3 & & $\begin{array}{r}\text { Last Updat } \\
\text { Print }\end{array}$ & $\begin{array}{l}\text { ed on Tue } 7 / 3 / 07 \\
\text { ed on Fri 8/17/07 }\end{array}$ \\
\hline
\end{tabular}




\section{\begin{tabular}{|ll}
\hline AEDR Construction Phase I Project Plan & AED
\end{tabular}}

\begin{tabular}{|c|c}
\hline ID \\
\hline 139 \\
\hline 140 \\
\hline 141 \\
\hline 142 \\
\hline 143 \\
\hline 144 \\
\hline 145 \\
\hline 146 \\
\hline 147 \\
\hline 148 \\
\hline 149 \\
\hline 150 \\
\hline 151 \\
\hline 152 \\
\hline 153 \\
\hline 154 \\
\hline 155 \\
\hline 156 \\
\hline 157 \\
\hline 158 \\
\hline 159 \\
\hline 160 \\
\hline 161 \\
\hline 162 \\
\hline 163 \\
\hline 164 \\
\hline 165 \\
\hline 166 \\
\hline 167 \\
\hline 168 \\
\hline 169 \\
\hline 170 \\
\hline 171 \\
\hline 172 \\
\hline 173 \\
\hline 174 \\
\hline 175 \\
\hline 176 \\
\hline 177 \\
\hline 178 \\
\hline 179 \\
\hline 180 \\
\hline 181 \\
\hline 183 \\
\hline 184 \\
\hline 119 \\
\hline
\end{tabular}

Task Name

\section{Establish and Train the Data Rectification QA Team}

dentify 4 resources to assist in QA/QC of rectified maps

Verify raster can be displayed with AutoCAD Map software

Outline step-by-step procedures for clerks

Fri 8/13/04

Thu 8/19/04

Wed $8 / 11 / 04$

Outline Outfield QC's with checklist

Tue 8/24/04

Order extra monitors for clerk's desktop computers

Fri 8/27/04

Ensure video cards \& monitors are delivered and installed

Create training documentation for the rectification QA team

Create procedures for communicating rectification rules

Fri 8/27/04

Fri $8 / 27 / 04$

Fri 8/27/04

Document a process with deliverables for communicating errors to data migration vendor

Train the rectification QA record clerks

\section{"Fuzzy Edge" Tasks}

Create prototype drawing file that is re-projected

Test and document the impacts of re-projection / rectification on CADOPS

Test and document the impacts of re-projection / rectification on InfoBuilder

Test and document the impacts of re-projection / rectification on Facility Browser

Test and document the impacts of re-projection / rectification on Field Browser

Test and document the impacts of re-projection / rectification on SynerGEE

Communicate "fuzzy" edge issues to CADOPS, SynerGEE, et al

Review fuzzy edge results and determine next steps

Re-project all tiles - batch \#1 (over the weekend)

Re-project all tiles - batch \#2 (over the weekend)

Process all DOQQ

Re-process InfoBuilder

Review reprojected tiles back from data migration vendor

Place CE, CG, Cl back into production

Change author file for facility browser to accept new coordinate system

Review and process Elementool tickets for $\mathrm{CF}$ and $\mathrm{CH}$

Re-deliver $\mathrm{CF}$ and $\mathrm{CH}$ files

Final $\mathrm{QA}$ of $\mathrm{CF}$ and $\mathrm{CH}$ files

\section{Functional Testing / Debugging / Record Clerk Support}

Review and research Outfield functional issues related to rectification

Establish DWGVerify review procedures

Assist with rectification research issues

Copy DOQQ files to a server closer to project team

\section{Automated Edge Matching}

Automated edgematching QA for migration document review \& meeting

Validate technical assumptions

Create list of entities that need to be edge-matched

Review latest issues associated with edge matching

Fri 8/27/04

Tue $8 / 31 / 04$

Thu 6/24/04

Thu $6 / 24 / 04$

Thu $7 / 1 / 04$

Wed $6 / 30 / 04$

Wed $6 / 30 / 04$

Thu $7 / 1 / 04$

Thu $7 / 1 / 04$

Thu 7/1/04

Fri $7 / 30 / 04$

Fri 9/17/04

Fri 9/24/04

Tue $9 / 28 / 04$

Tue $10 / 5 / 04$

Mon 10/11/04

Wed 10/20/04

Mon 10/25/04

Mon $10 / 25 / 04$

Tue 10/26/04

Wed $10 / 27 / 04$

Mon 11/1/0

Mon 11/1/04

Wed 11/3/04

Wed $11 / 3 / 04$

Mon 11/8/04

Tue 10/19/04

Tue 10/19/04

Fri 10/22/04

Mon $10 / 25 / 04$

Tue $10 / 26 / 04$

Create and test the automated edgematch query

Fri 10/29/04

Thu 10/28/04

Fri 10/29/04

Prepare automated edge match options

Review automated edge match options and determine solution

Create remaining design for automated edge match functionality

Review design for the remaining automated edge match functionaility

Mon 11/1/04

Tue 11/2/04

Wed $11 / 3 / 04$
Mon 8/16/04

Thu $8 / 19 / 04$

Thu 8/19/04

Tue 8/24/04

Fri 8/27/04

Fri 8/27/04

Fri 8/27/04

Fri 8/27/04

Fri 8/27/04

Tue 8/31/04

Wed 10/27/04

Thu 6/24/04

Thu 7/1/04

Wed 6/30/04

Wed 6/30/04

Thu 7/1/04

Thu 7/1/04

Thu 7/1/04

Fri $7 / 30 / 04$

Mon 9/20/04

Mon 9/27/04

Wed 9/29/04

Mon 10/11/04

Mon 10/11/04

Wed 10/20/04

Wed 10/27/04

Mon 10/25/04

Tue 10/26/04

Wed 10/27/04

Fri 11/5/04

Fri 11/5/04

Thu 11/4/04

Wed 11/3/04

Mon 11/15/04

Wed 11/17/04

Tue 10/19/04

Fri 10/22/04

Mon 10/25/04

Tue 10/26/04

Fri 10/29/04

Fri 10/29/04

Fri 10/29/04

Mon 11/1/04

Tue 11/2/04

Wed 11/3/04 


\begin{tabular}{|c|c|c|c|c|c|}
\hline \multicolumn{6}{|c|}{ AEDR Construction Phase I Project Plan } \\
\hline ID & ( & Task Name & Start & Finish & \\
\hline 185 & $\checkmark$ & Approve remaining automated edge match functionality & Wed $11 / 3 / 04$ & Wed 11/3/04 & \\
\hline 186 & $\checkmark$ & Finish the development of automated edge match functionality & Wed $11 / 3 / 04$ & Thu 11/4/04 & \\
\hline 187 & $\checkmark$ & Deliver batch 2 rectified files to vendor for edge match testing & Fri 11/5/04 & Fri 11/5/04 & \\
\hline 188 & $\checkmark$ & Run the edge match QA against the batch 2 files & Mon 11/8/04 & Mon 11/8/04 & \\
\hline 189 & $\checkmark$ & Run the Edge Match application on batch 2 files & Wed 11/10/04 & Wed 11/10/04 & \\
\hline 190 & $\checkmark$ & Validate the automated edge match results & Thu $11 / 11 / 04$ & Thu 11/11/04 & \\
\hline 191 & $\checkmark$ & Apply final updates to the application & Tue $11 / 16 / 04$ & Tue 11/16/04 & \\
\hline 192 & $\checkmark$ & Deliver and install automated edge match code at NIPSCO & Wed 11/17/04 & Wed 11/17/04 & \\
\hline 193 & $\checkmark$ & Rectify Batch 0 (Pilot) - CE, CF, CG, CH, Cl & Mon 6/7/04 & Tue 11/2/04 & \\
\hline 194 & $\checkmark$ & Miscellaneous Pilot Area Tasks & Tue 9/7/04 & Fri 10/22/04 & \\
\hline 195 & $\checkmark$ & Create script to run Outfield QA routines against all DWGs in directory & Tue $9 / 7 / 04$ & Fri 9/10/04 & \\
\hline 196 & $\checkmark$ & Create script to count features for all DWGs in directory & Thu 9/9/04 & Fri 9/10/04 & \\
\hline 197 & $\checkmark$ & Deliver the final versions of the $\mathrm{CF}, \mathrm{CG}, \mathrm{CH}$ and $\mathrm{Cl}$ tiles & Mon 9/13/04 & Mon 9/13/04 & \\
\hline 198 & $\checkmark$ & Finish QA of the CE tiles & Fri 9/10/04 & Fri 9/24/04 & \\
\hline 199 & $\checkmark$ & Test batch scripts against production 1 tiles & Fri 9/10/04 & Fri 9/10/04 & \\
\hline 200 & $\checkmark$ & Install batch scripts at NIPSCO & Fri 10/22/04 & Fri 10/22/04 & \\
\hline 201 & $\checkmark$ & Develop procedures for cross tile workorder issues & Fri 9/17/04 & Fri 9/17/04 & \\
\hline 202 & $\checkmark$ & Finalize and sign-off on the rectification rules & Fri 10/8/04 & Fri 10/8/04 & \\
\hline 203 & $\checkmark$ & Create schedule for $\mathrm{CF}, \mathrm{CG}, \mathrm{CH}$, and $\mathrm{Cl}$ re-QA tasks & Mon 10/4/04 & Mon 10/4/04 & \\
\hline 204 & $\checkmark$ & Re-QA CFNE (1430 features) & Fri 10/15/04 & Tue 10/19/04 & \\
\hline 205 & $\checkmark$ & Re-QA CFNW (3148 features) & Mon 10/18/04 & Wed 10/20/04 & \\
\hline 206 & $\checkmark$ & Re-QA CFSE (1798 features) & Fri 10/22/04 & Fri 10/22/04 & \\
\hline 207 & $\checkmark$ & Re-QA CFSW (1835 features) & Fri 10/22/04 & Fri 10/22/04 & \\
\hline 208 & $\checkmark$ & Re-QA CGNE (705 features) & Wed 10/6/04 & Wed 10/6/04 & \\
\hline 209 & $\checkmark$ & Re-QA CGNW (912 features) & Wed 10/6/04 & Wed 10/6/04 & \\
\hline 210 & $\checkmark$ & Re-QA CGSE (1220 features) & Fri 10/15/04 & Wed 10/20/04 & \\
\hline 211 & $\checkmark$ & Re-QA CGSW (2387 features) & Fri 10/22/04 & Fri $10 / 22 / 04$ & \\
\hline 212 & $\checkmark$ & Re-QA CHNE (4270 features) & Wed 10/6/04 & Wed 10/6/04 & \\
\hline 213 & $\checkmark$ & Re-QA CHNW (2319 features) & Wed 10/6/04 & Wed 10/6/04 & \\
\hline 214 & $\checkmark$ & Re-QA CHSE (771 features) & Mon 10/18/04 & Wed 10/20/04 & \\
\hline 215 & $\checkmark$ & Re-QA CHSW (1377 features) & Mon 10/18/04 & Thu $10 / 21 / 04$ & \\
\hline 216 & $\checkmark$ & Re-QA CINE (571 features) & Wed 10/6/04 & Wed 10/6/04 & \\
\hline 217 & $\checkmark$ & Re-QA CINW (492 features) & Wed 10/6/04 & Wed 10/6/04 & \\
\hline 218 & $\checkmark$ & Re-QA CISE (209 features) & Wed 10/6/04 & Wed 10/6/04 & \\
\hline 219 & $\checkmark$ & Re-QA CISW (315 features) & Wed 10/6/04 & Wed 10/6/04 & \\
\hline 220 & $\checkmark$ & Deliver the final version of $\mathrm{CE}$ & Thu $9 / 30 / 04$ & Thu 9/30/04 & \\
\hline 221 & $\checkmark$ & Re-QA the final version of the CE tile & Wed 10/6/04 & Wed 10/6/04 & \\
\hline 222 & $\checkmark$ & Rectify Pilot Tiles $\mathrm{CH}$ and $\mathrm{Cl}$ & Mon 6/7/04 & Tue 8/10/04 & \\
\hline 223 & $\checkmark$ & Perform Rectification of Pilot Tiles $\mathrm{CH}$ and $\mathrm{Cl}$ & Mon 6/7/04 & Fri 7/9/04 & \\
\hline 224 & $\checkmark$ & Complete workorders in $\mathrm{CH}$ and $\mathrm{Cl}$ & Mon 6/7/04 & Fri 6/18/04 & \\
\hline 225 & $\checkmark$ & Freeze pilot tiles $\mathrm{CH}$ and $\mathrm{Cl}$ & Mon 6/21/04 & Mon 6/21/04 & \\
\hline 226 & $\checkmark$ & Send pilot tiles $\mathrm{CH}$ and $\mathrm{Cl}$ to migration vendor & Mon 6/21/04 & Mon 6/21/04 & \\
\hline 227 & $\checkmark$ & Rectify pilot tiles $\mathrm{CH}$ and $\mathrm{Cl}$ & Mon 6/21/04 & Fri 7/9/04 & \\
\hline 228 & $\checkmark$ & Deliver pilot tiles $\mathrm{CH}$ and $\mathrm{Cl}$ & Fri $7 / 9 / 04$ & Fri $7 / 9 / 04$ & \\
\hline 229 & $\checkmark$ & Perform QA on Pilot Tiles $\mathrm{CH}$ and $\mathrm{Cl}$ & Mon 7/12/04 & Fri 7/23/04 & \\
\hline 230 & $\checkmark$ & General quality checks / updates for pilot tiles $\mathrm{CH}$ and $\mathrm{Cl}$ & Mon $7 / 12 / 04$ & Mon $7 / 19 / 04$ & \\
\hline $\begin{array}{l}\text { NIPSC } \\
\text { Confid }\end{array}$ & $\begin{array}{l}\text { Co GIS } \\
\text { lential }\end{array}$ & Page 5 & & $\begin{array}{r}\text { Last Updat } \\
\text { Print }\end{array}$ & $\begin{array}{l}\text { ed on Tue } 7 / 3 / 07 \\
\text { ed on Fri 8/17/07 }\end{array}$ \\
\hline
\end{tabular}




\begin{tabular}{|c|c|c|c|c|c|}
\hline \multicolumn{6}{|c|}{ AEDR Construction Phase I Project Plan } \\
\hline ID & (a) & Task Name & Start & Finish & \\
\hline 231 & $\checkmark$ & General quality checks of $\mathrm{CH}$ and $\mathrm{Cl}$ pilot tiles & Mon 7/12/04 & Fri $7 / 16 / 04$ & \\
\hline 232 & $\checkmark$ & Check connectivity of pilot tiles $\mathrm{CH}$ and $\mathrm{Cl}$ & Tue $7 / 13 / 04$ & Mon $7 / 19 / 04$ & \\
\hline 233 & $\checkmark$ & Revise NIPSCO data receipt and acceptance procedures & Fri $7 / 23 / 04$ & Fri 7/23/04 & \\
\hline 234 & $\checkmark$ & Correct Pilot Tiles $\mathrm{CH}$ and $\mathrm{Cl}$ Based on QA Comments & Mon $7 / 26 / 04$ & Wed 7/28/04 & \\
\hline 235 & $\checkmark$ & Fix pilot tiles $\mathrm{CH}$ and $\mathrm{Cl}$ based on the QA feedback & Mon $7 / 26 / 04$ & Tue $7 / 27 / 04$ & \\
\hline 236 & $\checkmark$ & Re-deliver pilot tiles $\mathrm{CH}$ and $\mathrm{Cl}$ with fixes based on QA feedback & Wed $7 / 28 / 04$ & Wed $7 / 28 / 04$ & \\
\hline 237 & $\checkmark$ & Re-Test the Re-Delivered $\mathrm{CH}$ and CI Pilot Tiles & Mon 8/2/04 & Tue 8/10/04 & \\
\hline 238 & $\checkmark$ & Re-test pilot tile CH SE & Tue $8 / 3 / 04$ & Tue $8 / 3 / 04$ & \\
\hline 239 & $\checkmark$ & Re-test pilot tile CH SW & Tue 8/10/04 & Tue $8 / 10 / 04$ & \\
\hline 240 & $\checkmark$ & Complete the remaining 2 land scoring spreadsheets & Mon 8/2/04 & Mon 8/2/04 & \\
\hline 241 & $\checkmark$ & Send all scoring spreadsheets to migration vendor & Mon 8/2/04 & Mon 8/2/04 & \\
\hline 242 & $\checkmark$ & Rectify Pilot Tiles CG and CF & Mon 6/7/04 & Thu 8/19/04 & \\
\hline 243 & $\checkmark$ & Perform the Rectification of Pilot Tiles CG and CF & Mon 6/7/04 & Fri $7 / 23 / 04$ & \\
\hline 244 & $\checkmark$ & Complete workorders in CG and CF & Mon 6/7/04 & Thu $7 / 1 / 04$ & \\
\hline 245 & $\checkmark$ & Freeze pilot tiles CG and CF & Fri 7/2/04 & Fri $7 / 2 / 04$ & \\
\hline 246 & $\checkmark$ & Send pilot tiles CG and CF to migration vendor & Fri 7/2/04 & Fri $7 / 2 / 04$ & \\
\hline 247 & $\checkmark$ & Rectify pilot tiles CG and CF & Fri 7/2/04 & Fri $7 / 23 / 04$ & \\
\hline 248 & $\checkmark$ & Deliver pilot tiles CG and CF & Fri 7/23/04 & Fri $7 / 23 / 04$ & \\
\hline 249 & $\checkmark$ & Perform QA on Pilot Tiles CG and CF & Tue $7 / 27 / 04$ & Thu 8/12/04 & \\
\hline 250 & $\checkmark$ & Review and score CF NW & Thu $7 / 29 / 04$ & Thu $7 / 29 / 04$ & \\
\hline 251 & $\checkmark$ & Review and score CF NE & Tue 8/10/04 & Tue 8/10/04 & \\
\hline 252 & $\checkmark$ & Review and score CF SW & Wed 8/11/04 & Wed 8/11/04 & \\
\hline 253 & $\checkmark$ & Review and score CF SE & Thu 8/12/04 & Thu 8/12/04 & \\
\hline 254 & $\checkmark$ & Review and score CG NW & Tue $7 / 27 / 04$ & Tue $7 / 27 / 04$ & \\
\hline 255 & $\checkmark$ & Review and score CG SW & Wed $7 / 28 / 04$ & Wed $7 / 28 / 04$ & \\
\hline 256 & $\checkmark$ & Review and score CG NE & Thu 8/5/04 & Thu 8/5/04 & \\
\hline 257 & $\checkmark$ & Review and score CG SE & Fri 8/6/04 & Fri 8/6/04 & \\
\hline 258 & $\checkmark$ & Peer Review / Cross-Checks for CF and CG & Mon 8/9/04 & Thu 8/19/04 & \\
\hline 259 & $\checkmark$ & Peer review of CF NW & Tue 8/10/04 & Wed 8/11/04 & \\
\hline 260 & $\checkmark$ & Peer review of CG NW & Mon 8/9/04 & Tue 8/10/04 & \\
\hline 261 & $\checkmark$ & Peer review of CG NE & Tue $8 / 10 / 04$ & Wed 8/11/04 & \\
\hline 262 & $\checkmark$ & Revise NIPSCO data receipt and acceptance procedures & Thu 8/19/04 & Thu $8 / 19 / 04$ & \\
\hline 263 & $\checkmark$ & Correct Pilot Tiles CG and CF Based on QA Comments & Thu 8/12/04 & Mon 8/16/04 & \\
\hline 264 & $\checkmark$ & Elementool \#343 - CGSWE- ERROR & Thu 8/12/04 & Thu 8/12/04 & \\
\hline 265 & $\checkmark$ & Elementool \#345 - Rectification QA Results - CGNW & Thu 8/12/04 & Thu 8/12/04 & \\
\hline 266 & $\checkmark$ & Elementool \#351 - 5-EdgeMatch Errors CGSEE\&CHNEE & Thu 8/12/04 & Thu 8/12/04 & \\
\hline 267 & $\checkmark$ & Correct pilot tiles CG and CF based on the QA feedback & Thu 8/12/04 & Mon 8/16/04 & \\
\hline 268 & $\checkmark$ & Re-deliver pilot tiles CG and CF with fixes based on QA feedback & Mon 8/16/04 & Mon 8/16/04 & \\
\hline 269 & $\checkmark$ & Rectify Pilot Tiles CE & Fri 8/6/04 & Wed 8/25/04 & \\
\hline 270 & $\checkmark$ & Complete workorders for CE & Fri 8/6/04 & Fri 8/6/04 & \\
\hline 271 & $\checkmark$ & Freeze pilot tiles for CE & Fri 8/6/04 & Fri 8/6/04 & \\
\hline 272 & $\checkmark$ & Send pilot tiles for $C E$ to migration vendor & Fri 8/6/04 & Fri 8/6/04 & \\
\hline 273 & $\checkmark$ & Rectify pilot tiles for $\mathrm{CE}$ & Fri 8/6/04 & Tue $8 / 24 / 04$ & \\
\hline 274 & $\checkmark$ & Deliver pilot tiles for $\mathrm{CE}$ & Wed 8/25/04 & Wed 8/25/04 & \\
\hline 275 & $\checkmark$ & Determine how to handle the errors detected by DWGVerify & Mon 11/1/04 & Mon 11/1/04 & \\
\hline 276 & $\checkmark$ & Place $\mathrm{CF}$ and $\mathrm{CH}$ back into production & Tue 11/2/04 & Tue $11 / 2 / 04$ & \\
\hline $\begin{array}{l}\text { NIPSC } \\
\text { Confid }\end{array}$ & $\begin{array}{l}\text { Co GIS } \\
\text { lential }\end{array}$ & Page 6 & & $\begin{array}{r}\text { Last Updat } \\
\text { Print }\end{array}$ & $\begin{array}{l}\text { ed on Tue } 7 / 3 / 07 \\
\text { ed on Fri 8/17/07 }\end{array}$ \\
\hline
\end{tabular}




\section{\begin{tabular}{|ll}
\hline & AEDR Construction Phase I Project Plan
\end{tabular}}

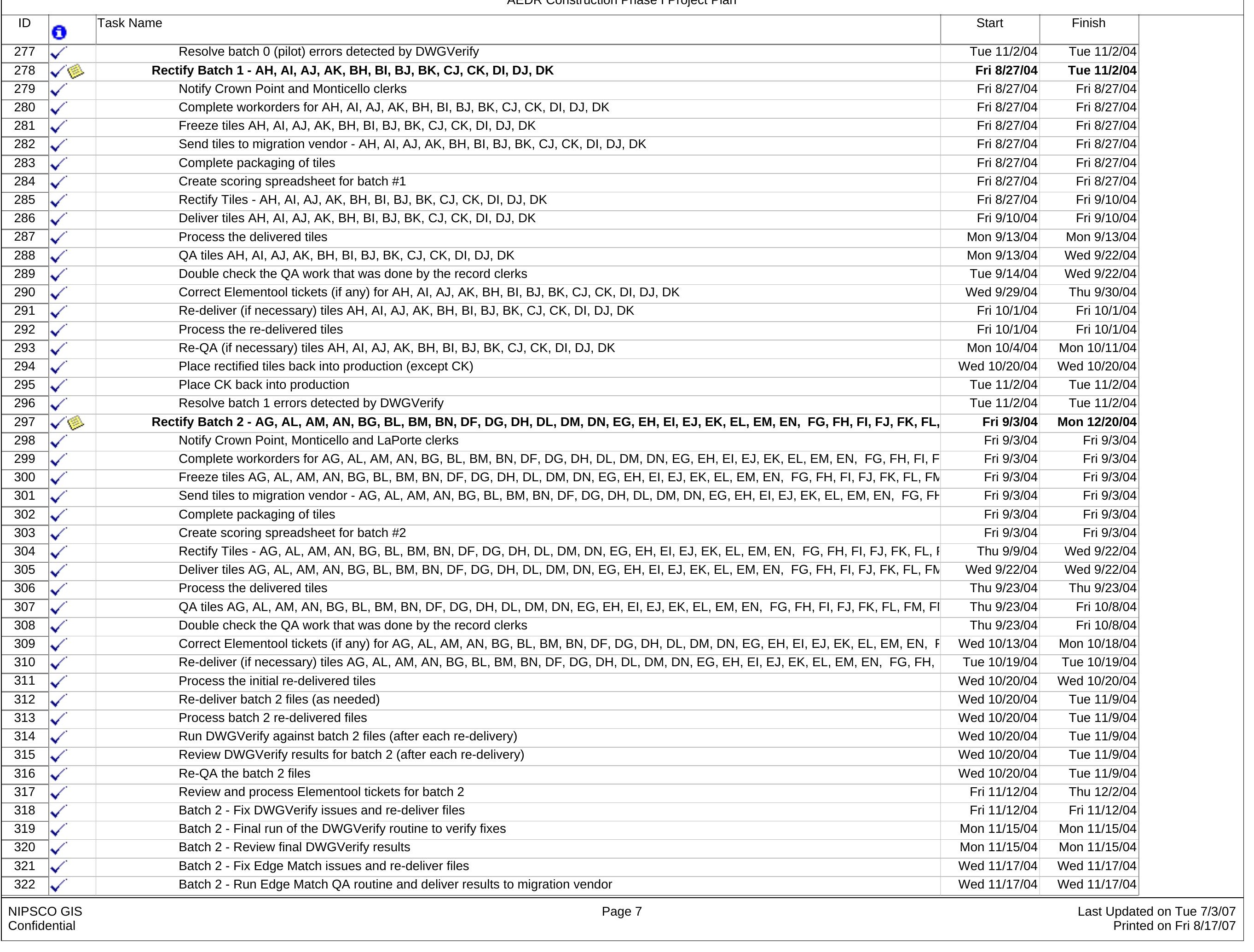




\section{\begin{tabular}{|ll}
\hline & AEDR Construction Phase I Project Plan
\end{tabular}}

\begin{tabular}{|l|l|l|}
\hline & & \\
\hline ID & $\mathbf{O}$ & Task Name \\
\hline 415 & $\checkmark$ \\
\hline 416 & $\checkmark$ \\
\hline 417 & $\checkmark$ \\
\hline 418 & $\checkmark$ \\
\hline 419 & $\checkmark$ \\
\hline 420 & $\checkmark$ \\
\hline 421 & $\checkmark$ \\
\hline 422 & $\checkmark$ \\
\hline 423 & $\checkmark$ \\
\hline 424 & $\checkmark$ \\
\hline 425 & $\checkmark$ \\
\hline 426 & $\checkmark$ \\
\hline 427 & $\checkmark$ \\
\hline 428 & $\checkmark$ \\
\hline 429 & $\checkmark$ \\
\hline 430 & $\checkmark$ \\
\hline 431 & $\checkmark$ \\
\hline 432 & $\checkmark$ \\
\hline 433 & $\checkmark$ \\
\hline 434 & $\checkmark$ \\
\hline 435 & $\checkmark$ \\
\hline 436 & $\checkmark$ \\
\hline 437 & $\checkmark$ \\
\hline 438 & $\checkmark$ \\
\hline 439 & $\checkmark$ & \\
\hline 440 & $\checkmark$ \\
\hline 441 & $\checkmark$ \\
\hline 442 & $\checkmark$ \\
\hline 443 & $\checkmark$ \\
\hline 444 & $\checkmark$ \\
\hline 445 & $\checkmark$ \\
\hline 446 & $\checkmark$ \\
\hline 447 & $\checkmark$ \\
\hline 448 & $\checkmark$ \\
\hline 449 & $\checkmark$ \\
\hline 450 & $\checkmark$ \\
\hline 451 & $\checkmark$ \\
\hline 452 & $\checkmark$ \\
\hline 453 & $\checkmark$ \\
\hline 454 & $\checkmark$ \\
\hline 455 & $\checkmark$ \\
\hline 456 & $\checkmark$ \\
\hline 457 & $\checkmark$ \\
\hline 458 & $\checkmark$ \\
\hline 459 & $\checkmark$ \\
\hline 460 & $\checkmark$ \\
\hline \hline
\end{tabular}

NIPSCO GIS Confidential

\section{Rectify the batch 5 tiles}

Start

Initial delivery of the batch 5 tiles

Add batch 5 to the scoring spreadsheet

Run initial DWGVerify checks against the batch 5 tiles

Review initial DWGVerify results for the batch 5 tiles

Batch 5 - Perform first pass QA

Batch 5 - Review and process Elementool tickets

Batch 5 - Add data migration operator info to the scoring spreadsheet

Batch 5 - Identify candidates for additional (second pass) QA

Batch 5 - Perform second pass QA on selected files

Batch 5 - Re-deliver files (as needed)

Batch 5 - Process re-delivered files

Batch 5 - Run DWGVerify against files (after each re-delivery)

Batch 5 - Review DWGVerify results (after each re-delivery)

Batch 5 - Run Edge Match QA (after each re-delivery)

Batch 5 - Review Edge Match results (after each re-delivery)

Batch 5 - Review and process Elementool tickets

Batch 5 - Create list of files that are ready to go back into production

Batch 5 - Place initial set of tiles back into production

Batch 5 - Cleanup issues on remaining tiles

Batch 5 - Place next batch of tiles back into production

Batch 5 - Rectification DWGVerify Reports

Batch 5 - Cleanup issues on remaining tiles

Batch 5 - Place final tiles back into production

\section{Rectify Batch 6 - AC, AD, KF, KG, KH, LA, LB, LC, LD, LE, LF, LG, LH, MC, MD, ME, MF, MG, MH}

Complete workorders for AC, AD, KF, KG, KH, LA, LB, LC, LD, LE, LF, LG, LH, MC, MD, ME, MF, MG, MH

Freeze tiles AC, AD, KF, KG, KH, LA, LB, LC, LD, LE, LF, LG, LH, MC, MD, ME, MF, MG, MH

Send tiles to data migration vendor - AC, AD, KF, KG, KH, LA, LB, LC, LD, LE, LF, LG, LH, MC, MD, ME, MF, MG, MH

Rectify the batch 6 tiles

Initial delivery of the batch 6 tiles

Batch 6 - Setup QA packages for QA clerks

Batch 6 - Run initial DWGVerify QA checks

Batch 6 - Review initial DWGVerify results and submit Elementool tickets

Batch 6 - Run initial Edge Match QA checks

Batch 6 - Review initial Edge Match results and submit Elementool tickets

Batch 6 - Setup the scoring spreadsheet with files / counts / formulas

Batch 6 - Add data migration vendor operator info to the scoring spreadsheet

Batch 6 - Perform first pass QA

Batch 6 - Review and process Elementool tickets

Batch 6 - Cleanup DWGVerify QA issues and re-deliver

Batch 6 - Cleanup Edge Match QA issues and re-delive

Batch 6 - Identify candidates for additional (second pass) QA

Batch 6 - Perform second pass QA on selected files

Batch 6 - Re-deliver files

Batch 6 - Process re-delivered files

Batch 6 - Run DWGVerify against files (after each re-delivery)
Mon 10/18/04

Mon $11 / 1 / 04$

Thu $11 / 4 / 04$

Thu $11 / 4 / 04$

Thu $11 / 4 / 04$

Tue 11/9/04

Tue 11/9/04

Wed $12 / 1 / 04$

Wed $12 / 1 / 04$

Wed $12 / 1 / 04$

Wed $12 / 1 / 04$

Wed $12 / 1 / 04$

Wed 12/1/04

Wed $12 / 1 / 04$

Wed $12 / 1 / 04$

Wed 12/1/04

Wed $12 / 1 / 04$

Tue 12/21/04

Wed $12 / 22 / 04$

Fri $1 / 7 / 05$

Mon $1 / 10 / 05$

Fri $1 / 7 / 05$

Thu 1/13/05

Wed $1 / 19 / 05$

Mon 10/25/04

Mon 10/25/04

Mon 10/25/04

Mon 10/25/04

Tue $10 / 26 / 04$

Tue 11/9/04

Wed 11/10/04

Thu 11/11/04

Thu 11/11/04

Wed 11/17/04

Wed 11/17/04

Fri $12 / 3 / 04$

Thu 12/2/04

Tue 11/9/04

Tue 11/9/04

Wed 12/8/04

Wed $12 / 8 / 04$

Thu 12/9/04

Fri 12/10/04

Fri 12/10/04

Fri 12/10/04

Fri 12/10/04

Thu 11/4/04

Thu 11/4/04

Thu 11/4/04

Fri 11/19/04

Fri 11/19/04

Wed $12 / 1 / 04$

Wed 12/1/04

Wed 12/15/04

Wed 12/15/04

Wed 12/15/04

Wed 12/15/04

Wed 12/15/04

Wed 12/15/04

Wed 12/15/04

Wed 12/15/04

Tue 12/21/04

Wed 12/22/04

Fri $1 / 7 / 05$

Mon 1/10/05

Fri $1 / 7 / 05$

Thu 1/13/05

Wed $1 / 19 / 05$

Fri 1/28/05

Mon 10/25/04

Mon 10/25/04

Mon 10/25/04

Tue 11/9/04

Tue 11/9/04

Wed $11 / 10 / 04$

Thu 11/11/04

Thu 11/11/04

Wed 11/17/04

Wed 11/17/04

Fri $12 / 3 / 04$

Thu 12/2/04

Tue $12 / 7 / 04$

Tue 12/7/04

Wed 12/8/04

Wed 12/8/04

Thu 12/9/04

Wed 12/22/04

Wed 12/22/04

Wed $12 / 22 / 04$

Wed 12/22/04

Last Updated on Tue 7/3/07 Printed on Fri 8/17/07 


\section{\begin{tabular}{|ll}
\hline & AEDR Construction Phase I Project Plan
\end{tabular}}

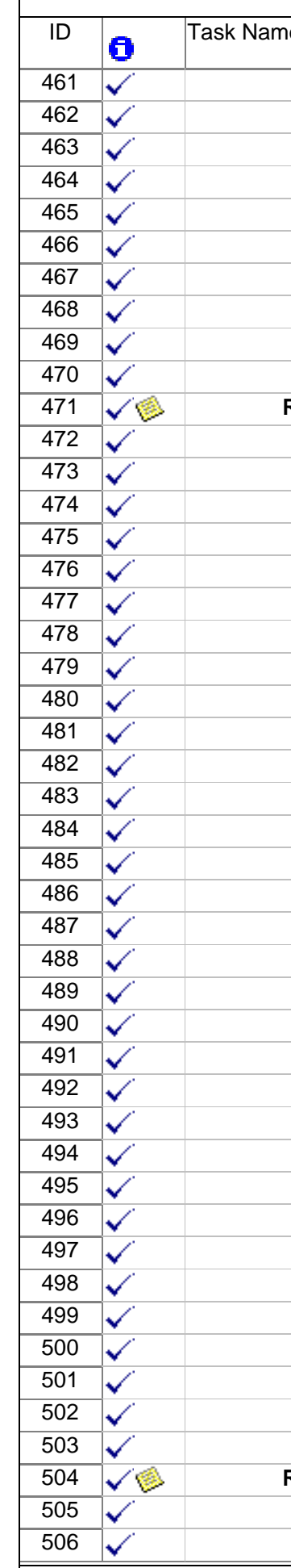

NIPSCO GIS Confidential
Batch 6 - Review DWGVerify results (after each re-delivery)

Batch 6 - Run Edge Match QA (after each re-delivery)

Batch 6 - Review Edge Match results (after each re-delivery)

Batch 6 - Review and process Elementool tickets

Batch 6 - Create list of files that are ready to go back into production

Batch 6 - Place initial set of tiles back into production

Batch 6 - Cleanup issues on subset of tiles

Batch 6 - Place the next subset of tiles back into production

Batch 6 - Cleanup issues on remaining tiles

Batch 6 - Place remaining tiles back into production

Rectify Batch 7 - BC, BD, LI, LJ, LK, LL, LM, MI, MJ, MK, ML, MN, NI, NJ, NK, NL, NM

Complete workorders for batch 7 tiles

Freeze tiles for batch 7

Send batch 7 tiles to migration vendor

Batch 7 - Rectify the tiles

Batch 7 - Initial delivery

Batch 7 - Setup QA packages for QA clerks

Batch 7 - Run initial DWGVerify QA checks

Batch 7 - Review initial DWGVerify results and submit Elementool tickets

Batch 7 - Run initial Edge Match QA checks

Batch 7 - Review initial Edge Match results and submit Elementool tickets

Batch 7 - Cleanup DWGVerify QA issues and re-deliver

Batch 7 - Cleanup Edge Match QA issues and re-deliver

Batch 7 - Setup the scoring spreadsheet with files / counts / formulas

Batch 7 - Add data migration vendor operator info to the scoring spreadsheet

Batch 7 - Perform first pass QA

Batch 7 - Review and process Elementool tickets

Batch 7 - Identify candidates for additional (second pass) QA

Batch 7 - Perform second pass QA on selected files

Batch 7 - Re-deliver files (complete re-delivery of BC and BD)

Batch 7 - Process re-delivered files - part 1

Batch 7 - Run Edge Match QA (after each re-delivery) - part 1

Batch 7 - Process re-delivered files - part 2

Batch 7 - Run DWGVerify against files (after each re-delivery)

Batch 7 - Review DWGVerify results (after each re-delivery)

Batch 7 - Run Edge Match QA (after each re-delivery) - part 2

Batch 7 - Review Edge Match results (after each re-delivery)

Batch 7 - Review and process Elementool tickets

Batch 7 - Place next set of tiles back into production

Batch 7 - Cleanup issues on a subset of tiles

Batch 7 - Place the next subset of tiles back into production

Batch 7 - Cleanup issues on remaining tiles

Batch 7 - Place remaining tiles back into production

Rectify Batch 8 - AA, AB, BA, BB, MA, MB, NA, NB, NH, OH, OI, OJ, OK, OL, OM, PH, PI, PJ, PK, PL, PM

Batch 8 - Complete workorders

Batch 8 - Freeze tiles
Thu $12 / 23 / 04$

Thu $12 / 23 / 04$

Thu $1 / 13 / 05$

Wed $1 / 19 / 05$

Thu 1/27/05

Fri 1/28/05

Thu 11/4/04

Thu 11/4/04

Thu 11/4/04

Thu 11/4/04

Fri 11/5/04

Mon 11/29/04

Tue 11/30/04

Tue 11/30/04

Tue 11/30/04

Tue 11/30/04

Tue 11/30/04

Wed 12/1/04

Wed 12/1/04

Thu 12/2/04

Fri 12/10/04

Thu 12/2/04

Thu 12/2/04

Tue 12/21/04

Tue 12/21/04

Tue 12/21/04

Tue 12/21/04

Tue 12/21/04

Mon 12/27/04

Tue 12/21/04

Tue 12/21/04

Tue 1/4/05

Tue 12/21/04

Tue 12/21/04

Mon 1/10/05

Thu 1/13/05

Wed 1/19/05

Thu 1/27/05

Fri 1/28/05

Mon 11/22/04

Mon 11/22/04

Mon 11/22/04

Wed 12/22/04

Wed 12/22/04

Thu 12/23/04

Thu 12/23/04

Thu 1/13/05

Wed $1 / 19 / 05$

Thu 1/27/05

Fri 1/28/05

Fri 1/28/05

Thu 11/4/04

Thu 11/4/04

Thu 11/4/04

Mon 11/29/04

Mon 11/29/04

Tue 11/30/04

Tue 11/30/04

Tue 11/30/04

Tue 11/30/04

Tue 11/30/04

Wed 12/1/04

Wed 12/1/04

Thu 12/2/04

Fri 12/10/04

Tue 12/21/04

Tue 12/21/04

Tue 12/21/04

Tue $1 / 4 / 05$

Tue 1/4/05

Fri 12/24/04

Fri 12/24/04

Thu 1/6/05

Thu $1 / 6 / 05$

Thu $1 / 6 / 05$

Thu $1 / 6 / 05$

Thu $1 / 6 / 05$

Thu $1 / 6 / 05$

Mon 1/10/05

Thu 1/13/05

Wed $1 / 19 / 05$

Thu 1/27/05

Fri 1/28/05

Fri 3/4/05

Mon 11/22/04

Mon 11/22/04 


\begin{tabular}{|c|c|c|c|c|c|}
\hline \multicolumn{6}{|c|}{ AEDR Construction Phase I Project Plan } \\
\hline ID & ( & Task Name & Start & Finish & \\
\hline 507 & $\checkmark$ & Batch 8 - Send tiles to data migration vendor & Mon 11/22/04 & Mon 11/22/04 & \\
\hline 508 & $\checkmark$ & Batch 8 - Rectify the tiles & Mon 11/22/04 & Tue $12 / 7 / 04$ & \\
\hline 509 & $\checkmark$ & Batch 8 - Initial delivery & Tue $12 / 7 / 04$ & Tue $12 / 7 / 04$ & \\
\hline 510 & $\checkmark$ & Batch 8 - Setup QA packages for QA clerks & Wed 12/8/04 & Wed 12/8/04 & \\
\hline 511 & $\checkmark$ & Batch 8 - Run initial DWGVerify QA checks & Wed 12/8/04 & Wed $12 / 8 / 04$ & \\
\hline 512 & $\checkmark$ & Batch 8 - Review initial DWGVerify results and submit Elementool tickets & Thu $12 / 9 / 04$ & Thu $12 / 9 / 04$ & \\
\hline 513 & $\checkmark$ & Batch 8 - Run initial Edge Match QA checks & Wed 12/8/04 & Wed 12/8/04 & \\
\hline 514 & $\checkmark$ & Batch 8 - Review initial Edge Match results and submit Elementool tickets & Thu $12 / 9 / 04$ & Thu $12 / 9 / 04$ & \\
\hline 515 & $\checkmark$ & Batch 8 - Cleanup DWGVerify QA issues and re-deliver & Fri 12/10/04 & Fri 12/10/04 & \\
\hline 516 & $\checkmark$ & Batch 8 - Cleanup Edge Match QA issues and re-deliver & Fri 12/10/04 & Fri $12 / 10 / 04$ & \\
\hline 517 & $\checkmark$ & Batch 8 - Setup the scoring spreadsheet with files / counts / formulas & Fri 12/10/04 & Fri 12/10/04 & \\
\hline 518 & $\checkmark$ & Batch 8 - Re-QA and Re-Deliver the entire batch & Mon 1/17/05 & Mon 1/17/05 & \\
\hline 519 & $\checkmark$ & Batch 8 - Setup the QA packages & Wed 1/19/05 & Wed 1/19/05 & \\
\hline 520 & $\checkmark$ & Batch 8 - Perform first pass QA & Thu $1 / 13 / 05$ & Fri $1 / 28 / 05$ & \\
\hline 521 & $\checkmark$ & Batch 8 - Review and process Elementool tickets & Wed 1/12/05 & Fri $1 / 28 / 05$ & \\
\hline 522 & $\checkmark$ & Batch 8 - Add data migration vendor operator info to the scoring spreadsheet & Fri 1/28/05 & Fri $1 / 28 / 05$ & \\
\hline 523 & $\checkmark$ & Batch 8 - Identify candidates for additional (second pass) QA & Fri $1 / 28 / 05$ & Mon 1/31/05 & \\
\hline 524 & $\checkmark$ & Batch 8 - Place first 2 tiles back into production & Fri $1 / 28 / 05$ & Fri 1/28/05 & \\
\hline 525 & $\checkmark$ & Batch 8 - Perform second pass QA on selected files & Mon 1/31/05 & Mon 2/14/05 & \\
\hline 526 & $\checkmark$ & Batch 8 - Re-deliver files with Elementool fixes & Tue $2 / 8 / 05$ & Tue $2 / 8 / 05$ & \\
\hline 527 & $\checkmark$ & Batch 8 - Process re-delivered files & Tue 2/8/05 & Tue $2 / 8 / 05$ & \\
\hline 528 & $\checkmark$ & Batch 8 - Test the Elementool fixes & Tue $2 / 8 / 05$ & Thu $2 / 10 / 05$ & \\
\hline 529 & $\checkmark$ & Batch 8 - Run DWGVerify against files (after each re-delivery) & Thu $2 / 10 / 05$ & Thu $2 / 10 / 05$ & \\
\hline 530 & $\checkmark$ & Batch 8 - Review DWGVerify results (after each re-delivery) & Thu $2 / 10 / 05$ & Thu $2 / 10 / 05$ & \\
\hline 531 & $\checkmark$ & Batch 8 - Run Edge Match QA (after each re-delivery) & Thu $2 / 10 / 05$ & Thu $2 / 10 / 05$ & \\
\hline 532 & $\checkmark$ & Batch 8 - Review Edge Match results (after each re-delivery) & Thu 2/10/05 & Thu $2 / 10 / 05$ & \\
\hline 533 & $\checkmark$ & Batch 8 - Prepare list of files to go back into production & Thu $2 / 10 / 05$ & Thu $2 / 10 / 05$ & \\
\hline 534 & $\checkmark$ & Batch 8 - Place next set of tiles back into production & Fri 2/11/05 & Fri 2/11/05 & \\
\hline 535 & $\checkmark$ & Batch 8 - Additional cleanup / fixes & Thu 2/17/05 & Thu 2/17/05 & \\
\hline 536 & $\checkmark$ & Batch 8 - Additional testing of the cleanup / fixes & Fri 2/18/05 & Fri 2/18/05 & \\
\hline 537 & $\checkmark$ & Batch 8 - Place next set of tiles back into production & Fri 2/18/05 & Fri 2/18/05 & \\
\hline 538 & $\checkmark$ & Batch 8 - Final edge match cleanup / fixes (2 tiles) & Wed 3/2/05 & Wed 3/2/05 & \\
\hline 539 & $\checkmark$ & Batch 8 - Additional testing of the cleanup / fixes ( 2 tiles) & Thu $3 / 3 / 05$ & Thu $3 / 3 / 05$ & \\
\hline 540 & $\checkmark$ & Batch 8 - Place final 2 tiles back into production & Fri 3/4/05 & Fri 3/4/05 & \\
\hline 541 & $\checkmark(6)$ & Rectify Batch 9 - NC, ND, NE, NF, NG, OA, OB, OC, OD, OE, OF, OG, QH, QI, QJ, QK, QL, QM, RH, RI, RJ, RK, RL, RM, SH, SI, SJ, Sk & Wed 12/1/04 & Fri 3/4/05 & \\
\hline 542 & $\checkmark$ & Batch 9 - Complete workorders - part 1 or 3 & Wed 12/1/04 & Wed 12/1/04 & \\
\hline 543 & $\checkmark$ & Batch 9 - Freeze tiles - part 1 of 3 & Wed 12/1/04 & Wed 12/1/04 & \\
\hline 544 & $\checkmark$ & Batch 9 - Send tiles to data migration vendor - part 1 of 3 & Wed 12/1/04 & Wed 12/1/04 & \\
\hline 545 & $\checkmark$ & Batch 9 - Complete workorders - part 2 or 3 & Tue $12 / 7 / 04$ & Tue $12 / 7 / 04$ & \\
\hline 546 & $\checkmark$ & Batch 9 - Freeze tiles - part 2 of 3 & Tue $12 / 7 / 04$ & Tue $12 / 7 / 04$ & \\
\hline 547 & $\checkmark$ & Batch 9 - Send tiles to data migration vendor - part 2 of 3 & Tue $12 / 7 / 04$ & Tue $12 / 7 / 04$ & \\
\hline 548 & $\checkmark$ & Batch 9 - Complete workorders - part 3 or 3 & Thu $12 / 9 / 04$ & Thu $12 / 9 / 04$ & \\
\hline 549 & $\checkmark$ & Batch 9 - Freeze tiles - part 3 of 3 & Thu $12 / 9 / 04$ & Thu 12/9/04 & \\
\hline 550 & $\checkmark$ & Batch 9 - Send tiles to data migration vendor - part 3 of 3 & Thu $12 / 9 / 04$ & Thu $12 / 9 / 04$ & \\
\hline 551 & $\checkmark$ & Batch 9 - Research and resolve DWG file with 0 features & Mon 12/13/04 & Fri $12 / 17 / 04$ & \\
\hline 552 & $\checkmark$ & Batch 9 - Rectify the tiles (including complete re-QA) & Thu 12/2/04 & Mon $1 / 17 / 05$ & \\
\hline $\begin{array}{l}\text { NIPSC } \\
\text { Confid }\end{array}$ & $\begin{array}{l}\text { Co GIS } \\
\text { lential }\end{array}$ & Page 12 & & $\begin{array}{r}\text { Last Updat } \\
\text { Print }\end{array}$ & $\begin{array}{l}\text { ed on Tue } 7 / 3 / 07 \\
\text { ed on Fri 8/17/07 }\end{array}$ \\
\hline
\end{tabular}




\begin{tabular}{|c|c|c|c|c|c|}
\hline \multicolumn{6}{|c|}{ AEDR Construction Phase I Project Plan } \\
\hline ID & ( & Task Name & Start & Finish & \\
\hline 553 & $\checkmark$ & Batch 9 - Initial delivery & Fri $1 / 21 / 05$ & Thu $1 / 27 / 05$ & \\
\hline 554 & $\checkmark$ & Batch 9 - Setup QA packages for QA clerks & Fri $1 / 28 / 05$ & Fri 1/28/05 & \\
\hline 555 & $\checkmark$ & Batch 9 - Run initial DWGVerify QA checks & Fri $1 / 28 / 05$ & Fri $1 / 28 / 05$ & \\
\hline 556 & $\checkmark$ & Batch 9 - Review initial DWGVerify results and submit Elementool tickets & Fri $1 / 28 / 05$ & Fri $1 / 28 / 05$ & \\
\hline 557 & $\checkmark$ & Batch 9 - Run initial Edge Match QA checks & Fri $1 / 28 / 05$ & Fri $1 / 28 / 05$ & \\
\hline 558 & $\checkmark$ & Batch 9 - Review initial Edge Match results and submit Elementool tickets & Fri $1 / 28 / 05$ & Fri $1 / 28 / 05$ & \\
\hline 559 & $\checkmark$ & Batch 9 - Cleanup DWGVerify QA issues and re-deliver & Fri $1 / 28 / 05$ & Fri $1 / 28 / 05$ & \\
\hline 560 & $\checkmark$ & Batch 9 - Cleanup Edge Match QA issues and re-deliver & Fri 1/28/05 & Mon 1/31/05 & \\
\hline 561 & $\checkmark$ & Batch 9 - Setup the scoring spreadsheet with files / counts / formulas & Mon 1/31/05 & Mon 1/31/05 & \\
\hline 562 & $\checkmark$ & Batch 9 - Perform first pass QA & Mon 1/31/05 & Mon 2/14/05 & \\
\hline 563 & $\checkmark$ & Batch 9 - Review and process Elementool tickets & Mon 1/31/05 & Mon 2/14/05 & \\
\hline 564 & $\checkmark$ & Batch 9 - Add data migration vendor operator info to the scoring spreadsheet & Wed 2/16/05 & Wed 2/16/05 & \\
\hline 565 & $\checkmark$ & Batch 9 - Identify candidates for additional (second pass) QA & Wed 2/16/05 & Wed 2/16/05 & \\
\hline 566 & $\checkmark$ & Batch 9 - Perform second pass QA on selected files & Wed 2/16/05 & Wed 3/2/05 & \\
\hline 567 & $\checkmark$ & Batch 9 - Re-deliver files & Wed 2/16/05 & Wed 2/23/05 & \\
\hline 568 & $\checkmark$ & Batch 9 - Process re-delivered files & Wed 2/16/05 & Wed 2/23/05 & \\
\hline 569 & $\checkmark$ & Batch 9 - Run DWGVerify against files (after each re-delivery) & Wed 2/16/05 & Wed 2/23/05 & \\
\hline 570 & $\checkmark$ & Batch 9 - Review DWGVerify results (after each re-delivery) & Wed 2/16/05 & Wed 2/23/05 & \\
\hline 571 & $\checkmark$ & Batch 9 - Run Edge Match QA (after each re-delivery) & Wed 2/16/05 & Wed 2/23/05 & \\
\hline 572 & $\checkmark$ & Batch 9 - Review Edge Match results (after each re-delivery) & Wed 2/16/05 & Wed 2/23/05 & \\
\hline 573 & $\checkmark$ & Batch 9 - Review and process Elementool tickets & Wed 2/16/05 & Wed 2/23/05 & \\
\hline 574 & $\checkmark$ & Batch 9 - Place next set of tiles back into production & Wed 2/23/05 & Wed 2/23/05 & \\
\hline 575 & $\checkmark$ & Batch 9 - Additional cleanup / fixes & Thu 3/3/05 & Thu $3 / 3 / 05$ & \\
\hline 576 & $\checkmark$ & Batch 9 - Additional testing of the cleanup / fixes & Fri 3/4/05 & Fri 3/4/05 & \\
\hline 577 & $\checkmark$ & Batch 9 - Place final tiles back into production & Fri 3/4/05 & Fri 3/4/05 & \\
\hline 578 & $\checkmark$ & Create New Rectification Batches and Schedule for 10 Through 14 & Tue $12 / 7 / 04$ & Fri 12/10/04 & \\
\hline 579 & $\checkmark$ & Revise rectification schedule - Identify critical tiles & Tue $12 / 7 / 04$ & Tue $12 / 7 / 04$ & \\
\hline 580 & $\checkmark$ & Revise rectification schedule - Create new draft of schedule for remaining batches & Wed 12/8/04 & Wed 12/8/04 & \\
\hline 581 & $\checkmark$ & Revise rectification schedule - Review draft schedule and provide comments & Thu $12 / 9 / 04$ & Thu 12/9/04 & \\
\hline 582 & $\checkmark$ & Revise rectification schedule - Revise schedule for remaining batches & Fri 12/10/04 & Fri 12/10/04 & \\
\hline 583 & $\checkmark$ & Revise rectification schedule - Update project plan with new schedule & Fri 12/10/04 & Fri 12/10/04 & \\
\hline 584 & $\checkmark b$ & Rectify Batch 10 - CB, DB, EB, PA, PB, PD, PE, PF, PG, UH, UI, UJ, UK, UL, UM, UN (17 tiles) & Fri 12/17/04 & Fri 3/25/05 & \\
\hline 585 & $\checkmark$ & Insert completed tasks here & Fri 12/17/04 & Fri 12/17/04 & \\
\hline 586 & $\checkmark$ & Batch 10 - Complete workorders & Fri 12/17/04 & Fri 12/17/04 & \\
\hline 587 & $\checkmark$ & Batch 10 - Freeze tiles & Fri 12/17/04 & Fri 12/17/04 & \\
\hline 588 & $\checkmark$ & Batch 10 - Send tiles to migration vendor & Fri 12/17/04 & Fri 12/17/04 & \\
\hline 589 & $\checkmark$ & Batch 10 - Rectify the tiles & Mon 12/20/04 & Wed $1 / 19 / 05$ & \\
\hline 590 & $\checkmark$ & Batch 10 - Initial delivery & Wed $1 / 26 / 05$ & Tue 2/8/05 & \\
\hline 591 & $\checkmark$ & Batch 10 - Setup QA packages for QA clerks & Wed 2/2/05 & Wed 2/2/05 & \\
\hline 592 & $\checkmark$ & Batch 10 - Run initial DWGVerify QA checks & Wed 2/2/05 & Wed 2/2/05 & \\
\hline 593 & $\checkmark$ & Batch 10 - Review initial DWGVerify results and submit Elementool tickets & Wed 2/2/05 & Wed 2/2/05 & \\
\hline 594 & $\checkmark$ & Batch 10 - Run initial Edge Match QA checks & Wed 2/2/05 & Wed 2/2/05 & \\
\hline 595 & $\checkmark$ & Batch 10 - Review initial Edge Match results and submit Elementool tickets & Wed 2/2/05 & Wed 2/2/05 & \\
\hline 596 & $\checkmark$ & Batch 10 - Cleanup DWGVerify QA issues and re-deliver & Wed 2/2/05 & Wed 2/2/05 & \\
\hline 597 & $\checkmark$ & Batch 10 - Cleanup Edge Match QA issues and re-deliver & Wed 2/2/05 & Thu $2 / 3 / 05$ & \\
\hline 598 & $\checkmark$ & Batch 10 - Setup the scoring spreadsheet with files / counts / formulas & Thu 2/3/05 & Thu $2 / 3 / 05$ & \\
\hline $\begin{array}{l}\text { NIPSC } \\
\text { Confid }\end{array}$ & $\begin{array}{l}\text { Co GIS } \\
\text { lential }\end{array}$ & Page 13 & & $\begin{array}{r}\text { Last Updat } \\
\text { Print }\end{array}$ & $\begin{array}{l}\text { ed on Tue } 7 / 3 / 07 \\
\text { ed on Fri 8/17/07 }\end{array}$ \\
\hline
\end{tabular}




\begin{tabular}{|c|c|c|c|c|c|}
\hline \multicolumn{6}{|c|}{ AEDR Construction Phase I Project Plan } \\
\hline ID & ( & Task Name & Start & Finish & \\
\hline 599 & $\checkmark$ & Batch 10 - Perform first pass QA & Mon 2/14/05 & Tue 3/1/05 & \\
\hline 600 & $\checkmark$ & Batch 10 - Review and process Elementool tickets & Mon 2/14/05 & Tue 3/1/05 & \\
\hline 601 & $\checkmark$ & Batch 10 - Add data migration vendor operator info to the scoring spreadsheet & Tue 3/1/05 & Tue 3/1/05 & \\
\hline 602 & $\checkmark$ & Batch 10 - Identify candidates for additional (second pass) QA & Tue 3/1/05 & Tue 3/1/05 & \\
\hline 603 & $\checkmark$ & Batch 10 - Perform second pass QA on selected files & Tue 3/1/05 & Tue $3 / 15 / 05$ & \\
\hline 604 & $\checkmark$ & Batch 10 - Re-deliver files & Tue 3/1/05 & Tue $3 / 15 / 05$ & \\
\hline 605 & $\checkmark$ & Batch 10 - Process re-delivered files & Tue 3/1/05 & Tue 3/15/05 & \\
\hline 606 & $\checkmark$ & Batch 10 - Run DWGVerify against files (after each re-delivery) & Tue 3/1/05 & Tue 3/15/05 & \\
\hline 607 & $\checkmark$ & Batch 10 - Review DWGVerify results (after each re-delivery) & Tue 3/1/05 & Tue $3 / 15 / 05$ & \\
\hline 608 & $\checkmark$ & Batch 10 - Run Edge Match QA (after each re-delivery) & Tue 3/1/05 & Tue $3 / 15 / 05$ & \\
\hline 609 & $\checkmark$ & Batch 10 - Review Edge Match results (after each re-delivery) & Tue 3/1/05 & Tue $3 / 15 / 05$ & \\
\hline 610 & $\checkmark$ & Batch 10 - Review and process Elementool tickets & Tue 3/1/05 & Tue $3 / 15 / 05$ & \\
\hline 611 & $\checkmark$ & Batch 10 - Place initial set of tiles back into production & Tue $3 / 15 / 05$ & Wed 3/16/05 & \\
\hline 612 & $\checkmark$ & Batch 10 - Additional cleanup / fixes & Wed 3/23/05 & Wed 3/23/05 & \\
\hline 613 & $\checkmark$ & Batch 10 - Additional testing of the cleanup / fixes & Thu $3 / 24 / 05$ & Thu $3 / 24 / 05$ & \\
\hline 614 & $\checkmark$ & Batch 10 - Place final tiles back into production & Fri 3/25/05 & Fri 3/25/05 & \\
\hline 615 & $\checkmark(6)$ & Rectify Batch 11 - FA, FB, FC, QA, QC, QD, QE, QF, QG, RA, RB, RC, RD, RE, RF, RG (17 files) & Mon $1 / 3 / 05$ & Thu 3/24/05 & \\
\hline 616 & $\checkmark$ & Batch 11 - Complete workorders & Tue $1 / 4 / 05$ & Tue $1 / 4 / 05$ & \\
\hline 617 & $\checkmark$ & Batch 11 - Freeze tiles & Tue $1 / 4 / 05$ & Tue $1 / 4 / 05$ & \\
\hline 618 & $\checkmark$ & Batch 11 - Send tiles to migration vendor & Tue $1 / 4 / 05$ & Tue $1 / 4 / 05$ & \\
\hline 619 & $\checkmark$ & Batch 11 - Setup the scoring spreadsheet with files / counts / formulas & Thu 2/24/05 & Thu $2 / 24 / 05$ & \\
\hline 620 & $\checkmark$ & Batch 11 - Rectify the tiles & Mon $1 / 3 / 05$ & Fri 1/21/05 & \\
\hline 621 & $\checkmark$ & Batch 11 - Initial delivery & Wed 2/16/05 & Tue 2/22/05 & \\
\hline 622 & $\checkmark$ & Batch 11 - Setup QA packages for QA clerks & Wed 2/23/05 & Wed 2/23/05 & \\
\hline 623 & $\checkmark$ & Batch 11 - Run initial DWGVerify QA checks & Wed 2/23/05 & Wed 2/23/05 & \\
\hline 624 & $\checkmark$ & Batch 11 - Review initial DWGVerify results and submit Elementool tickets & Wed 2/23/05 & Wed 2/23/05 & \\
\hline 625 & $\checkmark$ & Batch 11 - Run initial Edge Match QA checks & Wed 2/23/05 & Wed 2/23/05 & \\
\hline 626 & $\checkmark$ & Batch 11 - Review initial Edge Match results and submit Elementool tickets & Wed 2/23/05 & Wed 2/23/05 & \\
\hline 627 & $\checkmark$ & Batch 11 - Cleanup DWGVerify QA issues and re-deliver & Wed 2/23/05 & Wed 2/23/05 & \\
\hline 628 & $\checkmark$ & Batch 11 - Cleanup Edge Match QA issues and re-deliver & Wed 2/23/05 & Thu 2/24/05 & \\
\hline 629 & $\checkmark$ & Batch 11 - Perform first pass QA & Mon 2/28/05 & Wed 3/9/05 & \\
\hline 630 & $\checkmark$ & Batch 11 - Review and process Elementool tickets & Mon 2/28/05 & Wed 3/9/05 & \\
\hline 631 & $\checkmark$ & Batch 11 - Add data migration vendor operator info to the scoring spreadsheet & Wed 3/9/05 & Wed 3/9/05 & \\
\hline 632 & $\checkmark$ & Batch 11 - Identify candidates for additional (second pass) QA & Wed 3/9/05 & Wed 3/9/05 & \\
\hline 633 & $\checkmark$ & Batch 11 - Perform second pass QA on selected files & Wed 3/9/05 & Wed 3/23/05 & \\
\hline 634 & $\checkmark$ & Batch 11 - Re-deliver files & Wed 3/9/05 & Wed 3/23/05 & \\
\hline 635 & $\checkmark$ & Batch 11 - Process re-delivered files & Wed 3/9/05 & Wed 3/23/05 & \\
\hline 636 & $\checkmark$ & Batch 11 - Run DWGVerify against files (after each re-delivery) & Wed 3/9/05 & Wed 3/23/05 & \\
\hline 637 & $\checkmark$ & Batch 11 - Review DWGVerify results (after each re-delivery) & Wed 3/9/05 & Wed 3/23/05 & \\
\hline 638 & $\checkmark$ & Batch 11 - Run Edge Match QA (after each re-delivery) & Wed 3/9/05 & Wed 3/23/05 & \\
\hline 639 & $\checkmark$ & Batch 11 - Review Edge Match results (after each re-delivery) & Wed 3/9/05 & Wed 3/23/05 & \\
\hline 640 & $\checkmark$ & Batch 11 - Review and process Elementool tickets & Wed 3/9/05 & Wed 3/23/05 & \\
\hline 641 & $\checkmark$ & Batch 11 - Place tiles back into production & Wed 3/23/05 & Thu $3 / 24 / 05$ & \\
\hline 642 & $\checkmark(8)$ & Rectify Batch 12 - FD, SA, SB, SC, SD, SE, SF, SG, TA, TB, TC, TD, TE, TF, TG, UA, UB, UC, UD, UE, UF, UG (22 files) & Tue 1/11/05 & Thu 3/31/05 & \\
\hline 643 & $\checkmark$ & Batch 12 - Complete workorders & Tue 1/11/05 & Tue 1/11/05 & \\
\hline 644 & $\checkmark$ & Batch 12 - Freeze tiles & Tue 1/11/05 & Tue $1 / 11 / 05$ & \\
\hline $\begin{array}{l}\text { NIPSC } \\
\text { Confid }\end{array}$ & $\begin{array}{l}\text { Co GIS } \\
\text { lential }\end{array}$ & Page 14 & & $\begin{array}{r}\text { Last Updat } \\
\text { Print }\end{array}$ & $\begin{array}{l}\text { ed on Tue } 7 / 3 / 07 \\
\text { ed on Fri 8/17/07 }\end{array}$ \\
\hline
\end{tabular}




\section{\begin{tabular}{|ll}
\hline AEDR Construction Phase I Project Plan & AED
\end{tabular}}

\begin{tabular}{|l|l|l|}
\hline ID & $\mathbf{O}$ & Task Name \\
\hline 645 & $\checkmark$ \\
\hline 646 & $\checkmark$ \\
\hline 647 & $\checkmark$ \\
\hline 648 & $\checkmark$ \\
\hline 649 & $\checkmark$ \\
\hline 650 & $\checkmark$ \\
\hline 651 & $\checkmark$ \\
\hline 652 & $\checkmark$ \\
\hline 653 & $\checkmark$ \\
\hline 654 & $\checkmark$ \\
\hline 655 & $\checkmark$ \\
\hline 656 & $\checkmark$ \\
\hline 657 & $\checkmark$ \\
\hline 658 & $\checkmark$ \\
\hline 659 & $\checkmark$ \\
\hline 660 & $\checkmark$ \\
\hline 661 & $\checkmark$ \\
\hline 662 & $\checkmark$ \\
\hline 663 & $\checkmark$ \\
\hline 664 & $\checkmark$ \\
\hline 665 & $\checkmark$ \\
\hline 666 & $\checkmark$ \\
\hline 667 & $\checkmark$ \\
\hline 668 & $\checkmark$ \\
\hline 669 & $\checkmark$ & \\
\hline 670 & $\checkmark$ \\
\hline 671 & $\checkmark$ \\
\hline 672 & $\checkmark$ \\
\hline 673 & $\checkmark$ \\
\hline 674 & $\checkmark$ \\
\hline 675 & $\checkmark$ \\
\hline 676 & $\checkmark$ \\
\hline 677 & $\checkmark$ \\
\hline 678 & $\checkmark$ \\
\hline 679 & $\checkmark$ \\
\hline 680 & $\checkmark$ \\
\hline 681 & $\checkmark$ \\
\hline 682 & $\checkmark$ \\
\hline 683 & $\checkmark$ \\
\hline 684 & $\checkmark$ \\
\hline 685 & $\checkmark$ \\
\hline 686 & $\checkmark$ \\
\hline 687 & $\checkmark$ \\
\hline 688 & $\checkmark$ \\
\hline 689 & $\checkmark$ \\
\hline 690 & $\checkmark$ \\
\hline \hline N5Sco \\
\hline 6 \\
\hline 6
\end{tabular}

NIPSCO GIS Confidential

\section{Batch 12 - Send tiles to migration vendor}

Batch 12 - Rectify the tiles

Batch 12 - Initial delivery

Batch 12 - Setup QA packages for QA clerks

Batch 12 - Run initial DWGVerify QA checks

Batch 12 - Review initial DWGVerify results and submit Elementool tickets

Batch 12 - Run initial Edge Match QA checks

Batch 12 - Review initial Edge Match results and submit Elementool tickets

Batch 12 - Cleanup DWGVerify QA issues and re-deliver

Batch 12 - Cleanup Edge Match QA issues and re-deliver

Batch 12 - Setup the scoring spreadsheet with files / counts / formulas

Batch 12 - Perform first pass QA

Batch 12 - Review and process Elementool tickets

Batch 12 - Add data migration vendor operator info to the scoring spreadsheet

Batch 12 - Identify candidates for additional (second pass) QA

Batch 12 - Perform second pass QA on selected files

Batch 12 - Re-deliver files

Batch 12 - Process re-delivered files

Batch 12 - Run DWGVerify against files (after each re-delivery)

Batch 12 - Review DWGVerify results (after each re-delivery)

Batch 12 - Run Edge Match QA (after each re-delivery)

Batch 12 - Review Edge Match results (after each re-delivery)

Batch 12 - Review and process Elementool tickets

Batch 12 - Place tiles back into production

Rectify Batch 13 - VA, VB, VC, VD, VE, VF, VG, VH, VI, VJ, VK, VL, VM, VN, WA, WC, WD, WE, WF, WG, WH, WI, WJ, WK, WL, WM, $\mathrm{h}$

Batch 13 - Complete workorders

Batch 13 - Freeze tiles

Batch 13 - Send tiles to data migration vendor

Batch 13 - Rectify the tiles

Batch 13 - Initial delivery

Batch 13 - Setup QA packages for QA clerks

Batch 13 - Run initial DWGVerify QA checks

Batch 13 - Review initial DWGVerify results and submit Elementool tickets

Batch 13 - Run initial Edge Match QA checks

Batch 13 - Review initial Edge Match results and submit Elementool tickets

Batch 13 - Cleanup DWGVerify QA issues and re-deliver

Batch 13 - Cleanup Edge Match QA issues and re-deliver

Batch 13 - Setup the scoring spreadsheet with files / counts / formulas

Batch 13 - Perform first pass QA

Batch 13 - Review and process Elementool tickets

Batch 13 - Add data migration vendor operator info to the scoring spreadsheet

Batch 13 - Identify candidates for additional (second pass) QA

Batch 13 - Perform second pass QA on selected files

Batch 13 - Re-deliver files

Batch 13 - Process re-delivered files

Batch 13 - Run DWGVerify against files (after each re-delivery)

\section{Start}

Tue $1 / 11 / 05$

Wed $1 / 12 / 05$

Wed 2/23/05

Wed 3/2/05

Wed 3/2/05

Wed 3/2/05

Wed 3/2/05

Wed 3/2/05

Wed 3/2/05

Wed 3/2/05

Thu 3/3/05

Mon 3/7/05

Mon 3/7/05

Wed 3/16/05

Wed 3/16/05

Wed 3/16/05

Wed 3/16/05

Wed 3/16/05

Wed 3/16/05

Wed 3/16/05

Wed 3/16/05

Wed 3/16/05

Wed 3/16/05

Wed 3/30/05

Thu 1/20/05

Thu 1/20/05

Thu 1/20/05

Thu 1/20/05

Mon 1/31/05

Wed 3/2/05

Wed 3/9/05

Wed 3/9/05

Wed 3/9/05

Wed 3/9/05

Wed 3/9/05

Wed 3/9/05

Wed 3/9/05

Thu 3/10/05

Thu 3/10/05

Thu 3/10/05

Wed 3/23/05

Wed 3/23/05

Wed 3/23/05

Wed 3/23/05

Wed 3/23/05

Wed 3/23/05

Thu 1/20/05

Thu 1/20/05

Thu 1/20/05

Mon 2/21/05

Tue $3 / 8 / 05$

Wed 3/9/05

Wed 3/9/05

Wed 3/9/05

Wed 3/9/05

Wed 3/9/05

Wed 3/9/05

Thu 3/10/05

Thu 3/10/05

Wed 3/23/05

Wed 3/23/05

Wed 3/23/05

Wed 3/23/05

Wed 4/6/05

Wed 4/6/05

Wed 4/6/05

Wed 4/6/05 


\section{\begin{tabular}{|ll}
\hline & AEDR Construction Phase I Project Plan
\end{tabular}}

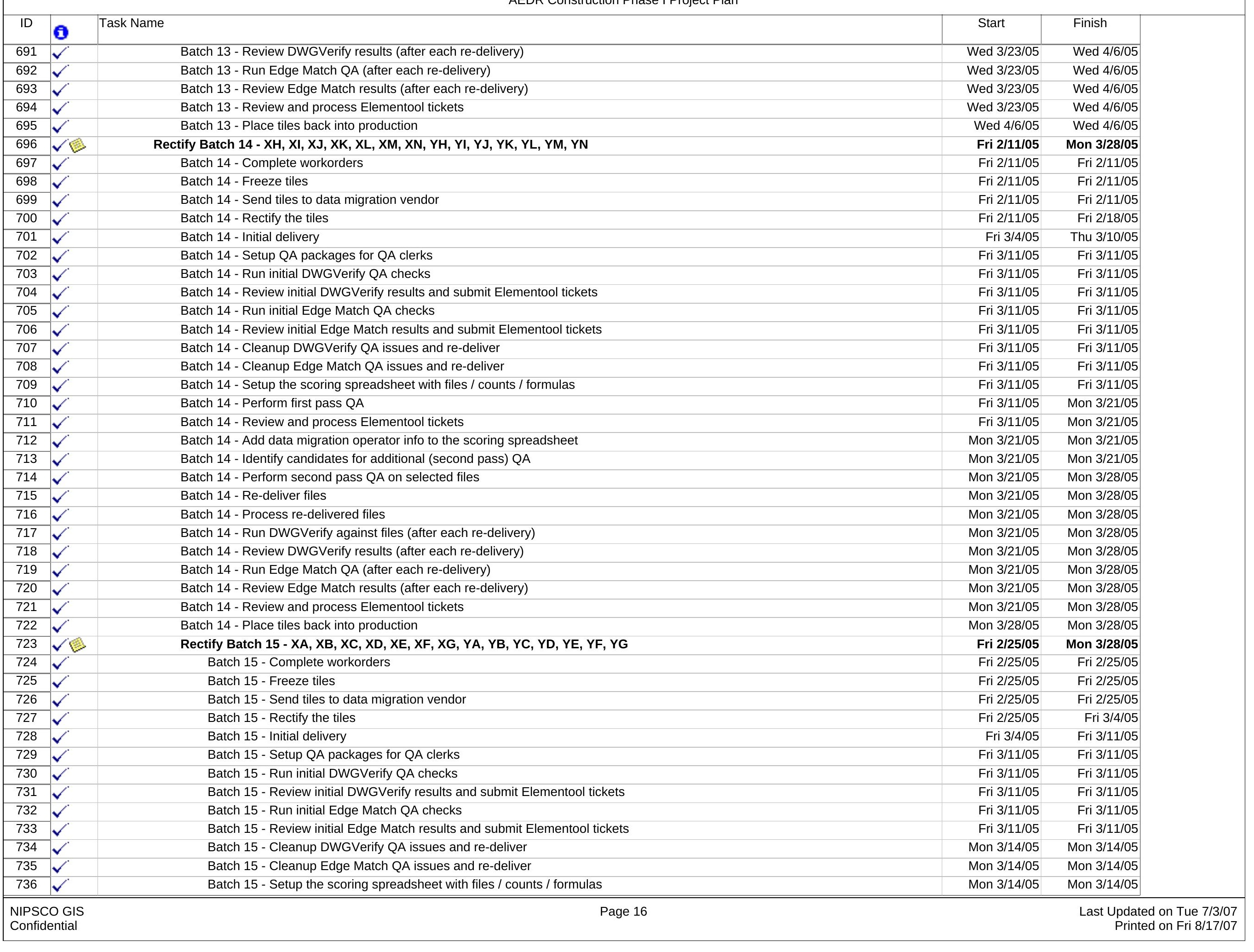




\begin{tabular}{|c|c|c|c|c|c|}
\hline \multicolumn{6}{|c|}{ AEDR Construction Phase I Project Plan } \\
\hline ID & ( & Task Name & Start & Finish & \\
\hline 737 & $\checkmark$ & Batch 15 - Perform first pass QA & Mon 3/14/05 & Mon 3/21/05 & \\
\hline 738 & $\checkmark$ & Batch 15 - Review and process Elementool tickets & Mon 3/14/05 & Mon 3/21/05 & \\
\hline 739 & $\checkmark$ & Batch 15 - Add data migration vendor operator info to the scoring spreadsheet & Mon 3/21/05 & Mon 3/21/05 & \\
\hline 740 & $\checkmark$ & Batch 15 - Identify candidates for additional (second pass) QA & Mon 3/21/05 & Mon 3/21/05 & \\
\hline 741 & $\checkmark$ & Batch 15 - Perform second pass QA on selected files & Mon 3/21/05 & Mon 3/28/05 & \\
\hline 742 & $\checkmark$ & Batch 15 - Re-deliver files & Mon 3/21/05 & Mon 3/28/05 & \\
\hline 743 & $\checkmark$ & Batch 15 - Process re-delivered files & Mon 3/21/05 & Mon 3/28/05 & \\
\hline 744 & $\checkmark$ & Batch 15 - Run DWGVerify against files (after each re-delivery) & Mon 3/21/05 & Mon 3/28/05 & \\
\hline 745 & $\checkmark$ & Batch 15 - Review DWGVerify results (after each re-delivery) & Mon 3/21/05 & Mon 3/28/05 & \\
\hline 746 & $\checkmark$ & Batch 15 - Run Edge Match QA (after each re-delivery) & Mon 3/21/05 & Mon 3/28/05 & \\
\hline 747 & $\checkmark$ & Batch 15 - Review Edge Match results (after each re-delivery) & Mon 3/21/05 & Mon 3/28/05 & \\
\hline 748 & $\checkmark$ & Batch 15 - Review and process Elementool tickets & Mon 3/21/05 & Mon 3/28/05 & \\
\hline 749 & $\checkmark$ & Batch 15 - Place tiles back into production & Mon 3/28/05 & Mon 3/28/05 & \\
\hline 750 & $\checkmark$ & Rectify Critical Tiles & Thu 1/20/05 & Thu 3/17/05 & \\
\hline 751 & $\checkmark$ & Rectify the Critical BC Tile & Tue 2/1/05 & Wed 2/2/05 & \\
\hline 752 & $\checkmark$ & BC Critical Tile - Finish the final QA tasks & Tue 2/1/05 & Tue 2/1/05 & \\
\hline 753 & $\checkmark$ & BC Critical Tile - Place the critical tile back into production & Wed 2/2/05 & Wed 2/2/05 & \\
\hline 754 & $\checkmark$ & Rectify the Critical PC Tile & Thu 1/20/05 & Fri 2/25/05 & \\
\hline 755 & $\checkmark$ & PC Critical Tile - Freeze and send the critical tile to the data migration vendor & Thu $1 / 20 / 05$ & Thu $1 / 20 / 05$ & \\
\hline 756 & $\checkmark$ & PC Critical Tile - Rectify the critical tile & Thu $1 / 20 / 05$ & Tue 2/1/05 & \\
\hline 757 & $\checkmark$ & PC Critical Tile - Review / answer data issues for data migration vendor & Tue $2 / 1 / 05$ & Tue 2/1/05 & \\
\hline 758 & $\checkmark$ & PC Critical Tile - Deliver the critical tile to NIPSCO & Tue 2/1/05 & Tue 2/1/05 & \\
\hline 759 & $\checkmark$ & PC Critical Tile - Create QA packages for the critical tile & Tue 2/1/05 & Tue 2/1/05 & \\
\hline 760 & $\checkmark$ & PC Critical Tile - Run DWGVerify against critical tile and review results & Tue 2/1/05 & Tue 2/1/05 & \\
\hline 761 & $\checkmark$ & PC Critical Tile - Run edge match against the critical tile and review results & Tue $2 / 1 / 05$ & Tue 2/1/05 & \\
\hline 762 & $\checkmark$ & PC Critical Tile - QA the critical tile, review issues, etc & Wed 2/2/05 & Tue 2/15/05 & \\
\hline 763 & $\checkmark$ & PC Critical Tile - Correct Elementool issues & Wed 2/16/05 & Mon 2/21/05 & \\
\hline 764 & $\checkmark$ & PC Critical Tile - Re-QA the Elementool issues & Tue 2/22/05 & Wed 2/23/05 & \\
\hline 765 & $\checkmark$ & PC Critical Tile - Place the critical tile back into production & Fri 2/25/05 & Fri 2/25/05 & \\
\hline 766 & $\checkmark$ & Rectify the Critical DD Tile & Wed 1/26/05 & Mon 2/21/05 & \\
\hline 767 & $\checkmark$ & DD Critical Tile - Freeze and send the critical tile to data migration vendor & Wed 1/26/05 & Wed $1 / 26 / 05$ & \\
\hline 768 & $\checkmark$ & DD Critical Tile - Rectify the critical tile & Wed 1/26/05 & Wed 2/2/05 & \\
\hline 769 & $\checkmark$ & DD Critical Tile - Review / answer data issues for data migration vendor & Wed 2/2/05 & Wed 2/2/05 & \\
\hline 770 & $\checkmark$ & DD Critical Tile - Deliver the critical tile to NIPSCO & Tue $2 / 8 / 05$ & Tue $2 / 8 / 05$ & \\
\hline 771 & $\checkmark$ & DD Critical Tile - Create QA packages for the critical tile & Tue 2/8/05 & Tue 2/8/05 & \\
\hline 772 & $\checkmark$ & DD Critical Tile - Run DWGVerify against critical tile and review results & Tue 2/8/05 & Tue $2 / 8 / 05$ & \\
\hline 773 & $\checkmark$ & DD Critical Tile - Run edge match against the critical tile and review results & Tue 2/8/05 & Tue 2/8/05 & \\
\hline 774 & $\checkmark$ & DD Critical Tile - QA the critical tile, review issues, etc & Tue $2 / 8 / 05$ & Thu $2 / 17 / 05$ & \\
\hline 775 & $\checkmark$ & DD Critical Tile - Correct Elementool issues & Thu $2 / 17 / 05$ & Fri 2/18/05 & \\
\hline 776 & $\checkmark$ & DD Critical Tile - Re-QA the Elementool issues & Fri $2 / 18 / 05$ & Mon 2/21/05 & \\
\hline 777 & $\checkmark$ & DD Critical Tile - Place the critical tile back into production & Fri 2/18/05 & Fri 2/18/05 & \\
\hline 778 & $\checkmark$ & Rectify the Critical QB Tile & Wed 1/26/05 & Fri 2/25/05 & \\
\hline 779 & $\checkmark$ & QB Critical Tile - Freeze and send the critical tile to data migration vendor & Wed 1/26/05 & Wed $1 / 26 / 05$ & \\
\hline 780 & $\checkmark$ & QB Critical Tile - Rectify the critical tile & Wed 1/26/05 & Wed 2/2/05 & \\
\hline 781 & $\checkmark$ & QB Critical Tile - Review / answer data issues for data migration vendor & Wed 2/2/05 & Wed 2/2/05 & \\
\hline 782 & $\checkmark$ & QB Critical Tile - Deliver the critical tile to NIPSCO & Fri 2/11/05 & Fri 2/11/05 & \\
\hline $\begin{array}{l}\text { NIPSC } \\
\text { Confid }\end{array}$ & $\begin{array}{l}\text { Co GIS } \\
\text { lential }\end{array}$ & Page 17 & & $\begin{array}{r}\text { Last Updat } \\
\text { Print }\end{array}$ & $\begin{array}{l}\text { ed on Tue } 7 / 3 / 07 \\
\text { ed on Fri 8/17/07 }\end{array}$ \\
\hline
\end{tabular}




\begin{tabular}{|c|c|c|c|c|c|}
\hline \multicolumn{6}{|c|}{ AEDR Construction Phase I Project Plan } \\
\hline ID & ( & Task Name & Start & Finish & \\
\hline 783 & $\checkmark$ & QB Critical Tile - Create QA packages for the critical tile & Fri 2/11/05 & Fri 2/11/05 & \\
\hline 784 & $\checkmark$ & QB Critical Tile - Run DWGVerify against critical tile and review results & Fri 2/11/05 & Fri 2/11/05 & \\
\hline 785 & $\checkmark$ & QB Critical Tile - Run edge match against the critical tile and review results & Fri 2/11/05 & Fri 2/11/05 & \\
\hline 786 & $\checkmark$ & QB Critical Tile - QA the critical tile, review issues, etc & Fri 2/11/05 & Tue 2/22/05 & \\
\hline 787 & $\checkmark$ & QB Critical Tile - Correct Elementool issues & Tue $2 / 22 / 05$ & Wed 2/23/05 & \\
\hline 788 & $\checkmark$ & QB Critical Tile - Re-QA the Elementool issues & Wed 2/23/05 & Thu 2/24/05 & \\
\hline 789 & $\checkmark$ & QB Critical Tile - Place the critical tile back into production & Fri 2/25/05 & Fri 2/25/05 & \\
\hline 790 & $\checkmark$ & Rectify the Critical CC Tile & Thu 2/3/05 & Mon 3/14/05 & \\
\hline 791 & $\checkmark$ & CC Critical Tile - Freeze and send the critical tile to data migration vendor & Thu $2 / 3 / 05$ & Thu 2/3/05 & \\
\hline 792 & $\checkmark$ & CC Critical Tile - Resolve edge matching with BC & Fri 2/18/05 & Fri 2/18/05 & \\
\hline 793 & $\checkmark$ & CC Critical Tile - Rectify the critical tile & Thu $2 / 3 / 05$ & Tue 2/15/05 & \\
\hline 794 & $\checkmark$ & CC Critical Tile - Review / answer data issues for data migration vendor & Fri 2/18/05 & Fri 2/18/05 & \\
\hline 795 & $\checkmark$ & CC Critical Tile - Deliver the critical tile to NIPSCO & Wed 2/23/05 & Wed 2/23/05 & \\
\hline 796 & $\checkmark$ & CC Critical Tile - Create QA packages for the critical tile & Wed 2/23/05 & Wed 2/23/05 & \\
\hline 797 & $\checkmark$ & CC Critical Tile - Run DWGVerify against critical tile and review results & Wed 2/23/05 & Wed 2/23/05 & \\
\hline 798 & $\checkmark$ & CC Critical Tile - Run edge match against the critical tile and review results & Wed 2/23/05 & Wed 2/23/05 & \\
\hline 799 & $\checkmark$ & CC Critical Tile - QA the critical tile, review issues, etc & Thu 2/24/05 & Wed 3/9/05 & \\
\hline 800 & $\checkmark$ & CC Critical Tile - Correct Elementool issues & Thu $3 / 10 / 05$ & Thu $3 / 10 / 05$ & \\
\hline 801 & $\checkmark$ & CC Critical Tile - Re-QA the Elementool issues & Fri 3/11/05 & Fri 3/11/05 & \\
\hline 802 & $\checkmark$ & CC Critical Tile - Place the critical tile back into production & Mon 3/14/05 & Mon 3/14/05 & \\
\hline 803 & $\checkmark$ & Rectify the Critical WB Tile & Fri 2/4/05 & Fri 3/4/05 & \\
\hline 804 & $\checkmark$ & WB Critical Tile - Freeze and send the critical tile to data migration vendor & Fri 2/4/05 & Fri 2/4/05 & \\
\hline 805 & $\checkmark$ & WB Critical Tile - Rectify the critical tile & Fri 2/4/05 & Fri 2/11/05 & \\
\hline 806 & $\checkmark$ & WB Critical Tile - Review / answer data issues for data migration vendor & Fri 2/11/05 & Fri 2/11/05 & \\
\hline 807 & $\checkmark$ & WB Critical Tile - Deliver the critical tile to NIPSCO & Fri 2/11/05 & Fri 2/11/05 & \\
\hline 808 & $\checkmark$ & WB Critical Tile - Create QA packages for the critical tile & Fri 2/11/05 & Fri 2/11/05 & \\
\hline 809 & $\checkmark$ & WB Critical Tile - Run DWGVerify against critical tile and review results & Fri 2/11/05 & Fri 2/11/05 & \\
\hline 810 & $\checkmark$ & WB Critical Tile - Run edge match against the critical tile and review results & Fri 2/11/05 & Fri 2/11/05 & \\
\hline 811 & $\checkmark$ & WB Critical Tile - QA the critical tile, review issues, etc & Fri 2/11/05 & Fri 2/18/05 & \\
\hline 812 & $\checkmark$ & WB Critical Tile - Correct Elementool issues & Thu 2/24/05 & Thu 2/24/05 & \\
\hline 813 & $\checkmark$ & WB Critical Tile - Re-QA the Elementool issues & Fri 2/25/05 & Fri 2/25/05 & \\
\hline 814 & $\checkmark$ & WB Critical Tile - Place the critical tile back into production & Fri 3/4/05 & Fri 3/4/05 & \\
\hline 815 & $\checkmark$ & Rectify the Critical EC Tile & Fri 2/4/05 & Thu 3/10/05 & \\
\hline 816 & $\checkmark$ & EC Critical Tile - Freeze and send the critical tile to data migration vendor & Fri 2/4/05 & Fri 2/4/05 & \\
\hline 817 & $\checkmark$ & EC Critical Tile - Rectify the critical tile & Fri 2/4/05 & Wed 2/16/05 & \\
\hline 818 & $\checkmark$ & EC Critical Tile - Review / answer data issues for data migration vendor & Wed 2/16/05 & Wed 2/16/05 & \\
\hline 819 & $\checkmark$ & EC Critical Tile - Deliver the critical tile to NIPSCO & Fri 2/25/05 & Fri 2/25/05 & \\
\hline 820 & $\checkmark$ & EC Critical Tile - Create QA packages for the critical tile & Wed 3/2/05 & Wed 3/2/05 & \\
\hline 821 & $\checkmark$ & EC Critical Tile - Run DWGVerify against critical tile and review results & Wed $3 / 2 / 05$ & Wed 3/2/05 & \\
\hline 822 & $\checkmark$ & EC Critical Tile - Run edge match against the critical tile and review results & Wed 3/2/05 & Wed 3/2/05 & \\
\hline 823 & $\checkmark$ & EC Critical Tile - Correct Elementool issues & Tue $3 / 8 / 05$ & Wed 3/9/05 & \\
\hline 824 & $\checkmark$ & EC Critical Tile - Re-QA the Elementool issues & Wed 3/9/05 & Thu $3 / 10 / 05$ & \\
\hline 825 & $\checkmark$ & EC Critical Tile - Place the critical tile back into production & Thu $3 / 10 / 05$ & Thu $3 / 10 / 05$ & \\
\hline 826 & $\checkmark$ & Rectify the Critical ED Tile & Fri 2/4/05 & Thu 3/10/05 & \\
\hline 827 & $\checkmark$ & ED Critical Tile - Freeze and send the critical tile to data migration vendor & Fri 2/4/05 & Fri 2/4/05 & \\
\hline 828 & $\checkmark$ & ED Critical Tile - Rectify the critical tile & Fri 2/4/05 & Wed 2/16/05 & \\
\hline $\begin{array}{l}\text { NIPSC } \\
\text { Confid }\end{array}$ & $\begin{array}{l}\text { Co GIS } \\
\text { lential }\end{array}$ & Page 18 & & $\begin{array}{r}\text { Last Updat } \\
\text { Print }\end{array}$ & $\begin{array}{l}\text { ed on Tue } 7 / 3 / 07 \\
\text { ed on Fri 8/17/07 }\end{array}$ \\
\hline
\end{tabular}




\section{\begin{tabular}{|ll}
\hline & AEDR Construction Phase I Project Plan
\end{tabular}}

\begin{tabular}{|c|c|c|c|c|c|}
\hline ID & (a) & Task Name & Start & Finish & \\
\hline 829 & $\checkmark$ & ED Critical Tile - Review / answer data issues for data migration vendor & Wed 2/16/05 & Wed 2/16/05 & \\
\hline 830 & $\checkmark$ & ED Critical Tile - Deliver the critical tile to NIPSCO & Fri 2/25/05 & Fri 2/25/05 & \\
\hline 831 & $\checkmark$ & ED Critical Tile - Create QA packages for the critical tile & Wed 3/2/05 & Wed 3/2/05 & \\
\hline 832 & $\checkmark$ & ED Critical Tile - Run DWGVerify against critical tile and review results & Wed 3/2/05 & Wed 3/2/05 & \\
\hline 833 & $\checkmark$ & ED Critical Tile - Run edge match against the critical tile and review results & Wed 3/2/05 & Wed 3/2/05 & \\
\hline 834 & $\checkmark$ & ED Critical Tile - QA the critical tile, review issues, etc & Wed 3/2/05 & Tue $3 / 8 / 05$ & \\
\hline 835 & $\checkmark$ & ED Critical Tile - Correct Elementool issues & Tue $3 / 8 / 05$ & Wed 3/9/05 & \\
\hline 836 & $\checkmark$ & ED Critical Tile - Re-QA the Elementool issues & Wed 3/9/05 & Thu 3/10/05 & \\
\hline 837 & $\checkmark$ & ED Critical Tile - Place the critical tile back into production & Thu $3 / 10 / 05$ & Thu 3/10/05 & \\
\hline 839 & $\checkmark$ & DC Critical Tile - Freeze and send the critical tile to data migration vendor & Tue $2 / 15 / 05$ & Tue $2 / 15 / 05$ & \\
\hline 840 & $\checkmark$ & DC Critical Tile - Rectify the critical tile & Tue 2/15/05 & Wed 2/23/05 & \\
\hline 841 & $\checkmark$ & DC Critical Tile - Review / answer data issues for data migration vendor & Wed 2/23/05 & Wed 2/23/05 & \\
\hline 842 & $\checkmark$ & DC Critical Tile - Deliver the critical tile to NIPSCO & Fri 3/4/05 & Fri 3/4/05 & \\
\hline 843 & $\checkmark$ & DC Critical Tile - Create QA packages for the critical tile & Mon 3/7/05 & Mon 3/7/05 & \\
\hline 844 & $\checkmark$ & DC Critical Tile - Run DWGVerify against critical tile and review results & Mon 3/7/05 & Mon 3/7/05 & \\
\hline 845 & $\checkmark$ & DC Critical Tile - Run edge match against the critical tile and review results & Mon 3/7/05 & Mon 3/7/05 & \\
\hline 846 & $\checkmark$ & DC Critical Tile - QA the critical tile, review issues, etc & Mon 3/7/05 & Fri 3/11/05 & \\
\hline 847 & $\checkmark$ & DC Critical Tile - Correct Elementool issues & Fri 3/11/05 & Mon 3/14/05 & \\
\hline 848 & $\checkmark$ & DC Critical Tile - Re-QA the Elementool issues & Mon 3/14/05 & Tue 3/15/05 & \\
\hline 850 & $\checkmark$ & Rectify the Critical FD Tile & Tue 2/15/05 & Tue 3/15/05 & \\
\hline 851 & $\checkmark$ & FD Critical Tile - Freeze and send the critical tile to data migration vendor & Tue 2/15/05 & Tue 2/15/05 & \\
\hline 852 & $\checkmark$ & FD Critical Tile - Rectify the critical tile & Tue 2/15/05 & Wed 2/23/05 & \\
\hline 853 & $\checkmark$ & FD Critical Tile - Review / answer data issues for data migration vendor & Wed 2/23/05 & Wed 2/23/05 & \\
\hline 854 & $\checkmark$ & FD Critical Tile - Deliver the critical tile to NIPSCO & Fri 3/4/05 & Fri 3/4/05 & \\
\hline 855 & $\checkmark$ & FD Critical Tile - Create QA packages for the critical tile & Mon 3/7/05 & Mon 3/7/05 & \\
\hline 856 & $\checkmark$ & FD Critical Tile - Run DWGVerify against critical tile and review results & Mon 3/7/05 & Mon 3/7/05 & \\
\hline 857 & $\checkmark$ & FD Critical Tile - Run edge match against the critical tile and review results & Mon 3/7/05 & Mon 3/7/05 & \\
\hline 858 & $\checkmark$ & FD Critical Tile - QA the critical tile, review issues, etc & Mon 3/7/05 & Fri 3/11/05 & \\
\hline 859 & $\checkmark$ & FD Critical Tile - Correct Elementool issues & Fri 3/11/05 & Mon 3/14/05 & \\
\hline 860 & $\checkmark$ & FD Critical Tile - Re-QA the Elementool issues & Mon 3/14/05 & Tue 3/15/05 & \\
\hline 861 & $\checkmark$ & FD Critical Tile - Place the critical tile back into production & Tue 3/15/05 & Tue 3/15/05 & \\
\hline 862 & $\checkmark$ & Rectify the Critical CD Tile & Wed 2/23/05 & Thu $3 / 17 / 05$ & \\
\hline 863 & $\checkmark$ & CD Critical Tile - Freeze and send the critical tile to data migration vendor & Wed 2/23/05 & Wed 2/23/05 & \\
\hline 864 & $\checkmark$ & CD Critical Tile - Rectify the critical tile & Wed 2/23/05 & Wed 3/2/05 & \\
\hline 865 & $\checkmark$ & CD Critical Tile - Review / answer data issues for data migration vendor & Wed 3/2/05 & Wed 3/2/05 & \\
\hline 866 & $\checkmark$ & CD Critical Tile - Deliver the critical tile to NIPSCO & Mon 3/7/05 & Mon 3/7/05 & \\
\hline 867 & $\checkmark$ & CD Critical Tile - Create QA packages for the critical tile & Wed 3/9/05 & Wed 3/9/05 & \\
\hline 868 & $\checkmark$ & CD Critical Tile - Run DWGVerify against critical tile and review results & Wed 3/9/05 & Wed 3/9/05 & \\
\hline 869 & $\checkmark$ & CD Critical Tile - Run edge match against the critical tile and review results & Wed 3/9/05 & Wed 3/9/05 & \\
\hline 870 & $\checkmark$ & CD Critical Tile - QA the critical tile, review issues, etc & Wed 3/9/05 & Tue 3/15/05 & \\
\hline 873 & $\checkmark$ & CD Critical Tile - Place the critical tile back into production & Thu 3/17/05 & Thu $3 / 17 / 05$ & \\
\hline 874 & $\checkmark$ & Data Corruption Management & Fri 5/27/05 & Fri 1/6/06 & \\
\hline $\begin{array}{l}\text { NIPSC } \\
\text { Confide }\end{array}$ & $\begin{array}{l}\text { O GIS } \\
\text { ential }\end{array}$ & Page 19 & & $\begin{array}{l}\text { Last Update } \\
\text { Print }\end{array}$ & $\begin{array}{l}\text { on Tue } 7 / 3 / 07 \\
\text { on Fri 8/17/07 }\end{array}$ \\
\hline
\end{tabular}




\begin{tabular}{|c|c|c|c|c|c|}
\hline \multicolumn{6}{|c|}{ AEDR Construction Phase I Project Plan } \\
\hline ID & ( & Task Name & Start & Finish & \\
\hline 875 & $\checkmark$ & Identify data migration vendor-introduced Data Corruption & Fri 5/27/05 & Tue $10 / 11 / 05$ & \\
\hline 876 & $\checkmark$ & Clean up the critical corrupted data & Fri 6/3/05 & Fri 1/6/06 & \\
\hline 877 & & GIS Data Migration & Mon 3/1/04 & Wed 5/2/07 & \\
\hline 878 & & Miscellaneous Data Migration Setup Tasks & Tue 9/28/04 & Tue $1 / 3 / 06$ & \\
\hline 879 & $\checkmark$ & Setup Automated Testing & Tue 9/28/04 & Wed 11/3/04 & \\
\hline 880 & $\checkmark$ & Get license key and install automated testing software on a common PC & Fri 10/1/04 & Fri 10/1/04 & \\
\hline 881 & $\checkmark$ & Apply initial automated testing software configuration to NIPSCO environment & Mon 10/18/04 & Mon 10/18/04 & \\
\hline 882 & $\checkmark$ & Download and install NIPSCO data from FTP server & Tue 9/28/04 & Tue 9/28/04 & \\
\hline 883 & $\checkmark$ & Finalize initial Landbase tests & Wed 9/29/04 & Wed 9/29/04 & \\
\hline 884 & $\checkmark$ & Create generated tests for landbase, gas and electric & Thu 9/30/04 & Thu 9/30/04 & \\
\hline 885 & $\checkmark$ & Deliver initial landbase tests, and generated tests for gas and electric & Mon 10/4/04 & Mon 10/4/04 & \\
\hline 886 & $\checkmark$ & Initial configuration of Nipsco-specific tests for electric & Mon 10/18/04 & Fri 10/22/04 & \\
\hline 887 & $\checkmark$ & Initial configuration of Nipsco-specific tests for gas & Mon 10/18/04 & Fri 10/22/04 & \\
\hline 888 & $\checkmark$ & Initial configuration Nipsco-specific tests for land & Tue 10/19/04 & Mon 10/25/04 & \\
\hline 889 & $\checkmark$ & Deliver Nipsco-specific tests for gas and electric & Wed 10/6/04 & Wed 10/6/04 & \\
\hline 890 & $\checkmark$ & Review tests and test results - Electric / log tickets & Thu 10/21/04 & Thu $10 / 21 / 04$ & \\
\hline 891 & $\checkmark$ & Review tests and test results - Gas / log tickets & Fri 10/22/04 & Fri 10/22/04 & \\
\hline 892 & $\checkmark$ & Review tests and test results - Land / log tickets & Mon 10/25/04 & Mon 10/25/04 & \\
\hline 893 & $\checkmark$ & Revise automated tests based on review comments & Mon 11/1/04 & Tue $11 / 2 / 04$ & \\
\hline 894 & $\checkmark$ & Deliver final automated testing software configuration to Nipsco & Wed 11/3/04 & Wed $11 / 3 / 04$ & \\
\hline 895 & $\checkmark$ & Finalize the Conductor Migration Specifications & Fri 10/8/04 & Fri 12/17/04 & \\
\hline 896 & $\checkmark$ & Coordinate meetings/logistics for all migration add-ons (secondary conductor, etc.) & Fri 10/8/04 & Fri 10/8/04 & \\
\hline 897 & $\checkmark$ & Complete the specifications for all migration add-ons (secondary conductor, etc.) & Thu $10 / 14 / 04$ & Fri 10/15/04 & \\
\hline 898 & $\checkmark$ & Provide conductor modeling details and data "preview" to data migration vendor & Tue 11/2/04 & Tue 11/2/04 & \\
\hline 899 & $\checkmark$ & Create draft secondary conductor creation specifications & Fri 11/5/04 & Fri 11/5/04 & \\
\hline 900 & $\checkmark$ & Review draft secondary conductor creation specifications & Tue $11 / 30 / 04$ & Tue 11/30/04 & \\
\hline 901 & $\checkmark$ & Finalize the secondary conductor creation specifications & Fri 12/17/04 & Fri $12 / 17 / 04$ & \\
\hline 902 & 国) & Write Scripts to create data cleanup reports of error codes on the full delivery of data & Fri 12/30/05 & Tue $1 / 3 / 06$ & \\
\hline 903 & $\checkmark$ & Data Migration Performance Issues & Fri 11/19/04 & Fri 11/18/05 & \\
\hline 904 & $\checkmark$ & Compare metrics & Fri 11/19/04 & Fri 11/19/04 & \\
\hline 905 & $\checkmark$ & Write-up next steps for the performance issues & Fri 11/19/04 & Fri 11/19/04 & \\
\hline 906 & $\checkmark$ & Data migration performance - next steps & Tue $12 / 7 / 04$ & Tue $12 / 7 / 04$ & \\
\hline 907 & $\checkmark$ & Reset Performance Metrics based on Delivery 6.0 & Fri 11/18/05 & Fri 11/18/05 & \\
\hline 908 & $\checkmark$ & Finalize the Source/Target Record Reconciliation Process & Wed 10/27/04 & Fri 12/17/04 & \\
\hline 909 & $\checkmark$ & Record Counting - Review record count comparison status & Wed 10/27/04 & Wed 10/27/04 & \\
\hline 910 & $\checkmark$ & Review reconciliation document and provide review comments & Wed 12/8/04 & Wed 12/8/04 & \\
\hline 911 & $\checkmark$ & Finalize the reconciliation document and project plan based on review comments & Fri 12/17/04 & Fri $12 / 17 / 04$ & \\
\hline 912 & $\checkmark$ & Develop Record Count Reconciliation Functionality & Wed $1 / 12 / 05$ & Wed 1/26/05 & \\
\hline 913 & $\checkmark$ & Develop specs / process for source (DWG File) record count report & Wed 1/12/05 & Wed 1/12/05 & \\
\hline 914 & $\checkmark$ & Develop source (DWG File) record count report & Wed 1/12/05 & Wed 1/12/05 & \\
\hline 915 & $\checkmark$ & Develop specs / process for target (ArcSDE) record count report & Wed 1/12/05 & Wed 1/12/05 & \\
\hline 916 & $\checkmark$ & Develop record count reconciliation checklist & Mon $1 / 24 / 05$ & Mon 1/24/05 & \\
\hline 917 & $\checkmark$ & Finalize the record count checklist & Wed $1 / 26 / 05$ & Wed 1/26/05 & \\
\hline 918 & $\checkmark$ & Determine Solution for Migration of Duplicate Centerlines Between Outfield Tiles & Thu 12/9/04 & Tue 1/25/05 & \\
\hline 919 & $\checkmark$ & Document options for handling duplicate centerlines & Thu $12 / 9 / 04$ & Thu 12/9/04 & \\
\hline 920 & $\checkmark$ & Review and determine option for handling duplicate features & Thu $1 / 6 / 05$ & Thu $1 / 6 / 05$ & \\
\hline $\begin{array}{l}\text { NIPSC } \\
\text { Confid }\end{array}$ & $\begin{array}{l}\text { Co GIS } \\
\text { lential }\end{array}$ & Page 20 & & $\begin{array}{r}\text { Last Updat } \\
\text { Print }\end{array}$ & $\begin{array}{l}\text { ed on Tue } 7 / 3 / 07 \\
\text { ed on Fri 8/17/07 }\end{array}$ \\
\hline
\end{tabular}




\begin{tabular}{|c|c|c|c|c|c|}
\hline \multicolumn{6}{|c|}{ AEDR Construction Phase I Project Plan } \\
\hline ID & ( & Task Name & Start & Finish & \\
\hline 921 & $\checkmark$ & Merge the Centerline and County Boundary files into a single map & Fri $1 / 14 / 05$ & Fri $1 / 14 / 05$ & \\
\hline 922 & $\checkmark$ & Review the merged Centerline / County map and identify $20-30$ candidates & Mon 1/24/05 & Mon $1 / 24 / 05$ & \\
\hline 923 & $\checkmark$ & Create a report of the results & Tue $1 / 25 / 05$ & Tue $1 / 25 / 05$ & \\
\hline 924 & $\checkmark$ & GIS Data Migration - Iteration 1 (Small Area and Subset of Feature Classes) & Mon 6/21/04 & Mon 10/25/04 & \\
\hline 925 & $\checkmark$ & GIS Data Migration - Additional AutoCAD File Sources & Wed 8/18/04 & Mon 8/23/04 & \\
\hline 926 & $\checkmark$ & Review additional sources for data migration matrix & Wed 8/18/04 & Wed 8/18/04 & \\
\hline 927 & $\checkmark$ & Send additional data source samples to data migration vendor & Mon 8/23/04 & Mon 8/23/04 & \\
\hline 928 & $\checkmark$ & Update the Data Matrix for Migration Iteration 1 & Fri 7/9/04 & Fri 8/20/04 & \\
\hline 929 & $\checkmark$ & High Priority Gas & Fri 7/9/04 & Fri 7/16/04 & \\
\hline 930 & $\checkmark$ & Meeting to review critical Gas matrix spreadsheets & Fri 7/9/04 & Fri $7 / 9 / 04$ & \\
\hline 931 & $\checkmark$ & Log Gas matrix changes into Elementool & Fri 7/9/04 & Fri $7 / 9 / 04$ & \\
\hline 932 & $\checkmark$ & Research miscellaneous gas matrix issues & Mon 7/12/04 & Mon 7/12/04 & \\
\hline 933 & $\checkmark$ & Apply the gas matrix changes & Fri 7/16/04 & Fri 7/16/04 & \\
\hline 934 & $\checkmark$ & Deliver the gas matrix spreadsheet(s) to data migration vendor & Fri 7/16/04 & Fri 7/16/04 & \\
\hline 935 & $\checkmark$ & High Priority Land (Critical Objects Only) & Fri 7/9/04 & Mon $7 / 19 / 04$ & \\
\hline 936 & $\checkmark$ & Prep for Land matrix review & Fri 7/9/04 & Fri 7/9/04 & \\
\hline 937 & $\checkmark$ & Meeting to review critical Land matrix spreadsheets & Fri 7/9/04 & Fri $7 / 9 / 04$ & \\
\hline 938 & $\checkmark$ & Log Land matrix changes into Elementool & Fri 7/9/04 & Fri $7 / 9 / 04$ & \\
\hline 939 & $\checkmark$ & Research miscellaneous land matrix issues & Mon 7/12/04 & Mon $7 / 12 / 04$ & \\
\hline 940 & $\checkmark$ & Apply the land matrix changes & Mon 7/19/04 & Mon $7 / 19 / 04$ & \\
\hline 941 & $\checkmark$ & Deliver the land matrix spreadsheet(s) to data migration vendor & Mon $7 / 19 / 04$ & Mon $7 / 19 / 04$ & \\
\hline 942 & $\checkmark$ & Update the Gas Matrix & Wed 7/21/04 & Fri $7 / 23 / 04$ & \\
\hline 943 & $\checkmark$ & Meeting to determine schedule for reviewing / updating gas matrix spreadsheets & Wed $7 / 21 / 04$ & Wed $7 / 21 / 04$ & \\
\hline 944 & $\checkmark$ & Update the Gas Casing matrix spreadsheet & Wed $7 / 21 / 04$ & Wed $7 / 21 / 04$ & \\
\hline 945 & $\checkmark$ & Update the Gas CP Rectifier matrix spreadsheet & Wed 7/21/04 & Wed $7 / 21 / 04$ & \\
\hline 946 & $\checkmark$ & Update the Gas Pipe Change matrix spreadsheet & Wed $7 / 21 / 04$ & Wed $7 / 21 / 04$ & \\
\hline 947 & $\checkmark$ & Update the Gas Pipe Exposure matrix spreadsheet & Thu $7 / 22 / 04$ & Thu $7 / 22 / 04$ & \\
\hline 948 & $\checkmark$ & Update the Gas Drip matrix spreadsheet & Fri 7/23/04 & Fri $7 / 23 / 04$ & \\
\hline 949 & $\checkmark$ & Update the Gas Insulated Coupling matrix spreadsheet & Fri 7/23/04 & Fri 7/23/04 & \\
\hline 950 & $\checkmark$ & Update the Gas Pressure Control matrix spreadsheet & Fri $7 / 23 / 04$ & Fri $7 / 23 / 04$ & \\
\hline 951 & $\checkmark$ & Update the Gas Dead End matrix spreadsheet & Fri $7 / 23 / 04$ & Fri $7 / 23 / 04$ & \\
\hline 952 & $\checkmark$ & Update the Gas Pipe Marker matrix spreadsheet & Fri 7/23/04 & Fri 7/23/04 & \\
\hline 953 & $\checkmark$ & Update the Gas Company Use matrix spreadsheet & Fri $7 / 23 / 04$ & Fri $7 / 23 / 04$ & \\
\hline 954 & $\checkmark$ & Update the Gas Leak Report matrix spreadsheet & Fri 7/23/04 & Fri $7 / 23 / 04$ & \\
\hline 955 & $\checkmark$ & Update the Gas Leak Survey Area matrix spreadsheet & Fri $7 / 23 / 04$ & Fri $7 / 23 / 04$ & \\
\hline 956 & $\checkmark$ & Update the Gas Service Stub matrix spreadsheet & Fri 7/23/04 & Fri $7 / 23 / 04$ & \\
\hline 957 & $\checkmark$ & Update the Gas Squeeze Off matrix spreadsheet & Fri $7 / 23 / 04$ & Fri $7 / 23 / 04$ & \\
\hline 958 & $\checkmark$ & Review and Finalize the Gas Matrix & Fri 7/30/04 & Fri 8/6/04 & \\
\hline 959 & $\checkmark$ & Review and verify gas matrix spreadsheets & Fri $7 / 30 / 04$ & Fri $7 / 30 / 04$ & \\
\hline 960 & $\checkmark$ & Meeting to review gas and electric customer issues & Fri 8/6/04 & Fri 8/6/04 & \\
\hline 961 & $\checkmark$ & Update the Gas Section matrix spreadsheet & Mon 8/2/04 & Mon 8/2/04 & \\
\hline 962 & $\checkmark$ & Update the Land Bridge Matrix & Thu 8/12/04 & Thu 8/12/04 & \\
\hline 963 & $\checkmark$ & Review Land Bridge matrix spreadsheet & Thu 8/12/04 & Thu 8/12/04 & \\
\hline 964 & $\checkmark$ & Log Land Bridge matrix changes into Elementool & Thu 8/12/04 & Thu 8/12/04 & \\
\hline 965 & $\checkmark$ & Apply the matrix changes for Land Bridge & Thu 8/12/04 & Thu 8/12/04 & \\
\hline 966 & $\checkmark$ & Initial delivery of the Land Bridge matrix & Thu 8/12/04 & Thu 8/12/04 & \\
\hline $\begin{array}{l}\text { NIPSC } \\
\text { Confid }\end{array}$ & $\begin{array}{l}\text { Co GIS } \\
\text { lential }\end{array}$ & Page 21 & & $\begin{array}{r}\text { Last Updat } \\
\text { Print }\end{array}$ & $\begin{array}{l}\text { ed on Tue } 7 / 3 / 07 \\
\text { ed on Fri 8/17/07 }\end{array}$ \\
\hline
\end{tabular}




\begin{tabular}{|c|c|c|c|c|c|}
\hline \multicolumn{6}{|c|}{ AEDR Construction Phase I Project Plan } \\
\hline ID & ( & Task Name & Start & Finish & \\
\hline 967 & $\checkmark$ & Update the Land County Matrix & Fri 8/13/04 & Mon 8/16/04 & \\
\hline 968 & $\checkmark$ & Review Land County matrix spreadsheet & Fri 8/13/04 & Fri 8/13/04 & \\
\hline 969 & $\checkmark$ & Log Land County matrix changes into Elementool & Fri 8/13/04 & Fri 8/13/04 & \\
\hline 970 & $\checkmark$ & Apply the matrix changes for Land County & Fri 8/13/04 & Fri 8/13/04 & \\
\hline 971 & $\checkmark$ & Initial delivery of the Land County matrix & Mon 8/16/04 & Mon 8/16/04 & \\
\hline 972 & $\checkmark$ & Update the Land DG Grid Matrix & Fri 8/13/04 & Fri 8/13/04 & \\
\hline 973 & $\checkmark$ & Review Land DG Grid matrix spreadsheet & Fri 8/13/04 & Fri 8/13/04 & \\
\hline 974 & $\checkmark$ & Log Land DG Grid matrix changes into Elementool & Fri 8/13/04 & Fri 8/13/04 & \\
\hline 975 & $\checkmark$ & Apply the matrix changes for Land DG Grid & Fri 8/13/04 & Fri 8/13/04 & \\
\hline 976 & $\checkmark$ & Initial delivery of the Land DG Grid matrix & Fri 8/13/04 & Fri 8/13/04 & \\
\hline 977 & $\checkmark$ & Update the Land Easement Matrix & Fri 8/13/04 & Mon 8/16/04 & \\
\hline 978 & $\checkmark$ & Review Land Easement matrix spreadsheet & Fri 8/13/04 & Fri 8/13/04 & \\
\hline 979 & $\checkmark$ & Log Land Easement matrix changes into Elementool & Mon 8/16/04 & Mon 8/16/04 & \\
\hline 980 & $\checkmark$ & Apply the matrix changes for Land Easement & Mon 8/16/04 & Mon 8/16/04 & \\
\hline 981 & $\checkmark$ & Initial delivery of the Land Easement matrix & Mon 8/16/04 & Mon 8/16/04 & \\
\hline 982 & $\checkmark$ & Update the Land Franchise BoundaryMatrix & Mon 8/16/04 & Mon 8/16/04 & \\
\hline 983 & $\checkmark$ & Review Land Franchise Boundary matrix spreadsheet & Mon 8/16/04 & Mon 8/16/04 & \\
\hline 984 & $\checkmark$ & Log Land Franchise Boundary matrix changes into Elementool & Mon 8/16/04 & Mon 8/16/04 & \\
\hline 985 & $\checkmark$ & Apply the matrix changes for Land Franchise Boundary & Mon 8/16/04 & Mon 8/16/04 & \\
\hline 986 & $\checkmark$ & Initial delivery of the Land Franchise Boundary matrix & Mon 8/16/04 & Mon 8/16/04 & \\
\hline 987 & $\checkmark$ & Update the Land Discrepancy Matrix & Mon 8/16/04 & Mon 8/16/04 & \\
\hline 988 & $\checkmark$ & Review Land Discrepancy matrix spreadsheet & Mon 8/16/04 & Mon 8/16/04 & \\
\hline 989 & $\checkmark$ & Apply the matrix changes for Land Discrepancy & Mon 8/16/04 & Mon 8/16/04 & \\
\hline 990 & $\checkmark$ & Initial delivery of the Land Discrepancy matrix & Mon 8/16/04 & Mon 8/16/04 & \\
\hline 991 & $\checkmark$ & Update the Land Linear Water Matrix & Mon 8/16/04 & Mon 8/16/04 & \\
\hline 992 & $\checkmark$ & Review Land Linear Water matrix spreadsheet & Mon 8/16/04 & Mon 8/16/04 & \\
\hline 993 & $\checkmark$ & Apply the matrix changes for Land Linear Water & Mon 8/16/04 & Mon 8/16/04 & \\
\hline 994 & $\checkmark$ & Initial delivery of the Land Linear Water matrix & Mon 8/16/04 & Mon 8/16/04 & \\
\hline 995 & $\checkmark$ & Update the Land LOA Matrix & Mon 8/16/04 & Tue $8 / 17 / 04$ & \\
\hline 996 & $\checkmark$ & Review Land LOA matrix spreadsheet & Mon 8/16/04 & Mon 8/16/04 & \\
\hline 997 & $\checkmark$ & Apply the matrix changes for Land LOA & Tue $8 / 17 / 04$ & Tue $8 / 17 / 04$ & \\
\hline 998 & $\checkmark$ & Initial delivery of the Land LOA matrix & Tue $8 / 17 / 04$ & Tue $8 / 17 / 04$ & \\
\hline 999 & $\checkmark$ & Update the Land Lotline Matrix & Tue 8/17/04 & Tue 8/17/04 & \\
\hline 1000 & $\checkmark$ & Review Land Lotline matrix spreadsheet & Tue $8 / 17 / 04$ & Tue $8 / 17 / 04$ & \\
\hline 1001 & $\checkmark$ & Apply the matrix changes for Land Lotline & Tue $8 / 17 / 04$ & Tue 8/17/04 & \\
\hline 1002 & $\checkmark$ & Initial delivery of the Land Lotline matrix & Tue 8/17/04 & Tue 8/17/04 & \\
\hline 1003 & $\checkmark$ & Update the Land Lot Number Matrix & Tue $8 / 17 / 04$ & Tue 8/17/04 & \\
\hline 1004 & $\checkmark$ & Review Land Lot Number matrix spreadsheet & Tue $8 / 17 / 04$ & Tue 8/17/04 & \\
\hline 1005 & $\checkmark$ & Apply the matrix changes for Land Lot Number & Tue $8 / 17 / 04$ & Tue 8/17/04 & \\
\hline 1006 & $\checkmark$ & Initial delivery of the Land Lot Number matrix & Tue $8 / 17 / 04$ & Tue 8/17/04 & \\
\hline 1007 & $\checkmark$ & Update the Land Minor Grid Matrix & Tue 8/17/04 & Tue 8/17/04 & \\
\hline 1008 & $\checkmark$ & Review Land Minor Grid matrix spreadsheet & Tue $8 / 17 / 04$ & Tue 8/17/04 & \\
\hline 1009 & $\checkmark$ & Apply the matrix changes for Land Minor Grid & Tue 8/17/04 & Tue 8/17/04 & \\
\hline 1010 & $\checkmark$ & Initial delivery of the Land Minor Grid matrix & Tue $8 / 17 / 04$ & Tue 8/17/04 & \\
\hline 1011 & $\checkmark$ & Update the Miscellaneous Linear Land Feature Matrix & Tue 8/17/04 & Tue 8/17/04 & \\
\hline 1012 & $\checkmark$ & Review Miscellaneous Linear Land Feature matrix spreadsheet & Tue 8/17/04 & Tue 8/17/04 & \\
\hline $\begin{array}{l}\text { NIPSC } \\
\text { Confid }\end{array}$ & $\begin{array}{l}\text { Co GIS } \\
\text { lential }\end{array}$ & Page 22 & & $\begin{array}{r}\text { Last Updat } \\
\text { Print }\end{array}$ & $\begin{array}{l}\text { ed on Tue } 7 / 3 / 07 \\
\text { ed on Fri 8/17/07 }\end{array}$ \\
\hline
\end{tabular}




\section{\begin{tabular}{|ll}
\hline & AEDR Construction Phase I Project Plan
\end{tabular}}

\begin{tabular}{|l|l|l|}
\hline $1 D$ & $\mathbf{C}$ & Task Name \\
\hline 1013 & $\checkmark$ \\
\hline 1014 & $\checkmark$ \\
\hline 1015 & $\checkmark$ \\
\hline 1016 & $\checkmark$ \\
\hline 1017 & $\checkmark$ \\
\hline 1018 & $\checkmark$ \\
\hline 1019 & $\checkmark$ \\
\hline 1020 & $\checkmark$ \\
\hline 1021 & $\checkmark$ \\
\hline 1022 & $\checkmark$ \\
\hline 1023 & $\checkmark$ \\
\hline 1024 & $\checkmark$ \\
\hline 1025 & $\checkmark$ \\
\hline 1026 & $\checkmark$ \\
\hline 1027 & $\checkmark$ \\
\hline 1028 & $\checkmark$ \\
\hline 1029 & $\checkmark$ \\
\hline 1030 & $\checkmark$ \\
\hline 1031 & $\checkmark$ \\
\hline 1032 & $\checkmark$ \\
\hline 1033 & $\checkmark$ \\
\hline 1034 & $\checkmark$ \\
\hline 1035 & $\checkmark$ \\
\hline 1036 & $\checkmark$ \\
\hline 1037 & $\checkmark$ \\
\hline 1038 & $\checkmark$ \\
\hline 1039 & $\checkmark$ \\
\hline 1040 & $\checkmark$ \\
\hline 1041 & $\checkmark$ \\
\hline 1042 & $\checkmark$ \\
\hline 1043 & $\checkmark$ \\
\hline 1044 & $\checkmark$ \\
\hline 1045 & $\checkmark$ \\
\hline 1046 & $\checkmark$ \\
\hline 1047 & $\checkmark$ \\
\hline 1048 & $\checkmark$ \\
\hline 1049 & $\checkmark$ \\
\hline 1050 & $\checkmark$ \\
\hline 1051 & $\checkmark$ & \\
\hline 1052 & $\checkmark$ \\
\hline 1053 & $\checkmark$ \\
\hline 1054 & $\checkmark$ \\
\hline 1055 & $\checkmark$ \\
\hline 1056 & $\checkmark$ \\
\hline 1058 & $\checkmark$ \\
\hline$N 1 P S C$ & \\
\hline
\end{tabular}

NIPSCO GIS Confidential
Apply the matrix changes for Miscellaneous Linear Land Feature

Initial delivery of the Miscellaneous Linear Land Feature matrix

Update the Miscellaneous Point Land Feature Matrix

Review Miscellaneous Point Land Feature matrix spreadsheet

Apply the matrix changes for Miscellaneous Point Land Feature

Initial delivery of the Miscellaneous Point Land Feature matrix

Update the Land Municipality Matrix

Review Land Municipality matrix spreadsheet

Apply the matrix changes for Land Municipality

Initial delivery of the Land Municipality matrix

Update the Land PLSS Section Matrix

Review Land PLSS Section matrix spreadsheet

Apply the matrix changes for Land PLSS Section

Initial delivery of the Land PLSS Section matrix

Update the Land Political Township Matrix

Review Land Political Township matrix spreadsheet

Apply the matrix changes for Land Political Township

Initial delivery of the Land Political Township matrix

Update the Land Polygon Water Matrix

Review Land Polygon Water matrix spreadsheet

Apply the matrix changes for Land Polygon Water

Initial delivery of the Land Polygon Water matrix

Update the Land Quarter Township Grid Matrix

Review Land Quarter Township Grid matrix spreadsheet

Apply the matrix changes for Land Quarter Township Grid

Initial delivery of the Land Quarter Township Grid matrix

Update the Land Railroad Matrix

Review Land Railroad matrix spreadsheet

Apply the matrix changes for Land Railroad

Initial delivery of the Land Railroad matrix

Update the Land Reference Line Matrix

Review Land Reference Line matrix spreadsheet

Apply the matrix changes for Land Reference Line

Initial delivery of the Land Reference Line matrix

Update the Land Reference Point Matrix

Review Land Reference Point matrix spreadsheet

Apply the matrix changes for Land Reference Poin

Initial delivery of the Land Reference Point matrix

Update the Land States Matrix

Review Land States matrix spreadsheet

Apply the matrix changes for Land States

Initial delivery of the Land States matrix

Update the Land Street ROW Matrix

Review Land Street ROW matrix spreadsheet

Apply the matrix changes for Land Street ROW

Initial delivery of the Land Street ROW matrix
Start

Tue 8/17/04

Tue 8/17/04

Tue 8/17/04

Tue 8/17/04

Tue 8/17/04

Tue 8/17/04

Wed 8/18/04

Wed 8/18/04

Wed 8/18/04

Wed $8 / 18 / 04$

Wed 8/18/04

Wed 8/18/04

Wed 8/18/04

Wed 8/18/04

Wed 8/18/04

Wed 8/18/04

Wed 8/18/04

Wed 8/18/04

Wed 8/18/04

Wed 8/18/04

Wed 8/18/04

Wed 8/18/04

Wed 8/18/04

Wed 8/18/04

Wed 8/18/04

Wed 8/18/04

Wed 8/18/04

Wed 8/18/04

Thu 8/19/04

Thu 8/19/04

Thu 8/19/04

Thu 8/19/04

Thu 8/19/04

Thu 8/19/04

Thu 8/19/04

Thu 8/19/04

Thu 8/19/04

Thu 8/19/04

Thu 8/19/04

Thu 8/19/04

Thu 8/19/04

Thu 8/19/04

Thu 8/19/04

Thu 8/19/04

Thu 8/19/04

Thu 8/19/04

Page 23

\section{Finish}

Tue 8/17/04

Tue 8/17/04

Tue 8/17/04

Tue 8/17/04

Tue 8/17/04

Tue 8/17/04

Wed 8/18/04

Wed 8/18/04

Wed 8/18/04

Wed 8/18/04

Wed 8/18/04

Wed 8/18/04

Wed 8/18/04

Wed 8/18/04

Wed 8/18/04

Wed 8/18/04

Wed 8/18/04

Wed 8/18/04

Wed 8/18/04

Wed 8/18/04

Wed 8/18/04

Wed 8/18/04

Wed 8/18/04

Wed 8/18/04

Wed 8/18/04

Wed 8/18/04

Thu 8/19/04

Thu 8/19/04

Thu 8/19/04

Thu 8/19/04

Thu 8/19/04

Thu 8/19/04

Thu 8/19/04

Thu 8/19/04

Thu 8/19/04

Thu 8/19/04

Thu 8/19/04

Thu 8/19/04

Thu 8/19/04

Thu 8/19/04

Thu 8/19/04

Thu 8/19/04

Thu 8/19/04

Thu 8/19/04

Thu 8/19/04

Thu 8/19/04

Last Updated on Tue 7/3/07 Printed on Fri 8/17/07 


\begin{tabular}{|c|c|c|c|c|c|}
\hline \multicolumn{6}{|c|}{ AEDR Construction Phase I Project Plan } \\
\hline ID & (a) & Task Name & Start & Finish & \\
\hline 1059 & $\checkmark$ & Update the Land Township Range Matrix & Thu 8/19/04 & Fri 8/20/04 & \\
\hline 1060 & $\checkmark$ & Review Land Township Range matrix spreadsheet & Thu 8/19/04 & Thu 8/19/04 & \\
\hline 1061 & $\checkmark$ & Apply the matrix changes for Land Township Range & Fri 8/20/04 & Fri 8/20/04 & \\
\hline 1062 & $\checkmark$ & Initial delivery of the Land Township Range matrix & Fri 8/20/04 & Fri 8/20/04 & \\
\hline 1063 & $\checkmark$ & Update the Electric Manhole Matrix & Tue $7 / 27 / 04$ & Fri $7 / 30 / 04$ & \\
\hline 1064 & $\checkmark$ & Review Manhole matrix spreadsheet & Tue $7 / 27 / 04$ & Tue $7 / 27 / 04$ & \\
\hline 1065 & $\checkmark$ & Log Manhole matrix changes into Elementool & Fri $7 / 30 / 04$ & Fri $7 / 30 / 04$ & \\
\hline 1066 & $\checkmark$ & Apply the matrix changes for Manhole & Fri $7 / 30 / 04$ & Fri $7 / 30 / 04$ & \\
\hline 1067 & $\checkmark$ & Initial delivery of the Manhole matrix & Fri 7/30/04 & Fri $7 / 30 / 04$ & \\
\hline 1068 & $\checkmark$ & Update the Electric Pedestal Matrix & Tue $7 / 27 / 04$ & Fri $7 / 30 / 04$ & \\
\hline 1069 & $\checkmark$ & Review Pedestal matrix spreadsheet & Tue $7 / 27 / 04$ & Tue $7 / 27 / 04$ & \\
\hline 1070 & $\checkmark$ & Log Pedestal matrix changes into Elementool & Fri $7 / 30 / 04$ & Fri $7 / 30 / 04$ & \\
\hline 1071 & $\checkmark$ & Apply the matrix changes for Pedestal & Fri $7 / 30 / 04$ & Fri $7 / 30 / 04$ & \\
\hline 1072 & $\checkmark$ & Initial delivery of the Pedestal matrix & Fri $7 / 30 / 04$ & Fri 7/30/04 & \\
\hline 1073 & $\checkmark$ & Update the Electric $\mathrm{OH}$ Transmission Matrix & Wed $7 / 28 / 04$ & Fri 7/30/04 & \\
\hline 1074 & $\checkmark$ & Review OH Transmission matrix spreadsheet & Wed $7 / 28 / 04$ & Wed $7 / 28 / 04$ & \\
\hline 1075 & $\checkmark$ & Apply the matrix changes for $\mathrm{OH}$ Transmission & Fri 7/30/04 & Fri 7/30/04 & \\
\hline 1076 & $\checkmark$ & Initial delivery of the $\mathrm{OH}$ Transmission matrix & Fri 7/30/04 & Fri $7 / 30 / 04$ & \\
\hline 1077 & $\checkmark$ & Update the Electric Substation Matrix & Wed 7/28/04 & Fri $7 / 30 / 04$ & \\
\hline 1078 & $\checkmark$ & Review Substation matrix spreadsheet & Wed $7 / 28 / 04$ & Wed $7 / 28 / 04$ & \\
\hline 1079 & $\checkmark$ & Apply the matrix changes for Substation & Fri $7 / 30 / 04$ & Fri 7/30/04 & \\
\hline 1080 & $\checkmark$ & Initial delivery of the Substation matrix & Fri $7 / 30 / 04$ & Fri $7 / 30 / 04$ & \\
\hline 1081 & $\checkmark$ & Update the Electric Switch Gear Matrix & Wed 7/28/04 & Fri $7 / 30 / 04$ & \\
\hline 1082 & $\checkmark$ & Review Switch Gear matrix spreadsheet & Wed $7 / 28 / 04$ & Wed $7 / 28 / 04$ & \\
\hline 1083 & $\checkmark$ & Apply the matrix changes for Switch Gear & Fri 7/30/04 & Fri $7 / 30 / 04$ & \\
\hline 1084 & $\checkmark$ & Initial delivery of the Switch Gear matrix & Fri $7 / 30 / 04$ & Fri $7 / 30 / 04$ & \\
\hline 1085 & $\checkmark$ & Update the Electric Substation Breaker Matrix & Wed 8/4/04 & Fri 8/20/04 & \\
\hline 1086 & $\checkmark$ & Review Substation Breaker matrix spreadsheet & Wed $8 / 4 / 04$ & Wed 8/4/04 & \\
\hline 1087 & $\checkmark$ & Apply the matrix changes for Substation Breaker & Fri 8/20/04 & Fri 8/20/04 & \\
\hline 1088 & $\checkmark$ & Initial delivery of the Substation Breaker matrix & Fri 8/20/04 & Fri 8/20/04 & \\
\hline 1089 & $\checkmark$ & Create and Deliver Initial Transformer Matrix & Thu $7 / 1 / 04$ & Wed $7 / 14 / 04$ & \\
\hline 1090 & $\checkmark$ & Meeting to review transformer matrix spreadsheet & Thu $7 / 1 / 04$ & Thu $7 / 1 / 04$ & \\
\hline 1091 & $\checkmark$ & Log transformer matrix changes into Elementool & Wed $7 / 7 / 04$ & Wed 7/7/04 & \\
\hline 1092 & $\checkmark$ & Apply the approved matrix changes for transformer bank & Mon 7/12/04 & Mon 7/12/04 & \\
\hline 1093 & $\checkmark$ & Review initial matrix template & Tue $7 / 13 / 04$ & Tue $7 / 13 / 04$ & \\
\hline 1094 & $\checkmark$ & Revise and finalize the matrix template & Wed $7 / 14 / 04$ & Wed $7 / 14 / 04$ & \\
\hline 1095 & $\checkmark$ & Initial delivery of the transformer bank matrix - approved rows & Wed 7/14/04 & Wed 7/14/04 & \\
\hline 1096 & $\checkmark$ & Create and Deliver Initial Conductor Matrix & Thu $7 / 8 / 04$ & Tue $7 / 27 / 04$ & \\
\hline 1097 & $\checkmark$ & Meeting to review conductor matrix spreadsheet & Thu $7 / 8 / 04$ & Thu $7 / 8 / 04$ & \\
\hline 1098 & $\checkmark$ & Log conductor matrix changes into Elementool & Thu $7 / 8 / 04$ & Thu $7 / 8 / 04$ & \\
\hline 1099 & $\checkmark$ & Apply the approved matrix changes for conductor & Tue $7 / 13 / 04$ & Tue $7 / 13 / 04$ & \\
\hline 1100 & $\checkmark$ & Initial delivery of the conductor matrix - approved rows & Wed $7 / 14 / 04$ & Wed $7 / 14 / 04$ & \\
\hline 1101 & $\checkmark$ & Research miscellaneous conductor matrix issues & Tue $7 / 27 / 04$ & Tue $7 / 27 / 04$ & \\
\hline 1102 & $\checkmark$ & Create and Deliver Initial Support Structure Matrix & Thu $7 / 8 / 04$ & Wed $7 / 14 / 04$ & \\
\hline 1103 & $\checkmark$ & Review support structure matrix spreadsheet & Thu $7 / 8 / 04$ & Thu $7 / 8 / 04$ & \\
\hline 1104 & $\checkmark$ & Log support structure matrix changes into Elementool & Thu $7 / 8 / 04$ & Thu $7 / 8 / 04$ & \\
\hline $\begin{array}{l}\text { NIPSC } \\
\text { Confid }\end{array}$ & $\begin{array}{l}\text { Co GIS } \\
\text { lential }\end{array}$ & Page 24 & & $\begin{array}{r}\text { Last Updat } \\
\text { Print }\end{array}$ & $\begin{array}{l}\text { ed on Tue } 7 / 3 / 07 \\
\text { ed on Fri 8/17/07 }\end{array}$ \\
\hline
\end{tabular}




\begin{tabular}{|c|c|c|c|c|c|}
\hline \multicolumn{6}{|c|}{ AEDR Construction Phase I Project Plan } \\
\hline ID & (a) & Task Name & Start & Finish & \\
\hline 1105 & $\checkmark$ & Apply the approved matrix changes for support structure & Wed $7 / 14 / 04$ & Wed $7 / 14 / 04$ & \\
\hline 1106 & $\checkmark$ & Initial delivery of the support structure matrix - approved rows & Wed $7 / 14 / 04$ & Wed $7 / 14 / 04$ & \\
\hline 1107 & $\checkmark$ & Create and Deliver Initial Capacitor Matrix & Fri 7/9/04 & Fri 7/16/04 & \\
\hline 1108 & $\checkmark$ & Review capacitor matrix spreadsheet & Fri 7/9/04 & Fri $7 / 9 / 04$ & \\
\hline 1109 & $\checkmark$ & Log capacitor matrix changes into Elementool & Fri $7 / 9 / 04$ & Fri $7 / 9 / 04$ & \\
\hline 1110 & $\checkmark$ & Apply the approved matrix changes for capacitor & Fri 7/16/04 & Fri $7 / 16 / 04$ & \\
\hline 1111 & $\checkmark$ & Initial delivery of the capacitor matrix - approved rows & Fri $7 / 16 / 04$ & Fri $7 / 16 / 04$ & \\
\hline 1112 & $\checkmark$ & Create and Deliver Initial Switch/Fuse Matrix & Thu $7 / 15 / 04$ & Mon $7 / 19 / 04$ & \\
\hline 1113 & $\checkmark$ & Review switch/fuse matrix spreadsheet & Thu $7 / 15 / 04$ & Thu $7 / 15 / 04$ & \\
\hline 1114 & $\checkmark$ & Log switch/fuse matrix changes into Elementool & Thu 7/15/04 & Thu $7 / 15 / 04$ & \\
\hline 1115 & $\checkmark$ & Apply the approved matrix changes for switch/fuse & Mon 7/19/04 & Mon $7 / 19 / 04$ & \\
\hline 1116 & $\checkmark$ & Initial delivery of the switch/fuse matrix - approved rows & Mon 7/19/04 & Mon 7/19/04 & \\
\hline 1117 & $\checkmark$ & Update the Electric Primary Meter Matrix & Tue $7 / 27 / 04$ & Fri $7 / 30 / 04$ & \\
\hline 1118 & $\checkmark$ & Review Primary Meter matrix spreadsheet & Tue $7 / 27 / 04$ & Tue $7 / 27 / 04$ & \\
\hline 1119 & $\checkmark$ & Log Primary Meter matrix changes into Elementool & Fri $7 / 30 / 04$ & Fri 7/30/04 & \\
\hline 1120 & $\checkmark$ & Apply the matrix changes for Primary Meter & Fri $7 / 30 / 04$ & Fri $7 / 30 / 04$ & \\
\hline 1121 & $\checkmark$ & Initial delivery of the Primary Meter matrix & Fri $7 / 30 / 04$ & Fri $7 / 30 / 04$ & \\
\hline 1122 & $\checkmark$ & Update the Electric Recloser Bank Matrix & Tue $7 / 27 / 04$ & Fri $7 / 30 / 04$ & \\
\hline 1123 & $\checkmark$ & Review Recloser Bank matrix spreadsheet & Tue $7 / 27 / 04$ & Tue $7 / 27 / 04$ & \\
\hline 1124 & $\checkmark$ & Log Recloser Bank matrix changes into Elementool & Fri $7 / 30 / 04$ & Fri $7 / 30 / 04$ & \\
\hline 1125 & $\checkmark$ & Apply the Recloser Bank changes for conduit & Fri $7 / 30 / 04$ & Fri 7/30/04 & \\
\hline 1126 & $\checkmark$ & Initial delivery of the Recloser Bank matrix & Fri $7 / 30 / 04$ & Fri $7 / 30 / 04$ & \\
\hline 1127 & $\checkmark$ & Miscellaneous Data Migration / Matrix Tickets & Fri 7/2/04 & Wed 9/8/04 & \\
\hline 1128 & $\checkmark$ & Elementool ticket processing & Fri 7/2/04 & Wed $9 / 8 / 04$ & \\
\hline 1129 & $\checkmark$ & Update the Data Matrix based on the Data Model & Fri 7/2/04 & Fri 7/9/04 & \\
\hline 1130 & $\checkmark$ & Decide on approach to be used for data matrix & Fri 7/2/04 & Fri $7 / 2 / 04$ & \\
\hline 1131 & $\checkmark$ & Create new, blank electric matrix with the latest data model & Thu $7 / 8 / 04$ & Thu $7 / 8 / 04$ & \\
\hline 1132 & $\checkmark$ & Create new, blank gas matrix with the latest data model & Fri 7/9/04 & Fri 7/9/04 & \\
\hline 1133 & $\checkmark$ & Create new, blank land matrix with the latest data model & Fri 7/9/04 & Fri $7 / 9 / 04$ & \\
\hline 1134 & $\checkmark$ & Freeze the Data Matrix for Data Migration Iteration 1 & Fri 9/3/04 & Fri 9/3/04 & \\
\hline 1135 & $\checkmark$ & FreezeData Matrix - Electric & Fri 9/3/04 & Fri 9/3/04 & \\
\hline 1136 & $\checkmark$ & FreezeData Matrix - Gas & Fri 9/3/04 & Fri 9/3/04 & \\
\hline 1137 & $\checkmark$ & FreezeData Matrix - Land & Fri 9/3/04 & Fri 9/3/04 & \\
\hline 1138 & $\checkmark$ & Update the Electric Primary Open Point Matrix & Fri 9/17/04 & Fri 9/17/04 & \\
\hline 1139 & $\checkmark$ & Review Primary Open Point matrix spreadsheet & Fri 9/17/04 & Fri 9/17/04 & \\
\hline 1140 & $\checkmark$ & Apply the matrix changes for Primary Open Point & Fri 9/17/04 & Fri 9/17/04 & \\
\hline 1141 & $\checkmark$ & Initial delivery of the Primary Open Point matrix & Fri 9/17/04 & Fri 9/17/04 & \\
\hline 1142 & $\checkmark$ & Update the Electric Splice Matrix & Tue 9/21/04 & Tue 9/21/04 & \\
\hline 1143 & $\checkmark$ & Review Splice matrix spreadsheet & Tue 9/21/04 & Tue 9/21/04 & \\
\hline 1144 & $\checkmark$ & Apply the matrix changes for Splice & Tue 9/21/04 & Tue 9/21/04 & \\
\hline 1145 & $\checkmark$ & Initial delivery of the Splice matrix & Tue 9/21/04 & Tue 9/21/04 & \\
\hline 1146 & $\checkmark$ & Update the Electric Terminator Matrix & Tue 9/21/04 & Tue 9/21/04 & \\
\hline 1147 & $\checkmark$ & Review Terminator matrix spreadsheet & Tue 9/21/04 & Tue 9/21/04 & \\
\hline 1148 & $\checkmark$ & Note: The data matrix task above covers the time needed for the matrix updates below & Tue 9/21/04 & Tue 9/21/04 & \\
\hline 1149 & $\checkmark$ & Apply the matrix changes for Terminator & Tue 9/21/04 & Tue 9/21/04 & \\
\hline 1150 & $\checkmark$ & Initial delivery of the Terminator matrix & Tue 9/21/04 & Tue 9/21/04 & \\
\hline $\begin{array}{l}\text { NIPSC } \\
\text { Confid }\end{array}$ & $\begin{array}{l}\text { Co GIS } \\
\text { lential }\end{array}$ & Page 25 & & $\begin{array}{r}\text { Last Updat } \\
\text { Print }\end{array}$ & $\begin{array}{l}\text { ed on Tue } 7 / 3 / 07 \\
\text { ed on Fri 8/17/07 }\end{array}$ \\
\hline
\end{tabular}




\section{\begin{tabular}{|ll}
\hline AEDR Construction Phase I Project Plan & A
\end{tabular}

Review Tie Wire matrix spreadsheet

Apply the matrix changes for Tie Wire

ue $9 / 21 / 04$

Initial delivery of the Tie Wire matrix

Tue $9 / 21 / 04$

Tue 9/21/04

ue $9 / 21 / 04$

Update the Electric Voltage Regulator Matrix

Tue 9/21/04

Review Voltage Regulator matrix spreadsheet

Log Voltage Regulator matrix changes into Elementool

Apply the matrix changes for Voltage Regulato

Initial delivery of the Voltage Regulator matrix

\section{Define Data Migration Data Receipt and Acceptance Procedures}

Create initial draft of data migration receipt and acceptance procedures

Data migration automated QA tools/scripts should be in QA plan

Update the migration receipt and acceptance procedures based on initial review

Tue $9 / 21 / 04$

Tue $9 / 21 / 04$

Wed $9 / 22 / 04$

Tue 9/21/04

Mon $7 / 26 / 04$

Mon $7 / 26 / 04$

Mon $9 / 20 / 04$

Update the migration receipt and acceptance procedures based on interactive testing

Fri 10/1/04

Fri $10 / 1 / 04$

Update the migration receipt and acceptance procedures based on batch testing

\section{Select Batch Migration QA Product}

Demo of vendor automated testing software products

Compare the two products and decide on next steps

Purchase Automated QA Routine product

Develop the Migration Routines / Processes for Iteration 1

Data migration iteration 1 development tasks

Meeting to review the deliverables from data migration vendor

Migration iteration 1 delivery date

Install the SQL backup file at NIPSCO

Test the Migrated Data

\section{Perform Interactive Testing of Migrated Data}

Perform first pass interactive testing (test one of each feature type)

Review \& resolve errors from first pass interactive testing

Submit Elementool tickets for first pass interactive testing

Perform second pass interactive testing (test each subtype)

Submit Elementool tickets for second pass interactive testing

Fri 10/22/04

Mon 9/13/04

Mon $9 / 13 / 04$

Mon 9/27/04

Mon 9/27/04

Mon 6/21/04

Mon 6/21/04

Fri 9/17/04

Wed $9 / 15 / 04$

Wed $9 / 22 / 04$

Wed 9/22/04

Wed 9/22/04

Wed 9/22/04

Fri 10/1/04

Tue $9 / 28 / 04$

Fri 10/1/04

Fri 10/15/04

Wed 9/29/04

Wed 9/29/04

Run landbase tests against pilot data

Run initial tests against the pilot data and deliver results

Run Nipsco-specific tests for gas and electric and deliver results

Mon 10/4/04

Wed $10 / 6 / 04$

Fri 8/27/04

Fri $8 / 27 / 04$

Tue $9 / 28 / 04$

Fri 9/17/04

Determine final data migration data timeframe freeze

Elementool ticket processing

\section{Update the Electric Secondary Matrix}

Review secondary matrix

Log Secondary matrix changes into Elementool

Apply the matrix changes for Secondary

Initial delivery of the Secondary matrix

Mon 10/4/04

Mon 10/4/04

Mon 10/4/04

Mon 10/11/04

Mon 10/11/04

Update the Electric Sectionalizer Bank Matrix

Mon 10/11/04

Initial pass to populate spreadsheet

Mon 10/11

Tue $9 / 21 / 04$

Tue $9 / 21 / 04$

Wed 9/22/04

Tue $9 / 21 / 04$

Tue 9/21/04

Wed $9 / 22 / 04$

Tue 9/21/04

Fri 10/22/04

Wed 8/18/04

Mon 9/20/04

Fri 10/1/04

Fri 10/1/04

Fri 10/22/04

Mon 9/27/04

Mon $9 / 13 / 04$

Mon 9/27/04

Mon 9/27/04

Fri 10/8/04

Tue 9/14/04

Fri $9 / 17 / 04$

Wed 9/15/04

Fri $10 / 8 / 04$

Fri 10/15/04

Fri 10/15/04

Mon 9/27/04

Tue 10/5/04

Tue 9/28/04

Thu 10/14/04

Fri 10/15/04

Wed 10/6/04

Wed 9/29/04

Mon 10/4/04

Wed 10/6/04

Mon 10/25/04

Fri 8/27/04

Tue 9/28/04

Mon 10/25/04

Mon 10/11/04

Mon 10/4/04

Mon 10/4/04

Mon 10/11/04

Mon 10/11/04

Fri 10/15/04

Mon 10/11/04 


\section{\begin{tabular}{|ll}
\hline & AEDR Construction Phase I Project Plan
\end{tabular}}

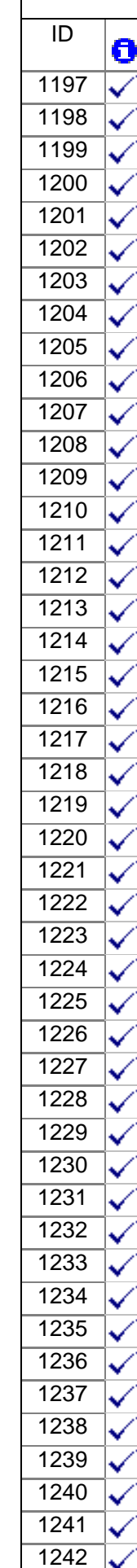

Task Name

$\checkmark$

Review Sectionalizer Bank matrix spreadsheet

Sectionalizer Bank matrix changes into Elementoo

Apply the matrix changes for Sectionalizer Bank

Initial delivery of the Sectionalizer Bank matrix

Update the Electric Discrepancy Matrix

Review Discrepancy matrix spreadsheet

Log Discrepancy matrix changes into Elementoo

Apply the matrix changes for Discrepancy

Initial delivery of the Discrepancy matrix

\section{Update the Electric Abandoned Conductor Matrix}

Review Abandoned Conductor matrix spreadsheet

Log Abandoned Conductor matrix changes into Elementool

Apply the matrix changes for Abandoned Conductor

Initial delivery of the Abandoned Conductor matrix

\section{Update the Padmount Matrix}

Review Padmount matrix spreadsheet

Log Padmount matrix changes into Elementool

Apply the matrix changes for Padmount

Initial delivery of the Padmount matrix

Update the CP Section Matrix

Review CP Section matrix spreadsheet

Log CP Section matrix changes into Elementool

Apply the matrix changes for CP Section

Initial delivery of the CP Section matrix

\section{Update the CP Test Point Matrix}

Review CP Test Point matrix spreadsheet

Log CP Test Point matrix changes into Elementoo

Apply the matrix changes for CP Test Point

Initial delivery of the CP Test Point matrix

\section{Update the Gas Discrepancy Matrix}

Review Gas Discrepancy matrix spreadsheet

Log Gas Discrepancy matrix changes into Elementoo

Apply the matrix changes for Gas Discrepancy

Initial delivery of the Gas Discrepancy matrix

Update the Gas Main Location Matrix

Review Gas Main Location matrix spreadsheet

Log Gas Main Location matrix changes into Elementoo

Apply the matrix changes for Gas Main Location

Initial delivery of the Gas Main Location matrix

Elementool ticket processing

Automated Testing Software Diagnostics Too

Evaluate Automated testing software diagnostics tool for reporting of deltas between data models

GIS Data Migration - Iteration 2 (Small Area and All Feature Classes)

Develop the Migration Routines / Processes for iteration 2

Data migration iteration 2 development tasks

Send data migration vendor a configured SDE SQL Backup file

Tue $10 / 12 / 04$

Wed $10 / 13 / 04$

Thu 10/14/04

Fri 10/15/04

Tue $10 / 12 / 04$

Tue 10/12/04

Tue $10 / 12 / 04$

Tue $10 / 12 / 04$

Tue 10/12/04

Wed 10/13/04

Wed 10/13/04

Wed 10/13/04

Wed 10/13/04

Wed 10/13/04

Wed 10/13/04

Wed 10/13/04

Wed 10/13/04

Wed 10/13/04

Wed 10/13/04

Thu 10/14/04

Thu 10/14/04

Thu 10/14/04

Thu 10/14/04

Thu 10/14/04

Thu 10/14/04

Thu 10/14/04

Thu 10/14/04

Thu 10/14/04

Thu 10/14/04

Fri 10/15/04

Fri 10/15/04

Fri 10/15/04

Fri 10/15/04

Fri 10/15/04

Fri 10/15/04

Fri 10/15/04

Fri 10/15/04

Fri 10/15/04

Fri 10/15/04

Fri 10/22/04

Mon 10/25/04

Mon 10/25/04

Thu 9/16/04

Thu 9/16/04

Thu 9/16/04

Wed 10/20/04

Tue 10/12/04

Tue 10/12/04

Tue 10/12/04

Wed 10/13/04

Wed 10/13/04

Wed 10/13/04

Wed 10/13/04

Wed 10/13/04

Wed 10/13/04

Wed 10/13/04

Wed 10/13/04

Wed 10/13/04

Wed 10/13/04

Thu 10/14/04

Thu 10/14/04

Thu 10/14/04

Thu 10/14/04

Thu $10 / 14 / 04$

Thu 10/14/04

Thu 10/14/04

Thu 10/14/04

Thu 10/14/04

Thu 10/14/04

Fri 10/15/04

Fri 10/15/04

Fri 10/15/04

Fri 10/15/04

Fri 10/15/04

Mon 10/25/04

Fri 10/15/04

Fri 10/15/04

Fri 10/15/04

Fri 10/15/04

Mon 10/25/04

Mon 10/25/04

Mon 10/25/04

Mon 11/22/04

Fri 11/5/04

Fri 10/29/04

Wed 10/20/04 


\begin{tabular}{|c|c|c|c|c|c|}
\hline \multicolumn{6}{|c|}{ AEDR Construction Phase I Project Plan } \\
\hline ID & ( & Task Name & Start & Finish & \\
\hline 1243 & $\checkmark$ & Process the data for migration delivery 2 & Mon 10/18/04 & Thu 11/4/04 & \\
\hline 1244 & $\checkmark$ & Migration iteration 2 delivery date (SQL server backup version) & Fri 11/5/04 & Fri 11/5/04 & \\
\hline 1245 & $\checkmark$ & Install the SQL backup file at NIPSCO & Fri 11/5/04 & Fri 11/5/04 & \\
\hline 1246 & $\checkmark$ & Test the Migrated Data (Batch and Interactive) & Mon 11/8/04 & Wed 11/17/04 & \\
\hline 1247 & $\checkmark$ & Perform batch QA of migration iteration 2 delivery & Mon 11/8/04 & Fri $11 / 12 / 04$ & \\
\hline 1248 & $\checkmark$ & Perform interactive QA of Gas for iteration 2 delivery & Fri 11/12/04 & Fri 11/12/04 & \\
\hline 1249 & $\checkmark$ & Perform interactive QA of Electric for iteration 2 delivery & Mon 11/15/04 & Tue 11/16/04 & \\
\hline 1250 & $\checkmark$ & Perform interactive QA of Land for iteration 2 delivery & Wed 11/17/04 & Wed 11/17/04 & \\
\hline 1251 & $\checkmark$ & Log Elementool issues for migration iteration 2 delivery & Mon 11/8/04 & Tue 11/16/04 & \\
\hline 1252 & $\checkmark$ & Review the Matrix Issues and Apply Changes & Thu 11/18/04 & Mon 11/22/04 & \\
\hline 1253 & $\checkmark$ & Process Elementool tickets & Thu $11 / 18 / 04$ & Mon 11/22/04 & \\
\hline 1254 & $\checkmark$ & GIS Data Migration - Iteration 3 (Entire Area and All Feature Classes) & Mon 11/1/04 & Fri 1/28/05 & \\
\hline 1255 & $\checkmark$ & Develop the Migration Routines / Processes for Iteration 3 & Mon 11/1/04 & Fri 12/17/04 & \\
\hline 1256 & $\checkmark$ & Data migration iteration 3 development tasks & Mon 11/1/04 & Tue $12 / 14 / 04$ & \\
\hline 1257 & $\checkmark$ & Migration iteration 3 delivery date & Wed 12/15/04 & Wed 12/15/04 & \\
\hline 1258 & $\checkmark$ & Install the SQL backup file at NIPSCO & Thu 12/16/04 & Fri 12/17/04 & \\
\hline 1259 & $\checkmark$ & Test the Migrated Data (via automated testing) & Thu 12/16/04 & Wed 1/5/05 & \\
\hline 1260 & $\checkmark$ & Run automated tests & Thu $12 / 16 / 04$ & Mon 12/20/04 & \\
\hline 1261 & $\checkmark$ & Install Beta Version / Re-Process / Testing & Fri 12/17/04 & Tue 12/21/04 & \\
\hline 1262 & $\checkmark$ & Evaluate Test Results & Fri 12/17/04 & Wed 12/22/04 & \\
\hline 1263 & $\checkmark$ & Log Elementool Ticket(s) & Thu $12 / 23 / 04$ & Thu 12/23/04 & \\
\hline 1264 & $\checkmark$ & Resolve critical Elementool matrix issues from the automated testing & Wed 1/5/05 & Wed $1 / 5 / 05$ & \\
\hline 1265 & $\checkmark$ & Test the Migrated Data (via Interactive testing) & Fri 12/17/04 & Fri 1/14/05 & \\
\hline 1266 & $\checkmark$ & Perform Electric Testing & Fri 12/17/04 & Mon 12/20/04 & \\
\hline 1267 & $\checkmark$ & Perform Gas Testing & Mon 12/20/04 & Tue 12/21/04 & \\
\hline 1268 & $\checkmark$ & Perform Land Testing & Wed 12/22/04 & Wed 12/22/04 & \\
\hline 1269 & $\checkmark$ & Log Elementool Ticket(s) & Wed $1 / 5 / 05$ & Wed 1/5/05 & \\
\hline 1270 & $\checkmark$ & Resolve critical Elementool matrix issues & Thu $1 / 6 / 05$ & Thu $1 / 6 / 05$ & \\
\hline 1271 & $\checkmark$ & Update Interactive testing spreadsheet from Migration Delivery \#2 & Fri $1 / 14 / 05$ & Fri 1/14/05 & \\
\hline 1272 & $\checkmark$ & Update Interactive testing spreadsheet & Fri 1/14/05 & Fri 1/14/05 & \\
\hline 1273 & $\checkmark$ & Test the Migrated Electric Data (via Feeder Manager testing) & Wed 1/12/05 & Thu $1 / 13 / 05$ & \\
\hline 1274 & $\checkmark$ & Build Electric Network for Feeder Manager & Wed 1/12/05 & Wed 1/12/05 & \\
\hline 1275 & $\checkmark$ & Test the Electric connectivity using Feeder Manager & Thu $1 / 13 / 05$ & Thu $1 / 13 / 05$ & \\
\hline 1276 & $\checkmark$ & Test the Migrated Data (via CADOPS / FeederAll testing) & Wed 1/12/05 & Wed 1/12/05 & \\
\hline 1277 & $\checkmark$ & Write script to populate the circuit source for CADOPS / Feederall & Wed 1/12/05 & Wed 1/12/05 & \\
\hline 1278 & $\checkmark$ & Test the Migrated Gas Data (via Gas Trace) & Mon 1/24/05 & Tue 1/25/05 & \\
\hline 1279 & $\checkmark$ & Build Gas Network for Gas Trace & Mon 1/24/05 & Mon $1 / 24 / 05$ & \\
\hline 1280 & $\checkmark$ & Document the process & Tue $1 / 25 / 05$ & Tue $1 / 25 / 05$ & \\
\hline 1281 & $\checkmark$ & Correct Data Migration Elementool Tickets & Thu $1 / 13 / 05$ & Fri 1/28/05 & \\
\hline 1282 & $\checkmark$ & Process data migration elementool tickets & Thu $1 / 13 / 05$ & Fri 1/28/05 & \\
\hline 1283 & $\checkmark$ & Data Migration Iteration Acceptance & Fri $\mathbf{1 / 7 / 0 5}$ & Fri 1/14/05 & \\
\hline 1284 & $\checkmark$ & Send notification of data migration iteration 1 and 2 acceptance & Fri 1/7/05 & Fri $1 / 7 / 05$ & \\
\hline 1285 & $\checkmark$ & Meeting to review migration testing status & Fri 1/7/05 & Fri 1/7/05 & \\
\hline 1286 & $\checkmark$ & Send notification of acceptance / rejection & Fri 1/14/05 & Fri 1/14/05 & \\
\hline 1287 & $\checkmark$ & GIS Data Migration - Delivery 4 (Entire Area with All EDFS Data Model Changes) & Mon 3/1/04 & Fri 3/11/05 & \\
\hline 1288 & $\checkmark$ & Data Migration Delivery 4 - Migrate the data & Mon 12/13/04 & Wed 2/2/05 & \\
\hline $\begin{array}{l}\text { NIPSC } \\
\text { Confid }\end{array}$ & $\begin{array}{l}\text { Co GIS } \\
\text { lential }\end{array}$ & Page 28 & & $\begin{array}{r}\text { Last Updat } \\
\text { Print }\end{array}$ & $\begin{array}{l}\text { ed on Tue } 7 / 3 / 07 \\
\text { ed on Fri 8/17/07 }\end{array}$ \\
\hline
\end{tabular}




\section{\begin{tabular}{ll}
\hline AEDR Construction Phase I Project Plan & A
\end{tabular}}

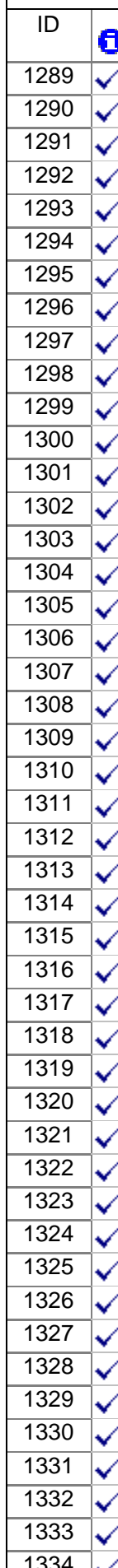

\begin{tabular}{|c|c|c|}
\hline ID & $\mathbf{B}$ & Task Name \\
\hline 1289 & $\checkmark$ & \\
\hline 1290 & $\checkmark$ & \\
\hline 1291 & $\checkmark$ & \\
\hline 1292 & $\checkmark$ \\
\hline 1293 & $\checkmark$ \\
\hline 1294 & $\checkmark$ \\
\hline 1295 & $\checkmark$ \\
\hline 1296 & $\checkmark$ \\
\hline 1297 & $\checkmark$ \\
\hline 1298 & $\checkmark$ \\
\hline 1299 & $\checkmark$
\end{tabular}

Provide landbase polygon data / research landbase polygon issues

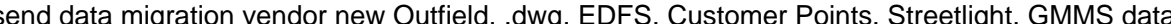

Mon 12/13/04

Fri $12 / 17 / 04$

Modify existing DWGVerify for use in Migration counts

Create Source counts for EDFS, .dwg, Outfield, GMMS, Sreetlights, Customer Points

Thu $1 / 13 / 05$

$1 / 13 / 05$

Development tasks

Fri $1 / 14 / 05$

Wed $1 / 12 / 05$

Fri $1 / 14 / 05$

Thu $12 / 16 / 04$

Tue $2 / 1 / 05$

Install the SQL backup file at NIPSCO

Reject Delivery due to unlinked feature anno \& missing disolve

Migr data delivery w/EDFS, Feature-linked anno \& dissolve from data migration vendor

Tue $2 / 1 / 05$

Tue $2 / 1 / 05$

Wed $2 / 2 / 05$

Install the SQL backup file at NIPSCO

Wed 2/2/05

Mon 3/1/04

Tue $2 / 1 / 05$

Pre-QA Review/identify updates to migration testing procedures

Develop QA Test Plan

Periodic updates of the QA Test Plan

Tue $2 / 1 / 05$

Mon $2 / 14 / 05$

Fri $2 / 18 / 05$

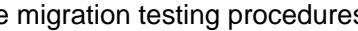

Wed $2 / 2 / 05$

Mon 3/1/04

Fri $2 / 18 / 05$

Automated Record Count Reconciliation QA

Wed $2 / 2 / 05$

Automated Attribute QA

Wed $2 / 16 / 05$

environment Setup

Thu 3/10/05

Tue $3 / 1 / 05$

Gas Trace QA

Interactive Feature Existence QA

Mon 2/14/05

Tue $2 / 8 / 05$

Interactive Detailed Attribute QA

Review / quantify all data-related issues

Post-QA Review / update the migration testing procedures

Data Migration - Daily Status Meetings

GIS Data Migration - Delivery 4.1 (1 small tile)

Data Migration 4.1 - Migrate the data

Development tasks/bug fixing

NIPSCO Create Delta Instance for data migration vendor

Create Source counts for EDFS, .dwg, Outfield, GMMS, Streetlights, Customer Points

ed $2 / 23 / 05$

Wed 3/9/05

Mon 2/7/05

Wed 4/13/05

Wed $4 / 13 / 05$

Wed $4 / 13 / 05$

Fri $4 / 15 / 05$

Tue 4/19/05

Tue $4 / 19 / 05$

Migration process tasks

Install the SQL backup file at NIPSCO

Fri $4 / 22 / 05$

Thu 4/21/05

Fri $4 / 22 / 05$

Environment Setup Tasks

Delivery Checklist Tasks

Thu 4/21/05

Fri $4 / 22 / 05$

Record Count Reconciliation QA

Thu 4/21/05

Automated Attribute QA

Review \& test exception reports

Fri 4/22/05

Preliminary Acceptance to move to Dev

DEV environment Setup

Mon 4/25/05

Mon 4/25/05

Mon $4 / 25 / 05$

Tue 4/26/05

Interactive Detailed Attribute QA

Wed $4 / 27 / 05$

Electric Connectivity QA

Gas Trace QA

Wed $4 / 27 / 05$

Mon 1/31/05

Tue 2/1/05

Tue $2 / 1 / 05$

Tue 2/1/05

Wed 2/2/05

Wed 2/2/05

Fri 3/11/05

Tue 2/1/05

Tue $2 / 1 / 05$

Fri 2/25/05

Fri $2 / 18 / 05$

Wed 2/2/05

Mon 3/1/04

Tue $3 / 1 / 05$

Fri 2/18/05

Fri 2/18/05

Fri 3/11/05

Tue 3/1/05

Tue 2/15/05

Fri 2/11/05

Mon 2/28/05

Wed 3/9/05

Fri 3/4/05

Thu 4/28/05

Fri 4/22/05

Fri 4/15/05

Tue $4 / 19 / 05$

Tue 4/19/05

Thu 4/21/05

Fri 4/22/05

Thu 4/28/05

Fri 4/22/05

Fri 4/22/05

Fri 4/22/05

Fri 4/22/05

Mon 4/25/05

Mon 4/25/05

Thu $4 / 28 / 05$

Tue $4 / 26 / 05$

Wed 4/27/05

Wed 4/27/05

Wed 4/27/05 


\begin{tabular}{|c|c|c|c|c|c|}
\hline \multicolumn{6}{|c|}{ AEDR Construction Phase I Project Plan } \\
\hline ID & (a) & Task Name & Start & Finish & \\
\hline 1335 & $\checkmark$ & Review / quantify all data-related issues & Wed 4/27/05 & Thu $4 / 28 / 05$ & \\
\hline 1336 & $\checkmark$ & Post-QA Review / update the migration testing procedures & Thu $4 / 28 / 05$ & Thu $4 / 28 / 05$ & \\
\hline 1337 & $\checkmark$ & Data Migration Delivery 4.1 Acceptance & Tue $4 / 26 / 05$ & Tue 4/26/05 & \\
\hline 1338 & $\checkmark$ & Send notification of acceptance/rejection & Tue $4 / 26 / 05$ & Tue $4 / 26 / 05$ & \\
\hline 1339 & $\checkmark$ & GIS Data Migration - Delivery 4.2 (1 small tile) & Wed 4/13/05 & Fri 5/13/05 & \\
\hline 1340 & $\checkmark$ & Data Migration 4.2 - Migrate the Data & Wed 4/13/05 & Fri 5/6/05 & \\
\hline 1341 & $\checkmark$ & Development tasks/bug fixing & Wed 4/13/05 & Fri 4/15/05 & \\
\hline 1342 & $\checkmark b$ & NIPSCO Create Delta Instance for data migration vendor & Fri 4/15/05 & Tue $4 / 19 / 05$ & \\
\hline 1343 & $\checkmark$ & Create Source counts for EDFS, .dwg, Outfield, GMMS, Streetlights, Customer Points & Tue $4 / 19 / 05$ & Tue $4 / 19 / 05$ & \\
\hline 1344 & $\checkmark$ & Migration process tasks & Tue $4 / 19 / 05$ & Thu $4 / 21 / 05$ & \\
\hline 1345 & $\checkmark$ & Install the SQL backup file at NIPSCO & Fri 5/6/05 & Fri 5/6/05 & \\
\hline 1346 & $\checkmark$ & Data Migration Delivery 4.2 - QA & Mon 4/18/05 & Fri 5/13/05 & \\
\hline 1347 & $\checkmark$ & Review/Update migration testing procedures & Mon 4/18/05 & Mon 4/18/05 & \\
\hline 1348 & $\checkmark$ & Environment Setup Tasks & Fri 5/6/05 & Fri 5/6/05 & \\
\hline 1349 & $\checkmark$ & Delivery Checklist Tasks & Fri 5/6/05 & Fri 5/6/05 & \\
\hline 1350 & $\checkmark$ & Record Count Reconciliation QA & Fri 5/6/05 & Fri 5/6/05 & \\
\hline 1351 & $\checkmark$ & Automated Attribute QA & Fri 5/6/05 & Fri 5/6/05 & \\
\hline 1352 & $\checkmark$ & Review \& test exception reports & Fri 5/6/05 & Mon 5/9/05 & \\
\hline 1353 & $\checkmark$ & Preliminary Acceptance to move to Dev & Mon 5/9/05 & Mon 5/9/05 & \\
\hline 1354 & $\checkmark$ & DEV environment Setup & Mon 5/9/05 & Thu 5/12/05 & \\
\hline 1355 & $\checkmark$ & Interactive Feature Existence QA & Mon 5/9/05 & Tue $5 / 10 / 05$ & \\
\hline 1356 & $\checkmark$ & Interactive Detailed Attribute QA & Tue $5 / 10 / 05$ & Wed 5/11/05 & \\
\hline 1357 & $\checkmark$ & Electric Connectivity QA & Wed 5/11/05 & Thu 5/12/05 & \\
\hline 1358 & $\checkmark$ & Gas Trace QA & Thu 5/12/05 & Thu 5/12/05 & \\
\hline 1359 & $\checkmark$ & Review / quantify all data-related issues & Thu 5/12/05 & Fri 5/13/05 & \\
\hline 1360 & $\checkmark$ & Post-QA Review / update the migration testing procedures & Fri 5/13/05 & Fri 5/13/05 & \\
\hline 1361 & $\checkmark$ & Data Migration Delivery 4.1 Acceptance & Fri 5/13/05 & Fri 5/13/05 & \\
\hline 1362 & $\checkmark$ & Meeting to review migration testing status & Fri 5/13/05 & Fri 5/13/05 & \\
\hline 1363 & $\checkmark$ & Send notification of acceptance / rejection & Fri 5/13/05 & Fri 5/13/05 & \\
\hline 1364 & $\checkmark$ & GIS Data Migration - Delivery 5 (Valpo only) & Mon 5/2/05 & Wed 8/31/05 & \\
\hline 1365 & $\checkmark$ & Data Migration Delivery 5 - Migrate the data & Mon 5/2/05 & Fri 8/5/05 & \\
\hline 1366 & $\checkmark$ & Development tasks/bug fixing/new data model \& matrix updates & Mon 5/2/05 & Fri 5/6/05 & \\
\hline 1367 & 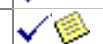 & NIPSCO Create Delta Instance for data migration vendor & Thu 5/5/05 & Fri 5/6/05 & \\
\hline 1368 & $\checkmark$ & Create Source counts for EDFS, .dwg, Outfield, GMMS, Sreetlights, Customer Points & Mon 5/9/05 & Mon 5/9/05 & \\
\hline 1369 & $\checkmark$ & Migration Pre-QA tasks & Mon 8/1/05 & Fri 8/5/05 & \\
\hline 1370 & $\checkmark$ & Receive complete migration data delivery from migration vendor & Sun $7 / 31 / 05$ & Sun $7 / 31 / 05$ & \\
\hline 1371 & $\checkmark$ & Install the SQL backup file at NIPSCO & Sun $7 / 31 / 05$ & Sun $7 / 31 / 05$ & \\
\hline 1372 & $\checkmark$ & Data Migration Delivery 5 - QA & Sun $7 / 31 / 05$ & Wed 8/31/05 & \\
\hline 1373 & $\checkmark$ & Environment Setup Tasks & Sun $7 / 31 / 05$ & Sun $7 / 31 / 05$ & \\
\hline 1374 & $\checkmark$ & Delivery Checklist Tasks & Sun $7 / 31 / 05$ & Mon 8/1/05 & \\
\hline 1375 & $\checkmark$ & Record Count Reconciliation QA & Sun $7 / 31 / 05$ & Mon 8/1/05 & \\
\hline 1376 & $\checkmark$ & Automated Attribute QA & Mon 8/1/05 & Thu 8/4/05 & \\
\hline 1377 & $\checkmark$ & Preliminary Acceptance to move to Dev & Mon 8/15/05 & Mon 8/15/05 & \\
\hline 1378 & $\checkmark$ & DEV environment Setup & Mon 8/15/05 & Thu 8/18/05 & \\
\hline 1379 & $\checkmark$ & Interactive Feature Existence QA & Mon 8/15/05 & Thu $8 / 18 / 05$ & \\
\hline 1380 & $\checkmark$ & Interactive Detailed Attribute QA & Thu 8/18/05 & Thu 8/25/05 & \\
\hline $\begin{array}{l}\text { NIPSC } \\
\text { Confid }\end{array}$ & $\begin{array}{l}\text { Co GIS } \\
\text { lential }\end{array}$ & Page 30 & & $\begin{array}{r}\text { Last Updat } \\
\text { Print }\end{array}$ & $\begin{array}{l}\text { ed on Tue } 7 / 3 / 07 \\
\text { ed on Fri 8/17/07 }\end{array}$ \\
\hline
\end{tabular}




\section{\begin{tabular}{|ll}
\hline & AEDR Construction Phase I Project Plan
\end{tabular}}

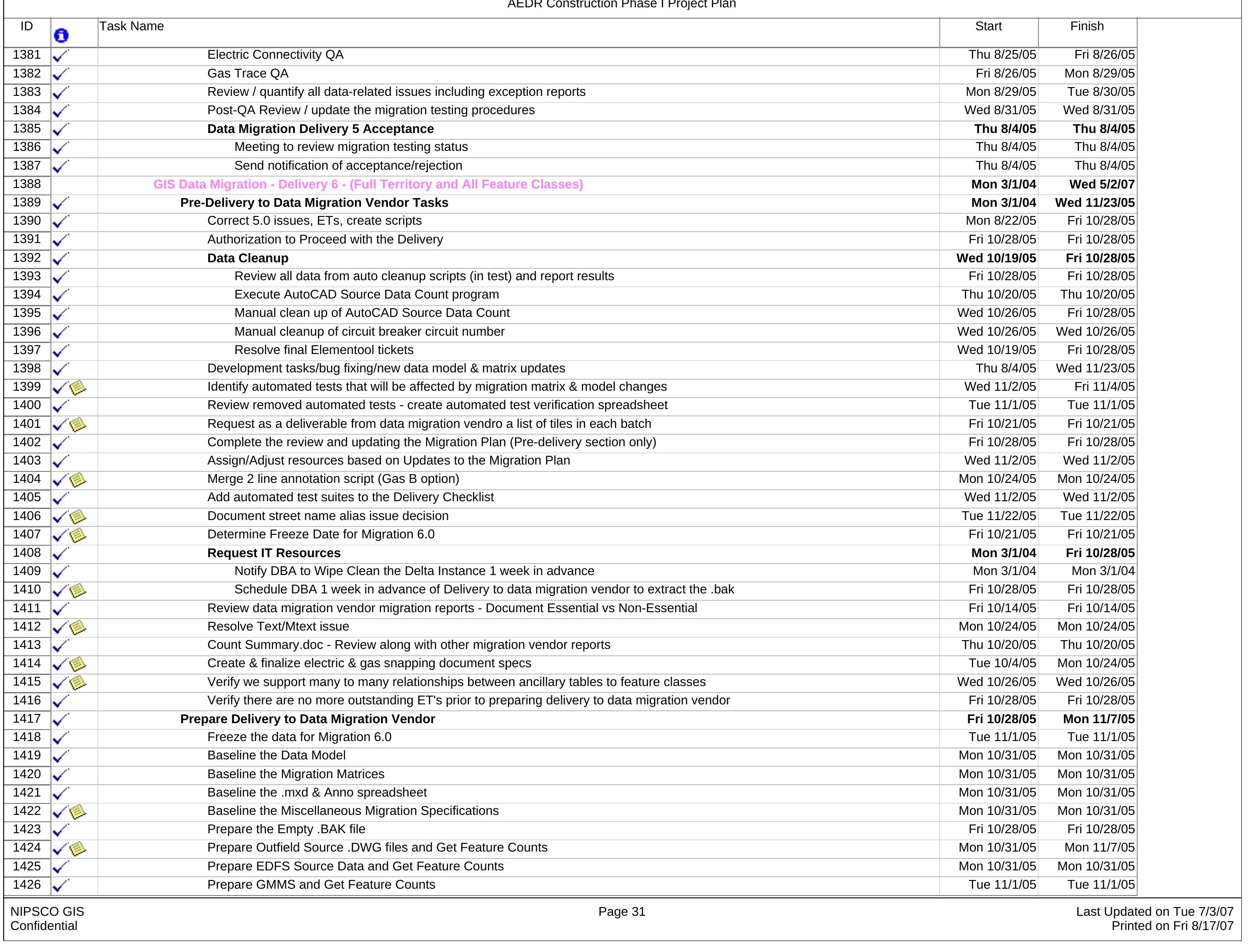




\section{\begin{tabular}{|ll}
\hline & AEDR Construction Phase I Project Plan
\end{tabular}}

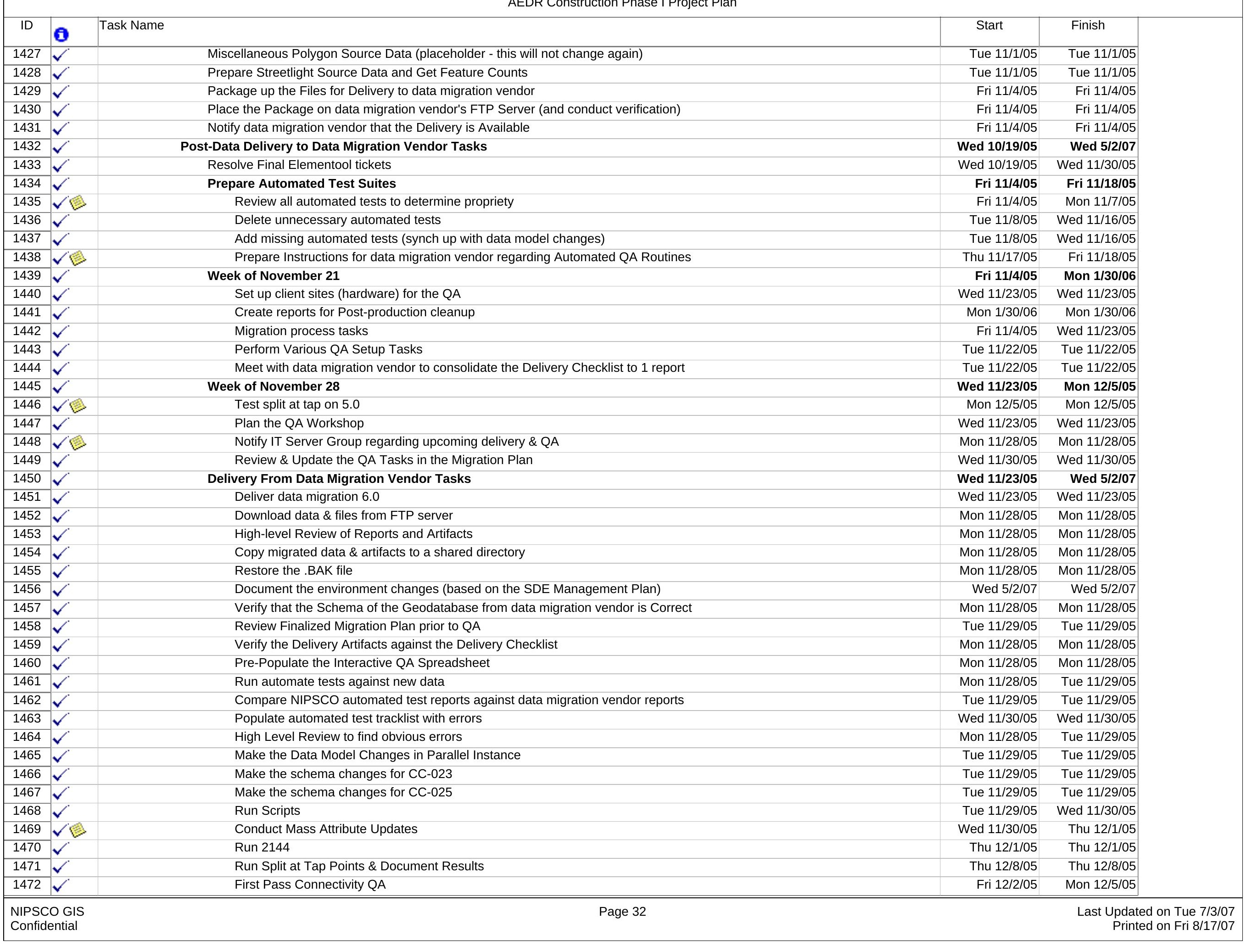




\section{\begin{tabular}{|ll}
\hline AEDR Construction Phase I Project Plan & AED
\end{tabular}}

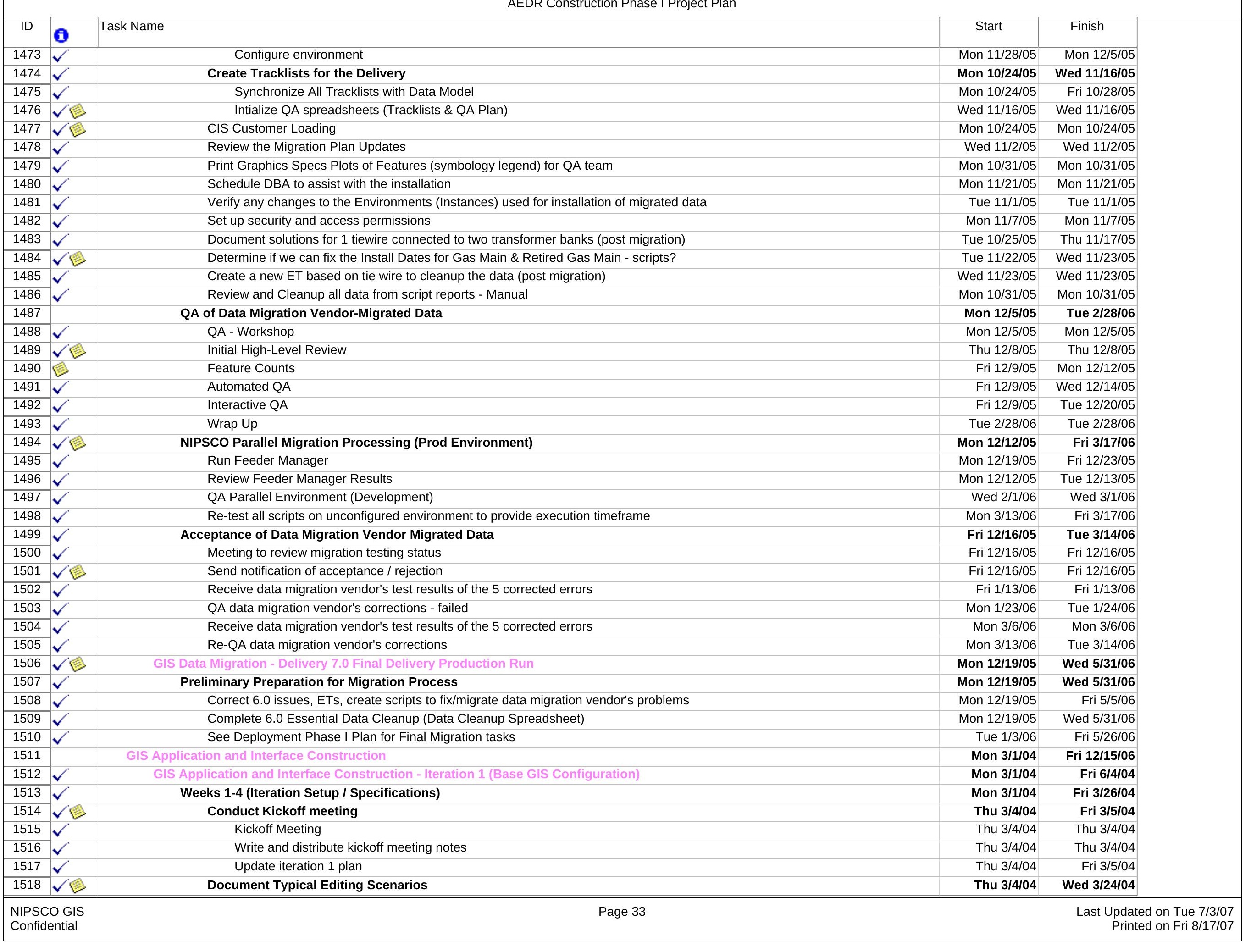




\begin{tabular}{|c|c|c|c|c|c|}
\hline \multicolumn{6}{|c|}{ AEDR Construction Phase I Project Plan } \\
\hline ID & (a) & Task Name & Start & Finish & \\
\hline 1519 & $\checkmark$ & Review initial draft of editing scenarios & Thu 3/4/04 & Thu $3 / 4 / 04$ & \\
\hline 1520 & $\checkmark$ & Document typical gas editing scenarios & Thu $3 / 4 / 04$ & Mon 3/8/04 & \\
\hline 1521 & $\checkmark$ & Document typical land editing scenarios & Mon $3 / 8 / 04$ & Tue 3/9/04 & \\
\hline 1522 & $\checkmark$ & Document typical electric editing scenarios (plus some for EDFS) & Tue 3/9/04 & Fri 3/12/04 & \\
\hline 1523 & $\checkmark$ & Review typical editing scenario documentation & Fri 3/12/04 & Tue $3 / 23 / 04$ & \\
\hline 1524 & $\checkmark$ & Update and finalize the typical editing scenario documentation & Tue $3 / 23 / 04$ & Wed 3/24/04 & \\
\hline 1525 & $\checkmark$ & Review and summarize editing scenario tasks for wrap-up report & Wed 3/24/04 & Wed 3/24/04 & \\
\hline 1526 & $\checkmark b$ & Setup for ArcFM Configuration & Thu $3 / 4 / 04$ & Mon 3/22/04 & \\
\hline 1527 & $\checkmark$ & Create primary display field document & Thu $3 / 4 / 04$ & Fri 3/5/04 & \\
\hline 1528 & $\checkmark$ & Create feature class / subtype / symbol definition & Fri 3/5/04 & Fri 3/12/04 & \\
\hline 1529 & $\checkmark$ & Create annotation / labeling documentation & Mon 3/15/04 & Wed 3/17/04 & \\
\hline 1530 & $\checkmark$ & Create class level automation configuration documentation & Fri 3/5/04 & Tue $3 / 9 / 04$ & \\
\hline 1531 & $\checkmark$ & Create field level automation configuration documentation & Tue 3/9/04 & Thu 3/11/04 & \\
\hline 1532 & $\checkmark$ & Define class and field model names & Thu $3 / 11 / 04$ & Mon 3/15/04 & \\
\hline 1533 & $\checkmark$ & Define snapping properties and connectivity & Mon 3/15/04 & Fri 3/19/04 & \\
\hline 1534 & $\checkmark$ & Review and summarize pre-config tasks for wrap-up report & Mon 3/22/04 & Mon 3/22/04 & \\
\hline 1535 & $\checkmark b$ & Finish Data Migration Matrix & Mon 3/1/04 & Fri 3/26/04 & \\
\hline 1536 & $\checkmark$ & Electric Matrix Tasks & Mon 3/1/04 & Fri 3/26/04 & \\
\hline 1537 & $\checkmark$ & PT Member1 Matrix Assignments & Mon 3/1/04 & Fri 3/19/04 & \\
\hline 1538 & $\checkmark$ & Miscellaneous electric matrix work & Mon 3/1/04 & Wed $3 / 17 / 04$ & \\
\hline 1539 & $\checkmark$ & Finish conduit matrix & Thu 3/18/04 & Thu $3 / 18 / 04$ & \\
\hline 1540 & $\checkmark$ & Finish electric discrepancy matrix & Thu 3/18/04 & Thu $3 / 18 / 04$ & \\
\hline 1541 & $\checkmark$ & Finish manhole matrix & Thu $3 / 18 / 04$ & Thu $3 / 18 / 04$ & \\
\hline 1542 & $\checkmark$ & Finish primary open point matrix & Thu $3 / 18 / 04$ & Thu $3 / 18 / 04$ & \\
\hline 1543 & $\checkmark$ & Finish splice matrix & Fri 3/19/04 & Fri 3/19/04 & \\
\hline 1544 & $\checkmark$ & Finish substation matrix & Fri 3/19/04 & Fri 3/19/04 & \\
\hline 1545 & $\checkmark$ & Finish Substation Breaker matrix & Fri 3/19/04 & Fri 3/19/04 & \\
\hline 1546 & $\checkmark$ & Finish terminator matrix & Fri 3/19/04 & Fri 3/19/04 & \\
\hline 1547 & $\checkmark$ & PT Member 2 Matrix Assignments & Mon 3/15/04 & Thu 3/25/04 & \\
\hline 1548 & $\checkmark$ & Finish transformer matrix & Mon 3/15/04 & Tue $3 / 16 / 04$ & \\
\hline 1549 & $\checkmark$ & Finish bus bar matrix & Tue $3 / 16 / 04$ & Wed 3/17/04 & \\
\hline 1550 & $\checkmark$ & Finish customer generator matrix & Wed 3/17/04 & Wed 3/17/04 & \\
\hline 1551 & $\checkmark$ & Finish electric company use matrix & Thu $3 / 18 / 04$ & Thu $3 / 18 / 04$ & \\
\hline 1552 & $\checkmark$ & Finish voltage regulator matrix & Thu $3 / 18 / 04$ & Fri 3/19/04 & \\
\hline 1553 & $\checkmark$ & Finish tie wire matrix & Fri 3/19/04 & Fri 3/19/04 & \\
\hline 1554 & $\checkmark$ & Finish recloser bank matrix & Mon 3/22/04 & Tue $3 / 23 / 04$ & \\
\hline 1555 & $\checkmark$ & Finish fuse cutout bank matrix & Tue $3 / 23 / 04$ & Thu $3 / 25 / 04$ & \\
\hline 1556 & $\checkmark$ & Finish switch matrix & Thu $3 / 25 / 04$ & Thu $3 / 25 / 04$ & \\
\hline 1557 & $\checkmark$ & PT Member3 Matrix Assignments & Fri 3/19/04 & Fri 3/26/04 & \\
\hline 1558 & $\checkmark$ & Finish primary meter matrix & Fri 3/19/04 & Fri 3/19/04 & \\
\hline 1559 & $\checkmark$ & Finish switch gear matrix & Wed 3/24/04 & Wed 3/24/04 & \\
\hline 1560 & $\checkmark$ & Finish $\mathrm{OH}$ primary matrix & Wed 3/24/04 & Thu $3 / 25 / 04$ & \\
\hline 1561 & $\checkmark$ & Finish secondary matrix & Thu $3 / 25 / 04$ & Fri 3/26/04 & \\
\hline 1562 & $\checkmark$ & PT Member4 Matrix Assignments & Thu 3/25/04 & Fri 3/26/04 & \\
\hline 1563 & $\checkmark$ & Finish support structure matrix & Thu 3/25/04 & Fri 3/26/04 & \\
\hline 1564 & $\checkmark$ & Gas Matrix Tasks & Mon 3/1/04 & Thu 3/25/04 & \\
\hline $\begin{array}{l}\text { NIPSC } \\
\text { Confid }\end{array}$ & $\begin{array}{l}\text { Co GIS } \\
\text { lential }\end{array}$ & Page 34 & & $\begin{array}{r}\text { Last Updat } \\
\text { Print }\end{array}$ & $\begin{array}{l}\text { ed on Tue } 7 / 3 / 07 \\
\text { ed on Fri 8/17/07 }\end{array}$ \\
\hline
\end{tabular}




\begin{tabular}{|c|c|c|c|c|c|}
\hline \multicolumn{6}{|c|}{ AEDR Construction Phase I Project Plan } \\
\hline ID & ( & Task Name & Start & Finish & \\
\hline 1565 & $\checkmark$ & PT Member5 Assignments & Mon 3/1/04 & Thu 3/18/04 & \\
\hline 1566 & $\checkmark$ & Miscellaneous gas matrix tasks & Mon 3/1/04 & Wed 3/10/04 & \\
\hline 1567 & $\checkmark$ & Finish drip matrix & Thu 3/11/04 & Thu $3 / 11 / 04$ & \\
\hline 1568 & $\checkmark$ & Finish pipeline marker matrix & Thu 3/11/04 & Thu $3 / 11 / 04$ & \\
\hline 1569 & $\checkmark$ & Finish service stub matrix & Thu 3/11/04 & Thu $3 / 11 / 04$ & \\
\hline 1570 & $\checkmark$ & Finish casing matrix & Thu 3/11/04 & Thu $3 / 11 / 04$ & \\
\hline 1571 & $\checkmark$ & Finish CP rectifier matrix & Thu 3/11/04 & Thu $3 / 11 / 04$ & \\
\hline 1572 & $\checkmark$ & Finish CP section matrix & Thu 3/11/04 & Fri 3/12/04 & \\
\hline 1573 & $\checkmark$ & Finish CP test point matrix & Fri 3/12/04 & Fri 3/12/04 & \\
\hline 1574 & $\checkmark$ & Finish emergency valve mark matrix & Fri 3/12/04 & Fri 3/12/04 & \\
\hline 1575 & $\checkmark$ & Finish gas discrepancy matrix & Fri 3/12/04 & Fri 3/12/04 & \\
\hline 1576 & $\checkmark$ & Finish squeeze off matrix & Fri 3/12/04 & Mon 3/15/04 & \\
\hline 1577 & $\checkmark$ & Finish fitting matrix & Mon 3/15/04 & Mon 3/15/04 & \\
\hline 1578 & $\checkmark$ & Finish the gas main matrix & Mon 3/15/04 & Tue $3 / 16 / 04$ & \\
\hline 1579 & $\checkmark$ & Finish the regulator station matrix & Tue $3 / 16 / 04$ & Thu 3/18/04 & \\
\hline 1580 & $\checkmark$ & PT Member6 Assignments & Mon 3/22/04 & Thu $3 / 25 / 04$ & \\
\hline 1581 & $\checkmark$ & Finish gas valve matrix & Mon 3/22/04 & Mon 3/22/04 & \\
\hline 1582 & $\checkmark$ & Finish distribution main matrix & Mon 3/22/04 & Tue $3 / 23 / 04$ & \\
\hline 1583 & $\checkmark$ & Finish regulator station matrix & Tue $3 / 23 / 04$ & Thu $3 / 25 / 04$ & \\
\hline 1584 & $\checkmark$ & Review / Cleanup Matrices & Thu 3/25/04 & Fri 3/26/04 & \\
\hline 1585 & $\checkmark b$ & Review/finalize gas data matrix & Thu $3 / 25 / 04$ & Fri 3/26/04 & \\
\hline 1586 & $\checkmark(1)$ & Review/finalize electric data matrix & Thu $3 / 25 / 04$ & Fri 3/26/04 & \\
\hline 1587 & $\checkmark$ & Review and summarize data matrix tasks for wrap-up report & Fri 3/26/04 & Fri 3/26/04 & \\
\hline 1588 & $\checkmark(1)$ & Migrate Sample Data Area & Thu 3/4/04 & Thu 3/25/04 & \\
\hline 1589 & $\checkmark$ & Create sample data migration delivery schedule & Thu $3 / 4 / 04$ & Thu $3 / 4 / 04$ & \\
\hline 1590 & $\checkmark$ & Migrate Sample Gas Data & Thu 3/4/04 & Fri 3/12/04 & \\
\hline 1591 & $\checkmark$ & Migrate sample gas data & Thu $3 / 4 / 04$ & Wed 3/10/04 & \\
\hline 1592 & $\checkmark$ & Test sample gas data & Wed 3/10/04 & Thu $3 / 11 / 04$ & \\
\hline 1593 & $\checkmark$ & Fix and re-deliver sample gas data & Thu $3 / 11 / 04$ & Fri 3/12/04 & \\
\hline 1594 & $\checkmark$ & Migrate Sample Electric Data & Thu 3/4/04 & Tue 3/16/04 & \\
\hline 1595 & $\checkmark$ & Migrate sample electric data & Thu $3 / 4 / 04$ & Fri 3/12/04 & \\
\hline 1596 & $\checkmark$ & Test sample electric data & Fri 3/12/04 & Mon 3/15/04 & \\
\hline 1597 & $\checkmark$ & Fix and re-deliver sample electric data & Mon 3/15/04 & Tue 3/16/04 & \\
\hline 1598 & $\checkmark$ & Migrate Sample Land Data & Thu $3 / 4 / 04$ & Fri 3/5/04 & \\
\hline 1599 & $\checkmark$ & Migrate sample land data & Thu $3 / 4 / 04$ & Thu $3 / 4 / 04$ & \\
\hline 1600 & $\checkmark$ & Test sample land data & Thu $3 / 4 / 04$ & Thu $3 / 4 / 04$ & \\
\hline 1601 & $\checkmark$ & Fix and re-deliver sample land data & Fri 3/5/04 & Fri 3/5/04 & \\
\hline 1602 & $\checkmark$ & Final Sample Data Migration Tasks & Tue 3/16/04 & Thu $3 / 25 / 04$ & \\
\hline 1603 & $\checkmark$ & Build Gas Network & Tue $3 / 16 / 04$ & Tue 3/23/04 & \\
\hline 1604 & $\checkmark b$ & QA gas data and address discrepancies & Tue $3 / 16 / 04$ & Fri 3/19/04 & \\
\hline 1605 & $\checkmark(3)$ & Build gas trace weights & Mon 3/22/04 & Mon 3/22/04 & \\
\hline 1606 & $\checkmark$ & Build gas geometric network & Tue $3 / 23 / 04$ & Tue $3 / 23 / 04$ & \\
\hline 1607 & $\checkmark$ & Review gas network build errors and address issues & Tue $3 / 23 / 04$ & Tue $3 / 23 / 04$ & \\
\hline 1608 & $\checkmark$ & Build Electric Network & Tue $3 / 16 / 04$ & Thu 3/25/04 & \\
\hline 1609 & $\checkmark b$ & QA electric data and address discrepancies & Tue $3 / 16 / 04$ & Tue $3 / 23 / 04$ & \\
\hline 1610 & $\sqrt{6}$ & Build electric trace weights & Wed 3/24/04 & Wed 3/24/04 & \\
\hline $\begin{array}{l}\text { NIPSC } \\
\text { Confid }\end{array}$ & $\begin{array}{l}\text { Co GIS } \\
\text { lential }\end{array}$ & Page 35 & & $\begin{array}{r}\text { Last Updat } \\
\text { Print }\end{array}$ & $\begin{array}{l}\text { ed on Tue } 7 / 3 / 07 \\
\text { ed on Fri 8/17/07 }\end{array}$ \\
\hline
\end{tabular}




\begin{tabular}{|c|c|c|c|c|c|}
\hline \multicolumn{6}{|c|}{ AEDR Construction Phase I Project Plan } \\
\hline ID & ( & Task Name & Start & Finish & \\
\hline 1611 & $\checkmark$ & Build electric geometric network & Thu 3/25/04 & Thu $3 / 25 / 04$ & \\
\hline 1612 & $\checkmark$ & Review electric network build errors and address issues & Thu $3 / 25 / 04$ & Thu $3 / 25 / 04$ & \\
\hline 1613 & $\checkmark b$ & Finalize the Data RFP & Thu 3/4/04 & Fri 3/26/04 & \\
\hline 1614 & $\checkmark$ & Add data migration to the RFP & Thu $3 / 4 / 04$ & Thu $3 / 18 / 04$ & \\
\hline 1615 & $\checkmark$ & Review the draft RFP & Fri 3/19/04 & Mon 3/22/04 & \\
\hline 1616 & $\checkmark$ & Notify the vendors that they will be receiving an RFP & Tue $3 / 23 / 04$ & Tue $3 / 23 / 04$ & \\
\hline 1617 & $\checkmark(1)$ & Meet to review/finalize data RFP / eval criteria & Tue $3 / 23 / 04$ & Tue $3 / 23 / 04$ & \\
\hline 1618 & $\checkmark$ & Revise the data RFP & Tue $3 / 23 / 04$ & Fri 3/26/04 & \\
\hline 1619 & $\checkmark$ & Weeks 5-6 (ArcFM Configuration) & Mon 3/29/04 & Fri 4/9/04 & \\
\hline 1620 & $\checkmark b$ & Re-Training / Refresher Course & Mon 3/29/04 & Tue $3 / 30 / 04$ & \\
\hline 1621 & $\checkmark$ & Re-training / refresher course & Mon 3/29/04 & Mon 3/29/04 & \\
\hline 1622 & $\checkmark$ & Document notes from the refresher class & Tue $3 / 30 / 04$ & Tue $3 / 30 / 04$ & \\
\hline 1623 & $\checkmark$ & Send RFP Out for Bid & Mon 3/29/04 & Tue 3/30/04 & \\
\hline 1624 & $\checkmark$ & Apply final RFP changes & Mon 3/29/04 & Mon 3/29/04 & \\
\hline 1625 & $\checkmark$ & Final review of the RFP & Mon 3/29/04 & Mon 3/29/04 & \\
\hline 1626 & $\checkmark$ & Send out the RFP & Mon 3/29/04 & Mon 3/29/04 & \\
\hline 1627 & $\checkmark$ & Review and summarize Data RFP tasks for wrap-up report & Tue $3 / 30 / 04$ & Tue $3 / 30 / 04$ & \\
\hline 1628 & $\checkmark$ & Final Sample Data Migration & Mon 3/29/04 & Wed $4 / 7 / 04$ & \\
\hline 1629 & $\checkmark$ & Final migration runs after matrix and data model freeze & Mon 3/29/04 & Thu 4/1/04 & \\
\hline 1630 & $\checkmark$ & Import personal geodatabase to SDE & Thu $4 / 1 / 04$ & Thu 4/1/04 & \\
\hline 1631 & $\checkmark$ & Apply miscellaneous data fixes & Thu $4 / 1 / 04$ & Wed $4 / 7 / 04$ & \\
\hline 1632 & $\checkmark$ & Review and summarize sample area data migration tasks for wrap-up report & Wed $4 / 7 / 04$ & Wed 4/7/04 & \\
\hline 1633 & $\checkmark y$ & Base ArcFM Configuration & Tue 3/30/04 & Fri 4/9/04 & \\
\hline 1634 & $\checkmark$ & Review Pre-Configuration Spreadsheets & Tue 3/30/04 & Fri 4/2/04 & \\
\hline 1635 & $\checkmark$ & Gas configuration spreadsheet review & Tue $3 / 30 / 04$ & Tue $3 / 30 / 04$ & \\
\hline 1636 & $\checkmark$ & Electric configuration spreadsheet review & Wed 3/31/04 & Wed 3/31/04 & \\
\hline 1637 & $\checkmark$ & Land configuration spreadsheet review & Thu $4 / 1 / 04$ & Thu $4 / 1 / 04$ & \\
\hline 1638 & $\checkmark$ & Symbology spreadsheet review & Thu $4 / 1 / 04$ & Thu 4/1/04 & \\
\hline 1639 & $\checkmark$ & Session manager configuration review & Fri 4/2/04 & Fri 4/2/04 & \\
\hline 1640 & $\checkmark$ & ArcFM Configuration Tasks & Mon 4/5/04 & Thu $4 / 8 / 04$ & \\
\hline 1641 & $\checkmark$ & ArcCatalog - Configure feature class properties based on specification & Mon 4/5/04 & Thu $4 / 8 / 04$ & \\
\hline 1642 & $\checkmark$ & ArcMap - Create initial set of stored displays & Mon 4/5/04 & Tue $4 / 6 / 04$ & \\
\hline 1643 & $\checkmark$ & ArcMap - Create initial set of page templates & Wed 4/7/04 & Thu $4 / 8 / 04$ & \\
\hline 1644 & $\checkmark$ & Final Review & Fri $4 / 9 / 04$ & Fri 4/9/04 & \\
\hline 1645 & $\checkmark$ & Review ArcMap feature class properties & Fri 4/9/04 & Fri 4/9/04 & \\
\hline 1646 & $\checkmark$ & Review data items identified during configuration & Fri 4/9/04 & Fri $4 / 9 / 04$ & \\
\hline 1647 & $\checkmark$ & Prep for functional analysis & Fri 4/9/04 & Fri 4/9/04 & \\
\hline 1648 & $\checkmark$ & Review and summarize ArcFM Config tasks for wrap-up report & Fri 4/9/04 & Fri $4 / 9 / 04$ & \\
\hline 1649 & $\checkmark b$ & Data RFP Week 5-6 Tasks & Mon 3/29/04 & Fri 4/9/04 & \\
\hline 1650 & $\checkmark$ & Vendor review of the RFP & Mon 3/29/04 & Fri 4/2/04 & \\
\hline 1651 & $\checkmark$ & Conduct data vendor conference call & Mon 4/5/04 & Mon 4/5/04 & \\
\hline 1652 & $\checkmark$ & Research of data vendor questions & Mon $4 / 5 / 04$ & Tue $4 / 6 / 04$ & \\
\hline 1653 & $\checkmark$ & Prepare Q/A summary sheets for data vendors & Tue 4/6/04 & Wed 4/7/04 & \\
\hline 1654 & $\checkmark$ & Vendors work on their proposals & Mon $4 / 5 / 04$ & Fri $4 / 9 / 04$ & \\
\hline 1655 & $\checkmark$ & Week 7 (Functional Analysis) & Mon 4/12/04 & Fri 4/16/04 & \\
\hline 1656 & $\checkmark 8$ & Base ArcFM Functional Analysis & Mon 4/12/04 & Fri 4/16/04 & \\
\hline $\begin{array}{l}\text { NIPSC } \\
\text { Confid }\end{array}$ & $\begin{array}{l}\text { Co GIS } \\
\text { lential }\end{array}$ & Page 36 & & $\begin{array}{r}\text { Last Updat } \\
\text { Print }\end{array}$ & $\begin{array}{l}\text { ed on Tue } 7 / 3 / 07 \\
\text { ed on Fri 8/17/07 }\end{array}$ \\
\hline
\end{tabular}




\begin{tabular}{|c|c|c|c|c|c|}
\hline \multicolumn{6}{|c|}{ AEDR Construction Phase I Project Plan } \\
\hline ID & ( & Task Name & Start & Finish & \\
\hline 1657 & $\checkmark$ & Review editing scenarios - Gas, Elec, Land & Mon 4/12/04 & Fri 4/16/04 & \\
\hline 1658 & $\checkmark$ & Review editing scenarios - EDFS & Fri 4/16/04 & Fri 4/16/04 & \\
\hline 1659 & $\checkmark$ & Data RFP Week 7 Tasks & Mon 4/12/04 & Fri 4/16/04 & \\
\hline 1660 & $\checkmark$ & Vendors work on their proposals & Mon 4/12/04 & Fri 4/16/04 & \\
\hline 1661 & $\checkmark$ & Notify internal audit & Thu 4/15/04 & Thu $4 / 15 / 04$ & \\
\hline 1662 & $\checkmark$ & Prepare for proposal delivery & Thu 4/15/04 & Thu $4 / 15 / 04$ & \\
\hline 1663 & $\checkmark$ & Week 8 (Proposal Review - Functional Analysis Follow-on) & Mon 4/19/04 & Fri 4/23/04 & \\
\hline 1664 & $\checkmark$ & Data RFP Week 8 Tasks & Mon 4/19/04 & Fri 4/23/04 & \\
\hline 1665 & $\checkmark$ & Vendors deliver proposals & Mon 4/19/04 & Mon 4/19/04 & \\
\hline 1666 & $\checkmark$ & Bid opening & Tue $4 / 20 / 04$ & Tue $4 / 20 / 04$ & \\
\hline 1667 & $\checkmark$ & Distribute proposals to the team & Tue $4 / 20 / 04$ & Tue $4 / 20 / 04$ & \\
\hline 1668 & $\checkmark$ & Prepare comparison spreadsheet & Tue $4 / 20 / 04$ & Thu 4/22/04 & \\
\hline 1669 & $\checkmark$ & Review all data proposals & Tue $4 / 20 / 04$ & Fri 4/23/04 & \\
\hline 1670 & $\checkmark$ & Meeting to determine reference checks & Thu $4 / 22 / 04$ & Thu $4 / 22 / 04$ & \\
\hline 1671 & $\checkmark$ & Start checking references & Thu $4 / 22 / 04$ & Thu $4 / 22 / 04$ & \\
\hline 1672 & $\checkmark$ & Week 8 Data RFP Status Meeting & Fri 4/23/04 & Fri 4/23/04 & \\
\hline 1673 & $\checkmark$ & Functional Analysis Follow-on Tasks & Mon 4/19/04 & Fri 4/23/04 & \\
\hline 1674 & $\checkmark$ & PT Member1 Week 8 Tasks & Mon 4/19/04 & Fri 4/23/04 & \\
\hline 1675 & $\checkmark$ & Review data model change candidates & Mon 4/19/04 & Mon 4/19/04 & \\
\hline 1676 & $\checkmark(1)$ & Configure the autoupdaters for transformers by sub-type & Tue $4 / 20 / 04$ & Tue $4 / 20 / 04$ & \\
\hline 1677 & $\checkmark$ & Review all relationship requirements not covered by func analysis & Thu $4 / 22 / 04$ & Fri 4/23/04 & \\
\hline 1678 & $\checkmark$ & PT Member2 Week 8 Tasks & Mon 4/19/04 & Fri 4/23/04 & \\
\hline 1679 & $\checkmark$ & Update field order for electric attributes & Mon 4/19/04 & Mon 4/19/04 & \\
\hline 1680 & $\checkmark$ & Add electric primary display fields & Mon 4/19/04 & Mon 4/19/04 & \\
\hline 1681 & $\checkmark$ & Finish annotation spreadsheet & Tue $4 / 20 / 04$ & Wed 4/21/04 & \\
\hline 1682 & $\checkmark$ & Add mask to Regulator symbols & Wed 4/21/04 & Wed $4 / 21 / 04$ & \\
\hline 1683 & $\checkmark$ & Add mask to Primary open point symbols & Wed 4/21/04 & Wed 4/21/04 & \\
\hline 1684 & $\checkmark$ & Remove rotation on capacitor bank & Wed 4/21/04 & Wed 4/21/04 & \\
\hline 1685 & $\checkmark$ & Apply "geometric" symbol rotation & Wed 4/21/04 & Thu $4 / 22 / 04$ & \\
\hline 1686 & $\checkmark$ & Update data matrix based on symbol rotation & Thu $4 / 22 / 04$ & Fri 4/23/04 & \\
\hline 1687 & $\checkmark$ & PT Member3 Week 8 Tasks & Mon 4/19/04 & Fri 4/23/04 & \\
\hline 1688 & $\checkmark$ & Prioritize the auto updater candidates & Mon 4/19/04 & Mon 4/19/04 & \\
\hline 1689 & $\checkmark$ & Investigate different ways to locate an intersection & Mon 4/19/04 & Mon 4/19/04 & \\
\hline 1690 & $\checkmark$ & Start implementation of annotation AU's & Tue $4 / 20 / 04$ & Tue $4 / 20 / 04$ & \\
\hline 1691 & $\checkmark$ & Document coding standards and guidelines & Wed $4 / 21 / 04$ & Thu $4 / 22 / 04$ & \\
\hline 1692 & $\checkmark$ & Implement selected AutoUpdaters & Fri 4/23/04 & Fri $4 / 23 / 04$ & \\
\hline 1693 & $\checkmark$ & PT Member4 Week 8 Tasks & Mon 4/19/04 & Fri 4/23/04 & \\
\hline 1694 & $\checkmark$ & Review data model change candidates & Mon 4/19/04 & Tue $4 / 20 / 04$ & \\
\hline 1695 & $\checkmark$ & Finish selected data mapping matrix sheets & Tue $4 / 20 / 04$ & Fri 4/23/04 & \\
\hline 1696 & $\checkmark$ & Add transformer jumper details to matrix & Fri 4/23/04 & Fri $4 / 23 / 04$ & \\
\hline 1697 & $\checkmark$ & PT Member5 Week 8 Tasks & Mon 4/19/04 & Wed 4/21/04 & \\
\hline 1698 & $\checkmark$ & Update field order for gas attributes & Mon 4/19/04 & Mon 4/19/04 & \\
\hline 1699 & $\checkmark$ & Update field order for land attributes & Mon 4/19/04 & Mon 4/19/04 & \\
\hline 1700 & $\checkmark$ & Add gas primary display fields & Mon 4/19/04 & Tue $4 / 20 / 04$ & \\
\hline 1701 & $\checkmark$ & Plot sample maps to test colors and page template & Tue $4 / 20 / 04$ & Wed 4/21/04 & \\
\hline 1702 & $\checkmark$ & Investigate whether or not reclosers can be connected to UG & Wed 4/21/04 & Wed 4/21/04 & \\
\hline $\begin{array}{l}\text { NIPSC } \\
\text { Confid }\end{array}$ & $\begin{array}{l}\text { Co GIS } \\
\text { lential }\end{array}$ & Page 37 & & $\begin{array}{r}\text { Last Updat } \\
\text { Print }\end{array}$ & $\begin{array}{l}\text { ed on Tue } 7 / 3 / 07 \\
\text { ed on Fri 8/17/07 }\end{array}$ \\
\hline
\end{tabular}




\begin{tabular}{|c|c|c|c|c|c|}
\hline \multicolumn{6}{|c|}{ AEDR Construction Phase I Project Plan } \\
\hline ID & (a) & Task Name & Start & Finish & \\
\hline 1703 & $\sqrt{b}$ & Add Add FuseUnit, TransformerUnit and PrimSecdCondlnfo to the template & Wed 4/21/04 & Wed 4/21/04 & \\
\hline 1704 & $\checkmark$ & Change snapping to be in DB units instead of pixels & Wed 4/21/04 & Wed $4 / 21 / 04$ & \\
\hline 1705 & $\checkmark$ & Week 9 (Vendor Shortlist / Func Analysis Follow-on) & Mon 4/26/04 & Fri $4 / 30 / 04$ & \\
\hline 1706 & $\checkmark$ & Data RFP Week 9 Tasks & Mon 4/26/04 & Fri $4 / 30 / 04$ & \\
\hline 1707 & $\checkmark$ & Create consolidated questions spreadsheet & Mon 4/26/04 & Mon 4/26/04 & \\
\hline 1708 & $\checkmark$ & Send clarification questions to the vendors & Mon 4/26/04 & Mon 4/26/04 & \\
\hline 1709 & $\checkmark$ & Vendors answer clarification questions & Mon 4/26/04 & Wed 4/28/04 & \\
\hline 1710 & $\checkmark$ & Continue reference checks & Mon 4/26/04 & Wed 4/28/04 & \\
\hline 1711 & $\checkmark$ & Review Proposals & Mon 4/26/04 & Wed 4/28/04 & \\
\hline 1712 & $\checkmark$ & Review vendors answers to clarification questions & Thu $4 / 29 / 04$ & Thu $4 / 29 / 04$ & \\
\hline 1713 & $\checkmark$ & Shortlist selection process & Thu $4 / 29 / 04$ & Fri 4/30/04 & \\
\hline 1714 & $\checkmark$ & Inform all vendors regarding their status & Fri 4/30/04 & Fri 4/30/04 & \\
\hline 1715 & $\checkmark$ & Functional Analysis Follow-on Tasks & Mon 4/26/04 & Fri $4 / 30 / 04$ & \\
\hline 1716 & $\checkmark$ & PT Member1 Week 9 Tasks & Mon 4/26/04 & Fri 4/30/04 & \\
\hline 1717 & $\checkmark$ & Update configuration spreadsheets with workshop notes & Mon 4/26/04 & Tue $4 / 27 / 04$ & \\
\hline 1718 & $\checkmark$ & Review and summarize Functional Analysis tasks for wrap-up report & Wed 4/28/04 & Thu $4 / 29 / 04$ & \\
\hline 1719 & $\checkmark$ & Review relationship requirements & Fri 4/30/04 & Fri 4/30/04 & \\
\hline 1720 & $\checkmark$ & Start Integration testing / cleanup & Mon 4/26/04 & Fri 4/30/04 & \\
\hline 1721 & $\checkmark$ & Defect tracking tool investigation & Wed 4/28/04 & Wed 4/28/04 & \\
\hline 1722 & $\checkmark$ & PT Member2 Week 9 Tasks & Mon 4/26/04 & Fri 4/30/04 & \\
\hline 1723 & $\checkmark$ & Research tools to apply data model changes to existing data & Mon 4/26/04 & Mon 4/26/04 & \\
\hline 1724 & $\checkmark$ & Apply selected data model edits & Tue $4 / 27 / 04$ & Tue $4 / 27 / 04$ & \\
\hline 1725 & $\checkmark$ & Resolve C\# install issues & Wed 4/28/04 & Wed $4 / 28 / 04$ & \\
\hline 1726 & $\checkmark$ & Add ref_point and ref_line feature classes & Wed 4/28/04 & Wed $4 / 28 / 04$ & \\
\hline 1727 & $\checkmark$ & Misc annotation cleanup / validation & Wed 4/28/04 & Wed $4 / 28 / 04$ & \\
\hline 1728 & $\checkmark$ & Finalize electric field order & Thu $4 / 29 / 04$ & Thu $4 / 29 / 04$ & \\
\hline 1729 & $\checkmark$ & Research font requirements & Fri $4 / 30 / 04$ & Fri 4/30/04 & \\
\hline 1730 & $\checkmark$ & PT Member3 Week 9 Tasks & Mon 4/26/04 & Fri $4 / 30 / 04$ & \\
\hline 1731 & $\checkmark$ & Complete programming standards and guidelines & Mon 4/26/04 & Mon 4/26/04 & \\
\hline 1732 & $\checkmark$ & Complete the Regulator Station edit task & Mon 4/26/04 & Tue $4 / 27 / 04$ & \\
\hline 1733 & $\checkmark$ & Organize remaining AU's and present to the team & Tue $4 / 27 / 04$ & Tue $4 / 27 / 04$ & \\
\hline 1734 & $\checkmark$ & Code selected high-priority AU's & Tue $4 / 27 / 04$ & Fri 4/30/04 & \\
\hline 1735 & $\checkmark$ & PT Member4 Week 9 Tasks & Mon 4/26/04 & Fri 4/30/04 & \\
\hline 1736 & $\checkmark$ & Data matrix - Manhole & Mon 4/26/04 & Tue $4 / 27 / 04$ & \\
\hline 1737 & $\checkmark$ & Data matrix - Field Inventory Light & Tue $4 / 27 / 04$ & Wed $4 / 28 / 04$ & \\
\hline 1738 & $\checkmark$ & Data matrix - Transmission Conductor & Wed 4/28/04 & Thu $4 / 29 / 04$ & \\
\hline 1739 & $\checkmark$ & Data matrix - Terminator & Thu 4/29/04 & Fri 4/30/04 & \\
\hline 1740 & $\checkmark$ & PT Member5 Week 9 Tasks & Mon 4/26/04 & Fri 4/30/04 & \\
\hline 1741 & $\checkmark$ & Recloser investigation & Mon 4/26/04 & Mon 4/26/04 & \\
\hline 1742 & $\checkmark$ & Cross-train on data model changes & Mon 4/26/04 & Mon 4/26/04 & \\
\hline 1743 & $\checkmark$ & Apply Gas data model changes & Tue $4 / 27 / 04$ & Tue $4 / 27 / 04$ & \\
\hline 1744 & $\checkmark$ & Finalize display order for gas and land object & Tue $4 / 27 / 04$ & Wed $4 / 28 / 04$ & \\
\hline 1745 & $\checkmark$ & Review hardcopy plots & Wed 4/28/04 & Wed 4/28/04 & \\
\hline 1746 & $\checkmark$ & Apply changes based on hardcopy review & Thu $4 / 29 / 04$ & Thu $4 / 29 / 04$ & \\
\hline 1747 & $\checkmark$ & Field Browser Monthly Update & Thu $4 / 29 / 04$ & Fri $4 / 30 / 04$ & \\
\hline 1748 & $\checkmark$ & Week 10 (RFP Review / Documentation) & Mon $5 / 3 / 04$ & Fri 5/7/04 & \\
\hline $\begin{array}{l}\text { NIPSC } \\
\text { Confid }\end{array}$ & $\begin{array}{l}\text { Co GIS } \\
\text { lential }\end{array}$ & Page 38 & & $\begin{array}{r}\text { Last Updat } \\
\text { Print }\end{array}$ & $\begin{array}{l}\text { ed on Tue } 7 / 3 / 07 \\
\text { ed on Fri 8/17/07 }\end{array}$ \\
\hline
\end{tabular}




\begin{tabular}{|c|c|c|c|c|c|}
\hline \multicolumn{6}{|c|}{ AEDR Construction Phase I Project Plan } \\
\hline ID & (a) & Task Name & Start & Finish & \\
\hline 1749 & $\checkmark$ & Miscellaneous Week 10 Tasks & Mon 5/3/04 & Fri 5/7/04 & \\
\hline 1750 & $\checkmark$ & Receive SynerGEE Interface proposals & Mon 5/3/04 & Mon 5/3/04 & \\
\hline 1751 & $\checkmark$ & Receive CADOPS / FeederAll Interface Proposals & Mon $5 / 3 / 04$ & Mon 5/3/04 & \\
\hline 1752 & $\checkmark$ & Investigate defect tracking systems & Mon 5/3/04 & Fri 5/7/04 & \\
\hline 1753 & $\checkmark$ & Investigate remote access for off-site vendors & Mon 5/3/04 & Fri 5/7/04 & \\
\hline 1754 & $\checkmark$ & Move Source Safe to the new server & Mon 5/3/04 & Wed 5/5/04 & \\
\hline 1755 & $\checkmark$ & C\# installation tasks & Mon 5/3/04 & Fri 5/7/04 & \\
\hline 1756 & $\checkmark$ & Functional analysis wrap-up report & Mon 5/3/04 & Mon 5/3/04 & \\
\hline 1757 & $\checkmark$ & Data RFP Week 10 Tasks & Mon 5/3/04 & Fri 5/7/04 & \\
\hline 1758 & $\checkmark$ & Vendors prepare for presentations & Mon $5 / 3 / 04$ & Fri 5/7/04 & \\
\hline 1759 & $\checkmark$ & Setup logistics for the vendor presentations & Mon $5 / 3 / 04$ & Fri 5/7/04 & \\
\hline 1760 & $\checkmark$ & Expert Choice workshops & Tue 5/4/04 & Wed 5/5/04 & \\
\hline 1761 & $\checkmark$ & Review data related risk list items & Wed 5/5/04 & Wed 5/5/04 & \\
\hline 1762 & $\checkmark$ & Create Documentation & Mon 5/3/04 & Fri 5/7/04 & \\
\hline 1763 & $\checkmark$ & Team meeting to determine documentation requirements & Tue 5/4/04 & Tue 5/4/04 & \\
\hline 1764 & $\checkmark$ & Start user guides / training manuals / on-line help & Mon 5/3/04 & Fri 5/7/04 & \\
\hline 1765 & $\checkmark$ & Start administrator's guide & Mon 5/3/04 & Fri 5/7/04 & \\
\hline 1766 & $\checkmark$ & Start testing documentation & Mon 5/3/04 & Fri 5/7/04 & \\
\hline 1767 & $\checkmark$ & Week 11 (Data Vendor Selection / Technical Wrap-Up) & Mon 5/10/04 & Fri 5/14/04 & \\
\hline 1768 & $\checkmark$ & Miscellaneous week 11 tasks & Mon 5/10/04 & Mon 5/10/04 & \\
\hline 1769 & $\checkmark$ & Review CADOPS/FeederAll proposals & Mon 5/10/04 & Mon 5/10/04 & \\
\hline 1770 & $\checkmark$ & Review SynerGEE proposals & Mon 5/10/04 & Mon 5/10/04 & \\
\hline 1771 & $\checkmark$ & Team meeting to review CADOPS/FeederAll/SynerGEE interface proposals & Mon 5/10/04 & Mon 5/10/04 & \\
\hline 1772 & $\checkmark(1)$ & Data RFP Week 11 Tasks & Tue 5/11/04 & Fri 5/14/04 & \\
\hline 1773 & $\checkmark$ & Vendor 1 presentation & Tue 5/11/04 & Tue $5 / 11 / 04$ & \\
\hline 1774 & $\checkmark$ & Vendor 1 wrap-up meeting & Tue 5/11/04 & Tue 5/11/04 & \\
\hline 1775 & $\checkmark$ & Vendor 2 presentation & Wed 5/12/04 & Wed 5/12/04 & \\
\hline 1776 & $\checkmark$ & Expert Choice - Vendor 1 vs 2 & Wed 5/12/04 & Wed 5/12/04 & \\
\hline 1777 & $\checkmark$ & Vendor 3 presentation & Thu 5/13/04 & Thu $5 / 13 / 04$ & \\
\hline 1778 & $\checkmark$ & Expert Choice - Vendor 1 vs 3 and 2 vs 3 & Thu 5/13/04 & Thu 5/13/04 & \\
\hline 1779 & $\checkmark$ & Prepare summary report / recommendation for data vendor & Thu 5/13/04 & Fri 5/14/04 & \\
\hline 1780 & $\checkmark$ & Final data vendor selection & Fri 5/14/04 & Fri 5/14/04 & \\
\hline 1781 & $\checkmark$ & Finish Iteration 1 Documentation & Mon 5/10/04 & Fri 5/14/04 & \\
\hline 1782 & $\checkmark$ & Finish user guides & Mon 5/10/04 & Fri 5/14/04 & \\
\hline 1783 & $\checkmark$ & Finish administrator's guide & Mon 5/10/04 & Fri 5/14/04 & \\
\hline 1784 & $\checkmark$ & Finish test case documentation & Mon 5/10/04 & Fri 5/14/04 & \\
\hline 1785 & $\sqrt{6}$ & Integration Testing / Rework & Mon 5/10/04 & Fri $5 / 14 / 04$ & \\
\hline 1786 & $\checkmark$ & Perform integration testing & Mon 5/10/04 & Fri 5/14/04 & \\
\hline 1787 & $\checkmark$ & Fix bugs that are found & Mon 5/10/04 & Fri 5/14/04 & \\
\hline 1788 & $\checkmark$ & Setup Production Servers & Tue 5/11/04 & Wed 5/12/04 & \\
\hline 1789 & $\checkmark$ & Install ESRI/M\&M software on new production server & Tue 5/11/04 & Tue 5/11/04 & \\
\hline 1790 & $\checkmark$ & Install / Configure SQL Server & Tue 5/11/04 & Wed $5 / 12 / 04$ & \\
\hline 1791 & $\checkmark$ & Document SQL Server Configuration & Wed 5/12/04 & Wed 5/12/04 & \\
\hline 1792 & $\checkmark$ & Week 12 (Iteration Wrap-Up) & Mon 5/17/04 & Fri 6/4/04 & \\
\hline 1793 & $\checkmark$ & Week 12 Data RFP Tasks & Mon 5/17/04 & Fri 5/21/04 & \\
\hline 1794 & $\checkmark$ & Answer vendor questions & Mon 5/17/04 & Mon 5/17/04 & \\
\hline $\begin{array}{l}\text { NIPSC } \\
\text { Confid }\end{array}$ & $\begin{array}{l}\text { Co GIS } \\
\text { lential }\end{array}$ & Page 39 & & $\begin{array}{r}\text { Last Updat } \\
\text { Print }\end{array}$ & $\begin{array}{l}\text { ed on Tue } 7 / 3 / 07 \\
\text { ed on Fri 8/17/07 }\end{array}$ \\
\hline
\end{tabular}




\begin{tabular}{|c|c|c|c|c|c|}
\hline \multicolumn{6}{|c|}{ AEDR Construction Phase I Project Plan } \\
\hline ID & (a) & Task Name & Start & Finish & \\
\hline 1795 & $\checkmark$ & Contract negotiations & Mon 5/17/04 & Fri 5/21/04 & \\
\hline 1796 & $\checkmark \vee$ & Miscellaneous Wrap-Up Tasks & Tue 5/18/04 & Fri 5/21/04 & \\
\hline 1797 & $\checkmark$ & Setup defect tracking software & Tue 5/18/04 & Tue $5 / 18 / 04$ & \\
\hline 1798 & $\checkmark$ & Review data model change candidates & Tue 5/18/04 & Wed 5/19/04 & \\
\hline 1799 & $\checkmark$ & Update data model & Wed 5/19/04 & Fri 5/21/04 & \\
\hline 1800 & $\checkmark$ & Review Vision document & Tue 5/18/04 & Tue $5 / 18 / 04$ & \\
\hline 1801 & $\checkmark$ & Review high level requirements & Tue 5/18/04 & Tue 5/18/04 & \\
\hline 1802 & $\checkmark$ & Review procedures and guidelines & Tue 5/18/04 & Tue 5/18/04 & \\
\hline 1803 & $\checkmark$ & Review sample data errors list & Tue 5/18/04 & Tue 5/18/04 & \\
\hline 1804 & $\checkmark$ & Review Source Safe directory structure & Tue 5/18/04 & Tue $5 / 18 / 04$ & \\
\hline 1805 & $\checkmark$ & Review team leads spreadsheet & Tue 5/18/04 & Tue 5/18/04 & \\
\hline 1806 & $\checkmark$ & Create Quick Start user's guide & Wed 5/19/04 & Wed 5/19/04 & \\
\hline 1807 & $\checkmark$ & Review data model issues with team & Wed 5/19/04 & Wed $5 / 19 / 04$ & \\
\hline 1808 & $\checkmark$ & Review risk list & Thu 5/20/04 & Thu 5/20/04 & \\
\hline 1809 & $\checkmark$ & Review communication plan & Thu 5/20/04 & Thu $5 / 20 / 04$ & \\
\hline 1810 & $\checkmark b$ & Iteration Wrap-Up Meeting & Fri 5/21/04 & Fri 5/21/04 & \\
\hline 1811 & $\checkmark$ & Iteration 1 wrap up meeting & Fri 5/21/04 & Fri 5/21/04 & \\
\hline 1812 & $\checkmark$ & Finalize the wrap-up report & Fri 5/21/04 & Fri 5/21/04 & \\
\hline 1813 & $\checkmark$ & Recurring Tasks & Mon 5/24/04 & Fri 6/4/04 & \\
\hline 1814 & $\checkmark(3)$ & Project management tasks & Mon 5/24/04 & Fri 6/4/04 & \\
\hline 1815 & $\checkmark$ & Iteration 2 Prep Tasks & Mon 5/24/04 & Fri 5/28/04 & \\
\hline 1816 & $\checkmark$ & Apply data model changes & Mon 5/24/04 & Fri 5/28/04 & \\
\hline 1817 & $\checkmark$ & CADOPS/FeederAll Planning & Mon 5/24/04 & Fri 5/28/04 & \\
\hline 1818 & $\checkmark$ & EDFS/ArcFM/MAPPS Planning & Mon 5/24/04 & Fri 5/28/04 & \\
\hline 1819 & $\checkmark$ & Data Migration / Rectification Planning & Mon 5/24/04 & Fri 5/28/04 & \\
\hline 1820 & $\checkmark$ & Setup VPN & Wed 5/26/04 & Wed 5/26/04 & \\
\hline 1821 & $\checkmark$ & Setup Defect Tracking & Thu $5 / 27 / 04$ & Thu $5 / 27 / 04$ & \\
\hline 1822 & $\checkmark$ & Finalize Team Leads Spreadsheet & Thu $5 / 27 / 04$ & Thu $5 / 27 / 04$ & \\
\hline 1823 & $\checkmark$ & Data review & Wed 5/26/04 & Thu 5/27/04 & \\
\hline 1824 & $\checkmark$ & Finish component specs & Mon 5/24/04 & Thu $5 / 27 / 04$ & \\
\hline 1825 & $\checkmark$ & GIS Application and Interface Construction - Iteration 2 (GIS Customizations and Interfaces) & Mon 6/7/04 & Mon 9/13/04 & \\
\hline 1826 & $\checkmark b$ & CADOPS / Feederall Interface & Mon 6/7/04 & Mon 8/16/04 & \\
\hline 1827 & $\checkmark(1)$ & Week 1 CADOPS / FeederAll Interface Tasks & Mon 6/7/04 & Fri 6/11/04 & \\
\hline 1828 & $\checkmark$ & Iteration 2 kickoff meeting & Mon 6/7/04 & Mon 6/7/04 & \\
\hline 1829 & $\checkmark$ & CADOPS / FeederAll Interface Workshops & Tue 6/8/04 & Thu 6/10/04 & \\
\hline 1830 & $\checkmark$ & Kickoff meeting & Tue 6/8/04 & Tue 6/8/04 & \\
\hline 1831 & $\checkmark$ & Review Network Adapter & Tue 6/8/04 & Tue 6/8/04 & \\
\hline 1832 & $\checkmark$ & Finalize Data Mapping Matrix (GIS-ABB) & Tue 6/8/04 & Wed 6/9/04 & \\
\hline 1833 & $\checkmark$ & Finalize Interface Specification & Thu $6 / 10 / 04$ & Thu 6/10/04 & \\
\hline 1834 & $\checkmark$ & Organize workshop notes & Fri 6/11/04 & Fri 6/11/04 & \\
\hline 1835 & $\checkmark$ & Deliver the notes to NIPSCO & Fri 6/11/04 & Fri 6/11/04 & \\
\hline 1836 & $\checkmark(1)$ & Week 2 CADOPS / FeederAll Interface Tasks & Mon 6/14/04 & Fri 6/18/04 & \\
\hline 1837 & $\checkmark$ & Prepare draft design and requirements documents & Mon 6/14/04 & Thu $6 / 17 / 04$ & \\
\hline 1838 & $\checkmark$ & Review with Analyst & Fri 6/18/04 & Fri 6/18/04 & \\
\hline 1839 & $\checkmark$ & Deliver requirements and design to NIPSCO & Fri 6/18/04 & Fri 6/18/04 & \\
\hline 1840 & $\checkmark 8$ & Week 3-4 CADOPS / FeederAll Interface Tasks & Mon 6/21/04 & Fri 7/2/04 & \\
\hline $\begin{array}{l}\text { NIPSC } \\
\text { Confid }\end{array}$ & $\begin{array}{l}\text { Co GIS } \\
\text { lential }\end{array}$ & Page 40 & & $\begin{array}{r}\text { Last Updat } \\
\text { Print }\end{array}$ & $\begin{array}{l}\text { ed on Tue } 7 / 3 / 07 \\
\text { ed on Fri 8/17/07 }\end{array}$ \\
\hline
\end{tabular}




\section{\begin{tabular}{|ll}
\hline & AEDR Construction Phase I Project Plan
\end{tabular}}

\begin{tabular}{|c|c|c|c|c|c|}
\hline ID & (a) & Task Name & Start & Finish & \\
\hline 1841 & $\checkmark$ & Review requirements and design & Mon 6/21/04 & Wed 6/23/04 & \\
\hline 1842 & $\checkmark$ & Update and finalize requirements and design & Thu 6/24/04 & Thu 6/24/04 & \\
\hline 1843 & $\checkmark$ & CADOPS / FeederAll requirements conference call & Thu $6 / 24 / 04$ & Thu 6/24/04 & \\
\hline 1844 & $\checkmark$ & Meeting to determine how to support new data requirements & Wed 6/30/04 & Wed 6/30/04 & \\
\hline 1845 & $\checkmark$ & Final review and approval of specs & Fri 7/2/04 & Fri 7/2/04 & \\
\hline 1846 & $\checkmark \theta$ & Weeks 5-9 CADOPS / FeederAll Interface Tasks & Mon $7 / 5 / 04$ & Tue 8/10/04 & \\
\hline 1847 & $\checkmark$ & Develop CADOPS I FeederAll Interfaces & Mon $7 / 5 / 04$ & Tue 8/10/04 & \\
\hline 1848 & $\checkmark$ & Geodatabase Export via Network Adapter & Mon $7 / 5 / 04$ & Wed $7 / 14 / 04$ & \\
\hline 1849 & $\checkmark$ & Create test data for Network Adapter functionality & Thu $7 / 15 / 04$ & Thu $7 / 15 / 04$ & \\
\hline 1851 & $\checkmark$ & Meeting to determine resolution to devices at the end point of an edge & Fri 7/23/04 & Fri 7/23/04 & \\
\hline 1852 & $\checkmark$ & Prep for code review meeting & Mon $7 / 26 / 04$ & Mon 7/26/04 & \\
\hline 1853 & $\checkmark$ & Code review for CADOPS / FeederAll interface code & Mon $7 / 26 / 04$ & Mon 7/26/04 & \\
\hline 1854 & $\checkmark$ & CADOPS / FeederAll Testing and Rework & Mon 7/26/04 & Wed $8 / 4 / 04$ & \\
\hline 1855 & $\checkmark$ & Documentation (user guide, training, test cases, admin guide) & Thu 8/5/04 & Tue 8/10/04 & \\
\hline 1856 & $\checkmark$ & Prepare for Delivery of the CADOPS / FeederAll interface & Thu $8 / 5 / 04$ & Thu 8/5/04 & \\
\hline 1857 & $\checkmark$ & Demonstrate the CADOPS and FeederAll Interfaces & Fri 7/23/04 & Fri 8/6/04 & \\
\hline 1858 & $\checkmark$ & Demo \#1 - CADOPS and FeederAll interface webcast demo / review & Fri $7 / 23 / 04$ & Fri $7 / 23 / 04$ & \\
\hline 1859 & $\checkmark$ & Demo \#2 - CADOPS and FeederAll interface webcast demo / review & Fri 7/30/04 & Fri 7/30/04 & \\
\hline 1860 & $\checkmark$ & Demo \#3 - CADOPS and FeederAll interface webcast demo / review & Fri 8/6/04 & Fri 8/6/04 & \\
\hline 1862 & $\checkmark$ & Install and Test the CADOPS / FeederAll Interface & Wed 8/11/04 & Mon 8/16/04 & \\
\hline 1863 & $\checkmark$ & Configure CADOPS / FeederAll interface at NIPSCO & Wed 8/11/04 & Wed 8/11/04 & \\
\hline 1864 & $\checkmark$ & Test CADOPS / FeederAll interface & Thu 8/12/04 & Thu 8/12/04 & \\
\hline 1865 & $\checkmark$ & Review CADOPS / FeederAll user documentation & Fri 8/13/04 & Fri 8/13/04 & \\
\hline 1866 & $\checkmark$ & Acceptance testing of the CADOPS / FeederAll interface & Mon 8/16/04 & Mon 8/16/04 & \\
\hline 1867 & $\checkmark$ & Complete CADOPS I FeederAll Open Issues & Mon 8/9/04 & Mon 8/9/04 & \\
\hline 1868 & $\checkmark$ & Ability to export assemblies on support structures & Mon 8/9/04 & Mon 8/9/04 & \\
\hline 1869 & $\checkmark$ & Additional issues as documented in the workshop notes & Mon 8/9/04 & Mon 8/9/04 & \\
\hline 1870 & $\checkmark$ & Data Modeling Tasks & Mon 6/14/04 & Fri 9/10/04 & \\
\hline 1871 & $\checkmark$ & Data Model Review & Thu 6/17/04 & Fri 7/2/04 & \\
\hline 1872 & $\checkmark$ & Gather the Data Model Requirements & Thu 6/17/04 & Fri 6/18/04 & \\
\hline 1873 & $\checkmark$ & Data model issue review meeting & Thu 6/17/04 & Thu $6 / 17 / 04$ & \\
\hline 1874 & $\checkmark$ & Review CADOPS / FeederAll data model issues & Thu $6 / 17 / 04$ & Thu 6/17/04 & \\
\hline 1875 & $\checkmark$ & Transmission versus distribution modeling - make consistent & Thu $6 / 17 / 04$ & Thu 6/17/04 & \\
\hline 1876 & $\checkmark$ & OH versus UG modeling - make consistent & Thu $6 / 17 / 04$ & Thu 6/17/04 & \\
\hline 1877 & $\checkmark$ & Look at linear features and related point data & Thu $6 / 17 / 04$ & Thu 6/17/04 & \\
\hline 1878 & $\checkmark$ & Decide if transmission switch feature class should be created or handled with existing model & Fri 6/18/04 & Fri 6/18/04 & \\
\hline 1879 & $\checkmark$ & Decide how to handle DOT Indicator for abandoned transmission main & Fri 6/18/04 & Fri 6/18/04 & \\
\hline 1880 & $\checkmark$ & Decide how to categorize primary and transmission conductors - e.g., $34 \mathrm{KV}$ & Fri 6/18/04 & Fri 6/18/04 & \\
\hline 1881 & $\checkmark$ & Decide if some common fields should be removed from the data model & Fri 6/18/04 & Fri 6/18/04 & \\
\hline 1882 & $\checkmark$ & Apply the Data Model Changes & Tue 6/22/04 & Fri 7/2/04 & \\
\hline 1885 & $\checkmark$ & Apply the gas and land data model normalization changes & Wed 6/30/04 & Wed 6/30/04 & \\
\hline 1886 & $\checkmark$ & Review the Elementool entries for duplicates & Thu $7 / 1 / 04$ & Thu $7 / 1 / 04$ & \\
\hline \multicolumn{2}{|c|}{$\begin{array}{l}\text { NIPSCO GIS } \\
\text { Confidential }\end{array}$} & Page 41 & & $\begin{array}{r}\text { Last Updat } \\
\text { Print }\end{array}$ & $\begin{array}{l}\text { on Tue } 7 / 3 / 07 \\
\text { on Fri 8/17/07 }\end{array}$ \\
\hline
\end{tabular}




\section{\begin{tabular}{|ll}
\hline & AEDR Construction Phase I Project Plan
\end{tabular}}

\begin{tabular}{|c|c|c|c|c|c|}
\hline ID & (a) & Task Name & Start & Finish & \\
\hline 1887 & $\checkmark$ & Review the data model normalization changes & Thu $7 / 1 / 04$ & Fri 7/2/04 & \\
\hline 1888 & $\checkmark$ & Finalize the data model normalization changes and check in the files & Fri $7 / 2 / 04$ & Fri 7/2/04 & \\
\hline 1889 & $\checkmark$ & Apply data model normalization changes to the sample data & Fri $7 / 2 / 04$ & Fri 7/2/04 & \\
\hline 1890 & $\checkmark$ & Custom Lighting Service & Thu 6/24/04 & Fri 7/2/04 & \\
\hline 1891 & $\checkmark$ & Gather the Data Model Requirements for Custom Lighting Services & Thu 6/24/04 & Mon 6/28/04 & \\
\hline 1892 & $\checkmark \bullet$ & Meet to determine lighting service requirements & Thu 6/24/04 & Thu 6/24/04 & \\
\hline 1893 & $\checkmark$ & Document custom lighting services requirements & Mon 6/28/04 & Mon 6/28/04 & \\
\hline 1894 & $\checkmark$ & Log the custom lighting service data model requirements in Elementool & Mon 6/28/04 & Mon 6/28/04 & \\
\hline 1895 & $\checkmark$ & Apply Data Model Changes for Custom Lighting Services & Tue 6/29/04 & Fri $7 / 2 / 04$ & \\
\hline 1897 & $\checkmark$ & Update the data model with the list of approved lighting service changes & Thu $7 / 1 / 04$ & Thu $7 / 1 / 04$ & \\
\hline 1898 & $\checkmark$ & Finalize the lighting service data model changes and check in the files & Fri $7 / 2 / 04$ & Fri $7 / 2 / 04$ & \\
\hline 1899 & $\checkmark$ & Apply the lighting service data model changes to the sample data & Fri $7 / 2 / 04$ & Fri 7/2/04 & \\
\hline 1900 & $\checkmark$ & EDFS & Mon 6/14/04 & Tue $7 / 6 / 04$ & \\
\hline 1901 & $\checkmark$ & Gather the Data Model Requirements for EDFS & Mon 6/14/04 & Mon 6/14/04 & \\
\hline 1902 & $\checkmark$ & Review the EDFS spreadsheet / requirements & Mon 6/14/04 & Mon 6/14/04 & \\
\hline 1903 & $\checkmark$ & Determine all EDFS data model requirements & Mon 6/14/04 & Mon 6/14/04 & \\
\hline 1904 & $\checkmark$ & Log the EDFS data model requirements in Elementool & Mon 6/14/04 & Mon 6/14/04 & \\
\hline 1905 & $\checkmark$ & Apply Data Model Changes for EDFS & Tue 6/29/04 & Tue $7 / 6 / 04$ & \\
\hline 1906 & $\checkmark$ & Review EDFS data model candidates in Elementool & Tue $6 / 29 / 04$ & Tue 6/29/04 & \\
\hline 1908 & $\checkmark$ & Finalize the EDFS data model changes and check in the files & Fri $7 / 2 / 04$ & Fri $7 / 2 / 04$ & \\
\hline 1909 & $\checkmark$ & Apply the EDFS data model changes to the sample data & Fri $7 / 2 / 04$ & Fri 7/2/04 & \\
\hline 1910 & $\checkmark$ & Review remaining EDFS data model candidates and determine next steps & Tue $7 / 6 / 04$ & Tue $7 / 6 / 04$ & \\
\hline 1911 & $\checkmark$ & Merge the Schemas Into ArcSDE & Mon $7 / 12 / 04$ & Fri 7/16/04 & \\
\hline 1912 & $\checkmark$ & Merge schemas from the three geodatabases into one SDE database & Mon 7/12/04 & Tue $7 / 13 / 04$ & \\
\hline 1913 & $\checkmark$ & Identify all single precision fields that need to be changed & Wed $7 / 14 / 04$ & Wed $7 / 14 / 04$ & \\
\hline 1914 & $\checkmark$ & Update single precision fields to double precision & Thu $7 / 15 / 04$ & Thu $7 / 15 / 04$ & \\
\hline 1915 & $\checkmark$ & Deliver merged data model to data migration vendor & Fri 7/16/04 & Fri 7/16/04 & \\
\hline 1916 & $\checkmark$ & Setup Tasks & Tue $7 / 6 / 04$ & Mon 8/16/04 & \\
\hline 1917 & $\checkmark$ & Review/assign all un-assigned data model candidates in Elementool & Tue $7 / 6 / 04$ & Wed $7 / 7 / 04$ & \\
\hline 1918 & $\checkmark$ & Update the logical data model & Fri 7/16/04 & Fri $7 / 23 / 04$ & \\
\hline 1919 & $\checkmark$ & Define the Data Model Rules & Mon 8/16/04 & Mon 8/16/04 & \\
\hline 1920 & $\checkmark$ & Legacy Data & Mon 8/16/04 & Fri 9/10/04 & \\
\hline 1921 & $\checkmark$ & Define Legacy elements & Mon 8/16/04 & Mon 8/16/04 & \\
\hline 1922 & $\checkmark$ & Review data model to verify Legacy elements \& identify any new legacy elements & Tue 8/17/04 & Tue 8/17/04 & \\
\hline 1923 & $\checkmark$ & Update data model for land legacy elements & Thu 8/19/04 & Thu 8/19/04 & \\
\hline 1924 & $\checkmark$ & Update data model for gas legacy elements & Wed 9/1/04 & Wed 9/1/04 & \\
\hline 1925 & $\checkmark$ & Update data model for electric legacy elements & Fri 9/10/04 & Fri 9/10/04 & \\
\hline 1926 & $\checkmark$ & Apply data model changes to the data migration matrix & Fri 9/10/04 & Fri 9/10/04 & \\
\hline 1927 & $\checkmark$ & Annotation & Thu 6/24/04 & Thu 9/2/04 & \\
\hline 1928 & $\checkmark$ & Gather the Data Model Requirements for Annotation & Thu 6/24/04 & Wed 7/28/04 & \\
\hline 1931 & $\checkmark$ & Log known annotation data model problems in Elementool & Mon $7 / 26 / 04$ & Wed $7 / 28 / 04$ & \\
\hline 1932 & $\checkmark$ & Apply data model changes for annotation & Tue 8/24/04 & Thu 9/2/04 & \\
\hline \multicolumn{2}{|c|}{$\begin{array}{l}\text { NIPSCO GIS } \\
\text { Confidential }\end{array}$} & Page 42 & & $\begin{array}{r}\text { Last Updat } \\
\text { Print }\end{array}$ & $\begin{array}{l}\text { ed on Tue } 7 / 3 / 07 \\
\text { ed on Fri 8/17/07 }\end{array}$ \\
\hline
\end{tabular}




\section{\begin{tabular}{|ll}
\hline & AEDR Construction Phase I Project Plan
\end{tabular}}

\begin{tabular}{|c|c|c|c|c|c|}
\hline ID & a & Task Name & Start & Finish & \\
\hline 1933 & $\checkmark$ & Process Annotation Elementool tickets & Tue $8 / 24 / 04$ & Thu $9 / 2 / 04$ & \\
\hline 1934 & $\checkmark$ & Process remaining data model Elementool tickets & Fri $7 / 30 / 04$ & Fri 9/10/04 & \\
\hline 1935 & $\checkmark$ & ArcFM customization / EDFS / MAPPS Planning & Mon 6/7/04 & Fri 9/10/04 & \\
\hline 1936 & $\checkmark$ & Design of EDFS Integration and MAPPS Interface & Mon 6/7/04 & Fri 9/10/04 & \\
\hline 1937 & $\checkmark$ & Process Miscellaneous EDFS Elementool Tickets & Wed 8/18/04 & Wed 8/18/04 & \\
\hline 1938 & $\checkmark$ & Finish Prep for EDFS & Mon 6/7/04 & Fri 6/25/04 & \\
\hline 1939 & $\checkmark$ & Review existing EDFS database and documentation & Mon 6/7/04 & Fri 6/11/04 & \\
\hline 1940 & $\checkmark$ & Demo EDFS & Thu 6/24/04 & Thu 6/24/04 & \\
\hline 1941 & $\checkmark$ & Review existing EDFS documentation & Wed 6/23/04 & Fri 6/25/04 & \\
\hline 1942 & $\checkmark$ & Document requirements for historical info & Mon $6 / 14 / 04$ & Mon 6/14/04 & \\
\hline 1943 & $\checkmark$ & Data mapping of EDFS to ArcGIS data model & Mon 6/14/04 & Tue $6 / 15 / 04$ & \\
\hline 1944 & $\checkmark$ & Draft EDFS/MAPPS Requirements Documents & Tue $6 / 15 / 04$ & Fri 9/10/04 & \\
\hline 1945 & $\checkmark$ & Create the EDFS Transformer / Regulator Requirements Document & Tue 6/15/04 & Mon 8/30/04 & \\
\hline 1946 & $\checkmark$ & Create the EDFS Transformer / Regulator document & Tue 6/15/04 & Wed $7 / 7 / 04$ & \\
\hline 1947 & $\checkmark$ & Create the EDFS requirements document template & Tue $6 / 15 / 04$ & Tue 6/15/04 & \\
\hline 1948 & $\checkmark$ & Review and finalize the EDFS requirements document template & Wed 6/16/04 & Wed 6/16/04 & \\
\hline 1949 & $\checkmark$ & Create requirements document for EDFS Unit of Property Management & Fri 6/18/04 & Mon 6/21/04 & \\
\hline 1950 & $\checkmark$ & Put the EDFS Unit of Property Management requirements into standard format & Wed 6/23/04 & Wed 6/23/04 & \\
\hline 1951 & $\checkmark$ & Review the EDFS standard format requirements document & Thu $6 / 24 / 04$ & Thu $6 / 24 / 04$ & \\
\hline 1952 & $\checkmark$ & Create standard design document template & Tue 6/29/04 & Tue 6/29/04 & \\
\hline 1953 & $\checkmark$ & Populate the standard design template with Unit of Property information & Wed 6/30/04 & Thu $7 / 1 / 04$ & \\
\hline 1954 & $\checkmark$ & Review Unit of Property document & Fri 7/2/04 & Fri $7 / 2 / 04$ & \\
\hline 1955 & $\checkmark$ & Decide on next steps for the EDFS requirements documents & Wed $7 / 7 / 04$ & Wed 7/7/04 & \\
\hline 1956 & $\checkmark$ & Additional review of Transformer / Regulator requirements document & Wed $7 / 28 / 04$ & Fri 8/13/04 & \\
\hline 1957 & $\checkmark$ & Finalize Transformer / Regulator requirements document & Fri 8/20/04 & Fri 8/27/04 & \\
\hline 1958 & $\checkmark$ & Send the final EDFS Transformer / Regulator Requirements Document to the vendors & Mon 8/30/04 & Mon 8/30/04 & \\
\hline 1959 & $\checkmark$ & EDFS Daily Report Requirements & Tue $7 / 27 / 04$ & Thu 8/26/04 & \\
\hline 1960 & $\checkmark$ & Create EDFS daily report requirement documents & Tue $7 / 27 / 04$ & Wed $7 / 28 / 04$ & \\
\hline 1961 & $\checkmark$ & Project Team Review of EDFS Daily Report Requirements Documents & Thu 8/26/04 & Thu 8/26/04 & \\
\hline 1962 & $\checkmark$ & Finalize the Daily Report requirements document & Thu 8/26/04 & Thu 8/26/04 & \\
\hline 1963 & $\checkmark$ & Send out the EDFS daily report requirements document to the vendors & Thu 8/26/04 & Thu $8 / 26 / 04$ & \\
\hline 1964 & $\checkmark$ & EDFS Annual Report Requirements & Tue $7 / 27 / 04$ & Thu 8/26/04 & \\
\hline 1965 & $\checkmark$ & Create the EDFS annual report requirements documents & Tue $7 / 27 / 04$ & Wed $7 / 28 / 04$ & \\
\hline 1966 & $\checkmark$ & Project Team Review of EDFS Annual Report Requirements Documents & Thu $7 / 29 / 04$ & Thu $7 / 29 / 04$ & \\
\hline 1967 & $\checkmark$ & Finalize the Annual Report requirements document & Thu $7 / 29 / 04$ & Thu $7 / 29 / 04$ & \\
\hline 1968 & $\checkmark$ & Send out the EDFS annual report requirements document to the vendors & Thu 8/26/04 & Thu $8 / 26 / 04$ & \\
\hline 1969 & $\checkmark$ & Create the EDFS Capcitor Requirements Document & Mon 8/30/04 & Fri 9/10/04 & \\
\hline 1970 & $\checkmark$ & Create EDFS capacitor requirement documents & Mon 8/30/04 & Fri 9/3/04 & \\
\hline 1971 & $\checkmark$ & Project Team Review of EDFS Capacitor Requirements Documents & Thu 9/9/04 & Thu 9/9/04 & \\
\hline 1972 & $\checkmark$ & Finalize the Capacitor requirements document & Thu 9/9/04 & Thu $9 / 9 / 04$ & \\
\hline 1973 & $\checkmark$ & Send approved EDFS Capacitor Requirements Document to the vendors & Fri 9/10/04 & Fri 9/10/04 & \\
\hline 1974 & $\checkmark$ & Miscellaneous Auto Updaters - Batch \#1 & Mon 6/7/04 & Thu $7 / 1 / 04$ & \\
\hline 1975 & $\checkmark$ & Previously Created Auto Updaters & Tue 6/8/04 & Wed 6/16/04 & \\
\hline 1976 & 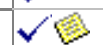 & Meeting to determine locator tool requirements & Wed 6/9/04 & Wed 6/9/04 & \\
\hline 1977 & $\checkmark$ & Re-install M\&M interop code on NIPSCO machines & Thu 6/10/04 & Thu $6 / 10 / 04$ & \\
\hline 1978 & $\checkmark$ & Resolve M\&M software issues with AU's (requires M\&M software re-install) & Tue 6/8/04 & Wed 6/16/04 & \\
\hline $\begin{array}{l}\text { NIPSC } \\
\text { Confid }\end{array}$ & $\begin{array}{l}\text { Co GIS } \\
\text { lential }\end{array}$ & Page 43 & & $\begin{array}{r}\text { Last Updat } \\
\text { Print }\end{array}$ & $\begin{array}{l}\text { ed on Tue } 7 / 3 / 07 \\
\text { ed on Fri 8/17/07 }\end{array}$ \\
\hline
\end{tabular}




\section{\begin{tabular}{|ll}
\hline AEDR Construction Phase I Project Plan & A
\end{tabular}}

\begin{tabular}{|c|c|c|c|c|c|}
\hline ID & (a) & Task Name & Start & Finish & \\
\hline 1979 & $\checkmark$ & New Auto Updaters & Mon 6/7/04 & Wed 6/23/04 & \\
\hline 1980 & $\checkmark$ & Review the "Elaboration" component specs & Mon 6/7/04 & Mon 6/7/04 & \\
\hline 1981 & $\checkmark$ & Update, finalize, and approve the Gas AU component specs & Thu $6 / 10 / 04$ & Thu 6/10/04 & \\
\hline 1982 & $\checkmark$ & Code / test / doc - Gas valve - valve number & Wed 6/16/04 & Thu 6/17/04 & \\
\hline 1983 & $\checkmark$ & Code / test / doc - Casing needs the related main OID & Thu $6 / 17 / 04$ & Fri 6/18/04 & \\
\hline 1984 & $\checkmark$ & Code / test / doc - Abandoned devices - device type (concat 2 fields) & Mon 6/21/04 & Wed 6/23/04 & \\
\hline 1985 & $\checkmark$ & Install All Auto Updaters & Mon 6/28/04 & Thu 7/1/04 & \\
\hline 1986 & $\checkmark$ & Resolve bug with installing AU on the client machine & Mon 6/28/04 & Mon 6/28/04 & \\
\hline 1987 & $\checkmark$ & Install AU's in NIPSCO dev environment (test / revise admin guides) & Thu $7 / 1 / 04$ & Thu $7 / 1 / 04$ & \\
\hline 1989 & $\checkmark$ & Admin / Misc AU tasks & Wed 8/11/04 & Tue 8/24/04 & \\
\hline 1990 & $\checkmark$ & Review Meeting for AutoUpdaters & Thu 8/12/04 & Thu 8/12/04 & \\
\hline 1991 & $\checkmark$ & Update customization candidate list spreadsheet \& check candidates into VSS \& Elementool & Wed 8/11/04 & Wed 8/11/04 & \\
\hline 1992 & $\checkmark$ & Review data model changes/determine effects on previously written AUs & Mon 8/23/04 & Mon 8/23/04 & \\
\hline 1993 & $\checkmark$ & Update component specs/code & Mon 8/23/04 & Tue 8/24/04 & \\
\hline 1994 & $\checkmark 6$ & ReferenceFeaturesRemovalTool & Mon 7/19/04 & Tue 7/27/04 & \\
\hline 1995 & $\checkmark$ & Create component spec for ReferenceFeaturesRemovalTool & Mon 7/19/04 & Mon 7/19/04 & \\
\hline 1996 & $\checkmark$ & Review component spec for ReferenceFeaturesRemovalTool & Thu $7 / 22 / 04$ & Thu $7 / 22 / 04$ & \\
\hline 1997 & $\checkmark$ & Finalize the component spec for ReferenceFeaturesRemovalTool & Mon $7 / 26 / 04$ & Mon 7/26/04 & \\
\hline 1998 & $\checkmark$ & Code / unit test for ReferenceFeaturesRemovalTool & Fri 7/23/04 & Tue $7 / 27 / 04$ & \\
\hline 2000 & $\checkmark$ & Create component spec for GasMainCrossoverHumpAU & Tue $7 / 20 / 04$ & Tue $7 / 20 / 04$ & \\
\hline 2001 & $\checkmark$ & Review component spec for GasMainCrossoverHumpAU & Thu $7 / 22 / 04$ & Thu $7 / 22 / 04$ & \\
\hline 2002 & $\checkmark$ & Finalize the component spec for GasMainCrossoverHumpAU & Tue $7 / 27 / 04$ & Tue $7 / 27 / 04$ & \\
\hline 2003 & $\checkmark$ & Code / unit test for GasMainCrossoverHumpAU & Tue $7 / 27 / 04$ & Wed $7 / 28 / 04$ & \\
\hline 2004 & $\checkmark y$ & GasMainPipeChangeIndicatorAU & Fri 7/16/04 & Mon 8/9/04 & \\
\hline 2005 & $\checkmark$ & Create component spec for GasMainPipeChangeIndicatorAU & Fri 7/16/04 & Fri 7/16/04 & \\
\hline 2006 & $\checkmark$ & Review component spec for GasMainPipeChangeIndicatorAU & Tue $7 / 20 / 04$ & Tue $7 / 20 / 04$ & \\
\hline 2007 & $\checkmark$ & Finalize the component spec for GasMainPipeChangeIndicatorAU & Thu $7 / 22 / 04$ & Thu $7 / 22 / 04$ & \\
\hline 2008 & $\checkmark$ & Code / unit test for GasMainPipeChangeIndicatorAU & Thu $7 / 29 / 04$ & Tue $8 / 3 / 04$ & \\
\hline 2009 & $\checkmark$ & Update GasMainPipeChangeIndicatorAU based on data model changes for Elementool \#367 & Mon 8/9/04 & Mon 8/9/04 & \\
\hline 2010 & $\checkmark 8$ & CPSectionCorrosionControINumberAU & Mon 7/19/04 & Fri 8/6/04 & \\
\hline 2011 & $\checkmark$ & Create component spec for CPSectionCorrosionControlNumberAU & Mon 7/19/04 & Mon $7 / 19 / 04$ & \\
\hline 2012 & $\checkmark$ & Review component spec for CPSectionCorrosionControlNumberAU & Thu $7 / 22 / 04$ & Thu $7 / 22 / 04$ & \\
\hline 2013 & $\checkmark$ & Resolve issue of what to do when Gas Main crossed more than 1 DG Grid & Fri 7/30/04 & Fri 7/30/04 & \\
\hline 2014 & $\checkmark$ & Update and finalize the component spec based on what to do when main crosses more than 1 DG Grid & Mon 8/2/04 & Mon 8/2/04 & \\
\hline 2015 & $\checkmark$ & Code / unit test for CPSectionCorrosionControlNumberAU & Wed $8 / 4 / 04$ & Fri 8/6/04 & \\
\hline 2016 & $\checkmark y$ & CPSectionMaintainenceTool (specs only) & Wed 7/21/04 & Fri 8/6/04 & \\
\hline 2017 & $\checkmark$ & Create component spec for CPSectionMaintainenceTool & Wed $7 / 21 / 04$ & Wed 7/21/04 & \\
\hline 2018 & $\checkmark$ & Review component spec for CPSectionMaintainenceTool & Fri 7/23/04 & Fri 7/23/04 & \\
\hline 2019 & $\checkmark$ & Finalize the component spec for CPSectionMaintainenceTool & Mon $7 / 26 / 04$ & Mon 7/26/04 & \\
\hline 2020 & $\checkmark$ & Code / unit test for CPSectionMaintainance Tool AU & Mon 8/2/04 & Fri 8/6/04 & \\
\hline 2023 & $\checkmark$ & Review component spec for UniqueDistribRefNumberCheckerAU & Thu $7 / 22 / 04$ & Thu $7 / 22 / 04$ & \\
\hline 2024 & $\checkmark$ & Finalize the component spec for UniqueDistribRefNumberCheckerAU & Wed 7/28/04 & Wed 7/28/04 & \\
\hline $\begin{array}{l}\text { NIPSC } \\
\text { Confid }\end{array}$ & ential & Page 44 & & $\begin{array}{r}\text { Last Updat } \\
\text { Print }\end{array}$ & $\begin{array}{l}\text { ed on Tue } 7 / 3 / 07 \\
\text { ed on Fri 8/17/07 }\end{array}$ \\
\hline
\end{tabular}




\section{\begin{tabular}{|ll}
\hline & AEDR Construction Phase I Project Plan
\end{tabular}}

\begin{tabular}{|c|c|c|c|c|c|}
\hline ID & (a) & Task Name & Start & Finish & \\
\hline 2025 & $\checkmark b$ & UniqueDistribRefNumberValidationRule (specs only) & Wed 7/21/04 & Thu $7 / 29 / 04$ & \\
\hline 2026 & $\checkmark$ & Create component spec for UniqueDistribRefNumberValidationRule & Wed 7/21/04 & Wed $7 / 21 / 04$ & \\
\hline 2027 & $\checkmark$ & Review component spec for UniqueDistribRefNumberValidationRule & Thu $7 / 22 / 04$ & Thu $7 / 22 / 04$ & \\
\hline 2028 & $\checkmark$ & Finalize component spec for UniqueDistribRefNumberValidationRule & Thu $7 / 29 / 04$ & Thu $7 / 29 / 04$ & \\
\hline 2029 & $\checkmark b$ & DistribRefNumber8DigitsAU (specs only) & Thu 7/22/04 & Tue 8/3/04 & \\
\hline 2030 & $\checkmark$ & Create component spec for DistribRefNumber8DigitsAU & Thu $7 / 22 / 04$ & Thu $7 / 22 / 04$ & \\
\hline 2031 & $\checkmark$ & Review component spec for DistribRefNumber8DigitsAU & Fri 7/23/04 & Fri $7 / 23 / 04$ & \\
\hline 2032 & $\checkmark$ & Finalize component spec for DistribRefNumber8DigitsAU & Tue $8 / 3 / 04$ & Tue 8/3/04 & \\
\hline 2033 & $\checkmark \theta$ & Location Description Custom Field Editor (specs only) & Thu 7/22/04 & Mon 8/2/04 & \\
\hline 2035 & $\checkmark$ & Review component spec for LocationDescriptionCustomFieldEditor & Fri 7/23/04 & Fri 7/23/04 & \\
\hline 2036 & $\checkmark$ & Finalize component spec for LocationDescriptionCustomFieldEditor & Mon 8/2/04 & Mon 8/2/04 & \\
\hline 2037 & $\checkmark$ & Code Review for All Batch $\# 2$ Auto Updaters & Wed 8/18/04 & Fri 8/20/04 & \\
\hline 2038 & $\checkmark$ & Code review of all batch \#2 AU's & Wed 8/18/04 & Wed 8/18/04 & \\
\hline 2039 & $\checkmark$ & Finalize and check-in the AU's & Fri 8/20/04 & Fri 8/20/04 & \\
\hline 2040 & $\checkmark$ & Miscellaneous AU & Fri 8/13/04 & Tue 8/24/04 & \\
\hline 2041 & $\checkmark$ & Configuration related Elementool Tickets & Fri 8/13/04 & Fri 8/13/04 & \\
\hline 2042 & $\checkmark y$ & Implement the AU for Valves/Symbology Attributes & Mon 8/23/04 & Tue 8/24/04 & \\
\hline 2043 & $\checkmark$ & Create component spec & Mon 8/23/04 & Mon 8/23/04 & \\
\hline 2044 & $\checkmark$ & Review component spec & Mon 8/23/04 & Mon 8/23/04 & \\
\hline 2046 & $\checkmark$ & Elementool Setup & Wed 6/9/04 & Fri $7 / 2 / 04$ & \\
\hline 2047 & $\checkmark$ & Create the Elementool Account & Wed 6/9/04 & Wed 6/9/04 & \\
\hline 2048 & $\checkmark$ & Upgrade our Elementool account \& check configuration & Wed 6/9/04 & Wed 6/9/04 & \\
\hline 2049 & $\checkmark$ & Load Elementool with the data model spreadsheet info & Fri 6/11/04 & Tue $6 / 15 / 04$ & \\
\hline 2050 & $\checkmark$ & Review the Elementool procedures and forms (related to TYPE field) & Tue $6 / 29 / 04$ & Tue $6 / 29 / 04$ & \\
\hline 2051 & $\checkmark$ & Evaluate how to update Elementool forms & Tue $6 / 29 / 04$ & Tue $6 / 29 / 04$ & \\
\hline 2052 & $\checkmark$ & Update the Elementool procedures and forms based on TYPE field & Tue $6 / 29 / 04$ & Tue 6/29/04 & \\
\hline 2053 & $\checkmark$ & Decide on team lead for Elementool admin tasks & Thu $7 / 1 / 04$ & Thu $7 / 1 / 04$ & \\
\hline 2054 & $\checkmark$ & Create draft of Elementool backup procedures & Wed 6/30/04 & Wed 6/30/04 & \\
\hline 2055 & $\checkmark$ & Review and finalize the Elementool backup procedures & Fri 7/2/04 & Fri $7 / 2 / 04$ & \\
\hline 2056 & $\checkmark$ & Field Browser Interface Preparation & Mon $7 / 19 / 04$ & Fri 7/23/04 & \\
\hline 2057 & $\checkmark$ & Test the existing demo environment & Mon 7/19/04 & Mon $7 / 19 / 04$ & \\
\hline 2058 & $\checkmark$ & Determine Field Browser hardware replacement & Mon 7/19/04 & Mon 7/19/04 & \\
\hline 2059 & $\checkmark$ & Demo the ArcReader prototype to the team & Tue $7 / 20 / 04$ & Tue $7 / 20 / 04$ & \\
\hline 2060 & $\checkmark$ & Verify hardware rollout schedule & Fri 7/23/04 & Fri 7/23/04 & \\
\hline 2061 & $\checkmark$ & $\mathrm{CH}$ Interface Tasks & Mon 6/14/04 & Wed 8/18/04 & \\
\hline 2062 & $\checkmark$ & Completed CH Interface Tasks & Mon 6/14/04 & Wed 8/18/04 & \\
\hline 2063 & $\checkmark 8$ & $\mathrm{CH}$ interface planning meeting & Mon 6/14/04 & Mon 6/14/04 & \\
\hline 2064 & $\checkmark 8$ & $\mathrm{CH}$ interface kickoff meeting & Tue $6 / 15 / 04$ & Tue 6/15/04 & \\
\hline 2065 & $\checkmark(-3$ & $\mathrm{CH}$ interface data model and interface workshop meetings & Mon 6/28/04 & Thu $7 / 1 / 04$ & \\
\hline 2066 & $\checkmark$ & Enter Elementool issues from the $\mathrm{CH}$ workshops & Fri 7/2/04 & Fri 7/2/04 & \\
\hline 2069 & $\checkmark$ & Get the $\mathrm{CH}$ project plan & Wed $7 / 28 / 04$ & Wed $7 / 28 / 04$ & \\
\hline 2070 & $\checkmark$ & Meet to solve the reserved word issue & Fri 8/6/04 & Fri 8/6/04 & \\
\hline $\begin{array}{l}\text { NIPSC } \\
\text { Confid }\end{array}$ & ential & Page 45 & & $\begin{array}{r}\text { Last Updat } \\
\text { Print }\end{array}$ & $\begin{array}{l}d \text { on Tue } 7 / 3 / 07 \\
\text { d on Fri 8/17/07 }\end{array}$ \\
\hline
\end{tabular}




\begin{tabular}{|c|c|c|c|c|c|}
\hline \multicolumn{6}{|c|}{ AEDR Construction Phase I Project Plan } \\
\hline ID & ( & Task Name & Start & Finish & \\
\hline 2071 & $\checkmark$ & CH Update Meeting & Wed 8/18/04 & Wed 8/18/04 & \\
\hline 2072 & $\checkmark$ & Source Safe Tasks & Mon 6/14/04 & Wed 8/4/04 & \\
\hline 2073 & $\checkmark$ & Miscellaneous Source Safe Setup Tasks & Mon 6/14/04 & Wed 6/23/04 & \\
\hline 2074 & $\checkmark$ & Check-in all AU code and technical documents & Mon 6/14/04 & Mon 6/14/04 & \\
\hline 2075 & $\checkmark$ & Find / document duplicate files in Source Safe & Tue 6/22/04 & Tue 6/22/04 & \\
\hline 2076 & $\checkmark$ & Send out details regarding duplicate file issues & Wed 6/23/04 & Wed 6/23/04 & \\
\hline 2077 & $\checkmark$ & Establish list of Source Safe directory review assignments based on Team Leads / Backups & Wed 6/23/04 & Wed 6/23/04 & \\
\hline 2078 & $\checkmark$ & Delete duplicate Source Safe Files & Mon 6/28/04 & Fri 7/30/04 & \\
\hline 2079 & $\checkmark$ & Send out the information regarding duplicate Source Safe files & Mon 6/28/04 & Mon 6/28/04 & \\
\hline 2080 & $\checkmark$ & Delete duplicate Source Safe files based on information from the team & Fri 7/30/04 & Fri 7/30/04 & \\
\hline 2081 & $\checkmark$ & Review Source Safe Directory Structure (Each team lead reviews their own areas) & Fri $7 / 23 / 04$ & Wed 8/4/04 & \\
\hline 2082 & $\checkmark$ & ArcFM configuration & Mon 8/2/04 & Mon 8/2/04 & \\
\hline 2083 & $\checkmark$ & ArcFM customization & Mon 8/2/04 & Mon 8/2/04 & \\
\hline 2084 & $\checkmark$ & Data migration / rectification & Fri 7/23/04 & Fri 7/23/04 & \\
\hline 2085 & $\checkmark$ & Data modeling & Mon $7 / 26 / 04$ & Mon $7 / 26 / 04$ & \\
\hline 2086 & $\checkmark$ & Dev/test/prod env & Fri 7/30/04 & Fri $7 / 30 / 04$ & \\
\hline 2087 & $\checkmark$ & Documentation & Wed $8 / 4 / 04$ & Wed 8/4/04 & \\
\hline 2088 & $\checkmark$ & Elementool & Mon 7/26/04 & Mon 7/26/04 & \\
\hline 2089 & $\checkmark$ & Interface development & Mon 8/2/04 & Mon 8/2/04 & \\
\hline 2090 & $\checkmark$ & Project management & Fri 7/30/04 & Fri $7 / 30 / 04$ & \\
\hline 2091 & $\checkmark$ & Source Safe admin & Fri $7 / 23 / 04$ & Fri 7/23/04 & \\
\hline 2092 & $\checkmark$ & System architecture & Fri $7 / 30 / 04$ & Fri 7/30/04 & \\
\hline 2093 & $\checkmark$ & Testing / QA / QC & Mon $7 / 26 / 04$ & Mon 7/26/04 & \\
\hline 2094 & $\checkmark$ & Training & Mon $7 / 26 / 04$ & Mon $7 / 26 / 04$ & \\
\hline 2095 & $\checkmark$ & GIS to DXF Export (CAD Interoperability) & Wed 6/30/04 & Fri $7 / 9 / 04$ & \\
\hline 2096 & $\checkmark$ & Get license costs / options & Wed 6/30/04 & Wed 6/30/04 & \\
\hline 2097 & $\checkmark$ & Purchase one license of FME \& install on a common machine & Fri 7/9/04 & Fri $7 / 9 / 04$ & \\
\hline 2098 & $\checkmark$ & Documentation wrap-up tasks & Tue 6/8/04 & Mon 9/13/04 & \\
\hline 2099 & $\checkmark$ & Create Test Cases for Iteration 1 Functionality & Tue 6/8/04 & Tue 8/17/04 & \\
\hline 2100 & $\checkmark$ & Finalize the test plan ("Vision" doc for testing) & Tue 6/8/04 & Tue 6/8/04 & \\
\hline 2101 & $\checkmark$ & Review and approve the Test Plan & Wed 6/9/04 & Wed 6/9/04 & \\
\hline 2102 & $\checkmark$ & Meeting to determine the sample Regulator Station test case & Tue $7 / 13 / 04$ & Tue $7 / 13 / 04$ & \\
\hline 2103 & $\checkmark$ & Create initial test case template based on Regulator Station test case & Tue $7 / 13 / 04$ & Tue $7 / 13 / 04$ & \\
\hline 2104 & $\checkmark$ & Finalize the test case template & Thu $7 / 15 / 04$ & Thu $7 / 15 / 04$ & \\
\hline 2105 & $\checkmark$ & Estimate time needed to create all test cases & Tue $7 / 20 / 04$ & Tue $7 / 20 / 04$ & \\
\hline 2106 & $\checkmark$ & Create the test case spreadsheet for Iteration 1 functionality & Tue $7 / 20 / 04$ & Wed $7 / 28 / 04$ & \\
\hline 2107 & $\checkmark$ & Review the test case spreadsheet for Iteration 1 functionality & Thu $7 / 29 / 04$ & Wed 8/4/04 & \\
\hline 2108 & $\checkmark$ & Update and finalize the test case spreadsheet & Tue 8/17/04 & Tue 8/17/04 & \\
\hline 2109 & $\checkmark$ & User Guides I On-Line Help for Iteration 1 Functionality & Tue 6/8/04 & Mon 9/13/04 & \\
\hline 2110 & $\checkmark$ & Establish Procedures/Guidelines for User Guides / On-Line Help & Wed 6/9/04 & Thu 6/17/04 & \\
\hline 2111 & $\checkmark$ & Determine / document procedure for updating help files & Wed 6/9/04 & Wed 6/9/04 & \\
\hline 2112 & $\checkmark$ & Update guidelines and procedures for on-line help & Thu 6/10/04 & Thu 6/10/04 & \\
\hline 2113 & $\checkmark$ & Determine NIPSCO standard for HTML authoring & Fri 6/11/04 & Fri 6/11/04 & \\
\hline 2114 & $\checkmark$ & Determine authoring tool to use & Fri 6/11/04 & Fri 6/11/04 & \\
\hline 2115 & $\checkmark$ & Create procedure for submitting new documents & Thu $6 / 17 / 04$ & Thu 6/17/04 & \\
\hline 2116 & $\checkmark$ & Establish Prototype/Sample On-Line Help System & Tue $6 / 8 / 04$ & Thu $7 / 1 / 04$ & \\
\hline $\begin{array}{l}\text { NIPSC } \\
\text { Confid }\end{array}$ & $\begin{array}{l}\text { Co GIS } \\
\text { lential }\end{array}$ & Page 46 & & $\begin{array}{r}\text { Last Updat } \\
\text { Print }\end{array}$ & $\begin{array}{l}\text { ed on Tue } 7 / 3 / 07 \\
\text { ed on Fri 8/17/07 }\end{array}$ \\
\hline
\end{tabular}




\begin{tabular}{|c|c|c|c|c|c|}
\hline \multicolumn{6}{|c|}{ AEDR Construction Phase I Project Plan } \\
\hline ID & (a) & Task Name & Start & Finish & \\
\hline 2117 & $\checkmark$ & Demo the on-line help prototype / sample & Tue 6/8/04 & Tue $6 / 8 / 04$ & \\
\hline 2118 & $\checkmark$ & Research how to invoke the NIPSCO help from within ArcMap & Mon 6/14/04 & Thu 6/17/04 & \\
\hline 2119 & $\checkmark$ & Update the 'Help Menu Item' Component Spec & Wed 6/16/04 & Wed 6/16/04 & \\
\hline 2120 & $\checkmark$ & Update the demo/sample on-line help with final standards and templates & Wed 6/23/04 & Wed 6/23/04 & \\
\hline 2121 & $\checkmark$ & Knowledge transfer regarding on-line help standards and templates & Wed 6/23/04 & Wed 6/23/04 & \\
\hline 2122 & $\checkmark$ & Self-study on DreamWeaver and the current on-line help prototype & Fri 6/25/04 & Thu $7 / 1 / 04$ & \\
\hline 2123 & $\checkmark$ & Create Templates for User Guides I On-Line Help & Wed 6/9/04 & Thu 6/10/04 & \\
\hline 2124 & $\checkmark$ & Review and comment on sample Regulator Station user guide & Wed 6/9/04 & Wed 6/9/04 & \\
\hline 2125 & $\checkmark$ & Finalize user guide template & Thu 6/10/04 & Thu 6/10/04 & \\
\hline 2126 & $\checkmark$ & Create Initial User Guides for Iteration 1 & Fri 6/25/04 & Mon 9/13/04 & \\
\hline 2127 & $\checkmark$ & Define SDE & Fri 6/25/04 & Fri 6/25/04 & \\
\hline 2128 & $\checkmark$ & Define ArcMap & Fri 6/25/04 & Fri 6/25/04 & \\
\hline 2129 & $\checkmark$ & How to start an edit session & Fri 6/25/04 & Fri $6 / 25 / 04$ & \\
\hline 2130 & $\checkmark$ & How to open ArcMap & Thu $7 / 1 / 04$ & Thu $7 / 1 / 04$ & \\
\hline 2131 & $\checkmark$ & Define ESRI & Thu $7 / 1 / 04$ & Thu 7/1/04 & \\
\hline 2132 & $\checkmark$ & Define ArcGIS & Thu $7 / 1 / 04$ & Thu $7 / 1 / 04$ & \\
\hline 2133 & $\checkmark$ & Define SessionManager.htm & Tue $7 / 13 / 04$ & Tue $7 / 13 / 04$ & \\
\hline 2134 & $\checkmark$ & Define AboutNIPSCOGIS.htm & Tue $7 / 13 / 04$ & Tue $7 / 13 / 04$ & \\
\hline 2135 & $\checkmark$ & Build the Help System Index & Mon 9/13/04 & Mon 9/13/04 & \\
\hline 2136 & $\checkmark$ & Re-organize the Help System Contents tab & Mon 9/13/04 & Mon 9/13/04 & \\
\hline 2137 & $\checkmark$ & Incorporate new user guides into the on-line help & Wed 9/1/04 & Fri 9/10/04 & \\
\hline 2138 & $\checkmark$ & Create Admin Guides for Iteration 1 functionality & Wed $7 / 14 / 04$ & Fri 9/10/04 & \\
\hline 2139 & $\checkmark$ & First Draft Admin Guides for Iteration 1 & Wed 7/14/04 & Wed 7/14/04 & \\
\hline 2140 & $\checkmark$ & Create database configuration admin guide & Wed $7 / 14 / 04$ & Wed $7 / 14 / 04$ & \\
\hline 2141 & $\checkmark$ & Create database permissions admin guide & Wed $7 / 14 / 04$ & Wed $7 / 14 / 04$ & \\
\hline 2142 & $\checkmark$ & Second Draft Admin Guides for Iteration 1 & Thu $9 / 9 / 04$ & Fri 9/10/04 & \\
\hline 2143 & $\checkmark$ & Database Configuration & Thu 9/9/04 & Thu 9/9/04 & \\
\hline 2144 & $\checkmark$ & Database Permissions & Thu 9/9/04 & Thu 9/9/04 & \\
\hline 2145 & $\checkmark$ & Visual Source Safe (VSS) & Thu 9/9/04 & Thu $9 / 9 / 04$ & \\
\hline 2146 & $\checkmark$ & Database SDE & Thu 9/9/04 & Thu $9 / 9 / 04$ & \\
\hline 2147 & $\checkmark$ & Help Resources & Fri 9/10/04 & Fri 9/10/04 & \\
\hline 2148 & $\checkmark$ & Software Requirements & Fri 9/10/04 & Fri 9/10/04 & \\
\hline 2149 & $\checkmark$ & Custom Components & Fri 9/10/04 & Fri 9/10/04 & \\
\hline 2150 & $\checkmark$ & Miscellaneous Tasks & Thu 6/10/04 & Fri 8/27/04 & \\
\hline 2151 & $\checkmark$ & Install all ESRI/M\&M components needed for .NET environment & Thu $6 / 10 / 04$ & Thu 6/10/04 & \\
\hline 2152 & $\checkmark$ & Determine position of transformer & Mon $7 / 12 / 04$ & Mon 7/12/04 & \\
\hline 2153 & $\checkmark$ & Identify corporate standards for historical information & Fri 8/13/04 & Fri 8/13/04 & \\
\hline 2154 & $\checkmark$ & Process miscellaneous Elementool tickets & Tue 7/13/04 & Tue $7 / 27 / 04$ & \\
\hline 2155 & $\checkmark$ & Configure cardinality for land & Fri 8/27/04 & Fri 8/27/04 & \\
\hline 2156 & $\checkmark b$ & Iteration 2 Integration Testing / Rework & Fri 8/20/04 & Fri 8/20/04 & \\
\hline 2157 & $\checkmark$ & Determine what needs to be tested & Fri 8/20/04 & Fri 8/20/04 & \\
\hline 2158 & $\checkmark$ & Iteration 2 Performance Tuning and Environment Setup & Mon 6/7/04 & Fri 9/10/04 & \\
\hline 2159 & $\checkmark$ & Performance Tuning & Fri 9/10/04 & Fri 9/10/04 & \\
\hline 2160 & $\checkmark$ & Note: No performance tuning will be done in this iteration & Fri 9/10/04 & Fri 9/10/04 & \\
\hline 2161 & $\checkmark$ & Environment Setup Tasks & Mon 6/7/04 & Fri 8/27/04 & \\
\hline 2162 & $\checkmark$ & Environment - Initial Setup Tasks & Mon 6/7/04 & Wed $7 / 21 / 04$ & \\
\hline $\begin{array}{l}\text { NIPSC } \\
\text { Confid }\end{array}$ & $\begin{array}{l}\text { Co GIS } \\
\text { lential }\end{array}$ & Page 47 & & $\begin{array}{r}\text { Last Updat } \\
\text { Print }\end{array}$ & $\begin{array}{l}\text { ed on Tue } 7 / 3 / 07 \\
\text { ed on Fri 8/17/07 }\end{array}$ \\
\hline
\end{tabular}




\section{\begin{tabular}{|ll}
\hline & AEDR Construction Phase I Project Plan
\end{tabular}}

\begin{tabular}{|c|c|c|c|c|c|}
\hline ID & ( & Task Name & Start & Finish & \\
\hline 2163 & $\checkmark$ & Investigate Source Safe to see if we can take snapshot at a later date & Mon 6/7/04 & Mon 6/7/04 & \\
\hline 2164 & $\checkmark$ & Set up new desktop in vacant cubicle with Maps \& Records software & Fri 6/18/04 & Fri 6/18/04 & \\
\hline 2165 & $\checkmark$ & Determine how off-site development will be managed & Wed 7/21/04 & Wed 7/21/04 & \\
\hline 2166 & $\checkmark$ & Environment - Workshop & Mon 8/23/04 & Fri 8/27/04 & \\
\hline 2167 & $\checkmark$ & Prep for workshop & Mon 8/23/04 & Mon 8/23/04 & \\
\hline 2168 & $\checkmark$ & Attend Workshop with Miner \& Miner & Tue $8 / 24 / 04$ & Fri 8/27/04 & \\
\hline 2169 & $\checkmark$ & GIS Application and Interface Construction - Iteration 3 (GIS Customizations and Interfaces) & Mon 9/13/04 & Mon 4/10/06 & \\
\hline 2170 & $\checkmark \theta$ & Iteration 2 Wrap-Up / Iteration 3 Kick-off & Tue 9/14/04 & Fri 10/29/04 & \\
\hline 2171 & $\checkmark$ & Iteration 2 wrap up / Iteration 3 kick-off meeting & Tue 9/14/04 & Tue 9/14/04 & \\
\hline 2173 & $\checkmark$ & Cadops \& Feederall Demo & Wed 9/29/04 & Wed 9/29/04 & \\
\hline 2174 & $\checkmark$ & GIS Product Demo includes AutoUpdaters - Install-Configure & Wed 9/29/04 & Wed 9/29/04 & \\
\hline 2175 & $\checkmark$ & Create Wrap-up Report & Fri 10/29/04 & Fri 10/29/04 & \\
\hline 2176 & $\checkmark$ & Create, Review, finalize and distribute the wrap-up report & Fri 10/29/04 & Fri 10/29/04 & \\
\hline 2177 & $\checkmark$ & CADOPS I FeederAll interface & Mon 9/13/04 & Mon 9/27/04 & \\
\hline 2178 & $\checkmark$ & ABB issues resolution & Mon 9/13/04 & Mon 9/20/04 & \\
\hline 2179 & $\checkmark$ & Update ArcFM Configuration for FeederAllOpenPoint & Mon 9/27/04 & Mon 9/27/04 & \\
\hline 2180 & $\checkmark$ & Final approval / sign-off on application & Mon 9/20/04 & Mon 9/20/04 & \\
\hline 2181 & $\checkmark 9$ & Iteration 2 Integration Testing / Rework & Wed 9/29/04 & Tue 10/19/04 & \\
\hline 2182 & $\checkmark$ & Install \& Configure demo AUs on test box & Wed 9/29/04 & Wed 9/29/04 & \\
\hline 2184 & $\checkmark$ & Configure ArcFM Deltas to match current data model - electric & Mon 10/18/04 & Tue 10/19/04 & \\
\hline 2185 & $\checkmark$ & Environment Setup Tasks & Tue 10/5/04 & Tue 12/7/04 & \\
\hline 2186 & $\checkmark$ & Environment Maintenance Tasks & Thu 10/21/04 & Mon 10/25/04 & \\
\hline 2187 & $\checkmark$ & Update the Schema for Development instance & Fri 10/22/04 & Mon 10/25/04 & \\
\hline 2188 & $\checkmark$ & Update configuration for Development instance & Thu 10/21/04 & Thu 10/21/04 & \\
\hline 2189 & $\checkmark$ & Install \& Configure AU's on Project Team Machines & Mon 11/8/04 & Mon 11/8/04 & \\
\hline 2190 & $\checkmark$ & Install \& configure AU's on all computers & Mon 11/8/04 & Mon 11/8/04 & \\
\hline 2191 & $\checkmark$ & Development Instance & Tue 10/5/04 & Thu 11/18/04 & \\
\hline 2192 & $\checkmark$ & Create SQL user accounts for ArcSDE Development & Tue $10 / 5 / 04$ & Tue $10 / 5 / 04$ & \\
\hline 2193 & $\checkmark$ & Create a list of user and roles for ArCSDE Development & Tue $10 / 5 / 04$ & Tue $10 / 5 / 04$ & \\
\hline 2194 & $\checkmark$ & Lock down user accounts for Development & Wed 10/13/04 & Wed 10/13/04 & \\
\hline 2195 & $\checkmark$ & Initialize ArcSDE Development User accounts & Thu 10/14/04 & Thu 10/14/04 & \\
\hline 2196 & $\checkmark$ & Export configuration from development instance (XML export) & Mon 11/15/04 & Mon 11/15/04 & \\
\hline 2197 & $\checkmark$ & Wipe the database clean / re-create SDE tables & Mon 11/15/04 & Mon 11/15/04 & \\
\hline 2198 & $\checkmark$ & Restore the backup file & Mon 11/15/04 & Mon 11/15/04 & \\
\hline 2199 & $\checkmark$ & Update the schema from Visio based on the latest data model changes & Mon 11/15/04 & Mon 11/15/04 & \\
\hline 2200 & $\checkmark$ & Build all ArcFM tables & Mon 11/15/04 & Mon 11/15/04 & \\
\hline 2201 & $\checkmark$ & Convert to ArcFM objects - expands out of the box ArcGIS objects & Mon 11/15/04 & Mon 11/15/04 & \\
\hline 2202 & $\checkmark$ & Check and/or build and re-link user roles & Mon 11/15/04 & Mon 11/15/04 & \\
\hline 2203 & $\checkmark$ & Grant permissions to the M\&M system tables & Mon 11/15/04 & Mon 11/15/04 & \\
\hline 2204 & $\checkmark$ & Import all configurations (XML import) & Tue 11/16/04 & Tue $11 / 16 / 04$ & \\
\hline 2207 & $\checkmark$ & Test Instance & Tue 10/5/04 & Tue 12/7/04 & \\
\hline 2208 & $\checkmark$ & Create SQL user accounts for ArcSDE Test & Wed 10/13/04 & Wed 10/13/04 & \\
\hline $\begin{array}{l}\text { NIPSC } \\
\text { Confid }\end{array}$ & ential & \multicolumn{2}{|l|}{ Page 48} & \multicolumn{2}{|c|}{$\begin{array}{r}\text { Last Updated on Tue } 7 / 3 / 07 \\
\text { Printed on Fri } 8 / 17 / 07\end{array}$} \\
\hline
\end{tabular}




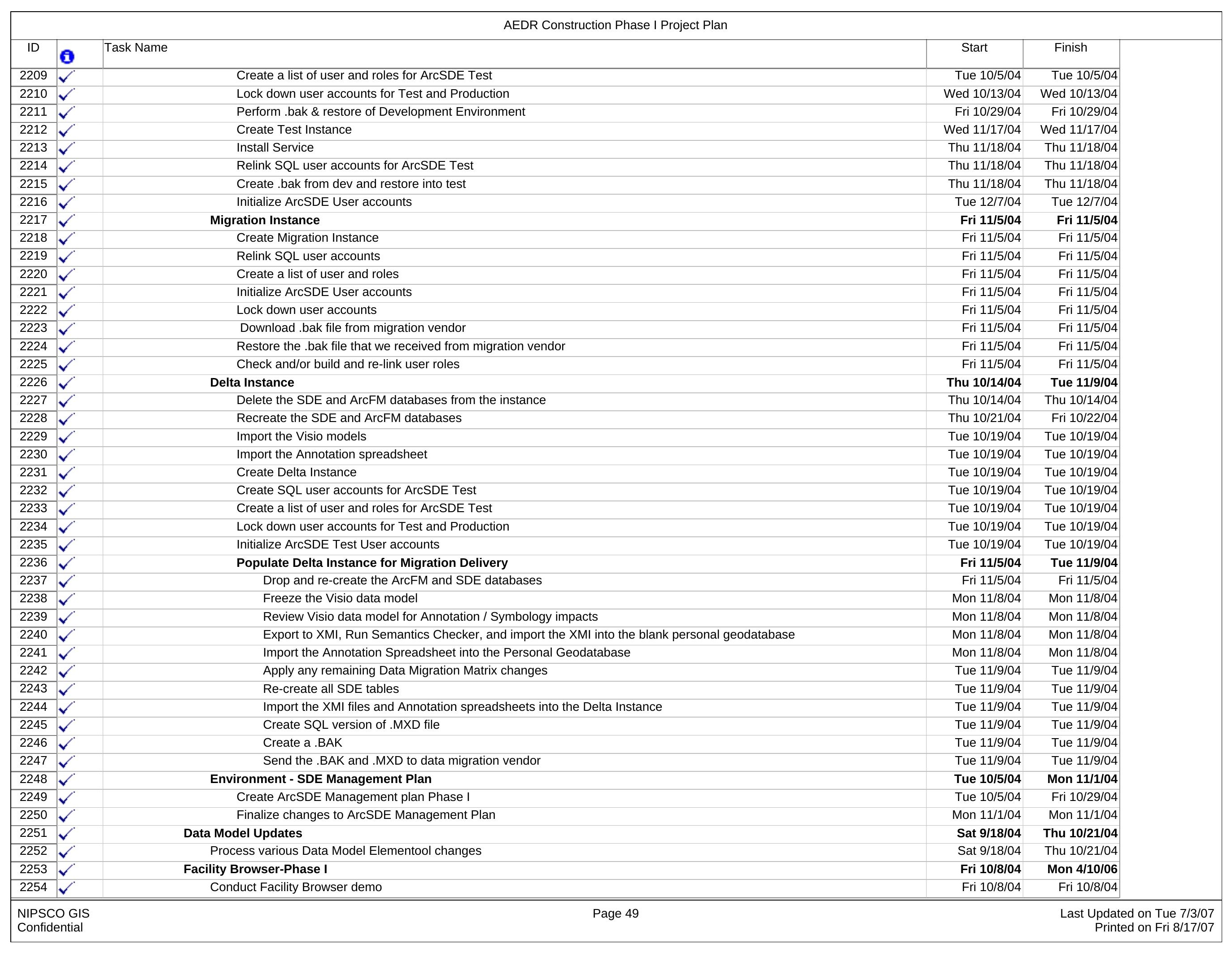




\begin{tabular}{|c|c|c|c|c|c|}
\hline \multicolumn{6}{|c|}{ AEDR Construction Phase I Project Plan } \\
\hline ID & ( & Task Name & Start & Finish & \\
\hline 2255 & $\checkmark$ & Make decision on direction for Facility Browser & Fri 10/8/04 & Fri 10/8/04 & \\
\hline 2256 & $\checkmark$ & Contact vendor for project initiation & Wed 10/20/04 & Wed 10/20/04 & \\
\hline 2257 & $\checkmark$ & Document requirements & Wed 10/20/04 & Wed 10/20/04 & \\
\hline 2258 & $\checkmark$ & Review Facility Browser vendor overview & Mon 10/25/04 & Mon 10/25/04 & \\
\hline 2259 & $\checkmark$ & Create Project Plan / Schedule for the work & Fri 11/5/04 & Fri 11/5/04 & \\
\hline 2260 & $\checkmark$ & Normalize Gas Service Cards - ArclMS Facility Browser - Create Backup of Data & Fri 11/19/04 & Fri 11/19/04 & \\
\hline 2261 & $\checkmark$ & Facility Browser Miscellaneous Setup Tasks & Wed 12/22/04 & Mon 4/10/06 & \\
\hline 2262 & $\checkmark$ & Research hardware needed for ArcIMS & Wed 12/22/04 & Wed 12/22/04 & \\
\hline 2263 & $\checkmark$ & Provide $12 / 17$.BAK file to vendor & Mon 1/10/05 & Mon 1/10/05 & \\
\hline 2264 & $\checkmark$ & Notify vendor of the CIS interface changes & Fri 1/21/05 & Fri 1/21/05 & \\
\hline 2265 & $\checkmark$ & Reconfig of the application based on 12/17 .BAK & Thu $1 / 27 / 05$ & Fri 1/28/05 & \\
\hline 2266 & $\checkmark$ & Build CIS schema into Visio diagram & Thu $2 / 10 / 05$ & Thu 2/10/05 & \\
\hline 2267 & $\checkmark$ & Generate Customer Data (Sample Data for Testing) & Fri 2/18/05 & Fri 2/18/05 & \\
\hline 2268 & $\checkmark$ & Provide 5.0 .BAK file to vendor & Fri 8/19/05 & Fri 8/19/05 & \\
\hline 2269 & $\checkmark$ & Reconfig of the application based on 5.0 .BAK & Fri 8/19/05 & Tue 8/23/05 & \\
\hline 2270 & $\checkmark$ & Update Data Model Change Interface Impact Grid & Fri 3/31/06 & Fri 3/31/06 & \\
\hline 2271 & $\checkmark$ & Review ArclMS Symbology document and decide on direction & Tue 2/7/06 & Tue 2/7/06 & \\
\hline 2272 & $\checkmark$ & Create Intersection Point Layer custom code & Wed 3/8/06 & Tue $3 / 14 / 06$ & \\
\hline 2273 & $\checkmark$ & Run Intersection Point Layert \& distribute the data & Fri 3/17/06 & Fri 3/17/06 & \\
\hline 2274 & $\checkmark$ & Decision on run frequency \& batch management & Mon 4/10/06 & Mon 4/10/06 & \\
\hline 2275 & $\checkmark$ & ArcIMS Facility Browser & Wed 11/10/04 & Fri 3/31/06 & \\
\hline 2276 & $\checkmark$ & Review/Design & Wed 11/10/04 & Wed 11/10/04 & \\
\hline 2277 & $\checkmark$ & Development Part 1 & Thu $11 / 11 / 04$ & Wed 11/24/04 & \\
\hline 2278 & $\checkmark$ & Development Part 2 & Mon 12/13/04 & Fri $1 / 7 / 05$ & \\
\hline 2279 & $\checkmark$ & Development Part 3 (updates for .BAK and CIS Data) & Mon 2/14/05 & Fri 2/25/05 & \\
\hline 2280 & $\checkmark$ & Development Part 4 (Updates to correct deficiencies) & Thu 2/16/06 & Fri 3/31/06 & \\
\hline 2281 & $\checkmark>$ & Normalize Gas Service Cards & Mon 12/13/04 & Mon 4/10/06 & \\
\hline 2282 & $\checkmark$ & Design Review & Mon 12/13/04 & Mon 12/13/04 & \\
\hline 2283 & $\checkmark$ & Development & Mon 12/27/04 & Wed 1/5/05 & \\
\hline 2284 & $\checkmark$ & Run Application 1-time (throw-away code) / QAQC & Wed $1 / 26 / 05$ & Fri $1 / 28 / 05$ & \\
\hline 2285 & $\checkmark$ & QA/QC Results & Mon 2/14/05 & Mon 2/14/05 & \\
\hline 2286 & $\checkmark b$ & Restore Gas Service Cards to Server & Fri 3/31/06 & Fri 3/31/06 & \\
\hline 2287 & $\checkmark$ & Integrate scanned backlog service cards & Mon 4/10/06 & Mon 4/10/06 & \\
\hline 2288 & $\checkmark$ & CIS to GIS Interface for Customers, XY's and Service Cards & Fri 12/17/04 & Mon 4/10/06 & \\
\hline 2289 & $\checkmark$ & Evaluate common data across interfaces to eliminate redundancy & Fri 12/17/04 & Fri $12 / 17 / 04$ & \\
\hline 2290 & $\checkmark$ & Evaluate current interface data and applications for validity & Fri 1/21/05 & Fri 1/21/05 & \\
\hline 2291 & $\checkmark$ & Define methods for data transfer & Mon 1/24/05 & Mon $1 / 24 / 05$ & \\
\hline 2292 & $\checkmark$ & Create design framework \& data model design & Mon 1/31/05 & Wed 2/9/05 & \\
\hline 2293 & $\checkmark$ & Update Data Model Change Interface Impact Grid & Wed 2/9/05 & Wed 2/9/05 & \\
\hline 2294 & $\checkmark$ & Create design specs & Tue 2/8/05 & Tue $2 / 8 / 05$ & \\
\hline 2295 & $\checkmark$ & Review \& approve the design specs & Mon 2/28/05 & Mon 2/28/05 & \\
\hline 2296 & $\checkmark$ & Notify CIS that June 12 is our go-live date and set up final meeting & Thu $3 / 30 / 06$ & Thu 3/30/06 & \\
\hline 2297 & $\checkmark$ & Finalize the design specs & Mon 2/28/05 & Mon 2/28/05 & \\
\hline 2298 & $\checkmark$ & Create test plans \& documentation & Fri 3/17/06 & Mon 3/20/06 & \\
\hline 2299 & $\checkmark$ & Code \& unit test applications / database triggers & Tue $3 / 1 / 05$ & Wed 3/2/05 & \\
\hline 2300 & $\checkmark$ & Install application at NIPSCO & Mon 4/10/06 & Mon 4/10/06 & \\
\hline $\begin{array}{l}\text { NIPSC } \\
\text { Confid }\end{array}$ & $\begin{array}{l}\text { Co GIS } \\
\text { lential }\end{array}$ & Page 50 & & $\begin{array}{r}\text { Last Updat } \\
\text { Print }\end{array}$ & $\begin{array}{l}\text { ed on Tue } 7 / 3 / 07 \\
\text { ed on Fri 8/17/07 }\end{array}$ \\
\hline
\end{tabular}




\begin{tabular}{|c|c|c|c|c|c|}
\hline \multicolumn{6}{|c|}{ AEDR Construction Phase I Project Plan } \\
\hline ID & ( & Task Name & Start & Finish & \\
\hline 2301 & $\checkmark$ & System test application & Mon 4/10/06 & Mon 4/10/06 & \\
\hline 2302 & $\checkmark$ & Field Browser - Phase I & Fri 11/12/04 & Fri 11/19/04 & \\
\hline 2303 & $\checkmark$ & Field Browser Requirements and Design & Fri 11/12/04 & Fri 11/19/04 & \\
\hline 2304 & $\checkmark$ & Identify end users & Fri 11/12/04 & Fri 11/12/04 & \\
\hline 2305 & $\checkmark$ & Send candidate list of end users out for review & Thu 11/18/04 & Thu 11/18/04 & \\
\hline 2306 & $\checkmark$ & Approve end user list & Fri 11/19/04 & Fri 11/19/04 & \\
\hline 2307 & $\checkmark$ & EDFS / MAPPS Requirements & Mon 9/13/04 & Fri 10/8/04 & \\
\hline 2308 & $\checkmark$ & EDFS Overhead and Underground/Luminaire Requirements Document & Mon 9/13/04 & Mon 9/20/04 & \\
\hline 2309 & $\checkmark$ & Create EDFS Overhead and Underground requirement documents & Mon 9/13/04 & Mon 9/13/04 & \\
\hline 2310 & $\checkmark$ & Review the EDFS Overhead and Underground requirement documents & Mon 9/13/04 & Mon 9/13/04 & \\
\hline 2311 & $\checkmark$ & Finalize the EDFS Overhead and Underground requirements document based on review comments & Mon 9/13/04 & Mon 9/13/04 & \\
\hline 2312 & $\checkmark$ & Send out the EDFS OH and UG requirements document to the vendors & Mon 9/20/04 & Mon 9/20/04 & \\
\hline 2313 & $\checkmark$ & Vendor Selection & Tue 10/5/04 & Fri 10/8/04 & \\
\hline 2314 & $\checkmark$ & Proposals are due & Tue $10 / 5 / 04$ & Tue $10 / 5 / 04$ & \\
\hline 2315 & $\checkmark$ & Review EDFS Integration Proposals & Tue 10/5/04 & Wed 10/6/04 & \\
\hline 2316 & $\checkmark$ & Vendor walk-through & Thu $10 / 7 / 04$ & Thu 10/7/04 & \\
\hline 2317 & $\checkmark$ & Select Vendor & Fri 10/8/04 & Fri 10/8/04 & \\
\hline 2318 & $\checkmark$ & EDFS Integration \#1 & Mon 10/11/04 & Mon 12/13/04 & \\
\hline 2319 & $\checkmark$ & Design Phase & Mon 10/11/04 & Mon 12/13/04 & \\
\hline 2320 & $\checkmark$ & Prep for workshops & Mon 10/11/04 & Thu 10/21/04 & \\
\hline 2321 & $\checkmark$ & Prepare for Workshop & Mon 10/11/04 & Fri 10/15/04 & \\
\hline 2322 & $\checkmark$ & Client Server vs Web and conductor meeting & Thu $10 / 21 / 04$ & Thu 10/21/04 & \\
\hline 2323 & $\checkmark$ & Design Workshops & Mon 10/18/04 & Mon 12/13/04 & \\
\hline 2324 & $\checkmark$ & Transformer/Regulator & Mon 10/18/04 & Tue 10/19/04 & \\
\hline 2325 & $\checkmark$ & Data Modeling \& Migration Planning & Mon 10/18/04 & Mon 10/18/04 & \\
\hline 2326 & $\checkmark$ & Sage Tool \& Batch Design & Mon 10/18/04 & Tue 10/19/04 & \\
\hline 2327 & $\checkmark$ & Capacitor & Tue 10/19/04 & Wed 10/20/04 & \\
\hline 2328 & $\checkmark$ & Data Modeling \& Migration Planning & Tue $10 / 19 / 04$ & Tue 10/19/04 & \\
\hline 2329 & $\checkmark$ & Sage Tool \& Batch Design & Wed 10/20/04 & Wed 10/20/04 & \\
\hline 2330 & $\checkmark$ & Overhead \& Underground & Thu 10/21/04 & Fri 10/29/04 & \\
\hline 2331 & $\checkmark$ & Data Modeling \& Migration Planning & Thu 10/21/04 & Thu 10/28/04 & \\
\hline 2332 & $\checkmark$ & Sage Tool \& Batch Design & Fri 10/29/04 & Fri 10/29/04 & \\
\hline 2333 & $\checkmark$ & Validation \& Domain Management & Fri 10/29/04 & Wed $11 / 3 / 04$ & \\
\hline 2334 & $\checkmark$ & Data Modeling \& Migration Planning & Fri 10/29/04 & Wed $11 / 3 / 04$ & \\
\hline 2335 & $\checkmark$ & Coordinator Management Tools & Tue 11/2/04 & Fri 11/5/04 & \\
\hline 2336 & $\checkmark$ & Sage Tool Design & Tue $11 / 2 / 04$ & Fri 11/5/04 & \\
\hline 2337 & $\checkmark$ & Reports & Tue 11/2/04 & Thu 11/4/04 & \\
\hline 2338 & $\checkmark$ & Choose Presentation Technology & Wed 11/3/04 & Wed 11/3/04 & \\
\hline 2339 & $\checkmark$ & Review Data Sources & Tue $11 / 2 / 04$ & Thu $11 / 4 / 04$ & \\
\hline 2340 & $\checkmark$ & Create Detailed Design (Component Specs) & Mon 11/8/04 & Mon 12/13/04 & \\
\hline 2341 & $\checkmark$ & Data Model Changes & Mon 11/8/04 & Fri 11/12/04 & \\
\hline 2342 & $\checkmark$ & Application Components: SAGE \& ArcFM & Mon 11/15/04 & Wed 12/1/04 & \\
\hline 2343 & $\checkmark$ & Design Interface for MLOG, MAPPS, General Ledger & Wed 12/8/04 & Wed 12/8/04 & \\
\hline 2344 & $\checkmark$ & Review Detailed Design & Mon 12/6/04 & Fri 12/10/04 & \\
\hline 2345 & $\checkmark$ & Review EDFS Interface Design & Fri 12/10/04 & Fri 12/10/04 & \\
\hline 2346 & $\checkmark$ & Review Data Model candidates & Mon 12/13/04 & Mon 12/13/04 & \\
\hline $\begin{array}{l}\text { NIPSC } \\
\text { Confid }\end{array}$ & $\begin{array}{l}\text { Co GIS } \\
\text { lential }\end{array}$ & Page 51 & & $\begin{array}{l}\text { Last Update } \\
\text { Print }\end{array}$ & $\begin{array}{l}\text { ed on Tue } 7 / 3 / 07 \\
\text { ed on Fri 8/17/07 }\end{array}$ \\
\hline
\end{tabular}




\begin{tabular}{|c|c|c|c|c|c|}
\hline \multicolumn{6}{|c|}{ AEDR Construction Phase I Project Plan } \\
\hline ID & ( & Task Name & Start & Finish & \\
\hline 2347 & $\checkmark$ & Non-EDFS AutoUpdaters / Miscellaneous Customizations & Mon 9/13/04 & Thu 12/16/04 & \\
\hline 2348 & $\checkmark$ & Distrib Ref Number - 8 Digit AU & Mon 9/13/04 & Tue 9/14/04 & \\
\hline 2349 & $\checkmark$ & Create component spec & Mon 9/13/04 & Mon 9/13/04 & \\
\hline 2350 & $\checkmark$ & Review component spec & Mon 9/13/04 & Mon 9/13/04 & \\
\hline 2351 & $\checkmark$ & Finalize component spec & Mon 9/13/04 & Mon 9/13/04 & \\
\hline 2352 & $\checkmark$ & Code / unit test & Mon 9/13/04 & Tue $9 / 14 / 04$ & \\
\hline 2353 & $\checkmark$ & Transformer - Prevent Deletion if Units Related AU & Wed 9/22/04 & Thu $9 / 23 / 04$ & \\
\hline 2354 & $\checkmark$ & Create component spec & Wed 9/22/04 & Thu $9 / 23 / 04$ & \\
\hline 2355 & $\checkmark$ & Review component spec & Wed 9/22/04 & Wed 9/22/04 & \\
\hline 2356 & $\checkmark$ & Code / unit test & Thu 9/23/04 & Thu 9/23/04 & \\
\hline 2357 & $\checkmark$ & Transformer - Temporary Install Field AU & Thu 9/23/04 & Fri 9/24/04 & \\
\hline 2358 & $\checkmark$ & Create component spec & Thu $9 / 23 / 04$ & Thu $9 / 23 / 04$ & \\
\hline 2359 & $\checkmark$ & Review component spec & Thu 9/23/04 & Thu 9/23/04 & \\
\hline 2360 & $\checkmark$ & Code / unit test & Fri 9/24/04 & Fri 9/24/04 & \\
\hline 2361 & $\checkmark$ & Capacitor - Prevent Delete AU & Mon 9/27/04 & Mon 9/27/04 & \\
\hline 2362 & $\checkmark$ & Create component spec & Mon 9/27/04 & Mon 9/27/04 & \\
\hline 2363 & $\checkmark$ & Review component spec & Mon 9/27/04 & Mon 9/27/04 & \\
\hline 2364 & $\checkmark$ & Code / unit test & Mon 9/27/04 & Mon 9/27/04 & \\
\hline 2365 & $\checkmark$ & Switch - Symbology Configuration Code AU & Tue 9/14/04 & Wed 9/15/04 & \\
\hline 2366 & $\checkmark$ & Create component spec & Tue $9 / 14 / 04$ & Wed 9/15/04 & \\
\hline 2367 & $\checkmark$ & Review component spec & Tue 9/14/04 & Tue $9 / 14 / 04$ & \\
\hline 2368 & $\checkmark$ & Code / unit test & Wed 9/15/04 & Wed 9/15/04 & \\
\hline 2369 & $\checkmark$ & Fuse Cutout Bank - Symbology Configuration Code AU & Wed 9/15/04 & Thu $9 / 16 / 04$ & \\
\hline 2370 & $\checkmark$ & Create component spec & Wed 9/15/04 & Wed 9/15/04 & \\
\hline 2371 & $\checkmark$ & Review component spec & Wed 9/15/04 & Wed 9/15/04 & \\
\hline 2372 & $\checkmark$ & Code / unit test & Thu 9/16/04 & Thu 9/16/04 & \\
\hline 2373 & $\checkmark$ & Sectionalizer Bank - Symbology Configuration Code AU & Thu 9/16/04 & Thu $9 / 16 / 04$ & \\
\hline 2374 & $\checkmark$ & Create component spec & Thu $9 / 16 / 04$ & Thu $9 / 16 / 04$ & \\
\hline 2375 & $\checkmark$ & Review component spec & Thu 9/16/04 & Thu 9/16/04 & \\
\hline 2376 & $\checkmark$ & Code / unit test & Thu $9 / 16 / 04$ & Thu 9/16/04 & \\
\hline 2377 & $\checkmark$ & Conductors - Cross Over Arc AU & Thu 10/21/04 & Fri 10/22/04 & \\
\hline 2378 & $\checkmark$ & Create component spec & Thu 10/21/04 & Thu 10/21/04 & \\
\hline 2379 & $\checkmark$ & Review component spec & Thu 10/21/04 & Thu 10/21/04 & \\
\hline 2380 & $\checkmark$ & Code / unit test & Fri 10/22/04 & Fri 10/22/04 & \\
\hline 2381 & $\checkmark$ & Voltage Regulator & Mon 10/4/04 & Mon 10/4/04 & \\
\hline 2382 & $\checkmark$ & Create component spec & Mon 10/4/04 & Mon 10/4/04 & \\
\hline 2383 & $\checkmark$ & Review component spec & Mon 10/4/04 & Mon 10/4/04 & \\
\hline 2384 & $\checkmark$ & Code / unit test & Mon 10/4/04 & Mon 10/4/04 & \\
\hline 2385 & $\checkmark$ & Fuse CutoutBank - Offset AU & Mon 10/4/04 & Wed 10/20/04 & \\
\hline 2386 & $\checkmark$ & Create component spec & Mon 10/4/04 & Mon 10/4/04 & \\
\hline 2387 & $\checkmark$ & Review component spec & Tue $10 / 12 / 04$ & Fri 10/15/04 & \\
\hline 2388 & $\checkmark$ & Code / unit test & Wed 10/20/04 & Wed 10/20/04 & \\
\hline 2389 & $\checkmark$ & Voltage Regulator - Offset AU & Tue 10/12/04 & Wed 10/20/04 & \\
\hline 2390 & $\checkmark$ & Create component spec & Tue $10 / 12 / 04$ & Tue 10/12/04 & \\
\hline 2391 & $\checkmark$ & Review component spec & Tue $10 / 12 / 04$ & Tue $10 / 12 / 04$ & \\
\hline 2392 & $\checkmark$ & Code / unit test & Wed 10/20/04 & Wed 10/20/04 & \\
\hline $\begin{array}{l}\text { NIPSC } \\
\text { Confid }\end{array}$ & $\begin{array}{l}\text { Co GIS } \\
\text { lential }\end{array}$ & & & $\begin{array}{r}\text { Last Updat } \\
\text { Print }\end{array}$ & $\begin{array}{l}\text { ed on Tue } 7 / 3 / 07 \\
\text { ed on Fri 8/17/07 }\end{array}$ \\
\hline
\end{tabular}




\begin{tabular}{|c|c|c|c|c|c|}
\hline \multicolumn{6}{|c|}{ AEDR Construction Phase I Project Plan } \\
\hline ID & ( & Task Name & Start & Finish & \\
\hline 2393 & $\checkmark$ & Switch Offset AU & Tue 10/26/04 & Tue 10/26/04 & \\
\hline 2394 & $\checkmark$ & Create component spec & Tue $10 / 26 / 04$ & Tue $10 / 26 / 04$ & \\
\hline 2395 & $\checkmark$ & Review component spec & Tue $10 / 26 / 04$ & Tue $10 / 26 / 04$ & \\
\hline 2396 & $\checkmark$ & Code / unit test & Tue $10 / 26 / 04$ & Tue $10 / 26 / 04$ & \\
\hline 2397 & $\checkmark$ & Capacitor Bank Offset AU & Tue 10/12/04 & Tue 10/26/04 & \\
\hline 2398 & $\checkmark$ & Create component spec & Tue $10 / 12 / 04$ & Tue 10/12/04 & \\
\hline 2399 & $\checkmark$ & Review component spec & Wed 10/20/04 & Wed 10/20/04 & \\
\hline 2400 & $\checkmark$ & Code / unit test & Tue 10/26/04 & Tue $10 / 26 / 04$ & \\
\hline 2401 & $\checkmark$ & Reclosure Bank Offset AU & Tue 10/26/04 & Tue 10/26/04 & \\
\hline 2402 & $\checkmark$ & Create component spec & Tue $10 / 26 / 04$ & Tue $10 / 26 / 04$ & \\
\hline 2403 & $\checkmark$ & Review component spec & Tue $10 / 26 / 04$ & Tue $10 / 26 / 04$ & \\
\hline 2404 & $\checkmark$ & Code / unit test & Tue $10 / 26 / 04$ & Tue $10 / 26 / 04$ & \\
\hline 2405 & $\checkmark$ & Gas Main - Split On Tap AU & Mon 9/13/04 & Thu $10 / 7 / 04$ & \\
\hline 2406 & $\checkmark$ & Create component spec & Mon 9/13/04 & Mon 9/13/04 & \\
\hline 2407 & $\checkmark$ & Review component spec & Mon 9/13/04 & Mon 9/13/04 & \\
\hline 2408 & $\checkmark$ & Resolve M\&M core software bug & Wed 9/29/04 & Wed 9/29/04 & \\
\hline 2409 & $\checkmark$ & Code / unit test & Wed 10/6/04 & Thu $10 / 7 / 04$ & \\
\hline 2410 & $\checkmark$ & Transformer Lead Creation AU & Wed 9/29/04 & Wed 11/3/04 & \\
\hline 2411 & $\checkmark$ & Research with M\&M to come up with estimates & Wed 9/29/04 & Wed 9/29/04 & \\
\hline 2412 & $\checkmark$ & Create component spec & Mon 10/25/04 & Mon 10/25/04 & \\
\hline 2413 & $\checkmark$ & Review component spec & Mon 10/25/04 & Mon 10/25/04 & \\
\hline 2414 & $\checkmark$ & Code / unit test & Mon 11/1/04 & Wed $11 / 3 / 04$ & \\
\hline 2415 & $\checkmark$ & Conductor - Split at Tap Point AU & Mon 10/25/04 & Thu 10/28/04 & \\
\hline 2416 & $\checkmark$ & Create component spec & Mon 10/25/04 & Mon 10/25/04 & \\
\hline 2417 & $\checkmark$ & Review component spec & Mon 10/25/04 & Mon 10/25/04 & \\
\hline 2418 & $\checkmark$ & Code / unit test & Wed 10/27/04 & Thu 10/28/04 & \\
\hline 2419 & $\checkmark$ & Configure ArcFM to Include all AUs & Thu 10/21/04 & Thu 11/4/04 & \\
\hline 2420 & $\checkmark$ & Import into ArcSDE & Thu $10 / 21 / 04$ & Thu 10/21/04 & \\
\hline 2421 & $\checkmark$ & Install and configure the AUs for land & Mon 11/1/04 & Mon 11/1/04 & \\
\hline 2422 & $\checkmark$ & Install and configure the AUs for gas & Mon 11/1/04 & Mon 11/1/04 & \\
\hline 2423 & $\checkmark$ & Install and configure the AUs for electric & Mon 11/1/04 & Mon 11/1/04 & \\
\hline 2424 & $\checkmark$ & Review and resolve $\mathrm{AU}$ installation issues & Wed 11/3/04 & Wed 11/3/04 & \\
\hline 2425 & $\checkmark$ & Additional AU configuration work based on changes made for data model updates, etc & Wed 11/3/04 & Thu $11 / 4 / 04$ & \\
\hline 2426 & $\checkmark$ & Miscellaneous Customizations & Tue 9/28/04 & Mon 10/25/04 & \\
\hline 2427 & $\checkmark$ & Fix CPSection Maintenance Tool bug & Tue 9/28/04 & Tue 9/28/04 & \\
\hline 2428 & $\checkmark$ & ArcFm Properties Manager Fieldlnfo Settings & Mon 10/4/04 & Mon 10/4/04 & \\
\hline 2429 & $\checkmark$ & Determine how to prevent stored displays from being changed & Mon 10/25/04 & Mon 10/25/04 & \\
\hline 2430 & $\checkmark$ & Conductor - Prevent Delete AU & Tue 11/2/04 & Thu 11/4/04 & \\
\hline 2431 & $\checkmark$ & Create component spec & Tue 11/2/04 & Tue 11/2/04 & \\
\hline 2432 & $\checkmark$ & Review component spec & Tue 11/2/04 & Tue 11/2/04 & \\
\hline 2433 & $\checkmark$ & Code / unit test & Thu $11 / 4 / 04$ & Thu $11 / 4 / 04$ & \\
\hline 2434 & $\checkmark$ & Configure Cardinality Rules & Tue 11/2/04 & Tue 11/2/04 & \\
\hline 2435 & $\checkmark$ & Modify cardinality rule information (gas only) & Tue 11/2/04 & Tue 11/2/04 & \\
\hline 2436 & $\checkmark$ & Configure cardinality for gas & Tue 11/2/04 & Tue $11 / 2 / 04$ & \\
\hline 2437 & $\checkmark$ & Change for AbandonedGasDeviceAU & Fri 11/19/04 & Fri 11/19/04 & \\
\hline 2438 & $\checkmark$ & Review and approve the change & Fri 11/19/04 & Fri 11/19/04 & \\
\hline $\begin{array}{l}\text { NIPSC } \\
\text { Confid }\end{array}$ & $\begin{array}{l}\text { Co GIS } \\
\text { lential }\end{array}$ & Page 53 & & $\begin{array}{l}\text { Last Update } \\
\text { Print }\end{array}$ & $\begin{array}{l}\text { ed on Tue } 7 / 3 / 07 \\
\text { ed on Fri 8/17/07 }\end{array}$ \\
\hline
\end{tabular}




\begin{tabular}{|c|c|c|c|c|c|}
\hline \multicolumn{6}{|c|}{ AEDR Construction Phase I Project Plan } \\
\hline ID & ( & Task Name & Start & Finish & \\
\hline 2439 & $\checkmark$ & Update the AbandonedGasDeviceAU & Fri 11/19/04 & Fri 11/19/04 & \\
\hline 2440 & $\checkmark$ & Meta Data Tasks & Tue 11/23/04 & Thu 12/9/04 & \\
\hline 2441 & $\checkmark$ & Identify SOX requirements & Tue $11 / 23 / 04$ & Tue $11 / 23 / 04$ & \\
\hline 2442 & $\checkmark$ & Meeting to determine the additional metadata that needs to be tracked & Wed 12/8/04 & Wed $12 / 8 / 04$ & \\
\hline 2443 & $\checkmark$ & Compile list of data that must be manually generated, auto generated, and not collected & Wed 12/8/04 & Thu 12/9/04 & \\
\hline 2444 & $\checkmark$ & Distrib Ref Number AU & Tue $9 / 28 / 04$ & Thu $12 / 16 / 04$ & \\
\hline 2445 & $\checkmark$ & Document solutions for DistribRefNumber-Uniqueness & Tue 9/28/04 & Tue 9/28/04 & \\
\hline 2446 & $\checkmark$ & Review DistribRefNum solutions document & Wed 10/6/04 & Wed 10/6/04 & \\
\hline 2447 & $\checkmark$ & Approve DistribRefNum document & Mon 11/1/04 & Mon 11/1/04 & \\
\hline 2448 & $\checkmark$ & Create component spec & Wed 11/3/04 & Wed $11 / 3 / 04$ & \\
\hline 2449 & $\checkmark$ & Review component spec & Mon 11/8/04 & Mon 11/8/04 & \\
\hline 2450 & $\checkmark$ & Revise component spec based on decisions & Fri 11/19/04 & Fri $11 / 19 / 04$ & \\
\hline 2451 & $\checkmark$ & Code / unit test population, etc & Wed 12/15/04 & Thu $12 / 16 / 04$ & \\
\hline 2452 & $\checkmark$ & OH Prim - Conductor Offset AU & Tue $9 / 28 / 04$ & Wed 10/20/04 & \\
\hline 2453 & $\checkmark$ & Research with M\&M to come up with estimates & Tue 9/28/04 & Tue $9 / 28 / 04$ & \\
\hline 2454 & $\checkmark$ & Create component spec & Tue 10/19/04 & Tue $10 / 19 / 04$ & \\
\hline 2455 & $\checkmark$ & Code / unit test population, etc & Tue $10 / 19 / 04$ & Wed 10/20/04 & \\
\hline 2456 & $\checkmark$ & Reference Features - Do Not Post To Parent & Fri 11/19/04 & Fri 12/3/04 & \\
\hline 2457 & $\checkmark$ & Demo ArcFM Session Manager and document options & Fri 11/19/04 & Fri $11 / 19 / 04$ & \\
\hline 2458 & $\checkmark$ & Make decision on Session Manager option to use & Mon 11/22/04 & Mon 11/22/04 & \\
\hline 2459 & $\checkmark$ & Create component spec & Wed 12/1/04 & Wed 12/1/04 & \\
\hline 2460 & $\checkmark$ & Code / unit test population, etc & Thu 12/2/04 & Fri 12/3/04 & \\
\hline 2461 & $\checkmark$ & Gas OMS & Fri 10/8/04 & Fri 10/8/04 & \\
\hline 2462 & $\checkmark$ & Prototype the Gas OMS application & Fri $10 / 8 / 04$ & Fri 10/8/04 & \\
\hline 2463 & $\checkmark$ & Conduct Gas OMS demo & Fri 10/8/04 & Fri 10/8/04 & \\
\hline 2464 & $\checkmark$ & CIS Interface & Wed 10/27/04 & Fri 10/29/04 & \\
\hline 2465 & $\checkmark$ & Create CIS Interface Spreadsheet & Wed 10/27/04 & Wed 10/27/04 & \\
\hline 2466 & $\checkmark$ & Evaluate CIS Interface Options & Fri 10/29/04 & Fri 10/29/04 & \\
\hline 2467 & $\checkmark$ & SynerGEE interface \#1 & Wed 10/20/04 & Fri $12 / 17 / 04$ & \\
\hline 2468 & $\checkmark$ & Review Overview & Mon 10/25/04 & Mon 10/25/04 & \\
\hline 2469 & $\checkmark$ & Export personal GDB & Wed 10/27/04 & Wed 10/27/04 & \\
\hline 2470 & $\checkmark$ & Analysis Phase & Wed 10/20/04 & Mon 10/25/04 & \\
\hline 2471 & $\checkmark$ & Create DataPrep Install (First Half) & Fri 11/5/04 & Fri 11/5/04 & \\
\hline 2472 & $\checkmark$ & Package up latest design docs for checkpoint review & Fri 12/10/04 & Fri $12 / 10 / 04$ & \\
\hline 2473 & $\checkmark$ & Review existing data model and suggest data model changes needed for SynerGEE & Fri 11/5/04 & Fri 12/17/04 & \\
\hline 2474 & $\checkmark$ & Develop DataPrep Design Document & Fri 11/5/04 & Fri 12/17/04 & \\
\hline 2475 & $\checkmark$ & Application Interface Design - Part 1 & Mon 11/8/04 & Fri 12/10/04 & \\
\hline 2476 & $\checkmark$ & DataPrep Development - Part 1 & Mon 11/8/04 & Fri 12/10/04 & \\
\hline 2477 & $\checkmark$ & Review / Revise the User Guides & Thu 9/16/04 & Tue $12 / 7 / 04$ & \\
\hline 2478 & $\checkmark$ & Completed User Guide Tasks & Thu 9/16/04 & Tue $12 / 7 / 04$ & \\
\hline 2479 & $\checkmark$ & How to relate Features/Objects & Fri 9/17/04 & Fri 9/17/04 & \\
\hline 2480 & $\checkmark$ & Demo the updated sample on-line help system & Thu 9/16/04 & Thu $9 / 16 / 04$ & \\
\hline 2481 & $\checkmark$ & Review the user guides for iteration $1,2,3$ & Fri 9/24/04 & Fri 10/15/04 & \\
\hline 2482 & $\checkmark$ & Revise the user guides for iteration $1,2,3$ & Mon 10/18/04 & Mon 10/18/04 & \\
\hline 2483 & $\checkmark$ & Provide full list of remaining user guide documents to be created & Mon 10/25/04 & Mon 10/25/04 & \\
\hline 2484 & $\checkmark$ & Create 'How to Install' template and 'How to Retire' template & Fri 9/24/04 & Fri 9/24/04 & \\
\hline $\begin{array}{l}\text { NIPSC } \\
\text { Confid }\end{array}$ & $\begin{array}{l}\text { Co GIS } \\
\text { lential }\end{array}$ & Page 54 & & $\begin{array}{r}\text { Last Updat } \\
\text { Print }\end{array}$ & $\begin{array}{l}\text { ed on Tue } 7 / 3 / 07 \\
\text { ed on Fri 8/17/07 }\end{array}$ \\
\hline
\end{tabular}




\section{\begin{tabular}{|ll}
\hline & AEDR Construction Phase I Project Plan
\end{tabular}}

Create initial "How to Login to ArcMap" user quide

Create initial "How to Select Features" user guide

Create initial "How to Sketch using ESRI Sketch tools" user guide

Mon $11 / 22 / 04$

Tue $11 / 23 / 04$

Create initial "How to Perform a Cathodic Protection Trace" user guide

Tue $11 / 23 / 04$

Create initial "How to Install a Pressure Control Fitting" user guide

Mon 11/29/04

Create initial "How to Install Gas Transmission Mains" user guide

Tue $11 / 30 / 04$

Create initial "How to Install Gas Distribution Mains" user guide

Tue $11 / 30 / 04$

Create initial "How to Install Gas Service Stub" user guide

Tue $11 / 30 / 04$

Create initial "How to Install a Capacitor" user guide

Create initial "How to Install a Splice" user guide

Create initial "How to Install an Overhead Transformer" user guide

Create initial "How to Install an Overhead Conductor" user guide

Create initial "How to Install Street Centerlines (details of all attributes/sketch etc)" user quide

Fri $12 / 3 / 04$

Fri $12 / 3 / 04$

Fri $12 / 3 / 04$

Mon $12 / 6 / 04$

Tue $12 / 7 / 04$

Create initial "How to Install Street Right-of-Way" user guide

Create initial "How to Install Railroad Centerline" user guide

Create initial "How to Railroad Right-of-Way" user guide

Create initial "How to Install an Electric Deadend" user guide

Create initial "How to Install an Underground Conductor" user guide

Create initial "How to Install a Tie Wire" user guide

Iteration $\mathbf{3}$ Integration Testing / Rework

Perform the Integration Testing

Perform configuration changes on the ArcSDE Database

Prepare AUs for integration testing

Test AUs, log bugs, work on resolutions

Special testing of UniqueDistribRefNumbersAU

Re-testing of all AU updates

Testing / rework support

Conduct integration testing on the ArcSDE database

\section{Iteration 3 Performance Tuning}

Conduct tuning workshop - day 1 (Create Perf. Test Scripts)

Conduct tuning workshop - day 2 (Spatial Tuning, Indexes)

Tue $12 / 7 / 04$

Tue $12 / 7 / 04$

Tue $12 / 7 / 04$

Tue $12 / 7 / 04$

Tue $12 / 7 / 04$

Tue $12 / 7 / 04$

Mon 11/22/04

Mon 11/22/04

Mon 11/22/04

Fri 11/26/04

Mon 11/29/04

Fri $12 / 3 / 04$

Tue $12 / 7 / 04$

Mon 11/29/04

Wed $12 / 8 / 04$

Wed 12/1/04

Wed $12 / 1 / 04$

Thu 12/2/04

Mon 3/1/04

Mon 2/13/06

Mon 12/5/05

Populate the feature class for customers in the geodatabase

Mon $12 / 13 / 04$

Mon 12/13/04

Fri $12 / 24 / 04$

Wed 1/5/05

Wed 1/5/05

Thu 1/6/05

Wed 1/12/05

Mon 3/1/04

Mon 6/20/05

Mon 6/20/05

Mon 6/27/05

Mon $11 / 22 / 04$

Tue 11/23/04

Tue 11/23/04

Tue 11/30/04

Tue 11/30/04

Tue $11 / 30 / 04$

Wed 12/1/04

Fri 12/3/04

Fri $12 / 3 / 04$

Fri 12/3/04

Mon 12/6/04

Tue 12/7/04

Tue $12 / 7 / 04$

Tue $12 / 7 / 04$

Tue $12 / 7 / 04$

Tue 12/7/04

Tue 12/7/04

Tue 12/7/04

Fri 12/10/04

Fri 12/10/04

Thu 11/25/04

Fri $11 / 26 / 04$

Fri 12/3/04

Fri 12/3/04

Tue $12 / 7 / 04$

Tue 12/7/04

Fri 12/10/04

Fri 12/3/04

Wed $12 / 1 / 04$

Fri 12/3/04

Fri 12/15/06

Mon 2/13/06

Mon 12/5/05

Fri 12/24/04

Thu 12/23/04

Fri 12/24/04

Wed 1/12/05

Wed 1/5/05

Thu 1/6/05

Wed 1/12/05

Fri 8/19/05

Fri 8/19/05

Mon 6/20/05

Mon 6/27/05 


\begin{tabular}{|c|c|c|c|c|c|}
\hline \multicolumn{6}{|c|}{ AEDR Construction Phase I Project Plan } \\
\hline ID & ( & Task Name & Start & Finish & \\
\hline 2531 & $\checkmark b$ & Create a Performance Plan & Mon 6/27/05 & Mon $7 / 18 / 05$ & \\
\hline 2532 & $\checkmark$ & Develop base tuning configuration & Fri 8/5/05 & Fri 8/19/05 & \\
\hline 2533 & $\checkmark$ & Performance Testing & Mon 3/1/04 & Wed 12/22/04 & \\
\hline 2534 & $\sqrt{8}$ & Generate data for use in future performance tests & Thu $12 / 16 / 04$ & Wed 12/22/04 & \\
\hline 2535 & $\checkmark$ & Hardware Setup & Mon 3/1/04 & Mon 3/1/04 & \\
\hline 2536 & $\checkmark$ & Exercise the test plan - 1 user & Mon 3/1/04 & Fri 3/5/04 & \\
\hline 2537 & $\checkmark$ & Performance Tuning Re-Work & Mon 3/1/04 & Fri 3/5/04 & \\
\hline 2538 & $\checkmark$ & Rework configuration based on results of scripts & Mon 3/1/04 & Fri 3/5/04 & \\
\hline 2539 & $\checkmark$ & Performance Maintentance Strategies & Mon 3/1/04 & Fri $1 / 7 / 05$ & \\
\hline 2540 & $\checkmark$ & Version Management Decisions & Mon 3/1/04 & Mon 3/1/04 & \\
\hline 2541 & $\checkmark$ & Integrate Perf Tuning into SDE management plan & Fri $1 / 7 / 05$ & Fri $1 / 7 / 05$ & \\
\hline 2542 & $\checkmark$ & Conductor Information Utility Tools & Fri 1/14/05 & Fri $1 / 14 / 05$ & \\
\hline 2543 & $\checkmark$ & Review documentation and update & Fri 1/14/05 & Fri $1 / 14 / 05$ & \\
\hline 2544 & $\checkmark$ & Data Model / Matrix Updates & Mon 12/13/04 & Tue $4 / 12 / 05$ & \\
\hline 2545 & $\checkmark$ & Research issues on transmission main & Mon 12/13/04 & Mon 12/13/04 & \\
\hline 2546 & $\checkmark$ & Research questions on CPSection and GasMain & Fri 12/17/04 & Fri $12 / 17 / 04$ & \\
\hline 2547 & $\checkmark$ & Move CisSite feature class and Cis Services table to a new Data Model & Tue $12 / 28 / 04$ & Tue $12 / 28 / 04$ & \\
\hline 2548 & $\checkmark$ & Review spatial extent and precision & Tue $12 / 28 / 04$ & Tue $12 / 28 / 04$ & \\
\hline 2549 & $\checkmark$ & Review OhConductor / UgConductor - LegacyCircuitNumber & Tue $12 / 28 / 04$ & Tue $12 / 28 / 04$ & \\
\hline 2550 & $\checkmark$ & Generate pick lists for SupportStructure & Tue $12 / 28 / 04$ & Tue $12 / 28 / 04$ & \\
\hline 2551 & $\checkmark$ & Streetlights & Fri 12/31/04 & Fri 12/31/04 & \\
\hline 2552 & $\checkmark$ & New table to track Assembly History & Fri 12/31/04 & Fri 12/31/04 & \\
\hline 2553 & $\checkmark$ & Create new domain for Cable Company & Fri 12/31/04 & Fri 12/31/04 & \\
\hline 2554 & $\checkmark$ & Create new domain for conductor attribution & Fri 12/31/04 & Fri 12/31/04 & \\
\hline 2555 & $\checkmark$ & Track installation history for Capacitors & Fri 12/31/04 & Fri 12/31/04 & \\
\hline 2556 & $\checkmark$ & Create table to track removed capacitors & Fri 12/31/04 & Fri 12/31/04 & \\
\hline 2557 & $\checkmark$ & Create table to track transfers of capacitors between central stores & Fri 12/31/04 & Fri 12/31/04 & \\
\hline 2558 & $\checkmark$ & Create table to track SIN numbers for capacitors & Fri 12/31/04 & Fri 12/31/04 & \\
\hline 2559 & $\checkmark$ & Ttrack CompanyLocation on TransformerUnit & Fri 12/31/04 & Fri 12/31/04 & \\
\hline 2560 & $\checkmark$ & Create domain for Storeroom Numbers & Fri 12/31/04 & Fri 12/31/04 & \\
\hline 2561 & $\checkmark$ & Create domain for Phone Companies & Fri 12/31/04 & Fri $12 / 31 / 04$ & \\
\hline 2562 & $\checkmark$ & Provide solution for condemnation of a transformer & Fri 12/31/04 & Fri 12/31/04 & \\
\hline 2563 & $\checkmark$ & Create new lookup table for SIN numbers for transformers & Fri 12/31/04 & Fri 12/31/04 & \\
\hline 2564 & $\checkmark$ & Modify Field Inventory Light Feature Class & Fri 12/31/04 & Fri 12/31/04 & \\
\hline 2565 & $\checkmark$ & Overhead Primary Conductor Data Migration Matrix Review Issue & Fri 12/31/04 & Fri 12/31/04 & \\
\hline 2566 & $\checkmark$ & Transformer ticket & Fri 12/31/04 & Fri 12/31/04 & \\
\hline 2567 & $\checkmark$ & Allow nulls to ActiveRetiredServiceCd & Fri 12/31/04 & Fri $12 / 31 / 04$ & \\
\hline 2568 & $\checkmark$ & Link streetlights to an SAA & Fri 12/31/04 & Fri 12/31/04 & \\
\hline 2569 & $\checkmark$ & Track information on manhole mountings & Fri 12/31/04 & Fri 12/31/04 & \\
\hline 2570 & $\checkmark$ & Track support structures where there are two poles & Fri 12/31/04 & Fri $12 / 31 / 04$ & \\
\hline 2571 & $\checkmark$ & Add releationship between service wire and transformer unit or bank & Fri 12/31/04 & Fri 12/31/04 & \\
\hline 2572 & $\checkmark$ & Move traffic light demand to a separate table & Fri 12/31/04 & Fri $12 / 31 / 04$ & \\
\hline 2573 & $\checkmark$ & Manage transformer install and remove events & Wed 12/15/04 & Wed 12/15/04 & \\
\hline 2574 & $\checkmark$ & Manage sale and lease of transformers & Fri 12/31/04 & Fri $12 / 31 / 04$ & \\
\hline 2575 & $\checkmark$ & Track work request information & Fri 12/31/04 & Fri $12 / 31 / 04$ & \\
\hline 2576 & $\checkmark$ & Create field specs for the EdfsCapacitorStoreltem & Fri 12/31/04 & Fri 12/31/04 & \\
\hline $\begin{array}{l}\text { NIPSC } \\
\text { Confid }\end{array}$ & $\begin{array}{l}\text { Co GIS } \\
\text { lential }\end{array}$ & Page 56 & & $\begin{array}{r}\text { Last Updat } \\
\text { Print }\end{array}$ & $\begin{array}{l}\text { ed on Tue } 7 / 3 / 07 \\
\text { ed on Fri 8/17/07 }\end{array}$ \\
\hline
\end{tabular}




\section{\begin{tabular}{|ll}
\hline & AEDR Construction Phase I Project Plan
\end{tabular}}

\begin{tabular}{|r|l|l|}
\hline ID & $\mathbf{O}$ & Task Name \\
\hline 2577 & $\checkmark$ \\
\hline 2578 & $\checkmark$ \\
\hline 2579 & $\checkmark$ \\
\hline 2580 & $\checkmark$ \\
\hline 2581 & $\checkmark$ \\
\hline 2582 & $\checkmark$ \\
\hline 2583 & $\checkmark$ \\
\hline 2584 & $\checkmark$ \\
\hline 2585 & $\checkmark$ \\
\hline 2586 & $\checkmark$ \\
\hline 2587 & $\checkmark$ \\
\hline 2588 & $\checkmark$ \\
\hline 2589 & $\checkmark$ \\
\hline 2590 & $\checkmark$ \\
\hline 2591 & $\checkmark$ \\
\hline 2592 & $\checkmark$ \\
\hline 2593 & $\checkmark$ \\
\hline 2594 & $\checkmark$ \\
\hline 2595 & $\checkmark$ \\
\hline 2596 & $\checkmark$ \\
\hline 2597 & $\checkmark$ \\
\hline 2598 & $\checkmark$ \\
\hline 2599 & $\checkmark$ \\
\hline 2600 & $\checkmark$ \\
\hline 2601 & $\checkmark$ \\
\hline 2602 & $\checkmark$ \\
\hline 2603 & $\checkmark$ \\
\hline 2604 & $\checkmark$ \\
\hline 2605 & $\checkmark$ \\
\hline 2606 & $\checkmark$ \\
\hline 2607 & $\checkmark$ \\
\hline 2608 & $\checkmark$ \\
\hline 2609 & $\checkmark$ \\
\hline 2610 & $\checkmark$ \\
\hline 2611 & $\checkmark$ \\
\hline 2612 & $\checkmark$ \\
\hline 2613 & $\checkmark$ \\
\hline 2614 & $\checkmark$ \\
\hline 2615 & $\checkmark$ \\
\hline 2616 & $\checkmark$ \\
\hline 2617 & $\checkmark$ \\
\hline 2618 & $\checkmark$ \\
\hline 2619 & $\checkmark$ \\
\hline 2620 & $\checkmark$ \\
\hline 2621 & $\checkmark$ \\
\hline
\end{tabular}

NIPSCO GIS Confidential
Create field Specs for the EdfsConductor Table

Create field specs for the EdfsCapacitorInstall table

Create spec for the ConductorMaterialCd field

Create field specs for the FieldInventoryLight table

Create field specs for TransformerStoreltem table

Create field specs for EdfsRetiredAssembly table

Investigate solutions for RecloserUnit table questions

Investigate solutions for JointUseAttachment table questions

Secondary Conductor

Create proposal for handling pole symbol classification

Resolve Assembly Object Class-EDFS issue

Apply sequence number for Blanket WO's

Perform TransformerUnit attribute verification

Resolve issue with TransformerUnit-LegacyDistribRefNumber

Create a domain for FuseUnit.CutoutAmperageValue

JointUseAttachment AttachmentCompanyName \& AttachmentTypeCd

Apply data model Change for Conductors

Annotation Review - Padmount Transformer

Landbase - verify layers

Investigate solution for Transmission Primary Meter

Apply data model change for DmSecondaryOperatingVoltage and DmPrimaryOperatingVoltage

Resolve issues with Padmount - LegacyDistribRefNumTermld

Create a new Annotation Class: SectionalizerBank_Anno

Investigate solution for RetireWorkOrderNumber

Delete column legacyCompanyLocationID in CapacitorUnit

AbstractClass:UnitObject on Objects Page, set PhaseDesignation - AllowNulls=True

Set RetiredPadMount.LegacyDistribRefNumTermld AllowNulls=True

Recreate values in DmConductorOHSizeCd

Create Data Model Change Interface Impact Grid

Review existing annotation and identify required changes

Log the annotation data model requirements

Apply Taxing Unit data model changes

Create field specs for the CircuitSource table

Apply data model change for TransformerBank

Apply data model changes to Pedestal - ShortPedestalNumber

Apply data model changes to LotNumber

Create domain for CapacitorBank.UnitNumber

Create a domain for SectionalizerBank

AbstractClass:UnitObject on Objects Page, set PhaseDesignation - AllowNulls=True

Miscellaneous Gas Data Model Changes

Fields to be removed from RetiredSupportStructure

Additional logic for PhaseDesignationCd field - Various Features

Update domains on SupportStructure

OpenClosedStatusCd field needs Precision tag value

OhConductor subtype - Moved to new Package OhConductorlnfo

CapacitorUnit - Delete Column LegacyCompanyLocationld
Fri 12/31/04

Fri $1 / 7 / 05$

Fri 1/7/05

Fri $1 / 7 / 05$

Fri 1/7/05

Fri $1 / 7 / 05$

Fri $1 / 7 / 05$

Fri 1/7/05

Fri $1 / 7 / 05$

Fri $1 / 7 / 05$

Wed 1/5/05

Tue 1/4/05

Thu 1/6/05

Fri 1/7/05

Fri 1/7/05

Fri 1/7/05

Mon 1/10/05

Mon 1/10/05

Wed 1/12/05

Fri 1/14/05

Tue 1/11/05

Mon 12/20/04

Thu $1 / 6 / 05$

Thu 1/13/05

Thu 1/13/05

Thu $1 / 6 / 05$

Tue 1/11/05

Wed 1/12/05

Wed 1/12/05

Thu $1 / 6 / 05$

Tue 1/11/05

Mon 1/10/05

Fri $1 / 7 / 05$

Tue 1/11/05

Mon 1/10/05

Fri $1 / 7 / 05$

Mon 1/10/05

Wed 12/15/04

Fri 12/31/04

Fri 12/31/04

Fri 12/31/04

Fri $1 / 7 / 05$

Fri $1 / 7 / 05$

Fri $1 / 7 / 05$

Fri $1 / 7 / 05$

Fri 1/7/05

Mon 1/10/05

Fri $1 / 7 / 05$

Fri $1 / 7 / 05$

Fri $1 / 7 / 05$

Wed $1 / 5 / 05$

Tue $1 / 4 / 05$

Thu 1/6/05

Fri 1/7/05

Fri $1 / 7 / 05$

Fri 1/7/05

Mon 1/10/05

Mon 1/10/05

Wed $1 / 12 / 05$

Fri 1/14/05

Tue 1/11/05

Mon 12/20/04

Thu $1 / 6 / 05$

Thu 1/13/05

Thu 1/13/05

Thu $1 / 6 / 05$

Tue 1/11/05

Wed 1/12/05

Wed 1/12/05

Fri 1/21/05

Wed 1/12/05

Mon 1/10/05

Fri $1 / 7 / 05$

Tue 1/11/05

Mon 1/10/05

Fri 1/7/05

Mon 1/10/05

Page 57

Last Updated on Tue 7/3/07 Printed on Fri 8/17/07 


\section{\begin{tabular}{|ll}
\hline & AEDR Construction Phase I Project Plan
\end{tabular}}

\begin{tabular}{|c|c|c|}
\hline ID & (i) & Task Name \\
\hline 2623 & $\checkmark$ & \\
\hline 2624 & $\checkmark$ & \\
\hline 2625 & $\checkmark$ & \\
\hline 2626 & 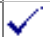 & \\
\hline 2627 & 4 & \\
\hline 2628 & $\checkmark$ & \\
\hline 2629 & $\checkmark$ & \\
\hline 2630 & & \\
\hline 2631 & $\checkmark$ & \\
\hline 2632 & & \\
\hline 2633 & 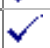 & \\
\hline 2634 & $\checkmark$ & \\
\hline 2635 & & \\
\hline 2636 & $\checkmark$ & \\
\hline 2637 & & \\
\hline 2638 & $\checkmark$ & \\
\hline 2639 & & \\
\hline 2640 & 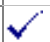 & \\
\hline 2641 & & \\
\hline 2642 & & \\
\hline 2643 & 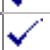 & \\
\hline 2644 & & \\
\hline 2645 & $\checkmark$ & \\
\hline 2646 & & \\
\hline 2647 & $\checkmark$ & \\
\hline 2648 & & \\
\hline 2649 & $\checkmark$ & \\
\hline 2650 & & \\
\hline 2651 & & \\
\hline 2652 & 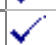 & \\
\hline 2653 & & \\
\hline 2654 & 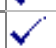 & \\
\hline 2655 & & \\
\hline 2656 & $\checkmark$ & \\
\hline 2657 & $\checkmark$ & \\
\hline 2658 & $\checkmark$ & \\
\hline 2659 & 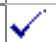 & \\
\hline 2660 & $\checkmark$ & \\
\hline 2661 & $\checkmark$ & \\
\hline 2662 & $\checkmark$ & \\
\hline 2663 & $\checkmark$ & \\
\hline 2664 & $\checkmark$ & \\
\hline 2665 & $\checkmark$ & \\
\hline 2666 & $\checkmark$ & \\
\hline 2667 & $\checkmark$ & \\
\hline 2668 & $\checkmark$ & \\
\hline
\end{tabular}

NIPSCO GIS Confidential
Update domain logic for CapacitorUnit.ManufacturerName

Add value to Domain DmCapacitorUnitKVAR

AbstactClass ObjectCommonData on the Objects page determine WOSequenceNumbers

AbstractClass:UnitObject on Objects Page, set PhaseDesignation - AllowNulls=True

Update migration logic for CISAccountNumber

Remove PadMount fields from matrix

Set RetiredPadMount.LegacyDistribRefNumTermld AllowNulls=True

Recreate values in DmConductorOHSizeCd

Assign DmConductorOHMaterialCd to ConductorInfo and LegacyConductorInfo WireMaterialCd fields

Update Migration Logic for ConductorDefinition.CategoryCd

TransformerRegulatorStoresltem - Field datatype changes

Add a subtype to SwitchGear feature class

Document determining factor for a domain to be defined as an Integer type verses a String type

Added tags to Relationship Classes

Rename the VoltageReg_VoltageRegInstall Relationship Class

Data Model Change for SubstationBreaker

Update Matrix - Value for JointUseAttachment.DataSourceCd

Update migration logic for Assembly

Update Assembly.InstallWorkOrderNumber - AllowNulls=true

Update domain for RetiredSupportStructure.PoleMaterialTypecd

Update AssemblyNumber to AllowNulls for RetiredSwitchGear and SwitchGear

Update matrix logic for TransformerUnit.ManufacturerName

Update matrix for TransformerKvaValue --> KvaValue

Set Terminator.PhaseDesignation default value to 7

Apply data model change for DmCapacitorBankKVAR

CADOPS/FeederAll missing fields LoaNumber and GridCd

CADOPS/FeederAll missing field: SectionalizerBank.SectionalizerAmpRating

Added WireSizeCd and WireMaterialCd to Conductor Features

Annotation - multiple annotations on features

Apply changes for DmForeignOwnerNameCd, DmPhoneCompanyCd, DmUtilityCompanyCd, DmCableCompanyCd

Establish GMMS links to GasMain feature

Apply data model changes to UgConductor - WireMaterialCd

\section{Review the Data Model \& Matrix}

Full data model / matrix review - Day \#1

Full data model / matrix review - Day \#2

Full data model / matrix review - Day \#3

Full data model / matrix review - Day \#4

Full data model / matrix review - Day \#5

Full data model / matrix review - Day \#6

Full data model / matrix review - Day \#7

Full data model / matrix review - Day \#8

Full data model / matrix review - Day \#9

Full data model / matrix review - Day $\# 10$

Full data model / matrix review - Day \#11

Summarize data model changes

Review and finalize data model changes
Mon $1 / 10 / 05$

Mon $1 / 10 / 05$

Thu $1 / 6 / 05$

Thu $1 / 6 / 05$

Mon 1/10/05

Tue $1 / 11 / 05$

Tue $1 / 11 / 05$

Thu $1 / 6 / 05$

Fri $1 / 7 / 05$

Mon $1 / 10 / 05$

Tue 1/11/05

Fri $1 / 7 / 05$

Fri $1 / 7 / 05$

Tue 1/11/05

Tue 1/11/05

Tue 1/11/05

Wed 1/12/05

Wed 1/12/05

Wed 1/12/05

Tue 1/11/05

Tue 1/11/05

Fri 1/14/05

Fri 1/14/05

Fri 1/14/05

Fri 1/14/05

Fri 1/14/05

Fri 1/14/05

Fri 1/21/05

Fri 1/21/05

Fri 1/21/05

Tue 2/22/05

Tue 2/22/05

Wed 2/23/05

Thu 2/24/05

Fri 2/25/05

Mon 2/28/05

Tue 3/1/05

Wed 3/2/05

Thu 3/3/05

Fri 3/4/05

Tue 3/8/05

Wed 3/9/05

Thu 3/10/05

Tue 3/15/05

Thu 2/24/05

Fri 2/25/05

Mon 2/28/05

Tue 3/1/05

Wed 3/2/05

Thu 3/3/05

Fri $3 / 4 / 05$

Tue 3/8/05

Wed 3/9/05

Mon 3/14/05

Tue 3/15/05 


\begin{tabular}{|c|c|c|c|c|c|}
\hline \multicolumn{6}{|c|}{ AEDR Construction Phase I Project Plan } \\
\hline ID & (a) & Task Name & Start & Finish & \\
\hline 2669 & $\checkmark$ & Send finalized data model to vendors \& request impact estimates & Wed 3/16/05 & Wed $3 / 16 / 05$ & \\
\hline 2670 & $\checkmark$ & Receive Impact Estimates from Vendors by noon & Mon 3/21/05 & Mon 3/21/05 & \\
\hline 2671 & $\checkmark$ & Review impact estimates from vendors \& approve changes & Mon 3/21/05 & Mon 3/21/05 & \\
\hline 2672 & $\checkmark$ & Apply the approved data model changes & Mon 3/21/05 & Wed 3/23/05 & \\
\hline 2673 & $\checkmark$ & Apply the approved matrix changes & Wed 3/23/05 & Fri 3/25/05 & \\
\hline 2674 & $\checkmark$ & Final freeze of the data model & Tue $4 / 12 / 05$ & Tue $4 / 12 / 05$ & \\
\hline 2675 & $\checkmark$ & Send the final data model/matrix to all vendors & Tue $4 / 12 / 05$ & Tue $4 / 12 / 05$ & \\
\hline 2676 & $\checkmark$ & Environment Setup Tasks & Mon 12/13/04 & Fri 6/9/06 & \\
\hline 2677 & $\checkmark$ & SDE Management Plan Remaining Tasks & Thu 12/16/04 & Tue $5 / 3 / 05$ & \\
\hline 2678 & $\checkmark$ & Review the Plan and provide comments & Thu $12 / 16 / 04$ & Thu $12 / 16 / 04$ & \\
\hline 2679 & $\checkmark$ & Finalize the Plan & Wed 1/12/05 & Wed $1 / 12 / 05$ & \\
\hline 2680 & $\checkmark$ & SDE Management Plan Updates & Tue $5 / 3 / 05$ & Tue $5 / 3 / 05$ & \\
\hline 2681 & $\checkmark$ & Environment Planning / Research / Setup / Testing Tasks & Fri 1/7/05 & Fri 6/9/06 & \\
\hline 2682 & $\checkmark$ & Create a list of users and roles for ArcSDE Production & Tue 2/1/05 & Tue 2/1/05 & \\
\hline 2683 & $\checkmark$ & Upgrade ArcSDE to SP2 & Fri 1/7/05 & Fri $1 / 7 / 05$ & \\
\hline 2684 & $\checkmark$ & Upgrade SQL Server to SP3a & Fri 1/7/05 & Fri $1 / 7 / 05$ & \\
\hline 2685 & $\checkmark$ & Create list of environment research topics & Fri 1/14/05 & Fri 1/14/05 & \\
\hline 2686 & $\checkmark$ & Upgrade to ArcFM 9.01 (server side tasks) & Mon 1/10/05 & Tue $1 / 11 / 05$ & \\
\hline 2687 & $\checkmark$ & Review misc env tasks / assign resource and estimates & Tue $1 / 18 / 05$ & Tue $1 / 18 / 05$ & \\
\hline 2688 & $\checkmark$ & Upgrade to ArcFM 9.01 (all remaining client machines) & Fri 1/21/05 & Fri 1/21/05 & \\
\hline 2689 & $\checkmark$ & Determine the mix of users thick client and citrix & Wed 1/19/05 & Wed $1 / 19 / 05$ & \\
\hline 2690 & $\checkmark$ & Finalize Citrix ArcFM upgrades to 9.0.1 and test the ICA client for refresh improvement & Mon 1/24/05 & Mon 1/24/05 & \\
\hline 2691 & $\checkmark$ & Finalize Citrix ArcFM upgrades to 9.0.1 for all other machines & Fri 2/4/05 & Fri 2/4/05 & \\
\hline 2692 & $\checkmark$ & Create Production Control Procedures & Tue 2/21/06 & Tue 2/21/06 & \\
\hline 2693 & $\checkmark$ & Inform IT server group of architecture and support roles / requirements & Tue 2/21/06 & Tue 2/21/06 & \\
\hline 2694 & $\checkmark$ & Define DBA and SDE administrator roles for production and SOX & Tue 2/21/06 & Tue 2/21/06 & \\
\hline 2695 & $\checkmark$ & Identify \& document production control procedures & Tue 2/21/06 & Tue 2/21/06 & \\
\hline 2696 & $\checkmark$ & Installation Tasks & Wed 2/2/05 & Fri 6/9/06 & \\
\hline 2697 & $\checkmark$ & Determine distribution of ICA clients and software installs & Wed 2/2/05 & Wed 2/2/05 & \\
\hline 2698 & $\checkmark$ & Document Installation Procedures & Fri 6/9/06 & Fri 6/9/06 & \\
\hline 2699 & $\checkmark$ & AEDRDBSQLP01 - Production Instance - Repeating Refresh Steps & Fri 1/7/05 & Fri 1/28/05 & \\
\hline 2700 & $\checkmark$ & Create .bak from dev and restore into Prod & Fri 1/7/05 & Fri $1 / 7 / 05$ & \\
\hline 2701 & $\checkmark$ & Initialize ArcSDE User accounts & Tue $1 / 11 / 05$ & Tue $1 / 11 / 05$ & \\
\hline 2702 & $\checkmark$ & Lock down user accounts for Prod & Tue $1 / 11 / 05$ & Tue $1 / 11 / 05$ & \\
\hline 2703 & $\checkmark$ & Check on impact of not locking down accounts for other instances & Fri 1/28/05 & Fri 1/28/05 & \\
\hline 2704 & $\checkmark$ & AEDRDBSQLP01 - Test Instance - Repeating Refresh Steps & Fri $1 / 7 / 05$ & Fri $1 / 7 / 05$ & \\
\hline 2705 & $\checkmark$ & Create .bak from dev and restore into prod & Fri 1/7/05 & Fri $1 / 7 / 05$ & \\
\hline 2706 & $\checkmark$ & AEDRMAPGIS01 - Development Instance Repeating Refresh Steps & Mon 12/13/04 & Wed 1/5/05 & \\
\hline 2707 & $\checkmark$ & Export any configuration from development instance (XML export) & Mon 12/13/04 & Mon 12/13/04 & \\
\hline 2708 & $\checkmark$ & Wipe the database clean / re-create SDE tables & Mon 12/13/04 & Mon 12/13/04 & \\
\hline 2709 & $\checkmark$ & Restore the backup file & Mon 12/13/04 & Mon 12/13/04 & \\
\hline 2710 & $\checkmark$ & Update the schema from Visio based on the latest data model changes & Fri 12/17/04 & Mon 12/20/04 & \\
\hline 2711 & $\checkmark$ & Build all ArcFM tables & Mon 12/20/04 & Mon 12/20/04 & \\
\hline 2712 & $\checkmark$ & Convert to ArcFM objects - expands out of the box ArcGIS objects & Mon 12/20/04 & Mon 12/20/04 & \\
\hline 2713 & $\checkmark$ & Import all configurations (XML import) & Mon 12/20/04 & Mon 12/20/04 & \\
\hline 2714 & $\checkmark$ & Import symbology (.mxd) to create Stored Displays & Mon 12/20/04 & Mon 12/20/04 & \\
\hline $\begin{array}{l}\text { NIPSC } \\
\text { Confid }\end{array}$ & $\begin{array}{l}\text { Co GIS } \\
\text { lential }\end{array}$ & Page 59 & & $\begin{array}{r}\text { Last Updat } \\
\text { Print }\end{array}$ & $\begin{array}{l}\text { ed on Tue } 7 / 3 / 07 \\
\text { ed on Fri 8/17/07 }\end{array}$ \\
\hline
\end{tabular}




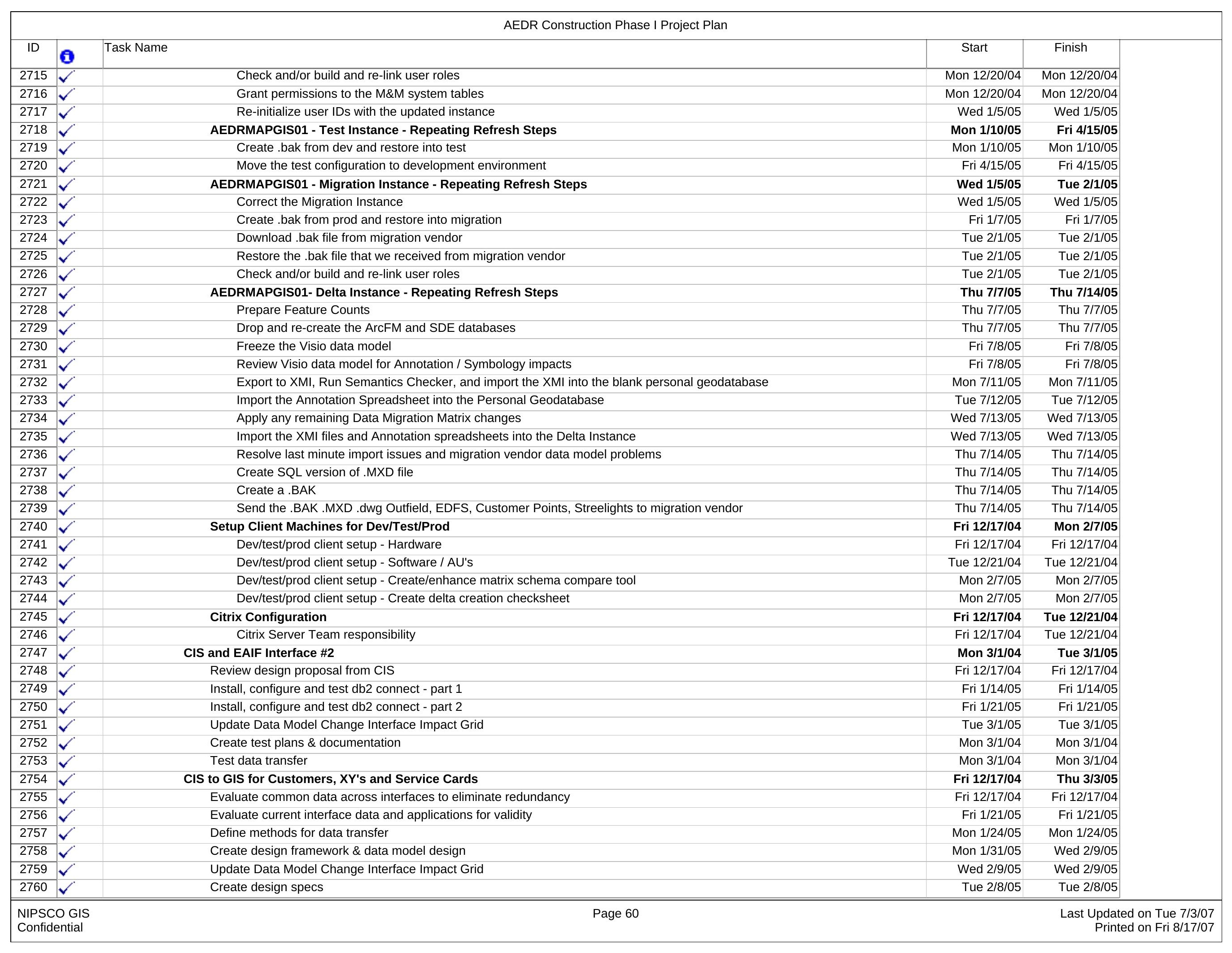




\begin{tabular}{|c|c|c|c|c|c|}
\hline \multicolumn{6}{|c|}{ AEDR Construction Phase I Project Plan } \\
\hline ID & (a) & Task Name & Start & Finish & \\
\hline 2761 & $\checkmark$ & Review \& approve the design specs & Mon 2/28/05 & Mon 2/28/05 & \\
\hline 2762 & $\checkmark$ & Finalize the design specs & Mon 2/28/05 & Mon 2/28/05 & \\
\hline 2763 & $\checkmark$ & Map Data fields to the form & Mon 2/28/05 & Mon 2/28/05 & \\
\hline 2764 & $\checkmark$ & Create test plans \& documentation & Mon 2/28/05 & Tue 3/1/05 & \\
\hline 2765 & $\checkmark$ & Code \& unit test applications or database triggers & Tue 3/1/05 & Wed 3/2/05 & \\
\hline 2766 & $\checkmark$ & Install application at NIPSCO & Thu 3/3/05 & Thu $3 / 3 / 05$ & \\
\hline 2767 & $\checkmark$ & System test application & Thu 3/3/05 & Thu $3 / 3 / 05$ & \\
\hline 2768 & $\checkmark$ & CIS to GIS for Life Support by Circuit & Fri 12/17/04 & Mon 3/7/05 & \\
\hline 2769 & $\checkmark$ & Evaluate current interface data and applications for validity & Fri 12/17/04 & Fri 12/17/04 & \\
\hline 2770 & $\checkmark$ & Evaluate common data across interfaces to eliminate redundancy & Fri 12/17/04 & Fri $12 / 17 / 04$ & \\
\hline 2771 & $\checkmark$ & Define methods for data transfer & Fri 1/28/05 & Fri 1/28/05 & \\
\hline 2772 & $\checkmark$ & Create design framework \& data model design & Wed 2/9/05 & Wed 2/9/05 & \\
\hline 2773 & $\checkmark$ & Update Data Model Change Interface Impact Grid & Wed 2/9/05 & Wed 2/9/05 & \\
\hline 2774 & $\checkmark$ & Create design specs & Wed 3/2/05 & Wed 3/2/05 & \\
\hline 2775 & $\checkmark$ & Review \& approve the design specs & Thu $3 / 3 / 05$ & Thu $3 / 3 / 05$ & \\
\hline 2776 & $\checkmark$ & Code \& unit test applications or database triggers & Thu $3 / 3 / 05$ & Fri 3/4/05 & \\
\hline 2777 & $\checkmark$ & Create test plans \& documentation & Fri 3/4/05 & Mon 3/7/05 & \\
\hline 2778 & $\checkmark$ & Install application at NIPSCO & Mon 3/7/05 & Mon 3/7/05 & \\
\hline 2779 & $\checkmark$ & System acceptance test application & Mon 3/7/05 & Mon 3/7/05 & \\
\hline 2780 & $\checkmark$ & EDFS Integration \#2 & Mon 3/1/04 & Thu 5/5/05 & \\
\hline 2781 & $\checkmark$ & Update Design with any changes & Mon 12/13/04 & Tue $12 / 14 / 04$ & \\
\hline 2782 & $\checkmark$ & MILESTONE: Design review and signoff & Mon 12/20/04 & Tue $12 / 21 / 04$ & \\
\hline 2783 & $\checkmark$ & Review permissions for ArcFM to only allow a coordinator to delete & Wed 2/9/05 & Wed 2/9/05 & \\
\hline 2784 & $\checkmark$ & Review permissions information & Mon 2/28/05 & Mon 2/28/05 & \\
\hline 2785 & $\checkmark$ & Develop Phase & Mon 3/1/04 & Thu 5/5/05 & \\
\hline 2786 & $\checkmark$ & NIPSCO compile \& deliver Final Geodatabase \& EDFS Data & Fri 12/17/04 & Fri 12/17/04 & \\
\hline 2787 & $\checkmark$ & Data Tasks & Mon 12/20/04 & Tue 3/1/05 & \\
\hline 2788 & $\checkmark$ & Implement Data Model Updates & Mon 12/20/04 & Wed 12/29/04 & \\
\hline 2789 & $\checkmark$ & Migrate EDFS Data into Geodatabase & Thu $12 / 30 / 04$ & Wed $1 / 12 / 05$ & \\
\hline 2790 & $\checkmark$ & Create Matrices for migration vendor & Mon 12/20/04 & Tue 12/21/04 & \\
\hline 2791 & $\checkmark$ & Update Data Model Change Interface Impact Grid & Tue 3/1/05 & Tue 3/1/05 & \\
\hline 2792 & $\checkmark$ & ArcFM Auto Updaters & Tue $1 / 4 / 05$ & Fri 1/28/05 & \\
\hline 2793 & $\checkmark$ & Design, Infrastructure, Reuse & Tue $1 / 4 / 05$ & Mon 1/10/05 & \\
\hline 2794 & $\checkmark$ & 1-OnCreate - ReplicateSinKva & Tue $1 / 11 / 05$ & Wed $1 / 12 / 05$ & \\
\hline 2795 & $\checkmark$ & 2-OnUpdate - UpdateSinKva & Wed 1/12/05 & Thu $1 / 13 / 05$ & \\
\hline 2796 & $\checkmark$ & 3-OnCreate - AssetInstallation & Wed 1/12/05 & Thu $1 / 13 / 05$ & \\
\hline 2797 & $\checkmark$ & 4-OnUpdate - AssetRemoval & Thu $1 / 13 / 05$ & Fri $1 / 14 / 05$ & \\
\hline 2798 & $\checkmark$ & 5-OnUpdate - CapacitorRemoval & Fri 1/14/05 & Mon 1/17/05 & \\
\hline 2799 & $\checkmark$ & 6-OnDelete - ResetAssetToStock & Fri $1 / 14 / 05$ & Mon 1/17/05 & \\
\hline 2800 & $\checkmark$ & 7-OnDelete - ResetAssetToInstalled & Mon 1/17/05 & Tue $1 / 18 / 05$ & \\
\hline 2801 & $\checkmark$ & 11-OnCreate, OnUpdate - ValidateConductorProperties & Thu $1 / 20 / 05$ & Fri 1/21/05 & \\
\hline 2802 & $\checkmark$ & 12-OnRetire, OnDelete - PreventDeletionlfRelatedAssets & Fri 1/21/05 & Mon $1 / 24 / 05$ & \\
\hline 2803 & $\checkmark$ & 13-OnRetire - SupportStructureRetirement & Mon 1/24/05 & Tue $1 / 25 / 05$ & \\
\hline 2804 & $\checkmark$ & 8-OnCreate, OnUpdate - ValidateWorkOrderNumber & Tue $1 / 18 / 05$ & Wed $1 / 19 / 05$ & \\
\hline 2805 & $\checkmark$ & 9-OnCreate, OnUpdate - ValidateAssemblyNumber & Wed 1/19/05 & Thu $1 / 20 / 05$ & \\
\hline 2806 & $\checkmark$ & 10-OnCreate, OnUpdate - ValidateReferencedAssembly & Wed $1 / 19 / 05$ & Thu $1 / 20 / 05$ & \\
\hline $\begin{array}{l}\text { NIPSC } \\
\text { Confid }\end{array}$ & $\begin{array}{l}\text { Co GIS } \\
\text { lential }\end{array}$ & Page 61 & & $\begin{array}{l}\text { Last Update } \\
\text { Print }\end{array}$ & $\begin{array}{l}\text { ed on Tue } 7 / 3 / 07 \\
\text { ed on Fri 8/17/07 }\end{array}$ \\
\hline
\end{tabular}




\begin{tabular}{|c|c|c|c|c|c|}
\hline \multicolumn{6}{|c|}{ AEDR Construction Phase I Project Plan } \\
\hline ID & (a) & Task Name & Start & Finish & \\
\hline 2807 & $\checkmark$ & 14-OnRetire - ReplicateRetirementData & Tue $1 / 25 / 05$ & Wed $1 / 26 / 05$ & \\
\hline 2808 & $\checkmark$ & 15-OnRetire - ReplicateSpatialData & Thu $1 / 27 / 05$ & Fri 1/28/05 & \\
\hline 2809 & $\checkmark$ & ArcFM GIS Tools & Thu $2 / 17 / 05$ & Mon 2/21/05 & \\
\hline 2810 & $\checkmark$ & SupportStructure and PadMount Replace Tool & Thu $2 / 17 / 05$ & Mon 2/21/05 & \\
\hline 2811 & $\checkmark$ & SAGE & Thu $12 / 23 / 04$ & Thu 3/3/05 & \\
\hline 2812 & $\checkmark$ & Infrastructure & Thu $12 / 23 / 04$ & Mon 1/3/05 & \\
\hline 2813 & $\checkmark$ & Authentication \& Authorization & Tue $1 / 4 / 05$ & Tue $1 / 4 / 05$ & \\
\hline 2814 & $\checkmark$ & Screen Development & Wed 1/19/05 & Thu $3 / 3 / 05$ & \\
\hline 2815 & $\checkmark$ & Coordinator Dashboard Screens & Wed $1 / 19 / 05$ & Thu $1 / 27 / 05$ & \\
\hline 2816 & $\checkmark$ & Coordinator Access Screens & Tue $2 / 8 / 05$ & Thu $2 / 10 / 05$ & \\
\hline 2817 & $\checkmark$ & Transformer Screens & Thu 2/10/05 & Thu $2 / 17 / 05$ & \\
\hline 2818 & $\checkmark$ & Regulator Screens & Thu $2 / 17 / 05$ & Thu $2 / 24 / 05$ & \\
\hline 2819 & $\checkmark$ & Capacitor Screens & Thu 2/24/05 & Wed 3/2/05 & \\
\hline 2820 & $\checkmark$ & Reports Screens & Wed 3/2/05 & Thu $3 / 3 / 05$ & \\
\hline 2821 & $\checkmark$ & Reports & Wed 1/26/05 & Wed 2/16/05 & \\
\hline 2822 & $\checkmark$ & Template Creation (Formatting \& Data Sources) & Wed $1 / 26 / 05$ & Wed $2 / 16 / 05$ & \\
\hline 2823 & $\checkmark$ & Conductor Info Management Tools & Mon 2/7/05 & Fri 3/18/05 & \\
\hline 2824 & $\checkmark$ & Create Component Spec & Mon 2/7/05 & Wed 2/9/05 & \\
\hline 2825 & $\checkmark$ & NIPSCO Review & Wed 2/9/05 & Wed 2/9/05 & \\
\hline 2826 & $\checkmark$ & Update Component Spec & Wed 2/9/05 & Thu $2 / 10 / 05$ & \\
\hline 2827 & $\checkmark$ & Coding & Thu $2 / 10 / 05$ & Fri 2/18/05 & \\
\hline 2828 & $\checkmark$ & User Guide & Fri 2/18/05 & Mon 2/21/05 & \\
\hline 2829 & $\checkmark$ & Operations Guide & Mon 2/21/05 & Tue 2/22/05 & \\
\hline 2830 & $\checkmark$ & Deploy & Mon 3/7/05 & Fri 3/18/05 & \\
\hline 2831 & $\checkmark$ & Batch Data Management and Reporting & Tue $1 / 11 / 05$ & Thu $3 / 3 / 05$ & \\
\hline 2832 & $\checkmark$ & Batch Reconcile and Post for SAGE Version & Tue $1 / 11 / 05$ & Thu $1 / 13 / 05$ & \\
\hline 2833 & $\checkmark$ & MAPPS/GIS Transaction Processing & Tue 2/22/05 & Mon 2/28/05 & \\
\hline 2834 & $\checkmark$ & Batch Report Generator & Wed 2/16/05 & Tue 2/22/05 & \\
\hline 2835 & $\checkmark$ & Structure to Conductor Join & Thu $2 / 24 / 05$ & Wed 3/2/05 & \\
\hline 2836 & $\checkmark$ & Duplicate Domains for SAGE Use & Tue $3 / 1 / 05$ & Thu $3 / 3 / 05$ & \\
\hline 2837 & $\checkmark$ & Establish External System Interfaces & Mon 3/1/04 & Fri 3/11/05 & \\
\hline 2838 & $\checkmark$ & MAPPS & Mon 1/31/05 & Thu $3 / 10 / 05$ & \\
\hline 2839 & $\checkmark$ & GL & Mon 1/31/05 & Fri 3/11/05 & \\
\hline 2840 & $\checkmark$ & MLOG & Mon 1/31/05 & Fri 3/11/05 & \\
\hline 2841 & $\checkmark$ & $\mathrm{CIS}$ & Mon 1/31/05 & Fri 3/11/05 & \\
\hline 2842 & $\checkmark$ & EAIF & Mon 3/1/04 & Mon 3/1/04 & \\
\hline 2843 & $\checkmark$ & Tax Department (This is not an interface, it is a 1-time dump) & Mon 1/31/05 & Fri 3/11/05 & \\
\hline 2844 & $\checkmark$ & Documentation & Thu $3 / 3 / 05$ & Mon 3/28/05 & \\
\hline 2845 & $\checkmark$ & AU Documentation & Thu $3 / 3 / 05$ & Fri 3/11/05 & \\
\hline 2846 & $\checkmark$ & Reports Documentation & Fri 3/11/05 & Fri 3/18/05 & \\
\hline 2847 & $\checkmark$ & SAGE Documentation & Fri 3/18/05 & Mon 3/28/05 & \\
\hline 2848 & $\checkmark$ & Code Reviews & Mon 2/28/05 & Mon 2/28/05 & \\
\hline 2849 & $\checkmark$ & AU Code Review & Mon 2/28/05 & Mon 2/28/05 & \\
\hline 2850 & $\checkmark$ & SAGE Code Review & Mon 2/28/05 & Mon 2/28/05 & \\
\hline 2851 & $\checkmark$ & Webcast Demos & Fri 3/4/05 & Fri $3 / 4 / 05$ & \\
\hline 2852 & $\checkmark$ & AU Webcast Demo & Fri 3/4/05 & Fri $3 / 4 / 05$ & \\
\hline $\begin{array}{l}\text { NIPSC } \\
\text { Confid }\end{array}$ & $\begin{array}{l}\text { Co GIS } \\
\text { lential }\end{array}$ & Page 62 & & $\begin{array}{r}\text { Last Updat } \\
\text { Print }\end{array}$ & $\begin{array}{l}\text { ed on Tue } 7 / 3 / 07 \\
\text { ed on Fri 8/17/07 }\end{array}$ \\
\hline
\end{tabular}




\begin{tabular}{|c|c|c|c|c|c|}
\hline \multicolumn{6}{|c|}{ AEDR Construction Phase I Project Plan } \\
\hline ID & (a) & Task Name & Start & Finish & \\
\hline 2853 & $\checkmark$ & Reports Webcast Demo & Fri 3/4/05 & Fri 3/4/05 & \\
\hline 2854 & $\checkmark$ & SAGE Webcast Demo & Fri 3/4/05 & Fri 3/4/05 & \\
\hline 2855 & $\checkmark$ & Integration Testing & Tue $3 / 29 / 05$ & Thu $4 / 7 / 05$ & \\
\hline 2856 & $\checkmark$ & MILESTONE: Development Complete & Thu $4 / 7 / 05$ & Thu $4 / 7 / 05$ & \\
\hline 2857 & $\checkmark$ & Deploy Phase & Fri $4 / 8 / 05$ & Thu 5/5/05 & \\
\hline 2858 & $\checkmark$ & Prepare for Delivery (Installers, Scripts, etc) & Fri $4 / 8 / 05$ & Thu $4 / 14 / 05$ & \\
\hline 2859 & $\checkmark$ & Create Acceptance Test Plans & Fri $4 / 8 / 05$ & Thu $4 / 14 / 05$ & \\
\hline 2860 & $\checkmark$ & Install Onsite at NIPSCO & Fri 4/15/05 & Tue $4 / 19 / 05$ & \\
\hline 2861 & $\checkmark$ & Onsite Testing & Wed $4 / 20 / 05$ & Thu $4 / 21 / 05$ & \\
\hline 2862 & $\checkmark$ & Onsite SAT & Fri 4/22/05 & Thu $4 / 28 / 05$ & \\
\hline 2863 & $\checkmark$ & Train Users & Fri 4/29/05 & Thu 5/5/05 & \\
\hline 2864 & $\checkmark$ & MILESTONE: Deployment Signoff & Thu 5/5/05 & Thu 5/5/05 & \\
\hline 2865 & $\checkmark$ & EDFS Related AU's & Tue 1/11/05 & Tue 1/11/05 & \\
\hline 2866 & $\checkmark$ & Transformer Unit - Location History AU & Tue $1 / 11 / 05$ & Tue 1/11/05 & \\
\hline 2867 & $\checkmark$ & Create component spec & Tue $1 / 11 / 05$ & Tue $1 / 11 / 05$ & \\
\hline 2868 & $\checkmark$ & Review component spec & Tue $1 / 11 / 05$ & Tue $1 / 11 / 05$ & \\
\hline 2869 & $\checkmark$ & Code / unit test & Tue $1 / 11 / 05$ & Tue $1 / 11 / 05$ & \\
\hline 2870 & $\checkmark$ & Capacitor Unit - Location History AU & Tue $1 / 11 / 05$ & Tue 1/11/05 & \\
\hline 2871 & $\checkmark$ & Create component spec & Tue $1 / 11 / 05$ & Tue $1 / 11 / 05$ & \\
\hline 2872 & $\checkmark$ & Review component spec & Tue $1 / 11 / 05$ & Tue $1 / 11 / 05$ & \\
\hline 2873 & $\checkmark$ & Code / unit test & Tue $1 / 11 / 05$ & Tue $1 / 11 / 05$ & \\
\hline 2874 & $\checkmark$ & Voltage Regulator Unit - Location History AU & Tue 1/11/05 & Tue 1/11/05 & \\
\hline 2875 & $\checkmark$ & Create component spec & Tue $1 / 11 / 05$ & Tue $1 / 11 / 05$ & \\
\hline 2876 & $\checkmark$ & Review component spec & Tue $1 / 11 / 05$ & Tue $1 / 11 / 05$ & \\
\hline 2877 & $\checkmark$ & Code / unit test & Tue $1 / 11 / 05$ & Tue $1 / 11 / 05$ & \\
\hline 2878 & $\checkmark$ & EDFS Integration Reconfiguration (Based on new frozen data model changes) & Mon 2/13/06 & Fri 12/15/06 & \\
\hline 2879 & $\checkmark$ & EDFS coding \& rework & Mon 11/20/06 & Fri 12/15/06 & \\
\hline 2880 & $\checkmark$ & EDFS Integration System Acceptance Testing & Mon 2/13/06 & Fri 2/17/06 & \\
\hline 2881 & $\checkmark(1)$ & Field Browser Application - Part 1 & Mon 1/10/05 & Wed 4/26/06 & \\
\hline 2882 & $\checkmark$ & Requirements and Design & Mon 1/10/05 & Tue 2/8/05 & \\
\hline 2883 & $\checkmark$ & Contact users to setup demo day/time & Mon 1/10/05 & Mon 1/10/05 & \\
\hline 2884 & $\checkmark$ & Prepare for demo & Mon 1/24/05 & Mon 1/24/05 & \\
\hline 2885 & $\checkmark$ & Demo ArcReader to select end users / set expectations & Fri 1/28/05 & Fri $1 / 28 / 05$ & \\
\hline 2886 & $\checkmark$ & Research Field Browser requirements & Mon 2/7/05 & Tue 2/8/05 & \\
\hline 2887 & $\checkmark y$ & Component Specifications & Wed 2/9/05 & Mon 4/17/06 & \\
\hline 2888 & $\checkmark$ & Document current Field Browser & Wed 2/9/05 & Wed 2/9/05 & \\
\hline 2889 & $\checkmark$ & Document required fields & Thu $2 / 17 / 05$ & Fri 2/18/05 & \\
\hline 2890 & $\checkmark$ & Research and configure GDB extraction tool & Thu $2 / 17 / 05$ & Fri 2/18/05 & \\
\hline 2891 & $\checkmark$ & Document extraction tool process & Mon 2/28/05 & Mon 2/28/05 & \\
\hline 2892 & $\checkmark$ & Document fields destined for removal & Fri 4/14/06 & Fri 4/14/06 & \\
\hline 2893 & $\checkmark$ & Review and approve the Field Browser component specs & Mon 4/17/06 & Mon 4/17/06 & \\
\hline 2894 & $\checkmark$ & Apply Configuration Changes & Mon 4/17/06 & Fri 4/21/06 & \\
\hline 2895 & $\checkmark$ & Populate PGD with SDE fields per component spec & Mon 4/17/06 & Wed 4/19/06 & \\
\hline 2896 & $\checkmark$ & Unit test Field Browser & Wed 4/19/06 & Thu $4 / 20 / 06$ & \\
\hline 2897 & $\checkmark$ & Performance tune Field Browser & Thu $4 / 20 / 06$ & Fri 4/21/06 & \\
\hline 2898 & $\checkmark$ & Demo for the Field Supervisors & Fri 4/21/06 & Wed 4/26/06 & \\
\hline $\begin{array}{l}\text { NIPSC } \\
\text { Confid }\end{array}$ & $\begin{array}{l}\text { Co GIS } \\
\text { lential }\end{array}$ & Page 63 & & $\begin{array}{r}\text { Last Updat } \\
\text { Print }\end{array}$ & $\begin{array}{l}\text { ed on Tue } 7 / 3 / 07 \\
\text { ed on Fri 8/17/07 }\end{array}$ \\
\hline
\end{tabular}




\begin{tabular}{|c|c|c|c|c|c|}
\hline \multicolumn{6}{|c|}{ AEDR Construction Phase I Project Plan } \\
\hline ID & ( & Task Name & Start & Finish & \\
\hline 2899 & $\checkmark$ & Prepare for demo & Fri 4/21/06 & Mon 4/24/06 & \\
\hline 2900 & $\checkmark$ & Document additional requirements during demo & Mon 4/24/06 & Mon 4/24/06 & \\
\hline 2901 & $\checkmark$ & Prioritize additional requirements with field supervisors during demo & Mon 4/24/06 & Mon 4/24/06 & \\
\hline 2902 & $\checkmark$ & Populate PGD with SDE fields per additional requirements doc & Mon 4/24/06 & Tue $4 / 25 / 06$ & \\
\hline 2903 & $\checkmark$ & Unit test/performance tune Field Browser & Tue $4 / 25 / 06$ & Wed 4/26/06 & \\
\hline 2904 & $\checkmark$ & Update component specs with results & Wed 4/26/06 & Wed 4/26/06 & \\
\hline 2905 & 6 & Mapping / Hardcopy & Mon 3/1/04 & Tue 3/29/05 & \\
\hline 2906 & $\checkmark$ & Develop list of paper maps to be generated - None Needed & Tue $3 / 29 / 05$ & Tue $3 / 29 / 05$ & \\
\hline 2907 & 6 & Develop templates for title blocks & Mon 3/1/04 & Mon 3/1/04 & \\
\hline 2908 & $\checkmark b$ & CADOPS / FeederAll Reconfiguration (based on new frozen data model) & Mon 3/1/04 & Fri 12/23/05 & \\
\hline 2909 & $\checkmark$ & Re-work CADOPS code for data model changes & Mon 12/19/05 & Fri 12/23/05 & \\
\hline 2910 & $\checkmark(1)$ & Research with MM any performance improvements for Network Adapter & Mon 3/1/04 & Mon 3/1/04 & \\
\hline 2911 & & SynerGEE Interface \#2 & Mon 12/13/04 & Thu $3 / 3 / 05$ & \\
\hline 2912 & $\checkmark$ & Completed Tasks & Mon 12/13/04 & Thu $1 / 27 / 05$ & \\
\hline 2913 & $\checkmark$ & SynerGEE - Remaining data model changes & Mon 12/13/04 & Wed 12/22/04 & \\
\hline 2914 & $\checkmark$ & SynerGEE - Remaining DataPrep Design document & Mon 12/13/04 & Wed 12/22/04 & \\
\hline 2915 & $\checkmark$ & SynerGEE - Remaining Application Interface Design - Part 1 & Mon 12/13/04 & Wed 12/22/04 & \\
\hline 2916 & $\checkmark$ & SynerGEE - Remaining DataPrep Development - Part 1 & Mon 12/13/04 & Wed 12/22/04 & \\
\hline 2917 & $\checkmark$ & SynerGEE - Application Interface Design - Part 2 & Wed 12/22/04 & Wed 12/29/04 & \\
\hline 2918 & $\checkmark$ & SynerGEE - DataPrep Development - Part 2 & Wed 12/22/04 & Wed 12/29/04 & \\
\hline 2919 & $\checkmark$ & SynerGEE - Review DataPrep Design Document & Fri 12/31/04 & Fri $12 / 31 / 04$ & \\
\hline 2920 & $\checkmark$ & SynerGEE - Revise \& Finalize DataPrep Design Document & Fri 12/31/04 & Fri 12/31/04 & \\
\hline 2921 & $\checkmark$ & SynerGEE - Approve DataPrep Design Document & Fri 12/31/04 & Fri 12/31/04 & \\
\hline 2922 & $\checkmark$ & SynerGEE - DataPrep Development Tasks - Week of 1/17 & Wed 1/19/05 & Thu $1 / 20 / 05$ & \\
\hline 2923 & $\checkmark$ & SynerGEE - DataPrep Development Tasks - Week of 1/24 & Wed 1/26/05 & Thu $1 / 27 / 05$ & \\
\hline 2924 & & Remaining Tasks & Mon 2/28/05 & Thu $3 / 3 / 05$ & \\
\hline 2925 & 国 & SynerGEE - Code Review & Mon 2/28/05 & Mon 2/28/05 & \\
\hline 2926 & $\checkmark$ & SynerGEE - DataPrep Testing & Mon 2/28/05 & Mon 2/28/05 & \\
\hline 2927 & $\checkmark$ & SynerGEE - Create Acceptance Testing Criteria & Mon 2/28/05 & Tue 3/1/05 & \\
\hline 2928 & $\checkmark$ & SynerGEE - Develop DataPrep Documentation & Tue 3/1/05 & Thu 3/3/05 & \\
\hline 2929 & $\checkmark$ & SynerGEE - Create DataPrep Install (Second Half) & Thu 3/3/05 & Thu 3/3/05 & \\
\hline 2930 & $\checkmark$ & Business Process (Maps \& Records Dept.) Change & Mon 4/3/06 & Mon 4/3/06 & \\
\hline 2931 & $\checkmark$ & Business process change - Update IRTH \& IUPPS buffer creation procedures for ESRI & Mon 4/3/06 & Mon 4/3/06 & \\
\hline 2932 & $\checkmark b$ & Business process change - Identify all Maps \& Records existing business processes for potential change & Mon 4/3/06 & Mon 4/3/06 & \\
\hline 2933 & & ArcFM Configuration Updates / Fixes & Wed 12/15/04 & Tue 5/2/06 & \\
\hline 2934 & $\checkmark$ & Change Annotation Color & Wed $1 / 12 / 05$ & Wed 1/12/05 & \\
\hline 2935 & $\checkmark$ & Configuration changes for RecloserBank & Wed 1/12/05 & Wed 1/12/05 & \\
\hline 2936 & $\checkmark$ & Configuration changes for Voltage Regulators & Wed 1/12/05 & Wed $1 / 12 / 05$ & \\
\hline 2937 & $\checkmark$ & Mass Attribute Update - correct problems & Wed 1/12/05 & Wed $1 / 12 / 05$ & \\
\hline 2938 & $\checkmark$ & Configuration change for Gas Network & Tue $1 / 18 / 05$ & Tue $1 / 18 / 05$ & \\
\hline 2939 & $\checkmark$ & Configuration Changes for Switches & Tue $1 / 18 / 05$ & Tue $1 / 18 / 05$ & \\
\hline 2940 & $\checkmark$ & Configuration Changes for Fuses & Tue $1 / 18 / 05$ & Tue $1 / 18 / 05$ & \\
\hline 2941 & $\checkmark$ & Phase of the device should match the phase of the conductor & Tue $1 / 18 / 05$ & Tue $1 / 18 / 05$ & \\
\hline 2942 & $\checkmark$ & Configuration Changes for TransformerBank & Tue $1 / 18 / 05$ & Tue $1 / 18 / 05$ & \\
\hline 2943 & $\checkmark$ & TransformerUnit creation for TransformerBank & Tue $1 / 18 / 05$ & Tue $1 / 18 / 05$ & \\
\hline 2944 & $\checkmark$ & Configuration Change for SupportStructure & Tue $1 / 18 / 05$ & Tue $1 / 18 / 05$ & \\
\hline $\begin{array}{l}\text { NIPSC } \\
\text { Confid }\end{array}$ & $\begin{array}{l}\text { Co GIS } \\
\text { lential }\end{array}$ & Page 64 & & $\begin{array}{l}\text { Last Update } \\
\text { Print }\end{array}$ & $\begin{array}{l}\text { ed on Tue } 7 / 3 / 07 \\
\text { ed on Fri 8/17/07 }\end{array}$ \\
\hline
\end{tabular}




\begin{tabular}{|c|c|c|c|c|c|}
\hline \multicolumn{6}{|c|}{ AEDR Construction Phase I Project Plan } \\
\hline ID & ( & Task Name & Start & Finish & \\
\hline 2945 & $\checkmark$ & Configuration Change for PrimaryMeter & Tue $1 / 18 / 05$ & Tue $1 / 18 / 05$ & \\
\hline 2946 & $\checkmark$ & Configuration Changes for SectionalizerBank & Tue $1 / 18 / 05$ & Tue 1/18/05 & \\
\hline 2947 & $\checkmark$ & Configuration Changes for TieWire & Tue $1 / 18 / 05$ & Tue $1 / 18 / 05$ & \\
\hline 2948 & $\checkmark$ & Error when creating a new Tie Wire & Tue $1 / 18 / 05$ & Tue $1 / 18 / 05$ & \\
\hline 2949 & $\checkmark$ & Configuration Changes for Terminator & Tue $1 / 18 / 05$ & Tue $1 / 18 / 05$ & \\
\hline 2950 & $\checkmark$ & Configuration Changes for PadMount & Tue $1 / 18 / 05$ & Tue $1 / 18 / 05$ & \\
\hline 2951 & $\checkmark$ & Configuration Changes for OpenPoint & Tue $1 / 18 / 05$ & Tue $1 / 18 / 05$ & \\
\hline 2952 & $\checkmark$ & Configuration Changes for Splice & Tue $1 / 18 / 05$ & Tue $1 / 18 / 05$ & \\
\hline 2953 & $\checkmark$ & Configuration Changes for Pedestals & Tue $1 / 18 / 05$ & Tue $1 / 18 / 05$ & \\
\hline 2954 & $\checkmark$ & Configuration Changes for ElectricDeadEnd & Tue $1 / 18 / 05$ & Tue $1 / 18 / 05$ & \\
\hline 2955 & $\checkmark$ & Configuration Changes for Manhole & Tue $1 / 18 / 05$ & Tue $1 / 18 / 05$ & \\
\hline 2956 & $\checkmark$ & Configuration Changes for Secondary & Tue $1 / 18 / 05$ & Tue 5/2/06 & \\
\hline 2957 & $\checkmark$ & Configuration Changes for SwitchGear & Tue 1/18/05 & Tue $1 / 18 / 05$ & \\
\hline 2958 & $\checkmark$ & Configuration Changes for GasMain & Mon $1 / 24 / 05$ & Mon $1 / 24 / 05$ & \\
\hline 2959 & $\checkmark$ & Configuration Changes for GasValves & Mon $1 / 24 / 05$ & Mon $1 / 24 / 05$ & \\
\hline 2960 & $\checkmark$ & Configuration Changes for Fittings & Mon $1 / 24 / 05$ & Mon $1 / 24 / 05$ & \\
\hline 2961 & $\checkmark$ & Configuration Changes for GasMainLocation & Mon $1 / 24 / 05$ & Mon 1/24/05 & \\
\hline 2962 & $\checkmark$ & Configuration Changes for PipeExposure & Mon $1 / 24 / 05$ & Mon $1 / 24 / 05$ & \\
\hline 2963 & $\checkmark$ & Configuration Changes for PipelineMarker & Mon $1 / 24 / 05$ & Mon $1 / 24 / 05$ & \\
\hline 2964 & $\checkmark$ & Configuration Changes for Drip & Mon $1 / 24 / 05$ & Mon $1 / 24 / 05$ & \\
\hline 2965 & $\checkmark$ & Configuration Changes for Casing & Mon $1 / 24 / 05$ & Mon $1 / 24 / 05$ & \\
\hline 2966 & $\checkmark$ & Configuration Changes for GasDeadEnd & Mon $1 / 24 / 05$ & Mon 1/24/05 & \\
\hline 2967 & $\checkmark$ & Configuration Changes for Regulator Station & Fri $1 / 28 / 05$ & Fri $1 / 28 / 05$ & \\
\hline 2968 & $\checkmark$ & Configuration Changes for TakeStation & Fri 1/28/05 & Fri 1/28/05 & \\
\hline 2969 & $\checkmark$ & Configuration Changes for StreetCenterLine & Fri $1 / 28 / 05$ & Fri $1 / 28 / 05$ & \\
\hline 2970 & $\checkmark$ & Configuration changes for StreetROW & Fri $1 / 28 / 05$ & Fri 1/28/05 & \\
\hline 2971 & $\checkmark$ & Configuration changes for Easement & Fri $1 / 28 / 05$ & Fri $1 / 28 / 05$ & \\
\hline 2972 & $\checkmark$ & Configuration Changes for Railroad. & Fri $1 / 28 / 05$ & Fri $1 / 28 / 05$ & \\
\hline 2973 & $\checkmark$ & Configuration Changes for LotLine & Fri $1 / 28 / 05$ & Fri $1 / 28 / 05$ & \\
\hline 2974 & $\checkmark$ & Configuration Chhanges for LotNumber & Fri $1 / 28 / 05$ & Fri $1 / 28 / 05$ & \\
\hline 2975 & $\checkmark$ & Configuration Changes for Bridge & Fri $1 / 28 / 05$ & Fri $1 / 28 / 05$ & \\
\hline 2976 & $\checkmark$ & Configuration Changes for Linear Water & Fri $1 / 28 / 05$ & Fri 1/28/05 & \\
\hline 2977 & $\checkmark$ & Configuration Changes for LOA & Fri $1 / 28 / 05$ & Fri 1/28/05 & \\
\hline 2978 & $\checkmark$ & Miscellaneous Land and Point Feature could not be created & Fri 1/28/05 & Fri 1/28/05 & \\
\hline 2979 & $\checkmark$ & Error Running automated tests & Fri 1/21/05 & Tue 5/2/06 & \\
\hline 2980 & $\checkmark$ & Log new base configuration Elementool tickets based on the test results & Fri 1/28/05 & Fri 1/28/05 & \\
\hline 2981 & $\checkmark$ & Part 1 Check Annotation for all feature classes - Integration testing & Fri 1/21/05 & Fri 1/21/05 & \\
\hline 2982 & $\checkmark$ & Symbol Offset for TransformerBank & Mon $1 / 31 / 05$ & Mon 1/31/05 & \\
\hline 2983 & $\checkmark$ & Add WireSizeCd and WireMaterialCd to Conductor Features & Fri 2/11/05 & Fri 2/11/05 & \\
\hline 2984 & $\checkmark$ & Pad number Annotation - Problems with Annotation & Fri 2/11/05 & Fri 2/11/05 & \\
\hline 2985 & $\checkmark$ & Part 2 Check Annotation for all feature classes - Integration testing & Mon 2/28/05 & Mon 2/28/05 & \\
\hline 2986 & $\checkmark$ & Troubleshoot all annotation related issues on the DEV instance & Mon 2/14/05 & Tue 2/15/05 & \\
\hline 2987 & $\checkmark$ & Build \& document all Stored Displays & Tue 5/2/06 & Tue 5/2/06 & \\
\hline 2988 & 国 & Correct Symbol Rotation Configuration to be compatible with both migration and ArcFM & Mon 2/28/05 & Mon 2/28/05 & \\
\hline 2989 & $\checkmark$ & Symbology for FuseCutoutBanks & Wed 1/11/06 & Wed 1/11/06 & \\
\hline 2990 & $\checkmark$ & Symbology for OpenPoint & Wed 1/11/06 & Wed 1/11/06 & \\
\hline $\begin{array}{l}\text { NIPSC } \\
\text { Confid }\end{array}$ & $\begin{array}{l}\text { Co GIS } \\
\text { lential }\end{array}$ & Page 65 & & $\begin{array}{r}\text { Last Updat } \\
\text { Print }\end{array}$ & $\begin{array}{l}\text { ed on Tue } 7 / 3 / 07 \\
\text { ed on Fri 8/17/07 }\end{array}$ \\
\hline
\end{tabular}




\begin{tabular}{|c|c|c|c|c|c|}
\hline \multicolumn{6}{|c|}{ AEDR Construction Phase I Project Plan } \\
\hline ID & (i & Task Name & Start & Finish & \\
\hline 2991 & $\checkmark$ & Symbology for TakeStation & Wed 1/11/06 & Wed 1/11/06 & \\
\hline 2992 & $\checkmark$ & Symbology for Fittings & Wed 1/11/06 & Wed 1/11/06 & \\
\hline 2993 & $\checkmark$ & Annotation \& Symbol review of Migration 3 & Fri 9/2/05 & Fri 9/2/05 & \\
\hline 2994 & $\checkmark$ & Test 1 - Iteration 3 QA - Land NIPSCO Migration issues & Tue $7 / 5 / 05$ & Tue $7 / 5 / 05$ & \\
\hline 2995 & $\checkmark$ & Test 3 \& 4 - Iteration 3 QA - Land NIPSCO Migration issues & Tue $7 / 5 / 05$ & Tue $7 / 5 / 05$ & \\
\hline 2996 & $\checkmark$ & AbandonGasDevice & Mon 4/4/05 & Mon 4/4/05 & \\
\hline 2997 & $\checkmark$ & Annotation Not Updating & Mon 1/16/06 & Mon 1/16/06 & \\
\hline 2998 & $\checkmark$ & Correct the Configuration Requirements for CPRectifier & Wed 3/1/06 & Wed 3/1/06 & \\
\hline 2999 & $\checkmark$ & Correct configuration requirements for CPTestPoint & Wed 12/15/04 & Wed 12/15/04 & \\
\hline 3000 & $\checkmark$ & Correct configuration requirements for ServiceStub & Wed 3/2/05 & Wed 3/2/05 & \\
\hline 3001 & $\checkmark$ & Identify Composite Favorites & Wed 3/2/05 & Wed 3/2/05 & \\
\hline 3002 & $\checkmark$ & Correct configuration on FeederAllOpenPoint & Wed 3/2/05 & Wed 3/2/05 & \\
\hline 3003 & $\checkmark$ & ArcFM Configuration Testing & Fri 2/18/05 & Thu $3 / 3 / 05$ & \\
\hline 3004 & $\checkmark$ & Test updated configuration & Fri 2/18/05 & Wed 2/23/05 & \\
\hline 3005 & $\checkmark$ & Generate ET's based on configuration testing & Thu 2/24/05 & Tue 3/1/05 & \\
\hline 3006 & $\checkmark$ & Resolve ETs & Wed 3/2/05 & Thu $3 / 3 / 05$ & \\
\hline 3007 & $\checkmark$ & Auto Updater / Miscellaneous Customization Tasks & Tue 12/14/04 & Fri 1/20/06 & \\
\hline 3008 & $\checkmark$ & FuseCutoutBank - SymbologyConfigurationCd AU & Thu $1 / 20 / 05$ & Wed 1/11/06 & \\
\hline 3009 & $\checkmark$ & Create component spec & Thu $1 / 20 / 05$ & Thu $1 / 20 / 05$ & \\
\hline 3010 & $\checkmark$ & Review component spec & Fri 1/21/05 & Fri 1/21/05 & \\
\hline 3011 & $\checkmark$ & Code / unit test & Fri 1/21/05 & Fri 1/21/05 & \\
\hline 3012 & $\checkmark$ & Acceptance test & Wed 1/11/06 & Wed 1/11/06 & \\
\hline 3013 & $\checkmark)$ & All LOA fields - LOA AU & Fri $1 / 7 / 05$ & Mon 1/16/06 & \\
\hline 3014 & $\checkmark$ & Create component spec & Fri $1 / 7 / 05$ & Fri $1 / 7 / 05$ & \\
\hline 3015 & $\checkmark$ & Review component spec & Fri $1 / 7 / 05$ & Fri $1 / 7 / 05$ & \\
\hline 3016 & $\checkmark$ & Code / unit test & Fri 1/7/05 & Fri $1 / 7 / 05$ & \\
\hline 3017 & $\checkmark$ & Determine the general LOA population approach & Tue $1 / 18 / 05$ & Tue $1 / 18 / 05$ & \\
\hline 3018 & $\checkmark$ & Determine objectclasses that need LOA information & Tue $1 / 18 / 05$ & Tue $1 / 18 / 05$ & \\
\hline 3019 & $\checkmark$ & Acceptance Test & Mon 1/16/06 & Mon 1/16/06 & \\
\hline 3020 & $\checkmark$ & Reference Features - Do Not Post To Parent & Tue 12/14/04 & Fri 1/20/06 & \\
\hline 3021 & $\checkmark$ & Review component spec & Tue $12 / 14 / 04$ & Tue $12 / 14 / 04$ & \\
\hline 3022 & $\checkmark$ & Code / unit test (depends on Session Mgr) & Tue $12 / 14 / 04$ & Fri 12/17/04 & \\
\hline 3023 & $\checkmark$ & Acceptance test & Fri 1/20/06 & Fri 1/20/06 & \\
\hline 3024 & $\checkmark$ & Elementool \#165 - FeederAllOpenPoint Deletions & Thu 1/27/05 & Fri 1/20/06 & \\
\hline 3025 & $\checkmark$ & Create component spec & Thu $1 / 27 / 05$ & Thu $1 / 27 / 05$ & \\
\hline 3026 & $\checkmark$ & Review component spec & Fri 2/4/05 & Fri 2/4/05 & \\
\hline 3027 & $\checkmark$ & Code / unit test & Fri 2/11/05 & Fri 2/11/05 & \\
\hline 3028 & $\checkmark$ & Acceptance Test & Fri 1/20/06 & Fri $1 / 20 / 06$ & \\
\hline 3029 & $\checkmark$ & DistribRefNumber AU & Fri 2/4/05 & Mon 1/16/06 & \\
\hline 3030 & $\checkmark$ & Code / unit test - admin tool & Fri $2 / 4 / 05$ & Fri $2 / 4 / 05$ & \\
\hline 3031 & $\checkmark$ & Acceptance Test & Mon 1/16/06 & Mon 1/16/06 & \\
\hline 3032 & $\checkmark$ & NIPSCO Custom Toolbar & Mon 2/7/05 & Mon 1/16/06 & \\
\hline 3033 & $\checkmark$ & Create component spec & Mon 2/7/05 & Mon 2/7/05 & \\
\hline 3034 & $\checkmark$ & Review component spec & Mon 2/7/05 & Mon 2/7/05 & \\
\hline 3035 & $\checkmark$ & Code / unit test & Mon 2/7/05 & Mon 2/7/05 & \\
\hline 3036 & $\checkmark$ & Acceptance Test & Mon 1/16/06 & Mon 1/16/06 & \\
\hline $\begin{array}{l}\text { NIPSC } \\
\text { Confid }\end{array}$ & $\begin{array}{l}\text { Co GIS } \\
\text { lential }\end{array}$ & Page 66 & & $\begin{array}{r}\text { Last Updat } \\
\text { Print }\end{array}$ & $\begin{array}{l}\text { ed on Tue } 7 / 3 / 07 \\
\text { ed on Fri 8/17/07 }\end{array}$ \\
\hline
\end{tabular}




\begin{tabular}{|c|c|c|c|c|}
\hline \multicolumn{5}{|c|}{ AEDR Construction Phase I Project Plan } \\
\hline ID & (1) & Task Name & Start & Finish \\
\hline 3037 & $\checkmark$ & AU UpstreamGasMainOID & Wed 2/2/05 & Mon 1/16/06 \\
\hline 3038 & $\checkmark$ & Create component spec & Wed $2 / 2 / 05$ & Wed 2/2/05 \\
\hline 3039 & $\checkmark$ & Review component spec & Thu 2/3/05 & Thu 2/3/05 \\
\hline 3040 & $\checkmark$ & Code / unit test & Fri 2/4/05 & Fri 2/4/05 \\
\hline 3041 & $\checkmark$ & Acceptance Test & Mon 1/16/06 & Mon 1/16/06 \\
\hline 3042 & $\checkmark$ & AU On-site Work \#1 - Install AU's on test machine & Tue $1 / 4 / 05$ & Tue $1 / 4 / 05$ \\
\hline 3043 & $\checkmark$ & AU On-site Work \#1 - Configure at NIPSCO & Tue $1 / 4 / 05$ & Wed $1 / 5 / 05$ \\
\hline 3044 & $\checkmark$ & AU On-site Work \#1 - Create AU tracking spreadsheet & Wed $1 / 5 / 05$ & Wed $1 / 5 / 05$ \\
\hline 3045 & $\checkmark$ & AU On-site Work \#1 - Review AU tracking spreadsheet & Wed $1 / 5 / 05$ & Wed 1/5/05 \\
\hline 3046 & $\checkmark$ & AU On-site Work \#1 - Test AU's at NIPSCO & Wed $1 / 5 / 05$ & Fri $1 / 7 / 05$ \\
\hline 3047 & $\checkmark$ & AU On-site Work \#1 - Follow-on tasks from site visit & Tue $1 / 11 / 05$ & Thu 1/13/05 \\
\hline 3048 & $\checkmark$ & AU On-site Work \#1 - Fix SDE Maximum Streams Exceeded Error (All AU's) & Wed $1 / 19 / 05$ & Wed 1/19/05 \\
\hline 3049 & $\checkmark$ & AU On-site Work \#1 - Fix SplitAtTap problem & Fri 1/21/05 & Fri $1 / 21 / 05$ \\
\hline 3050 & $\checkmark$ & AU On-site Work \#2 - Configure remaining AU's & Mon 1/24/05 & Mon 1/24/05 \\
\hline 3051 & $\checkmark$ & AU On-site Work \#2 - Test AU's and update tracking spreadsheet & Mon $1 / 24 / 05$ & Thu $1 / 27 / 05$ \\
\hline 3052 & $\checkmark$ & AU On-site Work \#2 - Resolve AU Elementool tickets & Fri $1 / 28 / 05$ & Fri $1 / 28 / 05$ \\
\hline 3053 & $\checkmark$ & AU On-site Work \#2 - Follow-on tasks from visit & Mon 2/7/05 & Tue $2 / 8 / 05$ \\
\hline 3054 & $\checkmark$ & Elementool Ticket Review \& Work & Mon 4/17/06 & Wed $4 / 19 / 06$ \\
\hline 3055 & $\checkmark$ & Review all critical and high priority ETs & Mon 4/17/06 & Tue $4 / 18 / 06$ \\
\hline 3056 & $\checkmark$ & Resolve the critical \& high priority ETs. & Wed 4/19/06 & Wed $4 / 19 / 06$ \\
\hline 3057 & $\checkmark$ & Session Manager Tasks & Mon 4/5/04 & Fri 1/28/05 \\
\hline 3058 & $\checkmark$ & Configuration & Mon 1/10/05 & Fri $1 / 14 / 05$ \\
\hline 3059 & $\checkmark$ & Installation at NIPSCO & Mon 1/17/05 & Tue $1 / 18 / 05$ \\
\hline 3060 & $\checkmark$ & Finalize documentation \& store in Source Safe & Fri $1 / 28 / 05$ & Fri $1 / 28 / 05$ \\
\hline 3061 & $\checkmark$ & Design Business Process Using Session Manager / Versions & Mon 4/5/04 & Wed 4/7/04 \\
\hline 3062 & $\checkmark$ & Design workflow including roles (QA/QC) & Mon 4/5/04 & Mon 4/5/04 \\
\hline 3063 & $\checkmark$ & Document business process \& procedures & Tue $4 / 6 / 04$ & Wed $4 / 7 / 04$ \\
\hline 3064 & $\checkmark$ & Create User Guides & Mon 1/16/06 & Mon 4/24/06 \\
\hline 3065 & $\checkmark$ & Create Online User Guides & Mon 1/16/06 & Mon 4/24/06 \\
\hline 3066 & $\checkmark$ & Define requirements & Mon $1 / 16 / 06$ & Wed $1 / 18 / 06$ \\
\hline 3067 & $\checkmark$ & Create NIMS Online Help & Wed 2/8/06 & Mon 3/6/06 \\
\hline 3068 & $\checkmark$ & Document Online Help & Thu $1 / 19 / 06$ & Wed $2 / 1 / 06$ \\
\hline 3069 & $\checkmark$ & Add Screenshots to Online Help & Fri 4/21/06 & Mon 4/24/06 \\
\hline
\end{tabular}


Appendix A-3

AEDR Deployment Phase I Project Plan 


\section{AEDR Deployment Phase I Project Plan}

\begin{tabular}{|c|c|c|c|c|}
\hline ID & a & Task Name & Start & Finish \\
\hline 1 & & NIPSCO AEDR Phase I Deployment & Mon 2/2/04 & Mon 3/26/07 \\
\hline 2 & $\checkmark$ & GIS Data Migration - Final Delivery & Tue 1/3/06 & Wed 6/7/06 \\
\hline 3 & $\checkmark$ & Pre-Delivery to Migration Vendor Tasks & Tue 1/3/06 & Fri 4/21/06 \\
\hline 4 & $\checkmark$ & Pre-final migration data cleanup & Tue 3/28/06 & Mon 4/10/06 \\
\hline 5 & $\checkmark$ & Authorization to proceed with the delivery of data to migration vendor & Fri 3/31/06 & Fri 3/31/06 \\
\hline 6 & $\checkmark$ & Verify the deliverable from data migration vendor; a list of tiles in each batch & Fri 3/31/06 & Fri 3/31/06 \\
\hline 7 & $\checkmark$ & Review and update the migration plan & Wed 3/29/06 & Wed 3/29/06 \\
\hline 8 & $\checkmark$ & Assign/adjust resources based on updates to the migration plan & Tue 4/11/06 & Tue $4 / 11 / 06$ \\
\hline 9 & $\checkmark$ & Correct critical 6.0 issues, ETs, create scripts & Tue $1 / 3 / 06$ & Mon $4 / 17 / 06$ \\
\hline 10 & $\checkmark$ & Complete 6.1 essential data cleanup (Data Cleanup Spreadsheet) & Fri 4/21/06 & Fri 4/21/06 \\
\hline 11 & $\checkmark$ & Run QA reports & Mon 3/20/06 & Mon 3/20/06 \\
\hline 12 & $\checkmark$ & Finalize all automated QA from data and migration scripts & Tue $1 / 3 / 06$ & Tue $1 / 24 / 06$ \\
\hline 13 & $\checkmark$ & Prepare automated test suites for migration vendor - see Migration Plan & Thu $4 / 20 / 06$ & Thu $4 / 20 / 06$ \\
\hline 14 & $\checkmark$ & Prepare instructions for the automated QA Routines -Mig Plan & Thu $4 / 20 / 06$ & Thu $4 / 20 / 06$ \\
\hline 15 & $\checkmark$ & Complete the review and updating the Migration Plan (Pre-delivery section only) & Mon 4/10/06 & Mon 4/10/06 \\
\hline 16 & $\checkmark$ & Identify resources for migration QA effort & Mon 3/27/06 & Mon 3/27/06 \\
\hline 17 & $\checkmark$ & Add automated test suites to the Delivery Checklist & Fri 4/21/06 & Fri 4/21/06 \\
\hline 18 & $\checkmark$ & Determine freeze date for Final Migration & Mon 3/27/06 & Mon 3/27/06 \\
\hline 19 & $\checkmark$ & Notify record clerks \& other users of impending freeze date $-4 / 24$ & Fri 3/31/06 & Fri 3/31/06 \\
\hline 20 & $\checkmark$ & Request IT resources & Thu 4/13/06 & Thu $4 / 13 / 06$ \\
\hline 21 & $\checkmark$ & Verify there are no more outstanding medium/critical ET's prior to preparing delivery to migration vendor & Mon 4/17/06 & Mon 4/17/06 \\
\hline 22 & $\checkmark$ & Prepare Delivery to Migration Vendor - Mig Plan & Mon 4/17/06 & Mon 5/1/06 \\
\hline 23 & $\checkmark$ & Notification to record clerks to finalize all work orders \& announce freeze date again & Mon 4/17/06 & Mon 4/17/06 \\
\hline 24 & $\checkmark$ & Freeze the AutoCAD/EDFS data \& applications & Mon 4/24/06 & Mon 4/24/06 \\
\hline 25 & $\checkmark$ & Set up Source Safe files & Mon 4/24/06 & Mon 4/24/06 \\
\hline 26 & $\checkmark$ & Baseline the data model (Visio file) & Mon 4/24/06 & Mon 4/24/06 \\
\hline 27 & $\checkmark$ & Baseline the data migration matrices & Mon $4 / 24 / 06$ & Mon $4 / 24 / 06$ \\
\hline 28 & $\checkmark$ & Baseline the .MXD file \& annotation spreadsheet & Mon 4/24/06 & Mon 4/24/06 \\
\hline 29 & $\checkmark$ & Baseline the miscellaneous migration specifications & Mon 4/24/06 & Mon 4/24/06 \\
\hline 30 & $\checkmark$ & Prepare Source Data \& Counts & Mon 4/24/06 & Thu 4/27/06 \\
\hline 31 & $\checkmark$ & Prepare Outfield source DWG files and prepare feature counts & Mon $4 / 24 / 06$ & Wed 4/26/06 \\
\hline 32 & $\checkmark$ & Prepare EDFS source data and feature counts & Wed $4 / 26 / 06$ & Thu $4 / 27 / 06$ \\
\hline 33 & $\checkmark$ & Prepare GMMS and feature counts & Thu $4 / 27 / 06$ & Thu $4 / 27 / 06$ \\
\hline 34 & $\checkmark$ & Prepare Streetlight source data and feature counts & Thu $4 / 27 / 06$ & Thu $4 / 27 / 06$ \\
\hline 35 & $\checkmark$ & Delivery / Notification Tasks & Fri 4/28/06 & Mon 5/1/06 \\
\hline 36 & $\checkmark$ & Package up the files for delivery & Fri 4/28/06 & Fri $4 / 28 / 06$ \\
\hline 37 & $\checkmark$ & Place the package on data migration vendor's FTP server & Mon 5/1/06 & Mon 5/1/06 \\
\hline 38 & $\checkmark$ & Notify migration vendor that the delivery is available & Fri 4/28/06 & Fri 4/28/06 \\
\hline 39 & $\checkmark$ & Post-Delivery to Data Migration Vendor Tasks & Mon 5/1/06 & Mon 6/5/06 \\
\hline 40 & $\checkmark$ & Perform various QA set-up tasks & Thu 5/11/06 & Thu 5/11/06 \\
\hline 41 & $\checkmark$ & Create tracklists for the delivery & Thu 5/18/06 & Thu 5/18/06 \\
\hline 42 & $\checkmark$ & Print graphic specs plot for the features (symbology legend) & Tue 5/16/06 & Fri 5/19/06 \\
\hline 43 & $\checkmark$ & Update feature count tracklists & Fri 5/19/06 & Fri 5/19/06 \\
\hline
\end{tabular}




\section{AEDR Deployment Phase I Project Plan}

\begin{tabular}{|c|c|c|c|c|}
\hline ID & (i) & Task Name & Start & Finish \\
\hline 44 & $\checkmark$ & Set Up X: drive folders & Mon 5/1/06 & Thu 5/18/06 \\
\hline 45 & $\checkmark$ & Schedule DBA to assist with the installation & Mon 5/1/06 & Tue 5/16/06 \\
\hline 46 & $\checkmark$ & Verify any changes to the environments (instances) & Fri 5/12/06 & Fri 5/12/06 \\
\hline 47 & $\checkmark$ & Setup hardware for the QA team & Wed $5 / 3 / 06$ & Mon 5/22/06 \\
\hline 48 & $\checkmark$ & Notify IT server group regarding upcoming delivery \& QA & Mon 5/1/06 & Tue 5/16/06 \\
\hline 49 & $\checkmark$ & CIS customer loading (ref ET 2146) & Mon 6/5/06 & Mon 6/5/06 \\
\hline 50 & $\checkmark$ & Verify with CIS that they have now implemented the incremental updates & Fri 6/2/06 & Fri 6/2/06 \\
\hline 51 & $\checkmark$ & Migration Vendor Tasks & Wed 4/26/06 & Tue 5/16/06 \\
\hline 52 & $\checkmark$ & Migrate and create new data & Wed 4/26/06 & Tue 5/16/06 \\
\hline 53 & $\checkmark$ & Notify NIPSCO of the delivery of migrated data & Tue 5/16/06 & Tue 5/16/06 \\
\hline 54 & $\checkmark$ & Install and Conduct High Level QA & Tue 5/16/06 & Fri 5/19/06 \\
\hline 55 & $\checkmark$ & Installation Tasks & Tue 5/16/06 & Wed 5/17/06 \\
\hline 56 & $\checkmark$ & Download data and files from FTP server & Tue $5 / 16 / 06$ & Tue $5 / 16 / 06$ \\
\hline 57 & $\checkmark$ & High-level review of reports and artifacts & Wed 5/17/06 & Wed 5/17/06 \\
\hline 58 & $\checkmark$ & Copy migrated data and artifacts to a shared directory & Wed 5/17/06 & Wed 5/17/06 \\
\hline 59 & $\checkmark$ & Restore the .BAK file & Wed 5/17/06 & Wed 5/17/06 \\
\hline 60 & $\checkmark$ & Final Delivery Compliance QA & Wed 5/17/06 & Fri 5/19/06 \\
\hline 61 & $\checkmark$ & Check for missing tiles & Wed 5/17/06 & Wed 5/17/06 \\
\hline 62 & $\checkmark$ & Document the environment change per the SDE Management Plan & Wed 5/17/06 & Wed 5/17/06 \\
\hline 63 & $\checkmark$ & Verify correct schema & Wed 5/17/06 & Wed 5/17/06 \\
\hline 64 & $\checkmark$ & Verify delivery artifacts against checklist & Wed 5/17/06 & Wed 5/17/06 \\
\hline 65 & $\checkmark$ & Review and process EDFS/Outfield mismatch report & Wed 5/17/06 & Wed 5/17/06 \\
\hline 66 & $\checkmark$ & Run the source count compare script & Fri 5/19/06 & Fri 5/19/06 \\
\hline 67 & $\checkmark$ & Pre-populate the interactive QA spreadsheet & Fri 5/19/06 & Fri 5/19/06 \\
\hline 68 & $\checkmark$ & Conduct object existence test & Thu 5/18/06 & Fri 5/19/06 \\
\hline 69 & $\checkmark$ & Run automated testing against new data & Wed 5/17/06 & Thu 5/18/06 \\
\hline 70 & $\checkmark$ & Compare NIPSCO automated reports against migration vendor's same automated reports & Wed $5 / 17 / 06$ & Thu 5/18/06 \\
\hline 71 & $\checkmark$ & NIPSCO Parallel Migration Processing & Wed 5/17/06 & Wed 6/7/06 \\
\hline 72 & $\checkmark$ & Build the parallel instance & Wed 5/17/06 & Sat $5 / 20 / 06$ \\
\hline 73 & $\checkmark$ & Run data cleanup and NIPSCO migration scripts & Thu 5/25/06 & Thu 5/25/06 \\
\hline 74 & $\checkmark$ & Run data cleanup and NIPSCO migration scripts - manual effort & Thu 5/25/06 & Thu 5/25/06 \\
\hline 75 & $\checkmark$ & Configure the geodatabase & Thu 5/25/06 & Fri 6/2/06 \\
\hline 76 & $\checkmark$ & QA of Parallel Migration Processing & Fri 6/2/06 & Wed $6 / 7 / 06$ \\
\hline 77 & $\checkmark$ & Conduct Ready for Review & Fri 6/2/06 & Tue $6 / 6 / 06$ \\
\hline 78 & $\checkmark$ & Conduct QA of data cleanup \& test scripts & Wed 6/7/06 & Wed 6/7/06 \\
\hline 79 & $\checkmark$ & QA of Migrated Data from Migration Vendor & Thu 5/18/06 & Wed 6/7/06 \\
\hline 80 & $\checkmark$ & QA - Remaining High-Level review & Wed 6/7/06 & Wed $6 / 7 / 06$ \\
\hline 81 & $\checkmark$ & QA - Conduct Ready for Review & Thu 6/1/06 & Wed $6 / 7 / 06$ \\
\hline 82 & $\checkmark$ & QA - Feature Counts & Fri 5/19/06 & Wed 5/24/06 \\
\hline 83 & $\checkmark$ & QA - Automated QA & Thu 5/18/06 & Wed 5/24/06 \\
\hline 84 & $\checkmark$ & QA - Interactive QA of critical features & Tue $5 / 23 / 06$ & Wed 5/24/06 \\
\hline 85 & $\checkmark$ & QA - First Pass Connectivity QA & Mon 5/22/06 & Fri 5/26/06 \\
\hline 86 & $\checkmark$ & QA - Second Pass Connectivity QA & Mon 6/5/06 & Mon 6/5/06 \\
\hline
\end{tabular}


AEDR Deployment Phase I Project Plan

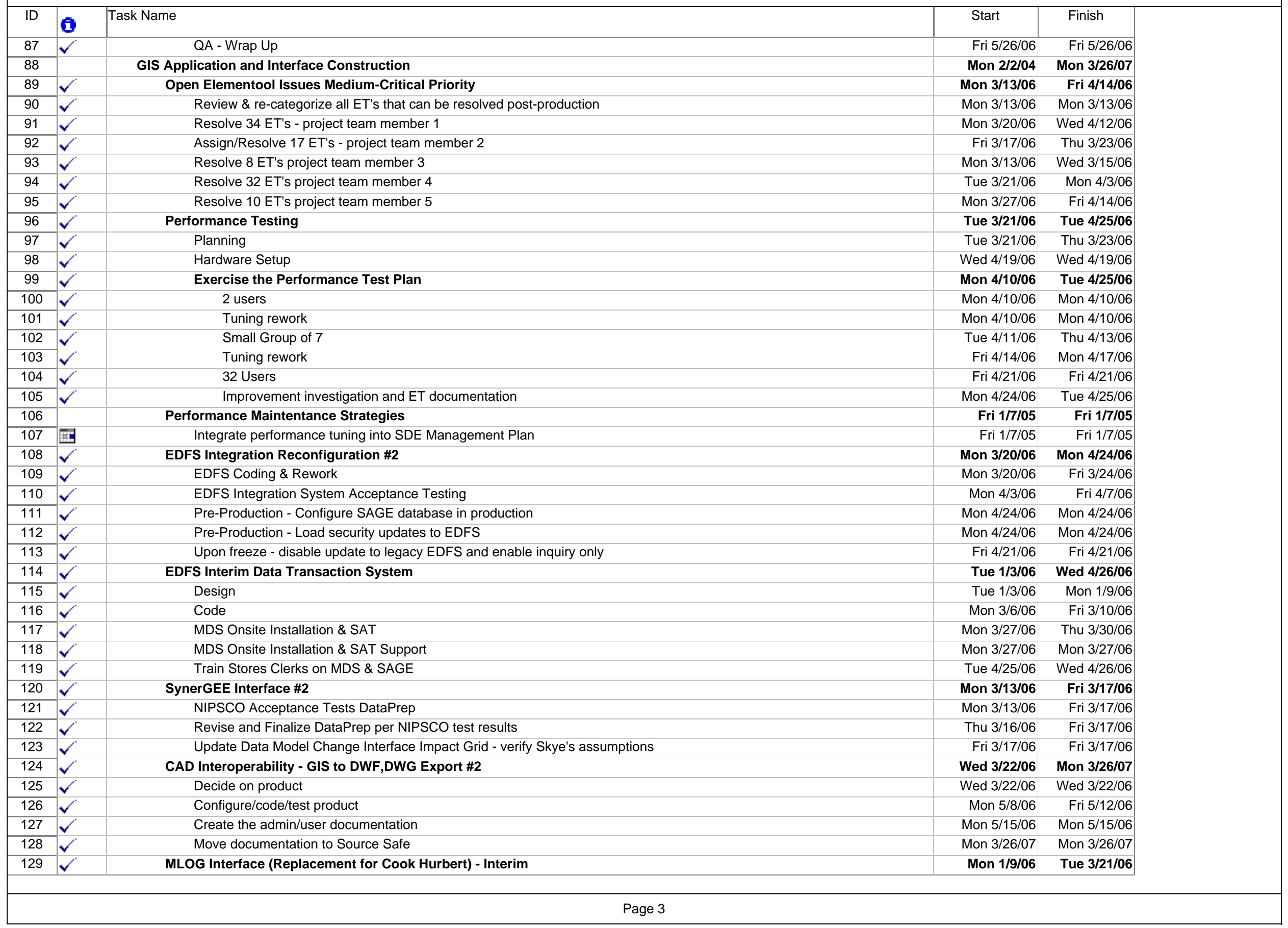




\section{AEDR Deployment Phase I Project Plan}

\begin{tabular}{|c|c|c|c|c|}
\hline ID & (i) & Task Name & Start & Finish \\
\hline 130 & $\checkmark$ & Re-design the AEDR Phase I interface to MLOG & Mon $1 / 9 / 06$ & Mon 1/9/06 \\
\hline 131 & $\checkmark$ & Develop the interface & Mon 1/9/06 & Mon 1/9/06 \\
\hline 132 & $\checkmark$ & Unit test the Interface & Tue 3/21/06 & Tue $3 / 21 / 06$ \\
\hline 133 & $\checkmark$ & Facility Browser Application - Phase I & Mon 3/13/06 & Mon 3/13/06 \\
\hline 134 & $\checkmark$ & Moved to AEDR Phase II & Mon 3/13/06 & Mon 3/13/06 \\
\hline 135 & $\checkmark$ & Gas \& Elec Service Request Application & Tue 2/15/05 & Tue 2/15/05 \\
\hline 136 & $\checkmark$ & Moved to AEDR Phase II & Tue $2 / 15 / 05$ & Tue $2 / 15 / 05$ \\
\hline 137 & $\checkmark$ & Field Browser Application & Mon 3/13/06 & Mon 3/13/06 \\
\hline 138 & $\checkmark$ & Moved to AEDR Phase II & Mon 3/13/06 & Mon 3/13/06 \\
\hline 139 & $\checkmark$ & CADOPS Reconfiguration (based on new frozen data model) & Mon 1/23/06 & Thu 5/25/06 \\
\hline 140 & $\checkmark$ & Install the reconfigured CADOPS / FeederAll Onsite & Mon 1/23/06 & Mon 1/23/06 \\
\hline 141 & $\checkmark$ & Test the reconfigured CADOPS Onsite & Mon 1/23/06 & Mon 1/30/06 \\
\hline 142 & $\checkmark$ & Complete the cleanup and finalize the territory for NORS & Mon 1/30/06 & Mon 3/20/06 \\
\hline 143 & $\checkmark$ & Support User Data Changes for NORS & Tue 3/21/06 & Thu $5 / 25 / 06$ \\
\hline 144 & $\checkmark$ & FeederAll Reconfiguration (based on new frozen data model) & Fri 10/6/06 & Fri 10/6/06 \\
\hline 145 & $\checkmark$ & Moved to AEDR Phase II & Fri 10/6/06 & Fri 10/6/06 \\
\hline 146 & $\checkmark$ & ArcFM Configuration Updates / Fixes & Mon 3/20/06 & Mon 5/1/06 \\
\hline 147 & $\checkmark$ & Miscellaneous & Fri 3/31/06 & Mon 5/1/06 \\
\hline 148 & $\checkmark$ & Modify Stored Displays as needed for performance & Mon 5/1/06 & Mon 5/1/06 \\
\hline 149 & $\checkmark$ & Symbology Rotation Problem Resolution & Fri 3/31/06 & Fri 3/31/06 \\
\hline 150 & $\checkmark$ & Re-write the scripts that adjust the rotation to back out the adjustments & Fri 3/31/06 & Fri 3/31/06 \\
\hline 151 & $\checkmark$ & Disable AutoAngle Setter AU & Fri 3/31/06 & Fri 3/31/06 \\
\hline 152 & $\checkmark$ & Run the new scripts in the Dev environment & Fri 3/31/06 & Fri 3/31/06 \\
\hline 153 & $\checkmark$ & ArcFM Configuration Testing & Mon 3/20/06 & Thu 4/6/06 \\
\hline 154 & $\checkmark$ & Test configuration using the Online Help Documents - ET any errors & Mon 3/20/06 & Mon 3/20/06 \\
\hline 155 & $\checkmark$ & Test configuration using the Online Help Documents - ET any errors & Tue 3/21/06 & Fri 3/24/06 \\
\hline 156 & $\checkmark$ & Create new test cases for configuration not addressed by Online Help & Mon $4 / 3 / 06$ & Tue $4 / 4 / 06$ \\
\hline 157 & $\checkmark$ & Test configuration residing outside the online files - ET any errors & Wed $4 / 5 / 06$ & Wed $4 / 5 / 06$ \\
\hline 158 & $\checkmark$ & Export baseline configuration and check into SourceSafe & Wed $4 / 5 / 06$ & Wed $4 / 5 / 06$ \\
\hline 159 & $\checkmark$ & Review \& correct configuration errors and synchronize Online Help & Mon 3/27/06 & Thu 4/6/06 \\
\hline 160 & $\checkmark$ & ArcFM Configuration Review & Fri 4/7/06 & Fri 4/7/06 \\
\hline 161 & $\checkmark$ & Final configuration review of custom code components & Fri 4/7/06 & Fri $4 / 7 / 06$ \\
\hline 162 & $\checkmark$ & Remaining Install and Configure AU tasks & Mon 1/16/06 & Mon 3/27/06 \\
\hline 163 & $\checkmark$ & AU On-site Work \#3 - Develop/test installation process that will work via e-mail & Mon 1/16/06 & Mon 1/16/06 \\
\hline 164 & $\checkmark$ & AU On-site Work \#3 - Make sure AU's are installed on all machines & Mon 1/16/06 & Mon 1/16/06 \\
\hline 165 & $\checkmark$ & AU On-site Work \#3 - Configure and test remaining AUs & Tue $1 / 17 / 06$ & Fri $1 / 20 / 06$ \\
\hline 166 & $\checkmark$ & AU On-site Work \#3 - Acceptance test entire suite of Aus & Mon 1/16/06 & Fri $1 / 20 / 06$ \\
\hline 167 & $\checkmark$ & Configure AU resulting from ET 2433 & Mon 3/6/06 & Mon 3/6/06 \\
\hline 168 & $\checkmark$ & Install \& Test AU from ET 2433 & Mon 3/27/06 & Mon 3/27/06 \\
\hline 169 & $\checkmark$ & Miscellaneous Customizations & Wed 3/30/05 & Wed 3/30/05 \\
\hline 170 & $\checkmark$ & Remaining Tasks & Wed 3/30/05 & Wed 3/30/05 \\
\hline 171 & $\checkmark$ & Customize the ArcGIS toolbar so that it has the functions needed by the record clerks & Wed 3/30/05 & Wed $3 / 30 / 05$ \\
\hline 172 & $\checkmark$ & System Acceptance Testing (SAT) Tasks Reside in Each Component Section & Mon 3/13/06 & Mon 3/13/06 \\
\hline
\end{tabular}




\section{AEDR Deployment Phase I Project Plan}

\begin{tabular}{|c|c|c|c|c|}
\hline ID & ( ) & Task Name & Start & Finish \\
\hline 173 & $\checkmark$ & See individual component sections & Mon 3/13/06 & Mon 3/13/06 \\
\hline 174 & & Integration Testing (IT) / Rework on Fully Configured Environment & Mon 3/13/06 & Fri 5/19/06 \\
\hline 175 & $\checkmark$ & IT - Standard configuration (create / maintain features) & Mon 5/8/06 & Fri 5/12/06 \\
\hline 176 & $\checkmark$ & IT - Auto Updaters & Mon 5/8/06 & Fri 5/12/06 \\
\hline 177 & $\checkmark$ & IT - CADOPS (Tested previously) & Fri 5/19/06 & Fri 5/19/06 \\
\hline 178 & & IT - FeederAll -Not tested - delayed by IT interface until post-AEDR implementation & Mon 3/13/06 & Mon 3/13/06 \\
\hline 179 & $\checkmark$ & IT - Gas \& Elec Service Cards & Thu 5/11/06 & Thu 5/11/06 \\
\hline 180 & 国 & IT - Facility Browser - Not tested - delayed to Phase II & Thu 5/11/06 & Thu 5/11/06 \\
\hline 181 & & IT - Gas \& Elec Service Card Application - Not tested - delayed to Phase II & Thu 5/11/06 & Fri 5/12/06 \\
\hline 182 & 国 & IT - Field Browser Export - Not tested - Delayed to Phase II & Fri 5/12/06 & Fri 5/12/06 \\
\hline 183 & $\checkmark$ & IT - Session Manager & Tue 5/9/06 & Thu 5/11/06 \\
\hline 184 & $\checkmark$ & Integration testing - EDFS \& Interfaces & Mon 5/8/06 & Fri 5/19/06 \\
\hline 185 & $\checkmark$ & IT - EDFS SAGE \& ArcFM & Mon 5/8/06 & Fri 5/19/06 \\
\hline 186 & $\checkmark$ & IT - MLOG Interface & Mon 5/8/06 & Fri 5/19/06 \\
\hline 187 & $\checkmark$ & IT - GL Interface & Mon 5/8/06 & Fri 5/19/06 \\
\hline 188 & $\checkmark$ & IT - MAPPS Interface & Mon 5/8/06 & Fri 5/19/06 \\
\hline 189 & $\checkmark$ & IT - EAIF Interface & Mon 5/8/06 & Fri 5/19/06 \\
\hline 190 & $\checkmark$ & IT - CIS Interface & Mon 5/8/06 & Fri 5/19/06 \\
\hline 191 & $\checkmark$ & IT - GIS to DWG Export (tested stand-alone in CAD Converter section) & Fri 5/12/06 & Fri 5/12/06 \\
\hline 192 & $\checkmark$ & IT - NPMS & Fri 5/12/06 & Fri 5/12/06 \\
\hline 193 & & User Acceptance Testing (UAT) on Fully Configured Environment & Mon 5/15/06 & Fri 5/19/06 \\
\hline 194 & $\checkmark$ & UAT - Standard configuration (create/maintain features) & Mon 5/15/06 & Fri 5/19/06 \\
\hline 195 & $\checkmark$ & UAT - Auto Updaters & Mon 5/15/06 & Fri 5/19/06 \\
\hline 196 & $\checkmark$ & UAT - CADOPS - Tested Previously & Mon 5/15/06 & Mon 5/15/06 \\
\hline 197 & 国 & UAT - FeederAll - Not tested - delayed to Phase II & Tue $5 / 16 / 06$ & Tue 5/16/06 \\
\hline 198 & 国 & UAT - Facility Browser - Not tested - delayed to Phase II & Tue 5/16/06 & Tue 5/16/06 \\
\hline 199 & 国 & UAT - Field Browser - Not tested - delayed to Phase II & Wed 5/17/06 & Wed 5/17/06 \\
\hline 200 & $\checkmark$ & UAT - SynerGEE - Tested Previously & Tue 5/16/06 & Tue 5/16/06 \\
\hline 201 & $\checkmark$ & UAT - EDFS Replacement & Tue 5/16/06 & Fri 5/19/06 \\
\hline 202 & $\checkmark$ & UAT - EDFS SAGE \& ArCFM & Tue 5/16/06 & Wed 5/17/06 \\
\hline 203 & $\checkmark$ & UAT - MLOG Interface & Thu 5/18/06 & Thu 5/18/06 \\
\hline 204 & $\checkmark$ & UAT - GL Interface & Thu 5/18/06 & Thu 5/18/06 \\
\hline 205 & $\checkmark$ & UAT - MAPPS Interface & Thu 5/18/06 & Thu 5/18/06 \\
\hline 206 & $\checkmark$ & UAT - EAIF Interface & Thu $5 / 18 / 06$ & Thu 5/18/06 \\
\hline 207 & $\checkmark$ & UAT - CIS Interface & Fri 5/19/06 & Fri 5/19/06 \\
\hline 208 & $\checkmark$ & UAT - GIS to DWG Export & Tue 5/16/06 & Tue 5/16/06 \\
\hline 209 & $\checkmark$ & Data Model Changes Based on Testing Re-Work & Mon 2/2/04 & Mon 5/22/06 \\
\hline 210 & $\checkmark$ & Completion of electric domain approvals & Mon 2/2/04 & Fri 6/4/04 \\
\hline 211 & $\checkmark$ & Completion of land and gas domain verification & Wed 3/22/06 & Fri 5/19/06 \\
\hline 212 & $\checkmark$ & Apply Data Model Changes & Mon 4/10/06 & Mon 5/22/06 \\
\hline 213 & $\checkmark$ & Work Arounds & Mon 5/22/06 & Mon 5/22/06 \\
\hline 214 & $\checkmark$ & Domain Changes & Mon 5/22/06 & Mon 5/22/06 \\
\hline 215 & $\checkmark$ & Aerial Photography & Mon 3/13/06 & Mon 3/13/06 \\
\hline
\end{tabular}


AEDR Deployment Phase I Project Plan

\begin{tabular}{|c|c|c|c|c|}
\hline ID & (i) & Task Name & Start & Finish \\
\hline 216 & $\checkmark$ & Determine where these files will permanently reside & Mon 3/13/06 & Mon 3/13/06 \\
\hline 217 & & Disaster Recovery Plan & Mon 5/15/06 & Mon 5/15/06 \\
\hline 218 & 国 & Develop the disaster recovery plan & Mon 5/15/06 & Mon 5/15/06 \\
\hline 219 & $\checkmark$ & Training Program & Mon 10/17/05 & Tue $6 / 13 / 06$ \\
\hline 220 & $\checkmark$ & Design - Develop Training Plan & Mon 10/17/05 & Wed 10/19/05 \\
\hline 221 & $\checkmark$ & Conduct training needs assessment & Mon 2/20/06 & Wed 2/22/06 \\
\hline 222 & $\checkmark$ & Assign userlD's to SQL instance & Mon 4/10/06 & Fri 4/28/06 \\
\hline 223 & $\checkmark$ & Test hardware \& document recommendation & Mon 4/3/06 & Fri 4/28/06 \\
\hline 224 & $\checkmark$ & Determine training instance $-(\mathrm{CITRIX})$ & Mon 4/3/06 & Fri 4/28/06 \\
\hline 225 & $\checkmark$ & Create \& deliver training manuals for GIS Team & Mon 2/27/06 & Mon 4/17/06 \\
\hline 226 & $\checkmark$ & Develop training environment & Mon 4/17/06 & Tue 5/2/06 \\
\hline 227 & $\checkmark$ & Train GIS staff & Mon 4/17/06 & Thu $4 / 20 / 06$ \\
\hline 228 & $\checkmark$ & Train record clerks week 1 & Mon 5/1/06 & Fri 5/5/06 \\
\hline 229 & $\checkmark$ & Train record clerks week 2 & Mon 5/15/06 & Fri 5/19/06 \\
\hline 230 & $\checkmark$ & Assist record clerks in Practice 1 & Mon 5/15/06 & Thu 5/18/06 \\
\hline 231 & $\checkmark$ & Assist record clerks in Practice 2 & Fri 5/19/06 & Tue $6 / 13 / 06$ \\
\hline 232 & $\checkmark$ & Implementation Plan & Wed 12/21/05 & Fri 5/26/06 \\
\hline 233 & $\checkmark$ & Develop first draft of the plan & Wed 12/21/05 & Wed 12/21/05 \\
\hline 234 & $\checkmark$ & Review \& adjust the Implementation Plan tasks & Mon 3/13/06 & Mon 3/13/06 \\
\hline 235 & $\checkmark$ & Develop white board of Implementation Plan tasks & Mon 3/20/06 & Mon $3 / 20 / 06$ \\
\hline 236 & $\checkmark$ & On-going implementation plan adjustments & Mon 3/20/06 & Fri 5/26/06 \\
\hline 237 & $\checkmark$ & Transition Phase - Production Rollout & Mon 4/24/06 & Fri 12/29/06 \\
\hline 238 & $\checkmark$ & Execute the Implementation Plan & Mon 4/24/06 & Fri 6/30/06 \\
\hline 239 & $\checkmark$ & Address data cleanup concerns from critical mismatch reports & Fri 12/29/06 & Fri 12/29/06 \\
\hline 240 & $\checkmark$ & Install SynerGEE on users PC and run Data Prep & Mon 6/12/06 & Mon 6/12/06 \\
\hline 241 & $\checkmark$ & Application production rollout support & Mon 6/12/06 & Mon 8/7/06 \\
\hline
\end{tabular}


Appendix B

Migration QA Plan Checklist 


\begin{tabular}{|c|c|c|c|c|c|}
\hline Start & End & $\begin{array}{c}\% \\
\text { Complete }\end{array}$ & Test Steps & Resources & Notes / Results \\
\hline & & & Pre-QA Setup & & $\begin{array}{l}\text { The purpose for this task is to prepare both the } \\
\text { QA environment and the QA team. }\end{array}$ \\
\hline $5 / 16$ - 8:00am & $5 / 18-$ noon & $0 \%$ & Perform various QA setup tasks & & \\
\hline $5 / 12-$ noon & 5/19 - 9:00pm & $0 \%$ & Create tracklists for the delivery & & \\
\hline $5 / 19-2: 00 p m$ & $5 / 19-2: 30 p m$ & $0 \%$ & $\begin{array}{l}\text { Print graphic specs plot for the features } \\
\text { (symbology legend) }\end{array}$ & & \\
\hline $5 / 19$ - 8:00am & $5 / 19-5: 00 p m$ & $0 \%$ & Update feature count $Q A$ tracklists with the counts & & \\
\hline $5 / 18-8: 00 a m$ & $5 / 18-$ noon & $0 \%$ & Set up $X$ drive folders & & \\
\hline $5 / 16-11: 30 a m$ & $5 / 16-11: 30 a m$ & $0 \%$ & Schedule DBA to assist with the installation & & \\
\hline $5 / 12-$ noon & $5 / 12-$ noon & $0 \%$ & $\begin{array}{l}\text { Verify any changes to the instances used for the } \\
\text { installation of migrated data }\end{array}$ & & \\
\hline $5 / 19-12: 30 p m$ & $5 / 22-11: 25 a m$ & $0 \%$ & Set up the hardware for QA team & & \\
\hline $5 / 16-8: 30 a m$ & $5 / 16-8: 30 a m$ & $0 \%$ & $\begin{array}{l}\text { Notify IT server group regarding upcoming delivery } \\
\text { and QA }\end{array}$ & & \\
\hline STEP 1 & & & $\begin{array}{l}\text { Install the Data in the Test/Migration Instance \& } \\
\text { Prod/Test Instance }\end{array}$ & & $\begin{array}{l}\text { The purpose for this task is to prepare the data } \\
\text { for QA processing. }\end{array}$ \\
\hline $5 / 16$ - 8:30am & $5 / 16-$ noon & $0 \%$ & Download data and files from FTP server & & \\
\hline $5 / 16-$ noon & $5 / 17-3: 00$ & $0 \%$ & High level review of reports \& artifacts & & \\
\hline $5 / 16-8: 30$ & $5 / 17-$ noon & $0 \%$ & $\begin{array}{l}\text { Copy migrated data and artifacts to a shared } \\
\text { directory }\end{array}$ & & \\
\hline $5 / 16-4: 00 a m$ & $5 / 17-$ noon & $0 \%$ & Restore the BAK file to Mig \& Prod & & \\
\hline STEP 2 & & & High Level/Preliminary QA & & $\begin{array}{l}\text { The purpose for this set of tasks is to make sure } \\
\text { that vendor delivered everything that will be } \\
\text { required for the migration QA. }\end{array}$ \\
\hline 5/17-am & 5/17-am & $0 \%$ & $\begin{array}{l}\text { Document the environment change per the SDE } \\
\text { Management Plan }\end{array}$ & & \\
\hline $5 / 18$ - 7:00am & 5/18 - 7:15am & $0 \%$ & $\begin{array}{l}\text { Verify that the schema of the geodatabase from } \\
\text { vendor is correct }\end{array}$ & & \\
\hline
\end{tabular}


Migration QA Plan Checklist

\begin{tabular}{|c|c|c|c|c|c|}
\hline Start & End & $\begin{array}{c}\% \\
\text { Complete } \\
\end{array}$ & Test Steps & Resources & Notes / Results \\
\hline 5/17-am & 5/17-am & $0 \%$ & $\begin{array}{l}\text { Verify the delivery artifacts against the Delivery } \\
\text { Checklist }\end{array}$ & & \\
\hline $5 / 19-a m$ & $5 / 19-a m$ & $0 \%$ & Run the source count compare script & & \\
\hline $5 / 18-p m$ & 5/19 - 9:00pm & $0 \%$ & $\begin{array}{l}\text { Pre-populate the Interactive \& Object Existence QA } \\
\text { spreadsheets }\end{array}$ & & \\
\hline $5 / 17-8: 30 a m$ & $5 / 17-4: 00 p m$ & $0 \%$ & Check for missing tiles & & \\
\hline $5 / 18-8: 45 a m$ & $5 / 19-4: 00 p m$ & $0 \%$ & Conduct object existence test & & \\
\hline 5/17 - 11:30am & $5 / 18-9: 30 a m$ & $0 \%$ & Run automated QA routines against new data & & \\
\hline $5 / 17-11: 30 a m$ & $5 / 18$ - 9:30am & $0 \%$ & $\begin{array}{l}\text { Compare NIPSCO automated QA reports against } \\
\text { vendor automated QA reports }\end{array}$ & & \\
\hline \multirow[t]{3}{*}{$5 / 22$ - 7:30am } & $5 / 22-8: 00$ & $0 \%$ & $\begin{array}{l}\text { Setup individual QA folders \& assignment } \\
\text { spreadsheet }\end{array}$ & & \\
\hline & & $0 \%$ & $\begin{array}{l}\text { CHECKPOINT: Authorization to proceed with QA } \\
\text { or rejection of the delivery }\end{array}$ & & \\
\hline & & & Build Production Instance & & $\begin{array}{l}\text { The purpose for this set of tasks is to prepare } \\
\text { the production instance for parallel processing }\end{array}$ \\
\hline $5 / 18-$ noon & $5 / 20-11: 00$ & $0 \%$ & Build the parallel production instance & & \\
\hline $5 / 20-11: 00 \mathrm{am}$ & $5 / 25-8: 55 \mathrm{am}$ & $0 \%$ & Run data cleanup and NIPSCO migration scripts & & \\
\hline $5 / 25-9: 00 \mathrm{am}$ & $6 / 2-7: 30 \mathrm{am}$ & $0 \%$ & Configure the geodatabase & & \\
\hline
\end{tabular}




\begin{tabular}{|c|c|c|c|c|c|}
\hline Start & End & $\begin{array}{c}\% \\
\text { Complete } \\
\end{array}$ & Test Steps & Resources & Notes / Results \\
\hline 6/2 - 9:30am & $6 / 7-5: 00$ & $0 \%$ & QA parallel production instance & & \\
\hline STEP 3 & & & Detail Level QA & & $\begin{array}{l}\text { The purpose for this set of tasks is to verify the } \\
\text { accuracy of the delivered data using multiple } \\
\text { types of QA processes }\end{array}$ \\
\hline \multirow[t]{2}{*}{$5 / 22-9: 00$} & 6/7 - 12:30pm & $0 \%$ & $\begin{array}{l}\text { Ready for review of corrected items from previous } \\
\text { deliveries }\end{array}$ & & \\
\hline & & & Feature Count QA - Electric & & $\begin{array}{l}\text { The purpose of this task is to provide a baseline } \\
\text { count of electric data sent for comparison of } \\
\text { data returned to ensure data is not missing nor } \\
\text { added }\end{array}$ \\
\hline 5/19 - 10:00am & $5 / 21-1: 00 p m$ & $0 \%$ & $\begin{array}{l}\text { Count ArcGIS objects from Outfield and update the } \\
\text { tracklist }\end{array}$ & & \\
\hline $5 / 22-1: 00 p m$ & $5 / 24-3: 00 p m$ & $0 \%$ & $\begin{array}{l}\text { Count ArcGIS objects from EDFS and update the } \\
\text { tracklist }\end{array}$ & & \\
\hline \multirow[t]{2}{*}{$5 / 22-4: 00 p m$} & $5 / 22-5: 30$ & $0 \%$ & $\begin{array}{l}\text { Count ArcGIS objects from annotation and update } \\
\text { the tracklist }\end{array}$ & & \\
\hline & & & Feature Count QA - Gas & & $\begin{array}{l}\text { The purpose of this task is to provide a baseline } \\
\text { count of gas data sent for comparison of data } \\
\text { returned to ensure data is not missing nor } \\
\text { added }\end{array}$ \\
\hline $5 / 22$ - 9:30am & $5 / 22$ - 3:00pm & $0 \%$ & $\begin{array}{l}\text { Count ArcGIS objects from Outfield and update } \\
\text { tracklist }\end{array}$ & & \\
\hline \multirow[t]{2}{*}{$5 / 22$ - 3:00pm } & $5 / 23-1: 30 p m$ & $0 \%$ & $\begin{array}{l}\text { Count ArcGIS objects from annotation and update } \\
\text { the tracklist }\end{array}$ & & \\
\hline & & & Feature Count QA - Land & & $\begin{array}{l}\text { The purpose of this task is to provide a baseline } \\
\text { count of land data sent for comparison of data } \\
\text { returned to ensure data is not missing nor } \\
\text { added }\end{array}$ \\
\hline
\end{tabular}




\begin{tabular}{|c|c|c|c|c|c|}
\hline Start & End & $\begin{array}{c}\% \\
\text { Complete } \\
\end{array}$ & Test Steps & Resources & Notes / Results \\
\hline $5 / 23-1: 30 p m$ & $5 / 24$ - 10:30am & $0 \%$ & $\begin{array}{l}\text { Count ArcGIS objects from Outfield and update } \\
\text { tracklist }\end{array}$ & & \\
\hline \multirow[t]{2}{*}{$5 / 23-4: 00 p m$} & $5 / 24$ - 10:30am & $0 \%$ & $\begin{array}{l}\text { Count ArcGIS objects from annotation and update } \\
\text { the tracklist }\end{array}$ & & \\
\hline & & & Feature Count QA - Reconcile Counts & & $\begin{array}{l}\text { Verify vendor delivered data is not missing } \\
\text { features or introducing features not previously } \\
\text { sent }\end{array}$ \\
\hline $5 / 24$ - 11:00am & $5 / 24-11: 30 a m$ & $0 \%$ & Apply the dissolve and split counts to the tracklist & & \\
\hline \multirow[t]{2}{*}{$5 / 24$ - 11:00am } & $5 / 24-11: 30 a m$ & $0 \%$ & $\begin{array}{l}\text { Compare internal source target counts and } \\
\text { compare with vendor counts }\end{array}$ & & \\
\hline & & & Automated QA & & $\begin{array}{l}\text { The purpose of this task is to quickly identify } \\
\text { data anamolies though the use of automated } \\
\text { routines. Data may fail an automated routine, } \\
\text { yet may still be valid, therefore visual inspection } \\
\text { is necessary. }\end{array}$ \\
\hline $5 / 18-10: 00 a m$ & $5 / 19-12: 00 p m$ & $0 \%$ & Copy all failed electric test info into the tracklist & & \\
\hline 5/18 - 10:00am & $5 / 19-12: 00 p m$ & $0 \%$ & Copy all failed gas test info into the tracklist & & \\
\hline $5 / 18-10: 00 a m$ & $5 / 19-12: 00 p m$ & $0 \%$ & Copy all failed land test info into the tracklist & & \\
\hline $5 / 22-10: 00 a m$ & $5 / 25-6: 00 p m$ & $0 \%$ & Automated QA - Electric & & \\
\hline $5 / 22$ - 10:00am & $5 / 24-6: 00 p m$ & $0 \%$ & Automated QA - Gas & & \\
\hline \multirow[t]{2}{*}{$5 / 22-2: 30 p m$} & $5 / 24-6: 00 p m$ & $0 \%$ & Automated QA - Land & & \\
\hline & & & First Pass Connectivity & & $\begin{array}{l}\text { The purpose of this task is to determine } \\
\text { geometric connectivity. (High Level) }\end{array}$ \\
\hline $5 / 22-1: 00$ & $5 / 26$ - 5:30 PM & $0 \%$ & $\begin{array}{l}\text { Determine sample set of gas and electric networks } \\
\text { across LOAs and batches }\end{array}$ & & \\
\hline
\end{tabular}




\begin{tabular}{|c|c|c|c|c|c|}
\hline Start & End & $\begin{array}{c}\% \\
\text { Complete } \\
\end{array}$ & Test Steps & Resources & Notes / Results \\
\hline $5 / 22-1: 00$ & $5 / 26-5: 30$ PM & $0 \%$ & $\begin{array}{l}\text { Trace each sample network and record the total } \\
\text { features traced in the tracklist }\end{array}$ & & \\
\hline \multirow[t]{2}{*}{$5 / 22-1: 00$} & $5 / 26$ - 5:30 PM & $0 \%$ & $\begin{array}{l}\text { Test each stop point and determine if the stop } \\
\text { points are valid }\end{array}$ & & \\
\hline & & & Second Pass Connectivity & & $\begin{array}{l}\text { The purpose of this task is to determine } \\
\text { geometric connectivity. (Low Level) }\end{array}$ \\
\hline $6 / 5-1: 00$ PM & 6/5 - 4:30 PM & $0 \%$ & $\begin{array}{l}\text { Determine sample set of gas and electric networks } \\
\text { across LOAs and batches }\end{array}$ & & \\
\hline \multirow[t]{2}{*}{$6 / 5-1: 00 \mathrm{PM}$} & 6/5 - 4:30 PM & $0 \%$ & Examine each sample network & & \\
\hline & & & Interactive QA & & $\begin{array}{l}\text { The purpose of this task is to visually inspect } \\
\text { the appropriate sample of the data to determine } \\
\text { accuracy and completeness }\end{array}$ \\
\hline 5/23 - 9:00am & $5 / 26-1: 30 p m$ & $0 \%$ & Interactive QA Electric & & \\
\hline $5 / 23$ - 10:00am & $5 / 24$ - 6:00pm & $0 \%$ & Interactive QA Gas & & \\
\hline $5 / 24$ - 8:00am & $5 / 24-6: 00 p m$ & $0 \%$ & Interactive QA Land & & \\
\hline STEP 8 & & & Miscellaneous & & \\
\hline $5 / 26-$ noon & & $0 \%$ & $\begin{array}{l}\text { Team summary review of all QA testing and } \\
\text { determination if any additional items or issues } \\
\text { need to be investigated. }\end{array}$ & & \\
\hline
\end{tabular}


Appendix C

GIS System Performance Testing 


\section{Table of Contents}

1. Introduction

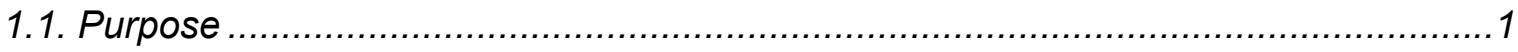

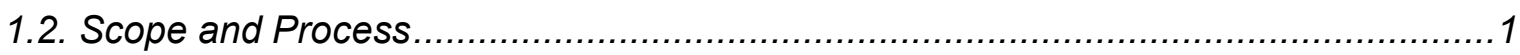

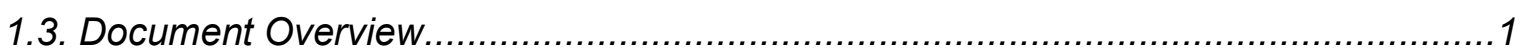

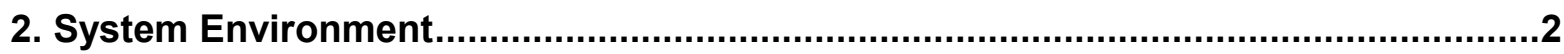

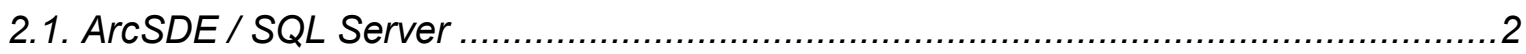

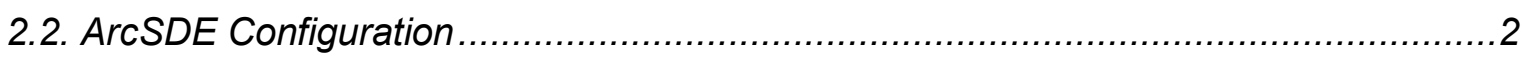

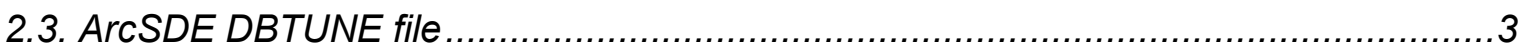

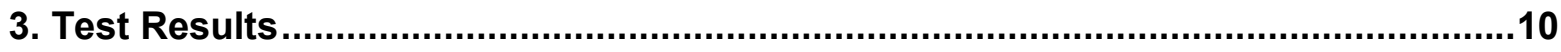

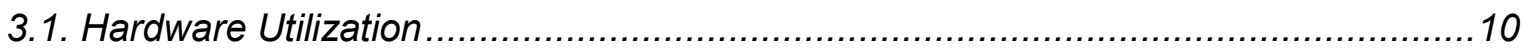

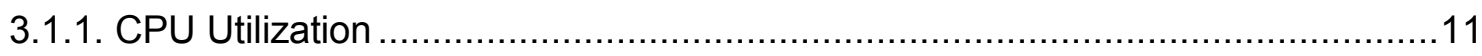

3.1.2. Physical Disk Queue ...............................................................................

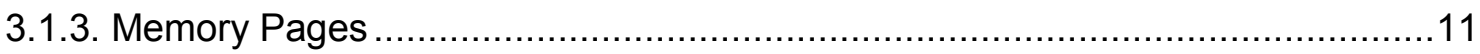

3.2. SQL Performance Indicators.......................................................................... 11

3.2.1. Buffer Cache Hit Ratio..........................................................................

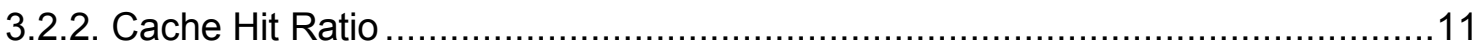

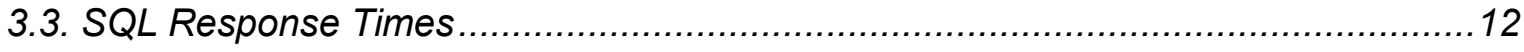

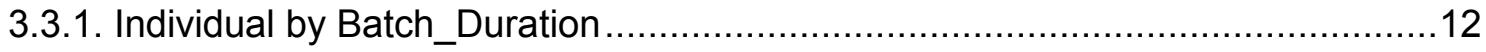

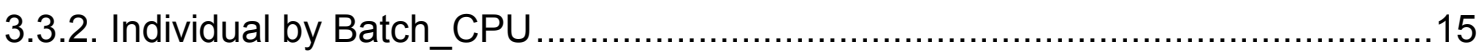

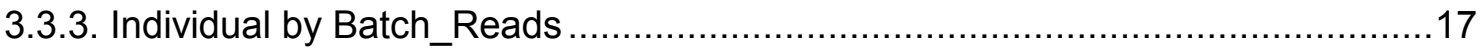

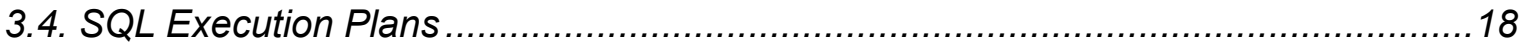

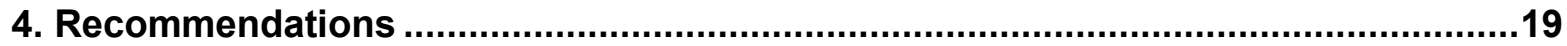

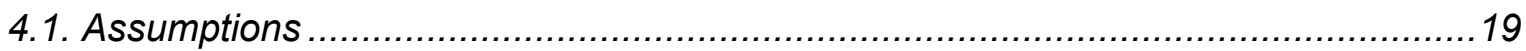

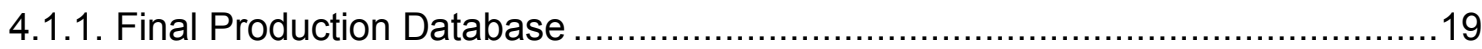




\section{Introduction}

\subsection{Purpose}

The purpose of this document is to present the results of a GIS Performance Testing initiative for the NIPSCO AEDR implementation. The NIPSCO AEDR will utilize Microsoft's SQL Server Relational Database Management System (RDBMS) with ESRI's ArcSDE for spatial database management. The AEDR will incorporate ESRI's ArcGIS and Miner and Miner's ArcFM products for the client front-end, as well as an ASP.NET Web application for non-graphical data updates.

\subsection{Scope and Process}

The scope of this document is limited to the performance testing of the SDE / SQL Server database server.

For this GIS Performance Testing initiative, NIPSCO gathered 32 users to simulate the workload that is expected once the system is fully utilized in production. Several test scripts were written by NIPSCO project team personnel, and these scripts were randomly given to the test users. All testing occurred on Friday, April 21, 2006 during an all-day testing event.

The performance tuning test period was broken down into two sessions. The first session (from 8am until lunch) was a "training" session for the users. Since most of the users had not been trained on ArcGIS or ArcFM, this session allowed them to become acquainted with the system and to practice their test scripts. The second session (from 1pm until 4pm) was the live "testing" session where performance statistics were gathered on the servers while users performed the actions in their test scripts.

Performance statistics were gathered using Microsoft's PERFMON utility, as well as SQL Server's PROFILER application. PERFMON allows statistics to be gathered against any user-defined criteria on the server, measuring hardware and software performance. The SQL Profiler allows a DBA to capture all SQL calls performed by the database, as well as database execution plans and response times for those SQL queries.

\subsection{Document Overview}

This document is divided into the following sections:

- Section 1 Introduction - States the document's purpose and scope, and provides a high-level overview of the rest of the document.

- Section 2 System Environment - defines the system environment under which the System Performance Testing was performed.

- Section 3 Test Results - presents the performance testing initiative results

- Section 4 Recommendations - presents any recommendations for tuning the ArcSDE / SQL Server database server. 


\section{System Environment}

\subsection{ArcSDE / SQL Server}

The system specifications of the ArcSDE / SQL Server production server are as follows:

\begin{tabular}{|l|l|}
\hline Characteristic & Value \\
\hline \hline Server Name & aedrdbsqlp01 \\
\hline Server Make/Model & IBM X445 \\
\hline Server CPUs & 4 CPUs @ 2.8 GHz \\
\hline Physical RAM & 4 GB \\
\hline OS & Windows 2003 Server with Service Pack 1 \\
\hline SQL Server & Microsoft SQL Server 2000 with Service Pack 4 \\
\hline Physical Disks & $2 \times 36 G B$ mirrored for Operating System. \\
\hline Data files & All data files located on SAN \\
\hline ArcSDE Version & 8.3 with Service Pack 3 \\
\hline
\end{tabular}

\subsection{ArcSDE Configuration}

The following file contains the ArcSDE configuration parameters:

LAYERS $\quad 500$ - maximum number of layers

REGISTRATIONS 1000 - maximum number of registered tables

CONNECTIONS 64 - maximum number of connections

STATELOCKS 10000 - maximum number of state locks

MAXTABLELOCKS 10000 - maximum number of table locks

OBJECTLOCKS 10000 - maximum number of object locks

RASTERCOLUMNS 500 - maximum number of raster columns

RASTERBUFSIZE 102400 - maximum raster buffer size

Increase the MINBUFSIZE and the MAXBUFSIZE when mass loading data. These values will provide optimum loading performance for most SDE servers.

MINBUFSIZE 409600 - minimum buffer size $>4096$

MAXBUFSIZE 819200 - maximum buffer size > MINBUFSIZE

Decrease the MINBUFSIZE and MAXBUFSIZE after the database has been loaded and before it is opened to the general public. Take caution when increasing the size of MAXBUFSIZE since SDE allocates MAXBUFSIZE bytes for each stream a SDE client has opened. For instance, if 50 users are connected to SDE and each has 5 streams opened and the MAXBUFSIZE is set 64 kilobytes, 16000 kilobytes of server memory are allocated to the transport buffers. 


\begin{tabular}{|c|c|}
\hline \\
\hline \multicolumn{2}{|c|}{$\begin{array}{l}\text { MAXBUFSIZE } 65536 \text { - maximum buffer size > MINBUFSIZE } \\
\text { MINBUFOBJECTS } 512 \text { - minimum objects per buffer }\end{array}$} \\
\hline & 00 - total number of locks for the system \\
\hline MAXTIMEDIFF & 1 - maximum client/server time difference (seconds) \\
\hline MAXBLOBSIZE & 100000 \\
\hline BLOBMEM & 00000 - Maximum BLO \\
\hline AUTOCOMMIT & 1000 - Auto-commit frequency within user transaction \\
\hline MAXINITIALFEA & 10000 - Max. Initial Features/Layer \\
\hline MAXDISTINCT & Max. number of unique values for attribute stats \\
\hline MAXSTREAMS & umber of streams \\
\hline SHAPEPTSBUFSIZE & TS array \\
\hline ATTRBUFSIZE & fer size \\
\hline MAXARRAYSIZE & 100 - Max. array fe \\
\hline MAXARRAYBYTES & Max. array bytes allocated per stream \\
\hline STREAMPOOLSIZE & \\
\hline STATECACHING & E - Toggles state cachi \\
\hline $\begin{array}{lr}\text { TCPKEEPALIVE } & F \\
\text { READONLY } & \text { FA }\end{array}$ & $\begin{array}{l}\text { FALSE - TCP keepalive on client connections } \\
\text { ALSE - ArcSDE service is or is not read only. }\end{array}$ \\
\hline
\end{tabular}

\subsection{ArcSDE DBTUNE file}

The following lists the ArcSDE DBTUNE parameters. These parameters define storage characteristics for the SDE data stored in SQL Server:

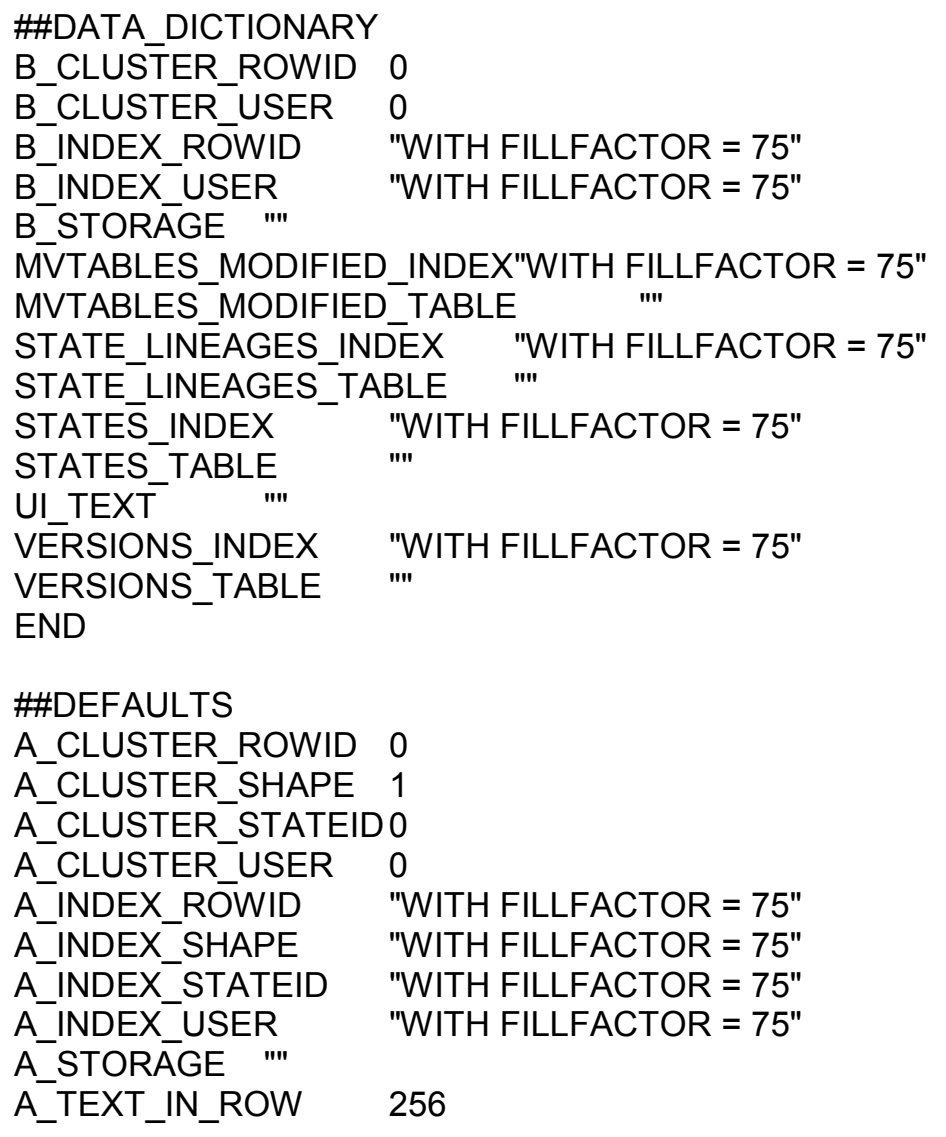




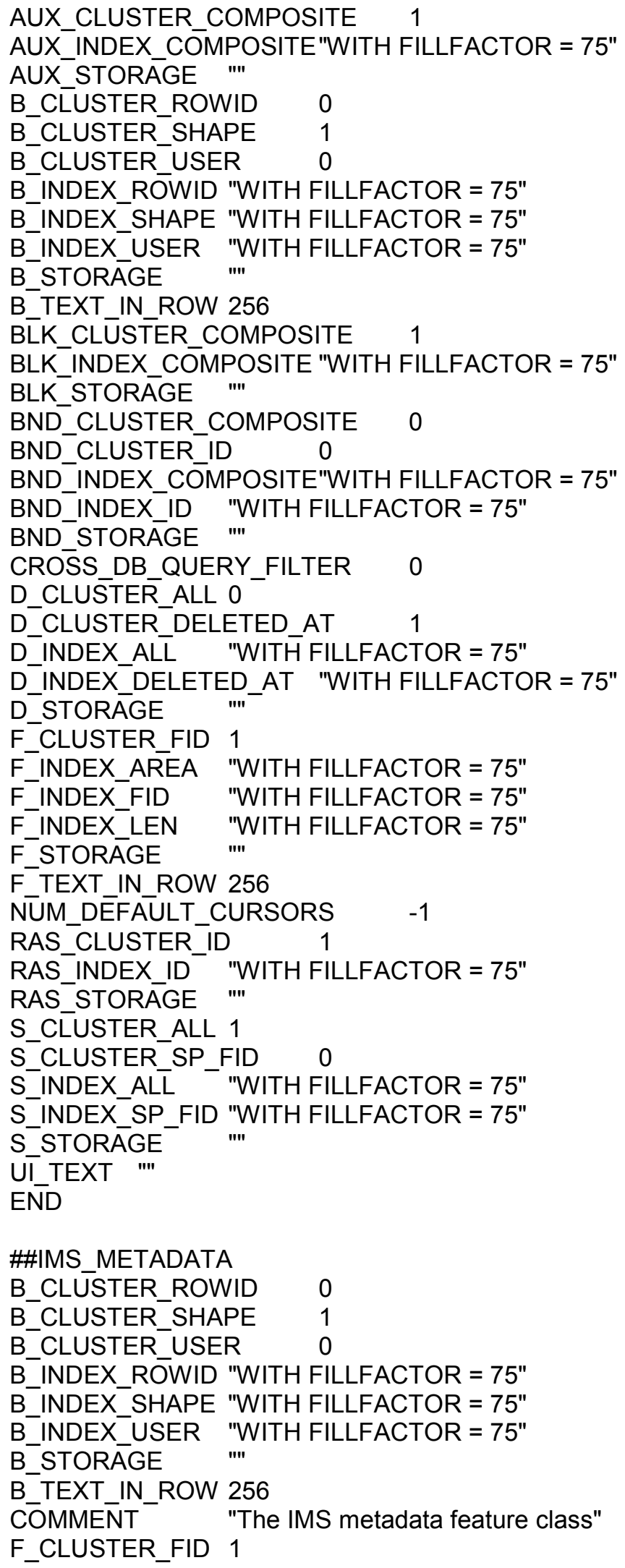




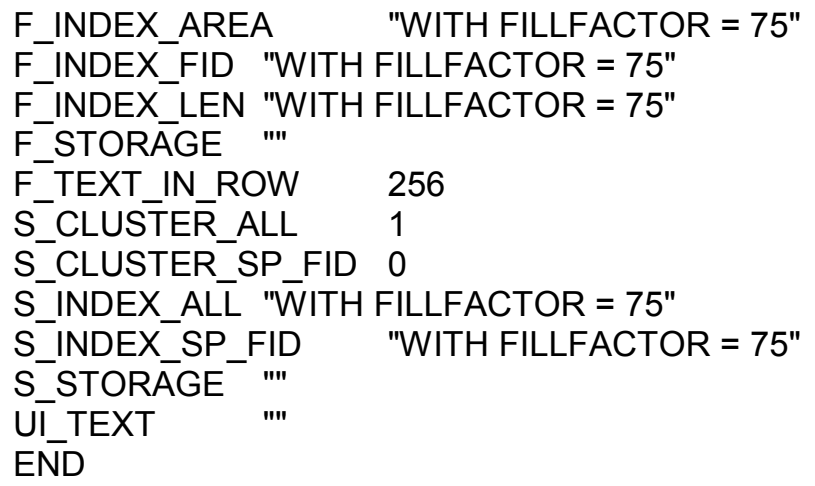




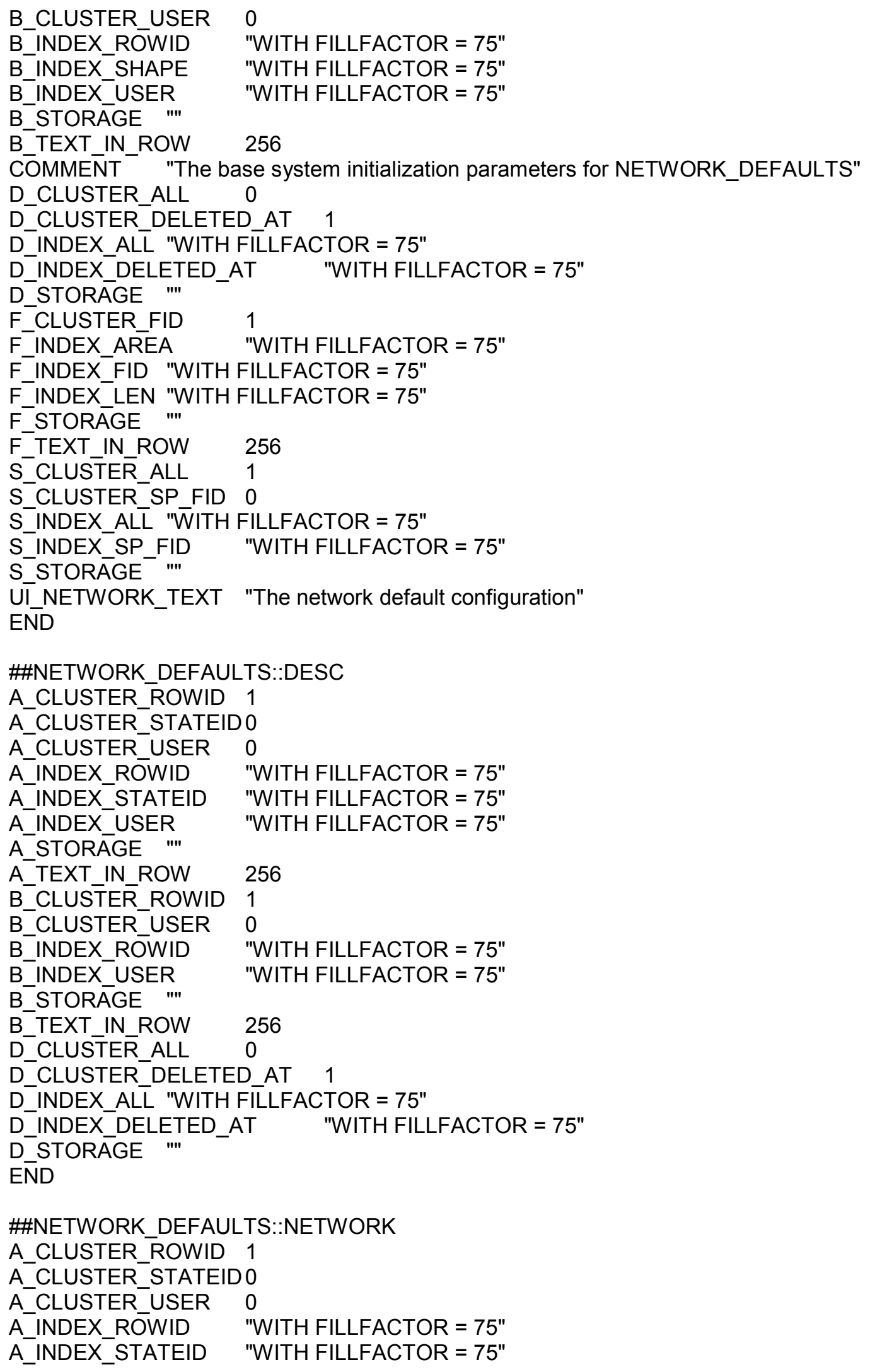




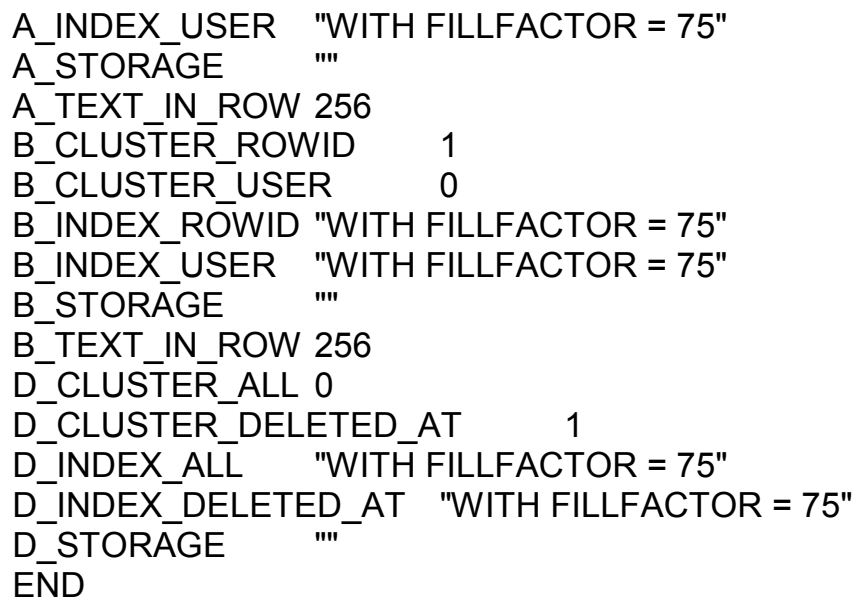


S_STORAGE "'"

UI_TOPOLOGY_TEXT "The topology default configuration" END

\#\#TOPOLOGY_DEFAULTS::DIRTYAREAS

A_CLUSTER_ROWID 0

A_CLUSTER_SHAPE 1

A_CLUSTER_STATEID 0

A CLUSTER USER 0

A_INDEX_ROWWID "WITH FILLFACTOR $=75 "$

A_INDEX_SHAPE "WITH FILLFACTOR $=75 "$

A_INDEX_STATEID "WITH FILLFACTOR $=75 "$

A_INDEX_USER "WITH FILLFACTOR $=75 "$

A STORAGE "'"

A_TEXT_IN_ROW 256

B_CLUSTER_ROWID 0

B_CLUSTER_SHAPE 1

B_CLUSTER_USER 0

B_INDEX_RŌWID "WITH FILLFACTOR $=75 "$

B_INDEX_SHAPE "WITH FILLFACTOR $=75 "$

B_INDEX_USER "WITH FILLFACTOR $=75 "$

B_STORAGE "'"

B_TEXT_IN_ROW 256

D_CLUSTETER_ALL 0

D_CLUSTER_DELETED_AT 1

D_INDEX_ALL "WITH FILLFACTOR $=75$ "

D_INDEX_DELETED_AT "WITH FILLFACTOR $=90 "$

D STORAGE "'"

F_CLUSTER_FID 1

F_INDEX_AREA "WITH FILLFACTOR $=75 "$

F INDEX_FID "WITH FILLFACTOR $=75$ "

F_INDEX_LEN "WITH FILLFACTOR $=75$ "

F_STORAGE

F_TEXT_IN_ROW 256

S_CLUSTER_ALL 1

S_CLUSTER_SP_FID 0

$S$ INDEX ALL "W'ITH FILLFACTOR $=75$ "

S_INDEX_SP_FID "WITH FILLFACTOR $=75 "$

S STORAGE' "'"

ENDD 


\section{Test Results}

Based on the statistics collected during the performance testing period, the ArcSDE / SQL Server RDBMS server named aedrdbsqlp01 is already adequately tuned and is currently responding to queries as well as it can. Below are several performance indicators that show how well the server and database is performing.

\subsection{Hardware Utilization}

Figure C-1 shows three key hardware performance monitoring statistics that were captured during the performance testing period. Each of these statistics are described and analyzed below.

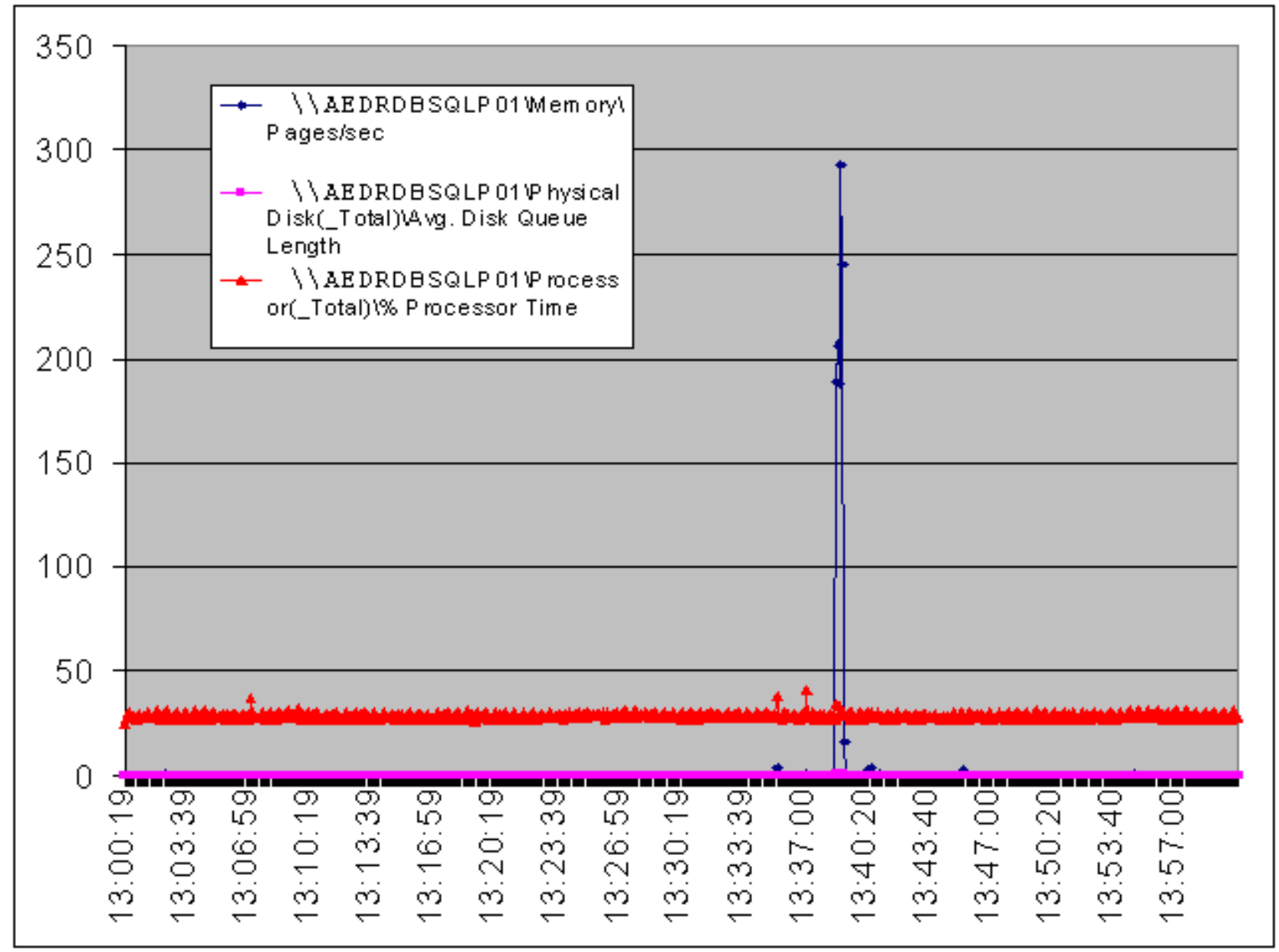

Figure C-1. Hardware performance monitoring statistics 


\subsubsection{CPU Utilization}

The red line shown in Figure C-1 illustrates the CPU utilization during the performance testing period. This is a measure of how "active" the CPUs in the server are during our performance testing. Between the hours of 1:00PM and 2:00pm on our testing day, CPU utilization averaged $28.8 \%$. This shows that the server is not over-utilized, and it actually has the capacity to perform a much higher workload.

\subsubsection{Physical Disk Queue}

The magenta line shown in Figure C-1 describes the Physical Disk Queue during the performance testing period. This is a measure of how many disk requests (both read and write requests) are sent to a queue while waiting on previous disks requests to complete. Essentially, this statistic indicates whether there is contention for the hard drives in the server. Between the hours of 1:00PM and 2:00PM on our testing day, there was no disk contention, as our average physical disk queue was 0.0138 .

\subsubsection{Memory Pages}

The blue line shown in Figure C-1 represents the Memory Pages / Second during the performance testing period. This performance indicator represents the rate at which memory pages are written to or read from disk. While there was one brief spike in the data (at approximately $1: 39 \mathrm{PM}$ ), there is no cause for concern. The average memory pages per second during our 1:00PM to 2:00PM time slice was only 1.6 pages per second. This indicates the server is utilizing its physical RAM properly and not writing memory pages to disk.

All three hardware performance indicators described above signify that the server (AEDRDBSQLP01) is performing well and is properly utilizing all major hardware subsystems (CPU, physical disk and physical memory).

\subsection{SQL Performance Indicators}

Figure C-2 shows two key SQL performance indicators captured on AEDRDBSQLP01 during our performance testing period. These two indicators are described and analyzed below.

\subsubsection{Buffer Cache Hit Ratio}

The first key indicator portrayed in Figure C-2 is the Buffer Cache Hit Ratio. This ratio defines what percentage of database pages were already found in memory (as opposed to being read from disk) and is recommended to run $>90 \%$ for best performance results. During our testing period, our Buffer Cache Hit Ratio averaged $>98 \%$. Figure 3.2 illustrates the period between 1:00PM and 2:00PM, where the Buffer Cache Hit Ratio averaged $99.858 \%$.

\subsubsection{Cache Hit Ratio}

The second key indicator in the graph below is the Cache Hit Ratio. This ratio is recommended to run $>70 \%$ for best performance results. The Cache Hit Ratio indicates how well the SQL that ArcSDE sends to the database can be "re-used", rather than "looked up". During our test period, the Cache Hit Ratio averaged 72.4\%. 


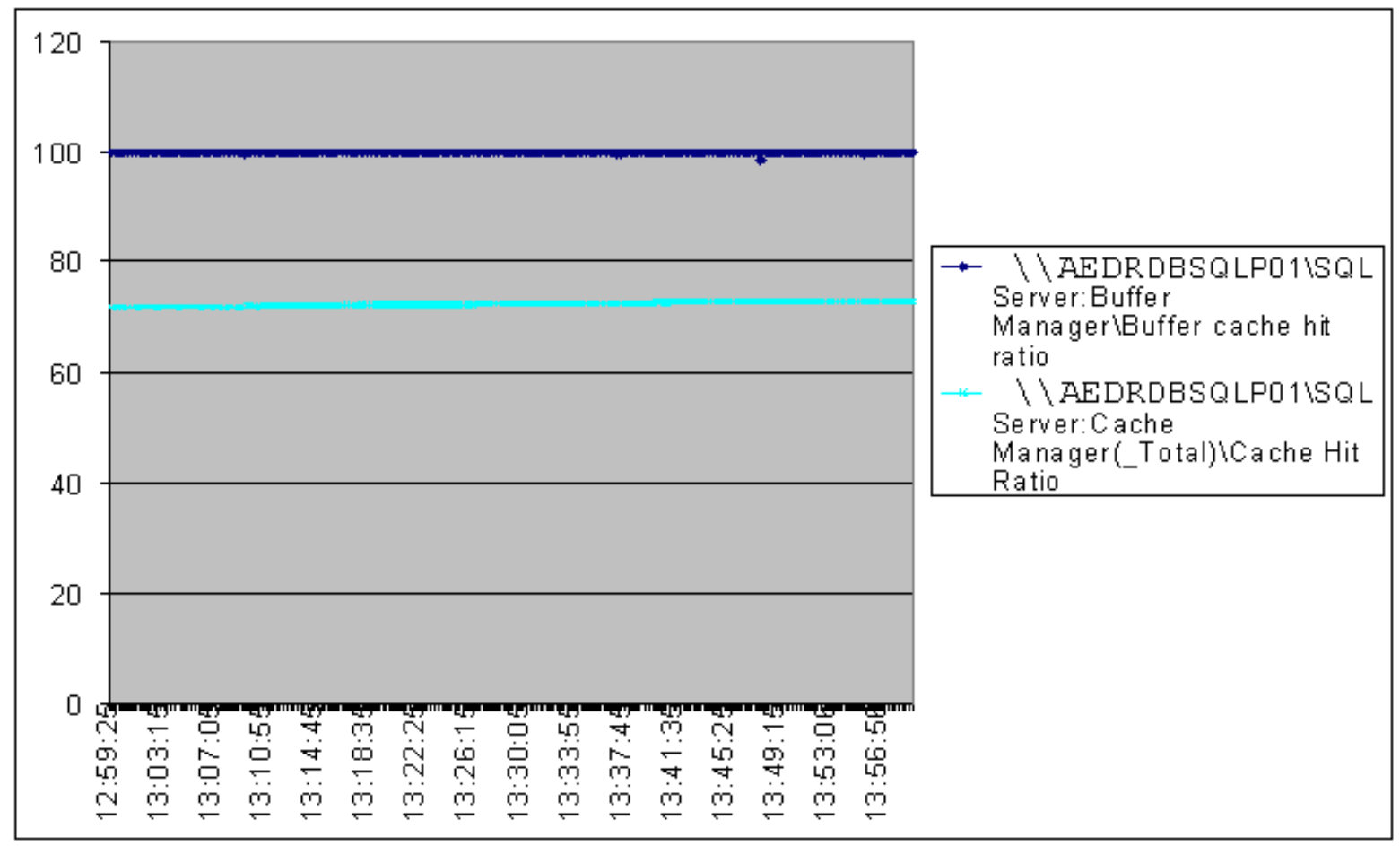

Figure C.2 - SQL Performance monitoring statistics

Both of these SQL performance metrics indicate that the SQL Server database is utilizing physical memory correctly.

\subsection{SQL Response Times}

During one period of testing, the SQL Profiler application was enabled, and all SQL queries into the database were monitored for execution plans and response times. The SQL Profiler output trace file was analyzed using a utility called "Read80Trace", a Microsoft utility available for download from their website.

\subsubsection{Individual by Batch_Duration}

Table C-1 shows individual SQL:BatchCompleted/RPC:Completed events with the longest duration. During the SQL Profiler tracing period, the longest running SQL statement was 110 milliseconds, or 0.110 seconds. This is an excellent query response time.

\begin{tabular}{|l|l|l|l|l|}
\hline Duration $(\mathbf{m s})$ & CPU (ms) & Reads & Writes & Query Format \\
\hline 110 & 94 & 15576 & 0 & $\begin{array}{l}\text { DELETE FROM ARCFM8.LANDBASE.S144 WHERE SP_FID IN(SELECT ) AND NOT } \\
\text { EXISTS (SELECT SHAPE FROM } \\
\text { ARCFM8.LANDBASE.QUARTERTOWNSHIPGRID_NUMBER_ANNO WHERE } \\
\text { ARCFM8.LANDBASE.QUARTERTOWNSHIPGRID_NUMBER_ANNO.SHAPE = } \\
\text { ARCFM8.LANDBASE.S144.SP_FID) AND NOT EXISTS (SELECT SHAPE FROM } \\
\text { ARCFM8.LANDBASE.A250 WHERE ARCFM8.LANDBASE.A250.SHAPE = } \\
\text { ARCFM8.LANDBASE.S144.SP_FID) }\end{array}$ \\
\hline 96 & 0 & 121 & 0 & SDE.SDE.SDE_TABLE_LOCK_DEF_INSERT \\
\hline 93 & 15 & 6083 & 2 & $\begin{array}{l}\text { DELETE FROM ARCFM8.LANDBASE.S143 WHERE SP_FID IN(SELECT ) AND NOT } \\
\text { EXISTS (SELECT SHAPE FROM }\end{array}$ \\
\hline
\end{tabular}




\begin{tabular}{|c|c|c|c|c|}
\hline & & & & $\begin{array}{l}\text { ARCFM8.LANDBASE.PLSSSECTION_SECTIONNUMBER_ANNO WHERE } \\
\text { ARCFM8.LANDBASE.PLSSSECTION_SECTIONNUMBER_ANNO.SHAPE = } \\
\text { ARCFM8.LANDBASE.S143.SP_FID) AND NOT EXISTS (SELECT SHAPE FROM } \\
\text { ARCFM8.LANDBASE.A249 WHERE ARCFM8.LANDBASE.A249.SHAPE = } \\
\text { ARCFM8.LANDBASE.S143.SP_FID) }\end{array}$ \\
\hline 80 & 63 & 303 & 0 & $\begin{array}{l}\text { UPDATE A SET SDE_STATE_ID = \{\#\#\} FROM ARCFM8.ELECTRIC.A203 A WHERE } \\
\text { A.SDE_STATE_ID IN(SELECT ) AND NOT EXISTS (SELECT * FROM (SELECT } \\
\text { SDE_DELETES_ROW_ID,SDE_STATE_ID FROM ARCFM8.ELECTRIC.D203 WHERE } \\
\text { DELETED_AT IN }(\text { SELECT )) D WHERE A.OBJECTID = D.SDE_DELETES_ROW_ID } \\
\text { AND A.SDE_STATE_ID = D.SDE_STATE_ID) }\end{array}$ \\
\hline 76 & 62 & 315 & 0 & $\begin{array}{l}\text { UPDATE A SET SDE_STATE_ID = \{\#\#\} FROM ARCFM8.ELECTRIC.A109 A WHERE } \\
\text { A.SDE_STATE_ID IN(SELECT ) AND NOT EXISTS (SELECT * FROM (SELECT } \\
\text { SDE_DELETES_ROW_ID,SDE_STATE_ID FROM ARCFM8.ELECTRIC.D109 WHERE } \\
\text { DELETED_AT IN (SELECT )) D WHERE A.OBJECTID = D.SDE_DELETES_ROW_ID } \\
\text { AND A.SDE_STATE_ID = D.SDE_STATE_ID) }\end{array}$ \\
\hline 66 & 62 & 379 & 0 & $\begin{array}{l}\text { UPDATE A SET SDE_STATE_ID = \{\#\#\} FROM ARCFM8.LANDBASE.A187 A WHERE } \\
\text { A.SDE_STATE_ID IN(SELECT ) AND NOT EXISTS (SELECT * FROM (SELECT } \\
\text { SDE_DELETES_ROW_ID,SDE_STATE_ID FROM ARCFM8.LANDBASE.D187 } \\
\text { WHERE DELETED_AT IN(SELECT )) D WHERE A.OBJECTID = } \\
\text { D.SDE_DELETES_ROW_ID AND A.SDE_STATE_ID = D.SDE_STATE_ID) }\end{array}$ \\
\hline 66 & 62 & 589 & 0 & $\begin{array}{l}\text { UPDATE A SET SDE_STATE_ID = \{\#\#\} FROM ARCFM8.LANDBASE.A178 A WHERE } \\
\text { A.SDE_STATE_ID IN(SELECT ) AND NOT EXISTS (SELECT * FROM (SELECT } \\
\text { SDE_DELETES_ROW_ID,SDE_STATE_ID FROM ARCFM8.LANDBASE.D178 } \\
\text { WHERE DELETED_AT IN(SELECT )) D WHERE A.OBJECTID = } \\
\text { D.SDE_DELETES_ROW_ID AND A.SDE_STATE_ID = D.SDE_STATE_ID) }\end{array}$ \\
\hline 66 & 31 & 5045 & 0 & $\begin{array}{l}\text { DELETE FROM ARCFM8.LANDBASE.S146 WHERE SP_FID IN(SELECT ) AND NOT } \\
\text { EXISTS (SELECT SHAPE FROM ARCFM8.LANDBASE.DGGRID_NUMBER_ANNO } \\
\text { WHERE ARCFM8.LANDBASE.DGGRID_NUMBER_ANNO.SHAPE = } \\
\text { ARCFM8.LANDBASE.S146.SP_FID) AND NOT EXISTS (SELECT SHAPE FROM } \\
\text { ARCFM8.LANDBASE.A252 WHERE ARCFM8.LANDBASE.A252.SHAPE = } \\
\text { ARCFM8.LANDBASE.S146.SP_FID) }\end{array}$ \\
\hline 66 & 46 & 199 & 0 & $\begin{array}{l}\text { DELETE FROM ARCFM8.LANDBASE.S84 WHERE SP_FID IN(SELECT ) AND NOT } \\
\text { EXISTS (SELECT SHAPE FROM ARCFM8.LANDBASE.LOTLINE WHERE } \\
\text { ARCFM8.LANDBASE.LOTLINE.SHAPE = ARCFM8.LANDBASE.S84.SP_FID) AND } \\
\text { NOT EXISTS (SELECT SHAPE FROM ARCFM8.LANDBASE.A190 WHERE } \\
\text { ARCFM8.LANDBASE.A190.SHAPE = ARCFM8.LANDBASE.S84.SP_FID) }\end{array}$ \\
\hline 66 & 46 & 221 & 0 & $\begin{array}{l}\text { DELETE FROM ARCFM8.ELECTRIC.S27 WHERE SP_FID IN(SELECT ) AND NOT } \\
\text { EXISTS (SELECT SHAPE FROM ARCFM8.ELECTRIC.SUBSTATION WHERE } \\
\text { ARCFM8.ELECTRIC.SUBSTATION.SHAPE = ARCFM8.ELECTRIC.S27.SP_FID) AND } \\
\text { NOT EXISTS (SELECT SHAPE FROM ARCFM8.ELECTRIC.A109 WHERE } \\
\text { ARCFM8.ELECTRIC.A109.SHAPE = ARCFM8.ELECTRIC.S27.SP_FID) }\end{array}$ \\
\hline 63 & 62 & 377 & 0 & $\begin{array}{l}\text { UPDATE A SET SDE_STATE_ID = \{\#\#\} FROM ARCFM8.LANDBASE.A252 A WHERE } \\
\text { A.SDE_STATE_ID IN(SELECT ) AND NOT EXISTS (SELECT * FROM (SELECT } \\
\text { SDE_DELETES_ROW_ID,SDE_STATE_ID FROM ARCFM8.LANDBASE.D252 } \\
\text { WHERE DELETED_AT IN(SELECT )) D WHERE A.OBJECTID = } \\
\text { D.SDE_DELETES_ROW_ID AND A.SDE_STATE_ID = D.SDE_STATE_ID) }\end{array}$ \\
\hline 63 & 46 & 365 & 0 & $\begin{array}{l}\text { UPDATE A SET SDE_STATE_ID = \{\#\#\} FROM ARCFM8.LANDBASE.A171 A WHERE } \\
\text { A.SDE_STATE_ID IN(SELECT ) AND NOT EXISTS (SELECT * FROM (SELECT } \\
\text { SDE_DELETES_ROW_ID,SDE_STATE_ID FROM ARCFM8.LANDBASE.D171 } \\
\text { WHERE DELETED_AT IN(SELECT )) D WHERE A.OBJECTID = } \\
\text { D.SDE_DELETES_ROW_ID AND A.SDE_STATE_ID = D.SDE_STATE_ID) }\end{array}$ \\
\hline 63 & 47 & 365 & 0 & $\begin{array}{l}\text { UPDATE A SET SDE_STATE_ID = \{\#\#\} FROM ARCFM8.LANDBASE.A175 A WHERE } \\
\text { A.SDE_STATE_ID IN(SELECT ) AND NOT EXISTS (SELECT * FROM (SELECT } \\
\text { SDE_DELETES_ROW_ID,SDE_STATE_ID FROM ARCFM8.LANDBASE.D175 } \\
\text { WHERE DELETED_AT IN(SELECT )) D WHERE A.OBJECTID = } \\
\text { D.SDE_DELETES_ROW_ID AND A.SDE_STATE_ID = D.SDE_STATE_ID) }\end{array}$ \\
\hline 63 & 63 & 365 & 0 & $\begin{array}{l}\text { UPDATE A SET SDE_STATE_ID = \{\#\#\} FROM ARCFM8.LANDBASE.A249 A WHERE } \\
\text { A.SDE_STATE_ID IN(SELECT ) AND NOT EXISTS (SELECT * FROM (SELECT } \\
\text { SDE_DELETES_ROW_ID,SDE_STATE_ID FROM ARCFM8.LANDBASE.D249 } \\
\text { WHERE DELETED_AT IN(SELECT )) D WHERE A.OBJECTID = } \\
\text { D.SDE_DELETES_ROW_ID AND A.SDE_STATE_ID = D.SDE_STATE_ID) }\end{array}$ \\
\hline 63 & 62 & 365 & 0 & $\begin{array}{l}\text { UPDATE A SET SDE_STATE_ID = \{\#\#\} FROM ARCFM8.LANDBASE.A247 A WHERE } \\
\text { A.SDE_STATE_ID IN(SELECT ) AND NOT EXISTS (SELECT * FROM (SELECT } \\
\text { SDE_DELETES_ROW_ID,SDE_STATE_ID FROM ARCFM8.LANDBASE.D247 } \\
\text { WHERE DELETED_AT IN(SELECT )) D WHERE A.OBJECTID = }\end{array}$ \\
\hline
\end{tabular}


D.SDE_DELETES_ROW_ID AND A.SDE_STATE_ID = D.SDE_STATE_ID)

Table C-1 Events with the longest duration 


\subsubsection{Individual by Batch_CPU}

Table C-2 shows individual SQL:BatchCompleted/RPC:Completed events with the highest CPU utilization. During the SQL Profiler tracing period, the highest CPU utilization was 94 milliseconds, or 0.094 seconds of CPU usage. This represents very low CPU utilization for such complex queries.

\begin{tabular}{|c|c|c|c|c|}
\hline Duration (ms) & CPU (ms) & Reads & Writes & Query Format \\
\hline 110 & 94 & 15576 & 0 & $\begin{array}{l}\text { DELETE FROM ARCFM8.LANDBASE.S144 WHERE SP_FID IN(SELECT ) AND NOT } \\
\text { EXISTS (SELECT SHAPE FROM } \\
\text { ARCFM8.LANDBASE.QUARTERTOWNSHIPGRID_NUMBER_ANNO WHERE } \\
\text { ARCFM8.LANDBASE.QUARTERTOWNSHIPGRID_NUMBER_ANNO.SHAPE = } \\
\text { ARCFM8.LANDBASE.S144.SP_FID) AND NOT EXISTS (SELECT SHAPE FROM } \\
\text { ARCFM8.LANDBASE.A250 WHERE ARCFM8.LANDBASE.A250.SHAPE = } \\
\text { ARCFM8.LANDBASE.S144.SP_FID) }\end{array}$ \\
\hline 80 & 63 & 303 & 0 & $\begin{array}{l}\text { UPDATE A SET SDE_STATE_ID = \{\#\#\} FROM ARCFM8.ELECTRIC.A203 A WHERE } \\
\text { A.SDE_STATE_ID IN(SELECT ) AND NOT EXISTS (SELECT * FROM (SELECT } \\
\text { SDE_DELETES_ROW_ID,SDE_STATE_ID FROM ARCFM8.ELECTRIC.D203 WHERE } \\
\text { DELETED_AT IN(SELECT )) D WHERE A.OBJECTID = D.SDE_DELETES_ROW_ID } \\
\text { AND A.SDE_STATE_ID = D.SDE_STATE_ID) }\end{array}$ \\
\hline 63 & 63 & 262 & 0 & $\begin{array}{l}\text { UPDATE A SET SDE_STATE_ID = \{\#\#\} FROM ARCFM8.LANDBASE.A189 A WHERE } \\
\text { A.SDE_STATE_ID IN(SELECT }) \text { AND NOT EXISTS (SELECT * FROM (SELECT } \\
\text { SDE_DELETES_ROW_ID,SDE_STATE_ID FROM ARCFM8.LANDBASE.D189 } \\
\text { WHERE DELETED_AT IN(SELECT )) D WHERE A.OBJECTID = } \\
\text { D.SDE_DELETES_ROW_ID AND A.SDE_STATE_ID = D.SDE_STATE_ID) }\end{array}$ \\
\hline 63 & 63 & 365 & 0 & $\begin{array}{l}\text { UPDATE A SET SDE_STATE_ID = \{\#\#\} FROM ARCFM8.LANDBASE.A180 A WHERE } \\
\text { A.SDE_STATE_ID IN(SELECT ) AND NOT EXISTS (SELECT * FROM (SELECT } \\
\text { SDE_DELETES_ROW_ID,SDE_STATE_ID FROM ARCFM8.LANDBASE.D180 } \\
\text { WHERE DELETED_AT IN(SELECT )) D WHERE A.OBJECTID = } \\
\text { D.SDE_DELETES_ROW_ID AND A.SDE_STATE_ID = D.SDE_STATE_ID) }\end{array}$ \\
\hline 63 & 63 & 377 & 0 & $\begin{array}{l}\text { UPDATE A SET SDE_STATE_ID = \{\#\#\} FROM ARCFM8.LANDBASE.A185 A WHERE } \\
\text { A.SDE_STATE_ID IN(SELECT }) \text { AND NOT EXISTS (SELECT * FROM (SELECT } \\
\text { SDE_DELETES_ROW_ID,SDE_STATE_ID FROM ARCFM8.LANDBASE.D185 } \\
\text { WHERE DELETED_AT IN(SELECT )) D WHERE A.OBJECTID = } \\
\text { D.SDE_DELETES_ROW_ID AND A.SDE_STATE_ID = D.SDE_STATE_ID) }\end{array}$ \\
\hline 63 & 63 & 365 & 0 & $\begin{array}{l}\text { UPDATE A SET SDE_STATE_ID = \{\#\#\} FROM ARCFM8.LANDBASE.A249 A WHERE } \\
\text { A.SDE_STATE_ID IN(SELECT }) \text { AND NOT EXISTS (SELECT * FROM (SELECT } \\
\text { SDE_DELETES_ROW_ID,SDE_STATE_ID FROM ARCFM8.LANDBASE.D249 } \\
\text { WHERE DELETED_AT IN(SELECT )) D WHERE A.OBJECTID = } \\
\text { D.SDE_DELETES_ROW_ID AND A.SDE_STATE_ID = D.SDE_STATE_ID) }\end{array}$ \\
\hline 76 & 62 & 315 & 0 & $\begin{array}{l}\text { UPDATE A SET SDE_STATE_ID = \{\#\#\} FROM ARCFM8.ELECTRIC.A109 A WHERE } \\
\text { A.SDE_STATE_ID IN(SELECT }) \text { AND NOT EXISTS (SELECT * FROM (SELECT } \\
\text { SDE_DELETES_ROW_ID,SDE_STATE_ID FROM ARCFM8.ELECTRIC.D109 WHERE } \\
\text { DELETED_AT IN }(\text { SELECT )) D WHERE A.OBJECTID = D.SDE_DELETES_ROW_ID } \\
\text { AND A.SDE_STATE_ID = D.SDE_STATE_ID) }\end{array}$ \\
\hline 63 & 62 & 365 & 0 & $\begin{array}{l}\text { UPDATE A SET SDE_STATE_ID = \{\#\#\} FROM ARCFM8.LANDBASE.A247 A WHERE } \\
\text { A.SDE_STATE_ID IN(SELECT ) AND NOT EXISTS (SELECT * FROM (SELECT } \\
\text { SDE_DELETES_ROW_ID,SDE_STATE_ID FROM ARCFM8.LANDBASE.D247 } \\
\text { WHERE DELETED_AT IN(SELECT )) D WHERE A.OBJECTID = } \\
\text { D.SDE_DELETES_ROW_ID AND A.SDE_STATE_ID = D.SDE_STATE_ID) }\end{array}$ \\
\hline 66 & 62 & 379 & 0 & $\begin{array}{l}\text { UPDATE A SET SDE_STATE_ID = \{\#\#\} FROM ARCFM8.LANDBASE.A187 A WHERE } \\
\text { A.SDE_STATE_ID IN(SELECT }) \text { AND NOT EXISTS (SELECT * FROM (SELECT } \\
\text { SDE_DELETES_ROW_ID,SDE_STATE_ID FROM ARCFM8.LANDBASE.D187 } \\
\text { WHERE DELETED_AT IN(SELECT )) D WHERE A.OBJECTID = } \\
\text { D.SDE_DELETES_ROW_ID AND A.SDE_STATE_ID = D.SDE_STATE_ID) }\end{array}$ \\
\hline 60 & 62 & 363 & 0 & $\begin{array}{l}\text { UPDATE A SET SDE_STATE_ID = \{\#\#\} FROM ARCFM8.LANDBASE.A183 A WHERE } \\
\text { A.SDE_STATE_ID IN(SELECT ) AND NOT EXISTS (SELECT * FROM (SELECT } \\
\text { SDE_DELETES_ROW_ID,SDE_STATE_ID FROM ARCFM8.LANDBASE.D183 } \\
\text { WHERE DELETED_AT IN(SELECT )) D WHERE A.OBJECTID = } \\
\text { D.SDE_DELETES_ROW_ID AND A.SDE_STATE_ID = D.SDE_STATE_ID) }\end{array}$ \\
\hline
\end{tabular}




\begin{tabular}{|c|c|c|c|c|}
\hline 63 & 62 & 375 & 0 & $\begin{array}{l}\text { UPDATE A SET SDE_STATE_ID = \{\#\#\} FROM ARCFM8.LANDBASE.A184 A WHERE } \\
\text { A.SDE_STATE_ID IN(SELECT ) AND NOT EXISTS (SELECT * FROM (SELECT } \\
\text { SDE_DELETES_ROW_ID,SDE_STATE_ID FROM ARCFM8.LANDBASE.D184 } \\
\text { WHERE DELETED_AT IN(SELECT )) D WHERE A.OBJECTID = } \\
\text { D.SDE_DELETES_ROW_ID AND A.SDE_STATE_ID = D.SDE_STATE_ID) }\end{array}$ \\
\hline 66 & 62 & 589 & 0 & $\begin{array}{l}\text { UPDATE A SET SDE_STATE_ID = \{\#\#\} FROM ARCFM8.LANDBASE.A178 A WHERE } \\
\text { A.SDE_STATE_ID IN(SELECT ) AND NOT EXISTS (SELECT * FROM (SELECT } \\
\text { SDE_DELETES_ROW_ID,SDE_STATE_ID FROM ARCFM8.LANDBASE.D178 } \\
\text { WHERE DELETED_AT IN(SELECT )) D WHERE A.OBJECTID = } \\
\text { D.SDE_DELETES_ROW_ID AND A.SDE_STATE_ID = D.SDE_STATE_ID) }\end{array}$ \\
\hline 63 & 62 & 377 & 0 & $\begin{array}{l}\text { UPDATE A SET SDE_STATE_ID = \{\#\#\} FROM ARCFM8.LANDBASE.A252 A WHERE } \\
\text { A.SDE_STATE_ID IN(SELECT }) \text { AND NOT EXISTS (SELECT * FROM (SELECT } \\
\text { SDE_DELETES_ROW_ID,SDE_STATE_ID FROM ARCFM8.LANDBASE.D252 } \\
\text { WHERE DELETED_AT IN(SELECT )) D WHERE A.OBJECTID = } \\
\text { D.SDE_DELETES_ROW_ID AND A.SDE_STATE_ID = D.SDE_STATE_ID) }\end{array}$ \\
\hline 46 & 47 & 197 & 0 & $\begin{array}{l}\text { DELETE FROM ARCFM8.LANDBASE.S83 WHERE SP_FID IN(SELECT ) AND NOT } \\
\text { EXISTS (SELECT SHAPE FROM ARCFM8.LANDBASE.STREETROW WHERE } \\
\text { ARCFM8.LANDBASE.STREETROW.SHAPE = ARCFM8.LANDBASE.S83.SP_FID) } \\
\text { AND NOT EXISTS (SELECT SHAPE FROM ARCFM8.LANDBASE.A189 WHERE } \\
\text { ARCFM8.LANDBASE.A189.SHAPE = ARCFM8.LANDBASE.S83.SP_FID) }\end{array}$ \\
\hline 46 & 47 & 299 & 0 & $\begin{array}{l}\text { UPDATE A SET SDE_STATE_ID = \{\#\#\} FROM ARCFM8.LANDBASE.A189 A WHERE } \\
\text { A.SDE_STATE_ID IN(SELECT }) \text { AND NOT EXISTS (SELECT * FROM (SELECT } \\
\text { SDE_DELETES_ROW_ID,SDE_STATE_ID FROM ARCFM8.LANDBASE.D189 } \\
\text { WHERE DELETED_AT IN(SELECT )) D WHERE A.OBJECTID = } \\
\text { D.SDE_DELETES_ROW_ID AND A.SDE_STATE_ID = D.SDE_STATE_ID) }\end{array}$ \\
\hline
\end{tabular}

Table C-2 Events with the highest CPU utilization 


\subsubsection{Individual by Batch_Reads}

Table C-3 shows individual SQL:BatchCompleted/RPC:Completed events with the most logical reads. Even though several queries had very large numbers of reads, they were still executed in only 110 milliseconds.

\begin{tabular}{|c|c|c|c|c|}
\hline Duration (ms) & CPU (ms) & Reads & Writes & Query Format \\
\hline 110 & 94 & 15576 & 0 & $\begin{array}{l}\text { DELETE FROM ARCFM8.LANDBASE.S144 WHERE SP_FID IN(SELECT ) AND NOT } \\
\text { EXISTS (SELECT SHAPE FROM } \\
\text { ARCFM8.LANDBASE.QUARTERTOWNSHIPGRID_NUMBER_ANNO WHERE } \\
\text { ARCFM8.LANDBASE.QUARTERTOWNSHIPGRID_NUMBER_ANNO.SHAPE = } \\
\text { ARCFM8.LANDBASE.S144.SP_FID) AND NOT EXISTS (SELECT SHAPE FROM } \\
\text { ARCFM8.LANDBASE.A250 WHERE ARCFM8.LANDBASE.A250.SHAPE = } \\
\text { ARCFM8.LANDBASE.S144.SP_FID) }\end{array}$ \\
\hline 93 & 15 & 6083 & 2 & $\begin{array}{l}\text { DELETE FROM ARCFM8.LANDBASE.S143 WHERE SP_FID IN(SELECT ) AND NOT } \\
\text { EXISTS (SELECT SHAPE FROM } \\
\text { ARCFM8.LANDBASE.PLSSSECTION_SECTIONNUMBER_ANNO WHERE } \\
\text { ARCFM8.LANDBASE.PLSSSECTION_SECTIONNUMBER_ANNO.SHAPE = } \\
\text { ARCFM8.LANDBASE.S143.SP_FID) AND NOT EXISTS (SELECT SHAPE FROM } \\
\text { ARCFM8.LANDBASE.A249 WHERE ARCFM8.LANDBASE.A249.SHAPE = } \\
\text { ARCFM8.LANDBASE.S143.SP_FID) }\end{array}$ \\
\hline 66 & 31 & 5045 & 0 & $\begin{array}{l}\text { DELETE FROM ARCFM8.LANDBASE.S146 WHERE SP_FID IN(SELECT ) AND NOT } \\
\text { EXISTS (SELECT SHAPE FROM ARCFM8.LANDBASE.DGGRID_NUMBER_ANNO } \\
\text { WHERE ARCFM8.LANDBASE.DGGRID_NUMBER_ANNO.SHAPE = } \\
\text { ARCFM8.LANDBASE.S146.SP_FID) AND NOT EXISTS (SELECT SHAPE FROM } \\
\text { ARCFM8.LANDBASE.A252 WHERE ARCFM8.LANDBASE.A252.SHAPE = } \\
\text { ARCFM8.LANDBASE.S146.SP_FID) }\end{array}$ \\
\hline 13 & 0 & 1101 & 0 & JOININDEXSEARCH \\
\hline 0 & 0 & 1101 & 0 & JOININDEXSEARCH \\
\hline 16 & 0 & 1101 & 0 & JOININDEXSEARCH \\
\hline 16 & 0 & 1101 & 0 & JOININDEXSEARCH \\
\hline 16 & 0 & 1101 & 0 & JOININDEXSEARCH \\
\hline 20 & 0 & 1101 & 0 & JOININDEXSEARCH \\
\hline 16 & 0 & 1101 & 0 & JOININDEXSEARCH \\
\hline 0 & 0 & 1101 & 0 & JOININDEXSEARCH \\
\hline 13 & 0 & 1101 & 0 & JOININDEXSEARCH \\
\hline 13 & 0 & 1101 & 0 & JOININDEXSEARCH \\
\hline 16 & 0 & 1101 & 0 & JOININDEXSEARCH \\
\hline 13 & 0 & 1101 & 0 & JOININDEXSEARCH \\
\hline
\end{tabular}

Table C-3 Events containing the most logical reads 


\subsection{SQL Execution Plans}

A database Execution Plan shows how the database will perform a given query. For example, SQL Server can JOIN a table using three different techniques: nested loop, hash, and merge. Generally, the fastest type of join is a nested loop, but if that is not feasible, then a hash JOIN or merge JOIN is used (as appropriate), both of which tend to be slower than the nested JOIN.

Several SQL Execution plans were pulled from the SQL Profiler output. These execution plans were examined to ensure that the SQL Server query optimizer was taking the most efficient route to get the requested data.

While it is not possible to examine every single SQL query and its execution plan, several hundred queries were analyzed for this report. No specific query execution plans were shown to be deficient in how they were accessing the requested data. The majority of execution plans utilized the most efficient "Nested Loops" JOIN.

Shown below is an example of such an execution plan.

\section{Execution Tree}

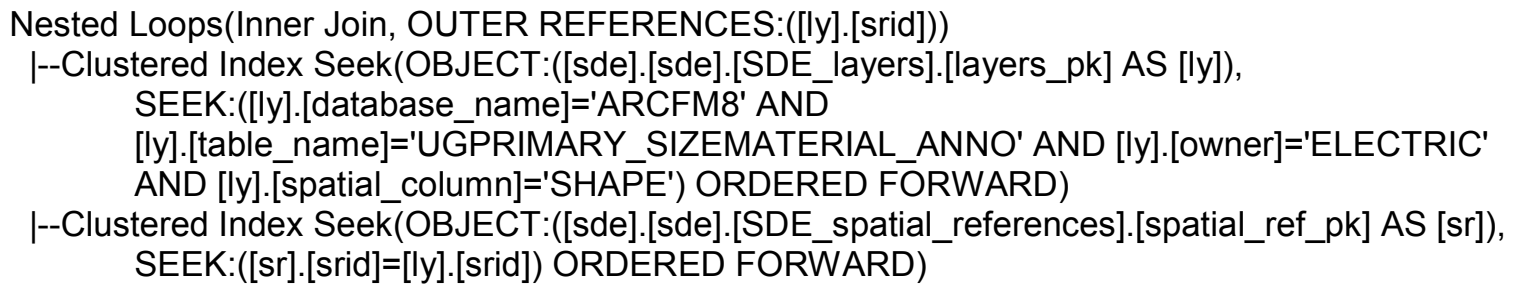




\section{Recommendations}

Based on the results presented in section 3, the ArcSDE / SQL Server database server (AEDRDBSQLP01) is currently processing SQL statements as efficiently as possible.

\section{There are no recommendations for making any configuration changes to} AEDRDBSQLP01 at this time.

\subsection{Assumptions}

The fact that there are no recommended configuration changes is based on a couple of assumptions. These assumptions can also be taken as things to monitor before going live into production with this system.

\subsubsection{Final Production Database}

The database that was utilized during this performance testing initiative was NOT the final, production database that will be delivered from the conversion vendor. Therefore, some items should be considered when the final production database is delivered.

\subsubsection{Spatial Indexes}

The spatial index Grid Size should be analyzed on the production database. This grid size can have a significant impact on performance, and is calculated per layer. For example, here are the spatial index statistics for the OHCONDUCTOR layer shown in Table C-4.

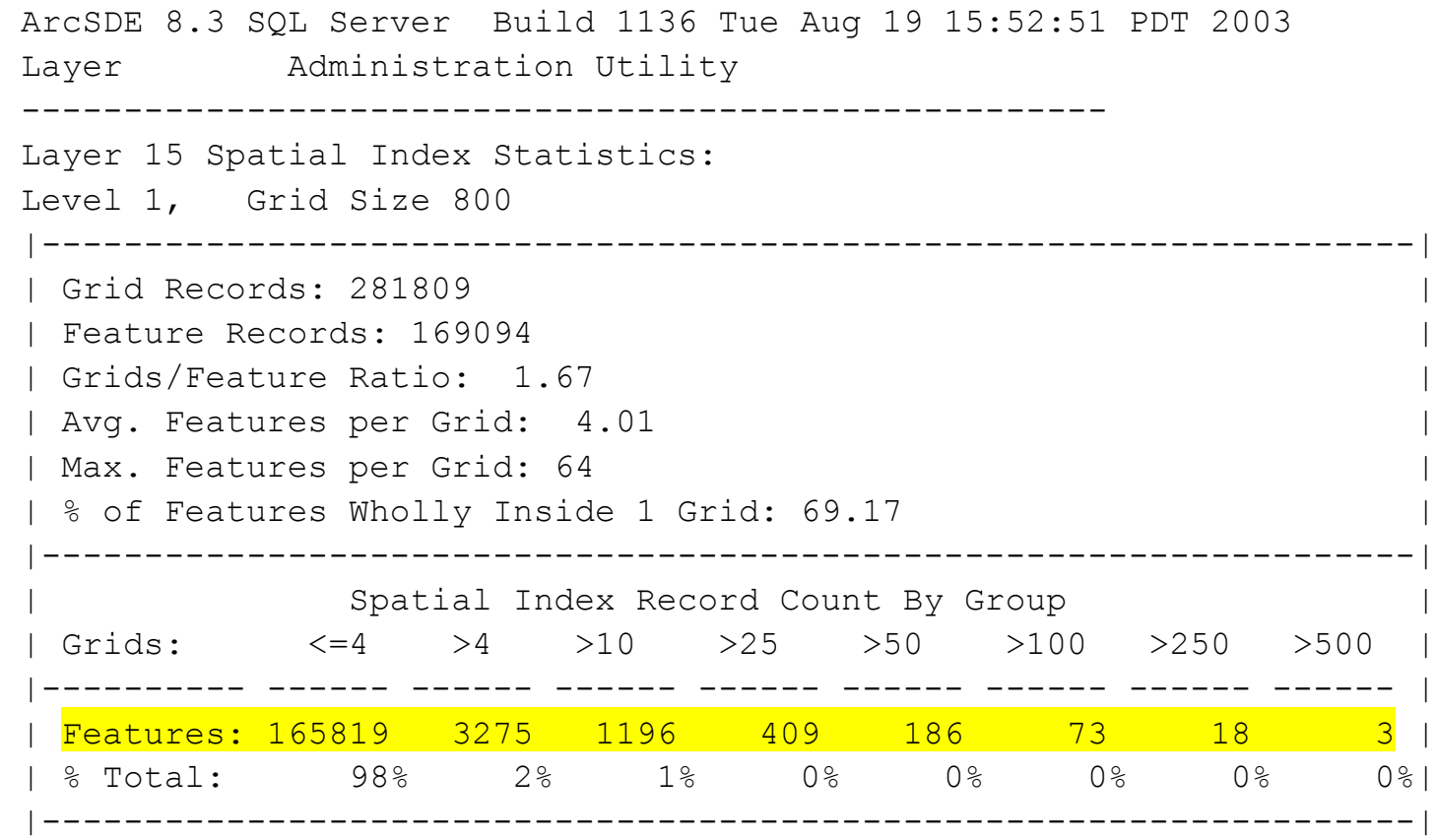

Table C-4. Spatial index statistics 
These statistics indicate that this layer is currently using a grid size of 800 . Based on the number of features that are located in more than 4 grids, this grid size *may* be too small. The only way to determine for sure is to benchmark some queries at the grid size of 800 , alter the grid size to a larger number (ie, 2000 would be reasonable), and benchmark the same queries.

The proper spatial index grid size for all feature classes should be calculated/set once the final production database is available.

\subsubsection{Data Model}

One item to consider is the potential to break the Landbase, Electric and Gas data sets into separate physical SQL databases. This would allow more SQL file groups to be utilized to help distribute the I/O load even more. Since we did not see any serious disk contention during the testing period, this is not a critical issue, but just something to consider moving forward. 


\section{Appendix D}

\section{AutoCAD (Outfield)}

The new GIS will replace all mapping requirements from the AutoCAD based Outfield system.

\section{EDFS Replacement - Electric Distribution Facility (Asset) System}

The new GIS will encompass all EDFS asset management and reporting functionality.

Table D-1. EDFS functional requirements

\begin{tabular}{|c|c|}
\hline ID & Requirements \\
\hline RE-EDFS-001 & Supply Record Clerks with one application to perform all tasks. \\
\hline RE-EDFS-002 & $\begin{array}{l}\text { The GIS electric data model must support retirement and history records } \\
\text { equivalent to those currently in EDFS for migration of existing EDFS } \\
\text { records. This is a legal requirement. Records must be kept for } \\
\text { Support Structure (Poles, Tower, H-Frames) } \\
\text { Pedestals } \\
\text { SwitchGear (Box) } \\
\text { Manhole } \\
\text { Voltage Regulators } \\
\text { Transformers } \\
\text { Regulators } \\
\text { Reclosers } \\
\text { Fuse Cutouts } \\
\text { Sectionalizer } \\
\text { Capacitors } \\
\text { Switches } \\
\text { Primary Conductor } \\
\text { Transmission Conductor } \\
\text { Secondary Conductor } \\
\text { Street Lights }\end{array}$ \\
\hline RE-EDFS-003 & $\begin{array}{l}\text { Creation of a retirement/history record shall be triggered by replacement or } \\
\text { removal of the feature and a change to an assembly. }\end{array}$ \\
\hline RE-EDFS-004 & $\begin{array}{l}\text { The GIS must allow a feature to be retired and have its attributes placed } \\
\text { into a retirement record. }\end{array}$ \\
\hline RE-EDFS-005 & $\begin{array}{l}\text { The GIS electric data model must define a relationship between a } \\
\text { transformer bank and the pole on which it is located. }\end{array}$ \\
\hline RE-EDFS-006 & $\begin{array}{l}\text { The GIS electric data model must define a relationship between a conductor } \\
\text { record and the poles to which it is attached. }\end{array}$ \\
\hline RE-EDFS-007 & $\begin{array}{l}\text { The GIS electric data model must define a relationship between a primary } \\
\text { conductor record and the poles to which it is attached. }\end{array}$ \\
\hline RE-EDFS-008 & $\begin{array}{l}\text { The GIS electric data model must define a relationship between a } \\
\text { secondary conductor record and the poles to which it is attached. }\end{array}$ \\
\hline
\end{tabular}




\begin{tabular}{|c|c|}
\hline RE-EDFS-009 & $\begin{array}{l}\text { The GIS must provide efficient tools that allow updates within } 24 \text { hours of } \\
\text { receipt of the as-built drawing in the records department. }\end{array}$ \\
\hline RE-EDFS-010 & $\begin{array}{l}\text { The GIS must provide the capability to condemn and reverse condemned } \\
\text { transformers. }\end{array}$ \\
\hline RE-EDFS-011 & $\begin{array}{l}\text { The GIS must provide the capability to replace a transformer while } \\
\text { maintaining the transformer location ID and related records. }\end{array}$ \\
\hline RE-EDFS-012 & $\begin{array}{l}\text { The GIS must provide the capability to replace a capacitor while maintaining } \\
\text { the capacitor location ID and related records. }\end{array}$ \\
\hline RE-EDFS-013 & $\begin{array}{l}\text { The GIS must provide the capability to replace a pole while maintaining the } \\
\text { pole location ID and related records. }\end{array}$ \\
\hline RE-EDFS-014 & $\begin{array}{l}\text { The GIS must provide the capability to track assembly units for poles and } \\
\text { other features. }\end{array}$ \\
\hline RE-EDFS-015 & $\begin{array}{l}\text { The GIS must provide the capability to import pole inspection information } \\
\text { received from the inspection contractor. }\end{array}$ \\
\hline RE-EDFS-016 & $\begin{array}{l}\text { The GIS must support the transfer of features between LOA's when LOA } \\
\text { boundaries change. }\end{array}$ \\
\hline RE-EDFS-017 & $\begin{array}{l}\text { The GIS electric data model must support the ability to track multiple cable } \\
\text { TV companies. }\end{array}$ \\
\hline RE-EDFS-018 & $\begin{array}{l}\text { The GIS electric data model and the GIS must support Circuit ID's for } \\
\text { features that are part of the electric network. This includes the automatic re- } \\
\text { assignment of Circuit ID's when feeders undergo a configuration change } \\
\text { due to switching. }\end{array}$ \\
\hline RE-EDFS-019 & $\begin{array}{l}\text { Where appropriate, the GIS electric data model must have "stores item } \\
\text { number" as an attribute. }\end{array}$ \\
\hline RE-EDFS-020 & $\begin{array}{l}\text { Where appropriate, the GIS electric data model must have "installed cost" } \\
\text { as an attribute. }\end{array}$ \\
\hline RE-EDFS-021 & $\begin{array}{l}\text { Where appropriate, the GIS electric data model must have "company } \\
\text { number" as an attribute. }\end{array}$ \\
\hline RE-EDFS-022 & $\begin{array}{l}\text { Where appropriate, the GIS electric data model must have "serial number" } \\
\text { as an attribute. }\end{array}$ \\
\hline RE-EDFS-023 & $\begin{array}{l}\text { Where appropriate, the GIS electric data model must have "adjustment } \\
\text { dollars" as an attribute. }\end{array}$ \\
\hline RE-EDFS-024 & $\begin{array}{l}\text { The GIS electric data model must have "SF number" as an attribute for } \\
\text { switches. }\end{array}$ \\
\hline RE-EDFS-025 & The GIS must support the generation of "Year End - FERC Form 1". \\
\hline RE-EDFS-026 & $\begin{array}{l}\text { The GIS must support the daily reconciliation reporting between Stores and } \\
\text { the GIS. Every removal in the GIS must have a corresponding stores entry } \\
\text { or condemnation record. }\end{array}$ \\
\hline RE-EDFS-027 & $\begin{array}{l}\text { The GIS must require the entry of a valid work order number before a } \\
\text { change to a capital item can be made. }\end{array}$ \\
\hline
\end{tabular}




\begin{tabular}{|l|l|} 
RE-EDFS-028 & $\begin{array}{l}\text { The GIS must support the generation of the PadMount transformer } \\
\text { inspection form. }\end{array}$ \\
\hline RE-EDFS-029 & The GIS must support limited access by storeroom personnel. \\
\hline RE-EDFS-030 & $\begin{array}{l}\text { The GIS electric data model must support the Company Sites such as } \\
\text { substations, storerooms, and the training center. }\end{array}$ \\
\hline
\end{tabular}

\section{CADOPS - NIPSCO Outage and Restoration System (NORS)}

Table D-2. NORS functional requirements

\begin{tabular}{|c|c|}
\hline ID & Requirements \\
\hline RE-NORS-001 & $\begin{array}{l}\text { The application shall create a series of comma delimited files for line, } \\
\text { device, load, node, capacitor, and source (i.e. CADOPS input files) } \\
\text { representing distribution features and the network hierarchy from the } \\
\text { NIPSCO MS SQL Server geodatabase. }\end{array}$ \\
\hline RE-NORS-002 & The application shall be capable of supporting an Oracle RDBMS. \\
\hline RE-NORS-003 & $\begin{array}{l}\text { The application shall be capable of utilizing the existing Oracle scripts to } \\
\text { bulk load the CADOPS input files. }\end{array}$ \\
\hline RE-NORS-004 & $\begin{array}{l}\text { The application shall be capable of bulk loading the CADOPS input files } \\
\text { manually. }\end{array}$ \\
\hline RE-NORS-005 & $\begin{array}{l}\text { The application shall be capable of bulk loading the CADOPS input files via } \\
\text { an automated process. }\end{array}$ \\
\hline RE-NORS-006 & $\begin{array}{l}\text { The application shall be capable of supplying tiled DXF files containing land } \\
\text { base features extracted from the GIS. }\end{array}$ \\
\hline RE-NORS-007 & $\begin{array}{l}\text { The application shall provide Phasing and KVAR rating (i.e. Size) as input } \\
\text { to the MAPNAME.CAP table. }\end{array}$ \\
\hline RE-NORS-008 & The application must support CADOPS 2003. \\
\hline RE-NORS-009 & The application must support an Oracle RDBMS \\
\hline RE-NORS-010 & $\begin{array}{l}\text { The GIS electric data model must support the ability to de-energize one } \\
\text { phase of a three phase line. }\end{array}$ \\
\hline RE-NORS-011 & $\begin{array}{l}\text { The application must assign Type Numbers to conductors and devices to } \\
\text { enable CADOPS to determine the electrical characteristics of each entity. } \\
\text { The type numbers are based on specific attributes of each GIS feature and } \\
\text { must match the Type Numbers found in the DEVTYPE, LINETYPE, and } \\
\text { REGTYPE CADOPS tables. }\end{array}$ \\
\hline
\end{tabular}




\section{FeederAll - Electric Distribution Planning System (Load Study)}

The GIS should provide an export of the GIS electric network data.

Table D-3. FeederAll functional requirements

\begin{tabular}{|c|c|}
\hline ID & Requirements \\
\hline RE-FDRA-001 & $\begin{array}{l}\text { The application shall extract distribution and transmission data from the } \\
\text { NIPSCO MS SQL Server geodatabase and import it into the FeederAll } \\
\text { Oracle database tables. }\end{array}$ \\
\hline RE-FDRA-002 & $\begin{array}{l}\text { The GIS electric data model shall support the ability to determine conductor } \\
\text { configuration }\end{array}$ \\
\hline RE-FDRA-003 & $\begin{array}{l}\text { The application shall support the ability to extract conductor configuration } \\
\text { data from the NIPSCO MS SQL Server geodatabase and import it into the } \\
\text { appropriate FeederAll Oracle database table. }\end{array}$ \\
\hline RE-FDRA-004 & $\begin{array}{l}\text { The GIS electric data model shall support the representation of a } 3 \text { single } \\
\text { phase regulator setting versus the current representation as } 3 \text { individual } \\
\text { regulator settings }\end{array}$ \\
\hline RE-FDRA-005 & $\begin{array}{l}\text { The GIS electric data model shall store "Source" attributes such as positive } \\
\text { and negative sequence resistances in a database table that can be related } \\
\text { to either a breaker or a substation transformer. }\end{array}$ \\
\hline RE-FDRA-006 & $\begin{array}{l}\text { The application shall support the ability to extract "Source" data from the } \\
\text { NIPSCO MS SQL Server geodatabase and import it into the appropriate } \\
\text { FeederAll Oracle database table. }\end{array}$ \\
\hline RE-FDRA-007 & The GIS electric data model shall represent substation transformers. \\
\hline RE-FDRA-008 & The GIS electric data model shall represent customer owned generation. \\
\hline RE-FDRA-009 & $\begin{array}{l}\text { In addition to data currently extracted from CADOPS, the application shall } \\
\text { extract Fuse Size, Regulator Bank Size, Capacitor Bank Size and Type } \\
\text { (fixed vs. switched), and Transformer Bank Size from the NIPSCO MS SQL } \\
\text { Server geodatabase. }\end{array}$ \\
\hline RE-FDRA-010 & $\begin{array}{l}\text { The GIS electric data model shall represent both switched and fixed } \\
\text { capacitor banks. }\end{array}$ \\
\hline
\end{tabular}




\section{EAIF (Engineering Accounts Information File)}

EAIF is a transformer load and analysis program. The GIS should provide current transformer installation data via an interface.

Table D-4. EAIF functional requirements

\begin{tabular}{|l|l|}
\hline \multicolumn{1}{|c|}{ ID } & \multicolumn{1}{c|}{ Requirements } \\
\hline RE-EAIF-001 & $\begin{array}{l}\text { The application shall extract customer load information from CIS for a } \\
\text { transformer using "Load Number" as the basis for the match between GIS } \\
\text { and CIS. }\end{array}$ \\
\hline RE-EAIF-002 & $\begin{array}{l}\text { The application shall use the extracted load information to determine a KVA } \\
\text { demand for transformers. }\end{array}$ \\
\hline RE-EAIF-003 & $\begin{array}{l}\text { The application shall support an EAIF report showing the KWH for each } \\
\text { service point on a transformer or set of transformers along with a sum of the } \\
\text { KWH values for the transformer(s) based on a month provided by the user. }\end{array}$ \\
\hline RE-EAIF-004 & $\begin{array}{l}\text { The application shall support selection of transformers for load analysis by } \\
\text { an on-screen pick, a locate, a trace, or any other method that results in a } \\
\text { selected set of transformers. }\end{array}$ \\
\hline RE-EAIF-005 & $\begin{array}{l}\text { The application shall support the ability to determine usage by phase for a } \\
\text { selected subset of a circuit or an entire circuit. }\end{array}$ \\
\hline RE-EAIF-006 & $\begin{array}{l}\text { The application shall support an EAIF report showing overloaded } \\
\text { transformers. }\end{array}$ \\
\hline
\end{tabular}

\section{SynerGEE}

SynerGEE is a gas distribution planning (load study) system. The GIS should provide an export of the GIS gas network data.

Table D-5. SynerGEE functional requirements

\begin{tabular}{|l|l|}
\hline \multicolumn{1}{|c|}{ ID } & \multicolumn{1}{c|}{ Requirements } \\
\hline RE-SYNG-001 & $\begin{array}{l}\text { The application shall support the extraction of the entire gas distribution } \\
\text { system from the NIPSCO MS SQL Server geodatabase and import it into } \\
\text { the SYNERGEE Gas modeling application. }\end{array}$ \\
\hline RE-SYNG-002 & $\begin{array}{l}\text { The GIS gas data model must support the ability to determine correct flow } \\
\text { direction through regulator stations. }\end{array}$ \\
\hline
\end{tabular}




\section{Facility Browser}

Facility Browser provides a web based representation of the GIS. The GIS should provide functionality to supply spatial data to the greater organization via the intranet.

Table D-6. Facility Browser functional requirements

\begin{tabular}{|l|l|}
\hline \multicolumn{1}{|c|}{ ID } & \multicolumn{1}{c|}{ Requirements } \\
\hline RE-FABR-001 & $\begin{array}{l}\text { The application shall support the extraction of the entire electric, land, and } \\
\text { gas distribution system from the NIPSCO MS SQL Server geodatabase and } \\
\text { import it into the a web based application that can be made available to the } \\
\text { entire NIPSCO organization. }\end{array}$ \\
\hline RE- FABR -002 & $\begin{array}{l}\text { The application shall allow the user to view the GIS maps with standard } \\
\text { pan, zoom, and navigation functions. }\end{array}$ \\
\hline RE- FABR -003 & $\begin{array}{l}\text { The application shall provide layer visibility toggles to allow the user to de- } \\
\text { clutter the graphics in congested areas. }\end{array}$ \\
\hline RE- FABR -004 & $\begin{array}{l}\text { The application shall allow the user to search for CIS installed services } \\
\text { records. }\end{array}$ \\
\hline RE- FABR -005 & $\begin{array}{l}\text { The application shall allow the user to view scanned images of Gas Service } \\
\text { cards that are related to CIS installed services. }\end{array}$ \\
\hline
\end{tabular}

\section{Field Browser}

The Field Browser resides on a stand alone laptop containing a recent version of the GIS data. The GIS shall provide functionality to supply spatial data to a stand alone application that can be distributed onto field laptops. The application must also be distributable to individuals requiring installation of the Field Browser application on nonstand alone Field Browser laptops/desktops.

\section{Table D-7. Field Browser functional requirements}

\begin{tabular}{|l|l|}
\hline \multicolumn{1}{|c|}{ ID } & \multicolumn{1}{c|}{ Requirements } \\
\hline RE-FIBR-001 & $\begin{array}{l}\text { The application shall support the extraction of the entire electric, land, and } \\
\text { gas distribution system from the NIPSCO MS SQL Server geodatabase and } \\
\text { import it into the a stand alone application that can be run disconnected } \\
\text { from the NIPSCO network. }\end{array}$ \\
\hline RE- FIBR -002 & $\begin{array}{l}\text { The application shall allow the user to view the GIS maps with standard } \\
\text { pan, zoom, and navigation functions. }\end{array}$ \\
\hline RE- FIBR -003 & $\begin{array}{l}\text { The application shall provide layer visibility toggles to allow the user to de- } \\
\text { clutter the graphics in congested areas. }\end{array}$ \\
\hline
\end{tabular}




\section{MAPPS (Material Accounts Payable and Purchasing) Interface}

Material accounts payable and purchasing. The new GIS shall maintain an interface to MAPPS equivalent to the EDFS-MAPPS interface including handling capital asset add, issue, removal, condemn, reverse-condemn, and transfer transactions.

Table D-8. MAPPS functional requirements

\begin{tabular}{|l|l|}
\hline \multicolumn{1}{|c|}{ ID } & \multicolumn{1}{c|}{ Requirements } \\
\hline RE-MAPP-001 & $\begin{array}{l}\text { The MAPPS/GIS interface must support the Add (Receipt) Transaction: Add } \\
\text { new item into inventory. }\end{array}$ \\
\hline RE-MAPP-002 & $\begin{array}{l}\text { The MAPPS/GIS interface must support the Condemn Transaction: } \\
\text { Removal from the field. }\end{array}$ \\
\hline RE-MAPP-003 & $\begin{array}{l}\text { The MAPPS/GIS interface must support the Issue Transaction: Move from } \\
\text { inventory to the field. }\end{array}$ \\
\hline RE-MAPP-004 & The MAPPS/GIS interface must support the Removal Transaction. \\
\hline RE-MAPP-005 & $\begin{array}{l}\text { The MAPPS/GIS interface must support the Return Transaction: Move from } \\
\text { field to inventory. }\end{array}$ \\
\hline RE-MAPP-006 & $\begin{array}{l}\text { The MAPPS/GIS interface must support the Transfer Transaction: } \\
\text { Move from one inventory to another inventory. }\end{array}$ \\
\hline RE-MAPP-007 & The MAPPS/GIS interface must support the Reverse Condemn Transaction \\
\hline
\end{tabular}

\section{IRTH One Call}

IRTH One Call is an application used by Indiana Underground Plant Protection Services (IUPPS) to support the location and delivery of One Call tickets. The application is also used by NIPSCO to aid the locate screening group. The GIS should provide data including buffers to aid this interface.

Table D-9. IRTH functional requirements

\begin{tabular}{|c|l|}
\hline ID & \multicolumn{1}{|c|}{ Requirements } \\
\hline RE-IRTH-001 & IRTH is currently using ArcView 8.3 and all requirements are being met. \\
\hline
\end{tabular}

\section{MLOG (Material and Labor Online Guide) Interface}

The Material and Labor Online Guide manages assemblies. The GIS must support the assignment of MLOG assembly numbers to various features and should allow for the real time validation of these numbers against the MLOG system.

Table D-10. MLOG functional requirements

\begin{tabular}{|l|l|}
\hline \multicolumn{1}{|c|}{ ID } & \multicolumn{1}{c|}{ Requirements } \\
\hline RE-MLOG-001 & $\begin{array}{l}\text { The application shall support the assignment of assemblies to GIS features } \\
\text { (SupportStructure, Pedestals, Switchgear, and Manholes) during the entry } \\
\text { of work order information into the GIS. }\end{array}$ \\
\hline RE-MLOG-002 & $\begin{array}{l}\text { The GIS electric data model must support the storage of assembly } \\
\text { information in an object table. }\end{array}$ \\
\hline
\end{tabular}




\begin{tabular}{|l|l|} 
RE-MLOG-003 & $\begin{array}{l}\text { The GIS electric data model must support a relationship between assembly } \\
\text { object records and features. }\end{array}$ \\
\hline RE-MLOG-004 & $\begin{array}{l}\text { The GIS electric data model must support up to } 8 \text { assemblies per instance } \\
\text { of a feature. }\end{array}$ \\
\hline RE-MLOG-005 & $\begin{array}{l}\text { The GIS must validate entered assembly records against MLOG assembly } \\
\text { records in real time. }\end{array}$ \\
\hline
\end{tabular}

\section{CIS (Customer Information System) Interface}

The new GIS should maintain an interface to the CIS equivalent to the EDFS-CIS interface including transfer of service account, site, pole, pad, and transformer data.

Table D-11. CIS functional requirements

\begin{tabular}{|l|l|}
\hline \multicolumn{1}{|c|}{ ID } & \multicolumn{1}{c|}{ Requirements } \\
\hline RE-CISI-001 & $\begin{array}{l}\text { The interface shall be based on the attribute "Load Number" that is common } \\
\text { to both systems. }\end{array}$ \\
\hline RE-CISI-002 & $\begin{array}{l}\text { The interface shall support the ability to enter a Load Number in the GIS } \\
\text { and return a list of customer accounts from the CIS. }\end{array}$ \\
\hline RE-CISI-003 & $\begin{array}{l}\text { The interface shall support the ability to enter a street light service account } \\
\text { in the GIS and return a customer name. }\end{array}$ \\
\hline RE-CISI-004 & $\begin{array}{l}\text { The interface shall populate the GIS pole location description for Load } \\
\text { Numbers in the CIS. }\end{array}$ \\
\hline RE-CISI-005 & $\begin{array}{l}\text { The interface shall continue to maintain the link between the customer and } \\
\text { the transformer serving the transformer. }\end{array}$ \\
\hline
\end{tabular}

\section{DXF Export for Design Engineering}

The new GIS must be able to export AutoCAD DXF files with enough detail for the engineering department to complete its work design process.

Table D-12. DXF export functional requirements

\begin{tabular}{|l|l|}
\hline \multicolumn{1}{|c|}{ ID } & \multicolumn{1}{c|}{ Requirements } \\
\hline RE-DXFE-001 & The tool must produce a DXF file from the ArcSDE Geodatabase. \\
\hline RE-DXFE-002 & $\begin{array}{l}\text { The tool must export CAD Layer Name information from a } \\
\text { "CADLayerName" field. }\end{array}$ \\
\hline RE-DXFE-003 & $\begin{array}{l}\text { The tool must export CAD Block Name information from a } \\
\text { "CADBlockName" field. }\end{array}$ \\
\hline RE-DXFE-004 & $\begin{array}{l}\text { The tool must export Block Rotation values from a "CADBlockRotation" } \\
\text { field. }\end{array}$ \\
\hline RE-DXFE-005 & The tool must export Block scale from a "CADBlockScale" field. \\
\hline RE-DXFE-006 & The tool must be configurable. \\
\hline
\end{tabular}




\begin{tabular}{|l|l|} 
RE-DXFE-007 & $\begin{array}{l}\text { The tool must be able to schedule the export of the DXF files from the GIS } \\
\text { (i.e., must support batch processing). }\end{array}$ \\
\hline RE-DXFE-008 & The tool must specify color as "By Layer" and "By Block". \\
\hline RE-DXFE-009 & The tool must support Line Weight. \\
\hline RE-DXFE-010 & The tool must be able to store and process multiple configurations. \\
\hline RE-DXFE-011 & $\begin{array}{l}\text { The tool must support the export of True Arc Information a.k.a. "bulge } \\
\text { factor" (i.e., "hops" over a gas main). AutoCAD and ArcSDE treat arcs and } \\
\text { polylines differently. }\end{array}$ \\
\hline
\end{tabular}

\section{Pipeline Integrity}

The new GIS shall handle the externally mandated requirements of managing the integrity of NIPSCO's gas pipelines.

Table D-13. Pipeline integrity functional requirements

\begin{tabular}{|l|l|}
\hline \multicolumn{1}{|c|}{ ID } & \multicolumn{1}{c|}{ Requirements } \\
\hline RE-PINT-001 & $\begin{array}{l}\text { The application shall support landbase features, recreational areas, total } \\
\text { impact zones, and high consequence area (HCA) definitions. }\end{array}$ \\
\hline RE-PINT-002 & $\begin{array}{l}\text { The application shall support the recording of inline inspection (ILI) data } \\
\text { results in GIS. }\end{array}$ \\
\hline RE-PINT-003 & $\begin{array}{l}\text { The application shall support recording of direct assessment (DA) data } \\
\text { results into GIS. }\end{array}$ \\
\hline RE-PINT-004 & The application shall support emergency response planning. \\
\hline RE-PINT-005 & The application shall support survey planning. \\
\hline RE-PINT-006 & The application shall support corrosion control management. \\
\hline RE-PINT-007 & The application shall support land management and rights of way. \\
\hline
\end{tabular}




\section{Appendix E}

Table E-1. Electric ArcFM configuration

arcfm8.ELECTRIC.AbandonedConductor

ArcFM Display Field: OBJECTID

Create Edit Task:

On Create Event:

On Update Event:

On Delete Event: ArcFM Delete Related Conductor Info

Before Split Event: ArcFM Save Related Objects

On Split Event:

After Split Event: ArcFM Restore Related Objects

Metadata Editor:

Custom Configuration Editor:

Extended Data Definition Table:

On Abandon Event:

Abandon Feature Class:

Abandon Subtype:

Remove Feature Class:

Remove Subtype:

\begin{tabular}{|c|c|c|c|c|c|c|}
\hline Field Alias & Visible & Editable & $\begin{array}{c}\text { Allow } \\
\text { Null } \\
\text { Values }\end{array}$ & $\begin{array}{l}\text { Clear } \\
\text { After } \\
\text { Create }\end{array}$ & $\begin{array}{c}\text { On Feature } \\
\text { Create }\end{array}$ & $\begin{array}{c}\text { On Feature } \\
\text { Update }\end{array}$ \\
\hline OBJECTID & TRUE & FALSE & FALSE & FALSE & & \\
\hline Subtype Code & TRUE & TRUE & FALSE & FALSE & & \\
\hline INSTALLDATE & TRUE & TRUE & FALSE & FALSE & & \\
\hline Install Work Order & TRUE & TRUE & FALSE & FALSE & & \\
\hline RETIREDATE & TRUE & TRUE & TRUE & FALSE & & \\
\hline Retire Work Order & TRUE & TRUE & TRUE & FALSE & & \\
\hline LOA Name & TRUE & FALSE & FALSE & FALSE & $\begin{array}{l}\text { NIPSCO } \\
\text { LoaName } \\
\end{array}$ & $\begin{array}{l}\text { NIPSCO } \\
\text { LoaName } \\
\end{array}$ \\
\hline Tax Unit & TRUE & TRUE & FALSE & FALSE & & \\
\hline Grid Code & TRUE & TRUE & FALSE & FALSE & & \\
\hline In Conduit & TRUE & TRUE & TRUE & FALSE & & \\
\hline Shape & FALSE & TRUE & TRUE & FALSE & & \\
\hline CREATIONDATE & FALSE & TRUE & FALSE & FALSE & \begin{tabular}{|l|} 
ArcFM \\
Current \\
Date \\
\end{tabular} & \\
\hline Created By & FALSE & TRUE & FALSE & FALSE & \begin{tabular}{|l|} 
ArcFM \\
User Name
\end{tabular} & \\
\hline UPDATEDATE & FALSE & FALSE & TRUE & FALSE & & $\begin{array}{l}\text { ArcFM Current } \\
\text { Date }\end{array}$ \\
\hline Updated By & FALSE & FALSE & TRUE & FALSE & & $\begin{array}{l}\text { ArcFM User } \\
\text { Name }\end{array}$ \\
\hline Legacy Ees Number & FALSE & TRUE & TRUE & FALSE & & \\
\hline Enabled & FALSE & TRUE & TRUE & FALSE & & \\
\hline SHAPE.len & FALSE & & & & & \\
\hline
\end{tabular}

arcfm8.ELECTRIC.Assembly

ArcFM Display Field: ASSEMBLYID

Create Edit Task:

On Create Event: EDFS Validate Assembly Number

On Create Event: EDFS Validate Work Order Number 2

On Update Event: EDFS Validate Work Order Number 2

On Update Event: EDFS Validate Assembly Number

On Delete Event:

On Abandon Event:

Abandon Feature Class:

Abandon Subtype: 
Remove Feature Class:

Remove Subtype:

\begin{tabular}{|c|c|c|c|c|c|c|}
\hline Field Alias & Visible & Editable & $\begin{array}{c}\text { Allow } \\
\text { Null } \\
\text { Values }\end{array}$ & $\begin{array}{l}\text { Clear } \\
\text { After } \\
\text { Create }\end{array}$ & $\begin{array}{c}\text { On Feature } \\
\text { Create }\end{array}$ & $\begin{array}{c}\text { On Feature } \\
\text { Update }\end{array}$ \\
\hline Subtype & TRUE & TRUE & FALSE & FALSE & & \\
\hline Assembly ID & TRUE & TRUE & FALSE & FALSE & & \\
\hline Assembly Count & TRUE & TRUE & FALSE & FALSE & & \\
\hline Primary Assembly & TRUE & TRUE & FALSE & FALSE & & \\
\hline Install WO Number & TRUE & TRUE & FALSE & FALSE & & \\
\hline Install Misc Order Id & TRUE & TRUE & FALSE & FALSE & & \\
\hline Retire WO Number & FALSE & TRUE & TRUE & FALSE & & \\
\hline Retire Misc Order Id & FALSE & TRUE & TRUE & FALSE & & \\
\hline Related SupportStructure Object Id & TRUE & FALSE & TRUE & FALSE & & \\
\hline Pole Number & FALSE & TRUE & TRUE & FALSE & & \\
\hline OBJECTID & TRUE & FALSE & FALSE & FALSE & & \\
\hline CreationDate & FALSE & TRUE & TRUE & FALSE & \begin{tabular}{|l} 
ArcFM \\
Current \\
Date \\
\end{tabular} & \\
\hline Created By & FALSE & TRUE & TRUE & FALSE & $\begin{array}{l}\text { ArcFM } \\
\text { User Name } \\
\end{array}$ & \\
\hline UPDATEDATE & FALSE & TRUE & TRUE & FALSE & & $\begin{array}{l}\text { ArcFM Current } \\
\text { Date }\end{array}$ \\
\hline Updated By & FALSE & TRUE & TRUE & FALSE & & \begin{tabular}{|l|} 
ArcFM User \\
Name
\end{tabular} \\
\hline Work Order Number Sequence Number & FALSE & TRUE & TRUE & FALSE & & \\
\hline ASSOCIATIONTIMESTAMP & FALSE & TRUE & TRUE & FALSE & & \\
\hline LEGACYWRINSTALLTIMESTAMP & FALSE & TRUE & TRUE & FALSE & & \\
\hline LEGACYWRREMOVALTIMESTAMP & FALSE & TRUE & TRUE & FALSE & & \\
\hline
\end{tabular}

arcfm8.ELECTRIC.CapacitorBank

ArcFM Display Field: CAPACITORTYPECD

Create Edit Task: NIPSCO.Electric.FeatureOffset

On Create Event: ArcFM Segment Split

On Create Event: NIPSCO Structure Relate

On Create Event: ArcFM Create Feeder Object

On Update Event: NIPSCO Structure Relate

On Update Event: ArcFM Update Feeder Object

On Update Event: NIPSCO.LOANamePropogation

On Delete Event: NIPSCO.Prevent Delete If Units Related

On Delete Event: ArcFM Delete Feeder Object

On Abandon Event:

Abandon Feature Class:

Abandon Subtype:

Remove Feature Class:

Remove Subtype:

\begin{tabular}{|l|l|l|c|c|c|c|}
\hline Field Alias & Visible & Editable & $\begin{array}{c}\text { Allow } \\
\text { Null } \\
\text { Values }\end{array}$ & $\begin{array}{c}\text { Clear } \\
\text { After } \\
\text { Create }\end{array}$ & $\begin{array}{c}\text { On Feature } \\
\text { Create }\end{array}$ & $\begin{array}{c}\text { On Feature } \\
\text { Update }\end{array}$ \\
\hline Subtype & TRUE & TRUE & FALSE & FALSE & & \\
\hline Phase & TRUE & TRUE & FALSE & FALSE & & \\
\hline Bank KVAR & TRUE & TRUE & FALSE & FALSE & & \\
\hline Unit Number & TRUE & TRUE & FALSE & FALSE & & \\
\hline Capacitor Type & TRUE & TRUE & FALSE & FALSE & & \\
\hline Related Support Structure Object Id & FALSE & TRUE & TRUE & FALSE & & \\
\hline Symbol Rotation & FALSE & FALSE & FALSE & FALSE & & \\
\hline Shape & FALSE & TRUE & TRUE & FALSE & & \\
\hline ANCILLARYROLE & FALSE & TRUE & TRUE & FALSE & & \\
\hline
\end{tabular}




\begin{tabular}{|l|l|l|l|l|l|l|}
\hline Enabled & FALSE & TRUE & TRUE & FALSE & & ArcFM \\
\hline CreationDate & FALSE & TRUE & FALSE & FALSE $\begin{array}{l}\text { Current } \\
\text { Date }\end{array}$ & \\
\hline Created By & FALSE & TRUE & FALSE & FALSE & $\begin{array}{l}\text { ArcFM } \\
\text { User Name }\end{array}$ & $\begin{array}{l}\text { ArcFM Current } \\
\text { Date }\end{array}$ \\
\hline UPDATEDATE & FALSE & FALSE & TRUE & FALSE & & $\begin{array}{l}\text { ArcFM User } \\
\text { Name }\end{array}$ \\
\hline Updated By & FALSE & FALSE & TRUE & FALSE & & \\
\hline Electric Trace Weight & FALSE & TRUE & TRUE & FALSE & & \\
\hline Feeder Manager Non-Traceable & FALSE & TRUE & TRUE & FALSE & & \\
\hline Circuit Number & TRUE & FALSE & TRUE & FALSE & & \\
\hline Circuit Number 2 & FALSE & TRUE & TRUE & FALSE & & \\
\hline Feeder Information & FALSE & TRUE & TRUE & FALSE & & \\
\hline Legacy Node 1 & FALSE & TRUE & TRUE & FALSE & & \\
\hline Legacy Node 2 & FALSE & TRUE & TRUE & FALSE & & \\
\hline Legacy Ees Number & FALSE & TRUE & TRUE & FALSE & & \\
\hline Legacy Pole Number & FALSE & TRUE & TRUE & FALSE & & \\
\hline DCSID & FALSE & TRUE & TRUE & FALSE & & \\
\hline HANDLE & FALSE & TRUE & TRUE & FALSE & & \\
\hline TILENAME & FALSE & TRUE & TRUE & FALSE & & \\
\hline LOA & TRUE & FALSE & TRUE & FALSE & $\begin{array}{l}\text { NIPSCO } \\
\text { LoaName }\end{array}$ & $\begin{array}{l}\text { NIPSCO Loaname } \\
\text { Lo }\end{array}$ \\
\hline Tax Unit & TRUE & TRUE & FALSE & FALSE & & \\
\hline Grid & TRUE & TRUE & FALSE & FALSE & & \\
\hline P_L & FALSE & TRUE & TRUE & FALSE & & \\
\hline SIZE & FALSE & TRUE & TRUE & FALSE & & \\
\hline OBJECTID & TRUE & FALSE & FALSE & FALSE & & \\
\hline
\end{tabular}

arcfm8.ELECTRIC.CapacitorStoresltem

ArcFM Display Field: STORESITEMNUMBER

Create Edit Task:

On Create Event:

On Update Event:

On Delete Event:

On Abandon Event:

Abandon Feature Class:

Abandon Subtype:

Remove Feature Class:

Remove Subtype:

\begin{tabular}{|l|l|l|l|l|l|l|}
\hline Field Alias & Visible & Editable & $\begin{array}{c}\text { Allow } \\
\text { Null } \\
\text { Values }\end{array}$ & $\begin{array}{c}\text { Clear } \\
\text { After } \\
\text { Create }\end{array}$ & $\begin{array}{l}\text { On Feature } \\
\text { Create }\end{array}$ & $\begin{array}{c}\text { On Feature } \\
\text { Update }\end{array}$ \\
\hline SIN & TRUE & FALSE & FALSE & FALSE & & \\
\hline Bushings & TRUE & FALSE & TRUE & FALSE & & \\
\hline KVAR & TRUE & FALSE & TRUE & FALSE & & \\
\hline OBJECTID & TRUE & FALSE & TRUE & FALSE & & \\
\hline CreationDate & TRUE & FALSE & FALSE & FALSE & & \\
\hline Created By & FALSE & TRUE & TRUE & FALSE & $\begin{array}{l}\text { ArcFM } \\
\text { Current } \\
\text { Date }\end{array}$ & \\
\hline UPDATEDATE & FALSE & TRUE & FALSE & FALSE & $\begin{array}{l}\text { ArcFM } \\
\text { User Name }\end{array}$ & \\
\hline Updated By & FALSE & FALSE & TRUE & FALSE & & $\begin{array}{l}\text { ArcFM Current } \\
\text { Date }\end{array}$ \\
\hline
\end{tabular}

arcfm8.ELECTRIC.CapacitorUnit

ArcFM Display Field: COMPANYNUMBER

Create Edit Task:

On Create Event:

On Update Event: 
On Delete Event:

On Abandon Event:

Abandon Feature Class:

Abandon Subtype:

Remove Feature Class:

Remove Subtype:

\begin{tabular}{|l|l|l|l|l|l|l|}
\hline Field Alias & Visible & Editable & $\begin{array}{c}\text { Allow } \\
\text { Null } \\
\text { Values }\end{array}$ & $\begin{array}{c}\text { Clear } \\
\text { After } \\
\text { Create }\end{array}$ & $\begin{array}{c}\text { On Feature } \\
\text { Create }\end{array}$ & $\begin{array}{c}\text { On Feature } \\
\text { Update }\end{array}$ \\
\hline Company Number & TRUE & FALSE & FALSE & FALSE & & \\
\hline SIN & TRUE & FALSE & TRUE & FALSE & & \\
\hline Status & TRUE & FALSE & TRUE & FALSE & & \\
\hline Disposition & TRUE & FALSE & TRUE & FALSE & & \\
\hline P.O. Number & TRUE & FALSE & TRUE & FALSE & & \\
\hline RECEIVEDATE & TRUE & FALSE & TRUE & FALSE & & \\
\hline Manufacturer & TRUE & FALSE & TRUE & FALSE & & \\
\hline Serial Number & TRUE & FALSE & TRUE & FALSE & & \\
\hline Fluid Type & TRUE & FALSE & TRUE & FALSE & & \\
\hline Warranty & TRUE & FALSE & TRUE & FALSE & & \\
\hline OBJECTID & TRUE & FALSE & FALSE & FALSE & & \\
\hline STATUSTIMESTAMP & FALSE & TRUE & FALSE & FALSE & & \\
\hline CreationDate & FALSE & TRUE & TRUE & FALSE & $\begin{array}{l}\text { ArcFM } \\
\text { Current } \\
\text { Date }\end{array}$ & \\
\hline Created By & FALSE & TRUE & TRUE & FALSE & $\begin{array}{l}\text { ArcFM } \\
\text { User Name }\end{array}$ & \\
\hline UPDATEDATE & FALSE & FALSE & TRUE & FALSE & & $\begin{array}{l}\text { ArcFM Current } \\
\text { Date }\end{array}$ \\
\hline Updated By & FALSE & FALSE & TRUE & FALSE & & $\begin{array}{l}\text { ArcFM User } \\
\text { Name }\end{array}$ \\
\hline
\end{tabular}

arcfm8.ELECTRIC.CapacitorUnitlnstall

ArcFM Display Field: RELCAPACITORUNITCOMPANYNUMBER

Create Edit Task:

On Create Event: EDFS Validate Work Order Number 1

On Update Event: EDFS Validate Work Order Number 1

On Delete Event: EDFS Reset Asset To Stock

On Abandon Event:

Abandon Feature Class:

Abandon Subtype:

Remove Feature Class:

Remove Subtype:

\begin{tabular}{|l|c|c|c|c|c|c|}
\hline Field Alias & Visible & Editable & $\begin{array}{c}\text { Allow } \\
\text { Null } \\
\text { Values }\end{array}$ & $\begin{array}{c}\text { Clear } \\
\text { After } \\
\text { Create }\end{array}$ & $\begin{array}{c}\text { On Feature } \\
\text { Create }\end{array}$ & $\begin{array}{c}\text { On Feature } \\
\text { Update }\end{array}$ \\
\hline Company Number & TRUE & FALSE & FALSE & FALSE & & \\
\hline Substation Id & TRUE & TRUE & TRUE & FALSE & & \\
\hline INSTALLDATE & TRUE & TRUE & FALSE & FALSE & & \\
\hline WO Number & TRUE & TRUE & FALSE & FALSE & & \\
\hline Misc Order Id & TRUE & TRUE & FALSE & FALSE & & \\
\hline Phase & TRUE & TRUE & TRUE & FALSE & & \\
\hline Direction & TRUE & TRUE & TRUE & FALSE & & \\
\hline Voltage & TRUE & TRUE & TRUE & FALSE & & \\
\hline Stage Number & TRUE & TRUE & TRUE & FALSE & & \\
\hline Installation Status & TRUE & TRUE & FALSE & FALSE & & \\
\hline Oil Switch & TRUE & TRUE & FALSE & FALSE & & \\
\hline LOA & TRUE & TRUE & FALSE & FALSE & & \\
\hline Tax Unit & TRUE & TRUE & FALSE & FALSE & & \\
\hline Grid & TRUE & TRUE & FALSE & FALSE & & \\
\hline Related Capacitor Bank Object Id & TRUE & FALSE & TRUE & FALSE & & \\
\hline Pole Number & TRUE & TRUE & TRUE & FALSE & & \\
\hline Circuit Number & FALSE & TRUE & TRUE & FALSE & & \\
\hline OBJECTID & TRUE & FALSE & FALSE & FALSE & & \\
\hline
\end{tabular}




\begin{tabular}{|l|l|l|l|l|l|l|}
\hline INSTALLTIMESTAMP & FALSE & TRUE & FALSE & FALSE & & \\
\hline CreationDate & FALSE & FALSE & FALSE & FALSE & $\begin{array}{l}\text { ArcFM } \\
\text { Darrent } \\
\text { Date }\end{array}$ & \\
\hline Created By & FALSE & FALSE & FALSE & FALSE & $\begin{array}{l}\text { ArcFM } \\
\text { User Name }\end{array}$ & \\
\hline UPDATEDATE & FALSE & FALSE & TRUE & FALSE & & $\begin{array}{l}\text { ArcFM Current } \\
\text { Date }\end{array}$ \\
\hline Updated By & FALSE & FALSE & TRUE & FALSE & & $\begin{array}{l}\text { ArcFM User } \\
\text { Name }\end{array}$ \\
\hline
\end{tabular}

arcfm8.ELECTRIC.CapacitorUnitRemove

ArcFM Display Field: REMOVALDATE

Create Edit Task:

On Create Event: EDFS Validate Work Order Number 1

On Update Event: EDFS Validate Work Order Number 1

On Delete Event: EDFS Reset Capacitor Asset To Installed

On Abandon Event:

Abandon Feature Class:

Abandon Subtype:

Remove Feature Class:

Remove Subtype:

\begin{tabular}{|l|l|l|l|l|l|l|}
\hline Field Alias & Visible & Editable & $\begin{array}{c}\text { Allow } \\
\text { Null } \\
\text { Values }\end{array}$ & $\begin{array}{c}\text { Clear } \\
\text { After } \\
\text { Create }\end{array}$ & $\begin{array}{c}\text { On Feature } \\
\text { Create }\end{array}$ & $\begin{array}{c}\text { On Feature } \\
\text { Update }\end{array}$ \\
\hline Company Number & TRUE & FALSE & FALSE & FALSE & & \\
\hline REMOVALDATE & TRUE & FALSE & FALSE & FALSE & & \\
\hline Reason & TRUE & FALSE & TRUE & FALSE & & \\
\hline WO Number & TRUE & FALSE & FALSE & FALSE & & \\
\hline Misc Order Id & TRUE & FALSE & FALSE & FALSE & & \\
\hline OBJECTID & TRUE & FALSE & FALSE & FALSE & & \\
\hline CreationDate & FALSE & TRUE & TRUE & FALSE & $\begin{array}{l}\text { ArcFM } \\
\text { Current } \\
\text { Date }\end{array}$ & \\
\hline Created By & FALSE & TRUE & FALSE & FALSE & $\begin{array}{l}\text { ArcFM } \\
\text { User Name }\end{array}$ & \\
\hline UPDATEDATE & FALSE & FALSE & TRUE & FALSE & & $\begin{array}{l}\text { ArcFM Current } \\
\text { Date }\end{array}$ \\
\hline Updated By & FALSE & FALSE & TRUE & FALSE & & $\begin{array}{l}\text { ArcFM User } \\
\text { Name }\end{array}$ \\
\hline REMOVALTIMESTAMP & FALSE & TRUE & FALSE & FALSE & & \\
\hline LEGACYINSTALLTIMESTAMP & FALSE & TRUE & TRUE & FALSE & & \\
\hline
\end{tabular}

arcfm8.ELECTRIC.CapacitorUnitStock

ArcFM Display Field: STOCKDATE

Create Edit Task:

On Create Event:

On Update Event:

On Delete Event:

On Abandon Event:

Abandon Feature Class:

Abandon Subtype:

Remove Feature Class:

Remove Subtype:

\begin{tabular}{|l|c|c|c|c|c|c|}
\hline Field Alias & Visible & Editable & $\begin{array}{c}\text { Allow } \\
\text { Null } \\
\text { Values }\end{array}$ & $\begin{array}{c}\text { Clear } \\
\text { After } \\
\text { Create }\end{array}$ & $\begin{array}{c}\text { On Feature } \\
\text { Create }\end{array}$ & $\begin{array}{c}\text { On Feature } \\
\text { Update }\end{array}$ \\
\hline Company Number & TRUE & FALSE & FALSE & FALSE & & \\
\hline STOCKDATE & TRUE & FALSE & TRUE & FALSE & & \\
\hline Store Room Number & TRUE & FALSE & TRUE & FALSE & & \\
\hline Transfer Number & TRUE & FALSE & TRUE & FALSE & & \\
\hline OBJECTID & TRUE & FALSE & FALSE & FALSE & & \\
\hline STOCKTIMESTAMP & FALSE & TRUE & FALSE & FALSE & & \\
\hline CreationDate & FALSE & TRUE & TRUE & FALSE & ArcFM & \\
\hline
\end{tabular}




\begin{tabular}{|l|l|l|l|l|l|l|}
\hline & & & & & $\begin{array}{l}\text { lurrent } \\
\text { Date }\end{array}$ & \\
\hline Created By & FALSE & TRUE & FALSE & FALSE & $\begin{array}{l}\text { ArcFM } \\
\text { User Name }\end{array}$ & \\
\hline UPDATEDATE & FALSE & FALSE & TRUE & FALSE & & $\begin{array}{l}\text { ArcFM Current } \\
\text { Date }\end{array}$ \\
\hline Updated By & FALSE & FALSE & TRUE & FALSE & & $\begin{array}{l}\text { ArcFM User } \\
\text { Name }\end{array}$ \\
\hline
\end{tabular}

arcfm8.ELECTRIC.CircuitSource

ArcFM Display Field: CIRCUITSOURCEID

Create Edit Task:

On Create Event:

On Update Event:

On Delete Event:

On Abandon Event:

Abandon Feature Class:

Abandon Subtype:

Remove Feature Class:

Remove Subtype:

\begin{tabular}{|c|c|c|c|c|c|c|}
\hline Field Alias & Visible & Editable & $\begin{array}{c}\text { Allow } \\
\text { Null } \\
\text { Values }\end{array}$ & $\begin{array}{c}\text { Clear } \\
\text { After } \\
\text { Create }\end{array}$ & $\begin{array}{c}\text { On Feature } \\
\text { Create }\end{array}$ & $\begin{array}{c}\text { On Feature } \\
\text { Update }\end{array}$ \\
\hline Circuit Number & TRUE & TRUE & FALSE & FALSE & & \\
\hline Circuit Name & TRUE & TRUE & TRUE & FALSE & & \\
\hline Substation Name & TRUE & TRUE & TRUE & FALSE & & \\
\hline CreationDate & FALSE & TRUE & TRUE & FALSE & \begin{tabular}{|l|} 
ArcFM \\
Current \\
Date
\end{tabular} & \\
\hline Created By & FALSE & TRUE & TRUE & FALSE & \begin{tabular}{|l|} 
ArcFM \\
User Name
\end{tabular} & \\
\hline UPDATEDATE & FALSE & FALSE & TRUE & FALSE & & $\begin{array}{l}\text { ArcFM Current } \\
\text { Date }\end{array}$ \\
\hline Updated By & FALSE & FALSE & TRUE & FALSE & & $\begin{array}{l}\text { ArcFM User } \\
\text { Name }\end{array}$ \\
\hline Nominal Voltage & TRUE & TRUE & TRUE & FALSE & & \\
\hline Operating Voltage & TRUE & TRUE & TRUE & FALSE & & \\
\hline Connection Configuration & TRUE & TRUE & TRUE & FALSE & & \\
\hline Emergency Capacity kW & TRUE & TRUE & TRUE & FALSE & & \\
\hline Ground Reactance & TRUE & TRUE & TRUE & FALSE & & \\
\hline Ground Resistance & TRUE & TRUE & TRUE & FALSE & & \\
\hline Maximum Capacity kW & TRUE & TRUE & TRUE & FALSE & & \\
\hline Maximum kVAR & TRUE & TRUE & TRUE & FALSE & & \\
\hline Minimum kVAR & TRUE & TRUE & TRUE & FALSE & & \\
\hline Maximum Positive Sequence Reactance & TRUE & TRUE & TRUE & FALSE & & \\
\hline Maximum Positive Sequence Resistance & TRUE & TRUE & TRUE & FALSE & & \\
\hline Maximum Zero Sequence Impedance & TRUE & TRUE & TRUE & FALSE & & \\
\hline Maximum Zero Sequence Resistance & TRUE & TRUE & TRUE & FALSE & & \\
\hline Negative Sequence Reactance & TRUE & TRUE & TRUE & FALSE & & \\
\hline Voltage Angle & TRUE & TRUE & TRUE & FALSE & & \\
\hline Feeder Source Information & TRUE & TRUE & TRUE & FALSE & & \\
\hline Related Substation Breaker Object Id & TRUE & FALSE & TRUE & FALSE & & \\
\hline OBJECTID & TRUE & FALSE & FALSE & FALSE & & \\
\hline
\end{tabular}

arcfm8.ELECTRIC.CompanyStreetlight

ArcFM Display Field: ASSEMBLYID

Create Edit Task:

On Create Event: 
On Update Event:

On Delete Event:

On Abandon Event:

Abandon Feature Class:

Abandon Subtype:

Remove Feature Class:

Remove Subtype:

\begin{tabular}{|c|c|c|c|c|c|c|}
\hline Field Alias & Visible & Editable & $\begin{array}{c}\text { Allow } \\
\text { Null } \\
\text { Values }\end{array}$ & $\begin{array}{c}\text { Clear } \\
\text { After } \\
\text { Create }\end{array}$ & $\begin{array}{c}\text { On Feature } \\
\text { Create }\end{array}$ & $\begin{array}{l}\text { On Feature } \\
\text { Update }\end{array}$ \\
\hline Subtype & TRUE & TRUE & FALSE & FALSE & & \\
\hline Assembly Id & TRUE & TRUE & FALSE & FALSE & & \\
\hline Assembly Count & TRUE & TRUE & FALSE & FALSE & & \\
\hline Construction & TRUE & TRUE & FALSE & FALSE & & \\
\hline \begin{tabular}{|l} 
INSTALLDATE \\
\end{tabular} & TRUE & TRUE & FALSE & FALSE & & \\
\hline Install WO Number & TRUE & TRUE & FALSE & FALSE & & \\
\hline Install Misc Order Id & TRUE & TRUE & FALSE & FALSE & & \\
\hline Rate Schedule & TRUE & TRUE & FALSE & FALSE & & \\
\hline CIS Account Number & TRUE & TRUE & FALSE & FALSE & & \\
\hline Legacy XRef & FALSE & TRUE & TRUE & FALSE & & \\
\hline Related Support Structure Object Id & TRUE & FALSE & TRUE & FALSE & & \\
\hline OBJECTID & TRUE & FALSE & FALSE & FALSE & & \\
\hline CreationDate & FALSE & TRUE & TRUE & FALSE & \begin{tabular}{|l} 
ArcFM \\
Current \\
Date
\end{tabular} & \\
\hline Created By & FALSE & TRUE & FALSE & FALSE & $\begin{array}{l}\text { ArcFM } \\
\text { User Name }\end{array}$ & \\
\hline UPDATEDATE & FALSE & FALSE & TRUE & FALSE & & $\begin{array}{l}\text { ArcFM Current } \\
\text { Date }\end{array}$ \\
\hline Updated By & FALSE & FALSE & TRUE & FALSE & & $\begin{array}{l}\text { ArcFM User } \\
\text { Name }\end{array}$ \\
\hline RETIREDATE & FALSE & TRUE & TRUE & FALSE & & \\
\hline Retire WO Number & FALSE & TRUE & TRUE & FALSE & & \\
\hline Retire Misc Order Id & FALSE & TRUE & TRUE & FALSE & & \\
\hline LEGACYWRINSTALLTIMESTAMP & FALSE & TRUE & TRUE & FALSE & & \\
\hline LEGACYWRREMOVALTIMESTAMP & FALSE & TRUE & TRUE & FALSE & & \\
\hline ASSOCIATIONTIMESTAMP & FALSE & TRUE & TRUE & FALSE & & \\
\hline Pole Number & FALSE & TRUE & TRUE & FALSE & & \\
\hline
\end{tabular}

arcfm8.ELECTRIC.CompanyUseLocation

ArcFM Display Field: COMPANYLOCATIONTYPECD

Create Edit Task:

On Create Event:

On Update Event:

On Delete Event:

On Abandon Event:

Abandon Feature Class:

Abandon Subtype:

Remove Feature Class:

Remove Subtype:

\begin{tabular}{|l|c|c|c|c|c|c|}
\hline Field Alias & Visible & Editable & $\begin{array}{c}\text { Allow Null } \\
\text { Values }\end{array}$ & $\begin{array}{c}\text { Clear } \\
\text { After } \\
\text { Create }\end{array}$ & $\begin{array}{c}\text { On Feature } \\
\text { Create }\end{array}$ & $\begin{array}{c}\text { On Feature } \\
\text { Update }\end{array}$ \\
\hline Location Id & TRUE & TRUE & FALSE & FALSE & & \\
\hline Location Type & TRUE & TRUE & FALSE & FALSE & & \\
\hline Description & TRUE & TRUE & TRUE & FALSE & & \\
\hline Tax Unit & TRUE & TRUE & TRUE & FALSE & & \\
\hline Grid & TRUE & TRUE & TRUE & FALSE & & \\
\hline OBJECTID & TRUE & FALSE & FALSE & FALSE & & \\
\hline CreationDate & FALSE & TRUE & TRUE & FALSE & ArcFM & \\
\hline
\end{tabular}




\begin{tabular}{|l|c|c|c|c|l|l|}
\hline & & & & & $\begin{array}{l}\text { Current } \\
\text { Date }\end{array}$ & \\
\hline Created By & FALSE & TRUE & FALSE & FALSE & $\begin{array}{l}\text { ArcFM } \\
\text { User Name }\end{array}$ & \\
\hline UPDATEDATE & FALSE & FALSE & TRUE & FALSE & & $\begin{array}{l}\text { ArcFM Current } \\
\text { Date }\end{array}$ \\
\hline Updated By & FALSE & FALSE & TRUE & FALSE & & $\begin{array}{l}\text { ArcFM User } \\
\text { Name }\end{array}$ \\
\hline
\end{tabular}

arcfm8.ELECTRIC.ConductorDefinition

ArcFM Display Field: CATEGORYCD

Create Edit Task:

On Create Event:

On Update Event:

On Delete Event:

On Abandon Event:

Abandon Feature Class:

Abandon Subtype:

Remove Feature Class:

Remove Subtype:

\begin{tabular}{|l|l|l|l|l|l|l|}
\hline Field Alias & Visible & Editable & $\begin{array}{c}\text { Allow Null } \\
\text { Values }\end{array}$ & $\begin{array}{c}\text { Clear } \\
\text { After } \\
\text { Create }\end{array}$ & $\begin{array}{c}\text { On Feature } \\
\text { Create }\end{array}$ & $\begin{array}{c}\text { On Feature } \\
\text { Update }\end{array}$ \\
\hline Category & TRUE & FALSE & TRUE & FALSE & & \\
\hline Location & TRUE & FALSE & TRUE & FALSE & & \\
\hline Wire Type & TRUE & FALSE & TRUE & FALSE & & \\
\hline Wire Size & TRUE & FALSE & TRUE & FALSE & & \\
\hline Wire Material & TRUE & FALSE & TRUE & FALSE & & \\
\hline Legacy Calculation Cd & FALSE & TRUE & TRUE & FALSE & & \\
\hline LegJECTID Conductor Number & FALSE & TRUE & TRUE & FALSE & & \\
\hline CreationDate & TRUE & FALSE & FALSE & FALSE & & \\
\hline Created By & FALSE & TRUE & TRUE & FALSE & $\begin{array}{l}\text { ArcFM } \\
\text { Current } \\
\text { Date }\end{array}$ & \\
\hline UPDATEDATE & FALSE & TRUE & FALSE & FALSE & $\begin{array}{l}\text { ArcFM } \\
\text { User Name }\end{array}$ & \\
\hline Updated By & FALSE & FALSE & TRUE & FALSE & & $\begin{array}{l}\text { ArcFM Current } \\
\text { Date }\end{array}$ \\
\hline
\end{tabular}

arcfm8.ELECTRIC.CustomerGenerator

ArcFM Display Field: OBJECTID

Create Edit Task:

On Create Event: NIPSCO.NormalPositionSymbol

On Update Event: NIPSCO.NormalPositionSymbol

On Delete Event:

On Abandon Event:

Abandon Feature Class:

Abandon Subtype:

Remove Feature Class:

Remove Subtype:

\begin{tabular}{|l|c|c|c|c|c|c|}
\hline Field Alias & Visible & Editable & $\begin{array}{c}\text { Allow Null } \\
\text { Values }\end{array}$ & $\begin{array}{c}\text { Clear } \\
\text { After } \\
\text { Create }\end{array}$ & $\begin{array}{c}\text { On Feature } \\
\text { Create }\end{array}$ & $\begin{array}{c}\text { On Feature } \\
\text { Update }\end{array}$ \\
\hline LOA & TRUE & TRUE & TRUE & FALSE & & \\
\hline OBJECTID & TRUE & FALSE & FALSE & FALSE & & \\
\hline Subtype & TRUE & TRUE & FALSE & FALSE & & \\
\hline Shape & FALSE & TRUE & TRUE & FALSE & & \\
\hline AncillaryRole & FALSE & TRUE & TRUE & FALSE & & \\
\hline Enabled & FALSE & TRUE & TRUE & FALSE & & \\
\hline CreationDate & FALSE & TRUE & TRUE & FALSE & $\begin{array}{l}\text { ArcFM } \\
\text { Current }\end{array}$ & \\
\hline
\end{tabular}




\begin{tabular}{|c|c|c|c|c|c|c|}
\hline & & & & & Date & \\
\hline Created By & FALSE & TRUE & FALSE & FALSE & \begin{tabular}{|l|} 
ArcFM \\
User Name
\end{tabular} & \\
\hline UPDATEDATE & FALSE & FALSE & TRUE & FALSE & & $\begin{array}{l}\text { ArcFM Current } \\
\text { Date }\end{array}$ \\
\hline Updated By & FALSE & FALSE & TRUE & FALSE & & $\begin{array}{l}\text { ArcFM User } \\
\text { Name }\end{array}$ \\
\hline Electric Trace Weight & FALSE & TRUE & TRUE & FALSE & & \\
\hline Feeder Manager Non-Traceable & FALSE & TRUE & TRUE & FALSE & & \\
\hline Circuit Number & TRUE & FALSE & TRUE & FALSE & & \\
\hline Circuit Number 2 & FALSE & TRUE & TRUE & FALSE & & \\
\hline Feeder Information & FALSE & TRUE & TRUE & FALSE & & \\
\hline Phase & TRUE & TRUE & FALSE & FALSE & & \\
\hline Symbol Rotation & FALSE & TRUE & FALSE & FALSE & & \\
\hline Graphics Scale Factor & FALSE & TRUE & TRUE & FALSE & & \\
\hline Disconnect Location & TRUE & TRUE & TRUE & FALSE & & \\
\hline A Phase Normal Position & TRUE & TRUE & FALSE & FALSE & & \\
\hline B Phase Normal Position & TRUE & TRUE & FALSE & FALSE & & \\
\hline C Phase Normal Position & TRUE & TRUE & FALSE & FALSE & & \\
\hline Grid & TRUE & TRUE & TRUE & FALSE & & \\
\hline Customer Name & TRUE & TRUE & TRUE & FALSE & & \\
\hline Nominal Voltage & TRUE & TRUE & TRUE & FALSE & & \\
\hline Customer Substation ID & TRUE & TRUE & TRUE & FALSE & & \\
\hline Maximum Positive Sequence Resistance & TRUE & TRUE & TRUE & FALSE & & \\
\hline Maximum Positive Sequence Reactance & TRUE & TRUE & TRUE & FALSE & & \\
\hline Negative Sequence Reactance & TRUE & TRUE & TRUE & FALSE & & \\
\hline Maximum Zero Sequence Resistance & TRUE & TRUE & TRUE & FALSE & & \\
\hline Maximum Zero Sequence Impedance & TRUE & TRUE & TRUE & FALSE & & \\
\hline Maximum KVAR & TRUE & TRUE & TRUE & FALSE & & \\
\hline Minimum KVAR & TRUE & TRUE & TRUE & FALSE & & \\
\hline Primary Operating Voltage & TRUE & TRUE & TRUE & FALSE & & \\
\hline Symbol Configuration Cd & FALSE & TRUE & TRUE & FALSE & & \\
\hline \multicolumn{7}{|l|}{$\begin{array}{l}\text { arcfm8.ELECTRIC.Custo } \\
\text { ArcFM Display Field: LUn } \\
\text { Create Edit Task: } \\
\text { On Create Event: } \\
\text { On Update Event: } \\
\text { On Delete Event: } \\
\text { On Abandon Event: } \\
\text { Abandon Feature Class: } \\
\text { Abandon Subtype: } \\
\text { Remove Feature Class: } \\
\text { Remove Subtype: }\end{array}$} \\
\hline Field Alias & Visible & Editable & $\begin{array}{c}\text { Allow Null } \\
\text { Values }\end{array}$ & \begin{tabular}{|c|} 
Clear \\
After \\
Create \\
\end{tabular} & $\begin{array}{c}\text { On Feature } \\
\text { Create }\end{array}$ & $\begin{array}{c}\text { On Feature } \\
\text { Update }\end{array}$ \\
\hline Pole Type & TRUE & TRUE & FALSE & FALSE & & \\
\hline Luminaire Type & TRUE & TRUE & FALSE & FALSE & & \\
\hline Luminaire Count & TRUE & TRUE & FALSE & FALSE & & \\
\hline Wattage & TRUE & TRUE & FALSE & FALSE & & \\
\hline INVENTORYDATE & TRUE & TRUE & FALSE & FALSE & & \\
\hline CIS Account Number & TRUE & TRUE & FALSE & FALSE & & \\
\hline Load Number & FALSE & TRUE & TRUE & FALSE & & \\
\hline Related Pad Mount Object Id & TRUE & FALSE & TRUE & FALSE & & \\
\hline Related Support Structure Object Id & TRUE & FALSE & TRUE & FALSE & & \\
\hline
\end{tabular}




\begin{tabular}{|c|c|c|c|c|c|c|}
\hline OBJECTID & TRUE & FALSE & FALSE & FALSE & & \\
\hline CreationDate & FALSE & TRUE & TRUE & FALSE & \begin{tabular}{|l|} 
ArcFM \\
Current \\
Date
\end{tabular} & \\
\hline Created By & FALSE & TRUE & FALSE & FALSE & $\begin{array}{l}\text { ArcFM } \\
\text { User Name }\end{array}$ & \\
\hline UPDATEDATE & FALSE & FALSE & TRUE & FALSE & & $\begin{array}{l}\text { ArcFM Current } \\
\text { Date }\end{array}$ \\
\hline Updated By & FALSE & FALSE & TRUE & FALSE & & $\begin{array}{l}\text { ArcFM User } \\
\text { Name }\end{array}$ \\
\hline ASSOCIATIONTIMESTAMP & FALSE & TRUE & TRUE & FALSE & & \\
\hline Legacy XRef & FALSE & TRUE & TRUE & FALSE & & \\
\hline
\end{tabular}

arcfm8.ELECTRIC.DeadEndElectric

ArcFM Display Field: OBJECTID

Create Edit Task:

On Create Event:

On Update Event:

On Delete Event:

On Abandon Event:

Abandon Feature Class:

Abandon Subtype:

Remove Feature Class:

Remove Subtype:

\begin{tabular}{|c|c|c|c|c|c|c|}
\hline Field Alias & Visible & Editable & $\begin{array}{l}\text { Allow Null } \\
\text { Values }\end{array}$ & $\begin{array}{l}\text { Clear } \\
\text { After } \\
\text { Create }\end{array}$ & $\begin{array}{c}\text { On Feature } \\
\text { Create }\end{array}$ & $\begin{array}{c}\text { On Feature } \\
\text { Update }\end{array}$ \\
\hline Subtype & TRUE & TRUE & FALSE & FALSE & & \\
\hline Shape & FALSE & TRUE & TRUE & FALSE & & \\
\hline CreationDate & FALSE & TRUE & FALSE & FALSE & \begin{tabular}{|l|} 
ArcFM \\
Current \\
Date
\end{tabular} & \\
\hline Created By & FALSE & TRUE & FALSE & FALSE & $\begin{array}{l}\text { ArcFM } \\
\text { User Name }\end{array}$ & \\
\hline UPDATEDATE & FALSE & FALSE & TRUE & FALSE & & $\begin{array}{l}\text { ArcFM Current } \\
\text { Date }\end{array}$ \\
\hline Updated By & FALSE & FALSE & TRUE & FALSE & & $\begin{array}{l}\text { ArcFM User } \\
\text { Name }\end{array}$ \\
\hline Symbol Rotation & FALSE & FALSE & FALSE & FALSE & & \\
\hline HANDLE & FALSE & TRUE & TRUE & FALSE & & \\
\hline TILENAME & FALSE & TRUE & TRUE & FALSE & & \\
\hline OBJECTID & TRUE & FALSE & FALSE & FALSE & & \\
\hline
\end{tabular}

arcfm8.ELECTRIC.ElectricDiscrepancy

ArcFM Display Field: OBJECTID

Create Edit Task:

On Create Event:

On Update Event:

On Delete Event:

Metadata Editor:

Custom Configuration Editor:

Extended Data Definition Table:

On Abandon Event:

Abandon Feature Class:

Abandon Subtype:

Remove Feature Class:

Remove Subtype:

\begin{tabular}{|l|c|c|c|c|c|c|}
\hline Field Alias & Visible & Editable & $\begin{array}{c}\text { Allow Null } \\
\text { Values }\end{array}$ & $\begin{array}{c}\text { Clear } \\
\text { After } \\
\text { Create }\end{array}$ & $\begin{array}{c}\text { On Feature } \\
\text { Create }\end{array}$ & $\begin{array}{c}\text { On Feature } \\
\text { Update }\end{array}$ \\
\hline OBJECTID & TRUE & FALSE & FALSE & FALSE & & \\
\hline Subtype & TRUE & TRUE & FALSE & FALSE & & \\
\hline Error Description & TRUE & TRUE & TRUE & FALSE & & \\
\hline
\end{tabular}




\begin{tabular}{|c|c|c|c|c|c|c|}
\hline Shape & FALSE & TRUE & TRUE & FALSE & & \\
\hline CREATIONDATE & FALSE & TRUE & FALSE & FALSE & \begin{tabular}{|l|} 
ArcFM \\
Current \\
Date
\end{tabular} & \\
\hline Created By & FALSE & TRUE & FALSE & FALSE & $\begin{array}{l}\text { ArcFM } \\
\text { User Name }\end{array}$ & \\
\hline UPDATEDATE & FALSE & FALSE & TRUE & FALSE & & $\begin{array}{l}\text { ArcFM Current } \\
\text { Date }\end{array}$ \\
\hline Updated By & FALSE & FALSE & TRUE & FALSE & & $\begin{array}{l}\text { ArcFM User } \\
\text { Name }\end{array}$ \\
\hline Legacy Ees Number & FALSE & TRUE & TRUE & FALSE & & \\
\hline
\end{tabular}

arcfm8.ELECTRIC.FeederAllOpenPoint

ArcFM Display Field: OBJECTID

Create Edit Task:

On Create Event: ArcFM Segment Split

On Update Event:

On Delete Event:

On Abandon Event:

Abandon Feature Class:

Abandon Subtype:

Remove Feature Class:

Remove Subtype:

\begin{tabular}{|l|l|l|l|l|l|l|}
\hline Field Alias & Visible & Editable & $\begin{array}{c}\text { Allow Null } \\
\text { Values }\end{array}$ & $\begin{array}{l}\text { Clear } \\
\text { After } \\
\text { Create }\end{array}$ & $\begin{array}{l}\text { On Feature } \\
\text { Create }\end{array}$ & $\begin{array}{c}\text { On Feature } \\
\text { Update }\end{array}$ \\
\hline Shape & FALSE & TRUE & TRUE & FALSE & & \\
\hline AncillaryRole & FALSE & TRUE & TRUE & FALSE & & \\
\hline Enabled & FALSE & TRUE & TRUE & FALSE & & \\
\hline CreationDate & FALSE & TRUE & FALSE & FALSE & $\begin{array}{l}\text { ArcFM } \\
\text { Current } \\
\text { Date }\end{array}$ & $\begin{array}{l}\text { FarcF } \\
\text { User Name }\end{array}$ \\
\hline Created By & FALSE & TRUE & FALSE & FALSE & $\begin{array}{l}\text { ArcFM Current } \\
\text { Date }\end{array}$ \\
\hline UPDATEDATE & FALSE & FALSE & TRUE & FALSE & & $\begin{array}{l}\text { ArcFM User } \\
\text { Name }\end{array}$ \\
\hline Updated By & FALSE & FALSE & TRUE & FALSE & & \\
\hline OBJECTID & TRUE & FALSE & FALSE & FALSE & & \\
\hline
\end{tabular}

arcfm8.ELECTRIC.FuseCutoutBank

ArcFM Display Field: SUBTYPECD

Create Edit Task:

On Create Event: ArcFM Segment Split

On Create Event: NIPSCO Structure Relate

On Create Event: ArcFM Create Feeder Object

On Create Event: NIPSCO.NormalPositionSymbol

On Update Event: NIPSCO Structure Relate

On Update Event: ArcFM Update Feeder Object

On Update Event: NIPSCO.NormalPositionSymbol

On Delete Event: NIPSCO.Prevent Delete If Units Related

On Delete Event: ArcFM Delete Feeder Object

On Abandon Event:

Abandon Feature Class:

Abandon Subtype:

Remove Feature Class:

Remove Subtype:

\begin{tabular}{|l|c|c|c|c|c|c|}
\hline Field Alias & Visible & Editable & $\begin{array}{c}\text { Allow Null } \\
\text { Values }\end{array}$ & $\begin{array}{c}\text { Clear } \\
\text { After } \\
\text { Create }\end{array}$ & $\begin{array}{c}\text { On Feature } \\
\text { Create }\end{array}$ & $\begin{array}{c}\text { On Feature } \\
\text { Update }\end{array}$ \\
\hline Subtype & TRUE & TRUE & FALSE & FALSE & & \\
\hline UPDATEDATE & FALSE & FALSE & TRUE & FALSE & & $\begin{array}{l}\text { ArcFM Current } \\
\text { Date }\end{array}$ \\
\hline
\end{tabular}




\begin{tabular}{|c|c|c|c|c|c|c|}
\hline Updated By & FALSE & FALSE & TRUE & FALSE & & $\begin{array}{l}\text { ArcFM User } \\
\text { Name }\end{array}$ \\
\hline Electric Trace Weight & FALSE & TRUE & TRUE & FALSE & & \\
\hline Feeder Manager Non-Traceable & FALSE & TRUE & TRUE & FALSE & & \\
\hline Phase & TRUE & TRUE & FALSE & FALSE & & \\
\hline Circuit Number & TRUE & FALSE & TRUE & FALSE & & \\
\hline Fuse Link Amperage Rating & TRUE & TRUE & FALSE & FALSE & & \\
\hline A Phase Normal Position & TRUE & TRUE & FALSE & FALSE & & \\
\hline B Phase Normal Position & TRUE & TRUE & FALSE & FALSE & & \\
\hline C Phase Normal Position & TRUE & TRUE & FALSE & FALSE & & \\
\hline Section Fuse Number & TRUE & TRUE & TRUE & FALSE & & \\
\hline Circuit Number 2 & FALSE & TRUE & TRUE & FALSE & & \\
\hline Feeder Information & FALSE & TRUE & TRUE & FALSE & & \\
\hline AncillaryRole & FALSE & TRUE & TRUE & FALSE & & \\
\hline Legacy Node 1 & FALSE & TRUE & TRUE & FALSE & & \\
\hline Enabled & FALSE & TRUE & TRUE & FALSE & & \\
\hline Legacy Node 2 & FALSE & TRUE & TRUE & FALSE & & \\
\hline CreationDate & FALSE & TRUE & FALSE & FALSE & \begin{tabular}{|l|} 
ArcFM \\
Current \\
Date
\end{tabular} & \\
\hline Legacy Ees Number & FALSE & TRUE & TRUE & FALSE & & \\
\hline Legacy Pole Number & FALSE & TRUE & TRUE & FALSE & & \\
\hline Created By & FALSE & TRUE & FALSE & FALSE & \begin{tabular}{|l|} 
ArcFM \\
User Name \\
\end{tabular} & \\
\hline Related Support Structure Object Id & FALSE & TRUE & TRUE & FALSE & & \\
\hline Related SwitchGear Object Id & FALSE & TRUE & TRUE & FALSE & & \\
\hline Symbol Rotation & FALSE & FALSE & TRUE & FALSE & & \\
\hline Shape & FALSE & TRUE & TRUE & FALSE & & \\
\hline DCSID & FALSE & TRUE & TRUE & FALSE & & \\
\hline HANDLE & FALSE & TRUE & TRUE & FALSE & & \\
\hline TILENAME & FALSE & TRUE & TRUE & FALSE & & \\
\hline LAYER & FALSE & TRUE & TRUE & FALSE & & \\
\hline LOA & TRUE & FALSE & TRUE & FALSE & $\begin{array}{l}\text { NIPSCO } \\
\text { LoaName }\end{array}$ & $\begin{array}{l}\text { NIPSCO } \\
\text { LoaName }\end{array}$ \\
\hline Tax Unit & TRUE & TRUE & FALSE & FALSE & & \\
\hline Grid & TRUE & TRUE & FALSE & FALSE & & \\
\hline Symbol Configuration Cd & FALSE & TRUE & TRUE & FALSE & & \\
\hline FUSNUM & FALSE & TRUE & TRUE & FALSE & & \\
\hline$P \mathrm{PL}$ & FALSE & TRUE & TRUE & FALSE & & \\
\hline OBJECTID & TRUE & FALSE & FALSE & FALSE & & \\
\hline
\end{tabular}

arcfm8.ELECTRIC.FuseUnit

ArcFM Display Field: PHASEDESIGNATION

Create Edit Task:

On Create Event: EDFS Validate Work Order Number 2

On Update Event: EDFS Validate Work Order Number 2

On Delete Event: ArcFM Delete Feeder Object

On Abandon Event:

Abandon Feature Class:

Abandon Subtype:

Remove Feature Class:

Remove Subtype:

\begin{tabular}{|l|c|c|c|c|c|c|}
\hline Field Alias & Visible & Editable & $\begin{array}{c}\text { Allow Null } \\
\text { Values }\end{array}$ & $\begin{array}{c}\text { Clear } \\
\text { After } \\
\text { Create }\end{array}$ & $\begin{array}{c}\text { On Feature } \\
\text { Create }\end{array}$ & $\begin{array}{c}\text { On Feature } \\
\text { Update }\end{array}$ \\
\hline Subtype & TRUE & TRUE & FALSE & FALSE & & \\
\hline Cutout Amperage & TRUE & TRUE & FALSE & FALSE & & \\
\hline Fuse Link Amperage & TRUE & TRUE & FALSE & FALSE & & \\
\hline Phase & TRUE & TRUE & FALSE & FALSE & & \\
\hline Sectionalizer Fuse Number & TRUE & TRUE & TRUE & FALSE & & \\
\hline
\end{tabular}




\begin{tabular}{|c|c|c|c|c|c|c|}
\hline INSTALLDATE & TRUE & TRUE & FALSE & FALSE & & \\
\hline Install WO Number & TRUE & TRUE & FALSE & FALSE & & \\
\hline Install Misc Order Id & TRUE & TRUE & FALSE & FALSE & & \\
\hline RETIREDATE & FALSE & TRUE & TRUE & FALSE & & \\
\hline Retire WO Number & FALSE & TRUE & TRUE & FALSE & & \\
\hline Retire Misc Order Id & FALSE & TRUE & TRUE & FALSE & & \\
\hline Pole/Pad Number & FALSE & TRUE & TRUE & FALSE & & \\
\hline Circuit Number & FALSE & TRUE & TRUE & FALSE & & \\
\hline Related Fuse Cutout Object Id & TRUE & FALSE & TRUE & FALSE & & \\
\hline OBJECTID & TRUE & FALSE & FALSE & FALSE & & \\
\hline CreationDate & FALSE & TRUE & TRUE & FALSE & \begin{tabular}{|l|} 
ArcFM \\
Current \\
Date
\end{tabular} & \\
\hline Created By & FALSE & TRUE & TRUE & FALSE & \begin{tabular}{|l} 
ArcFM \\
User Name
\end{tabular} & \\
\hline UPDATEDATE & FALSE & FALSE & TRUE & FALSE & & $\begin{array}{l}\text { ArcFM Current } \\
\text { Date }\end{array}$ \\
\hline Updated By & FALSE & FALSE & TRUE & FALSE & & $\begin{array}{l}\text { ArcFM User } \\
\text { Name }\end{array}$ \\
\hline LEGACYWRINSTALLTIMESTAMP & FALSE & TRUE & TRUE & FALSE & & \\
\hline LEGACYWRREMOVALTIMESTAMP & FALSE & TRUE & TRUE & FALSE & & \\
\hline ASSOCIATIONTIMESTAMP & FALSE & TRUE & TRUE & FALSE & & \\
\hline
\end{tabular}

arcfm8.ELECTRIC.GisMappsTransactionQueue

ArcFM Display Field: OBJECTID

Create Edit Task:

On Create Event:

On Update Event:

On Delete Event:

On Abandon Event:

Abandon Feature Class:

Abandon Subtype:

Remove Feature Class:

Remove Subtype:

\begin{tabular}{|l|l|l|l|l|l|l|}
\hline Field Alias & Visible & Editable & $\begin{array}{c}\text { Allow Null } \\
\text { Values }\end{array}$ & $\begin{array}{c}\text { Clear } \\
\text { After } \\
\text { Create }\end{array}$ & $\begin{array}{c}\text { On Feature } \\
\text { Create }\end{array}$ & $\begin{array}{c}\text { On Feature } \\
\text { Update }\end{array}$ \\
\hline OBJECTID & TRUE & FALSE & FALSE & FALSE & & \\
\hline Company Number & TRUE & FALSE & FALSE & FALSE & & \\
\hline Transaction Type & TRUE & FALSE & FALSE & FALSE & & \\
\hline LOA & TRUE & FALSE & FALSE & FALSE & & \\
\hline SIN & TRUE & FALSE & FALSE & FALSE & & \\
\hline TRANSACTIONDATE & TRUE & FALSE & TRUE & FALSE & & \\
\hline Condemnation Number & TRUE & FALSE & FALSE & FALSE & & \\
\hline
\end{tabular}

arcfm8.ELECTRIC.JointUseAttachment

ArcFM Display Field: SUBTYPECD

Create Edit Task:

On Create Event: ArcFM Relate/Create Feeder Object

On Update Event: ArcFM Relate/Update Feeder Object

On Delete Event: ArcFM Delete Feeder Object

On Abandon Event:

Abandon Feature Class:

Abandon Subtype:

Remove Feature Class:

Remove Subtype:

\begin{tabular}{|l|c|c|c|c|c|c|}
\hline Field Alias & Visible & Editable & $\begin{array}{c}\text { Allow Null } \\
\text { Values }\end{array}$ & $\begin{array}{c}\text { Clear } \\
\text { After } \\
\text { Create }\end{array}$ & $\begin{array}{c}\text { On Feature } \\
\text { Create }\end{array}$ & $\begin{array}{c}\text { On Feature } \\
\text { Update }\end{array}$ \\
\hline Subtype & TRUE & TRUE & FALSE & FALSE & & \\
\hline
\end{tabular}




\begin{tabular}{|l|c|c|c|l|l|l|}
\hline Company Name & TRUE & TRUE & FALSE & FALSE & & \\
\hline Bound Indicator & TRUE & TRUE & FALSE & FALSE & & \\
\hline Related SupportStructure Object ID & TRUE & FALSE & TRUE & FALSE & & \\
\hline Legacy Pole Number & FALSE & TRUE & TRUE & FALSE & & \\
\hline OBJECTID & TRUE & FALSE & FALSE & FALSE & & \\
\hline CreationDate & FALSE & TRUE & TRUE & FALSE & $\begin{array}{l}\text { ArcFM } \\
\text { Datrent }\end{array}$ & \\
\hline Created By & FALSE & TRUE & TRUE & FALSE & $\begin{array}{l}\text { ArcFM } \\
\text { User Name }\end{array}$ & \\
\hline UPDATEDATE & FALSE & FALSE & TRUE & FALSE & & $\begin{array}{l}\text { ArcFM Current } \\
\text { Date }\end{array}$ \\
\hline Updated By & FALSE & FALSE & TRUE & FALSE & & $\begin{array}{l}\text { ArcFM User } \\
\text { Name }\end{array}$ \\
\hline
\end{tabular}

arcfm8.ELECTRIC.LegacyOhConductorInfo

ArcFM Display Field: WIRETYPECD

Create Edit Task:

On Create Event: EDFS Validate OH Primary Properties

On Create Event: EDFS Validate Work Order Number 2

On Update Event: EDFS Validate OH Primary Properties

On Update Event: EDFS Validate Work Order Number 2

On Delete Event:

On Abandon Event:

Abandon Feature Class:

Abandon Subtype:

Remove Feature Class:

Remove Subtype:

\begin{tabular}{|c|c|c|c|c|c|c|}
\hline Field Alias & Visible & Editable & $\begin{array}{c}\text { Allow Null } \\
\text { Values }\end{array}$ & $\begin{array}{l}\text { Clear } \\
\text { After } \\
\text { Create }\end{array}$ & $\begin{array}{c}\text { On Feature } \\
\text { Create }\end{array}$ & $\begin{array}{l}\text { On Feature } \\
\text { Update }\end{array}$ \\
\hline Subtype & TRUE & FALSE & FALSE & FALSE & & \\
\hline From Pole Number & TRUE & FALSE & FALSE & FALSE & & \\
\hline To Pole Number & TRUE & TRUE & FALSE & FALSE & & \\
\hline \begin{tabular}{|l|} 
INSTALLDATE \\
\end{tabular} & TRUE & FALSE & TRUE & FALSE & & \\
\hline Install WO Number & TRUE & FALSE & TRUE & FALSE & & \\
\hline Install Misc Order Id & TRUE & FALSE & TRUE & FALSE & & \\
\hline RETIREDATE & FALSE & FALSE & TRUE & FALSE & & \\
\hline Retire WO Number & FALSE & FALSE & TRUE & FALSE & & \\
\hline Retire Misc Order Id & FALSE & FALSE & TRUE & FALSE & & \\
\hline Wire Size & TRUE & FALSE & TRUE & FALSE & & \\
\hline Wire Material & TRUE & FALSE & TRUE & FALSE & & \\
\hline Wire Type & TRUE & FALSE & TRUE & FALSE & & \\
\hline Circuit Number & TRUE & TRUE & FALSE & FALSE & & \\
\hline Conductor Span Count & TRUE & TRUE & FALSE & FALSE & & \\
\hline Span Length & FALSE & TRUE & FALSE & FALSE & & \\
\hline OBJECTID & TRUE & FALSE & FALSE & FALSE & & \\
\hline Legacy Conductor Number & FALSE & TRUE & TRUE & FALSE & & \\
\hline CreationDate & FALSE & TRUE & TRUE & FALSE & & \\
\hline Created By & FALSE & TRUE & FALSE & FALSE & & \\
\hline UPDATEDATE & FALSE & FALSE & TRUE & FALSE & & $\begin{array}{l}\text { ArcFM Current } \\
\text { Date }\end{array}$ \\
\hline Updated By & FALSE & FALSE & TRUE & FALSE & & $\begin{array}{l}\text { ArcFM User } \\
\text { Name }\end{array}$ \\
\hline LEGACYWRINSTALLTIMESTAMP & FALSE & TRUE & TRUE & FALSE & & \\
\hline LEGACYWRREMOVALTIMESTAMP & FALSE & TRUE & TRUE & FALSE & & \\
\hline ASSOCIATIONTIMESTAMP & FALSE & TRUE & TRUE & FALSE & & \\
\hline
\end{tabular}

arcfm8.ELECTRIC.LegacyUgConductorInfo

ArcFM Display Field: WIRETYPECD 
Create Edit Task:

On Create Event: EDFS Validate UG Primary Properties

On Create Event: EDFS Validate Work Order Number 2

On Update Event: EDFS Validate UG Primary Properties

On Update Event: EDFS Validate Work Order Number 2

On Delete Event:

On Abandon Event:

Abandon Feature Class:

Abandon Subtype:

Remove Feature Class:

Remove Subtype:

\begin{tabular}{|c|c|c|c|c|c|c|}
\hline Field Alias & Visible & Editable & $\begin{array}{l}\text { Allow Null } \\
\text { Values }\end{array}$ & $\begin{array}{l}\text { Clear } \\
\text { After } \\
\text { Create }\end{array}$ & $\begin{array}{c}\text { On Feature } \\
\text { Create }\end{array}$ & $\begin{array}{c}\text { On Feature } \\
\text { Update }\end{array}$ \\
\hline Subtype & TRUE & FALSE & FALSE & FALSE & & \\
\hline From Pole/Pad Number & TRUE & FALSE & FALSE & FALSE & & \\
\hline To Structure Id & TRUE & TRUE & FALSE & FALSE & & \\
\hline INSTALLDATE & TRUE & FALSE & TRUE & FALSE & & \\
\hline Install WO Number & TRUE & FALSE & TRUE & FALSE & & \\
\hline Install Misc Order Id & TRUE & FALSE & TRUE & FALSE & & \\
\hline RETIREDATE & FALSE & FALSE & TRUE & FALSE & & \\
\hline Retire WO Number & FALSE & FALSE & TRUE & FALSE & & \\
\hline Retire Misc Order Id & FALSE & FALSE & TRUE & FALSE & & \\
\hline Wire Size & TRUE & FALSE & TRUE & FALSE & & \\
\hline Wire Material & TRUE & FALSE & TRUE & FALSE & & \\
\hline Wire Type & TRUE & FALSE & TRUE & FALSE & & \\
\hline \begin{tabular}{|l} 
In Conduit \\
\end{tabular} & TRUE & FALSE & TRUE & FALSE & & \\
\hline Circuit Number & TRUE & TRUE & FALSE & FALSE & & \\
\hline Conductor Span Count & TRUE & TRUE & FALSE & FALSE & & \\
\hline Span Length & FALSE & TRUE & TRUE & FALSE & & \\
\hline OBJECTID & TRUE & FALSE & FALSE & FALSE & & \\
\hline CreationDate & FALSE & TRUE & TRUE & FALSE & & \\
\hline Created By & FALSE & TRUE & FALSE & FALSE & & \\
\hline UPDATEDATE & FALSE & FALSE & TRUE & FALSE & & $\begin{array}{l}\text { ArcFM Current } \\
\text { Date }\end{array}$ \\
\hline Updated By & FALSE & FALSE & TRUE & FALSE & & $\begin{array}{l}\text { ArcFM User } \\
\text { Name }\end{array}$ \\
\hline LEGACYWRINSTALLTIMESTAMP & FALSE & TRUE & TRUE & FALSE & & \\
\hline LEGACYWRREMOVALTIMESTAMP & FALSE & TRUE & TRUE & FALSE & & \\
\hline ASSOCIATIONTIMESTAMP & FALSE & TRUE & TRUE & FALSE & & \\
\hline Legacy Conductor Number & FALSE & TRUE & TRUE & FALSE & & \\
\hline
\end{tabular}

arcfm8.ELECTRIC.Manhole

ArcFM Display Field: OBJECTID

Create Edit Task:

On Create Event:

On Update Event:

On Delete Event:

On Abandon Event:

Abandon Feature Class:

Abandon Subtype:

Remove Feature Class:

Remove Subtype:

\begin{tabular}{|l|l|l|l|l|l|l|}
\hline Field Alias & Visible & Editable & $\begin{array}{c}\text { Allow Null } \\
\text { Values }\end{array}$ & $\begin{array}{c}\text { Clear } \\
\text { After } \\
\text { Create }\end{array}$ & $\begin{array}{l}\text { On Feature } \\
\text { Create }\end{array}$ & $\begin{array}{c}\text { On Feature } \\
\text { Update }\end{array}$ \\
\hline Subtype & TRUE & TRUE & FALSE & FALSE & & \\
\hline LOA & TRUE & FALSE & FALSE & FALSE & $\begin{array}{l}\text { NIPSCO.Lo } \\
\text { aName }\end{array}$ & $\begin{array}{l}\text { NIPSCO.LOaNa } \\
\text { me }\end{array}$ \\
\hline
\end{tabular}




\begin{tabular}{|l|l|l|l|l|l|l|}
\hline Tax Unit & TRUE & TRUE & FALSE & FALSE & & \\
\hline Grid & TRUE & TRUE & FALSE & FALSE & & \\
\hline Manhole Number & TRUE & TRUE & TRUE & FALSE & & \\
\hline Shape & FALSE & TRUE & TRUE & FALSE & & \\
\hline CreationDate & FALSE & TRUE & FALSE & FALSE & $\begin{array}{l}\text { ArcFM } \\
\text { Darrent } \\
\text { Date }\end{array}$ & \\
\hline Created By & FALSE & TRUE & FALSE & FALSE & $\begin{array}{l}\text { ArcFM } \\
\text { User Name }\end{array}$ & \\
\hline UPDATEDATE & FALSE & FALSE & TRUE & FALSE & & $\begin{array}{l}\text { ArcFM Current } \\
\text { Date }\end{array}$ \\
\hline Updated By & FALSE & FALSE & TRUE & FALSE & & $\begin{array}{l}\text { ArcFM User } \\
\text { Name }\end{array}$ \\
\hline Legacy Ees Number & FALSE & TRUE & TRUE & FALSE & & \\
\hline Symbol Rotation & FALSE & FALSE & TRUE & FALSE & & \\
\hline HANDLE & FALSE & TRUE & TRUE & FALSE & & \\
\hline TILENAME & FALSE & TRUE & TRUE & FALSE & & \\
\hline MTGNUM & FALSE & TRUE & TRUE & FALSE & & \\
\hline OBJECTID & TRUE & FALSE & FALSE & FALSE & & \\
\hline
\end{tabular}

arcfm8.ELECTRIC.OhConductor

ArcFM Display Field: OBJECTID

Create Edit Task:

On Create Event: ArcFM Create Feeder Object

On Create Event: NIPSCO.SplitAtTapPoint

On Update Event: ArcFM Update Feeder Object

On Delete Event: NIPSCO.Prevent Delete If Units Related

On Delete Event: ArcFM OH - Delete Feeder Conductor/Units

On Split Event: ArcFM Split Feeder Object

On Abandon Event:

Before Split Event: EDFS Turn WOMO Validation Off

Before Split Event: ArcFM Save Related Objects

Before Split Event: EDFS Turn WOMO Validation On

After Split Event: EDFS Turn WOMO Validation Off

After Split Event: ArcFM Restore Related Objects

After Split Event: EDFS Turn WOMO Validation On

Abandon Feature Class:

Abandon Subtype:

Remove Feature Class:

Remove Subtype:

\begin{tabular}{|c|c|c|c|c|c|c|}
\hline Field Alias & Visible & Editable & $\begin{array}{l}\text { Allow Null } \\
\text { Values }\end{array}$ & $\begin{array}{l}\text { Clear } \\
\text { After } \\
\text { Create }\end{array}$ & $\begin{array}{c}\text { On Feature } \\
\text { Create }\end{array}$ & $\begin{array}{c}\text { On Feature } \\
\text { Update }\end{array}$ \\
\hline Subtype & TRUE & TRUE & FALSE & FALSE & & \\
\hline LOA Number & TRUE & FALSE & TRUE & FALSE & $\begin{array}{l}\text { NIPSCO.Lo } \\
\text { aName }\end{array}$ & $\begin{array}{l}\text { NIPSCO.LoaNa } \\
\text { me }\end{array}$ \\
\hline Grid & TRUE & TRUE & FALSE & FALSE & & \\
\hline Phase & TRUE & TRUE & FALSE & FALSE & & \\
\hline Circuit Number & TRUE & FALSE & TRUE & FALSE & & \\
\hline Primary Operating Voltage & TRUE & TRUE & FALSE & FALSE & & \\
\hline Shape & FALSE & TRUE & TRUE & FALSE & & \\
\hline Enabled & FALSE & TRUE & TRUE & FALSE & & \\
\hline CreationDate & FALSE & TRUE & FALSE & FALSE & \begin{tabular}{|l|} 
ArcFM \\
Current \\
Date \\
\end{tabular} & \\
\hline Created By & FALSE & TRUE & FALSE & FALSE & $\begin{array}{l}\text { ArcFM } \\
\text { User Name }\end{array}$ & \\
\hline UPDATEDATE & FALSE & FALSE & TRUE & FALSE & & $\begin{array}{l}\text { ArcFM Current } \\
\text { Date }\end{array}$ \\
\hline Updated By & FALSE & FALSE & TRUE & FALSE & & $\begin{array}{l}\text { ArcFM User } \\
\text { Name }\end{array}$ \\
\hline Legacy Node 1 & FALSE & TRUE & TRUE & FALSE & & \\
\hline
\end{tabular}




\begin{tabular}{|c|c|c|c|c|c|c|}
\hline Legacy Node 2 & FALSE & TRUE & TRUE & FALSE & & \\
\hline Measured Length & FALSE & TRUE & TRUE & FALSE & & \\
\hline Legacy Ees Number & FALSE & TRUE & TRUE & FALSE & & \\
\hline Electric Trace Weight & FALSE & TRUE & TRUE & FALSE & & \\
\hline Feeder Manager Non-Traceable & FALSE & TRUE & TRUE & FALSE & & \\
\hline Feeder Information & FALSE & TRUE & TRUE & FALSE & & \\
\hline Circuit Number 2 & FALSE & TRUE & TRUE & FALSE & & \\
\hline SHAPE.len & FALSE & & & & & \\
\hline Owner & TRUE & TRUE & FALSE & FALSE & & \\
\hline Legacy Circuit Number & FALSE & TRUE & TRUE & FALSE & & \\
\hline Wire Size & TRUE & TRUE & FALSE & FALSE & & \\
\hline Wire Material & TRUE & TRUE & FALSE & FALSE & & \\
\hline OBJECTID & TRUE & FALSE & FALSE & FALSE & & \\
\hline
\end{tabular}

arcfm8.ELECTRIC.OhConductorlnfo

ArcFM Display Field: WIREMATERIALCD

Create Edit Task:

On Create Event: ArcFM Create Feeder Object

On Create Event: EDFS Validate OH Primary Properties

On Create Event: EDFS Validate Work Order Number 2

On Update Event: ArcFM Update Feeder Object

On Update Event: EDFS Validate OH Primary Properties

On Update Event: EDFS Validate Work Order Number 2

On Delete Event: ArcFM Delete Feeder Object

On Abandon Event:

Abandon Feature Class:

Abandon Subtype:

Remove Feature Class:

Remove Subtype:

\begin{tabular}{|l|l|l|l|l|l|l|}
\hline Field Alias & Visible & Editable & $\begin{array}{c}\text { Allow Null } \\
\text { Values }\end{array}$ & $\begin{array}{c}\text { Clear } \\
\text { After } \\
\text { Create }\end{array}$ & $\begin{array}{c}\text { On Feature } \\
\text { Create }\end{array}$ & $\begin{array}{c}\text { On Feature } \\
\text { Update }\end{array}$ \\
\hline Subtype & TRUE & TRUE & FALSE & FALSE & & \\
\hline Wire Size & TRUE & TRUE & FALSE & FALSE & & \\
\hline Wire Material & TRUE & TRUE & FALSE & FALSE & & \\
\hline Wire Type & TRUE & TRUE & FALSE & FALSE & & \\
\hline Measured Length & FALSE & TRUE & TRUE & FALSE & & \\
\hline INSTALLDATE & TRUE & TRUE & FALSE & FALSE & & \\
\hline Install WO Number & TRUE & TRUE & FALSE & FALSE & & \\
\hline Install Misc Order Id & TRUE & TRUE & FALSE & FALSE & & \\
\hline RETIREDATE & FALSE & TRUE & TRUE & FALSE & & \\
\hline Retire WO Number & FALSE & TRUE & TRUE & FALSE & & \\
\hline Retire Misc Order Id & FALSE & TRUE & TRUE & FALSE & & \\
\hline From Pole Number & FALSE & TRUE & TRUE & FALSE & & \\
\hline To Pole Number & FALSE & TRUE & TRUE & FALSE & & \\
\hline Circuit Number & FALSE & TRUE & TRUE & FALSE & & \\
\hline Related Oh Conductor Object Id & TRUE & FALSE & TRUE & FALSE & & \\
\hline OBJECTID & TRUE & FALSE & FALSE & FALSE & & \\
\hline CreationDate & FALSE & TRUE & TRUE & FALSE & $\begin{array}{l}\text { ArcFM } \\
\text { Durrent }\end{array}$ & \\
\hline Created By & FALSE & TRUE & TRUE & FALSE & $\begin{array}{l}\text { ArcFM } \\
\text { User Name }\end{array}$ & ArcFM Current \\
\hline UPDATEDATE & FALSE & FALSE & TRUE & FALSE & & $\begin{array}{l}\text { ArcFM User } \\
\text { Name }\end{array}$ \\
\hline Updated By & FALSE & FALSE & TRUE & FALSE & & \\
\hline
\end{tabular}

arcfm8.ELECTRIC.OpenPoint

ArcFM Display Field: OBJECTID

Create Edit Task:

On Delete Event: ArcFM Delete Feeder Object 
On Abandon Event:

On Create Event: ArcFM Segment Split

On Create Event: ArcFM Create Feeder Object

On Update Event: ArcFM Update Feeder Object

Abandon Feature Class:

Abandon Subtype:

Remove Feature Class:

Remove Subtype:

\begin{tabular}{|c|c|c|c|c|c|c|}
\hline Field Alias & Visible & Editable & $\begin{array}{c}\text { Allow Null } \\
\text { Values }\end{array}$ & $\begin{array}{l}\text { Clear } \\
\text { After } \\
\text { Create }\end{array}$ & $\begin{array}{c}\text { On Feature } \\
\text { Create }\end{array}$ & $\begin{array}{c}\text { On Feature } \\
\text { Update }\end{array}$ \\
\hline Subtype & TRUE & TRUE & FALSE & FALSE & & \\
\hline LOA & TRUE & FALSE & TRUE & FALSE & $\begin{array}{l}\text { NIPSCO } \\
\text { LoaName }\end{array}$ & $\begin{array}{l}\text { NIPSCO } \\
\text { LoaName }\end{array}$ \\
\hline Grid & TRUE & TRUE & FALSE & FALSE & & \\
\hline Phase & TRUE & TRUE & FALSE & FALSE & & \\
\hline Circuit Number & TRUE & FALSE & TRUE & FALSE & & \\
\hline A Phase Normal Position & TRUE & TRUE & FALSE & FALSE & & \\
\hline B Phase Normal Position & TRUE & TRUE & FALSE & FALSE & & \\
\hline C Phase Normal Position & TRUE & TRUE & FALSE & FALSE & & \\
\hline Shape & FALSE & TRUE & TRUE & FALSE & & \\
\hline AncillaryRole & FALSE & TRUE & TRUE & FALSE & & \\
\hline Enabled & FALSE & TRUE & TRUE & FALSE & & \\
\hline CreationDate & FALSE & TRUE & FALSE & FALSE & \begin{tabular}{|l} 
ArcFM \\
Current \\
Date
\end{tabular} & \\
\hline Created By & FALSE & TRUE & FALSE & FALSE & \begin{tabular}{|l|} 
ArcFM \\
User Name
\end{tabular} & \\
\hline UPDATEDATE & FALSE & FALSE & TRUE & FALSE & & $\begin{array}{l}\text { ArcFM Current } \\
\text { Date }\end{array}$ \\
\hline Updated By & FALSE & FALSE & TRUE & FALSE & & $\begin{array}{l}\text { ArcFM User } \\
\text { Name }\end{array}$ \\
\hline Electric Trace Weight & FALSE & TRUE & TRUE & FALSE & & \\
\hline Feeder Manager Non-Traceable & FALSE & TRUE & TRUE & FALSE & & \\
\hline Circuit Number 2 & FALSE & TRUE & TRUE & FALSE & & \\
\hline Feeder Information & FALSE & TRUE & TRUE & FALSE & & \\
\hline Symbol Rotation & FALSE & FALSE & FALSE & FALSE & & \\
\hline Legacy Node 1 & FALSE & TRUE & TRUE & FALSE & & \\
\hline Legacy Node 2 & FALSE & TRUE & TRUE & FALSE & & \\
\hline Legacy Ees Number & FALSE & TRUE & TRUE & FALSE & & \\
\hline HANDLE & FALSE & TRUE & TRUE & FALSE & & \\
\hline TILENAME & FALSE & TRUE & TRUE & FALSE & & \\
\hline DCSID & FALSE & TRUE & TRUE & FALSE & & \\
\hline LAYER & FALSE & TRUE & TRUE & FALSE & & \\
\hline OBJECTID & TRUE & FALSE & FALSE & FALSE & & \\
\hline
\end{tabular}

arcfm8.ELECTRIC.PadMount

ArcFM Display Field: DISTRIBREFNUMBER

Create Edit Task:

On Create Event: EDFS Validate Work Order Number 2

On Create Event: NIPSCO.UniqueDistribRefNumber-OnCreate

On Delete Event: EDFS Prevent Deletion if Related Assets

On Abandon Event:

On Update Event: EDFS Validate Work Order Number 2

On Update Event: NIPSCO.UniqueDistribRefNumber-OnUpdate

Abandon Feature Class:

Abandon Subtype:

Remove Feature Class: arcfm8.ELECTRIC.RetiredPadMount

Remove Subtype:

\begin{tabular}{|l|l|l|l|l|l|l|}
\hline Field Alias & Visible & Editable & $\begin{array}{c}\text { Allow Null } \\
\text { Values }\end{array}$ & $\begin{array}{c}\text { Clear } \\
\text { After } \\
\text { Create }\end{array}$ & $\begin{array}{c}\text { On Feature } \\
\text { Create }\end{array}$ & $\begin{array}{c}\text { On Feature } \\
\text { Update }\end{array}$ \\
\hline
\end{tabular}




\begin{tabular}{|c|c|c|c|c|c|c|}
\hline Subtype & TRUE & TRUE & FALSE & FALSE & & \\
\hline Long Pad Number & TRUE & TRUE & FALSE & FALSE & & \\
\hline Location Description & TRUE & TRUE & FALSE & FALSE & & \\
\hline Print Id & TRUE & TRUE & FALSE & FALSE & & \\
\hline Owner Indicator & TRUE & TRUE & FALSE & FALSE & & \\
\hline INSTALLDATE & TRUE & TRUE & FALSE & FALSE & & \\
\hline Install WO Number & TRUE & TRUE & FALSE & FALSE & & \\
\hline Install Misc Order Id & TRUE & TRUE & FALSE & FALSE & & \\
\hline RETIREDATE & FALSE & TRUE & TRUE & FALSE & & \\
\hline Retire WO Number & FALSE & TRUE & TRUE & FALSE & & \\
\hline Retire Misc Order Id & FALSE & TRUE & TRUE & FALSE & & \\
\hline LOA & TRUE & FALSE & TRUE & FALSE & $\begin{array}{l}\text { NIPSCO } \\
\text { LoaName }\end{array}$ & $\begin{array}{l}\text { NIPSCO } \\
\text { LoaName }\end{array}$ \\
\hline Tax Unit & TRUE & TRUE & FALSE & FALSE & & \\
\hline Grid & TRUE & TRUE & FALSE & FALSE & & \\
\hline Type & TRUE & TRUE & TRUE & FALSE & & \\
\hline Service PointType & TRUE & TRUE & TRUE & FALSE & & \\
\hline OBJECTID & TRUE & FALSE & FALSE & FALSE & & \\
\hline Shape & FALSE & TRUE & TRUE & FALSE & & \\
\hline CreationDate & FALSE & TRUE & TRUE & FALSE & \begin{tabular}{|l|} 
ArcFM \\
Current \\
Date
\end{tabular} & \\
\hline Created By & FALSE & TRUE & TRUE & FALSE & \begin{tabular}{|l|} 
ArcFM \\
User Name
\end{tabular} & \\
\hline UPDATEDATE & FALSE & FALSE & TRUE & FALSE & & $\begin{array}{l}\text { ArcFM Current } \\
\text { Date }\end{array}$ \\
\hline Updated By & FALSE & FALSE & TRUE & FALSE & & $\begin{array}{l}\text { ArcFM User } \\
\text { Name }\end{array}$ \\
\hline Legacy Ees Number & FALSE & TRUE & TRUE & FALSE & & \\
\hline Symbol Rotation & FALSE & FALSE & FALSE & FALSE & & \\
\hline Graphics Scale Factor & FALSE & TRUE & TRUE & FALSE & & \\
\hline Legacy Termination Pole Id & FALSE & TRUE & TRUE & FALSE & & \\
\hline LEGACYWRREMOVALTIMESTAMP & FALSE & TRUE & TRUE & FALSE & & \\
\hline LEGACYWRINSTALLTIMESTAMP & FALSE & TRUE & TRUE & FALSE & & \\
\hline REMOVALTIMESTAMP & FALSE & TRUE & TRUE & FALSE & & \\
\hline INSTALLTIMESTAMP & FALSE & TRUE & TRUE & FALSE & $\begin{array}{l}\text { ArcFM } \\
\text { Current } \\
\text { Date } \\
\end{array}$ & \\
\hline
\end{tabular}

arcfm8.ELECTRIC.Pedestal

ArcFM Display Field: LONGPEDESTALNUMBER

Create Edit Task:

On Create Event: EDFS Validate Work Order Number 2

On Update Event: EDFS Validate Work Order Number 2

On Delete Event: EDFS Prevent Deletion if Related Assets

On Abandon Event:

Abandon Feature Class:

Abandon Subtype:

Remove Feature Class:

Remove Subtype:

\begin{tabular}{|l|c|c|c|c|c|c|}
\hline Field Alias & Visible & Editable & $\begin{array}{c}\text { Allow Null } \\
\text { Values }\end{array}$ & $\begin{array}{c}\text { Clear } \\
\text { After } \\
\text { Create }\end{array}$ & $\begin{array}{c}\text { On Feature } \\
\text { Create }\end{array}$ & $\begin{array}{c}\text { On Feature } \\
\text { Update }\end{array}$ \\
\hline Subtype & TRUE & TRUE & FALSE & FALSE & & \\
\hline Long Pedestal Number & TRUE & TRUE & FALSE & FALSE & & \\
\hline INSTALLDATE & TRUE & TRUE & FALSE & FALSE & & \\
\hline Install WO Number & TRUE & TRUE & FALSE & FALSE & & \\
\hline Install Misc Order Id & TRUE & TRUE & FALSE & FALSE & & \\
\hline RETIREDATE & FALSE & TRUE & TRUE & FALSE & & \\
\hline Retire WO Number & FALSE & TRUE & TRUE & FALSE & & \\
\hline Retire Misc Order Id & FALSE & TRUE & TRUE & FALSE & & \\
\hline LOA & TRUE & FALSE & FALSE & FALSE & NIPSCO & NIPSCO \\
\hline
\end{tabular}




\begin{tabular}{|l|l|l|l|l|l|l|}
\hline & & & & & LoaName & LoaName \\
\hline Tax Unit & TRUE & TRUE & FALSE & FALSE & & \\
\hline Grid & TRUE & TRUE & FALSE & FALSE & & \\
\hline CreationDate & FALSE & TRUE & TRUE & FALSE & & \\
\hline Created By & FALSE & TRUE & FALSE & FALSE & $\begin{array}{l}\text { ArcFM } \\
\text { Current } \\
\text { Date }\end{array}$ & \\
\hline UPDATEDATE & FALSE & TRUE & FALSE & FALSE & $\begin{array}{l}\text { ArcFM } \\
\text { User Name }\end{array}$ & \\
\hline Updated By & FALSE & FALSE & TRUE & FALSE & & $\begin{array}{l}\text { ArcFM Current } \\
\text { Date }\end{array}$ \\
\hline Legacy Ees Number & FALSE & FALSE & TRUE & FALSE & & $\begin{array}{l}\text { ArcFM User } \\
\text { Name }\end{array}$ \\
\hline Symbol Rotation & FALSE & TRUE & TRUE & FALSE & & \\
\hline LEGACYWRINSTALLTIMESTAMP & FALSE & FALSE & TRUE & FALSE & & \\
\hline LEGACYWRREMOVALTIMESTAMP & FALSE & TRUE & TRUE & FALSE & & \\
\hline OBJECTID & FALSE & TRUE & TRUE & FALSE & & \\
\hline
\end{tabular}

arcfm8.ELECTRIC.PowerTransformer

ArcFM Display Field: OBJECTID

Create Edit Task:

On Create Event:

On Update Event:

On Delete Event:

On Abandon Event:

Abandon Feature Class:

Abandon Subtype:

Remove Feature Class:

Remove Subtype:

\begin{tabular}{|l|c|c|c|c|c|c|}
\hline Field Alias & Visible & Editable & $\begin{array}{c}\text { Allow Null } \\
\text { Values }\end{array}$ & $\begin{array}{c}\text { Clear } \\
\text { After } \\
\text { Create }\end{array}$ & $\begin{array}{c}\text { On Feature } \\
\text { Create }\end{array}$ & $\begin{array}{c}\text { On Feature } \\
\text { Update }\end{array}$ \\
\hline OBJECTID & TRUE & FALSE & FALSE & FALSE & & \\
\hline Shape & FALSE & TRUE & TRUE & FALSE & & \\
\hline
\end{tabular}

arcfm8.ELECTRIC.PrimaryMeter

ArcFM Display Field: OBJECTID

Create Edit Task:

On Delete Event: ArcFM Delete Feeder Object

On Abandon Event:

On Create Event: EDFS Validate Work Order Number 1

On Create Event: NIPSCO Structure Relate

On Create Event: ArcFM Create Feeder Object

On Update Event: EDFS Validate Work Order Number 1

On Update Event: NIPSCO Structure Relate

On Update Event: ArcFM Update Feeder Object

Abandon Feature Class:

Abandon Subtype:

Remove Feature Class:

Remove Subtype:

\begin{tabular}{|l|l|l|l|l|l|l|}
\hline Field Alias & Visible & Editable & $\begin{array}{c}\text { Allow Null } \\
\text { Values }\end{array}$ & $\begin{array}{c}\text { Clear } \\
\text { After } \\
\text { Create }\end{array}$ & $\begin{array}{c}\text { On Feature } \\
\text { Create }\end{array}$ & $\begin{array}{c}\text { On Feature } \\
\text { Update }\end{array}$ \\
\hline Subtype & TRUE & TRUE & FALSE & FALSE & & \\
\hline INSTALLDATE & TRUE & TRUE & FALSE & FALSE & & \\
\hline Install WO Number & TRUE & TRUE & FALSE & FALSE & & \\
\hline Install Misc Order Id & TRUE & TRUE & FALSE & FALSE & & \\
\hline RETIREDATE & FALSE & TRUE & TRUE & FALSE & & \\
\hline Retire WO Number & FALSE & TRUE & TRUE & FALSE & & \\
\hline Retire Misc Order Id & FALSE & TRUE & TRUE & FALSE & & \\
\hline
\end{tabular}




\begin{tabular}{|c|c|c|c|c|c|c|}
\hline LOA & TRUE & FALSE & TRUE & FALSE & $\begin{array}{l}\text { NIPSCO } \\
\text { LoaName }\end{array}$ & $\begin{array}{l}\text { NIPSCO } \\
\text { LoaName }\end{array}$ \\
\hline Grid & TRUE & TRUE & FALSE & FALSE & & \\
\hline Phase & TRUE & TRUE & FALSE & FALSE & & \\
\hline Circuit Number & TRUE & FALSE & TRUE & FALSE & & \\
\hline Related Support Structure Object Id & FALSE & TRUE & TRUE & FALSE & & \\
\hline Shape & FALSE & TRUE & TRUE & FALSE & & \\
\hline AncillaryRole & FALSE & TRUE & TRUE & FALSE & & \\
\hline Enabled & FALSE & TRUE & TRUE & FALSE & & \\
\hline CreationDate & FALSE & TRUE & FALSE & FALSE & $\begin{array}{l}\text { ArcFM } \\
\text { Current } \\
\text { Date } \\
\end{array}$ & \\
\hline Created By & FALSE & TRUE & FALSE & FALSE & \begin{tabular}{|l|} 
ArcFM \\
User Name
\end{tabular} & \\
\hline UPDATEDATE & FALSE & FALSE & TRUE & FALSE & & $\begin{array}{l}\text { ArcFM Current } \\
\text { Date }\end{array}$ \\
\hline Updated By & FALSE & FALSE & TRUE & FALSE & & $\begin{array}{l}\text { ArcFM User } \\
\text { Name }\end{array}$ \\
\hline Electric Trace Weight & FALSE & TRUE & TRUE & FALSE & & \\
\hline Feeder Manager Non-Traceable & FALSE & TRUE & TRUE & FALSE & & \\
\hline Circuit Number 2 & FALSE & TRUE & TRUE & FALSE & & \\
\hline Feeder Information & FALSE & TRUE & TRUE & FALSE & & \\
\hline Legacy Node 1 & FALSE & TRUE & TRUE & FALSE & & \\
\hline Legacy Node 2 & FALSE & TRUE & TRUE & FALSE & & \\
\hline Legacy Ees Number & FALSE & TRUE & TRUE & FALSE & & \\
\hline Legacy Distribution Reference Number & FALSE & TRUE & TRUE & FALSE & & \\
\hline Symbol Rotation & FALSE & FALSE & TRUE & FALSE & & \\
\hline Related Pad Mount Object Id & FALSE & TRUE & TRUE & FALSE & & \\
\hline OBJECTID & TRUE & FALSE & FALSE & FALSE & & \\
\hline
\end{tabular}

arcfm8.ELECTRIC.RecloserBank

ArcFM Display Field: OBJECTID

Create Edit Task: NIPSCO.Electric.FeatureOffset

On Abandon Event:

On Create Event: ArcFM Segment Split

On Create Event: NIPSCO Structure Relate

On Create Event: ArcFM Create Feeder Object

On Update Event: NIPSCO Structure Relate

On Update Event: ArcFM Update Feeder Object

On Delete Event: NIPSCO.Prevent Delete If Units Related

On Delete Event: ArcFM Delete Feeder Object

Abandon Feature Class:

Abandon Subtype:

Remove Feature Class:

Remove Subtype:

\begin{tabular}{|l|l|l|l|l|l|l|}
\hline Field Alias & Visible & Editable & $\begin{array}{c}\text { Allow Null } \\
\text { Values }\end{array}$ & $\begin{array}{c}\text { Clear } \\
\text { After } \\
\text { Create }\end{array}$ & $\begin{array}{c}\text { On Feature } \\
\text { Create }\end{array}$ & $\begin{array}{c}\text { On Feature } \\
\text { Update }\end{array}$ \\
\hline Subtype & TRUE & TRUE & FALSE & FALSE & & \\
\hline Recloser Type & TRUE & TRUE & FALSE & FALSE & & \\
\hline Recloser Size & TRUE & TRUE & FALSE & FALSE & & \\
\hline Bypass Status & TRUE & TRUE & FALSE & FALSE & & \\
\hline Phase & TRUE & TRUE & FALSE & FALSE & & \\
\hline Circuit Number & TRUE & FALSE & TRUE & FALSE & & \\
\hline A Phase Normal Position & TRUE & TRUE & FALSE & FALSE & & \\
\hline B Phase Normal Position & TRUE & TRUE & FALSE & FALSE & & \\
\hline C Phase Normal Position & TRUE & TRUE & FALSE & FALSE & & \\
\hline Related Support Structure Object Id & FALSE & TRUE & TRUE & FALSE & & \\
\hline Shape & FALSE & TRUE & TRUE & FALSE & & \\
\hline
\end{tabular}




\begin{tabular}{|c|c|c|c|c|c|c|}
\hline AncillaryRole & FALSE & TRUE & TRUE & FALSE & & \\
\hline Enabled & FALSE & TRUE & TRUE & FALSE & & \\
\hline CreationDate & FALSE & TRUE & FALSE & FALSE & \begin{tabular}{|l|} 
ArcFM \\
Current \\
Date \\
\end{tabular} & \\
\hline Created By & FALSE & TRUE & FALSE & FALSE & \begin{tabular}{|l|} 
ArcFM \\
User Name
\end{tabular} & \\
\hline UPDATEDATE & FALSE & FALSE & TRUE & FALSE & & $\begin{array}{l}\text { ArcFM Current } \\
\text { Date } \\
\end{array}$ \\
\hline Updated By & FALSE & FALSE & TRUE & FALSE & & $\begin{array}{l}\text { ArcFM User } \\
\text { Name }\end{array}$ \\
\hline Electric Trace Weight & FALSE & TRUE & TRUE & FALSE & & \\
\hline Feeder Manager Non-Traceable & FALSE & TRUE & TRUE & FALSE & & \\
\hline Circuit Number 2 & FALSE & TRUE & TRUE & FALSE & & \\
\hline Feeder Information & FALSE & TRUE & TRUE & FALSE & & \\
\hline Graphics Scale Factor & FALSE & TRUE & TRUE & FALSE & & \\
\hline Legacy Node 1 & FALSE & TRUE & TRUE & FALSE & & \\
\hline Legacy Node 2 & FALSE & TRUE & TRUE & FALSE & & \\
\hline Legacy Ees Number & FALSE & TRUE & TRUE & FALSE & & \\
\hline Legacy Pole Number & FALSE & TRUE & TRUE & FALSE & & \\
\hline Symbol Rotation & FALSE & FALSE & TRUE & FALSE & & \\
\hline Setting & TRUE & TRUE & TRUE & FALSE & & \\
\hline DCSID & FALSE & TRUE & TRUE & FALSE & & \\
\hline HANDLE & FALSE & TRUE & TRUE & FALSE & & \\
\hline TILENAME & FALSE & TRUE & TRUE & FALSE & & \\
\hline LOA & TRUE & FALSE & TRUE & FALSE & $\begin{array}{l}\text { NIPSCO } \\
\text { LoaName } \\
\end{array}$ & \begin{tabular}{|l} 
NIPSCO \\
LoaName \\
\end{tabular} \\
\hline Tax Unit & TRUE & TRUE & FALSE & FALSE & & \\
\hline Grid & TRUE & TRUE & TRUE & FALSE & & \\
\hline $\mathrm{P} \_\mathrm{L}$ & FALSE & TRUE & TRUE & FALSE & & \\
\hline OBJECTID & TRUE & FALSE & FALSE & FALSE & & \\
\hline
\end{tabular}

arcfm8.ELECTRIC.RecloserUnit

ArcFM Display Field: COMPANYNUMBER

Create Edit Task:

On Create Event: EDFS Validate Work Order Number 2

On Update Event: EDFS Validate Work Order Number 2

On Delete Event:

On Abandon Event:

Abandon Feature Class:

Abandon Subtype:

Remove Feature Class:

Remove Subtype:

\begin{tabular}{|l|c|c|c|c|c|c|}
\hline Field Alias & Visible & Editable & $\begin{array}{c}\text { Allow Null } \\
\text { Values }\end{array}$ & $\begin{array}{c}\text { Clear } \\
\text { After } \\
\text { Create }\end{array}$ & $\begin{array}{c}\text { On Feature } \\
\text { Create }\end{array}$ & $\begin{array}{c}\text { On Feature } \\
\text { Update }\end{array}$ \\
\hline Subtype & TRUE & TRUE & FALSE & FALSE & & \\
\hline Company Number & TRUE & TRUE & FALSE & FALSE & & \\
\hline Recloser Amperage & TRUE & TRUE & FALSE & FALSE & & \\
\hline Phase & TRUE & TRUE & FALSE & FALSE & & \\
\hline Sectionalizer Fuse Number & TRUE & TRUE & TRUE & FALSE & & \\
\hline Pole/Pad Number & FALSE & TRUE & TRUE & FALSE & & \\
\hline INSTALLDATE & TRUE & TRUE & FALSE & FALSE & & \\
\hline Install WO Number & TRUE & TRUE & FALSE & FALSE & & \\
\hline Install Misc Order Id & TRUE & TRUE & FALSE & FALSE & & \\
\hline RETIREDATE & FALSE & TRUE & TRUE & FALSE & & \\
\hline Retire WO Number & FALSE & TRUE & TRUE & FALSE & & \\
\hline Retire Misc Order Id & FALSE & TRUE & TRUE & FALSE & & \\
\hline Circuit Number & FALSE & TRUE & TRUE & FALSE & & \\
\hline Related Recloser Bank Object Id & TRUE & FALSE & TRUE & FALSE & & \\
\hline OBJECTID & TRUE & FALSE & FALSE & FALSE & & \\
\hline CreationDate & FALSE & TRUE & TRUE & FALSE & ArcFM & \\
\hline
\end{tabular}




\begin{tabular}{|l|c|c|c|l|l|l|}
\hline & & & & & $\begin{array}{l}\text { lurrent } \\
\text { Date }\end{array}$ & \\
\hline Created By & FALSE & TRUE & TRUE & FALSE & $\begin{array}{l}\text { ArcFM } \\
\text { User Name }\end{array}$ & \\
\hline UPDATEDATE & FALSE & FALSE & TRUE & FALSE & & $\begin{array}{l}\text { ArcFM Current } \\
\text { Date }\end{array}$ \\
\hline Updated By & FALSE & FALSE & TRUE & FALSE & & $\begin{array}{l}\text { ArcFM User } \\
\text { Name }\end{array}$ \\
\hline LEGACYWRINSTALLTIMESTAMP & FALSE & TRUE & TRUE & FALSE & & \\
\hline LEGACYWRREMOVALTIMESTAMP & FALSE & TRUE & TRUE & FALSE & & \\
\hline ASSOCIATIONTIMESTAMP & FALSE & TRUE & TRUE & FALSE & & \\
\hline
\end{tabular}

arcfm8.ELECTRIC.RegulatorFunctionTest

ArcFM Display Field: FUNCTIONTESTDATE

Create Edit Task:

On Create Event:

On Update Event:

On Delete Event:

On Abandon Event:

Abandon Feature Class:

Abandon Subtype:

Remove Feature Class:

Remove Subtype:

\begin{tabular}{|l|c|c|c|c|c|c|}
\hline Field Alias & Visible & Editable & $\begin{array}{c}\text { Allow Null } \\
\text { Values }\end{array}$ & $\begin{array}{c}\text { Clear } \\
\text { After } \\
\text { Create }\end{array}$ & $\begin{array}{c}\text { On Feature } \\
\text { Create }\end{array}$ & $\begin{array}{c}\text { On Feature } \\
\text { Update }\end{array}$ \\
\hline Company Number & TRUE & FALSE & FALSE & FALSE & & \\
\hline FUNCTIONTESTDATE & TRUE & FALSE & FALSE & FALSE & & \\
\hline OBJECTID & TRUE & FALSE & FALSE & FALSE & & \\
\hline CreationDate & FALSE & TRUE & TRUE & FALSE & & \\
\hline Created By & FALSE & TRUE & FALSE & FALSE & & \\
\hline UPDATEDATE & FALSE & TRUE & TRUE & FALSE & & \\
\hline Updated By & FALSE & TRUE & FALSE & FALSE & & \\
\hline
\end{tabular}

arcfm8.ELECTRIC.RegulatorOilTest

ArcFM Display Field: OILTESTDATE

Create Edit Task:

On Create Event:

On Update Event:

On Delete Event:

On Abandon Event:

Abandon Feature Class:

Abandon Subtype:

Remove Feature Class:

Remove Subtype:

\begin{tabular}{|l|c|c|c|c|c|c|}
\hline Field Alias & Visible & Editable & $\begin{array}{c}\text { Allow Null } \\
\text { Values }\end{array}$ & $\begin{array}{c}\text { Clear } \\
\text { After } \\
\text { Create }\end{array}$ & $\begin{array}{c}\text { On Feature } \\
\text { Create }\end{array}$ & $\begin{array}{c}\text { On Feature } \\
\text { Update }\end{array}$ \\
\hline Company Number & TRUE & FALSE & FALSE & FALSE & & \\
\hline OILTESTDATE & TRUE & FALSE & FALSE & FALSE & & \\
\hline Test Type & TRUE & FALSE & FALSE & FALSE & & \\
\hline PPM & TRUE & FALSE & FALSE & FALSE & & \\
\hline OBJECTID & TRUE & FALSE & FALSE & FALSE & & \\
\hline CreationDate & FALSE & TRUE & TRUE & FALSE & & \\
\hline Created By & FALSE & TRUE & FALSE & FALSE & & \\
\hline UPDATEDATE & FALSE & TRUE & TRUE & FALSE & & \\
\hline Updated By & FALSE & TRUE & FALSE & FALSE & & \\
\hline
\end{tabular}

arcfm8.ELECTRIC.RegulatorUnit

ArcFM Display Field: COMPANYNUMBER

Create Edit Task: 
On Create Event:

On Update Event:

On Delete Event:

On Abandon Event:

Abandon Feature Class:

Abandon Subtype:

Remove Feature Class:

Remove Subtype:

\begin{tabular}{|l|l|l|l|l|l|l|}
\hline Field Alias & Visible & Editable & $\begin{array}{c}\text { Allow Null } \\
\text { Values }\end{array}$ & $\begin{array}{c}\text { Clear } \\
\text { After } \\
\text { Create }\end{array}$ & $\begin{array}{c}\text { On Feature } \\
\text { Create }\end{array}$ & $\begin{array}{c}\text { On Feature } \\
\text { Update }\end{array}$ \\
\hline Company Number & TRUE & FALSE & FALSE & FALSE & & \\
\hline SIN & TRUE & FALSE & TRUE & FALSE & & \\
\hline Status & TRUE & FALSE & TRUE & FALSE & & \\
\hline Disposition & TRUE & FALSE & TRUE & FALSE & & \\
\hline P.O. Number & TRUE & FALSE & TRUE & FALSE & & \\
\hline RECEIVEDATE & TRUE & FALSE & TRUE & FALSE & & \\
\hline Impedance & TRUE & FALSE & TRUE & FALSE & & \\
\hline Weight & TRUE & FALSE & TRUE & FALSE & & \\
\hline Installation Cost & TRUE & FALSE & TRUE & FALSE & & \\
\hline Polarity & TRUE & FALSE & TRUE & FALSE & & \\
\hline Gallons of Oil & TRUE & FALSE & TRUE & FALSE & & \\
\hline Manufacturer & TRUE & FALSE & TRUE & FALSE & & \\
\hline Serial Number & TRUE & FALSE & TRUE & FALSE & & \\
\hline Warranty & TRUE & FALSE & TRUE & FALSE & & \\
\hline OBJECTID & TRUE & FALSE & FALSE & FALSE & & \\
\hline DISPOSITIONTIMESTAMP & FALSE & TRUE & TRUE & FALSE & & \\
\hline STATUSTIMESTAMP & FALSE & TRUE & FALSE & FALSE & & \\
\hline CreationDate & FALSE & TRUE & TRUE & FALSE & $\begin{array}{l}\text { ArcFM } \\
\text { Current } \\
\text { Date }\end{array}$ & \\
\hline Created By & FALSE & TRUE & FALSE & FALSE & $\begin{array}{l}\text { ArcFM } \\
\text { User Name }\end{array}$ & \\
\hline UPDATEDATE & FALSE & FALSE & TRUE & FALSE & & $\begin{array}{l}\text { ArcFM Current } \\
\text { Date }\end{array}$ \\
\hline Updated By & FALSE & FALSE & TRUE & FALSE & & $\begin{array}{l}\text { ArcFM User } \\
\text { Name }\end{array}$ \\
\hline
\end{tabular}

arcfm8.ELECTRIC.RegulatorUnitCondemn

ArcFM Display Field: CONDEMNDATE

Create Edit Task:

On Create Event:

On Update Event:

On Delete Event:

On Abandon Event:

Abandon Feature Class:

Abandon Subtype:

Remove Feature Class:

Remove Subtype:

\begin{tabular}{|l|c|c|c|c|c|c|}
\hline Field Alias & Visible & Editable & $\begin{array}{c}\text { Allow Null } \\
\text { Values }\end{array}$ & $\begin{array}{c}\text { Clear } \\
\text { After } \\
\text { Create }\end{array}$ & $\begin{array}{c}\text { On Feature } \\
\text { Create }\end{array}$ & $\begin{array}{c}\text { On Feature } \\
\text { Update }\end{array}$ \\
\hline Company Number & TRUE & FALSE & FALSE & FALSE & & \\
\hline CONDEMNDATE & TRUE & FALSE & TRUE & FALSE & & \\
\hline Condemn Number & TRUE & FALSE & TRUE & FALSE & & \\
\hline Type & TRUE & FALSE & TRUE & FALSE & & \\
\hline Reason & TRUE & FALSE & TRUE & FALSE & & \\
\hline Approved & TRUE & FALSE & TRUE & FALSE & & \\
\hline OBJECTID & TRUE & FALSE & FALSE & FALSE & & \\
\hline CreationDate & FALSE & TRUE & TRUE & FALSE & $\begin{array}{l}\text { ArcFM } \\
\text { Current } \\
\text { Date }\end{array}$ & \\
\hline
\end{tabular}




\begin{tabular}{|l|c|c|c|c|l|l|}
\hline Created By & FALSE & TRUE & FALSE & FALSE & $\begin{array}{l}\text { ArcFM } \\
\text { User Name }\end{array}$ & \\
\hline UPDATEDATE & FALSE & FALSE & TRUE & FALSE & & $\begin{array}{l}\text { ArcFM Current } \\
\text { Date }\end{array}$ \\
\hline Updated By & FALSE & FALSE & TRUE & FALSE & & $\begin{array}{l}\text { ArcFM User } \\
\text { Name }\end{array}$ \\
\hline CONDEMNTIMESTAMP & FALSE & TRUE & FALSE & FALSE & & \\
\hline
\end{tabular}

arcfm8.ELECTRIC.RegulatorUnitInstall

ArcFM Display Field: RELREGULATORUNITCOMPANYNUMBER

Create Edit Task:

On Create Event:

On Update Event:

On Delete Event: EDFS Reset Asset To Stock

On Abandon Event:

Abandon Feature Class:

Abandon Subtype:

Remove Feature Class:

Remove Subtype:

\begin{tabular}{|c|c|c|c|c|c|c|}
\hline Field Alias & Visible & Editable & \begin{tabular}{|c|} 
Allow \\
Null \\
Values
\end{tabular} & $\begin{array}{c}\text { Clear After } \\
\text { Create }\end{array}$ & $\begin{array}{c}\text { On Feature } \\
\text { Create }\end{array}$ & $\begin{array}{l}\text { On Feature } \\
\text { Update }\end{array}$ \\
\hline Company Number & TRUE & FALSE & FALSE & FALSE & & \\
\hline Company Use Location Id & TRUE & TRUE & TRUE & FALSE & & \\
\hline INSTALLDATE & TRUE & FALSE & TRUE & FALSE & & \\
\hline Material Ticket & TRUE & TRUE & FALSE & FALSE & & \\
\hline Circuit Number & FALSE & FALSE & TRUE & FALSE & & \\
\hline Phase & TRUE & TRUE & FALSE & FALSE & & \\
\hline KVA & TRUE & FALSE & TRUE & FALSE & & \\
\hline \begin{tabular}{|l|} 
Installation Status \\
\end{tabular} & TRUE & TRUE & FALSE & FALSE & & \\
\hline Installation Type & TRUE & TRUE & FALSE & FALSE & & \\
\hline LOA & TRUE & FALSE & TRUE & FALSE & & \\
\hline Tax Unit & TRUE & TRUE & FALSE & FALSE & & \\
\hline Grid & TRUE & TRUE & FALSE & FALSE & & \\
\hline Pole Number & TRUE & TRUE & FALSE & FALSE & & \\
\hline Related Voltage Regulator Object Id & TRUE & FALSE & TRUE & FALSE & & \\
\hline OBJECTID & TRUE & FALSE & FALSE & FALSE & & \\
\hline INSTALLTIMESTAMP & FALSE & TRUE & FALSE & FALSE & & \\
\hline CreationDate & FALSE & TRUE & TRUE & FALSE & \begin{tabular}{|l} 
ArcFM \\
Current \\
Date
\end{tabular} & \\
\hline Created By & FALSE & TRUE & FALSE & FALSE & \begin{tabular}{|l|} 
ArcFM \\
User Name
\end{tabular} & \\
\hline UPDATEDATE & FALSE & FALSE & TRUE & FALSE & & $\begin{array}{l}\text { ArcFM Current } \\
\text { Date }\end{array}$ \\
\hline Updated By & FALSE & FALSE & TRUE & FALSE & & $\begin{array}{l}\text { ArcFM User } \\
\text { Name }\end{array}$ \\
\hline
\end{tabular}

arcfm8.ELECTRIC.RegulatorUnitRemove

ArcFM Display Field: REMOVALDATE

Create Edit Task:

On Create Event:

On Update Event:

On Delete Event: EDFS Reset Transformer/Regulator Asset To Installed

On Abandon Event:

Abandon Feature Class:

Abandon Subtype:

Remove Feature Class:

Remove Subtype:

\begin{tabular}{|l|l|l|l|l|l|l|}
\hline Field Alias & Visible & Editable & $\begin{array}{c}\text { Allow } \\
\text { Null } \\
\text { Values }\end{array}$ & $\begin{array}{c}\text { Clear After } \\
\text { Create }\end{array}$ & $\begin{array}{c}\text { On Feature } \\
\text { Create }\end{array}$ & $\begin{array}{c}\text { On Feature } \\
\text { Update }\end{array}$ \\
\hline
\end{tabular}




\begin{tabular}{|l|l|l|l|l|l|l|}
\hline Company Number & TRUE & FALSE & FALSE & FALSE & & \\
\hline REMOVALDATE & TRUE & FALSE & TRUE & FALSE & & \\
\hline Reason & TRUE & FALSE & TRUE & FALSE & & \\
\hline Material Ticket & TRUE & FALSE & TRUE & FALSE & & \\
\hline OBJECTID & TRUE & FALSE & FALSE & FALSE & & \\
\hline CreationDate & FALSE & TRUE & TRUE & FALSE & $\begin{array}{l}\text { ArcFM } \\
\text { Darrent }\end{array}$ & \\
\hline Created By & FALSE & TRUE & FALSE & FALSE & $\begin{array}{l}\text { ArcFM } \\
\text { User Name }\end{array}$ & \\
\hline UPDATEDATE & FALSE & FALSE & TRUE & FALSE & & $\begin{array}{l}\text { ArcFM Current } \\
\text { Date }\end{array}$ \\
\hline Updated By & FALSE & FALSE & TRUE & FALSE & & $\begin{array}{l}\text { ArcFM User } \\
\text { Name }\end{array}$ \\
\hline REMOVALTIMESTAMP & FALSE & TRUE & FALSE & FALSE & & \\
\hline LEGACYINSTALLTIMESTAMP & FALSE & TRUE & TRUE & FALSE & & \\
\hline
\end{tabular}

arcfm8.ELECTRIC.RegulatorUnitSoldOrLeased

ArcFM Display Field: SOLDLEASEDDATE

Create Edit Task:

On Create Event:

On Update Event:

On Delete Event:

On Abandon Event:

Abandon Feature Class:

Abandon Subtype:

Remove Feature Class:

Remove Subtype:

\begin{tabular}{|l|l|l|l|l|l|l|}
\hline Field Alias & Visible & Editable & $\begin{array}{c}\text { Allow } \\
\text { Vull }\end{array}$ & $\begin{array}{c}\text { Clear After } \\
\text { Create }\end{array}$ & $\begin{array}{l}\text { On Feature } \\
\text { Create }\end{array}$ & $\begin{array}{c}\text { On Feature } \\
\text { Update }\end{array}$ \\
\hline Company Number & TRUE & FALSE & FALSE & FALSE & & \\
\hline Sold/Leased & TRUE & FALSE & TRUE & FALSE & & \\
\hline SOLDLEASEDDATE & TRUE & FALSE & TRUE & FALSE & & \\
\hline Material Ticket & TRUE & FALSE & TRUE & FALSE & & \\
\hline Receiver & TRUE & FALSE & TRUE & FALSE & & \\
\hline Lease Terminated & TRUE & FALSE & TRUE & FALSE & & \\
\hline LEASETERMINATEDDATE & TRUE & FALSE & TRUE & FALSE & & \\
\hline OBJECTID & TRUE & FALSE & FALSE & FALSE & & \\
\hline CreationDate & FALSE & TRUE & TRUE & FALSE & $\begin{array}{l}\text { ArcFM } \\
\text { Current } \\
\text { Date }\end{array}$ & \\
\hline Created By & FALSE & TRUE & FALSE & FALSE & $\begin{array}{l}\text { ArcFM } \\
\text { User Name }\end{array}$ & \\
\hline UPDATEDATE & FALSE & FALSE & TRUE & FALSE & & $\begin{array}{l}\text { ArcFM Current } \\
\text { Date }\end{array}$ \\
\hline Updated By & FALSE & FALSE & TRUE & FALSE & & $\begin{array}{l}\text { ArcFM User } \\
\text { Name }\end{array}$ \\
\hline SOLDLEASEDTIMESTAMP & FALSE & TRUE & FALSE & FALSE & & \\
\hline
\end{tabular}

arcfm8.ELECTRIC.RegulatorUnitStock

ArcFM Display Field: STOCKDATE

Create Edit Task:

On Create Event:

On Update Event:

On Delete Event:

On Abandon Event:

Abandon Feature Class:

Abandon Subtype:

Remove Feature Class:

Remove Subtype: 


\begin{tabular}{|l|l|l|l|l|l|l|}
\hline Field Alias & Visible & Editable & $\begin{array}{c}\text { Allow } \\
\text { Null } \\
\text { Values }\end{array}$ & $\begin{array}{c}\text { Clear After } \\
\text { Create }\end{array}$ & $\begin{array}{c}\text { On Feature } \\
\text { Create }\end{array}$ & $\begin{array}{c}\text { On Feature } \\
\text { Update }\end{array}$ \\
\hline Company Number & TRUE & FALSE & FALSE & FALSE & & \\
\hline Store Room Number & TRUE & FALSE & TRUE & FALSE & & \\
\hline STOCKDATE & TRUE & FALSE & TRUE & FALSE & & \\
\hline Transfer Number & TRUE & FALSE & TRUE & FALSE & & \\
\hline OBJECTID & TRUE & FALSE & FALSE & FALSE & & \\
\hline STOCKTIMESTAMP & FALSE & TRUE & FALSE & FALSE & & \\
\hline CreationDate & FALSE & TRUE & TRUE & FALSE & $\begin{array}{l}\text { ArcFM } \\
\text { Current } \\
\text { Date }\end{array}$ & \\
\hline Created By & FALSE & TRUE & FALSE & FALSE & $\begin{array}{l}\text { ArcFM } \\
\text { User Name }\end{array}$ & \\
\hline UPDATEDATE & FALSE & FALSE & TRUE & FALSE & & $\begin{array}{l}\text { ArcFM Current } \\
\text { Date }\end{array}$ \\
\hline Updated By & FALSE & FALSE & TRUE & FALSE & & $\begin{array}{l}\text { ArcFM User } \\
\text { Name }\end{array}$ \\
\hline
\end{tabular}

arcfm8.ELECTRIC.RetiredPadMount

ArcFM Display Field: DISTRIBREFNUMBER

Create Edit Task:

On Create Event:

On Update Event:

On Delete Event:

On Abandon Event:

Abandon Feature Class:

Abandon Subtype:

Remove Feature Class:

Remove Subtype:

\begin{tabular}{|c|c|c|c|c|c|c|}
\hline Field Alias & Visible & Editable & $\begin{array}{c}\text { Allow } \\
\text { Null } \\
\text { Values }\end{array}$ & $\begin{array}{c}\text { Clear After } \\
\text { Create }\end{array}$ & $\begin{array}{c}\text { On Feature } \\
\text { Create }\end{array}$ & $\begin{array}{l}\text { On Feature } \\
\text { Update }\end{array}$ \\
\hline Pad Number & TRUE & TRUE & TRUE & FALSE & & \\
\hline Subtype & TRUE & TRUE & TRUE & FALSE & & \\
\hline CreationDate & FALSE & TRUE & TRUE & FALSE & & \\
\hline Created By & FALSE & TRUE & TRUE & FALSE & & \\
\hline UPDATEDATE & FALSE & TRUE & TRUE & FALSE & & \\
\hline Updated By & FALSE & TRUE & TRUE & FALSE & & \\
\hline LOA & TRUE & TRUE & TRUE & FALSE & & \\
\hline Tax Unit & TRUE & TRUE & TRUE & FALSE & & \\
\hline Grid & TRUE & TRUE & TRUE & FALSE & & \\
\hline INSTALLDATE & TRUE & TRUE & TRUE & FALSE & & \\
\hline Install WO Number & TRUE & TRUE & TRUE & FALSE & & \\
\hline Install Misc Order Id & TRUE & TRUE & TRUE & FALSE & & \\
\hline RETIREDATE & TRUE & TRUE & TRUE & FALSE & & \\
\hline Retire WO Number & TRUE & TRUE & TRUE & FALSE & & \\
\hline Retire Misc Order Id & TRUE & TRUE & TRUE & FALSE & & \\
\hline LEGACYWRINSTALLTIMESTAMP & FALSE & TRUE & TRUE & FALSE & & \\
\hline LEGACYWRREMOVALTIMESTAMP & FALSE & TRUE & TRUE & FALSE & & \\
\hline Type & TRUE & TRUE & TRUE & FALSE & & \\
\hline Owner Indicator & TRUE & TRUE & TRUE & FALSE & & \\
\hline Long Pad Number & FALSE & TRUE & TRUE & FALSE & & \\
\hline $\begin{array}{l}\text { Legacy Distribution Reference Number } \\
\text { Termination Id }\end{array}$ & FALSE & TRUE & TRUE & FALSE & & \\
\hline INSTALLTIMESTAMP & FALSE & TRUE & TRUE & FALSE & & \\
\hline LEGACYREMOVALTIMESTAMP & FALSE & TRUE & TRUE & FALSE & & \\
\hline Print Id & TRUE & TRUE & TRUE & FALSE & & \\
\hline Location Description & TRUE & TRUE & TRUE & FALSE & & \\
\hline Service PointType & TRUE & TRUE & TRUE & FALSE & & \\
\hline OBJECTID & TRUE & FALSE & FALSE & FALSE & & \\
\hline
\end{tabular}


arcfm8.ELECTRIC.RetiredSupportStructure

ArcFM Display Field: DISTRIBREFNUMBER

Create Edit Task:

On Update Event: EDFS Validate Work Order Number 2

On Update Event: NIPSCO.UniqueDistribRefNumber-

OnCreate

On Delete Event:

On Abandon Event:

On Create Event:

Abandon Feature Class:

Abandon Subtype:

Remove Feature Class:

Remove Subtype:

\begin{tabular}{|c|c|c|c|c|c|c|}
\hline Field Alias & Visible & Editable & \begin{tabular}{|c|} 
Allow \\
Null \\
Values
\end{tabular} & $\begin{array}{c}\text { Clear After } \\
\text { Create }\end{array}$ & $\begin{array}{c}\text { On Feature } \\
\text { Create }\end{array}$ & $\begin{array}{c}\text { On Feature } \\
\text { Update }\end{array}$ \\
\hline Subtype & TRUE & TRUE & FALSE & FALSE & & \\
\hline Pole Number & TRUE & FALSE & FALSE & FALSE & & \\
\hline INSTALLDATE & TRUE & TRUE & TRUE & FALSE & & \\
\hline Install WO Number & TRUE & TRUE & TRUE & FALSE & & \\
\hline Install Misc Order Id & TRUE & TRUE & TRUE & FALSE & & \\
\hline RETIREDATE & TRUE & TRUE & TRUE & FALSE & & \\
\hline Retire WO Number & TRUE & TRUE & TRUE & FALSE & & \\
\hline Retire Misc Order Id & TRUE & TRUE & TRUE & FALSE & & \\
\hline LOA & TRUE & TRUE & TRUE & FALSE & & \\
\hline Tax Unit & TRUE & TRUE & TRUE & FALSE & & \\
\hline Grid & TRUE & TRUE & TRUE & FALSE & & \\
\hline Owner Type & TRUE & TRUE & TRUE & FALSE & & \\
\hline Location Description & TRUE & TRUE & TRUE & FALSE & & \\
\hline Pole Height & TRUE & TRUE & TRUE & FALSE & & \\
\hline Pole Material & TRUE & TRUE & \begin{tabular}{|l|} 
FALSE \\
\end{tabular} & FALSE & & \\
\hline Transmission Structure Number & TRUE & TRUE & TRUE & FALSE & & \\
\hline LEGACYWRINSTALLTIMESTAMP & FALSE & TRUE & TRUE & FALSE & & \\
\hline LEGACYWRREMOVALTIMESTAMP & FALSE & TRUE & TRUE & FALSE & & \\
\hline Primary Pole Number & TRUE & TRUE & TRUE & FALSE & & \\
\hline INSTALLTIMESTAMP & FALSE & TRUE & TRUE & FALSE & & \\
\hline REMOVALTIMESTAMP & FALSE & TRUE & TRUE & FALSE & & \\
\hline Telephone Company & TRUE & TRUE & TRUE & FALSE & & \\
\hline First Cable Company & TRUE & TRUE & TRUE & FALSE & & \\
\hline Second Cable Company & TRUE & TRUE & TRUE & FALSE & & \\
\hline Utility Company & TRUE & TRUE & TRUE & FALSE & & \\
\hline Fiber Company & TRUE & TRUE & TRUE & FALSE & & \\
\hline Other Company & TRUE & TRUE & TRUE & FALSE & & \\
\hline Transformer Company Number 1 & FALSE & TRUE & TRUE & FALSE & & \\
\hline Transformer Company Number 2 & FALSE & TRUE & TRUE & FALSE & & \\
\hline Transformer Company Number 3 & FALSE & TRUE & TRUE & FALSE & & \\
\hline Transformer Company Number 4 & FALSE & TRUE & TRUE & FALSE & & \\
\hline Foreign Owner Name & TRUE & TRUE & FALSE & FALSE & & \\
\hline OBJECTID & TRUE & FALSE & FALSE & FALSE & & \\
\hline CreationDate & FALSE & TRUE & TRUE & FALSE & & \\
\hline Created By & FALSE & TRUE & TRUE & FALSE & & \\
\hline UPDATEDATE & FALSE & FALSE & TRUE & FALSE & & $\begin{array}{l}\text { ArcFM Current } \\
\text { Date }\end{array}$ \\
\hline Updated By & FALSE & FALSE & TRUE & FALSE & & $\begin{array}{l}\text { ArcFM User } \\
\text { Name }\end{array}$ \\
\hline Service Point Type & TRUE & TRUE & TRUE & FALSE & & \\
\hline
\end{tabular}


arcfm8.ELECTRIC.RetiredSwitchGear

ArcFM Display Field: SUBTYPECD

Create Edit Task:

On Create Event:

On Update Event: EDFS Validate Work Order Number 2

On Delete Event:

On Abandon Event:

Abandon Feature Class:

Abandon Subtype:

Remove Feature Class:

Remove Subtype:

\begin{tabular}{|c|c|c|c|c|c|c|}
\hline Field Alias & Visible & Editable & $\begin{array}{c}\text { Allow } \\
\text { Null } \\
\text { Values }\end{array}$ & $\begin{array}{l}\text { Clear After } \\
\text { Create }\end{array}$ & $\begin{array}{c}\text { On Feature } \\
\text { Create }\end{array}$ & $\begin{array}{c}\text { On Feature } \\
\text { Update }\end{array}$ \\
\hline Subtype & TRUE & TRUE & FALSE & FALSE & & \\
\hline KV & TRUE & TRUE & TRUE & FALSE & & \\
\hline Operation Type & TRUE & TRUE & TRUE & FALSE & & \\
\hline Front Kind & TRUE & TRUE & TRUE & FALSE & & \\
\hline Used Compartment Count & TRUE & TRUE & TRUE & FALSE & & \\
\hline Pad Number & TRUE & TRUE & FALSE & FALSE & & \\
\hline Assembly Number & TRUE & TRUE & TRUE & FALSE & & \\
\hline INSTALLDATE & TRUE & TRUE & TRUE & FALSE & & \\
\hline Install WO Number & TRUE & TRUE & TRUE & FALSE & & \\
\hline Install Misc Order Id & TRUE & TRUE & TRUE & FALSE & & \\
\hline RETIREDATE & TRUE & TRUE & TRUE & FALSE & & \\
\hline Retire WO Number & TRUE & TRUE & TRUE & FALSE & & \\
\hline Retire Misc Order Id & TRUE & TRUE & TRUE & FALSE & & \\
\hline LOA & TRUE & TRUE & TRUE & FALSE & & \\
\hline Tax Unit & TRUE & TRUE & TRUE & FALSE & & \\
\hline Grid & TRUE & TRUE & TRUE & FALSE & & \\
\hline OBJECTID & TRUE & FALSE & FALSE & FALSE & & \\
\hline LEGACYWRINSTALLTIMESTAMP & FALSE & TRUE & TRUE & FALSE & & \\
\hline LEGACYWRREMOVALTIMESTAMP & FALSE & TRUE & TRUE & FALSE & & \\
\hline ASSOCIATIONTIMESTAMP & FALSE & TRUE & TRUE & FALSE & & \\
\hline CreationDate & FALSE & TRUE & TRUE & FALSE & & \\
\hline Created By & FALSE & TRUE & FALSE & FALSE & & \\
\hline UPDATEDATE & FALSE & FALSE & TRUE & FALSE & & $\begin{array}{l}\text { ArcFM Current } \\
\text { Date }\end{array}$ \\
\hline Updated By & FALSE & FALSE & TRUE & FALSE & & $\begin{array}{l}\text { ArcFM User } \\
\text { Name }\end{array}$ \\
\hline
\end{tabular}

arcfm8.ELECTRIC.RetiredWoodPoleEvents

ArcFM Display Field: OBJECTID

arcfm8.ELECTRIC.RetiredWoodPolelnfo

ArcFM Display Field: OBJECTID

arcfm8.ELECTRIC.Secondary

ArcFM Display Field: OBJECTID

Create Edit Task:

On Create Event:

On Update Event:

On Delete Event: NIPSCO.Prevent Delete If Units Related

On Split Event:

On Abandon Event:

Before Split Event: EDFS Turn WOMO Validation Off

Before Split Event: ArcFM Save Related Objects

Before Split Event: EDFS Turn WOMO Validation On

After Split Event: EDFS Turn WOMO Validation Off

After Split Event: ArcFM Restore Related Objects 
After Split Event: EDFS Turn WOMO Validation On

Abandon Feature Class:

Abandon Subtype:

Remove Feature Class:

Remove Subtype:

\begin{tabular}{|l|l|l|l|l|l|l|}
\hline Field Alias & Visible & Editable & $\begin{array}{c}\text { Allow } \begin{array}{l}\text { Null } \\
\text { Values }\end{array} \\
\text { Subtype }\end{array}$ & $\begin{array}{c}\text { Clear After } \\
\text { Create }\end{array}$ & $\begin{array}{l}\text { On Feature } \\
\text { Create }\end{array}$ & $\begin{array}{c}\text { On Feature } \\
\text { Update }\end{array}$ \\
\hline Secondary Operating Voltage & TRUE & TRUE & FALSE & FALSE & & \\
\hline LOA & TRUE & FALSE & FALSE & FALSE & $\begin{array}{l}\text { NIPSCO } \\
\text { LoaName }\end{array}$ & $\begin{array}{l}\text { NIPSCO } \\
\text { LoaName }\end{array}$ \\
\hline Grid & TRUE & TRUE & FALSE & FALSE & & \\
\hline Streetlight Indicator & TRUE & TRUE & TRUE & FALSE & & \\
\hline From Outfield Indicator & TRUE & TRUE & TRUE & FALSE & & \\
\hline Shape & FALSE & TRUE & TRUE & FALSE & & \\
\hline CreationDate & FALSE & TRUE & FALSE & FALSE & $\begin{array}{l}\text { ArcFM } \\
\text { Current } \\
\text { Date }\end{array}$ & \\
\hline Created By & FALSE & TRUE & FALSE & FALSE & $\begin{array}{l}\text { ArcFM } \\
\text { User Name }\end{array}$ & \\
\hline UPDATEDATE & FALSE & FALSE & TRUE & FALSE & & $\begin{array}{l}\text { ArcFM Current } \\
\text { Date }\end{array}$ \\
\hline Updated By & FALSE & FALSE & TRUE & FALSE & & $\begin{array}{l}\text { ArcFM User } \\
\text { Name }\end{array}$ \\
\hline Legacy Ees Number & FALSE & TRUE & TRUE & FALSE & & \\
\hline SHAPE.len & FALSE & & & & & \\
\hline Wire Size & TRUE & TRUE & FALSE & FALSE & & \\
\hline Wire Material & TRUE & TRUE & FALSE & FALSE & & \\
\hline OBJECTID & TRUE & FALSE & FALSE & FALSE & & \\
\hline
\end{tabular}

arcfm8.ELECTRIC.SECONDARYCONDUCTORINFO

ArcFM Display Field: WIREMATERIALCD

Create Edit Task:

On Create Event: EDFS Validate Secondary Properties

On Create Event: EDFS Validate Work Order Number 2

On Update Event: EDFS Validate Secondary Properties

On Update Event: EDFS Validate Work Order Number 2

On Delete Event: NIPSCO.Prevent Delete If Units Related

On Abandon Event:

Abandon Feature Class:

Abandon Subtype:

Remove Feature Class:

Remove Subtype:

\begin{tabular}{|l|c|c|c|c|c|c|}
\hline Field Alias & Visible & Editable & $\begin{array}{c}\text { Allow } \begin{array}{c}\text { Null } \\
\text { Values }\end{array} \\
\text { Subtype }\end{array} \begin{array}{c}\text { Clear After } \\
\text { Create }\end{array}$ & $\begin{array}{c}\text { On Feature } \\
\text { Create }\end{array}$ & $\begin{array}{c}\text { On Feature } \\
\text { Update }\end{array}$ \\
\hline Wire Size & TRUE & TRUE & FALSE & FALSE & & \\
\hline Wire Material & TRUE & TRUE & FALSE & FALSE & & \\
\hline Wire Type & TRUE & TRUE & FALSE & FALSE & & \\
\hline In Conduit & TRUE & TRUE & FALSE & FALSE & & \\
\hline Measured Length & TRUE & TRUE & FALSE & FALSE & & \\
\hline Secondary Operating VoltageValue & FALSE & TRUE & TRUE & FALSE & & \\
\hline Conductor Span Count & TRUE & TRUE & FALSE & FALSE & & \\
\hline Span Length & TRUE & FALSE & TRUE & FALSE & & \\
\hline Install Date & FALSE & TRUE & TRUE & FALSE & & \\
\hline Install WO Number & TRUE & TRUE & FALSE & FALSE & & \\
\hline Install Misc Order Id & TRUE & TRUE & FALSE & FALSE & & \\
\hline Retire Date & TRUE & TRUE & FALSE & FALSE & & \\
\hline Retire WO Number & FALSE & TRUE & TRUE & FALSE & & \\
\hline Retire Misc Order Id & FALSE & TRUE & TRUE & FALSE & & \\
\hline Rel Secondary Object Id & FALSE & TRUE & TRUE & FALSE & & \\
\hline
\end{tabular}




\begin{tabular}{|l|l|l|l|l|l|l|}
\hline From Pole/Pad Number & TRUE & FALSE & TRUE & FALSE & & \\
\hline To Pole/Pad Number & TRUE & TRUE & TRUE & FALSE & & \\
\hline To Alternate Number & TRUE & TRUE & TRUE & FALSE & & \\
\hline OBJECTID & TRUE & FALSE & FALSE & FALSE & & \\
\hline Date Created & FALSE & TRUE & TRUE & FALSE & $\begin{array}{l}\text { ArcFM } \\
\text { Darrent } \\
\text { Date }\end{array}$ & \\
\hline Created By & FALSE & TRUE & TRUE & FALSE & $\begin{array}{l}\text { ArcFM } \\
\text { User Name }\end{array}$ & \\
\hline Date Updated & FALSE & FALSE & TRUE & FALSE & & $\begin{array}{l}\text { ArcFM Current } \\
\text { Date }\end{array}$ \\
\hline Updated By & FALSE & FALSE & TRUE & FALSE & & $\begin{array}{l}\text { ArcFM User } \\
\text { Name }\end{array}$ \\
\hline Legacy WR Install Timestamp & FALSE & TRUE & TRUE & FALSE & & \\
\hline Legacy WR Removal Timestamp & FALSE & TRUE & TRUE & FALSE & & \\
\hline Timestamp & FALSE & TRUE & TRUE & FALSE & & \\
\hline Legacy Conductor Number & FALSE & TRUE & TRUE & FALSE & & \\
\hline AVI_STATCD & FALSE & TRUE & TRUE & FALSE & & \\
\hline AVI_LOCCD & FALSE & TRUE & TRUE & FALSE & & \\
\hline
\end{tabular}

arcfm8.ELECTRIC.SectionalizerBank

ArcFM Display Field: OBJECTID

Create Edit Task: NIPSCO.Electric.FeatureOffset

On Create Event: ArcFM Segment Split

On Create Event: NIPSCO Structure Relate

On Create Event: ArcFM Create Feeder Object

On Create Event: NIPSCO.NormalPositionSymbol

On Update Event: NIPSCO Structure Relate

On Update Event: ArcFM Update Feeder Object

On Update Event: NIPSCO.NormalPositionSymbol

On Delete Event: NIPSCO.Prevent Delete If Units Related

On Delete Event: ArcFM Delete Feeder Object

On Abandon Event:

Abandon Feature Class:

Abandon Subtype:

Remove Feature Class:

Remove Subtype:

\begin{tabular}{|c|c|c|c|c|c|c|}
\hline Field Alias & Visible & Editable & $\begin{array}{c}\text { Allow } \\
\text { Null } \\
\text { Values }\end{array}$ & $\begin{array}{c}\text { Clear After } \\
\text { Create }\end{array}$ & $\begin{array}{c}\text { On Feature } \\
\text { Create }\end{array}$ & $\begin{array}{c}\text { On Feature } \\
\text { Update }\end{array}$ \\
\hline Subtype & TRUE & TRUE & FALSE & FALSE & & \\
\hline Phase & TRUE & TRUE & FALSE & FALSE & & \\
\hline Circuit Number & TRUE & FALSE & TRUE & FALSE & & \\
\hline A Phase Normal Position & TRUE & TRUE & FALSE & FALSE & & \\
\hline B Phase Normal Position & TRUE & TRUE & FALSE & FALSE & & \\
\hline C Phase Normal Position & TRUE & TRUE & FALSE & FALSE & & \\
\hline Section Fuse Number & TRUE & TRUE & TRUE & FALSE & & \\
\hline Sectionalizer Count & TRUE & TRUE & FALSE & FALSE & & \\
\hline Rated Amperage & TRUE & TRUE & FALSE & FALSE & & \\
\hline Related Support Structure Object Id & FALSE & TRUE & TRUE & FALSE & & \\
\hline Shape & FALSE & TRUE & TRUE & FALSE & & \\
\hline AncillaryRole & FALSE & TRUE & TRUE & FALSE & & \\
\hline Enabled & FALSE & TRUE & TRUE & FALSE & & \\
\hline CreationDate & FALSE & TRUE & FALSE & FALSE & \begin{tabular}{|l} 
ArcFM \\
Current \\
Date
\end{tabular} & \\
\hline Created By & FALSE & TRUE & FALSE & FALSE & \begin{tabular}{|l|} 
ArcFM \\
User Name
\end{tabular} & \\
\hline UPDATEDATE & FALSE & FALSE & TRUE & FALSE & & $\begin{array}{l}\text { ArcFM Current } \\
\text { Date }\end{array}$ \\
\hline
\end{tabular}




\begin{tabular}{|l|c|c|c|c|c|l|}
\hline Updated By & FALSE & FALSE & TRUE & FALSE & & $\begin{array}{l}\text { ArcFM User } \\
\text { Name }\end{array}$ \\
\hline Electric Trace Weight & FALSE & TRUE & TRUE & FALSE & & \\
\hline Feeder Manager Non-Traceable & FALSE & TRUE & TRUE & FALSE & & \\
\hline Circuit Number 2 & FALSE & TRUE & TRUE & FALSE & & \\
\hline Feeder Information & FALSE & TRUE & TRUE & FALSE & & \\
\hline Legacy Node 1 & FALSE & TRUE & TRUE & FALSE & & \\
\hline Legacy Node 2 & FALSE & TRUE & TRUE & FALSE & & \\
\hline Legacy Ees Number & FALSE & TRUE & TRUE & FALSE & & \\
\hline Legacy Pole Number & FALSE & TRUE & TRUE & FALSE & & \\
\hline Symbol Rotation & FALSE & FALSE & TRUE & FALSE & & \\
\hline HANDLE & FALSE & TRUE & TRUE & FALSE & & \\
\hline TILENAME & FALSE & TRUE & TRUE & FALSE & & \\
\hline LOA & TRUE & FALSE & TRUE & FALSE & NIPSCO & NIPSCO \\
LoaName & LoaName \\
\hline Tax Unit & TRUE & TRUE & FALSE & FALSE & & \\
\hline Grid & TRUE & TRUE & FALSE & FALSE & & \\
\hline Symbol Configuration Cd & FALSE & TRUE & TRUE & FALSE & & \\
\hline DCSID & FALSE & TRUE & TRUE & FALSE & & \\
\hline P_L & FALSE & TRUE & TRUE & FALSE & & \\
\hline FUS_NUM & FALSE & TRUE & TRUE & FALSE & & \\
\hline OBJECTID & TRUE & FALSE & FALSE & FALSE & & \\
\hline
\end{tabular}

arcfm8.ELECTRIC.SectionalizerUnit

ArcFM Display Field: PHASEDESIGNATION

Create Edit Task: ArcFM Manual Angle Setter

On Create Event: EDFS Validate Work Order Number 2

On Update Event: EDFS Validate Work Order Number 2

On Delete Event:

On Abandon Event:

Abandon Feature Class:

Abandon Subtype:

Remove Feature Class:

Remove Subtype:

\begin{tabular}{|c|c|c|c|c|c|c|}
\hline Field Alias & Visible & Editable & $\begin{array}{c}\text { Allow } \\
\text { Null } \\
\text { Values }\end{array}$ & $\begin{array}{c}\text { Clear After } \\
\text { Create }\end{array}$ & $\begin{array}{c}\text { On Feature } \\
\text { Create }\end{array}$ & $\begin{array}{c}\text { On Feature } \\
\text { Update }\end{array}$ \\
\hline Subtype & TRUE & TRUE & FALSE & FALSE & & \\
\hline INSTALLDATE & TRUE & TRUE & FALSE & FALSE & & \\
\hline Install WO Number & TRUE & TRUE & FALSE & FALSE & & \\
\hline Install Misc Order Id & TRUE & TRUE & FALSE & FALSE & & \\
\hline RETIREDATE & FALSE & TRUE & TRUE & FALSE & & \\
\hline Retire WO Number & FALSE & TRUE & TRUE & FALSE & & \\
\hline Retire Misc Order Id & FALSE & TRUE & TRUE & FALSE & & \\
\hline Pole/Pad Number & FALSE & TRUE & TRUE & FALSE & & \\
\hline CreationDate & FALSE & TRUE & TRUE & FALSE & $\begin{array}{l}\text { ArcFM } \\
\text { Current } \\
\text { Date } \\
\end{array}$ & \\
\hline Created By & FALSE & FALSE & TRUE & FALSE & \begin{tabular}{|l|} 
ArcFM \\
User Name
\end{tabular} & \\
\hline UPDATEDATE & FALSE & FALSE & TRUE & FALSE & & $\begin{array}{l}\text { ArcFM Current } \\
\text { Date }\end{array}$ \\
\hline Updated By & FALSE & FALSE & TRUE & FALSE & & $\begin{array}{l}\text { ArcFM User } \\
\text { Name }\end{array}$ \\
\hline LEGACYWRINSTALLTIMESTAMP & FALSE & TRUE & TRUE & FALSE & & \\
\hline LEGACYWRREMOVALTIMESTAMP & FALSE & TRUE & TRUE & FALSE & & \\
\hline ASSOCIATIONTIMESTAMP & FALSE & TRUE & TRUE & FALSE & & \\
\hline Phase & TRUE & TRUE & FALSE & FALSE & & \\
\hline Circuit Number & FALSE & TRUE & TRUE & FALSE & & \\
\hline Related Sectionalizer Bank Object Id & TRUE & FALSE & TRUE & FALSE & & \\
\hline
\end{tabular}




\begin{tabular}{|l|l|l|l|l|l|l|}
\hline Section Fuse Number & TRUE & TRUE & TRUE & FALSE & & \\
\hline OBJECTID & TRUE & FALSE & FALSE & FALSE & & \\
\hline
\end{tabular}

arcfm8.ELECTRIC.ServiceConductorlnfo

ArcFM Display Field: WIREMATERIALCD

Create Edit Task:

On Create Event: EDFS Validate Work Order Number 2

On Create Event: EDFS Validate Service Properties

On Update Event: EDFS Validate Work Order Number 2

On Update Event: EDFS Validate Service Properties

On Delete Event:

On Abandon Event:

Abandon Feature Class:

Abandon Subtype:

Remove Feature Class:

Remove Subtype:

\begin{tabular}{|c|c|c|c|c|c|c|}
\hline Field Alias & Visible & Editable & $\begin{array}{c}\text { Allow } \\
\text { Null } \\
\text { Values }\end{array}$ & $\begin{array}{l}\text { Clear After } \\
\text { Create }\end{array}$ & $\begin{array}{c}\text { On Feature } \\
\text { Create }\end{array}$ & $\begin{array}{l}\text { On Feature } \\
\text { Update }\end{array}$ \\
\hline Subtype & TRUE & TRUE & FALSE & FALSE & & \\
\hline Wire Size & TRUE & TRUE & FALSE & FALSE & & \\
\hline Wire Material & TRUE & TRUE & FALSE & FALSE & & \\
\hline Wire Type & TRUE & TRUE & FALSE & FALSE & & \\
\hline In Conduit & TRUE & TRUE & FALSE & FALSE & & \\
\hline \begin{tabular}{|l} 
Load Number \\
\end{tabular} & FALSE & TRUE & TRUE & FALSE & & \\
\hline Take Off Structure Number & FALSE & TRUE & TRUE & FALSE & & \\
\hline \begin{tabular}{|l} 
INSTALLDATE \\
\end{tabular} & TRUE & TRUE & FALSE & FALSE & & \\
\hline Install WO Number & TRUE & TRUE & FALSE & FALSE & & \\
\hline Install Misc Order Id & TRUE & TRUE & FALSE & FALSE & & \\
\hline \begin{tabular}{|l} 
RETIREDATE \\
\end{tabular} & FALSE & TRUE & \begin{tabular}{|l} 
TRULL \\
TRUE
\end{tabular} & FALSE & & \\
\hline Retire WO Number & FALSE & TRUE & TRUE & FALSE & & \\
\hline Retire Misc Order Id & FALSE & TRUE & \begin{tabular}{|l|} 
TRUE \\
\end{tabular} & FALSE & & \\
\hline Conductor Span Count & TRUE & TRUE & FALSE & FALSE & & \\
\hline Span Length & TRUE & TRUE & FALSE & FALSE & & \\
\hline OBJECTID & TRUE & FALSE & FALSE & FALSE & & \\
\hline Legacy Conductor Number & FALSE & TRUE & TRUE & FALSE & & \\
\hline Related Load Pad Mount Object Id & FALSE & TRUE & TRUE & FALSE & & \\
\hline Related Take Off Pad Mount Object Id & FALSE & TRUE & TRUE & FALSE & & \\
\hline Related Take Off Pedestal Object Id & FALSE & TRUE & TRUE & FALSE & & \\
\hline Related Load Support Structure Object Id & FALSE & TRUE & TRUE & FALSE & & \\
\hline Related Take Off Support Structure Object Id & FALSE & TRUE & TRUE & FALSE & & \\
\hline CreationDate & FALSE & TRUE & TRUE & FALSE & $\begin{array}{l}\text { ArcFM } \\
\text { Current } \\
\text { Date }\end{array}$ & \\
\hline Created By & FALSE & TRUE & FALSE & FALSE & \begin{tabular}{|l|} 
ArcFM \\
User Name
\end{tabular} & \\
\hline UPDATEDATE & FALSE & FALSE & TRUE & FALSE & & $\begin{array}{l}\text { ArcFM Current } \\
\text { Date }\end{array}$ \\
\hline Updated By & FALSE & FALSE & TRUE & FALSE & & \begin{tabular}{|l|} 
ArcFM User \\
Name
\end{tabular} \\
\hline LEGACYWRINSTALLTIMESTAMP & FALSE & TRUE & TRUE & FALSE & & \\
\hline LEGACYWRREMOVALTIMESTAMP & FALSE & TRUE & TRUE & FALSE & & \\
\hline ASSOCIATIONTIMESTAMP & FALSE & TRUE & TRUE & FALSE & & \\
\hline
\end{tabular}

arcfm8.ELECTRIC.Splice

ArcFM Display Field: OBJECTID 
Create Edit Task:

On Create Event: ArcFM Create Feeder Object

On Update Event: ArcFM Update Feeder Object

On Delete Event: ArcFM Delete Feeder Object

On Abandon Event:

Abandon Feature Class:

Abandon Subtype:

Remove Feature Class:

Remove Subtype:

\begin{tabular}{|l|l|l|l|l|l|l|}
\hline Field Alias & Visible & Editable & $\begin{array}{c}\text { Allow } \\
\text { Values }\end{array}$ & $\begin{array}{c}\text { Clear After } \\
\text { Create }\end{array}$ & $\begin{array}{c}\text { On Feature } \\
\text { Create }\end{array}$ & $\begin{array}{c}\text { On Feature } \\
\text { Update }\end{array}$ \\
\hline Subtype & TRUE & TRUE & FALSE & FALSE & & \\
\hline Shape & FALSE & TRUE & TRUE & FALSE & & \\
\hline ANCILLARYROLE & FALSE & TRUE & TRUE & FALSE & & \\
\hline Enabled & FALSE & TRUE & TRUE & FALSE & & \\
\hline CreationDate & FALSE & TRUE & FALSE & FALSE & $\begin{array}{l}\text { ArcFM } \\
\text { Datrent }\end{array}$ & \\
\hline Created By & FALSE & TRUE & FALSE & FALSE & $\begin{array}{l}\text { ArcFM } \\
\text { User Name }\end{array}$ & \\
\hline UPDATEDATE & FALSE & FALSE & TRUE & FALSE & & $\begin{array}{l}\text { ArcFM Current } \\
\text { Date }\end{array}$ \\
\hline Updated By & FALSE & FALSE & TRUE & FALSE & & $\begin{array}{l}\text { ArcFM User } \\
\text { Name }\end{array}$ \\
\hline Electric Trace Weight & FALSE & TRUE & TRUE & FALSE & & \\
\hline Feeder Manager Non-Traceable & FALSE & TRUE & TRUE & FALSE & & \\
\hline Circuit Number 2 & FALSE & TRUE & TRUE & FALSE & & \\
\hline Feeder Information & FALSE & TRUE & TRUE & FALSE & & \\
\hline Phase & TRUE & TRUE & FALSE & FALSE & & \\
\hline Circuit Number & TRUE & FALSE & TRUE & FALSE & & \\
\hline Legacy Node 1 & FALSE & TRUE & TRUE & FALSE & & \\
\hline Legacy Node 2 & FALSE & TRUE & TRUE & FALSE & & \\
\hline Legacy Ees Number & FALSE & TRUE & TRUE & FALSE & & \\
\hline Symbol Rotation & FALSE & FALSE & TRUE & FALSE & & \\
\hline HANDLE & FALSE & TRUE & TRUE & FALSE & & \\
\hline TILENAME & FALSE & TRUE & TRUE & FALSE & & \\
\hline C L & FALSE & TRUE & TRUE & FALSE & & \\
\hline OBJECTID & TRUE & FALSE & FALSE & FALSE & & \\
\hline arffy & & & & \\
\hline
\end{tabular}

arcfm8.ELECTRIC.StoreRoom

ArcFM Display Field: STOREROOMNUMBER

Create Edit Task:

On Create Event:

On Update Event:

On Delete Event:

On Abandon Event:

Abandon Feature Class:

Abandon Subtype:

Remove Feature Class:

Remove Subtype:

\begin{tabular}{|l|c|c|c|c|c|c|}
\hline Field Alias & Visible & Editable & $\begin{array}{c}\text { Allow } \\
\text { Null } \\
\text { Values }\end{array}$ & $\begin{array}{c}\text { Clear After } \\
\text { Create }\end{array}$ & $\begin{array}{l}\text { On Feature } \\
\text { Create }\end{array}$ & $\begin{array}{c}\text { On Feature } \\
\text { Update }\end{array}$ \\
\hline Store Room Number & TRUE & TRUE & FALSE & FALSE & & \\
\hline LOA & TRUE & TRUE & FALSE & FALSE & & \\
\hline Tax Unit & TRUE & TRUE & FALSE & FALSE & & \\
\hline Grid & TRUE & TRUE & FALSE & FALSE & & \\
\hline OBJECTID & TRUE & FALSE & FALSE & FALSE & & \\
\hline CreationDate & FALSE & TRUE & TRUE & FALSE & $\begin{array}{l}\text { ArcFM } \\
\text { Current } \\
\text { Date }\end{array}$ & \\
\hline
\end{tabular}




\begin{tabular}{|l|l|l|l|l|l|l|}
\hline Created By & FALSE & TRUE & FALSE & FALSE & $\begin{array}{l}\text { ArcFM } \\
\text { User Name }\end{array}$ \\
\hline UPDATEDATE & FALSE & FALSE & TRUE & FALSE & & $\begin{array}{l}\text { ArcFM Current } \\
\text { Date }\end{array}$ \\
\hline Updated By & FALSE & FALSE & TRUE & FALSE & & $\begin{array}{l}\text { ArcFM User } \\
\text { Name }\end{array}$ \\
\hline
\end{tabular}

arcfm8.ELECTRIC.StreetlightSwitch

ArcFM Display Field: AMPERAGEVALUE

Create Edit Task:

On Create Event: EDFS Validate Work Order Number 2

On Update Event: EDFS Validate Work Order Number 2

On Delete Event:

On Abandon Event:

Abandon Feature Class:

Abandon Subtype:

Remove Feature Class:

Remove Subtype:

\begin{tabular}{|l|l|l|l|l|l|l|}
\hline Field Alias & Visible & Editable & $\begin{array}{c}\text { Allow } \\
\text { Vall }\end{array}$ & $\begin{array}{c}\text { Clear After } \\
\text { Create }\end{array}$ & $\begin{array}{c}\text { On Feature } \\
\text { Create }\end{array}$ & $\begin{array}{c}\text { On Feature } \\
\text { Update }\end{array}$ \\
\hline Subtype & TRUE & TRUE & FALSE & FALSE & & \\
\hline Type & TRUE & TRUE & TRUE & FALSE & & \\
\hline Amperage & TRUE & TRUE & TRUE & FALSE & & \\
\hline Voltage & TRUE & TRUE & TRUE & FALSE & & \\
\hline Pole Number & FALSE & TRUE & TRUE & FALSE & & \\
\hline INSTALLDATE & TRUE & TRUE & FALSE & FALSE & & \\
\hline Install WO Number & TRUE & TRUE & FALSE & FALSE & & \\
\hline Install Misc Order Id & TRUE & TRUE & FALSE & FALSE & & \\
\hline RETIREDATE & FALSE & TRUE & TRUE & FALSE & & \\
\hline Retire WO Number & FALSE & TRUE & TRUE & FALSE & & \\
\hline Retire Misc Order Id & FALSE & TRUE & TRUE & FALSE & & \\
\hline Related Support Structure Object Id & TRUE & FALSE & TRUE & FALSE & & \\
\hline OBJECTID & TRUE & FALSE & FALSE & FALSE & & \\
\hline CreationDate & FALSE & TRUE & TRUE & FALSE & $\begin{array}{l}\text { ArcFM } \\
\text { Current } \\
\text { Date }\end{array}$ & \\
\hline Created By & FALSE & TRUE & TRUE & FALSE & $\begin{array}{l}\text { ArcFM } \\
\text { User Name }\end{array}$ & \\
\hline UPDATEDATE & FALSE & FALSE & TRUE & FALSE & & $\begin{array}{l}\text { ArcFM Current } \\
\text { Date }\end{array}$ \\
\hline Updated By & FALSE & FALSE & TRUE & FALSE & & $\begin{array}{l}\text { ArcFM User } \\
\text { Name }\end{array}$ \\
\hline LEGACYWRINSTALLTIMESTAMP & FALSE & TRUE & TRUE & FALSE & & \\
\hline LEGACYWRREMOVALTIMESTAMP & FALSE & TRUE & TRUE & FALSE & & \\
\hline ASSOCIATIONTIMESTAMP & FALSE & TRUE & TRUE & FALSE & & \\
\hline
\end{tabular}

arcfm8.Electric.StructureToStructureLength

ArcFM Display Field:

Create Edit Task:

On Abandon Event:

Abandon Feature Class:

Abandon Subtype:

Remove Feature Class:

Remove Subtype:

\begin{tabular}{|l|c|c|c|c|c|c|}
\hline Field Alias & Visible & Editable & $\begin{array}{c}\text { Allow } \\
\text { Null } \\
\text { Values }\end{array}$ & $\begin{array}{c}\text { Clear After } \\
\text { Create }\end{array}$ & $\begin{array}{c}\text { On Feature } \\
\text { Create }\end{array}$ & $\begin{array}{c}\text { On Feature } \\
\text { Update }\end{array}$ \\
\hline OBJECTID & TRUE & TRUE & TRUE & FALSE & & \\
\hline CREATIONDATE & TRUE & TRUE & TRUE & FALSE & & \\
\hline CREATIONUSERID & TRUE & TRUE & FALSE & FALSE & & \\
\hline
\end{tabular}




\begin{tabular}{|l|c|c|c|c|c|c|}
\hline UPDATEDATE & TRUE & TRUE & TRUE & FALSE & & \\
\hline UPDATEUSERID & TRUE & TRUE & TRUE & FALSE & & \\
\hline REMOVALTIMESTAMP & FALSE & TRUE & FALSE & FALSE & & \\
\hline REMOVALDATE & TRUE & TRUE & TRUE & FALSE & & \\
\hline REMOVALCD & TRUE & TRUE & TRUE & FALSE & & \\
\hline MATERIALTICKETNUMBER & TRUE & TRUE & TRUE & FALSE & & \\
\hline LEGACYINSTALLTIMESTAMP & TRUE & TRUE & TRUE & FALSE & & \\
\hline $\begin{array}{l}\text { RELTRANSFORMERUNITCOMPANYNUMBE } \\
\text { R }\end{array}$ & TRUE & TRUE & TRUE & FALSE & & \\
\hline SDE_STATE_ID & TRUE & TRUE & TRUE & FALSE & & \\
\hline
\end{tabular}

arcfm8.ELECTRIC.Substation

ArcFM Display Field: SUBSTATIONNAME

Create Edit Task:

On Create Event:

On Update Event:

On Delete Event:

On Abandon Event:

Abandon Feature Class:

Abandon Subtype:

Remove Feature Class:

Remove Subtype:

\begin{tabular}{|l|l|l|l|l|l|l|}
\hline Field Alias & Visible & Editable & $\begin{array}{c}\text { Allow } \\
\text { Vull } \\
\text { Values }\end{array}$ & $\begin{array}{c}\text { Clear After } \\
\text { Create }\end{array}$ & $\begin{array}{c}\text { On Feature } \\
\text { Create }\end{array}$ & $\begin{array}{c}\text { On Feature } \\
\text { Update }\end{array}$ \\
\hline Subtype & TRUE & TRUE & FALSE & FALSE & & \\
\hline Substation Name & TRUE & TRUE & FALSE & FALSE & & \\
\hline Substation Number & TRUE & TRUE & FALSE & FALSE & & \\
\hline Substation Owner & TRUE & TRUE & FALSE & FALSE & & \\
\hline Shape & FALSE & TRUE & TRUE & FALSE & & \\
\hline CreationDate & FALSE & TRUE & FALSE & FALSE & $\begin{array}{l}\text { ArcFM } \\
\text { Current } \\
\text { Date }\end{array}$ & \\
\hline Created By & FALSE & TRUE & FALSE & FALSE & $\begin{array}{l}\text { ArcFM } \\
\text { User Name }\end{array}$ & \\
\hline UPDATEDATE & FALSE & FALSE & TRUE & FALSE & & $\begin{array}{l}\text { ArcFM Current } \\
\text { Date }\end{array}$ \\
\hline Updated By & FALSE & FALSE & TRUE & FALSE & & $\begin{array}{l}\text { ArcFM User } \\
\text { Name }\end{array}$ \\
\hline Symbol Rotation & FALSE & TRUE & TRUE & FALSE & & \\
\hline Legacy EES Number & FALSE & TRUE & TRUE & FALSE & & \\
\hline HANDLE & FALSE & TRUE & TRUE & FALSE & & \\
\hline TILENAME & FALSE & TRUE & TRUE & FALSE & & \\
\hline DCSID & FALSE & TRUE & TRUE & FALSE & & \\
\hline OBJECTID & TRUE & FALSE & FALSE & FALSE & & \\
\hline
\end{tabular}

arcfm8.ELECTRIC.SubstationBreaker

ArcFM Display Field: FEEDERID

Create Edit Task:

On Create Event: NIPSCO Substation Relate

On Create Event: ArcFM Create Feeder Object

On Update Event: NIPSCO Substation Relate

On Update Event: ArcFM Update Feeder Object

On Delete Event: ArcFM Delete Feeder Object

On Abandon Event:

Abandon Feature Class:

Abandon Subtype:

Remove Feature Class:

Remove Subtype:

\begin{tabular}{|l|l|l|l|l|l|l|}
\hline Field Alias & Visible & Editable & $\begin{array}{c}\text { Allow } \\
\text { Null } \\
\text { Values }\end{array}$ & $\begin{array}{c}\text { Clear After } \\
\text { Create }\end{array}$ & $\begin{array}{c}\text { On Feature } \\
\text { Create }\end{array}$ & $\begin{array}{c}\text { On Feature } \\
\text { Update }\end{array}$ \\
\hline
\end{tabular}




\begin{tabular}{|c|c|c|c|c|c|c|}
\hline Subtype & TRUE & TRUE & FALSE & FALSE & & \\
\hline LOA & TRUE & FALSE & TRUE & FALSE & $\begin{array}{l}\text { NIPSCO } \\
\text { LoaName }\end{array}$ & $\begin{array}{l}\text { NIPSCO } \\
\text { LoaName }\end{array}$ \\
\hline Grid & TRUE & TRUE & FALSE & FALSE & & \\
\hline Normal Position & TRUE & TRUE & FALSE & FALSE & & \\
\hline Related Substation Object Id & FALSE & TRUE & TRUE & FALSE & & \\
\hline Related Power Transformer Object Id & FALSE & TRUE & TRUE & FALSE & & \\
\hline Shape & FALSE & TRUE & TRUE & FALSE & & \\
\hline AncillaryRole & FALSE & TRUE & TRUE & FALSE & & \\
\hline Enabled & FALSE & TRUE & TRUE & FALSE & & \\
\hline CreationDate & FALSE & TRUE & FALSE & FALSE & $\begin{array}{l}\text { ArcFM } \\
\text { Current } \\
\text { Date }\end{array}$ & \\
\hline Created By & FALSE & TRUE & FALSE & FALSE & \begin{tabular}{|l|} 
ArcFM \\
User Name
\end{tabular} & \\
\hline UPDATEDATE & FALSE & FALSE & TRUE & FALSE & & $\begin{array}{l}\text { ArcFM Current } \\
\text { Date }\end{array}$ \\
\hline Updated By & FALSE & FALSE & TRUE & FALSE & & $\begin{array}{l}\text { ArcFM User } \\
\text { Name }\end{array}$ \\
\hline Electric Trace Weight & FALSE & TRUE & TRUE & FALSE & & \\
\hline Feeder Manager Non-Traceable & FALSE & TRUE & TRUE & FALSE & & \\
\hline Circuit Number 2 & FALSE & TRUE & TRUE & FALSE & & \\
\hline Feeder Information & FALSE & TRUE & TRUE & FALSE & & \\
\hline Circuit Number & TRUE & FALSE & TRUE & FALSE & & \\
\hline Phase & TRUE & TRUE & FALSE & FALSE & & \\
\hline Legacy Node 1 & FALSE & TRUE & TRUE & FALSE & & \\
\hline Legacy Node 2 & FALSE & TRUE & TRUE & FALSE & & \\
\hline Legacy Ees Number & FALSE & TRUE & TRUE & FALSE & & \\
\hline Symbol Rotation & FALSE & TRUE & TRUE & FALSE & & \\
\hline HANDLE & FALSE & TRUE & TRUE & FALSE & & \\
\hline TILENAME & FALSE & TRUE & TRUE & FALSE & & \\
\hline Legacy Feeder ID & FALSE & TRUE & TRUE & FALSE & & \\
\hline S_L & FALSE & TRUE & TRUE & FALSE & & \\
\hline S_L2 & FALSE & TRUE & TRUE & FALSE & & \\
\hline SUBNUM & FALSE & TRUE & TRUE & FALSE & & \\
\hline OBJECTID & TRUE & FALSE & FALSE & FALSE & & \\
\hline
\end{tabular}

arcfm8.ELECTRIC.SupportStructure

ArcFM Display Field: DISTRIBREFNUMBER

Create Edit Task: ArcFM Linear Point

On Create Event: EDFS Validate Work Order Number 2

On Create Event: NIPSCO.UniqueDistribRefNumber-OnCreate

On Delete Event: EDFS Prevent Deletion if Related Assets

On Abandon Event:

On Update Event: EDFS Validate Work Order Number 2

On Update Event: NIPSCO.UniqueDistribRefNumber-

OnUpdate

Abandon Feature Class:

Abandon Subtype:

Remove Feature Class: arcfm8.ELECTRIC.RetiredSupportStructure

Remove Subtype:

\begin{tabular}{|c|c|c|c|c|c|c|}
\hline Field Alias & Visible & Editable & $\begin{array}{c}\text { Allow } \\
\text { Null } \\
\text { Values }\end{array}$ & $\begin{array}{l}\text { Clear After } \\
\text { Create }\end{array}$ & $\begin{array}{c}\text { On Feature } \\
\text { Create }\end{array}$ & $\begin{array}{c}\text { On Feature } \\
\text { Update }\end{array}$ \\
\hline Subtype & TRUE & TRUE & FALSE & FALSE & & \\
\hline Pole Number & TRUE & TRUE & FALSE & FALSE & & \\
\hline Primary Pole Number & TRUE & TRUE & TRUE & FALSE & & \\
\hline Location Description & TRUE & TRUE & FALSE & FALSE & & \\
\hline Foreign Owner Name & TRUE & TRUE & FALSE & FALSE & & \\
\hline INSTALLDATE & TRUE & TRUE & FALSE & FALSE & & \\
\hline Install WO Number & TRUE & TRUE & FALSE & FALSE & & \\
\hline
\end{tabular}




\begin{tabular}{|c|c|c|c|c|c|c|}
\hline Install Misc Order Id & TRUE & TRUE & FALSE & FALSE & & \\
\hline RETIREDATE & FALSE & TRUE & TRUE & FALSE & & \\
\hline Retire WO Number & FALSE & TRUE & TRUE & FALSE & & \\
\hline Retire Misc Order Id & FALSE & TRUE & TRUE & FALSE & & \\
\hline LOA & TRUE & FALSE & TRUE & FALSE & $\begin{array}{l}\text { NIPSCO } \\
\text { LoaName } \\
\end{array}$ & \begin{tabular}{|l|} 
NIPSCO \\
LoaName \\
\end{tabular} \\
\hline Tax Unit & TRUE & TRUE & FALSE & FALSE & & \\
\hline Grid & TRUE & TRUE & FALSE & FALSE & & \\
\hline Pole Material & TRUE & TRUE & FALSE & FALSE & & \\
\hline Pole Height & TRUE & TRUE & FALSE & FALSE & & \\
\hline Transmission Structure Number & TRUE & TRUE & TRUE & FALSE & & \\
\hline Service PointType & TRUE & TRUE & TRUE & FALSE & & \\
\hline Shape & FALSE & TRUE & TRUE & FALSE & & \\
\hline CreationDate & FALSE & TRUE & FALSE & FALSE & $\begin{array}{l}\text { ArcFM } \\
\text { Current } \\
\text { Date } \\
\end{array}$ & \\
\hline Created By & FALSE & TRUE & FALSE & FALSE & \begin{tabular}{|l|} 
ArcFM \\
User Name
\end{tabular} & \\
\hline UPDATEDATE & FALSE & FALSE & TRUE & FALSE & & $\begin{array}{l}\text { ArcFM Current } \\
\text { Date } \\
\end{array}$ \\
\hline Updated By & FALSE & FALSE & TRUE & FALSE & & $\begin{array}{l}\text { ArcFM User } \\
\text { Name }\end{array}$ \\
\hline Legacy Ees Number & FALSE & TRUE & TRUE & FALSE & & \\
\hline Graphics Scale Factor & FALSE & TRUE & TRUE & FALSE & & \\
\hline Symbol Rotation & FALSE & TRUE & TRUE & FALSE & & \\
\hline LEGACYWRINSTALLTIMESTAMP & FALSE & TRUE & TRUE & FALSE & & \\
\hline LEGACYWRREMOVALTIMESTAMP & FALSE & TRUE & TRUE & FALSE & & \\
\hline INSTALLTIMESTAMP & FALSE & TRUE & TRUE & FALSE & & \\
\hline REMOVALTIMESTAMP & FALSE & TRUE & TRUE & FALSE & & \\
\hline OBJECTID & TRUE & FALSE & FALSE & FALSE & & \\
\hline
\end{tabular}

arcfm8.ELECTRIC.Switch

ArcFM Display Field: OBJECTID

Create Edit Task:

On Create Event: ArcFM Segment Split

On Create Event: NIPSCO Structure Relate

On Create Event: ArcFM Create Feeder Object

On Create Event: NIPSCO.NormalPositionSymbol

On Update Event: NIPSCO Structure Relate

On Update Event: ArcFM Update Feeder Object

On Update Event: NIPSCO.NormalPositionSymbol

On Delete Event: NIPSCO.Prevent Delete If Units Related

On Delete Event: ArcFM Delete Feeder Object

On Abandon Event:

Abandon Feature Class:

Abandon Subtype:

Remove Feature Class:

Remove Subtype:

\begin{tabular}{|l|c|c|c|c|c|c|}
\hline Field Alias & Visible & Editable & $\begin{array}{c}\text { Allow } \\
\text { Null } \\
\text { Values }\end{array}$ & $\begin{array}{c}\text { Clear After } \\
\text { Create }\end{array}$ & $\begin{array}{c}\text { On Feature } \\
\text { Create }\end{array}$ & $\begin{array}{c}\text { On Feature } \\
\text { Update }\end{array}$ \\
\hline Subtype & TRUE & TRUE & FALSE & FALSE & & \\
\hline Switch ID Number & TRUE & TRUE & FALSE & FALSE & & \\
\hline Phase & TRUE & TRUE & FALSE & FALSE & & \\
\hline A Phase Normal Position & TRUE & TRUE & FALSE & FALSE & & \\
\hline B Phase Normal Position & TRUE & TRUE & FALSE & FALSE & & \\
\hline C Phase Normal Position & TRUE & TRUE & FALSE & FALSE & & \\
\hline Primary Operating Voltage & TRUE & TRUE & FALSE & FALSE & & \\
\hline Tie Switch Indicator & TRUE & TRUE & TRUE & FALSE & & \\
\hline Load Break Indicator & TRUE & TRUE & TRUE & FALSE & & \\
\hline
\end{tabular}




\begin{tabular}{|c|c|c|c|c|c|c|}
\hline Circuit Number & TRUE & FALSE & TRUE & FALSE & & \\
\hline Circuit Number 2 & TRUE & FALSE & TRUE & FALSE & & \\
\hline Shape & FALSE & $\begin{array}{l}\text { TRUE } \\
\end{array}$ & TRUE & FALSE & & \\
\hline AncillaryRole & FALSE & TRUE & TRUE & FALSE & & \\
\hline Enabled & FALSE & TRUE & TRUE & FALSE & & \\
\hline CreationDate & FALSE & TRUE & FALSE & FALSE & \begin{tabular}{|l|} 
ArcFM \\
Current \\
Date
\end{tabular} & \\
\hline Created By & FALSE & TRUE & FALSE & FALSE & $\begin{array}{l}\text { ArcFM } \\
\text { User Name }\end{array}$ & \\
\hline UPDATEDATE & FALSE & FALSE & TRUE & FALSE & & $\begin{array}{l}\text { ArcFM Current } \\
\text { Date }\end{array}$ \\
\hline Updated By & FALSE & FALSE & TRUE & FALSE & & $\begin{array}{l}\text { ArcFM User } \\
\text { Name }\end{array}$ \\
\hline Electric Trace Weight & FALSE & TRUE & TRUE & FALSE & & \\
\hline Feeder Manager Non-Traceable & FALSE & TRUE & TRUE & FALSE & & \\
\hline Feeder Information & FALSE & TRUE & TRUE & FALSE & & \\
\hline Legacy Node 1 & FALSE & TRUE & TRUE & FALSE & & \\
\hline Legacy Node 2 & FALSE & TRUE & TRUE & FALSE & & \\
\hline Legacy Ees Number & FALSE & TRUE & TRUE & FALSE & & \\
\hline Legacy Pole/Pad Number & FALSE & TRUE & TRUE & FALSE & & \\
\hline Related Substation Object Id & FALSE & TRUE & TRUE & FALSE & & \\
\hline Related Switch Gear Object Id & FALSE & TRUE & TRUE & FALSE & & \\
\hline Related Support Structure Object Id & FALSE & TRUE & TRUE & FALSE & & \\
\hline Symbol Rotation & FALSE & FALSE & TRUE & FALSE & & \\
\hline DCSID & FALSE & TRUE & TRUE & FALSE & & \\
\hline HANDLE & FALSE & TRUE & TRUE & FALSE & & \\
\hline TILENAME & FALSE & TRUE & TRUE & FALSE & & \\
\hline LAYER & FALSE & TRUE & TRUE & FALSE & & \\
\hline LOA & TRUE & FALSE & TRUE & FALSE & $\begin{array}{l}\text { NIPSCO } \\
\text { LoaName }\end{array}$ & $\begin{array}{l}\text { NIPSCO } \\
\text { LoaName }\end{array}$ \\
\hline Tax Unit & TRUE & TRUE & FALSE & FALSE & & \\
\hline Grid & TRUE & TRUE & FALSE & FALSE & & \\
\hline Symbol Configuration Cd & FALSE & TRUE & TRUE & FALSE & & \\
\hline$P_{2} L$ & FALSE & TRUE & TRUE & FALSE & & \\
\hline$P \_L T$ & FALSE & TRUE & TRUE & FALSE & & \\
\hline C_L & FALSE & TRUE & TRUE & FALSE & & \\
\hline $\bar{C}$ CLT & FALSE & TRUE & TRUE & FALSE & & \\
\hline$\overline{\text { FDR }}$ & FALSE & TRUE & TRUE & FALSE & & \\
\hline FDR_T & FALSE & TRUE & TRUE & FALSE & & \\
\hline OBJECTID & TRUE & FALSE & FALSE & FALSE & & \\
\hline LOA & FALSE & TRUE & TRUE & FALSE & & \\
\hline
\end{tabular}

\section{arcfm8.ELECTRIC.SwitchGear}

ArcFM Display Field: OBJECTID

Create Edit Task:

On Create Event: NIPSCO Structure Relate

On Update Event: NIPSCO Structure Relate

On Delete Event:

On Abandon Event:

Abandon Feature Class:

Abandon Subtype:

Remove Feature Class: arcfm8.ELECTRIC.RetiredSwitchGear

Remove Subtype:

\begin{tabular}{|l|c|c|c|c|c|c|}
\hline Field Alias & Visible & Editable & $\begin{array}{c}\text { Allow } \\
\text { Null } \\
\text { Values }\end{array}$ & $\begin{array}{c}\text { Clear After } \\
\text { Create }\end{array}$ & $\begin{array}{c}\text { On Feature } \\
\text { Create }\end{array}$ & $\begin{array}{c}\text { On Feature } \\
\text { Update }\end{array}$ \\
\hline Subtype & TRUE & TRUE & FALSE & FALSE & & \\
\hline Operation Type & TRUE & TRUE & TRUE & FALSE & & \\
\hline Front Kind & TRUE & TRUE & TRUE & FALSE & & \\
\hline
\end{tabular}




\begin{tabular}{|c|c|c|c|c|c|c|}
\hline Used Compartment Count & TRUE & TRUE & TRUE & FALSE & & \\
\hline Voltage Rating Value & TRUE & TRUE & FALSE & FALSE & & \\
\hline Assembly Number & TRUE & TRUE & TRUE & FALSE & & \\
\hline INSTALLDATE & TRUE & TRUE & FALSE & FALSE & & \\
\hline Install WO Number & TRUE & TRUE & FALSE & FALSE & & \\
\hline Install Misc Order Id & TRUE & TRUE & FALSE & FALSE & & \\
\hline RETIREDATE & FALSE & TRUE & TRUE & FALSE & & \\
\hline Retire WO Number & FALSE & TRUE & TRUE & FALSE & & \\
\hline Retire Misc Order Id & FALSE & TRUE & TRUE & FALSE & & \\
\hline LOA & TRUE & FALSE & TRUE & FALSE & $\begin{array}{l}\text { NIPSCO } \\
\text { LoaName }\end{array}$ & \begin{tabular}{|l} 
NIPSCO \\
LoaName \\
\end{tabular} \\
\hline Tax Unit & TRUE & TRUE & FALSE & FALSE & & \\
\hline Grid & TRUE & TRUE & FALSE & FALSE & & \\
\hline Related Pad Mount Object Id & TRUE & FALSE & TRUE & FALSE & & \\
\hline OBJECTID & TRUE & FALSE & FALSE & FALSE & & \\
\hline Legacy Pad Number & FALSE & TRUE & TRUE & TRUE & & \\
\hline Shape & FALSE & TRUE & TRUE & FALSE & & \\
\hline CreationDate & FALSE & TRUE & FALSE & FALSE & \begin{tabular}{|l|} 
ArcFM \\
Current \\
Date \\
\end{tabular} & \\
\hline Created By & FALSE & TRUE & FALSE & FALSE & \begin{tabular}{|l|} 
ArcFM \\
User Name
\end{tabular} & \\
\hline UPDATEDATE & FALSE & FALSE & TRUE & FALSE & & $\begin{array}{l}\text { ArcFM Current } \\
\text { Date } \\
\end{array}$ \\
\hline Updated By & FALSE & FALSE & TRUE & FALSE & & $\begin{array}{l}\text { ArcFM User } \\
\text { Name }\end{array}$ \\
\hline Legacy Ees Number & FALSE & TRUE & TRUE & FALSE & & \\
\hline Graphics Scale Factor & FALSE & TRUE & TRUE & FALSE & & \\
\hline Symbol Rotation & FALSE & FALSE & TRUE & FALSE & & \\
\hline LEGACYWRINSTALLTIMESTAMP & FALSE & TRUE & TRUE & FALSE & & \\
\hline LEGACYWRREMOVALTIMESTAMP & FALSE & TRUE & TRUE & FALSE & & \\
\hline ASSOCIATIONTIMESTAMP & FALSE & TRUE & TRUE & FALSE & & \\
\hline
\end{tabular}

arcfm8.ELECTRIC.SwitchUnit

ArcFM Display Field: PHASEDESIGNATION

Create Edit Task:

On Create Event: EDFS Validate Work Order Number 2

On Update Event: EDFS Validate Work Order Number 2

On Delete Event:

On Abandon Event:

Abandon Feature Class:

Abandon Subtype:

Remove Feature Class:

Remove Subtype:

\begin{tabular}{|l|c|c|c|c|c|c|}
\hline Field Alias & Visible & Editable & $\begin{array}{c}\text { Allow } \\
\text { Null } \\
\text { Values }\end{array}$ & $\begin{array}{c}\text { Clear After } \\
\text { Create }\end{array}$ & $\begin{array}{c}\text { On Feature } \\
\text { Create }\end{array}$ & $\begin{array}{c}\text { On Feature } \\
\text { Update }\end{array}$ \\
\hline Subtype & TRUE & TRUE & FALSE & FALSE & & \\
\hline Type & FALSE & TRUE & TRUE & FALSE & & \\
\hline Phase & TRUE & TRUE & FALSE & FALSE & & \\
\hline Amperage & TRUE & TRUE & FALSE & FALSE & & \\
\hline Switch ID Number & TRUE & TRUE & FALSE & FALSE & & \\
\hline INSTALLDATE & TRUE & TRUE & FALSE & FALSE & & \\
\hline Install WO Number & TRUE & TRUE & FALSE & FALSE & & \\
\hline Install Misc Order Id & TRUE & TRUE & FALSE & FALSE & & \\
\hline RETIREDATE & FALSE & TRUE & TRUE & FALSE & & \\
\hline Retire WO Number & FALSE & TRUE & TRUE & FALSE & & \\
\hline Retire Misc Order Id & FALSE & TRUE & TRUE & FALSE & & \\
\hline Pole/Pad Number & FALSE & TRUE & TRUE & FALSE & & \\
\hline Circuit Number & FALSE & TRUE & TRUE & FALSE & & \\
\hline
\end{tabular}




\begin{tabular}{|c|c|c|c|c|c|c|}
\hline Related Switch Object Id & TRUE & FALSE & TRUE & FALSE & & \\
\hline OBJECTID & TRUE & FALSE & FALSE & FALSE & & \\
\hline LEGACYWRINSTALLTIMESTAMP & FALSE & TRUE & TRUE & FALSE & & \\
\hline LEGACYWRREMOVALTIMESTAMP & FALSE & TRUE & TRUE & FALSE & & \\
\hline ASSOCIATIONTIMESTAMP & FALSE & TRUE & TRUE & FALSE & & \\
\hline CreationDate & FALSE & TRUE & TRUE & FALSE & \begin{tabular}{|l|} 
ArcFM \\
Current \\
Date \\
\end{tabular} & \\
\hline Created By & FALSE & TRUE & TRUE & FALSE & \begin{tabular}{|l|} 
ArcFM \\
User Name
\end{tabular} & \\
\hline UPDATEDATE & FALSE & FALSE & TRUE & FALSE & & $\begin{array}{l}\text { ArcFM Current } \\
\text { Date }\end{array}$ \\
\hline Updated By & FALSE & FALSE & TRUE & FALSE & & $\begin{array}{l}\text { ArcFM User } \\
\text { Name }\end{array}$ \\
\hline
\end{tabular}

arcfm8.ELECTRIC.Terminator

ArcFM Display Field: OBJECTID

Create Edit Task:

On Create Event: ArcFM Create Feeder Object

On Update Event: ArcFM Update Feeder Object

On Delete Event: ArcFM Delete Feeder Object

On Abandon Event:

Abandon Feature Class:

Abandon Subtype:

Remove Feature Class:

Remove Subtype:

\begin{tabular}{|c|c|c|c|c|c|c|}
\hline Field Alias & Visible & Editable & \begin{tabular}{|c|} 
Allow \\
Null \\
Values
\end{tabular} & $\begin{array}{c}\text { Clear After } \\
\text { Create }\end{array}$ & $\begin{array}{c}\text { On Feature } \\
\text { Create }\end{array}$ & $\begin{array}{c}\text { On Feature } \\
\text { Update }\end{array}$ \\
\hline Subtype & TRUE & TRUE & FALSE & FALSE & & \\
\hline Phase & TRUE & TRUE & FALSE & FALSE & & \\
\hline Circuit Number & TRUE & FALSE & TRUE & FALSE & & \\
\hline Shape & FALSE & TRUE & TRUE & FALSE & & \\
\hline ANCILLARYROLE & FALSE & TRUE & TRUE & FALSE & & \\
\hline Enabled & FALSE & TRUE & TRUE & FALSE & & \\
\hline CreationDate & FALSE & TRUE & FALSE & FALSE & \begin{tabular}{|l|} 
ArcFM \\
Current \\
Date
\end{tabular} & \\
\hline Created By & FALSE & TRUE & FALSE & FALSE & \begin{tabular}{|l|} 
ArcFM \\
User Name
\end{tabular} & \\
\hline UPDATEDATE & FALSE & FALSE & TRUE & FALSE & & $\begin{array}{l}\text { ArcFM Current } \\
\text { Date }\end{array}$ \\
\hline Updated By & FALSE & FALSE & TRUE & FALSE & & $\begin{array}{l}\text { ArcFM User } \\
\text { Name }\end{array}$ \\
\hline \begin{tabular}{|l} 
Electric Trace Weight \\
\end{tabular} & FALSE & TRUE & TRUE & FALSE & & \\
\hline Feeder Manager Non-Traceable & FALSE & TRUE & TRUE & FALSE & & \\
\hline Circuit Number 2 & FALSE & TRUE & TRUE & FALSE & & \\
\hline Feeder Information & FALSE & TRUE & TRUE & FALSE & & \\
\hline Legacy Node 1 & FALSE & TRUE & TRUE & FALSE & & \\
\hline Legacy Node 2 & FALSE & TRUE & TRUE & FALSE & & \\
\hline Legacy Ees Number & FALSE & TRUE & TRUE & FALSE & & \\
\hline Symbol Rotation & FALSE & FALSE & TRUE & FALSE & & \\
\hline HANDLE & FALSE & TRUE & TRUE & FALSE & & \\
\hline TILENAME & FALSE & TRUE & TRUE & FALSE & & \\
\hline C_L & FALSE & TRUE & TRUE & FALSE & & \\
\hline OBJECTID & TRUE & FALSE & FALSE & FALSE & & \\
\hline
\end{tabular}

arcfm8.ELECTRIC.TieBus

ArcFM Display Field: OBJECTID

Create Edit Task:

On Create Event: NIPSCO.SplitAtTapPoint 
On Create Event: ArcFM Create Feeder Object

On Update Event: ArcFM Update Feeder Object

On Delete Event: ArcFM Delete Feeder Object

Before Split Event:

On Split Event: ArcFM Split Feeder Object

After Split Event:

On Abandon Event:

Abandon Feature Class:

Abandon Subtype:

Remove Feature Class:

Remove Subtype:

\begin{tabular}{|c|c|c|c|c|c|c|}
\hline Field Alias & Visible & Editable & \begin{tabular}{|c} 
Allow \\
Null \\
Values
\end{tabular} & $\begin{array}{c}\text { Clear After } \\
\text { Create }\end{array}$ & $\begin{array}{c}\text { On Feature } \\
\text { Create }\end{array}$ & $\begin{array}{c}\text { On Feature } \\
\text { Update }\end{array}$ \\
\hline Subtype & TRUE & TRUE & FALSE & FALSE & & \\
\hline LOA & TRUE & FALSE & TRUE & FALSE & $\begin{array}{l}\text { NIPSCO } \\
\text { LoaName }\end{array}$ & $\begin{array}{l}\text { NIPSCO } \\
\text { LoaName }\end{array}$ \\
\hline Phase & TRUE & TRUE & FALSE & FALSE & & \\
\hline Primary Operating Voltage & TRUE & TRUE & FALSE & FALSE & & \\
\hline Shape & FALSE & TRUE & TRUE & FALSE & & \\
\hline Enabled & FALSE & TRUE & TRUE & FALSE & & \\
\hline CreationDate & FALSE & TRUE & FALSE & FALSE & \begin{tabular}{|l|} 
ArcFM \\
Current \\
Date \\
\end{tabular} & \\
\hline Created By & FALSE & TRUE & FALSE & FALSE & \begin{tabular}{|l} 
ArcFM \\
User Name
\end{tabular} & \\
\hline UPDATEDATE & FALSE & FALSE & TRUE & FALSE & & $\begin{array}{l}\text { ArcFM Current } \\
\text { Date } \\
\end{array}$ \\
\hline Updated By & FALSE & FALSE & TRUE & FALSE & & $\begin{array}{l}\text { ArcFM User } \\
\text { Name }\end{array}$ \\
\hline \begin{tabular}{|l|} 
Legacy Node 1 \\
\end{tabular} & FALSE & TRUE & TRUE & FALSE & & \\
\hline Legacy Node 2 & FALSE & TRUE & TRUE & FALSE & & \\
\hline Legacy Ees Number & FALSE & TRUE & TRUE & FALSE & & \\
\hline Electric Trace Weight & FALSE & TRUE & TRUE & FALSE & & \\
\hline Feeder Manager Non-Traceable & FALSE & TRUE & TRUE & FALSE & & \\
\hline Feeder Information & FALSE & TRUE & TRUE & FALSE & & \\
\hline Circuit Number & TRUE & FALSE & TRUE & FALSE & & \\
\hline Circuit Number 2 & FALSE & TRUE & \begin{tabular}{|l|l|} 
TRUE \\
\end{tabular} & FALSE & & \\
\hline SHAPE.Ien & FALSE & & & & & \\
\hline HANDLE & FALSE & TRUE & TRUE & FALSE & & \\
\hline TILENAME & FALSE & TRUE & TRUE & FALSE & & \\
\hline OBJECTID & TRUE & FALSE & FALSE & FALSE & & \\
\hline
\end{tabular}

arcfm8.ELECTRIC.TrafficLightDemand

ArcFM Display Field: DEMANDWATTAGEVALUE

Create Edit Task:

On Create Event:

On Update Event:

On Delete Event:

On Abandon Event:

Abandon Feature Class:

Abandon Subtype:

Remove Feature Class:

Remove Subtype:

\begin{tabular}{|l|c|c|c|c|c|c|}
\hline Field Alias & Visible & Editable & $\begin{array}{c}\text { Allow } \\
\text { Null } \\
\text { Values }\end{array}$ & $\begin{array}{c}\text { Clear After } \\
\text { Create }\end{array}$ & $\begin{array}{c}\text { On Feature } \\
\text { Create }\end{array}$ & $\begin{array}{c}\text { On Feature } \\
\text { Update }\end{array}$ \\
\hline Load Number & FALSE & TRUE & TRUE & FALSE & & \\
\hline DEMANDDATE & TRUE & TRUE & FALSE & FALSE & & \\
\hline Demand Wattage & TRUE & TRUE & FALSE & FALSE & & \\
\hline CIS Account Number & TRUE & TRUE & FALSE & FALSE & & \\
\hline
\end{tabular}




\begin{tabular}{|l|c|c|l|l|l|l|}
\hline Related PadMount Object Id & FALSE & FALSE & TRUE & FALSE & & \\
\hline Related SupportStructure Object Id & FALSE & FALSE & TRUE & FALSE & & \\
\hline OBJECTID & TRUE & FALSE & FALSE & FALSE & & \\
\hline Legacy XRef & FALSE & TRUE & TRUE & FALSE & & \\
\hline CreationDate & FALSE & TRUE & TRUE & FALSE & $\begin{array}{l}\text { ArcFM } \\
\text { Darrent }\end{array}$ & \\
\hline Created By & FALSE & TRUE & FALSE & FALSE & $\begin{array}{l}\text { ArcFM } \\
\text { User Name }\end{array}$ & $\begin{array}{l}\text { ArcFM Current } \\
\text { Date }\end{array}$ \\
\hline UPDATEDATE & FALSE & FALSE & TRUE & FALSE & & $\begin{array}{l}\text { ArcFM User } \\
\text { Name }\end{array}$ \\
\hline Updated By & FALSE & FALSE & TRUE & FALSE & & \\
\hline
\end{tabular}

arcfm8.ELECTRIC.TransformerBank

ArcFM Display Field: OBJECTID

Create Edit Task:

On Create Event: ArcFM Segment Split

On Create Event: NIPSCO Structure Relate

On Create Event: ArcFM Create Feeder Object

On Update Event: NIPSCO Structure Relate

On Update Event: ArcFM Update Feeder Object

On Update Event: NIPSCO.LOANamePropogation

On Abandon Event:

On Delete Event: NIPSCO.Prevent Delete If Units Related

On Delete Event: NIPSCO Delete Attached Transformer Lead

On Delete Event: ArcFM Delete Feeder Object

Abandon Feature Class:

Abandon Subtype:

Remove Feature Class:

Remove Subtype:

\begin{tabular}{|c|c|c|c|c|c|c|}
\hline Field Alias & Visible & Editable & \begin{tabular}{|c|} 
Allow \\
Null \\
Values
\end{tabular} & $\begin{array}{l}\text { Clear After } \\
\text { Create }\end{array}$ & $\begin{array}{c}\text { On Feature } \\
\text { Create }\end{array}$ & $\begin{array}{l}\text { On Feature } \\
\text { Update }\end{array}$ \\
\hline Subtype & TRUE & TRUE & FALSE & FALSE & & \\
\hline Phase & TRUE & TRUE & FALSE & FALSE & & \\
\hline Bank KVA & TRUE & TRUE & FALSE & FALSE & & \\
\hline Live Front Indicator & TRUE & TRUE & FALSE & FALSE & & \\
\hline Non-Metered Service Point Indicator & TRUE & TRUE & FALSE & FALSE & & \\
\hline Fault Indicator Present Indicator & TRUE & TRUE & FALSE & FALSE & & \\
\hline Circuit Number & TRUE & FALSE & TRUE & FALSE & & \\
\hline Shape & FALSE & TRUE & TRUE & FALSE & & \\
\hline AncillaryRole & FALSE & TRUE & TRUE & FALSE & & \\
\hline Enabled & FALSE & TRUE & TRUE & FALSE & & \\
\hline CreationDate & FALSE & TRUE & FALSE & FALSE & \begin{tabular}{|l|} 
ArcFM \\
Current \\
Date \\
\end{tabular} & \\
\hline Created By & FALSE & TRUE & FALSE & FALSE & \begin{tabular}{|l|} 
ArcFM \\
User Name
\end{tabular} & \\
\hline UPDATEDATE & FALSE & FALSE & TRUE & FALSE & & $\begin{array}{l}\text { ArcFM Current } \\
\text { Date }\end{array}$ \\
\hline Updated By & FALSE & FALSE & TRUE & FALSE & & $\begin{array}{l}\text { ArcFM User } \\
\text { Name }\end{array}$ \\
\hline Electric Trace Weight & FALSE & TRUE & TRUE & FALSE & & \\
\hline Feeder Manager Non-Traceable & FALSE & TRUE & TRUE & FALSE & & \\
\hline Circuit Number 2 & FALSE & TRUE & TRUE & FALSE & & \\
\hline Feeder Information & FALSE & TRUE & TRUE & FALSE & & \\
\hline Legacy Node 1 & FALSE & TRUE & TRUE & FALSE & & \\
\hline Legacy Node 2 & FALSE & TRUE & TRUE & FALSE & & \\
\hline
\end{tabular}




\begin{tabular}{|l|c|c|c|c|c|c|}
\hline Legacy Ees Number & FALSE & TRUE & TRUE & FALSE & & \\
\hline Secondary Pad Metering Indicator & TRUE & TRUE & FALSE & FALSE & & \\
\hline Related Support Structure Object Id & FALSE & TRUE & TRUE & FALSE & & \\
\hline Symbol Rotation & FALSE & TRUE & TRUE & FALSE & & \\
\hline LegacyPole/Pad Number & FALSE & FALSE & TRUE & FALSE & & \\
\hline LOA & TRUE & FALSE & TRUE & FALSE & $\begin{array}{l}\text { NIPSCO } \\
\text { LoaName }\end{array}$ & $\begin{array}{l}\text { NIPSCO } \\
\text { LoaName }\end{array}$ \\
\hline Tax Unit & TRUE & TRUE & FALSE & FALSE & & \\
\hline Grid & TRUE & TRUE & FALSE & FALSE & & \\
\hline Related Pad Mount Object Id & FALSE & TRUE & TRUE & FALSE & & \\
\hline OBJECTID & TRUE & FALSE & FALSE & FALSE & & \\
\hline
\end{tabular}

arcfm8.ELECTRIC.TransformerFunctionTest

ArcFM Display Field: FUNCTIONTESTDATE

Create Edit Task:

On Create Event:

On Update Event:

On Delete Event:

On Abandon Event:

Abandon Feature Class:

Abandon Subtype:

Remove Feature Class:

Remove Subtype:

\begin{tabular}{|l|c|c|c|c|c|c|}
\hline Field Alias & Visible & Editable & $\begin{array}{c}\text { Allow } \\
\text { Null } \\
\text { Values }\end{array}$ & $\begin{array}{c}\text { Clear After } \\
\text { Create }\end{array}$ & $\begin{array}{l}\text { On Feature } \\
\text { Create }\end{array}$ & $\begin{array}{c}\text { On Feature } \\
\text { Update }\end{array}$ \\
\hline Company Number & TRUE & FALSE & FALSE & FALSE & & \\
\hline FUNCTIONTESTDATE & TRUE & FALSE & FALSE & FALSE & & \\
\hline OBJECTID & TRUE & FALSE & FALSE & FALSE & & \\
\hline CreationDate & FALSE & TRUE & TRUE & FALSE & $\begin{array}{l}\text { ArcFM } \\
\text { Current } \\
\text { Date }\end{array}$ & \\
\hline Created By & FALSE & TRUE & TRUE & FALSE & $\begin{array}{l}\text { ArcFM } \\
\text { User Name }\end{array}$ & \\
\hline UPDATEDATE & FALSE & FALSE & TRUE & FALSE & & $\begin{array}{l}\text { ArcFM Current } \\
\text { Date }\end{array}$ \\
\hline Updated By & FALSE & TRUE & FALSE & FALSE & & $\begin{array}{l}\text { ArcFM User } \\
\text { Name }\end{array}$ \\
\hline
\end{tabular}

arcfm8.ELECTRIC.TransformerOilTest

ArcFM Display Field: OILTESTDATE

Create Edit Task:

On Create Event:

On Update Event:

On Delete Event:

On Abandon Event:

Abandon Feature Class:

Abandon Subtype:

Remove Feature Class:

Remove Subtype:

\begin{tabular}{|l|l|l|l|l|l|l|}
\hline Field Alias & Visible & Editable & $\begin{array}{c}\text { Allow } \\
\text { Null } \\
\text { Values }\end{array}$ & $\begin{array}{c}\text { Clear After } \\
\text { Create }\end{array}$ & $\begin{array}{l}\text { On Feature } \\
\text { Create }\end{array}$ & $\begin{array}{c}\text { On Feature } \\
\text { Update }\end{array}$ \\
\hline Company Number & TRUE & FALSE & FALSE & FALSE & & \\
\hline OILTESTDATE & TRUE & FALSE & FALSE & FALSE & & \\
\hline Test Type & TRUE & FALSE & FALSE & FALSE & & \\
\hline PPM & TRUE & FALSE & FALSE & FALSE & & \\
\hline OBJECTID & TRUE & FALSE & FALSE & FALSE & & \\
\hline CreationDate & FALSE & TRUE & TRUE & FALSE & $\begin{array}{l}\text { ArcFM } \\
\text { Current } \\
\text { Date }\end{array}$ & \\
\hline
\end{tabular}




\begin{tabular}{|l|c|c|c|c|l|l|}
\hline Created By & FALSE & TRUE & TRUE & FALSE & $\begin{array}{l}\text { ArcFM } \\
\text { User Name }\end{array}$ \\
\hline UPDATEDATE & FALSE & FALSE & TRUE & FALSE & & $\begin{array}{l}\text { ArcFM Current } \\
\text { Date }\end{array}$ \\
\hline Updated By & FALSE & TRUE & FALSE & FALSE & & $\begin{array}{l}\text { ArcFM User } \\
\text { Name }\end{array}$ \\
\hline
\end{tabular}

arcfm8.ELECTRIC.TransformerRegulatorStoresItem

ArcFM Display Field: STORESITEMNUMBER

Create Edit Task:

On Create Event:

On Update Event:

On Delete Event:

On Abandon Event:

Abandon Feature Class:

Abandon Subtype:

Remove Feature Class:

Remove Subtype:

\begin{tabular}{|l|l|l|l|l|l|l|}
\hline Field Alias & Visible & Editable & $\begin{array}{c}\text { Allow } \\
\text { Null } \\
\text { Values }\end{array}$ & $\begin{array}{c}\text { Clear After } \\
\text { Create }\end{array}$ & $\begin{array}{c}\text { On Feature } \\
\text { Create }\end{array}$ & $\begin{array}{c}\text { On Feature } \\
\text { Update }\end{array}$ \\
\hline SIN & TRUE & FALSE & FALSE & FALSE & & \\
\hline Type & TRUE & FALSE & FALSE & FALSE & & \\
\hline Location & TRUE & FALSE & FALSE & FALSE & & \\
\hline Phase Count & TRUE & FALSE & FALSE & FALSE & & \\
\hline KVA & TRUE & FALSE & FALSE & FALSE & & \\
\hline Amperage & TRUE & FALSE & FALSE & FALSE & & \\
\hline Primary Voltage & TRUE & FALSE & FALSE & FALSE & & \\
\hline OBJECTID & TRUE & FALSE & FALSE & FALSE & & \\
\hline CreationDate & TRUE & FALSE & FALSE & FALSE & & \\
\hline Created By & FALSE & TRUE & TRUE & FALSE & $\begin{array}{l}\text { ArcFM } \\
\text { Current } \\
\text { Date }\end{array}$ & \\
\hline UPDATEDATE & FALSE & TRUE & FALSE & FALSE & $\begin{array}{l}\text { ArcFM } \\
\text { User Name }\end{array}$ & \\
\hline Updated By & FALSE & FALSE & TRUE & FALSE & & $\begin{array}{l}\text { ArcFM Current } \\
\text { Date }\end{array}$ \\
\hline
\end{tabular}

arcfm8.ELECTRIC.TransformerUnit

ArcFM Display Field: COMPANYNUMBER

Create Edit Task:

On Create Event:

On Update Event: EDFS Update SIN KVA

On Delete Event:

On Abandon Event:

Abandon Feature Class:

Abandon Subtype:

Remove Feature Class:

Remove Subtype:

\begin{tabular}{|l|c|c|c|c|c|c|}
\hline Field Alias & Visible & Editable & $\begin{array}{c}\text { Allow } \\
\text { Null } \\
\text { Values }\end{array}$ & $\begin{array}{c}\text { Clear After } \\
\text { Create }\end{array}$ & $\begin{array}{c}\text { On Feature } \\
\text { Create }\end{array}$ & $\begin{array}{c}\text { On Feature } \\
\text { Update }\end{array}$ \\
\hline Company Number & TRUE & FALSE & FALSE & FALSE & & \\
\hline SIN & TRUE & FALSE & FALSE & FALSE & & \\
\hline Manufacturer & TRUE & FALSE & FALSE & FALSE & & \\
\hline Serial Number & TRUE & FALSE & FALSE & FALSE & & \\
\hline Status & TRUE & FALSE & TRUE & FALSE & & \\
\hline Disposition & TRUE & FALSE & TRUE & FALSE & & \\
\hline RECEIVEDATE & TRUE & FALSE & FALSE & FALSE & & \\
\hline P.O. Number & TRUE & FALSE & FALSE & FALSE & & \\
\hline Impedance & TRUE & FALSE & FALSE & FALSE & & \\
\hline Weight & TRUE & FALSE & FALSE & FALSE & & \\
\hline
\end{tabular}




\begin{tabular}{|l|c|c|c|c|l|l|}
\hline Installation Cost & TRUE & FALSE & FALSE & FALSE & & \\
\hline Polarity & TRUE & FALSE & FALSE & FALSE & & \\
\hline Gallons of Oil & TRUE & FALSE & FALSE & FALSE & & \\
\hline Warranty & TRUE & FALSE & FALSE & FALSE & & \\
\hline OBJECTID & TRUE & FALSE & FALSE & FALSE & & \\
\hline STATUSTIMESTAMP & FALSE & TRUE & FALSE & FALSE & & \\
\hline DISPOSITIONTIMESTAMP & FALSE & TRUE & TRUE & FALSE & & \\
\hline CreationDate & FALSE & TRUE & FALSE & FALSE & $\begin{array}{l}\text { ArcFM } \\
\text { Darrent } \\
\text { Date }\end{array}$ & $\begin{array}{l}\text { FrcFM } \\
\text { User Name }\end{array}$ \\
\hline Created By & FALSE & FALSE & FALSE & FALSE & $\begin{array}{l}\text { ArcFM Current } \\
\text { Date }\end{array}$ \\
\hline UPDATEDATE & FALSE & FALSE & TRUE & FALSE & & $\begin{array}{l}\text { ArcFM User } \\
\text { Name }\end{array}$ \\
\hline Updated By & FALSE & FALSE & TRUE & FALSE & \\
\hline
\end{tabular}

arcfm8.ELECTRIC.TransformerUnitCondemn

ArcFM Display Field: CONDEMNDATE

Create Edit Task:

On Create Event:

On Update Event:

On Delete Event:

On Abandon Event:

Abandon Feature Class:

Abandon Subtype:

Remove Feature Class:

Remove Subtype:

\begin{tabular}{|l|l|l|l|l|l|l|}
\hline Field Alias & Visible & Editable & $\begin{array}{c}\text { Allow } \\
\text { Null } \\
\text { Values }\end{array}$ & $\begin{array}{c}\text { Clear After } \\
\text { Create }\end{array}$ & $\begin{array}{l}\text { On Feature } \\
\text { Create }\end{array}$ & $\begin{array}{c}\text { On Feature } \\
\text { Update }\end{array}$ \\
\hline Company Number & TRUE & FALSE & FALSE & FALSE & & \\
\hline Condemn Number & TRUE & FALSE & TRUE & FALSE & & \\
\hline Type & TRUE & FALSE & FALSE & FALSE & & \\
\hline CONDEMNDATE & TRUE & FALSE & FALSE & FALSE & & \\
\hline Reason & TRUE & FALSE & FALSE & FALSE & & \\
\hline Approved & TRUE & FALSE & FALSE & FALSE & & \\
\hline CONDECID & TRUE & FALSE & FALSE & FALSE & & \\
\hline CreationDate & FALSE & TRUE & FALSE & FALSE & & \\
\hline Created By & FALSE & TRUE & TRUE & FALSE & $\begin{array}{l}\text { ArcFM } \\
\text { Darrent }\end{array}$ & \\
\hline UPDATEDATE & FALSE & TRUE & FALSE & FALSE & $\begin{array}{l}\text { ArcFM } \\
\text { User Name }\end{array}$ & ArcFM Current \\
\hline Updated By & FALSE & FALSE & TRUE & FALSE & & $\begin{array}{l}\text { ArcFM User } \\
\text { Name }\end{array}$ \\
\hline
\end{tabular}

arcfm8.ELECTRIC.TransformerUnitlnstall

ArcFM Display Field: RELTRANSFORMERUNITCOMPANYNUMBER

Create Edit Task:

On Create Event:

On Update Event:

On Delete Event: EDFS Reset Asset To Stock

On Abandon Event:

Abandon Feature Class:

Abandon Subtype:

Remove Feature Class:

Remove Subtype:

\begin{tabular}{|l|c|l|c|c|c|c|}
\hline Field Alias & Visible & Editable & $\begin{array}{c}\text { Allow } \\
\text { Null }\end{array}$ & $\begin{array}{c}\text { Clear After } \\
\text { Create }\end{array}$ & $\begin{array}{c}\text { On Feature } \\
\text { Create }\end{array}$ & $\begin{array}{c}\text { On Feature } \\
\text { Update }\end{array}$ \\
\hline
\end{tabular}




\begin{tabular}{|l|l|l|l|l|l|l|}
\hline & & & Values & & & \\
\hline Company Number & TRUE & FALSE & FALSE & FALSE & & \\
\hline Company Use Location Id & TRUE & TRUE & TRUE & FALSE & & \\
\hline INSTALLDATE & TRUE & FALSE & FALSE & FALSE & & \\
\hline Material Ticket & TRUE & TRUE & FALSE & FALSE & & \\
\hline Circuit Number & FALSE & TRUE & TRUE & FALSE & & \\
\hline Phase & TRUE & TRUE & FALSE & FALSE & & \\
\hline Installation Status & TRUE & TRUE & FALSE & FALSE & & \\
\hline Installation Type & TRUE & TRUE & FALSE & FALSE & & \\
\hline KVA & TRUE & FALSE & TRUE & FALSE & & \\
\hline LOA & TRUE & FALSE & TRUE & FALSE & & \\
\hline Tax Unit & TRUE & TRUE & FALSE & FALSE & & \\
\hline Grid & TRUE & TRUE & FALSE & FALSE & & \\
\hline Pole/Pad Number & TRUE & TRUE & FALSE & FALSE & & \\
\hline Related Transformer Bank Object Id & TRUE & FALSE & TRUE & FALSE & & \\
\hline OBJECTID & TRUE & FALSE & FALSE & FALSE & & \\
\hline INSTALLTIMESTAMP & FALSE & TRUE & FALSE & FALSE & & \\
\hline CreationDate & FALSE & TRUE & TRUE & FALSE & $\begin{array}{l}\text { ArcFM } \\
\text { Current } \\
\text { Date }\end{array}$ & \\
\hline Created By & FALSE & TRUE & FALSE & FALSE & $\begin{array}{l}\text { ArcFM } \\
\text { User Name }\end{array}$ & \\
\hline UPDATEDATE & FALSE & FALSE & TRUE & FALSE & & $\begin{array}{l}\text { ArcFM Current } \\
\text { Date }\end{array}$ \\
\hline Updated By & FALSE & FALSE & TRUE & FALSE & & $\begin{array}{l}\text { ArcFM User } \\
\text { Name }\end{array}$ \\
\hline
\end{tabular}

arcfm8.ELECTRIC.TransformerUnitRemove

ArcFM Display Field: REMOVALDATE

Create Edit Task:

On Create Event:

On Update Event:

On Delete Event: EDFS Reset Transformer/Regulator Asset To Installed

On Abandon Event:

Abandon Feature Class:

Abandon Subtype:

Remove Feature Class:

Remove Subtype:

\begin{tabular}{|l|l|l|l|l|l|l|}
\hline Field Alias & Visible & Editable & $\begin{array}{c}\text { Allow } \\
\text { Null } \\
\text { Values }\end{array}$ & $\begin{array}{c}\text { Clear After } \\
\text { Create }\end{array}$ & $\begin{array}{c}\text { On Feature } \\
\text { Create }\end{array}$ & $\begin{array}{c}\text { On Feature } \\
\text { Update }\end{array}$ \\
\hline Company Number & TRUE & FALSE & FALSE & FALSE & & \\
\hline REMOVALDATE & TRUE & FALSE & FALSE & FALSE & & \\
\hline Reason & TRUE & FALSE & FALSE & FALSE & & \\
\hline Material Ticket & TRUE & FALSE & FALSE & FALSE & & \\
\hline OBJECTID & TRUE & FALSE & FALSE & FALSE & & \\
\hline CreationDate & FALSE & TRUE & TRUE & FALSE & $\begin{array}{l}\text { ArcFM } \\
\text { Darrent } \\
\text { Date }\end{array}$ & \\
\hline Created By & FALSE & TRUE & FALSE & FALSE & $\begin{array}{l}\text { ArcFM } \\
\text { User Name }\end{array}$ & \\
\hline UPDATEDATE & FALSE & FALSE & TRUE & FALSE & & $\begin{array}{l}\text { ArcFM Current } \\
\text { Date }\end{array}$ \\
\hline Updated By & FALSE & FALSE & TRUE & FALSE & & $\begin{array}{l}\text { ArcFM User } \\
\text { Name }\end{array}$ \\
\hline REMOVALTIMESTAMP & FALSE & TRUE & FALSE & FALSE & & \\
\hline LEGACYINSTALLTIMESTAMP & FALSE & TRUE & TRUE & FALSE & & \\
\hline
\end{tabular}

arcfm8.ELECTRIC.TransformerUnitSoldOrLeased

ArcFM Display Field: SOLDLEASEDDATE

Create Edit Task:

On Create Event: 
On Update Event:

On Delete Event:

On Abandon Event:

Abandon Feature Class:

Abandon Subtype:

Remove Feature Class:

Remove Subtype:

\begin{tabular}{|l|l|l|l|l|l|l|}
\hline Field Alias & Visible & Editable & $\begin{array}{c}\text { Allow } \\
\text { Null } \\
\text { Values }\end{array}$ & $\begin{array}{c}\text { Clear After } \\
\text { Create }\end{array}$ & $\begin{array}{c}\text { On Feature } \\
\text { Create }\end{array}$ & $\begin{array}{c}\text { On Feature } \\
\text { Update }\end{array}$ \\
\hline Company Number & TRUE & FALSE & FALSE & FALSE & & \\
\hline SOLDLEASEDTIMESTAMP & FALSE & TRUE & FALSE & FALSE & & \\
\hline Sold/Leased & TRUE & FALSE & FALSE & FALSE & & \\
\hline SOLDLEASEDDATE & TRUE & FALSE & FALSE & FALSE & & \\
\hline Material Ticket & TRUE & FALSE & FALSE & FALSE & & \\
\hline Receiver & TRUE & FALSE & FALSE & FALSE & & \\
\hline Lease Terminated & TRUE & FALSE & TRUE & FALSE & & \\
\hline LEASETERMINATEDDATE & TRUE & FALSE & TRUE & FALSE & & \\
\hline OBJECTID & TRUE & FALSE & FALSE & FALSE & & \\
\hline CreationDate & FALSE & TRUE & TRUE & FALSE & $\begin{array}{l}\text { ArcFM Current } \\
\text { Date }\end{array}$ & \\
\hline Created By & FALSE & TRUE & FALSE & FALSE & $\begin{array}{l}\text { ArcFM } \\
\text { User Name }\end{array}$ & \\
\hline UPDATEDATE & FALSE & FALSE & TRUE & FALSE & & $\begin{array}{l}\text { ArcFM Current } \\
\text { Date }\end{array}$ \\
\hline Updated By & FALSE & FALSE & TRUE & FALSE & & $\begin{array}{l}\text { ArcFM User } \\
\text { Name }\end{array}$ \\
\hline
\end{tabular}

arcfm8.ELECTRIC.TransformerUnitStock

ArcFM Display Field: STOCKDATE

Create Edit Task:

On Create Event:

On Update Event:

On Delete Event:

On Abandon Event:

Abandon Feature Class:

Abandon Subtype:

Remove Feature Class:

Remove Subtype:

\begin{tabular}{|l|c|c|c|c|l|l|}
\hline Field Alias & Visible & Editable & $\begin{array}{c}\text { Allow } \\
\text { Null } \\
\text { Values }\end{array}$ & $\begin{array}{c}\text { Clear After } \\
\text { Create }\end{array}$ & $\begin{array}{c}\text { On Feature } \\
\text { Create }\end{array}$ & $\begin{array}{c}\text { On Feature } \\
\text { Update }\end{array}$ \\
\hline Company Number & TRUE & FALSE & FALSE & FALSE & & \\
\hline STOCKDATE & TRUE & FALSE & FALSE & FALSE & & \\
\hline Store Room Number & TRUE & FALSE & FALSE & FALSE & & \\
\hline Transfer Number & TRUE & FALSE & FALSE & FALSE & & \\
\hline OBJECTID & TRUE & FALSE & FALSE & FALSE & & \\
\hline CrOCKTIMESTAMP & FALSE & TRUE & FALSE & FALSE & & \\
\hline CreationDate & FALSE & TRUE & TRUE & FALSE & $\begin{array}{l}\text { ArcFM } \\
\text { Current } \\
\text { Date }\end{array}$ & \\
\hline UPDATEDATE & FALSE & TRUE & FALSE & FALSE & $\begin{array}{l}\text { ArcFM } \\
\text { User Name }\end{array}$ & \\
\hline Updated By & FALSE & FALSE & TRUE & FALSE & & $\begin{array}{l}\text { ArcFM Current } \\
\text { Date }\end{array}$ \\
\hline
\end{tabular}

arcfm8.ELECTRIC.UgConductor

ArcFM Display Field: OBJECTID

Create Edit Task: 
On Create Event: ArcFM Create Feeder Object

On Create Event: NIPSCO.SplitAtTapPoint

On Update Event: ArcFM Update Feeder Object

On Delete Event: NIPSCO.Prevent Delete If Units Related

On Delete Event: NIPSCO.FeederAllOpenPointDelete

On Delete Event: ArcFM OH - Delete Feeder Conductor/Units

On Split Event: ArcFM Split Feeder Object

On Abandon Event:

Before Split Event: EDFS Turn WOMO Validation Off

Before Split Event: ArcFM Save Related Objects

Before Split Event: EDFS Turn WOMO Validation On

After Split Event: EDFS Turn WOMO Validation Off

After Split Event: ArcFM Restore Related Objects

After Split Event: EDFS Turn WOMO Validation On

Abandon Feature Class: arcfm8.ELECTRIC.AbandonedConductor

Abandon Subtype: 1

Remove Feature Class:

Remove Subtype:

\begin{tabular}{|c|c|c|c|c|c|c|}
\hline Field Alias & Visible & Editable & \begin{tabular}{|c|} 
Allow \\
Null \\
Values
\end{tabular} & $\begin{array}{c}\text { Clear After } \\
\text { Create }\end{array}$ & $\begin{array}{c}\text { On Feature } \\
\text { Create }\end{array}$ & $\begin{array}{c}\text { On Feature } \\
\text { Update }\end{array}$ \\
\hline Subtype & TRUE & TRUE & FALSE & FALSE & & \\
\hline LOA Number & TRUE & FALSE & FALSE & FALSE & $\begin{array}{l}\text { NIPSCO } \\
\text { LoaName } \\
\end{array}$ & $\begin{array}{l}\text { NIPSCO } \\
\text { LoaName }\end{array}$ \\
\hline Grid & TRUE & TRUE & FALSE & FALSE & & \\
\hline Primary Operating Voltage & TRUE & TRUE & FALSE & FALSE & & \\
\hline Circuit Number & TRUE & FALSE & TRUE & FALSE & & \\
\hline Phase & TRUE & TRUE & FALSE & FALSE & & \\
\hline In Conduit & TRUE & TRUE & TRUE & FALSE & & \\
\hline Shape & FALSE & TRUE & TRUE & FALSE & & \\
\hline Enabled & FALSE & TRUE & TRUE & FALSE & & \\
\hline CreationDate & FALSE & TRUE & FALSE & FALSE & \begin{tabular}{|l|} 
ArcFM \\
Current \\
Date \\
\end{tabular} & \\
\hline Created By & FALSE & TRUE & FALSE & FALSE & \begin{tabular}{|l|} 
ArcFM \\
User Name
\end{tabular} & \\
\hline UPDATEDATE & FALSE & FALSE & TRUE & FALSE & & $\begin{array}{l}\text { ArcFM Current } \\
\text { Date }\end{array}$ \\
\hline Updated By & FALSE & FALSE & TRUE & FALSE & & $\begin{array}{l}\text { ArcFM User } \\
\text { Name }\end{array}$ \\
\hline Legacy Node 1 & FALSE & TRUE & TRUE & FALSE & & \\
\hline Legacy Node 2 & FALSE & TRUE & TRUE & FALSE & & \\
\hline Measured Length & FALSE & TRUE & TRUE & FALSE & & \\
\hline Legacy Ees Number & FALSE & TRUE & TRUE & FALSE & & \\
\hline Electric Trace Weight & FALSE & TRUE & TRUE & FALSE & & \\
\hline Feeder Manager Non-Traceable & FALSE & TRUE & TRUE & FALSE & & \\
\hline Feeder Information & FALSE & TRUE & TRUE & FALSE & & \\
\hline Circuit Number 2 & FALSE & TRUE & TRUE & FALSE & & \\
\hline FeederAll Phase & FALSE & TRUE & TRUE & FALSE & & \\
\hline SHAPE.len & FALSE & & & & & \\
\hline Owner & TRUE & TRUE & FALSE & FALSE & & \\
\hline Legacy Circuit Number & FALSE & TRUE & TRUE & FALSE & & \\
\hline Wire Size & TRUE & TRUE & FALSE & FALSE & & \\
\hline Wire Material & TRUE & TRUE & FALSE & FALSE & & \\
\hline OBJECTID & TRUE & FALSE & FALSE & FALSE & & \\
\hline
\end{tabular}

arcfm8.ELECTRIC.UgConductorInfo

ArcFM Display Field: WIREMATERIALCD

Create Edit Task:

On Create Event: EDFS Validate UG Primary Properties

On Create Event: EDFS Validate Work Order Number 2 
On Update Event: EDFS Validate UG Primary Properties

On Update Event: EDFS Validate Work Order Number 2

On Delete Event:

On Abandon Event:

Abandon Feature Class:

Abandon Subtype:

Remove Feature Class:

Remove Subtype:

\begin{tabular}{|l|l|l|l|l|l|l|}
\hline Field Alias & Visible & Editable & $\begin{array}{c}\text { Allow } \begin{array}{l}\text { Null } \\
\text { Values }\end{array} \\
\text { Subtype }\end{array}$ & $\begin{array}{c}\text { Clear After } \\
\text { Create }\end{array}$ & $\begin{array}{c}\text { On Feature } \\
\text { Create }\end{array}$ & $\begin{array}{c}\text { On Feature } \\
\text { Update }\end{array}$ \\
\hline Wire Size & TRUE & TRUE & FALSE & FALSE & & \\
\hline Wire Material & TRUE & TRUE & FALSE & FALSE & & \\
\hline Wire Type & TRUE & TRUE & FALSE & FALSE & & \\
\hline In Conduit & TRUE & TRUE & FALSE & FALSE & & \\
\hline Measured Length & TRUE & TRUE & FALSE & FALSE & & \\
\hline INSTALLDATE & FALSE & TRUE & TRUE & FALSE & & \\
\hline Install WO Number & TRUE & TRUE & FALSE & FALSE & & \\
\hline Install Misc Order Id & TRUE & TRUE & FALSE & FALSE & & \\
\hline RETIREDATE & TRUE & TRUE & FALSE & FALSE & & \\
\hline Retire WO Number & FALSE & TRUE & TRUE & FALSE & & \\
\hline Retire Misc Order Id & FALSE & TRUE & TRUE & FALSE & & \\
\hline Circuit Number & FALSE & TRUE & TRUE & FALSE & & \\
\hline From Pole/Pad Number & FALSE & TRUE & TRUE & FALSE & & \\
\hline To Pole/Pad Number & FALSE & TRUE & TRUE & FALSE & & \\
\hline Related Ug Conductor Object Id & FALSE & TRUE & TRUE & FALSE & & \\
\hline OBJECTID & TRUE & FALSE & TRUE & FALSE & & \\
\hline CreationDate & TRUE & FALSE & FALSE & FALSE & & \\
\hline Created By & FALSE & TRUE & TRUE & FALSE & $\begin{array}{l}\text { ArcFM } \\
\text { Current } \\
\text { Date }\end{array}$ & \\
\hline UPDATEDATE & FALSE & TRUE & TRUE & FALSE & $\begin{array}{l}\text { ArcFM } \\
\text { User Name }\end{array}$ & \\
\hline Updated By & FALSE & FALSE & TRUE & FALSE & & $\begin{array}{l}\text { ArcFM Current } \\
\text { Date }\end{array}$ \\
\hline
\end{tabular}

arcfm8.ELECTRIC.VoltageRegulator

ArcFM Display Field: OBJECTID

Create Edit Task: NIPSCO.Electric.FeatureOffset

On Create Event: ArcFM Segment Split

On Create Event: NIPSCO Structure Relate

On Create Event: ArcFM Create Feeder Object

On Update Event: NIPSCO Structure Relate

On Update Event: ArcFM Update Feeder Object

On Update Event: NIPSCO.LOANamePropogation

On Delete Event: NIPSCO.Prevent Delete If Units Related

On Delete Event: ArcFM Delete Feeder Object

On Abandon Event:

Abandon Feature Class:

Abandon Subtype:

Remove Feature Class:

Remove Subtype:

\begin{tabular}{|l|c|c|c|c|c|c|}
\hline Field Alias & Visible & Editable & $\begin{array}{c}\text { Allow } \\
\text { Null } \\
\text { Values }\end{array}$ & $\begin{array}{c}\text { Clear After } \\
\text { Create }\end{array}$ & $\begin{array}{l}\text { On Feature } \\
\text { Create }\end{array}$ & $\begin{array}{c}\text { On Feature } \\
\text { Update }\end{array}$ \\
\hline Subtype & TRUE & TRUE & FALSE & FALSE & & \\
\hline LOA & TRUE & FALSE & TRUE & FALSE & $\begin{array}{l}\text { NIPSCO } \\
\text { LoaName }\end{array}$ & $\begin{array}{l}\text { NIPSCO } \\
\text { LoaName }\end{array}$ \\
\hline Tax Unit & TRUE & TRUE & FALSE & FALSE & & \\
\hline Grid & TRUE & TRUE & FALSE & FALSE & & \\
\hline
\end{tabular}




\begin{tabular}{|c|c|c|c|c|c|c|}
\hline Phase & TRUE & TRUE & FALSE & FALSE & & \\
\hline KVA & TRUE & TRUE & FALSE & FALSE & & \\
\hline Regulator Type & TRUE & TRUE & FALSE & FALSE & & \\
\hline Shape & FALSE & TRUE & TRUE & FALSE & & \\
\hline ANCILLARYROLE & FALSE & TRUE & TRUE & FALSE & & \\
\hline Enabled & FALSE & TRUE & TRUE & FALSE & & \\
\hline CreationDate & FALSE & TRUE & FALSE & FALSE & \begin{tabular}{|l|} 
ArcFM \\
Current \\
Date \\
\end{tabular} & \\
\hline Created By & FALSE & TRUE & FALSE & FALSE & $\begin{array}{l}\text { ArcFM } \\
\text { User Name }\end{array}$ & \\
\hline UPDATEDATE & FALSE & FALSE & TRUE & FALSE & & $\begin{array}{l}\text { ArcFM Current } \\
\text { Date }\end{array}$ \\
\hline Updated By & FALSE & FALSE & TRUE & FALSE & & $\begin{array}{l}\text { ArcFM User } \\
\text { Name }\end{array}$ \\
\hline Electric Trace Weight & FALSE & TRUE & TRUE & FALSE & & \\
\hline Feeder Manager Non-Traceable & FALSE & TRUE & TRUE & FALSE & & \\
\hline Circuit Number & TRUE & FALSE & TRUE & FALSE & & \\
\hline Circuit Number 2 & FALSE & TRUE & TRUE & FALSE & & \\
\hline Feeder Information & FALSE & TRUE & TRUE & FALSE & & \\
\hline Graphics Scale Factor & FALSE & TRUE & TRUE & FALSE & & \\
\hline Legacy Node 1 & FALSE & TRUE & TRUE & FALSE & & \\
\hline Legacy Node 2 & FALSE & TRUE & TRUE & FALSE & & \\
\hline Legacy Ees Number & FALSE & TRUE & TRUE & FALSE & & \\
\hline Legacy Pole Number & FALSE & TRUE & TRUE & FALSE & & \\
\hline Related Support Structure Object Id & FALSE & TRUE & TRUE & FALSE & & \\
\hline Related Regulator Bank Object Id & FALSE & TRUE & TRUE & FALSE & & \\
\hline Symbol Rotation & FALSE & FALSE & TRUE & FALSE & & \\
\hline DCSID & FALSE & TRUE & TRUE & FALSE & & \\
\hline HANDLE & FALSE & TRUE & TRUE & FALSE & & \\
\hline TILENAME & FALSE & TRUE & TRUE & FALSE & & \\
\hline REGTYPE & FALSE & TRUE & TRUE & FALSE & & \\
\hline Rated Amperage & TRUE & TRUE & TRUE & FALSE & & \\
\hline$P \_L$ & FALSE & TRUE & TRUE & FALSE & & \\
\hline OBJECTID & TRUE & FALSE & FALSE & FALSE & & \\
\hline
\end{tabular}

arcfm8.ELECTRIC.VoltageRegulatorBank

ArcFM Display Field: BANKKVAVALUE

Create Edit Task:

On Create Event:

On Update Event:

On Delete Event: ArcFM Delete Related Conductor Info

On Abandon Event:

Abandon Feature Class:

Abandon Subtype:

Remove Feature Class:

Remove Subtype:

\begin{tabular}{|l|c|c|c|c|l|l|}
\hline Field Alias & Visible & Editable & $\begin{array}{c}\text { Allow } \\
\text { Null } \\
\text { Values }\end{array}$ & $\begin{array}{l}\text { Clear After } \\
\text { Create }\end{array}$ & $\begin{array}{l}\text { On Feature } \\
\text { Create }\end{array}$ & $\begin{array}{c}\text { On Feature } \\
\text { Update }\end{array}$ \\
\hline Bank KVA & TRUE & TRUE & FALSE & FALSE & & \\
\hline OBJECTID & TRUE & FALSE & FALSE & FALSE & & \\
\hline CreationDate & FALSE & TRUE & TRUE & FALSE & $\begin{array}{l}\text { ArcFM } \\
\text { Current } \\
\text { Date }\end{array}$ & \\
\hline Created By & FALSE & TRUE & TRUE & FALSE & $\begin{array}{l}\text { ArcFM } \\
\text { User Name }\end{array}$ & \\
\hline UPDATEDATE & FALSE & FALSE & TRUE & FALSE & & $\begin{array}{l}\text { ArcFM Current } \\
\text { Date }\end{array}$ \\
\hline Updated By & FALSE & FALSE & TRUE & FALSE & & $\begin{array}{l}\text { ArcFM User } \\
\text { Name }\end{array}$ \\
\hline
\end{tabular}

arcfm8.ELECTRIC.WoodPoleEvents 
ArcFM Display Field: EVENTTYPECD

Create Edit Task:

On Create Event:

On Update Event:

On Delete Event:

On Abandon Event:

Abandon Feature Class:

Abandon Subtype:

Remove Feature Class:

Remove Subtype:

\begin{tabular}{|l|c|c|c|c|c|c|}
\hline Field Alias & Visible & Editable & $\begin{array}{c}\text { Allow } \\
\text { Null } \\
\text { Values }\end{array}$ & $\begin{array}{c}\text { Clear After } \\
\text { Create }\end{array}$ & $\begin{array}{c}\text { On Feature } \\
\text { Create }\end{array}$ & $\begin{array}{c}\text { On Feature } \\
\text { Update }\end{array}$ \\
\hline Related Distribution Refrence Number & FALSE & TRUE & TRUE & FALSE & & \\
\hline Event Type & TRUE & TRUE & FALSE & FALSE & & \\
\hline Pole Inspection Year & TRUE & TRUE & TRUE & FALSE & & \\
\hline Pole Inspection Pass/Fail Code & TRUE & TRUE & TRUE & FALSE & & \\
\hline WOODPOLEREINFORCEDDATE & TRUE & TRUE & TRUE & FALSE & & \\
\hline WOODPOLEGNDTREATDATE & TRUE & TRUE & TRUE & FALSE & & \\
\hline OBJECTID & TRUE & FALSE & FALSE & FALSE & & \\
\hline
\end{tabular}

arcfm8.ELECTRIC.WoodPolelnfo

ArcFM Display Field: RELDISTRIBREFNUMBER

Create Edit Task:

On Create Event:

On Update Event:

On Delete Event:

On Abandon Event:

Abandon Feature Class:

Abandon Subtype:

Remove Feature Class:

Remove Subtype:

\begin{tabular}{|l|c|c|c|c|c|c|}
\hline Field Alias & Visible & Editable & $\begin{array}{c}\text { Allow } \\
\text { Null } \\
\text { Values }\end{array}$ & $\begin{array}{c}\text { Clear After } \\
\text { Create }\end{array}$ & $\begin{array}{c}\text { On Feature } \\
\text { Create }\end{array}$ & $\begin{array}{c}\text { On Feature } \\
\text { Update }\end{array}$ \\
\hline Related Distribution Reference Number & FALSE & TRUE & TRUE & FALSE & & \\
\hline Pole Class & TRUE & TRUE & FALSE & FALSE & & \\
\hline Pole Type & TRUE & TRUE & FALSE & FALSE & & \\
\hline Pole Original Treatment Type & TRUE & TRUE & FALSE & FALSE & & \\
\hline Wood Setting & TRUE & TRUE & FALSE & FALSE & & \\
\hline OBJECTID & TRUE & FALSE & FALSE & FALSE & & \\
\hline
\end{tabular}


Table E-2. Electric Relationship Class configuration. Classes that did not have any configuration have been excluded

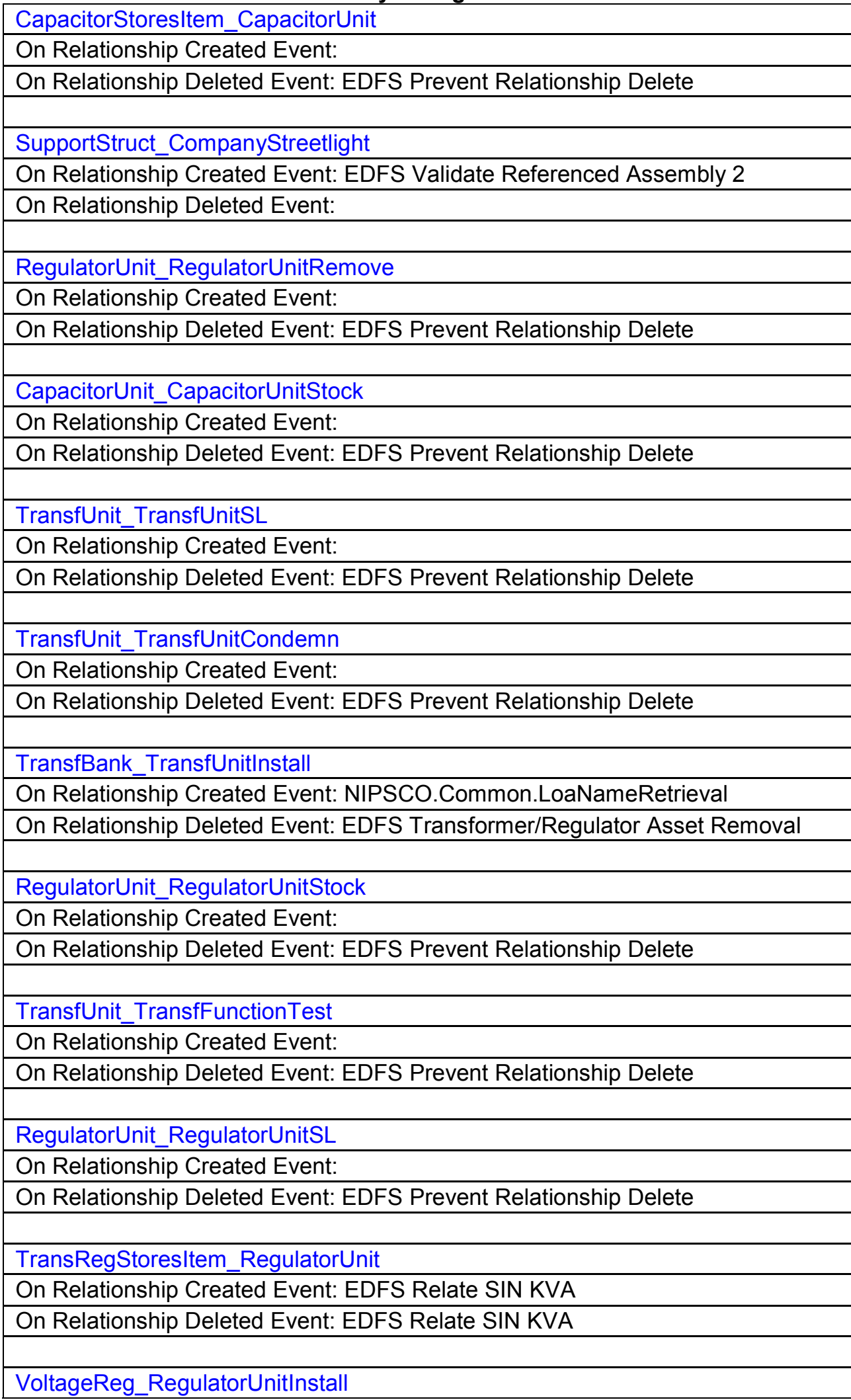




\begin{tabular}{|c|}
\hline On Relationship Created Event: NIPSCO.Common.LoaNameRetrieval \\
\hline On Relationship Deleted Event: EDFS Transformer/Regulator Asset Removal \\
\hline RegUnit_RegFunctionTest \\
\hline On Relationship Created Event: \\
\hline On Relationship Deleted Event: EDFS Prevent Relationship Delete \\
\hline SubBreaker_CircuitSource \\
\hline On Relationship Created Event: ArcFM Relate Feeder Object \\
\hline On Relationship Deleted Event: ArcFM Relate Feeder Object \\
\hline TransfUnit_TransfUnitInstall \\
\hline On Relationship Created Event: \\
\hline On Relationship Deleted Event: EDFS Prevent Relationship Delete \\
\hline CapacitorUnit_CapacitorUnitRemove \\
\hline On Relationship Created Event: \\
\hline On Relationship Deleted Event: EDFS Prevent Relationship Delete \\
\hline TransfUnit_TransfUnitStock \\
\hline On Relationship Created Event: \\
\hline On Relationship Deleted Event: EDFS Prevent Relationship Delete \\
\hline RegUnit_RegOilTest \\
\hline On Relationship Created Event: \\
\hline On Relationship Deleted Event: EDFS Prevent Relationship Delete \\
\hline TransfRegStoresItem_TransfUnit \\
\hline On Relationship Created Event: EDFS Relate SIN KVA \\
\hline On Relationship Deleted Event: EDFS Relate SIN KVA \\
\hline CapacitorUnit_CapacitorUnitInstall \\
\hline On Relationship Created Event: \\
\hline On Relationship Deleted Event: EDFS Prevent Relationship Delete \\
\hline CapacitorBank_CapacitorUnitInstall \\
\hline On Relationship Created Event: NIPSCO.Common.LoaNameRetrieval \\
\hline On Relationship Deleted Event: EDFS Capacitor Asset Removal \\
\hline TransfUnit_TransfOilTest \\
\hline On Relationship Created Event: \\
\hline On Relationship Deleted Event: EDFS Prevent Relationship Delete \\
\hline RegulatorUnit_RegulatorUnitCondemn \\
\hline On Relationship Created Event: \\
\hline On Relationship Deleted Event: EDFS Prevent Relationship Delete \\
\hline
\end{tabular}




\begin{tabular}{|l|}
\hline RegulatorUnit_RegulatorUnitlnstall \\
\hline On Relationship Created Event: \\
\hline On Relationship Deleted Event: EDFS Prevent Relationship Delete \\
\hline \\
\hline TransfUnit_TransfUnitRemove \\
\hline On Relationship Created Event: \\
\hline On Relationship Deleted Event: EDFS Prevent Relationship Delete \\
\hline
\end{tabular}


Table E-3. Contains all ArcFM Model Name assignments for both the base and custom configuration

AbandonedConductor

Object Class Model Names

\begin{tabular}{|l|l|}
\hline Object Class & Model Name \\
\hline AbandonedConductor & MMABANDONED \\
\hline AbandonedConductor & LOCATABLEOBJECT \\
\hline AbandonedConductor & MMREMOVABLE \\
\hline
\end{tabular}

Field Model Names

\begin{tabular}{|l|l|}
\hline Field & Model Name \\
\hline OBJECTID & LOCATABLEFIELD \\
\hline CREATIONDATE & AT_CREATIONDATE \\
\hline CREATIONUSERID & AT_CREATIONUSER \\
\hline UPDATEDATE & AT_UPDATEDATE \\
\hline UPDATEUSERID & AT_UPDATEUSERID \\
\hline LOANUMBER & AT_1 \\
\hline GRIDCD & AT_2 \\
\hline INCONDUITIND & AT_3 \\
\hline SHAPE & AT_SHAPE \\
\hline
\end{tabular}

Assembly

Object Class Model Names

\begin{tabular}{|l|l|}
\hline Object Class & Model Name \\
\hline Assembly & ASSEMBLY \\
\hline Assembly & EDFS RETIREMENT INFO \\
\hline Assembly & EDFS WORKORDER 2 \\
\hline
\end{tabular}

Field Model Names

\begin{tabular}{|l|l|}
\hline Field & Model Name \\
\hline UPDATEDATE & RETIREDATE \\
\hline SUBTYPECD & SUBTYPECD \\
\hline DISTRIBREFNUMBER & DISTRIBREFNUMBER \\
\hline ASSEMBLYID & ASSEMBLYID \\
\hline ASSEMBLYCOUNT & ASSEMBLYCOUNT \\
\hline INSTALLWORKORDERNUMBER & INSTALLWORKORDERNUMBER \\
\hline INSTALLMISCORDERID & INSTALLMISCORDERID \\
\hline RETIREWORKORDERNUMBER & RETIREWORKORDERNUMBER \\
\hline RETIREMISCORDERID & RETIREMISCORDERID \\
\hline RELSUPPORTSTRUCTUREOBJECTID & RELSUPPORTSTRUCTUREOBJECTID \\
\hline
\end{tabular}

CapacitorBank

Object Class Model Names

\begin{tabular}{|l|l|}
\hline Object Class & Model Name \\
\hline CapacitorBank & CADOPS \\
\hline CapacitorBank & CAPACITORBANK \\
\hline CapacitorBank & EDFS ASSETFEATURE \\
\hline CapacitorBank & EDFS STRUCTURE PREVENT DELETION \\
\hline
\end{tabular}


CapacitorBank

CapacitorBank

Field Model Names

\begin{tabular}{|l|l|}
\hline Field & Model Name \\
\hline MMELECTRICTRACEWEIGHT & MMELECTRICTRACEWEIGHT \\
\hline FDRMGRNONTRACEABLE & FDRMGRNONTRACEABLE \\
\hline FEEDERID & CADOPS \\
\hline FEEDERID & FEEDERALL \\
\hline FEEDERID & FEEDERID \\
\hline FEEDERID2 & FEEDERID2 \\
\hline FEEDERINFO & FEEDERINFO \\
\hline PHASEDESIGNATIONCD & CADOPS \\
\hline PHASEDESIGNATIONCD & FEEDERALL \\
\hline PHASEDESIGNATIONCD & PHASEDESIGNATION \\
\hline SYMBOLROTATIONVALUE & SYMBOLROTATION \\
\hline LOANUMBER & LOANAME \\
\hline LOANUMBER & LOANUMBER \\
\hline GRIDCD & CADOPS \\
\hline GRIDCD & FEEDERALL \\
\hline SUBTYPECD & CADOPS \\
\hline SUBTYPECD & FEEDERALL \\
\hline LEGACYDISTRIBREFNUMBER & LOCATABLEFIELD \\
\hline BANKKVARVALUE & CADOPS \\
\hline BANKKVARVALUE & FEEDERALL \\
\hline CAPACITORTYPECD & CADOPS \\
\hline CAPACITORTYPECD & FEEDERALL \\
\hline
\end{tabular}

FEEDERALL

LOCATABLEOBJECT

CapacitorStores/tem

Object Class Model Names

\begin{tabular}{|l|l|}
\hline Object Class & Model Name \\
\hline CapacitorStoresItem & CADOPS \\
\hline CapacitorStoresItem & FEEDERALL \\
\hline CapacitorStoresItem & NABACKWARD \\
\hline
\end{tabular}

Field Model Names

\begin{tabular}{|l|l|}
\hline Field & Model Name \\
\hline KVARVALUE & FEEDERALL \\
\hline KVARVALUE & CADOPS \\
\hline
\end{tabular}

CapacitorUnit

Object Class Model Names

\begin{tabular}{|l|l|}
\hline Object Class & Model Name \\
\hline CapacitorUnit & FEEDERALL \\
\hline CapacitorUnit & LOCATABLEOBJECT \\
\hline CapacitorUnit & NABACKWARD \\
\hline CapacitorUnit & CADOPS \\
\hline CapacitorUnit & CAPACITORUNIT \\
\hline CapacitorUnit & EDFS ASSETUNIT \\
\hline
\end{tabular}


Field Model Names

\begin{tabular}{|l|l|}
\hline Field & Model Name \\
\hline RELSTORESITEMNUMBER & RELSTORESITEMNUMBER \\
\hline COMPANYNUMBER & COMPANYNUMBER \\
\hline STATUSCD & STATUSCD \\
\hline STATUSTIMESTAMP & STATUSTIMESTAMP \\
\hline DISPOSITIONCD & DISPOSITIONCD \\
\hline
\end{tabular}

CapacitorUnitInstall

Object Class Model Names

\begin{tabular}{|l|l|}
\hline Object Class & Model Name \\
\hline CapacitorUnitInstall & EDFS WORKORDER \\
\hline CapacitorUnitInstall & FEEDERALL \\
\hline CapacitorUnitInstall & CADOPS \\
\hline CapacitorUnitInstall & CAPACITORUNITINSTALL \\
\hline CapacitorUnitInstall & EDFS ASSETINSTALL \\
\hline
\end{tabular}

Field Model Names

\begin{tabular}{|l|l}
\hline Field & \\
\hline LOANUMBER & \\
\hline LOANUMBER & \\
\hline TAXUNITCD & \\
\hline GRIDCD & \\
\hline INSTALLTIMESTAMP & \\
\hline INSTALLDATE & \\
\hline PHASEDESIGNATION & \\
\hline PHASEDESIGNATION & \\
\hline PHASEDESIGNATION & \\
\hline INSTALLATIONSTATUSCD & \\
\hline WORKORDERNUMBER & \\
\hline MISCORDERID & \\
\hline DISTRIBREFNUMBER & \\
\hline CIRCUITNUMBER & RE \\
\hline RELCAPACITORBANKOBJECTID & \\
\hline RELCAPACITORUNITCOMPANYNUMBER & \\
\hline
\end{tabular}

\begin{tabular}{|l|}
\hline Model Name \\
\hline LOANAME \\
\hline LOANUMBER \\
\hline TAXUNITCD \\
\hline GRIDCD \\
\hline INSTALLTIMESTAMP \\
\hline INSTALLDATE \\
\hline CADOPS \\
\hline FEEDERALL \\
\hline PHASEDESIGNATION \\
\hline INSTALLATIONSTATUSCD \\
\hline WORKORDERNUMBER \\
\hline MISCORDERID \\
\hline DISTRIBREFNUMBER \\
\hline CIRCUITNUMBER \\
\hline RELATEDBANKOID \\
\hline COMPANYNUMBER \\
\hline
\end{tabular}

CapacitorUnitRemove

Object Class Model Names

\begin{tabular}{|l|l|}
\hline Object Class & Model Name \\
\hline CapacitorUnitRemove & CAPACITORUNITREMOVE \\
\hline CapacitorUnitRemove & EDFS ASSETREMOVE \\
\hline CapacitorUnitRemove & EDFS WORKORDER \\
\hline
\end{tabular}

Field Model Names

\begin{tabular}{|l|l|}
\hline Field & Model Name \\
\hline REMOVALTIMESTAMP & REMOVALTIMESTAMP \\
\hline REMOVALDATE & REMOVALDATE \\
\hline REMOVALCD & REMOVALCD \\
\hline
\end{tabular}


MISCORDERID

WORKORDERNUMBER

RELCAPACITORUNITCOMPANYNUMBER

CapacitorUnitStock

Object Class Model Names

Object Class

CapacitorUnitStock

Field Model Names

\section{Field}

STOCKTIMESTAMP

STOCKTIMESTAMP

STOCKDATE

RELCAPACITORUNITCOMPANYNUMBER

RELSTOREROOMNUMBER
MISCORDERID

WORKORDERNUMBER

COMPANYNUMBER

CircuitSource

Object Class Model Names

Object Class

CircuitSource

CircuitSource

CircuitSource

CircuitSource

Model Name

EDFS ASSETSTOCK

Field Model Names

\begin{tabular}{|l|l|}
\hline Field & Model Name \\
\hline NOMINALVOLTAGE & CADOPS \\
\hline NOMINALVOLTAGE & FEEDERALL \\
\hline CIRCUITSOURCEID & FEEDERALL \\
\hline CIRCUITSOURCEID & FEEDERID \\
\hline CIRCUITSOURCEID & CADOPS \\
\hline CIRCUITSOURCENAME & FEEDERALL \\
\hline CIRCUITSOURCENAME & FEEDERNAME \\
\hline CIRCUITSOURCENAME & CADOPS \\
\hline SUBSTATIONID & CADOPS \\
\hline SUBSTATIONID & FEEDERALL \\
\hline SUBSTATIONID & SUBSTATIONID \\
\hline EMERGENCYCAPACITYKW & CADOPS \\
\hline EMERGENCYCAPACITYKW & FEEDERALL \\
\hline MAXKVAR & CADOPS \\
\hline MAXKVAR & FEEDERALL \\
\hline MINKVAR & CADOPS \\
\hline MINKVAR & FEEDERALL \\
\hline MAXPOSITIVESEQUENCEREACTANCE & CADOPS \\
\hline MAXPOSITIVESEQUENCEREACTANCE & FEEDERALL \\
\hline MAXPOSITIVESEQUENCERESISTANCE & CADOPS \\
\hline MAXPOSITIVESEQUENCERESISTANCE & FEEDERALL \\
\hline MAXZEROSEQUENCEIMPEDANCE & CADOPS \\
\hline
\end{tabular}


MAXZEROSEQUENCEIMPEDANCE MAXZEROSEQUENCERESISTANCE MAXZEROSEQUENCERESISTANCE NEGATIVESEQUENCEREACTANCE NEGATIVESEQUENCEREACTANCE VOLTAGEANGLE

VOLTAGEANGLE

FEEDERSOURCEINFO
FEEDERALL CADOPS FEEDERALL CADOPS FEEDERALL CADOPS

FEEDERALL FEEDERSOURCEINFO

Company Streetlight

Object Class Model Names

\begin{tabular}{|l|l|}
\hline Object Class & Model Name \\
\hline CompanyStreetlight & COMPANYSTREETLIGHT \\
\hline CompanyStreetlight & EDFS RETIREMENT INFO \\
\hline CompanyStreetlight & EDFS STRUCTURE PREVENT DELETION \\
\hline CompanyStreetlight & EDFS WORKORDER 2 \\
\hline
\end{tabular}

Field Model Names

\begin{tabular}{|l|l|}
\hline Field & Model Name \\
\hline INSTALLWORKORDERNUMBER & INSTALLWORKORDERNUMBER \\
\hline INSTALLMISCORDERID & INSTALLMISCORDERID \\
\hline RETIREDATE & RETIREDATE \\
\hline RETIREWORKORDERNUMBER & RETIREWORKORDERNUMBER \\
\hline RETIREMISCORDERID & RETIREMISCORDERID \\
\hline SUBTYPECD & SUBTYPECD \\
\hline DISTRIBREFNUMBER & DISTRIBREFNUMBER \\
\hline ASSEMBLYID & ASSEMBLYID \\
\hline ASSEMBLYIDCOUNT & ASSEMBLYCOUNT \\
\hline ASSEMBLYIDCOUNT & ASSEMBLYIDCOUNT \\
\hline RELSUPPORTSTRUCTUREOBJECTID & RELSUPPORTSTRUCTUREOBJECTID \\
\hline
\end{tabular}

ConductorDefinition

Object Class Model Names

\begin{tabular}{|l|l|}
\hline Object Class & Model Name \\
\hline ConductorDefinition & CONDUCTORDEFINITION \\
\hline
\end{tabular}

Field Model Names

\begin{tabular}{|l|l|}
\hline Field & Model Name \\
\hline CATEGORYCD & CATEGORYCD \\
\hline LOCATIONCD & LOCATIONCD \\
\hline WIRETYPECD & WIRETYPECD \\
\hline WIRESIZECD & WIRESIZECD \\
\hline WIREMATERIALCD & WIREMATERIALCD \\
\hline
\end{tabular}

CustomerGenerator

Object Class Model Names

\section{Object Class}

CustomerGenerator

CustomerGenerator

\section{Model Name}

CADOPS

FEEDERALL 
Field Model Names

\begin{tabular}{|c|c|}
\hline Field & Model Name \\
\hline MMELECTRICTRACEWEIGHT & MMELECTRICTRACEWEIGHT \\
\hline FDRMGRNONTRACEABLE & FDRMGRNONTRACEABLE \\
\hline FEEDERID & CADOPS \\
\hline FEEDERID & FEEDERALL \\
\hline FEEDERID & FEEDERID \\
\hline FEEDERID2 & FEEDERID2 \\
\hline FEEDERINFO & FEEDERINFO \\
\hline PHASEDESIGNATIONCD & CADOPS \\
\hline PHASEDESIGNATIONCD & FEEDERALL \\
\hline PHASEDESIGNATIONCD & PHASEDESIGNATION \\
\hline SYMBOLROTATIONVALUE & SYMBOLROTATION \\
\hline NORMALPOSITIONA & CADOPS \\
\hline NORMALPOSITIONA & FEEDERALL \\
\hline NORMALPOSITIONA & NORMALPOSITION_A \\
\hline NORMALPOSITIONB & CADOPS \\
\hline NORMALPOSITIONB & FEEDERALL \\
\hline NORMALPOSITIONB & NORMALPOSITION_B \\
\hline NORMALPOSITIONC & CADOPS \\
\hline NORMALPOSITIONC & FEEDERALL \\
\hline NORMALPOSITIONC & NORMALPOSITION_C \\
\hline LOANUMBER & CADOPS \\
\hline LOANUMBER & FEEDERALL \\
\hline GRIDCD & CADOPS \\
\hline GRIDCD & FEEDERALL \\
\hline SYMBOLCONFIGURATIONCD & SYMBOLOGYCONFIGURATIONCD \\
\hline CUSTOMERNAME & CADOPS \\
\hline CUSTOMERNAME & FEEDERALL \\
\hline NOMINALVOLTAGE & CADOPS \\
\hline NOMINALVOLTAGE & FEEDERALL \\
\hline CUSTOMERSUBSTATIONID & CADOPS \\
\hline CUSTOMERSUBSTATIONID & FEEDERALL \\
\hline MAXPOSITIVESEQUENCERESISTANCE & CADOPS \\
\hline MAXPOSITIVESEQUENCERESISTANCE & FEEDERALL \\
\hline MAXPOSITIVESEQUENCEREACTANCE & CADOPS \\
\hline MAXPOSITIVESEQUENCEREACTANCE & FEEDERALL \\
\hline NEGATIVESEQUENCEREACTANCE & CADOPS \\
\hline NEGATIVESEQUENCEREACTANCE & FEEDERALL \\
\hline MAXZEROSEQUENCERESISTANCE & CADOPS \\
\hline MAXZEROSEQUENCERESISTANCE & FEEDERALL \\
\hline MAXZEROSEQUENCEIMPEDANCE & CADOPS \\
\hline MAXZEROSEQUENCEIMPEDANCE & FEEDERALL \\
\hline MAXKVAR & CADOPS \\
\hline MAXKVAR & FEEDERALL \\
\hline MINKVAR & CADOPS \\
\hline
\end{tabular}


CustomerStreetlight

Object Class Model Names

\begin{tabular}{|l|l|}
\hline Object Class & Model Name \\
\hline CustomerStreetlight & EDFS PADMOUNT PREVENT DELETION \\
\hline CustomerStreetlight & EDFS STRUCTURE PREVENT DELETION \\
\hline CustomerStreetlight & LOCATABLEOBJECT \\
\hline
\end{tabular}

Field Model Names

Field Model Name

DeadEndElectric

Object Class Model Names

\begin{tabular}{|l|l|}
\hline Object Class & Model Name \\
\hline DeadEndElectric & DEADENDELECTRIC
\end{tabular}

Field Model Names

\begin{tabular}{|l|l}
\hline Field & Model Name \\
\hline
\end{tabular}

ElectricDiscrepancy

Object Class Model Names

\begin{tabular}{|l|l|}
\hline Object Class & Model Name \\
\hline ElectricDiscrepancy & LOCATABLEOBJECT \\
\hline
\end{tabular}

Field Model Names

\begin{tabular}{|l|l|}
\hline Field & Model Name \\
\hline ERRORDESCRIPTION & LOCATABLEFIELD \\
\hline
\end{tabular}

ElectricNetwork_Junctions

Object Class Model Names

\section{Object Class}

ElectricNetwork_Junctions

ElectricNetwork_Junctions

Model Name
CADOPS
FEEDERALL

Field Model Names

\begin{tabular}{|l|l|}
\hline Field & Model Name \\
\hline ENABLED & CADOPS \\
\hline ENABLED & FEEDERALL \\
\hline
\end{tabular}

FeederAllOpenPoint

Object Class Model Names

\begin{tabular}{|l|}
\hline Object Class \\
\hline FeederAllOpenPoin \\
\hline FeederAllOpenPoint \\
\hline FeederAllOpenPoin \\
\hline
\end{tabular}

Field Model Names

\section{Field}

Model Name


OBJECTID

ENABLED

FuseCutoutBank

Object Class Model Names

\begin{tabular}{|l|l|}
\hline Object Class & Model Name \\
\hline FuseCutoutBank & CADOPS \\
\hline FuseCutoutBank & EDFS STRUCTURE PREVENT DELETION \\
\hline FuseCutoutBank & FDRMGRPROTECTIVE \\
\hline FuseCutoutBank & FEEDERALL \\
\hline FuseCutoutBank & FUSE \\
\hline FuseCutoutBank & FUSECUTOUTBANK \\
\hline FuseCutoutBank & LOCATABLEOBJECT \\
\hline FuseCutoutBank & PROTECTIVE \\
\hline
\end{tabular}

Field Model Names

\begin{tabular}{|l|l|}
\hline Field & ModeI Name \\
\hline MMELECTRICTRACEWEIGHT & MMELECTRICTRACEWEIGHT \\
\hline FDRMGRNONTRACEABLE & FDRMGRNONTRACEABLE \\
\hline FEEDERID & CADOPS \\
\hline FEEDERID & FEEDERALL \\
\hline FEEDERID & FEEDERID \\
\hline FEEDERID2 & FEEDERID2 \\
\hline FEEDERINFO & FEEDERINFO \\
\hline PHASEDESIGNATIONCD & CADOPS \\
\hline PHASEDESIGNATIONCD & FEEDERALL \\
\hline PHASEDESIGNATIONCD & PHASEDESIGNATION \\
\hline SYMBOLROTATIONVALUE & SYMBOLROTATION \\
\hline LOANUMBER & CADOPS \\
\hline LOANUMBER & FEEDERALL \\
\hline LOANUMBER & LOANAME \\
\hline GRIDCD & CADOPS \\
\hline GRIDCD & FEEDERALL \\
\hline LEGACYDISTRIBREFNUMBER & LOCATABLEFIELD \\
\hline FUSELINKAMPRATINGVALUE & CADOPS \\
\hline FUSELINKAMPRATINGVALUE & FEEDERALL \\
\hline NORMALPOSITIONA & CADOPS \\
\hline NORMALPOSITIONA & FEEDERALL \\
\hline NORMALPOSITIONA & NORMALPOSITION_A \\
\hline NORMALPOSITIONB & CADOPS \\
\hline NORMALPOSITIONB & FEEDERALL \\
\hline NORMALPOSITIONB & NORMALPOSITION_B \\
\hline NORMALPOSITIONC & CADOPS \\
\hline NORMALPOSITIONC & FEEDERALL \\
\hline NORMALPOSITIONC & NORMALPOSITION_C \\
\hline SYMBOLCONFIGURATIONCD & SYMBOLOGYCONFIGURATIONCD \\
\hline & \\
\hline
\end{tabular}

FuseUnit

Object Class Model Names
LOCATABLEFIELD

FEEDERALL
PROTECTIVE 


\begin{tabular}{|l|l|} 
Object Class & Model Name \\
\hline FuseUnit & CADOPS \\
\hline FuseUnit & EDFS RETIREMENT INFO \\
\hline FuseUnit & EDFS WORKORDER 2 \\
\hline FuseUnit & FEEDERALL \\
\hline FuseUnit & FUSEUNIT \\
\hline FuseUnit & LOCATABLEOBJECT \\
\hline
\end{tabular}

Field Model Names

\begin{tabular}{|l|l|}
\hline Field & Model Name \\
\hline INSTALLWORKORDERNUMBER & INSTALLWORKORDERNUMBER \\
\hline INSTALLMISCORDERID & INSTALLMISCORDERID \\
\hline RETIREDATE & RETIREDATE \\
\hline RETIREWORKORDERNUMBER & RETIREWORKORDERNUMBER \\
\hline RETIREMISCORDERID & RETIREMISCORDERID \\
\hline PHASEDESIGNATION & PHASEDESIGNATION \\
\hline DISTRIBREFNUMBER & DISTRIBREFNUMBER \\
\hline CIRCUITNUMBER & FEEDERID \\
\hline SUBTYPECD & SUBTYPECD \\
\hline CUTOUTAMPERAGEVALUE & CADOPS \\
\hline CUTOUTAMPERAGEVALUE & FEEDERALL \\
\hline
\end{tabular}

GisMappsTransactionQueue

Object Class Model Names

\begin{tabular}{|l|l|}
\hline Object Class & Model Name \\
\hline GisMappsTransactionQueue & GISMAPPSTRANSACTIONQUEUE \\
\hline
\end{tabular}

Field Model Names

\begin{tabular}{|l|l|}
\hline Field & Model Name \\
\hline COMPANYNUMBER & COMPANYNUMBER \\
\hline TRANSACTIONTYPE & TRANSACTIONTYPE \\
\hline LOANUMBER & LOANUMBER \\
\hline STORESITEMNUMBER & STORESITEMNUMBER \\
\hline TRANSACTIONDATE & TRANSACTIONDATE \\
\hline
\end{tabular}

InterfaceCHAssemblyNumber

Object Class Model Names

\begin{tabular}{|l}
\hline Object Class \\
\hline InterfaceCHAssemblyNumber \\
\hline
\end{tabular}

Model Name

CHASSEMBLYVIEW

Field Model Names

\begin{tabular}{|l|l|}
\hline Field & Model Name \\
\hline AssemblyNumber & ASSEMBLYID \\
\hline \\
$\begin{array}{l}\text { InterfaceCISLifeSupport } \\
\text { Object Class Model Names }\end{array}$ \\
\hline Object Class & Model Name \\
\hline InterfaceCISLifeSupport & CISLIFESUPPORT \\
\hline
\end{tabular}


Field Model Names

\begin{tabular}{|l|l|}
\hline Field & Model Name \\
\hline LoadDistribRefNumber & DISTRIBREFNUMBER \\
\hline LifeSupportYesNolnd & LIFESUPPORTINDICATOR \\
\hline
\end{tabular}

InterfaceGLWorkOrderNumber

Object Class Model Names

Object Class

InterfaceGLWorkOrderNumber

Model Name

GENERALLEDGERVIEW

Field Model Names

\begin{tabular}{|l|l|}
\hline Field & Model Name \\
\hline WorkOrderNumber & WORKORDERNUMBER \\
\hline
\end{tabular}

JointUseAttachment

Object Class Model Names

\begin{tabular}{|l|l|}
\hline Object Class & Model Name \\
\hline JointUseAttachment & JOINTUSEATTACHMENT \\
\hline JointUseAttachment & LOCATABLEOBJECT \\
\hline
\end{tabular}

Field Model Names

\begin{tabular}{|l|l|}
\hline Field & Model Name \\
\hline SUBTYPECD & SUBTYPECD \\
\hline ATTACHMENTCOMPANYNAME & ATTACHMENTCOMPANYNAME \\
\hline
\end{tabular}

LegacyOhConductorInfo

Object Class Model Names

\begin{tabular}{|l|l|}
\hline Object Class & Model Name \\
\hline LegacyOhConductorInfo & CONDUCTORINFO \\
\hline LegacyOhConductorInfo & EDFS CONDUCTORINFO \\
\hline LegacyOhConductorInfo & EDFS RETIREMENT INFO \\
\hline LegacyOhConductorInfo & EDFS WORKORDER 2 \\
\hline LegacyOhConductorInfo & LEGACYOHCONDUCTORINFO \\
\hline LegacyOhConductorInfo & LEGACYPRIMARYCONDUCTORINFO \\
\hline
\end{tabular}

Field Model Names

\begin{tabular}{|l|l|}
\hline Field & Model Name \\
\hline INSTALLDATE & INSTALLDATE \\
\hline INSTALLWORKORDERNUMBER & INSTALLWORKORDERNUMBER \\
\hline INSTALLMISCORDERID & INSTALLMISCORDERID \\
\hline RETIREDATE & RETIREDATE \\
\hline RETIREWORKORDERNUMBER & RETIREWORKORDERNUMBER \\
\hline RETIREMISCORDERID & RETIREMISCORDERID \\
\hline SUBTYPECD & SUBTYPECD \\
\hline FROMDISTRIBREFNUMBER & FROMDISTRIBREF \\
\hline TODISTRIBREFNUMBER & TODISTRIBREF \\
\hline WIRESIZECD & WIRESIZECD \\
\hline WIREMATERIALCD & WIREMATERIALCD \\
\hline WIRETYPECD & WIRETYPECD \\
\hline
\end{tabular}


CIRCUITNUMBER CONDUCTORSPANCOUNT

SPANLENGTHVALUE

LegacyUgConductorInfo

Object Class Model Names

\section{Object Class}

LegacyUgConductorInfo

LegacyUgConductorInfo

LegacyUgConductorInfo

LegacyUgConductorInfo

LegacyUgConductorlnfo

LegacyUgConductorInfo

LegacyUgConductorInfo
CIRCUITNUMBER

SPANCOUNT

SPANLENGTH

Field Model Names

\begin{tabular}{|l|l|}
\hline Field & Model Name \\
\hline INSTALLDATE & INSTALLDATE \\
\hline INSTALLWORKORDERNUMBER & INSTALLWORKORDERNUMBER \\
\hline INSTALLMISCORDERID & INSTALLMISCORDERID \\
\hline RETIREDATE & RETIREDATE \\
\hline RETIREWORKORDERNUMBER & RETIREWORKORDERNUMBER \\
\hline RETIREMISCORDERID & RETIREMISCORDERID \\
\hline SUBTYPECD & SUBTYPECD \\
\hline WIRESIZECD & WIRESIZECD \\
\hline WIREMATERIALCD & WIREMATERIALCD \\
\hline WIRETYPECD & WIRETYPECD \\
\hline INCONDUITCD & INCONDUITCD \\
\hline CIRCUITNUMBER & CIRCUITNUMBER \\
\hline FROMDISTRIBREFNUMBER & FROMDISTRIBREF \\
\hline TOSTRUCTUREID & TODISTRIBREF \\
\hline CONDUCTORSPANCOUNT & SPANCOUNT \\
\hline SPANLENGTHVALUE & SPANLENGTH \\
\hline
\end{tabular}

Manhole

Object Class Model Names

\section{Object Class}

Manhole

\section{Model Name}

CONDUCTORINFO

EDFS CONDUCTORINFO

EDFS RETIREMENT INFO

EDFS WORKORDER 2

LEGACYPRIMARYCONDUCTORINFO

LEGACYPRIMARYUGCONDUCTORINFO

LEGACYUGCONDUCTORINFO

Field Model Names

\begin{tabular}{|l|l|}
\hline Field & Model Name \\
\hline LOANUMBER & LOANAME \\
\hline SYMBOLROTATIONVALUE & SYMBOLROTATION \\
\hline
\end{tabular}

OhConductor

Object Class Model Names

Object Class

OhConductor

OhConductor

\section{Model Name}

LOCATABLEOBJECT 
OhConductor

OhConductor

OhConductor

OhConductor

OhConductor

OhConductor

OhConductor
EDFS CONDUCTORINFO

FEEDERALL

LINE

LOCATABLEOBJECT

OHCONDUCTOR

OVERHEADCONDUCTOR

SPLITTARGET

Field Model Names

\section{Field}

OBJECTID

LOANUMBER

LOANUMBER

LOANUMBER

GRIDCD

GRIDCD

MEASUREDLENGTH

MEASUREDLENGTH

MEASUREDLENGTH

MMELECTRICTRACEWEIGHT

FDRMGRNONTRACEABLE

FEEDERINFO

FEEDERID

FEEDERID

FEEDERID

FEEDERID2

PRIMARYOPERATINGVOLTAGEVALUE

PRIMARYOPERATINGVOLTAGEVALUE

PRIMARYOPERATINGVOLTAGEVALUE

PHASEDESIGNATIONCD

PHASEDESIGNATIONCD

PHASEDESIGNATIONCD

SUBTYPECD

SUBTYPECD

WIRESIZECD

WIRESIZECD

WIREMATERIALCD

WIREMATERIALCD

\section{Model Name}

LOCATABLEFIELD

CADOPS

FEEDERALL

LOANAME

CADOPS

FEEDERALL

CADOPS

FEEDERALL

MEASUREDLENGTH

MMELECTRICTRACEWEIGHT

FDRMGRNONTRACEABLE

FEEDERINFO

CADOPS

FEEDERALL

FEEDERID

FEEDERID2

CADOPS

FEEDERALL

OPERATINGVOLTAGE

CADOPS

FEEDERALL

PHASEDESIGNATION

CADOPS

FEEDERALL

CADOPS

FEEDERALL

CADOPS

FEEDERALL

OhConductorInfo

Object Class Model Names

\begin{tabular}{|l|l|}
\hline Object Class & Model Name \\
\hline OhConductorlnfo & CADOPS \\
\hline OhConductorlnfo & CONDUCTORINFO \\
\hline OhConductorlnfo & EDFS CONDUCTORINFO \\
\hline OhConductorInfo & EDFS RETIREMENT INFO \\
\hline OhConductorlnfo & EDFS WORKORDER 2 \\
\hline OhConductorlnfo & FEEDERALL \\
\hline OhConductorInfo & LOCATABLEOBJECT \\
\hline
\end{tabular}


OhConductorInfo

OhConductorlnfo

OhConductorInfo
NIPSCOTARGETCLASS

OHCONDUCTORINFO

PRIMARYCONDUCTORINFO

Field Model Names

\begin{tabular}{|l|l|}
\hline Field & Model Name \\
\hline INSTALLDATE & INSTALLDATE \\
\hline INSTALLWORKORDERNUMBER & INSTALLWORKORDERNUMBER \\
\hline INSTALLMISCORDERID & INSTALLMISCORDERID \\
\hline RETIREDATE & RETIREDATE \\
\hline RETIREWORKORDERNUMBER & RETIREWORKORDERNUMBER \\
\hline RETIREMISCORDERID & RETIREMISCORDERID \\
\hline SUBTYPECD & CADOPS \\
\hline SUBTYPECD & FEEDERALL \\
\hline SUBTYPECD & SUBTYPECD \\
\hline FROMDISTRIBREFNUMBER & FROMDISTRIBREF \\
\hline TODISTRIBREFNUMBER & TODISTRIBREF \\
\hline WIRESIZECD & CADOPS \\
\hline WIRESIZECD & FEEDERALL \\
\hline WIRESIZECD & WIRESIZECD \\
\hline WIREMATERIALCD & CADOPS \\
\hline WIREMATERIALCD & FEEDERALL \\
\hline WIREMATERIALCD & WIREMATERIALCD \\
\hline WIRETYPECD & WIRETYPECD \\
\hline WIRETYPECD & CADOPS \\
\hline WIRETYPECD & FEEDERALL \\
\hline CIRCUITNUMBER & CIRCUITNUMBER \\
\hline CIRCUITNUMBER & FEEDERID \\
\hline MEASUREDLENGTH & MEASUREDLENGTH \\
\hline MEASUREDLENGTH & SPANLENGTH \\
\hline
\end{tabular}

OpenPoint

Object Class Model Names

\begin{tabular}{|l|l|}
\hline Object Class & Model Name \\
\hline OpenPoint & SWITCH \\
\hline OpenPoint & CADOPS \\
\hline OpenPoint & FEEDERALL \\
\hline OpenPoint & LOCATABLEOBJECT \\
\hline
\end{tabular}

Field Model Names

\begin{tabular}{|l|l|}
\hline Field & Model Name \\
\hline OBJECTID & LOCATABLEFIELD \\
\hline MMELECTRICTRACEWEIGHT & MMELECTRICTRACEWEIGHT \\
\hline FDRMGRNONTRACEABLE & FDRMGRNONTRACEABLE \\
\hline FEEDERID & CADOPS \\
\hline FEEDERID & FEEDERALL \\
\hline FEEDERID & FEEDERID \\
\hline FEEDERID2 & FEEDERID2 \\
\hline FEEDERINFO & FEEDERINFO \\
\hline
\end{tabular}




\begin{tabular}{|l|l|} 
PHASEDESIGNATIONCD & CADOPS \\
\hline PHASEDESIGNATIONCD & FEEDERALL \\
\hline PHASEDESIGNATIONCD & PHASEDESIGNATION \\
\hline LOANUMBER & CADOPS \\
\hline LOANUMBER & FEEDERALL \\
\hline LOANUMBER & LOANAME \\
\hline LOANUMBER & LOANUMBER \\
\hline GRIDCD & CADOPS \\
\hline GRIDCD & FEEDERALL \\
\hline NORMALPOSITIONA & CADOPS \\
\hline NORMALPOSITIONA & FEEDERALL \\
\hline NORMALPOSITIONA & NORMALPOSITION_A \\
\hline NORMALPOSITIONB & CADOPS \\
\hline NORMALPOSITIONB & FEEDERALL \\
\hline NORMALPOSITIONB & NORMALPOSITION_B \\
\hline NORMALPOSITIONC & CADOPS \\
\hline NORMALPOSITIONC & FEEDERALL \\
\hline NORMALPOSITIONC & NORMALPOSITION_C \\
\hline
\end{tabular}

PadMount

Object Class Model Names

\begin{tabular}{|l|l|}
\hline Object Class & Model Name \\
\hline PadMount & EDFS ASSETSTRUCTURE \\
\hline PadMount & EDFS RETIREMENT INFO \\
\hline PadMount & EDFS WORKORDER 2 \\
\hline PadMount & FEEDERALL \\
\hline PadMount & MMREMOVABLE \\
\hline PadMount & NABACKWARD \\
\hline PadMount & PADMOUNT \\
\hline PadMount & STRUCTURE \\
\hline PadMount & UNIQUEDISTRIBREFNUMBERCLASS \\
\hline PadMount & CADOPS \\
\hline
\end{tabular}

Field Model Names

\begin{tabular}{|l|l|}
\hline Field & Model Name \\
\hline CREATIONDATE & AT_CREATIONDATE \\
\hline CREATIONUSERID & AT_CREATIONUSER \\
\hline UPDATEDATE & AT_1 \\
\hline UPDATEUSERID & AT_2 \\
\hline LOANUMBER & AT_3 \\
\hline LOANUMBER & LOANAME \\
\hline LOANUMBER & LOANUMBER \\
\hline GRIDCD & AT_5 \\
\hline GRIDCD & GRIDCD \\
\hline INSTALLDATE & AT_6 \\
\hline INSTALLDATE & INSTALLDATE \\
\hline INSTALLWORKORDERNUMBER & AT_7 \\
\hline INSTALLWORKORDERNUMBER & INSTALLWORKORDERNUMBER \\
\hline INSTALLMISCORDERID & AT_12 \\
\hline
\end{tabular}




\begin{tabular}{|l|l|} 
INSTALLMISCORDERID & INSTALLMISCORDERID \\
\hline RETIREDATE & AT_8 \\
\hline RETIREDATE & RETIREDATE \\
\hline RETIREWORKORDERNUMBER & AT_9 \\
\hline RETIREWORKORDERNUMBER & RETIREWORKORDERNUMBER \\
\hline RETIREMISCORDERID & AT_13 \\
\hline RETIREMISCORDERID & RETIREMISCORDERID \\
\hline TAXUNITCD & AT_4 \\
\hline TAXUNITCD & TAXUNITCD \\
\hline LEGACYWRINSTALLTIMESTAMP & AT_10 \\
\hline LEGACYWRREMOVALTIMESTAMP & AT_11 \\
\hline SUBTYPECD & AT_SUBTYPECD \\
\hline SUBTYPECD & SUBTYPECD \\
\hline TYPECD & AT_14 \\
\hline OWNERIND & AT_15 \\
\hline OWNERIND & OWNER \\
\hline LEGACYDISTRIBREFNUMTERMID & AT_17 \\
\hline INSTALLTIMESTAMP & AT_18 \\
\hline INSTALLTIMESTAMP & INSTALLTIMESTAMP \\
\hline REMOVALTIMESTAMP & AT_19 \\
\hline PRINTID & AT_20 \\
\hline DISTRIBREFNUMBER & AT_21 \\
\hline DISTRIBREFNUMBER & CADOPS \\
\hline DISTRIBREFNUMBER & DISTRIBREFNUMBER \\
\hline DISTRIBREFNUMBER & FEEDERALL \\
\hline DISTRIBREFNUMBER & FROMDISTRIBREF \\
\hline DISTRIBREFNUMBER & LOADDISTRIBREFNUMBER \\
\hline DISTRIBREFNUMBER & TAKEOFFREFNUMBER \\
\hline DISTRIBREFNUMBER & TODISTRIBREF \\
\hline LOCATIONDESCRIPTION & AT_22 \\
\hline SERVICEPOINTTYPECD & AT_23 \\
\hline & \\
\hline
\end{tabular}

\section{Pedestal}

Object Class Model Names

\begin{tabular}{|l|}
\hline Object Class \\
\hline Pedestal \\
\hline Pedestal \\
\hline Pedestal \\
\hline Pedestal \\
\hline Pedestal \\
\hline Pedestal \\
\hline
\end{tabular}

\begin{tabular}{|l|}
\hline Model Name \\
\hline COMPANYSTREETLIGHT \\
\hline EDFS WORKORDER 2 \\
\hline LOCATABLEOBJECT \\
\hline PEDESTAL \\
\hline STRUCTURE \\
\hline UNIQUEDISTRIBREFNUMBERCLASS \\
\hline
\end{tabular}

Field Model Names

\begin{tabular}{|l|l|}
\hline Field & Model Name \\
\hline LOANUMBER & LOANAME \\
\hline LOANUMBER & LOANUMBER \\
\hline INSTALLWORKORDERNUMBER & INSTALLWORKORDERNUMBER \\
\hline INSTALLMISCORDERID & INSTALLMISCORDERID \\
\hline RETIREDATE & RETIREDATE \\
\hline
\end{tabular}


RETIREWORKORDERNUMBER

RETIREMISCORDERID

SYMBOLROTATIONVALUE

LONGPEDESTALNUMBER

LONGPEDESTALNUMBER

LONGPEDESTALNUMBER

LONGPEDESTALNUMBER

LONGPEDESTALNUMBER
RETIREWORKORDERNUMBER

RETIREMISCORDERID

SYMBOLROTATION

DISTRIBREFNUMBER

FROMDISTRIBREF

LOADDISTRIBREFNUMBER

TAKEOFFREFNUMBER

TODISTRIBREF

\section{PowerTransformer}

Object Class Model Names

\begin{tabular}{|l|l|}
\hline Object Class & Model Name \\
\hline PowerTransformer & FEEDERALL \\
\hline PowerTransformer & NAGETSHAPE \\
\hline
\end{tabular}

Field Model Names

\begin{tabular}{|l|l}
\hline Field & Model Name \\
\hline
\end{tabular}

PrimaryMeter

Object Class Model Names

\section{Object Class}

PrimaryMeter

PrimaryMeter

PrimaryMeter

PrimaryMeter

PrimaryMeter

\begin{tabular}{|l|}
\hline Model Name \\
\hline CADOPS \\
\hline EDFS WORKORDER \\
\hline FEEDERALL \\
\hline LOCATABLEOBJECT \\
\hline NABACKWARD \\
\hline
\end{tabular}

Field Model Names

\begin{tabular}{|l|l|}
\hline Field & Model Name \\
\hline MMELECTRICTRACEWEIGHT & MMELECTRICTRACEWEIGHT \\
\hline FDRMGRNONTRACEABLE & FDRMGRNONTRACEABLE \\
\hline FEEDERID & CADOPS \\
\hline FEEDERID & FEEDERALL \\
\hline FEEDERID & FEEDERID \\
\hline FEEDERID2 & FEEDERID2 \\
\hline FEEDERINFO & FEEDERINFO \\
\hline PHASEDESIGNATIONCD & CADOPS \\
\hline PHASEDESIGNATIONCD & FEEDERALL \\
\hline PHASEDESIGNATIONCD & PHASEDESIGNATION \\
\hline SYMBOLROTATIONVALUE & SYMBOLROTATION \\
\hline LOANUMBER & CADOPS \\
\hline LOANUMBER & FEEDERALL \\
\hline LOANUMBER & LOANAME \\
\hline GRIDCD & CADOPS \\
\hline GRIDCD & FEEDERALL \\
\hline INSTALLWORKORDERNUMBER & WORKORDERNUMBER \\
\hline SUBTYPECD & CADOPS \\
\hline SUBTYPECD & FEEDERALL \\
\hline LEGACYDISTRIBREFNUMBER & LOCATABLEFIELD \\
\hline
\end{tabular}


RecloserBank

Object Class Model Names

\begin{tabular}{|l|l|}
\hline Object Class & Model Name \\
\hline RecloserBank & CADOPS \\
\hline RecloserBank & DYNAMICPROTECTIVEDEVICE \\
\hline RecloserBank & EDFS STRUCTURE PREVENT DELETION \\
\hline RecloserBank & FDRMGRPROTECTIVE \\
\hline RecloserBank & FEEDERALL \\
\hline RecloserBank & LOCATABLEOBJECT \\
\hline RecloserBank & PROTECTIVE \\
\hline RecloserBank & RECLOSERBANK \\
\hline
\end{tabular}

Field Model Names

\begin{tabular}{|l|l|}
\hline Field & Model Name \\
\hline MMELECTRICTRACEWEIGHT & MMELECTRICTRACEWEIGHT \\
\hline FDRMGRNONTRACEABLE & FDRMGRNONTRACEABLE \\
\hline FEEDERID & CADOPS \\
\hline FEEDERID & FEEDERALL \\
\hline FEEDERID & FEEDERID \\
\hline FEEDERID2 & FEEDERID2 \\
\hline FEEDERINFO & FEEDERINFO \\
\hline PHASEDESIGNATIONCD & CADOPS \\
\hline PHASEDESIGNATIONCD & FEEDERALL \\
\hline PHASEDESIGNATIONCD & PHASEDESIGNATION \\
\hline SYMBOLROTATIONVALUE & SYMBOLROTATION \\
\hline LOANUMBER & CADOPS \\
\hline LOANUMBER & FEEDERALL \\
\hline LOANUMBER & LOANAME \\
\hline GRIDCD & FEEDERALL \\
\hline GRIDCD & CADOPS \\
\hline LEGACYDISTRIBREFNUMBER & LOCATABLEFIELD \\
\hline RECLOSERTYPECD & CADOPS \\
\hline RECLOSERTYPECD & FEEDERALL \\
\hline RECLOSERSIZEVALUE & CADOPS \\
\hline RECLOSERSIZEVALUE & FEEDERALL \\
\hline NORMALPOSITIONA & CADOPS \\
\hline NORMALPOSITIONA & FEEDERALL \\
\hline NORMALPOSITIONA & NORMALPOSITION_A \\
\hline NORMALPOSITIONB & CADOPS \\
\hline NORMALPOSITIONB & FEEDERALL_ \\
\hline NORMALPOSITIONB & NORMALPOSITION_B \\
\hline NORMALPOSITIONC & CADOPS \\
\hline NORMALPOSITIONC & FEEDERALL \\
\hline NORMALPOSITIONC & NORMALPOSITION_C \\
\hline &
\end{tabular}

RecloserUnit

Object Class Model Names 


\begin{tabular}{|l|} 
Object Class \\
\hline RecloserUnit \\
\hline RecloserUnit \\
\hline RecloserUnit \\
\hline RecloserUnit \\
\hline RecloserUnit \\
\hline RecloserUnit \\
\hline
\end{tabular}

Model Name

CADOPS

EDFS RETIREMENT INFO

EDFS WORKORDER 2

FEEDERALL

LOCATABLEOBJECT

RECLOSERUNIT

Field Model Names

\begin{tabular}{|l|l}
\hline Field & \\
\hline INSTALLWORKORDERNUMBER & \\
\hline INSTALLMISCORDERID & \\
\hline RETIREDATE & RE \\
\hline RETIREWORKORDERNUMBER & RE \\
\hline RETIREMISCORDERID & \\
\hline PHASEDESIGNATION & \\
\hline DISTRIBREFNUMBER & \\
\hline CIRCUITNUMBER & \\
\hline CIRCUITNUMBER & \\
\hline SUBTYPECD & \\
\hline
\end{tabular}

Model Name

INSTALLWORKORDERNUMBER

INSTALLMISCORDERID

RETIREDATE

RETIREWORKORDERNUMBER

RETIREMISCORDERID

PHASEDESIGNATION

DISTRIBREFNUMBER

CIRCUITNUMBER

FEEDERID

SUBTYPECD

RegulatorFunctionTest

Object Class Model Names

\begin{tabular}{|l|l|}
\hline Object Class & Model Name \\
\hline RegulatorFunctionTest & LOCATABLEOBJECT \\
\hline
\end{tabular}

Field Model Names

\section{Field}

RegulatorUnit

Object Class Model Names

\begin{tabular}{|l|l|}
\hline Object Class & Model Name \\
\hline RegulatorUnit & CADOPS \\
\hline RegulatorUnit & EDFS ASSETUNIT \\
\hline RegulatorUnit & FEEDERALL \\
\hline RegulatorUnit & LOCATABLEOBJECT \\
\hline
\end{tabular}

Field Model Names

\section{Field}

COMPANYNUMBER

STATUSTIMESTAMP

DISPOSITIONCD

STATUSCD

RELSTORESITEMNUMBER

\section{Model Name}

\begin{tabular}{|l|}
\hline Model Name \\
\hline COMPANYNUMBER \\
\hline STATUSTIMESTAMP \\
\hline DISPOSITIONCD \\
\hline STATUSCD \\
\hline RELSTORESITEMNUMBER \\
\hline
\end{tabular}

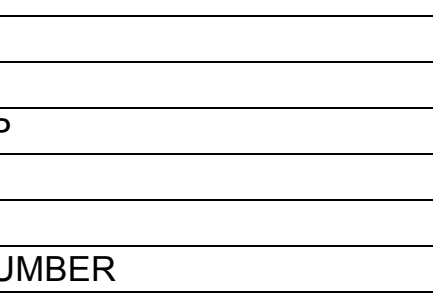

RegulatorUnitInstall

Object Class Model Names

Object Class

RegulatorUnitInstall 
RegulatorUnitInstall

RegulatorUnitInstall

RegulatorUnitInstall

RegulatorUnitInstall
EDFS ASSETINSTALL

FEEDERALL

LOCATABLEOBJECT

REGULATORUNITINSTALL

Field Model Names

\begin{tabular}{|l|l|}
\hline Field & Model Name \\
\hline LOANUMBER & LOANAME \\
\hline LOANUMBER & LOANUMBER \\
\hline TAXUNITCD & TAXUNITCD \\
\hline GRIDCD & GRIDCD \\
\hline INSTALLTIMESTAMP & INSTALLTIMESTAMP \\
\hline INSTALLDATE & INSTALLDATE \\
\hline CIRCUITNUMBER & CIRCUITNUMBER \\
\hline PHASEDESIGNATION & PHASEDESIGNATION \\
\hline INSTALLATIONSTATUSCD & INSTALLATIONSTATUSCD \\
\hline MATERIALTICKETNUMBER & MATERIALTICKETNUMBER \\
\hline DISTRIBREFNUMBER & DISTRIBREFNUMBER \\
\hline RELREGULATORUNITCOMPANYNUMBER & COMPANYNUMBER \\
\hline RELVOLTAGEREGULATOROBJECTID & RELATEDBANKOID \\
\hline
\end{tabular}

RegulatorUnitRemove

Object Class Model Names

\begin{tabular}{|l|l|}
\hline Object Class & Model Name \\
\hline RegulatorUnitRemove & EDFS ASSETREMOVE \\
\hline RegulatorUnitRemove & REGULATORUNITREMOVE \\
\hline
\end{tabular}

Field Model Names

\begin{tabular}{|l|l|}
\hline Field & Model Name \\
\hline REMOVALTIMESTAMP & REMOVALTIMESTAMP \\
\hline REMOVALDATE & REMOVALDATE \\
\hline REMOVALCD & REMOVALCD \\
\hline MATERIALTICKETNUMBER & MATERIALTICKETNUMBER \\
\hline RELREGULATORUNITCOMPANYNUMBER & COMPANYNUMBER \\
\hline
\end{tabular}

RegulatorUnitStock

Object Class Model Names

\section{Object Class}

RegulatorUnitStock

Model Name
EDFS ASSETSTOCK

Field Model Names

\begin{tabular}{|l|l|}
\hline Field & Model Name \\
\hline STOCKTIMESTAMP & STOCKTIMESTAMP \\
\hline STOCKTIMESTAMP & STATUSTIMESTAMP \\
\hline STOCKDATE & STOCKDATE \\
\hline RELREGULATORUNITCOMPANYNUMBER & COMPANYNUMBER \\
\hline RELSTOREROOMNUMBER & STOREROOMNUMBER \\
\hline
\end{tabular}

RetiredPadMount 
Object Class Model Names

\begin{tabular}{|l|l|}
\hline Object Class & Model Name \\
\hline RetiredPadMount & EDFS RETIRED ASSETSTRUCTURE \\
\hline RetiredPadMount & EDFS WORKORDER 2 \\
\hline RetiredPadMount & MMREMOVED \\
\hline
\end{tabular}

Field Model Names

\begin{tabular}{|l|l|}
\hline Field & Model Name \\
\hline CREATIONDATE & AT_CREATIONDATE \\
\hline CREATIONUSERID & AT_CREATIONUSER \\
\hline UPDATEDATE & AT_1 \\
\hline UPDATEUSERID & AT_2 \\
\hline LOANUMBER & AT_3 \\
\hline TAXUNITCD & AT_4 \\
\hline GRIDCD & AT_5 \\
\hline INSTALLDATE & AT_6 \\
\hline INSTALLDATE & INSTALLDATE \\
\hline INSTALLWORKORDERNUMBER & AT_7 \\
\hline INSTALLWORKORDERNUMBER & INSTALLWORKORDERNUMBER \\
\hline INSTALLMISCORDERID & AT_12 \\
\hline INSTALLMISCORDERID & INSTALLMISCORDERID \\
\hline RETIREDATE & AT_8 \\
\hline RETIREDATE & RETIREDATE \\
\hline RETIREWORKORDERNUMBER & AT_9 \\
\hline RETIREWORKORDERNUMBER & RETIREWORKORDERNUMBER \\
\hline RETIREMISCORDERID & AT_13 \\
\hline RETIREMISCORDERID & RETIREMISCORDERID \\
\hline LEGACYWRINSTALLTIMESTAMP & AT_10 \\
\hline LEGACYWRREMOVALTIMESTAMP & AT_11 \\
\hline SUBTYPECD & AT_SUBTYPECD \\
\hline TYPECD & AT_14 \\
\hline OWNERIND & AT_15 \\
\hline LEGACYDISTRIBREFNUMTERMID & AT_17 \\
\hline INSTALLTIMESTAMP & AT_18 \\
\hline LEGACYREMOVALTIMESTAMP & AT_19 \\
\hline PRINTID & AT_20 \\
\hline DISTRIBREFNUMBER & AT_21 \\
\hline LOCATIONDESCRIPTION & AT_22 \\
\hline SERVICEPOINTTYPECD & AT_23 \\
\hline
\end{tabular}

RetiredSupportStructure

Object Class Model Names

\section{Object Class}

RetiredSupportStructure

RetiredSupportStructure

RetiredSupportStructure

RetiredSupportStructure

RetiredSupportStructure

RetiredSupportStructure

\begin{tabular}{|l|}
\hline Model Name \\
\hline EDFS RETIRED ASSETSTRUCTURE \\
\hline EDFS WORKORDER 2 \\
\hline LOCATABLEOBJECT \\
\hline MMREMOVED \\
\hline RETIREDSUPPORTSTRUCTURE \\
\hline UNIQUEDISTRIBREFNUMBERCLASS \\
\hline
\end{tabular}


Field Model Names

\begin{tabular}{|l|l|}
\hline Field & Model Name \\
\hline CREATIONDATE & AT_CREATIONDATE \\
\hline CREATIONUSERID & AT_CREATIONUSER \\
\hline UPDATEDATE & AT_1 \\
\hline UPDATEUSERID & AT_2 \\
\hline LOANUMBER & AT_3 \\
\hline TAXUNITCD & AT_4 \\
\hline GRIDCD & AT_5 \\
\hline INSTALLDATE & AT_6 \\
\hline INSTALLDATE & INSTALLDATE \\
\hline INSTALLWORKORDERNUMBER & AT_7 \\
\hline INSTALLWORKORDERNUMBER & INSTALLWORKORDERNUMBER \\
\hline INSTALLMISCORDERID & AT_12 \\
\hline INSTALLMISCORDERID & INSTALLMISCORDERID \\
\hline RETIREDATE & AT_8 \\
\hline RETIREDATE & RETIREDATE \\
\hline RETIREWORKORDERNUMBER & AT_9 \\
\hline RETIREWORKORDERNUMBER & RETIREWORKORDERNUMBER \\
\hline RETIREMISCORDERID & AT_13 \\
\hline RETIREMISCORDERID & RETIREMISCORDERID \\
\hline LEGACYWRINSTALLTIMESTAMP & AT_10 \\
\hline LEGACYWRREMOVALTIMESTAMP & AT_11 \\
\hline SUBTYPECD & AT_SUBTYPECD \\
\hline DISTRIBREFNUMBER & AT_14 \\
\hline DISTRIBREFNUMBER & DISTRIBREFNUMBER \\
\hline LOCATIONDESCRIPTION & AT_17 \\
\hline POLEHEIGHTVALUE & AT_18 \\
\hline POLEMATERIALCD & AT__19 \\
\hline PRIMARYDISTRIBREFNUMBER & AT__20 \\
\hline SERVICEPOINTTYPECD & AT_28 \\
\hline TRANSMISSIONSTRUCTURENUMBER & AT_29 \\
\hline INSTALLTIMESTAMP & AT_30 \\
\hline REMOVALTIMESTAMP & AT_31 \\
\hline TELEPHONECOMPANYCD & TELEPHONECOMPANYCD \\
\hline FIRSTCABLECOMPANYCD & FIRSTCABLECOMPANYCD \\
\hline SECONDCABLECOMPANYCD & SECONDCABLECOMPANYCD \\
\hline UTILITYCOMPANYCD & UTILITYCOMPANYCD \\
\hline FIBERCOMPANYCD & FIBERCOMPANYCD \\
\hline OTHERCOMPANYCD & OTHERCOMPANYCD \\
\hline FOREIGNOWNERNAME & AT_33 \\
\hline & \\
\hline
\end{tabular}

RetiredSwitchGear

Object Class Model Names

\begin{tabular}{|l|l|}
\hline Object Class & Model Name \\
\hline RetiredSwitchGear & EDFS WORKORDER 2 \\
\hline RetiredSwitchGear & MMREMOVED \\
\hline
\end{tabular}


Field Model Names

\begin{tabular}{|l|l|}
\hline Field & Model Name \\
\hline CREATIONDATE & AT_CREATIONUSER \\
\hline CREATIONUSERID & AT_CREATIONUSER \\
\hline UPDATEDATE & AT_1 \\
\hline UPDATEUSERID & AT_2 \\
\hline LOANUMBER & AT_3 \\
\hline TAXUNITCD & AT_4 \\
\hline GRIDCD & AT_5 \\
\hline INSTALLDATE & AT_6 \\
\hline INSTALLWORKORDERNUMBER & AT_7 \\
\hline INSTALLWORKORDERNUMBER & INSTALLWORKORDERNUMBER \\
\hline INSTALLMISCORDERID & AT_12 \\
\hline INSTALLMISCORDERID & INSTALLMISCORDERID \\
\hline RETIREDATE & AT_8 \\
\hline RETIREWORKORDERNUMBER & AT_99 \\
\hline RETIREWORKORDERNUMBER & RETIREWORKORDERNUMBER \\
\hline RETIREMISCORDERID & AT_13 \\
\hline RETIREMISCORDERID & RETIREMISCORDERID \\
\hline LEGACYWRINSTALLTIMESTAMP & AT_10 \\
\hline LEGACYWRREMOVALTIMESTAMP & AT_11 \\
\hline SUBTYPECD & AT_SUBTYPECD \\
\hline SWITCHGEARKV & AT_14 \\
\hline OPERATIONTYPECD & AT_15 \\
\hline FRONTKINDCD & AT_16 \\
\hline USEDCOMPARTMENTCOUNT & AT_17 \\
\hline DISTRIBREFNUMBER & AT_18 \\
\hline ASSEMBLYNUMBER & AT_19 \\
\hline ASSOCIATIONTIMESTAMP & AT_20 \\
\hline
\end{tabular}

RetiredWoodPoleEvents

Object Class Model Names

Object Class

Model Name

RetiredWoodPoleEvents

RETIREDWOODPOLEEVENTS

Field Model Names

\begin{tabular}{|l|l|}
\hline Field & Model Name \\
\hline WOODPOLEINSPECTIONYEAR & INSPECTIONYEAR \\
\hline WOODPOLEINSPECTIONPASSFAILCD & INSPECTIONPASSFAILCD \\
\hline WOODPOLEREINFORCEDDATE & REINFORCEDDATE \\
\hline WOODPOLEGNDTREATDATE & GROUNDTREATMENTDATE \\
\hline RELDISTRIBREFNUMBER & DISTRIBREFNUMBER \\
\hline EVENTTYPECD & EVENTTYPECD \\
\hline
\end{tabular}

RetiredWoodPolelnfo

Object Class Model Names

\begin{tabular}{|l|l}
\hline Object Class & Model Name \\
\hline RetiredWoodPolelnfo & RETIREDWOODPOLEINFO
\end{tabular}


Field Model Names

\begin{tabular}{|l|l|}
\hline Field & Model Name \\
\hline WOODPOLECLASSCD & CLASSCD \\
\hline WOODPOLETYPECD & TYPECD \\
\hline WOODSETTINGCD & SETTINGCD \\
\hline RELDISTRIBREFNUMBER & DISTRIBREFNUMBER \\
\hline WOODPOLEORIGINALTREATMNTTYPECD & ORIGINALTREATMENTTYPECD \\
\hline
\end{tabular}

Secondary

Object Class Model Names

\begin{tabular}{|l|l|}
\hline Object Class & Model Name \\
\hline Secondary & LINE \\
\hline Secondary & LOCATABLEOBJECT \\
\hline Secondary & MMABANDONABLE \\
\hline Secondary & SECONDARY \\
\hline
\end{tabular}

Field Model Names

\begin{tabular}{|l|l|}
\hline Field & Model Name \\
\hline OBJECTID & LOCATABLEFIELD \\
\hline CREATIONDATE & AT_CREATIONDATE \\
\hline CREATIONUSERID & AT_CREATIONUSER \\
\hline UPDATEDATE & AT_UPDATEDATE \\
\hline UPDATEUSERID & AT_UPDATEUSERID \\
\hline LOANUMBER & AT_1 \\
\hline LOANUMBER & LOANAME \\
\hline GRIDCD & AT_2 \\
\hline SHAPE & AT_SHAPE \\
\hline
\end{tabular}

\section{SECONDARYCONDUCTORINFO}

Object Class Model Names

\begin{tabular}{|l|l|}
\hline Object Class & Model Name \\
\hline SECONDARYCONDUCTORINFO & CONDUCTORINFO \\
\hline SECONDARYCONDUCTORINFO & EDFS CONDUCTORINFO \\
\hline SECONDARYCONDUCTORINFO & EDFS RETIREMENT INFO \\
\hline SECONDARYCONDUCTORINFO & EDFS WORKORDER 2 \\
\hline SECONDARYCONDUCTORINFO & LOCATABLEOBJECT \\
\hline SECONDARYCONDUCTORINFO & SECONDARYCONDUCTORINFO \\
\hline
\end{tabular}

Field Model Names

\begin{tabular}{|l|l|}
\hline Field & Model Name \\
\hline INSTALLWORKORDERNUMBER & INSTALLWORKORDERNUMBER \\
\hline INSTALLMISCORDERID & INSTALLMISCORDERID \\
\hline RETIREWORKORDERNUMBER & RETIREWORKORDERNUMBER \\
\hline RETIREDATE & RETIREDATE \\
\hline RETIREMISCORDERID & RETIREMISCORDERID \\
\hline SUBTYPECD & SUBTYPECD \\
\hline WIRESIZECD & WIRESIZE \\
\hline WIRESIZECD & WIRESIZECD \\
\hline WIREMATERIALCD & WIREMATERIALCD \\
\hline
\end{tabular}


WIRETYPECD

FROMDISTRIBREFNUMBER

TODISTRIBREFNUMBER

SectionalizerBank

Object Class Model Names

\section{Object Class}

SectionalizerBank

SectionalizerBank

SectionalizerBank

SectionalizerBank

SectionalizerBank

SectionalizerBank

SectionalizerBank
WIRETYPECD

FROMDISTRIBREF

TODISTRIBREF

Field Model Names

\begin{tabular}{|l|l|}
\hline Field & Model Name \\
\hline MMELECTRICTRACEWEIGHT & MMELECTRICTRACEWEIGHT \\
\hline FDRMGRNONTRACEABLE & FDRMGRNONTRACEABLE \\
\hline FEEDERID & CADOPS \\
\hline FEEDERID & FEEDERALL \\
\hline FEEDERID & FEEDERID \\
\hline FEEDERID2 & FEEDERID2 \\
\hline FEEDERINFO & FEEDERINFO \\
\hline PHASEDESIGNATIONCD & CADOPS \\
\hline PHASEDESIGNATIONCD & FEEDERALL \\
\hline PHASEDESIGNATIONCD & PHASEDESIGNATION \\
\hline SYMBOLROTATIONVALUE & SYMBOLROTATION \\
\hline LOANUMBER & CADOPS \\
\hline LOANUMBER & FEEDERALL \\
\hline LOANUMBER & LOANAME \\
\hline GRIDCD & CADOPS \\
\hline GRIDCD & FEEDERALL \\
\hline LEGACYDISTRIBREFNUMBER & LOCATABLEFIELD \\
\hline NORMALPOSITIONA & CADOPS \\
\hline NORMALPOSITIONA & FEEDERALL \\
\hline NORMALPOSITIONA & NORMALPOSITION_A \\
\hline NORMALPOSITIONB & CADOPS \\
\hline NORMALPOSITIONB & FEEDERALL \\
\hline NORMALPOSITIONB & NORMALPOSITION_B \\
\hline NORMALPOSITIONC & CADOPS \\
\hline NORMALPOSITIONC & FEEDERALL \\
\hline NORMALPOSITIONC & NORMALPOSITION_C \\
\hline SECTIONFUSENUMBER & CADOPS \\
\hline SECTIONFUSENUMBER & FEEDERALL \\
\hline SYMBOLCONFIGURATIONCD & SYMBOLOGYCONFIGURATIONCD \\
\hline SECTIONALIZERAMPRATING & CADOPS \\
\hline SECTIONALIZERAMPRATING & FEEDERALL \\
\hline
\end{tabular}


SectionalizerUnit

Object Class Model Names

\begin{tabular}{|l|}
\hline Object Class \\
\hline SectionalizerUnit \\
\hline SectionalizerUnit \\
\hline SectionalizerUnit \\
\hline SectionalizerUnit \\
\hline SectionalizerUnit \\
\hline SectionalizerUnit \\
\hline
\end{tabular}

\begin{tabular}{|l|}
\hline Model Name \\
\hline CADOPS \\
\hline EDFS RETIREMENT INFO \\
\hline EDFS WORKORDER 2 \\
\hline FEEDERALL \\
\hline LOCATABLEOBJECT \\
\hline SECTIONALIZERUNIT \\
\hline
\end{tabular}

Field Model Names

\begin{tabular}{|l|l|}
\hline Field & Model Name \\
\hline OBJECTID & CADOPS \\
\hline OBJECTID & FEEDERALL \\
\hline INSTALLWORKORDERNUMBER & INSTALLWORKORDERNUMBER \\
\hline INSTALLMISCORDERID & INSTALLMISCORDERID \\
\hline RETIREDATE & RETIREDATE \\
\hline RETIREWORKORDERNUMBER & RETIREWORKORDERNUMBER \\
\hline RETIREMISCORDERID & RETIREMISCORDERID \\
\hline PHASEDESIGNATION & CADOPS \\
\hline PHASEDESIGNATION & FEEDERALL \\
\hline PHASEDESIGNATION & PHASEDESIGNATION \\
\hline DISTRIBREFNUMBER & DISTRIBREFNUMBER \\
\hline CIRCUITNUMBER & FEEDERID \\
\hline SUBTYPECD & SUBTYPECD \\
\hline
\end{tabular}

ServiceConductorlnfo

Object Class Model Names

\begin{tabular}{|l|}
\hline Object Class \\
\hline ServiceConductorlnfo \\
\hline
\end{tabular}

ServiceConductorlnfo

ServiceConductorlnfo

ServiceConductorlnfo

ServiceConductorInfo

ServiceConductorInfo

ServiceConductorlnfo

ServiceConductorlnfo

\begin{tabular}{|l|}
\hline Model Name \\
\hline SERVICECONDUCTORINFO \\
CONDUCTORINFO \\
\hline EDFS CONDUCTORINFO \\
\hline EDFS PADMOUNT PREVENT DELETION \\
\hline EDFS PEDESTAL PREVENT DELETION \\
\hline EDFS RETIREMENT INFO \\
\hline EDFS STRUCTURE PREVENT DELETION \\
\hline EDFS WORKORDER 2 \\
\hline
\end{tabular}

Field Model Names

\begin{tabular}{|l|l|}
\hline Field & Model Name \\
\hline INSTALLWORKORDERNUMBER & INSTALLWORKORDERNUMBER \\
\hline INSTALLMISCORDERID & INSTALLMISCORDERID \\
\hline RETIREDATE & RETIREDATE \\
\hline RETIREWORKORDERNUMBER & RETIREWORKORDERNUMBER \\
\hline RETIREMISCORDERID & RETIREMISCORDERID \\
\hline SUBTYPECD & SUBTYPECD \\
\hline WIRESIZECD & WIRESIZE \\
\hline WIRESIZECD & WIRESIZECD \\
\hline WIREMATERIALCD & WIREMATERIALCD \\
\hline
\end{tabular}


WIRETYPECD

LOADDISTRIBREFNUMBER

TAKEOFFREFNUMBER

Splice

Object Class Model Names

Object Class

Splice

Field Model Names

\section{Field}

OBJECTID

MMELECTRICTRACEWEIGHT

FDRMGRNONTRACEABLE

FEEDERID

FEEDERID2

FEEDERINFO

PHASEDESIGNATIONCD

SYMBOLROTATIONVALUE
WIRETYPECD

LOADDISTRIBREFNUMBER

TAKEOFFREFNUMBER

StreetlightSwitch

Object Class Model Names

\begin{tabular}{|l|l|}
\hline Object Class & Model Name \\
\hline StreetlightSwitch & EDFS RETIREMENT INFO \\
\hline StreetlightSwitch & EDFS STRUCTURE PREVENT DELETION \\
\hline StreetlightSwitch & EDFS WORKORDER 2 \\
\hline StreetlightSwitch & LOCATABLEOBJECT \\
\hline StreetlightSwitch & STREETLIGHTSWITCH \\
\hline
\end{tabular}

Field Model Names

\begin{tabular}{|l|l|}
\hline Field & Model Name \\
\hline INSTALLWORKORDERNUMBER & INSTALLWORKORDERNUMBER \\
\hline INSTALLMISCORDERID & INSTALLMISCORDERID \\
\hline RETIREDATE & RETIREDATE \\
\hline RETIREWORKORDERNUMBER & RETIREWORKORDERNUMBER \\
\hline RETIREMISCORDERID & RETIREMISCORDERID \\
\hline SUBTYPECD & SUBTYPECD \\
\hline DISTRIBREFNUMBER & DISTRIBREFNUMBER \\
\hline
\end{tabular}

Substation

Object Class Model Names

\begin{tabular}{|l|l|}
\hline Object Class & Model Name \\
\hline Substation & CADOPS \\
\hline Substation & FEEDERALL \\
\hline Substation & LOCATABLEOBJECT \\
\hline Substation & NABACKWARD \\
\hline Substation & STRUCTURE \\
\hline Substation & SUBSTATION \\
\hline
\end{tabular}


Field Model Names

\begin{tabular}{|l|l|}
\hline Field & Model Name \\
\hline SUBSTATIONNAME & CADOPS \\
\hline SUBSTATIONNAME & FEEDERALL \\
\hline SUBSTATIONNAME & LOCATABLEFIELD \\
\hline SUBSTATIONNUMBER & CADOPS \\
\hline SUBSTATIONNUMBER & FEEDERALL \\
\hline SYMBOLROTATIONVALUE & SYMBOLROTATION \\
\hline
\end{tabular}

\section{SubstationBreaker}

Object Class Model Names

\begin{tabular}{|l|l|}
\hline Object Class & Model Name \\
\hline SubstationBreaker & PROTECTIVE \\
\hline SubstationBreaker & CADOPS \\
\hline SubstationBreaker & DYNAMICPROTECTIVEDEVICE \\
\hline SubstationBreaker & FDRMGRPROTECTIVE \\
\hline SubstationBreaker & FEEDERALL \\
\hline SubstationBreaker & LOCATABLEOBJECT \\
\hline
\end{tabular}

Field Model Names

\begin{tabular}{|l|l|}
\hline Field & Model Name \\
\hline MMELECTRICTRACEWEIGHT & MMELECTRICTRACEWEIGHT \\
\hline FDRMGRNONTRACEABLE & FDRMGRNONTRACEABLE \\
\hline FEEDERID & CADOPS \\
\hline FEEDERID & FEEDERALL \\
\hline FEEDERID & FEEDERID \\
\hline FEEDERID & LOCATABLEFIELD \\
\hline FEEDERID2 & FEEDERID2 \\
\hline FEEDERINFO & FEEDERINFO \\
\hline PHASEDESIGNATIONCD & CADOPS \\
\hline PHASEDESIGNATIONCD & FEEDERALL \\
\hline PHASEDESIGNATIONCD & PHASEDESIGNATION \\
\hline SYMBOLROTATIONVALUE & SYMBOLROTATION \\
\hline LOANUMBER & CADOPS \\
\hline LOANUMBER & FEEDERALL \\
\hline LOANUMBER & LOANAME \\
\hline LOANUMBER & LOANUMBER \\
\hline GRIDCD & CADOPS \\
\hline GRIDCD & FEEDERALL \\
\hline SUBTYPECD & CADOPS \\
\hline SUBTYPECD & FEEDERALL \\
\hline NORMALPOSITIONABC & CADOPS \\
\hline NORMALPOSITIONABC & FEEDERALL \\
\hline NORMALPOSITIONABC & NORMALPOSITION_A \\
\hline NORMALPOSITIONABC & NORMALPOSITION_B \\
\hline NORMALPOSITIONABC & NORMALPOSITION_C \\
\hline
\end{tabular}

SupportStructure

Object Class Model Names 
Object Class

SupportStructure

SupportStructure

SupportStructure

SupportStructure

SupportStructure

SupportStructure

SupportStructure

SupportStructure

SupportStructure

SupportStructure

SupportStructure
Model Name

UNIQUEDISTRIBREFNUMBERCLASS

CADOPS

EDFS ASSETSTRUCTURE

EDFS RETIREMENT INFO

EDFS WORKORDER 2

FEEDERALL

LOCATABLEOBJECT

MMREMOVABLE

NABACKWARD

STRUCTURE

SUPPORTSTRUCTURE

Field Model Names

\begin{tabular}{|l|l|}
\hline Field & Model Name \\
\hline CREATIONDATE & AT_CREATIONDATE \\
\hline CREATIONUSERID & AT_CREATIONUSER \\
\hline UPDATEDATE & AT_1 \\
\hline UPDATEUSERID & AT_2 \\
\hline LOANUMBER & AT_3 \\
\hline LOANUMBER & LOANAME \\
\hline LOANUMBER & LOANUMBER \\
\hline GRIDCD & AT_5 \\
\hline GRIDCD & GRIDCD \\
\hline INSTALLDATE & AT_6 \\
\hline INSTALLDATE & INSTALLDATE \\
\hline INSTALLWORKORDERNUMBER & AT_7 \\
\hline INSTALLWORKORDERNUMBER & INSTALLWORKORDERNUMBER \\
\hline INSTALLMISCORDERID & AT_12 \\
\hline INSTALLMISCORDERID & INSTALLMISCORDERID \\
\hline RETIREDATE & AT_8 \\
\hline RETIREDATE & RETIREDATE \\
\hline RETIREWORKORDERNUMBER & AT_9 \\
\hline RETIREWORKORDERNUMBER & RETIREWORKORDERNUMBER \\
\hline RETIREMISCORDERID & AT_13 \\
\hline RETIREMISCORDERID & RETIREMISCORDERID \\
\hline TAXUNITCD & AT_4 \\
\hline TAXUNITCD & TAXUNITCD \\
\hline LEGACYWRINSTALLTIMESTAMP & AT_10 \\
\hline LEGACYWRREMOVALTIMESTAMP & AT_11 \\
\hline SYMBOLROTATIONVALUE & SYMBOLROTATION \\
\hline SUBTYPECD & AT_SUBTYPECD \\
\hline LOCATIONDESCRIPTION & AT_17 \\
\hline POLEHEIGHTVALUE & AT_18 \\
\hline POLEMATERIALCD & AT_19 \\
\hline PRIMARYDISTRIBREFNUMBER & AT_20 \\
\hline PRIMARYDISTRIBREFNUMBER & CADOPS \\
\hline PRIMARYDISTRIBREFNUMBER & FEEDERALL \\
\hline
\end{tabular}




\begin{tabular}{|l|l|} 
FOREIGNOWNERNAME & AT_33 \\
\hline SERVICEPOINTTYPECD & AT_28 \\
\hline TRANSMISSIONSTRUCTURENUMBER & AT_29 \\
\hline INSTALLTIMESTAMP & AT_30 \\
\hline INSTALLTIMESTAMP & INSTALLTIMESTAMP \\
\hline REMOVALTIMESTAMP & AT_31 \\
\hline DISTRIBREFNUMBER & AT_14 \\
\hline DISTRIBREFNUMBER & CADOPS \\
\hline DISTRIBREFNUMBER & DISTRIBREFNUMBER \\
\hline DISTRIBREFNUMBER & FEEDERALL \\
\hline DISTRIBREFNUMBER & FROMDISTRIBREF \\
\hline DISTRIBREFNUMBER & LOADDISTRIBREFNUMBER \\
\hline DISTRIBREFNUMBER & LOCATABLEFIELD \\
\hline DISTRIBREFNUMBER & TAKEOFFREFNUMBER \\
\hline DISTRIBREFNUMBER & TODISTRIBREF \\
\hline
\end{tabular}

\section{Switch}

Object Class Model Names

\begin{tabular}{|l|l|}
\hline Object Class & Model Name \\
\hline Switch & CADOPS \\
\hline Switch & EDFS STRUCTURE PREVENT DELETION \\
\hline Switch & FEEDERALL \\
\hline Switch & LOCATABLEOBJECT \\
\hline Switch & SWITCH \\
\hline
\end{tabular}

Field Model Names

\begin{tabular}{|l|l|}
\hline Field & Model Name \\
\hline MMELECTRICTRACEWEIGHT & MMELECTRICTRACEWEIGHT \\
\hline FDRMGRNONTRACEABLE & FDRMGRNONTRACEABLE \\
\hline FEEDERID & CADOPS \\
\hline FEEDERID & FEEDERALL \\
\hline FEEDERID & FEEDERID \\
\hline FEEDERID2 & FEEDERID2 \\
\hline FEEDERINFO & FEEDERINFO \\
\hline PHASEDESIGNATIONCD & CADOPS \\
\hline PHASEDESIGNATIONCD & FEEDERALL \\
\hline PHASEDESIGNATIONCD & PHASEDESIGNATION \\
\hline SYMBOLROTATIONVALUE & SYMBOLROTATION \\
\hline LOANUMBER & CADOPS \\
\hline LOANUMBER & FEEDERALL \\
\hline LOANUMBER & LOANAME \\
\hline LOANUMBER & LOANUMBER \\
\hline GRIDCD & CADOPS \\
\hline GRIDCD & FEEDERALL \\
\hline SUBTYPECD & CADOPS \\
\hline SUBTYPECD & FEEDERALL \\
\hline SUBTYPECD & SWITCHTYPECD \\
\hline NORMALPOSITIONA & CADOPS \\
\hline NORMALPOSITIONA & FEEDERALL \\
\hline
\end{tabular}


NORMALPOSITIONA

NORMALPOSITIONB

NORMALPOSITIONB

NORMALPOSITIONB

NORMALPOSITIONC

NORMALPOSITIONC

NORMALPOSITIONC

SWITCHIDNUMBER

SWITCHIDNUMBER

SWITCHIDNUMBER

SYMBOLCONFIGURATIONCD
NORMALPOSITION_A

CADOPS

FEEDERALL

NORMALPOSITION_B

CADOPS

FEEDERALL

NORMALPOSITION C

CADOPS

FEEDERALL

LOCATABLEFIELD

SYMBOLOGYCONFIGURATIONCD

SwitchGear

Object Class Model Names

\begin{tabular}{|l|l|}
\hline Object Class & Model Name \\
\hline SwitchGear & STRUCTURE \\
\hline SwitchGear & SWITCHGEAR \\
\hline SwitchGear & EDFS PADMOUNT PREVENT DELETION \\
\hline SwitchGear & EDFS RETIREMENT INFO \\
\hline SwitchGear & EDFS WORKORDER 2 \\
\hline SwitchGear & LOCATABLEOBJECT \\
\hline SwitchGear & MMREMOVABLE \\
\hline
\end{tabular}

Field Model Names

\begin{tabular}{|l|l|}
\hline Field & Model Name \\
\hline CREATIONDATE & AT_CREATIONDATE \\
\hline CREATIONUSERID & AT_CREATIONUSER \\
\hline UPDATEDATE & AT_1 \\
\hline UPDATEUSERID & AT_2 \\
\hline LOANUMBER & AT_3 \\
\hline LOANUMBER & LOANAME \\
\hline GRIDCD & AT_5 \\
\hline INSTALLDATE & AT_6 \\
\hline INSTALLWORKORDERNUMBER & AT_7 \\
\hline INSTALLWORKORDERNUMBER & INSTALLWORKORDERNUMBER \\
\hline INSTALLMISCORDERID & AT_12 \\
\hline INSTALLMISCORDERID & INSTALLMISCORDERID \\
\hline RETIREDATE & AT_8 \\
\hline RETIREDATE & RETIREDATE \\
\hline RETIREWORKORDERNUMBER & AT_9 \\
\hline RETIREWORKORDERNUMBER & RETIREWORKORDERNUMBER \\
\hline RETIREMISCORDERID & AT_13 \\
\hline RETIREMISCORDERID & RETIREMISCORDERID \\
\hline TAXUNITCD & AT_4 \\
\hline LEGACYWRINSTALLTIMESTAMP & AT_10 \\
\hline LEGACYWRREMOVALTIMESTAMP & AT_11 \\
\hline SYMBOLROTATIONVALUE & SYMBOLROTATION \\
\hline SUBTYPECD & AT_SUBTYPECD \\
\hline SWITCHGEARVOLTAGERATINGVALUE & AT_14 \\
\hline
\end{tabular}




\begin{tabular}{|l|l|} 
OPERATIONTYPECD & AT_15 \\
\hline FRONTKINDCD & AT_16 \\
\hline USEDCOMPARTMENTCOUNT & AT_17 \\
\hline LEGACYDISTRIBREFNUMBER & DISTRIBREFNUMBER \\
\hline LEGACYDISTRIBREFNUMBER & AT_18 \\
\hline ASSEMBLYNUMBER & AT_19 \\
\hline ASSOCIATIONTIMESTAMP & AT_20 \\
\hline
\end{tabular}

\section{SwitchUnit}

Object Class Model Names

\begin{tabular}{|l|c|}
\hline Object Class & \\
\hline SwitchUnit & Ch \\
\hline SwitchUnit & \\
\hline SwitchUnit & \\
\hline SwitchUnit & FE \\
\hline SwitchUnit & LC \\
\hline SwitchUnit & SV \\
\hline
\end{tabular}

\begin{tabular}{|l|}
\hline Model Name \\
\hline CADOPS \\
\hline EDFS RETIREMENT INFO \\
\hline EDFS WORKORDER 2 \\
\hline FEEDERALL \\
\hline LOCATABLEOBJECT \\
\hline SWITCHUNIT \\
\hline
\end{tabular}

Field Model Names

\begin{tabular}{|l|l|}
\hline Field & Model Name \\
\hline INSTALLWORKORDERNUMBER & INSTALLWORKORDERNUMBER \\
\hline INSTALLMISCORDERID & INSTALLMISCORDERID \\
\hline RETIREDATE & RETIREDATE \\
\hline RETIREWORKORDERNUMBER & RETIREWORKORDERNUMBER \\
\hline RETIREMISCORDERID & RETIREMISCORDERID \\
\hline PHASEDESIGNATION & PHASEDESIGNATION \\
\hline DISTRIBREFNUMBER & DISTRIBREFNUMBER \\
\hline CIRCUITNUMBER & FEEDERID \\
\hline SUBTYPECD & SUBTYPECD \\
\hline SWITCHAMPERAGEVALUE & CADOPS \\
\hline SWITCHAMPERAGEVALUE & FEEDERALL \\
\hline SWITCHTYPECD & SWITCHTYPECD \\
\hline
\end{tabular}

Terminator

Object Class Model Names

\begin{tabular}{|l|l|}
\hline Object Class & Model Name \\
\hline Terminator & LOCATABLEOBJECT \\
\hline
\end{tabular}

Field Model Names

\begin{tabular}{|l|l|}
\hline Field & Model Name \\
\hline OBJECTID & LOCATABLEFIELD \\
\hline MMELECTRICTRACEWEIGHT & MMELECTRICTRACEWEIGHT \\
\hline FDRMGRNONTRACEABLE & FDRMGRNONTRACEABLE \\
\hline FEEDERID & FEEDERID \\
\hline FEEDERID2 & FEEDERID2 \\
\hline FEEDERINFO & FEEDERINFO \\
\hline PHASEDESIGNATIONCD & PHASEDESIGNATION \\
\hline SYMBOLROTATIONVALUE & SYMBOLROTATION \\
\hline
\end{tabular}


TieBus

Object Class Model Names

\begin{tabular}{|l|l|}
\hline Object Class & Model Name \\
\hline TieBus & TRANSFORMERLEAD \\
\hline TieBus & CADOPS \\
\hline TieBus & CONDUCTOR \\
\hline TieBus & FEEDERALL \\
\hline TieBus & LOCATABLEOBJECT \\
\hline TieBus & OHCONDUCTOR \\
\hline TieBus & SPLITTARGET \\
\hline
\end{tabular}

Field Model Names

\begin{tabular}{|l|l|}
\hline Field & Model Name \\
\hline OBJECTID & LOCATABLEFIELD \\
\hline SUBTYPECD & CADOPS \\
\hline SUBTYPECD & FEEDERALL \\
\hline SUBTYPECD & SUBTYPECD \\
\hline MMELECTRICTRACEWEIGHT & MMELECTRICTRACEWEIGHT \\
\hline FDRMGRNONTRACEABLE & FDRMGRNONTRACEABLE \\
\hline FEEDERINFO & FEEDERINFO \\
\hline FEEDERID & CADOPS \\
\hline FEEDERID & FEEDERALL \\
\hline FEEDERID & FEEDERID \\
\hline FEEDERID2 & FEEDERID2 \\
\hline PRIMARYOPERATINGVOLTAGEVALUE & CADOPS \\
\hline PRIMARYOPERATINGVOLTAGEVALUE & FEEDERALL \\
\hline PRIMARYOPERATINGVOLTAGEVALUE & OPERATINGVOLTAGE \\
\hline PRIMARYOPERATINGVOLTAGEVALUE & PRIMARYOPERATINGVOLTAGE \\
\hline PHASEDESIGNATIONCD & CADOPS \\
\hline PHASEDESIGNATIONCD & FEEDERALL \\
\hline PHASEDESIGNATIONCD & PHASEDESIGNATION \\
\hline PHASEDESIGNATIONCD & PHASEDESIGNATIONCD \\
\hline LOANUMBER & CADOPS \\
\hline LOANUMBER & FEEDERALL \\
\hline LOANUMBER & LOANAME \\
\hline
\end{tabular}

TrafficLightDemand

Object Class Model Names

\begin{tabular}{|l|l}
\hline Object Class & Model Name \\
\hline TrafficLightDemand & LOCATABLEOBJECT
\end{tabular}

Field Model Names

\begin{tabular}{|l|l|}
\hline Field & Model Name \\
\hline
\end{tabular}

TransformerBank Object Class Model Names

\section{Object Class}

TransformerBank

TransformerBank

\begin{tabular}{|l|}
\hline Model Name \\
\hline CADOPS \\
\hline DISTRIBUTIONTRANSFORMER \\
\hline
\end{tabular}


TransformerBank

TransformerBank

TransformerBank

TransformerBank

TransformerBank

TransformerBank

TransformerBank
EDFS ASSETFEATURE

EDFS PADMOUNT PREVENT DELETION

EDFS STRUCTURE PREVENT DELETION

FEEDERALL

LOCATABLEOBJECT

TRANSFORMER

TRANSFORMERBANK

Field Model Names

\begin{tabular}{|l|l|}
\hline Field & Model Name \\
\hline MMELECTRICTRACEWEIGHT & MMELECTRICTRACEWEIGHT \\
\hline FDRMGRNONTRACEABLE & FDRMGRNONTRACEABLE \\
\hline FEEDERID & CADOPS \\
\hline FEEDERID & FEEDERALL \\
\hline FEEDERID & FEEDERID \\
\hline FEEDERID2 & FEEDERID2 \\
\hline FEEDERINFO & FEEDERINFO \\
\hline PHASEDESIGNATIONCD & CADOPS \\
\hline PHASEDESIGNATIONCD & FEEDERALL \\
\hline PHASEDESIGNATIONCD & PHASEDESIGNATION \\
\hline SYMBOLROTATIONVALUE & SYMBOLROTATION \\
\hline SYMBOLROTATIONVALUE & SYMBOLROTATIONVALUE \\
\hline LOANUMBER & CADOPS \\
\hline LOANUMBER & FEEDERALL \\
\hline LOANUMBER & LOANAME \\
\hline LOANUMBER & LOANUMBER \\
\hline GRIDCD & CADOPS \\
\hline GRIDCD & FEEDERALL \\
\hline SUBTYPECD & CADOPS \\
\hline SUBTYPECD & FEEDERALL \\
\hline LEGACYDISTRIBREFNUMBER & DISTRIBREFNUMBER \\
\hline LEGACYDISTRIBREFNUMBER & LOCATABLEFIELD \\
\hline TRANSFORMERBANKKVA & CADOPS \\
\hline TRANSFORMERBANKKVA & FEEDERALL \\
\hline TRANSFORMERBANKKVA & RATEDKVA \\
\hline
\end{tabular}

TransformerFunctionTest

Object Class Model Names

\begin{tabular}{|l|l}
\hline Object Class & Model Name \\
\hline TransformerFunctionTest & LOCATABLEOBJECT
\end{tabular}

Field Model Names

Field

Model Name

TransformerOilTest

Object Class Model Names

\begin{tabular}{|l|l|}
\hline Object Class & Model Name \\
\hline TransformerOilTest & LOCATABLEOBJECT \\
\hline
\end{tabular}


Field Model Names

Field

Model Name

TransformerRegulatorStores/tem

Object Class Model Names

\begin{tabular}{|l|l|}
\hline Object Class & Model Name \\
\hline TransformerRegulatorStoresltem & TRANSFORMERREGULATORSTORESITEM \\
\hline
\end{tabular}

Field Model Names

Field

KVAVALUE

Model Name

KVA

TransformerUnit

Object Class Model Names

\begin{tabular}{|l|l|}
\hline Object Class & Model Name \\
\hline TransformerUnit & CADOPS \\
\hline TransformerUnit & EDFS ASSETUNIT \\
\hline TransformerUnit & FEEDERALL \\
\hline TransformerUnit & LOCATABLEOBJECT \\
\hline TransformerUnit & TRANSFORMERUNIT \\
\hline
\end{tabular}

Field Model Names

\begin{tabular}{|l|l|}
\hline Field & Model Name \\
\hline COMPANYNUMBER & COMPANYNUMBER \\
\hline COMPANYNUMBER & LOCATABLEFIELD \\
\hline STATUSTIMESTAMP & STATUSTIMESTAMP \\
\hline DISPOSITIONCD & DISPOSITIONCD \\
\hline STATUSCD & STATUSCD \\
\hline RELSTORESITEMNUMBER & RELSTORESITEMNUMBER \\
\hline
\end{tabular}

TransformerUnitInstall

Object Class Model Names

Object Class

TransformerUnitInstall

TransformerUnitInstall

TransformerUnitInstall

TransformerUnitInstall
Model Name

CADOPS

EDFS ASSETINSTALL

FEEDERALL

TRANSFORMERUNITINSTALL

Field Model Names

\begin{tabular}{|l|l|}
\hline Field & Model Name \\
\hline LOANUMBER & LOANAME \\
\hline LOANUMBER & LOANUMBER \\
\hline TAXUNITCD & TAXUNITCD \\
\hline GRIDCD & GRIDCD \\
\hline INSTALLTIMESTAMP & INSTALLTIMESTAMP \\
\hline INSTALLDATE & INSTALLDATE \\
\hline CIRCUITNUMBER & CIRCUITNUMBER \\
\hline PHASEDESIGNATION & CADOPS \\
\hline PHASEDESIGNATION & FEEDERALL \\
\hline
\end{tabular}




\begin{tabular}{|c|}
\hline PHASEDESIGNATION \\
\hline KVAVALUE \\
\hline KVAVALUE \\
\hline KVAVALUE \\
\hline KVAVALUE \\
\hline INSTALLATIONSTATUSCD \\
\hline MATERIALTICKETNUMBER \\
\hline DISTRIBREFNUMBER \\
\hline RELTRANSFORMERBANKOBJECTID \\
\hline RELTRANSFORMERUNITCOMPANYNUMBER \\
\hline
\end{tabular}

PHASEDESIGNATION

CADOPS

FEEDERALL

KVA

RATEDKVA

INSTALLATIONSTATUSCD

MATERIALTICKETNUMBER

DISTRIBREFNUMBER

RELATEDBANKOID

COMPANYNUMBER

TransformerUnitRemove

Object Class Model Names

\begin{tabular}{|l|l|}
\hline Object Class & Model Name \\
\hline TransformerUnitRemove & EDFS ASSETREMOVE \\
\hline TransformerUnitRemove & TRANSFORMERUNITREMOVE \\
\hline
\end{tabular}

Field Model Names

\begin{tabular}{|l|l|}
\hline Field & Model Name \\
\hline REMOVALTIMESTAMP & REMOVALTIMESTAMP \\
\hline REMOVALDATE & REMOVALDATE \\
\hline REMOVALCD & REMOVALCD \\
\hline MATERIALTICKETNUMBER & MATERIALTICKETNUMBER \\
\hline RELTRANSFORMERUNITCOMPANYNUMBER & COMPANYNUMBER \\
\hline
\end{tabular}

TransformerUnitStock

Object Class Model Names

Object Class

TransformerUnitStock

Model Name

EDFS ASSETSTOCK

Field Model Names

\begin{tabular}{|l|l|}
\hline Field & Model Name \\
\hline STOCKTIMESTAMP & STOCKTIMESTAMP \\
\hline STOCKDATE & STOCKDATE \\
\hline RELSTOREROOMNUMBER & STOREROOMNUMBER \\
\hline RELTRANSFORMERUNITCOMPANYNUMBER & COMPANYNUMBER \\
\hline
\end{tabular}

UgConductor

Object Class Model Names

\section{Object Class}

UgConductor

UgConductor

UgConductor

UgConductor

UgConductor

UgConductor

UgConductor

UgConductor

\begin{tabular}{|l}
\hline Model Name \\
\hline CADOPS \\
\hline CONDUCTOR \\
FEEDERALL \\
\hline LINE \\
\hline LOCATABLEOBJECT \\
MMABANDONABLE \\
\hline SPLITTARGET \\
\hline UGCONDUCTOR \\
\hline
\end{tabular}


Field Model Names

\begin{tabular}{|l|l|}
\hline Field & Model Name \\
\hline OBJECTID & LOCATABLEFIELD \\
\hline CREATIONDATE & AT_CREATIONDATE \\
\hline CREATIONUSERID & AT_CREATIONUSER \\
\hline UPDATEDATE & AT_UPDATEDATE \\
\hline UPDATEUSERID & AT_UPDATEUSERID \\
\hline LOANUMBER & AT_1 \\
\hline LOANUMBER & CADOPS \\
\hline LOANUMBER & FEEDERALL \\
\hline LOANUMBER & LOANAME \\
\hline LOANUMBER & LOANUMBER \\
\hline GRIDCD & AT_2 \\
\hline GRIDCD & CADOPS \\
\hline GRIDCD & FEEDERALL \\
\hline MEASUREDLENGTH & CADOPS \\
\hline MEASUREDLENGTH & FEEDERALL \\
\hline MEASUREDLENGTH & MEASUREDLENGTH \\
\hline MMELECTRICTRACEWEIGHT & MMELECTRICTRACEWEIGHT \\
\hline FDRMGRNONTRACEABLE & FDRMGRNONTRACEABLE \\
\hline FEEDERINFO & FEEDERINFO \\
\hline FEEDERID & CADOPS \\
\hline FEEDERID & FEEDERALL \\
\hline FEEDERID & FEEDERID \\
\hline FEEDERID2 & FEEDERID2 \\
\hline PRIMARYOPERATINGVOLTAGEVALUE & CADOPS \\
\hline PRIMARYOPERATINGVOLTAGEVALUE & FEEDERALL \\
\hline PRIMARYOPERATINGVOLTAGEVALUE & OPERATINGVOLTAGE \\
\hline PHASEDESIGNATIONCD & CADOPS \\
\hline PHASEDESIGNATIONCD & FEEDERALL \\
\hline PHASEDESIGNATIONCD & PHASEDESIGNATION \\
\hline SUBTYPECD & CADOPS \\
\hline SUBTYPECD & FEEDERALL \\
\hline INCONDUITIND & AT_3 \\
\hline INCONDUITIND & INCONDUITCD \\
\hline INCONDUITIND & INCONDUITIND \\
\hline FEEDERALLPHASE & CADOPS \\
\hline FEEDERALLPHASE & FEEDERALL \\
\hline WIRESIZECD & CADOPS \\
\hline WIRESIZECD & FEEDERALL \\
\hline WIREMATERIALCD & CADOPS \\
\hline WIREMATERIALCD & FEEDERALL \\
\hline SHAPE & AT_SHAPE \\
\hline & \\
\hline
\end{tabular}

UgConductorInfo

Object Class Model Names

\begin{tabular}{|l|l|}
\hline Object Class & Model Name \\
\hline UgConductorlnfo & CADOPS \\
\hline
\end{tabular}


UgConductorlnfo

UgConductorInfo

UgConductorInfo

UgConductorInfo

UgConductorlnfo

UgConductorInfo

UgConductorlnfo

UgConductorlnfo

UgConductorInfo
CONDUCTORINFO

EDFS CONDUCTORINFO

EDFS RETIREMENT INFO

EDFS WORKORDER 2

FEEDERALL

LOCATABLEOBJECT

PRIMARYCONDUCTORINFO

PRIMARYUGCONDUCTORINFO

UGCONDUCTORINFO

Field Model Names

\begin{tabular}{|l|l|}
\hline Field & Model Name \\
\hline INSTALLDATE & INSTALLDATE \\
\hline INSTALLWORKORDERNUMBER & INSTALLWORKORDERNUMBER \\
\hline INSTALLMISCORDERID & INSTALLMISCORDERID \\
\hline RETIREDATE & RETIREDATE \\
\hline RETIREWORKORDERNUMBER & RETIREWORKORDERNUMBER \\
\hline RETIREMISCORDERID & RETIREMISCORDERID \\
\hline SUBTYPECD & CADOPS \\
\hline SUBTYPECD & FEEDERALL \\
\hline SUBTYPECD & SUBTYPECD \\
\hline WIRESIZECD & CADOPS \\
\hline WIRESIZECD & FEEDERALL \\
\hline WIRESIZECD & WIRESIZE \\
\hline WIRESIZECD & WIRESIZECD \\
\hline WIREMATERIALCD & CADOPS \\
\hline WIREMATERIALCD & FEEDERALL \\
\hline WIREMATERIALCD & WIREMATERIALCD \\
\hline WIRETYPECD & CADOPS \\
\hline WIRETYPECD & FEEDERALL \\
\hline WIRETYPECD & WIRETYPECD \\
\hline INCONDUITCD & INCONDUITCD \\
\hline INCONDUITCD & INCONDUITIND \\
\hline CIRCUITNUMBER & CIRCUITNUMBER \\
\hline CIRCUITNUMBER & FEEDERID \\
\hline FROMDISTRIBREFNUMBER & FROMDISTRIBREF \\
\hline TODISTRIBREFNUMBER & TODISTRIBREF \\
\hline MEASUREDLENGTH & MEASUREDLENGTH \\
\hline MEASUREDLENGTH & SPANLENGTH \\
\hline
\end{tabular}

UniqueDistribRefNumbersTable

Object Class Model Names

\section{Object Class}

UniqueDistribRefNumbersTable

Model Name

UNIQUEDISTRIBREFNUMBERSTABLE

Field Model Names

\section{Field}

UniqueDistribRefNumber

Model Name

DISTRIBREFNUMBER 
VoltageRegulator

Object Class Model Names

\begin{tabular}{|l|l|}
\hline Object Class & Model Name \\
\hline VoltageRegulator & CADOPS \\
\hline VoltageRegulator & EDFS ASSETFEATURE \\
\hline VoltageRegulator & EDFS STRUCTURE PREVENT DELETION \\
\hline VoltageRegulator & FEEDERALL \\
\hline VoltageRegulator & LOCATABLEOBJECT \\
\hline VoltageRegulator & VOLTAGEREGULATORUNIT \\
\hline
\end{tabular}

Field Model Names

\begin{tabular}{|l|l|}
\hline Field & Model Name \\
\hline MMELECTRICTRACEWEIGHT & MMELECTRICTRACEWEIGHT \\
\hline FDRMGRNONTRACEABLE & FDRMGRNONTRACEABLE \\
\hline FEEDERID & CADOPS \\
\hline FEEDERID & FEEDERALL \\
\hline FEEDERID & FEEDERID \\
\hline FEEDERID2 & FEEDERID2 \\
\hline FEEDERINFO & FEEDERINFO \\
\hline PHASEDESIGNATIONCD & FEEDERALL \\
\hline PHASEDESIGNATIONCD & CADOPS \\
\hline PHASEDESIGNATIONCD & PHASEDESIGNATION \\
\hline SYMBOLROTATIONVALUE & SYMBOLROTATION \\
\hline LOANUMBER & CADOPS \\
\hline LOANUMBER & FEEDERALL \\
\hline LOANUMBER & LOANAME \\
\hline LOANUMBER & LOANUMBER \\
\hline GRIDCD & CADOPS \\
\hline GRIDCD & FEEDERALL \\
\hline LEGACYDISTRIBREFNUMBER & LOCATABLEFIELD \\
\hline REGULATORKVA & CADOPS \\
\hline REGULATORKVA & FEEDERALL \\
\hline VOLTAGEREGULATORAMPRATING & CADOPS \\
\hline VOLTAGEREGULATORAMPRATING & FEEDERALL \\
\hline
\end{tabular}

VoltageRegulatorBank

Object Class Model Names

\section{Object Class}

VoltageRegulatorBank

VoltageRegulatorBank

VoltageRegulatorBank

VoltageRegulatorBank

VoltageRegulatorBank

\begin{tabular}{|l|}
\hline Model Name \\
\hline CADOPS \\
\hline FEEDERALL \\
\hline LOCATABLEOBJECT \\
\hline NABACKWARD \\
\hline VOLTAGEREGULATOR \\
\hline
\end{tabular}

Field Model Names

\begin{tabular}{|l|l|}
\hline Field & Model Name \\
\hline BANKKVAVALUE & CADOPS \\
\hline BANKKVAVALUE & FEEDERALL \\
\hline
\end{tabular}


WoodPoleEvents

Object Class Model Names

\begin{tabular}{|l|l}
\hline Object Class & Model Name \\
\hline WoodPoleEvents & WOODPOLEEVENTS \\
\hline
\end{tabular}

Field Model Names

\begin{tabular}{|l|l|}
\hline Field & Model Name \\
\hline WOODPOLEINSPECTIONYEAR & INSPECTIONYEAR \\
\hline WOODPOLEINSPECTIONPASSFAILCD & INSPECTIONPASSFAILCD \\
\hline WOODPOLEREINFORCEDDATE & REINFORCEDDATE \\
\hline WOODPOLEGNDTREATDATE & GROUNDTREATMENTDATE \\
\hline RELDISTRIBREFNUMBER & DISTRIBREFNUMBER \\
\hline EVENTTYPECD & EVENTTYPECD \\
\hline
\end{tabular}

WoodPolelnfo

Object Class Model Names

\begin{tabular}{|l|l}
\hline Object Class & Model Name \\
\hline WoodPolnfo & WOODPOLEIFF
\end{tabular}

\begin{tabular}{|l|l}
\hline WoodPolelnfo & WOODPOLEINFO
\end{tabular}

Field Model Names

\begin{tabular}{|l|l|}
\hline Field & Model Name \\
\hline WOODPOLECLASSCD & CLASSCD \\
\hline WOODPOLETYPECD & TYPECD \\
\hline WOODPOLEORIGINALTREATMNTTYPECD & ORIGINALTREATMENTTYPECD \\
\hline WOODSETTINGCD & SETTINGCD \\
\hline RELDISTRIBREFNUMBER & DISTRIBREFNUMBER \\
\hline
\end{tabular}


arcfm8.GAS.Casing

Table E-4. Gas ArcFM configuration

ArcFM Display Field:

SUBTYPECD

Create Edit Task:

On Delete Event:

Before Split Event:

On Split Event:

After Split Event:

On Abandon Event:

On Create Event: EDFS Validate Work Order

Number 1

On Update Event: EDFS Validate Work Order

Number 1

Abandon Feature Class:

arcfm8.GAS.RetiredCasing

Abandon Subtype:

Remove Feature

Class:

Remove Subtype:

\begin{tabular}{|l|l|l|c|l|l|}
\hline Field Alias & Visible & Editable & $\begin{array}{c}\text { Allow } \\
\text { Null } \\
\text { Values }\end{array}$ & On Feature Create & On Feature Update \\
\hline OBJECTID & TRUE & FALSE & FALSE & & \\
\hline Subtype Code & TRUE & TRUE & FALSE & & \\
\hline INSTALLDATE & TRUE & TRUE & FALSE & & \\
\hline Install Work Order & TRUE & TRUE & FALSE & & \\
\hline Install Misc Order Id & TRUE & TRUE & FALSE & & \\
\hline LOA Name & TRUE & FALSE & TRUE & & \\
\hline Tax Unit & TRUE & TRUE & FALSE & & \\
\hline Grid Code & TRUE & TRUE & FALSE & & \\
\hline Casing Diameter & TRUE & TRUE & FALSE & & \\
\hline Installed Length & TRUE & TRUE & FALSE & & \\
\hline Length Source & TRUE & TRUE & TRUE & & \\
\hline $\begin{array}{l}\text { RR Crossing Agreement } \\
\text { Number }\end{array}$ & TRUE & TRUE & TRUE & & OIDSCO.Casing.RelatedMain \\
\hline Related Main OID & FALSE & FALSE & TRUE & NIPSCO.Casing.RelatedMain & OID \\
\hline CP Section OID & FALSE & FALSE & TRUE & & \\
\hline Shape & FALSE & TRUE & TRUE & & \\
\hline CreationDate & FALSE & TRUE & FALSE & ArcFM Current Date & \\
\hline Created By & FALSE & TRUE & FALSE & ArcFM User Name & \\
\hline UPDATEDATE & FALSE & FALSE & TRUE & & ArcFM Current Date \\
\hline Updated By & FALSE & FALSE & TRUE & & ArcFM User Name \\
\hline Legacy Main Link Value & FALSE & TRUE & TRUE & & \\
\hline Legacy Ees Number & FALSE & TRUE & TRUE & & \\
\hline SHAPE.len & FALSE & & & & \\
\hline
\end{tabular}

arcfm8.GAS.CpRectifier

ArcFM Display Field: RECTIFIERNUMBER

Create Edit Task:

On Delete Event:

On Abandon Event:

On Create Event: EDFS Validate Work Order Number 1

On Update Event: EDFS Validate Work Order Number 1

Abandon Feature Class:

Abandon Subtype:

Remove Feature Class:

Remove Subtype: 


\begin{tabular}{|l|c|c|c|l|l|}
\hline Field Alias & Visible & Editable & $\begin{array}{c}\text { Allow Null } \\
\text { Values }\end{array}$ & On Feature Create & On Feature Update \\
\hline OBJECTID & TRUE & FALSE & FALSE & & \\
\hline Subtype Code & TRUE & TRUE & FALSE & & \\
\hline INSTALLDATE & TRUE & TRUE & FALSE & & \\
\hline Install Work Order & TRUE & TRUE & FALSE & & \\
\hline Install Misc Order Id & TRUE & TRUE & FALSE & & NIPSCO.LoaName \\
\hline LOA Name & TRUE & FALSE & FALSE & NIPSCO.LoaName & \\
\hline Tax Unit & TRUE & TRUE & FALSE & & \\
\hline Grid Code & TRUE & TRUE & FALSE & & \\
\hline Rectifier Number & TRUE & TRUE & TRUE & & \\
\hline Related CP Section OID & FALSE & TRUE & TRUE & & \\
\hline Shape & FALSE & TRUE & TRUE & & \\
\hline CreationDate & FALSE & TRUE & FALSE & ArcFM Current Date & \\
\hline Created By & FALSE & TRUE & FALSE & ArcFM User Name & \\
\hline UPDATEDATE & FALSE & TRUE & TRUE & & ArcFM Current Date \\
\hline Updated By & FALSE & FALSE & TRUE & & \\
\hline Symbol Rotation & FALSE & TRUE & FALSE & & \\
\hline Legacy Main Link Value & FALSE & TRUE & TRUE & & \\
\hline Legacy Ees Number & FALSE & TRUE & TRUE & & \\
\hline
\end{tabular}

arcfm8.GAS.CpSection

ArcFM Display Field: OBJECTID

Create Edit Task:

On Create Event: NIPSCO.CPSection.CorrosionControlNumber

On Update Event: NIPSCO.CPSection.CorrosionControlNumber

On Delete Event:

On Abandon Event:

Abandon Feature Class:

Abandon Subtype:

Remove Feature Class:

Remove Subtype:

\begin{tabular}{|l|l|l|c|l|l|}
\hline Field Alias & Visible & Editable & $\begin{array}{c}\text { Allow } \\
\text { Null } \\
\text { Values }\end{array}$ & On Feature Create & On Feature Update \\
\hline OBJECTID & TRUE & FALSE & FALSE & & \\
\hline Subtype Code & TRUE & TRUE & FALSE & & \\
\hline LOA Name & TRUE & FALSE & FALSE & NIPSCO.LoaName & NIPSCO.LoaName \\
\hline Tax Unit & TRUE & TRUE & FALSE & & \\
\hline Grid Code & TRUE & TRUE & FALSE & & \\
\hline $\begin{array}{l}\text { Corrosion Section } \\
\text { Number }\end{array}$ & TRUE & TRUE & FALSE & & \\
\hline $\begin{array}{l}\text { Corrosion Control } \\
\text { Number }\end{array}$ & TRUE & FALSE & TRUE & & \\
\hline Shape & FALSE & TRUE & TRUE & & \\
\hline CreationDate & FALSE & TRUE & FALSE & ArcFM Current Date & \\
\hline Created By & FALSE & TRUE & FALSE & ArcFM User Name & ArcFM Current Date \\
\hline UPDATEDATE & FALSE & TRUE & TRUE & & ArcFM User Name \\
\hline Updated By & FALSE & TRUE & TRUE & & \\
\hline Legacy Main Link Value & FALSE & TRUE & TRUE & & \\
\hline Legacy Ees Number & FALSE & TRUE & TRUE & & \\
\hline HANDLE & FALSE & TRUE & TRUE & & \\
\hline TILENAME & FALSE & TRUE & TRUE & & \\
\hline DCSID & FALSE & TRUE & TRUE & & \\
\hline GLINK & FALSE & TRUE & TRUE & & \\
\hline
\end{tabular}

arcfm8.GAS.CpTestPoint

ArcFM Display Field:

SUBTYPECD

Create Edit Task:

On Delete Event:

On Abandon Event:

On Create Event: EDFS Validate Work Order Number 1 
On Update Event: EDFS Validate Work Order Number 1

Abandon Feature Class:

Abandon Subtype:

Remove Feature Class:

Remove Subtype:

\begin{tabular}{|l|c|c|c|l|l|}
\hline Field Alias & Visible & Editable & $\begin{array}{c}\text { Allow } \\
\text { Null } \\
\text { Values }\end{array}$ & On Feature Create & On Feature Update \\
\hline OBJECTID & TRUE & FALSE & FALSE & & \\
\hline Subtype Code & TRUE & TRUE & FALSE & & \\
\hline INSTALLDATE & TRUE & TRUE & FALSE & & \\
\hline Install Work Order & TRUE & TRUE & FALSE & & \\
\hline Install Misc Order Id & TRUE & TRUE & FALSE & & NIPSCO.LoaName \\
\hline LOA Name & TRUE & FALSE & FALSE & NIPSCO.LoaName & \\
\hline Tax Unit & TRUE & TRUE & FALSE & & \\
\hline Grid Code & TRUE & TRUE & FALSE & & \\
\hline Location Description 1 & TRUE & TRUE & FALSE & & \\
\hline Location Description 2 & TRUE & TRUE & FALSE & & \\
\hline Graphics Scale Factor & FALSE & TRUE & FALSE & & \\
\hline Related CP Section OID & FALSE & TRUE & TRUE & & \\
\hline Shape & FALSE & TRUE & TRUE & & \\
\hline CreationDate & FALSE & TRUE & FALSE & ArcFM Current Date & \\
\hline Created By & FALSE & TRUE & FALSE & ArcFM User Name & ArcFM Current Date \\
\hline UPDATEDATE & FALSE & FALSE & TRUE & & \\
\hline Updated By & FALSE & FALSE & TRUE & & \\
\hline Legacy Ees Number & FALSE & TRUE & TRUE & & \\
\hline
\end{tabular}

arcfm8.GAS.DeadEndGas

ArcFM Display Field: SUBTYPECD

Create Edit Task:

On Delete Event:

On Abandon Event:

On Create Event: EDFS Validate Work Order Number 1

On Update Event: EDFS Validate Work Order Number 1

Abandon Feature Class: arcfm8.GAS.RetiredDeadEndGas

Abandon Subtype:

Remove Feature Class:

Remove Subtype:

\begin{tabular}{|l|c|c|c|l|l|}
\hline Field Alias & Visible & Editable & $\begin{array}{c}\text { Allow } \\
\text { Null } \\
\text { Values }\end{array}$ & On Feature Create & On Feature Update \\
\hline OBJECTID & TRUE & FALSE & FALSE & & \\
\hline Subtype Field & TRUE & TRUE & FALSE & & \\
\hline INSTALLDATE & TRUE & TRUE & FALSE & & \\
\hline Install Work Order & TRUE & TRUE & FALSE & & \\
\hline Install Misc Order Id & TRUE & TRUE & FALSE & & NIPSCO.LoaName \\
\hline LOA Name & TRUE & FALSE & FALSE & NIPSCO.LoaName & \\
\hline Tax Unit & TRUE & TRUE & FALSE & & \\
\hline Grid Code & TRUE & TRUE & FALSE & & \\
\hline Location Description 1 & TRUE & TRUE & FALSE & & \\
\hline Location Description 2 & TRUE & TRUE & FALSE & & \\
\hline Shape & FALSE & TRUE & TRUE & & ArcFM Current Date \\
\hline CreationDate & FALSE & TRUE & FALSE & ArcFM Current Date & ArcFM User Name \\
\hline Created By & FALSE & TRUE & FALSE & ArcFM User Name & \\
\hline UPDATEDATE & FALSE & FALSE & TRUE & & \\
\hline Updated By & FALSE & FALSE & TRUE & & \\
\hline Main Link Value & FALSE & TRUE & TRUE & & \\
\hline Symbol Rotation & FALSE & TRUE & FALSE & & \\
\hline Legacy Ees Number & FALSE & TRUE & TRUE & & \\
\hline Legacy Node 1 & FALSE & TRUE & TRUE & & \\
\hline Legacy Node 2 & FALSE & TRUE & TRUE & & \\
\hline
\end{tabular}

arcfm8.GAS.Drip

ArcFM Display Field: 
DRIPPOTTYPE

Create Edit Task:

On Delete Event:

On Abandon Event:

On Create Event: EDFS Validate Work Order Number 1

On Update Event: EDFS Validate Work Order Number 1

Abandon Feature Class:

arcfm8.GAS.RetiredDrip

Abandon Subtype:

Remove Feature Class:

Remove Subtype:

\begin{tabular}{|c|c|c|c|c|c|}
\hline Field Alias & Visible & Editable & $\begin{array}{c}\text { Allow } \\
\text { Null } \\
\text { Values }\end{array}$ & On Feature Create & On Feature Update \\
\hline OBJECTID & TRUE & FALSE & FALSE & & \\
\hline Subtype Code & TRUE & TRUE & FALSE & & \\
\hline INSTALLDATE & TRUE & TRUE & FALSE & & \\
\hline Install Work Order & TRUE & TRUE & FALSE & & \\
\hline Install Misc Order Id & TRUE & TRUE & FALSE & & \\
\hline LOA Name & TRUE & FALSE & FALSE & NIPSCO.LoaName & NIPSCO.LoaName \\
\hline Tax Unit & TRUE & TRUE & FALSE & & \\
\hline Grid Code & TRUE & TRUE & FALSE & & \\
\hline Drip Pot Type & TRUE & TRUE & FALSE & & \\
\hline Shape & FALSE & TRUE & TRUE & & \\
\hline CreationDate & FALSE & TRUE & FALSE & ArcFM Current Date & \\
\hline Created By & FALSE & TRUE & FALSE & ArcFM User Name & \\
\hline UPDATEDATE & FALSE & FALSE & TRUE & & ArcFM Current Date \\
\hline Updated By & FALSE & FALSE & TRUE & & ArcFM User Name \\
\hline Legacy Main Link Value & FALSE & TRUE & TRUE & & \\
\hline Symbol Rotation & FALSE & TRUE & FALSE & & \\
\hline Legacy Ees Number & FALSE & TRUE & TRUE & & \\
\hline Related Main OID & FALSE & FALSE & TRUE & $\begin{array}{l}\text { NIPSCO.RetrieveOIDofClosest } \\
\text { GasMain }\end{array}$ & $\begin{array}{l}\text { NIPSCO.RetrieveOIDofClosest } \\
\text { GasMain }\end{array}$ \\
\hline
\end{tabular}

arcfm8.GAS.EmergencyValveMarker

ArcFM Display Field: SUBTYPECD

Create Edit Task: ArcFM Manual Angle Setter

On Create Event:

On Update Event:

On Delete Event:

On Abandon Event:

Abandon Feature Class:

Abandon Subtype:

Remove Feature Class:

Remove Subtype:

\begin{tabular}{|l|c|c|c|l|l|}
\hline Field Alias & Visible & Editable & $\begin{array}{c}\text { Allow } \\
\text { Null } \\
\text { Values }\end{array}$ & On Feature Create & On Feature Update \\
\hline OBJECTID & TRUE & FALSE & FALSE & & \\
\hline Subtype Code & TRUE & TRUE & FALSE & & \\
\hline Graphics Scale Factor & FALSE & TRUE & FALSE & & \\
\hline Related Valve OID & FALSE & TRUE & TRUE & & \\
\hline Shape & FALSE & TRUE & TRUE & & \\
\hline CreationDate & FALSE & TRUE & FALSE & ArcFM Current Date & \\
\hline Created By & FALSE & TRUE & FALSE & ArcFM User Name & \\
\hline UPDATEDATE & FALSE & FALSE & TRUE & & ArcFM Current Date \\
\hline Updated By & FALSE & FALSE & TRUE & & ArcFM User Name \\
\hline Symbol Rotation & FALSE & TRUE & FALSE & & \\
\hline HANDLE & FALSE & TRUE & TRUE & & \\
\hline TILENAME & FALSE & TRUE & TRUE & & \\
\hline DCSID & FALSE & TRUE & TRUE & & \\
\hline Label Text & FALSE & TRUE & TRUE & & \\
\hline
\end{tabular}




\begin{tabular}{|c|c|c|c|c|c|}
\hline EES & FALSE & TRUE & TRUE & & \\
\hline \multicolumn{6}{|c|}{$\begin{array}{l}\text { arcfm8.GAS.GasDiscrepancy } \\
\text { ArcFM Display Field: ERRORDESCRIPTION } \\
\text { Create Edit Task: } \\
\text { On Create Event: } \\
\text { On Update Event: } \\
\text { On Delete Event: } \\
\text { On Abandon Event: } \\
\text { Abandon Feature Class: } \\
\text { Abandon Subtype: } \\
\text { Remove Feature Class: } \\
\text { Remove Subtype: }\end{array}$} \\
\hline Field Alias & Visible & Editable & $\begin{array}{c}\text { Allow } \\
\text { Null } \\
\text { Values }\end{array}$ & On Feature Create & On Feature Update \\
\hline OBJECTID & TRUE & FALSE & FALSE & & \\
\hline Subtype & TRUE & TRUE & FALSE & & \\
\hline Error Description & TRUE & TRUE & FALSE & & \\
\hline Shape & FALSE & TRUE & TRUE & & \\
\hline CreationDate & FALSE & TRUE & FALSE & ArcFM Current Date & \\
\hline Created By & FALSE & TRUE & FALSE & ArcFM User Name & \\
\hline UPDATEDATE & FALSE & FALSE & TRUE & & ArcFM Current Date \\
\hline Updated By & FALSE & FALSE & TRUE & & ArcFM User Name \\
\hline Legacy Ees Number & FALSE & TRUE & TRUE & & \\
\hline HANDLE & FALSE & TRUE & TRUE & & \\
\hline TILENAME & FALSE & TRUE & TRUE & & \\
\hline DCSID & FALSE & TRUE & TRUE & & \\
\hline
\end{tabular}

arcfm8.GAS.GasMain

ArcFM Display Field: SUBTYPECD

Create Edit Task:

On Update Event: EDFS Validate Work Order Number 1

On Update Event: ArcFM Check for Pinchable Pipe

On Update Event: NIPSCO.GasMain.PipeChangeAU

On Delete Event:

On Split Event:

On Abandon Event:

Before Split Event: EDFS Turn WOMO Validation Off

Before Split Event: EDFS Keep WOMO Validation Off For Split

After Split Event: EDFS WOMO Validation Split Finished

After Split Event: EDFS Turn WOMO Validation On

On Create Event: EDFS Validate Work Order Number 1

On Create Event: ArcFM Check for Pinchable Pipe

On Create Event: EDFS Turn WOMO Validation Off

On Create Event: NIPSCO.SplitAtTapPoint

On Create Event: EDFS Turn WOMO Validation On

On Create Event: NIPSCO.GasMain.PipeChangeAU

Abandon Feature Class: arcfm8.GAS.RetiredGasMain

Abandon Subtype:

Remove Feature Class:

Remove Subtype:

\begin{tabular}{|l|c|c|c|c|c|}
\hline Field Alias & Visible & Editable & $\begin{array}{c}\text { Allow } \\
\text { Null } \\
\text { Values }\end{array}$ & On Feature Create & On Feature Update \\
\hline Subtype Code & TRUE & TRUE & FALSE & & \\
\hline INSTALLDATE & TRUE & TRUE & FALSE & & \\
\hline Install Work Order & TRUE & TRUE & FALSE & & \\
\hline Install Misc Order Id & TRUE & TRUE & FALSE & & NIPSCO.LoaName \\
\hline LOA Name & TRUE & FALSE & TRUE & NIPSCO.LoaName & \\
\hline Tax Unit & TRUE & TRUE & FALSE & & \\
\hline
\end{tabular}




\begin{tabular}{|c|c|c|c|c|c|}
\hline Grid Code & TRUE & TRUE & FALSE & & \\
\hline Main Diameter & TRUE & TRUE & FALSE & & \\
\hline Main Material & TRUE & TRUE & FALSE & & \\
\hline ArcFm Main Material & TRUE & TRUE & TRUE & & \\
\hline \begin{tabular}{|l} 
Measured Length \\
\end{tabular} & TRUE & TRUE & FALSE & & \\
\hline Length Source & TRUE & TRUE & FALSE & & \\
\hline Insert Indicator & TRUE & TRUE & FALSE & & \\
\hline Bonded Indicator & TRUE & TRUE & FALSE & & \\
\hline Maintained By & TRUE & TRUE & TRUE & & \\
\hline Calculated Flow & TRUE & TRUE & TRUE & & \\
\hline $\begin{array}{l}\text { Upstream Calculated } \\
\text { Pressure }\end{array}$ & TRUE & TRUE & TRUE & & \\
\hline $\begin{array}{l}\text { Downstream Calculated } \\
\text { Pressure }\end{array}$ & TRUE & TRUE & TRUE & & \\
\hline System Name & TRUE & TRUE & TRUE & & \\
\hline Line Section Number & TRUE & TRUE & TRUE & & \\
\hline Shape & FALSE & TRUE & TRUE & & \\
\hline Enabled & FALSE & TRUE & TRUE & & \\
\hline CreationDate & FALSE & TRUE & FALSE & ArcFM Current Date & \\
\hline Created By & FALSE & TRUE & FALSE & ArcFM User Name & \\
\hline UPDATEDATE & FALSE & FALSE & TRUE & & ArcFM Current Date \\
\hline Updated By & FALSE & FALSE & TRUE & & \\
\hline Gas Trace Weight & FALSE & FALSE & TRUE & $\begin{array}{l}\text { ArcFM Gas Distribution Main } \\
\text { Trace Weight }\end{array}$ & $\begin{array}{l}\text { ArcFM Gas Distribution Main } \\
\text { Trace Weight }\end{array}$ \\
\hline CP System OID & FALSE & TRUE & TRUE & & \\
\hline Legacy Node 1 & FALSE & TRUE & TRUE & & \\
\hline Legacy Node 2 & FALSE & TRUE & TRUE & & \\
\hline Legacy Ees Number & FALSE & TRUE & TRUE & & \\
\hline SHAPE.Ien & FALSE & & & & \\
\hline $\begin{array}{l}\text { Gas System Status } \\
\text { Code }\end{array}$ & TRUE & TRUE & FALSE & & \\
\hline $\begin{array}{l}\text { Pressure System Status } \\
\text { Code }\end{array}$ & TRUE & TRUE & FALSE & & \\
\hline $\begin{array}{l}\text { Emergency Isolation } \\
\text { System Status Code }\end{array}$ & TRUE & TRUE & FALSE & & \\
\hline OBJECTID & TRUE & FALSE & FALSE & & \\
\hline
\end{tabular}

arcfm8.GAS.GasMainLocation

ArcFM Display Field: OBJECTID

Create Edit Task: NIPSCO.Gas.PlaceAndRelateGasMain

On Create Event: NIPSCO.Gas.RelatedPipeObjectld

On Update Event: NIPSCO.Gas.RelatedPipeObjectld

On Delete Event:

On Abandon Event:

Abandon Feature Class: arcfm8.GAS.RetiredGasMainLocation

Abandon Subtype:

Remove Feature Class:

Remove Subtype:

\begin{tabular}{|l|c|c|c|l|l|}
\hline Field Alias & Visible & Editable & $\begin{array}{c}\text { Allow } \\
\text { Null } \\
\text { Values }\end{array}$ & On Feature Create & On Feature Update \\
\hline OBJECTID & TRUE & FALSE & FALSE & & \\
\hline Shape & FALSE & TRUE & TRUE & & \\
\hline Location Description 1 & TRUE & TRUE & FALSE & & \\
\hline Location Description 2 & TRUE & TRUE & TRUE & & \\
\hline CreationDate & FALSE & TRUE & FALSE & ArcFM Current Date & \\
\hline Created By & FALSE & TRUE & FALSE & ArcFM User Name & \\
\hline UPDATEDATE & FALSE & FALSE & TRUE & & ArcFM Current Date \\
\hline Updated By & FALSE & FALSE & TRUE & & ArcFM User Name \\
\hline Legacy Main Link Value & FALSE & TRUE & TRUE & & \\
\hline $\begin{array}{l}\text { Related Distribution } \\
\text { Main ObjectID }\end{array}$ & FALSE & FALSE & FALSE & $\begin{array}{l}\text { NIPSCO.RetrieveOIDofClosest } \\
\text { GasMain }\end{array}$ & $\begin{array}{l}\text { NIPSCO.RetrieveOIDofClosest } \\
\text { GasMain }\end{array}$ \\
\hline HANDLE & FALSE & TRUE & TRUE & & \\
\hline TILENAME & FALSE & TRUE & TRUE & & \\
\hline
\end{tabular}




\begin{tabular}{|c|c|c|c|c|c|}
\hline DCSID & FALSE & TRUE & TRUE & & \\
\hline $\begin{array}{l}\text { arcfm8.GAS.Gas } \\
\text { ArcFM Display Fi } \\
\text { Create Edit Task: } \\
\text { On Delete Event: } \\
\text { Before Split Even } \\
\text { On Split Event: } \\
\text { After Split Event: } \\
\text { On Abandon Eve } \\
\text { On Create Event: } \\
\text { On Update Event } \\
\text { Abandon Feature } \\
\text { Abandon Subtype } \\
\text { Remove Feature } \\
\text { Remove Subtype }\end{array}$ & $\begin{array}{l}\text { FS Vali } \\
\text { JFS Val } \\
\text { ass: } \\
\text { ss: }\end{array}$ & $\begin{array}{l}\text { ate Wor } \\
\text { date Wo }\end{array}$ & $\begin{array}{l}\text { Order } \\
\text { Order }\end{array}$ & $\begin{array}{l}\text { ber } 1 \\
\text { ber } 1\end{array}$ & \\
\hline Field Alias & Visible & Editable & $\begin{array}{c}\text { Allow } \\
\text { Null } \\
\text { Values }\end{array}$ & On Feature Create & On Feature Update \\
\hline OBJECTID & TRUE & FALSE & FALSE & & \\
\hline Subtype Code & TRUE & TRUE & FALSE & & \\
\hline Shape & FALSE & TRUE & TRUE & & \\
\hline CreationDate & FALSE & TRUE & TRUE & & \\
\hline Created By & FALSE & TRUE & FALSE & & \\
\hline UPDATEDATE & FALSE & TRUE & TRUE & & \\
\hline Updated By & FALSE & TRUE & TRUE & & \\
\hline Legacy Ees Number & FALSE & TRUE & TRUE & & \\
\hline LOA Name & TRUE & FALSE & TRUE & & \\
\hline Tax Unit & TRUE & TRUE & FALSE & & \\
\hline Grid Code & TRUE & TRUE & FALSE & & \\
\hline INSTALLDATE & TRUE & TRUE & FALSE & & \\
\hline Install Work Order & TRUE & TRUE & FALSE & & \\
\hline Install Misc Order Id & TRUE & TRUE & FALSE & & \\
\hline Main Material & TRUE & TRUE & TRUE & & \\
\hline Main Diameter & TRUE & TRUE & TRUE & & \\
\hline Insert Indicator & TRUE & TRUE & FALSE & & \\
\hline DG Grid & FALSE & TRUE & TRUE & & \\
\hline Maintained By & TRUE & TRUE & TRUE & & \\
\hline Bonded Indicator & TRUE & TRUE & FALSE & & \\
\hline Length Source & TRUE & TRUE & TRUE & & \\
\hline Measured Length & TRUE & TRUE & TRUE & & \\
\hline SHAPE.Ien & FALSE & & & & \\
\hline
\end{tabular}

arcfm8.GAS.GasValve

ArcFM Display Field:

VALVENUMBER

Create Edit Task:

On Delete Event:

On Abandon Event:

On Update Event: EDFS Validate Work Order Number 1

On Update Event: NIPSCO.Generic.FieldConcatination

On Update Event:

NIPSCO.CopyValueToRelatedObject

On Create Event: EDFS Validate Work Order Number 1

On Create Event: ArcFM Segment

Split

On Create Event: NIPSCO.Generic.FieldConcatination

On Create Event: NIPSCO.CopyValueToRelatedObject

Abandon Feature Class: arcfm8.GAS.RetiredGasValve

Abandon Subtype:

Remove Feature Class: 
Remove Subtype:

\begin{tabular}{|c|c|c|c|c|c|}
\hline Field Alias & Visible & Editable & $\begin{array}{c}\text { Allow } \\
\text { Null } \\
\text { Values }\end{array}$ & On Feature Create & On Feature Update \\
\hline OBJECTID & TRUE & FALSE & FALSE & & \\
\hline Subtype Code & TRUE & TRUE & FALSE & & \\
\hline INSTALLDATE & TRUE & TRUE & FALSE & & \\
\hline Install Work Order & TRUE & TRUE & FALSE & & \\
\hline Install Misc Order Id & TRUE & TRUE & FALSE & & \\
\hline LOA Name & TRUE & FALSE & TRUE & & \\
\hline Tax Unit & TRUE & TRUE & FALSE & & \\
\hline Grid Code & TRUE & TRUE & FALSE & & \\
\hline Valve Size & TRUE & TRUE & FALSE & & \\
\hline Valve Number & TRUE & TRUE & TRUE & & \\
\hline Open/Closed & TRUE & TRUE & FALSE & & \\
\hline Installation Type & TRUE & TRUE & FALSE & & \\
\hline Location Description 1 & TRUE & TRUE & FALSE & & \\
\hline Location Description 2 & TRUE & TRUE & FALSE & & \\
\hline Emergency Valve & TRUE & TRUE & FALSE & $\begin{array}{l}\text { NIPSCO.EmergencyValvelnd } \\
\text { Field }\end{array}$ & $\begin{array}{l}\text { NIPSCO.EmergencyValvelnd } \\
\text { Field }\end{array}$ \\
\hline Insulated Indicator & TRUE & TRUE & FALSE & & \\
\hline Graphics Scale Factor & FALSE & TRUE & TRUE & & \\
\hline Shape & FALSE & TRUE & TRUE & & \\
\hline AncillaryRole & FALSE & TRUE & TRUE & & \\
\hline Enabled & FALSE & TRUE & TRUE & & \\
\hline CreationDate & FALSE & TRUE & FALSE & ArcFM Current Date & \\
\hline Created By & FALSE & TRUE & FALSE & ArcFM User Name & \\
\hline UPDATEDATE & FALSE & FALSE & TRUE & & ArcFM Current Date \\
\hline Updated By & FALSE & FALSE & TRUE & & ArcFM User Name \\
\hline Gas Trace Weight & FALSE & FALSE & TRUE & $\begin{array}{l}\text { ArcFM Gas Valve Trace } \\
\text { Weight }\end{array}$ & ArcFM Gas Valve Trace Weight \\
\hline Symbol Rotation & FALSE & TRUE & FALSE & & \\
\hline Legacy Node 1 & FALSE & TRUE & TRUE & & \\
\hline Legacy Node 2 & FALSE & TRUE & TRUE & & \\
\hline Legacy Ees Number & FALSE & TRUE & TRUE & & \\
\hline $\begin{array}{l}\text { Symbol Configuration } \\
\text { Code }\end{array}$ & FALSE & TRUE & TRUE & & \\
\hline $\begin{array}{l}\text { Pressure System Status } \\
\text { Code }\end{array}$ & TRUE & TRUE & FALSE & & \\
\hline $\begin{array}{l}\text { Emergency Isolation } \\
\text { System Status Code }\end{array}$ & TRUE & TRUE & FALSE & & \\
\hline $\begin{array}{l}\text { Gas System Status } \\
\text { Code }\end{array}$ & TRUE & TRUE & FALSE & & \\
\hline
\end{tabular}

arcfm8.GAS.GMMS

ArcFM Display Field: SYSTEMNAME

Create Edit Task: ArcFM Manual Angle Setter

On Create Event:

On Update Event:

On Delete Event:

On Abandon Event:

Abandon Feature Class:

Abandon Subtype:

Remove Feature Class:

Remove Subtype:

\begin{tabular}{|l|c|c|c|l|l|}
\hline Field Alias & Visible & Editable & $\begin{array}{c}\text { Allow } \\
\text { Null } \\
\text { Values }\end{array}$ & On Feature Create & On Feature Update \\
\hline Line Section Number & TRUE & TRUE & TRUE & & \\
\hline System Name & TRUE & TRUE & TRUE & & \\
\hline HCA Number & TRUE & TRUE & TRUE & & \\
\hline $\begin{array}{l}\text { Foreign Key to Gas Main } \\
\text { Object Id }\end{array}$ & TRUE & FALSE & TRUE & & \\
\hline OBJECTID & TRUE & FALSE & FALSE & & \\
\hline
\end{tabular}




\begin{tabular}{|l|c|c|c|l|l|}
\hline CreationDate & FALSE & TRUE & TRUE & ArcFM Current Date & \\
\hline Created By & FALSE & TRUE & TRUE & ArcFM User Name & \\
\hline UPDATEDATE & FALSE & FALSE & TRUE & & ArcFM Current Date \\
\hline Updated By & FALSE & FALSE & TRUE & & ArcFM User Name \\
\hline
\end{tabular}

arcfm8.GAS.InsulatedCoupling

ArcFM Display Field: OBJECTID

Create Edit Task:

On Delete Event:

On Abandon Event:

On Update Event: EDFS Validate Work Order Number 1

On Create Event: EDFS Validate Work Order Number 1

On Create Event: ArcFM Segment

Split

Abandon Feature Class: arcfm8.GAS.RetiredInsulatedCoupling

Abandon Subtype:

Remove Feature Class:

Remove Subtype:

\begin{tabular}{|l|c|c|c|l|l|}
\hline Field Alias & Visible & Editable & $\begin{array}{c}\text { Allow } \\
\text { Null } \\
\text { Values }\end{array}$ & On Feature Create & On Feature Update \\
\hline OBJECTID & TRUE & FALSE & FALSE & & \\
\hline Subtype Code & TRUE & TRUE & FALSE & & \\
\hline Shape & FALSE & TRUE & TRUE & & \\
\hline AncillaryRole & FALSE & TRUE & TRUE & & \\
\hline Enabled & FALSE & TRUE & TRUE & & \\
\hline CreationDate & FALSE & TRUE & TRUE & & \\
\hline Created By & FALSE & TRUE & FALSE & & \\
\hline UPDATEDATE & FALSE & TRUE & TRUE & & \\
\hline Updated By & FALSE & TRUE & TRUE & & \\
\hline Legacy Node 1 & FALSE & TRUE & TRUE & & \\
\hline Legacy Node 2 & FALSE & TRUE & TRUE & & \\
\hline Legacy Ees Number & FALSE & TRUE & TRUE & & \\
\hline INSTALLDATE & TRUE & TRUE & FALSE & & NIPSCO.LoaName \\
\hline Install Work Order & TRUE & TRUE & FALSE & & \\
\hline Install Misc Order Id & TRUE & TRUE & FALSE & & \\
\hline LOA Name & TRUE & FALSE & TRUE & NIPSCO.LoaName & \\
\hline Tax Unit & TRUE & TRUE & FALSE & & ArcFM Gas Non-controllable \\
\hline Grid Code & TRUE & TRUE & FALSE & & \\
\hline Gas Trace Weight & FALSE & FALSE & TRUE & $\begin{array}{l}\text { ArcFM Gas Non-controllable } \\
\text { Fitting Trace Weight }\end{array}$ \\
\hline Symbol Rotation & FALSE & FALSE & FALSE & & \\
\hline $\begin{array}{l}\text { Pressure System Status } \\
\text { Code }\end{array}$ & TRUE & TRUE & FALSE & & \\
\hline $\begin{array}{l}\text { Emergency Isolation } \\
\text { System Status Code }\end{array}$ & TRUE & TRUE & FALSE & & \\
\hline $\begin{array}{l}\text { Gas System Status } \\
\text { Code }\end{array}$ & TRUE & TRUE & FALSE & & \\
\hline Graphics Scale Factor & TRUE & TRUE & FALSE & & \\
\hline Insulated Indicator & TRUE & TRUE & FALSE & & \\
\hline
\end{tabular}

arcfm8.GAS.LeakReport

ArcFM Display Field: LEAKID

Create Edit Task:

On Delete Event:

On Abandon Event:

On Create Event: EDFS Validate Work Order Number 1

On Update Event: EDFS Validate Work Order Number 1

Abandon Feature Class:

Abandon Subtype:

Remove Feature Class:

Remove Subtype: 


\begin{tabular}{|c|c|c|c|c|c|}
\hline Field Alias & Visible & Editable & $\begin{array}{c}\text { Allow } \\
\text { Null } \\
\text { Values }\end{array}$ & On Feature Create & On Feature Update \\
\hline OBJECTID & TRUE & FALSE & FALSE & & \\
\hline Subtype Code & TRUE & TRUE & FALSE & & \\
\hline $\begin{array}{l}\text { Leak Identification } \\
\text { Number }\end{array}$ & TRUE & TRUE & TRUE & & \\
\hline INSTALLDATE & TRUE & TRUE & FALSE & & \\
\hline Install Work Order & TRUE & TRUE & FALSE & & \\
\hline Install Misc Order Id & TRUE & TRUE & FALSE & & \\
\hline LOA Name & TRUE & FALSE & FALSE & NIPSCO.LoaName & NIPSCO.LoaName \\
\hline Tax Unit & TRUE & TRUE & FALSE & & \\
\hline Grid Code & TRUE & TRUE & FALSE & & \\
\hline Graphics Scale Factor & FALSE & TRUE & FALSE & & \\
\hline Shape & FALSE & TRUE & TRUE & & \\
\hline CreationDate & FALSE & TRUE & FALSE & ArcFM Current Date & \\
\hline Created By & FALSE & TRUE & FALSE & ArcFM User Name & \\
\hline Updated By & FALSE & FALSE & TRUE & & ArcFM User Name \\
\hline UPDATEDATE & FALSE & FALSE & TRUE & & ArcFM Current Date \\
\hline Symbol Rotation & FALSE & TRUE & FALSE & & \\
\hline Legacy Ees Number & FALSE & TRUE & TRUE & & \\
\hline Related Main OID & FALSE & FALSE & FALSE & $\begin{array}{l}\text { NIPSCO.RetrieveOIDofClosest } \\
\text { GasMain }\end{array}$ & $\begin{array}{l}\text { NIPSCO.RetrieveOIDofClosest } \\
\text { GasMain }\end{array}$ \\
\hline
\end{tabular}

arcfm8.GAS.LeakSurveyArea

ArcFM Display Field: SURVEYAREAID

Create Edit Task:

On Delete Event:

On Abandon Event:

On Create Event: EDFS Validate Work Order Number 1

On Update Event: EDFS Validate Work Order Number 1

Abandon Feature Class:

Abandon Subtype:

Remove Feature Class:

Remove Subtype:

\begin{tabular}{|l|c|c|c|l|l|}
\hline Field Alias & Visible & Editable & $\begin{array}{c}\text { Allow } \\
\text { Null } \\
\text { Values }\end{array}$ & On Feature Create & On Feature Update \\
\hline OBJECTID & TRUE & FALSE & FALSE & & \\
\hline INSTALLDATE & TRUE & TRUE & FALSE & & \\
\hline Install Work Order & TRUE & TRUE & FALSE & & \\
\hline Install Misc Order Id & TRUE & TRUE & FALSE & & NIPSCO.LoaName \\
\hline LOA Name & TRUE & FALSE & FALSE & NIPSCO.LoaName & \\
\hline Tax Unit & TRUE & TRUE & FALSE & & \\
\hline Grid Code & TRUE & TRUE & FALSE & & \\
\hline COMMENTS & TRUE & TRUE & TRUE & & \\
\hline SURVEYDUEDATE & TRUE & TRUE & TRUE & & \\
\hline Survey Area ID & TRUE & TRUE & TRUE & & \\
\hline Survey Frequency & TRUE & TRUE & TRUE & & \\
\hline Area Type & TRUE & TRUE & TRUE & & \\
\hline TARGETDATE & TRUE & TRUE & TRUE & & \\
\hline DATECOMPLETED1 & TRUE & TRUE & TRUE & & \\
\hline DATECOMPLETED2 & TRUE & TRUE & TRUE & & \\
\hline Completed By & TRUE & TRUE & TRUE & & \\
\hline Shape & FALSE & TRUE & TRUE & & ArcFM Current Date \\
\hline CreationDate & FALSE & TRUE & FALSE & ArcFM Current Date & \\
\hline Created By & FALSE & TRUE & FALSE & ArcFM User Name & \\
\hline UPDATEDATE & FALSE & FALSE & TRUE & & \\
\hline Updated By & FALSE & FALSE & TRUE & & \\
\hline Legacy Ees Number & FALSE & TRUE & TRUE & & \\
\hline SHAPE.area & FALSE & & & & \\
\hline SHAPE.len & FALSE & & & & \\
\hline
\end{tabular}

arcfm8.GAS.Meter

ArcFM Display Field: OBJECTID 
Create Edit Task:

On Delete Event:

On Abandon Event:

On Create Event: EDFS Validate Work Order Number 1

On Update Event: EDFS Validate Work Order Number 1

Abandon Feature Class:

Abandon Subtype:

Remove Feature Class:

Remove Subtype:

\begin{tabular}{|l|c|c|c|l|l|}
\hline Field Alias & Visible & Editable & $\begin{array}{c}\text { Allow } \\
\text { Null } \\
\text { Values }\end{array}$ & On Feature Create & On Feature Update \\
\hline INSTALLDATE & TRUE & TRUE & FALSE & & \\
\hline Install Work Order & TRUE & TRUE & FALSE & & \\
\hline Install Misc Order Id & TRUE & TRUE & FALSE & & \\
\hline LOA Name & TRUE & TRUE & FALSE & & \\
\hline Tax Unit & TRUE & TRUE & FALSE & & \\
\hline Grid Code & TRUE & TRUE & FALSE & & \\
\hline Regulator Station OID & FALSE & TRUE & TRUE & & \\
\hline Regulator Station OID & FALSE & TRUE & TRUE & & \\
\hline OBJECTID & TRUE & FALSE & FALSE & & \\
\hline CreationDate & FALSE & TRUE & TRUE & ArcFM Current Date & \\
\hline Created By & FALSE & TRUE & FALSE & ArcFM User Name & \\
\hline UPDATEDATE & FALSE & FALSE & TRUE & & ArcFM Current Date \\
\hline Updated By & FALSE & FALSE & FALSE & & \\
\hline
\end{tabular}

arcfm8.GAS.PipeChange

ArcFM Display Field: OBJECTID

Create Edit Task:

On Delete Event:

On Abandon Event:

On Create Event: NIPSCO.PipeChange.RotateRelate

On Update Event: NIPSCO.PipeChange.RotateRelate

Abandon Feature Class: arcfm8.GAS.RetiredPipeChange

Abandon Subtype:

Remove Feature Class:

Remove Subtype:

\begin{tabular}{|l|c|c|c|l|l|}
\hline Field Alias & Visible & Editable & $\begin{array}{c}\text { Allow } \\
\text { Null } \\
\text { Values }\end{array}$ & On Feature Create & On Feature Update \\
\hline OBJECTID & TRUE & FALSE & FALSE & & \\
\hline Subtype Code & TRUE & TRUE & FALSE & & \\
\hline Shape & FALSE & TRUE & TRUE & & \\
\hline Symbol Rotation & FALSE & TRUE & FALSE & & \\
\hline Related Main OID & FALSE & TRUE & FALSE & & \\
\hline $\begin{array}{l}\text { Legacy Main Vertex } \\
\text { Value }\end{array}$ & FALSE & TRUE & TRUE & & \\
\hline EES & FALSE & TRUE & TRUE & & \\
\hline HANDLE & FALSE & TRUE & TRUE & & \\
\hline TILENAME & FALSE & TRUE & TRUE & & \\
\hline
\end{tabular}

arcfm8.GAS.PipeExposure

ArcFM Display Field: LOCATIONDESCRIPTION

Create Edit Task:

On Delete Event:

On Abandon Event:

On Create Event: EDFS Validate Work Order Number 1

On Update Event: EDFS Validate Work Order Number 1

Abandon Feature Class:

Abandon Subtype: 
Remove Feature Class:

Remove Subtype:

\begin{tabular}{|l|c|c|c|l|l|}
\hline Field Alias & Visible & Editable & $\begin{array}{c}\text { Allow } \\
\text { Null } \\
\text { Values }\end{array}$ & On Feature Create & On Feature Update \\
\hline OBJECTID & TRUE & FALSE & FALSE & & \\
\hline Subtype Code & TRUE & TRUE & FALSE & & \\
\hline INSTALLDATE & TRUE & TRUE & FALSE & & \\
\hline Install Work Order & TRUE & TRUE & FALSE & & \\
\hline Install Misc Order Id & TRUE & TRUE & FALSE & & NIPSCO.LoaName \\
\hline LOA Name & TRUE & FALSE & FALSE & NIPSCO.LoaName & \\
\hline Tax Unit & TRUE & TRUE & FALSE & & \\
\hline Grid Code & TRUE & TRUE & FALSE & & \\
\hline Location Description & TRUE & TRUE & FALSE & & \\
\hline Graphics Scale Factor & FALSE & TRUE & FALSE & & \\
\hline Shape & FALSE & TRUE & TRUE & & ArcFM Current Date \\
\hline CreationDate & FALSE & TRUE & FALSE & ArcFM Current Date & ArcFM User Name \\
\hline Created By & FALSE & TRUE & FALSE & ArcFM User Name & \\
\hline UPDATEDATE & FALSE & FALSE & TRUE & & \\
\hline Updated By & FALSE & FALSE & TRUE & & NasCO.RetrieveOIDofClosest \\
\hline Symbol Rotation & FALSE & TRUE & FALSE & & GasMain \\
\hline Legacy Ees Number & FALSE & TRUE & TRUE & & $\begin{array}{l}\text { NIPSCO.RetrieveOIDofClosest } \\
\text { RelPS }\end{array}$ \\
\hline Related Main OID & FALSE & FALSE & FALSE & GasMain & \\
\hline
\end{tabular}

arcfm8.GAS.PipelineMarker

ArcFM Display Field: MARKERTYPECD

Create Edit Task: NIPSCO.Gas.PlaceAndRelateGasMain

On Delete Event:

On Abandon Event:

On Create Event: EDFS Validate Work Order Number 1

On Create Event: NIPSCO.Gas. RelatedPipeObjectld

On Update Event: EDFS Validate Work Order Number 1

On Update Event: NIPSCO.Gas.RelatedPipeObjectld

Abandon Feature Class:

Abandon Subtype:

Remove Feature Class:

Remove Subtype:

\begin{tabular}{|c|c|c|c|c|c|}
\hline Field Alias & Visible & Editable & $\begin{array}{c}\text { Allow } \\
\text { Null } \\
\text { Values }\end{array}$ & On Feature Create & On Feature Update \\
\hline OBJECTID & TRUE & FALSE & FALSE & & \\
\hline Subtype Code & TRUE & TRUE & FALSE & & \\
\hline INSTALLDATE & TRUE & TRUE & FALSE & & \\
\hline Install Work Order & TRUE & TRUE & FALSE & & \\
\hline Install Misc Order Id & TRUE & TRUE & FALSE & & \\
\hline LOA Name & TRUE & FALSE & FALSE & NIPSCO.LoaName & NIPSCO.LoaName \\
\hline Tax Unit & TRUE & TRUE & FALSE & & \\
\hline Grid Code & TRUE & TRUE & FALSE & & \\
\hline Marker Type & TRUE & TRUE & FALSE & & \\
\hline Marker Number & TRUE & TRUE & TRUE & & \\
\hline Shape & FALSE & TRUE & TRUE & & \\
\hline CreationDate & FALSE & TRUE & FALSE & ArcFM Current Date & \\
\hline Created By & FALSE & TRUE & FALSE & ArcFM User Name & \\
\hline UPDATEDATE & FALSE & FALSE & TRUE & & ArcFM Current Date \\
\hline Updated By & FALSE & FALSE & TRUE & & ArcFM User Name \\
\hline Symbol Rotation & FALSE & TRUE & TRUE & & \\
\hline Legacy Main Link Value & FALSE & TRUE & TRUE & & \\
\hline Legacy Ees Number & FALSE & TRUE & TRUE & & \\
\hline Related Gas Main OID & FALSE & FALSE & FALSE & $\begin{array}{l}\text { NIPSCO.RetrieveOIDofClosest } \\
\text { GasMain }\end{array}$ & $\begin{array}{l}\text { NIPSCO.RetrieveOIDofClosest } \\
\text { GasMain }\end{array}$ \\
\hline
\end{tabular}

arcfm8.GAS.PressureControlFitting

ArcFM Display Field: OBJECTID 
Create Edit Task:

On Delete Event:

On Abandon Event:

On Update Event: EDFS Validate Work Order Number 1

On Create Event: EDFS Validate Work Order Number 1

On Create Event: ArcFM Segment Split

Abandon Feature Class: arcfm8.GAS.RetiredPressureControlFitting

Abandon Subtype:

Remove Feature Class:

Remove Subtype:

\begin{tabular}{|c|c|c|c|c|c|}
\hline Field Alias & Visible & Editable & $\begin{array}{c}\text { Allow } \\
\text { Null } \\
\text { Values }\end{array}$ & On Feature Create & On Feature Update \\
\hline OBJECTID & TRUE & FALSE & FALSE & & \\
\hline Subtype Code & TRUE & TRUE & FALSE & & \\
\hline Shape & FALSE & TRUE & TRUE & & \\
\hline AncillaryRole & FALSE & TRUE & TRUE & & \\
\hline Enabled & FALSE & TRUE & TRUE & & \\
\hline CreationDate & FALSE & TRUE & TRUE & & \\
\hline Created By & FALSE & TRUE & FALSE & & \\
\hline UPDATEDATE & FALSE & TRUE & TRUE & & \\
\hline Updated By & FALSE & TRUE & TRUE & & \\
\hline Legacy Node 1 & FALSE & TRUE & TRUE & & \\
\hline Legacy Node 2 & FALSE & TRUE & TRUE & & \\
\hline Legacy Ees Number & FALSE & TRUE & TRUE & & \\
\hline INSTALLDATE & TRUE & TRUE & FALSE & & \\
\hline Install Work Order & TRUE & TRUE & FALSE & & \\
\hline Install Misc Order Id & TRUE & TRUE & FALSE & & \\
\hline LOA Name & TRUE & FALSE & TRUE & NIPSCO.LoaName & NIPSCO.LoaName \\
\hline Tax Unit & TRUE & TRUE & FALSE & & \\
\hline Grid Code & TRUE & TRUE & FALSE & & \\
\hline Gas Trace Weight & FALSE & FALSE & TRUE & $\begin{array}{l}\text { ArcFM Gas Non-controllable } \\
\text { Fitting Trace Weight }\end{array}$ & $\begin{array}{l}\text { ArcFM Gas Non-controllable } \\
\text { Fitting Trace Weight }\end{array}$ \\
\hline Symbol Rotation & FALSE & FALSE & FALSE & & \\
\hline $\begin{array}{l}\text { Pressure System Status } \\
\text { Code }\end{array}$ & TRUE & TRUE & FALSE & & \\
\hline $\begin{array}{l}\text { Emergency Isolation } \\
\text { System Status Code }\end{array}$ & TRUE & TRUE & FALSE & & \\
\hline $\begin{array}{l}\text { Gas System Status } \\
\text { Code }\end{array}$ & TRUE & TRUE & FALSE & & \\
\hline Graphics Scale Factor & TRUE & TRUE & FALSE & & \\
\hline Location Description 1 & TRUE & TRUE & TRUE & & \\
\hline Location Description 2 & TRUE & TRUE & TRUE & & \\
\hline
\end{tabular}

arcfm8.GAS.Regulator

ArcFM Display Field: OBJECTID

Create Edit Task:

On Delete Event:

On Abandon Event:

On Create Event: EDFS Validate Work Order Number 1

On Update Event: EDFS Validate Work Order Number 1

Abandon Feature Class:

Abandon Subtype:

Remove Feature Class:

Remove Subtype:

\begin{tabular}{|l|c|c|c|c|c|}
\hline Field Alias & Visible & Editable & $\begin{array}{c}\text { Allow } \\
\text { Null } \\
\text { Values }\end{array}$ & On Feature Create & On Feature Update \\
\hline INSTALLDATE & TRUE & TRUE & FALSE & & \\
\hline Install Work Order & TRUE & TRUE & FALSE & & \\
\hline Install Misc Order Id & TRUE & TRUE & FALSE & & \\
\hline LOA Name & TRUE & TRUE & FALSE & & \\
\hline Tax Unit & TRUE & TRUE & FALSE & & \\
\hline
\end{tabular}




\begin{tabular}{|l|c|c|c|l|l|}
\hline Grid Code & TRUE & TRUE & FALSE & & \\
\hline Regulator Station OID & FALSE & TRUE & TRUE & & \\
\hline Take Station OID & FALSE & TRUE & TRUE & & \\
\hline OBJECTID & TRUE & FALSE & FALSE & & \\
\hline CreationDate & FALSE & TRUE & TRUE & ArcFM Current Date & \\
\hline Created By & FALSE & TRUE & FALSE & ArcFM User Name & \\
\hline UPDATEDATE & FALSE & FALSE & TRUE & & ArcFM Current Date \\
\hline Updated By & FALSE & FALSE & FALSE & & ArcFM User Name \\
\hline
\end{tabular}

arcfm8.GAS.RegulatorStation

ArcFM Display Field: REGULATORSTATIONNUMBER

Create Edit Task: NIPSCO.Gas.PlaceAndRelateGasMain

On Create Event: EDFS Validate Work Order Number 1

On Create Event: ArcFM Segment

Split

On Create Event: NIPSCO.Gas.RelatedPipeObjectld

On Update Event: EDFS Validate Work Order Number 1

On Update Event: ArcFM Segment

Split

On Update Event: NIPSCO.Gas.RelatedPipeObjectld

On Delete Event:

On Abandon Event:

Abandon Feature Class: arcfm8.GAS.RetiredRegulatorStation

Abandon Subtype:

Remove Feature Class:

Remove Subtype:

\begin{tabular}{|c|c|c|c|c|c|}
\hline Field Alias & Visible & Editable & \begin{tabular}{|c|} 
Allow \\
Null \\
Values \\
\end{tabular} & On Feature Create & On Feature Update \\
\hline OBJECTID & TRUE & FALSE & FALSE & & \\
\hline Subtype Code & TRUE & TRUE & FALSE & & \\
\hline INSTALLDATE & TRUE & TRUE & FALSE & & \\
\hline Install Work Order & TRUE & TRUE & FALSE & & \\
\hline Install Misc Order Id & TRUE & TRUE & \begin{tabular}{|l|} 
FALSE \\
\end{tabular} & & \\
\hline LOA Name & FALSE & TRUE & TRUE & & \\
\hline Tax Unit & TRUE & TRUE & FALSE & & \\
\hline Grid Code & TRUE & TRUE & FALSE & & \\
\hline $\begin{array}{l}\text { Regulator Station } \\
\text { Number }\end{array}$ & TRUE & TRUE & FALSE & & \\
\hline Seq Num & TRUE & TRUE & FALSE & & \\
\hline Installation Type & TRUE & TRUE & TRUE & & \\
\hline Graphics Scale Factor & FALSE & TRUE & TRUE & & \\
\hline Inlet Pressure & FALSE & TRUE & TRUE & & \\
\hline Outlet Pressure & FALSE & TRUE & TRUE & & \\
\hline Shape & FALSE & TRUE & TRUE & & \\
\hline AncillaryRole & FALSE & TRUE & TRUE & & \\
\hline Enabled & FALSE & TRUE & TRUE & & \\
\hline CreationDate & FALSE & TRUE & TRUE & ArcFM Current Date & \\
\hline Created By & FALSE & TRUE & FALSE & ArcFM User Name & \\
\hline UPDATEDATE & FALSE & FALSE & \begin{tabular}{|l|} 
TRUE \\
\end{tabular} & & ArcFM Current Date \\
\hline Updated By & FALSE & FALSE & FALSE & & ArcFM User Name \\
\hline Gas Trace Weight & FALSE & FALSE & TRUE & $\begin{array}{l}\text { ArcFM Gas Regulator Trace } \\
\text { Weight }\end{array}$ & $\begin{array}{l}\text { ArcFM Gas Regulator Trace } \\
\text { Weight }\end{array}$ \\
\hline Symbol Rotation & FALSE & FALSE & FALSE & & \\
\hline Legacy Node 1 & FALSE & TRUE & TRUE & & \\
\hline Legacy Node 2 & FALSE & TRUE & TRUE & & \\
\hline Legacy Ees Number & FALSE & TRUE & \begin{tabular}{|l|} 
TRUE \\
\end{tabular} & & \\
\hline $\begin{array}{l}\text { Related Upstream Gas } \\
\text { Main OID }\end{array}$ & FALSE & TRUE & TRUE & & \\
\hline $\begin{array}{l}\text { Pressure System Status } \\
\text { Code }\end{array}$ & TRUE & TRUE & FALSE & & \\
\hline \begin{tabular}{|l|} 
Emergency Isolation \\
System Status Code
\end{tabular} & TRUE & TRUE & FALSE & & \\
\hline
\end{tabular}




\begin{tabular}{|l|c|c|c|c|c|}
\hline $\begin{array}{l}\text { Gas System Status } \\
\text { Code }\end{array}$ & TRUE & TRUE & FALSE & & \\
\hline Insulated Indicator & TRUE & TRUE & FALSE & & \\
\hline Customer Name & TRUE & TRUE & TRUE & & \\
\hline
\end{tabular}

arcfm8.GAS.RetiredCasing

ArcFM Display Field: OBJECTID

Create Edit Task:

On Create Event:

On Update Event:

On Delete Event:

Before Split Event:

On Split Event:

After Split Event:

On Abandon Event:

Abandon Feature Class:

Abandon Subtype:

Remove Feature Class:

Remove Subtype:

\begin{tabular}{|l|c|c|c|c|c|}
\hline Field Alias & Visible & Editable & $\begin{array}{c}\text { Allow } \\
\text { Null } \\
\text { Values }\end{array}$ & On Feature Create & On Feature Update \\
\hline OBJECTID & TRUE & FALSE & FALSE & & \\
\hline Subtype Code & TRUE & TRUE & TRUE & & \\
\hline Shape & TRUE & TRUE & TRUE & & \\
\hline CreationDate & TRUE & TRUE & TRUE & & \\
\hline Created By & TRUE & TRUE & TRUE & & \\
\hline UPDATEDATE & TRUE & TRUE & TRUE & & \\
\hline Updated By & TRUE & TRUE & TRUE & & \\
\hline Legacy Ees Number & TRUE & TRUE & TRUE & & \\
\hline LOA Name & TRUE & TRUE & TRUE & & \\
\hline Tax Unit & TRUE & TRUE & TRUE & & \\
\hline Grid Code & TRUE & TRUE & TRUE & & \\
\hline INSTALLDATE & TRUE & TRUE & TRUE & & \\
\hline Install Work Order & TRUE & TRUE & TRUE & & \\
\hline Install Misc Order Id & TRUE & TRUE & TRUE & & \\
\hline RETIREDATE & TRUE & TRUE & TRUE & & \\
\hline Retire Work Order & TRUE & TRUE & TRUE & & \\
\hline Retire Misc Order Id & TRUE & TRUE & TRUE & & \\
\hline RetireType & TRUE & TRUE & TRUE & & \\
\hline Legacy Main Link Value & TRUE & TRUE & TRUE & & \\
\hline Casing Diameter & TRUE & TRUE & TRUE & & \\
\hline Installed Length & TRUE & TRUE & TRUE & & \\
\hline Length Source & TRUE & TRUE & TRUE & & \\
\hline $\begin{array}{l}\text { RR Crossing Agreement } \\
\text { Number }\end{array}$ & TRUE & TRUE & TRUE & & \\
\hline Related Main OID & TRUE & TRUE & TRUE & & \\
\hline CP Section OID & TRUE & TRUE & TRUE & & \\
\hline SHAPE.len & FALSE & & & & \\
\hline
\end{tabular}

arcfm8.GAS.RetiredDeadEndGas

ArcFM Display Field: OBJECTID

Create Edit Task:

On Create Event:

On Update Event:

On Delete Event:

On Abandon Event:

Abandon Feature Class:

Abandon Subtype:

Remove Feature Class:

Remove Subtype: 


\begin{tabular}{|l|l|l|l|l|l|}
\hline Field Alias & Visible & Editable & $\begin{array}{c}\text { Allow } \\
\text { Null } \\
\text { Values }\end{array}$ & On Feature Create & On Feature Update \\
\hline OBJECTID & TRUE & FALSE & FALSE & & \\
\hline Subtype Field & TRUE & TRUE & TRUE & & \\
\hline Shape & TRUE & TRUE & TRUE & & \\
\hline CreationDate & TRUE & TRUE & TRUE & & \\
\hline Created By & TRUE & TRUE & TRUE & & \\
\hline UPDATEDATE & TRUE & TRUE & TRUE & & \\
\hline Updated By & TRUE & TRUE & TRUE & & \\
\hline Legacy Ees Number & TRUE & TRUE & TRUE & & \\
\hline LOA Name & TRUE & TRUE & TRUE & & \\
\hline Tax Unit & TRUE & TRUE & TRUE & & \\
\hline Grid Code & TRUE & TRUE & TRUE & & \\
\hline INSTALLDATE & TRUE & TRUE & TRUE & & \\
\hline Install Work Order & TRUE & TRUE & TRUE & & \\
\hline Install Misc Order Id & TRUE & TRUE & TRUE & & \\
\hline RETIREDATE & TRUE & TRUE & TRUE & & \\
\hline Retire Work Order & TRUE & TRUE & TRUE & & \\
\hline Retire Misc Order Id & TRUE & TRUE & TRUE & & \\
\hline RetireType & TRUE & TRUE & TRUE & & \\
\hline Main Link Value & TRUE & TRUE & TRUE & & \\
\hline Location Description 1 & TRUE & TRUE & TRUE & & \\
\hline Location Description 2 & TRUE & TRUE & TRUE & & \\
\hline Symbol Rotation & TRUE & TRUE & TRUE & & \\
\hline Legacy Node 1 & TRUE & TRUE & TRUE & & \\
\hline Legacy Node 2 & TRUE & TRUE & TRUE & & \\
\hline
\end{tabular}

arcfm8.GAS.RetiredDrip

ArcFM Display Field: OBJECTID

Create Edit Task:

On Create Event:

On Update Event:

On Delete Event:

On Abandon Event:

Abandon Feature Class:

Abandon Subtype:

Remove Feature Class:

Remove Subtype:

\begin{tabular}{|l|l|l|l|l|l|}
\hline Field Alias & Visible & Editable & $\begin{array}{c}\text { Allow } \\
\text { Null } \\
\text { Values }\end{array}$ & On Feature Create & On Feature Update \\
\hline OBJECTID & TRUE & FALSE & FALSE & & \\
\hline Subtype Code & TRUE & TRUE & TRUE & & \\
\hline Shape & TRUE & TRUE & TRUE & & \\
\hline CreationDate & TRUE & TRUE & TRUE & & \\
\hline Created By & TRUE & TRUE & TRUE & & \\
\hline UPDATEDATE & TRUE & TRUE & TRUE & & \\
\hline Updated By & TRUE & TRUE & TRUE & & \\
\hline Legacy Ees Number & TRUE & TRUE & TRUE & & \\
\hline LOA Name & TRUE & TRUE & TRUE & & \\
\hline Tax Unit & TRUE & TRUE & TRUE & & \\
\hline Grid Code & TRUE & TRUE & TRUE & & \\
\hline INSTALLDATE & TRUE & TRUE & TRUE & & \\
\hline Install Work Order & TRUE & TRUE & TRUE & & \\
\hline Install Misc Order Id & TRUE & TRUE & TRUE & & \\
\hline RETIREDATE & TRUE & TRUE & TRUE & & \\
\hline Retire Work Order & TRUE & TRUE & TRUE & & \\
\hline Retire Misc Order Id & TRUE & TRUE & TRUE & & \\
\hline RetireType & TRUE & TRUE & TRUE & & \\
\hline Legacy Main Link Value & TRUE & TRUE & TRUE & & \\
\hline Drip Pot Type & TRUE & TRUE & TRUE & & \\
\hline Symbol Rotation & TRUE & TRUE & TRUE & & \\
\hline Related Main OID & TRUE & TRUE & TRUE & & \\
\hline
\end{tabular}


arcfm8.GAS.RetiredGasMain

Create Edit Task:

On Create Event:

On Update Event:

On Delete Event:

Before Split Event:

On Split Event:

After Split Event:

On Abandon Event:

Abandon Feature Class:

Abandon Subtype:

Remove Feature Class:

Remove Subtype:

\begin{tabular}{|c|c|c|c|c|c|}
\hline Field Alias & Visible & Editable & \begin{tabular}{|c|} 
Allow \\
Null \\
Values
\end{tabular} & On Feature Create & On Feature Update \\
\hline OBJECTID & TRUE & FALSE & FALSE & & \\
\hline Subtype Code & TRUE & TRUE & FALSE & & \\
\hline Shape & TRUE & TRUE & TRUE & & \\
\hline CreationDate & TRUE & TRUE & TRUE & & \\
\hline Created By & TRUE & TRUE & FALSE & & \\
\hline UPDATEDATE & TRUE & TRUE & TRUE & & \\
\hline Updated By & TRUE & TRUE & TRUE & & \\
\hline Legacy Ees Number & TRUE & TRUE & TRUE & & \\
\hline LOA Name & TRUE & TRUE & TRUE & & \\
\hline Tax Unit & TRUE & TRUE & TRUE & & \\
\hline Grid Code & TRUE & TRUE & TRUE & & \\
\hline INSTALLDATE & TRUE & TRUE & TRUE & & \\
\hline Install Work Order & TRUE & TRUE & FALSE & & \\
\hline Install Misc Order Id & TRUE & TRUE & TRUE & & \\
\hline RETIREDATE & TRUE & TRUE & TRUE & & \\
\hline Retire Work Order & TRUE & TRUE & TRUE & & \\
\hline Retire Misc Order Id & TRUE & TRUE & TRUE & & \\
\hline RetireType & TRUE & TRUE & TRUE & & \\
\hline Legacy Node 1 & TRUE & TRUE & TRUE & & \\
\hline Legacy Node 2 & TRUE & TRUE & TRUE & & \\
\hline $\begin{array}{l}\text { Upstream Calculated } \\
\text { Pressure }\end{array}$ & TRUE & TRUE & TRUE & & \\
\hline CP System OID & TRUE & TRUE & \begin{tabular}{|l|} 
TRUE \\
\end{tabular} & & \\
\hline Measured Length & TRUE & TRUE & TRUE & & \\
\hline Maintained By & TRUE & TRUE & TRUE & & \\
\hline Main Material & TRUE & TRUE & FALSE & & \\
\hline Main Diameter & TRUE & TRUE & TRUE & & \\
\hline Length Source & TRUE & TRUE & TRUE & & \\
\hline Insert Indicator & TRUE & TRUE & FALSE & & \\
\hline $\begin{array}{l}\text { Downstream Calculated } \\
\text { Pressure }\end{array}$ & TRUE & TRUE & TRUE & & \\
\hline Calculated Flow & TRUE & TRUE & TRUE & & \\
\hline Bonded Indicator & TRUE & TRUE & FALSE & & \\
\hline SHAPE.len & FALSE & & & & \\
\hline
\end{tabular}

arcfm8.GAS.RetiredGasMainLocation

ArcFM Display Field: OBJECTID

arcfm8.GAS. RetiredGasValve

ArcFM Display Field: OBJECTID

Create Edit Task:

On Create Event:

On Update Event:

On Delete Event:

On Abandon Event:

Abandon Feature Class:

Abandon Subtype: 
Remove Feature Class:

Remove Subtype:

\begin{tabular}{|c|c|c|c|c|c|}
\hline Field Alias & Visible & Editable & $\begin{array}{c}\text { Allow } \\
\text { Null } \\
\text { Values }\end{array}$ & On Feature Create & On Feature Update \\
\hline OBJECTID & TRUE & FALSE & FALSE & & \\
\hline Subtype Code & TRUE & TRUE & TRUE & & \\
\hline Shape & TRUE & TRUE & TRUE & & \\
\hline CreationDate & TRUE & TRUE & \begin{tabular}{|l|} 
TRUE \\
\end{tabular} & & \\
\hline Created By & TRUE & TRUE & TRUE & & \\
\hline UPDATEDATE & TRUE & TRUE & TRUE & & \\
\hline Updated By & TRUE & TRUE & \begin{tabular}{|l|} 
TRUE \\
\end{tabular} & & \\
\hline Legacy Ees Number & TRUE & TRUE & TRUE & & \\
\hline LOA Name & TRUE & TRUE & TRUE & & \\
\hline Tax Unit & TRUE & TRUE & TRUE & & \\
\hline Grid Code & TRUE & TRUE & TRUE & & \\
\hline \begin{tabular}{|l} 
INSTALLDATE \\
\end{tabular} & TRUE & TRUE & TRUE & & \\
\hline Install Work Order & TRUE & TRUE & TRUE & & \\
\hline Install Misc Order Id & TRUE & TRUE & TRUE & & \\
\hline RETIREDATE & TRUE & TRUE & \begin{tabular}{|l|} 
TRUE \\
\end{tabular} & & \\
\hline Retire Work Order & TRUE & TRUE & TRUE & & \\
\hline Retire Misc Order Id & TRUE & TRUE & TRUE & & \\
\hline RetireType & TRUE & TRUE & TRUE & & \\
\hline Legacy Node 1 & TRUE & TRUE & \begin{tabular}{|l|} 
TRUE \\
\end{tabular} & & \\
\hline \begin{tabular}{|l|} 
Legacy Node 2 \\
\end{tabular} & TRUE & TRUE & TRUE & & \\
\hline Symbol Rotation & TRUE & TRUE & TRUE & & \\
\hline Graphics Scale Factor & TRUE & TRUE & TRUE & & \\
\hline Valve Number & TRUE & TRUE & TRUE & & \\
\hline Valve Size & TRUE & TRUE & TRUE & & \\
\hline Location Description 1 & TRUE & TRUE & \begin{tabular}{|l|} 
TRUE \\
\end{tabular} & & \\
\hline Location Description 2 & TRUE & TRUE & TRUE & & \\
\hline Insulated Indicator & TRUE & TRUE & TRUE & & \\
\hline Open/Closed & TRUE & TRUE & TRUE & & \\
\hline Emergency Valve & TRUE & TRUE & TRUE & & \\
\hline $\begin{array}{l}\text { Symbol Configuration } \\
\text { Code }\end{array}$ & TRUE & TRUE & TRUE & & \\
\hline \begin{tabular}{|l} 
Installation Type \\
\end{tabular} & TRUE & TRUE & TRUE & & \\
\hline
\end{tabular}

arcfm8.GAS.RetiredlnsulatedCoupling

ArcFM Display Field: OBJECTID

Create Edit Task:

On Create Event:

On Update Event:

On Delete Event:

On Abandon Event:

Abandon Feature Class:

Abandon Subtype:

Remove Feature Class:

Remove Subtype:

\begin{tabular}{|l|c|c|c|c|c|}
\hline Field Alias & Visible & Editable & $\begin{array}{c}\text { Allow } \\
\text { Null } \\
\text { Values }\end{array}$ & On Feature Create & On Feature Update \\
\hline OBJECTID & TRUE & FALSE & FALSE & & \\
\hline Subtype Code & TRUE & TRUE & TRUE & & \\
\hline Shape & TRUE & TRUE & TRUE & & \\
\hline CreationDate & TRUE & TRUE & TRUE & & \\
\hline Created By & TRUE & TRUE & TRUE & & \\
\hline UPDATEDATE & TRUE & TRUE & TRUE & & \\
\hline Updated By & TRUE & TRUE & TRUE & & \\
\hline Legacy Ees Number & TRUE & TRUE & TRUE & & \\
\hline LOA Name & TRUE & TRUE & TRUE & & \\
\hline Tax Unit & TRUE & TRUE & TRUE & & \\
\hline Grid Code & TRUE & TRUE & TRUE & & \\
\hline INSTALLDATE & TRUE & TRUE & TRUE & & \\
\hline
\end{tabular}




\begin{tabular}{|l|c|c|c|l|l|}
\hline Install Work Order & TRUE & TRUE & TRUE & & \\
\hline Install Misc Order Id & TRUE & TRUE & TRUE & & \\
\hline RETIREDATE & TRUE & TRUE & TRUE & & \\
\hline Retire Work Order & TRUE & TRUE & TRUE & & \\
\hline Retire Misc Order Id & TRUE & TRUE & TRUE & & \\
\hline RetireType & TRUE & TRUE & TRUE & & \\
\hline Legacy Node 1 & TRUE & TRUE & TRUE & & \\
\hline Legacy Node 2 & TRUE & TRUE & TRUE & & \\
\hline Symbol Rotation & TRUE & TRUE & TRUE & & \\
\hline Graphics Scale Factor & TRUE & TRUE & TRUE & & \\
\hline Insulated Indicator & TRUE & TRUE & TRUE & & \\
\hline
\end{tabular}

arcfm8.GAS.RetiredPipeChange

ArcFM Display Field: OBJECTID

arcfm8.GAS. RetiredPressureControlFitting

ArcFM Display Field: OBJECTID

Create Edit Task:

On Create Event:

On Update Event:

On Delete Event:

On Abandon Event:

Abandon Feature Class:

Abandon Subtype:

Remove Feature Class:

Remove Subtype:

\begin{tabular}{|l|l|l|l|l|l|}
\hline Field Alias & Visible & Editable & $\begin{array}{c}\text { Allow } \\
\text { Null } \\
\text { Values }\end{array}$ & On Feature Create & On Feature Update \\
\hline OBJECTID & TRUE & FALSE & FALSE & & \\
\hline Subtype Code & TRUE & TRUE & TRUE & & \\
\hline Shape & TRUE & TRUE & TRUE & & \\
\hline CreationDate & TRUE & TRUE & TRUE & & \\
\hline Created By & TRUE & TRUE & TRUE & & \\
\hline UPDATEDATE & TRUE & TRUE & TRUE & & \\
\hline Updated By & TRUE & TRUE & TRUE & & \\
\hline Legacy Ees Number & TRUE & TRUE & TRUE & & \\
\hline LOA Name & TRUE & TRUE & TRUE & & \\
\hline Tax Unit & TRUE & TRUE & TRUE & & \\
\hline Grid Code & TRUE & TRUE & TRUE & & \\
\hline INSTALLDATE & TRUE & TRUE & TRUE & & \\
\hline Install Work Order & TRUE & TRUE & TRUE & & \\
\hline Install Misc Order Id & TRUE & TRUE & TRUE & & \\
\hline RETIREDATE & TRUE & TRUE & TRUE & & \\
\hline Retire Work Order & TRUE & TRUE & TRUE & & \\
\hline Retire Misc Order Id & TRUE & TRUE & TRUE & & \\
\hline RetireType & TRUE & TRUE & TRUE & & \\
\hline Legacy Node 1 & TRUE & TRUE & TRUE & & \\
\hline Legacy Node 2 & TRUE & TRUE & TRUE & & \\
\hline Symbol Rotation & TRUE & TRUE & TRUE & & \\
\hline Graphics Scale Factor & TRUE & TRUE & TRUE & & \\
\hline Location Description 1 & TRUE & TRUE & TRUE & & \\
\hline Location Description 2 & TRUE & TRUE & TRUE & & \\
\hline
\end{tabular}

arcfm8.GAS.RetiredRegulatorStation

ArcFM Display Field: OBJECTID

Create Edit Task:

On Create Event:

On Update Event:

On Delete Event:

On Abandon Event:

Abandon Feature Class:

Abandon Subtype:

Remove Feature Class: 
Remove Subtype:

\begin{tabular}{|c|c|c|c|c|c|}
\hline Field Alias & Visible & Editable & $\begin{array}{c}\text { Allow } \\
\text { Null } \\
\text { Values }\end{array}$ & On Feature Create & On Feature Update \\
\hline OBJECTID & TRUE & FALSE & FALSE & & \\
\hline Subtype Code & TRUE & TRUE & TRUE & & \\
\hline Shape & TRUE & TRUE & TRUE & & \\
\hline CreationDate & TRUE & TRUE & TRUE & & \\
\hline Created By & TRUE & TRUE & TRUE & & \\
\hline UPDATEDATE & TRUE & TRUE & TRUE & & \\
\hline Updated By & TRUE & TRUE & TRUE & & \\
\hline Legacy Ees Number & TRUE & TRUE & TRUE & & \\
\hline LOA Name & TRUE & TRUE & TRUE & & \\
\hline Tax Unit & TRUE & TRUE & TRUE & & \\
\hline Grid Code & TRUE & TRUE & TRUE & & \\
\hline \begin{tabular}{|l|} 
INSTALLDATE \\
\end{tabular} & TRUE & TRUE & TRUE & & \\
\hline Install Work Order & TRUE & TRUE & TRUE & & \\
\hline Install Misc Order Id & TRUE & TRUE & TRUE & & \\
\hline RETIREDATE & TRUE & TRUE & TRUE & & \\
\hline Retire Work Order & TRUE & TRUE & TRUE & & \\
\hline Retire Misc Order Id & TRUE & TRUE & TRUE & & \\
\hline RetireType & TRUE & TRUE & TRUE & & \\
\hline Legacy Node 1 & TRUE & TRUE & TRUE & & \\
\hline Legacy Node 2 & TRUE & TRUE & TRUE & & \\
\hline Symbol Rotation & TRUE & TRUE & TRUE & & \\
\hline Graphics Scale Factor & TRUE & TRUE & TRUE & & \\
\hline Reg Sta Num & TRUE & TRUE & TRUE & & \\
\hline Seq Num & TRUE & TRUE & TRUE & & \\
\hline Inlet Pressure & TRUE & TRUE & TRUE & & \\
\hline Outlet Pressure & TRUE & TRUE & TRUE & & \\
\hline Installation Type & TRUE & TRUE & TRUE & & \\
\hline Insulated Indicator & TRUE & TRUE & TRUE & & \\
\hline $\begin{array}{l}\text { Related Upstream Gas } \\
\text { Main OID }\end{array}$ & TRUE & TRUE & TRUE & & \\
\hline Customer Name & TRUE & TRUE & TRUE & & \\
\hline
\end{tabular}

arcfm8.GAS.RetiredTakeStation

ArcFM Display Field: OBJECTID

Create Edit Task:

On Create Event:

On Update Event:

On Delete Event:

On Abandon Event:

Abandon Feature Class:

Abandon Subtype:

Remove Feature Class:

Remove Subtype:

\begin{tabular}{|l|c|c|c|c|c|}
\hline Field Alias & Visible & Editable & $\begin{array}{c}\text { Allow } \\
\text { Null } \\
\text { Values }\end{array}$ & On Feature Create & On Feature Update \\
\hline OBJECTID & TRUE & FALSE & FALSE & & \\
\hline Subtype Code & TRUE & TRUE & TRUE & & \\
\hline Shape & TRUE & TRUE & TRUE & & \\
\hline CreationDate & TRUE & TRUE & TRUE & & \\
\hline Created By & TRUE & TRUE & TRUE & & \\
\hline UPDATEDATE & TRUE & TRUE & TRUE & & \\
\hline Updated By & TRUE & TRUE & TRUE & & \\
\hline Legacy Ees Number & TRUE & TRUE & TRUE & & \\
\hline LOA Name & TRUE & TRUE & TRUE & & \\
\hline Tax Unit & TRUE & TRUE & TRUE & & \\
\hline Grid Code & TRUE & TRUE & TRUE & & \\
\hline INSTALLDATE & TRUE & TRUE & TRUE & & \\
\hline Install Work Order & TRUE & TRUE & TRUE & & \\
\hline Install Misc Order Id & TRUE & TRUE & TRUE & & \\
\hline
\end{tabular}




\begin{tabular}{|l|c|c|c|l|l|}
\hline RETIREDATE & TRUE & TRUE & TRUE & & \\
\hline Retire Work Order & TRUE & TRUE & TRUE & & \\
\hline Retire Misc Order Id & TRUE & TRUE & TRUE & & \\
\hline RetireType & TRUE & TRUE & TRUE & & \\
\hline Legacy Node 1 & TRUE & TRUE & TRUE & & \\
\hline Legacy Node 2 & TRUE & TRUE & TRUE & & \\
\hline Symbol Rotation & TRUE & TRUE & TRUE & & \\
\hline Graphics Scale Factor & TRUE & TRUE & TRUE & & \\
\hline Reg Sta Num & TRUE & TRUE & TRUE & & \\
\hline Seq Num & TRUE & TRUE & TRUE & & \\
\hline Inlet Pressure & TRUE & TRUE & TRUE & & \\
\hline Outlet Pressure & TRUE & TRUE & TRUE & & \\
\hline Installation Type & TRUE & TRUE & TRUE & & \\
\hline Insulated Indicator & TRUE & TRUE & TRUE & & \\
\hline $\begin{array}{l}\text { Pipeline Operator } \\
\text { Number }\end{array}$ & TRUE & TRUE & TRUE & & \\
\hline Supplier Name & TRUE & TRUE & TRUE & & \\
\hline
\end{tabular}

arcfm8.GAS.RetiredWorkOrderLeaderLine

ArcFM Display Field: OBJECTID

arcfm8.GAS.SqueezeOff

ArcFM Display Field: SubtypeCd

Create Edit Task:

On Create Event: ArcFM Create Squeeze Off

On Update Event:

On Delete Event:

Metadata Editor:

Custom Configuration

Editor:

Extended Data Definition Table:

On Abandon Event:

Abandon Feature Class:

Abandon Subtype:

Remove Feature Class:

Remove Subtype:

\begin{tabular}{|l|c|c|c|l|l|}
\hline Field Alias & Visible & Editable & $\begin{array}{c}\text { Allow } \\
\text { Null } \\
\text { Values }\end{array}$ & On Feature Create & On Feature Update \\
\hline OBJECTID & TRUE & FALSE & FALSE & & \\
\hline Subtype Code & TRUE & TRUE & FALSE & & \\
\hline Gas Trace Weight & FALSE & FALSE & TRUE & $\begin{array}{l}\text { ArcFM Gas Squeeze Off Trace } \\
\text { Weight }\end{array}$ & $\begin{array}{l}\text { ArcFM Gas Squeeze Off Trace } \\
\text { Weight }\end{array}$ \\
\hline Open/Closed & TRUE & TRUE & FALSE & & \\
\hline Symbol Rotation & FALSE & FALSE & FALSE & ArcFM Auto Angle Setter & ArcFM Auto Angle Setter \\
\hline Shape & FALSE & TRUE & TRUE & & \\
\hline AncillaryRole & FALSE & TRUE & TRUE & & \\
\hline Enabled & FALSE & TRUE & TRUE & & \\
\hline CreationDate & FALSE & TRUE & FALSE & ArcFM Current Date & \\
\hline Created By & FALSE & TRUE & FALSE & ArcFM User Name & \\
\hline UpdateDate & FALSE & FALSE & TRUE & & ArcFM Current Date \\
\hline Updated By & FALSE & FALSE & FALSE & & ArcFM User Name \\
\hline Graphics Scale Factor & FALSE & TRUE & TRUE & & \\
\hline
\end{tabular}

arcfm8.GAS.TakeStation

ArcFM Display Field: REGULATORSTATIONNUMBER

Create Edit Task:

On Delete Event:

On Abandon Event:

On Create Event: EDFS Validate Work Order Number 1

On Create Event: ArcFM Segment

Split

On Update Event: EDFS Validate Work Order Number 1

Abandon Feature Class: arcfm8.GAS.RetiredTakeStation 


\section{Abandon Subtype:}

Remove Feature

Class:

Remove Subtype:

\begin{tabular}{|c|c|c|c|c|c|}
\hline Field Alias & Visible & Editable & $\begin{array}{c}\text { Allow } \\
\text { Null } \\
\text { Values }\end{array}$ & On Feature Create & On Feature Update \\
\hline OBJECTID & TRUE & FALSE & FALSE & & \\
\hline Subtype Code & TRUE & TRUE & FALSE & & \\
\hline INSTALLDATE & TRUE & TRUE & FALSE & & \\
\hline Install Work Order & TRUE & TRUE & FALSE & & \\
\hline InstallMiscOrderld & TRUE & TRUE & FALSE & & \\
\hline LOA Name & TRUE & FALSE & TRUE & NIPSCO.LoaName & NIPSCO.LoaName \\
\hline Tax Unit & TRUE & TRUE & FALSE & & \\
\hline Grid Code & TRUE & TRUE & FALSE & & \\
\hline Reg Sta Num & TRUE & TRUE & FALSE & & \\
\hline Seq Num & TRUE & TRUE & FALSE & & \\
\hline Installation Type & TRUE & TRUE & TRUE & & \\
\hline Graphics Scale Factor & FALSE & TRUE & TRUE & & \\
\hline Inlet Pressure & FALSE & TRUE & TRUE & & \\
\hline Outlet Pressure & FALSE & TRUE & TRUE & & \\
\hline $\begin{array}{l}\text { Pipeline Operator } \\
\text { Number }\end{array}$ & TRUE & TRUE & TRUE & & \\
\hline Supplier Name & TRUE & TRUE & TRUE & & \\
\hline Shape & FALSE & TRUE & TRUE & & \\
\hline AncillaryRole & FALSE & TRUE & TRUE & & \\
\hline Enabled & FALSE & TRUE & TRUE & & \\
\hline CreationDate & FALSE & TRUE & TRUE & ArcFM Current Date & \\
\hline Created By & FALSE & TRUE & FALSE & ArcFM User Name & \\
\hline UPDATEDATE & FALSE & FALSE & TRUE & & ArcFM Current Date \\
\hline Updated By & FALSE & FALSE & TRUE & & ArcFM User Name \\
\hline Gas Trace Weight & FALSE & FALSE & TRUE & ArcFM Gas TBS Trace Weight & ArcFM Gas TBS Trace Weight \\
\hline Symbol Rotation & FALSE & FALSE & FALSE & & \\
\hline Legacy Node 1 & FALSE & TRUE & TRUE & & \\
\hline Legacy Node 2 & FALSE & TRUE & TRUE & & \\
\hline Legacy Ees Number & FALSE & TRUE & TRUE & & \\
\hline $\begin{array}{l}\text { Pressure System Status } \\
\text { Code }\end{array}$ & TRUE & TRUE & FALSE & & \\
\hline $\begin{array}{l}\text { Emergency Isolation } \\
\text { System Status Code }\end{array}$ & TRUE & TRUE & FALSE & & \\
\hline $\begin{array}{l}\text { Gas System Status } \\
\text { Code }\end{array}$ & TRUE & TRUE & FALSE & & \\
\hline Insulated Indicator & TRUE & TRUE & FALSE & & \\
\hline
\end{tabular}


Table E-5. Contains all gas ArcFM model name assignments for both the base and custom configuration

Casing

Object Class Model Names

\begin{tabular}{|l|l|}
\hline Object Class & Model Name \\
\hline Casing & CASING \\
\hline Casing & EDFS WORKORDER \\
\hline Casing & LOCATABLEOBJECT \\
\hline Casing & MMABANDONABLE \\
\hline
\end{tabular}

Field Model Names

\begin{tabular}{|l|l|}
\hline Field & Model Name \\
\hline CREATIONDATE & AT_CREATIONDATE \\
\hline CREATIONUSERID & AT_CREATIONUSERID \\
\hline UPDATEDATE & AT_UPDATEDATE \\
\hline UPDATEUSERID & AT_UPDATEUSERID \\
\hline LOANUMBER & AT_LOANUMBER \\
\hline TAXUNITCD & AT_TAXUNITCD \\
\hline GRIDCD & AT_GRIDCD \\
\hline INSTALLDATE & AT_INSTALLDATE \\
\hline INSTALLWORKORDERNUMBER & AT_INSTALLWORKORDERNUMBER \\
\hline INSTALLWORKORDERNUMBER & WORKORDERNUMBER \\
\hline SUBTYPECD & AT_SUBTYPECD \\
\hline SUBTYPECD & SUBTYPECD \\
\hline CASINGDIAMETERVALUE & AT_1 \\
\hline INSTALLEDLENGTHVALUE & AT_2 \\
\hline LENGTHSOURCECD & AT_3 \\
\hline RRCROSSINGAGREEMENTNUMBER & AT_4 \\
\hline RRCROSSINGAGREEMENTNUMBER & LOCATABLEFIELD \\
\hline RELMAINOBJECTID & RELMAINOBJECTID \\
\hline SHAPE & AT_SHAPE \\
\hline INSTALLMISCORDERID & AT_INSTALLMISCORDERID \\
\hline INSTALLMISCORDERID & MISCORDERID \\
\hline
\end{tabular}

Casing_Anno

Object Class Model Names

\begin{tabular}{|l|l|}
\hline Object Class & Model Name \\
\hline Casing_Anno & MMABANDONABLE \\
\hline
\end{tabular}

Field Model Names

\begin{tabular}{|l|l|}
\hline Field & Model Name \\
\hline FEATUREID & AT_1 \\
\hline ZORDER & AT_2 \\
\hline ANNOTATIONCLASSID & AT_3 \\
\hline ELEMENT & AT_4 \\
\hline SHAPE & AT_SHAPE \\
\hline
\end{tabular}

CpRectifier 
Object Class Model Names

\begin{tabular}{|l|l|}
\hline Object Class & Model Name \\
\hline CpRectifier & EDFS WORKORDER \\
\hline CpRectifier & LOCATABLEOBJECT \\
\hline CpRectifier & RECTIFIER \\
\hline
\end{tabular}

Field Model Names

\begin{tabular}{|l|l|}
\hline Field & Model Name \\
\hline OBJECTID & LOCATABLEFIELD \\
\hline LOANUMBER & LOANAME \\
\hline INSTALLWORKORDERNUMBER & WORKORDERNUMBER \\
\hline SUBTYPECD & SUBTYPECD \\
\hline INSTALLMISCORDERID & MISCORDERID \\
\hline
\end{tabular}

CpSection

Object Class Model Names

\begin{tabular}{|l|l|}
\hline Object Class & Model Name \\
\hline CpSection & CPSECTION \\
\hline CpSection & LOCATABLEOBJECT \\
\hline
\end{tabular}

Field Model Names

\begin{tabular}{|l|l|}
\hline Field & Model Name \\
\hline LOANUMBER & LOANAME \\
\hline GRIDCD & DGGRIDCD \\
\hline SUBTYPECD & SUBTYPECD \\
\hline CORROSIONSECTIONNUMBER & CORROSIONSECTIONNUMBER \\
\hline CORROSIONCONTROLNUMBER & CORROSIONCONTROLNUMBER \\
\hline
\end{tabular}

CpTestPoint

Object Class Model Names

\begin{tabular}{|l|l|}
\hline Object Class & Model Name \\
\hline CpTestPoint & EDFS WORKORDER \\
\hline CpTestPoint & LOCATABLEOBJECT \\
\hline
\end{tabular}

Field Model Names

\begin{tabular}{|l|l|}
\hline Field & Model Name \\
\hline OBJECTID & LOCATABLEFIELD \\
\hline LOANUMBER & LOANAME \\
\hline INSTALLWORKORDERNUMBER & WORKORDERNUMBER \\
\hline SUBTYPECD & SUBTYPECD \\
\hline INSTALLMISCORDERID & MISCORDERID \\
\hline
\end{tabular}

DeadEnd_Location_Anno

Object Class Model Names

\begin{tabular}{|l|l|}
\hline Object Class & Model Name \\
\hline DeadEnd_Location_Anno & MMABANDONABLE \\
\hline
\end{tabular}

Field Model Names

\begin{tabular}{|l|l|}
\hline Field & Model Name \\
\hline
\end{tabular}




\begin{tabular}{|l|l|}
\hline FEATUREID & AT_1 \\
\hline ZORDER & AT_2 \\
\hline ANNOTATIONCLASSID & AT_3 \\
\hline ELEMENT & AT_4 \\
\hline SHAPE & AT_SHAPE \\
\hline
\end{tabular}

DeadEndGas

Object Class Model Names

Object Class

DeadEndGas

DeadEndGas

DeadEndGas

\begin{tabular}{|l|}
\hline Model Name \\
\hline DEADEND \\
\hline EDFS WORKORDER \\
\hline MMABANDONABLE \\
\hline
\end{tabular}

Field Model Names

\begin{tabular}{|l|l|}
\hline Field & Model Name \\
\hline CREATIONDATE & AT_CREATIONDATE \\
\hline CREATIONUSERID & AT_CREATIONUSER \\
\hline UPDATEDATE & AT_UPDATEDATE \\
\hline UPDATEUSERID & AT_UPDATEUSERID \\
\hline LOANUMBER & AT_LOANUMBER \\
\hline LOANUMBER & LOANAME \\
\hline TAXUNITCD & AT_TAXUNITCD \\
\hline GRIDCD & AT_GRIDCD \\
\hline INSTALLDATE & AT_INSTALLDATE \\
\hline INSTALLWORKORDERNUMBER & AT_INSTALLWORKORDERNUMBER \\
\hline INSTALLWORKORDERNUMBER & WORKORDERNUMBER \\
\hline SUBTYPECD & AT_SUBTYPECD \\
\hline LOCATIONDESC1 & AT_LOCATIONDESC1 \\
\hline LOCATIONDESC2 & AT_LOCATIONDESC2 \\
\hline SYMBOLROTATIONVALUE & AT_SYMBOLROTATIONVALUE \\
\hline SHAPE & AT_SHAPE \\
\hline INSTALLMISCORDERID & AT_INSTALLMISCORDERID \\
\hline INSTALLMISCORDERID & MISCORDERID \\
\hline
\end{tabular}

DistributionMain_Size_Anno

Object Class Model Names

Object Class

DistributionMain_Size_Anno

\section{Model Name}

MMABANDONABLE

Field Model Names

\begin{tabular}{|l|l|}
\hline Field & Model Name \\
\hline FEATUREID & AT_1 \\
\hline ZORDER & AT_2 \\
\hline ANNOTATIONCLASSID & AT_3 \\
\hline ELEMENT & AT_4 \\
\hline SHAPE & AT_SHAPE \\
\hline
\end{tabular}

Drip

Object Class Model Names 


\begin{tabular}{|l|l|}
\hline Object Class & Model Name \\
\hline Drip & DRIP \\
\hline Drip & EDFS WORKORDER \\
\hline Drip & LOCATABLEOBJECT \\
\hline Drip & MMABANDONABLE \\
\hline
\end{tabular}

Field Model Names

\begin{tabular}{|l|l|}
\hline Field & Model Name \\
\hline OBJECTID & LOCATABLEFIELD \\
\hline CREATIONDATE & AT_CREATIONDATE \\
\hline CREATIONUSERID & AT_CREATIONUSERID \\
\hline UPDATEDATE & AT_UPDATEDATE \\
\hline UPDATEUSERID & AT_UPDATEUSERID \\
\hline LOANUMBER & AT_LOANUMBER \\
\hline LOANUMBER & LOANAME \\
\hline LOANUMBER & LOANUMBER \\
\hline TAXUNITCD & AT_TAXUNITCD \\
\hline GRIDCD & AT_GRIDCD \\
\hline INSTALLDATE & AT_INSTALLDATE \\
\hline INSTALLWORKORDERNUMBER & AT_INSTALLWORKORDERNUMBER \\
\hline INSTALLWORKORDERNUMBER & WORKORDERNUMBER \\
\hline SUBTYPECD & AT_SUBTYPECD \\
\hline SUBTYPECD & SUBTYPECD \\
\hline DRIPPOTTYPE & AT_1 \\
\hline SYMBOLROTATIONVALUE & AT_SYMBOLROTATIONVALUE \\
\hline SHAPE & AT_SHAPE \\
\hline INSTALLMISCORDERID & AT_INSTALLMISCORDERID \\
\hline INSTALLMISCORDERID & MISCORDERID \\
\hline
\end{tabular}

EmergencyValveMarker

Object Class Model Names

\begin{tabular}{|l|l|}
\hline Object Class & Model Name \\
\hline EmergencyValveMarker & EMERGENCYVALVEMARKER \\
\hline EmergencyValveMarker & NIPSCOTARGETCLASS \\
\hline
\end{tabular}

Field Model Names

\begin{tabular}{|l|l|}
\hline Field & Model Name \\
\hline SUBTYPECD & SUBTYPECD \\
\hline SYMBOLROTATIONVALUE & SYMBOLROTATIONVALUE \\
\hline GRAPHICSSCALEFACTORVALUE & GRAPHICSSCALEFACTOR \\
\hline LABELTEXT & NIPSCOTARGETFIELD \\
\hline LABELTEXT & LABELTEXT \\
\hline SHAPE & SHAPE \\
\hline
\end{tabular}

EmergencyValveMarker_Anno

Object Class Model Names

\begin{tabular}{|l|l|}
\hline Object Class & Model Name \\
\hline EmergencyValveMarker_Anno & EMERGENCYVALVEMARKERANNO
\end{tabular}


Field Model Names

Field

Model Name

GasDiscrepancy

Object Class Model Names

\begin{tabular}{|l|l|}
\hline Object Class & Model Name \\
\hline GasDiscrepancy & LOCATABLEOBJECT \\
\hline
\end{tabular}

Field Model Names

\begin{tabular}{|l|l|}
\hline Field & Model Name \\
\hline SUBTYPECD & SUBTYPECD \\
\hline ERRORDESCRIPTION & LOCATABLEFIELD \\
\hline
\end{tabular}

GasMain

Object Class Model Names

\begin{tabular}{|l|l|}
\hline Object Class & Model Name \\
\hline GasMain & DISTRIBUTIONMAIN \\
\hline GasMain & EDFS WORKORDER \\
\hline GasMain & GASMAIN \\
\hline GasMain & LINE \\
\hline GasMain & LOCATABLEOBJECT \\
\hline GasMain & MMABANDONABLE \\
\hline GasMain & PIPE \\
\hline GasMain & SERVICE \\
\hline GasMain & SPLITTARGET \\
\hline
\end{tabular}

Field Model Names

\begin{tabular}{|l|l|}
\hline Field & Model Name \\
\hline OBJECTID & LOCATABLEFIELD \\
\hline CREATIONDATE & AT_CREATIONDATE \\
\hline CREATIONUSERID & AT_CREATIONUSERID \\
\hline UPDATEDATE & AT_UPDATEDATE \\
\hline UPDATEUSERID & AT_UPDATEUSERID \\
\hline INSTALLDATE & AT_INSTALLDATE \\
\hline INSTALLWORKORDERNUMBER & AT_INSTALLWORKORDERNUMBER \\
\hline INSTALLWORKORDERNUMBER & WORKORDERNUMBER \\
\hline LOANUMBER & AT_LOANUMBER \\
\hline LOANUMBER & LOANAME \\
\hline TAXUNITCD & AT_TAXUNITCD \\
\hline GRIDCD & AT_GRIDCD \\
\hline SUBTYPECD & SUBTYPECD \\
\hline SUBTYPECD & AT_SUBTYPECD \\
\hline UPSTREAMCALCPRESSUREVALUE & AT_1 \\
\hline RELCPSECTIONOBJECTID & RELCPSECTIONOBJECTID \\
\hline MEASUREDLENGTHVALUE & AT_2 \\
\hline MAINTAINEDBYNAME & AT_MAINTAINEDBYNAME \\
\hline MAINMATERIALCD & AT_MAINMATERIALCD \\
\hline MAINMATERIALCD & PIPECHANGEMATERIAL \\
\hline MAINDIAMETERVALUE & AT_MAINDIAMETERVALUE \\
\hline
\end{tabular}




\begin{tabular}{|l|l|}
\hline MAINDIAMETERVALUE & PIPEDIAMETER \\
\hline MAINDIAMETERVALUE & SIZE \\
\hline LENGTHSOURCECD & AT_3 \\
\hline INSERTIND & AT_INSERTIND \\
\hline GASTRACEWEIGHT & GASTRACEWEIGHT \\
\hline DOWNSTREAMCALCPRESSUREVALUE & AT_4 \\
\hline CALCULATEDFLOWVALUE & AT_5 \\
\hline BONDEDIND & AT_6 \\
\hline BONDEDIND & BONDEDINDICATOR \\
\hline SHAPE & AT_SHAPE \\
\hline ARCFMMAINMATERIALTYPE & MATERIAL \\
\hline INSTALLMISCORDERID & AT_INSTALLMISCORDERID \\
\hline INSTALLMISCORDERID & MISCORDERID \\
\hline
\end{tabular}

GasMainLocation

Object Class Model Names

\section{Object Class}

GasMainLocation

GasMainLocation

Model Name
GASMAINLOCATION
MMABANDONABLE

Field Model Names

\begin{tabular}{|l|l|}
\hline Field & Model Name \\
\hline CREATIONDATE & AT_CREATIONDATE \\
\hline CREATIONUSERID & AT_CREATIONUSER \\
\hline UPDATEDATE & AT_UPDATEDATE \\
\hline UPDATEUSERID & AT_UPDATEUSERID \\
\hline LOCATIONDESC1 & AT_LOCATIONDESC1 \\
\hline LOCATIONDESC2 & AT_LOCATIONDESC2 \\
\hline RELGASMAINOID & RELATEDGASMAINOID \\
\hline SHAPE & AT_SHAPE \\
\hline
\end{tabular}

GasMainLocation_Anno

Object Class Model Names

\begin{tabular}{|l|l|}
\hline Object Class & Model Name \\
\hline GasMainLocation_Anno & MMABANDONABLE \\
\hline
\end{tabular}

Field Model Names

\begin{tabular}{|l|l|}
\hline Field & Model Name \\
\hline FEATUREID & AT_1 \\
\hline ZORDER & AT_2 \\
\hline ANNOTATIONCLASSID & AT_3 \\
\hline ELEMENT & AT_4 \\
\hline SHAPE & AT_SHAPE \\
\hline
\end{tabular}

\section{GasService}

Object Class Model Names

\begin{tabular}{|l|l|}
\hline Object Class & Model Name \\
\hline GasService & EDFS WORKORDER \\
\hline
\end{tabular}


Field Model Names

\begin{tabular}{|l|l|}
\hline Field & Model Name \\
\hline INSTALLWORKORDERNUMBER & WORKORDERNUMBER \\
\hline INSTALLMISCORDERID & MISCORDERID \\
\hline
\end{tabular}

GasValve

Object Class Model Names

\begin{tabular}{|l|l|}
\hline Object Class & Model Name \\
\hline GasValve & EDFS WORKORDER \\
\hline GasValve & GASVALVE \\
\hline GasValve & LOCATABLEOBJECT \\
\hline GasValve & MMABANDONABLE \\
\hline GasValve & VALVE \\
\hline
\end{tabular}

Field Model Names

\begin{tabular}{|l|l|}
\hline Field & Model Name \\
\hline CREATIONDATE & AT_CREATIONDATE \\
\hline CREATIONUSERID & AT_CREATIONUSERID \\
\hline UPDATEDATE & AT_UPDATEDATE \\
\hline UPDATEUSERID & AT_UPDATEUSERID \\
\hline INSTALLDATE & AT_INSTALLDATE \\
\hline INSTALLWORKORDERNUMBER & AT_INSTALLWORKORDERNUMBER \\
\hline INSTALLWORKORDERNUMBER & WORKORDERNUMBER \\
\hline LOANUMBER & AT_LOANUMBER \\
\hline TAXUNITCD & AT_TAXUNITCD \\
\hline GRIDCD & AT_GRIDCD \\
\hline GASTRACEWEIGHT & GASTRACEWEIGHT \\
\hline SYMBOLROTATIONVALUE & AT_SYMBOLROTATIONVALUE \\
\hline SYMBOLROTATIONVALUE & SYMBOLROTATION \\
\hline SYMBOLROTATIONVALUE & SYMBOLROTATIONVALUE \\
\hline GASPRESSURESYSTEMSTATUSCD & GASPRESSURESYSTEMSTATUS \\
\hline EMERISOLATIONSYSTEMSTATUSCD & EMERISOLATIONSYSSTATUS \\
\hline GASSYSTEMSTATUSCD & GASSYSTEMSTATUS \\
\hline GRAPHICSSCALEFACTORVALUE & AT_GRAPHICSCALEFACTORVALUE \\
\hline GRAPHICSSCALEFACTORVALUE & GRAPHICSSCALEFACTOR \\
\hline SUBTYPECD & SUBTYPECD \\
\hline SUBTYPECD & AT_SUBTYPECD \\
\hline VALVENUMBER & AT_1 \\
\hline VALVENUMBER & LOCATABLEFIELD \\
\hline VALVENUMBER & NIPSCOSOURCEFIELD \\
\hline VALVENUMBER & VALVENUMBER \\
\hline VALVESIZEVALUE & AT_2 \\
\hline LOCATIONDESC1 & AT_LOCATIONDESC1 \\
\hline LOCATIONDESC2 & AT_LOCATIONDESC2 \\
\hline INSULATEDIND & AT_3 \\
\hline INSULATEDIND & CPSYSTEMSTATUS \\
\hline INSULATEDIND & INPUTFIELD4 \\
\hline INSULATEDIND & INSULATEDIND \\
\hline OPENCLOSEDSTATUSCD & AT_4 \\
\hline
\end{tabular}




\begin{tabular}{|l|l|}
\hline OPENCLOSEDSTATUSCD & INPUTFIELD1 \\
\hline OPENCLOSEDSTATUSCD & NORMALPOSITION \\
\hline OPENCLOSEDSTATUSCD & OPENCLOSEDSTATUSCD \\
\hline EMERGENCYVALVEIND & AT_5 \\
\hline EMERGENCYVALVEIND & EMERGENCYVALVEIND \\
\hline EMERGENCYVALVEIND & INPUTFIELD3 \\
\hline SYMBOLCONFIGURATIONCD & AT_6 \\
\hline SYMBOLCONFIGURATIONCD & OUTPUTFIELD \\
\hline SYMBOLCONFIGURATIONCD & SYMBOLOGYCONFIGURATIONCD \\
\hline INSTALLATIONTYPECD & AT_7 \\
\hline INSTALLATIONTYPECD & INPUTFIELD2 \\
\hline INSTALLATIONTYPECD & INSTALLATIONTYPECD \\
\hline SHAPE & AT_SHAPE \\
\hline SHAPE & SHAPE \\
\hline INSTALLMISCORDERID & AT_INSTALLMISCORDERID \\
\hline INSTALLMISCORDERID & MISCORDERID \\
\hline
\end{tabular}

GasValve_Location_Anno

Object Class Model Names

\section{Object Class}

GasValve_Location_Anno

\section{Model Name}

MMABANDONABLE

Field Model Names

\begin{tabular}{|l|l|}
\hline Field & Model Name \\
\hline FEATUREID & AT_1 \\
\hline ZORDER & AT_2 \\
\hline ANNOTATIONCLASSID & AT_3 \\
\hline ELEMENT & AT_4 \\
\hline SHAPE & AT_SHAPE \\
\hline
\end{tabular}

GasValve_Size_Anno

Object Class Model Names

Object Class

GasValve_Size_Anno

Model Name

MMABANDONABLE

Field Model Names

\begin{tabular}{|l|l|}
\hline Field & Model Name \\
\hline FEATUREID & AT_1 \\
\hline ZORDER & AT_2 \\
\hline ANNOTATIONCLASSID & AT_3 \\
\hline ELEMENT & AT_4 \\
\hline SHAPE & AT_SHAPE \\
\hline
\end{tabular}

InsulatedCoupling

Object Class Model Names

\section{Object Class}

InsulatedCoupling

InsulatedCoupling

InsulatedCoupling

\begin{tabular}{l} 
Model Name \\
\hline EDFS WORKORDER \\
\hline FITTING \\
\hline LOCATABLEOBJECT \\
\hline
\end{tabular}




\begin{tabular}{|l|l|}
\hline InsulatedCoupling & MMABANDONABLE \\
\hline InsulatedCoupling & NONCONTROLFITTING \\
\hline
\end{tabular}

Field Model Names

\begin{tabular}{|l|l|}
\hline Field & Model Name \\
\hline OBJECTID & LOCATABLEFIELD \\
\hline CREATIONDATE & AT_CREATIONDATE \\
\hline CREATIONDATE & DATE \\
\hline CREATIONUSERID & AT_CREATIONUSERID \\
\hline UPDATEDATE & AT_UPDATEDATE \\
\hline UPDATEUSERID & AT_UPDATEUSERID \\
\hline INSTALLDATE & AT_INSTALLDATE \\
\hline INSTALLWORKORDERNUMBER & AT_INSTALLWORKORDERNUMBER \\
\hline INSTALLWORKORDERNUMBER & WORKORDERNUMBER \\
\hline LOANUMBER & AT_LOANUMBER \\
\hline LOANUMBER & LOANAME \\
\hline TAXUNITCD & AT_TAXUNITCD \\
\hline GRIDCD & AT_GRIDCD \\
\hline GASTRACEWEIGHT & GASTRACEWEIGHT \\
\hline SYMBOLROTATIONVALUE & AT_SYMBOLROTATIONVALUE \\
\hline SYMBOLROTATIONVALUE & SYMBOLROTATION \\
\hline GASPRESSURESYSTEMSTATUSCD & GASPRESSURESYSTEMSTATUS \\
\hline EMERISOLATIONSYSTEMSTATUSCD & EMERISOLATIONSYSSTATUS \\
\hline GASSYSTEMSTATUSCD & GASSYSTEMSTATUS \\
\hline GRAPHICSSCALEFACTORVALUE & AT_GRAPHICSCALEFACTORVALUE \\
\hline SUBTYPECD & AT_SUBTYPECD \\
\hline SUBTYPECD & SUBTYPECD \\
\hline INSULATEDIND & AT_1 \\
\hline INSULATEDIND & CPSYSTEMSTATUS \\
\hline SHAPE & AT_SHAPE \\
\hline INSTALLMISCORDERID & AT_INSTALLMISCORDERID \\
\hline INSTALLMISCORDERID & MISCORDERID \\
\hline
\end{tabular}

LeakReport

Object Class Model Names

\begin{tabular}{|l|l|}
\hline Object Class & Model Name \\
\hline LeakReport & EDFS WORKORDER \\
\hline LeakReport & LOCATABLEOBJECT \\
\hline
\end{tabular}

Field Model Names

\begin{tabular}{|l|l|}
\hline Field & Model Name \\
\hline OBJECTID & LOCATABLEFIELD \\
\hline LOANUMBER & LOANAME \\
\hline INSTALLWORKORDERNUMBER & WORKORDERNUMBER \\
\hline SUBTYPECD & SUBTYPECD \\
\hline INSTALLMISCORDERID & MISCORDERID \\
\hline
\end{tabular}




\begin{tabular}{|l|l|}
\hline Object Class & Model Name \\
\hline LeakSurveyArea & EDFS WORKORDER \\
\hline LeakSurveyArea & LEAKSURVEYAREA \\
\hline
\end{tabular}

Field Model Names

\begin{tabular}{|l|l|}
\hline Field & Model Name \\
\hline INSTALLWORKORDERNUMBER & WORKORDERNUMBER \\
\hline COMMENTS & COMMENTS \\
\hline INSTALLMISCORDERID & MISCORDERID \\
\hline
\end{tabular}

Meter

Object Class Model Names

\begin{tabular}{|l|l|}
\hline Object Class & Model Name \\
\hline Meter & EDFS WORKORDER \\
\hline
\end{tabular}

Field Model Names

\begin{tabular}{|l|l|}
\hline Field & Model Name \\
\hline INSTALLWORKORDERNUMBER & WORKORDERNUMBER \\
\hline INSTALLMISCORDERID & MISCORDERID \\
\hline
\end{tabular}

PCF_Location_Anno

Object Class Model Names

\begin{tabular}{|l|l|}
\hline Object Class & Model Name \\
\hline PCF_Location_Anno & MMABANDONABLE \\
\hline
\end{tabular}

Field Model Names

\begin{tabular}{|l|l|}
\hline Field & Model Name \\
\hline FEATUREID & AT_1 \\
\hline ZORDER & AT_2 \\
\hline ANNOTATIONCLASSID & AT_3 \\
\hline ELEMENT & AT_4 \\
\hline SHAPE & AT_SHAPE \\
\hline
\end{tabular}

PipeChange

Object Class Model Names

\begin{tabular}{|l|l|}
\hline Object Class & Model Name \\
\hline PipeChange & LOCATABLEOBJECT \\
\hline PipeChange & MMABANDONABLE \\
\hline PipeChange & PIPECHANGE \\
\hline
\end{tabular}

Field Model Names

\begin{tabular}{|l|l|}
\hline Field & Model Name \\
\hline OBJECTID & LOCATABLEFIELD \\
\hline SUBTYPECD & AT_SUBTYPECD \\
\hline SUBTYPECD & SUBTYPECD \\
\hline SYMBOLROTATIONVALUE & AT_SYMBOLROTATIONVALUE \\
\hline SYMBOLROTATIONVALUE & SYMBOLROTATION \\
\hline RELGASMAINOBJECTID & RELGASMAINOBJECTID \\
\hline SHAPE & AT_SHAPE \\
\hline
\end{tabular}


PipeExposure

Object Class Model Names

\begin{tabular}{|l|l|}
\hline Object Class & Model Name \\
\hline PipeExposure & EDFS WORKORDER \\
\hline PipeExposure & LOCATABLEOBJECT \\
\hline
\end{tabular}

Field Model Names

\begin{tabular}{|l|l|}
\hline Field & Model Name \\
\hline OBJECTID & LOCATABLEFIELD \\
\hline INSTALLWORKORDERNUMBER & WORKORDERNUMBER \\
\hline SUBTYPECD & SUBTYPECD \\
\hline INSTALLMISCORDERID & MISCORDERID \\
\hline
\end{tabular}

PipelineMarker

Object Class Model Names

\begin{tabular}{|l|l|}
\hline Object Class & Model Name \\
\hline PipelineMarker & EDFS WORKORDER \\
\hline PipelineMarker & LOCATABLEOBJECT \\
\hline
\end{tabular}

Field Model Names

\begin{tabular}{|l|l|}
\hline Field & Model Name \\
\hline OBJECTID & LOCATABLEFIELD \\
\hline LOANUMBER & LOANAME \\
\hline INSTALLDATE & DATE \\
\hline INSTALLWORKORDERNUMBER & WORKORDERNUMBER \\
\hline SUBTYPECD & SUBTYPECD \\
\hline SYMBOLROTATIONVALUE & SYMBOLROTATION \\
\hline RELGASMAINOBJECTID & RELATEDGASMAINOID \\
\hline INSTALLMISCORDERID & MISCORDERID \\
\hline
\end{tabular}

PressureControlFitting

Object Class Model Names

\section{Object Class}

PressureControlFitting

PressureControlFitting

PressureControlFitting

PressureControlFitting

PressureControlFitting

\begin{tabular}{|l|}
\hline Model Name \\
\hline EDFS WORKORDER \\
\hline FITTING \\
\hline LOCATABLEOBJECT \\
\hline MMABANDONABLE \\
\hline NONCONTROLFITTING \\
\hline
\end{tabular}

Field Model Names

\begin{tabular}{|l|l|}
\hline Field & Model Name \\
\hline OBJECTID & LOCATABLEFIELD \\
\hline CREATIONDATE & AT_CREATIONDATE \\
\hline CREATIONDATE & DATE \\
\hline CREATIONUSERID & AT_CREATIONUSERID \\
\hline INSTALLDATE & AT_INSTALLDATE \\
\hline INSTALLWORKORDERNUMBER & AT_INSTALLWORKORDERNUMBER \\
\hline INSTALLWORKORDERNUMBER & WORKORDERNUMBER \\
\hline
\end{tabular}




\begin{tabular}{|l|l|}
\hline LOANUMBER & AT_LOANUMBER \\
\hline LOANUMBER & LOANAME \\
\hline TAXUNITCD & AT_TAXUNITCD \\
\hline GRIDCD & AT_GRIDCD \\
\hline GASTRACEWEIGHT & GASTRACEWEIGHT \\
\hline SYMBOLROTATIONVALUE & AT_SYMBOLROTATIONVALUE \\
\hline SYMBOLROTATIONVALUE & SYMBOLROTATION \\
\hline GASPRESSURESYSTEMSTATUSCD & GASPRESSURESYSTEMSTATUS \\
\hline EMERISOLATIONSYSTEMSTATUSCD & EMERISOLATIONSYSSTATUS \\
\hline GASSYSTEMSTATUSCD & GASPRESSURESYSTEMSTATUS \\
\hline GRAPHICSSCALEFACTORVALUE & AT_GRAPHICSCALEFACTORVALUE \\
\hline SUBTYPECD & SUBTYPECD \\
\hline SUBTYPECD & AT_SUBTYPECD \\
\hline LOCATIONDESC1 & AT_LOCATIONDESC1 \\
\hline LOCATIONDESC2 & AT_LOCATIONDESC2 \\
\hline SHAPE & AT_SHAPE \\
\hline INSTALLMISCORDERID & MISCORDERID \\
\hline INSTALLMISCORDERID & AT_INSTALLMISCORDERID \\
\hline
\end{tabular}

Regulator

Object Class Model Names

Object Class

Regulator

Regulator

Model Name

EDFS WORKORDER

REGULATOR

Field Model Names

\begin{tabular}{|l|l|}
\hline Field & Model Name \\
\hline INSTALLWORKORDERNUMBER & WORKORDERNUMBER \\
\hline INSTALLMISCORDERID & MISCORDERID \\
\hline
\end{tabular}

RegulatorStation

Object Class Model Names

\begin{tabular}{|l|l|}
\hline Object Class & Model Name \\
\hline RegulatorStation & EDFS WORKORDER \\
\hline RegulatorStation & LOCATABLEOBJECT \\
\hline RegulatorStation & MMABANDONABLE \\
\hline RegulatorStation & REGSTN \\
\hline RegulatorStation & REGULATOR \\
\hline
\end{tabular}

Field Model Names

\begin{tabular}{|l|l|}
\hline Field & Model Name \\
\hline CREATIONDATE & AT_CREATIONDATE \\
\hline CREATIONUSERID & AT_CREATIONUSERID \\
\hline UPDATEDATE & AT_UPDATEDATE \\
\hline UPDATEUSERID & AT_UPDATEUSERID \\
\hline INSTALLDATE & AT_INSTALLDATE \\
\hline INSTALLWORKORDERNUMBER & AT_INSTALLWORKORDERNUMBER \\
\hline INSTALLWORKORDERNUMBER & WORKORDERNUMBER \\
\hline LOANUMBER & AT_LOANUMBER \\
\hline
\end{tabular}




\begin{tabular}{|l|l|}
\hline TAXUNITCD & AT_TAXUNITCD \\
\hline GRIDCD & AT_GRIDCD \\
\hline GASTRACEWEIGHT & GASTRACEWEIGHT \\
\hline SYMBOLROTATIONVALUE & AT_SYMBOLROTATIONVALUE \\
\hline GASPRESSURESYSTEMSTATUSCD & GASPRESSURESYSTEMSTATUS \\
\hline EMERISOLATIONSYSTEMSTATUSCD & EMERISOLATIONSYSSTATUS \\
\hline GASSYSTEMSTATUSCD & GASSYSTEMSTATUS \\
\hline GRAPHICSSCALEFACTORVALUE & AT_GRAPHICSCALEFACTORVALUE \\
\hline REGULATORSTATIONNUMBER & AT_1 \\
\hline REGULATORSTATIONNUMBER & LOCATABLEFIELD \\
\hline REGULATORSTATIONSEQUENCECD & AT_2 \\
\hline INLETPRESSUREVALUE & AT_3 \\
\hline INLETPRESSUREVALUE & SOPIN \\
\hline OUTLETPRESSUREVALUE & AT_4 \\
\hline OUTLETPRESSUREVALUE & SOPOUT \\
\hline INSTALATIONTYYECD & AT_5 \\
\hline INSULATEDIND & AT_6 \\
\hline INSULATEDIND & CPSYSTEMSTATUS \\
\hline SUBTYPECD & AT_SUBTYPECD \\
\hline SUBTYPECD & SUBTYPECD \\
\hline RELUPSTREAMGASMAINOID & RELATEDGASMAINOID \\
\hline CUSTOMERNAME & AT_7 \\
\hline SHAPE & AT_SHAPE \\
\hline INSTALLMISCORDERID & AT_INSTALLMISCORDERID \\
\hline INSTALLMISCORDERID & MISCORDERID \\
\hline
\end{tabular}

RegulatorStation_Anno

Object Class Model Names

\begin{tabular}{|l|l|}
\hline Object Class & Model Name \\
\hline RegulatorStation_Anno & MMABANDONABLE \\
\hline
\end{tabular}

Field Model Names

\begin{tabular}{|l|l|}
\hline Field & Model Name \\
\hline FEATUREID & AT_1 \\
\hline ZORDER & AT_2 \\
\hline ANNOTATIONCLASSID & AT_3 \\
\hline ELEMENT & AT_4 \\
\hline SHAPE & AT_SHAPE \\
\hline
\end{tabular}

RetiredCasing

Object Class Model Names

Object Class

RetiredCasing

Model Name

MMABANDONED

Field Model Names

\begin{tabular}{|l|l|}
\hline Field & Model Name \\
\hline CREATIONDATE & AT_CREATIONDATE \\
\hline CREATIONUSERID & AT_CREATIONUSER \\
\hline UPDATEDATE & AT_UPDATEDATE \\
\hline
\end{tabular}




\begin{tabular}{|l|l|}
\hline UPDATEUSERID & AT_UPDATEUSERID \\
\hline LOANUMBER & AT_LOANUMBER \\
\hline TAXUNITCD & AT_TAXUNITCD \\
\hline GRIDCD & AT_GRIDCD \\
\hline INSTALLDATE & AT_INSTALLDATE \\
\hline INSTALLWORKORDERNUMBER & AT_INSTALLWORKORDERNUMBER \\
\hline RETIREDATE & RETIREDATE \\
\hline RETIREWORKORDERNUMBER & RETIREWORKORDERNUMBER \\
\hline RETIRETYPE & RETIRETYPE \\
\hline SUBTYPECD & AT_SUBTYPECD \\
\hline CASINGDIAMETERVALUE & AT_1 \\
\hline INSTALLEDLENGTHVALUE & AT_2 \\
\hline LENGTHSOURCECD & AT_3 \\
\hline RRCROSSINGAGREEMENTNUMBER & AT_4 \\
\hline SHAPE & AT_SHAPE \\
\hline INSTALLMISCORDERID & AT_INSTALLMISCORDERID \\
\hline
\end{tabular}

RetiredCasing_Anno

Object Class Model Names

\begin{tabular}{|l|l|}
\hline Object Class & Model Name \\
\hline RetiredCasing_Anno & MMABANDONED \\
\hline
\end{tabular}

Field Model Names

\begin{tabular}{|l|l|}
\hline Field & Model Name \\
\hline FEATUREID & AT_1 \\
\hline ZORDER & AT_2 \\
\hline ANNOTATIONCLASSID & AT_3 \\
\hline ELEMENT & AT_4 \\
\hline SHAPE & AT_SHAPE \\
\hline
\end{tabular}

RetiredDeadEnd_Location_Anno

Object Class Model Names

Object Class

RetiredDeadEnd_Location_Anno

Model Name

MMABANDONED

Field Model Names

\begin{tabular}{|l|l|}
\hline Field & Model Name \\
\hline FEATUREID & AT_1 \\
\hline ZORDER & AT_2 \\
\hline ANNOTATIONCLASSID & AT_3 \\
\hline ELEMENT & AT_4 \\
\hline SHAPE & AT_SHAPE \\
\hline
\end{tabular}

RetiredDeadEndGas

Object Class Model Names

Object Class

RetiredDeadEndGas

Model Name

MMABANDONED

Field Model Names 


\begin{tabular}{|l|l|}
\hline Field & Model Name \\
\hline CREATIONDATE & AT_CREATIONDATE \\
\hline CREATIONUSERID & AT_CREATIONUSER \\
\hline UPDATEDATE & AT_UPDATEDATE \\
\hline UPDATEUSERID & AT_UPDATEUSERID \\
\hline LOANUMBER & AT_LOANUMBER \\
\hline TAXUNITCD & AT_TAXUNITCD \\
\hline GRIDCD & AT_GRIDCD \\
\hline INSTALLDATE & AT_INSTALLDATE \\
\hline INSTALLWORKORDERNUMBER & AT_INSTALLWORKORDERNUMBER \\
\hline RETIREDATE & RETIREDATE \\
\hline RETIREWORKORDERNUMBER & RETIREWORKORDERNUMBER \\
\hline RETIRETYYE & RETIRETYPE \\
\hline SUBTYPECD & AT_SUBTYPECD \\
\hline LOCATIONDESC1 & AT_LOCATIONDESC1 \\
\hline LOCATIONDESC2 & AT_LOCATIONDESC2 \\
\hline SYMBOLROTATIONVALUE & AT_SYMBOLROTATIONVALUE \\
\hline SHAPE & AT_SHAPE \\
\hline INSTALLMISCORDERID & AT_INSTALLMISCORDERID \\
\hline
\end{tabular}

RetiredDistribMain_Size_Anno

Object Class Model Names

\section{Object Class}

RetiredDistribMain_Size_Anno

\section{Model Name}

MMABANDONED

Field Model Names

\begin{tabular}{|l|l|}
\hline Field & Model Name \\
\hline FEATUREID & AT_1 \\
\hline ZORDER & AT_2 \\
\hline ANNOTATIONCLASSID & AT_3 \\
\hline ELEMENT & AT_4 \\
\hline SHAPE & AT_SHAPE \\
\hline
\end{tabular}

RetiredDrip

Object Class Model Names

Object Class

RetiredDrip

\begin{tabular}{|l} 
Model Name \\
MMABANDONED
\end{tabular}

Field Model Names

\begin{tabular}{|l|l|}
\hline Field & Model Name \\
\hline CREATIONDATE & AT_CREATIONDATE \\
\hline CREATIONUSERID & AT_CREATIONUSER \\
\hline UPDATEDATE & AT_UPDATEDATE \\
\hline UPDATEUSERID & AT_UPDATEUSERID \\
\hline LOANUMBER & AT_LOANUMBER \\
\hline TAXUNITCD & AT_TAXUNITCD \\
\hline GRIDCD & AT_GRIDCD \\
\hline INSTALLDATE & AT_INSTALLDATE \\
\hline INSTALLWORKORDERNUMBER & AT_INSTALLWORKORDERNUMBER \\
\hline
\end{tabular}




\begin{tabular}{|l|l|}
\hline RETIREDATE & RETIREDATE \\
\hline RETIREWORKORDERNUMBER & RETIREWORKORDERNUMBER \\
\hline RETIRETYPE & RETIRETYPE \\
\hline SUBTYPECD & AT_SUBTYPECD \\
\hline DRIPPOTTYPE & AT_1 \\
\hline SYMBOLROTATIONVALUE & AT_SYMBOLROTATIONVALUE \\
\hline SHAPE & AT_SHAPE \\
\hline INSTALLMISCORDERID & AT_INSTALLMISCORDERID \\
\hline
\end{tabular}

RetiredGasMain

Object Class Model Names

Object Class

RetiredGasMain

Model Name

MMABANDONED

Field Model Names

\begin{tabular}{|l|l|}
\hline Field & Model Name \\
\hline CREATIONDATE & AT_CREATIONDATE \\
\hline CREATIONUSERID & AT_CREATIONUSER \\
\hline UPDATEDATE & AT_UPDATEDATE \\
\hline UPDATEUSERID & AT_UPDATEUSERID \\
\hline LOANUMBER & AT_LOANUMBER \\
\hline TAXUNITCD & AT_TAXUNITCD \\
\hline GRIDCD & AT_GRIDCD \\
\hline INSTALLDATE & AT_INSTALLDATE \\
\hline INSTALLWORKORDERNUMBER & AT_INSTALLWORKORDERNUMBER \\
\hline RETIREDATE & RETIREDATE \\
\hline RETIREWORKORDERNUMBER & RETIREWORKORDERNUMBER \\
\hline RETIRETYPE & RETIRETYPE \\
\hline SUBTYPECD & AT_SUBTYPECD \\
\hline UPSTREAMCALCPRESSUREVALUE & AT_1 \\
\hline MEASUREDLENGTHVALUE & AT_2 \\
\hline MAINTAINEDBYNAME & AT_MAINTAINEDBYNAME \\
\hline MAINMATERIALCD & AT_MAINMATERIALCD \\
\hline MAINDIAMETERVALUE & AT_MAINDIAMETERVALUE \\
\hline LENGTHSOURCECD & AT_3 \\
\hline INSERTIND & AT_INSERTIND \\
\hline DOWNSTREAMCALCPRESSUREVALUE & AT_4 \\
\hline CALCULATEDFLOWVALUE & AT_5 \\
\hline BONDEDIND & AT_6 \\
\hline SHAPE & AT_SHAPE \\
\hline INSTALLMISCORDERID & AT_INSTALLMISCORDERID \\
\hline
\end{tabular}

RetiredGasMainLocation

Object Class Model Names

Object Class

RetiredGasMainLocation

Model Name
MMABANDONED

Field Model Names

\begin{tabular}{|l|l|}
\hline Field & Model Name \\
\hline
\end{tabular} 


\begin{tabular}{|l|l|}
\hline CREATIONDATE & AT_CREATIONDATE \\
\hline CREATIONUSERID & AT_CREATIONUSERID \\
\hline UPDATEDATE & AT_UPDATEDATE \\
\hline UPDATEUSERID & AT_UPDATEUSERID \\
\hline LOCATIONDESC1 & AT_LOCATIONDESC1 \\
\hline LOCATIONDESC2 & AT_LOCATIONDESC2 \\
\hline RETIRETYPE & RETIRETYPE \\
\hline SHAPE & AT_SHAPE \\
\hline
\end{tabular}

RetiredGasMainLocation_Anno

Object Class Model Names

\section{Object Class}

RetiredGasMainLocation_Anno

Model Name

MMABANDONED

Field Model Names

\begin{tabular}{|l|l|}
\hline Field & Model Name \\
\hline FEATUREID & AT_1 \\
\hline ZORDER & AT_2 \\
\hline ANNOTATIONCLASSID & AT_3 \\
\hline ELEMENT & AT_4 \\
\hline SHAPE & AT_SHAPE \\
\hline
\end{tabular}

RetiredGasValve

Object Class Model Names

\section{Object Class}

RetiredGasValve

\section{Model Name}

MMABANDONED

Field Model Names

\begin{tabular}{|l|l|}
\hline Field & Model Name \\
\hline CREATIONDATE & AT_CREATIONDATE \\
\hline CREATIONUSERID & AT_CREATIONUSER \\
\hline UPDATEDATE & AT_UPDATEDATE \\
\hline UPDATEUSERID & AT_UPDATEUSERID \\
\hline LOANUMBER & AT_LOANUMBER \\
\hline TAXUNITCD & AT_TAXUNITCD \\
\hline GRIDCD & AT_GRIDCD \\
\hline INSTALLDATE & AT_INSTALLDATE \\
\hline INSTALLWORKORDERNUMBER & AT_INSTALLWORKORDERNUMBER \\
\hline RETIREDATE & RETIREDATE \\
\hline RETIREWORKORDERNUMBER & RETIREWORKORDERNUMBER \\
\hline RETIRETYPE & RETIRETYPE \\
\hline SYMBOLROTATIONVALUE & AT_SYMBOLROTATIONVALUE \\
\hline GRAPHICSSCALEFACTORVALUE & AT_GRAPHICSCALEFACTORVALUE \\
\hline SUBTYPECD & AT_SUBTYPECD \\
\hline VALVENUMBER & AT_1 \\
\hline VALVESIZEVALUE & AT_2 \\
\hline LOCATIONDESC1 & AT_LOCATIONDESC1 \\
\hline LOCATIONDESC2 & AT_LOCATIONDESC2 \\
\hline INSULATEDIND & AT_3 \\
\hline
\end{tabular}




\begin{tabular}{|l|l|}
\hline OPENCLOSEDSTATUSCD & AT_4 \\
\hline EMERGENCYVALVEIND & AT_5 \\
\hline SYMBOLCONFIGURATIONCD & AT_6 \\
\hline INSTALLATIONTYPECD & AT_7 \\
\hline SHAPE & AT_SHAPE \\
\hline INSTALLMISCORDERID & AT_INSTALLMISCORDERID \\
\hline
\end{tabular}

RetiredGasValve_Location_Anno

Object Class Model Names

\section{Object Class}

RetiredGasValve_Location_Anno

\section{Model Name}

MMABANDONED

Field Model Names

\begin{tabular}{|l|l|}
\hline Field & Model Name \\
\hline FEATUREID & AT_1 \\
\hline ZORDER & AT_2 \\
\hline ANNOTATIONCLASSID & AT_3 \\
\hline ELEMENT & AT_4 \\
\hline SHAPE & AT_SHAPE \\
\hline
\end{tabular}

RetiredGasValve_Size_Anno

Object Class Model Names

\begin{tabular}{|l|l|}
\hline Object Class & Model Name \\
\hline RetiredGasValve_Size_Anno & MMABANDONED \\
\hline
\end{tabular}

Field Model Names

\begin{tabular}{|l|l|}
\hline Field & Model Name \\
\hline FEATUREID & AT_1 \\
\hline ZORDER & AT_2 \\
\hline ANNOTATIONCLASSID & AT_3 \\
\hline ELEMENT & AT_4 \\
\hline SHAPE & AT_SHAPE \\
\hline
\end{tabular}

RetiredInsulatedCoupling

Object Class Model Names

\begin{tabular}{|l|l|}
\hline Object Class & Model Name \\
\hline RetiredInsulatedCoupling & MMABANDONED \\
\hline
\end{tabular}

Field Model Names

\begin{tabular}{|l|l|}
\hline Field & Model Name \\
\hline CREATIONDATE & AT_CREATIONDATE \\
\hline CREATIONUSERID & AT_CREATIONUSER \\
\hline UPDATEDATE & AT_UPDATEDATE \\
\hline UPDATEUSERID & AT_UPDATEUSERID \\
\hline LOANUMBER & AT_LOANUMBER \\
\hline TAXUNITCD & AT_TAXUNITCD \\
\hline GRIDCD & AT_GRIDCD \\
\hline INSTALLDATE & AT_INSTALLDATE \\
\hline INSTALLWORKORDERNUMBER & AT_INSTALLWORKORDERNUMBER \\
\hline
\end{tabular}




\begin{tabular}{|l|l|}
\hline RETIREDATE & RETIREDATE \\
\hline RETIREWORKORDERNUMBER & RETIREWORKORDERNUMBER \\
\hline RETIRETYPE & RETIRETYPE \\
\hline SYMBOLROTATIONVALUE & AT_SYMBOLROTATIONVALUE \\
\hline GRAPHICSSCALEFACTORVALUE & AT_GRAPHICSCALEFACTORVALUE \\
\hline SUBTYPECD & AT_SUBTYPECD \\
\hline INSULATEDIND & AT_1 \\
\hline SHAPE & AT_SHAPE \\
\hline INSTALLMISCORDERID & AT_INSTALLMISCORDERID \\
\hline
\end{tabular}

RetiredPCF_Location_Anno

Object Class Model Names

\section{Object Class}

RetiredPCF_Location_Anno

Model Name

MMABANDONED

Field Model Names

\begin{tabular}{|l|l|}
\hline Field & Model Name \\
\hline FEATUREID & AT_1 \\
\hline ZORDER & AT_2 \\
\hline ANNOTATIONCLASSID & AT_3 \\
\hline ELEMENT & AT_4 \\
\hline SHAPE & AT_SHAPE \\
\hline
\end{tabular}

RetiredPipeChange

Object Class Model Names

\section{Object Class}

RetiredPipeChange

Model Name

MMABANDONED

Field Model Names

\begin{tabular}{|c|}
\hline Field \\
\hline SUBTYPECD \\
\hline SYMBOLROTATIONVALUE \\
\hline RETIREDATE \\
\hline RETIREWORKORDERNUMBER \\
\hline RETIRETYPE \\
\hline SHAPE \\
\hline
\end{tabular}

Model Name

AT_SUBTYPECD

AT_SYMBOLROTATIONVALUE

RETIREDATE

RETIREWORKORDERNUMBER

RETIRETYPE

AT_SHAPE

RetiredPressureControlFitting

Object Class Model Names

\begin{tabular}{|l|l|}
\hline Object Class & Model Name \\
\hline RetiredPressureControlFitting & MMABANDONED \\
\hline
\end{tabular}

Field Model Names

\begin{tabular}{|l|l|}
\hline Field & Model Name \\
\hline CREATIONDATE & AT_CREATIONDATE \\
\hline CREATIONUSERID & AT_CREATIONUSER \\
\hline UPDATEDATE & AT_UPDATEDATE \\
\hline UPDATEUSERID & AT_UPDATEUSERID \\
\hline LOANUMBER & AT_LOANUMBER \\
\hline
\end{tabular}




\begin{tabular}{|l|l|}
\hline TAXUNITCD & AT_TAXUNITCD \\
\hline GRIDCD & AT_GRIDCD \\
\hline INSTALLDATE & AT_INSTALLDATE \\
\hline INSTALLWORKORDERNUMBER & AT_INSTALLWORKORDERNUMBER \\
\hline SYMBOLROTATIONVALUE & AT_SYMBOLROTATIONVALUE \\
\hline GRAPHICSSCALEFACTORVALUE & AT_GRAPHICSCALEFACTORVALUE \\
\hline SUBTYPECD & AT_SUBTYPECD \\
\hline LOCATIONDESC1 & AT_LOCATIONDESC1 \\
\hline LOCATIONDESC2 & AT_LOCATIONDESC2 \\
\hline SHAPE & AT_SHAPE \\
\hline INSTALLMISCORDERID & AT_INSTALLMISCORDERID \\
\hline
\end{tabular}

RetiredRegulatorStation

Object Class Model Names

\section{Object Class}

RetiredRegulatorStation

Model Name

MMABANDONED

Field Model Names

\begin{tabular}{|l|l|}
\hline Field & Model Name \\
\hline CREATIONDATE & AT_CREATIONDATE \\
\hline CREATIONUSERID & AT_CREATIONUSER \\
\hline UPDATEDATE & AT_UPDATEDATE \\
\hline UPDATEUSERID & AT_UPDATEUSERID \\
\hline LOANUMBER & AT_LOANUMBER \\
\hline TAXUNITCD & AT_TAXUNITCD \\
\hline GRIDCD & AT_GRIDCD \\
\hline INSTALLDATE & AT_INSTALLDATE \\
\hline INSTALLWORKORDERNUMBER & AT_INSTALLWORKORDERNUMBER \\
\hline RETIREDATE & RETIREDATE \\
\hline RETIREWORKORDERNUMBER & RETIREWORKORDERNUMBER \\
\hline RETIRETYPE & RETIRETYPE \\
\hline SYMBOLROTATIONVALUE & AT_SYMBOLROTATIONVALUE \\
\hline GRAPHICSSCALEFACTORVALUE & AT_GRAPHICSCALEFACTORVALUE \\
\hline REGULATORSTATIONNUMBER & AT_1 \\
\hline REGULATORSTATIONSEQUENCECD & AT_2 \\
\hline INLETPRESSUREVALUE & AT_3 \\
\hline OUTLETPRESSUREVALUE & AT_4 \\
\hline INSTALLATIONTYPECD & AT_5 \\
\hline INSULATEDIND & AT_6 \\
\hline SUBTYPECD & AT_SUBTYPECD \\
\hline CUSTOMERNAME & AT_7 \\
\hline SHAPE & AT_SHAPE \\
\hline INSTALLMISCORDERID & AT_INSTALLMISCORDERID \\
\hline
\end{tabular}

RetiredRegulatorStation_Anno

Object Class Model Names

Object Class

RetiredRegulatorStation_Anno

Model Name

MMABANDONED 
Field Model Names

\begin{tabular}{|l|l|}
\hline Field & Model Name \\
\hline FEATUREID & AT_1 \\
\hline ZORDER & AT_2 \\
\hline ANNOTATIONCLASSID & AT_3 \\
\hline ELEMENT & AT_4 \\
\hline SHAPE & AT_SHAPE \\
\hline
\end{tabular}

RetiredTakeStation

Object Class Model Names

Object Class

RetiredTakeStation

Model Name
MMABANDONED

Field Model Names

\begin{tabular}{|l|l|}
\hline Field & Model Name \\
\hline CREATIONDATE & AT_CREATIONDATE \\
\hline CREATIONUSERID & AT_CREATIONUSER \\
\hline UPDATEDATE & AT_UPDATEDATE \\
\hline UPDATEUSERID & AT_UPDATEUSERID \\
\hline RETIREDATE & RETIREDATE \\
\hline RETIREWORKORDERNUMBER & RETIREWORKORDERNUMBER \\
\hline RETIRETYPE & RETIRETYPE \\
\hline REGULATORSTATIONNUMBER & AT_1 \\
\hline REGULATORSTATIONSEQUENCECD & AT_2 \\
\hline INLETPRESSUREVALUE & AT_3 \\
\hline OUTLETPRESSUREVALUE & AT_4 \\
\hline INSTALLATIONTYPECD & AT_5 \\
\hline INSULATEDIND & AT_6 \\
\hline SUBTYPECD & AT_SUBTYPECD \\
\hline PIPELINEOPERATORNUMBER & AT_7 \\
\hline SUPPLIERNAME & AT_8 \\
\hline INSTALLMISCORDERID & AT_INSTALLMISCORDERID \\
\hline
\end{tabular}

RetiredTransMain_Name_Anno

Object Class Model Names

\section{Object Class}

RetiredTransMain_Name_Anno

Model Name

MMABANDONED

Field Model Names

\begin{tabular}{|l|l|}
\hline Field & Model Name \\
\hline FEATUREID & AT_1 \\
\hline ZORDER & AT_2 \\
\hline ANNOTATIONCLASSID & AT_3 \\
\hline ELEMENT & AT_4 \\
\hline SHAPE & AT_SHAPE \\
\hline
\end{tabular}

RetiredTransMain_SizeMat_Anno

Object Class Model Names

Object Class

Model Name 
Field Model Names

\begin{tabular}{|l|l|}
\hline Field & Model Name \\
\hline FEATUREID & AT_1 \\
\hline ZORDER & AT_2 \\
\hline ANNOTATIONCLASSID & AT_3 \\
\hline ELEMENT & AT_4 \\
\hline SHAPE & AT_SHAPE \\
\hline
\end{tabular}

RetiredWorkOrder_Anno

Object Class Model Names

\begin{tabular}{|l|l|}
\hline Object Class & Model Name \\
\hline RetiredWorkOrder_Anno & MMABANDONED \\
\hline
\end{tabular}

Field Model Names

\begin{tabular}{|l|l|}
\hline Field & Model Name \\
\hline
\end{tabular}

RetiredWorkOrderLeaderLine

Object Class Model Names

Object Class

RetiredWorkOrderLeaderLine

Model Name

MMABANDONED

Field Model Names

\begin{tabular}{|c|c|}
\hline Field & Model Name \\
\hline \multicolumn{2}{|c|}{$\begin{array}{l}\text { SqueezeOff } \\
\text { Object Class Model Names }\end{array}$} \\
\hline Object Class & Model Name \\
\hline SqueezeOff & LOCATABLEOBJECT \\
\hline SqueezeOff & SQUEEZEOFF \\
\hline
\end{tabular}

Field Model Names

\begin{tabular}{|l|l|}
\hline Field & Model Name \\
\hline OBJECTID & LOCATABLEFIELD \\
\hline SUBTYPECD & SUBTYPECD \\
\hline GASTRACEWEIGHT & GASTRACEWEIGHT \\
\hline OPENCLOSEDSTATUSCD & NORMALPOSITION \\
\hline
\end{tabular}

TakeStation

Object Class Model Names

\begin{tabular}{|l|l|}
\hline Object Class & Model Name \\
\hline TakeStation & EDFS WORKORDER \\
\hline TakeStation & LOCATABLEOBJECT \\
\hline TakeStation & MMABANDONABLE \\
\hline TakeStation & TBS \\
\hline
\end{tabular}

Field Model Names

\section{Field}

Model Name 


\begin{tabular}{|l|l|}
\hline CREATIONDATE & AT_CREATIONDATE \\
\hline CREATIONUSERID & AT_CREATIONUSER \\
\hline UPDATEDATE & AT_UPDATEDATE \\
\hline UPDATEUSERID & AT_UPDATEUSERID \\
\hline INSTALLDATE & AT_INSTALLDATE \\
\hline INSTALLWORKORDERNUMBER & AT_INSTALLWORKORDERNUMBER \\
\hline INSTALLWORKORDERNUMBER & WORKORDERNUMBER \\
\hline LOANUMBER & AT_LOANUMBER \\
\hline LOANUMBER & LOANAME \\
\hline TAXUNITCD & AT_TAXUNITCD \\
\hline GRIDCD & AT_GRIDCD \\
\hline GASTRACEWEIGHT & GASTRACEWEIGHT \\
\hline SYMBOLROTATIONVALUE & AT_SYMBOLROTATIONVALUE \\
\hline GASPRESSURESYSTEMSTATUSCD & GASPRESSURESYSTEMSTATUS \\
\hline EMERISOLATIONSYSTEMSTATUSCD & EMERISOLATIONSYSSTATUS \\
\hline GASSYSTEMSTATUSCD & GASSYSTEMSTATUS \\
\hline GRAPHICSSCALEFACTORVALUE & AT_GRAPHICSCALEFACTORVALUE \\
\hline REGULATORSTATIONNUMBER & AT_1 \\
\hline REGULATORSTATIONNUMBER & LOCATABLEFIELD \\
\hline REGULATORSTATIONSEQUENCECD & AT_2 \\
\hline INLETPRESSUREVALUE & AT_3 \\
\hline INLETPRESSUREVALUE & SOPIN \\
\hline OUTLETPRESSUREVALUE & AT_4 \\
\hline OUTLETPRESSUREVALUE & SOPOUT \\
\hline INSTALLATIONTYPECD & AT_5 \\
\hline INSULATEDIND & AT_6 \\
\hline INSULATEDIND & CPSYSTEMSTATUS \\
\hline SUBTYPECD & SUBTYPECD \\
\hline SUBTYPECD & AT_SUBTYPECD \\
\hline PIPELINEOPERATORNUMBER & AT_7 \\
\hline SUPPLIERNAME & AT_8 \\
\hline SHAPE & AT_SHAPE \\
\hline INSTALLMISCORDERID & MISCORDERID \\
\hline INSTALLMISCORDERID & AT_INSTALLMISCORDERID \\
\hline & \\
\hline
\end{tabular}

TransmissionMain_Name_Anno

Object Class Model Names

Object Class

TransmissionMain_Name_Anno

Model Name

MMABANDONABLE

Field Model Names

\begin{tabular}{|l|l|}
\hline Field & Model Name \\
\hline FEATUREID & AT_1 \\
\hline ZORDER & AT_2 \\
\hline ANNOTATIONCLASSID & AT_3 \\
\hline ELEMENT & AT_4 \\
\hline SHAPE & AT_SHAPE \\
\hline
\end{tabular}

TransmissionMain_SizeMat_Anno 
Object Class Model Names

\begin{tabular}{|l|l|}
\hline Object Class & Model Name \\
\hline TransmissionMain_SizeMat_Anno & MMABANDONABLE \\
\hline
\end{tabular}

Field Model Names

\begin{tabular}{|l|l|}
\hline Field & Model Name \\
\hline FEATUREID & AT_1 \\
\hline ZORDER & AT_2 \\
\hline ANNOTATIONCLASSID & AT_3 \\
\hline ELEMENT & AT_4 \\
\hline SHAPE & AT_SHAPE \\
\hline
\end{tabular}


Table E-6. Land ArcFM configuration

arcfm8.LANDBASE.Bridge

ArcFM Display Field: OBJECTID

Create Edit Task:

On Create Event:

On Update Event:

On Delete Event:

Before Split Event:

On Split Event:

After Split Event:

On Abandon Event:

Abandon Feature Class:

Abandon Subtype:

Remove Feature Class:

Remove Subtype:

\begin{tabular}{|l|c|c|c|c|c|}
\hline Field Alias & Visible & Editable & $\begin{array}{c}\text { Allow Null } \\
\text { Values }\end{array}$ & $\begin{array}{c}\text { On } \\
\text { Feature } \\
\text { Create }\end{array}$ & On Feature Update \\
\hline Subtype & TRUE & TRUE & FALSE & & \\
\hline OBJECTID & TRUE & FALSE & FALSE & & \\
\hline HANDLE & FALSE & TRUE & TRUE & & \\
\hline TILENAME & FALSE & TRUE & TRUE & & \\
\hline SHAPE.len & FALSE & & & & \\
\hline Shape & FALSE & TRUE & TRUE & & \\
\hline CreationDate & FALSE & TRUE & FALSE & $\begin{array}{l}\text { ArcFM } \\
\text { Current } \\
\text { Date }\end{array}$ & \\
\hline Created By & FALSE & TRUE & FALSE & $\begin{array}{l}\text { ArcFM } \\
\text { User } \\
\text { Name }\end{array}$ & \\
\hline UPDATEDATE & FALSE & FALSE & TRUE & & ArcFM Current Date \\
\hline Updated By & FALSE & FALSE & TRUE & & ArcFM User Name \\
\hline
\end{tabular}

arcfm8.LANDBASE.County

ArcFM Display Field: OBJECTID

Create Edit Task:

On Create Event:

On Update Event:

On Delete Event:

On Abandon Event:

Abandon Feature Class:

Abandon Subtype:

Remove Feature Class:

Remove Subtype:

\begin{tabular}{|l|c|c|c|l|l|}
\hline Field Alias & Visible & Editable & $\begin{array}{c}\text { Allow Null } \\
\text { Values }\end{array}$ & $\begin{array}{c}\text { On } \\
\text { Feature } \\
\text { Create }\end{array}$ & On Feature Update \\
\hline Subtype & TRUE & TRUE & FALSE & & \\
\hline State Name & TRUE & TRUE & FALSE & & \\
\hline County Name & TRUE & TRUE & FALSE & & \\
\hline OBJECTID & TRUE & FALSE & FALSE & & \\
\hline Shape & FALSE & TRUE & TRUE & & \\
\hline CreationDate & FALSE & TRUE & FALSE & $\begin{array}{l}\text { ArcFM } \\
\text { Current } \\
\text { Date }\end{array}$ & \\
\hline Created By & FALSE & TRUE & FALSE & $\begin{array}{l}\text { ArcFM } \\
\text { User } \\
\text { Name }\end{array}$ & \\
\hline UPDATEDATE & FALSE & FALSE & TRUE & & ArcFM Current Date \\
\hline Updated By & FALSE & FALSE & TRUE & & ArcFM User Name \\
\hline
\end{tabular}




\begin{tabular}{|c|c|c|c|c|c|}
\hline SHAPE.area & FALSE & & & & \\
\hline SHAPE.Ien & FALSE & & & & \\
\hline $\begin{array}{l}\text { arcfm8.LANDE } \\
\text { ArcFM Display } \\
\text { Create Edit Ta } \\
\text { On Create Eve } \\
\text { On Update Ev } \\
\text { On Delete Eve } \\
\text { On Abandon E } \\
\text { Abandon Feat } \\
\text { Abandon Subt } \\
\text { Remove Featu } \\
\text { Remove Subty }\end{array}$ & CTID & & & & \\
\hline Field Alias & Visible & Editable & $\begin{array}{l}\text { Allow Null } \\
\text { Values }\end{array}$ & $\begin{array}{c}\text { On } \\
\text { Feature } \\
\text { Create }\end{array}$ & On Feature Update \\
\hline Subtype & TRUE & TRUE & FALSE & & \\
\hline DG Grid Number & TRUE & TRUE & FALSE & & \\
\hline OBJECTID & TRUE & FALSE & FALSE & & \\
\hline Shape & FALSE & TRUE & TRUE & & \\
\hline CreationDate & FALSE & TRUE & TRUE & $\begin{array}{l}\text { ArcFM } \\
\text { Current } \\
\text { Date } \\
\end{array}$ & \\
\hline Created By & FALSE & TRUE & FALSE & $\begin{array}{l}\text { ArcFM } \\
\text { User } \\
\text { Name }\end{array}$ & \\
\hline UPDATEDATE & FALSE & FALSE & TRUE & & ArcFM Current Date \\
\hline Updated By & FALSE & FALSE & TRUE & & ArcFM User Name \\
\hline SHAPE.area & FALSE & & & & \\
\hline SHAPE.Ien & FALSE & & & & \\
\hline DCSID & FALSE & TRUE & TRUE & & \\
\hline
\end{tabular}

\section{arcfm8.LANDBASE.Easement}

ArcFM Display Field: OBJECTID

Create Edit Task:

On Create Event:

On Update Event:

On Delete Event:

Before Split Event:

On Split Event:

After Split Event:

On Abandon Event:

Abandon Feature Class:

Abandon Subtype:

Remove Feature Class:

Remove Subtype:

\begin{tabular}{|l|c|c|c|c|c|}
\hline Field Alias & Visible & Editable & $\begin{array}{c}\text { Allow Null } \\
\text { Values }\end{array}$ & $\begin{array}{c}\text { On } \\
\text { Feature } \\
\text { Create }\end{array}$ & On Feature Update \\
\hline Subtype & TRUE & TRUE & FALSE & & \\
\hline Recorded Plat ? & TRUE & TRUE & FALSE & & \\
\hline Easement Number & TRUE & TRUE & TRUE & & \\
\hline Easement Width & TRUE & TRUE & FALSE & & \\
\hline OBJECTID & TRUE & FALSE & FALSE & & \\
\hline Shape & FALSE & TRUE & TRUE & & \\
\hline CreationDate & FALSE & TRUE & FALSE & $\begin{array}{l}\text { ArcFM } \\
\text { Current } \\
\text { Date }\end{array}$ & \\
\hline Created By & FALSE & TRUE & FALSE & $\begin{array}{l}\text { ArcFM } \\
\text { User } \\
\text { Name }\end{array}$ & \\
\hline UPDATEDATE & FALSE & FALSE & TRUE & & ArcFM Current Date \\
\hline Updated By & FALSE & FALSE & TRUE & & ArcFM User Name \\
\hline
\end{tabular}




\begin{tabular}{|l|c|c|c|l|l|} 
HANDLE & FALSE & TRUE & TRUE & & \\
\hline TILENAME & FALSE & TRUE & TRUE & & \\
\hline DCSID & FALSE & TRUE & TRUE & & \\
\hline SHAPE.len & FALSE & & & & \\
\hline AVLINK & FALSE & TRUE & TRUE & & \\
\hline
\end{tabular}

arcfm8.LANDBASE.FranchiseBoundary

ArcFM Display Field: OBJECTID

Create Edit Task:

On Create Event:

On Update Event:

On Delete Event:

On Abandon Event:

Abandon Feature Class:

Abandon Subtype:

Remove Feature Class:

Remove Subtype:

\begin{tabular}{|l|c|c|c|c|c|}
\hline Field Alias & Visible & Editable & $\begin{array}{c}\text { Allow Null } \\
\text { Values }\end{array}$ & $\begin{array}{c}\text { On } \\
\text { Feature } \\
\text { Create }\end{array}$ & On Feature Update \\
\hline Subtype & TRUE & TRUE & FALSE & & \\
\hline UtilityName & TRUE & TRUE & FALSE & & \\
\hline OBJECTID & TRUE & FALSE & FALSE & & \\
\hline Shape & FALSE & TRUE & TRUE & & \\
\hline CreationDate & FALSE & TRUE & FALSE & $\begin{array}{l}\text { ArcFM } \\
\text { Current } \\
\text { Date }\end{array}$ & \\
\hline Created By & FALSE & TRUE & FALSE & $\begin{array}{l}\text { ArcFM } \\
\text { Nser } \\
\text { Name }\end{array}$ & \\
\hline UPDATEDATE & FALSE & FALSE & TRUE & & ArcFM Current Date \\
\hline Updated By & FALSE & FALSE & TRUE & & ArcFM User Name \\
\hline SHAPE.area & FALSE & & & & \\
\hline SHAPE.len & FALSE & & & & \\
\hline
\end{tabular}

arcfm8.LANDBASE.LandDiscrepancy

ArcFM Display Field: OBJECTID

Create Edit Task:

On Create Event:

On Update Event:

On Delete Event:

On Abandon Event:

Abandon Feature Class:

Abandon Subtype:

Remove Feature Class:

Remove Subtype:

\begin{tabular}{|l|c|c|c|c|c|}
\hline Field Alias & Visible & Editable & $\begin{array}{c}\text { Allow Null } \\
\text { Values }\end{array}$ & $\begin{array}{c}\text { On } \\
\text { Feature } \\
\text { Create }\end{array}$ & On Feature Update \\
\hline Subtype & TRUE & TRUE & FALSE & & \\
\hline Error Description & TRUE & TRUE & FALSE & & \\
\hline OBJECTID & TRUE & FALSE & FALSE & & \\
\hline Shape & FALSE & TRUE & TRUE & & \\
\hline CreationDate & FALSE & TRUE & FALSE & $\begin{array}{l}\text { ArcFM } \\
\text { Current } \\
\text { Date }\end{array}$ & \\
\hline Created By & FALSE & TRUE & FALSE & $\begin{array}{l}\text { ArcFM } \\
\text { User } \\
\text { Name }\end{array}$ & \\
\hline UPDATEDATE & FALSE & FALSE & TRUE & & ArcFM Current Date \\
\hline Updated By & FALSE & FALSE & TRUE & & ArcFM User Name \\
\hline HANDLE & FALSE & TRUE & TRUE & & \\
\hline TILENAME & FALSE & TRUE & TRUE & & \\
\hline DCSID & FALSE & TRUE & TRUE & & \\
\hline
\end{tabular}

arcfm8.LANDBASE.LegacyStreetCenterLine 
ArcFM Display Field: OBJECTID

Create Edit Task:

On Create Event:

On Update Event:

On Delete Event:

Metadata Editor:

Custom Configuration Editor:

Extended Data Definition Table:

On Abandon Event:

Abandon Feature Class:

Abandon Subtype:

Remove Feature Class:

Remove Subtype:

\begin{tabular}{|c|c|c|c|c|c|}
\hline Field Alias & Visible & Editable & $\begin{array}{c}\text { Allow } \\
\text { Null } \\
\text { Values }\end{array}$ & $\begin{array}{c}\text { On } \\
\text { Feature } \\
\text { Create }\end{array}$ & On Feature Update \\
\hline OBJECTID & TRUE & FALSE & FALSE & & \\
\hline NM1 & TRUE & TRUE & TRUE & & \\
\hline NM2 & TRUE & TRUE & TRUE & & \\
\hline PRE & TRUE & TRUE & TRUE & & \\
\hline TYP & TRUE & TRUE & TRUE & & \\
\hline AD1 & TRUE & TRUE & TRUE & & \\
\hline AD2 & TRUE & TRUE & TRUE & & \\
\hline AD3 & TRUE & TRUE & TRUE & & \\
\hline AD4 & TRUE & TRUE & TRUE & & \\
\hline ZIP1 & TRUE & TRUE & TRUE & & \\
\hline ZIP2 & TRUE & TRUE & TRUE & & \\
\hline $\mathrm{CDE}$ & TRUE & TRUE & TRUE & & \\
\hline SUF & TRUE & TRUE & TRUE & & \\
\hline AD5 & TRUE & TRUE & TRUE & & \\
\hline AD6 & TRUE & TRUE & TRUE & & \\
\hline AD7 & TRUE & TRUE & TRUE & & \\
\hline AD8 & TRUE & TRUE & TRUE & & \\
\hline ZIP3 & TRUE & TRUE & TRUE & & \\
\hline ZIP4 & TRUE & TRUE & TRUE & & \\
\hline Related Street C/L Object ID & TRUE & TRUE & TRUE & & \\
\hline Legacy EES Number & FALSE & TRUE & TRUE & & \\
\hline CreationDate & TRUE & TRUE & TRUE & $\begin{array}{l}\text { ArcFM } \\
\text { Current } \\
\text { Date }\end{array}$ & \\
\hline Created By & TRUE & TRUE & FALSE & $\begin{array}{l}\text { ArcFM } \\
\text { User } \\
\text { Name }\end{array}$ & \\
\hline UpdateDate & TRUE & FALSE & TRUE & & ArcFM Current Date \\
\hline Updated By & FALSE & FALSE & FALSE & & ArcFM User Name \\
\hline
\end{tabular}

arcfm8.LANDBASE.LinearWater

ArcFM Display Field: OBJECTID

Create Edit Task:

On Create Event:

On Update Event:

On Delete Event:

Before Split Event:

On Split Event:

After Split Event:

On Abandon Event:

Abandon Feature Class:

Abandon Subtype:

Remove Feature Class:

Remove Subtype: 


\begin{tabular}{|l|c|c|c|c|c|}
\hline Field Alias & Visible & Editable & $\begin{array}{c}\text { Allow } \\
\text { Null } \\
\text { Values }\end{array}$ & $\begin{array}{c}\text { On } \\
\text { Feature } \\
\text { Create }\end{array}$ & On Feature Update \\
\hline Subtype & TRUE & TRUE & FALSE & & \\
\hline Name & TRUE & TRUE & TRUE & & \\
\hline OBJECTID & TRUE & FALSE & FALSE & & \\
\hline Shape & FALSE & TRUE & TRUE & & \\
\hline CreationDate & FALSE & TRUE & FALSE & $\begin{array}{l}\text { ArcFM } \\
\text { Current } \\
\text { Date }\end{array}$ & \\
\hline Created By & FALSE & TRUE & FALSE & $\begin{array}{l}\text { ArcFM } \\
\text { User } \\
\text { Name }\end{array}$ & \\
\hline UPDATEDATE & FALSE & FALSE & TRUE & & ArcFM Current Date \\
\hline Updated By & FALSE & FALSE & TRUE & & ArcFM User Name \\
\hline HANDLE & FALSE & TRUE & TRUE & & \\
\hline TILENAME & FALSE & TRUE & TRUE & & \\
\hline DCSID & FALSE & TRUE & TRUE & & \\
\hline SHAPE.len & FALSE & & & & \\
\hline AVLINK & FALSE & TRUE & TRUE & & \\
\hline
\end{tabular}

arcfm8.LANDBASE.Loa

ArcFM Display Field: OBJECTID

Create Edit Task:

On Create Event:

On Update Event:

On Delete Event:

On Abandon Event:

Abandon Feature Class:

Abandon Subtype:

Remove Feature Class:

Remove Subtype:

\begin{tabular}{|l|c|c|c|l|l|}
\hline Field Alias & Visible & Editable & $\begin{array}{c}\text { Allow } \\
\text { Null } \\
\text { Values }\end{array}$ & $\begin{array}{c}\text { On } \\
\text { Feature } \\
\text { Create }\end{array}$ & On Feature Update \\
\hline Subtype & TRUE & TRUE & FALSE & & \\
\hline LOA Number & TRUE & TRUE & FALSE & & \\
\hline OBJECTID & TRUE & FALSE & FALSE & & \\
\hline Shape & FALSE & TRUE & TRUE & & \\
\hline CreationDate & FALSE & TRUE & FALSE & $\begin{array}{l}\text { ArcFM } \\
\text { Current } \\
\text { Date }\end{array}$ & \\
\hline Created By & FALSE & TRUE & FALSE & $\begin{array}{l}\text { ArcFM } \\
\text { User } \\
\text { Name }\end{array}$ & \\
\hline UPDATEDATE & FALSE & FALSE & TRUE & & ArcFM Current Date \\
\hline Updated By & FALSE & FALSE & TRUE & & ArcFM User Name \\
\hline SHAPE.area & FALSE & & & & \\
\hline SHAPE.len & FALSE & & & & \\
\hline
\end{tabular}

arcfm8.LANDBASE.LotLine

ArcFM Display Field: OBJECTID

Create Edit Task:

On Create Event:

On Update Event:

On Delete Event:

Before Split Event:

On Split Event:

After Split Event:

On Abandon Event:

Abandon Feature Class:

Abandon Subtype:

Remove Feature Class:

Remove Subtype: 


\begin{tabular}{|l|c|c|c|c|c|}
\hline Field Alias & Visible & Editable & $\begin{array}{c}\text { Allow } \\
\text { Null } \\
\text { Values }\end{array}$ & $\begin{array}{c}\text { On } \\
\text { Feature } \\
\text { Create }\end{array}$ & On Feature Update \\
\hline Subtype & TRUE & TRUE & FALSE & & \\
\hline OBJECTID & TRUE & FALSE & FALSE & & \\
\hline Shape & FALSE & TRUE & TRUE & & \\
\hline CreationDate & FALSE & TRUE & FALSE & $\begin{array}{l}\text { ArcFM } \\
\text { Current } \\
\text { Date }\end{array}$ & \\
\hline Created By & FALSE & TRUE & FALSE & $\begin{array}{l}\text { ArcFM } \\
\text { User } \\
\text { Name }\end{array}$ & \\
\hline UPDATEDATE & FALSE & FALSE & TRUE & & ArcFM Current Date \\
\hline Updated By & FALSE & FALSE & TRUE & & ArcFM User Name \\
\hline HANDLE & FALSE & TRUE & TRUE & & \\
\hline TILENAME & FALSE & TRUE & TRUE & & \\
\hline DCSID & FALSE & TRUE & TRUE & & \\
\hline SHAPE.len & FALSE & & & & \\
\hline AVLINK & FALSE & TRUE & TRUE & & \\
\hline
\end{tabular}

arcfm8.LANDBASE.LotNumber

ArcFM Display Field: OBJECTID

Create Edit Task:

On Create Event:

On Update Event:

On Delete Event:

On Abandon Event:

Abandon Feature Class:

Abandon Subtype:

Remove Feature Class:

Remove Subtype:

\begin{tabular}{|l|c|c|c|c|c|}
\hline Field Alias & Visible & Editable & $\begin{array}{c}\text { Allow } \\
\text { Null } \\
\text { Values }\end{array}$ & $\begin{array}{c}\text { On } \\
\text { Feature } \\
\text { Create }\end{array}$ & On Feature Update \\
\hline Subtype & TRUE & TRUE & FALSE & & \\
\hline Lot Number & FALSE & TRUE & TRUE & & \\
\hline Lot Description & TRUE & TRUE & FALSE & & \\
\hline ADDRESS & TRUE & TRUE & TRUE & & \\
\hline OBJECTID & TRUE & FALSE & FALSE & & \\
\hline Shape & FALSE & TRUE & TRUE & & \\
\hline CreationDate & FALSE & TRUE & FALSE & $\begin{array}{l}\text { ArcFM } \\
\text { Current } \\
\text { Date }\end{array}$ & \\
\hline Created By & FALSE & TRUE & FALSE & $\begin{array}{l}\text { ArcFM } \\
\text { User } \\
\text { Name }\end{array}$ & \\
\hline UPDATEDATE & FALSE & FALSE & TRUE & & ArcFM Current Date \\
\hline Updated By & FALSE & FALSE & TRUE & & ArcFM User Name \\
\hline Symbol Rotation & FALSE & TRUE & FALSE & & \\
\hline HANDLE & FALSE & TRUE & TRUE & & \\
\hline TILENAME & FALSE & TRUE & TRUE & & \\
\hline DCSID & FALSE & TRUE & TRUE & & \\
\hline AVLINK & FALSE & TRUE & TRUE & & \\
\hline
\end{tabular}

arcfm8.LANDBASE.MinorGrid

ArcFM Display Field: OBJECTID

Create Edit Task:

On Create Event:

On Update Event:

On Delete Event:

On Abandon Event:

Abandon Feature Class:

Abandon Subtype:

Remove Feature Class:

Remove Subtype: 


\begin{tabular}{|l|l|l|c|l|l|}
\hline Field Alias & Visible & Editable & $\begin{array}{c}\text { Allow } \\
\text { Null } \\
\text { Values }\end{array}$ & $\begin{array}{c}\text { On } \\
\text { Feature } \\
\text { Create }\end{array}$ & On Feature Update \\
\hline Subtype & TRUE & TRUE & FALSE & & \\
\hline Township & TRUE & TRUE & FALSE & & \\
\hline Range & TRUE & TRUE & FALSE & & \\
\hline Major Grid & TRUE & TRUE & FALSE & & \\
\hline Section/Intermediate Grid & TRUE & TRUE & FALSE & & \\
\hline Minor Grid & TRUE & TRUE & FALSE & & \\
\hline OBJECTID & TRUE & FALSE & FALSE & & \\
\hline Shape & FALSE & TRUE & TRUE & & \\
\hline CreationDate & FALSE & TRUE & TRUE & $\begin{array}{l}\text { ArcFM } \\
\text { Current } \\
\text { Date }\end{array}$ & \\
\hline Created By & FALSE & TRUE & FALSE & $\begin{array}{l}\text { ArcFM } \\
\text { User } \\
\text { Name }\end{array}$ & \\
\hline UPDATEDATE & FALSE & FALSE & TRUE & & ArcFM Current Date \\
\hline Updated By & FALSE & FALSE & TRUE & & ArcFM User Name \\
\hline DCSID & FALSE & TRUE & TRUE & & \\
\hline SHAPE.area & FALSE & & & & \\
\hline SHAPE.len & FALSE & & & & \\
\hline
\end{tabular}

arcfm8.LANDBASE.MiscellaneousLinearLandFeature

ArcFM Display Field: OBJECTID

Create Edit Task:

On Create Event:

On Update Event:

On Delete Event:

Before Split Event:

On Split Event:

After Split Event:

On Abandon Event:

Abandon Feature Class:

Abandon Subtype:

Remove Feature Class:

Remove Subtype:

\begin{tabular}{|l|c|c|c|c|c|}
\hline Field Alias & Visible & Editable & $\begin{array}{c}\text { Allow } \\
\text { Null } \\
\text { Values }\end{array}$ & $\begin{array}{c}\text { On } \\
\text { Feature } \\
\text { Create }\end{array}$ & On Feature Update \\
\hline Subtype & TRUE & TRUE & FALSE & & \\
\hline Identifier & TRUE & TRUE & TRUE & & \\
\hline Classification & TRUE & TRUE & TRUE & & \\
\hline Label Text & TRUE & TRUE & TRUE & & \\
\hline OBJECTID & TRUE & FALSE & FALSE & & \\
\hline Shape & FALSE & TRUE & FALSE & & \\
\hline CreationDate & FALSE & TRUE & TRUE & $\begin{array}{l}\text { ArcFM } \\
\text { Current } \\
\text { Date }\end{array}$ & \\
\hline Created By & FALSE & TRUE & FALSE & $\begin{array}{l}\text { ArcFM } \\
\text { User Name }\end{array}$ & \\
\hline UPDATEDATE & FALSE & FALSE & TRUE & & ArcFM Current Date \\
\hline Updated By & FALSE & FALSE & TRUE & & ArcFM User Name \\
\hline HANDLE & FALSE & TRUE & TRUE & & \\
\hline TILENAME & FALSE & TRUE & TRUE & & \\
\hline SHAPE.len & FALSE & & & & \\
\hline
\end{tabular}

arcfm8.LANDBASE.MiscellaneousPointLandFeature

ArcFM Display Field: OBJECTID

Create Edit Task:

On Create Event:

On Update Event:

On Delete Event:

On Abandon Event: 
Abandon Feature Class:

Abandon Subtype:

Remove Feature Class:

Remove Subtype:

\begin{tabular}{|l|c|c|c|c|c|}
\hline Field Alias & Visible & Editable & $\begin{array}{c}\text { Allow Null } \\
\text { Values }\end{array}$ & $\begin{array}{c}\text { On } \\
\text { Feature } \\
\text { Create }\end{array}$ & On Feature Update \\
\hline Subtype & TRUE & TRUE & FALSE & & \\
\hline Identifier & TRUE & TRUE & TRUE & & \\
\hline Classification & TRUE & TRUE & TRUE & & \\
\hline Label Text & TRUE & TRUE & TRUE & & \\
\hline OBJECTID & TRUE & FALSE & FALSE & & \\
\hline Shape & FALSE & TRUE & TRUE & & \\
\hline CreationDate & FALSE & TRUE & FALSE & $\begin{array}{l}\text { ArcFM } \\
\text { Current } \\
\text { Date }\end{array}$ & \\
\hline Created By & FALSE & TRUE & FALSE & $\begin{array}{l}\text { ArcFM } \\
\text { User } \\
\text { Name }\end{array}$ & \\
\hline UPDATEDATE & FALSE & FALSE & TRUE & & ArcFM Current Date \\
\hline Updated By & FALSE & FALSE & TRUE & & ArcFM User Name \\
\hline Symbol Rotation & FALSE & TRUE & FALSE & & \\
\hline Graphics Scale Factor & FALSE & TRUE & TRUE & & \\
\hline
\end{tabular}

arcfm8.LANDBASE.Municipality

ArcFM Display Field: OBJECTID

Create Edit Task:

On Create Event:

On Update Event:

On Delete Event:

On Abandon Event:

Abandon Feature Class:

Abandon Subtype:

Remove Feature Class:

Remove Subtype:

\begin{tabular}{|l|c|c|c|c|c|}
\hline Field Alias & Visible & Editable & $\begin{array}{c}\text { Allow Null } \\
\text { Values }\end{array}$ & $\begin{array}{c}\text { On } \\
\text { Feature } \\
\text { Create }\end{array}$ & On Feature Update \\
\hline Subtype & TRUE & TRUE & FALSE & & \\
\hline State Name & TRUE & TRUE & FALSE & & \\
\hline Municipality Name & TRUE & TRUE & FALSE & & \\
\hline OBJECTID & TRUE & FALSE & FALSE & & \\
\hline Shape & FALSE & TRUE & TRUE & & \\
\hline CreationDate & FALSE & TRUE & FALSE & $\begin{array}{l}\text { ArcFM } \\
\text { Current } \\
\text { Date }\end{array}$ & \\
\hline Created By & FALSE & TRUE & FALSE & $\begin{array}{l}\text { ArcFM } \\
\text { User } \\
\text { Name }\end{array}$ & \\
\hline UPDATEDATE & FALSE & FALSE & TRUE & & ArcFM Current Date \\
\hline Updated By & FALSE & FALSE & TRUE & & ArcFM User Name \\
\hline SHAPE.area & FALSE & & & & \\
\hline SHAPE.len & FALSE & & & & \\
\hline
\end{tabular}

arcfm8.LANDBASE.PIssSection

ArcFM Display Field: OBJECTID

Create Edit Task:

On Create Event:

On Update Event:

On Delete Event:

On Abandon Event:

Abandon Feature Class:

Abandon Subtype:

Remove Feature Class: 
Remove Subtype:

\begin{tabular}{|l|c|c|c|c|c|}
\hline Field Alias & Visible & Editable & $\begin{array}{c}\text { Allow Null } \\
\text { Values }\end{array}$ & $\begin{array}{c}\text { On } \\
\text { Feature } \\
\text { Create }\end{array}$ & On Feature Update \\
\hline Subtype & TRUE & TRUE & FALSE & & \\
\hline Township & TRUE & TRUE & FALSE & & \\
\hline Range & TRUE & TRUE & FALSE & & \\
\hline Major Grid & TRUE & TRUE & FALSE & & \\
\hline Section/Intermediate Grid & TRUE & TRUE & FALSE & & \\
\hline OBJECTID & TRUE & FALSE & FALSE & & \\
\hline Shape & FALSE & TRUE & TRUE & & \\
\hline CreationDate & FALSE & TRUE & FALSE & $\begin{array}{l}\text { ArcFM } \\
\text { Current } \\
\text { Date }\end{array}$ & \\
\hline Created By & FALSE & TRUE & FALSE & $\begin{array}{l}\text { ArcFM } \\
\text { User } \\
\text { Name }\end{array}$ & \\
\hline UPDATEDATE & FALSE & FALSE & TRUE & & ArcFM Current Date \\
\hline Updated By & FALSE & FALSE & TRUE & & ArcFM User Name \\
\hline DCSID & FALSE & TRUE & TRUE & & \multicolumn{1}{|l|}{} \\
\hline SHAPE.area & FALSE & & & & \\
\hline SHAPE.len & FALSE & & & & \\
\hline
\end{tabular}

arcfm8.LANDBASE.PoliticalTownship

ArcFM Display Field: OBJECTID

Create Edit Task:

On Create Event:

On Update Event:

On Delete Event:

On Abandon Event:

Abandon Feature Class:

Abandon Subtype:

Remove Feature Class:

Remove Subtype:

\begin{tabular}{|l|c|c|c|c|c|}
\hline Field Alias & Visible & Editable & $\begin{array}{c}\text { Allow Null } \\
\text { Values }\end{array}$ & $\begin{array}{c}\text { On } \\
\text { Feature } \\
\text { Create }\end{array}$ & On Feature Update \\
\hline Subtype & TRUE & TRUE & FALSE & & \\
\hline State Name & TRUE & TRUE & FALSE & & \\
\hline County Name & TRUE & TRUE & FALSE & & \\
\hline Township Name & TRUE & TRUE & FALSE & & \\
\hline OBJECTID & TRUE & FALSE & FALSE & & \\
\hline Shape & FALSE & TRUE & TRUE & & \\
\hline CreationDate & FALSE & TRUE & FALSE & $\begin{array}{l}\text { ArcFM } \\
\text { Current } \\
\text { Date }\end{array}$ & \\
\hline Created By & FALSE & TRUE & FALSE & $\begin{array}{l}\text { ArcFM } \\
\text { User } \\
\text { Name }\end{array}$ & \\
\hline UPDATEDATE & FALSE & FALSE & TRUE & & ArcFM Current Date \\
\hline Updated By & FALSE & FALSE & TRUE & & ArcFM User Name \\
\hline SHAPE.area & FALSE & & & & \\
\hline SHAPE.len & FALSE & & & & \\
\hline
\end{tabular}

arcfm8.LANDBASE.QuarterTownshipGrid

ArcFM Display Field: OBJECTID

Create Edit Task:

On Create Event:

On Update Event:

On Delete Event:

On Abandon Event:

Abandon Feature Class:

Abandon Subtype:

Remove Feature Class: 
Remove Subtype:

\begin{tabular}{|l|l|l|c|l|l|}
\hline Field Alias & Visible & Editable & $\begin{array}{c}\text { Allow } \\
\text { Null } \\
\text { Values }\end{array}$ & $\begin{array}{c}\text { On } \\
\text { Feature } \\
\text { Create }\end{array}$ & On Feature Update \\
\hline Subtype & TRUE & TRUE & FALSE & & \\
\hline Township & TRUE & TRUE & FALSE & & \\
\hline Major Grid & TRUE & TRUE & FALSE & & \\
\hline Quarter Township Grid Number & TRUE & TRUE & FALSE & & \\
\hline Range & TRUE & TRUE & FALSE & & \\
\hline OBJECTID & TRUE & FALSE & FALSE & & \\
\hline Shape & FALSE & TRUE & TRUE & & \\
\hline CreationDate & FALSE & TRUE & FALSE & $\begin{array}{l}\text { ArcFM } \\
\text { Current } \\
\text { Date }\end{array}$ & \\
\hline Created By & FALSE & TRUE & FALSE & $\begin{array}{l}\text { ArcFM } \\
\text { User } \\
\text { Name }\end{array}$ & \\
\hline UPDATEDATE & FALSE & FALSE & TRUE & & ArcFM Current Date \\
\hline Updated By & FALSE & FALSE & TRUE & & ArcFM User Name \\
\hline DCSID & FALSE & TRUE & TRUE & & \\
\hline SHAPE.area & FALSE & & & & \\
\hline SHAPE.len & FALSE & & & & \\
\hline
\end{tabular}

arcfm8.LANDBASE.Railroad

ArcFM Display Field: OBJECTID

Create Edit Task:

On Create Event:

On Update Event:

On Delete Event:

Before Split Event:

On Split Event:

After Split Event:

On Abandon Event:

Abandon Feature Class:

Abandon Subtype:

Remove Feature Class:

Remove Subtype:

\begin{tabular}{|l|c|c|c|c|c|}
\hline Field Alias & Visible & Editable & $\begin{array}{c}\text { Allow } \\
\text { Null } \\
\text { Values }\end{array}$ & $\begin{array}{c}\text { On } \\
\text { Feature } \\
\text { Create }\end{array}$ & On Feature Update \\
\hline Subtype & TRUE & TRUE & FALSE & & \\
\hline Name & TRUE & TRUE & TRUE & & \\
\hline OBJECTID & TRUE & FALSE & FALSE & & \\
\hline Shape & FALSE & TRUE & TRUE & & \\
\hline CreationDate & FALSE & TRUE & FALSE & $\begin{array}{l}\text { ArcFM } \\
\text { Current } \\
\text { Date }\end{array}$ & \\
\hline Created By & FALSE & TRUE & FALSE & $\begin{array}{l}\text { ArcFM } \\
\text { User } \\
\text { Name }\end{array}$ & \\
\hline UPDATEDATE & FALSE & FALSE & TRUE & & ArcFM Current Date \\
\hline Updated By & FALSE & FALSE & TRUE & & ArcFM User Name \\
\hline HANDLE & FALSE & TRUE & TRUE & & \\
\hline TILENAME & FALSE & TRUE & TRUE & & \\
\hline DCSID & FALSE & TRUE & TRUE & & \\
\hline SHAPE.len & FALSE & & & & \\
\hline AVLINK & FALSE & TRUE & TRUE & & \\
\hline
\end{tabular}

arcfm8.LANDBASE.ReferenceLine

ArcFM Display Field: OBJECTID

arcfm8.LANDBASE.ReferencePoint

ArcFM Display Field: OBJECTID

Create Edit Task: ArcFM Linear Point

On Create Event: 
On Update Event:

On Delete Event:

On Abandon Event:

Abandon Feature Class:

Abandon Subtype:

Remove Feature Class:

Remove Subtype:

\begin{tabular}{|l|c|c|c|c|c|}
\hline Field Alias & Visible & Editable & $\begin{array}{c}\text { Allow } \\
\text { Null } \\
\text { Values }\end{array}$ & $\begin{array}{c}\text { On } \\
\text { Feature } \\
\text { Create }\end{array}$ & On Feature Update \\
\hline OBJECTID & TRUE & FALSE & FALSE & & \\
\hline Shape & TRUE & TRUE & TRUE & & \\
\hline
\end{tabular}

arcfm8.LANDBASE.States

ArcFM Display Field: OBJECTID

Create Edit Task:

On Create Event:

On Update Event:

On Delete Event:

On Abandon Event:

Abandon Feature Class:

Abandon Subtype:

Remove Feature Class:

Remove Subtype:

\begin{tabular}{|l|c|c|c|c|c|}
\hline Field Alias & Visible & Editable & $\begin{array}{c}\text { Allow } \\
\text { Null } \\
\text { Values }\end{array}$ & $\begin{array}{c}\text { On } \\
\text { Feature } \\
\text { Create }\end{array}$ & On Feature Update \\
\hline Subtype & TRUE & TRUE & FALSE & & \\
\hline State Name & TRUE & TRUE & FALSE & & \\
\hline OBJECTID & TRUE & FALSE & FALSE & & \\
\hline Shape & FALSE & TRUE & TRUE & & \\
\hline CreationDate & FALSE & TRUE & FALSE & $\begin{array}{l}\text { ArcFM } \\
\text { Current } \\
\text { Date }\end{array}$ & \\
\hline Created By & FALSE & TRUE & FALSE & $\begin{array}{l}\text { ArcFM } \\
\text { User } \\
\text { Name }\end{array}$ & \\
\hline UPDATEDATE & FALSE & FALSE & TRUE & & ArcFM Current Date \\
\hline Updated By & FALSE & FALSE & TRUE & & ArcFM User Name \\
\hline SHAPE.area & FALSE & & & & \\
\hline SHAPE.len & FALSE & & & & \\
\hline
\end{tabular}

arcfm8.LANDBASE.StreetCenterLine

ArcFM Display Field: OBJECTID

Create Edit Task:

On Create Event:

On Update Event:

On Delete Event:

Before Split Event:

On Split Event:

After Split Event:

On Abandon Event:

Abandon Feature Class:

Abandon Subtype:

Remove Feature Class:

Remove Subtype:

\begin{tabular}{|l|l|l|c|c|c|}
\hline Field Alias & Visible & Editable & $\begin{array}{c}\text { Allow } \\
\text { Null } \\
\text { Values }\end{array}$ & $\begin{array}{c}\text { On } \\
\text { Feature } \\
\text { Create }\end{array}$ & On Feature Update \\
\hline Subtype & TRUE & TRUE & FALSE & & \\
\hline Road Name & TRUE & TRUE & FALSE & & \\
\hline Road Type Suffix & TRUE & TRUE & FALSE & & \\
\hline Road Direction Prefix & TRUE & TRUE & TRUE & & \\
\hline
\end{tabular}




\begin{tabular}{|l|c|c|c|l|l|} 
Road Direction Suffix & TRUE & TRUE & TRUE & & \\
\hline Left Low Address Range & TRUE & TRUE & TRUE & & \\
\hline Left High Address Range & TRUE & TRUE & TRUE & & \\
\hline Right Low Address Range & TRUE & TRUE & TRUE & & \\
\hline Right High Address Range & TRUE & TRUE & TRUE & & \\
\hline Left Zip Code & TRUE & TRUE & TRUE & & \\
\hline Right Zip Code & TRUE & TRUE & TRUE & & \\
\hline Alternate Name 1 & TRUE & TRUE & TRUE & & \\
\hline Alternate Name 2 & TRUE & TRUE & TRUE & & \\
\hline Alternate Name 3 & TRUE & TRUE & TRUE & & \\
\hline Alternate Name 4 & TRUE & TRUE & TRUE & & \\
\hline OBJECTID & TRUE & FALSE & FALSE & & \\
\hline Shape & FALSE & TRUE & TRUE & & \\
\hline CreationDate & FALSE & TRUE & FALSE & $\begin{array}{l}\text { ArcFM } \\
\text { Current } \\
\text { Date }\end{array}$ & \\
\hline Created By & FALSE & TRUE & FALSE & $\begin{array}{l}\text { ArcFM } \\
\text { Name }\end{array}$ & \\
\hline UPDATEDATE & FALSE & FALSE & TRUE & & ArcFM Current Date \\
\hline Updated By & FALSE & FALSE & TRUE & & ArcFM User Name \\
\hline Legacy EES Number & FALSE & TRUE & TRUE & & \\
\hline SHAPE.len & FALSE & & & & \\
\hline
\end{tabular}

arcfm8.LANDBASE.StreetCenterlinelntersections

ArcFM Display Field: OBJECTID

Create Edit Task:

On Create Event:

On Update Event:

On Delete Event:

On Abandon Event:

Abandon Feature Class:

Abandon Subtype:

Remove Feature Class:

Remove Subtype:

\begin{tabular}{|l|c|c|c|c|c|}
\hline Field Alias & Visible & Editable & $\begin{array}{c}\text { Allow } \\
\text { Null } \\
\text { Values }\end{array}$ & $\begin{array}{c}\text { On } \\
\text { Feature } \\
\text { Create }\end{array}$ & On Feature Update \\
\hline NAME1 & TRUE & TRUE & TRUE & & \\
\hline NAME2 & TRUE & TRUE & TRUE & & \\
\hline ZIPCODES & FALSE & TRUE & TRUE & & \\
\hline XCOORD & FALSE & TRUE & TRUE & & \\
\hline YCOORD & FALSE & TRUE & TRUE & & \\
\hline OBJECTID & TRUE & FALSE & FALSE & & \\
\hline SHAPE & FALSE & TRUE & TRUE & & \\
\hline ROADNAME & FALSE & TRUE & TRUE & & \\
\hline
\end{tabular}

arcfm8.LANDBASE.StreetRow

ArcFM Display Field: OBJECTID

Create Edit Task:

On Create Event:

On Update Event:

On Delete Event:

Before Split Event:

On Split Event:

After Split Event:

On Abandon Event:

Abandon Feature Class:

Abandon Subtype:

Remove Feature Class:

Remove Subtype: 


\begin{tabular}{|l|c|c|c|c|c|}
\hline Field Alias & Visible & Editable & $\begin{array}{c}\text { Allow } \\
\text { Null } \\
\text { Values }\end{array}$ & $\begin{array}{c}\text { On } \\
\text { Feature } \\
\text { Create }\end{array}$ & On Feature Update \\
\hline Subtype & TRUE & TRUE & FALSE & & \\
\hline OBJECTID & TRUE & FALSE & FALSE & & \\
\hline Shape & FALSE & TRUE & TRUE & & \\
\hline CreationDate & FALSE & TRUE & FALSE & $\begin{array}{l}\text { ArcFM } \\
\text { Current } \\
\text { Date }\end{array}$ & \\
\hline Created By & FALSE & TRUE & FALSE & $\begin{array}{l}\text { ArcFM } \\
\text { User } \\
\text { Name }\end{array}$ & \\
\hline UPDATEDATE & FALSE & FALSE & TRUE & & ArcFM Current Date \\
\hline Updated By & FALSE & FALSE & TRUE & & ArcFM User Name \\
\hline HANDLE & FALSE & TRUE & TRUE & & \\
\hline TILENAME & FALSE & TRUE & TRUE & & \\
\hline SHAPE.len & FALSE & & & & \\
\hline
\end{tabular}

arcfm8.LANDBASE.TownshipRange

ArcFM Display Field: OBJECTID

Create Edit Task:

On Create Event:

On Update Event:

On Delete Event:

On Abandon Event:

Abandon Feature Class:

Abandon Subtype:

Remove Feature Class:

Remove Subtype:

\begin{tabular}{|l|c|c|c|c|c|}
\hline Field Alias & Visible & Editable & $\begin{array}{c}\text { Allow } \\
\text { Null } \\
\text { Values }\end{array}$ & $\begin{array}{c}\text { On } \\
\text { Feature } \\
\text { Create }\end{array}$ & On Feature Update \\
\hline Subtype & TRUE & TRUE & FALSE & & \\
\hline Township & TRUE & TRUE & FALSE & & \\
\hline Range & TRUE & TRUE & FALSE & & \\
\hline Major Grid & TRUE & TRUE & FALSE & & \\
\hline OBJECTID & TRUE & FALSE & FALSE & & \\
\hline Shape & FALSE & TRUE & TRUE & & \\
\hline CreationDate & FALSE & TRUE & FALSE & $\begin{array}{l}\text { ArcFM } \\
\text { Current } \\
\text { Date }\end{array}$ & \\
\hline Created By & FALSE & TRUE & FALSE & $\begin{array}{l}\text { ArcFM } \\
\text { User } \\
\text { Name }\end{array}$ & \\
\hline UPDATEDATE & FALSE & FALSE & TRUE & & ArcFM Current Date \\
\hline Updated By & FALSE & FALSE & TRUE & & ArcFM User Name \\
\hline DCSID & FALSE & TRUE & TRUE & & \\
\hline SHAPE.area & FALSE & & & & \\
\hline SHAPE.len & FALSE & & & & \\
\hline
\end{tabular}


Table E-7. Contains all land ArcFM model name assignments for both the base and custom configuration

Bridge

Object Class Model Names

Object Class

Bridge

Model Name

BRIDGE

Field Model Names

\begin{tabular}{|l|l}
\hline Field & Model Name
\end{tabular}

County

Object Class Model Names

\begin{tabular}{|l|l|}
\hline Object Class & Model Name \\
\hline County & LOCATABLEOBJECT \\
\hline
\end{tabular}

Field Model Names

\begin{tabular}{|l|l|}
\hline Field & Model Name \\
\hline COUNTYNAME & LOCATABLEFIELD \\
\hline
\end{tabular}

DgGrid

Object Class Model Names

\begin{tabular}{|l|l|}
\hline Object Class & Model Name \\
\hline DgGrid & DGGRID \\
\hline DgGrid & LOCATABLEOBJECT \\
\hline
\end{tabular}

Field Model Names

\begin{tabular}{|l|l|}
\hline Field & Model Name \\
\hline DGGRIDNUMBER & DGGRIDNUMBER \\
\hline DGGRIDNUMBER & LOCATABLEFIELD \\
\hline
\end{tabular}

Easement

Object Class Model Names

\begin{tabular}{|l|l|}
\hline Object Class & Model Name \\
\hline Easement & LOCATABLEOBJECT \\
\hline
\end{tabular}

Field Model Names

\begin{tabular}{|l|l|}
\hline Field & Model Name \\
\hline EASEMENTNUMBER & LOCATABLEFIELD \\
\hline
\end{tabular}

FranchiseBoundary

Object Class Model Names

\begin{tabular}{|l|l}
\hline Object Class & Model Name \\
\hline FranchiseBoundary & LOCATABLEOBJECT \\
\hline
\end{tabular}

Field Model Names

\begin{tabular}{|l|l|}
\hline Field & Model Name \\
\hline UTILITYNAME & LOCATABLEFIELD \\
\hline
\end{tabular}


LandDiscrepancy

Object Class Model Names

\begin{tabular}{l|l}
\hline Object Class & Model Name \\
\hline LandDiscrepancy & LOCATABLEOBJECT
\end{tabular}

Field Model Names

\begin{tabular}{|l|l|}
\hline Field & Model Name \\
\hline ERRORDESCRIPTION & LOCATABLEFIELD \\
\hline
\end{tabular}

LegacyStreetCenterLine

Object Class Model Names

\begin{tabular}{|l|l|}
\hline Object Class & Model Name \\
\hline LegacyStreetCenterLine & LOCATABLEOBJECT \\
\hline
\end{tabular}

Field Model Names

\begin{tabular}{|l|l|}
\hline Field & Model Name \\
\hline LEGACYEESNUMBER & LOCATABLEFIELD \\
\hline
\end{tabular}

LinearWater

Object Class Model Names

\begin{tabular}{l|l}
\hline Object Class & Model Name \\
\hline LinearWater & LOCATABLEOBJECT \\
\hline
\end{tabular}

Field Model Names

\begin{tabular}{|l|l|}
\hline Field & Model Name \\
\hline WATERFEATURENAME & LOCATABLEFIELD \\
\hline
\end{tabular}

Loa

Object Class Model Names

\begin{tabular}{|l|l|}
\hline Object Class & Model Name \\
\hline Loa & LOA \\
\hline Loa & LOCATABLEOBJECT \\
\hline
\end{tabular}

Field Model Names

\begin{tabular}{|l|l|}
\hline Field & Model Name \\
\hline LOANUMBER & LOANAME \\
\hline LOANUMBER & LOANUMBER \\
\hline LOANUMBER & LOCATABLEFIELD \\
\hline
\end{tabular}

LotLine

Object Class Model Names

\begin{tabular}{|l|l|}
\hline Object Class & Model Name \\
\hline LotLine & LOCATABLEOBJECT \\
\hline
\end{tabular}

Field Model Names

\begin{tabular}{|l|l|}
\hline Field & Model Name \\
\hline OBJECTID & LOCATABLEFIELD \\
\hline
\end{tabular}

LotNumber 
Object Class Model Names

\begin{tabular}{|l|l|}
\hline Object Class & Model Name \\
\hline LotNumber & LOCATABLEOBJECT \\
\hline
\end{tabular}

Field Model Names

\begin{tabular}{|l|l|}
\hline Field & Model Name \\
\hline LOTNUMBER & LOCATABLEFIELD \\
\hline
\end{tabular}

MinorGrid

Object Class Model Names

\begin{tabular}{|l|l|}
\hline Object Class & Model Name \\
\hline MinorGrid & LOCATABLEOBJECT \\
\hline
\end{tabular}

Field Model Names

\begin{tabular}{|l|l|}
\hline Field & Model Name \\
\hline MINORGRIDNUMBER & LOCATABLEFIELD \\
\hline
\end{tabular}

MiscellaneousLinearLandFeature

Object Class Model Names

\begin{tabular}{|l|l}
\hline Object Class & Model Name \\
\hline MiscellaneousLinearLandFeature & LOCATABLEOBJECT \\
\hline
\end{tabular}

Field Model Names

\begin{tabular}{|l|l|}
\hline Field & Model Name \\
\hline FEATUREID & LOCATABLEFIELD \\
\hline
\end{tabular}

MiscellaneousPointLandFeature

Object Class Model Names

\begin{tabular}{|l|l|}
\hline Object Class & Model Name \\
\hline MiscellaneousPointLandFeature & LOCATABLEOBJECT \\
\hline
\end{tabular}

Field Model Names

\begin{tabular}{|l|l|}
\hline Field & Model Name \\
\hline FEATUREID & LOCATABLEFIELD \\
\hline
\end{tabular}

Municipality

Object Class Model Names

Object Class

Model Name

Municipality

LOCATABLEOBJECT

Field Model Names

\begin{tabular}{|l|l|}
\hline Field & Model Name \\
\hline MUNICIPALITYNAME & LOCATABLEFIELD \\
\hline & \\
PIssSection & \\
Object Class Model Names & Model Name \\
\hline Object Class & LOCATABLEOBJECT \\
\hline PlssSection &
\end{tabular}


Field Model Names

\begin{tabular}{|l|l|}
\hline Field & Model Name \\
\hline PLSSSECTIONNUMBER & LOCATABLEFIELD \\
\hline
\end{tabular}

PoliticalTownship

Object Class Model Names

Object Class

PoliticalTownship

Model Name

LOCATABLEOBJECT

Field Model Names

\begin{tabular}{|l|l|}
\hline Field & Model Name \\
\hline TOWNSHIPNAME & LOCATABLEFIELD \\
\hline
\end{tabular}

QuarterTownshipGrid

Object Class Model Names

\begin{tabular}{|l|l}
\hline Object Class & Model Name \\
\hline QuarterTownshipGrid & LOCATABLEOBJECT
\end{tabular}

Field Model Names

\begin{tabular}{|l|l|}
\hline Field & Model Name \\
\hline QUARTERTOWNSHIPGRIDNUMBER & LOCATABLEFIELD \\
\hline
\end{tabular}

Railroad

Object Class Model Names

\begin{tabular}{|l|l}
\hline Object Class & Model Name \\
\hline Railroad & LOCATABLEOBJECT
\end{tabular}

Field Model Names

\begin{tabular}{|l|l|}
\hline Field & Model Name \\
\hline RAILROADNAME & LOCATABLEFIELD \\
\hline
\end{tabular}

ReferenceLine

Object Class Model Names

\begin{tabular}{|l|l}
\hline Object Class & Model Name \\
\hline ReferenceLine & REFERENCEFEATURE \\
\hline ReferenceLine & DONOTPOST \\
\hline
\end{tabular}

Field Model Names

\section{Field}

Model Name

ReferencePoint

Object Class Model Names

\begin{tabular}{|l|l|}
\hline Object Class & Model Name \\
\hline ReferencePoint & DONOTPOST \\
\hline ReferencePoint & REFERENCEFEATURE \\
\hline
\end{tabular}

Field Model Names

Field

Model Name 
States

Object Class Model Names

\begin{tabular}{|l|l|}
\hline Object Class & Model Name \\
\hline States & LOCATABLEOBJECT \\
\hline States & GDBEXTRACTION \\
\hline
\end{tabular}

Field Model Names

\begin{tabular}{|l|l|}
\hline Field & Model Name \\
\hline STATENAME & GDBEXTRACTION \\
\hline STATENAME & LOCATABLEFIELD \\
\hline
\end{tabular}

StreetCenterLine

Object Class Model Names

\begin{tabular}{|l|l|}
\hline Object Class & Model Name \\
\hline StreetCenterLine & LOCATABLEOBJECT \\
\hline
\end{tabular}

Field Model Names

\begin{tabular}{|l|l|}
\hline Field & Model Name \\
\hline ROADNAME & LOCATABLEFIELD \\
\hline
\end{tabular}

StreetRow

Object Class Model Names

\begin{tabular}{|l|l|}
\hline Object Class & Model Name \\
\hline StreetRow & LOCATABLEOBJECT \\
\hline
\end{tabular}

Field Model Names

\begin{tabular}{|l|l|}
\hline Field & Model Name \\
\hline OBJECTID & LOCATABLEFIELD \\
\hline
\end{tabular}

TownshipRange

Object Class Model Names

\begin{tabular}{|l|l|}
\hline Object Class & Model Name \\
\hline TownshipRange & LOCATABLEOBJECT \\
\hline
\end{tabular}

Field Model Names

\begin{tabular}{|l|l|}
\hline Field & Model Name \\
\hline MAJORGRIDNUMBER & LOCATABLEFIELD \\
\hline
\end{tabular}


Table E-8. Electric snapping configuration

CapacitorBank

\begin{tabular}{|c|c|c|c|}
\hline Subtype & Snap To Feature & Hit Type & Tolerance \\
\hline SinglePhaseCapacitor & OhConductor & Vertex, Edge, End & 10 \\
\hline ThreePhaseCapacitor & OhConductor & Vertex, Edge, End & 10 \\
\hline \multicolumn{4}{|l|}{ CustomerGenerator } \\
\hline Subtype & Snap To Feature & Hit Type & Tolerance \\
\hline Generator & OhConductor & Vertex, Edge, End & 10 \\
\hline Generator & UgConductor & Vertex, Edge, End & 10 \\
\hline Generator & Secondary & Vertex, Edge, End & 10 \\
\hline
\end{tabular}

DeadEndElectric

\begin{tabular}{l|l|c|c|}
\hline Subtype & Snap To Feature & Hit Type & Tolerance \\
\hline DeadEnd & OhConductor & End & 10 \\
\hline DeadEnd & UgConductor & End & 10 \\
\hline FeederAllOpenPoint & Snap To Feature & Hit Type & Tolerance \\
\hline Subtype & UgConductor & Vertex, Edge, End & 10 \\
\hline FeederAllOpenPoint
\end{tabular}

FuseCutoutBank

\begin{tabular}{|l|l|l|c|}
\hline Subtype & Snap To Feature & Hit Type & Tolerance \\
\hline SinglePhaseUgFuse & UgConductor & Vertex, Edge, End & 10 \\
\hline TwoPhaseUgFuse & TieBus & Vertex, Edge, End & 10 \\
\hline TwoPhaseUgFuse & UgConductor & Vertex, Edge, End & 10 \\
\hline ThreePhaseUgFuse & TieBus & Vertex, Edge, End & 10 \\
\hline ThreePhaseUgFuse & UgConductor & Vertex, Edge, End & 10 \\
\hline ThreePhaseOhFuse & OhConductor & Vertex, Edge, End & 10 \\
\hline ThreePhaseOhFuse & TieBus & Vertex, Edge, End & 10 \\
\hline TwoPhaseTripleShot & OhConductor & Vertex, Edge, End & 10 \\
\hline TwoPhaseTripleShot & TieBus & Vertex, Edge, End & 10 \\
\hline TwoPhaseOhFuse & OhConductor & Vertex, Edge, End & 10 \\
\hline TwoPhaseOhFuse & TieBus & Vertex, Edge, End & 10 \\
\hline SinglePhaseTripleShot & OhConductor & Vertex, Edge, End & 10 \\
\hline SinglePhaseTripleShot & TieBus & Vertex, Edge, End & 10 \\
\hline ThreePhaseTripleShot & OhConductor & Vertex, Edge, End & 10 \\
\hline ThreePhaseTripleShot & TieBus & Vertex, Edge, End & 10 \\
\hline SinglePhaseOhFuse & OhConductor & Vertex, Edge, End & 10 \\
\hline SinglePhaseOhFuse & TieBus & Vertex, Edge, End & 10 \\
\hline SinglePhaseUgFuse & TieBus & Vertex, Edge, End & 10 \\
\hline \\
\begin{tabular}{|l|l|l|c|}
\hline Manhole & \multicolumn{1}{|c|}{ Hit Type } & Tolerance \\
\hline Mantype & Snap To Feature & Vertex, Edge, End & 10 \\
\hline
\end{tabular} OhConductor & UgConductor & Hit Type & Tolerance \\
\hline Subtype & Snap To Feature & Vertex, Edge, End & 10 \\
\hline OhTransmission & OhConductor & Vertex, Edge, End & 10 \\
\hline OhTransmission & SubstationBreaker & Vertex, Edge, End & 10 \\
\hline ThreePhaseOverheadPrimary & FuseCutoutBank & Vertex, Edge, End & 10 \\
\hline ThreePhaseOverheadPrimary & OhConductor & 10 \\
\hline ThreePhaseOverheadPrimary & PrimaryMeter & Vertex, Edge, End & 10 \\
\hline ThreePhaseOverheadPrimary & RecloserBank & Vertex, Edge, End & 10 \\
\hline ThreePhaseOverheadPrimary & SectionalizerBank & \\
\hline
\end{tabular}




\begin{tabular}{|l|l|l|c|} 
ThreePhaseOverheadPrimary & Switch & Vertex, Edge, End & 10 \\
\hline ThreePhaseOverheadPrimary & TransformerBank & Vertex, Edge, End & 10 \\
\hline ThreePhaseOverheadPrimary & SubstationBreaker & Vertex, Edge, End & 10 \\
\hline ThreePhaseOverheadPrimary & TieBus & Vertex, Edge, End & 10 \\
\hline ThreePhaseOverheadPrimary & CustomerGenerator & Vertex, Edge, End & 10 \\
\hline SinglePhaseOverheadPrimary & FuseCutoutBank & Vertex, Edge, End & 10 \\
\hline SinglePhaseOverheadPrimary & TransformerBank & Vertex, Edge, End & 10 \\
\hline SinglePhaseOverheadPrimary & CapacitorBank & Vertex, Edge, End & 10 \\
\hline SinglePhaseOverheadPrimary & CustomerGenerator & Vertex, Edge, End & 10 \\
\hline SinglePhaseOverheadPrimary & OhConductor & Vertex, Edge, End & 10 \\
\hline SinglePhaseOverheadPrimary & RecloserBank & Vertex, Edge, End & 10 \\
\hline SinglePhaseOverheadPrimary & SectionalizerBank & Vertex, Edge, End & 10 \\
\hline SinglePhaseOverheadPrimary & Switch & Vertex, Edge, End & 10 \\
\hline SinglePhaseOverheadPrimary & TieBus & Vertex, Edge, End & 10 \\
\hline SinglePhaseOverheadPrimary & VoltageRegulator & Vertex, Edge, End & 10 \\
\hline SinglePhaseOverheadPrimary & PrimaryMeter & Vertex, Edge, End & 10 \\
\hline SinglePhaseOverheadPrimary & SubstationBreaker & Vertex, Edge, End & 10 \\
\hline TwoPhaseOverheadPrimary & CapacitorBank & Vertex, Edge, End & 10 \\
\hline TwoPhaseOverheadPrimary & CustomerGenerator & Vertex, Edge, End & 10 \\
\hline TwoPhaseOverheadPrimary & FuseCutoutBank & Vertex, Edge, End & 10 \\
\hline TwoPhaseOverheadPrimary & OhConductor & Vertex, Edge, End & 10 \\
\hline TwoPhaseOverheadPrimary & PrimaryMeter & Vertex, Edge, End & 10 \\
\hline TwoPhaseOverheadPrimary & RecloserBank & Vertex, Edge, End & 10 \\
\hline TwoPhaseOverheadPrimary & SectionalizerBank & Vertex, Edge, End & 10 \\
\hline TwoPhaseOverheadPrimary & SubstationBreaker & Vertex, Edge, End & 10 \\
\hline TwoPhaseOverheadPrimary & Switch & Vertex, Edge, End & 10 \\
\hline TwoPhaseOverheadPrimary & TieBus & Vertex, Edge, End & 10 \\
\hline TwoPhaseOverheadPrimary & TransformerBank & Vertex, Edge, End & 10 \\
\hline TwoPhaseOverheadPrimary & VoltageRegulator & Vertex, Edge, End & 10 \\
\hline OpenPont & & \\
\hline
\end{tabular}

OpenPoint

\begin{tabular}{|l|l|c|c|}
\hline Subtype & Snap To Feature & Hit Type & Tolerance \\
\hline OpenPoint & UgConductor & Vertex, Edge, End & 10 \\
\hline OpenPoint & OhConductor & Vertex, Edge, End & 10 \\
\hline OpenPoint & Secondary & Vertex, Edge, End & 10 \\
\hline
\end{tabular}

PadMount

\begin{tabular}{|l|l|c|c|}
\hline Subtype & Snap To Feature & Hit Type & Tolerance \\
\hline Pad & UgConductor & Vertex, Edge, End & 10 \\
\hline Vault & UgConductor & Vertex, Edge, End & 10 \\
\hline
\end{tabular}

Pedestal

\begin{tabular}{|c|c|c|c|}
\hline Subtype & Snap To Feature & Hit Type & Tolerance \\
\hline FusePedestal & UgConductor & Vertex, Edge, End & 10 \\
\hline PrimaryPedestal & UgConductor & Vertex, Edge, End & 10 \\
\hline SwitchPedestal & UgConductor & Vertex, Edge, End & 10 \\
\hline SecondaryPedestal & UgConductor & Vertex, Edge, End & 10 \\
\hline SecondaryPedestal & Secondary & Vertex, Edge, End & 10 \\
\hline \multicolumn{4}{|l|}{ PrimaryMeter } \\
\hline Subtype & Snap To Feature & Hit Type & Tolerance \\
\hline Overhead & OhConductor & Vertex, Edge, End & 10 \\
\hline
\end{tabular}




\begin{tabular}{|c|c|c|c|}
\hline Underground & UgConductor & Vertex, Edge, End & 10 \\
\hline \multicolumn{4}{|l|}{ RecloserBank } \\
\hline Subtype & Snap To Feature & Hit Type & Tolerance \\
\hline HybridRecloser & OhConductor & Vertex, Edge, End & 10 \\
\hline ThreePhaseRecloser & OhConductor & Vertex, Edge, End & 10 \\
\hline SinglePhaseRecloser & OhConductor & Vertex, Edge, End & 10 \\
\hline \multicolumn{4}{|l|}{ Secondary } \\
\hline Subtype & Snap To Feature & Hit Type & Tolerance \\
\hline OhSecondary & Secondary & Vertex, Edge, End & 10 \\
\hline OhSecondary & SupportStructure & Vertex, Edge, End & 10 \\
\hline UgSecondary & Secondary & Vertex, Edge, End & 10 \\
\hline UgSecondary & SupportStructure & Vertex, Edge, End & 10 \\
\hline UgSecondary & PadMount & Vertex, Edge, End & 10 \\
\hline UgSecondary & Pedestal & Vertex, Edge, End & 10 \\
\hline \multicolumn{4}{|l|}{ SectionalizerBank } \\
\hline Subtype & Snap To Feature & Hit Type & Tolerance \\
\hline Sectionalizer & OhConductor & Vertex, Edge, End & 10 \\
\hline Sectionalizer & \begin{tabular}{|l|} 
TieBus \\
\end{tabular} & Vertex, Edge, End & 10 \\
\hline \multicolumn{4}{|l|}{ Splice } \\
\hline Subtype & Snap To Feature & Hit Type & Tolerance \\
\hline UgSplice & UgConductor & Vertex, Edge, End & 10 \\
\hline \multicolumn{4}{|l|}{ SupportStructure } \\
\hline Subtype & Snap To Feature & Hit Type & Tolerance \\
\hline Tower & SupportStructure & Vertex, Edge, End & 10 \\
\hline \multicolumn{4}{|l|}{ Switch } \\
\hline Subtype & Snap To Feature & Hit Type & Tolerance \\
\hline GoabSwitch & OhConductor & Vertex, Edge, End & 10 \\
\hline HookSwitchUnderhung & OhConductor & Vertex, Edge, End & 10 \\
\hline DistributionAutomationSwitch & OhConductor & Vertex, Edge, End & 10 \\
\hline HookSwitchlnLine & OhConductor & Vertex, Edge, End & 10 \\
\hline UgSwitch & TieBus & Vertex, Edge, End & 10 \\
\hline UgSwitch & UgConductor & Vertex, Edge, End & 10 \\
\hline UgDistributionAutomationSwitch & UgConductor & Vertex, Edge, End & 10 \\
\hline UgDistributionAutomationSwitch & TieBus & Vertex, Edge, End & 10 \\
\hline OhDistributionAutomationSwitch & OhConductor & Vertex, Edge, End & 10 \\
\hline OhDistributionAutomationSwitch & TieBus & Vertex, Edge, End & 10 \\
\hline \multicolumn{4}{|l|}{ SwitchGear } \\
\hline Subtype & Snap To Feature & Hit Type & Tolerance \\
\hline SwitchGear & UgConductor & Vertex, Edge, End & 10 \\
\hline \multicolumn{4}{|l|}{ Terminator } \\
\hline Subtype & Snap To Feature & Hit Type & Tolerance \\
\hline PoleTerminator & TieBus & End & 10 \\
\hline PoleTerminator & UgConductor & Vertex, Edge, End & 10 \\
\hline \multicolumn{4}{|l|}{ TieBus } \\
\hline Subtype & Snap To Feature & Hit Type & Tolerance \\
\hline DistributionTieWire & OhConductor & Vertex, Edge, End & 10 \\
\hline DistributionTieWire & UgConductor & Vertex, Edge, End & 10 \\
\hline TransmissionTieWire & OhConductor & Vertex, Edge, End & 10 \\
\hline
\end{tabular}




\begin{tabular}{|l|l|l|c|} 
TransmissionTieWire & UgConductor & Vertex, Edge, End & 10 \\
\hline SwitchGearBusBar & Switch & Vertex, Edge, End & 10 \\
\hline SwitchGearBusBar & FuseCutoutBank & Vertex, Edge, End & 10 \\
\hline SubstationBusBar & PowerTransformer & Vertex, Edge, End & 10 \\
\hline SubstationBusBar & SubstationBreaker & Vertex, Edge, End & 10 \\
\hline TransformerLead & OhConductor & Vertex, Edge, End & 10 \\
\hline TransformerLead & TransformerBank & Vertex, Edge, End & 10 \\
\hline
\end{tabular}

TransformerBank

\begin{tabular}{|l|l|l|c|}
\hline Subtype & Snap To Feature & Hit Type & Tolerance \\
\hline TwoPhaseOverhead & SupportStructure & Vertex, Edge, End & 10 \\
\hline TwoPhaseOverhead & OhConductor & Vertex, Edge, End & 10 \\
\hline SinglePhaseStep & SupportStructure & Vertex, Edge, End & 10 \\
\hline SinglePhaseStep & OhConductor & Vertex, Edge, End & 10 \\
\hline ThreePhaseOverhead & SupportStructure & Vertex, Edge, End & 10 \\
\hline ThreePhaseOverhead & OhConductor & Vertex, Edge, End & 10 \\
\hline ThreePhaseStep & SupportStructure & Vertex, Edge, End & 10 \\
\hline ThreePhaseStep & OhConductor & Vertex, Edge, End & 10 \\
\hline SinglePhaseOverhead & SupportStructure & Vertex, Edge, End & 10 \\
\hline SinglePhaseOverhead & OhConductor & Vertex, Edge, End & 10 \\
\hline ThreePhaseUnderground & UgConductor & Vertex, Edge, End & 10 \\
\hline SinglePhaseUnderground & UgConductor & Vertex, Edge, End & 10 \\
\hline ThreePhaseOverheadInVault & PadMount & Vertex, Edge, End & 10 \\
\hline ThreePhaseOverheadInVault & SupportStructure & Vertex, Edge, End & 10 \\
\hline ThreePhaseOverheadInVault & OhConductor & Vertex, Edge, End & 10 \\
\hline TwoPhaseOverheadlnVault & PadMount & Vertex, Edge, End & 10 \\
\hline TwoPhaseOverheadInVault & SupportStructure & Vertex, Edge, End & 10 \\
\hline TwoPhaseOverheadInVault & OhConductor & Vertex, Edge, End & 10 \\
\hline SinglePhaseOverheadInVault & PadMount & Vertex, Edge, End & 10 \\
\hline SinglePhaseOverheadInVault & SupportStructure & Vertex, Edge, End & 10 \\
\hline SinglePhaseOverheadInVault & OhConductor & Vertex, Edge, End & 10 \\
\hline
\end{tabular}

\section{UgConductor}

\begin{tabular}{|l|l|l|c|}
\hline Subtype & Snap To Feature & Hit Type & Tolerance \\
\hline ThreePhasePrimaryUnderground & VoltageRegulator & Vertex, Edge, End & 10 \\
\hline ThreePhasePrimaryUnderground & CapacitorBank & Vertex, Edge, End & 10 \\
\hline ThreePhasePrimaryUnderground & CustomerGenerator & Vertex, Edge, End & 10 \\
\hline ThreePhasePrimaryUnderground & FuseCutoutBank & Vertex, Edge, End & 10 \\
\hline ThreePhasePrimaryUnderground & OhConductor & Vertex, Edge, End & 10 \\
\hline ThreePhasePrimaryUnderground & PrimaryMeter & Vertex, Edge, End & 10 \\
\hline ThreePhasePrimaryUnderground & RecloserBank & Vertex, Edge, End & 10 \\
\hline ThreePhasePrimaryUnderground & SectionalizerBank & Vertex, Edge, End & 10 \\
\hline ThreePhasePrimaryUnderground & Splice & Vertex, Edge, End & 10 \\
\hline ThreePhasePrimaryUnderground & SubstationBreaker & Vertex, Edge, End & 10 \\
\hline ThreePhasePrimaryUnderground & Switch & Vertex, Edge, End & 10 \\
\hline ThreePhasePrimaryUnderground & Terminator & Vertex, Edge, End & 10 \\
\hline ThreePhasePrimaryUnderground & TieBus & Vertex, Edge, End & 10 \\
\hline ThreePhasePrimaryUnderground & TransformerBank & Vertex, Edge, End & 10 \\
\hline ThreePhasePrimaryUnderground & UgConductor & Vertex, Edge, End & 10 \\
\hline TwoPhasePrimaryUnderground & VoltageRegulator & Vertex, Edge, End & 10 \\
\hline
\end{tabular}




\begin{tabular}{|l|l|l|c|} 
TwoPhasePrimaryUnderground & CapacitorBank & Vertex, Edge, End & 10 \\
\hline TwoPhasePrimaryUnderground & CustomerGenerator & Vertex, Edge, End & 10 \\
\hline TwoPhasePrimaryUnderground & FuseCutoutBank & Vertex, Edge, End & 10 \\
\hline TwoPhasePrimaryUnderground & OhConductor & Vertex, Edge, End & 10 \\
\hline TwoPhasePrimaryUnderground & PrimaryMeter & Vertex, Edge, End & 10 \\
\hline TwoPhasePrimaryUnderground & RecloserBank & Vertex, Edge, End & 10 \\
\hline TwoPhasePrimaryUnderground & SectionalizerBank & Vertex, Edge, End & 10 \\
\hline TwoPhasePrimaryUnderground & Splice & Vertex, Edge, End & 10 \\
\hline TwoPhasePrimaryUnderground & SubstationBreaker & Vertex, Edge, End & 10 \\
\hline TwoPhasePrimaryUnderground & Switch & Vertex, Edge, End & 10 \\
\hline TwoPhasePrimaryUnderground & Terminator & Vertex, Edge, End & 10 \\
\hline TwoPhasePrimaryUnderground & TieBus & Vertex, Edge, End & 10 \\
\hline TwoPhasePrimaryUnderground & UgConductor & Vertex, Edge, End & 10 \\
\hline UgTransmission & UgConductor & Vertex, Edge, End & 10 \\
\hline SinglePhasePrimaryUnderground & VoltageRegulator & Vertex, Edge, End & 10 \\
\hline SinglePhasePrimaryUnderground & CapacitorBank & Vertex, Edge, End & 10 \\
\hline SinglePhasePrimaryUnderground & CustomerGenerator & Vertex, Edge, End & 10 \\
\hline SinglePhasePrimaryUnderground & FuseCutoutBank & Vertex, Edge, End & 10 \\
\hline SinglePhasePrimaryUnderground & OhConductor & Vertex, Edge, End & 10 \\
\hline SinglePhasePrimaryUnderground & PrimaryMeter & Vertex, Edge, End & 10 \\
\hline SinglePhasePrimaryUnderground & RecloserBank & Vertex, Edge, End & 10 \\
\hline SinglePhasePrimaryUnderground & Switch & Vertex, Edge, End & 10 \\
\hline SinglePhasePrimaryUnderground & TieBus & Vertex, Edge, End & 10 \\
\hline SinglePhasePrimaryUnderground & TransformerBank & Vertex, Edge, End & 10 \\
\hline SinglePhasePrimaryUnderground & UgConductor & Vertex, Edge, End & 10 \\
\hline SinglePhasePrimaryUnderground & SubstationBreaker & Vertex, Edge, End & 10 \\
\hline VoltageRegulator & Snap To Feature & \multicolumn{1}{|c|}{ Hit Type } & Tolerance \\
\hline Subtype & OhConductor & Vertex, Edge, End & 10 \\
\hline ThreePhase & Vertex, Edge, End & 10 \\
\hline SinglePhase & \multicolumn{3}{|c|}{} \\
\hline
\end{tabular}


Table E-9. Gas snapping configurations

Casing

\begin{tabular}{|l|l|c|c|}
\hline Subtype & Snap To Feature & Hit Type & Tolerance \\
\hline TransCasing & GasMain & Vertex, Edge, End & 10 \\
\hline DistCasing & GasMain & Vertex, Edge, End & 10 \\
\hline
\end{tabular}

CpRectifier

\begin{tabular}{|l|l|c|c|}
\hline Subtype & Snap To Feature & Hit Type & Tolerance \\
\hline Rectifier & GasMain & Vertex, Edge, End & 10 \\
\hline
\end{tabular}

DeadEndGas

\begin{tabular}{|l|l|c|c|}
\hline Subtype & Snap To Feature & Hit Type & Tolerance \\
\hline DeadEnd & GasMain & Vertex, Edge, End & 10 \\
\hline \\
Drip & Snap To Feature & Hit Type & Tolerance \\
\hline Subtype & GasMain & Vertex, Edge, End & 10 \\
\hline Drip
\end{tabular}

GasMain

\begin{tabular}{|l|l|c|c|}
\hline Subtype & Snap To Feature & Hit Type & Tolerance \\
\hline DistributionHighPressure & GasMain & Vertex, Edge, End & 10 \\
\hline DistributionLowPressure & GasMain & Vertex, Edge, End & 10 \\
\hline TransmissionDOT & GasMain & Vertex, Edge, End & 10 \\
\hline TransmissionNIPSCO & GasMain & Vertex, Edge, End & 10 \\
\hline DistributionMediumPressure & GasMain & Vertex, Edge, End & 10 \\
\hline
\end{tabular}

GasMainLocation

\begin{tabular}{|l|l|c|c|}
\hline Subtype & Snap To Feature & Hit Type & Tolerance \\
\hline GasMainLocation & GasMain & Vertex, Edge, End & 10 \\
\hline
\end{tabular}

GasService

\begin{tabular}{|l|l|l|c|}
\hline Subtype & Snap To Feature & Hit Type & Tolerance \\
\hline Service & GasMain & Vertex, Edge, End & 10 \\
\hline Service & GasService & Vertex, Edge, End & 10 \\
\hline ServiceStub & GasMain & Vertex, Edge, End & 10 \\
\hline ServiceStub & GasService & Vertex, Edge, End & 10 \\
\hline
\end{tabular}

GasValve

\begin{tabular}{|l|l|c|c|}
\hline Subtype & Snap To Feature & Hit Type & Tolerance \\
\hline DistributionValve & GasMain & Vertex, Edge, End & 10 \\
\hline TransmissionValve & GasMain & Vertex, Edge, End & 10 \\
\hline
\end{tabular}

InsulatedCoupling

\begin{tabular}{|c|c|c|c|}
\hline Subtype & Snap To Feature & Hit Type & Tolerance \\
\hline InsulatedCoupling & GasMain & Vertex, Edge, End & 10 \\
\hline \multicolumn{4}{|l|}{ PipeExposure } \\
\hline Subtype & Snap To Feature & Hit Type & Tolerance \\
\hline PipeExposure & GasMain & Vertex, Edge, End & 10 \\
\hline \multicolumn{4}{|l|}{ PressureControlFitting } \\
\hline Subtype & Snap To Feature & Hit Type & Tolerance \\
\hline PressureControlFitting & GasMain & Vertex, Edge, End & 10 \\
\hline \multicolumn{4}{|l|}{ RegulatorStation } \\
\hline Subtype & Snap To Feature & Hit Type & Tolerance \\
\hline MeterStation & GasMain & Vertex, Edge, End & 10 \\
\hline SingleCustomer & & Vertex, Edge, End & 10 \\
\hline RegulatorStation & GasMain & Vertex, Edge, End & 10 \\
\hline
\end{tabular}

TakeStation 


\begin{tabular}{|l|l|c|c|}
\hline Subtype & Snap To Feature & Hit Type & Tolerance \\
\hline TakeStation & GasMain & Vertex, Edge, End & 10 \\
\hline
\end{tabular}


Table E-10. Land snapping configuration

Bridge

\begin{tabular}{|c|c|c|c|}
\hline Subtype & Snap To Feature & Hit Type & Tolerance \\
\hline Bridge & StreetRow & Vertex, Edge, End & 10 \\
\hline \multicolumn{4}{|l|}{ Easement } \\
\hline Subtype & Snap To Feature & Hit Type & Tolerance \\
\hline Easement & StreetRow & Vertex, Edge, End & 10 \\
\hline Easement & LotLine & Vertex, Edge, End & 10 \\
\hline Easement & Easement & Vertex, Edge, End & 10 \\
\hline \multicolumn{4}{|l|}{ LinearWater } \\
\hline Subtype & Snap To Feature & Hit Type & Tolerance \\
\hline MinorWater & LinearWater & Vertex, Edge, End & 10 \\
\hline MajorWater & LinearWater & Vertex, Edge, End & 10 \\
\hline \multicolumn{4}{|l|}{ Loa } \\
\hline Subtype & Snap To Feature & Hit Type & Tolerance \\
\hline Loa & Loa & Vertex, Edge, End & 10 \\
\hline \multicolumn{4}{|l|}{ LotLine } \\
\hline Subtype & Snap To Feature & Hit Type & Tolerance \\
\hline LotLine & StreetRow & Vertex, Edge, End & 10 \\
\hline LotLine & LotLine & Vertex, Edge, End & 10 \\
\hline LotLine & Easement & Vertex, Edge, End & 10 \\
\hline \multicolumn{4}{|c|}{$\begin{array}{l}\text { MiscellaneousLinearLand } \\
\text { Feature }\end{array}$} \\
\hline Subtype & Snap To Feature & Hit Type & Tolerance \\
\hline LinearLandFeature & $\begin{array}{l}\text { MiscellaneousLinearLand } \\
\text { Feature }\end{array}$ & Vertex, Edge, End & 10 \\
\hline \multicolumn{4}{|l|}{ Railroad } \\
\hline Subtype & Snap To Feature & Hit Type & Tolerance \\
\hline RailroadRow & Railroad & Vertex, Edge, End & 10 \\
\hline RailroadCenterline & Railroad & Vertex, Edge, End & 10 \\
\hline \multicolumn{4}{|l|}{ StreetCenterLine } \\
\hline Subtype & Snap To Feature & Hit Type & Tolerance \\
\hline MajorRoad & StreetCenterLine & Vertex, Edge, End & 10 \\
\hline Interstate & StreetCenterLine & Vertex, Edge, End & 10 \\
\hline Highway & StreetCenterLine & Vertex, Edge, End & 10 \\
\hline Alley & StreetCenterLine & Vertex, Edge, End & 10 \\
\hline MinorRoad & StreetCenterLine & Vertex, Edge, End & 10 \\
\hline \multicolumn{4}{|l|}{ StreetRow } \\
\hline Subtype & Snap To Feature & Hit Type & Tolerance \\
\hline Recorded & StreetRow & Vertex, Edge, End & 10 \\
\hline Unrecorded & StreetRow & Vertex, Edge, End & 10 \\
\hline
\end{tabular}


Table E-11. Electric relationship rules

\section{CapacitorBank_CapacitorUnitInstall}

Composite No

Cardinality One To Many

Origin

ObjectClass CapacitorBank

Key

Labels

OBJECTID (Origin

Primary Key)

CapacitorUnitInstall

Rules

\section{Subtype}

SinglePhaseCapacitor

ThreePhaseCapacitor
Destination

CapacitorUnitInstall

RelCapacitorBankObjectld

(Origin Foreign Key)

CapacitorBank

Origin Cardinality

$0 . .1$

$0 . .1$

\section{Subtype}

CapacitorUnitInstall

CapacitorUnitInstall
Destination

Cardinality

1..1

3.. 12

\section{FuseCutoutBank_FuseUnit}

Composite No

Cardinality One To Many

$\begin{array}{ll} & \text { Origin } \\ \text { ObjectClass } & \text { FuseCutoutBank } \\ \text { Key } & \text { OBJECTID (Origin } \\ \text { Labels } & \text { Primary Key) } \\ & \text { FuseUnit }\end{array}$

Subtype

SinglePhaseUgFuse

TwoPhaseOhFuse

SinglePhaseOhFuse

Rules

ThreePhaseOhFuse

SinglePhaseTripleShot

ThreePhaseUgFuse

ThreePhaseTripleShot

TwoPhaseUgFuse

TwoPhaseTripleShot

\section{Destination \\ FuseUnit \\ RelFuseCutoutBankObjectld \\ (Origin Foreign Key) \\ FuseCutoutBank}

\section{Origin Cardinality}

$0 . .1$

0.1

0.1

0.1

0.1

0.1

0.1

0.1

0.1

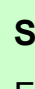

\section{Fuse}

Fuse

Fuse

Fuse

Fuse

Fuse

Fuse

Fuse

Fuse

Destination Cardinality

1..1

2..2

1..1

3.3

$1 . .1$

3.3

3.. 3

2.. 2

$2 . .2$

\section{OhConductor_OhConductorInfo \\ Composite No \\ Cardinality One To Many \\ Origin \\ ObjectClass OhConductor \\ Key \\ OBJECTID (Origin \\ Primary Key) \\ Labels \\ OhConductorInfo \\ Subtype \\ SinglePhaseOverhead \\ Rules

$$
\text { Primary }
$$ \\ TwoPhaseOverhead \\ Primary \\ ThreePhaseOverhead \\ Primary \\ OhTransmission}

\section{Destination}

OhConductorlnfo

RelOhConductorObjectld

(Origin Foreign Key)

OhConductor

Origin Cardinality

0.1

Subtype

Destination

$0 . .1$

OhConductor

Cardinality

$0 . .1$

OhConductor

$1 . .2$

OhConductor

2..3

0.1

OhConductor

3.. 4

$3 . .4$ 


\begin{tabular}{|c|c|c|c|c|}
\hline \multicolumn{5}{|c|}{ PadMount TransformerBank } \\
\hline \multirow{3}{*}{$\begin{array}{l}\text { Composite } \\
\text { Cardinality }\end{array}$} & No & & & \\
\hline & One To One & \multirow{2}{*}{ Destination } & & \\
\hline & & & & \\
\hline ObjectClass & PadMount & \multicolumn{3}{|l|}{ TransformerBank } \\
\hline Key & $\begin{array}{l}\text { OBJECTID (Origin } \\
\text { Primary Key) }\end{array}$ & \multicolumn{3}{|l|}{$\begin{array}{l}\text { RelPadMountObjectld } \\
\text { (Oriqin Foreian Kev) }\end{array}$} \\
\hline Labels & TransformerBank & \multicolumn{3}{|l|}{ PadMount } \\
\hline \multirow{8}{*}{ Rules } & Subtype & Origin Cardinality & Subtype & $\begin{array}{l}\text { Destination } \\
\text { Cardinality }\end{array}$ \\
\hline & Vault & $1 . .1$ & \multirow{3}{*}{$\begin{array}{l}\text { SinglePhase } \\
\text { OverheadlnVault } \\
\text { ThreePhase } \\
\text { Underground } \\
\text { SinglePhase } \\
\text { Underground }\end{array}$} & $0 . .1$ \\
\hline & Pad & $1 . .1$ & & $0 . .1$ \\
\hline & Pad & $1 . .1$ & & $0 . .1$ \\
\hline & Vault & $1 . .1$ & $\begin{array}{l}\text { TwoPhase } \\
\text { OverheadlnVault }\end{array}$ & $0 . .1$ \\
\hline & Vault & $1 . .1$ & $\begin{array}{l}\text { SinglePhase } \\
\text { Undergroundln } \\
\text { Vault }\end{array}$ & $0 . .1$ \\
\hline & Vault & $1 . .1$ & $\begin{array}{l}\text { ThreePhase } \\
\text { OverheadInVault }\end{array}$ & $0 . .1$ \\
\hline & Vault & $1 . .1$ & $\begin{array}{l}\text { ThreePhase } \\
\text { Undergroundln } \\
\text { Vault }\end{array}$ & $0 . .1$ \\
\hline
\end{tabular}

\begin{tabular}{|c|c|c|c|c|}
\hline \multicolumn{5}{|c|}{ RecloserBank RecloserUnit } \\
\hline Composite & Nó & & & \\
\hline Cardinality & One To Many & & & \\
\hline & Origin & Destination & & \\
\hline ObjectClass & RecloserBank & RecloserUnit & & \\
\hline Key & $\begin{array}{l}\text { OBJECTID (Origin } \\
\text { Primary Key) }\end{array}$ & $\begin{array}{l}\text { RelRecloserBankObjectld } \\
\text { (Origin Foreign Key) }\end{array}$ & & \\
\hline Labels & RecloserUnit & RecloserBank & & \\
\hline \multirow{5}{*}{ Rules } & Subtype & Origin Cardinality & Subtype & $\begin{array}{l}\text { Destination } \\
\text { Cardinality }\end{array}$ \\
\hline & SinglePhaseRecloser & 0.1 & Recloser & $1 . .1$ \\
\hline & ThreePhaseRecloser & 0.1 & Recloser & $3 . .3$ \\
\hline & HybridRecloser & 0.1 & Recloser & $1 . .1$ \\
\hline & TwoPhaseRecloser & 0.1 & Recloser & $2 . .2$ \\
\hline
\end{tabular}

Secondary_Conductorlnfo

Composite No

Cardinality One To Many

Origin

ObjectClass Secondary

$\begin{array}{ll}\text { Key } & \text { OBJECTID (Origin } \\ \text { Labels } & \text { Primary Key) } \\ \text { Rules } & \text { CONDUCTORINFO } \\ & \text { Subtype }\end{array}$

\section{Destination} SECONDARYCONDUCTOR INFO

RelSecondaryObjectld (Origin

Foreign Key)

Secondary

Origin Cardinality
Subtype

Destination 
OhSecondary

UgSecondary
0.1

0.1
OhSecondary

UgSecondary
Cardinality

$1 . .3$

$1 . .1$

\section{SectionalizerBank_SectUnit}

Composite No

Cardinality One To Many Origin

ObjectClass SectionalizerBank

Key

Labels

Rules

\section{OBJECTID (Origin}

Primary Key)

SectionalizerUnit

\section{Subtype}

Sectionalizer

\section{Destination}

SectionalizerUnit

RelSectionalizerBankObjectld (Origin Foreign Key)

SectionalizerBank

Origin Cardinality

$0 . .1$

\section{Subtype}

Sectionalizer
Destination Cardinality

$1 . .3$

\section{SubBreaker_CircuitSource \\ Composite No \\ Cardinality One To One \\ Origin}

ObjectClass SubstationBreaker

Key

Labels

Rules
OBJECTID (Origin

Primary Key)

CircuitSource

\section{Subtype}

DistributionBreaker TransmissionBreaker

\section{Destination}

CircuitSource

RelSubstationBreakerObject Id (Origin Foreign Key)

SubstationBreaker

Origin Cardinality

0.1

$0 . .1$

\section{Subtype}

CircuitSource

CircuitSource
Destination Cardinality

$1 . .1$

$1 . .1$

\section{SupportStruct_FuseCutoutBank \\ Composite No \\ Cardinality One To Many \\ Origin \\ ObjectClass SupportStructure \\ Key \\ Labels \\ Primary Key) \\ Fuse Cutout Bank \\ Destination \\ FuseCutoutBank \\ RelSupportStructureObjectld \\ (Origin Foreign Key) \\ SupportStructure}

Subtype

Pole

Pole

Rules

Pole

Pole

Pole

Pole
Origin Cardinality

$1 . .1$

$1 . .1$

$1 . .1$

1..1

$1 . .1$

$1 . .1$

\section{Subtype}

SinglePhaseOh

Fuse

TwoPhaseTriple

Shot

ThreePhaseOh

Fuse

ThreePhaseTriple

Shot

TwoPhaseOhFuse

SinglePhaseTriple

Shot
Destination

Cardinality

$0 . .3$

0.3

0.3

0.3

$0 . .3$

$0 . .3$

\section{SupportStruct_SectBank}




\begin{tabular}{|c|c|c|}
\hline $\begin{array}{l}\text { Composite } \\
\text { Cardinality }\end{array}$ & $\begin{array}{l}\text { No } \\
\text { One To Many } \\
\text { Oriqin }\end{array}$ & Destination \\
\hline $\begin{array}{l}\text { ObjectClass } \\
\text { Key } \\
\text { Labels }\end{array}$ & $\begin{array}{l}\text { SupportStructure } \\
\text { OBJECTID (Origin } \\
\text { Primary Key) } \\
\text { SectionalizerBank }\end{array}$ & $\begin{array}{l}\text { SectionalizerBank } \\
\text { RelSupportStructureObjectld } \\
\text { (Origin Foreign Key) } \\
\text { SupportStructure }\end{array}$ \\
\hline Rules & $\begin{array}{l}\text { Subtype } \\
\text { Pole }\end{array}$ & $\begin{array}{l}\text { Origin Cardinality } \\
1 . .1\end{array}$ \\
\hline $\begin{array}{l}\text { SupportStr } \\
\text { Composite } \\
\text { Cardinality }\end{array}$ & $\begin{array}{l}\text { Ict_Switch } \\
\text { No } \\
\text { One To Many }\end{array}$ & \\
\hline $\begin{array}{l}\text { ObjectClass } \\
\text { Key } \\
\text { Labels }\end{array}$ & $\begin{array}{l}\text { Origin } \\
\text { SupportStructure } \\
\text { OBJECTID (Origin } \\
\text { Primary Key) } \\
\text { Switch }\end{array}$ & $\begin{array}{l}\text { Destination } \\
\text { Switch } \\
\text { RelSupportStructureObjectl } \\
\text { d (Origin Foreign Key) } \\
\text { SupportStructure }\end{array}$ \\
\hline \multirow{3}{*}{ Rules } & $\begin{array}{l}\text { Subtype } \\
\text { Pole }\end{array}$ & $\begin{array}{l}\text { Origin Cardinality } \\
0 . .1\end{array}$ \\
\hline & $\begin{array}{l}\text { Pole } \\
\text { Pole }\end{array}$ & $\begin{array}{l}0 . .1 \\
0 . .1\end{array}$ \\
\hline & Pole & $0 . .1$ \\
\hline
\end{tabular}

$\begin{array}{ll}\text { Subtype } & \begin{array}{l}\text { Destination } \\ \text { Cardinality }\end{array} \\ \text { Sectionalizer } & 0 . .3\end{array}$

$\begin{array}{ll}\text { Subtype } & \begin{array}{l}\text { Destination } \\ \text { Cardinality }\end{array} \\ \text { HookSwitchUnderh } & 0.2 \\ \text { ung } & 0.2 \\ \text { GoabSwitch } & 0.2 \\ \text { HookSwitchInLine } & 0.2 \\ \text { OhDistribution } & 0 . .2 \\ \text { AutomationSwitch } & \end{array}$

\begin{tabular}{|c|c|c|c|c|}
\hline \multicolumn{3}{|c|}{ SupportStruct_TransformerBank } & & \\
\hline Composite & Nó & & & \\
\hline Cardinality & One To Many & & & \\
\hline & Origin & Destination & & \\
\hline ObjectClass & SupportStructure & TransformerBank & & \\
\hline Key & $\begin{array}{l}\text { OBJECTID (Origin } \\
\text { Primary Key) }\end{array}$ & $\begin{array}{l}\text { RelSupportStructureObjectl } \\
\text { d (Origin Foreign Key) }\end{array}$ & & \\
\hline Labels & Transformer Bank & SupportStructure & & \\
\hline \multirow{4}{*}{ Rules } & Subtype & Origin Cardinality & Subtype & $\begin{array}{l}\text { Destination } \\
\text { Cardinality }\end{array}$ \\
\hline & Pole & $1 . .1$ & \multirow{3}{*}{$\begin{array}{l}\text { TwoPhaseOverhea } \\
\text { d } \\
\text { SinglePhaseOverh } \\
\text { ead } \\
\text { ThreePhaseOverhe } \\
\text { ad }\end{array}$} & $0 . .2$ \\
\hline & Pole & $1 . .1$ & & $0 . .2$ \\
\hline & Pole & $1 . .1$ & & $0 . .2$ \\
\hline
\end{tabular}




$\begin{array}{lllll}\text { Labels } & \text { WoodPolelnfo } & \text { SupportStructure } & & \\ & \text { Subtype } & \text { Origin Cardinality } & \text { Subtype } & \begin{array}{l}\text { Destination } \\ \text { Cardinality }\end{array} \\ \text { Rules } & \text { Pole } & 0.1 & \text { WoodPolelnfo } & 0 . .1 \\ & \text { HFrame } & 0 . .1 & \text { WoodPolelnfo } & 0 . .1\end{array}$

\section{Switch_SwitchUnit}

$\begin{array}{lll}\text { Composite } & \text { No } & \\ \text { Cardinality } & \text { One To Many } & \\ & \text { Origin } & \text { Destination } \\ \text { ObjectClass } & \text { Switch } & \text { SwitchUnit } \\ \text { Key } & \text { OBJECTID (Origin } & \text { RelSwitchObjectld (Origin } \\ \text { Labels } & \text { Primary Key) } & \text { Foreign Key) } \\ \text { SwitchUnit } & \text { Switch }\end{array}$

\begin{tabular}{lllll} 
& Subtype & Origin Cardinality & Subtype & Destination \\
& HookSwitchUnderhung & $0 . .1$ & Switch & $1 . .3$ \\
\multirow{4}{*}{ Rules } & HookSwitchInLine & 0.1 & Switch & $1 . .3$ \\
& GoabSwitch & 0.1 & Switch & $1 . .3$ \\
& OhDistributionAutomatio & 0.1 & Switch & $1 . .3$ \\
nSwitch & 0.1 & Switch & $1 . .1$ \\
& UgSwitch & Switch & $1 . .1$
\end{tabular}

$\begin{array}{lll}\text { SwitchGear_FuseCutoutBank } & \\ \text { Composite } & \text { No } & \\ \text { Cardinality } & \text { One To Many } & \\ & \text { Origin } & \text { Destination } \\ \text { ObjectClass } & \text { SwitchGear } & \text { FuseCutoutBank } \\ \text { Key } & \text { OBJECTID (Origin } & \text { RelSwitchGearObjectld } \\ \text { Labels } & \text { Primary Key) } & \text { (Origin Foreign Key) } \\ & \text { Fuse Cutout Bank } & \text { SwitchGear }\end{array}$

\section{Subtype Origin Cardinality}

4 Compartment

Rules

$1 . .1$

$1 . .1$

$\begin{array}{ll}\text { Subtype } & \begin{array}{l}\text { Des } \\ \text { Cardin }\end{array} \\ \text { SinglePhaseUgFus } & 0 . .1 \\ \text { e } & \\ \text { ThreePhaseUgFus } & 0 . .1 \\ \text { e } & \\ \text { TwoPhaseUgFuse } & 0 . .1\end{array}$

\section{SwitchGear_Switch \\ Composite No \\ Cardinality One To Many \\ Origin \\ ObjectClass SwitchGear \\ Key \\ Labels \\ OBJECTID (Origin \\ Primary Key) \\ Switch \\ Destination \\ Switch \\ RelSwitchGearObjectld \\ (Origin Foreign Key) \\ SwitchGear}

Rules

Subtype
Origin Cardinality
Subtype
Destination

Cardinality 


$\begin{array}{llll}4 \text { Compartment } & 1 . .1 & \begin{array}{l}\text { UgDistributionAuto } \\ \text { mationSwitch }\end{array} & 0 . .4 \\ \begin{array}{l}\text { S Compartment } \\ \text { SwitchGear }\end{array} & 1 . .1 & \text { UgSwitch } & 0 . .4\end{array}$

\begin{tabular}{|c|c|c|c|c|}
\hline \multicolumn{5}{|c|}{ TransfBank TransfUnitInstall } \\
\hline Composite & No & & & \\
\hline \multirow[t]{2}{*}{ Cardinality } & One To Many & & & \\
\hline & Origin & Destination & & \\
\hline ObjectClass & TransformerBank & TransformerUnitInstall & & \\
\hline Key & $\begin{array}{l}\text { OBJECTID (Origin } \\
\text { Primary Key) }\end{array}$ & $\begin{array}{l}\text { RelTransformerBankObject } \\
\text { Id (Origin Foreign Key) }\end{array}$ & & \\
\hline \multirow[t]{6}{*}{ Labels } & TransformerUnitInstall & TransformerBank & & \\
\hline & Subtype & Origin Cardinality & Subtype & $\begin{array}{l}\text { Destination } \\
\text { Cardinality }\end{array}$ \\
\hline & SinglePhaseOverhead & 0.1 & $\begin{array}{l}\text { TransformerUnit } \\
\text { Install }\end{array}$ & $1 . .1$ \\
\hline & $\begin{array}{l}\text { ThreePhaseOverheadln } \\
\text { Vault }\end{array}$ & 0.1 & $\begin{array}{l}\text { TransformerUnit } \\
\text { Install }\end{array}$ & $3 . .3$ \\
\hline & SinglePhaseUnderground & 0.1 & $\begin{array}{l}\text { TransformerUnit } \\
\text { Install }\end{array}$ & $1 . .1$ \\
\hline & TwoPhaseOverhead & $0 . .1$ & $\begin{array}{l}\text { TransformerUnit } \\
\text { Install }\end{array}$ & $2 . .2$ \\
\hline \multirow[t]{6}{*}{ Rules } & ThreePhaseOverhead & 0.1 & $\begin{array}{l}\text { TransformerUnit } \\
\text { Install }\end{array}$ & $3 . .3$ \\
\hline & ThreePhaseUnderground & 0.1 & $\begin{array}{l}\text { TransformerUnit } \\
\text { Install }\end{array}$ & $1 . .1$ \\
\hline & $\begin{array}{l}\text { SinglePhaseUnderground } \\
\text { InVault }\end{array}$ & $0 . .1$ & $\begin{array}{l}\text { TransformerUnit } \\
\text { Install }\end{array}$ & $1 . .1$ \\
\hline & $\begin{array}{l}\text { ThreePhaseUnderground } \\
\text { InVault }\end{array}$ & $0 . .1$ & $\begin{array}{l}\text { TransformerUnit } \\
\text { Install }\end{array}$ & $1 . .1$ \\
\hline & $\begin{array}{l}\text { SinglePhaseOverheadln } \\
\text { Vault }\end{array}$ & $0 . .1$ & $\begin{array}{l}\text { TransformerUnit } \\
\text { Install }\end{array}$ & $1 . .1$ \\
\hline & $\begin{array}{l}\text { TwoPhaseOverheadln } \\
\text { Vault }\end{array}$ & $0 . .1$ & $\begin{array}{l}\text { TransformerUnit } \\
\text { Install }\end{array}$ & $2 . .2$ \\
\hline
\end{tabular}

\section{UgConductor_UgConductorlnfo Composite No \\ Cardinality One To Many Origin \\ ObjectClass UgConductor

$\begin{array}{ll}\text { Key } & \text { OBJECTID (Origin } \\ \text { Labels } & \text { Primary Key) } \\ & \text { Conductorlnfo }\end{array}$ \\ Destination \\ UgConductorInfo RelUgConductorObjectld (Origin Foreign Key) UgPrimary}

\section{Subtype}

SinglePhasePrimaryUnd erground

Rules TwoPhasePrimary

Underground

ThreePhasePrimary

Underground

UgTransmission

\section{Origin Cardinality}

0.1

$0 . .1$

0.1

$0 . .1$
Subtype

UgConductor

UgConductor

UgConductor

UgConductor
Destination

Cardinality

$1 . .1$

$1 . .2$

1..3

$1 . .3$ 


\section{VoltageReg_RegulatorUnitInstall \\ Composite No \\ Cardinality One To One

$$
\text { Origin }
$$

ObjectClass VoltageRegulator

Key

Labels OBJECTID (Origin

Primary Key)

RegulatorUnitInstall

Rules

\section{Destination}

RegulatorUnitInstall

RelVoltageRegulatorObject

Id (Origin Foreign Key)

VoltageRegulator

Origin Cardinality

0.1

0.1
Subtype

RegulatorUnitInstall

RegulatorUnitInstall
Destination Cardinality

1..1

$1 . .1$

\section{VoltRegBank_VoltRegulator}

Composite

Cardinality One To Many Origin

ObjectClass VoltageRegulatorBank

Key

Labels

Rules OBJECTID (Origin Primary Key)

VoltageRegulator

Subtype

VoltageRegulatorBank VoltageRegulatorBank

\section{Destination}

VoltageRegulator

RelVoltRegBankObjectld

(Origin Foreign Key)

VoltageRegulatorBank

Origin Cardinality

1..1

1..1
Subtype

SinglePhase

ThreePhase
Destination Cardinality

1.3

$1 . .1$ 
Table E-13. Electric connectivity Edge - Edge rules

From Edge

OhConductor OhConductor OhConductor OhConductor OhConductor OhConductor OhConductor OhConductor OhConductor OhConductor OhConductor OhConductor OhConductor OhConductor OhConductor OhConductor OhConductor OhConductor OhConductor OhConductor OhConductor OhConductor OhConductor OhConductor OhConductor OhConductor OhConductor OhConductor OhConductor OhConductor OhConductor OhConductor OhConductor OhConductor OhConductor OhConductor OhConductor OhConductor OhConductor OhConductor OhConductor OhConductor OhConductor OhConductor OhConductor OhConductor

OhConductor OhConductor OhConductor OhConductor OhConductor OhConductor OhConductor OhConductor OhConductor

OhConductor OhConductor OhConductor OhConductor TieBus

TieBus

TieBus

TieBus

TieBus
From Edge Subtype

SinglePhaseOverheadPrimary TwoPhaseOverheadPrimary ThreePhaseOverheadPrimary SinglePhaseOverheadPrimary TwoPhaseOverheadPrimary ThreePhaseOverheadPrimary OhTransmission ThreePhaseOverheadPrimary TwoPhaseOverheadPrimary OhTransmission

OhTransmission

OhTransmission

OhTransmission

ThreePhaseOverheadPrimary ThreePhaseOverheadPrimary ThreePhaseOverheadPrimary ThreePhaseOverheadPrimary ThreePhaseOverheadPrimary ThreePhaseOverheadPrimary ThreePhaseOverheadPrimary ThreePhaseOverheadPrimary ThreePhaseOverheadPrimary ThreePhaseOverheadPrimary ThreePhaseOverheadPrimary ThreePhaseOverheadPrimary ThreePhaseOverheadPrimary ThreePhaseOverheadPrimary ThreePhaseOverheadPrimary ThreePhaseOverheadPrimary ThreePhaseOverheadPrimary ThreePhaseOverheadPrimary ThreePhaseOverheadPrimary ThreePhaseOverheadPrimary OhTransmission SinglePhaseOverheadPrimary SinglePhaseOverheadPrimary SinglePhaseOverheadPrimary SinglePhaseOverheadPrimary SinglePhaseOverheadPrimary SinglePhaseOverheadPrimary SinglePhaseOverheadPrimary SinglePhaseOverheadPrimary SinglePhaseOverheadPrimary SinglePhaseOverheadPrimary SinglePhaseOverheadPrimary TwoPhaseOverheadPrimary TwoPhaseOverheadPrimary TwoPhaseOverheadPrimary TwoPhaseOverheadPrimary TwoPhaseOverheadPrimary TwoPhaseOverheadPrimary TwoPhaseOverheadPrimary TwoPhaseOverheadPrimary TwoPhaseOverheadPrimary TwoPhaseOverheadPrimary TwoPhaseOverheadPrimary TwoPhaseOverheadPrimary TwoPhaseOverheadPrimary TwoPhaseOverheadPrimary DistributionTieWire DistributionTieWire DistributionTieWire DistributionTieWire DistributionTieWire
To Edge

TieBus TieBus

TieBus

TieBus

OhConductor

OhConductor

TieBus

TieBus

TieBus

OhConductor

OhConductor

OhConductor

OhConductor

OhConductor

OhConductor

OhConductor

OhConductor

OhConductor

OhConductor

OhConductor

OhConductor

OhConductor

OhConductor

OhConductor

OhConductor

OhConductor

OhConductor

OhConductor

OhConductor

OhConductor

OhConductor

OhConductor

TieBus

TieBus

OhConductor

OhConductor

OhConductor

OhConductor

OhConductor

OhConductor

OhConductor

OhConductor

OhConductor

OhConductor

OhConductor

OhConductor

OhConductor

OhConductor

OhConductor

OhConductor

OhConductor

OhConductor

OhConductor

OhConductor

OhConductor

OhConductor

OhConductor

OhConductor

OhConductor

TieBus

TieBus

TieBus

TieBus

TieBus
To Edge Subtype

DistributionTieWire

TransformerLead

TransformerLead

TransformerLead

SinglePhaseOverheadPrimary

SinglePhaseOverheadPrimary

TransmissionTieWire

DistributionTieWire

DistributionTieWire

OhTransmission

OhTransmission

OhTransmission

OhTransmission

TwoPhaseOverheadPrimary

ThreePhaseOverheadPrimary

ThreePhaseOverheadPrimary

ThreePhaseOverheadPrimary

ThreePhaseOverheadPrimary

ThreePhaseOverheadPrimary

ThreePhaseOverheadPrimary

ThreePhaseOverheadPrimary

ThreePhaseOverheadPrimary

ThreePhaseOverheadPrimary

ThreePhaseOverheadPrimary

ThreePhaseOverheadPrimary

ThreePhaseOverheadPrimary

ThreePhaseOverheadPrimary

ThreePhaseOverheadPrimary

ThreePhaseOverheadPrimary

ThreePhaseOverheadPrimary

ThreePhaseOverheadPrimary

ThreePhaseOverheadPrimary

SubstationBusBar

SubstationBusBar

SinglePhaseOverheadPrimary

SinglePhaseOverheadPrimary

SinglePhaseOverheadPrimary

SinglePhaseOverheadPrimary

SinglePhaseOverheadPrimary

SinglePhaseOverheadPrimary

SinglePhaseOverheadPrimary

SinglePhaseOverheadPrimary

SinglePhaseOverheadPrimary

SinglePhaseOverheadPrimary

SinglePhaseOverheadPrimary

TwoPhaseOverheadPrimary

TwoPhaseOverheadPrimary

TwoPhaseOverheadPrimary

TwoPhaseOverheadPrimary

TwoPhaseOverheadPrimary

TwoPhaseOverheadPrimary

TwoPhaseOverheadPrimary

TwoPhaseOverheadPrimary

TwoPhaseOverheadPrimary

TwoPhaseOverheadPrimary

TwoPhaseOverheadPrimary

TwoPhaseOverheadPrimary

TwoPhaseOverheadPrimary

TwoPhaseOverheadPrimary

DistributionTieWire

DistributionTieWire

DistributionTieWire

DistributionTieWire

DistributionTieWire

Via Junction::Subtype (Default)

ElectricNetwork_Junctions

ectricNetwork_Junctions

ElectricNetwork_Junctions

ElectricNetwork_Junctions

ElectricNetwork_Junctions

ElectricNetwork Junctions

ElectricNetwork_Junctions

ElectricNetwork Junctions

ElectricNetwork_Junctions

ElectricNetwork Junctions

PrimaryMeter::Overhead

Switch::GoabSwitch

Switch::OhDistributionAutomationSwitch

ElectricNetwork_Junctions

CapacitorBank::SinglePhaseCapacitor

CapacitorBank::ThreePhaseCapacitor

ElectricNetwork Junctions

FuseCutoutBank::SinglePhaseOhFuse

FuseCutoutBank::SinglePhaseTripleShot

FuseCutoutBank::ThreePhaseOhFuse

FuseCutoutBank::ThreePhaseTripleShot

OpenPoint::OpenPoint

PrimaryMeter::Overhead

RecloserBank::HybridRecloser

RecloserBank::ThreePhaseRecloser

SectionalizerBank::Sectionalizer

Switch::GoabSwitch

Switch::HookSwitchInLine 


\begin{tabular}{|c|c|c|c|c|}
\hline TieBus & DistributionTieWire & TieBus & DistributionTieWire & Switch::HookSwitchUnderhung \\
\hline TieBus & SubstationBusBar & TieBus & SubstationBusBar & ElectricNetwork_Junctions \\
\hline TieBus & SwitchGearBusBar & TieBus & SwitchGearBusBar & ElectricNetwork_Junctions \\
\hline TieBus & SwitchGearBusBar & TieBus & SwitchGearBusBar & FuseCutoutBank::ThreePhaseUgFuse \\
\hline TieBus & SwitchGearBusBar & TieBus & SwitchGearBusBar & FuseCutoutBank::SinglePhaseUgFuse \\
\hline TieBus & SwitchGearBusBar & TieBus & SwitchGearBusBar & FuseCutoutBank::TwoPhaseUgFuse \\
\hline TieBus & SwitchGearBusBar & TieBus & SwitchGearBusBar & Switch::UgSwitch \\
\hline TieBus & SwitchGearBusBar & TieBus & SwitchGearBusBar & Switch::UgDistributionAutomationSwitch \\
\hline UgConductor & TwoPhasePrimaryUnderground & UgConductor & SinglePhasePrimaryUnderground & ElectricNetwork_Junctions \\
\hline UgConductor & SinglePhasePrimaryUnderground & UgConductor & ThreePhasePrimaryUnderground & ElectricNetwork_Junctions \\
\hline UgConductor & TwoPhasePrimaryUnderground & UgConductor & ThreePhasePrimaryUnderground & ElectricNetwork_Junctions \\
\hline UgConductor & SinglePhasePrimaryUnderground & TieBus & DistributionTieWire & Terminator::PoleTerminator \\
\hline UgConductor & SinglePhasePrimaryUnderground & TieBus & SwitchGearBusBar & ElectricNetwork_Junctions \\
\hline UgConductor & ThreePhasePrimaryUnderground & UgConductor & ThreePhasePrimaryUnderground & CustomerGenerator::Generator \\
\hline UgConductor & ThreePhasePrimaryUnderground & UgConductor & ThreePhasePrimaryUnderground & ElectricNetwork_Junctions \\
\hline UgConductor & ThreePhasePrimaryUnderground & UgConductor & ThreePhasePrimaryUnderground & FeederAllOpenPoint::FeederAllOpenPoint \\
\hline UgConductor & ThreePhasePrimaryUnderground & UgConductor & ThreePhasePrimaryUnderground & FuseCutoutBank::ThreePhaseUgFuse \\
\hline UgConductor & ThreePhasePrimaryUnderground & UgConductor & ThreePhasePrimaryUnderground & OpenPoint::OpenPoint \\
\hline UgConductor & ThreePhasePrimaryUnderground & UgConductor & ThreePhasePrimaryUnderground & PrimaryMeter::Underground \\
\hline UgConductor & ThreePhasePrimaryUnderground & UgConductor & ThreePhasePrimaryUnderground & Splice::UgSplice \\
\hline UgConductor & ThreePhasePrimaryUnderground & UgConductor & ThreePhasePrimaryUnderground & TransformerBank::SinglePhaseUnderground \\
\hline UgConductor & ThreePhasePrimaryUnderground & UgConductor & ThreePhasePrimaryUnderground & TransformerBank::ThreePhaseUnderground \\
\hline UgCon & Thr & $\mathrm{UgC}$ & Thi & $\begin{array}{l}\text { TransformerBank:: } \\
\text { ThreePhaseUndergroundlnVault }\end{array}$ \\
\hline UgConductor & SinglePhasePrimaryUnderground & UgConductor & SinglePhasePrimaryUnderground & CustomerGenerator::Generator \\
\hline UgConductor & SinglePhasePrimaryUnderground & UgConductor & SinglePhasePrimaryUnderground & ElectricNetwork_Junctions \\
\hline UgConductor & SinglePhasePrimaryUnderground & UgConductor & SinglePhasePrimaryUnderground & FeederAllOpenPoint::FeederAllOpenPoint \\
\hline UgConductor & SinglePhasePrimaryUnderground & UgConductor & SinglePhasePrimaryUnderground & FuseCutoutBank::SinglePhaseUgFuse \\
\hline UgConductor & SinglePhasePrimaryUnderground & UgConductor & SinglePhasePrimaryUnderground & OpenPoint::OpenPoint \\
\hline UgConductor & SinglePhasePrimaryUnderground & UgConductor & SinglePhasePrimaryUnderground & PrimaryMeter::Underground \\
\hline UgConductor & SinglePhasePrimaryUnderground & UgConductor & SinglePhasePrimaryUnderground & Splice::UgSplice \\
\hline UgConductor & SinglePhasePrimaryUnderground & UgConductor & SinglePhasePrimaryUnderground & TransformerBank::SinglePhaseUnderground \\
\hline UgConductor & SinglePhasePrin & uctor & ryUnderground & $\begin{array}{l}\text { TransformerBank:: } \\
\text { SinglePhaseUndergroundlnVault }\end{array}$ \\
\hline UgConductor & SinglePhasePrimaryUnderground & UgConductor & SinglePhasePrimaryU & $\begin{array}{l}\text { TransformerBank::SinglePhaseOverheadln } \\
\text { Vault }\end{array}$ \\
\hline UgConductor & ThreePhasePrimaryUnderground & TieBus & DistributionTieWire & Terminator::PoleTerminator \\
\hline UgConductor & ThreePhasePrimaryUnderground & TieBus & SubstationBusBar & SubstationBreaker::DistributionBreaker \\
\hline UgConductor & ThreePhasePrimaryUnderground & TieBus & SwitchGearBusBar & ElectricNetwork_Junctions \\
\hline UgConductor & TwoPhasePrimaryUnderground & UgConductor & TwoPhasePrimaryUnderground & CustomerGenerator::Generator \\
\hline UgConductor & TwoPhasePrimaryUnderground & UgConductor & TwoPhasePrimaryUnderground & ElectricNetwork_Junctions \\
\hline UgConductor & TwoPhasePrimaryUnderground & UgConductor & TwoPhasePrimaryUnderground & FeederAllOpenPoint::FeederAllOpenPoint \\
\hline UgConductor & TwoPhasePrimaryUnderground & UgConductor & TwoPhasePrimaryUnderground & FuseCutoutBank::TwoPhaseUgFuse \\
\hline UgConductor & TwoPhasePrimaryUnderground & UgConductor & TwoPhasePrimaryUnderground & OpenPoint::OpenPoint \\
\hline UgConductor & TwoPhasePrimaryUnderground & UgConductor & TwoPhasePrimaryUnderground & PrimaryMeter::Underground \\
\hline UgConductor & TwoPhasePrimaryUnderground & UgConductor & TwoPhasePrimaryUnderground & Splice::UgSplice \\
\hline UgConductor & TwoPhasePrimaryUnderground & UgConductor & TwoPhasePrimaryUnderground & TransformerBank::SinglePhaseUnderground \\
\hline UgConductor & TwoPhasePrimaryUnderground & UgConductor & TwoPhasePrimaryUnderground & $\begin{array}{l}\text { TransformerBank::TwoPhaseOverheadln } \\
\text { Vault }\end{array}$ \\
\hline UgConductor & TwoPhasePrimaryUnderground & TieBus & DistributionTieWire & Terminator::PoleTerminator \\
\hline UgConductor & TwoPhasePrimaryUnderground & TieBus & SwitchGearBusBar & ElectricNetwork_Junctions \\
\hline UgConductor & UgTransmission & TieBus & TransmissionTieWire & Terminator::PoleTerminator \\
\hline UgConductor & UgTransmission & TieBus & SubstationBusBar & SubstationBreaker::TransmissionBreaker \\
\hline
\end{tabular}


Table E-14. Edge - Electric connectivity junction rules

\begin{tabular}{|c|c|c|c|c|c|c|c|}
\hline From Edge & From Edge Subtype & To Junction & To Junction Subtype (default) & $\underset{\mathbf{E}}{\operatorname{Min}}$ & $\underset{\mathrm{E}}{\operatorname{Max}}$ & $\underset{\mathbf{J}}{\operatorname{Min}}$ & $\underset{\mathbf{J}}{\operatorname{Max}}$ \\
\hline OhConductor & OhTransmission & CustomerGenerator & Generator & - & - & - & - \\
\hline OhConductor & OhTransmission & ElectricNetwork_Junctions & - & - & - & - & - \\
\hline OhConductor & OhTransmission & PrimaryMeter & Overhead & 0 & 2 & 0 & 2 \\
\hline OhConductor & OhTransmission & Switch & GoabSwitch & 0 & 2 & 0 & 2 \\
\hline OhConductor & OhTransmission & Switch & HookSwitchInLine & 0 & 2 & 0 & 2 \\
\hline OhConductor & SinglePhaseOverheadPrimary & CapacitorBank & SinglePhaseCapacitor & 0 & 2 & 0 & 2 \\
\hline OhConductor & SinglePhaseOverheadPrimary & FuseCutoutBank & SinglePhaseOhFuse & 0 & 2 & 0 & 2 \\
\hline OhConductor & SinglePhaseOverheadPrimary & FuseCutoutBank & SinglePhaseTripleShot & 0 & 2 & 0 & 2 \\
\hline OhConductor & SinglePhaseOverheadPrimary & OpenPoint & OpenPoint & 0 & 2 & 0 & 2 \\
\hline OhConductor & SinglePhaseOverheadPrimary & PrimaryMeter & Overhead & 0 & 2 & 0 & 2 \\
\hline OhConductor & SinglePhaseOverheadPrimary & RecloserBank & SinglePhaseRecloser & 2 & 2 & 0 & 2 \\
\hline OhConductor & SinglePhaseOverheadPrimary & SectionalizerBank & Sectionalizer & 0 & 2 & 0 & 2 \\
\hline OhConductor & SinglePhaseOverheadPrimary & Switch & HookSwitchlnLine & 0 & 2 & 0 & 2 \\
\hline OhConductor & ThreePhaseOverheadPrimary & CustomerGenerator & Generator & - & - & - & - \\
\hline OhConductor & ThreePhaseOverheadPrimary & ElectricNetwork_Junctions & - & - & - & - & - \\
\hline OhConductor & ThreePhaseOverheadPrimary & FuseCutoutBank & ThreePhaseOhFuse & 0 & 2 & 0 & 2 \\
\hline OhConductor & ThreePhaseOverheadPrimary & FuseCutoutBank & ThreePhaseTripleShot & 2 & 2 & 0 & 2 \\
\hline OhConductor & ThreePhaseOverheadPrimary & FuseCutoutBank & SinglePhaseOhFuse & 0 & 2 & 0 & 2 \\
\hline OhConductor & ThreePhaseOverheadPrimary & FuseCutoutBank & SinglePhaseTripleShot & 0 & 2 & 0 & 2 \\
\hline OhConductor & ThreePhaseOverheadPrimary & OpenPoint & OpenPoint & 0 & 2 & 0 & 2 \\
\hline OhConductor & ThreePhaseOverheadPrimary & PrimaryMeter & Overhead & 0 & 2 & 0 & 2 \\
\hline OhConductor & ThreePhaseOverheadPrimary & RecloserBank & ThreePhaseRecloser & 2 & 2 & 0 & 2 \\
\hline OhConductor & ThreePhaseOverheadPrimary & RecloserBank & HybridRecloser & 2 & 2 & 0 & 2 \\
\hline OhConductor & ThreePhaseOverheadPrimary & SectionalizerBank & Sectionalizer & 0 & 2 & 0 & 2 \\
\hline OhConductor & ThreePhaseOverheadPrimary & SubstationBreaker & DistributionBreaker & 0 & 1 & 0 & 1 \\
\hline OhConductor & ThreePhaseOverheadPrimary & Switch & GoabSwitch & 0 & 2 & 0 & 2 \\
\hline OhConductor & TwoPhaseOverheadPrimary & FuseCutoutBank & SinglePhaseOhFuse & 0 & 2 & 0 & 2 \\
\hline OhConductor & TwoPhaseOverheadPrimary & FuseCutoutBank & SinglePhaseTripleShot & 0 & 2 & 0 & 2 \\
\hline OhConductor & TwoPhaseOverheadPrimary & FuseCutoutBank & TwoPhaseOhFuse & 0 & 2 & 0 & 2 \\
\hline OhConductor & TwoPhaseOverheadPrimary & FuseCutoutBank & TwoPhaseTripleShot & 2 & 2 & 0 & 2 \\
\hline OhConductor & TwoPhaseOverheadPrimary & OpenPoint & OpenPoint & 0 & 2 & 0 & 2 \\
\hline OhConductor & TwoPhaseOverheadPrimary & PrimaryMeter & Overhead & 0 & 2 & 0 & 2 \\
\hline OhConductor & TwoPhaseOverheadPrimary & RecloserBank & TwoPhaseRecloser & 2 & 2 & 0 & 2 \\
\hline OhConductor & TwoPhaseOverheadPrimary & RecloserBank & SinglePhaseRecloser & - & - & - & - \\
\hline OhConductor & TwoPhaseOverheadPrimary & SectionalizerBank & Sectionalizer & 0 & 2 & 0 & 2 \\
\hline OhConductor & TwoPhaseOverheadPrimary & Switch & HookSwitchInLine & 0 & 2 & 0 & 2 \\
\hline OhConductor & TwoPhaseOverheadPrimary & Switch & HookSwitchUnderhung & - & - & - & - \\
\hline OhConductor & TwoPhaseOverheadPrimary & VoltageRegulator & SinglePhase & 0 & 2 & 0 & 2 \\
\hline TieBus & DistributionTieWire & ElectricNetwork_Junctions & - & - & - & - & - \\
\hline TieBus & DistributionTieWire & FuseCutoutBank & ThreePhaseOhFuse & 0 & 2 & 0 & 1 \\
\hline TieBus & DistributionTieWire & FuseCutoutBank & TwoPhaseOhFuse & 0 & 2 & 0 & 2 \\
\hline TieBus & DistributionTieWire & FuseCutoutBank & SinglePhaseOhFuse & 0 & 2 & 0 & 2 \\
\hline TieBus & DistributionTieWire & SectionalizerBank & Sectionalizer & - & - & - & - \\
\hline TieBus & DistributionTieWire & Switch & HookSwitchlnLine & 0 & 2 & 0 & 2 \\
\hline TieBus & DistributionTieWire & Switch & HookSwitchUnderhung & 0 & 2 & 0 & 2 \\
\hline TieBus & DistributionTieWire & Terminator & PoleTerminator & 0 & 1 & 0 & 1 \\
\hline TieBus & SubstationBusBar & ElectricNetwork_Junctions & - & - & - & - & - \\
\hline TieBus & SubstationBusBar & SubstationBreaker & TransmissionBreaker & 0 & 1 & 0 & \\
\hline
\end{tabular}




\begin{tabular}{|c|c|c|c|c|c|c|c|}
\hline TieBus & SubstationBusBar & SubstationBreaker & DistributionBreaker & 0 & 1 & 0 & 1 \\
\hline TieBus & SwitchGearBusBar & ElectricNetwork_Junctions & - & - & - & - & - \\
\hline TieBus & SwitchGearBusBar & FuseCutoutBank & ThreePhaseUgFuse & 0 & 2 & 0 & 2 \\
\hline TieBus & SwitchGearBusBar & FuseCutoutBank & SinglePhaseUgFuse & 0 & 2 & - & - \\
\hline TieBus & SwitchGearBusBar & FuseCutoutBank & TwoPhaseUgFuse & 0 & 2 & 0 & 2 \\
\hline TieBus & SwitchGearBusBar & Switch & UgSwitch & 2 & 2 & 0 & 2 \\
\hline TieBus & SwitchGearBusBar & Switch & UgDistributionAutomationSwitch & 2 & 2 & 0 & 2 \\
\hline TieBus & TransformerLead & ElectricNetwork_Junctions & - & - & - & - & - \\
\hline TieBus & TransformerLead & TransformerBank & TwoPhaseOverhead & 1 & 1 & 0 & 1 \\
\hline TieBus & TransformerLead & TransformerBank & ThreePhaseOverhead & 1 & 1 & 0 & 1 \\
\hline TieBus & TransformerLead & TransformerBank & SinglePhaseOverhead & 1 & 1 & 0 & 1 \\
\hline TieBus & TransmissionTieWire & ElectricNetwork_Junctions & - & - & - & - & - \\
\hline TieBus & TransmissionTieWire & FuseCutoutBank & ThreePhaseOhFuse & 0 & 2 & - & - \\
\hline TieBus & TransmissionTieWire & Terminator & PoleTerminator & 0 & 1 & 0 & 1 \\
\hline UgConductor & SinglePhasePrimaryUnderground & CustomerGenerator & Generator & - & - & - & - \\
\hline UgConductor & SinglePhasePrimaryUnderground & ElectricNetwork_Junctions & - & - & - & - & - \\
\hline UgConductor & SinglePhasePrimaryUnderground & FeederAllOpenPoint & - & - & - & - & - \\
\hline UgConductor & SinglePhasePrimaryUnderground & FuseCutoutBank & SinglePhaseUgFuse & 0 & 2 & - & - \\
\hline UgConductor & SinglePhasePrimaryUnderground & OpenPoint & OpenPoint & 0 & 2 & 0 & 2 \\
\hline UgConductor & SinglePhasePrimaryUnderground & PrimaryMeter & Underground & 0 & 2 & 0 & 2 \\
\hline UgConductor & SinglePhasePrimaryUnderground & Splice & UgSplice & 0 & 2 & 0 & 2 \\
\hline UgConductor & SinglePhasePrimaryUnderground & Terminator & PoleTerminator & 0 & 1 & 0 & 1 \\
\hline UgConductor & SinglePhasePrimaryUnderground & TransformerBank & SinglePhaseUnderground & 0 & 4 & 0 & 2 \\
\hline UgConductor & SinglePhasePrimaryUnderground & TransformerBank & SinglePhaseOverheadInVault & 1 & 4 & 0 & 2 \\
\hline UgConductor & SinglePhasePrimaryUnderground & TransformerBank & SinglePhaseUndergroundInVault & 1 & 2 & 0 & 2 \\
\hline UgConductor & ThreePhasePrimaryUnderground & CustomerGenerator & Generator & - & - & - & - \\
\hline UgConductor & ThreePhasePrimaryUnderground & ElectricNetwork_Junctions & - & - & - & - & - \\
\hline UgConductor & ThreePhasePrimaryUnderground & FeederAllOpenPoint & - & - & - & - & - \\
\hline UgConductor & ThreePhasePrimaryUnderground & FuseCutoutBank & ThreePhaseUgFuse & 0 & 2 & 0 & 2 \\
\hline UgConductor & ThreePhasePrimaryUnderground & OpenPoint & OpenPoint & 0 & 2 & 0 & 2 \\
\hline UgConductor & ThreePhasePrimaryUnderground & PrimaryMeter & Underground & 0 & 2 & 0 & 2 \\
\hline UgConductor & ThreePhasePrimaryUnderground & Splice & UgSplice & 0 & 2 & 0 & 2 \\
\hline UgConductor & ThreePhasePrimaryUnderground & SubstationBreaker & DistributionBreaker & 0 & 1 & 0 & 1 \\
\hline UgConductor & ThreePhasePrimaryUnderground & Terminator & PoleTerminator & 0 & 1 & 0 & 1 \\
\hline UgConductor & ThreePhasePrimaryUnderground & TransformerBank & ThreePhaseUnderground & 1 & 4 & 0 & 2 \\
\hline UgConductor & ThreePhasePrimaryUnderground & TransformerBank & SinglePhaseUnderground & 0 & 4 & 0 & 2 \\
\hline UgConductor & ThreePhasePrimaryUnderground & TransformerBank & ThreePhaseOverheadInVault & 1 & 4 & 0 & 2 \\
\hline UgConductor & ThreePhasePrimaryUnderground & TransformerBank & ThreePhaseUndergroundlnVault & 1 & 4 & 0 & 2 \\
\hline UgConductor & TwoPhasePrimaryUnderground & CustomerGenerator & Generator & - & - & - & - \\
\hline UgConductor & TwoPhasePrimaryUnderground & ElectricNetwork_Junctions & - & - & - & - & - \\
\hline UgConductor & TwoPhasePrimaryUnderground & FeederAllOpenPoint & - & - & - & - & - \\
\hline UgConductor & TwoPhasePrimaryUnderground & FuseCutoutBank & TwoPhaseUgFuse & 0 & 2 & 0 & 2 \\
\hline UgConductor & TwoPhasePrimaryUnderground & OpenPoint & OpenPoint & 0 & 2 & 0 & 2 \\
\hline UgConductor & TwoPhasePrimaryUnderground & PrimaryMeter & Underground & 0 & 2 & 0 & 2 \\
\hline UgConductor & TwoPhasePrimaryUnderground & Splice & UgSplice & 0 & 2 & 0 & 2 \\
\hline UgConductor & TwoPhasePrimaryUnderground & Terminator & PoleTerminator & 0 & 1 & 0 & 1 \\
\hline UgConductor & TwoPhasePrimaryUnderground & TransformerBank & SinglePhaseUnderground & 0 & 4 & 0 & 2 \\
\hline UgConductor & TwoPhasePrimaryUnderground & TransformerBank & TwoPhaseOverheadlnVault & 1 & 4 & 0 & 2 \\
\hline UgConductor & UgTransmission & CustomerGenerator & Generator & - & - & - & - \\
\hline UgConductor & UgTransmission & ElectricNetwork Junctions & - & - & - & - & - \\
\hline UgConductor & UgTransmission & FeederAllOpenPoint & - & - & - & - & - \\
\hline UgConductor & UgTransmission & PrimaryMeter & Underground & 0 & 2 & 0 & 2 \\
\hline UgConductor & UgTransmission & SubstationBreaker & TransmissionBreaker & 0 & 1 & 0 & 1 \\
\hline UgConductor & UgTransmission & Terminator & PoleTerminator & 0 & 1 & 0 & 1 \\
\hline
\end{tabular}




\section{Table E-15. Gas connectivity Edge - Edge rules}

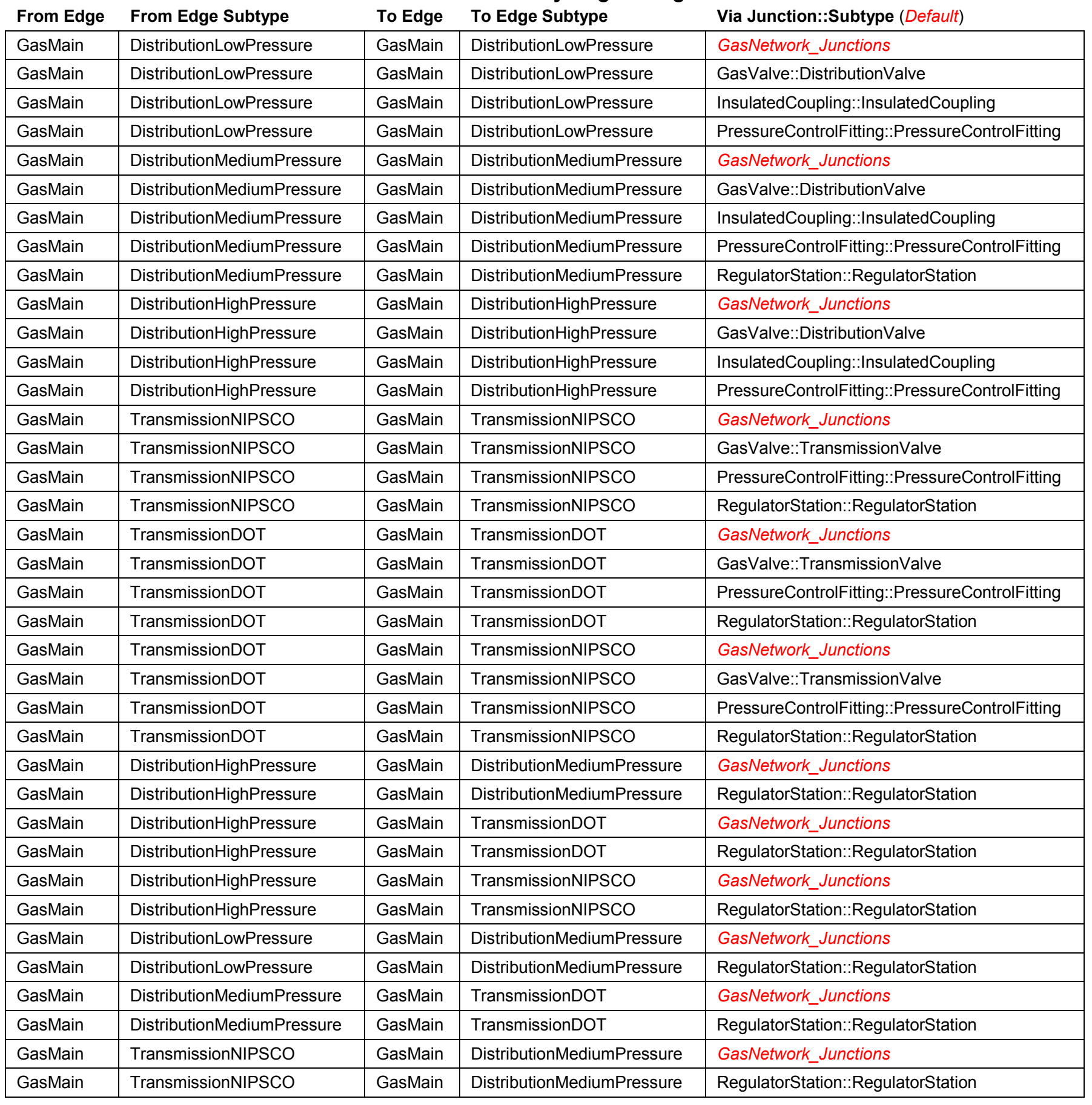


Table E-16. Edge - Edge gas junction rules

\begin{tabular}{|c|c|c|c|c|c|c|c|}
\hline From Edge & From Edge Subtype & To Junction & $\begin{array}{l}\text { To Junction Subtype } \\
\text { (default) }\end{array}$ & $\underset{E}{\operatorname{Min}}$ & $\underset{E}{\operatorname{Max}}$ & $\underset{J}{\operatorname{Min}}$ & $\operatorname{Max} \mathbf{J}$ \\
\hline GasMain & DistributionHighPressure & GasNetwork_Junctions & - & - & - & - & - \\
\hline GasMain & DistributionHighPressure & GasValve & DistributionValve & 0 & 2 & 0 & 2 \\
\hline GasMain & DistributionHighPressure & InsulatedCoupling & InsulatedCoupling & 0 & 2 & 0 & 2 \\
\hline GasMain & DistributionHighPressure & PressureControlFitting & PressureControlFitting & 0 & 3 & 0 & 2 \\
\hline GasMain & DistributionHighPressure & RegulatorStation & MeterStation & 0 & 2 & 0 & 2 \\
\hline GasMain & DistributionHighPressure & RegulatorStation & RegulatorStation & 0 & 2 & 0 & 2 \\
\hline GasMain & DistributionHighPressure & RegulatorStation & SingleCustomer & 0 & 2 & 0 & 2 \\
\hline GasMain & DistributionHighPressure & SqueezeOff & SqueezeOff & 0 & 2 & 0 & 2 \\
\hline GasMain & DistributionHighPressure & TakeStation & TakeStation & 0 & 1 & 0 & 1 \\
\hline GasMain & DistributionLowPressure & GasNetwork Junctions & - & - & - & - & - \\
\hline GasMain & DistributionLowPressure & GasValve & DistributionValve & 0 & 2 & 0 & 2 \\
\hline GasMain & DistributionLowPressure & InsulatedCoupling & InsulatedCoupling & 0 & 2 & 0 & 2 \\
\hline GasMain & DistributionLowPressure & PressureControlFitting & PressureControlFitting & 0 & 3 & 0 & 2 \\
\hline GasMain & DistributionLowPressure & RegulatorStation & MeterStation & 0 & 2 & 0 & 2 \\
\hline GasMain & DistributionLowPressure & RegulatorStation & RegulatorStation & 0 & 2 & 0 & 2 \\
\hline GasMain & DistributionLowPressure & SqueezeOff & SqueezeOff & 0 & 2 & 0 & 2 \\
\hline GasMain & DistributionMediumPressure & GasNetwork Junctions & - & - & - & - & - \\
\hline GasMain & DistributionMediumPressure & GasValve & DistributionValve & 0 & 2 & 0 & 2 \\
\hline GasMain & DistributionMediumPressure & InsulatedCoupling & InsulatedCoupling & 0 & 2 & 0 & 2 \\
\hline GasMain & DistributionMediumPressure & PressureControlFitting & PressureControlFitting & 0 & 3 & 0 & 2 \\
\hline GasMain & DistributionMediumPressure & RegulatorStation & MeterStation & 0 & 2 & 0 & 2 \\
\hline GasMain & DistributionMediumPressure & RegulatorStation & RegulatorStation & 0 & 2 & 0 & 2 \\
\hline GasMain & DistributionMediumPressure & RegulatorStation & SingleCustomer & 0 & 2 & 0 & 2 \\
\hline GasMain & DistributionMediumPressure & SqueezeOff & SqueezeOff & 0 & 2 & 0 & 2 \\
\hline GasMain & DistributionMediumPressure & TakeStation & TakeStation & 0 & 1 & 0 & 1 \\
\hline GasMain & TransmissionDOT & GasNetwork_Junctions & - & - & - & - & - \\
\hline GasMain & TransmissionDOT & GasValve & TransmissionValve & 0 & 2 & 0 & 2 \\
\hline GasMain & TransmissionDOT & PressureControlFitting & PressureControlFitting & 0 & 3 & 0 & 2 \\
\hline GasMain & TransmissionDOT & RegulatorStation & RegulatorStation & 0 & 2 & 0 & 2 \\
\hline GasMain & TransmissionDOT & RegulatorStation & SingleCustomer & 0 & 1 & 0 & 1 \\
\hline GasMain & TransmissionDOT & TakeStation & TakeStation & 0 & 1 & 0 & 1 \\
\hline GasMain & TransmissionNIPSCO & GasNetwork_Junctions & - & - & - & - & - \\
\hline GasMain & TransmissionNIPSCO & GasValve & TransmissionValve & 0 & 2 & 0 & 2 \\
\hline GasMain & TransmissionNIPSCO & PressureControlFitting & PressureControlFitting & 0 & 3 & 0 & 2 \\
\hline GasMain & TransmissionNIPSCO & RegulatorStation & RegulatorStation & 0 & 2 & 0 & 2 \\
\hline GasMain & TransmissionNIPSCO & RegulatorStation & SingleCustomer & 0 & 1 & 0 & 1 \\
\hline GasMain & TransmissionNIPSCO & TakeStation & TakeStation & 0 & 1 & 0 & 1 \\
\hline
\end{tabular}




\section{Appendix F}

Table F-1. Abstract class data model changes

\begin{tabular}{|c|c|c|}
\hline Table & Action & $\begin{array}{l}\text { Details of Data Model Requested } \\
\text { Change }\end{array}$ \\
\hline \multirow[t]{5}{*}{ ObjectLocationCommonData } & $\begin{array}{l}\text { Create } \\
\text { Abstract } \\
\text { Class }\end{array}$ & Copy ObjectCommonData \\
\hline & Inheritance & Inherit from UnitLocationCodes \\
\hline & $\begin{array}{l}\text { New } \\
\text { Columns }\end{array}$ & $\begin{array}{l}\text { LegacyWRInstallTimestamp - date } \\
\text { LegacyWRRemovalTimestamp - date } \\
\text { InstallMiscOrderld - string }(15) \\
\text { RetireMiscOrderld - string(15) }\end{array}$ \\
\hline & $\begin{array}{l}\text { Update } \\
\text { Columns }\end{array}$ & $\begin{array}{l}\text { Update InstallWorkOrderNumber to length } \\
\text { of } 15 \\
\text { Update RetireWorkOrderNumber to length } \\
\text { of } 15\end{array}$ \\
\hline & $\begin{array}{l}\text { Add } \\
\text { Subclasses }\end{array}$ & $\begin{array}{l}\text { Move RetiredSupportStructure subclass } \\
\text { from ObjectCommonData to } \\
\text { ObjectLocationCommonData }\end{array}$ \\
\hline \multirow[t]{3}{*}{ ObjectCommonData } & $\begin{array}{l}\text { Change } \\
\text { Inheritance }\end{array}$ & $\begin{array}{l}\text { Change Inheritance from } \\
\text { UnitLocationCodes to ObjectMetadata - All } \\
\text { EDFS objects except } \\
\text { RetiredSupportStructure underneath this } \\
\text { abstract class do not need to capture } \\
\text { LoaNumber, TaxUnitCd, or GridCd } \\
\text { because these values can be found by } \\
\text { tracing to the related structure. }\end{array}$ \\
\hline & $\begin{array}{l}\text { Update } \\
\text { Columns }\end{array}$ & $\begin{array}{l}\text { Update InstallWorkOrderNumber to length } \\
\text { of } 15 \\
\text { Update RetireWorkOrderNumber to length } \\
\text { of } 15\end{array}$ \\
\hline & $\begin{array}{l}\text { New } \\
\text { Columns }\end{array}$ & $\begin{array}{l}\text { InstallMiscOrderld - string(15) } \\
\text { RetireMiscOrderld - string(15) }\end{array}$ \\
\hline \multirow[t]{3}{*}{ EDFSTimeStamps } & $\begin{array}{l}\text { Create } \\
\text { Abstract } \\
\text { Class }\end{array}$ & $\begin{array}{l}\text { Create New Abstract Class under the } \\
\text { objects diagram }\end{array}$ \\
\hline & Inheritance & Inherit from ObjectCommonData \\
\hline & $\begin{array}{l}\text { New } \\
\text { Columns }\end{array}$ & $\begin{array}{l}\text { LegacyWRInstallTimestamp - date } \\
\text { LegacyWRRemovalTimestamp - date } \\
\text { AssociationTimestamp - date }\end{array}$ \\
\hline UnitObject & $\begin{array}{l}\text { New } \\
\text { Columns }\end{array}$ & $\begin{array}{l}\text { CircuitNumber (must be carried on retired } \\
\text { units AU captures on retirement) - string( } 7 \text { ) } \\
\text { SubtypeCd - integer }\end{array}$ \\
\hline
\end{tabular}




\begin{tabular}{|c|c|c|}
\hline & $\begin{array}{l}\text { Remove } \\
\text { Column }\end{array}$ & $\begin{array}{l}\text { ActiveRetireStatusCd (this is managed via } \\
\text { the subtypes) }\end{array}$ \\
\hline & $\begin{array}{l}\text { Rename } \\
\text { Column }\end{array}$ & $\begin{array}{l}\text { LegacyDistribRefNumber --> } \\
\text { DistribRefNumber (must be carried on } \\
\text { retired units, AU captures on retirement) }\end{array}$ \\
\hline & $\begin{array}{l}\text { Change } \\
\text { Inheritance }\end{array}$ & $\begin{array}{l}\text { Change inheritance from } \\
\text { ObjectCommonData to EDFSTimeStamps }\end{array}$ \\
\hline PointFeatureCommonData & $\begin{array}{l}\text { New } \\
\text { Columns }\end{array}$ & $\begin{array}{l}\text { LegacyWRInstallTimestamp - date } \\
\text { LegacyWRRemovalTimestamp - date }\end{array}$ \\
\hline \multirow[t]{2}{*}{ FeatureCommonData } & $\begin{array}{l}\text { Update } \\
\text { Columns }\end{array}$ & $\begin{array}{l}\text { Update InstallWorkOrderNumber to length } \\
\text { of } 15 \\
\text { Update RetireWorkOrderNumber to length } \\
\text { of } 15\end{array}$ \\
\hline & $\begin{array}{l}\text { New } \\
\text { Columns }\end{array}$ & $\begin{array}{l}\text { InstallMiscOrderld - string(15) } \\
\text { RetireMiscOrderld - string(15) }\end{array}$ \\
\hline
\end{tabular}


Table F-2. Transformer and regulator data model changes

\begin{tabular}{|c|c|c|}
\hline $\begin{array}{l}\text { Table } \\
\text { TransformerUnit }\end{array}$ & Action & Details of Data Model Requested Change \\
\hline \multirow[t]{5}{*}{ TransformerUnit } & $\begin{array}{l}\text { New } \\
\text { Column / } \\
\text { New } \\
\text { Domain }\end{array}$ & $\begin{array}{l}\text { Add new column StatusCd with a domain: } \\
\text { S, I, C : In Stock, Installed, Comdemned }\end{array}$ \\
\hline & $\begin{array}{l}\text { New } \\
\text { Domain }\end{array}$ & $\begin{array}{l}\text { Create Domain for DispositionCd: } \\
\text { C, L, S, R : Condemned, Leased, Sold, } \\
\text { Reverse-Condmned }\end{array}$ \\
\hline & $\begin{array}{l}\text { New } \\
\text { Domain }\end{array}$ & $\begin{array}{l}\text { Create Domain for ManufacturerName: } \\
\text { A.B. Chance } \\
\text { Allis Chalmers } \\
\text { Central Maloney } \\
\text { Dowzer Electric } \\
\text { Ferranti-Packar } \\
\text { General Electric } \\
\text { Howard Industries } \\
\text { Kentucky Rural } \\
\text { Kuhlman Electric } \\
\text { Line Material } \\
\text { McGraw Edison } \\
\text { Pauwells Chance } \\
\text { RTE Corporation } \\
\text { Siemens-Allis } \\
\text { Aolo Basis } \\
\text { Square D Company } \\
\text { Standard } \\
\text { Vantran Electric } \\
\text { Wagner electric } \\
\text { Westinghouse Electric } \\
\text { Other }\end{array}$ \\
\hline & & $\begin{array}{l}\text { Verify this with a query against the EDFS } \\
\text { data: } \\
\text { select distinct TRSFMR_MFR_NM from } \\
\text { dbo.ED_TRSFMR a, } \\
\text { dbo.ED_TRSFMR_STR_ITM b } \\
\text { where a.STR_ITM_NUM=b.STR_ITM_NUM } \\
\text { and b.TRSFMR_TYP_CD='T' } \\
\text { order by TRSFMR_MFR_NM }\end{array}$ \\
\hline & $\begin{array}{l}\text { Rename } \\
\text { Column }\end{array}$ & $\begin{array}{l}\text { StoresltemNumber --> } \\
\text { RelStoresItemNumber }\end{array}$ \\
\hline
\end{tabular}




\begin{tabular}{|c|c|c|}
\hline & $\begin{array}{l}\text { Remove } \\
\text { Column }\end{array}$ & $\begin{array}{l}\text { CondemnDate } \\
\text { RetireDate } \\
\text { InstallDate } \\
\text { LegacyDistribRefNumber } \\
\text { ActiveRetireStatusCd } \\
\text { PhaseDesignation } \\
\text { PrimaryOperatingVoltage } \\
\text { SecondaryOperatingVoltage } \\
\text { RelCompanyNumberObjectld } \\
\text { TransformerKvaVallue } \\
\text { TemporarylnstallInd } \\
\text { TransformerRemovalCd } \\
\text { RemovalMaterialTicketNumber } \\
\text { RelTransformerBankObjectld } \\
\text { TransformerCondmendationCd } \\
\text { CondemnationTicketNumber } \\
\text { InstallMaterialTicketNumber }\end{array}$ \\
\hline & $\begin{array}{l}\text { Remove } \\
\text { Relationship }\end{array}$ & $\begin{array}{l}\text { Delete the relationship between the Unit } \\
\text { table and the Bank table. The unit table } \\
\text { simply captures the asset information and } \\
\text { has nothing to do with the actual installation } \\
\text { of the unit. }\end{array}$ \\
\hline & $\begin{array}{l}\text { Change } \\
\text { Inheritance }\end{array}$ & $\begin{array}{l}\text { No longer inherit from abstract class } \\
\text { UnitLocationCodes. Instead inherit from } \\
\text { ObjectMetadata }\end{array}$ \\
\hline EdfsTransformerlnstall & $\begin{array}{l}\text { Rename } \\
\text { Table }\end{array}$ & Rename Table to TransformerUnitInstall \\
\hline \multirow[t]{4}{*}{ TransformerUnitInstall } & $\begin{array}{l}\text { Rename } \\
\text { Column }\end{array}$ & $\begin{array}{l}\text { InstallTimestamp } \\
\text { InstallDate } \\
\text { InstallationStatusCd } \\
\text { RelCompanyUseLocationld } \\
\text { TransformerlnstallationPhaseCd --> } \\
\text { PhaseDesignation } \\
\text { TransformerNumber --> } \\
\text { RelTransformerUnitCompanyNumber }\end{array}$ \\
\hline & $\begin{array}{l}\text { Assign } \\
\text { Domain }\end{array}$ & $\begin{array}{l}\text { Assign Phase Designation Domain to } \\
\text { PhaseDesignation Column }\end{array}$ \\
\hline & $\begin{array}{l}\text { Change } \\
\text { Inheritance }\end{array}$ & $\begin{array}{l}\text { Change to Inherit from Abstract Class } \\
\text { UnitLocationCodes to include LoaNumber, } \\
\text { TaxUnitCd, and GridCd }\end{array}$ \\
\hline & $\begin{array}{l}\text { New } \\
\text { Column / } \\
\text { New } \\
\text { Domain }\end{array}$ & $\begin{array}{l}\text { Add new column InstallationTypeCd with } \\
\text { new domain: } \\
\text { M - Pad Installation } \\
\text { V - Vault Installation } \\
\text { P - Pole Installation }\end{array}$ \\
\hline
\end{tabular}




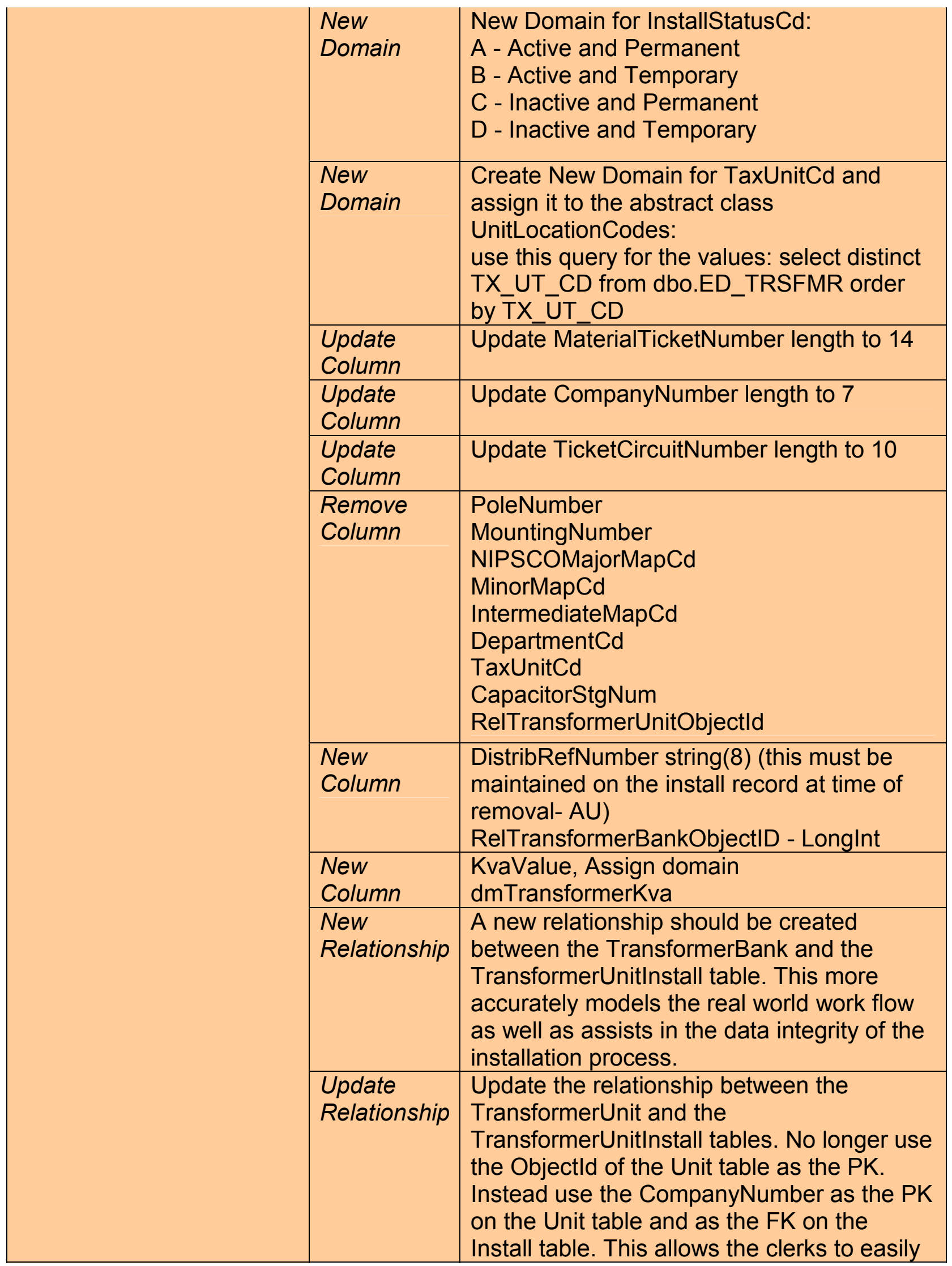




\begin{tabular}{|c|c|c|}
\hline & & $\begin{array}{l}\text { locate the transformer records upon } \\
\text { installation. }\end{array}$ \\
\hline \multirow[t]{3}{*}{ TransformerFunctionTest } & $\begin{array}{l}\text { Rename } \\
\text { Column }\end{array}$ & $\begin{array}{l}\text { CompanyNumber --> } \\
\text { RelTransformerUnitCompanyNumber }\end{array}$ \\
\hline & $\begin{array}{l}\text { Remove } \\
\text { Column }\end{array}$ & RelTransformerUnitObjectld \\
\hline & $\begin{array}{l}\text { Update } \\
\text { Relationship }\end{array}$ & $\begin{array}{l}\text { Update the relationship between the Test } \\
\text { Record and the TransformerUnit to use the } \\
\text { RelTransformerUnitCompanyNumber field }\end{array}$ \\
\hline \multirow[t]{4}{*}{ TransformerOilTest } & $\begin{array}{l}\text { Rename } \\
\text { Column }\end{array}$ & \begin{tabular}{|l|} 
CompanyNumber --> \\
RelTransformerUnitCompanyNumber
\end{tabular} \\
\hline & $\begin{array}{l}\text { Remove } \\
\text { Column }\end{array}$ & RelTransformerUnitObjectld \\
\hline & $\begin{array}{l}\text { Update } \\
\text { Relationship }\end{array}$ & $\begin{array}{l}\text { Update the relationship between the Test } \\
\text { Record and the TransformerUnit to use the } \\
\text { RelTransformerUnitCompanyNumber field }\end{array}$ \\
\hline & $\begin{array}{l}\text { New } \\
\text { Domain }\end{array}$ & $\begin{array}{l}\text { Create Domain for OilTestType: } \\
\text { S - Screen Test } \\
\text { L - Lab Test } \\
\text { R - Retrofill }\end{array}$ \\
\hline EdfsTransformerRemove & $\begin{array}{l}\text { Rename } \\
\text { Table }\end{array}$ & Rename Table to TransformerUnitRemove \\
\hline TransformerUnitRemove & $\begin{array}{l}\text { Rename } \\
\text { Column }\end{array}$ & $\begin{array}{l}\text { TransformerNumber --> } \\
\text { RelTransformerUnitCompanyNumber } \\
\text { RemovalTimestamp } \\
\text { RemovalDate } \\
\text { RemovalCd } \\
\text { LegacylnstallTimestamp }\end{array}$ \\
\hline
\end{tabular}




\begin{tabular}{|c|c|c|}
\hline & $\begin{array}{l}\text { New } \\
\text { Domain }\end{array}$ & $\begin{array}{l}\text { RemovalCd: } \\
\text { A - Inactive } \\
\text { B - Sold/Exchanged } \\
\text { C - Pole/Mounting Replaced or Removed } \\
\text { D - Voltage Conversion } \\
\text { E - Change in Capacity } \\
\text { F - Cracked Busshing } \\
\text { G - Oil Leak } \\
\text { H - Accident/Vandalism } \\
\text { I - Animals } \\
\text { J - Storm } \\
\text { K - Lightning } \\
\text { L - Overloaded } \\
\text { M - Secondary Short } \\
\text { N - Unknown }\end{array}$ \\
\hline & $\begin{array}{l}\text { Remove } \\
\text { Column }\end{array}$ & RelTransformerUnitObjectld \\
\hline & $\begin{array}{l}\text { Update } \\
\text { Relationship }\end{array}$ & $\begin{array}{l}\text { Update the relationship between the } \\
\text { Remove Record and the TransformerUnit to } \\
\text { use the } \\
\text { RelTransformerUnitCompanyNumber field }\end{array}$ \\
\hline & $\begin{array}{l}\text { Update } \\
\text { Column }\end{array}$ & $\begin{array}{l}\text { Update: } \\
\text { MaterialTicketNumber length to } 14 \\
\text { CompanyNumber to } 7\end{array}$ \\
\hline EdfsTransformerStock & $\begin{array}{l}\text { Rename } \\
\text { Table }\end{array}$ & Rename Table to TransformerUnitStock \\
\hline \multirow[t]{5}{*}{ TransformerUnitStock } & $\begin{array}{l}\text { Rename } \\
\text { Column }\end{array}$ & $\begin{array}{l}\text { TransformerNumber --> } \\
\text { RelTransformerUnitCompanyNumber } \\
\text { StockTimestamp } \\
\text { StockDate } \\
\text { TransferNumber }\end{array}$ \\
\hline & $\begin{array}{l}\text { Remove } \\
\text { Column }\end{array}$ & $\begin{array}{l}\text { RelTransformerUnitObjectld } \\
\text { DepartmentCd } \\
\text { NipscoMajorMapCd } \\
\text { IntermediateMapCd } \\
\text { MinorMapCd } \\
\text { TaxUnitCd }\end{array}$ \\
\hline & $\begin{array}{l}\text { New } \\
\text { Column }\end{array}$ & $\begin{array}{l}\text { RelStoreRoomNumber String(3) (This is a } \\
\text { lookup against the StoreRoom Table) }\end{array}$ \\
\hline & $\begin{array}{l}\text { Update } \\
\text { Relationship }\end{array}$ & $\begin{array}{l}\text { Update the relationship between the Stock } \\
\text { Record and the TransformerUnit to use the } \\
\text { RelTransformerUnitCompanyNumber field }\end{array}$ \\
\hline & $\begin{array}{l}\text { Update } \\
\text { Column }\end{array}$ & Update TransferNumber length to 7 \\
\hline
\end{tabular}




\begin{tabular}{|c|c|c|}
\hline EdfsTransformerCondemn & $\begin{array}{l}\text { Rename } \\
\text { Table }\end{array}$ & Rename Table to TransformerUnitCondemn \\
\hline \multirow[t]{7}{*}{ TransformerUnitCondemn } & $\begin{array}{l}\text { Rename } \\
\text { Column }\end{array}$ & $\begin{array}{l}\text { TransformerInstallNumber --> } \\
\text { RelTransformerUnitCompanyNumber } \\
\text { CondemnNumber } \\
\text { CondemnTimestamp } \\
\text { CondemnTypeCd } \\
\text { CondemnDate } \\
\text { CondemnCd } \\
\text { CondemnNumber } \\
\text { CondemnApproveCd }\end{array}$ \\
\hline & $\begin{array}{l}\text { New } \\
\text { Domain }\end{array}$ & $\begin{array}{l}\text { CondemnTypeCd: } \\
\text { C - Condemned } \\
\text { R - Reverse-Condemned }\end{array}$ \\
\hline & $\begin{array}{l}\text { New } \\
\text { Domain }\end{array}$ & $\begin{array}{l}\text { CondemnApproveCd: } \\
\text { Y - Approved } \\
\text { N - Not Approved } \\
\text { P - Printed for Approval }\end{array}$ \\
\hline & $\begin{array}{l}\text { New } \\
\text { Domain }\end{array}$ & $\begin{array}{l}\text { CondemnCd: } \\
\text { A - Per Company Standard E21-698T } \\
\text { B - PCB Contaminated } \\
\text { C - To be Sold } \\
\text { D - Lost or Stolen } \\
\text { E - Destroyed } \\
\text { F - Conveyed to REMC } \\
\text { G - Returned to REMC } \\
\text { J - Non-repairable/Sealed } \\
1 \text { - Returned from REMC } \\
2 \text { - Previously Lost or Stolen } \\
3 \text { - Condemnation Cancelled }\end{array}$ \\
\hline & $\begin{array}{l}\text { Remove } \\
\text { Column }\end{array}$ & RelTransformerUnitObjectld \\
\hline & $\begin{array}{l}\text { Update } \\
\text { Relationship }\end{array}$ & $\begin{array}{l}\text { Update the relationship between the } \\
\text { Condemn Record and the TransformerUnit } \\
\text { to use the } \\
\text { RelTransformerUnitCompanyNumber field }\end{array}$ \\
\hline & \begin{tabular}{|l|} 
Update \\
Column
\end{tabular} & Update: CondemnNumber length to 3 \\
\hline \multirow[t]{2}{*}{$\begin{array}{l}\text { TransformerUnitSold } \\
\text { OrLeased }\end{array}$} & \begin{tabular}{|l|} 
Create \\
Table \\
\end{tabular} & Create Table TransformerUnitSoldOrLeased \\
\hline & Inheritance & Inherit from ObjectMetaData \\
\hline
\end{tabular}




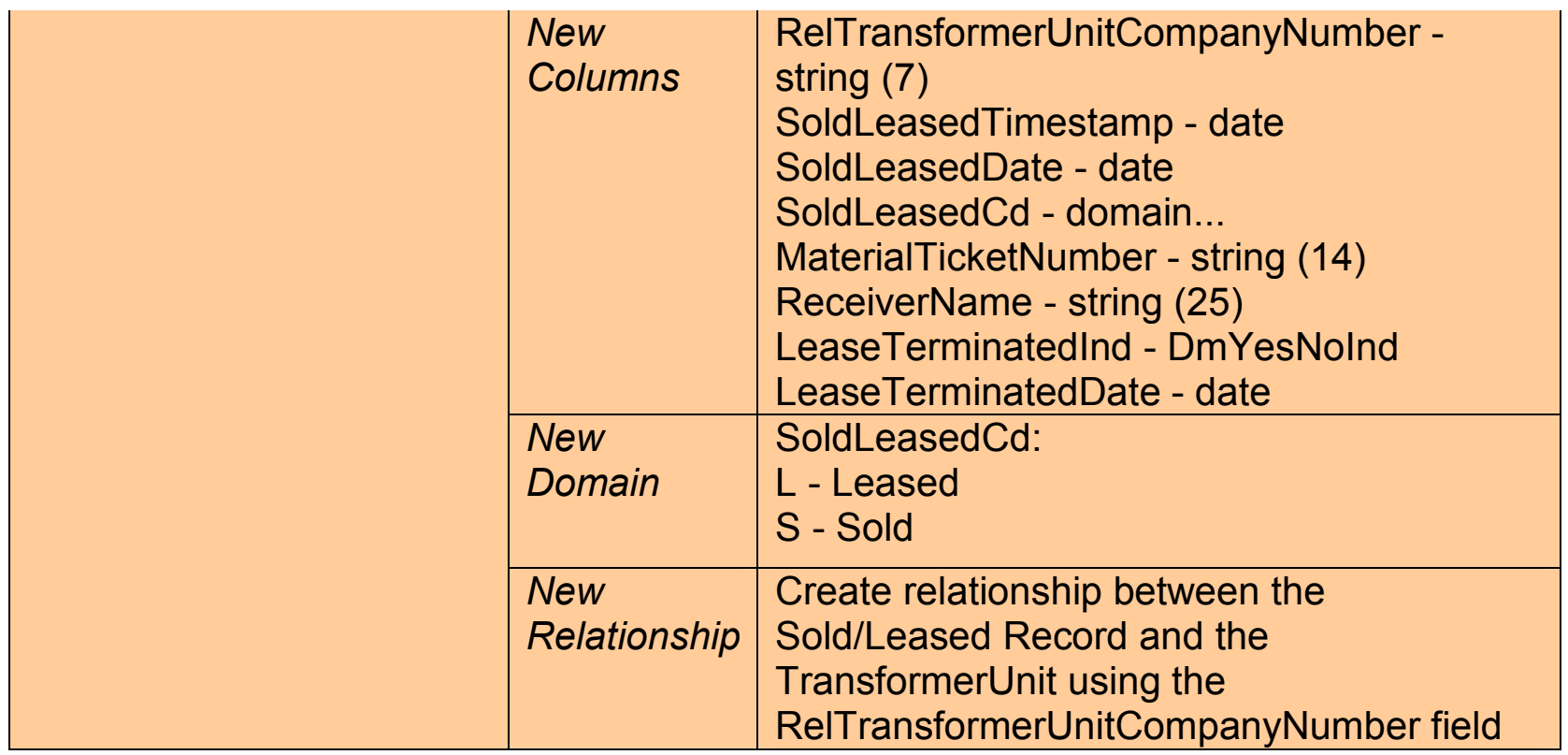


Table F-3. Capacitor data model changes

\begin{tabular}{|c|c|c|}
\hline Table & Action & Details of Data Model Requested Change \\
\hline \multirow[t]{8}{*}{ CapacitorUnit } & $\begin{array}{l}\text { Rename } \\
\text { Column }\end{array}$ & $\begin{array}{l}\text { CapacitorFluidTypeCode --> FluidTypeCd } \\
\text { StatusCd } \\
\text { RelStoresItemNumber }\end{array}$ \\
\hline & $\begin{array}{l}\text { New } \\
\text { Domain }\end{array}$ & $\begin{array}{l}\text { FluidTypeCd: } \\
\text { C - Compound } \\
\text { L - Liquid }\end{array}$ \\
\hline & \begin{tabular}{|l|} 
New \\
Domain
\end{tabular} & $\begin{array}{l}\text { StatusCd: } \\
\text { S - In Stock } \\
\text { I - Installed } \\
\text { C - Out of Service }\end{array}$ \\
\hline & $\begin{array}{l}\text { New } \\
\text { Domain }\end{array}$ & $\begin{array}{l}\text { ManufacturerName: } \\
\text { Federal Pacific Electric } \\
\text { General Electric } \\
\text { McGraw Edison } \\
\text { Sangamo Electric } \\
\text { Westinghouse ABB } \\
\text { Cooper Powerhouse Systems } \\
\text { Other }\end{array}$ \\
\hline & $\begin{array}{l}\text { New } \\
\text { Column }\end{array}$ & StatusTimeStamp - date \\
\hline & $\begin{array}{l}\text { New } \\
\text { Column / } \\
\text { New } \\
\text { Domain }\end{array}$ & $\begin{array}{l}\text { DispositionCd: } \\
\mathrm{N} \text { - N/A } \\
\text { L - Lost or Stolen } \\
\text { C - Conveyed to REMC }\end{array}$ \\
\hline & $\begin{array}{l}\text { Remove } \\
\text { Column }\end{array}$ & $\begin{array}{l}\text { KVARValue } \\
\text { InstallMaterialTicketNumber } \\
\text { CapacitorDirectionCd } \\
\text { CapacitorVoltageValue } \\
\text { CapacitorStageNumber } \\
\text { CapacitorlnstallationCd } \\
\text { OilSwitchIndicator } \\
\text { RelCapacitorBankObjectld } \\
\text { PhaseDesignation } \\
\text { LegacyDistribRefNumber } \\
\text { ActiveRetireStatusCd } \\
\text { RemoveMaterialTicketNumber } \\
\text { InstallDate } \\
\text { RetireDate }\end{array}$ \\
\hline & $\begin{array}{l}\text { Remove } \\
\text { Relationship }\end{array}$ & $\begin{array}{l}\text { Delete the relationship between the Unit } \\
\text { table and the Bank table. The unit table } \\
\text { simply captures the asset information and }\end{array}$ \\
\hline
\end{tabular}




\begin{tabular}{|c|c|c|}
\hline & & $\begin{array}{l}\text { has nothing to do with the acutal installation } \\
\text { of the unit. }\end{array}$ \\
\hline & $\begin{array}{l}\text { New } \\
\text { Relationship }\end{array}$ & $\begin{array}{l}\text { A new relationship should be created } \\
\text { between the CapacitorBank and the } \\
\text { CapacitorUnitlnstall }\end{array}$ \\
\hline & $\begin{array}{l}\text { New } \\
\text { Relationship }\end{array}$ & $\begin{array}{l}\text { New Relationship between CapacitorUnit } \\
\text { and CapacitorStoresltem based on } \\
\text { RelStoresltemNumber }\end{array}$ \\
\hline & $\begin{array}{l}\text { Change } \\
\text { Inheritance }\end{array}$ & $\begin{array}{l}\text { No longer inherit from abstract class } \\
\text { UnitLocationCodes. Instead inherit from } \\
\text { ObjectMetadata. This excludes the } \\
\text { LoaNumber, TaxUnitCd, and GridCd }\end{array}$ \\
\hline EdfsCapacitorInstall & $\begin{array}{l}\text { Rename } \\
\text { Table }\end{array}$ & Rename Table to CapacitorUnitInstall \\
\hline CapacitorUnitlnstall & $\begin{array}{l}\text { Rename } \\
\text { Column }\end{array}$ & $\begin{array}{l}\text { CapacitorNumber --> } \\
\text { RelCapacitorUnitCompanyNumber } \\
\text { InstallTimestamp } \\
\text { InstallDate } \\
\text { CircuitNumber --> TicketCircuitNumber } \\
\text { CapacitorInstallPhaseCd --> } \\
\text { PhaseDesignation } \\
\text { PoleNumber --> DistributionReferenceld } \\
\text { CoLocationld --> } \\
\text { RelCompanyUseLocationld } \\
\text { VoltageValue } \\
\text { InstallationStatusCd } \\
\text { OilSwitchYesNolnd } \\
\text { CapacitorDRTNCd --> DirectionCd } \\
\text { CapacitorStgNum --> StageNumber } \\
\text { LedgerAccountld --> WorkOrderNumber }\end{array}$ \\
\hline & $\begin{array}{l}\text { Assign } \\
\text { Domain }\end{array}$ & $\begin{array}{l}\text { Assign existing domain DmYesNolnd to } \\
\text { OilSwitchYesNolnd }\end{array}$ \\
\hline & $\begin{array}{l}\text { Change } \\
\text { Inheritance }\end{array}$ & $\begin{array}{l}\text { Change to Inherit from Abstract Class } \\
\text { UnitLocationCodes to include LoaNumber, } \\
\text { TaxUnitCd, and GridCd }\end{array}$ \\
\hline
\end{tabular}




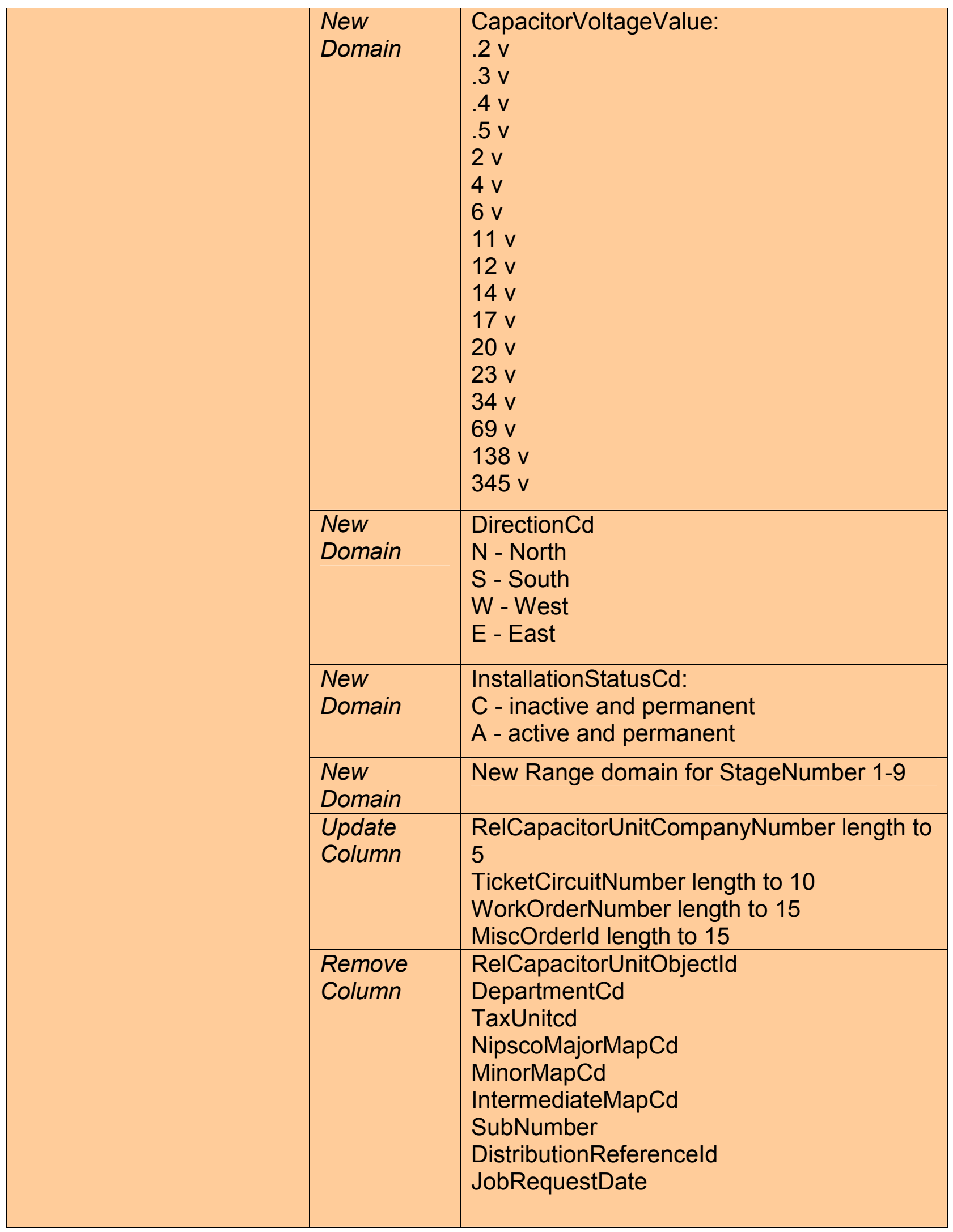




\begin{tabular}{|c|c|c|}
\hline & \begin{tabular}{|l} 
New \\
Column
\end{tabular} & $\begin{array}{l}\text { DistribRefNum string(8) (this must be } \\
\text { maintained on the install record at time of } \\
\text { removal- AU) } \\
\text { CircuitNumber string(7) (this must be } \\
\text { maintained on the install record at time of } \\
\text { removal- AU) } \\
\text { RelCapacitorBankObjectld Integer }\end{array}$ \\
\hline & \begin{tabular}{|l|} 
New \\
Relationship
\end{tabular} & $\begin{array}{l}\text { New Relationship between } \\
\text { CapacitorUnitInstall and the CapacitorBank } \\
\text { based on RelCapacitorBankObjectld }\end{array}$ \\
\hline & \begin{tabular}{|l|} 
Update \\
Relationship
\end{tabular} & $\begin{array}{l}\text { Update the relationship between the } \\
\text { CapacitorUnit and the CapacitorUnitlnstall } \\
\text { tables. No longer use the Objectld of the } \\
\text { Unit table as the PK. Instead use the } \\
\text { CompanyNumber as the PK on the Unit } \\
\text { table and as the FK on the Install table. This } \\
\text { allows the clerks to easily locate the } \\
\text { transformer records upon installation. }\end{array}$ \\
\hline EdfsCapacitorRemove & $\begin{array}{l}\text { Rename } \\
\text { Table }\end{array}$ & Rename Table to CapacitorUnitRemove \\
\hline \multirow[t]{4}{*}{ CapacitorUnitRemove } & $\begin{array}{l}\text { Rename } \\
\text { Column }\end{array}$ & $\begin{array}{l}\text { CapacitorNumber --> } \\
\text { RelCapacitorUnitCompanyNumber } \\
\text { RemovalTimestamp } \\
\text { RemovalDate } \\
\text { RemovalCd } \\
\text { LegacylnstallTimestamp }\end{array}$ \\
\hline & \begin{tabular}{|l|} 
New \\
Domain
\end{tabular} & $\begin{array}{l}\text { RemovalCd: } \\
\text { A - Inactive } \\
\text { B - Sold/Echanged REMC } \\
\text { C - Pole Replace/Removed } \\
\text { D - Scheduled } \\
\text { E - Lost/Stolen } \\
\text { F - Cracked Bushing } \\
\text { G - Oil Leak } \\
\text { H - Accident/Vandalism } \\
\text { I - Animals } \\
\text { J - Storm } \\
\text { K - Lightning } \\
\text { L Unknown }\end{array}$ \\
\hline & \begin{tabular}{|l|} 
New \\
Column
\end{tabular} & WorkOrderNumber String(15) \\
\hline & \begin{tabular}{|l|} 
Update \\
Column
\end{tabular} & MiscOrderld length to 15 \\
\hline
\end{tabular}




\begin{tabular}{|c|c|c|}
\hline & $\begin{array}{l}\text { Remove } \\
\text { Column }\end{array}$ & $\begin{array}{l}\text { CapacitorInstallDate } \\
\text { RelCapacitorUnitObjectld } \\
\text { CapacitorInstallDate } \\
\text { CapacitorJobRequestTimestamp }\end{array}$ \\
\hline & $\begin{array}{l}\text { Update } \\
\text { Relationship }\end{array}$ & $\begin{array}{l}\text { Update the relationship between the } \\
\text { Remove Record and the CapacitorUnit to } \\
\text { use the RelCapacitorUnitCompanyNumber } \\
\text { field }\end{array}$ \\
\hline EdfsCapacitorStock & $\begin{array}{l}\text { Rename } \\
\text { Table }\end{array}$ & Rename Table to CapacitorUnitStock \\
\hline \multirow[t]{5}{*}{ CapacitorUnitStock } & $\begin{array}{l}\text { Rename } \\
\text { Column }\end{array}$ & $\begin{array}{l}\text { CapacitorNumber --> } \\
\text { RelCapacitorUnitCompanyNumber } \\
\text { StockTimestamp } \\
\text { StockDate } \\
\text { TransferNumber }\end{array}$ \\
\hline & $\begin{array}{l}\text { Remove } \\
\text { Column }\end{array}$ & $\begin{array}{l}\text { NipscoMajorMapCd } \\
\text { IntermediateMapCd } \\
\text { MinorMapCd } \\
\text { TaxUnitCd } \\
\text { RelCapacitorUnitObjectld } \\
\text { DepartmentCd }\end{array}$ \\
\hline & $\begin{array}{l}\text { New } \\
\text { Column }\end{array}$ & $\begin{array}{l}\text { RelStoreRoomNumber String(3) (This is a } \\
\text { lookup against the StoreRoom Table) }\end{array}$ \\
\hline & $\begin{array}{l}\text { Update } \\
\text { Relationship }\end{array}$ & $\begin{array}{l}\text { Update the relationship between the Stock } \\
\text { Record and the CapacitorUnit to use the } \\
\text { RelCapacitorUnitCompanyNumber field }\end{array}$ \\
\hline & $\begin{array}{l}\text { Update } \\
\text { Column }\end{array}$ & Update TransferNumber length to 7 \\
\hline EdfsCapacitorStoreltem & $\begin{array}{l}\text { Rename } \\
\text { Table }\end{array}$ & Rename Table to CapacitorStoresltem \\
\hline \multirow[t]{4}{*}{ CapacitorStoresItem } & & \\
\hline & $\begin{array}{l}\text { Rename } \\
\text { Column }\end{array}$ & $\begin{array}{l}\text { StoresltemNumber } \\
\text { BushingNumber } \\
\text { KvarValue } \\
\text { KvValue }\end{array}$ \\
\hline & $\begin{array}{l}\text { Update } \\
\text { Column }\end{array}$ & $\begin{array}{l}\text { update KvValue tagged items with } \\
\text { Precision }=4 \text { and } S \text { cale }=2\end{array}$ \\
\hline & $\begin{array}{l}\text { Assign } \\
\text { Domain }\end{array}$ & $\begin{array}{l}\text { KvarValue: use existing } \\
\text { DmCapacitorUnitKvar }\end{array}$ \\
\hline
\end{tabular}


Table F-4. EDFS shared tables data model changes

\begin{tabular}{|c|c|c|}
\hline Table & Action & $\begin{array}{l}\text { Details of Data Model Requested } \\
\text { Change }\end{array}$ \\
\hline EdfsCompanyNumber & \begin{tabular}{|l} 
Rename \\
Table
\end{tabular} & Rename Table to CompanyUseLocation \\
\hline \multirow[t]{9}{*}{ CompanyUseLocation } & $\begin{array}{l}\text { Remove } \\
\text { Column }\end{array}$ & $\begin{array}{l}\text { NIPSCOMajorMapCd } \\
\text { MinorMapCd } \\
\text { IntermediateMapCd } \\
\text { RelTransformerUnitObjectld } \\
\text { RelCapacitorUnitObjectld }\end{array}$ \\
\hline & \begin{tabular}{|l|} 
New \\
Column
\end{tabular} & GridCd String(5) \\
\hline & \begin{tabular}{|l|} 
Update \\
Column \\
\end{tabular} & Update CompanyLocationld lentgh to 4 \\
\hline & $\begin{array}{l}\text { New } \\
\text { Domain }\end{array}$ & \begin{tabular}{l|} 
New domain for \\
CompanyLocationTypeCd: \\
G - Generating Station \\
S - Substation \\
T - Training Center \\
\end{tabular} \\
\hline & $\begin{array}{l}\text { Assign } \\
\text { Domain }\end{array}$ & $\begin{array}{l}\text { Assign domain to TaxUnit which was } \\
\text { created via the TransformerUnitlnstall } \\
\text { data model changes }\end{array}$ \\
\hline & $\begin{array}{l}\text { Remove } \\
\text { Relationship }\end{array}$ & $\begin{array}{l}\text { Delete the relationship beteween this } \\
\text { class and the TransformerUnit }\end{array}$ \\
\hline & \begin{tabular}{|l|} 
Remove \\
Relationship
\end{tabular} & $\begin{array}{l}\text { Delete the relationship beteween this } \\
\text { class and the CapacitorUnit }\end{array}$ \\
\hline & \begin{tabular}{|l|} 
New \\
Relationship
\end{tabular} & $\begin{array}{l}\text { Create new relationship between this } \\
\text { class and the TransformerUnitInstall } \\
\text { based on CompanyLocationld }\end{array}$ \\
\hline & $\begin{array}{l}\text { New } \\
\text { Relationship }\end{array}$ & $\begin{array}{l}\text { Create new relationship between this } \\
\text { class and the CapacitorUnitlnstall based } \\
\text { on CompanyLocationld }\end{array}$ \\
\hline EdfsTransformerStoreltem & $\begin{array}{l}\text { Rename } \\
\text { Table }\end{array}$ & $\begin{array}{l}\text { Rename Table to } \\
\text { TransformerRegulatorStoresItem }\end{array}$ \\
\hline $\begin{array}{l}\text { TransformerRegulator } \\
\text { Storesltem }\end{array}$ & $\begin{array}{l}\text { New } \\
\text { Relationship }\end{array}$ & $\begin{array}{l}\text { Create new relationship between this table } \\
\text { and TransformerUnit based on } \\
\text { StoresltemNumber. }\end{array}$ \\
\hline
\end{tabular}




\begin{tabular}{|c|c|c|}
\hline & $\begin{array}{l}\text { Rename } \\
\text { Column }\end{array}$ & $\begin{array}{l}\text { StoreltemNumber --> StoresltemNumber } \\
\text { TypeCd } \\
\text { LocationCd } \\
\text { PhaseTypeCd } \\
\text { KvaValue } \\
\text { AmpValue } \\
\text { PrimaryVoltageValue } \\
\text { SecondaryVoltageValue } \\
\text { PropertyRecordsInstallAmount } \\
\text { PropertyRecordsEffectiveDate }\end{array}$ \\
\hline & $\begin{array}{l}\text { Update } \\
\text { Column }\end{array}$ & Update StoresItemNumber length to 7 \\
\hline & $\begin{array}{l}\text { New } \\
\text { Domain }\end{array}$ & $\begin{array}{l}\text { TypeCd: } \\
\text { R - Regulator } \\
\text { T - Transformer }\end{array}$ \\
\hline & $\begin{array}{l}\text { New } \\
\text { Domain }\end{array}$ & $\begin{array}{l}\text { LocationCd: } \\
\text { O - Overhead } \\
\text { U - Underground }\end{array}$ \\
\hline & $\begin{array}{l}\text { New } \\
\text { Domain }\end{array}$ & $\begin{array}{l}\text { PhaseTypeCd: } \\
1 \text { - SinglePhase } \\
3 \text { - ThreePhase }\end{array}$ \\
\hline & $\begin{array}{l}\text { Update } \\
\text { Domain }\end{array}$ & $\begin{array}{l}\text { DmVoltageRegulatorAmpRating: } \\
\text { Remove UNK and add: } \\
\text { 50, } 75,100,150,219,438,548,668\end{array}$ \\
\hline & $\begin{array}{l}\text { New } \\
\text { Domain }\end{array}$ & $\begin{array}{l}\text { PrimaryVoltageValue: } \\
\text { select distinct TRSFMR_PRIM_VT_VAL } \\
\text { from ED TRSFMR STR ITM }\end{array}$ \\
\hline & $\begin{array}{l}\text { New } \\
\text { Domain }\end{array}$ & $\begin{array}{l}\text { SecondaryVoltageValue: } \\
\text { select distinct TRSFMR_SECD_VT_VAL } \\
\text { from ED_TRSFMR_STR_ITM }\end{array}$ \\
\hline & $\begin{array}{l}\text { Assign } \\
\text { Domain }\end{array}$ & $\begin{array}{l}\text { Assign DmVoltage } \bar{R} \text { egulatorAmpRating to } \\
\text { AmpValue }\end{array}$ \\
\hline & $\begin{array}{l}\text { Update } \\
\text { Domain }\end{array}$ & $\begin{array}{l}\text { DmTransformerKva (should be assigned } \\
\text { to KvaValue): } \\
\text { Remove UNK }\end{array}$ \\
\hline & $\begin{array}{l}\text { Update } \\
\text { Column }\end{array}$ & $\begin{array}{l}\text { Update PropertyRecordsInstallAmount to } \\
\text { be type double with precision of } 6 \text { and a } \\
\text { scale of } 0 \text {. }\end{array}$ \\
\hline EdfsStoreroom & $\begin{array}{l}\text { Rename } \\
\text { Table }\end{array}$ & Rename Table to StoreRoom \\
\hline
\end{tabular}




\begin{tabular}{|c|c|c|}
\hline \multirow[t]{6}{*}{ StoreRoom } & $\begin{array}{l}\text { Remove } \\
\text { Column }\end{array}$ & $\begin{array}{l}\text { NIPSCOMajorMapCd } \\
\text { MinorMapCd } \\
\text { IntermediateMapCd } \\
\text { TaxUnitCd }\end{array}$ \\
\hline & $\begin{array}{l}\text { Rename } \\
\text { Column }\end{array}$ & StoreRoomNumber \\
\hline & $\begin{array}{l}\text { New } \\
\text { Relationship }\end{array}$ & $\begin{array}{l}\text { Create a new relationship between } \\
\text { StoreRoom (PK - StoreRoomNumber) to } \\
\text { TransformerUnitStock (FK - } \\
\text { RelStoreRoomNumber) }\end{array}$ \\
\hline & $\begin{array}{l}\text { New } \\
\text { Relationship }\end{array}$ & $\begin{array}{l}\text { Create a new relationship between } \\
\text { StoreRoom (PK - StoreRoomNumber) to } \\
\text { CapacitorUnitStock (FK - } \\
\text { RelStoreRoomNumber) }\end{array}$ \\
\hline & $\begin{array}{l}\text { Update } \\
\text { Inheritance }\end{array}$ & $\begin{array}{l}\text { Change to Inherit from Abstract Class } \\
\text { UnitLocationCodes to include LoaNumber, } \\
\text { TaxUnitCd, and GridCd }\end{array}$ \\
\hline & $\begin{array}{l}\text { Update } \\
\text { Column }\end{array}$ & StoreRoomNumber length to 3 \\
\hline \multirow[t]{4}{*}{ GisMappsTransactionQueue } & $\begin{array}{l}\text { Create } \\
\text { Table }\end{array}$ & Inherit from Object \\
\hline & $\begin{array}{l}\text { New } \\
\text { Column }\end{array}$ & $\begin{array}{l}\text { CompanyNumber string(7) } \\
\text { TransactionType string(3) } \\
\text { LoaNumber string(3) } \\
\text { StoresItemNumber string(7) } \\
\text { TransactionDate date } \\
\text { CondemnationNumber varchar(3) }\end{array}$ \\
\hline & $\begin{array}{l}\text { New } \\
\text { Domain }\end{array}$ & $\begin{array}{l}\text { TransactionType: } \\
\text { INS - T\&R Install } \\
\text { RMV - T\&R Removal } \\
\text { CI - Capacitor Install } \\
\text { CND - T\&R Condemnation }\end{array}$ \\
\hline & $\begin{array}{l}\text { Assign } \\
\text { Domain }\end{array}$ & $\begin{array}{l}\text { Department: } \\
\text { DmLoa }\end{array}$ \\
\hline
\end{tabular}


Table F-5. Poles, attachments and assemblies data model changes

\begin{tabular}{|c|c|c|}
\hline Table & Action & Details of Data Model Requested Change \\
\hline \multirow[t]{6}{*}{ SupportStructure } & $\begin{array}{l}\text { New } \\
\text { Column }\end{array}$ & $\begin{array}{l}\text { InstallTimestamp } \\
\text { RemovalTimestamp } \\
\text { WoodSettingCd }\end{array}$ \\
\hline & $\begin{array}{l}\text { Rename } \\
\text { Column }\end{array}$ & $\begin{array}{l}\text { PrimaryReferenceNumber --> } \\
\text { PrimaryDistribRefNumber }\end{array}$ \\
\hline & $\begin{array}{l}\text { New } \\
\text { Domain }\end{array}$ & $\begin{array}{l}\text { NonMeteredServicePointTypeCd: } \\
\text { C - Cable } \\
\text { D - Dusk/Dawn } \\
\text { M - Multiple } \\
\text { O - Other } \\
\text { S - Streetlights } \\
\text { T - Traffic Lights } \\
\text { N - None }\end{array}$ \\
\hline & $\begin{array}{l}\text { Update } \\
\text { Domain }\end{array}$ & $\begin{array}{l}\text { DmStructureOwner: } \\
\text { Make sure all owners from EDFS are } \\
\text { included in list. Use query as follows: } \\
\text { "select distinct PL_OWN_NM from } \\
\text { ED_POLE_NUM" } \\
\text { And then weed out all the bad values... }\end{array}$ \\
\hline & $\begin{array}{l}\text { New } \\
\text { Domain }\end{array}$ & $\begin{array}{l}\text { WoodPoleTypeCd: } \\
\text { WF - Western Fir } \\
\text { NP - Northern Pine } \\
\text { EC - Northern White/Eastern Cedar } \\
\text { WP - Western Pine } \\
\text { DF - Douglas Fir } \\
\text { SP - Southern Pine } \\
\text { LP - Lodge Pole Pine } \\
\text { CH - Chestnut } \\
\text { YC - Alaskan Yellow Cedar } \\
\text { WL - Western Larch } \\
\text { WC - Western Cedar } \\
\text { WH - Western Hemlock } \\
\text { H1 - The material has to equal W, Pole Size } \\
\text { has to equal } 40\end{array}$ \\
\hline & $\begin{array}{l}\text { New } \\
\text { Domain }\end{array}$ & $\begin{array}{l}\text { WoodPoleClassCd: } \\
1,2,3,4,5,6,7,8,9,10\end{array}$ \\
\hline
\end{tabular}




\begin{tabular}{|c|c|c|}
\hline & \begin{tabular}{|l} 
New \\
Domain
\end{tabular} & $\begin{array}{l}\text { WoodPoleOriginalTreatmntTypeCd: } \\
\text { ACZA - Ammoniacal Copper Zinc Arsenate } \\
\text { C - Creosote } \\
\text { O - Other } \\
\text { P - Pentachlorophenol } \\
\text { CN - Copper Napthenate } \\
\text { CCA - Chromated Copper Arsenate }\end{array}$ \\
\hline & \begin{tabular}{|l|} 
New \\
Domain
\end{tabular} & $\begin{array}{l}\text { WoodSettingCd: } \\
\text { ASPH - Asphalt } \\
\text { WET - Wet } \\
\text { DRY - Dry } \\
\text { CONC - Concrete }\end{array}$ \\
\hline & \begin{tabular}{|l|} 
New \\
Domain
\end{tabular} & $\begin{array}{l}\text { WoodPolelnspectionPassFailCd: } \\
\text { N - No } \\
\text { Y - Yes } \\
\text { P - Pass }\end{array}$ \\
\hline \multirow[t]{6}{*}{ JointUseAttachment } & \begin{tabular}{|l|} 
New \\
Column
\end{tabular} & SubtypeCd \\
\hline & $\begin{array}{l}\text { New } \\
\text { Subtypes }\end{array}$ & $\begin{array}{l}\text { Telephone (subtypecd=1) } \\
\text { Cable }(\text { subtypecd=2) } \\
\text { Utility (subtypecd=3) } \\
\text { Fiber (subtypecd }=4) \\
\text { Other (subtypecd=5) }\end{array}$ \\
\hline & \begin{tabular}{|l|} 
Verify \\
Domains
\end{tabular} & $\begin{array}{l}\text { Verify DmPhoneCompanyCd against table } \\
\text { ED_TLPHON } \\
\text { Verify DmCableCompanyCd against table } \\
\text { ED_CABLE } \\
\text { Verify DmUtilityCompanyCd against table } \\
\text { ED_UTLTY }\end{array}$ \\
\hline & $\begin{array}{l}\text { Assign } \\
\text { Domains }\end{array}$ & $\begin{array}{l}\text { Assign Telephone, Cable, and Utility } \\
\text { domains to the appropriate subtypes for the } \\
\text { ComanyName Field }\end{array}$ \\
\hline & $\begin{array}{l}\text { Remove } \\
\text { Column }\end{array}$ & AttachmentTypeCd \\
\hline & $\begin{array}{l}\text { Change } \\
\text { Inheritance }\end{array}$ & $\begin{array}{l}\text { Change to inherit from ObjectMetadata } \\
\text { instead of ObjectCommonData to exclude } \\
\text { the install and retirement information }\end{array}$ \\
\hline \multirow[t]{3}{*}{ Assembly } & $\begin{array}{l}\text { Remove } \\
\text { Subtype }\end{array}$ & PadAssembly \\
\hline & $\begin{array}{l}\text { New } \\
\text { Subtype }\end{array}$ & RetiredPoleAssembly (subtypecd=2) \\
\hline & $\begin{array}{l}\text { Remove } \\
\text { Relationship }\end{array}$ & Remove Relationship to a PadMount \\
\hline
\end{tabular}




\begin{tabular}{|c|c|c|}
\hline & $\begin{array}{l}\text { Remove } \\
\text { Column }\end{array}$ & $\begin{array}{l}\text { RelPadObjectld } \\
\text { RelUgTransformerObjectld } \\
\text { RelSwitchGearObjectld } \\
\text { RelPedestalObjectld } \\
\text { RelManholeObjectld } \\
\text { AssemblyStatus }\end{array}$ \\
\hline & $\begin{array}{l}\text { New } \\
\text { Column }\end{array}$ & $\begin{array}{l}\text { AssociationTimestamp - date } \\
\text { InstallMiscOrderld - String }(15) \\
\text { RetireMiscOrderld - String }(15) \\
\text { RetireWorkOrderNumber - String(15) } \\
\text { LegacyWRInstallTimestamp - date } \\
\text { LegacyWRRemovalTimestamp -date }\end{array}$ \\
\hline & $\begin{array}{l}\text { Rename } \\
\text { Column }\end{array}$ & $\begin{array}{l}\text { WorkOrderNumber --> } \\
\text { InstallWorkOrderNumber } \\
\text { LegacyDistribRefNumber --> } \\
\text { DistribRefNumber } \\
\text { This field was first used for Migration } \\
\text { purposes. Going forward it will not be } \\
\text { populated on Active assemblies. However, } \\
\text { upon retirement, when the subtype is } \\
\text { updated to 'RetiredPoleAssembly', code will } \\
\text { grab the distribrefnumber of the related pole } \\
\text { and populate it on the record. }\end{array}$ \\
\hline & $\begin{array}{l}\text { Update } \\
\text { Column }\end{array}$ & InstallWorkOrderNumber length to 15 \\
\hline \multirow[t]{4}{*}{ RetiredSupportStructure } & $\begin{array}{l}\text { New } \\
\text { Column }\end{array}$ & $\begin{array}{l}\text { InstallTimestamp } \\
\text { RemovalTimestamp } \\
\text { WoodSettingCd } \\
\text { TelephoneCompanyCd } \\
\text { FirstCableCompanyCd } \\
\text { SecondCableCompanyCd } \\
\text { UtitlityCompanyCd } \\
\text { FiberCompanyCd } \\
\text { OtherCompanyCd } \\
\text { TransformerCompanyNumber1 } \\
\text { TransformerCompanyNumber2 } \\
\text { TransformerCompanyNumber3 } \\
\text { TransformerCompanyNumber4 }\end{array}$ \\
\hline & $\begin{array}{l}\text { Remove } \\
\text { Column }\end{array}$ & $\begin{array}{l}\text { PoleExtensionSizeValue } \\
\text { PoleExtensionTypeCd }\end{array}$ \\
\hline & $\begin{array}{l}\text { Rename } \\
\text { Column }\end{array}$ & $\begin{array}{l}\text { PrimaryReferenceNumber --> } \\
\text { PrimaryDistribRefNumber }\end{array}$ \\
\hline & $\begin{array}{l}\text { Update } \\
\text { Column }\end{array}$ & $\begin{array}{l}\text { Update RetireWorkOrderNumber length to } \\
15\end{array}$ \\
\hline
\end{tabular}


Table F-6. Pad mounts (pad / vaults) data model changes

\begin{tabular}{|c|c|c|}
\hline Table & Action & Details of Data Model Requested Change \\
\hline \multirow[t]{7}{*}{ PadMount } & $\begin{array}{l}\text { New } \\
\text { Column }\end{array}$ & $\begin{array}{l}\text { InstallTimestamp - date } \\
\text { RemovalTimestamp - date } \\
\text { Printld - string(6) } \\
\text { LegacyDistribRefTerminationld - string(8) } \\
\text { DistribRefNumber - String(8) } \\
\text { LocationDescription - String(100) }\end{array}$ \\
\hline & $\begin{array}{l}\text { Rename } \\
\text { Column }\end{array}$ & $\begin{array}{l}\text { MountingTypeCd --> TypeCd } \\
\text { MountingOwnerCd --> Ownerlnd }\end{array}$ \\
\hline & $\begin{array}{l}\text { Remove } \\
\text { Column }\end{array}$ & MountingStatusCd \\
\hline & $\begin{array}{l}\text { New } \\
\text { Column / } \\
\text { New } \\
\text { Domain }\end{array}$ & $\begin{array}{l}\text { NonMeteredServicePointTypeCd: } \\
\text { C - Cable } \\
\text { D - Dusk/Dawn } \\
\text { M - Multiple } \\
\text { O - Other } \\
\text { S - Streetlights } \\
\text { T - Traffic Lights } \\
\text { N - None }\end{array}$ \\
\hline & $\begin{array}{l}\text { New } \\
\text { Domain }\end{array}$ & $\begin{array}{l}\text { TypeCd: } \\
\text { F - Fiberglass } \\
\text { C - Concrete }\end{array}$ \\
\hline & $\begin{array}{l}\text { Assign } \\
\text { Domain }\end{array}$ & $\begin{array}{l}\text { Ownerlnd: } \\
\text { DmYesNolnd }\end{array}$ \\
\hline & $\begin{array}{l}\text { New } \\
\text { Subtype }\end{array}$ & Vault (subtypeCd=2) \\
\hline \multirow[t]{3}{*}{ RetiredPadMount } & $\begin{array}{l}\text { Create } \\
\text { Table }\end{array}$ & Copy PadMount, move to objects diagram \\
\hline & Inheritance & Inherit from ObjectLocationCommonData \\
\hline & $\begin{array}{l}\text { New } \\
\text { Subtypes }\end{array}$ & $\begin{array}{l}\text { RetiredPad (subtypecd=1) } \\
\text { RetiredVault (subtypecd=2) }\end{array}$ \\
\hline
\end{tabular}


Table F-7. Protective devices data model changes

\begin{tabular}{|l|l|l|}
\hline Table & Action & Details of Data Model Requested Change \\
\hline SwitchUnit & New & Switch (subtypecd=1) \\
Subtypes & RetiredSwitch (subtypecd=2)
\end{tabular}




\begin{tabular}{|c|c|c|}
\hline & $\begin{array}{l}\text { Rename } \\
\text { Column }\end{array}$ & $\begin{array}{l}\text { DistribRefNumber --> } \\
\text { LegacyDistribRefNumber (The } \\
\text { DistribRefNumber is able to be determined } \\
\text { by traversing the relationship to the } \\
\text { PadMount based on Objectld for Active } \\
\text { SwtichGears) }\end{array}$ \\
\hline & $\begin{array}{l}\text { New } \\
\text { Domain }\end{array}$ & $\begin{array}{l}\text { SwitchGearKv: } \\
12.0 \\
14.4\end{array}$ \\
\hline & $\begin{array}{l}\text { New } \\
\text { Relationship }\end{array}$ & $\begin{array}{l}\text { Create a relationship to PadMount based on } \\
\text { RelPadMountObjectld. } 1 \text { PadMount can } \\
\text { have } 0 \text { or } 1 \text { SwitchGear }\end{array}$ \\
\hline & $\begin{array}{l}\text { Update } \\
\text { Default }\end{array}$ & $\begin{array}{l}\text { Column - FrontKindCd: Update default from } \\
\text { D to } L\end{array}$ \\
\hline \multirow[t]{4}{*}{ RetiredSwitchGear } & $\begin{array}{l}\text { Create } \\
\text { Table }\end{array}$ & Create new object class RetiredSwitchGear \\
\hline & Inheritance & Inherit from ObjectLocationCommonData \\
\hline & $\begin{array}{l}\text { New } \\
\text { Columns }\end{array}$ & $\begin{array}{l}\text { SubtypeCd - integer } \\
\text { DistribRefNumber - string(8) } \\
\text { SwitchGearKv - domain (see SwitchGear) } \\
\text { OperationTypeCd - domain (see } \\
\text { SwitchGear) } \\
\text { FrondKindCd - domain (see SwitchGear) } \\
\text { UsedCompartmentCount - domain (see } \\
\text { SwitchGear) } \\
\text { AssociationTimestamp - date } \\
\text { AssemblyNumber - string(9) }\end{array}$ \\
\hline & $\begin{array}{l}\text { New } \\
\text { Subtype }\end{array}$ & RetiredSwitchGear (subtypecd=1) \\
\hline
\end{tabular}


Table F-8. Lighting data model changes

\begin{tabular}{|c|c|c|}
\hline Table & Action & Details of Data Model Requested Change \\
\hline EdfsCompanyStreetlight & $\begin{array}{l}\text { Rename } \\
\text { Table }\end{array}$ & Rename table to CompanyStreetlight \\
\hline \multirow[t]{6}{*}{ CompanyStreetlight } & New Column & $\begin{array}{l}\text { SubtypeCd - integer } \\
\text { NearestAddress - string } 20 \\
\text { StreetName - string - } 30 \\
\text { RateSchedule - double 5,2 } \\
\text { CISAccountNumber - integer } \\
\text { LegacyCustXrefCustAcctRefNumber - } \\
\text { integer }\end{array}$ \\
\hline & $\begin{array}{l}\text { Remove } \\
\text { Column }\end{array}$ & $\begin{array}{l}\text { RelUgTransformerObjectld } \\
\text { RelPedestalObjectld } \\
\text { RelFieldInvLightObjectld } \\
\text { CustomerAcctReferenceNumber }\end{array}$ \\
\hline & $\begin{array}{l}\text { New } \\
\text { Subtypes }\end{array}$ & $\begin{array}{l}\text { CompanyStreetlight (subtypecd=1) } \\
\text { RetiredCompanyStreetlight (subtypecd=2) }\end{array}$ \\
\hline & $\begin{array}{l}\text { Change } \\
\text { Inheritance }\end{array}$ & $\begin{array}{l}\text { Change to inherit from } \\
\text { LegacyWorkOrderTimestamps }\end{array}$ \\
\hline & $\begin{array}{l}\text { Remove } \\
\text { Relationships }\end{array}$ & $\begin{array}{l}\text { to FieldlnventoryLight } \\
\text { to Pedestal } \\
\text { to TransformerBank }\end{array}$ \\
\hline & $\begin{array}{l}\text { New } \\
\text { Relationship }\end{array}$ & $\begin{array}{l}\text { Create a relationship to SupportStructure } \\
\text { based on RelSupportStructureObjectld. } 1 \\
\text { SupportStructure can have } 0 \text { or many } \\
\text { CompanyStreetlights }\end{array}$ \\
\hline EdfsCustomerStreetlight & $\begin{array}{l}\text { Rename } \\
\text { Table }\end{array}$ & Rename table to CustomerStreetlight \\
\hline \multirow[t]{3}{*}{ CustomerStreetlight } & New Column & $\begin{array}{l}\text { RelPadMountObjectld - integer } \\
\text { AssociationTimestamp - date } \\
\text { CISAccountNumber - integer } \\
\text { LegacyCustXrefCustAcctRefNumber - } \\
\text { integer }\end{array}$ \\
\hline & $\begin{array}{l}\text { Rename } \\
\text { Column }\end{array}$ & $\begin{array}{l}\text { PoleTypeCd } \\
\text { LuminaireTypeCd } \\
\text { LuminaireCount } \\
\text { WattageValue } \\
\text { InventoryDate }\end{array}$ \\
\hline & $\begin{array}{l}\text { Remove } \\
\text { Column }\end{array}$ & $\begin{array}{l}\text { RelUgTransformerObjectld } \\
\text { RelPedestalObjectld } \\
\text { RelFieldInvLightObjectld } \\
\text { CustAccountReferenceNumber }\end{array}$ \\
\hline
\end{tabular}




\begin{tabular}{|c|c|c|}
\hline & $\begin{array}{l}\text { Remove } \\
\text { Relationships }\end{array}$ & $\begin{array}{l}\text { to FieldlnventoryLight } \\
\text { to Pedestal } \\
\text { to TransformerBank }\end{array}$ \\
\hline & $\begin{array}{l}\text { Change } \\
\text { Inheritance }\end{array}$ & $\begin{array}{l}\text { Change to inherit from ObjectMetaData } \\
\text { instead of ObjectCommonData to exclude } \\
\text { install and retire data }\end{array}$ \\
\hline & $\begin{array}{l}\text { New } \\
\text { Relationship }\end{array}$ & $\begin{array}{l}\text { Create a relationship to PadMount based on } \\
\text { RelPadMountObjectld. } 1 \text { PadMount can } \\
\text { have } 0 \text { or many CompanyStreetlights }\end{array}$ \\
\hline & $\begin{array}{l}\text { New } \\
\text { Relationship }\end{array}$ & $\begin{array}{l}\text { Create a relationship to SupportStructure } \\
\text { based on RelSupportStructureObjectld. } 1 \\
\text { SupportStructure can have } 0 \text { or many } \\
\text { CustomerStreetlights }\end{array}$ \\
\hline FieldInventoryLight & Delete Table & $\begin{array}{l}\text { No longer needed because its data has been } \\
\text { merged into the CompanyStreetLight object } \\
\text { class and the support structure feature class. }\end{array}$ \\
\hline \multirow[t]{7}{*}{ StreetlightSwitch } & New Column & SubtypeCd - integer \\
\hline & $\begin{array}{l}\text { Rename } \\
\text { Column }\end{array}$ & $\begin{array}{l}\text { LegacyDistribRefNumber --> } \\
\text { DistribRefNumber }\end{array}$ \\
\hline & $\begin{array}{l}\text { Remove } \\
\text { Column }\end{array}$ & StreetlightSwitchStatusCd \\
\hline & $\begin{array}{l}\text { New } \\
\text { Subtypes }\end{array}$ & $\begin{array}{l}\text { StreetlightSwitch (subtypecd=1) } \\
\text { RetiredStreetlightSwitch (subtypecd=2) }\end{array}$ \\
\hline & $\begin{array}{l}\text { Rename } \\
\text { Column }\end{array}$ & $\begin{array}{l}\text { StreetlightSwitchAmpereValue --> } \\
\text { AmperageValue } \\
\text { StreetlightSwitchVoltageValue --> } \\
\text { VoltageValue }\end{array}$ \\
\hline & $\begin{array}{l}\text { Change } \\
\text { Inheritance }\end{array}$ & $\begin{array}{l}\text { Change to inherit from } \\
\text { LegacyWorkOrderTimestamps }\end{array}$ \\
\hline & $\begin{array}{l}\text { Verify } \\
\text { Domain }\end{array}$ & $\begin{array}{l}\text { Verify that DmStreetlightSwAmpereValue } \\
\text { includes: } \\
20,30,35,60,75,100\end{array}$ \\
\hline EdfsTrafficLight & $\begin{array}{l}\text { Rename } \\
\text { Table }\end{array}$ & Rename table to TrafficLightDemand \\
\hline \multirow[t]{2}{*}{ TrafficLightDemand } & $\begin{array}{l}\text { Rename } \\
\text { Column }\end{array}$ & $\begin{array}{l}\text { DemandDate } \\
\text { DemandWattageValue }\end{array}$ \\
\hline & New Column & $\begin{array}{l}\text { RelPadMountObjectld - integer } \\
\text { CISAccountNumber - integer } \\
\text { LegacyCustXrefCustAcctRefNumber - } \\
\text { integer }\end{array}$ \\
\hline
\end{tabular}




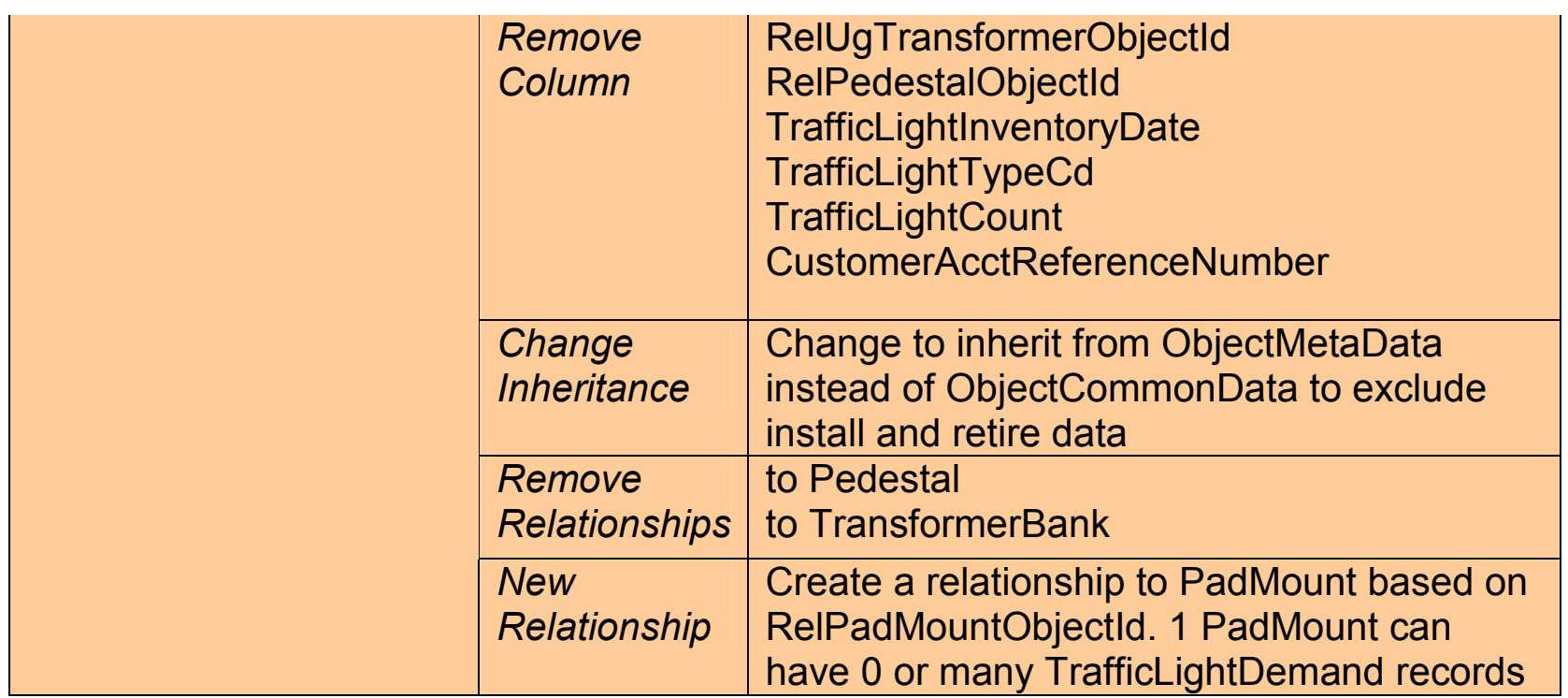


Table F-9. Conductor data model changes

\begin{tabular}{|c|c|c|}
\hline Table & Action & Details of Data Model Requested Change \\
\hline EdfsConductor & $\begin{array}{l}\text { Rename } \\
\text { Table }\end{array}$ & Rename Table to ConductorDefinition \\
\hline \multirow[t]{5}{*}{ ConductorDefinition } & $\begin{array}{l}\text { Verify } \\
\text { Inheritance }\end{array}$ & Should inherit from ObjectMetadata \\
\hline & $\begin{array}{l}\text { Rename } \\
\text { Column }\end{array}$ & $\begin{array}{l}\text { LegacyConductorNumber } \\
\text { CategoryCd - new domain } \\
\text { LocationCd - new domain } \\
\text { WireTypeCd - new domain } \\
\text { WireSizeCd - new domain } \\
\text { WireMaterialCd - new domain } \\
\text { LegacyCalculationCd }\end{array}$ \\
\hline & New Domain & $\begin{array}{l}\text { CategoryCd: } \\
1 \text { - Primary } \\
2 \text { - Secondary } \\
3 \text { - Service }\end{array}$ \\
\hline & New Domain & $\begin{array}{l}\text { LocationCd: } \\
\text { O - Overhead } \\
\text { U - Underground }\end{array}$ \\
\hline & New Domain & $\begin{array}{l}\text { WireSizeCd: } \\
\text { verify against old data with query: } \\
\text { select distinct CNDUCR_SZ_CD from } \\
\text { ED_CONDTR }\end{array}$ \\
\hline
\end{tabular}




\begin{tabular}{|c|c|c|}
\hline & New Domain & $\begin{array}{l}\text { WireTypeCd (may match } \\
\text { DmConductorTypeCd - this is the superset of } \\
\text { all types from prim, sec, svc, oh, ug, etc): } \\
\text { W - Solid Wire } \\
\text { WS - Stranded Wire } \\
\text { WN - Solid Wire as Neutral } \\
\text { WSN Stranded Wire as Neutral } \\
\text { WSS - Stranded Wire as Static or Sheild } \\
\text { WSU - Wire Size Unknown } \\
\text { CSU - Cable Size Unknown } \\
\text { CO - Customer Owned } \\
\text { B - Duplex } \\
\text { C - Triplex } \\
\text { CCU - Triplex with Copper Neutral } \\
\text { D - Quadruplex } \\
\text { UAS - Single Cable } \\
\text { UCS - Triplex Cable } \\
\text { UDS - Quadruplex } \\
\text { UA - Single Cable Concentric } \\
\text { UAJ - Single Cable Concentric Jacketed } \\
\text { UC - Triplex Cable Concentric } \\
\text { UCJ - Triplex Cable Concentric Jacketed } \\
\text { UP - Single Cable Power } \\
\text { UF - Single Cable Power XLP 5Kv } \\
\text { Verify against data with query: } \\
\text { select distinct CNDUCR_TYP_CD from } \\
\text { ED_CONDTR }\end{array}$ \\
\hline & New Domain & $\begin{array}{l}\text { WireMaterialCd (may match } \\
\text { DmConductorMaterialCd - this is the } \\
\text { superset of all types from prim, sec, svc, oh, } \\
\text { ug, etc): } \\
\text { AA - Aluminum Alloy } \\
\text { ACSR- Aluminum Coated Steel Reinforced } \\
\text { CU - Copper } \\
\text { CW - Copper Weld } \\
\text { AL - Aluminum } \\
\text { WSU - Wire Size Unknown } \\
\text { CSU - Cable Size Unknown } \\
\text { Verify against data with query: } \\
\text { select distinct CNDUCR_KND_CD from } \\
\text { ED_CONDTR }\end{array}$ \\
\hline OhConductorInfo & New Column & $\begin{array}{l}\text { MeasuredLength - } \\
\text { DmMeasuredLengthRange (to be populated }\end{array}$ \\
\hline
\end{tabular}




\begin{tabular}{|c|c|c|}
\hline & & $\begin{array}{l}\text { in retirement) } \\
\text { CircuitNumber string(20) (to be populated in } \\
\text { retirement) }\end{array}$ \\
\hline & $\begin{array}{l}\text { Rename } \\
\text { Column }\end{array}$ & $\begin{array}{l}\text { ConductorSizeCd --> WireSizeCd } \\
\text { ConductorMaterialCd --> WireMaterialCd } \\
\text { ConductorTypeCd --> WireTypeCd }\end{array}$ \\
\hline & $\begin{array}{l}\text { Remove } \\
\text { Column }\end{array}$ & $\begin{array}{l}\text { ConductorCalculationTypeCd } \\
\text { ConductorsPerPhaseCount } \\
\text { PrimaryOperatingVoltageValue } \\
\text { RelAbandonedConductorObjectld } \\
\text { ConductorSpanCount } \\
\text { SpanLengthValue } \\
\text { ConductorNumberCd }\end{array}$ \\
\hline & New Domain & $\begin{array}{l}\text { WireSizeCd: } \\
\text { select distinct CNDUCR_SZ_CD from } \\
\text { ED_CONDTR where } \\
\text { CNDUCR_LOC_CD='O' and } \\
\text { CNDUCR_CTGR_TYP_CD }=\text { ' } 1 \text { ' }\end{array}$ \\
\hline & New Domain & $\begin{array}{l}\text { WireTypeCd (OH ONLY): } \\
\text { W - Solid Wire } \\
\text { WS - Stranded Wire } \\
\text { WN - Solid Wire as Neutral } \\
\text { WSN Stranded Wire as Neutral } \\
\text { WSS - Stranded Wire as Static or Sheild } \\
\text { WSU - Wire Size Unknown } \\
\text { CSU - Cable Size Unknown } \\
\text { CO - Customer Owned }\end{array}$ \\
\hline & New Domain & $\begin{array}{l}\text { WireMaterialCd (OH ONLY): } \\
\text { AA - Aluminum Alloy } \\
\text { ACSR- Aluminum Coated Steel Reinforced } \\
\text { CU - Copper } \\
\text { CW - Copper Weld } \\
\text { WSU - Wire Size Unknown } \\
\text { CSU - Cable Size Unknown }\end{array}$ \\
\hline & $\begin{array}{l}\text { New } \\
\text { Subtype }\end{array}$ & RetiredOhConductor (subtypecd=2) \\
\hline & \begin{tabular}{|l|} 
Remove \\
Relationship
\end{tabular} & to AbandonedConductor \\
\hline LegacyOhConductorInfo & Create Table & Copy OhConductorInfo \\
\hline
\end{tabular}




\begin{tabular}{|l|l|l|}
\hline New Column & $\begin{array}{l}\text { ConductorSpanCount - integer } \\
\text { SpanLengthValue - integer } \\
\text { LegacyConductorNumber - integer }\end{array}$ \\
\hline Uemove & MeasuredLength \\
Column & RelOhConductorObjectld \\
\hline Inheritance & Inherit from EDFSTimeStamps \\
\hline New & OhConductor (subtypecd=1) \\
Subtypes & RetiredOhConductor (subtypecd=2)
\end{tabular}




\begin{tabular}{|c|c|c|}
\hline & New Domain & $\begin{array}{l}\text { WireMaterialCd (UG ONLY): } \\
\text { AL - Aluminum } \\
\text { AA - Aluminum Alloy } \\
\text { CU - Copper } \\
\text { WSU - Wire Size Unknown } \\
\text { CSU - Cable Size Unknown }\end{array}$ \\
\hline & New Domain & $\begin{array}{l}\text { InConduitCd: } \\
\text { Y - Yes } \\
\text { N - No } \\
\text { C - Customer }\end{array}$ \\
\hline & $\begin{array}{l}\text { New } \\
\text { Subtype }\end{array}$ & RetiredUgConductor (subtypecd=2) \\
\hline & $\begin{array}{l}\text { Remove } \\
\text { Relationship }\end{array}$ & to AbandonedConductor \\
\hline \multirow{6}{*}{ LegacyUgConductorlnfo } & Create Table & Copy UgConductorlnfo \\
\hline & New Column & $\begin{array}{l}\text { ConductorSpanCount - integer } \\
\text { SpanLengthValue - integer } \\
\text { LegacyConductorNumber - integer }\end{array}$ \\
\hline & $\begin{array}{l}\text { Remove } \\
\text { Column }\end{array}$ & $\begin{array}{l}\text { MeasuredLength } \\
\text { RelUgConductorObjectld }\end{array}$ \\
\hline & $\begin{array}{l}\text { Rename } \\
\text { Column }\end{array}$ & ToDistribRefNumber --> ToStructureld \\
\hline & Inheritance & Inherit from EDFSTimeStamps \\
\hline & $\begin{array}{l}\text { New } \\
\text { Subtypes }\end{array}$ & $\begin{array}{l}\text { UgConductor (subtypecd=1) } \\
\text { RetiredUgConductor (subtypecd=2) }\end{array}$ \\
\hline \multirow[t]{3}{*}{ SecondaryConductorInfo } & New Column & $\begin{array}{l}\text { MeasuredLength - } \\
\text { DmMeasuredLengthRange (to be populated } \\
\text { in retirement) } \\
\text { ToAlternateNumber string(8) }\end{array}$ \\
\hline & $\begin{array}{l}\text { Rename } \\
\text { Column }\end{array}$ & $\begin{array}{l}\text { ConductorSizeCd --> WireSizeCd } \\
\text { ConductorMaterialCd --> WireMaterialCd } \\
\text { ConductorTypeCd --> WireTypeCd } \\
\text { InConduitInd --> InConduitCd }\end{array}$ \\
\hline & $\begin{array}{l}\text { Remove } \\
\text { Column }\end{array}$ & $\begin{array}{l}\text { ConductorCateogoryTypeCd } \\
\text { ConductorCalculationTypeCd } \\
\text { RelAbandonedConductorObjectld } \\
\text { ConductorSpanCount } \\
\text { SpanLengthValue } \\
\text { ConductorNumberCd }\end{array}$ \\
\hline
\end{tabular}




\begin{tabular}{|l|l|} 
New Domain & WireSizeCd: \\
& select distinct CNDUCR_SZ_CD from \\
& ED_CONDTR where \\
& CNDUCR_CTGR_TYP_CD = '2' \\
\hline New Domain & SecondaryOperatingVoltage: \\
& $120 / 208$ \\
& $120 / 240$ \\
& 120 \\
& MS \\
& 230 \\
& $277 / 480$ \\
& $240 / 480$ \\
& SS \\
& 480 \\
& \\
\hline New Domain & WireTypeCd (SEC ONLY): \\
& OH - \\
& W - Solid Wire \\
& WS - Stranded Wire \\
& WN - Solid Wire as Neutral \\
& WSN Stranded Wire as Neutral \\
& WSU - Wire Size Unknown \\
& CSU - Cable Size Unknown \\
& CO - Customer Owned \\
& B - Duplex \\
& C - Triplex \\
& CCU - Triplex with Copper Neutral \\
D - Quadruplex & \\
& UG - \\
& UAS - single Cable \\
& UCS - Triple Cable \\
& UDS - Quadruplex \\
& WSU - Wire Size Unknown \\
& CSU - Cable Size Unknown \\
& CO - Customer Owned \\
& WireMaterialCd (SEC ONLY): \\
& AA = Aluminum Alloy \\
& ACSR- Aluminum Coated Steel Reinforced \\
& CU - Copper \\
& CW - Copper Weld \\
& AL - Aluminum \\
& \\
\hline New Domain & \\
&
\end{tabular}




\begin{tabular}{|c|c|c|}
\hline & New Domain & $\begin{array}{l}\text { InConduitCd: } \\
\text { Y - Yes } \\
\text { N - No } \\
\text { C - Customer }\end{array}$ \\
\hline & $\begin{array}{l}\text { Rename } \\
\text { Subtype }\end{array}$ & $\begin{array}{l}\text { Rename the single existing Subtype to } \\
\text { become OhSecondary (subtypecd=1) }\end{array}$ \\
\hline & $\begin{array}{l}\text { New } \\
\text { Subtypes }\end{array}$ & $\begin{array}{l}\text { UgSecondary (subtypecd=2) } \\
\text { RetiredOhSecondary (subtypecd=3) } \\
\text { RetiredUgSecondary (subtypecd=4) }\end{array}$ \\
\hline & $\begin{array}{l}\text { Domain via } \\
\text { Subtype }\end{array}$ & $\begin{array}{l}\text { Set up the domain for WireTypeCd to be } \\
\text { specific to } O H / U G \text { subtypes }\end{array}$ \\
\hline & $\begin{array}{l}\text { Remove } \\
\text { Relationship }\end{array}$ & to AbandonedConductor \\
\hline \multirow{5}{*}{$\begin{array}{l}\text { LegacySecondary } \\
\text { Conductorlnfo }\end{array}$} & Create Table & Copy SecondaryConductorlnfo \\
\hline & New Column & $\begin{array}{l}\text { ConductorSpanCount - integer } \\
\text { SpanLengthValue - integer } \\
\text { LegacyConductorNumber - integer }\end{array}$ \\
\hline & $\begin{array}{l}\text { Remove } \\
\text { Column }\end{array}$ & $\begin{array}{l}\text { MeasuredLength } \\
\text { RelSecondaryConductorObjectld }\end{array}$ \\
\hline & Inheritance & Inherit from EDFSTimeStamps \\
\hline & $\begin{array}{l}\text { New } \\
\text { Subtypes }\end{array}$ & $\begin{array}{l}\text { OhSecondary (subtypecd=1) } \\
\text { UgSecondary (subtypecd=2) } \\
\text { RetiredOhSecondary (subtypecd=3) } \\
\text { RetiredUgSecondary (subtypecd=4) }\end{array}$ \\
\hline $\begin{array}{l}\text { OhServiceConductor } \\
\text { UgServiceConductor }\end{array}$ & $\begin{array}{l}\text { Merge } \\
\text { Tables }\end{array}$ & $\begin{array}{l}\text { Merge these two object classes together as } \\
\text { follows: } \\
\text { 1) Rename OhServiceConductor to } \\
\text { ServiceConductorlnfo } \\
\text { 2) Delete UgServiceConductor \& related } \\
\text { subtypes \& relationships }\end{array}$ \\
\hline ServiceConductorlnfo & $\begin{array}{l}\text { Rename } \\
\text { Column }\end{array}$ & $\begin{array}{l}\text { ConductorNumberCd - } \\
\text { LegacyConductorNumber } \\
\text { ConductorSizeCd - WireSizeCd } \\
\text { ConductorMaterialCd - WireMaterialCd } \\
\text { ConductorTypeCd - WireTypeCd } \\
\text { LoadPoleDistribRefNumber - } \\
\text { LoadDistribRefNumber } \\
\text { RelSupportStructureObjectld - } \\
\text { RelLoadSupportStructureObjectld }\end{array}$ \\
\hline
\end{tabular}




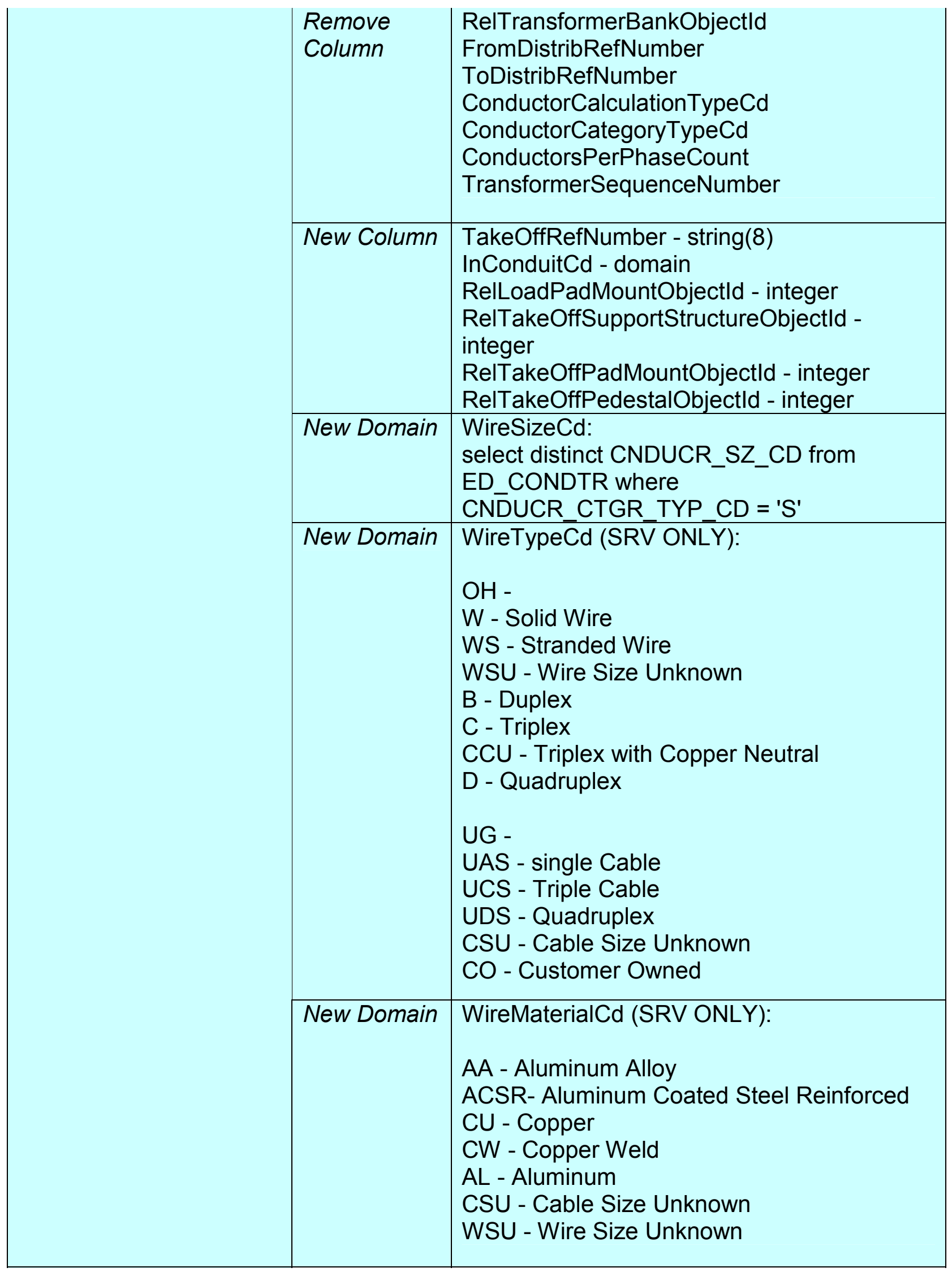




\begin{tabular}{|c|c|c|}
\hline & New Domain & $\begin{array}{l}\text { InConduitCd: } \\
\text { Y - Yes } \\
\text { N - No } \\
\text { C - Customer }\end{array}$ \\
\hline & $\begin{array}{l}\text { Change } \\
\text { Inheritance }\end{array}$ & Change to inherit from EDFSTimeStamps \\
\hline & $\begin{array}{l}\text { Update } \\
\text { Relationship }\end{array}$ & $\begin{array}{l}\text { Update existing relationship to support } \\
\text { structure to be based off of } \\
\text { RelLoadSupportStructureObjectld }\end{array}$ \\
\hline & $\begin{array}{l}\text { New } \\
\text { Relationship }\end{array}$ & $\begin{array}{l}\text { Create new relationship to SupportStructure } \\
\text { based on } \\
\text { RelTakeOffSupportStructureObjectld. }\end{array}$ \\
\hline & $\begin{array}{l}\text { New } \\
\text { Relationship }\end{array}$ & $\begin{array}{l}\text { Create new relationship to PadMount based } \\
\text { on RelLoadPadMountObjectld }\end{array}$ \\
\hline & $\begin{array}{l}\text { New } \\
\text { Relationship }\end{array}$ & $\begin{array}{l}\text { Create new relationship to PadMount based } \\
\text { on RelTakeOffPadMountObjectld }\end{array}$ \\
\hline & $\begin{array}{l}\text { New } \\
\text { Relationship }\end{array}$ & $\begin{array}{l}\text { Create new relationship to Pedestal based } \\
\text { on RelTakeOffPedestalObjectld }\end{array}$ \\
\hline & $\begin{array}{l}\text { New } \\
\text { Subtypes }\end{array}$ & $\begin{array}{l}\text { UgServiceConductor (subtypecd=2) } \\
\text { RetiredOhServiceConductor (subtypecd=3) } \\
\text { RetiredUgServiceConductor (subtypecd=4) }\end{array}$ \\
\hline & $\begin{array}{l}\text { Domain via } \\
\text { Subtype }\end{array}$ & $\begin{array}{l}\text { Set up the domain for WireTypeCd to be } \\
\text { specific to } O H / U \text { subtypes }\end{array}$ \\
\hline & $\begin{array}{l}\text { Remove } \\
\text { Relationship }\end{array}$ & to TransformerBank \\
\hline RetiredServiceConductor & Delete Table & $\begin{array}{l}\text { No longer needed because the retired } \\
\text { service conductor is handled by subtypes }\end{array}$ \\
\hline
\end{tabular}




\section{Appendix G}

\section{Glossary of Terms}

\begin{tabular}{|l|l|}
\hline Term & Definition \\
\hline .NET & $\begin{array}{l}\text { Microsoft's most recent Application Development } \\
\text { Environment for programming custom software. }\end{array}$ \\
\hline ADO (ActiveX Directory Object) & $\begin{array}{l}\text { Microsoft software which allows programmers to } \\
\text { create software which accesses data in a database. }\end{array}$ \\
\hline AEDR & $\begin{array}{l}\text { Automated Energy Distribution and Reliability } \\
\text { system. NIPSCO's Geographic Information System. }\end{array}$ \\
\hline Application Interface & $\begin{array}{l}\text { Text labels displayed on a map whose size, position, } \\
\text { color, and other properties are stored as a record in the } \\
\text { Geodatabase. }\end{array}$ \\
\hline ArcCatalog & $\begin{array}{l}\text { Regarding NIPSCO's GIS, communication of data } \\
\text { between the GIS and other applications typically } \\
\text { owned and maintained by another NIPSCO } \\
\text { department. }\end{array}$ \\
\hline ArcFM & $\begin{array}{l}\text { ESRI software for managing GIS databases. Often } \\
\text { referred to as "Windows Explorer for GIS". }\end{array}$ \\
\hline ArcMap & $\begin{array}{l}\text { Software from Miner \& Miner extending ArcGIS's } \\
\text { core functionality to support business processes } \\
\text { specific to the Utility Industry. }\end{array}$ \\
\hline ArcGIS & $\begin{array}{l}\text { A family of software from ESRI for building and } \\
\text { running GIS databases and applications. The ArcGIS } \\
\text { includes ArcMap, ArcCatalog, and ArcSDE. }\end{array}$ \\
\hline $\begin{array}{l}\text { ESRI software for building and distributing an Internet } \\
\text { Mapping Service, which displays map data in a web } \\
\text { browser such as Internet Explorer. }\end{array}$ \\
$\begin{array}{l}\text { ESRI software for viewing, analyzing, and editing GIS } \\
\text { datasets. }\end{array}$ \\
\hline ArciMS
\end{tabular}




\begin{tabular}{|c|c|}
\hline ArcMap Publisher Extension & $\begin{array}{l}\text { ESRI software for exporting GIS information to a } \\
\text { Published Map File which can be viewed using ESRI's } \\
\text { free ArcReader application. }\end{array}$ \\
\hline ArcObjects & $\begin{array}{l}\text { ESRI's framework of programming tools which allows } \\
\text { programmers to create software that employs GIS } \\
\text { specific functions. }\end{array}$ \\
\hline ArcReader & ESRI's free GIS data viewing application. \\
\hline $\operatorname{ArcSDE}$ & $\begin{array}{l}\text { ESRI's Spatial Database Engine connecting ArcGIS } \\
\text { applications to data stored in a RDBMS such as SQL } \\
\text { Server. }\end{array}$ \\
\hline ASP .NET & $\begin{array}{l}\text { Microsoft application development technology that } \\
\text { allows programmers to create interactive web pages, } \\
\text { such as a web page that collects user input and posts } \\
\text { the input to a database. }\end{array}$ \\
\hline Attribute Domain & See Domain. \\
\hline Attribute Query & $\begin{array}{l}\text { A technique for selecting a subset of records in } \\
\text { ArcMap by specifying a filter value for a given table } \\
\text { column. }\end{array}$ \\
\hline Auto Updaters (AU) & $\begin{array}{l}\text { Miner \& Miner technology that automatically } \\
\text { performs a predetermined activity when triggered by a } \\
\text { specific event. The event trigger may be the creating, } \\
\text { deleting, or updating a record in the database. }\end{array}$ \\
\hline AutoCAD & $\begin{array}{l}\text { AutoDesk's Computer Aided Drafting software which } \\
\text { provides tools for creating any type of drawing. }\end{array}$ \\
\hline Batch (process or application) & $\begin{array}{l}\text { A group of similar activities automatically executed in } \\
\text { sequence. Often batch processes or applications are } \\
\text { scheduled to run at repeated intervals, such as nightly } \\
\text { or weekly. }\end{array}$ \\
\hline Binary Data & $\begin{array}{l}\text { Information stored in a manner that only computer } \\
\text { software can interpret. In other words, data that is not } \\
\text { presented as letters or numbers. }\end{array}$ \\
\hline Buffer & $\begin{array}{l}\text { An area whose perimeter is a specified map distance } \\
\text { from a given geometry. For example, a } 10 \text { foot buffer }\end{array}$ \\
\hline
\end{tabular}




\begin{tabular}{|c|c|}
\hline & $\begin{array}{l}\text { around a point on a map is a circle with a radius of } 10 \\
\text { feet. }\end{array}$ \\
\hline C\#.NET & $\begin{array}{l}\text { Microsoft's Application Development Environment in } \\
\text { which programmers enter instructions for the } \\
\text { computer in the C\# programming language. }\end{array}$ \\
\hline CAD (Computer Aided Drafting) & $\begin{array}{l}\text { A type of software that allows the user to create any } \\
\text { type of drawing be it a design of automobile part or a } \\
\text { street map. }\end{array}$ \\
\hline CADOPS & $\begin{array}{l}\text { A software tool used to analyze outages in utility } \\
\text { network systems. }\end{array}$ \\
\hline $\begin{array}{l}\text { CICS (Customer Information } \\
\text { Control System) }\end{array}$ & $\begin{array}{l}\text { A mainframe application that controls a variety of } \\
\text { mainframe computer applications. }\end{array}$ \\
\hline CIS (Customer Information System) & $\begin{array}{l}\text { A client/mainframe based application that maintains } \\
\text { all utility customers billing and account information. }\end{array}$ \\
\hline CITRIX & $\begin{array}{l}\text { A software application that allows a user to control a } \\
\text { remote computer and application using their local } \\
\text { computer over a network, }\end{array}$ \\
\hline Client-Server Application & $\begin{array}{l}\text { A set of applications for which some function execute } \\
\text { on the local computer in front of the user, and other } \\
\text { functions or data are processed on a remote server. } \\
\text { The network provides communication between the } \\
\text { two. }\end{array}$ \\
\hline Commit & $\begin{array}{l}\text { Regarding database edits, the process that saves } \\
\text { database edits from the computer's temporary memory } \\
\text { to the physical drive space. }\end{array}$ \\
\hline Compress & $\begin{array}{l}\text { An ArcSDE Geodatabase process which reduces the } \\
\text { size of the versioning state tree by deleting all version } \\
\text { states that have been completely posted to the default } \\
\text { version. See Versioning for more information. }\end{array}$ \\
\hline Concatenate & $\begin{array}{l}\text { Combine two strings together, for example "hel" + } \\
\text { "lo" = "hello" }\end{array}$ \\
\hline Conflict & $\begin{array}{l}\text { A discrepancy among the data reported when } \\
\text { reconciling an ArcSDE version to a parent version. }\end{array}$ \\
\hline
\end{tabular}




\begin{tabular}{|c|c|}
\hline CPU (Central Processing Unit) & $\begin{array}{l}\text { The main logic engine of a computer through which } \\
\text { all instructions are read and executed. }\end{array}$ \\
\hline Data Migration & $\begin{array}{l}\text { A process where data is copied from one data set to } \\
\text { another data set. Typically the two datasets are of } \\
\text { different types. For example, NIPSCO moved data } \\
\text { stored in AutoCad Drawing files into an ArcSDE } \\
\text { Geodatabase. }\end{array}$ \\
\hline Data Model & $\begin{array}{l}\text { An organization of information types into tables, } \\
\text { records, relationships, subtypes, domains, etc. Defines } \\
\text { the structure of a database. }\end{array}$ \\
\hline DataPrep & $\begin{array}{l}\text { A conversion tool which exports the Gas Network to a } \\
\text { data format compatible with the SynerGEE gas } \\
\text { analysis software. }\end{array}$ \\
\hline DB2 & $\begin{array}{l}\text { A Relational Database Management System } \\
\text { (RDBMS). }\end{array}$ \\
\hline Distribution Reference Number & $\begin{array}{l}\text { A NIPSCO specific unique number assigned to all } \\
\text { electric poles, transformer pads, and pedestals. }\end{array}$ \\
\hline Domain & $\begin{array}{l}\text { Regarding database columns, a finite list or range of } \\
\text { values that are allowed to be assigned to a given } \\
\text { column for any table record. }\end{array}$ \\
\hline DOQQ & $\begin{array}{l}\text { Digital Orthophoto Quarter Quadrangle; A digital } \\
\text { raster image of an aerial photo mapped by the USGS, } \\
\text { specifically a "quarter quad", or the extent of one } \\
\text { fourth of a } 7.5 \text {-minute quadrangle, often used in GIS } \\
\text { applications. }\end{array}$ \\
\hline EAIF & $\begin{array}{l}\text { Engineering Accounts Information File. A mainframe } \\
\text { system that keeps load information on each } \\
\text { transformer. }\end{array}$ \\
\hline $\begin{array}{l}\text { EDFS (Electric Distribution Facility } \\
\text { Services) }\end{array}$ & $\begin{array}{l}\text { NIPSCO's legacy electric asset tracking system which } \\
\text { was migrated to and replaced by the GIS. }\end{array}$ \\
\hline $\begin{array}{l}\text { Electric Distribution Planning } \\
\text { System (Load Study) }\end{array}$ & See FeederALL. \\
\hline
\end{tabular}




\begin{tabular}{|c|c|}
\hline Enterprise Application & $\begin{array}{l}\text { A system of software, computer resources, and data } \\
\text { that is shared among many users. }\end{array}$ \\
\hline $\begin{array}{l}\text { ESRI (Environmental Systems } \\
\text { Research Institute) }\end{array}$ & $\begin{array}{l}\text { A GIS software and services provider which produces } \\
\text { the ArcGIS family of software. }\end{array}$ \\
\hline Extension to ArcGIS & $\begin{array}{l}\text { Software built with ArcObjects that adds functionality } \\
\text { to ESRI's ArcGIS. }\end{array}$ \\
\hline Fat-Client & $\begin{array}{l}\text { The client of a Client-Server Application where the } \\
\text { majority of processing is performed on the user's local } \\
\text { computer. }\end{array}$ \\
\hline Feature & $\begin{array}{l}\text { A record in a Geodatabase Feature Class which } \\
\text { models the attributes and geometry of a real work } \\
\text { object. }\end{array}$ \\
\hline Feature Class & $\begin{array}{l}\text { A table in the Geodatabase with a defined set of } \\
\text { attributes and a geometry type (point, line, polygon, or } \\
\text { annotation) that stores a collection of Features. }\end{array}$ \\
\hline FeederAll & $\begin{array}{l}\text { Package software that uses the computer model of the } \\
\text { electric network and actual load readings to determine } \\
\text { wire size requirements for the distribution network. }\end{array}$ \\
\hline FME (Feature Manipulation Engine) & $\begin{array}{l}\text { Software produced by Safe Software that converts } \\
\text { many types of GIS file formats. }\end{array}$ \\
\hline General Ledger & $\begin{array}{l}\text { Computer system that maintains a portion of the } \\
\text { financial information for NIPSCO }\end{array}$ \\
\hline Geodatabase & $\begin{array}{l}\text { An ESRI proprietary data structure for modeling and } \\
\text { storing real world map objects in a database such as } \\
\text { MS Access or MS SQL Server. }\end{array}$ \\
\hline Geometric Network & See Utility Network \\
\hline Geometry & $\begin{array}{l}\text { A line, point, or polygon that represents the trend, } \\
\text { location, or shape of a real world map object. }\end{array}$ \\
\hline $\begin{array}{l}\text { HTML (Hypertext Markup } \\
\text { Language) }\end{array}$ & $\begin{array}{l}\text { A collection of tags that allows for the formatting of } \\
\text { text and images. Internet Browsers are the most } \\
\text { common applications which read HTML formatted } \\
\text { documents. }\end{array}$ \\
\hline
\end{tabular}




\begin{tabular}{|c|c|}
\hline Interface & See Application Interface. \\
\hline IRTH & $\begin{array}{l}\text { A web based mapping application using GIS data to } \\
\text { manage facility locate (call before you dig) requests. }\end{array}$ \\
\hline JavaScript & $\begin{array}{l}\text { A programming scripting language used to add } \\
\text { dynamic content to internet pages. }\end{array}$ \\
\hline Join & $\begin{array}{l}\text { Regarding databases, merges the records of two or } \\
\text { more database tables using a column in each table } \\
\text { containing common data. }\end{array}$ \\
\hline $\begin{array}{l}\text { MAPPS (Materials, Purchasing and } \\
\text { Accounts Payable) }\end{array}$ & $\begin{array}{l}\text { Mainframe based system that manages the purchase, } \\
\text { inventory and payments of various goods and services. }\end{array}$ \\
\hline Migration & See Data Migration \\
\hline Miner \& Miner & $\begin{array}{l}\text { A GIS software and services provider focusing on the } \\
\text { utility industry. Produces ArcFM. }\end{array}$ \\
\hline MLOG & $\begin{array}{l}\text { Material and labor estimating system for distribution } \\
\text { and substation design. }\end{array}$ \\
\hline MVV (Multi-Versioned View) & $\begin{array}{l}\text { A presentation of versioned ESRI Geodatabase tables } \\
\text { for a specific version without the need for any ESRI } \\
\text { licensed software. }\end{array}$ \\
\hline Network & See Utility Network. \\
\hline Network Edge & See Utility Network. \\
\hline Network Junction & See Utility Network. \\
\hline NIPSCO & $\begin{array}{l}\text { Northern Indiana Public Service Company, a NiSource } \\
\text { company }\end{array}$ \\
\hline $\begin{array}{l}\text { NORS (NIPSCO Outage and } \\
\text { Restoration System) }\end{array}$ & $\begin{array}{l}\text { Packaged software from ABB that combines the } \\
\text { electric network connectivity and customer } \\
\text { information to determine the most likely point of } \\
\text { failure in the network for storm restoration work. }\end{array}$ \\
\hline Oracle & A RDMBS. \\
\hline
\end{tabular}




\begin{tabular}{|c|c|}
\hline Outfield & $\begin{array}{l}\text { NIPSCO's legacy automated mapping system which } \\
\text { stored all map data and attributes until it was replaced } \\
\text { by the GIS. }\end{array}$ \\
\hline OOTB (Out-Of-The-Box) & $\begin{array}{l}\text { Software functionality that does not require any } \\
\text { programming or customization. }\end{array}$ \\
\hline Performance & $\begin{array}{l}\text { The ability of a computer to perform a task within a } \\
\text { duration. }\end{array}$ \\
\hline Post & See Versioning. \\
\hline PLSS & Public Land Survey System \\
\hline $\begin{array}{l}\text { QAQC (Quality Assurance Quality } \\
\text { Control) }\end{array}$ & $\begin{array}{l}\text { The process of preventing inconsistent or inaccurate } \\
\text { data from entering the system. }\end{array}$ \\
\hline $\begin{array}{l}\text { RDBMS (Relational Database } \\
\text { Management System) }\end{array}$ & $\begin{array}{l}\text { A software for managing large collections of } \\
\text { structured data. }\end{array}$ \\
\hline Reconcile & See Versioning. \\
\hline Relationship Classes & $\begin{array}{l}\text { A structure of ESRI's Geodatabase which maintains a } \\
\text { relationship between records in two tables or feature } \\
\text { classes. }\end{array}$ \\
\hline ROW & Right of Way \\
\hline $\begin{array}{l}\text { SAGE (Stand Alone Geodatabase } \\
\text { Editor) }\end{array}$ & $\begin{array}{l}\text { A tool provided by Miner \& Miner which allow for } \\
\text { viewing and editing of data stored in a versioned ESRI } \\
\text { Geodatabase without the need for an ESRI software } \\
\text { license. }\end{array}$ \\
\hline SDE & See $\operatorname{ArcSDE}$. \\
\hline Session & $\begin{array}{l}\text { A Miner \& Miner invention that presents ESRI } \\
\text { versioning management in a user friendly manner. }\end{array}$ \\
\hline Spatial Query & $\begin{array}{l}\text { A technique for selecting a subset of data within } \\
\text { ArcGIS by comparing the geometry of the selection } \\
\text { candidate to a filter geometry. }\end{array}$ \\
\hline Split & $\begin{array}{l}\text { Division of a single linear feature into two shorter } \\
\text { linear features. }\end{array}$ \\
\hline
\end{tabular}




\begin{tabular}{|c|c|}
\hline SQL Query & $\begin{array}{l}\text { A database operation, such as Select, Insert, or Delete, } \\
\text { which is executed via syntax written in Structure } \\
\text { Query Language. }\end{array}$ \\
\hline SQL Server & A RDBMS. \\
\hline Symbology & $\begin{array}{l}\text { A definition of size, shape, color, and other parameters } \\
\text { used for displaying a feature's geometry on the map. }\end{array}$ \\
\hline SynerGEE & A software for analyzing gas utility networks. \\
\hline Tap & The point at which two network edges intersect. \\
\hline Thin-Client & $\begin{array}{l}\text { The client of a Client-Server Application where the } \\
\text { majority of processing is performed on the server } \\
\text { computer. }\end{array}$ \\
\hline $\begin{array}{l}\text { UNC Path (Universal Naming } \\
\text { Convention) }\end{array}$ & Syntax for identifying a file path on a network. \\
\hline Use Case & $\begin{array}{l}\text { A specific business process for which a software } \\
\text { component is designed to accomplish. }\end{array}$ \\
\hline Utility Network & $\begin{array}{l}\text { A collection of features that model a real world gas, } \\
\text { electric, or water system. Line features become } \\
\text { network edges which transmit the commodity through } \\
\text { the system. Point features become junctions which } \\
\text { control how the commodity moves through the system. }\end{array}$ \\
\hline Versioning & $\begin{array}{l}\text { An ESRI invention which allows multiple users to edit } \\
\text { a Geodatabase at the same time by assigning a copy of } \\
\text { the data to each user. The edits that are made within } \\
\text { the copy are posted back to the copy's parent when the } \\
\text { editing is complete. }\end{array}$ \\
\hline Visio & $\begin{array}{l}\text { A software for creating diagrams, such as a visual } \\
\text { diagram of a data model. }\end{array}$ \\
\hline Walker GL & $\begin{array}{l}\text { Computer system that maintains a portion of the } \\
\text { financial information for NIPSCO }\end{array}$ \\
\hline Web Server & $\begin{array}{l}\text { A computer which makes documents and applications } \\
\text { available to users via the internet. }\end{array}$ \\
\hline
\end{tabular}




\begin{tabular}{|l|l|}
\hline XML (Extensible Markup Language) & $\begin{array}{l}\text { A system of user defined tags that allows for the } \\
\text { formatting of text and images. Internet Browsers are } \\
\text { the most common applications which read XML } \\
\text { formatted documents. }\end{array}$ \\
\hline $\begin{array}{l}\text { XSLT (Extensible Stylesheet } \\
\text { Language Transformations) }\end{array}$ & $\begin{array}{l}\text { A document that defines how information in an XML } \\
\text { document should be converted or displayed in a web } \\
\text { browser. }\end{array}$ \\
\hline
\end{tabular}




\section{REPORT DOCUMENTATION PAGE}

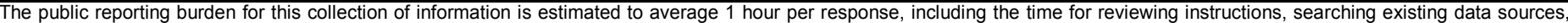

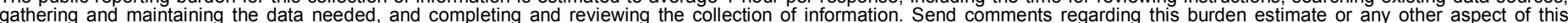

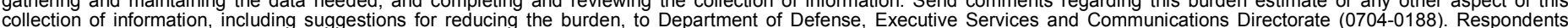

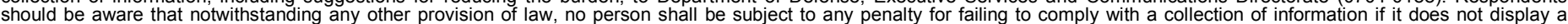

should be aware that notwithstandin

PLEASE DO NOT RETURN YOUR FORM TO THE ABOVE ORGANIZATION.

\begin{tabular}{l|l|l|} 
1. REPORT DATE $(D D-M M-Y Y Y Y)$ & 2. REPORT TYPE & 3. DATES COVERED (FrOm - TO)
\end{tabular}

October 2007

Subcontract Report

4. TITLE AND SUBTITLE

Automated Energy Distribution and Reliability System Status Report

5a. CONTRACT NUMBER

DE-AC36-99-GO10337

5b. GRANT NUMBER

5c. PROGRAM ELEMENT NUMBER

6. AUTHOR(S)

D.L. Buche and S. Perry

5d. PROJECT NUMBER

NREL/SR-581-42265

5e. TASK NUMBER

DP07.2001

5f. WORK UNIT NUMBER
7. PERFORMING ORGANIZATION NAME(S) AND ADDRESS(ES)

Northern Indiana Public Service Co.

801 E. $86^{\text {th }}$ Avenue

Merrillville, IN 46410
8. PERFORMING ORGANIZATION REPORT NUMBER

RAX-4-33652-01

9. SPONSORING/MONITORING AGENCY NAME(S) AND ADDRESS(ES)

National Renewable Energy Laboratory

1617 Cole Blvd.

Golden, CO 80401-3393

10. SPONSOR/MONITOR'S ACRONYM(S)

NREL

11. SPONSORING/MONITORING AGENCY REPORT NUMBER NREL/SR-581-42265

12. DISTRIBUTION AVAILABILITY STATEMENT

National Technical Information Service

U.S. Department of Commerce

5285 Port Royal Road

Springfield, VA 22161

\section{SUPPLEMENTARY NOTES}

14. ABSTRACT (Maximum 200 Words)

This report describes Northern Indiana Public Service Co. project efforts to develop an automated energy distribution and reliability system. The purpose of this project was to implement a database-driven GIS solution that would manage all of the company's gas, electric, and landbase objects.

15. SUBJECT TERMS

automated energy distribution and reliability system; AEDR; geographic information system; GIS; Northern Indiana Public Service Co.; NIPSCO; National Renewable Energy Laboratory; NREL

\begin{tabular}{|c|c|c|c|c|}
\hline 16. SECURIT & CLASSIFICATI & N OF: & 17. LIMITATION & 18. NUMBER \\
\hline $\begin{array}{l}\text { a. REPORT } \\
\text { Unclassified }\end{array}$ & $\begin{array}{l}\text { b. ABSTRACT } \\
\text { Unclassified }\end{array}$ & $\begin{array}{l}\text { c. THIS PAGE } \\
\text { Unclassified }\end{array}$ & $\begin{array}{l}\text { OF ABSTRACT } \\
\text { UL }\end{array}$ & \\
\hline
\end{tabular}

9b. TELEPHONE NUMBER (Include area code) 